SAND97-1880

Unlimited Release

UC-721

\title{
Waste Isolation Pilot Plant Salado Hydrology Program Data Report \#3
}

David A. Chace, Randall M. Roberts, Jeff B. Palmer, Matthias B. Kloska, Michael D. Fort, Greg J. Martin, Wayne A. Stensrud

INTERA, Inc.

1650 University Blvd. NE, Suite 300

Albuquerque, New Mexico 87102

Prepared by

Sandia National Laboratories

Albuquerque, New Mexico 87185 and Livermore, California 94550

Sandia is a multiprogram laboratory operated by Sandia Corporation,

a Lockheed Martin Company, for the United States Department of

Energy under Contract DE-AC04-94AL85000.

Approved for public release; distribution is unlimited.

Printed January 1998

\section{i. Sandia National Laboratories}


Issued by Sandia National Laboratories, operated for the United States Department of Energy by Sandia Corporation.

NOTICE: This report was prepared as an account of work sponsored by an agency of the United States Government. Neither the United States Government nor any agency thereof, nor any of their employees, nor any of their contractors, subcontractors, or their employees, makes any warranty, express or implied, or assumes any legal liability or responsibility for the accuracy, completeness, or usefulness of any information, apparatus, product, or process disclosed, or represents that its use would not infringe privately owned rights. Reference herein to any specific commercial product, process, or service by trade name, trademark, manufacturer, or otherwise, does not necessarily constitute or imply its endorsement, recommendation, or favoring by the United States Government, any agency thereof, or any of their contractors or subcontractors. The views and opinions expressed herein do not necessarily state or reflect those of the United States Government, any agency thereof, or any of their contractors.

Printed in the United States of America. This report has been reproduced directly from the best available copy.

Available to DOE and DOE contractors from

Office of Scientific and Technical Information

P.O. Box 62

Oak Ridge, TN 37831

Prices available from (615) 576-8401, FTS 626-8401

Available to the public from

National Technical Information Service

U.S. Department of Commerce

5285 Port Royal Rd

Springfield, VA 22161

NTIS price codes

Printed copy: A23

Microfiche copy: A01

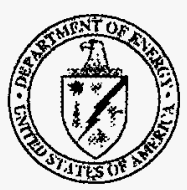




\section{DISCLAIMER}

Portions of this document may be illegible electronic image products. Images are produced from the best available original document. 
SAND97-1880

Unlimited Release

Printed January 1998

\author{
Category UC-721
}

$\infty$

\title{
Waste Isolation Pilot Plant Salado Hydrology Program Data Report \#3
}

\author{
David A. Chace, Randall M. Roberts, Jeff B. Palmer, Matthias B. Kloska, \\ Michael D. Fort, Greg J. Martin, and Wayne A. Stensrud \\ INTERA Inc. \\ 1650 University Blvd. NE, Suite 300 \\ Albuquerque, New Mexico 87102
}

Sandia Contract No. AP2242

\begin{abstract}
WIPP Salado Hydrology Program Data Report \#3 presents hydrologic data collected during permeability testing, coupled permeability and hydrofracture testing, and gas-threshold-pressure testing of the Salado Formation performed from November 1991 through October 1995. Fluid-pressure monitoring data representing August 1989 through May 1995 are also included. The report presents data from the drilling and testing of three boreholes associated with the permeability testing program, nine boreholes associated with the coupled permeability and hydrofracture testing program, and three boreholes associated with the gasthreshold-pressure testing program. The purpose of the permeability testing program was to provide data with which to interpret the disturbed and undisturbed permeability and pore pressure characteristics of the different Salado Formation lithologies. The purpose of the coupled permeability and hydrofracture testing program was to provide data with which to characterize the occurrence, propagation, and direction of pressure induced fractures in the Salado Formation lithologies, especially MB139. The purpose of the gas-threshold-pressure testing program was to provide data with which to characterize the conditions under which pressurized gas displaces fluid in the brine-saturated Salado Formation lithologies. All of the holes were drilled from the WIPP underground facility $655 \mathrm{~m}$ below ground surface in the Salado Formation. All testing was conducted using multipacker test tools with inflatable packers to allow formation pore-pressure buildup and subsequent test events to be carried out in isolated intervals. The testing sequences performed or monitored in boreholes C1H05, C1H06, C1H07, C1X05, C1X06, C1X10, C2H01, C2H02, DPD02, DPD03, L4P51, L4P52, SCP01, and S1P74 involved Marker Beds 138, 139, 140, a clean halite above Marker Bed 140, and an argillaceous halite approximately $10 \mathrm{~m}$ below Marker Bed 140 . Test data include pressures and temperatures from the brine-filled, packer-isolated test intervals, fluid production during constant-pressure-flow tests, nitrogeninjection rates during gas-threshold-pressure tests, and borehole-closure and axial test-tool movement measurements. The boreholes associated with the permeability and gas-threshold-pressure testing programs were drilled and/or cored to a nominal 4-inch $(10.2-\mathrm{cm})$ diameter using compressed air or brine circulation to remove drill cuttings. The two test boreholes associated with the coupled permeability and hydrofracture testing program were drilled and/or cored to a nominal 3-inch $(7.62-\mathrm{cm})$ diameter using similar drilling techniques. The observation boreholes associated with the coupled permeability and hydrofracture testing program were similar to those associated with the permeability and gas-threshold-pressure testing programs. Compliance tests were conducted on the test tools in sections of stainless-steel casing to evaluate the mechanical performance and behavior of the tools. Compliance testing sequences include leak tests and zone compressibility tests (zone compressibility test were sometimes conducted within a test borehole. Following permeability and/or gas-threshold-pressure testing, fluid pressures were monitored in packerisolated sections of test boreholes C2H01, C2H02, DPD01, DPD02, DPD03, L4P52, SCP01, S1P71, and S1P72. This was done in an effort to quantify the relationship between proximity to an excavation and change in formation pore pressure as a function of time.
\end{abstract}




\section{EXECUTIVE SUMMARY}

WIPP Salado Hydrology Program Data Report \#3 contains data collected during the drilling and permeability testing of boreholes L4P51, L4P52, and S1P74, the drilling and coupled permeability and hydrofracture testing of boreholes C1H05, C1H06, C1H07, C1X05, $\mathrm{C} 1 \mathrm{X} 06, \mathrm{C} 1 \mathrm{X} 10, \mathrm{C} 2 \mathrm{H} 01, \mathrm{DPD} 02$, and DPD03, and the drilling and gas-threshold-pressure testing of boreholes $\mathrm{C} 2 \mathrm{HO2}$, L4P52, and SCP01. All of the test boreholes were drilled with 4.0-inch $(10.2-\mathrm{cm})$ core and drill bits using compressed air or circulated brine to remove drill cuttings, except for the hydrofracture test boreholes $\mathrm{C} 1 \mathrm{X} 05$ and $\mathrm{C} 1 \mathrm{X} 10$, which were drilled with 3.0-inch $(7.62-\mathrm{cm})$ core and drill bits using similar techniques. Descriptions of the recovered core samples are presented in Appendix A.

Permeability tests were designed to meet three objectives: 1) to provide technically defensible data with which to interpret the permeability characteristics of the different lithologies of the Salado Formation that could contribute to fluid flow to and/or from the WIPP underground facility; 2) to delineate, if possible, the extent of hydrogeologic disturbance in the Salado Formation around the WIPP underground facility; and 3) to provide estimates of the formation pore pressure of the Salado Formation near the WIPP underground facility, and where possible, establish a pore-pressure profile from the surface of the excavation to undisturbed far-field conditions in the formation. Permeability testing boreholes were drilled in Room L4 and Waste Panel 1, Room 7. Borehole L4P51 was deepened from 10.06 to $22.35 \mathrm{~m}$ vertically downward from the floor in Room L4 to test Marker Bed (MB) 140 during permeability-testing sequence L4P51-C1 and to test the halite above MB140 during permeability-testing sequence L4P51-C2. Borehole L4P51 was later deepened to a total depth of $30.45 \mathrm{~m}$ to allow testing of an argillaceous halite from 29.62 to $30.40 \mathrm{~m}$ below the room floor during permeability-testing sequences L4P51-D1 and L4P51-D2. Borehole L4P52 was deepened from 5.56 to $14.18 \mathrm{~m}$ at an upward angle of $40^{\circ}$ from vertical from near the top of the west rib of Room L4 to test MB138 during permeability-testing sequence L4P52-B. Borehole S1P74 was drilled at an upward angle of $40^{\circ}$ from vertical from near the top of the east rib of Waste Panel 1, Room 7 to a depth of $7.69 \mathrm{~m}$ to test anhydrite "a" during permeability-testing sequence S1P74-A. Borehole S1P74 was later deepened to a total depth of $16.88 \mathrm{~m}$ to test MB138 and the argillaceous halite below MB138 during permeability-testing sequence S1P74-B.

Permeability testing was performed using multipacker test tools to isolate brine-filled sections of the boreholes for pressure buildup/falloff, pulse-withdrawal, and constantpressure-flow testing. The test tools utilized two or three 3.75 -inch- $(9.53-\mathrm{cm})$ diameter packers with $92-\mathrm{cm}$-(36.22-inch) long elements to isolate single or multiple test zones and guard zones of various lengths. Multiple isolated zones are useful for decreasing the pressure gradient across a packer, thereby decreasing the possibility for pressure bypass around a packer and also for detecting pressure bypass if it does occur. The principal test zones were the bottom-hole zones, although testing was also performed in selected middle zones that contained horizons of hydrologic interest.

The permeability test tools were equipped with pressure transducers to monitor fluid pressure in the zones and the inflation pressure of each of the packers. In some cases, 
thermocouple were used to monitor temperatures in the zones. Also, in some cases, the test tools were equipped with three radially oriented linear variable-differential transformers (LVDTs) to measure borehole closure, and an axially oriented LVDT to monitor test-tool movement in response to pressure changes in the isolated intervals as well as in the packers. A differential-pressure-transmitter (DPT) panel was used to measure brine production during zone compressibility tests and constant-pressure-flow tests. Calibration data for the pressure transducers, LVDTs, and DPT panel columns are stored in the Sandia WIPP Central Records File (SWCF) under WPO \#42269.

In most permeability-testing sequences, pulse-withdrawal tests and constant-pressure-flow tests were performed after fluid pressures in the zones to be tested had built up to relatively stable levels. Pulse-withdrawal tests were used because of the low permeability of the Salado Formation as indicated by other testing at the WIPP site (Beauheim et al., 1991). Pulse-withdrawal tests were performed in preference to pulse-injection tests to avoid potential hydrofracturing of the formation, which could occur if a pulse-injection were to exceed the formation's lithostatic pressure. Pulse-withdrawal tests were typically repeated to provide an indication of the reproducibility of the test results. In some cases, pulse injections were performed in order to increase the fluid pressure in isolated intervals in which little or no fluid-pressure responses were observed after shut in. Constantpressure-flow tests were performed in order to obtain brine-production information and data to allow for the evaluation of the formation's hydraulic properties. Permeability-testing results are presented in sequence plots of the parameters monitored versus time in Section 3.6.

Pulse-withdrawal and constant-pressure-flow tests were conducted in the isolated guard zone containing the halite above MB140 in borehole L4P51 during permeability testing sequence L4P51-C1. Several constant-pressure-withdrawal tests were conducted in the isolated test zone containing MB140 during the same testing sequence. Pulse-withdrawal tests, constant-pressure-injection tests, and constant-pressure-withdrawal tests were conducted in the isolated test zone containing the halite above MB140 in borehole L4P51 during permeability testing sequence L4P51-C2. In borehole L4P52, both constantpressure-injection tests and constant-pressure-withdrawal tests were conducted in the isolated test zone containing MB138 during permeability-testing sequence L4P52-B.

A pulse-withdrawal test and a constant-pressure-withdrawal test were conducted in the isolated test zone containing anhydrite " $a$ " in borehole S1P74 during permeability-testing sequence S1P74-A. Pulse-withdrawal tests were conducted in both the isolated test intervals containing MB138 and the argillaceous halite below MB138 (AH-1) in borehole S1P74 during permeability-testing sequence S1P74-B.

Unsuccessful attempts were made to perform permeability tests in the isolated test zone containing the argillaceous halite below MB140 in borehole L4P51 during permeabilitytesting sequence L4P51-D1. Testing was suspended for permeability-testing sequence L4P51-D1 when the test tool was determined to be unreliable. Pressure monitoring only was performed with tool configuration L4P51-D2. The following table notes the hydrologic units monitored in the test and guard zone intervals for the respective permeability-testing sequences. 
Summary of Hydrologic Units Tested During the Permeability Testing Program

\begin{tabular}{cccc}
\hline $\begin{array}{c}\text { PERMEABILITY } \\
\text { TESTING SEQUENCE }\end{array}$ & TEST ZONE 1 & TEST ZONE 2 & GUARD ZONE \\
\hline L4P51-C1 & Marker Bed 140 & N/A & halite \\
L4P51-C2 & Marker Bed 140 & halite & halite \\
L4P51-D1 & argillaceous halite & & Marker Bed 140 \\
L4P51-D2 & argillaceous halite & N/A & N/A \\
L4P52-B & Marker Bed 138 & N/A & $\begin{array}{c}\text { halite, clay J, } \\
\text { argillaceous halite } \\
\text { halite, anhydrite } \\
\text { S1P74-A }\end{array}$ \\
& anhydrite "a" & N/A & N/A \\
S1P74-B & Marker Bed 138 & argillaceous halite & .
\end{tabular}

The hydraulic fracturing program was designed to meet six objectives: 1) to determine the fluid pressures at which fracturing will occur in anhydrite interbeds, especially MB139, both in a potentially disturbed state and in its undisturbed state; 2) to determine whether fracturing would take place by the opening and interconnection of preexisting, partially healed fractures of by the formation of new fractures; 3 ) to determine at what induced pressure (liquid or gas) fracturing might be sustained; 4) to determine if fracturing in MB139 involved the development of new fractures, would the fracturing be confined to the anhydrite interbed, or would newly created fractured extend across the MB139 contacts; 5 ) to determine if the total stress state (matrix stress plus pore pressure) in MB139 and MB140 is isotropic or not, and can near-field stress measurements around excavations be used to infer the undisturbed state of stress in the interbeds; and 6) to determine the magnitude of the smallest principal stress in anhydrite interbeds, if the stress state is anisotropic. Complementary pre- and post-fracture hydrological measurements were intended to meet three objective: 1) to provide reliable values of formation (pore) fluid pressure; 2 ) to provide permeability values of the interbeds before and after hydraulic fracturing; and 3) to provide a comparison of permeability measurements in MB139 relatively near existing excavations with equivalent data in MB140, which was expected to be essentially undisturbed. Coupled permeability and hydrofracture testing and observation boreholes were drilled in Room C1. Boreholes in Room C2 and in the North 1420 drift were also monitored. Borehole $\mathrm{C} 1 \mathrm{X} 10$ was drilled vertically downward from the floor of Room C1 to a total depth of $10.16 \mathrm{~m}$ to test MB139 and clay E during testing sequence $\mathrm{C} 1 \mathrm{X} 10$. Testing sequence $\mathrm{C} 1 \mathrm{X} 10$ had five associated observation boreholes, all completed to allow monitoring of MB139 and clay E: C1H05, C1H06, C2H01, DPD02, and DPD03. Borehole $\mathrm{C} 1 \mathrm{H} 05$ was drilled vertically downward from the floor of Room $\mathrm{C} 1$ to a total depth of $8.26 \mathrm{~m}$. Borehole $\mathrm{C} 1 \mathrm{H} 06$ was drilled downward, $30^{\circ}$ from vertical, to a depth of $9.40 \mathrm{~m}$ at the intersection of the floor and the north rib of the North 1420 drift west of Room C1. Borehole $\mathrm{C} 2 \mathrm{HO} 1$ was drilled vertically downward into the floor of Room C2 
to a total depth of $8.97 \mathrm{~m}$. Boreholes DPD02, and DPD03 are located at the junction of the north rib and the floor of the North 1420 drift positioned between Room C1 and Room D. Borehole DPD02 was drilled downward, $46.8^{\circ}$ from vertical to a total depth of $13.11 \mathrm{~m}$. Borehole DPD03 was drilled downward, $45.8^{\circ}$ from vertical to a total depth of $13.11 \mathrm{~m}$.

Borehole C1X05 was drilled vertically downward from the floor of Room C1 to a depth of $9.14 \mathrm{~m}$ to test MB139 and clay $E$ during coupled permeability and hydrofracture-testing sequence C1X05-A. Testing sequence C1X05-A had six associated observation boreholes: $\mathrm{C} 1 \mathrm{H} 05, \mathrm{C} 1 \mathrm{H} 06, \mathrm{C} 1 \mathrm{H} 07, \mathrm{C} 1 \times 06, \mathrm{C} 1 \times 10$, and $\mathrm{C} 2 \mathrm{H} 01$. The configurations of boreholes $\mathrm{C} 1 \mathrm{H} 05, \mathrm{C} 1 \mathrm{H} 06, \mathrm{C} 1 \mathrm{X} 10$, and $\mathrm{C} 2 \mathrm{H} 01$ have already been described. Boreholes $\mathrm{C} 1 \mathrm{H} 07$ and $\mathrm{C} 1 \mathrm{X} 06$ were drilled vertically downward from the floor of Room $\mathrm{C} 1$ to depths of $8.18 \mathrm{~m}$ and $7.63 \mathrm{~m}$, respectively.

Borehole C1X05 was deepened vertically downward to a total depth of $30.20 \mathrm{~m}$ to test MB140 during coupled permeability and hydrofracture testing sequence $\mathrm{C} 1 \mathrm{X} 05-\mathrm{B}$. Testing sequence $\mathrm{C} 1 \mathrm{X} 05-\mathrm{B}$ had two associated observation borehole's completed to MB140: $\mathrm{C} 1 \mathrm{H} 07$ and $\mathrm{C} 1 \mathrm{X06}$. $\mathrm{C} 1 \mathrm{H} 07$ and $\mathrm{C} 1 \mathrm{X06}$ were deepened vertically downward to total depths of $27.88 \mathrm{~m}$ and $27.99 \mathrm{~m}$ from the floor of Room C1 respectively.

Hydraulic fracturing of the Salado Formation was conducted at three locations within Room C1. Section 4.3 gives a detailed description of the actual hydrofracture process. Pre- and post-hydrofracture permeability testing were performed in each of the testing sequences using multipacker test tools to isolate the individual sections of the test boreholes for pressure-buildup/falloff, pulse-withdrawal, and constant-pressure-flow testing. The test tools utilized two or three 2-5/8-inch- $(6.67-\mathrm{cm})$ diameter packers with 40 -inch- $(102-\mathrm{cm})$ long elements to isolate test and guard zones of various lengths. Pressure responses to hydrofracture events were also monitored in nearby boreholes during all three of the hydrofracture events. Single- and triple-packer test tools as described in Section 4.3 were used to monitor pressure, temperature, borehole closure, test-tool movement, and fluid production in the observation boreholes. The hydrofracture test and monitor tools were equipped similar to the permeability-testing tools.

In all of the coupled permeability and hydrofracture testing sequences, typical permeability tests (as described previously) were conducted both prior to and after hydraulic fracturing of the formation. By proceeding along these lines, the pre-fracture and post-fracture formation parameters could be characterized. Coupled permeability and hydrofracture testing results are presented in sequence plots of the parameters monitored versus time in Section 4.6.

In borehole $\mathrm{C} 1 \mathrm{X} 10$, constant-pressure-injection tests and constant-pressure-withdrawal tests were conducted in the isolated test zone containing MB139 during testing sequence $\mathrm{C} 1 \mathrm{X} 10$. Both pre- and post-fracture tests were conducted during this testing sequence. In borehole $\mathrm{C} 1 \mathrm{X} 05$, constant-pressure-injection tests and constant-pressure-withdrawal tests were conducted in the isolated test zone containing MB139 during testing sequence $\mathrm{C} 1 \mathrm{X} 05-\mathrm{A}$. Both pre- and post-fracture tests were conducted during this testing sequence as well. In borehole $\mathrm{C} 1 \mathrm{X} 05$, constant-pressure-injection tests, constant-pressurewithdrawal tests, and a pulse-withdrawal test were conducted in the test zone isolating a 
portion of MB140 during testing sequence C1X05-B. Both pre- and post-fracture constantpressure flow tests were conducted during this testing sequence and a pulse-withdrawal test was conducted post-fracture. The following table notes the hydrologic units monitored and the test-zone intervals for the coupled permeability and hydrofracture-testing sequences.

Summary of Hydrologic Units Tested During the Hydrofracture Testing Program

\begin{tabular}{ccc}
\hline $\begin{array}{c}\text { HYDROFRACTURE TESTING } \\
\text { SEQUENCE }\end{array}$ & TEST ZONE 1 & TEST ZONE 2 \\
\hline C1X10 & Marker Bed 139 & N/A \\
C1X05-A & Marker Bed 139 & N/A \\
C1X05-B & Marker Bed 140 & Marker Bed 140 \\
\hline
\end{tabular}

The gas-threshold-pressure testing (GTPT) program was designed to meet five objectives: 1) to provide information about the far-field gas-threshold pressure in the Salado Formation anhydrite interbeds, particularly MB139 and MB140;2) to determine, if possible, the farfield gas-threshold pressure in both argillaceous and pure halite beds of the Salado Formation; 3) to determine if the gas-threshold pressure varies with distance from the excavation in the WIPP underground facility; 4) to determine if the gas-threshold pressure is related to formation permeability; and 5) to determine if there will be sustained gas flow into the formation after gas-threshold pressure is reached, and at what rate pressure will the gas flow. GTPT was performed in boreholes previously drilled in Room C2, Room L4, and in the Core Storage Library. Borehole $\mathrm{C} 2 \mathrm{HO} 2$ was drilled at the intersection of the west rib and floor of Room C2 downward, $45^{\circ}$ from vertical to a total depth of $10.91 \mathrm{~m}$ and allowed testing of MB139 and clay E during testing sequence $\mathrm{C} 2 \mathrm{H} 02$. Borehole SCP01 was drilled downward, $77^{\circ}$ from vertical on the south rib of the Core Storage Library to a total depth of $15.39 \mathrm{~m}$ allowing testing of MB139 and clay E during testing sequences SCP01-1 and SCP01-2. The configuration of borehole L4P52-B has already been described.

GTPT was performed using three different types of multipacker test tools to isolate gasfilled sections of the boreholes. A detailed description of the three different types of multipacker test-tools used in the GTPT program is given in Section 5.3. All of the GTPTs were performed in the bottom-hole zones of the respective boreholes. The GTPT tools were equipped similar to the permeability-testing tools.

In GTPT sequences, the isolated test zone was allowed to reach stabilization pressure under typical permeability-testing conditions (brine-filled zones) at which point the brine was exchanged with gas (nitrogen) in preparation for the GTPT. Upon successful completion of the gas/brine exchange, additional gas was introduced into the isolated test zone at a constant rate, thereby increasing the test-zone pressure. When it was determined that the gas threshold pressure had been reached, gas injection was terminated and the test-zone pressure was allowed to decay continuously or by imposing 
step decreases by withdrawing a volume of the gas from the test zone. GTPT results are presented in sequence plots of the parameters monitored versus time in Section 5.6.

Constant-rate-injection, and pulse-withdrawal tests were conducted in the isolated test zone containing $\mathrm{MB} 139$ and clay $\mathrm{E}$ in borehole $\mathrm{C} 2 \mathrm{HO} 2$ during GTPT sequence $\mathrm{C} 2 \mathrm{HO} 2$. Also, a pulse-withdrawal test was conducted in the isolated test zone containing the halite above MB139 in borehole C2H02 during this same GTPT sequence. In borehole L4P52, constant-rate-injection and pulse-withdrawal tests were conducted in the isolated test zone containing MB138 during GTPT sequence L4P52-B. In borehole SCP01, only constantrate-injection tests were conducted in the isolated test zone containing MB139 and clay $E$ during GTPT sequence SCP01-2.

Unsuccessful attempts were made to perform GTPTs in the isolated test zone including MB139 and clay E in borehole SCP01 during GTPT sequence SCP01-1. Testing was suspended when it was determined that the test-zone packer was not properly sealed and the test-zone pressure was bypassing the test-zone packer. The following table notes the hydrologic units monitored and the test and guard zone intervals for the respective GTPT sequences:

Summary of Hydrologic Units Tested During the Gas Threshold-Pressure Testing Program

\begin{tabular}{|c|c|c|}
\hline GTPT SEQUENCE & TEST ZONE & GUARD ZONE \\
\hline SCP01-1 & Marker Bed 139 , clay $E$ & polyhalitic halite 4 \\
\hline $\mathrm{C} 2 \mathrm{HO} 2$ & Marker Bed 139 , clay E & polyhalitic halite 4 \\
\hline L4P52-B & Marker Bed 138 & $\begin{array}{c}\text { halite, clay J, argillaceous } \\
\text { halite }\end{array}$ \\
\hline SCP01-2 & Marker Bed 139 , clay E & polyhalitic halite 4 \\
\hline
\end{tabular}

In most cases, compliance testing of the multipacker test tools was performed before each testing sequence in order to achieve the following three objectives: 1) to characterize testtool performance, 2) to identify the responses due to test-tool movement, and 3) to identify changes in the size and shape of the elastic packer elements caused by changes in packer and zone pressures. During a typical compliance testing sequence, the multipacker test tool was installed in a sealed chamber and pressure checked to ensure that the tool's components were not leaking. Constant-pressure injection and withdrawal tests were performed in the isolated intervals in the chamber in order to determine zone compressibility (in some cases zone compressibility tests were performed in test boreholes). The responses of the pressure transducers, thermocouple, LVDTs, and DPTs were monitored over the entire duration of the testing sequence in order to determine the test-tool behavior. Compliance testing results are presented in Section 6 . 
The fluid-pressure monitoring program was designed to meet two objectives: 1) to determine if pore pressures in the various Salado Formation interbeds change as a function of time due to the underground excavation; and 2) to determine the relationship between proximity to an excavation and change in formation pore pressure as a function of time. As part of the fluid-pressure monitoring program, fluid-pressure data obtained during the various testing programs were supplemented with data collected from packerisolated intervals of test boreholes C2H01, C2H02, DPD01, DPD02, DPD03, L4P52, SCP01, S1P71, and S1P72. Borehole DPD01 is located at the junction of the north rib and the floor of the North 1420 drift positioned between Room C1 and Room D. Borehole DPD01 was drilled downward, $49.9^{\circ}$ from vertical to a total depth of $12.34 \mathrm{~m}$ to test MB139 and clay E. Borehole S1P71 was drilled vertically downward to a depth of $4.55 \mathrm{~m}$ and deepened to a total depth of $10.15 \mathrm{~m}$ from the floor of Waste Panel 1, Room 7 to test anhydrite "c" and clay B. Borehole S1P72 was drilled downward, $58^{\circ}$ from vertical to a total depth of $6.05 \mathrm{~m}$ to test MB139 and clay E. The configurations and locations of boreholes $\mathrm{C} 2 \mathrm{H} 01, \mathrm{C} 2 \mathrm{H02}$, DPD02, DPD03, L4P52, and SCP01 have already been described. The following table notes the hydrologic units monitored during the fluid-pressure monitoring.

Summary of Hydrologic Units Monitored During the Fluid-Pressure Monitoring Program

\begin{tabular}{cc}
\hline $\begin{array}{c}\text { FLUID-PRESSURE MONITORING } \\
\text { BOREHOLE }\end{array}$ & TEST ZONE \\
\hline C2H01 & Marker Bed 139, clay E \\
C2H02 & Marker Bed 139, clay E \\
DPD01 & Marker Bed 139, clay E \\
DPD02 & Marker Bed 139, clay E \\
DPD03 & Marker Bed 139, clay E \\
L4P52 & Marker Bed 138 \\
SCP01 & Marker Bed 139, clay E \\
S1P71 & anhydrite "c" \\
S1P72 & Marker Bed 139, clay E
\end{tabular}

Data from the fluid-pressure monitoring are shown graphically in Section 7. 


\section{TABLE OF CONTENTS}

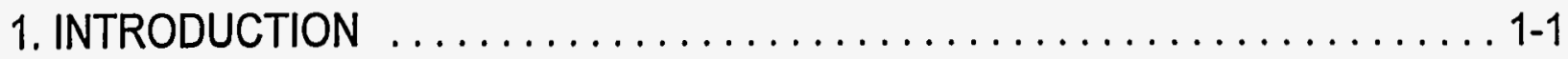

2. THE WIPP UNDERGROUND FACILITY $\ldots \ldots \ldots \ldots \ldots \ldots \ldots \ldots \ldots \ldots \ldots \ldots .2-1$

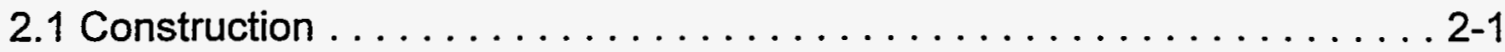

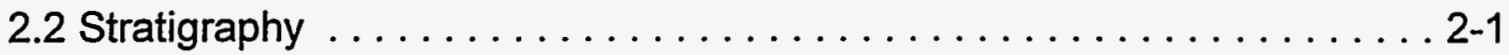

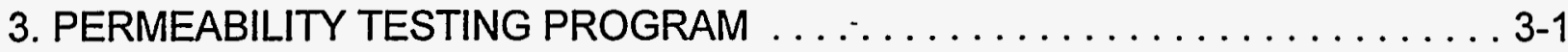

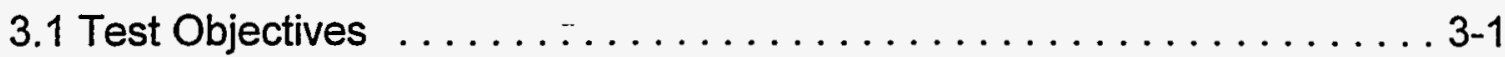

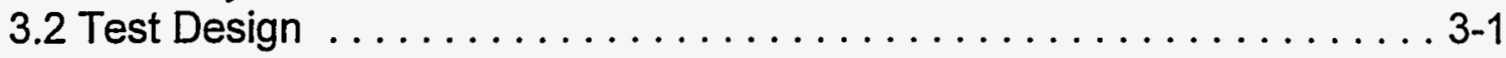

3.3 Test Equipment for Permeability Testing $\ldots \ldots \ldots \ldots \ldots \ldots \ldots \ldots .4$

3.3.1 Description of Equipment . . . . . . . . . . . . . . 3-4

3.3.1.1 Multipacker Test Tools ................ 3-4

3.3.1.2 Data-Acquisition System (DAS) $\ldots \ldots \ldots \ldots \ldots \ldots .3-10$

3.3.1.3 Pressure Transducers $\ldots \ldots \ldots \ldots \ldots \ldots \ldots .3-10$

3.3.1.4 Thermocouples ................... 3-14

3.3.1.5 Linear Variable-Differential Transformers (LVDTs) . . . 3-14

3.3.1.6 Differential Pressure-Transmitter Panel ... . . . . . 3-15

3.3.1.7 Pressurized-Brine-Sampling Apparatus . . . . . . . 3-19

3.3.1.8 Pressure-Maintenance System . . . . . . . . . . 3-19

3.3.2 Equipment Calibration . . . . . . . . . . . . . . 3-19

3.3.2.1 Data-Acquisition System . . . . . . . . . . . 3-23

3.3.2.2 Pressure Transducers $\ldots \ldots \ldots \ldots \ldots \ldots \ldots \ldots .3-23$

3.3.2.3 Thermocouples . . . . . . . . . . . . . . 3-25

3.3.2.4 Linear Variable-Differential Transformers . . . . . . . . 3-25

3.3.2.5 Differential-Pressure-Transmitter Panel . . . . . . . . 3-25

3.4 Procedures for Permeability Testing . . . . . . . . . . . . . . 3-27

3.4.1 Test-Tool-Installation Procedures $\ldots \ldots \ldots \ldots \ldots \ldots \ldots .3-27$

3.4.2 Pulse-Withdrawal Testing Procedures . . . . . . . . . . . . 3-31

3.4.3 Constant-Pressure-Injection/Withdrawal Testing Procedures . . 3-31

3.4.4 Pressurized Brine-Sampling Procedures . . . . . . . . . . 3-34

3.5 Boreholes and Test-Tool Configurations for Permeability Testing . . . . . 3-35

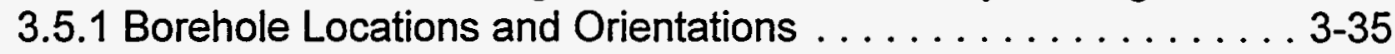

3.5.2 Borehole Drilling ................. 3. $\ldots \ldots \ldots \ldots \ldots$

3.5.3 Test-Tool Configurations . . . . . . . . . . . . . . 3-40

3.6 Permeability Test Data . . . . . . . . . . . . . . . . . . . 3-58

3.6.1 Permeability Testing in Room $L 4 \ldots \ldots . \ldots . . . . . . .3-59$

3.6.1.1 Borehole L4P51, Permeability-Testing Sequence L4P51-C1

3.6.1.2 Borehole L4P51, Permeability-Testing Sequence L4P51-C2

3-74

3.6.1.3 Borehole L4P51, Permeability-Testing Sequence L4P51-D1 


\section{TABLE OF CONTENTS (Continued)}

3.6.1.4 Borehole L4P51, Permeability-Testing Sequence L4P51-D2 . 3-94

3.6.1.5 Borehole L4P52, Permeability-Testing Sequence L4P52-B

3.6.2 Permeability Testing in Waste-Panel 1, Room 7 . . . . . . . 3-107

3.6.2.1 Borehole S1P74, Permeability-Testing Sequence S1P74-A

. . . . . . . . . . . . . . . . . . . . . . . . 3-107

3.6.2.2 Borehole S1P74, Permeability-Testing Sequence S1P74-B

3.6.3 Summary of Pressurized Brine-Sampling Conducted During the

Permeability-Testing Program ........... . . . 3-124

4. COUPLED PERMEABILITY AND HYDROFRACTURE-TESTING PROGRAM . . 4-1

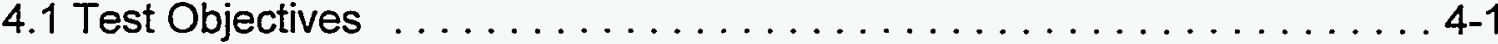

4.2 Test Design . . . . . . . . . . . . . . . . . . . . . . . . 4-2

4.3 Test Equipment for Coupled Permeability and Hydrofracture Testing . . . 4-4

4.3.1 Description of Equipment . . . . . . . . . . . . . . 4-4

4.3.1.1 Test Tools . . . . . . . . . . . . . . . . . . . 4-4

4.3.1.2 Other Equipment $\ldots \ldots \ldots \ldots \ldots \ldots \ldots$ 4-7

4.3.2 Equipment Calibration . . . . . . . . . . . . . . . . . 4-9

4.4 Procedures for Permeability Testing Associated with Hydrofracture Testing

4.5 Boreholes and Test-Tool Configurations for Coupled Permeability and

Hydrofracture Testing . . . . . . . . . . . . . . . . . . . 4-9

4.6 Test Data . . . . . . . . . . . . . . . . . . . . . . . . . . . . . 4-28

4.6.1 Borehole C1X10, Testing Sequence C1X10 . . . . . . . . . . 4-29

4.6.1.1 Observation Borehole C1H05, Testing Sequence C1X10

4.6.1.2 Observation Borehole C1H06, Testing Sequence C1X10

$\ldots \ldots \ldots \ldots \ldots \ldots \ldots \ldots \ldots \ldots . \ldots \ldots$. . . . . . . . . . . . . . . . . .

4.6.1.3 Observation Borehole DPD02, Testing Sequence C1X10

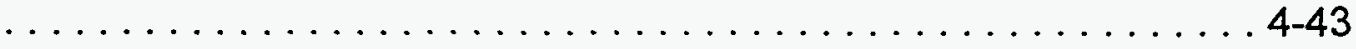

4.6.1.4 Observation Borehole DPD03, Testing Sequence C1X10

4.6.1.5 Observation Borehole $\mathrm{C} 2 \mathrm{H01}$, Testing Sequence $\mathrm{C} 1 \times 10$

4.6.2 Borehole C1X05, Testing Sequence C1X05-A . . . . . . . . . 4-49

4.6.2.1 Observation Borehole C1H05, Testing Sequence C1X05-A

4.6.2.2 Observation Borehole C1H06, Testing Sequence C1X05-A

4.6.2.3 Observation Borehole C1H07, Testing Sequence C1X05-A 


\section{TABLE OF CONTENTS (Continued)}

4.6.2.4 Observation Borehole C1X06, Testing Sequence C1X05-A ... . . . . . . . . . . . . . . . . . . . . . . . . . 4-69 4.6.2.5 Observation Borehole C1X10, Testing Sequence C1X05-A .................................4-73 4.6.2.6 Observation Borehole C2H01, Testing Sequence C1X05-A

4.6.3 Borehole C1X05, Testing Sequence C1X05-B . . . . . . . . 4-77 4.6.3.1 Observation Borehole C1H07, Testing Sequence C1X05-B

5. GAS-THRESHOLD-PRESSURE TESTING $\ldots \ldots \ldots \ldots \ldots \ldots \ldots \ldots \ldots$ 5-1

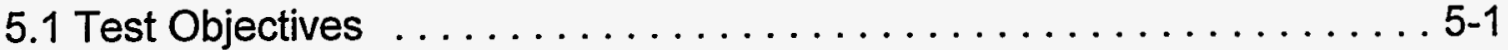

5.2 Test Design . . . . . . . . . . . . . . . . . . . . . . 5-1

5.3 Test Equipment for Gas-Threshold Pressure Testing . . . . . . . . 5-2

5.3.1 Description of Equipment ................ 5-2

5.3.1.1 Multipacker Test Tools .................. 5-2

5.3.1.2 Data-Acquisition System (DAS) $\ldots \ldots \ldots \ldots \ldots \ldots .5-4$

5.3.1.3 Mass Flow Meter . . . . . . . . . . . . . . . 5-5

5.3.2 Equipment Calibration ................... 5-5

5.3.2.1 Data-Acquisition System ............. 5-5

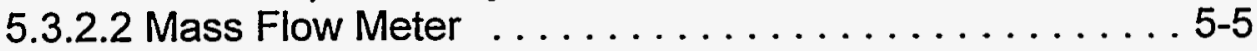

5.4 Procedures for Gas-Threshold-Pressure Testing . . . . . . . . . . . 5-7 5.5 Boreholes and Test-Tool Configurations for Gas-Threshold Pressure Testing

5.5.1 Borehole Drilling . ................ 5-15

5.6 Gas-Threshold-Pressure Test Data . . . . . . . . . . . . . . . 5-19

5.6.1 Gas-Threshold Pressure Testing in the Core Storage Library . . 5-20

5.6.1.1 Gas-Threshold-Pressure-Testing Sequence SCP01-1 . 5-20

5.6.1.2 Gas-Threshold-Pressure-Testing Sequence SCP01-2 . 5-32

5.6.2 Gas-Threshold-Pressure Testing in Room C2 . . . . . . 5-41

5.6.2.1 Observation Borehole C2H01 . . . . . . . . . . 5-50

5.6.3 Gas-Threshold-Pressure Testing in Room L4 . . . . . . . 5-50

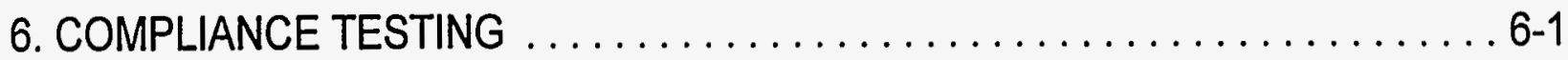

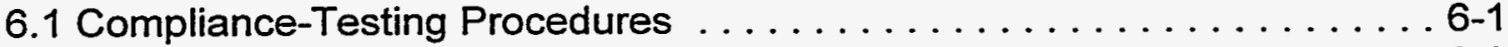

6.2 Compliance-Test Data ............................6. 6-3

6.2.1 Test Tool \#32A (Borehole C1H05, Coupled Permeability and Hydrofracture-Testing Sequences $C 1 \times 10$ and C1X05-A) . . . 6-5

6.2.2 Test Tool \#32B (Borehole C1H06, Coupled Permeability and Hydrofracture-Testing Sequences C1X10 and C1X05-A) . . . 6 6-9

6.2.3 Test Tool \#33A (Permeability-Testing Sequence L4P51-C1) . 6-12

6.2.4 Test Tool \#34 (Borehole C1H07, Coupled Permeability and Hydrofracture-Testing Sequence C1X05-A) . . . . . . . . 6-24 


\section{TABLE OF CONTENTS (Continued)}

6.2.5 Test Tool \#35 (Permeability-Testing Sequence S1P74-A) . . . . 6-27

6.2.6 Test Tool \#36 (Borehole C1X06, Coupled Permeability and Hydrofracture-Testing Sequence C1X05-A) . . . . . . . . . . 6-39

6.2.7 Test Tool \#37 (Permeability-Testing Sequence L4P52-B) . . . . 6-42

6.2.8 Test Tool \#38A (Gas-Threshold Pressure Testing Sequence SCP01-1) . . . . . . . . . . . . . . . . . . 6-51

6.2.9 Test Tool \#39 (Borehole C1H07, Coupled Permeability and Hydrofracture-Testing Sequence C1X05-B) . . . . . . . . . . 6-59

6.2.10 Test Tool \#40 (Borehole C1X06, Coupled.Permeability and Hydrofracture-Testing Sequence C1X05-B) . . . . . . . . . 6-63

6.2.11 Test Tool \#41 (Permeability-Testing Sequence L4P51-C2) . . 6-67

6.2.12 Test Tool \#BOT- 01 (Gas-Threshold Pressure-Testing Sequence SCP01-2) ....................... 6-72

6.2.13 Test Tool \#P51-D1A (Permeability-Testing Sequence L4P51-D1)

6.2.14 Test Tool \#P74-B (Permeability-Testing Sequence S1P74-B) . 6-88

7. LONG TERM FLUID-PRESSURE MONITORING $\ldots \ldots \ldots \ldots \ldots \ldots \ldots \ldots 7$ 7-1

7.1 Implementation $\ldots \ldots \ldots \ldots \ldots \ldots \ldots \ldots \ldots \ldots \ldots \ldots \ldots \ldots \ldots .2$

7.2 Equipment for Long-Term Fluid-Pressure Monitoring $\ldots \ldots \ldots \ldots \ldots 7-3$

7.2.1 Single-packer Test Tool . . . . . . . . . . . . . . . . . 7-3

7.2.2 Bourdon Tube Gages . . . . . . . . . . . . . . . . . . . . . . . 7-5

7.3 Procedures for Long-Term Fluid-Pressure Monitoring . . . . . . . . 7-5

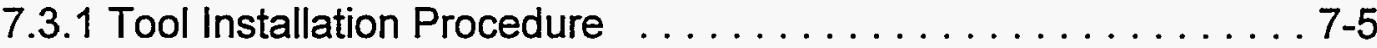

7.4 Fluid-Pressure Monitoring $\ldots \ldots \ldots \ldots \ldots \ldots \ldots \ldots \ldots \ldots \ldots \ldots \ldots$

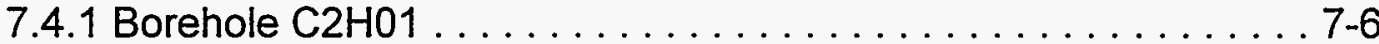

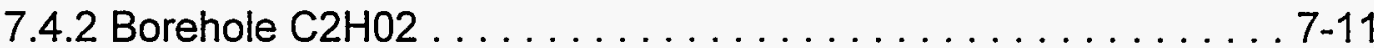

7.4.3 Borehole L4P52 . . . . . . . . . . . . . . . . . . . . . . 7-16

7.4.4 Borehole SCP01 . . . . . . . . . . . . . . . . . 7-19

7.4.5 Borehole S1P71 . . . . . . . . . . . . . . . . . . 7-24

7.4.6 Borehole S1P72 . . . . . . . . . . . . . . . . . . . . 7-28

7.5 Fluid-Pressure Monitoring in Other Boreholes . . . . . . . . . . 7-31

7.5.1 Borehole DPD01 . . . . . . . . . . . . . . . . . . 7-34

7.5.2 Borehole DPD02 … . . . . . . . . . . . . . . 7-37

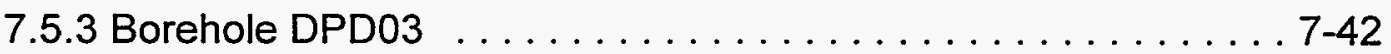

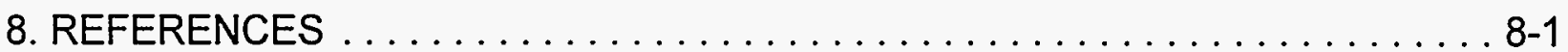

APPENDIX A (Core Descriptions of Test and Observation Boreholes) $\ldots \ldots \ldots$ A-1 


\section{LIST OF FIGURES}

Figure 1-1 Location of WIPP Site and associated access shafts. . . . . . . . . 1-2

Figure 2-1 Map of the WIPP Site underground facility and test and observation

borehole locations. . . . . . . . . . . . . . . . . . .

Figure 2-2a Detailed lithology of the Salado Formation and typical positions of WIPP

underground rooms. ..............................

Figure 2-2b Detailed lithology of the Salado Formation and typical positions of WIPP underground rooms (continued). . . . . . . . . . . . . . . . . 2-4

Figure 3-1 Stratigraphic positions of permeability-testing boreholes with test zones and guard zones indicated. . .........................

Figure 3-2 Generic double-packer test-tool configuration used for permeability testing.

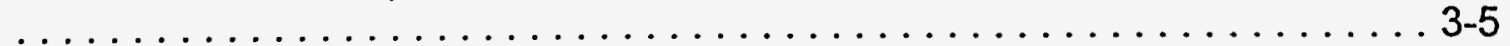

Figure 3-3 Generic triple-packer test-tool configuration used for permeability testing.

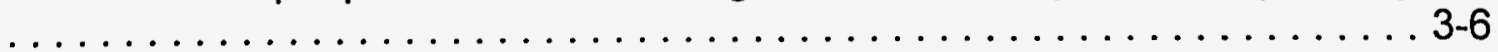

Figure 3-4 Detail of multipacker test tool. $\ldots \ldots \ldots \ldots \ldots \ldots \ldots \ldots \ldots \ldots \ldots \ldots \ldots$

Figure 3-5 Slip-type test-tool restraint device. . . . . . . . . . . . . . . . . 3-8

Figure 3-6 Generic split-view schematic illustration of a multipacker test tool used for

permeability testing. . . . . . . . . . . . . . . . . . . .

Figure 3-7a Intensifier pump system for packer inflation and fluid injection

(configuration \#1). . . . . . . . . . . . . . . . . . . . . .

Figure 3-7b Intensifier pump system for packer inflation and fluid injection

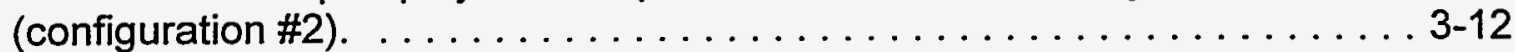

Figure 3-8 Schematic illustration of the data acquisition system. ......... 3-13

Figure 3-9a Differential-pressure-transmitter panel (configuration \#1). . . . . . . 3-16

Figure 3-9b Differential-pressure-transmitter panel (configuration \#2). . . . . . . . 3-17

Figure 3-9c Differential-pressure-transmitter panel (configuration \#3). . . . . . . 3-18

Figure $3-10$ Pressurized-brine-sampling apparatus. . . . . . . . . . . .

Figure 3-11 Typical stainless-steel vessel pressure maintenance system. . . . . . 3-21

Figure 3-12 Typical gas-bladder pressure-maintenance system. . . . . . . . . . 3-22

Figure 3-13 Schematic illustration of the pressure-transducer-calibration system. . 3-24

Figure 3-14 Radial LVDT Measurement Device. . . . . . . . . . . . . . . 3-26

Figure 3-15 Test-tool installation in a borehole oriented vertically downward. . . . . 3-28

Figure 3-16 Test-tool installation in a borehole oriented upward at an angle. . . . . 3-29

Figure 3-17 Test-tool installation in a borehole oriented vertically upward. . . . . . 3-30

Figure 3-18 Test-tool packer-inflation and fluid-injection configuration for vertically

upward and angled upward boreholes. . . . . . . . . . . . . . . $\ldots \ldots$

Figure $3-19$ Typical permeability-testing sequence. . . . . . . . . . . . . 3-33

Figure 3-20 Spatial representation of Room L4 showing the locations and orientations

of boreholes L4P51 and L4P52. . . . . . . . . . . . . . . . 3-38

Figure 3-21 Spatial representation of Waste Panel 1, Room 7 showing the locations

and orientations of boreholes S1P71, S1P72, and S1P74 . . . . . . 3-39

Figure 3-22 Configuration \#1 of permeability test tool for sequence L4P51-C1 . . . 3-41

Figure 3-23 Configuration \#2 of permeability test tool for sequence L4P51-C1. . . . 3-42

Figure 3-24 Configuration of permeability test tool for sequence L4P51-C2 . . . . . 3-43

Figure 3-25a Configuration \#1 of permeability test tool for sequence L4P51-D1. . . 3-44 


\section{LIST OF FIGURES (Continued)}

Figure 3-25b Configuration \#1 of permeability test tool for sequence L4P51-D1

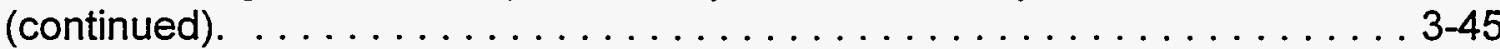

Figure 3-26a Configuration \#2 of permeability test tool for sequence L4P51-D1. . . 3-46

Figure 3-26b Configuration \#2 of permeability test tool for sequence L4P51-D1

(continued). . . . . . . . . . . . . . . . . . . . . . . . . . 3-47

Figure 3-27a Configuration \#3 of permeability test tool for sequence L4P51-D1. . . 3-48

Figure 3-27b Configuration \#3 of permeability test tool for sequence L4P51-D1

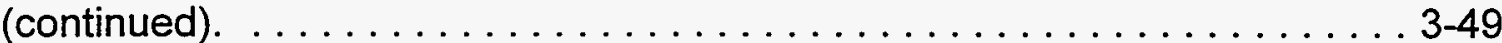

Figure 3-28a Configuration \#4 of permeability test tool for sequence L4P51-D1 . . 3-50

Figure 3-28b Configuration \#4 of permeability test tool for sequence L4P51-D1

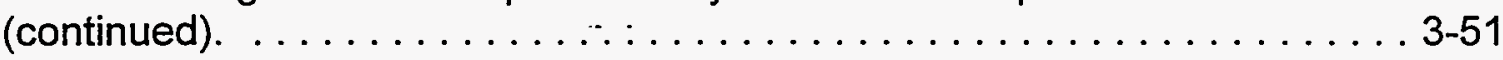

Figure 3-29a Configuration \#5 for permeability test tool for sequence L4P51-D1. . 3-52

Figure 3-29b Configuration \#5 for permeability test tool for sequence L4P51-D1

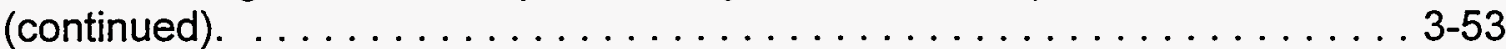

Figure 3-30 Configuration of permeability test tool for sequence L4P51-D2. . . . 3-54

Figure 3-31 Configuration of permeability test tool for sequence L4P52-B. . . . . 3-55

Figure 3-32 Configuration of permeability test tool for sequence S1P74-A. . . . . 3-56

Figure 3-33 Configuration of permeability test tool for sequence S1P74-B. . . . . . 3-57

Figure 3-34 Zone pressures during permeability-testing sequence L4P51-C1 . . . 3-65

Figure 3-35 Packer pressures during permeability-testing sequence L4P51-C1. . . 3-66

Figure 3-36 Zone temperatures during permeability-testing sequence L4P51-C1 . . 3-67

Figure 3-37 Axial-LVDT displacement during permeability-testing sequence L4P51-C1.

Figure 3-38 Radial-LVDT displacement during permeability-testing sequence L4P51-

C1. . . . . . . . . . . . . . . . . . . . . . . . . . . . . . 3-69

Figure 3-39a Fluid production during constant-pressure-flow tests in permeability-

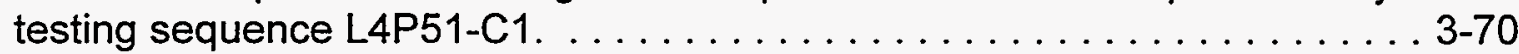

Figure 3-39b Fluid production during constant-pressure-flow tests in permeability-

testing sequence L4P51-C1 (continued). . . . . . . . . . . . . 3-71

Figure 3-40 Test-zone compressibility as measured during permeability-testing

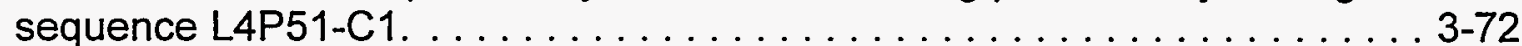

Figure 3-41 Zone pressures during permeability-testing sequence L4P51-C2 . . . . 3-77

Figure 3-42 Packer pressures during permeability-testing sequence L4P51-C2 . . 3-78

Figure 3-43 Test-zone 1 temperature during permeability-testing sequence L4P51-C2.

Figure 3-44 Fluid production during constant-pressure-flow tests in permeability-testing

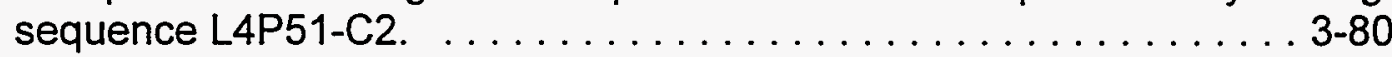

Figure 3-45 Zone pressures during permeability-testing sequence L4P51-D1 . . . . 3-87

Figure 3-46 Packer pressures during permeability-testing sequence L4P51-D1. . 3-88

Figure 3-47 Axial-LVDT displacement during permeability-testing sequence L4P51-D1.

. . . . . . . . . . . . . . . . . . . . . . . . . . . . . 3-89

Figure 3-48 Radial-LVDT displacement during permeability-testing sequence L4P51-

D1. . . . . . . . . . . . . . . . . . . . . . . . . . . . . . . . 3-90

Figure 3-49a Fluid production during constant-pressure-flow tests in permeabilitytesting sequence L4P51-D1. . . . . . . . . . . . . . . . . 3-91 


\section{LIST OF FIGURES (Continued)}

Figure 3-49b Fluid production during constant-pressure-flow tests in permeability-

testing sequence L4P51-D1 (continued). . . . . . . . . . . . . 3-92

Figure 3-50 Zone pressure during permeability-testing sequence L4P51-D2 . . . . 3-95

Figure 3-51 Packer pressure during permeability-testing sequence L4P51-D2 . . . 3-96

Figure 3-52 Zone pressures during permeability-testing sequence L4P52-B. . . . 3-100

Figure 3-53 Packer pressures during permeability-testing sequence L4P52-B. . . 3-101

Figure 3-54 Zone temperatures during permeability-testing sequence L4P52-B. . 3-102

Figure 3-55 Axial-LVDT displacement during permeability-testing sequence L4P52-B.

Figure 3-56 Radial-LVDT displacement during permeability-testing sequence L4P52-B.

. . . . . . . . . . . . . . . . . . . . . . . . . . . . . . . . . 3-104

Figure 3-57 Fluid production during constant-pressure-flow tests in permeability-testing

sequence L4P52-B. . . . . . . . . . . . . . . . . . . . . . 3-105

Figure 3-58 Zone pressure during permeability-testing sequence S1P74-A. . . . 3-110

Figure 3-59 Packer pressures during permeability-testing sequence S1P74-A. . . 3-111

Figure 3-60 Zone temperatures during permeability-testing sequence S1P74-A. . 3-112

Figure 3-61 Axial-LVDT displacement during permeability-testing sequence S1P74-A.

Figure 3-62 Radial-LVDT displacement during permeability-testing sequence S1P74-A.

Figure 3-63 Fluid production during constant-pressure-flow tests in permeability-testing sequence S1P74-A. . . . . . . . . . . . . . . . . . . 3115

Figure 3-64 Zone compressibility as measured during permeability-testing sequence

S1P74-A. .............................. 3-116

Figure 3-65 Zone pressures during permeability-testing sequence S1P74-B. . . . 3-121

Figure 3-66 Packer pressures during permeability-testing sequence S1P74-B. . . 3-122

Figure 3-67 Axial-LVDT displacement during permeability-testing sequence S1P74-B.

Figure 3-68 Cumulative production from the test zone of borehole L4P51 during the pressurized brine-sampling campaign (sample dates indicated). . . . . . 3-126

Figure 4-1 Stratigraphic positions of coupled permeability and hydrofracture test and observation boreholes with test zones indicated. . . . . . . . . . . . . 4-3

Figure 4-2 Generic double-packer test-tool configuration used for coupled permeability and hydrofracture testing. . . . . . . . . . . . . . . . .

Figure 4-3 Generic triple-packer test-tool configuration used for coupled permeability and hydrofracture testing. . . . . . . . . . . . . . . . .

Figure 4-4 Generic single-packer monitor-tool configuration used for coupled permeability and hydrofracture testing. . . . . . . . . . . . . . . .

Figure 4-5 Spatial representation of Room C1 showing the locations and orientations of test and observation boreholes. . . . . . . . . . . . . . .

Figure 4-6 Cross-sectional view of Room C1 showing the locations of test and observation boreholes. . . . . . . . . . . . . . . . . . . 4-12

Figure 4-7 Spatial representation of Room C2 showing the locations and orientations of GTPT test and observation boreholes $\mathrm{C} 2 \mathrm{H} 01$ and $\mathrm{C} 2 \mathrm{H} 02 . \ldots \ldots \ldots . . \ldots 4-13$ 


\section{LIST OF FIGURES (Continued)}

Figure 4-8 Configuration of the tool in test borehole $C 1 \times 10$ for testing sequences C1X10 and C1X05-A. . . . . . . . . . . . . . . . . . . 4-15

Figure 4-9 Configuration of the tool in observation borehole $\mathrm{C} 1 \mathrm{H} 05$ for testing

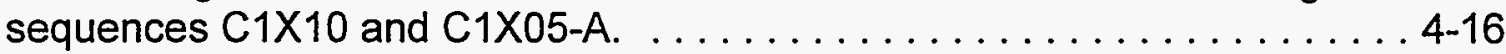

Figure 4-10 Configuration of the tool in observation borehole $\mathrm{C} 1 \mathrm{HO} 6$ for testing sequences $\mathrm{C} 1 \mathrm{X} 10$ and $\mathrm{C} 1 \mathrm{X} 05-\mathrm{A} . \ldots \ldots \ldots \ldots \ldots \ldots \ldots \ldots \ldots .17$

Figure 4-11 Configuration of the tool in test borehole $\mathrm{C} 1 \mathrm{X} 05$ for testing sequence C1X05-A. . . . . . . . . . . . . . . . . . . . . . . 4-18

Figure 4-12 Configuration of the tool in observation borehole $\mathrm{C} 1 \mathrm{HO}$ for testing sequence C1X05-A........................... 4-19

Figure 4-13 Configuration of the tool in observation borehole $\mathrm{C} 1 \mathrm{X} 06$ for testing sequence C1X05-A. . . . . . . . . . . . . . . . . . . . . . . 4-20

Figure 4-14 Configuration of the tool in test borehole $\mathrm{C} 1 \mathrm{X} 05$ for testing sequence C1X05-B. . . . . . . . . . . . . . . . . . . . . . . . . . . 4-21

Figure 4-15a Configuration \#1 of the tool in observation borehole $\mathrm{C} 1 \mathrm{H} 07$ for testing

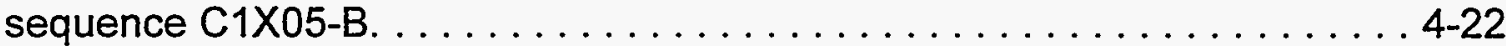

Figure 4-15b Configuration \#1 of the tool in observation borehole $\mathrm{C} 1 \mathrm{H} 07$ for testing sequence $\mathrm{C} 1 \mathrm{X} 05-\mathrm{B}$ (continued). . . . . . . . . . . . . . . 4-23

Figure 4-16a Configuration \#2 of the tool in observation borehole $\mathrm{C} 1 \mathrm{H} 07$ for testing

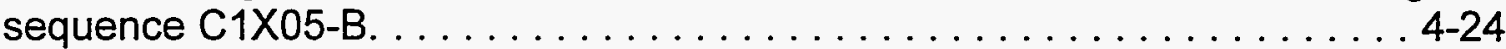

Figure 4-16b Configuration \#2 of the tool in observation borehole $\mathrm{C} 1 \mathrm{H} 07$ for testing sequence C1X05-B (continued). . . . . . . . . . . . . . . . 25

Figure 4-17a Configuration of the tool in observation borehole $\mathrm{C} 1 \mathrm{X} 06$ for testing sequence C1X05-B. . . . . . . . . . . . . . . . . . . . . . . . . 4-26

Figure 4-17b Configuration of the tool in observation borehole $\mathrm{C} 1 \mathrm{X} 06$ for testing sequence C1X05-B (continued). . . . . . . . . . . . . . . . . 4-27

Figure 4-18 Test-zone pressure in test borehole $\mathrm{C} 1 \mathrm{X} 10$ during testing sequence C1X10. .................................. 4-33

Figure 4-19a Fluid production during constant-pressure-flow tests during testing

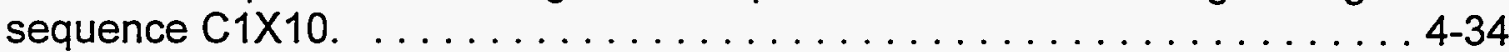

Figure 4-19b Fluid production during constant-pressure-flow tests during testing sequence $\mathrm{C} 1 \mathrm{X} 10$ (continued).

Figure 4-19c Fluid production during constant-pressure-flow tests during testing sequence $\mathrm{C} 1 \mathrm{X} 10$ (continued).

Figure 4-20 Test-zone pressure in observation borehole $\mathrm{C} 1 \mathrm{H} 05$ during testing sequence $\mathrm{C} 1 \mathrm{X} 10$.

Figure 4-21 Test-zone packer pressure in observation borehole $\mathrm{C} 1 \mathrm{H} 05$ during testing sequence $\mathrm{C} 1 \mathrm{X} 10 . \ldots \ldots \ldots \ldots \ldots \ldots \ldots \ldots \ldots \ldots \ldots . \ldots . . \ldots \ldots$

Figure 4-22 Test-zone pressure in observation borehole $\mathrm{C} 1 \mathrm{H} 06$ during testing sequence $\mathrm{C} 1 \times 10$.

Figure 4-23 Test-zone packer pressure in observation borehole $\mathrm{C} 1 \mathrm{HO} 6$ during testing sequence $\mathrm{C} 1 \mathrm{X} 10$.

Figure 4-24 Test-zone pressure in observation borehole DPD02 during testing sequence $\mathrm{C} 1 \mathrm{X} 10$. 


\section{LIST OF FIGURES (Continued)}

Figure 4-25 Test-zone pressure in observation borehole DPD03 during testing

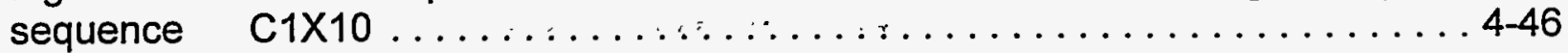

Figure 4-26 Test-zone pressure in observation borehole $\mathrm{C} 2 \mathrm{H} 01$ during testing sequence $\mathrm{C} 1 \times 10 \ldots \ldots \ldots \ldots \ldots \ldots \ldots \ldots \ldots \ldots . \ldots \ldots$ 4. 48

Figure 4-27 Test-zone pressure in test borehole $\mathrm{C} 1 \mathrm{X} 05$ during testing sequence C1X05-A. . . . . . . . . . . . . . . . . . . . . . . . 4-53

Figure 4-28a Fluid production during constant-pressure-flow tests in test borehole C1X05 during testing sequence $C 1 X 05-A . \ldots \ldots \ldots \ldots \ldots \ldots \ldots 4-54$

Figure 4-28b Fluid production during constant-pressure-flow tests in test borehole C1X05 during testing sequence $\mathrm{C} 1 \mathrm{X05}-\mathrm{A}$ (continued). . . . . . . . 4-55

Figure 4-28c Fluid production during constant-pressure-flow tests in test borehole C1X05 during testing sequence C1X05-A (continued). . . . . . . . 4-56

Figure 4-28d Fluid production during constant-pressure-flow tests in test borehole C1X05 during testing sequence C1X05-A (continued) ......... 4-57

Figure 4-28e Fluid production during constant-pressure-flow tests in test borehole C1X05 during testing sequence C1X05-A (continued). . . . . . . . 4-58

Figure 4-29 Zone pressure in observation borehole $\mathrm{C} 1 \mathrm{H} 05$ during testing sequence C1X05-A. . . ........................... 4-60

Figure 4-30 Packer pressure in observation borehole $\mathrm{C} 1 \mathrm{H} 05$ during testing sequence

C1X05-A. ............................. 4-61

Figure 4-31 Zone pressure in observation borehole $\mathrm{C} 1 \mathrm{H} 06$ during testing sequence

C1X05-A. . . ........................... 4-63

Figure 4-32 Packer pressure in observation borehole $\mathrm{C} 1 \mathrm{H} 06$ during testing sequence

C1X05-A. ............................... 4-64

Figure 4-33 Zone pressure in observation borehole $\mathrm{C} 1 \mathrm{H} 07$ during testing sequence

C1X05-A. . . . . . . . . . . . . . . . . . . . . . . 4-66

Figure 4-34 Packer pressure in observation borehole $\mathrm{C} 1 \mathrm{H} 07$ during testing sequence

C1X05-A. . . . . . . . . . . . . . . . . . . . . . . 4-67

Figure 4-35 Zone temperature in observation borehole $\mathrm{C} 1 \mathrm{H} 07$ during testing sequence

C1X05-A. . . . . . . . . . . . . . . . . . . . . . . . 4-68

Figure 4-36 Zone pressure in observation borehole $\mathrm{C} 1 \mathrm{X} 06$ during testing sequence

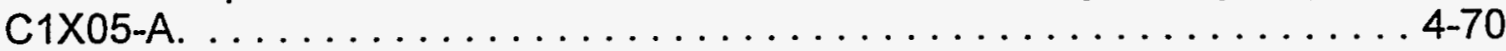

Figure 4-37 Packer pressure in observation borehole $\mathrm{C} 1 \mathrm{X} 06$ during testing sequence

C1X05-A. ............................ 4-71

Figure 4-38 Axial-LVDT displacement in observation borehole C1X06 during testing

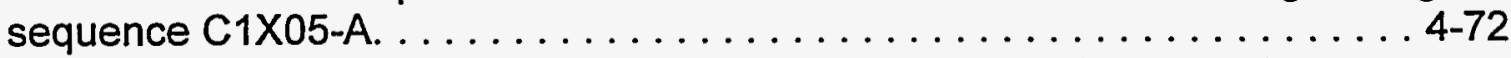

Figure 4-39 Test-zone pressure in observation borehole $\mathrm{C} 1 \mathrm{X} 10$ during testing sequence $C 1 \times 05-A \ldots \ldots \ldots \ldots \ldots \ldots \ldots \ldots \ldots \ldots .74$

Figure 4-40 Zone pressure in observation borehole $\mathrm{C} 2 \mathrm{H} 01$ during testing sequence

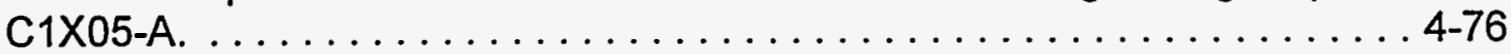

Figure 4-41 Zone pressures in test borehole $\mathrm{C} 1 \mathrm{X} 05$ during testing sequence $\mathrm{C} 1 \mathrm{X} 05-\mathrm{B}$

Figure 4-42a Fluid production during constant-pressure-flow tests in test borehole

$\mathrm{C} 1 \mathrm{X05}$ during testing sequence $\mathrm{C} 1 \mathrm{X} 05-\mathrm{B} . \ldots \ldots \ldots \ldots \ldots \ldots \ldots \ldots$ 


\section{LIST OF FIGURES (Continued)}

Figure 4-42b Fluid production during constant-pressure-flow tests in test borehole $\mathrm{C} 1 \mathrm{X} 05$ during testing sequence $\mathrm{C} 1 \mathrm{X05-B}$ (continued). . . . . . . . 4-84

Figure 4-42c Fluid production during constant-pressure-flow tests in test borehole

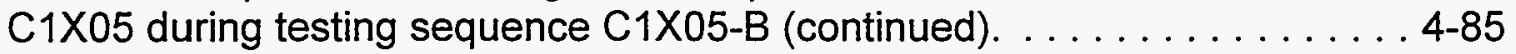

Figure 4-43 Zone pressures in observation borehole $\mathrm{C} 1 \mathrm{H} 07$ during testing sequence

C1X05-B. . . ............................ 4-89

Figure 4-44 Zone pressures in observation borehole $\mathrm{C} 1 \mathrm{X} 06$ during testing sequence

C1X05-B. . . .......................... 4-92

Figure 4-45 Axial-LVDT displacement in observation borehole C1X06 during testing

sequence C1X05-B. . . . . . . . . . . . . . . . . . . . . 4-93

Figure 5-1 Stratigraphic positions of GTPT boreholes with test zones indicated. . . 5-3

Figure 5-2 Schematic illustration of the constant-rate gas-injection system. . . . . . 5-6

Figure 5-3 Spatial representation of the Core Storage Library showing the location and

orientation of GTPT test borehole SCP01. . . . . . . . . . . . 5-9

Figure 5-4 Configuration \#1 of GTPT test tool for sequence SCP01-1. . . . . . 5-10

Figure 5-5 Configuration \#2 of GTPT test tool for sequence SCP01-1. . . . . . . 5-11

Figure 5-6 Configuration \#3 of GTPT test tool for sequence SCP01-1. . . . . . 5-12

Figure 5-7 Configuration \#1 of GTPT test tool for sequence SCP01-2. . . . . . . 5-13

Figure 5-8 Eonfiguration \#2 of GTPT test tool for sequence SCP01-2. . . . . . . 5-14

Figure 5-9 Configuration of monitor tool for sequence $\mathrm{C} 2 \mathrm{HO} 2$ in borehole $\mathrm{C} 2 \mathrm{H} 01$. 5-16

Figure 5-10 Configuration \#1 of test tool for sequence $\mathrm{C} 2 \mathrm{H} 02 . \ldots \ldots \ldots \ldots$ 5-17

Figure 5-11 Configuration \#2 of test tool for sequence $\mathrm{C} 2 \mathrm{H} 02 . \ldots \ldots \ldots \ldots .5-18$

Figure 5-12 Zone pressures during testing sequence SCP01-1 . . . . . . . 5-26

Figure 5-13 Packer pressures during testing sequence SCP01-1. . . . . . . . 5-27

Figure 5-14 Nitrogen-injection rate during testing sequence SCP01-1. . . . . . . 5-28

Figure 5-15 Test-zone temperature during testing sequence SCP01-1. . . . . . 5-29

Figure 5-16 Brine-injection volume of test-zone compressibility test during testing

sequence SCP01-1. . . . . . . . . . . . . . . . . . . . 5-30

Figure 5-17 Calculated test-zone compressibility as a function of pressure obtained

from test-zone compressibility test during testing sequence SCP01-1. . . 5-31

Figure 5-18 Zone pressures during testing sequence SCP01-2 . . . . . . 5-36

Figure 5-19 Packer pressures during testing sequence SCP01-2. . . . . . . 5-37

Figure 5-20 Nitrogen-injection rate during testing sequence SCP01-2. . . . . . . 5-38

Figure 5-21 Brine-injection volume of test-zone compressibility test during testing sequence SCP01-2. . . . . . . . . . . . . . . . . . . . 5-39

Figure 5-22 Calculated test-zone compressibility as a function of pressure obtained from test-zone compressibility test during testing sequence SCP01-2. . . . 5-40

Figure 5-23 Zone pressures during testing sequence $\mathrm{C} 2 \mathrm{HO} 2$ in test borehole $\mathrm{C} 2 \mathrm{HO} 2$

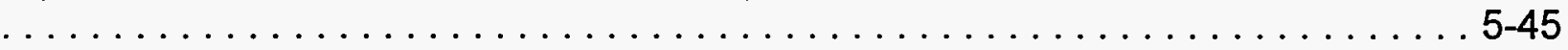

Figure 5-24 Packer pressures during testing sequence $\mathrm{C} 2 \mathrm{HO} 2$ in test borehole $\mathrm{C} 2 \mathrm{HO} 2$

Figure 5-25 Nitrogen-injection rate during testing sequence $\mathrm{C} 2 \mathrm{HO} 2$ in test borehole

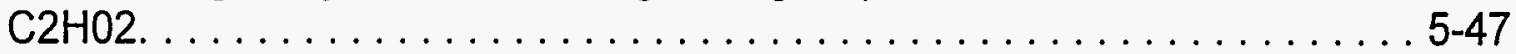

Figure 5-26 Test-zone temperature during testing sequence $\mathrm{C} 2 \mathrm{H} 02$ in test borehole C2H02. . . . . . . . . . . . . . . . . . . . . . . . . . . . . . . 5-48 


\section{LIST OF FIGURES (Continued)}

Figure 5-27 Zone pressure during testing sequence $\mathrm{C} 2 \mathrm{H} 02$ in observation borehole

C2H01................................... 5-49

Figure 5-28 Zone pressures during testing sequence L4P52-B. . . . . . . 5-54

Figure 5-29 Packer pressures during testing sequence L4P52-B. . . . . . 5-55

Figure 5-30 Nitrogen-injection rate during testing sequence L4P52-B. . . . . . . 5-56

Figure 5-31 Test-zone temperature during testing sequence L4P52-B. . . . . . 5-57

Figure 6-1 Cross-sectional view of stainless-steel chamber for compliance testing. . 6-2

Figure 6-2 Zone pressure during compliance testing of test tool \#32A........ 6-6

Figure 6-3 Packer pressure during compliance testing of test tool \#32A: . . . . . 6-7

Figure 6-4 Zone temperature during compliance testing of test tool \#32A. . . . . 6-8

Figure 6-5 Zone pressure during compliance testing of test tool \#32B. . . . . . 6-10

Figure 6-6 Packer pressure during compliance testing of test tool \#32B. . . . . 6-11

Figure 6-7 Zone pressures during compliance testing of test tool \#33A. . . . . . 6-15

Figure 6-8 Packer pressures during compliance testing of test tool \#33A. . . . 6-16

Figure 6-9 Zone temperatures during compliance testing of test tool \#33A. . . . . 6-17

Figure 6-10 Axial-LVDT displacement during compliance testing of test tool \#33A. 6-18

Figure 6-11 Radial-LVDT displacement during compliance testing of test tool \#33A.

Figure 6-12a Test-zone fluid-injection volumes during compliance testing of test tool $\# 33$ A. . . . . . . . . . . . . . . . . . . . . . . . . 6-20

Figure 6-12b Guard-zone fluid-injection volumes during compliance testing of test tool $\# 33 A \ldots \ldots \ldots \ldots \ldots \ldots \ldots \ldots \ldots \ldots \ldots \ldots \ldots .6 .21$

Figure 6-13a Test-zone compressibility as measured during compliance testing of test tool \#33A. . . . . . . . . . . . . . . . . . . . . . . . . 6-22

Figure 6-13b Guard-zone compressibility as measured during compliance testing of test tool \#33A. . . . . . . . . . . . . . . . . . . . . 6-23

Figure 6-14 Zone pressure during compliance testing of test tool \#34 . . . . 6 6-25

Figure 6-15 Packer pressure during compliance testing of test tool \#34. . . . . . 6-26

Figure 6-16 Zone pressures during compliance testing of test tool \#35. . . . . . 6-30

Figure 6-17 Packer pressures during compliance testing of test tool \#35. . . . . 6-31

Figure 6-18 Zone temperatures during compliance testing of test tool \#35. . . . 6 6-32

Figure 6-19 Axial-LVDT displacement during compliance testing of test tool \#35. . 6-33 Figure 6-20 Radial-LVDT displacement during compliance testing of test tool \#35. 6-34 Figure 6-21a Test-zone fluid-injection volumes during compliance testing of test tool

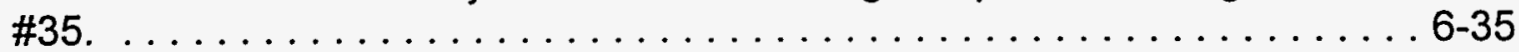

Figure 6-21b Guard-zone fluid-injection volumes during compliance testing of test tool

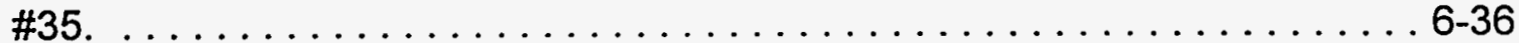

Figure $6-22 a$ Test-zone compressibility as measured during compliance testing of test

tool \#35. . . . . . . . . . . . . . . . . . . . . . . . . . . .6-37

Figure 6-22b Guard-zone compressibility as measured during compliance testing of

test tool \#35. . . . . . . . . . . . . . . . . . . . . . .6-38

Figure 6-23 Zone pressure during compliance testing of test tool \#36. . . . . . 6-40

Figure 6-24 Packer pressure during compliance testing of test tool \#36. . . . . 6 6-41

Figure 6-25 Zone pressures during compliance testing of test tool $\# 37 . \ldots \ldots 66-44$

Figure 6-26 Packer pressures during compliance testing of test tool \#37. . . . . 6-45 


\section{LIST OF FIGURES (Continued)}

Figure 6-27 Zone temperatures during compliance testing of test tool \#37. . . . 6-46

Figure 6-28 Axial-LVDT displacement during compliance testing of test tool \#37. . 6-47

Figure 6-29 Radial-LVDT displacement during compliance testing of test tool \#37. 6-48

Figure 6-30 Test-zone fluid-injection volumes during compliance testing of test tool \#37.

Figure 6-31 Test-zone compressibility as measured during compliance testing of test tool \#37. . . . . . . . . . . . . . . . . . . . . . . . . . . 6-50

Figure 6-32 Zone pressures during compliance testing of test tool \#38A. . . . 6 6-54

Figure 6-33 Packer pressures during compliance testing of test tool \#38A. . . . 6 6-55

Figure 6-34 Zone temperatures during compliance testing of test tool \#38A. . . . 6-56

Figure 6-35 Test-zone fluid-injection volumes during compliance testing of test tool \#38. . . . . . . . . . . . . . . . . . . . . . . . . . . . . . . . 6-57

Figure 6-36 Test-zone compressibility as measured during compliance testing of test

tool \#38A. . . . . . . . . . . . . . . . . . . . . . . . . . 6-58

Figure 6-37 Zone pressures during compliance testing of test tool \#39. . . . . 6 6-61

Figure 6-38 Packer pressures during compliance testing of test tool \#39. . . . . . 6-62

Figure 6-39 Zone pressures during compliance testing of test tool \#40. . . . . 6-65

Figure 6-40 Packer pressures during compliance testing of test tool \#40. . . . . 6-66

Figure 6-41 Zone pressures during compliance testing of test tool \#41. . . . . 6-69

Figure 6-42 Packer pressures during compliance testing of test tool \#41. . . . . . 6-70

Figure 6-43 Test-zone 1 temperature during compliance testing of test tool \#41. . 6-71

Figure 6-44 Zone pressures during compliance testing of test tool \#BOT-01. . . . 6-75

Figure 6-45 Packer pressures during compliance testing of test tool \#BOT-01. . . 6-76

Figure 6-46 Test-zone fluid-injection volumes during compliance testing of test tool

\#BOT-01. . . . . . . . . . . . . . . . . . . . . . . . 6-77

Figure 6-47 Test-zone compressibility as measured during compliance testing of test

tool \#BOT-01. . . . . . . . . . . . . . . . . . . . . . . . . . 6-78

Figure 6-48 Zone pressures during compliance testing of test tool \#P51-D1A. . . 6-82

Figure 6-49 Packer pressures during compliance testing of test tool \#P51-D1A. . . 6-83

Figure 6-50 Axial-LVDT displacement during compliance testing of test tool \#P51-D1A.

... . . . . . . . . . . . . . . . . . . . . . . . . . . . . 6-84

Figure 6-51 Radial-LVDT displacement during compliance testing of test tool \#P51-

D1A. . . . . . . . . . . . . . . . . . . . . . . . . 6-85

Figure 6-52 Fluid-injection volumes during compliance testing of test tool \#P51-D1A.

Figure 6-53 Test-zone compressibility as measured during compliance testing of test

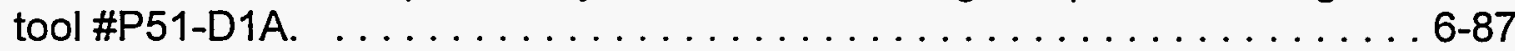

Figure 6-54 Zone pressures during compliance testing of test tool \#P74-B. . . . . 6-91

Figure 6-55 Packer pressures during compliance testing of test tool \#P74-B. . . . 6-92

Figure 6-56 Axial-LVDT displacement during compliance testing of test tool \#P74-B.

. . . . . . . . . . . . . . . . . . . . . . . . . . . . . . . . 6-93

Figure 6-57 Fluid-injection volumes during compliance testing of test tool \#P74-B.

Figure 6-58 Test-zone compressibility as measured during compliance testing of test tool \#P74-B. . . . . . . . . . . . . . . . . . . . . . . . . . . . . . 6-95 


\section{LIST OF FIGURES (Continued)}

Figure 7-1 Stratigraphic positions of long-term fluid-pressure monitoring boreholes with test zones indicated. ........................ $7-4$

Figure 7-2 Configuration \#1 of long-term fluid-pressure monitoring tool in borehole C2H01............................... 7-9

Figure 7-3 Long-term fluid-pressure in MB139 in borehole $\mathrm{C} 2 \mathrm{HO} 01 . \ldots \ldots \ldots 7-10$

Figure 7-4 Configuration \#1 of long-term fluid-pressure monitoring tool in borehole

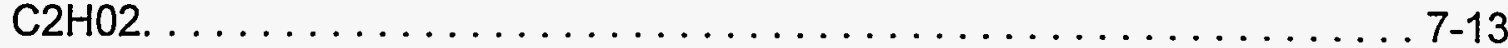

Figure 7-5 Configuration \#2 of long-term fluid-pressure monitoring tool in borehole

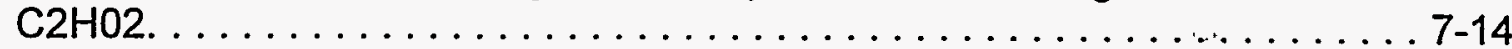

Figure 7-6 Long-term fluid-pressure in $\mathrm{MB} 139$ in borehole $\mathrm{C} 2 \mathrm{H02}$. . . . . . . 7-15

Figure 7-7 Configuration of long-term fluid-pressure monitoring tool in borehole L4P52

....................................... . . . 17

Figure 7-8 Long-term fluid-pressure in MB138 in borehole L4P52. . . . . . . . . 7-18

Figure 7-9 Configuration \#1 of long-term fluid-pressure monitoring tool in borehole SCP01.

Figure 7-10 Configuration \#3 of long-term fluid-pressure monitoring tool in borehole

SCP01.

Figure 7-11 Long-term fluid-pressure in MB139 in borehole SCP01. . . . . . . . 7-23

Figure 7-12 Configuration of long-term fluid-pressure monitoring tool in borehole

S1P71. ............................ 7-26

Figure 7-13 Long-term fluid-pressure in anhydrite "C" in borehole S1P71 . . . . . 7-27

Figure 7-14 Configuration of long-term fluid-pressure monitoring tool in borehole

S1P72. . . . . . . . . . . .

Figure 7-15 Long-term fluid-pressure in MB139 in borehole S1P72. . . . . . . . . 7-30

Figure 7-16 Locations and orientations of long-term fluid-pressure monitoring

boreholes DPD01, DPD02, and DPD03. ............... 7-33

Figure 7-17 Configuration of long-term fluid-pressure monitoring tool in borehole DPD01.

Figure 7-18 Long-term fluid-pressure in MB139 in borehole DPD01.

Figure 7-19 Configuration \#1 of long-term fluid-pressure monitoring tool in borehole DPD02.

Figure 7-20 Configuration \#2 of long-term fluid-pressure monitoring tool in borehole

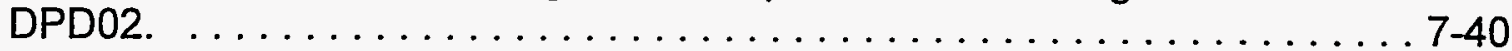

Figure 7-21 Long-term fluid-pressure in MB139 in borehole DPD02. . . . . . 7-41

Figure 7-22 Configuration \#1 of long-term fluid-pressure monitoring tool in borehole DPD03.

Figure 7-23 Configuration \#2 of long-term fluid-pressure monitoring tool in borehole DPD03.

Figure 7-24 Long-term fluid-pressure in MB139 in borehole DPD03. . . . . . . 7-46 


\section{LIST OF TABLES}

Table 3-1 Summary of Borehole Locations and Orientations . . . . . . . . . . 3-36

Table 3-2 Permeability-Testing Sequence Test-Zone Information . . . . . . . . . . 3-37

Table 3-3 Test Summary Associated with Permeability Testing . . . . . . . . . . 3-58

Table 3-4 Permeability-Testing Sequence L4P51-C1 Events $\ldots \ldots \ldots \ldots \ldots \ldots \ldots$ 3-60

Table 3-5 Permeability-Testing Sequence L4P51-C1 Equipment . . . . . . . . . . 3-73

Table 3-6 Permeability-Testing Sequence L4P51-C2 Events . . . . . . . . . . . 3-74

Table 3-7 Permeability-Testing Sequence L4P51-C2 Equipment . . . . . . . . . . 3-81

Table 3-8 Permeability-Testing Sequence L4P51-D1 Events . . . . . . . . . . . . . . 3-82

Table 3-9 Permeability-Testing Sequence L4P51-D1 Equipment . . . . . . . . . . . . 3-93

Table 3-10 Permeability-Testing Sequence L4P51-D2 Events $\ldots \ldots \ldots \ldots \ldots \ldots \ldots$. . . . . .

Table 3-11 Permeability-Testing Sequence L4P51-D2 Equipment . . . . . . . . . . . . . . 3-97

Table 3-12 Permeability-Testing Sequence L4P52-B Events . . . . . . . . . . . . 3-97

Table 3-13 Permeability-Testing Sequence L4P52-B Equipment . . . . . . . . . . 3-106

Table 3-14 Permeability-Testing Sequence S1P74-A Events . . . . . . . . . . . 3-107

Table 3-15 Permeability-Testing Sequence S1P74-A Equipment . . . . . . . . . . 3-117

Table 3-16 Permeability-Testing Sequence S1P74-B Events . . . . . . . . . . . . 3-118

Table 3-17 Permeability-Testing Sequence S1P74-B Equipment . . . . . . . . . . 3-124

Table 3-18 Pressurized Brine Sampling Campaign . . . . . . . . . . . . . . . . . 3-125

Table 4-1 Coupled Permeability and Hydrofracture Test Borehole Information . . 4-9

Table 4-2 Coupled Permeability and Hydrofracture Observation Borehole

Information . . . . . . . . . . . . . . . . . . . . . . . 4-10

Table 4-3 Summary of Test information from Test Boreholes . . . . . . . . . . 4-28

Table 4-4 Events Associated with Test Borehole C1X10 During Testing Sequence

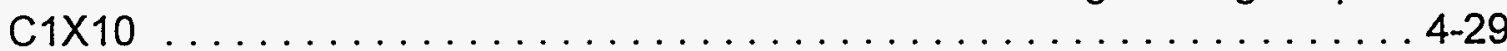

Table 4-5 Testing Sequence $\mathrm{C} 1 \times 10$ Equipment . . . . . . . . . . . . . . 4-32

Table 4-6 Events Associated with Observation Borehole C1H05 During Testing

Sequence $C 1 \times 10 \ldots \ldots \ldots \ldots \ldots \ldots \ldots \ldots \ldots \ldots$. . . . . . . . . . . . . . . . . . .

Table 4-7 Events Associated with Observation Borehole C1H06 During Testing Sequence $\mathrm{C} 1 \times 10 \ldots \ldots \ldots \ldots \ldots \ldots \ldots \ldots$. . . . . . . . . . . . . . . . . . . .

Table 4-8 Events Associated with Observation Borehole DPD02 During Testing Sequence $C 1 X 10 \ldots \ldots \ldots \ldots \ldots \ldots \ldots$. . . . . . . . . . . . . . . . . . . . . . . . . . .

Table 4-9 Events Associated with Observation Borehole DPD03 During Testing Sequence $\mathrm{C} 1 \times 10 \ldots \ldots \ldots \ldots \ldots \ldots \ldots \ldots \ldots$. . . . . . . . . . . . . . . . . . . .

Table 4-10 Events Associated with Observation Borehole $\mathrm{C} 2 \mathrm{H} 01$ During Testing Sequence $\mathrm{C} 1 \mathrm{X} 10 \ldots \ldots \ldots \ldots \ldots \ldots \ldots$. . . . . . . . . . . . . . . . . . . . .

Table 4-11 Events Associated with Test Borehole C1X05 During Testing Sequence C1X05-A . . . . . . . . . . . . . . . . . . . . . . . . 4-49

Table 4-12 Testing Sequence C1X05-A Equipment . . . . . . . . . . . . 4-51

Table 4-13 Events Associated with Observation Borehole C1H05 During Testing Sequence C1X05-A . . . . . . . . . . . . . . . . . . . . . . . . 4-59

Table 4-14 Events Associated with Observation Borehole C1H06 During Testing Sequence C1X05-A . . . . . . . . . . . . . . . . . . . 4-62

Table 4-15 Events Associated with Observation Borehole C1H07 During Testing Sequence C1X05-A . . . . . . . . . . . . . . . . . . . 65 


\section{LIST OF TABLES (Continued)}

Table 4-16 Events Associated with Observation Borehole C1X06 During Testing Sequence C1X05-A ............................ 4-69

Table 4-17 Events Associated with Öbservation Borehole C1X10 During Testing

Sequence C1X05-A . . . . . . . . . . . . . . . . . . . 4-73

Table 4-18 Events Associated with Observation Borehole C2H01 During Testing

Sequence C1X05-A . . . . . . . . . . . . . . . . . . . . . . . 4-75

Table 4-19 Events Associated with Test Borehole C1X05 During Testing Sequence

C1X05-B . . . . . . . . . . . . . . . . . . . . . . . . 4-77

Table 4-20 Testing Sequence C1X05-B Equipment .............. . 4-80

Table 4-21 Events Associated with Observation Borehole C1H07 During Testing

Sequence C1X05-B . ...................... 4-86

Table 4-22 Borehole C1H07 Equipment . . . . . . . . . . . . . . . 4-88

Table 4-23 Events Associated with Observation Borehole C1X06 During Testing

Sequence C1X05-B ......................... 4-90

Table 5-1 Borehole Test-Zone Information . . . . . . . . . . . . . . 5-8

Table 5-2 Borehole Locations, Orientations, and Drilling Information . . . . . . . . 5-8

Table 5-3 Summary of Tests Associated with the GTPT Program . . . . . . . 5-19

Table 5-4 Testing Sequence SCP01-1 Events .................. 5-21

Table 5-5 Testing Sequence SCP01-1 Equipment ............... 5-24

Table 5-6 Testing Sequence SCP01-2 Events . . . . . . . . . . . . . . 5-32

Table 5-7 Testing Sequence SCP01-2 Equipment ............... . 5-35

Table 5-8 Testing Sequence $\mathrm{C} 2 \mathrm{H} 02$ Events . . . . . . . . . . . . . . 5-41

Table 5-9 Testing Sequence $\mathrm{C} 2 \mathrm{H} 02$ Equipment ............... . 5-44

Table 5-10 Events in Borehole $\mathrm{C} 2 \mathrm{H} 01$ During Testing Sequence $\mathrm{C} 2 \mathrm{H} 02$. . . . 5-50

Table 5-11 Testing Sequence L4P52-B Events ................ . 5-50

Table 5-12 Testing Sequence L4P52-B Equipment . . . . . . . . . . . . 5-53

Table 6-1 Compliance-Testing Information ..................... 6-3

Table 6-2 Events Associated with Compliance Testing of Test Tool \#32A; Borehole

C1H05; Sequence C1X10 . ......................6.5

Table 6-3 Compliance Equipment Associated with Test Tool \#32A; Borehole C1H05;

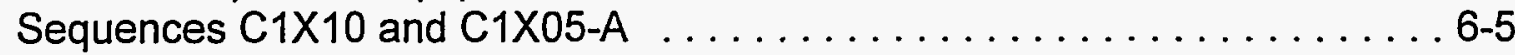

Table 6-4 Events Associated with Compliance Testing of Test Tool \#32B; Borehole

C1H06; Sequence C1X10 .........................6-9

Table 6-5 Compliance Equipment Associated with Test Tool \#32B; Borehole C1H06;

Sequences $\mathrm{C} 1 \mathrm{X} 10$ and $\mathrm{C} 1 \mathrm{X} 05-\mathrm{A} \ldots \ldots \ldots \ldots \ldots \ldots \ldots \ldots .6 .6 \ldots$

Table 6-6 Events Associated with Compliance Testing of Test Tool \#33A; Borehole

L4P51; Sequence L4P51-C1 . . . . . . . . . . . . . . . . . 6-12

Table 6-7 Compliance Equipment Associated with Test Tool \#33A; Borehole L4P51;

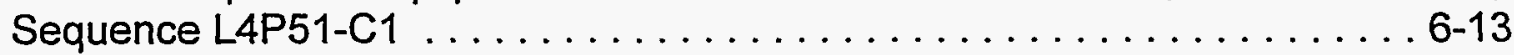

Table 6-8 Events Associated with Compliance Testing of Test Tool \#34; Borehole

C1H07; Sequence C1X05-A . . . . . . . . . . . . . . . . . . . . 6-24

Table 6-9 Compliance Equipment Associated with Test Tool \#34; Borehole C1H07;

Sequence C1X05-A ............................6-24

Table 6-10 Events Associated with Compliance Testing of Test Tool \#35; Borehole

S1P74; Sequence S1P74-A . . . . . . . . . . . . . . . . . 6-27 


\section{LIST OF TABLES (Continued)}

Table 6-11 Compliance Equipment Associated with Test Tool \#35; Borehole S1P74;

Sequence S1P74-A . . . . . . . . . . . . . . . . . . . . 6-28

Table 6-12 Events Associated with Compliance Testing of Test Tool \#36; Borehole

C1X06; Sequence C1X05-A . . . . . . . . . . . . . . . . . . 6-39

Table 6-13 Compliance Equipment Associated with Test Tool \#36; Borehole C1X06;

Sequence C1X05-A . . . . . . . . . . . . . . . . . . . . . . 6-39

Table 6-14 Events Associated with Compliance Testing of Test Tool \#37; Borehole

L4P52; Sequence L4P52-B . . . . . . . . . . . . . . . . . . . . . . . . . 6-42

Table 6-15 Compliance Equipment Associated with Test Tool \#37; Borehole L4P52;

Sequence $L 4$ P52-B . . . . . . . . . . . . . . . . . . . . . . . 6-43

Table 6-16 Events Associated with Compliance Testing of Test Tool \#38A; Borehole

SCP01; Sequence SCP01-1 . . . . . . . . . . . . . . . . . 6-51

Table 6-17 Compliance Equipment Associated with Test Tool \#38A; Borehole

SCP01; Sequence SCP01-1 . . . . . . . . . . . . . . . . 6-53

Table 6-18 Events Associated with Compliance Testing of Test Tool \#39; Borehole

C1H07; Sequence C1X05-B . . . ...................6-59

Table 6-19 Compliance Equipment Associated with Test Tool \#39, Borehole C1H07;

Sequence C1X05-B . . . . . . . . . . . . . . . . . . . . 6-60

Table 6-20 Events Associated with Compliance Testing of Test Tool \#40; Borehole

C1X06; Sequence C1X05-B . . . ....................6.63

Table 6-21 Compliance Equipment Associated with Test Tool \#40; Borehole C1X06;

Sequence C1X05-B . . . . . . . . . . . . . . . . . . . 6-64

Table 6-22 Events Associated with Compliance Testing of Test Tool \#41; Borehole

L4P51; Sequence L4P51-C2 . . . . . . . . . . . . . . . . . . . . 6-67

Table 6-23 Compliance Equipment Associated with Test Tool \#41; Borehole L4P51;

Sequence L4P51-C2 . . . . . . . . . . . . . . . . . . . . . . . . 6-68

Table 6-24 Events Associated with Compliance Testing of Test Tool \#BOT-01;

Borehole SCP01; Sequence SCP01-2 . . . . . . . . . . . . . . 6-72

Table 6-25 Compliance Equipment Associated with Test Tool \#BOT-01; Borehole

SCP01; Sequence SCP01-2 . . . . . . . . . . . . . . . 6-73

Table 6-26 Events Associated with Compliance Testing of Test Tool \#P51-D1A;

Borehole L4P51; Sequence L4P51-D1 . . . . . . . . . . . . . . 6-79

Table 6-27 Compliance Equipment Associated with Test Tool \#P51-D1A; Borehole

L4P51; Sequence L4P51-D1 . . . . . . . . . . . . . . . . . . . . 6 6-811

Table 6-28 Events Associated with Compliance Testing of Test Tool \#P74-B;

Borehole S1P74; Sequence S1P74-B . . . . . . . . . . . . . 6-88

Table 6-29 Compliance Equipment Associated with Test Tool \#P74-B; Borehole

S1P74; Sequence S1P74-B . . . . . . . . . . . . . . . . . . . 6-89

Table 7-1 Location and Dates of Testing Performed in Long-Term Fluid-Pressure

Monitoring Boreholes . . . . . . . . . . . . . . . . . . . .

Table 7-2 Fluid-Pressure Monitoring Boreholes; Test-Zone Information . . . . . . 7-3

Table 7-3 Long-Term Fluid-Pressure Monitoring C2H01 Events . . . . . . . . . . 7-6

Table 7-4 Long Term Fluid-Pressure Monitoring $\mathrm{C} 2 \mathrm{H} 02$ Events . . . . . . . . . 7-11

Table 7-5 Long-Term Fluid-Pressure Monitoring L4P52 Events . . . . . . . . . 7-16

Table 7-6 Long Term Fluid-Pressure Monitoring SCP01 Events . . . . . . . . 7-19 


\section{LIST OF TABLES (Continued)}

Table 7-7 Long-Term Fluid-Pressure Monitoring S1P71 Events . . . . . . . . 7-24

Table 7-8 Long-Term Fluid-Pressure Monitoring S1P72 Events . . . . . . . . 7-28

Table 7-9 Fluid-Pressure Monitoring in Other Boreholes; Test-Zone Information ....................................... 7-31

Table 7-10 Summary of Long-Term Fluid-Pressure Monitoring Borehole Locations and Orientations Not Previously Tested ................ 7-31

Table 7-11 Long-Term Fluid-Pressure Monitoring DPD01 Events $\ldots \ldots \ldots \ldots 7$ 7-34

Table 7-12 Long-Term Fluid-Pressure Monitoring DPD02 Events . . . . . . . 7-37

Table 7-13 Long Term Fluid-Pressure Monitoring DPD03 Events . ..... . . . . 7-42 
$x x v i$ 


\section{INTRODUCTION}

WIPP Salado Hydrology Program Data Report \#3 is the third and final in a series of reports that make available hydrologic data collected during drilling, permeability testing, coupled permeability and hydrofracture testing, gas-threshold-pressure testing (GTPT), and fluidpressure monitoring in the Salado Formation at the Waste Isolation Pilot Plant (WIPP) site near Carlsbad, New Mexico. The data presented in this report were collected between November 1991 and October 1995. The purpose of this series of reports is to disseminate basic hydrologic data to interested parties in a timely manner, often before data interpretation. The underground testing described in this report was carried out by INTERA Inc. under the direction of the Geohydrology Department of Sandia National Laboratories, Albuquerque, New Mexico.

This report presents data from the drilling and testing of fourteen boreholes drilled from the WIPP underground facility in the Salado Formation $655 \mathrm{~m}$ below ground surface (Figure 1-1). The boreholes were in the experimental, operations, and waste-storage areas of the underground facility. The boreholes are oriented vertically downward, angled downward, and angled upward. The data presented include the pressures and temperatures in brinefilled, packer-isolated test intervals, fluid production during constant-pressure-flow tests, nitrogen-injection rates during gas-threshold-pressure testing, and borehole-closure and test-tool movement measurements collected during pressure-buildup/falloff periods and in response to pressure-pulse and constant-pressure-flow tests. The report also contains fluid-pressure monitoring data from packer-isolated intervals of selected boreholes encompassing the time period from August 1989 to May 1995.

This report is written using standard SI units and abbreviations with the following exceptions. For convenience, liter, abbreviated $L$, is used instead of $0.001 \mathrm{~m}^{3}$. When certain equipment has been manufactured using materials specified in inch-pound units (such as standard tubular supplies), or the equipment is rated in inch-pound units (such as pressure equipment), the inch-pound units are used, followed by the SI equivalent in parentheses.

Information and data contained in this report is kept on file in electronic form in the Sandia WIPP Central File (SWCF) under WPO \#42269. Persons interested in obtaining this information should make inquiries to the SWCF of Sandia National Laboratories.

NOTE: The use of brand names in this report is for identification purposes only and does not imply any endorsement of specific products by Sandia National Laboratories or INTERA Inc. 

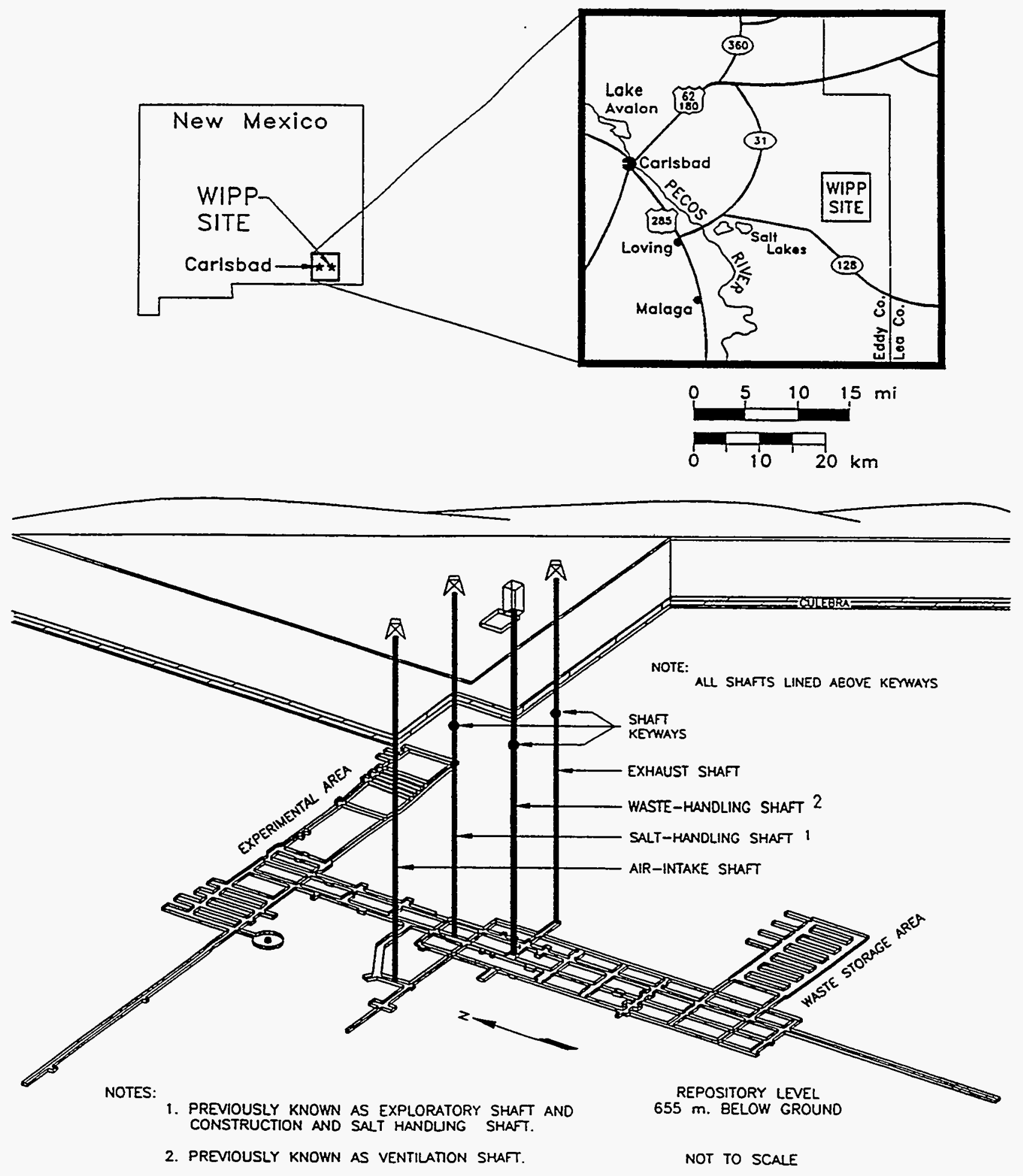

INTERA-6115-1-0

Figure 1.1 Location of WIPP Site and associated access shafts. 


\section{THE WIPP UNDERGROUND FACILITY}

\subsection{Construction}

Figure 2-1 diagrammatically illustrates the WIPP underground facility as it exists at the time of this report. The facility is being constructed in the Salado Formation approximately 655 $\mathrm{m}$ below ground surface. The Salado Formation is a bedded-evaporite sequence consisting primarily of bedded halite with associated interbeds composed principally of anhydrite, polyhalite, clay, and siltstone. As shown in Figure 1-1, the underground facility is accessed by four vertical shafts.

The underground rooms and drifts of the WIPP facility were excavated by continuousmining techniques. Blasting techniques were not employed in the WIPP underground facility. The drifts in the underground excavations are numbered according to their northsouth or east-west distance in feet from the salt-handling $(\mathrm{SH})$ shaft. These designations are used to identify locations such as the East 140 drift. Figures 2-2a and 2-2b show the geometry of typical rooms in the northern experimental area and in the southern wastestorage area, and the generalized local stratigraphy of the Salado Formation. The figure shows that the floors of the simulated waste experimental rooms are stratigraphically approximately $1.8 \mathrm{~m}$ above the backs of the rooms in the waste-storage area. Bechtel (1986) provides construction details for the WIPP underground facility and the locations of geomechanical instrumentation stations in both the experimental and waste-storage areas.

\subsection{Stratigraphy}

Figures $2-2 a$ and $2-2 b$ illustrate the typical stratigraphic sequence encountered in the Salado Formation near the WIPP underground facility and shows that the Salado contains evaporite-mineral and clay-rich interbeds above and below the repository level. Figures $2-2 a$ and $2-2 b$ also indicate the named clay units and anhydrite interbeds and shows mapunit designations according to terminology presented in Bechtel (1986) and Deal et al. (1989). Anhydrite marker beds and their associated clay seams, as well as other clay seams, are of particular interest to the testing programs. These stratigraphic interbeds are often the loci for stress-relief fractures and salt efflorescences noted in 10- to $90-\mathrm{cm}$ diameter boreholes drilled from the drifts and rooms in the underground facility (Bechtel, 1986).

During this reporting period, the anhydrite interbeds "a", "b", Marker Bed (MB) 138, MB139, MB140, the halite approximately $1 \mathrm{~m}$ above $\mathrm{MB140}$, and an argillaceous halite approximately $10 \mathrm{~m}$ below MB140 were tested under the permeability-testing program, the anhydrite interbeds MB139 and MB140 were tested under the coupled permeability and hydrofracture testing program, and the anhydrite interbeds MB138 and MB139 were tested under the gas-threshold-pressure testing (GTPT) program. Also, the anhydrite interbeds "c", MB138, and MB139 were monitored under the long-term fluid-pressure monitoring program. 


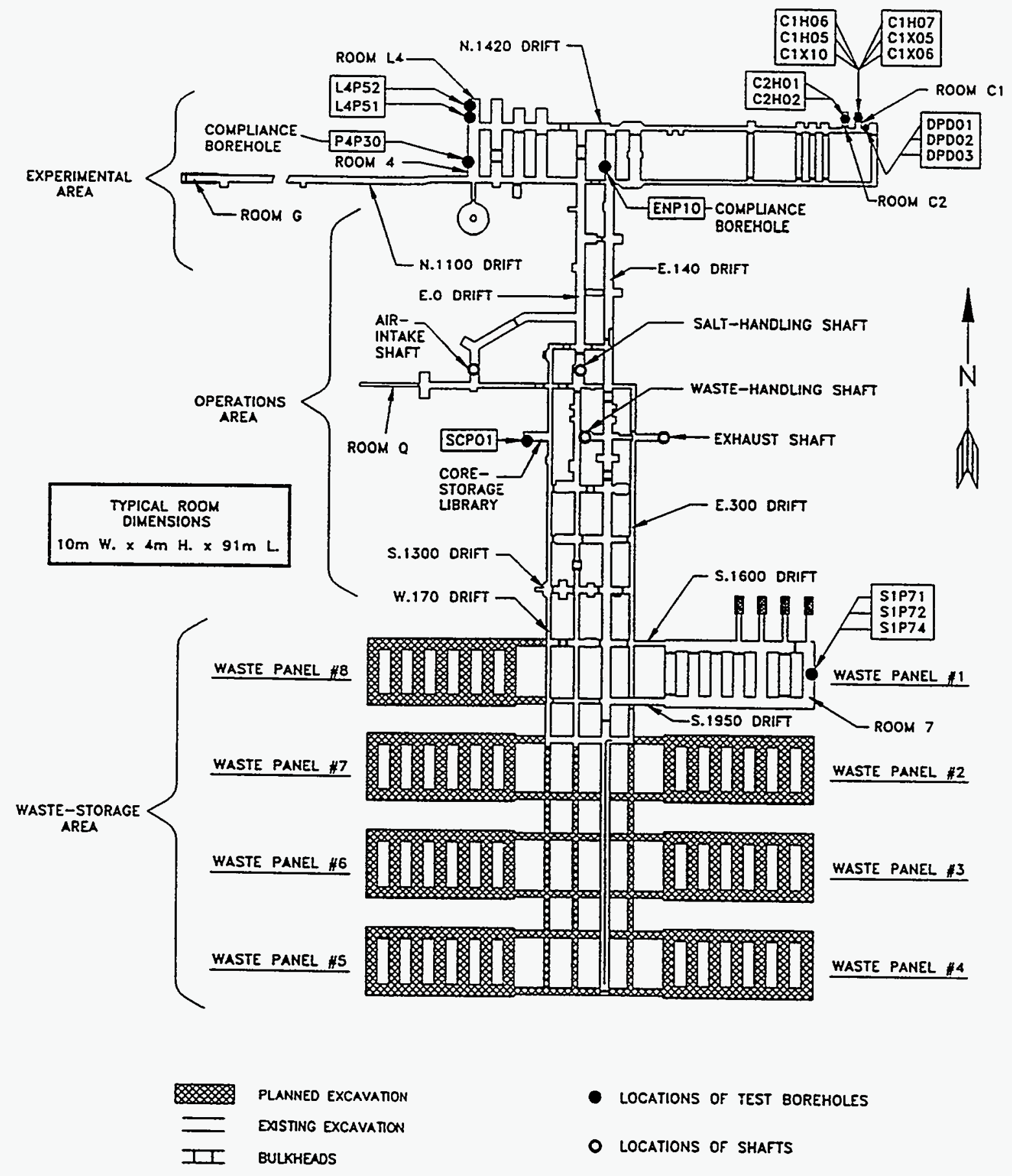

INTERA-61 15-2-0

Figure 2-1. Map of the WIPP Site underground facility and test and observation borehole locations. 


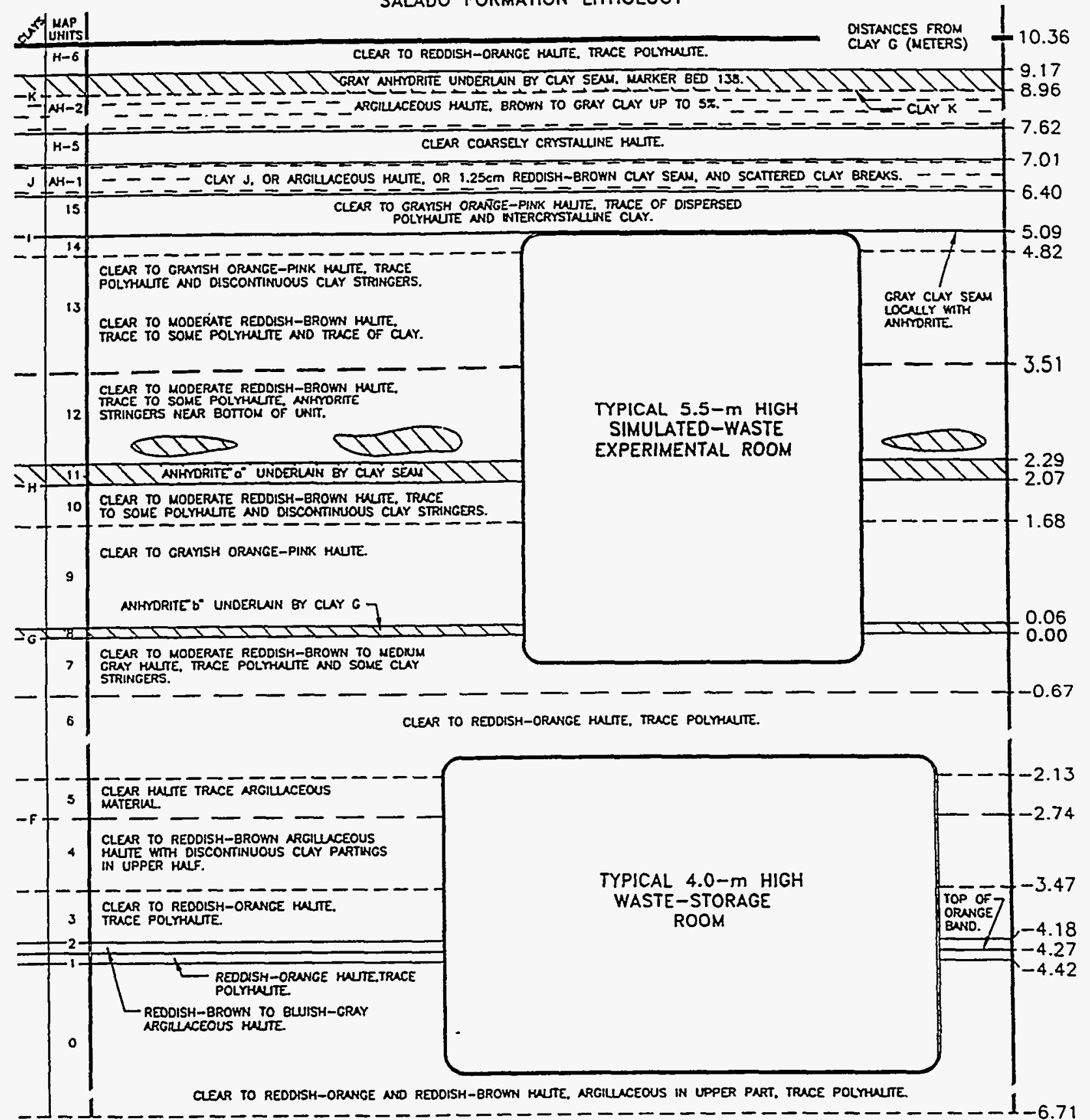

CONTINUED

WTERA-61 15-3-0

Figure 2-2a. Detailed lithology of the Salado Formation and typical positions of WIPP underground rooms. 


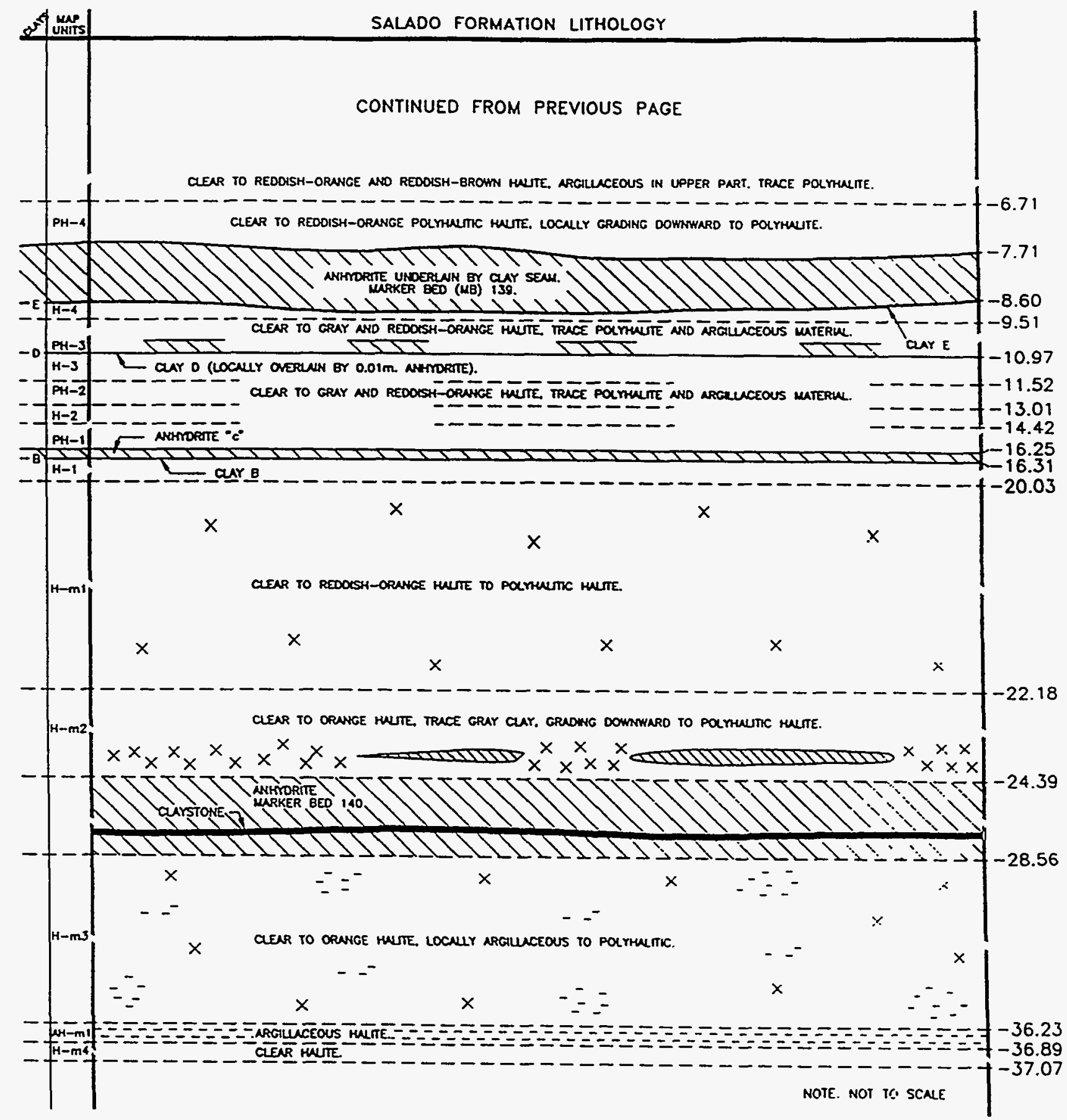

INIERA-6I15-4-0

Figure 2-2b. Detailed lithology of the Salado Formation and typical positions of WIPP underground rooms (continued). 


\section{PERMEABILITY TESTING PROGRAM}

The permeability-testing program for the WIPP underground facility was designed to provide data on the permeability and formation pore pressure of the Salado Formation. These data are required for estimating brine flow into and away from the repository, a factor that will affect the saturation state and pressurization of the sealed waste panels after closure of the repository. Analysis of the data provided by the Salado Hydrology Program reduces the level of uncertainty in permeability values used in performanceassessment calculations for the WIPP repository. The permeability-testing program was designed to provide hydraulic data both within the zone of influence of the WIPP excavations and in the undisturbed "far-field" parts of the Salado Formation. WIPP Procedure 483 describes the permeability testing procedure in detail.

\subsection{Test Objectives}

The design of effective plugs and seals for the repository's waste panels, proper evaluation of backfill, and performance-assessment calculations for the WIPP underground facility require knowledge of the distribution of permeability and formation pore pressure within the geologic media surrounding the facility. The far-field permeability may control the rate of brine flow into and away from the facility after closure. The amount of brine that enters the repository will affect the state of consolidation of the backfill and, therefore, the condition of the material in the sealed waste panels.

The specific objectives of the permeability-testing program are:

- to provide technically defensible data with which to interpret the permeability characteristics of the different lithologies of the Salado Formation that could contribute to fluid flow to and/or from the WIPP underground facility;

- to delineate, if possible, the extent of hydrogeologic disturbance in the Salado Formation around the WIPP underground facility; and

- to provide estimates of the formation pore pressure of the Salado Formation near the WIPP underground facility, and where possible, establish a pore-pressure profile from the surface of the excavation to undisturbed far-field conditions in the formation.

\subsection{Test Design}

The underground-permeability tests discussed in this data report were conducted in three boreholes drilled at two locations in the WIPP underground facility shown in Figure 2-1 and described in detail in Section 3.5. The boreholes were drilled vertically downward and angled upward. In the experimental area, boreholes L4P51 and L4P52 were drilled in Room L4. In the waste-storage area, borehole S1P74 was drilled in Waste Panel 1, Room 
7. The test locations were chosen to provide an adequate representation of permeability in the Salado Formation and its interbeds at and near the repository horizon and to provide comparisons with previously tested areas. Borehole locations and orientations are discussed in Section 3.5.1.

After drilling, a multipacker test tool (see Section 3.3) was installed in each borehole. The packers were used to isolate a bottom-hole test zone and a guard zone between the two packers in the case of a double-packer test tool, and to isolate a bottom-hole test zone, a middle zone between the bottom two packers, and a guard zone between the top two packers in the case of a triple-packer test tool. In a triple-packer configuration, the bottomhole zone is referred to as test-zone 1, the "middle zone" is referred to as test-zone 2, and the isolated zone closest to the repository is the guard zone. The zones were filled with $1.22 \mathrm{~kg} / \mathrm{L}$ sodium-chloride brine. The middle and/or guard zones were used to detect pressure leakage from the bottom-hole test zone transmitted either through the test-tool or through the formation and to reduce the pressure gradient across any one of the packers. The middle and/or guard zones were also used as a testing zone in some instances. After packer inflation, the zones were shut in and the fluid-pressure buildup was monitored with pressure transducers and recorded on a computer-controlled dataacquisition system (DAS). As fluid pressures stabilized, over periods ranging from weeks to months, permeability tests were performed on the isolated, bottom-hole test zones, as well as on the middle and/or guard zones of selected boreholes.

Pulse-withdrawal testing and constant-pressure-injection/withdrawal testing procedures are described in Section 3.4. The permeability tests were conducted in a manner similar to that used to measure permeability and pore pressure in the WIPP waste-handling shaft (Stensrud et al., 1988; Saulnier and Avis, 1988) and to that used to measure permeability and pore pressure in the Salado Formation (Saulnier et al., 1991; Stensrud et al., 1992). For the Salado Hydrology Program, permeability tests were conducted in halite, argillaceous halite, and in anhydrite interbeds, similar to those described in Saulnier et al. (1991) and in Stensrud et al. (1992), at depths of approximately 7 to $30 \mathrm{~m}$ from the ribs, floor, or back of the WIPP underground facility.

Pulse-withdrawal tests were performed in order to provide data with which to estimate the compressibility of the zone being tested as well as formation hydraulic conductivity $(K)$, formation pore pressure, and specific storage. Constant-pressure-injection/withdrawal tests were performed because this type of test allows a much larger radius of influence within the formation to be investigated than with pulse-withdrawal tests. Also, for testing sequences in which test-zone compressibility was not calculated, a constant-pressureinjection/withdrawal test is less sensitive to uncertainty in compressibility estimates.

Figure 3-1 illustrates the stratigraphic position of each of the permeability-testing boreholes and shows which strata were included in the test intervals. 


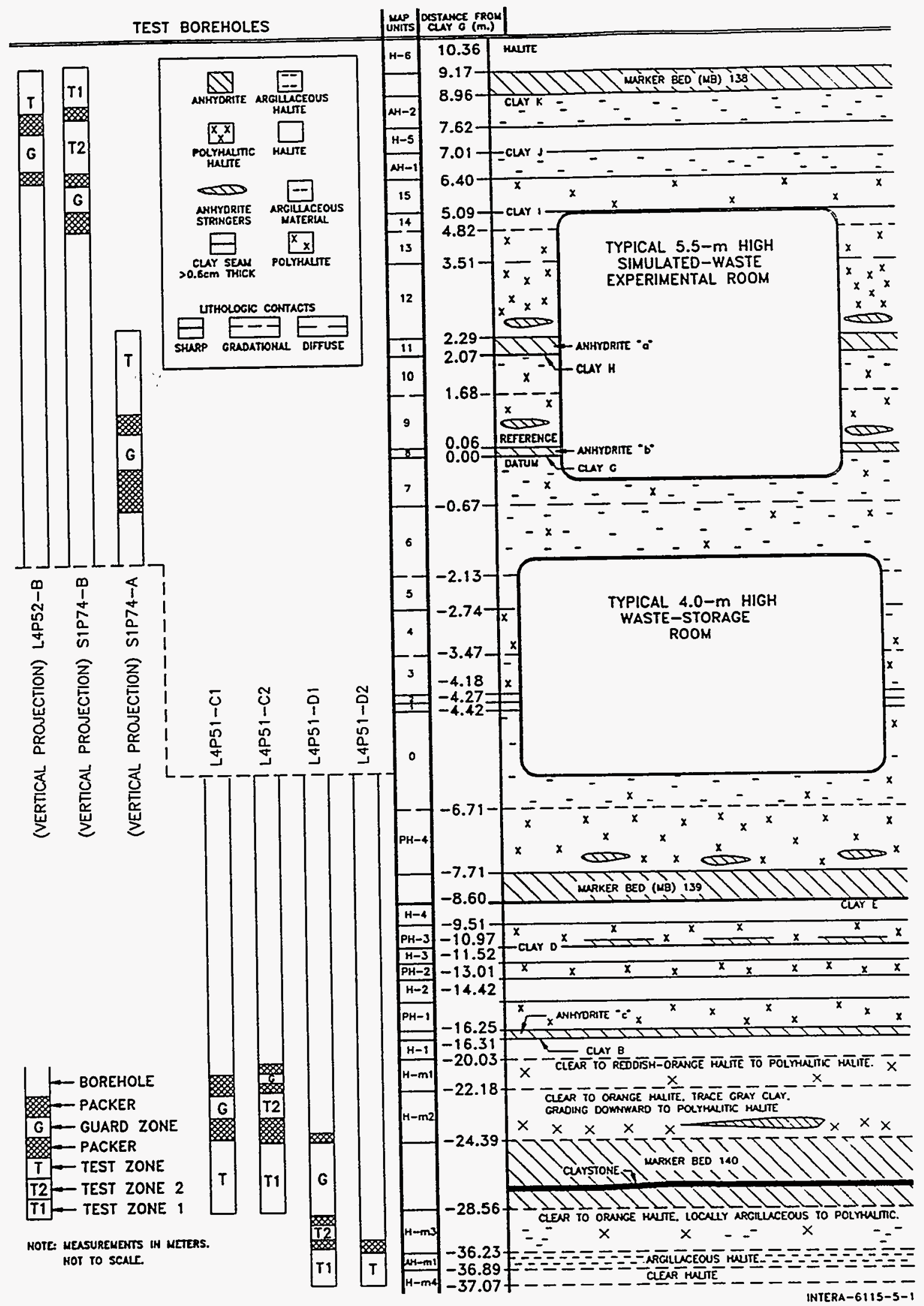

Figure 3-1. Stratigraphic positions of permeability-testing boreholes with test zones and guard zones indicated. 


\subsection{Test Equipment for Permeability Testing}

The following section (3.3.1) briefly describe the equipment used in the permeability-testing program in the WIPP underground facility. The equipment includes multipacker test-tools, data-acquisition systems, pressure transducers, thermocouples, linear variable-differential transformers, a differential-pressure-transmitter panel, and a constant-rate injection system. Equipment specific to individual testing sequences is provided in Section 3.6. More detailed descriptions of the testing equipment and the procedures are presented in Stensrud et al. (1992). Equipment calibration is discussed in Section 3.3.2.

\subsubsection{Description of Equipment}

\subsubsection{MULTIPACKER TEST TOOLS}

The multipacker test tools, shown in Figures 3-2 and 3-3, were designed and built specifically for the permeability-testing program by Baski Inc. in conjunction with SNL and INTERA Inc. The test tool in Figure 3-2 has two sliding-end, 3.75-inch $(9.53-\mathrm{cm})$ O.D. packers mounted on a 1.9-inch $(4.83-\mathrm{cm})$ mandrel with the fixed ends oriented toward the bottom-hole end of the test tool. The test tool in Figure 3-3 has three sliding-end, 3.75-inch $(9.53-\mathrm{cm})$ packers mounted on a 1.9-inch $(4.83-\mathrm{cm})$ mandrel with the fixed ends oriented toward the bottom-hole end of the test tool. Figure 3-4 shows the nominal dimensions of the test tools' parts. Packers were inflated using a Teledyne Sprague Engineering Model S-216-J series air-driven hydraulic pump. When inflated, the packers provide isolation of a bottom-hole test zone, and one or two other zones between packers depending on the test-tool configuration. Pipe extensions of different lengths can be added to the test tool to accommodate boreholes and test zones of varying lengths. Packers had either a $0.92-\mathrm{m}$ or $0.60-\mathrm{m}$ long, inflatable, synthetic rubber element which had seal lengths of $0.84 \mathrm{~m}$ and $0.51 \mathrm{~m}$, respectively, in a 4-inch- $(10.2-\mathrm{cm})$ diameter borehole.

In some cases, a volume displacement device was used during testing in order to minimize the effective borehole volume. The volume displacement device was constructed of 3.75inch $(9.53-\mathrm{cm})$ O.D. stainless steel of various lengths. Two or more of the individual volume displacement devices could be connected if necessary to fill a long portion of a borehole.

During permeability and compliance testing, the test tool was anchored to the borehole collar or compliance chamber using a slip-type restraining device. The slip-type restraining device (Figure 3-5) consisted of a set of radially oriented, tapered slips that tightened onto the test-tool's extension tubing as the test tool attempted to move out of the borehole in response to fluid-pressure buildup.

The multipacker test tools were equipped with pressure transducers and thermocouples, in most cases, to monitor the pressures and temperatures in the zones and the inflation pressure in each of the packers (Figure 3-6). The test tools had vent and/or injection ports for all of the zones. The vent and/or injection ports allow for the dissipation of squeeze 


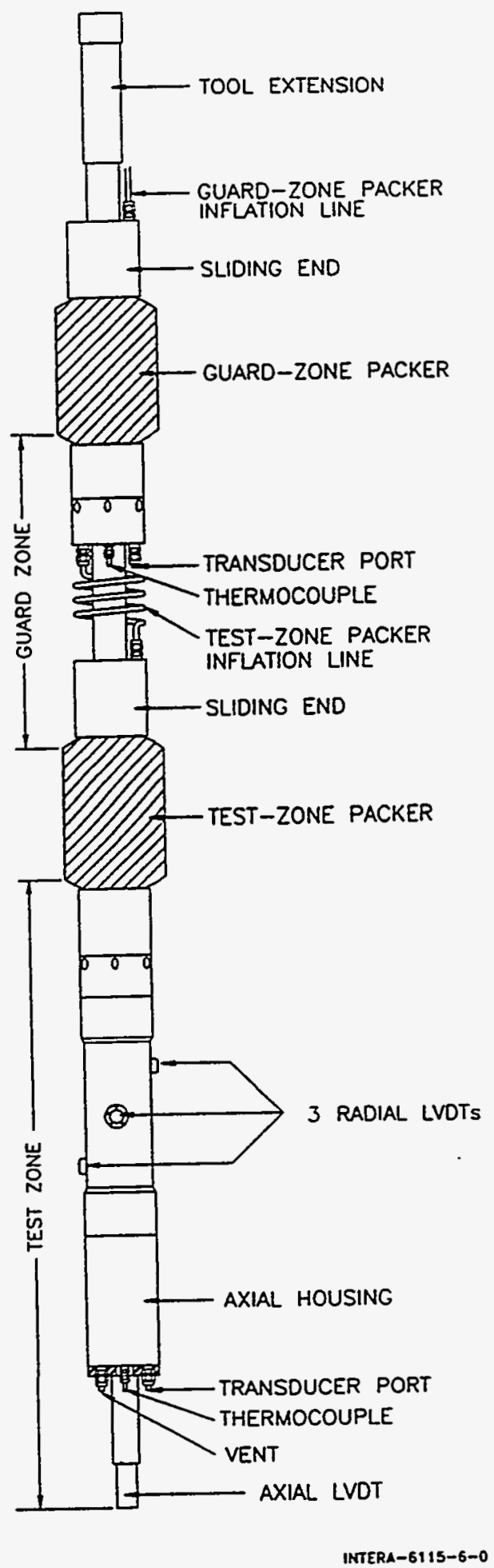

Figure 3-2. Generic double-packer test-tool configuration used for permeability testing. 
NOTE: SOME INSTALLATIONS USED INTERNAL INFLATION LINES. PACKER LENGTHS VARIED.

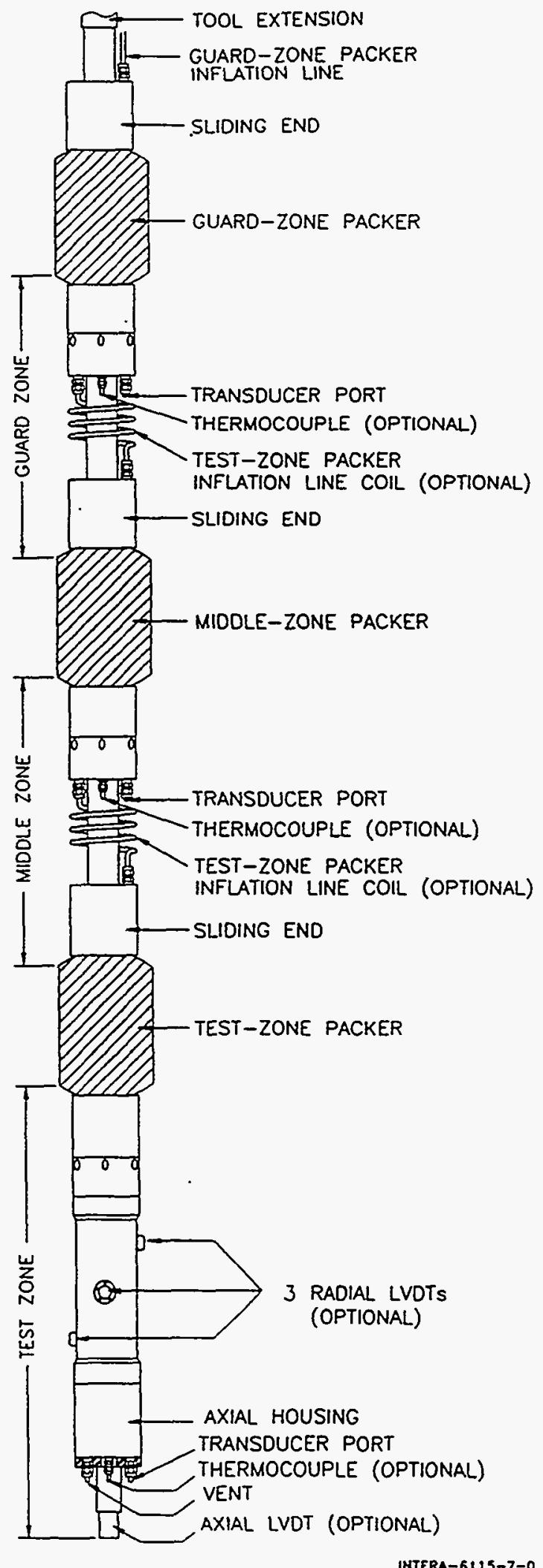

Figure 3-3. Generic triple-packer test-tool configuration used for permeability testing. 


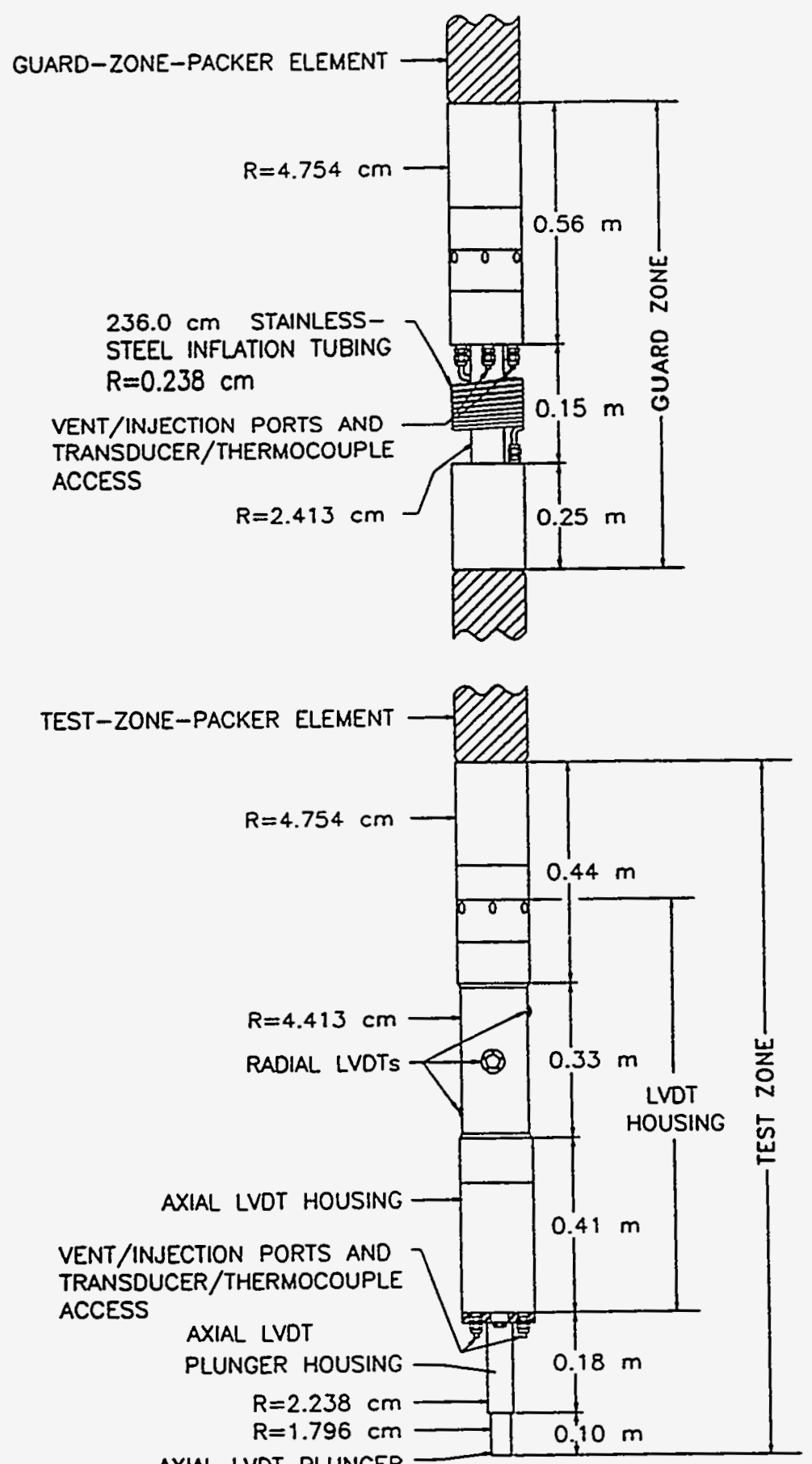

NOTE: TEST-ZONE AND GUARD-ZONE PACKER ELEMENTS $=0.92 \mathrm{~m}$ LONG.

INTERA-6115-8-0

Figure 3-4. Detail of multipacker test tool. 


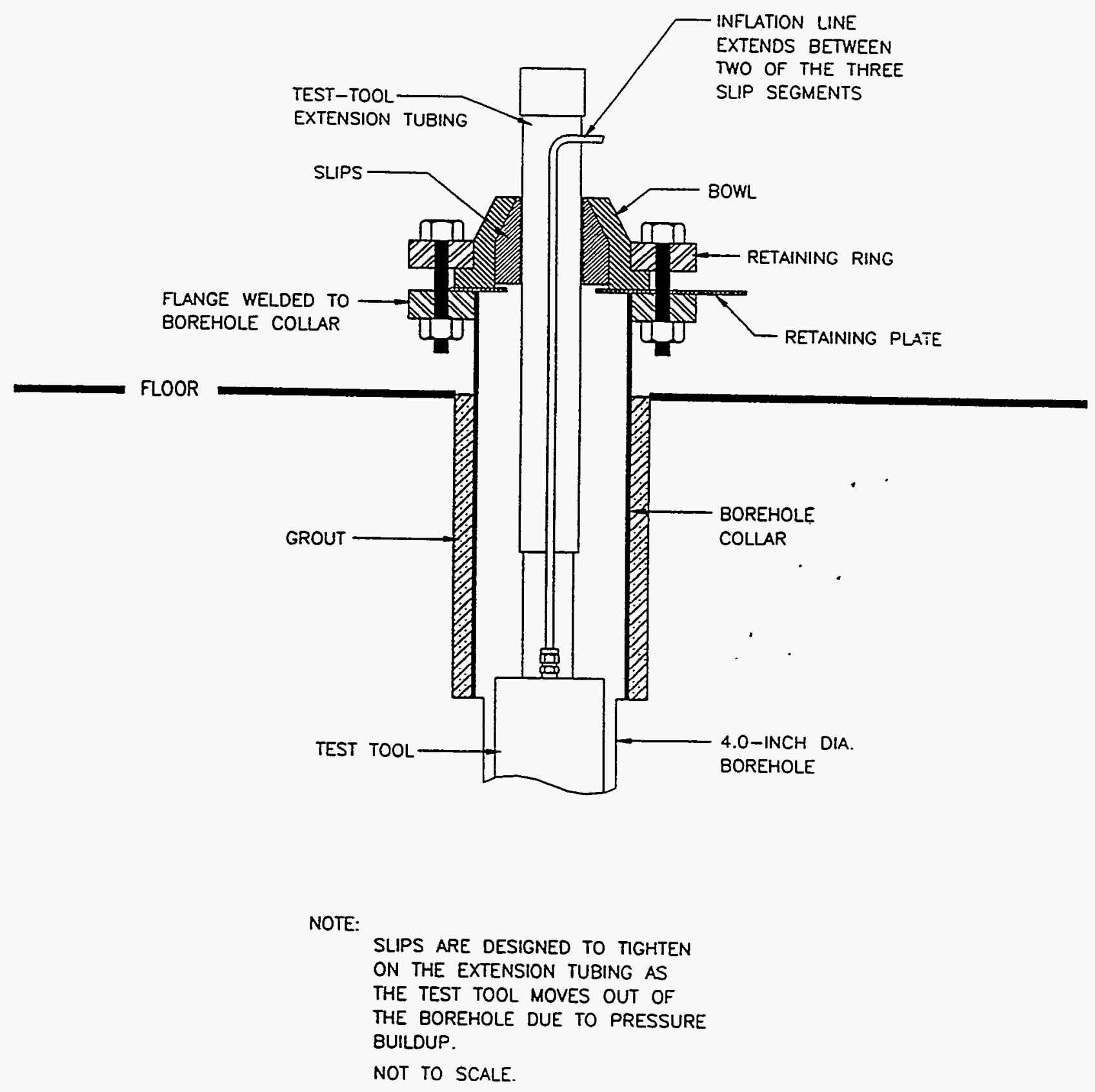

WTERA-6115-9-0

Figure 3-5. Slip-type test-tool restraint device. 


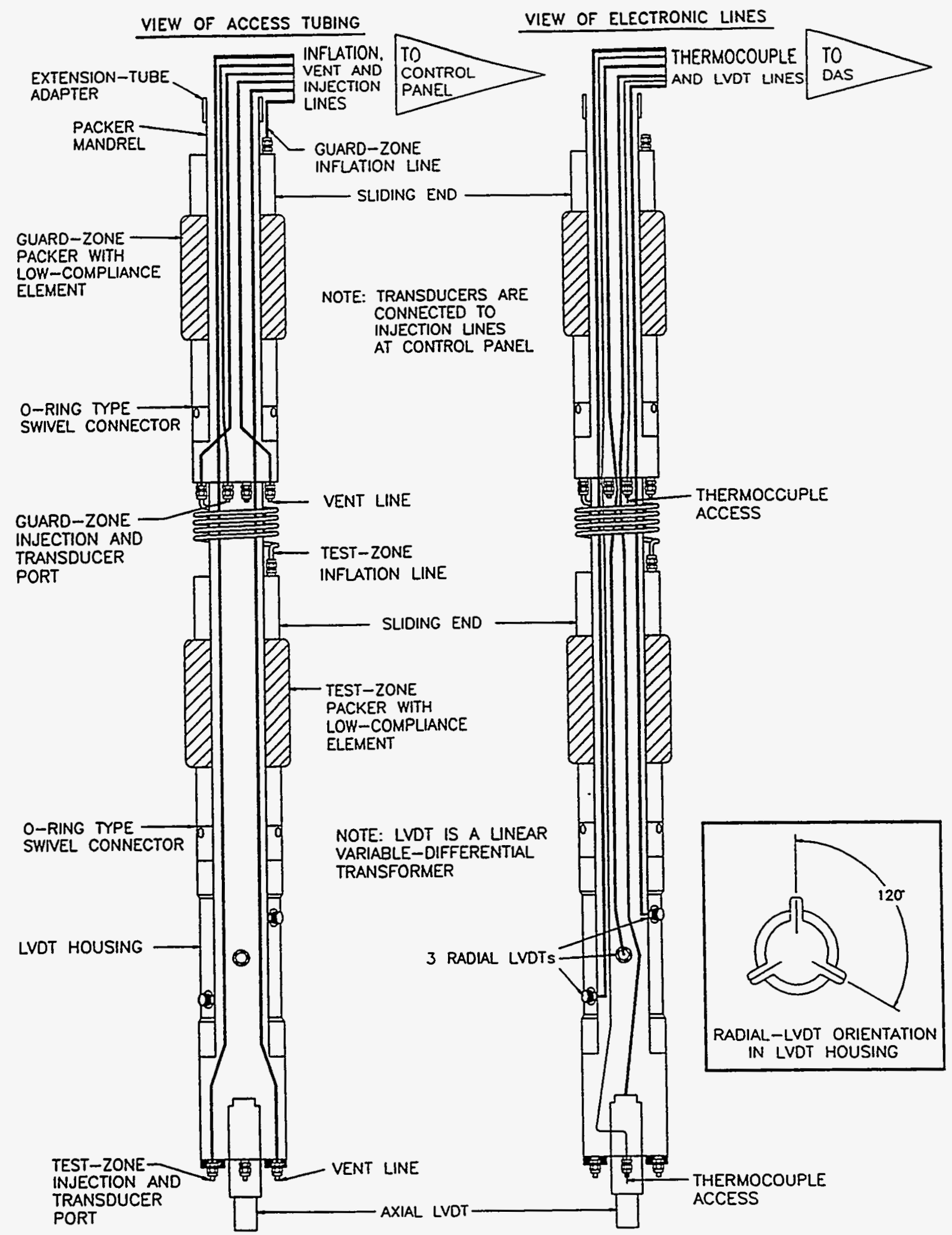

INTERA-51 IS-10-0

Figure 3-6. Generic split-view schematic illustration of a multipacker test tool used for permeability testing. 
pressure during packer inflation and for venting of entrapped air or formation-derived gas from the zones. When the test tools were assembled, the vent tubes for angled and vertically upward installations were left as long as possible in order to minimize air entrapment. The injection ports could also be used to apply pressure to the isolated zones. Under-pressures and over-pressures could be applied as pressure pulses or as constant-pressure-brine injection/withdrawal tests.

In most instances, the test-zone section of the test tool was equipped with LVDTs to measure radial-borehole deformation and/or test-tool movement during the permeability test. Three LVDTs were radially oriented $120^{\circ}$ apart, immediately below the test-zone packer (Figure 3-6) One axially oriented LVDT was mounted at the bottom end of the test tool and was used to measure axial borehole elongation and test-tool movement during permeability testing.

An control panel with pressure gages was sometimes used to monitor the progress of packer inflation and fluid injection/withdrawal directly (Figures 3-7a and 3-7b). Packer inflation and fluid injection were controlled by positive-displacement intensifier pumps which were activated using compressed air or pressurized nitrogen. The various intensifier pump configurations were designed to accommodate different needs during testing.

\subsubsection{DATA-ACQUISITION SYSTEM (DAS)}

A computer-controlled data-acquisition system (DAS) was used to monitor the progress of each test and recorded pressure, in some cases temperature, and in some cases radial borehole closure and axial tool movement (Figure 3-8). Each DAS consisted of an IBM compatible desktop computer for system control and data storage, a Hewlett Packard (HP) 3497A Data-Acquisition/Control Unit (DCU) containing power supplies to excite the instruments associated with a given test (transducers, thermocouples, differential-pressure transmitters (DPTs, and LVDTs), a signal scanner to switch and read channels, a 5-1/2 digit voltmeter to measure the output from the instruments associated with a given test (transducers, thermocouples, DPTs, and LVDTs), and an Elgar Model 6000B uninteruptable power supply. The HP-3497A was used with PERM data acquisition software (PERM4C and PERM4F). The data-acquisition software allowed sampling of the instrument output signals at user-specified time intervals ranging from approximately 10 seconds to several days. As data were acquired, they were stored both on the computer's hard disk and on a 3.5-inch diskette. Real-time listing of the data on an auxiliary printer and monitor and/or printer plots of the accumulated data were also possible.

\subsubsection{PRESSURE TRANSDUCERS}

During permeability testing, pressures in the test and guard zones and in the packers were monitored with various Druck strain-gage pressure transducers (models PDCR 10/D, 


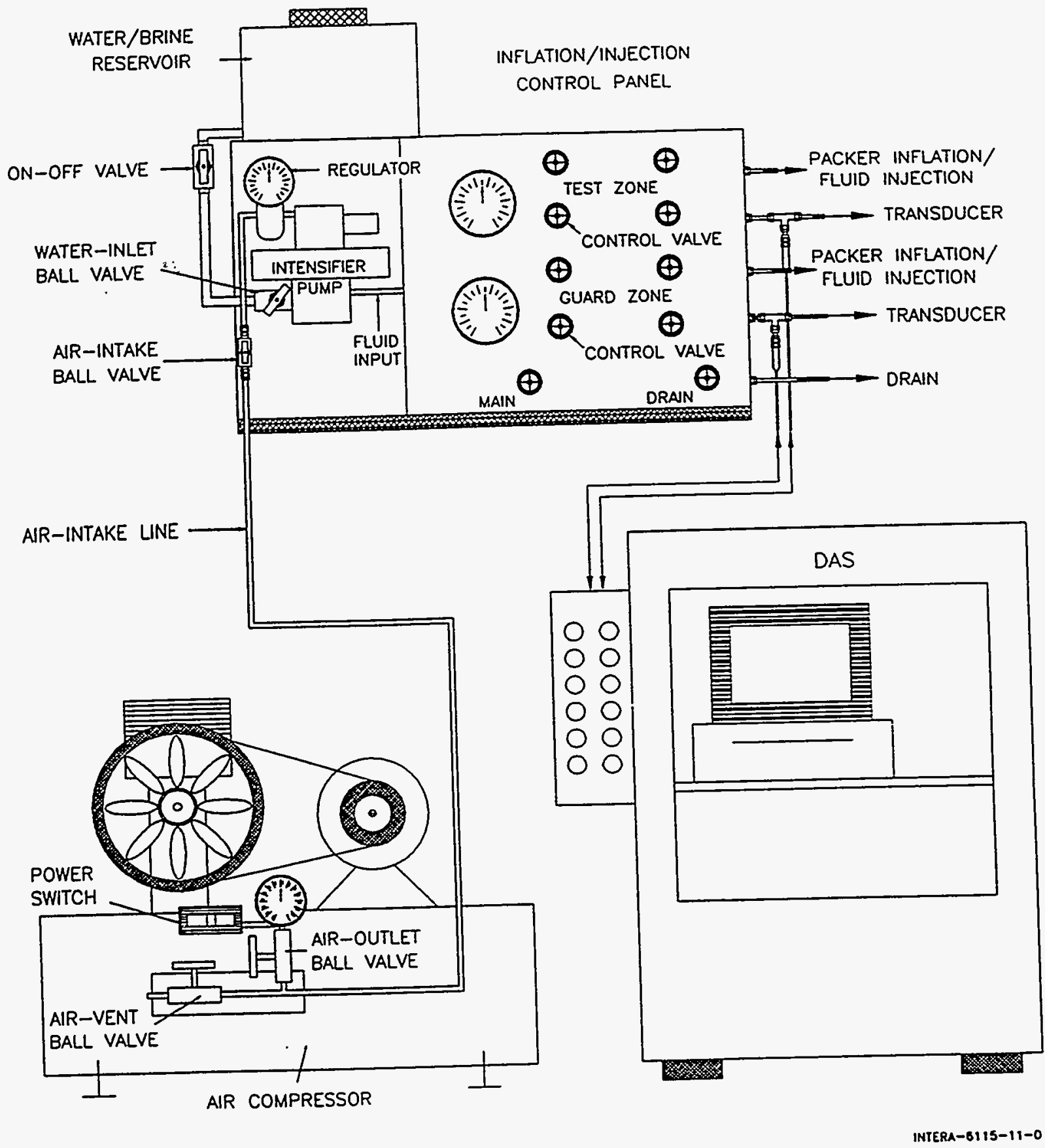

Figure 3-7a. Intensifier pump system for packer inflation and fluid injection (configuration \#1). 


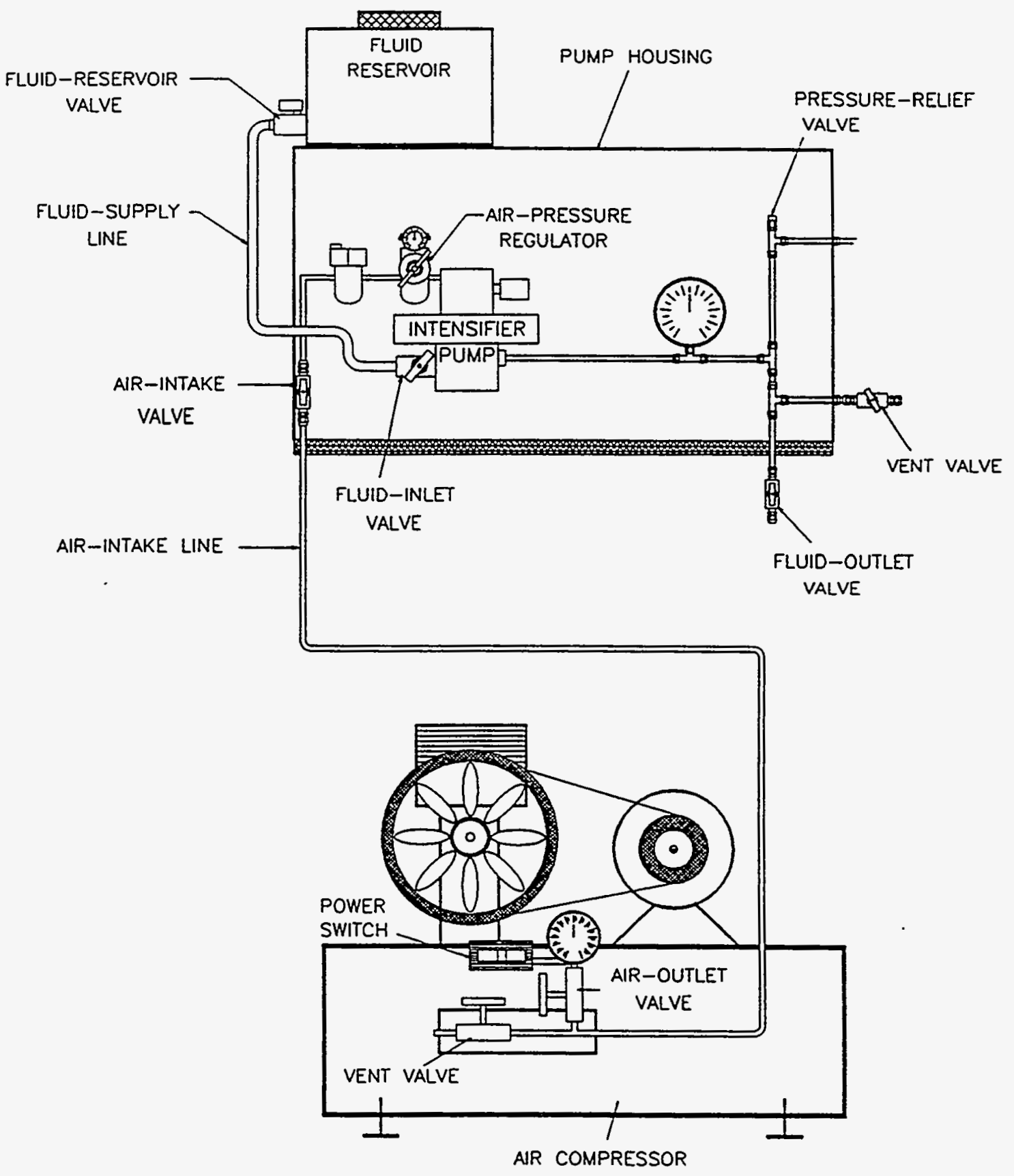

INTERA-6115-12-0

Figure 3-7b. Intensifier pump system for packer inflation and fluid injection (configuration \#2). 


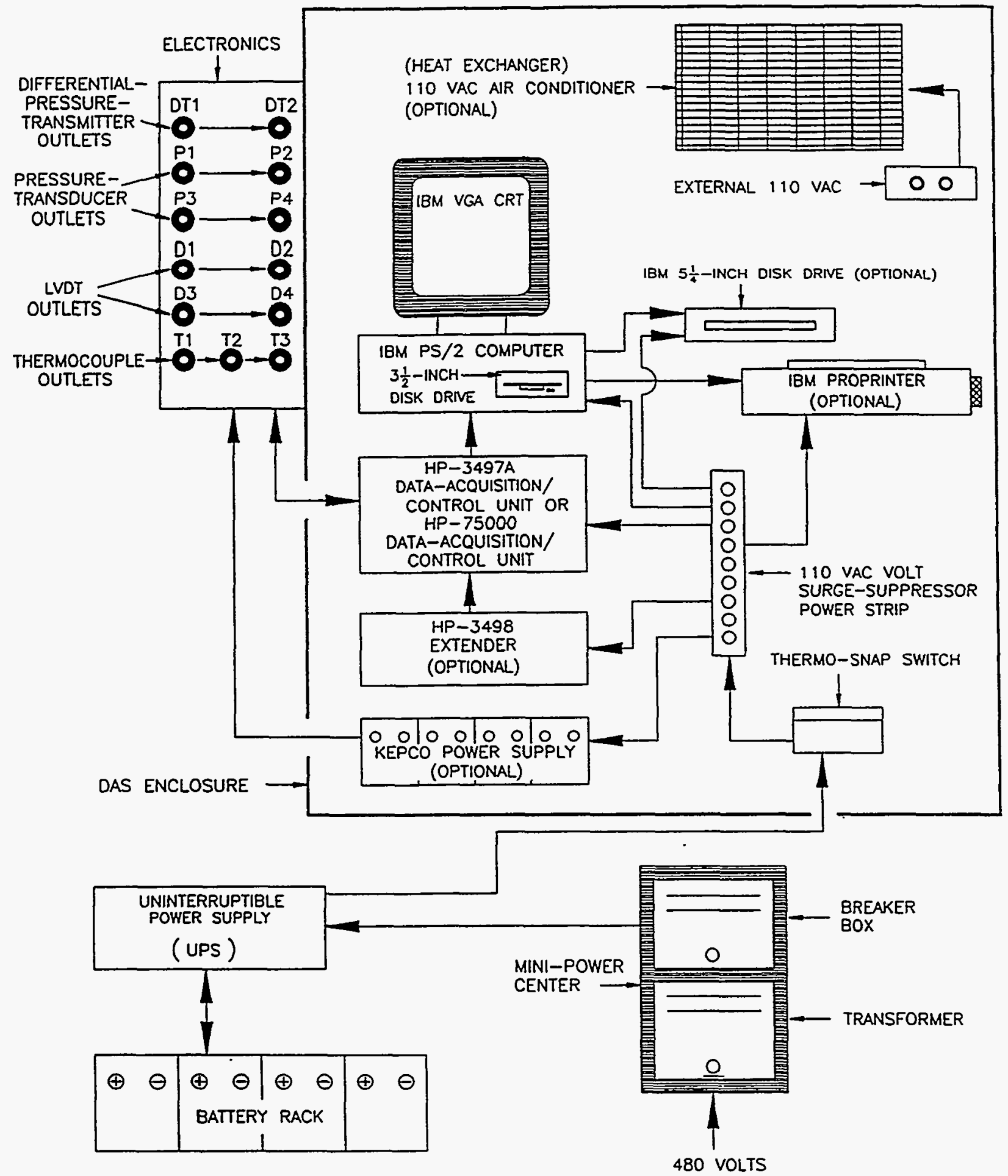

IKTERA-6I15-13-0

Figure 3-8. Schematic illustration of the data acquisition system. 
PDCR 830, PDCR 910, and D930-18) rated to monitor pressures from 0 to $3000 \mathrm{psi}$ (0 to 20.7 MPa). The transducers were mounted on instrument panels outside the boreholes and were connected to the isolated zones and the packers through $3 / 16$-inch $(0.48-\mathrm{cm})$ O.D. and/or 1/4-inch (0.64-cm) O.D. stainless-steel tubing which passed into and through the packer mandrels (Figure 3-6) to the respective termination points. The manufacturer's stated accuracy of the least accurate of these Druck transducers is $\pm 0.1 \%$ of full scale, or approximately \pm 3 psi $(0.021 \mathrm{MPa})$.

\subsubsection{THERMOCOUPLES}

Type E Chromel-Constantan thermocouples are used to monitor temperatures within the test and guard zones during permeability testing, in monitor boreholes during coupled permeability and hydrofracture testing, and in some test zones during GTPT's. The thermocouples are $1 / 8$ inch $(0.32 \mathrm{~cm})$ in diameter and are sheathed in Inconel 600 . The thermocouples are reported to be accurate to within $\pm 0.06^{\circ} \mathrm{C}$ by the manufacturer, $A R I$ Industries.

\subsubsection{LINEAR VARIABLE-DIFFERENTIAL TRANSFORMERS (LVDTS)}

Open boreholes, rooms, and drifts in the WIPP underground facility exhibit closure, deformation, and differential movement between halite and anhydrite beds (Bechtel, 1986). Measurable borehole closure (on the order of a few tenths-of-a-millimeter change in borehole diameter) in a shut-in, fluid-filled test interval could raise the pressure in the hole given the low compressibility of the fluid. Three Trans-Tek Model 241 LVDTs were radially mounted, with $120^{\circ}$ separation, on the test-interval part of the multipacker test tool to measure radial borehole deformation (Figure 3-6). These LVDTs can each measure a range of motion of $0.5 \mathrm{~cm}$. Axial movement of the multipacker test tool can occur due to changes in packer-inflation pressure, pressure buildup or withdrawal in the isolated intervals, and hole elongation resulting from creep closure of the excavations. The rate of rock creep decreases with increasing distance from an excavation (Westinghouse, 1990), causing boreholes drilled from an excavation to elongate. Axial movement of the test tool can change the test-zone volume, which, in low-permeability media, can affect the observed pressure response in an isolated borehole interval. An axially mounted TransTek Model 245 LVDT on the bottom of the test tool measures tool movement relative to the bottom of the borehole along the borehole axis (Figure 3-6). This LVDT has a range of motion of $10 \mathrm{~cm}$. The LVDT responses are reported by Trans-Tek to be linear within \pm $0.5 \%$ over their working ranges. Two Trans-Tek Model 242 LVDTs were used during permeability-testing sequence S1P74-B to measure fracture dilation as a function of pressure, however, this testing sequence was terminated prior to obtaining information about fracture dilation. These LVDTs were similar in design and specification to the TransTek Model 245 LVDTs. Jensen (1990) discusses in detail the design, calibration, and use of the LVDTs. LVDTs were used in order to account for any change in interval pressure caused by too movement and/or borehole closure. 


\subsubsection{DIFFERENTIAL PRESSURE-TRANSMITTER PANEL}

Fluid volumes produced from or injected into test zones during test-zone-compressibility tests and constant-pressúre-flow tests were measured using a diffêrential-pressuretransmitter panel (Figures 3-9a, 3-9b, and 3-9c). The panel consists of a differentialpressure transmitter (DPT) and injection/withdrawal columns which act as fluid reservoirs. Rosemount Alphaline Model 1151DP4E44M3B2 DPTs were used in the WIPP permeability-testing program.

The DPT panel can be used with several different cylindrical columns: 8 -inch $(20.32-\mathrm{cm})$, 4-inch $(10.16-\mathrm{cm}), 1$-inch $(2.54-\mathrm{cm}), 1 / 2$-inch $(1.27-\mathrm{cm})$, and 3/8-inch $(0.95-\mathrm{cm})$ O.D. stainless-steel columns, and a 1/4-inch $(0.64-\mathrm{cm})$ Lexan-column manometer (Figures 3-9a, $3-9 b$, and 3-9c) depending upon the volume of fluid that is withdrawn from or injected into the test zone. Ideally, the smaller the volume of fluid required, the smaller the diameter of the column to be used so that accuracy is not sacrificed when only small fluid volumes are required. Fluid level in a column increases as the fluid leaves the test zone and enters the column or decreases as the fluid leaves the column and enters the test zone. The DPT measures the difference in the pressure exerted on two sides of a sensing diaphragm. On one side of the diaphragm is the ambient test pressure. On the other side of the diaphragm is the pressure exerted by the fluid in the column, plus the ambient pressure. Therefore, the difference, or differential pressure, is equal to the pressure exerted by the fluid in the column. As the fluid level in the column changes (a change in fluid-column height corresponds to a linear change in the volume), the voltage output from the DPT changes proportionally. The DPT output voltage signal is recorded by the DAS.

During test-zone compressibility tests and constant-pressure-flow tests, it is necessary that the pressure inside the injection/withdrawal column remain under near constant-pressure conditions. In all testing sequences, a Victor SR4J-580 pressure regulator was used to regulate the pressure on the gas-side of the DPT panel. In some cases (testing sequences L4P51-C2 and L4P51-D1) an electronic pressure controller was used in conjunction with a Victor SR4J-580 pressure regulator, a TESCOM 54-2000 series pressure-reducing regulator, a TESCOM ER-2000 pressure controller, and a Druck D930-18 pressure transducer to maintain constant pressure conditions. To maintain constant pressure, the injection/withdrawal column is connected to a TESCOM ER-2000 pressure controller which is, in turn, connected to a pressurized nitrogen reservoir. As the fluid moves into or out of the injection/withdrawal column during testing, the pressure controller maintains a constant back-pressure on the fluid column. Pressure relief valves were incorporated into each of the differential pressure-transmitter panels (see Figures 3-9a, 3-9b, and 3-9c) in order to eliminate the possibility of over-pressurizing equipment.

The various differential pressure-transmitter panel configurations were designed to accommodate different needs during testing. 


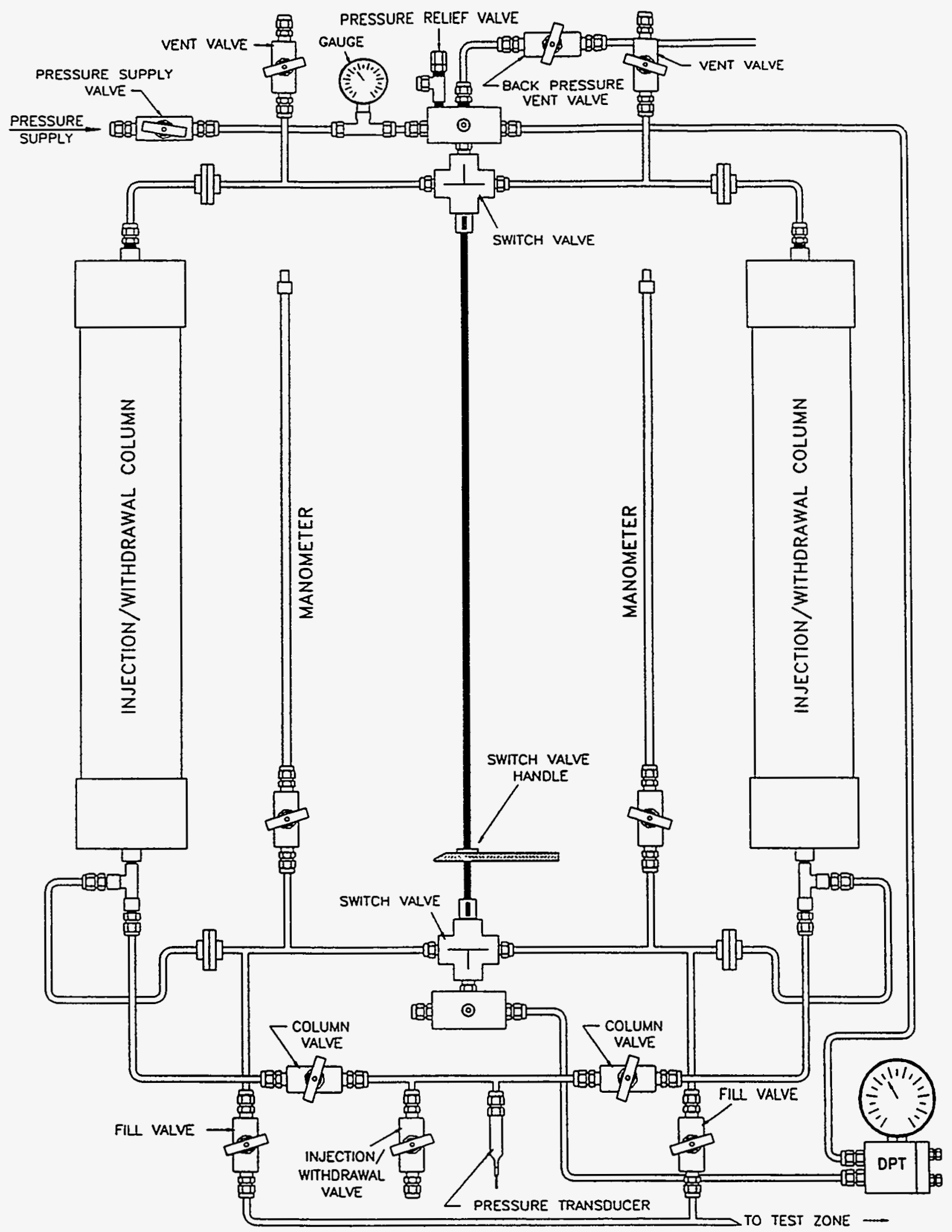

INTERA-6IIS-14-0

Figure 3-9a. Differential-pressure-transmitter panel (configuration \#1). 


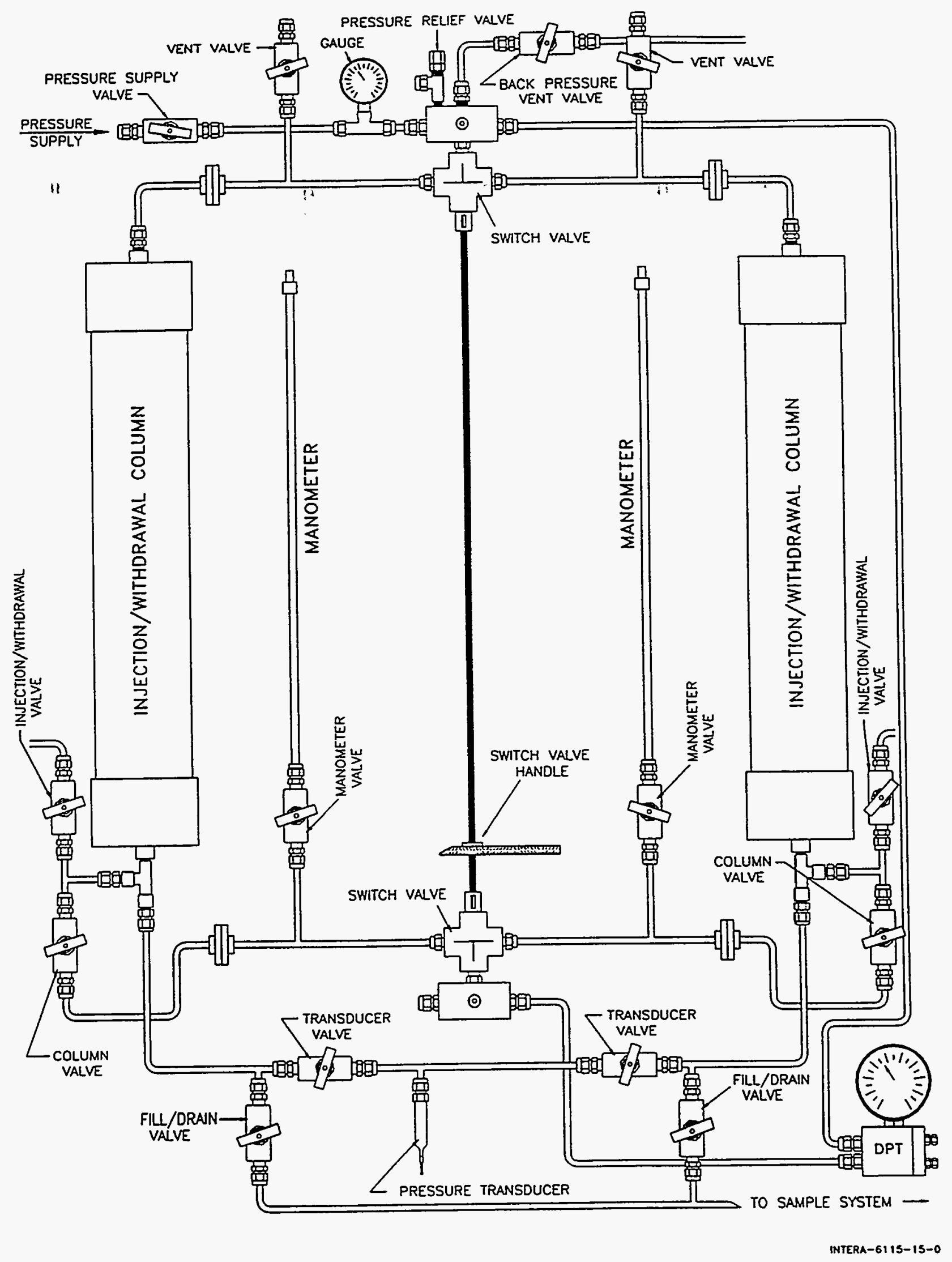

Figure 3-9b. Differential-pressure-transmitter panel (configuration \#2). 


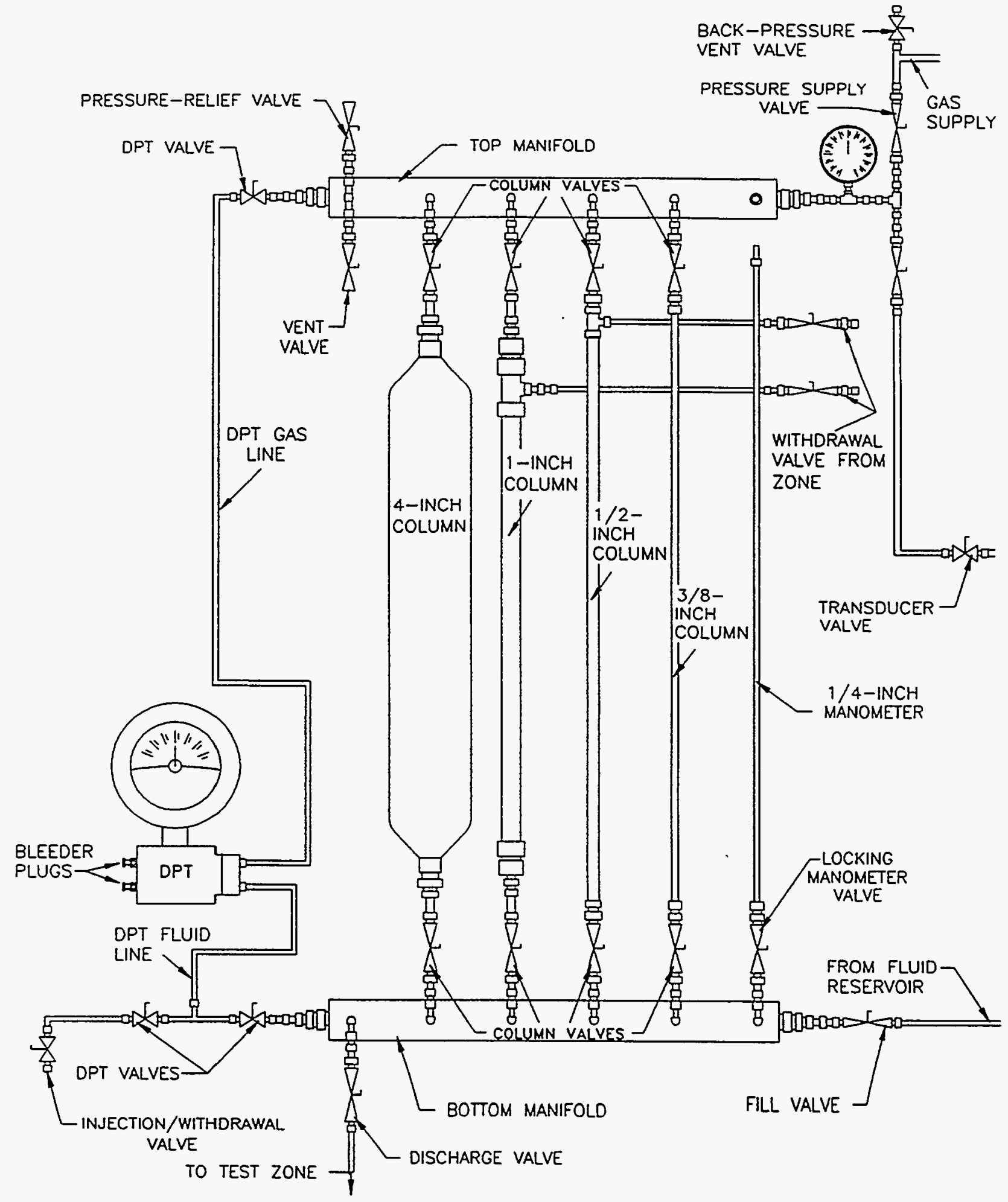

INTERA-6115-18-0

Figure 3-9c. Differential-pressure-transmitter panel (configuration \#3). 


\subsubsection{PRESSURIZED-BRINE-SAMPLING APPARATUS}

Brine/gas samples were collected during constant-pressure flow tests during permeabilitytesting sequence L4P51-C1 in order to help define the types, amounts, and origins of gas being produced by the tested strata. Figure 3-10 is a diagram of the pressurized-brinesampling apparatus used in the permeability-testing program. The sampling apparatus was incorporated into the DPT panel and consisted of a Whitey sample cylinder, two Nupro non-rotating stem valves, and two Nupro rising-plug valves. The sampling apparatus was incorporated into the DPT panel in such a way that fluid and/or gas fills the sample cylinder on its way to the designated fluid reservoir. When the sample is to be removed, the four Nupro valves were closed allowing the fluid and/or gas to bypass the sample cylinder. When a new sample cylinder is installed, the valves are opened and the sample cylinder is again filled. A vacuum pump is attached to the sampling apparatus prior to installation to evacuate all gas from the sample cylinder. After drawing a vacuum on the sample cylinder, the cylinder is flushed with argon gas and the two Nupro non-rotating stem valves are closed. Finally, the sampling apparatus is installed in the DPT panel.

\subsubsection{PRESSURE-MAINTENANCE SYSTEM}

Packer pressures steadily declined during some testing sequences, potentially jeopardizing the isolation of test and/or guard zones. In most testing sequences, a pressuremaintenance system (Figures 3-11 and 3-12) was attached to the packers associated with the test to hold the packer pressure nearly constant during testing. In some cases, a pressure-maintenance system was attached to zones in order to minimize pressure perturbations in the test zone. A Whitey 1-gallon cylinder was filled, with water when attached to packers and brine when attached to zones, and then pressurized with nitrogen to the desired pressure. The control valve between the cylinder and the nitrogen tank was left open, creating a large buffer volume. When the desired pressure in the cylinder was achieved, the control valve between the cylinder and the packer/zone was opened, allowing the pressures in the packer/zone and in the cylinder to equilibrate. Subsequent losses of fluid from the packer/zone and/or changes in packer/zone volume thereafter resulted in smaller changes in pressure than would have otherwise occurred. The operation and use of the pressure-maintenance system is described, in detail, in WIPP Procedure 470.

\subsubsection{Equipment Calibration}

This section discusses calibrations performed on equipment described in Section 3.3.1. Calibrations performed by the SNL. WIPP calibration laboratory or INTERA personnel were in accordance with SNL WIPP procedures. Calibrations performed by the SNL calibration laboratory in Albuquerque, NM were in accordance with that organization's policies and procedures. Calibration records associated with this equipment are provided in the SWCF under WPO \#42269. 


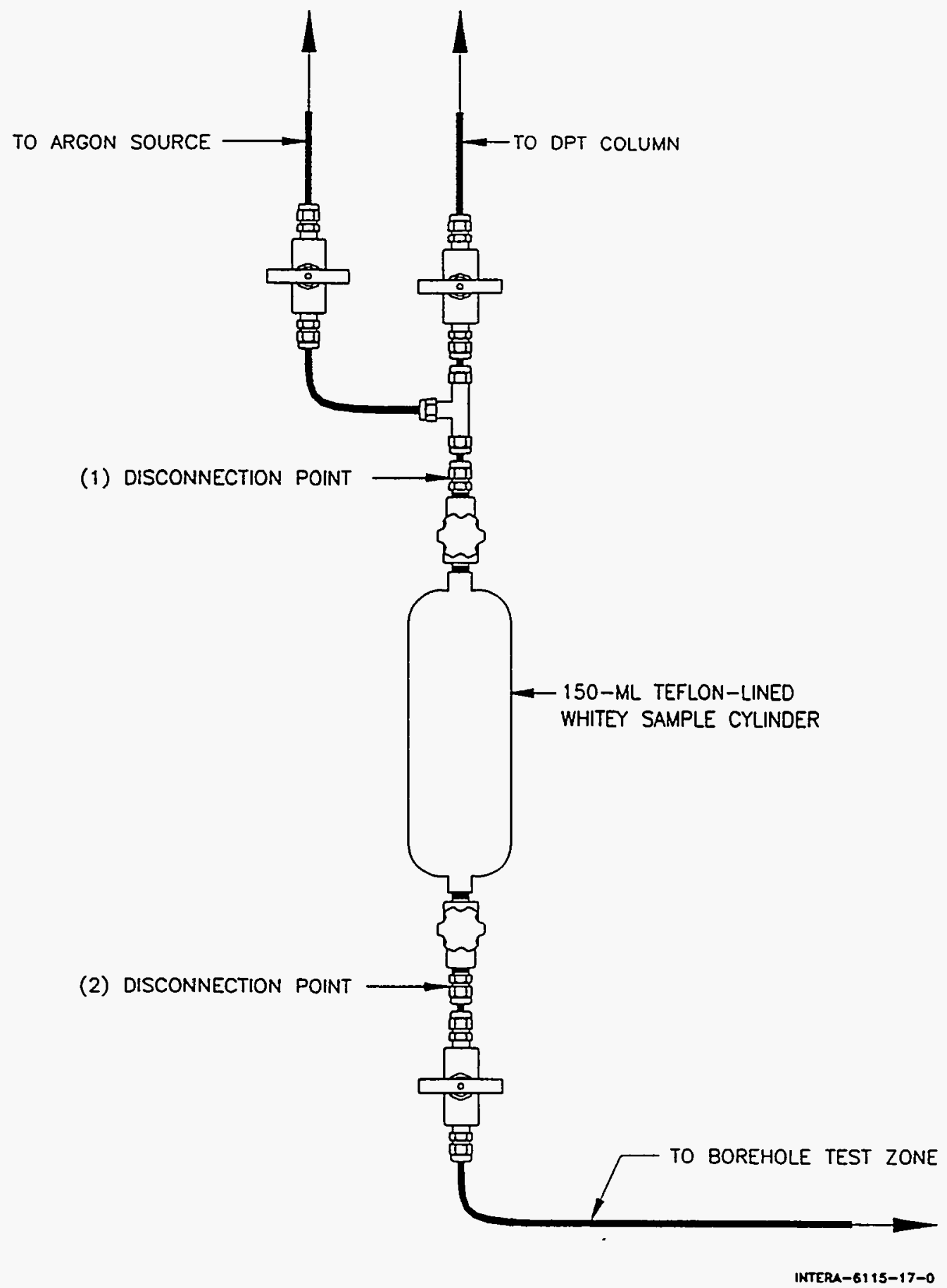

Figure 3-10. Pressurized-brine-sampling apparatus. 


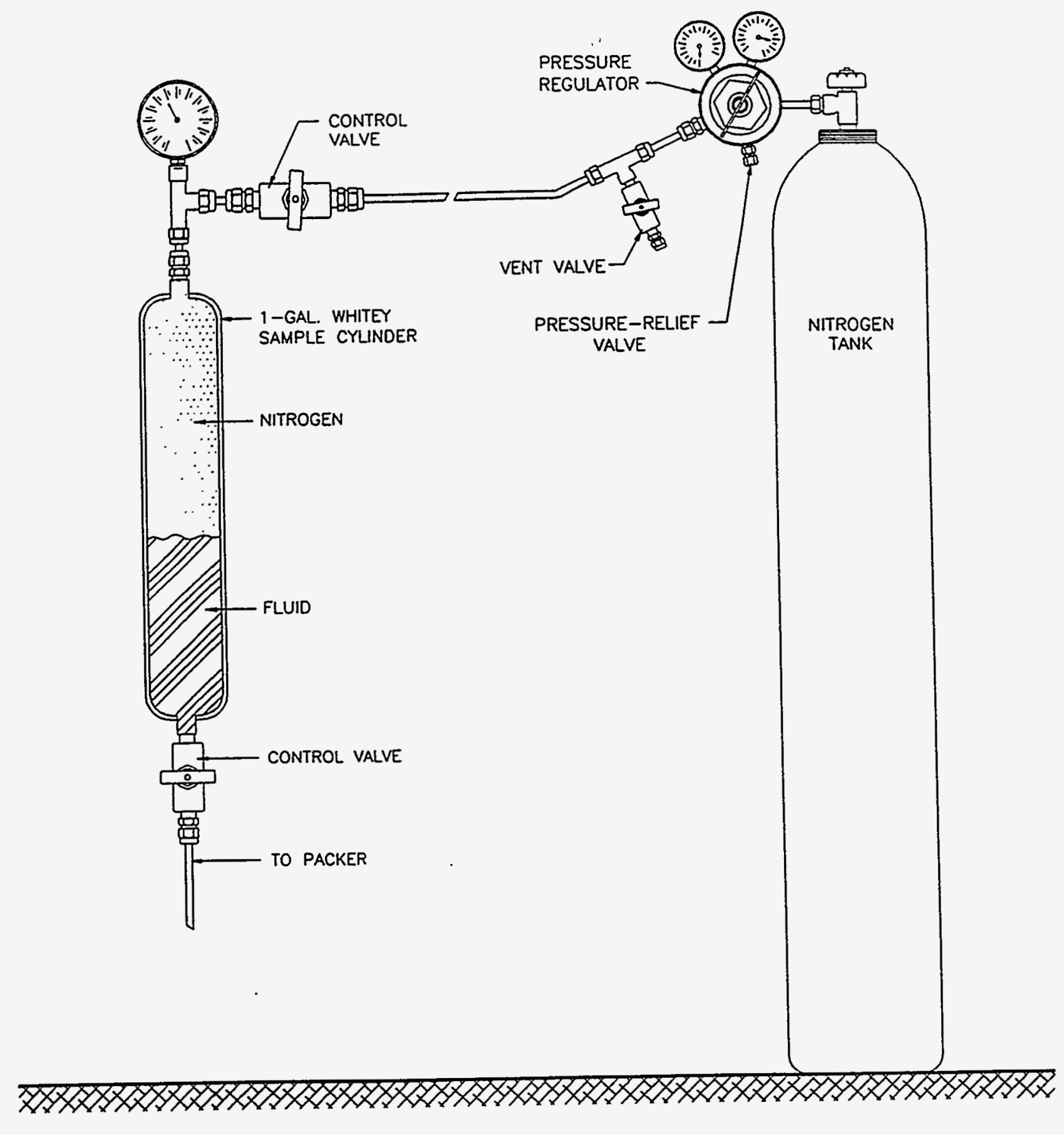

Figure 3-11. Typical stainless-steel vessel pressure-maintenance system. 


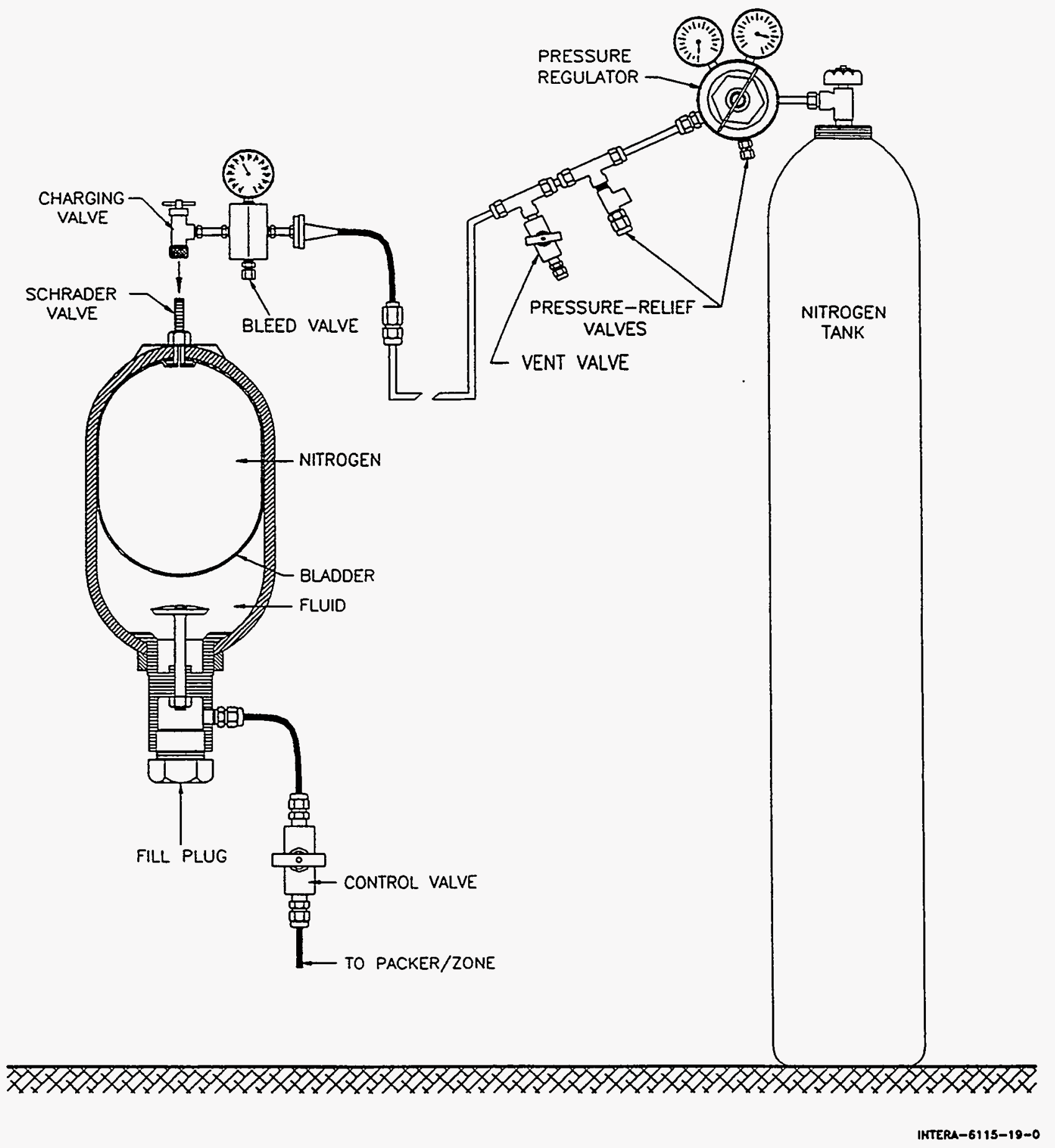

Figure 3-12. Typical gas-bladder pressure-maintenance system. 


\subsubsection{DATA-ACQUISITION SYSTEM}

The DAS equipment and associated software were tested prior to the start of the permeability-testing program to ensure proper operation. The procedure consisted of testing the response of the HP-3497A Data-Acquisition/Control Unit and the DAS software using known input signals to ensure proper signal conversion. The DAS software was designed and tested by SNL and INTERA Inc. The DAS software was baselined and controlled in accordance with the SNL approved INTERA WIPP Quality Assurance Manual. In addition, the HP-3497A DC voltmeter option was calibrated by SNL WIPP calibration laboratory using WIPP Procedure 182.

\subsubsection{PRESSURE TRANSDUCERS}

All pressure transducers associated with the permeability-testing program were calibrated before and after use for each permeability test. Two methods of calibration have been employed to calibrate pressure transducers for use in the permeability-testing program. In both methods, the pressure transducers were calibrated in a constant-temperature environment.

The first, as illustrated in Figure 3-13, was employed by INTERA personnel until November 11, 1993. In this method, the transducers were connected to a Heise pressuremeasurement gage (Model CMM) and to an intensifier pump with an air compressor used as the source of pressure. Pressure was applied to the transducers in stepwise increments, measured with the Heise gage, over the transducers' rated pressure range, typically 0-2000 psig. The transducers' responses to the applied pressures were then analyzed by linear-regression techniques to determine their sensitivity coefficients, which consisted of the slopes, expressed as pressure/mV, and the $y$-intercepts, expressed as pressure. The sensitivity coefficients were used to convert the transducers' millivolt output signals to pressure values. The Druck pressure transducers used in the permeabilitytesting program were pressure rated by the manufacturer in pounds per square inch, gage pressure (psig). The Heise gage used to calibrate the transducers by this method used a psig scale and was itself calibrated by the manufacturer and by the SNL calibration laboratory in Albuquerque, NM. Because the transducers' ratings and the calibration gage's scale are in psig, the test-tool pressure transducers are calibrated in psig. The calibrations that were performed following the method described above were done so using the transducer calibration code RAMBO1D, RAMBO2, or PERM4F. The DAS software converts the transducers' psig/mV sensitivity coefficients to MegaPascals (MPa) using the conversion factor $0.006895 \mathrm{MPa} / \mathrm{psig}$. More detailed descriptions of methods used to calibrate the equipment are presented in Stensrud et al. (1992).

The second method employed to calibrate pressure transducers used in the permeabilitytesting program was done so by the SNL WIPP calibration laboratory after November 11,1993 in accordance with WIPP Procedure 054. Here, a dead weight tester was used to apply pressures in stepwise increments, over the transducers' rated pressure range. This method also analyzed the transducers' responses to the applied pressures by linearregression in order to establish sensitivity coefficients for the transducers with the psig to $\mathrm{MPa}$ conversion incorporated into the coefficients. 


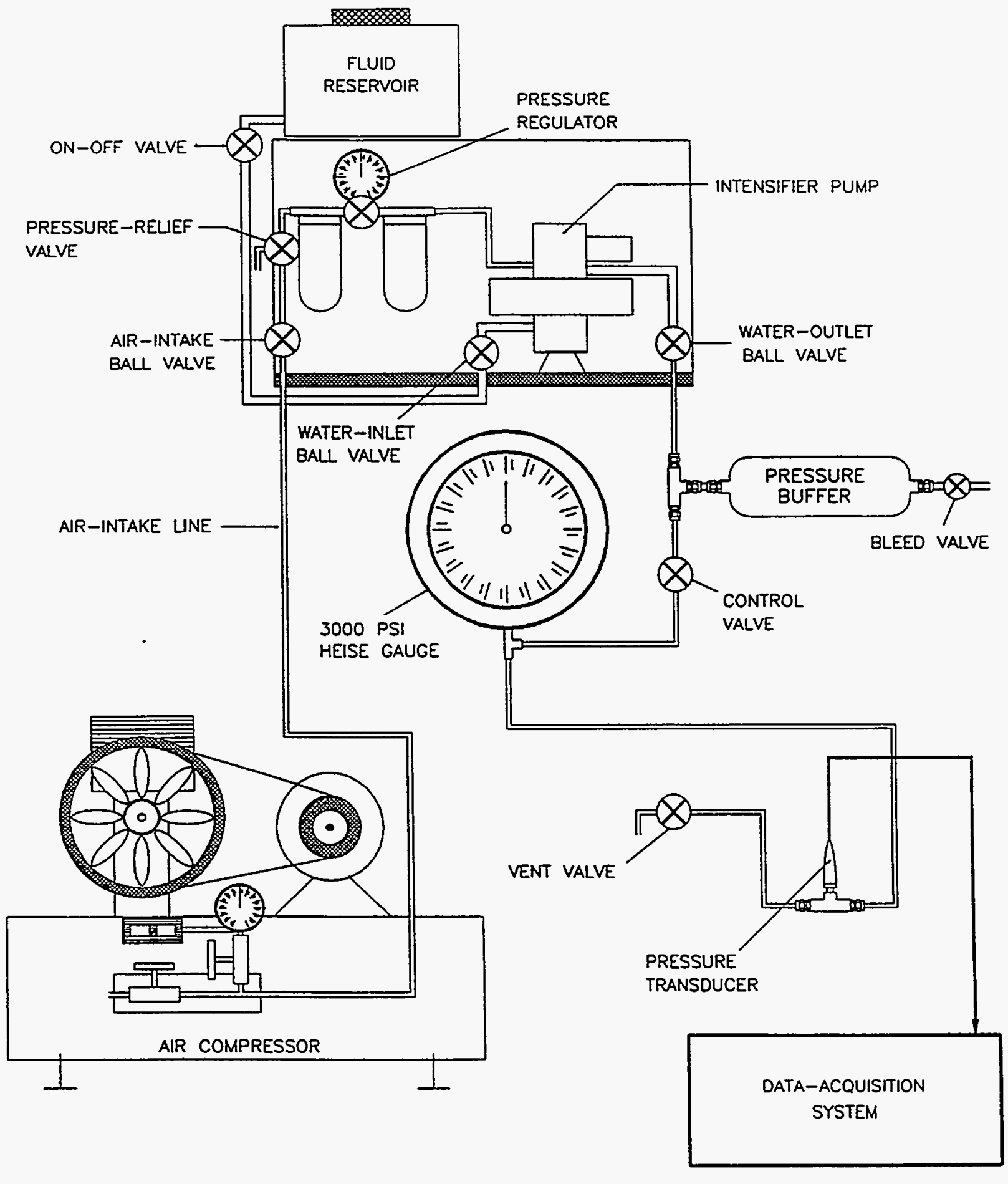

WNTERA-61 15-21-0

Figure 3-13. Schematic illustration of the pressure-transducer-calibration system. 


\subsubsection{THERMOCOUPLES}

Type E Chromel-Constantan thermocouples were used to monitor temperatures within the test and guard zones during permeability testing. The thermocouples were $1 / 8$-inch $(0.32$ $\mathrm{cm}$ ) in diameter and were sheathed in Inconel 600 . The thermocouples were reported to be accurate to within $\pm 0.06^{\circ} \mathrm{C}$ by the manufacturer, ARI Industries. The thermocouples were calibrated by SNL calibration laboratory in Albuquerque, NM.

\subsubsection{LINEAR VARIABLE-DIFFERENTIAL TRANSFORMERS}

Each of the radially-oriented LVDT plunger assemblies used to measure radial deformation was calibrated by the SNL WIPP calibration laboratory in accordance with WIPP Procedure 204. Linear-regression analysis of the calibration data provides slope (sensitivity) and offset ( $y$-intercept) values that are used by the DAS software to convert the DC voltage output to a linear dimension $(\mathrm{cm})$, starting near zero $(0)$ when the plungers are extended and increasing in magnitude as the plungers are depressed. The technique used to establish the LVDT response to a known radius is to use a Radial LVDT Measurement Device (Figure 3-14). Establishing a known reference radius for the radial LVDT assemblies was done in accordance with WIPP Procedure 473. The Radial LVDT Measurement Device provides an accurately machined radius of $4.9735 \pm 0.0005 \mathrm{~cm}$, and is designed to center and align the LVDT assembly. The Radial LVDT Measurement Device is also used at the end of a permeability testing sequence when the test tool is retrieved from the borehole to recalibrate the plunger depressions and provide a measure of instrument drift with respect to time. The average plunger depression value along with the known calibration sleeve radius is used to establish the initial borehole radius after installing the multipacker test tool in a test borehole and inflating the packers. Combining the average change in plunger depressions with the previously calculated borehole radius, the change in borehole radius is measured during permeability testing.

The LVDT plunger assembly used to measure axial test-tool movement is calibrated prior to installation using WIPP Procedure 205. Linear-regression analysis of the calibration data provides slope (sensitivity) and offset ( $y$-intercept) values that are used by the DAS software to convert the $D C$ voltage output to linear dimensions $(\mathrm{cm})$ starting near zero $(0)$ when the plunger is extended and increasing in magnitude as the plunger is depressed. These measurements correspond directly to the axial movements of the test tool relative to the bottom of the test borehole. Axial LVDT values decrease in magnitude if the testtool moves out of the borehole and increase in magnitude if the test tool moves into the borehole.

\subsubsection{DIFFERENTIAL-PRESSURE-TRANSMITTER PANEL}

The DPTs are calibrated from 0 to $100 \mathrm{~cm}$ of water $(0-9.8 \mathrm{kPa})$ by the SNL WIPP calibration laboratory in accordance with WIPP Procewdure 353 . The manufacturer's stated accuracy of the DPTs is $\pm 0.2 \%$ of the calibrated span, including the combined effects of hysteresis, repeatability, and independent linearity. The various injection/withdrawal columns associated with the DPT panels were verified in the field using 


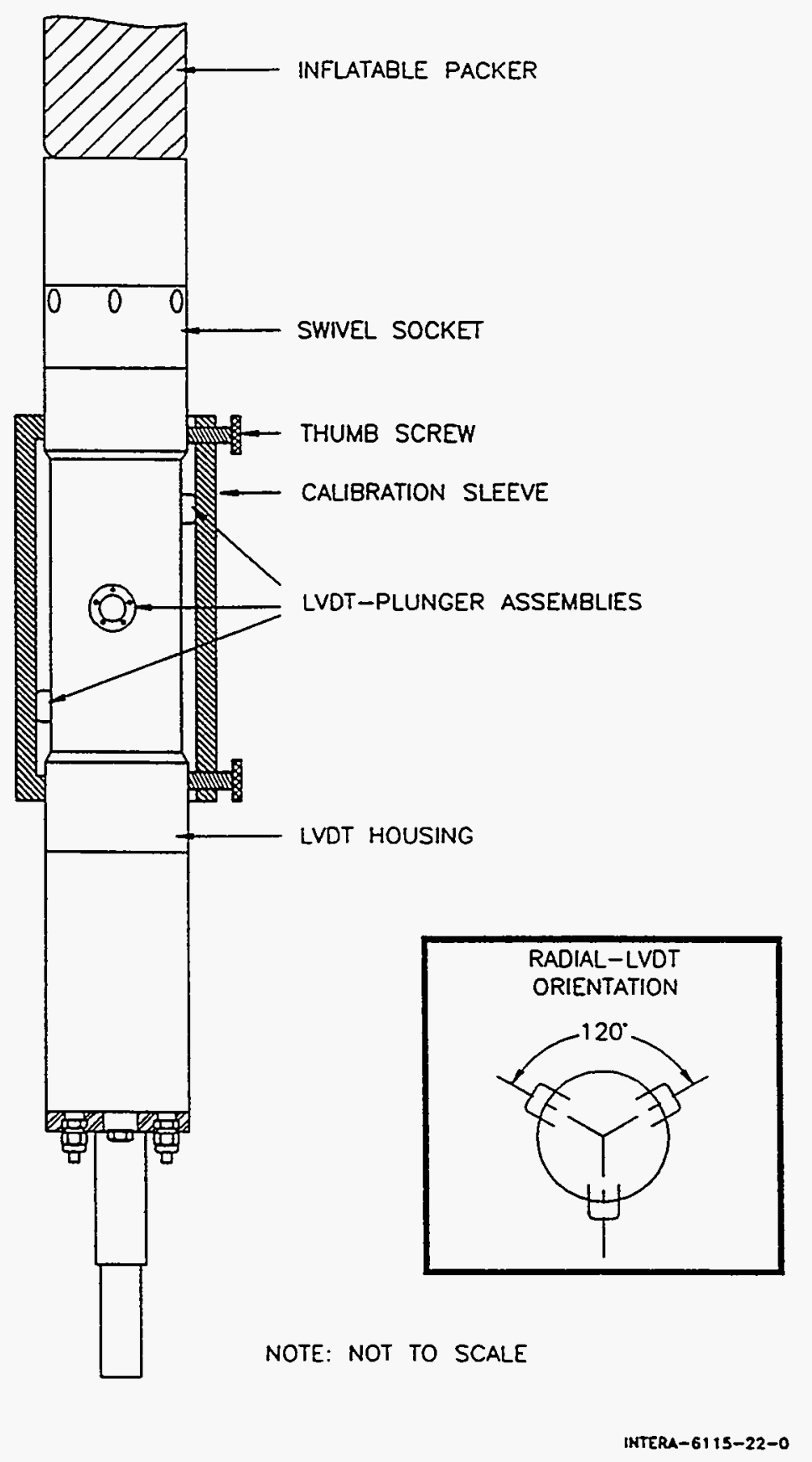

Figure 3-14. Radial-LVDT Measurement Device. 
the DPT. The verification procedure involves filling a column with the fluid to be used in a constant-pressure-injection/withdrawal test. The column is pressurized to the test pressure and a fixed volume of fluid is removed from the column, generating a voltage drop across the DPT. This process is repeated until the column has been emptied. Linearregression analysis of the DPT voltage output versus the cumulative volume of fluid removed from the column provides sensitivity values, which consist of the slope expressed as volume/volt, and the $y$-intercept expressed as volume. Sensitivity coefficients are used to convert the DPT voltage signal to a volume measurement for a given test pressure. $A$ step-by-step description of the verification procedure is presented in SOP INT-5 (INTERA, 1992) and in WIPP Procedure 476.

\subsection{Procedures for Permeability Testing}

\subsubsection{Test-Tool Installation Procedures}

The following paragraphs describe a typical test-tool-installation sequence. Multipacker test tools were installed in each of the test boreholes as soon after drilling as possible in order to minimize depressurization of the formation surrounding the borehole. All multipacker test-tools were installed following test tool installation procedures presented in SOP INT-5 (INTERA, 1992) and/or WIPP Procedures for multipacker-test-tool installation $(479,480$, and 481) (see Figures 3-15 through 3-17). Before test-tool installation, in vertically down or angled downward borehole orientations enough brine was added to the borehole to fill all of the zones, helping to minimize air entrapment. After the multipacker test tool was installed and the DAS activated, the packers were sequentially inflated to approximately $14 \mathrm{MPa}$, beginning with the uppermost packer and continuing until the bottom-hole packer was inflated. Pressures and, in some cases, temperatures were monitored until the observed values stabilized or the packers were put on a pressuremaintenance system.

If the packers were not put on a pressure maintenance system, the packer inflation pressures were monitored for 24 to 48 hours after inflation. If compliance-related pressure decreases of greater than $3 \mathrm{MPa}$ were observed, the packer inflation pressures were increased to approximately $14 \mathrm{MPa}$ and observed for an additional 24 hours. After achieving satisfactory packer inflation pressure response, the zones were shut in by closing all associated valves. The pressure buildups in the isolated zones were monitored for the duration of the testing sequence.

For boreholes oriented vertically or angled upward, the multipacker test tools were installed and the guard-zone packer inflated to approximately $14 \mathrm{MPa}$. The isolated borehole was then filled with brine while a vacuum pump was attached to the bottom-hole zone vent line to removed entrapped air. In some cases, $10 \mathrm{psi}(0.07 \mathrm{MPa})$ check valves were attached to the vent lines to prevent brine from draining from the isolated borehole interval, assuring that all zones were fluid filled (Figure 3-18). The next packer (going into the borehole) was then inflated to approximately $14 \mathrm{MPa}$. The in-line check valves prevented the zone 


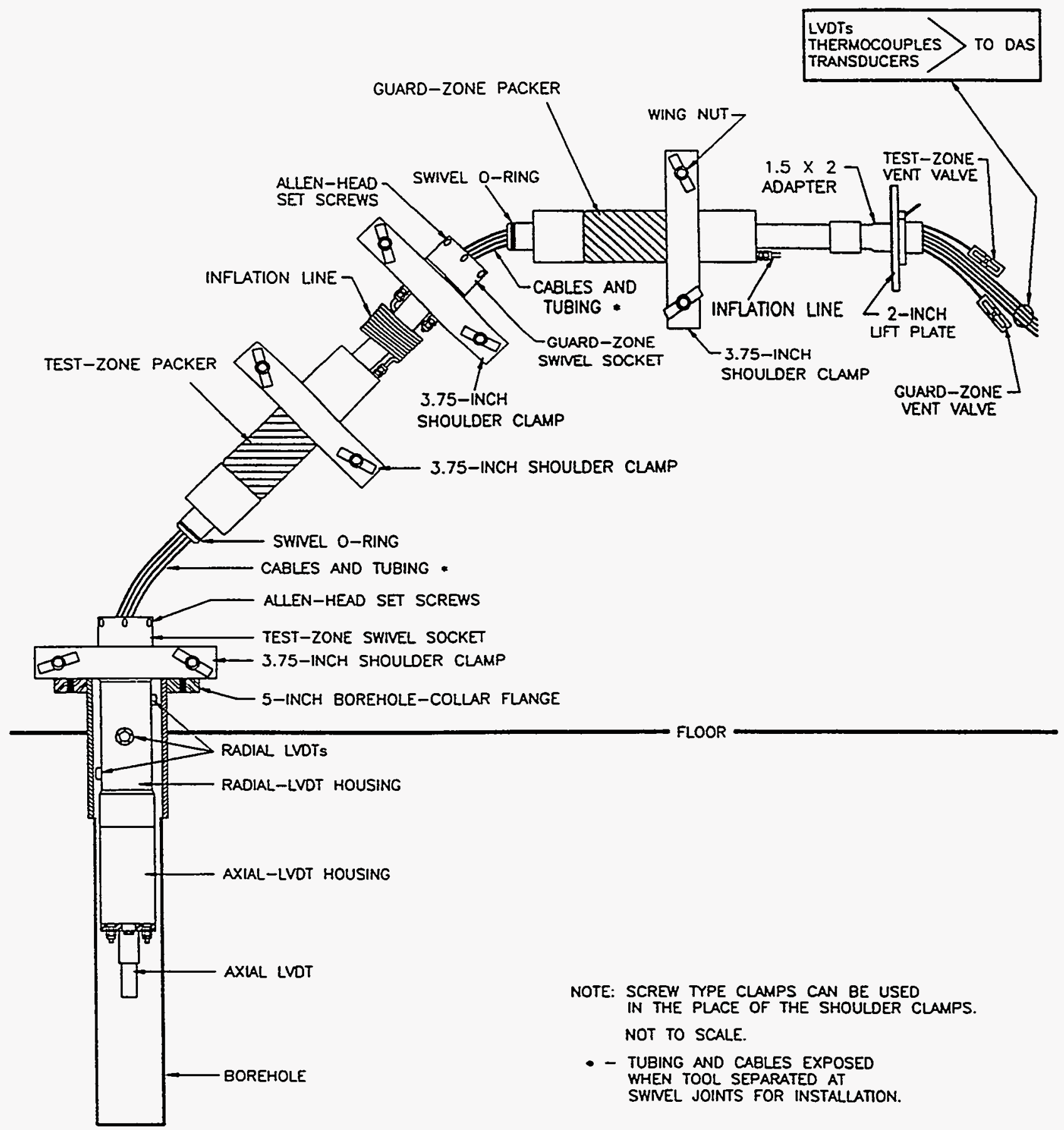

INIERA-6115-23-0

Figure 3-15. Test-tool installation in a borehole oriented vertically downward. 


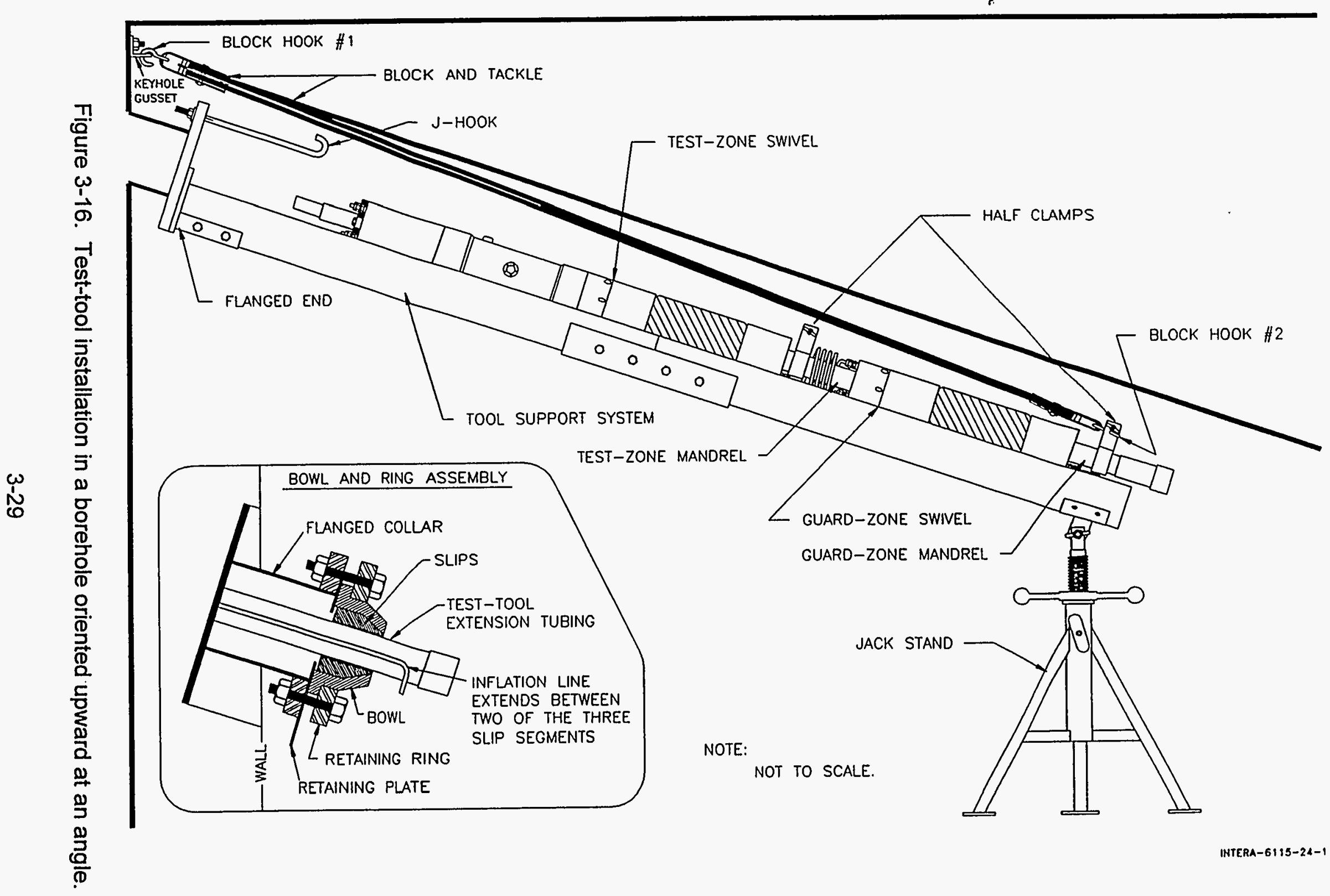




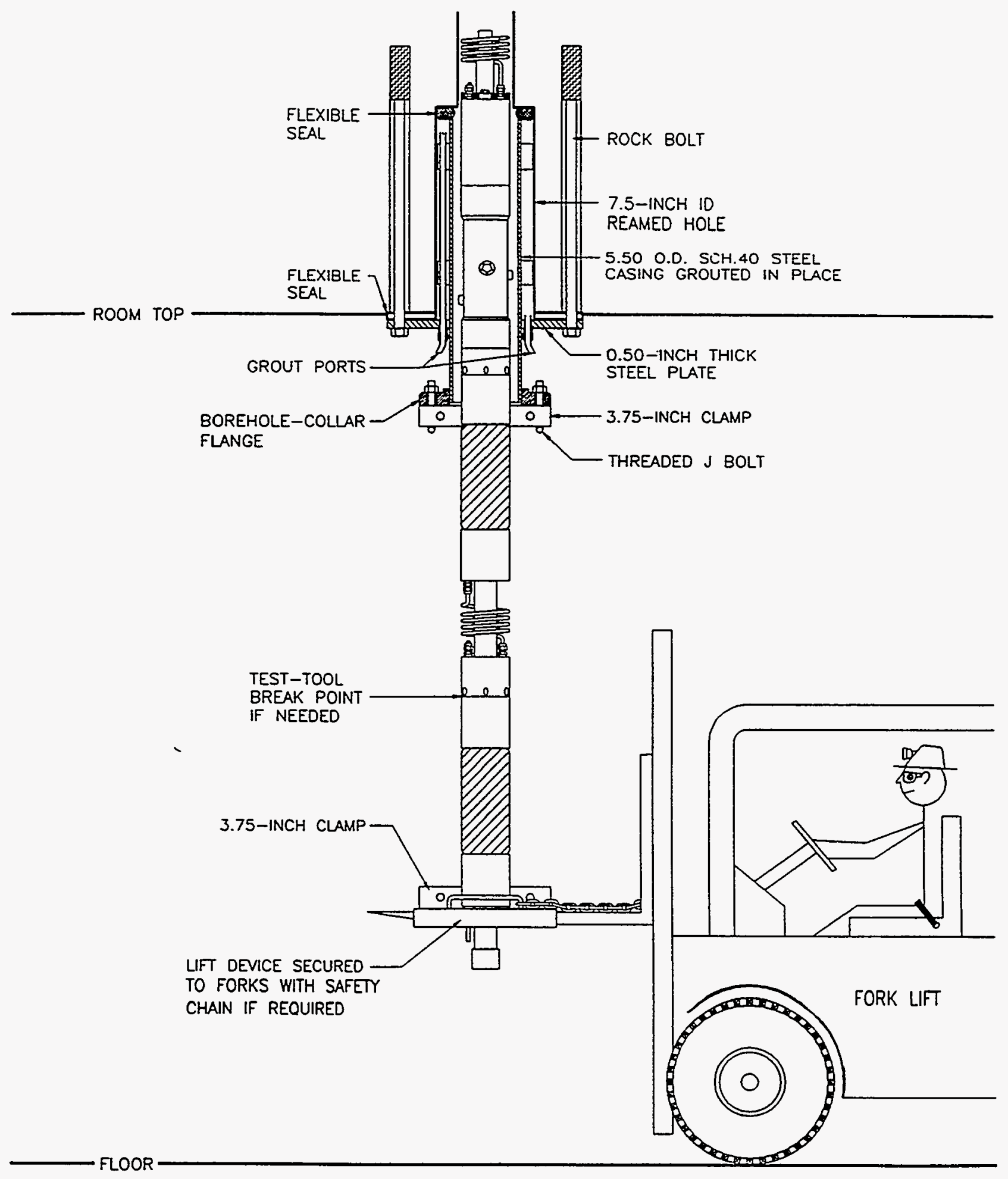

INTERA-6115-25-0

Figure 3-17. Test-tool installation in a borehole oriented vertically upward. 
pressures from exceeding $10 \mathrm{psi}(0.07 \mathrm{MPa})$ during packer inflation. This process was repeated until all of the packers were inflated. In instances when the check valves were not incorporated into the vent lines, all associated valves were shut in during packer inflation and the zone pressures were closely monitored as the packers were inflated. If the zone pressures showed an increase, the associated vent valves were opened slightly to relieve the pressure buildup. This process was repeated until all of the packers were inflated to the desired pressure.

\subsubsection{Pulse-Withdrawal Testing Procedures}

Pulse-withdrawal tests performed as part of the permeability-testing sequences were conducted after monitoring fluid-pressure buildups in the test zones until the rate of pressure increase had decreased to a satisfactory level and the pressure-recovery curves appeared to be on asymptotic trends toward stable values. For the majority of permeability-testing sequences, pulse-withdrawal tests were conducted in one or more of the zones after the fluid-pressure buildups in the zones had achieved relative stability. Pulse withdrawals rather than pulse injections were chosen for permeability-testing sequences because, in some cases, the pressure buildups in the zones approached lithostatic pressure. With zone pressures of this magnitude, pulse-injection pressures of sufficient magnitude to generate interpretable fluid-pressure responses would have approached the hydrofracture pressure of the Salado Formation. In this case, the boreholes would have been artificially stimulated, resulting in an invalid representation of formation conditions. Figure 3-19 illustrates the elements of a typical permeability-testing sequence including a pulse-withdrawal test.

The following procedures were used to conduct most of the pulse-withdrawal tests that are reported here. The DAS was set to scan the instruments' return signals at frequencies sufficient to monitor test responses adequately. Then the test-zone vent valve was opened and a portion of the shut-in pressure was allowed to dissipate by allowing fluid to leave the test zone. When the desired pressure decrease was achieved, the test zone was shut in. The resulting test-zone fluid-pressure buildup, packer-inflation pressure, and guard-zone pressure were all monitored with the DAS according to prescribed frequencies. The volume of fluid removed from the test zone was measured and recorded for each pulsewithdrawal test performed. Pulse-withdrawal tests were sometimes repeated, after the formation showed full recovery, to ensure that the fluid-pressure responses were reproducible and that they were representative of the actual formation responses. Pulsewithdrawal tests allowed for the determination of the compressibility of the zone being tested by measuring the volume of fluid required to increase/decrease the zone pressure a measured amount.

\subsubsection{Constant-Pressure-Injection/Withdrawal Testing Procedures}

Constant-pressure-injection and/or withdrawal tests were performed as part of the permeability-testing sequences were conducted after monitoring fluid-pressure buildups 


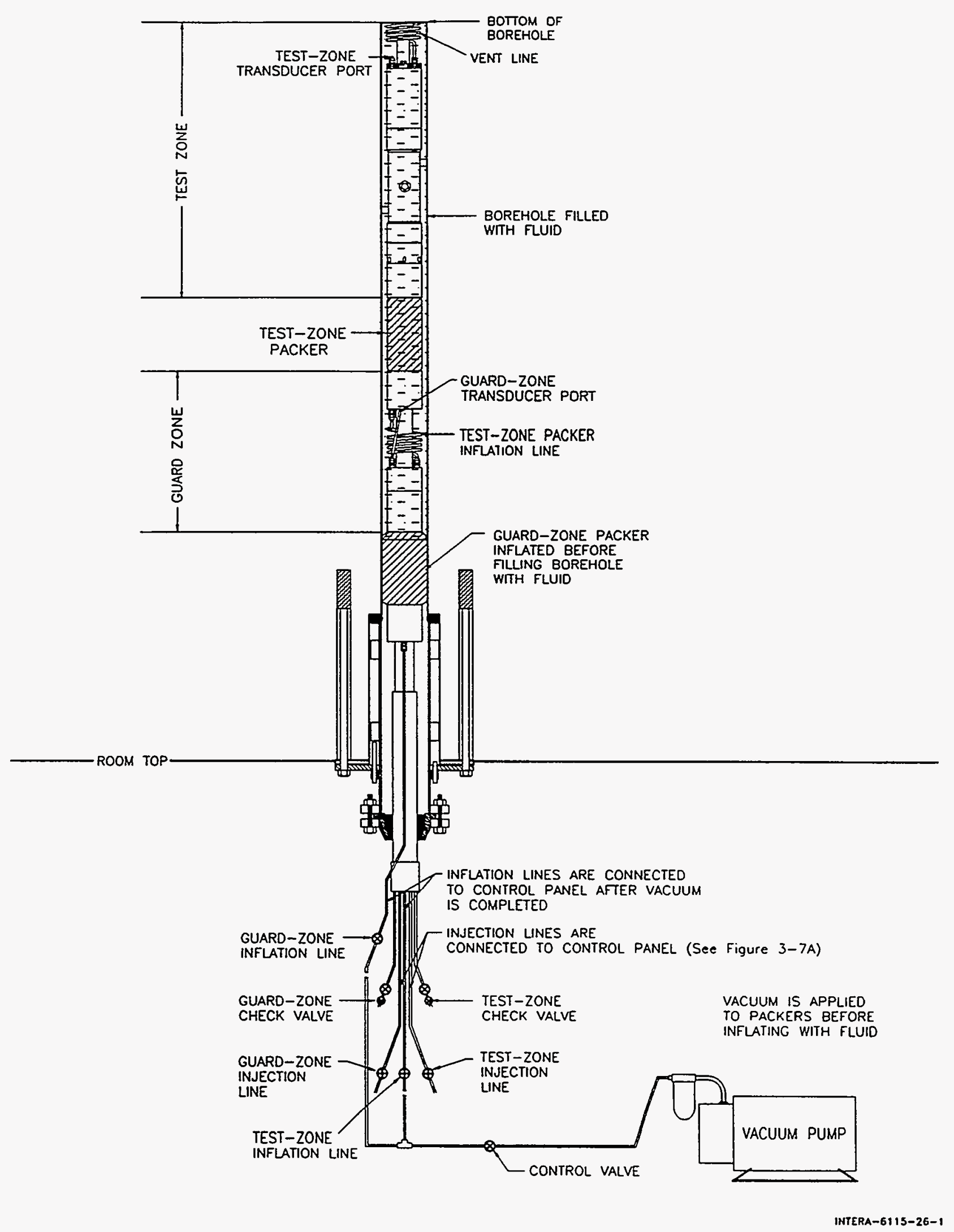

Figure 3-18. Test-tool packer-inflation and fluid-injection configuration for vertically upward and angled upward boreholes. 


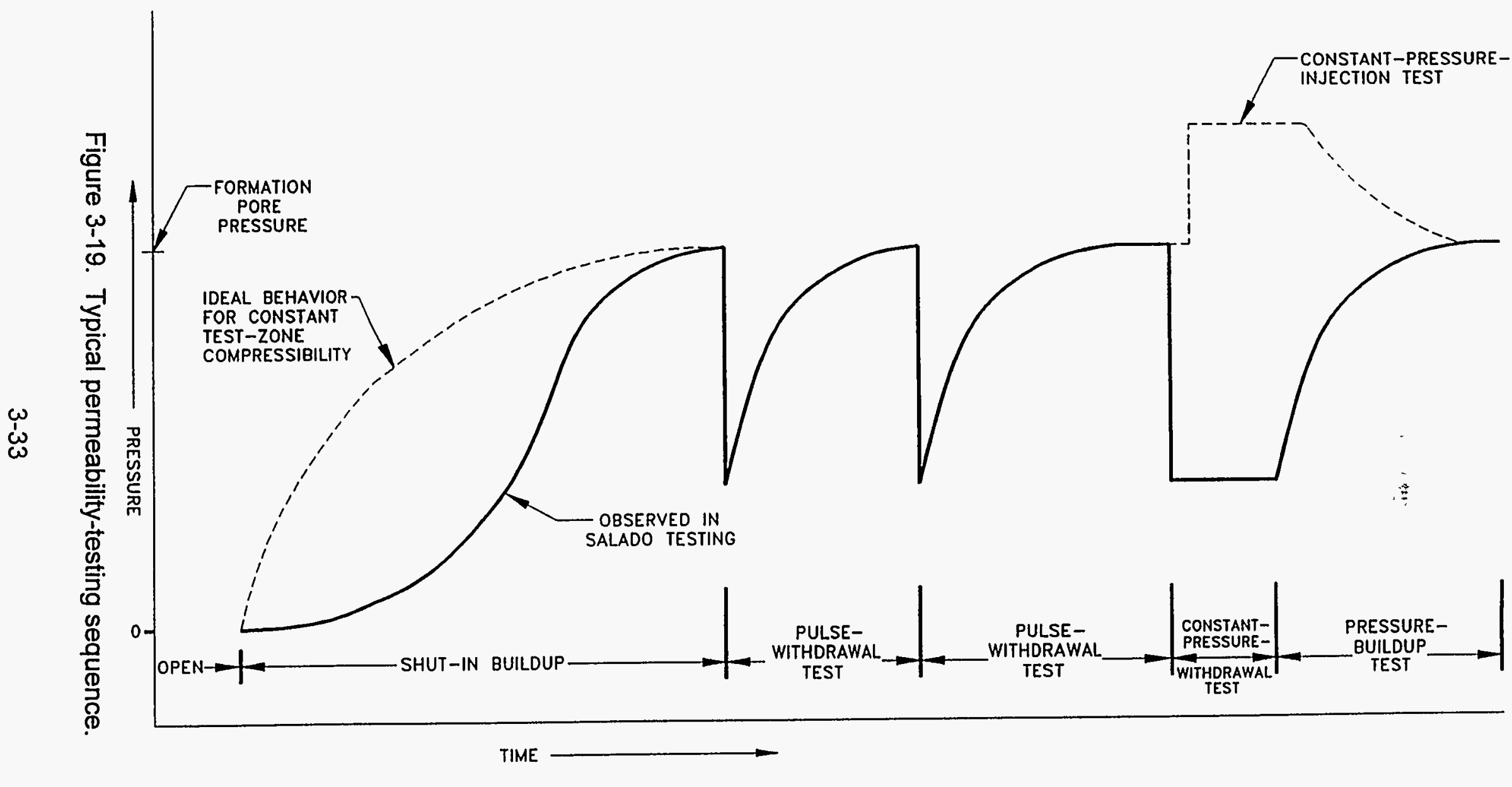


in the test zones until the rate of pressure increase had decreased to a satisfactory level and the pressure-recovery curves appeared to be on asymptotic trends toward stable values. The DPT panel, as described in Section 3.3.1.6 and illustrated in Figure 3-9, was used to perform the constant-pressure-injection/withdrawal tests. In some of the permeability-testing sequences, both constant-pressure injection and constant-pressure withdrawal tests were performed in order to evaluate the flow properties of the formation in the vicinity of the borehole as a function of pressure. Figure 3-19 illustrates the elements of a typical permeability-testing sequence. Due to the much longer period of time during a constant-pressure-injection/withdrawal test relative to the pulse tests when the formation is subjected to over- or under-pressure conditions, a much larger portion of the formation can be effectively tested. Another advantage of the constant-pressureinjection/withdrawal test is that it provides flow-rate data for the testing period which provide an additional constraint during analysis of the data. A detailed description of the steps that must be followed in order to conduct a constant-pressure-injection/withdrawal test successfully is given in SOP INT-5 (INTERA, 1992) and WIPP Procedure 476.

In some cases, a series of constant-pressure-injection or constant-pressure-withdrawal tests were performed at different pressures in succession. By conducting several tests at different pressures, the formation pressure and flow responses could be evaluated as a function of pressure without going through the intermediate step of pressure recovery after each individual flow period.

A variation of the constant-pressure injection/withdrawal test is a zone compressibility test. In a zone compressibility test the pressure within a given zone is increased from 0 to something approaching the maximum pressure that would be expected in that zone while the test tool is in the borehole. The pressure is increased slowly through the DPT panel in order to measure the fluid volume required to increase the zone pressure by a certain amount. A needle valve is used to regulate the rate at which the zone pressure is increases. When the maximum pressure is achieved, the procedure is repeated only the zone pressure is decreased back to 0 . The zone compressibility tests are typically conducted in a compliance chamber during compliance testing, however, in some cases they were conducted in test boreholes. A more detailed description of zone compressibility tests is given in Section 6.

\subsubsection{Pressurized Brine-Sampling Procedures}

Pressurized brine samples were collected as part of the permeability-testing program from MB140 during permeability-testing sequence L4P51-C1. These pressurized brine samples were collected in order to determine the volume and composition of dissolved gas contained in the formation fluid within MB140 at this location as well as the chemical composition of the fluid. Because nitrogen was expected to be one of the principal gases in solution, the sampling apparatus was pressurized with argon gas.

The following procedures were used to collect all of the pressurized brine samples associated with permeability-testing sequence L4P51-C1. Following a pre-determined schedule, samples were collected at approximately 14-day intervals. A sampling apparatus 
as shown in Figure 3-10 was used in conjunction with the differential-pressure-transmitter (DPT) panel to collect the pressurized brine samples as part of a constant-pressurewithdrawal test. During a constant-pressure-withdrawal test formation fluid flows through the sampling apparatus prior to entering the designated column on the DPT panel. When a sample is to be retrieved, a series of valves are opened and/or closed in a prescribed sequence in order to allow the formation fluid to bypass the sampling apparatus. When the sampling apparatus is effectively isolated, the sample is retrieved. WIPP Procedure 467 describes the steps required to collect and submit for analysis a pressurized brine sample to assure that an uncontaminated sample was supplied to the laboratory conducting the analysis.

The gas analysis that was performed on each of the samples was conducted by the geochemistry department at New Mexico Institute of Mining and Technology under the supervision of Dave Norman. The brine analysis on each of the samples was conducted by Chem-Nuclear Geotech Analytical Laboratory. A portion of the results from the brine analysis is presented in Section 3.6.3.

\subsection{Boreholes and Test-Tool Configurations for Permeability Testing}

\subsubsection{Borehole Locations and Orientations}

Figure 2-1 presents the locations of the boreholes associated with the permeability-testing program. A testing sequence is referred to by the borehole designation in which it was conducted. Modifications to this reference system were required when a borehole was deepened and additional tests performed in new (deeper) intervals or when a new test-tool configuration was used to conduct tests in borehole intervals that had been previously tested. When a borehole was deepened to test a new interval a letter designation was added to the borehole designation with " $A$ " referring to the initial testing sequence and all subsequent borehole deepenings proceeding alphabetically. An example of this is illustrated in the S1P74-A and S1P74-B testing sequences. The initial testing sequence was conducted in borehole S1P74 and was referred to by this designation. Then the borehole was deepened and the new interval was tested. Therefore, the initial testing sequence (S1P74) was redesignated S1P74-A and the new testing sequence (in the deeper borehole) was designated S1P74-B. In the case when a new test-tool configuration was used to conduct tests in a borehole that had been previously tested a number designation was added to the appropriate borehole and letter designation with " 1 " referring to the original test-tool configuration and all subsequent test-tool configurations proceeding numerically. An example of this is illustrated in the L4P51-C1 and L4P51-C2 testing sequences. The initial test-tool configuration used in testing sequence L4P51-C was a double-packer configuration and was referred to as L4P51-C and a testing sequence was conducted using this configuration. Later, it was decided that a triple-packer configuration was needed to better test a particular interval. Therefore, the original test tool was removed and the new one was installed and a second testing sequence was performed. Under these circumstances the first testing sequence was redesignated L4P51-C1 and the second testing sequence was designated L4P51-C2. 
The locations for the WIPP underground permeability-testing boreholes were based on the ages of excavations and access to Salado Formation lithologies of interest. Therefore, permeability testing results from boreholes located in more recently excavated areas of the underground facility could be compared with those from areas that had been excavated earlier, thus providing an opportunity to investigate the permeability of geologic media least affected by the pressure relief imposed on the Salado Formation by excavations. Table 3-1 summarizes the location and orientation of each of the boreholes associated with the permeability testing program. Borehole orientations at an underground location attempt to accomplish three objectives:

- to characterize the interbeds in close areal proximity;

- to test the same interbed at varying distances from an excavation while maintaining close areal proximity; and

- to characterize the interbeds at a maximum distance from the excavation.

Table 3-1. Summary of Borehole Locations and Orientations

\begin{tabular}{|c|c|c|c|c|c|}
\hline $\begin{array}{l}\text { Test Sequence } \\
\text { (Orientation) }\end{array}$ & $\begin{array}{l}\text { Drilling } \\
\text { Method }\end{array}$ & $\begin{array}{c}\text { Interval } \\
\text { Drilled/Cored (m) }\end{array}$ & $\begin{array}{l}\text { Date } \\
\text { Drilled/ } \\
\text { Cored }\end{array}$ & Location & $\begin{array}{l}\text { Excavation } \\
\text { Date of Room }\end{array}$ \\
\hline $\begin{array}{c}\text { L4P51-C1 } \\
\text { (vertical down) }\end{array}$ & $\begin{array}{l}\text { Brine-rotary } \\
\text { w/ coring bit }\end{array}$ & $10.06-23.35$ & $\begin{array}{c}4-1-92 \text { to } \\
4-15-92\end{array}$ & Room L4 & $\begin{array}{c}\text { February } \\
1989\end{array}$ \\
\hline $\begin{array}{c}\text { L4P51-C2 } \\
\text { (vertical down) }\end{array}$ & $\begin{array}{l}\text { Brine-rotary } \\
\text { w/ coring bit }\end{array}$ & $10.06-23.35$ & $\begin{array}{c}4-1-92 \text { to } \\
4-15-92\end{array}$ & Room L4 & $\begin{array}{c}\text { February } \\
1989\end{array}$ \\
\hline $\begin{array}{l}\text { L4P51-D1 } \\
\text { (vertical down) }\end{array}$ & $\begin{array}{l}\text { Brine-rotary } \\
\text { w/ coring bit }\end{array}$ & $23.35-30.45$ & $\begin{array}{l}9-20-94 \text { to } \\
9-22-94\end{array}$ & Room L4 & $\begin{array}{c}\text { February } \\
1989\end{array}$ \\
\hline $\begin{array}{c}\text { L4P51-D2 } \\
\text { (vertical down) }\end{array}$ & $\begin{array}{l}\text { Brine-rotary } \\
\text { w/ coring bit }\end{array}$ & $23.35-30.45$ & $\begin{array}{l}9-20-94 \text { to } \\
9-22-94\end{array}$ & Room L4 & $\begin{array}{c}\text { February } \\
1989\end{array}$ \\
\hline $\begin{array}{c}\text { L4P52-B } \\
\left.\text { (angled upward } 40^{\circ}\right) \\
\text { ( from vertical) }\end{array}$ & $\begin{array}{l}\text { Air-rotary } \\
\text { w/ coring bit }\end{array}$ & $9.02-14.12$ & $\begin{array}{c}12-10-92 \text { to } \\
12-14-92\end{array}$ & Room L4 & $\begin{array}{c}\text { February } \\
1989\end{array}$ \\
\hline $\begin{array}{c}\text { S1P74-A } \\
\text { (angled upward } 40^{\circ} \text { ) } \\
\text { (from vertical) }\end{array}$ & $\begin{array}{c}\text { Air-rotary } \\
\text { w/ coring bit }\end{array}$ & $0.00-7.69$ & $\begin{array}{l}7-27-92 \text { to } \\
7-29-92\end{array}$ & $\begin{array}{l}\text { Waste Panel 1, } \\
\text { Room } 7\end{array}$ & $\begin{array}{c}\text { March } \\
1988\end{array}$ \\
\hline $\begin{array}{c}\text { S1P74-B } \\
\text { (angled upward } 40^{\circ} \text { ) } \\
\text { (from vertical) }\end{array}$ & $\begin{array}{l}\text { Air-rotary } \\
\text { w/ coring bit }\end{array}$ & $7.69-16.88$ & $\begin{array}{l}1-26-95 \text { to } \\
1-31-95\end{array}$ & $\begin{array}{l}\text { Waste Panel } 1 \text {, } \\
\text { Room } 7\end{array}$ & $\begin{array}{r}\text { March } \\
1988\end{array}$ \\
\hline
\end{tabular}

Tables 3-2 provides pertinent information on the boreholes and test zones associated with the permeability-testing program. 
Table 3-2. Permeability-Testing Sequence Test-Zone Information

\begin{tabular}{|c|c|c|c|c|c|c|c|}
\hline $\begin{array}{c}\text { Test } \\
\text { Sequence } \\
\end{array}$ & $\begin{array}{c}\begin{array}{c}\text { Test } \\
\text { Horizon }\end{array} \\
\end{array}$ & $\begin{array}{c}\text { Test Horizon } \\
\text { Penetrated } \\
\end{array}$ & $\begin{array}{c}\text { Borehole } \\
\text { Diameter }(\mathrm{cm}) \\
\end{array}$ & $\begin{array}{c}\text { Test Interval } \\
\text { Depth }(m)\end{array}$ & $\begin{array}{l}\text { Borehole } \\
\text { Depth }(\mathrm{m})\end{array}$ & $\begin{array}{c}\text { Sequence } \\
\text { Started }\end{array}$ & $\begin{array}{c}\text { Sequence } \\
\text { Terminated }\end{array}$ \\
\hline L4P51-C1 & Halite & $\begin{array}{l}4-3-92 \\
(09: 00)\end{array}$ & 11.12 & $15.59-16.55$ & 22.20 & $4-24-92$ & $11-11-93$ \\
\hline L4P51-C1 & MB140 & $\begin{array}{l}4-14-92 \\
(14: 20)\end{array}$ & 10.33 & $17.80-21.97$ & 22.20 & $4-24-92$ & $11-11-93$ \\
\hline L4P51-C2 & Halite & $\begin{array}{l}4-3-92 \\
(09: 00)\end{array}$ & 11.12 & $15.59-16.53$ & 22.20 & $11-18-93$ & $7-19-94$ \\
\hline L4P51-D1 & $\begin{array}{l}\text { Argillaceous } \\
\text { Halite }\end{array}$ & $\begin{array}{l}9-21-94 \\
(14: 30)\end{array}$ & 10.33 & $29.62-30.40$ & 30.45 & $9-30-94$ & $3-21-95$ \\
\hline L4P51-D2 & $\begin{array}{l}\text { Argillaceous } \\
\text { Halite }\end{array}$ & $\begin{array}{l}9-21-94 \\
(14: 30)\end{array}$ & 10.33 & $29.62-30.40$ & 30.45 & $4-6-95$ & $10-25-95$ \\
\hline L4P52-B & MB138 & $\begin{array}{c}12-14-92 \\
(12: 00)\end{array}$ & 10.15 & $13.89 \cdot 14.02$ & 14.18 & $12-17-92$ & $12-29-93$ \\
\hline S1P74-A & Anhydrite "a" & $\begin{array}{l}7-29-92 \\
(09: 15)\end{array}$ & 10.35 & $7.15-7.41$ & 7.69 & $8-5-92$ & $8-9-93$ \\
\hline S1P74-B & MB138 (TZ1) & $\begin{array}{l}1-31-95 \\
(12: 45)\end{array}$ & 10.35 & $16.38-16.66$ & 16.88 & $2-7-95$ & $5-18-95$ \\
\hline S1P74-B & $\begin{array}{l}\text { Argillaceous } \\
\text { Halite (TZ2) }\end{array}$ & $\begin{array}{l}1-27-95 \\
(12: 15)\end{array}$ & 10.35 & $12.58-13.49$ & 16.88 & $2-7-95$ & $5-18-95$ \\
\hline
\end{tabular}

Figure 3-20 schematically depicts Room L4 in plan view and in cross section, showing the locations and orientations of boreholes L4P51 and L4P52. Figure 3-21 schematically depicts Waste Panel 1, Room 7 in plan view and in cross section, showing the location and orientation of borehole S1P74.

\subsubsection{Borehole Drilling}

Requests for borehole drilling and coring were made in accordance with WIPP Procedure 140 and WIPP Procedure 125. Drilling and coring operations were performed by Westinghouse including installation and load-testing of borehole-flanged collars and borehole surveys. Records of borehole drilling and coring activities are maintained by Westinghouse.

The boreholes for the permeability-testing program were drilled using two different procedures. The first of the two procedures consisted of an air-rotary drilling technique in which the following steps were followed:

- A Longyear D-65 compressed-air drill was set in place at each borehole location and used to drill the test and observation boreholes into the floors and ribs of the test rooms. The boreholes were drilled primarily with a coring bit and core barrel for sample recovery. Compressed air was the principal means used to remove drilling cuttings from the boreholes as they were being drilled. The bottom of each borehole was faced off with a flat bit to insure a smooth surface for proper test-tool seating and test-zone-volume calculations. 


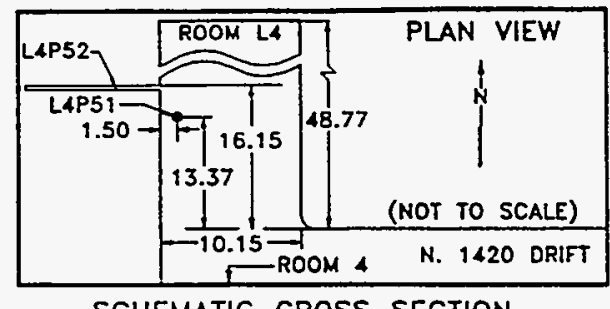

SCHEMATIC CROSS SECTION

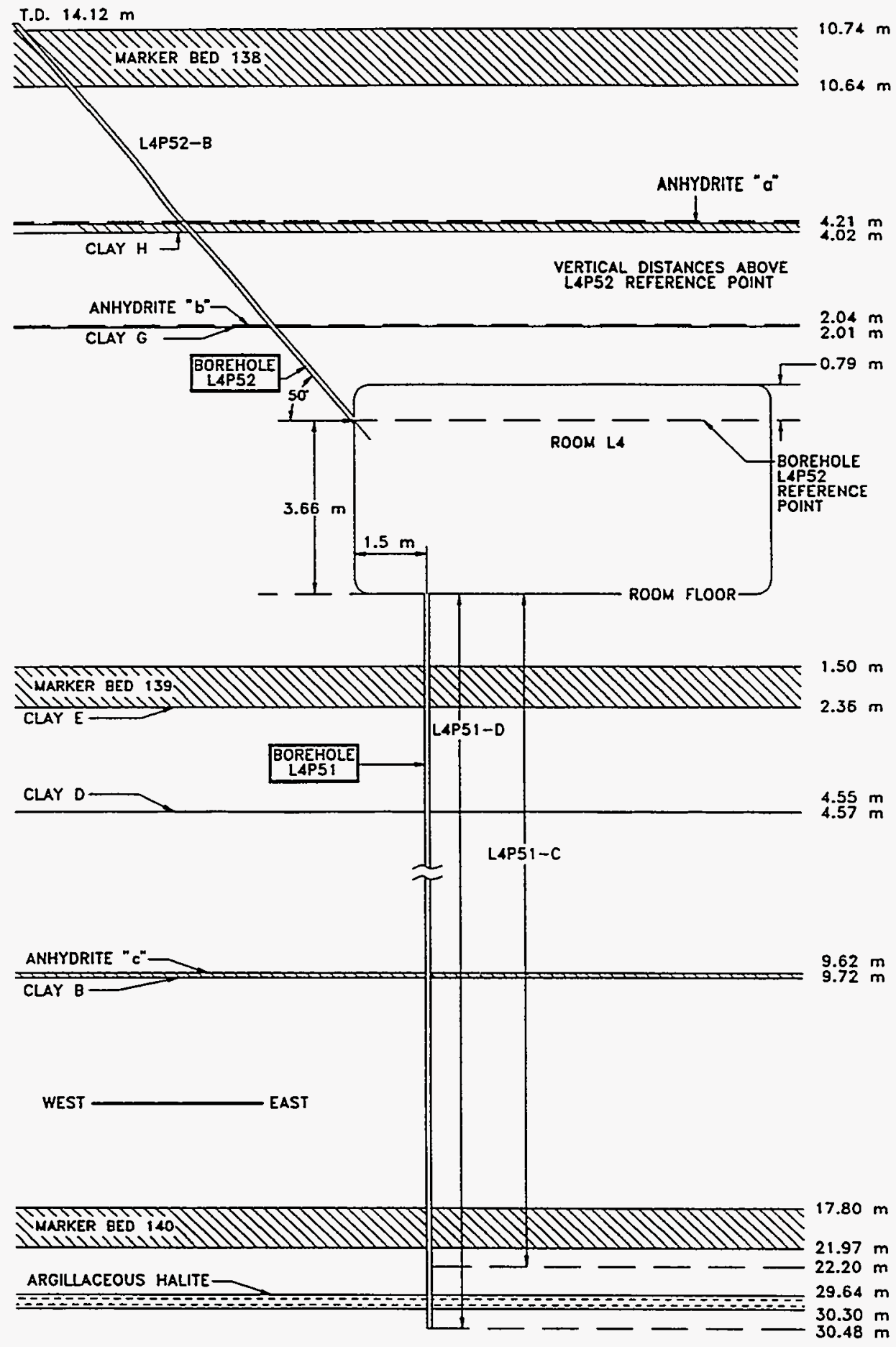

Figure 3-20. Spatial representation of Room L4 showing the locations and orientations of boreholes L4P51 and L4P52. 


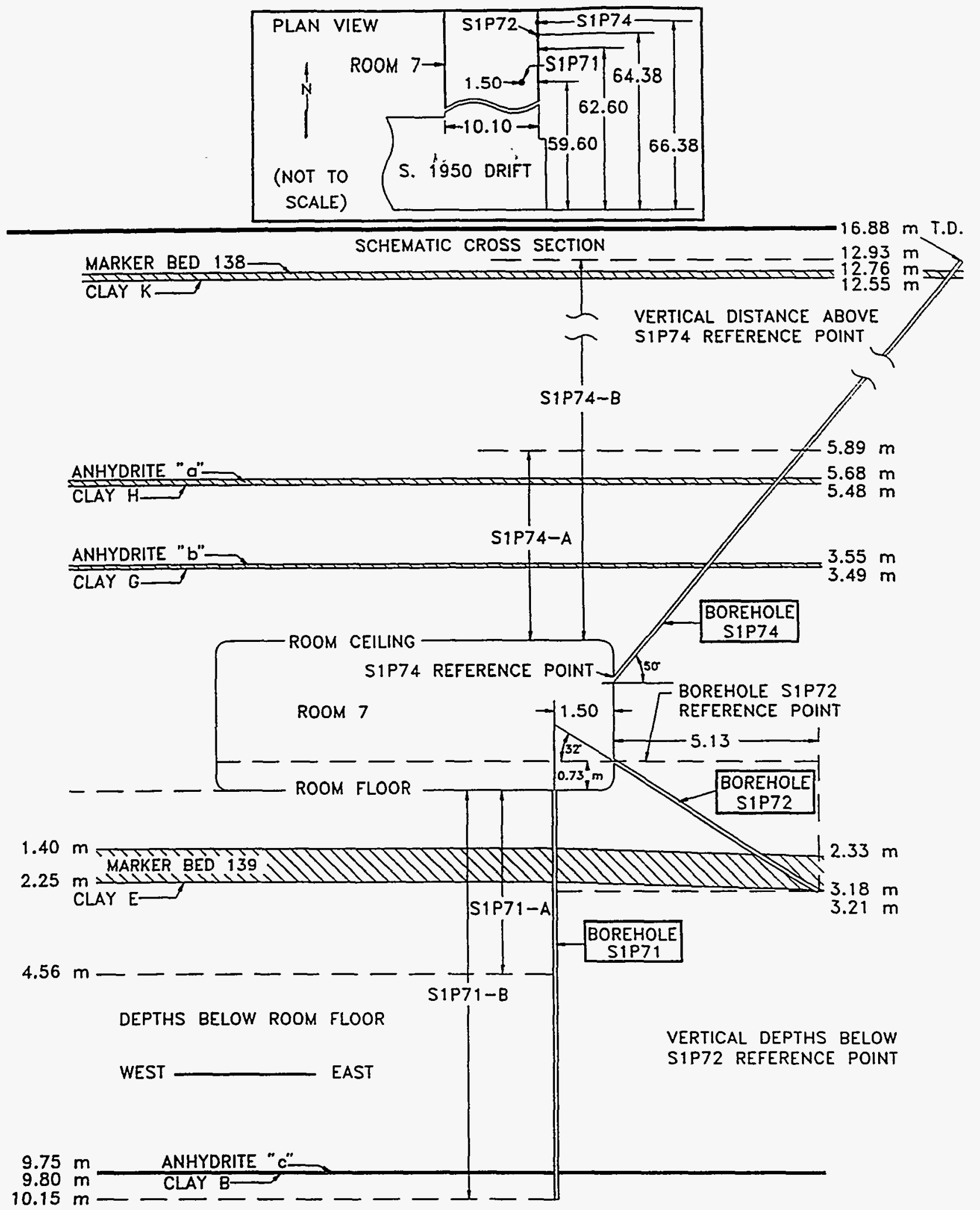

INTERA-6115-29-1

Figure 3-21. Spatial representation of Waste Panel 1, Room 7 showing the locations and orientations of boreholes S1P71, S1P72, and S1P74. 
- Using 4.0-inch (10.2-cm) drill and core bits, the test and observation boreholes were drilled and cored to depths of up to $10 \mathrm{~m}$ from the excavations. The following data and associated core logs and descriptions were recorded and are included in Appendix A of this report:

1) the time of the start of drilling and interval times for every $1 \mathrm{~m}$ penetrated;

2) the presence and depth of any brine produced by the formation;

3) the presence and depth of any significant fracturing; and

4) the time and depth at which the bottom of the borehole was reached.

- Core samples were recovered from over $95 \%$ of the cored sections and were marked, boxed, and described. The core samples were marked to indicate their proper orientation relative to the bottom of the boreholes and each $0.1-\mathrm{m}$ increment was marked on each core sample. Core was transferred to the WIPP underground Core Storage Library upon completion of core logging. Core logs are included in Appendix A for boreholes S1P74, C1H05, C1H06, C1H07, C1X05, C1X06, C1X10, L4P51 ( $C$ and D series), and L4P52 (B series). Additional documentation for boreholes L4P51 (A and B series) and L4P52 (A series) are provided in Stensrud et al. (1992).

- The boreholes were reamed to 7-inch $(17.8-\mathrm{cm})$ diameter to a depth of approximately 20 inches $(50.8 \mathrm{~cm})$. A 5-1/2-inch $(14.0-\mathrm{cm})$ I.D., 20-inch- $(50.8-\mathrm{cm})$ long, steel borehole collar was then installed and grouted to the surrounding formation material. The multipacker test tool was bolted to the borehole collar to help eliminate test-tool movement in response to packer inflation and fluid-pressure buildup.

The second of the two procedures consisted of a brine-rotary drilling technique in which a Longyear D-38 pressurized-fluid drill was set in place and used at the remaining borehole locations. In this drilling procedure, due to the depth of the boreholes, pressurized brine was the principal means used to remove drilling cuttings from the boreholes.

Boreholes L4P52 and S1P74-B were drilled using air drilling techniques. All other boreholes were drilled using brine as the circulation medium.

\subsubsection{Test-Tool Configurations}

Figures 3-20 and 3-21 schematically depict Room L4 and Waste Panel 1, Room 7 and show the positions of the boreholes associated with the testing program. Figures 3-22 through 3-33 show the test-tool configurations and associated installations that were used during the testing program. It should be noted that Figures 3-25, 3-26, 3-27, 3-28, and 329 each consist of two parts (ex. 3-25a and 3-25b). Specific test tools associated with each permeability-testing sequence are identified in the events tables (Tables 3-4, 3-6, 3-8, $3-10,3-12,3-14$, and 3-16). 


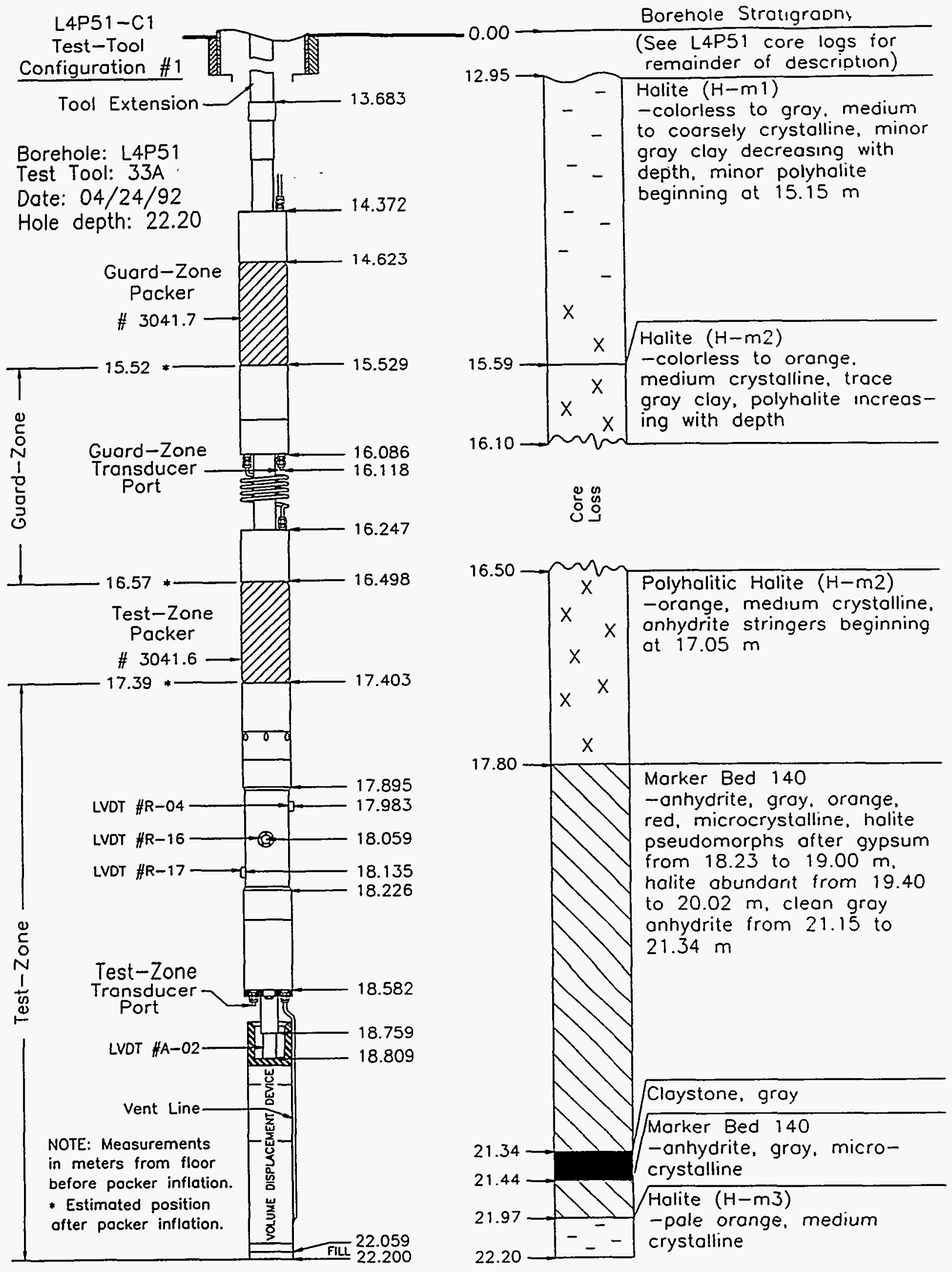

INTERA-6115-30-1

Figure 3-22. Configuration \#1 of permeability test tool for sequence L4P51-C1. 


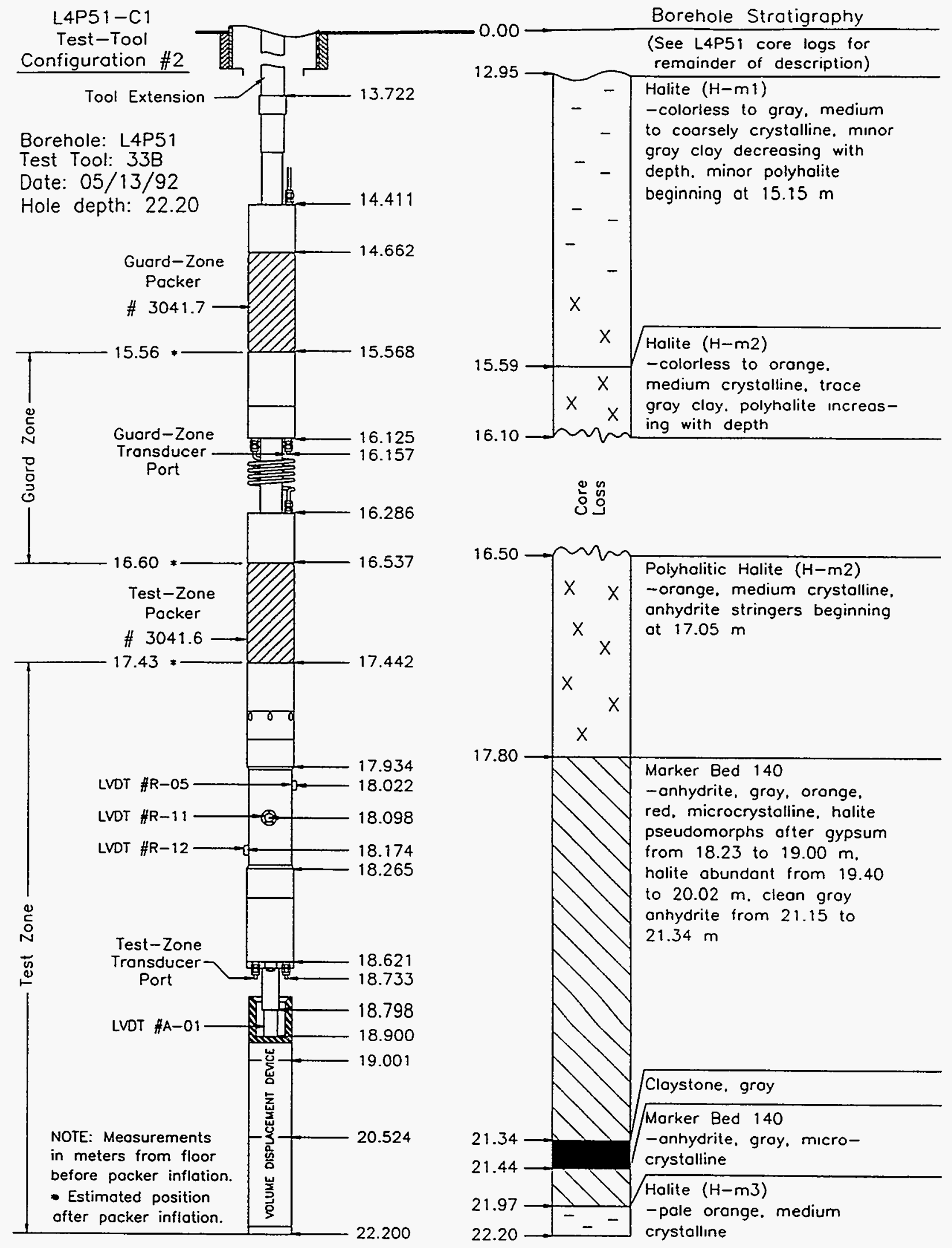

INIERA-61 15-31-1

Figure 3-23. Configuration \#2 of permeability test tool for sequence L4P51-C1. 


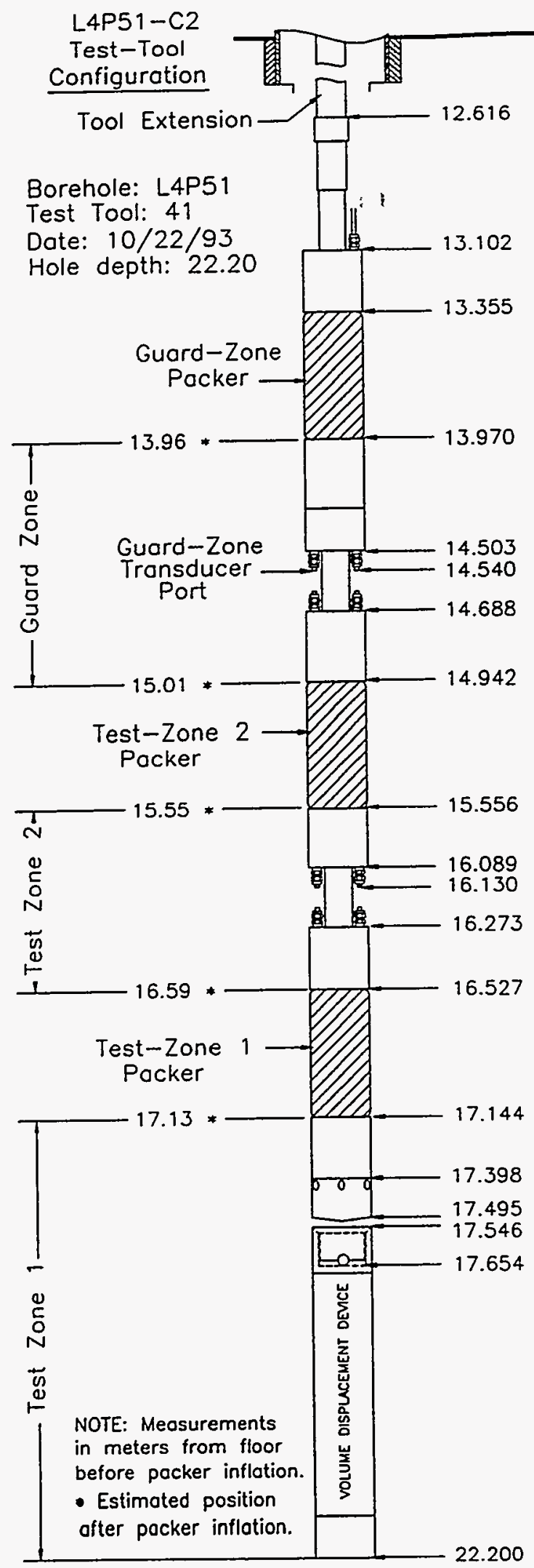

0.00

Borehole Stratigrophy

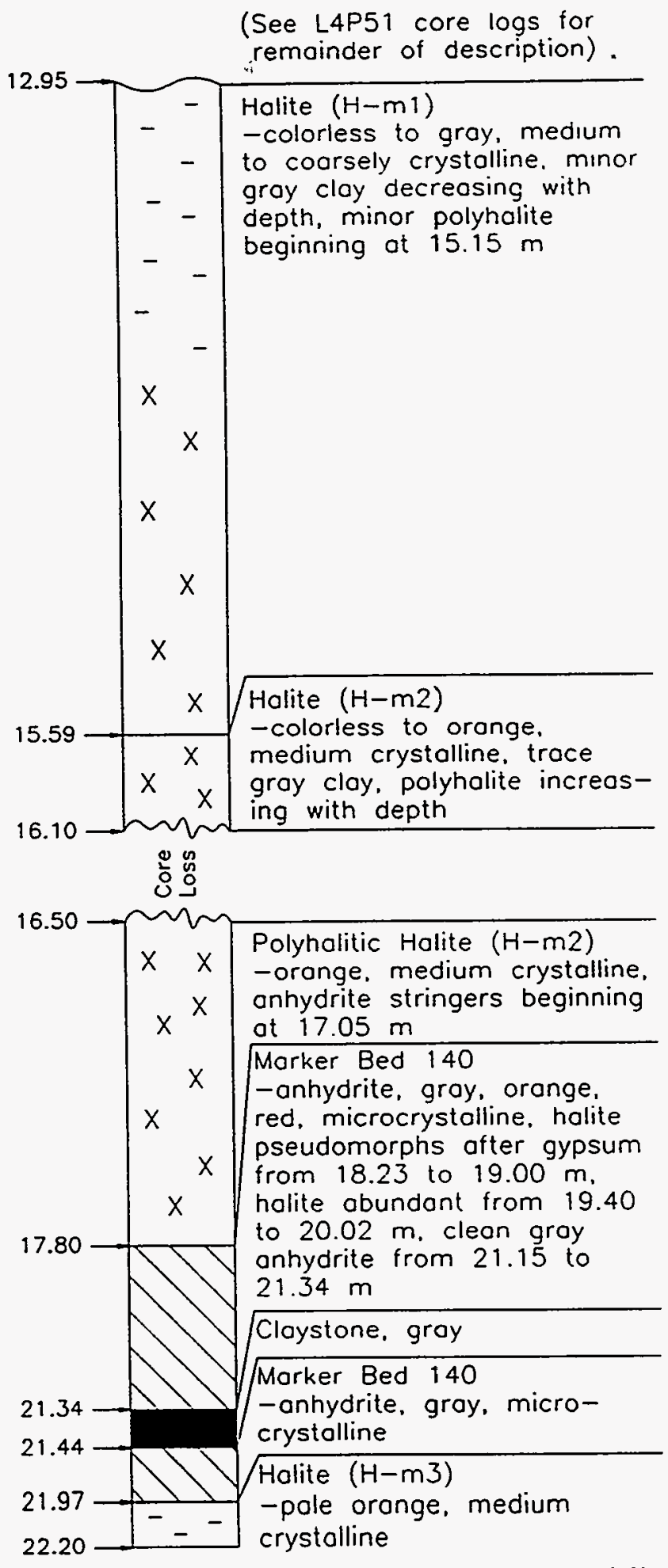

INTERA-6:15-32-1

Figure 3-24. Configuration of permeability test tool for sequence L4P51-C2. 


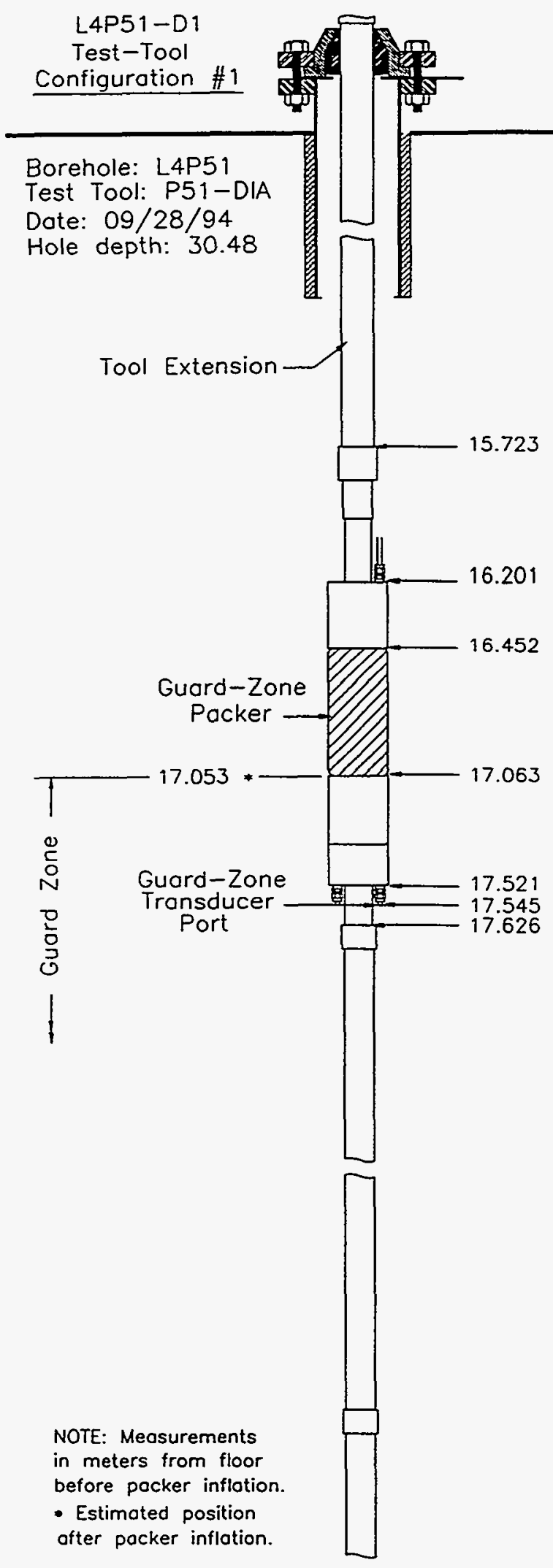

PAGE 1 OF 2

BOREHOLE STRATIGRAPHY
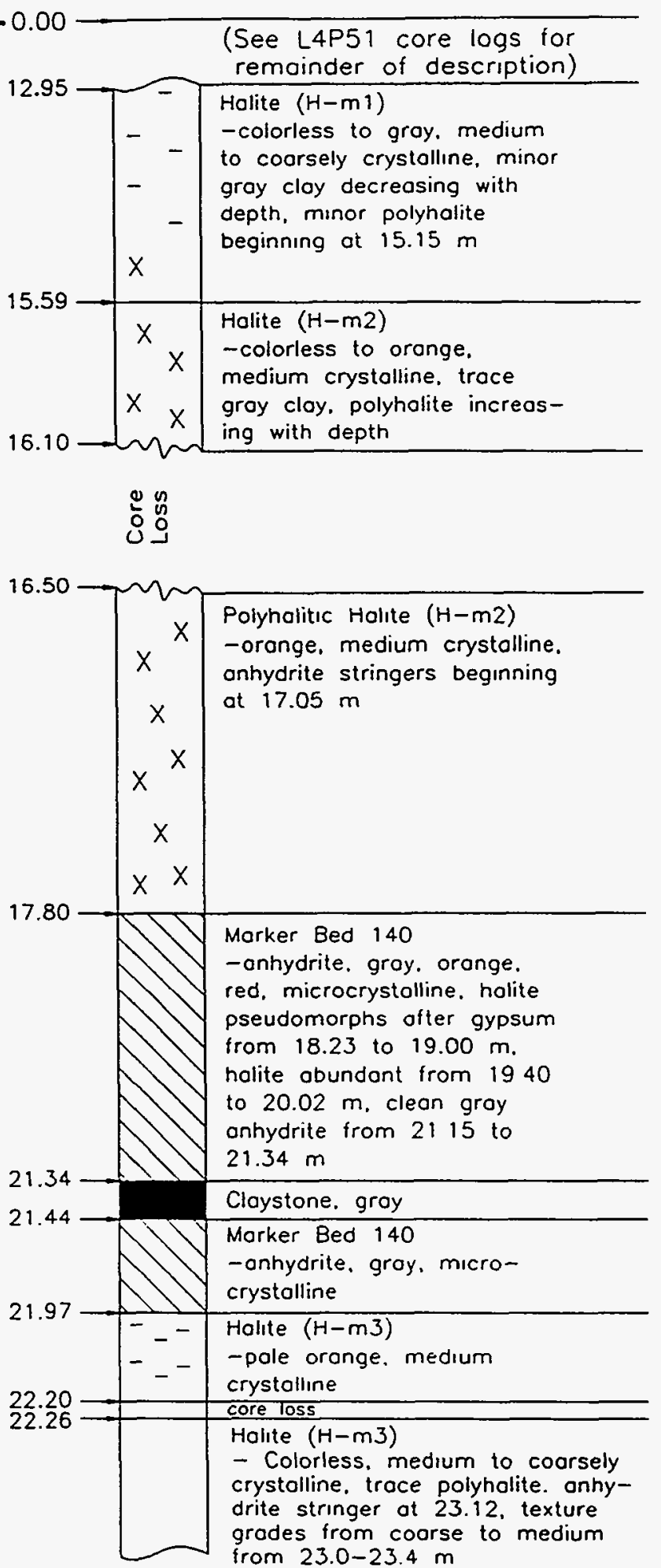

INTERA-6115-33-1

Figure 3-25a. Configuration \#1 of permeability test tool for sequence L4P51-D1. 
L4P51-D1

Test-Tool Configuration \#1

Borehole: L4P51

Test Tool: P51-DIA

Date: 09/28/94

Hole depth: 30.48
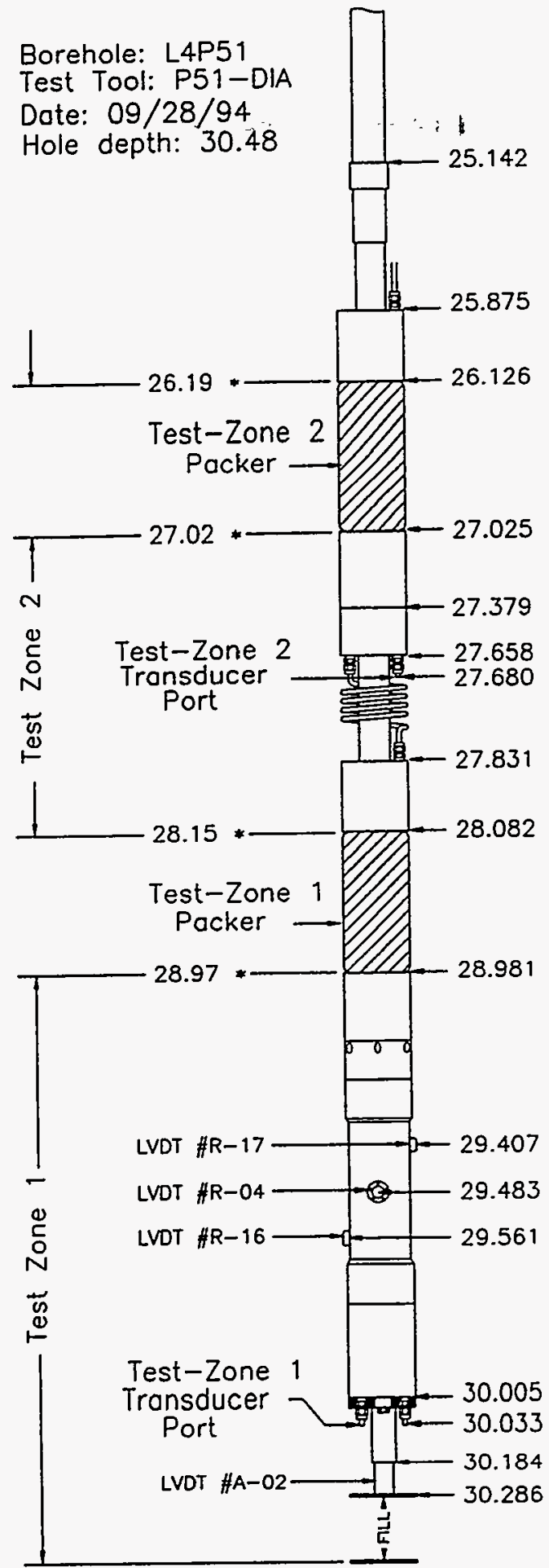

BOREHOLE STRATIGRAPHY

PAGE 2 OF =

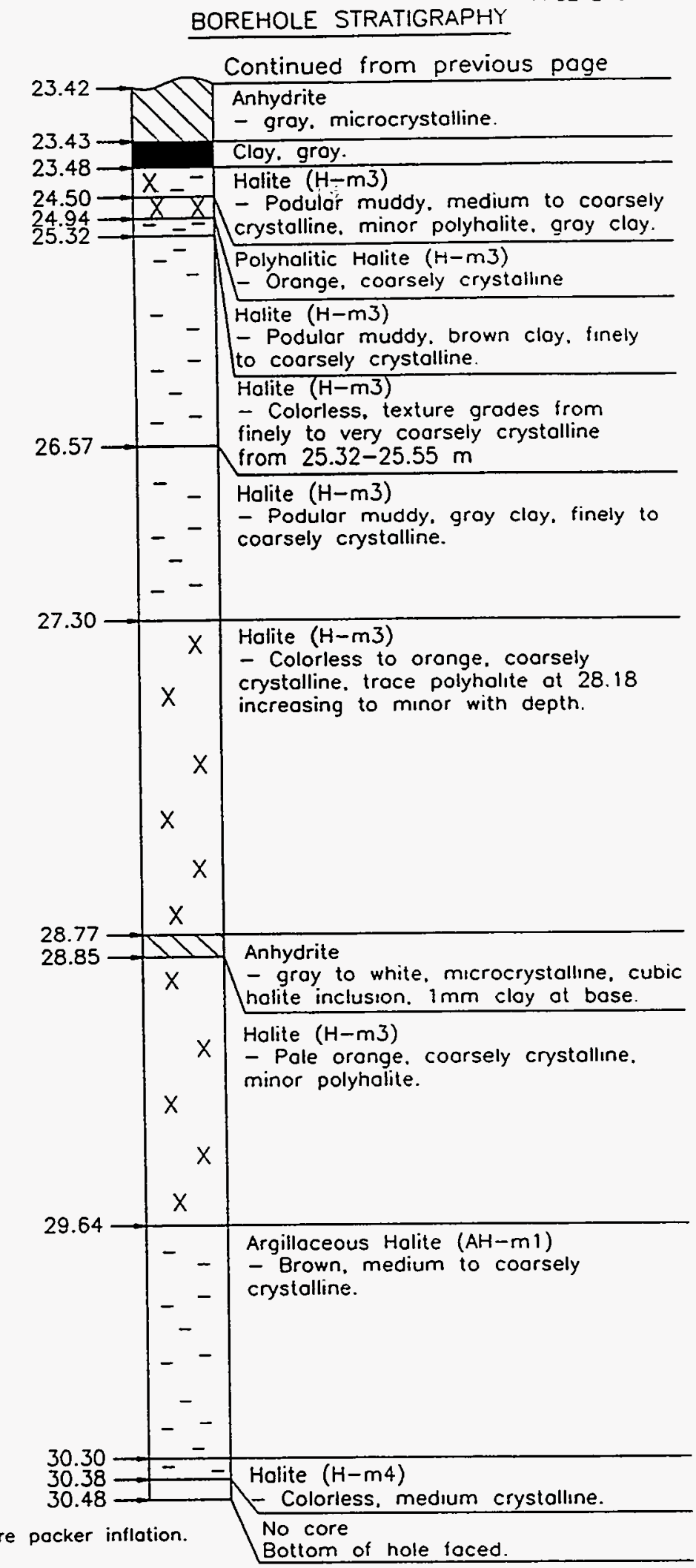

INTERA-61 15-34-1

\footnotetext{
- Estimated position ofter pocker inflotion.
}

Figure 3-25b. Configuration \#1 of permeability test tool for sequence L4P51-D1 (continued). 


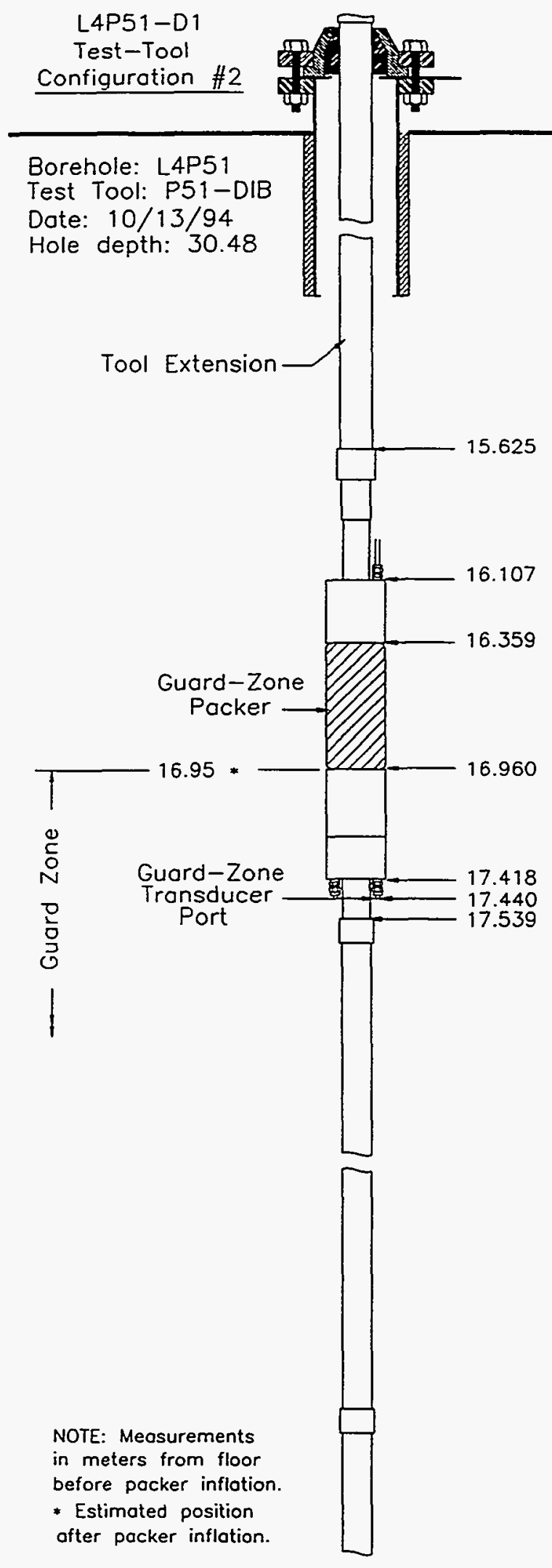

BOREHOLE STRATIGRAPHY

\begin{tabular}{|c|c|c|}
\hline & & $\begin{array}{l}\text { (See L4P51 core logs for } \\
\text { remainder of description) }\end{array}$ \\
\hline & $\begin{array}{l}- \\
- \\
- \\
x^{-}\end{array}$ & $\begin{array}{l}\text { Holite }(\mathrm{H}-\mathrm{m} 1) \\
\text { - colorless to groy, medium } \\
\text { to coorsely crystolline, minor } \\
\text { groy clay decreosing with } \\
\text { depth, minor polyholite } \\
\text { beginning ot } 15.15 \mathrm{~m}\end{array}$ \\
\hline & $\begin{array}{r}x \\
x \\
x\end{array}$ & $\begin{array}{l}\text { Halite (H-m2) } \\
\text { - colorless to oronge. } \\
\text { medium crystalline. troce } \\
\text { gray clay. polyholite increas- } \\
\text { ing with depth }\end{array}$ \\
\hline
\end{tabular}

过嵒

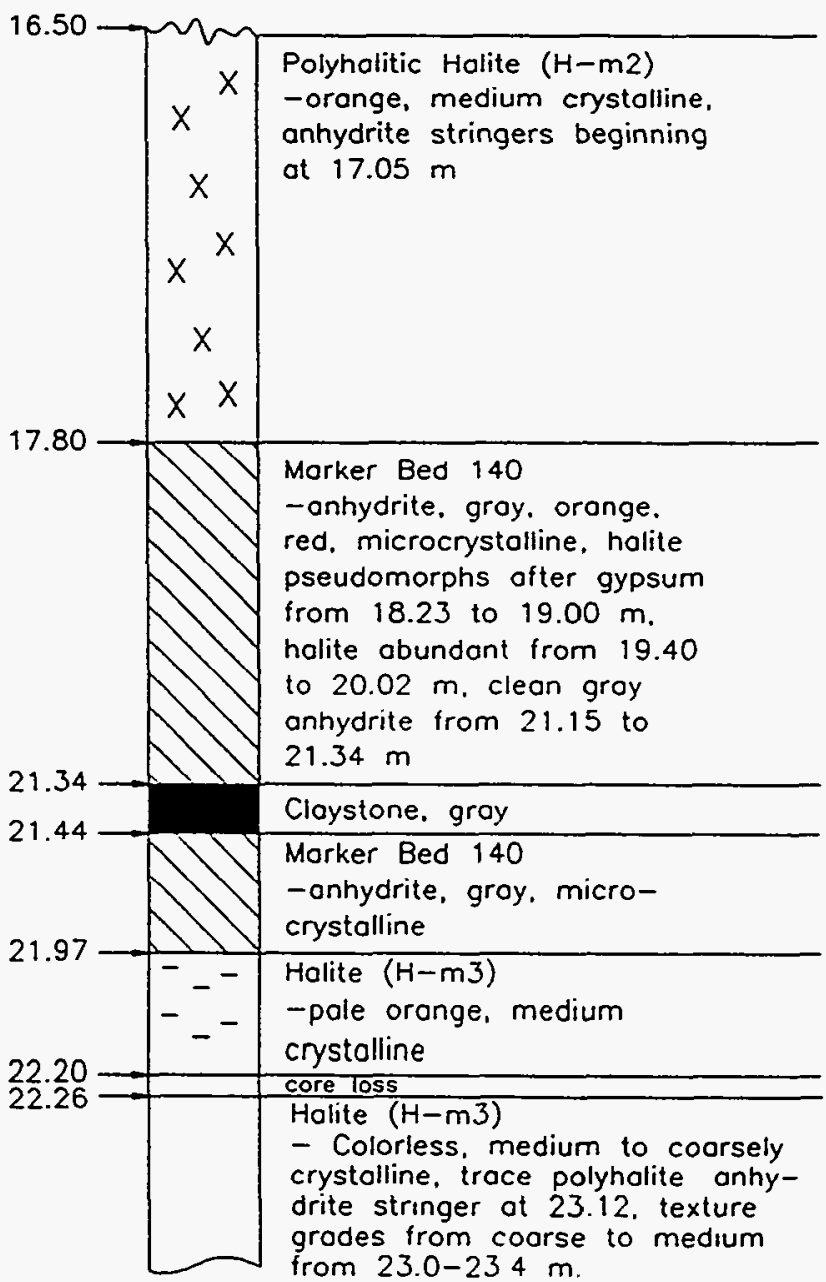

INIERA-6115-35- I

Figure 3-26a. Configuration \#2 of permeability test tool for sequence L4P51-D1. 
L4P51-D1

Test-Tool Configuration \#2

Borehole: L4P51

Test Tool: P51-DIB

Date: $10 / 13 / 94$

Hole depth: 30.48
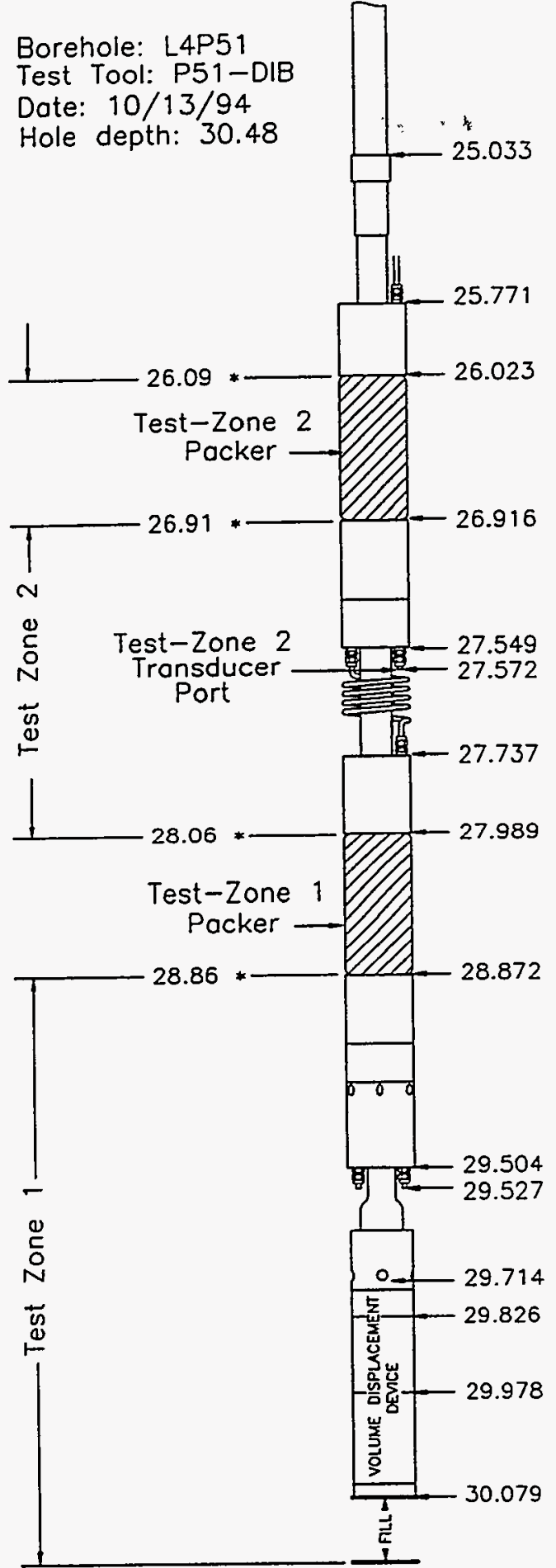

BOREHOLE STRATIGRAPHY

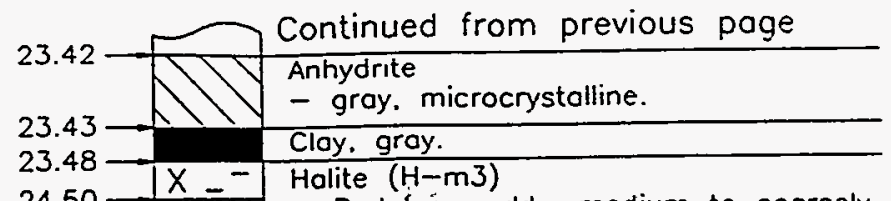

$24.50 \rightarrow X=X \quad$ Podular muddy. medium to coorsely

$24.94 \Rightarrow-1$ - crystolline, minor polyhalite. groy cloy.

Polyholitic Halite (H-m3)

- Orange, coarsely crystolline.

$-\quad-\int_{\text {Halite }(\mathrm{H}-\mathrm{m} 3)}$ - Podular muddy, brown clay. finely

$\begin{aligned} & - \\ & 26.57-\end{aligned}-\begin{aligned} & \text { Halite (H-m3) } \\ & - \text { Colorless, texture grades from } \\ & \text { finely to very coorsely crystalline }\end{aligned}$

$-\quad-\quad \begin{aligned} & \text { from 25.32-25.55 m. } \\ & \text { Holite }(\mathrm{H}-\mathrm{m} 3)\end{aligned}$

- Podular muddy, gray clay, finely to

- coorsely crystalline.

27

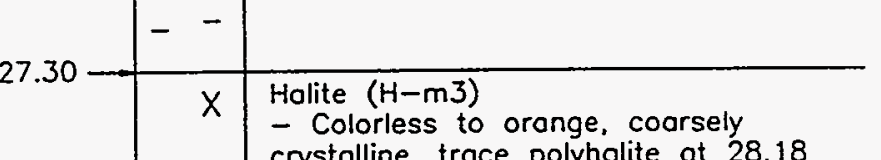

$X \quad$ crystolline, trace polyholite ot 28.18

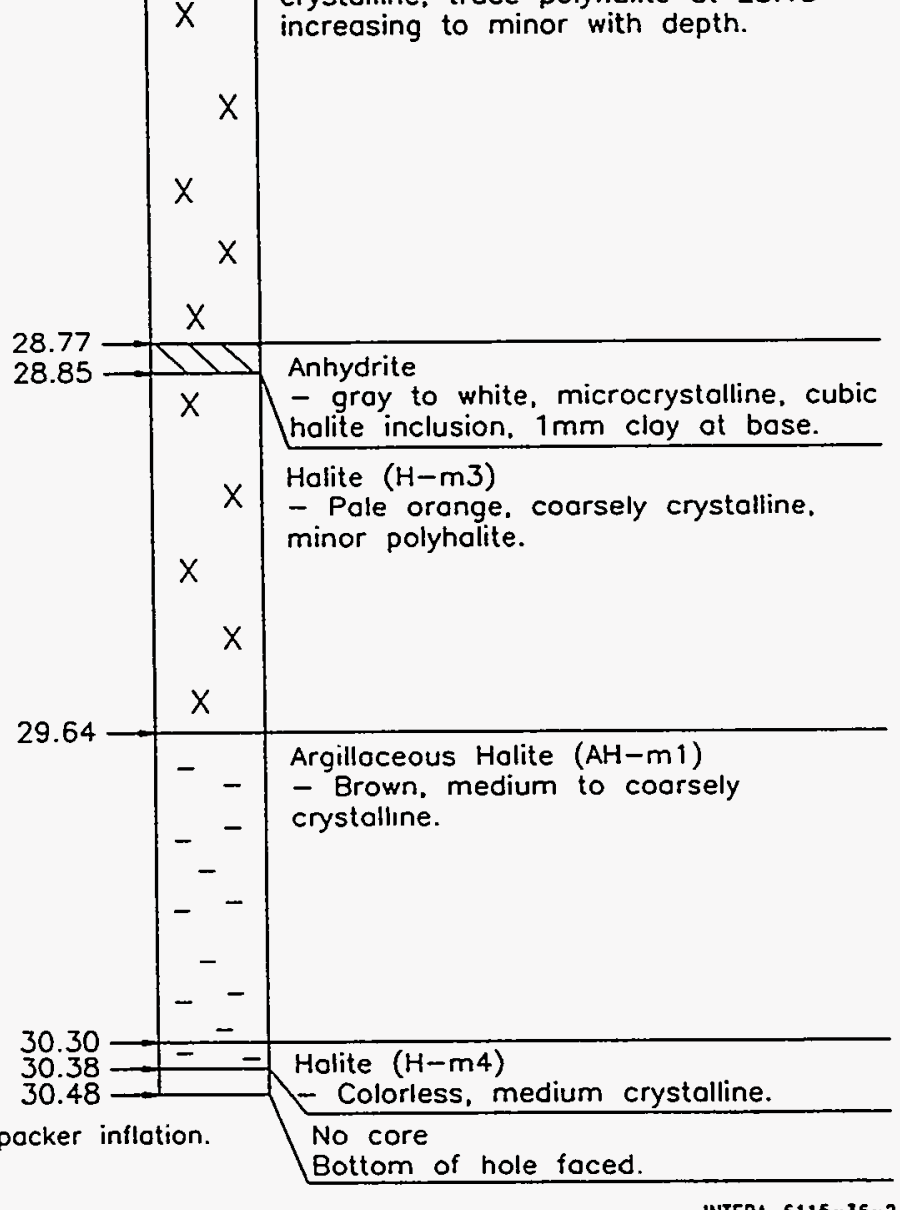

INTERA-6115-36-2

* Estimated position ofter pocker inflation.

Figure 3-26b. Configuration \#2 of permeability test tool for sequence L4P51-D1 (continued). 


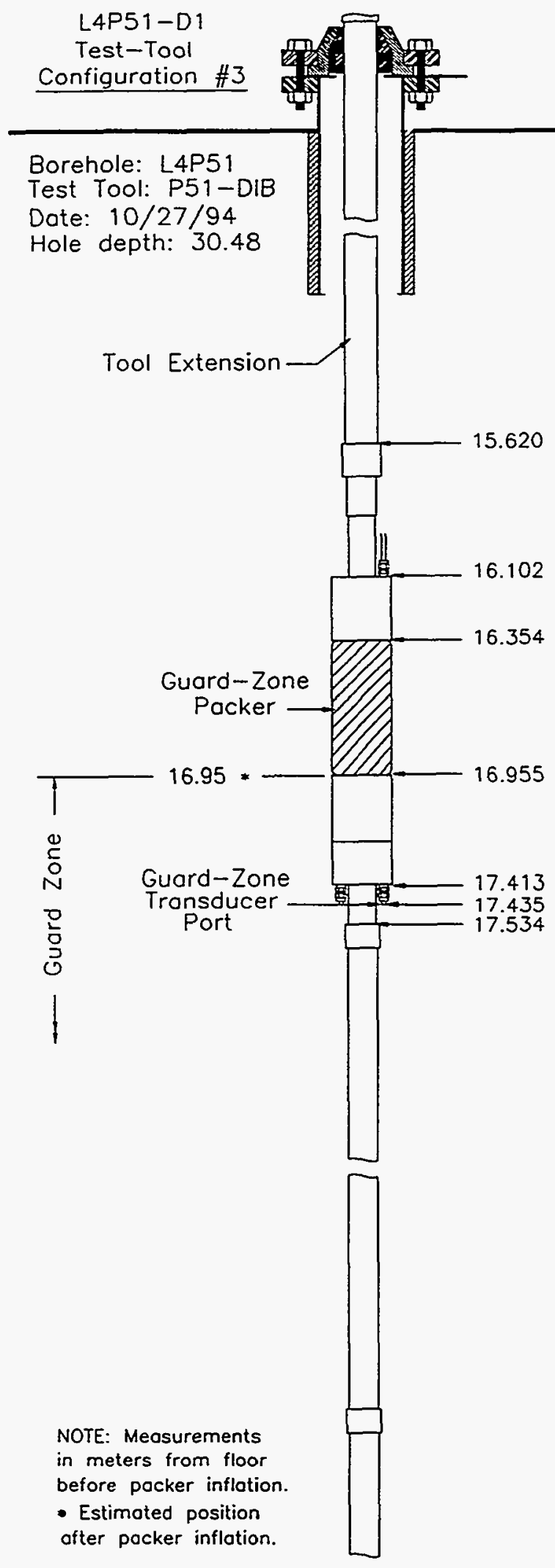

\begin{tabular}{|c|c|c|}
\hline & & $\begin{array}{l}\text { (See L4P51 core logs for } \\
\text { remainder of description) }\end{array}$ \\
\hline & $\begin{array}{l}- \\
- \\
-\end{array}$ & $\begin{array}{l}\text { Holite }(\mathrm{H}-\mathrm{m} 1) \\
\text { - colorless to groy, medium } \\
\text { to coorsely crystolline, minor } \\
\text { groy clay decreasing with } \\
\text { depth, minor polyhalite } \\
\text { beginning at } 15.15 \mathrm{~m}\end{array}$ \\
\hline & $\begin{array}{r}x \\
x \\
x\end{array}$ & $\begin{array}{l}\text { Halite (H-m2) } \\
\text { - colorless to oronge. } \\
\text { medium crystalline, troce } \\
\text { gray clay, polyhalite increas- } \\
\text { ing with depth }\end{array}$ \\
\hline
\end{tabular}

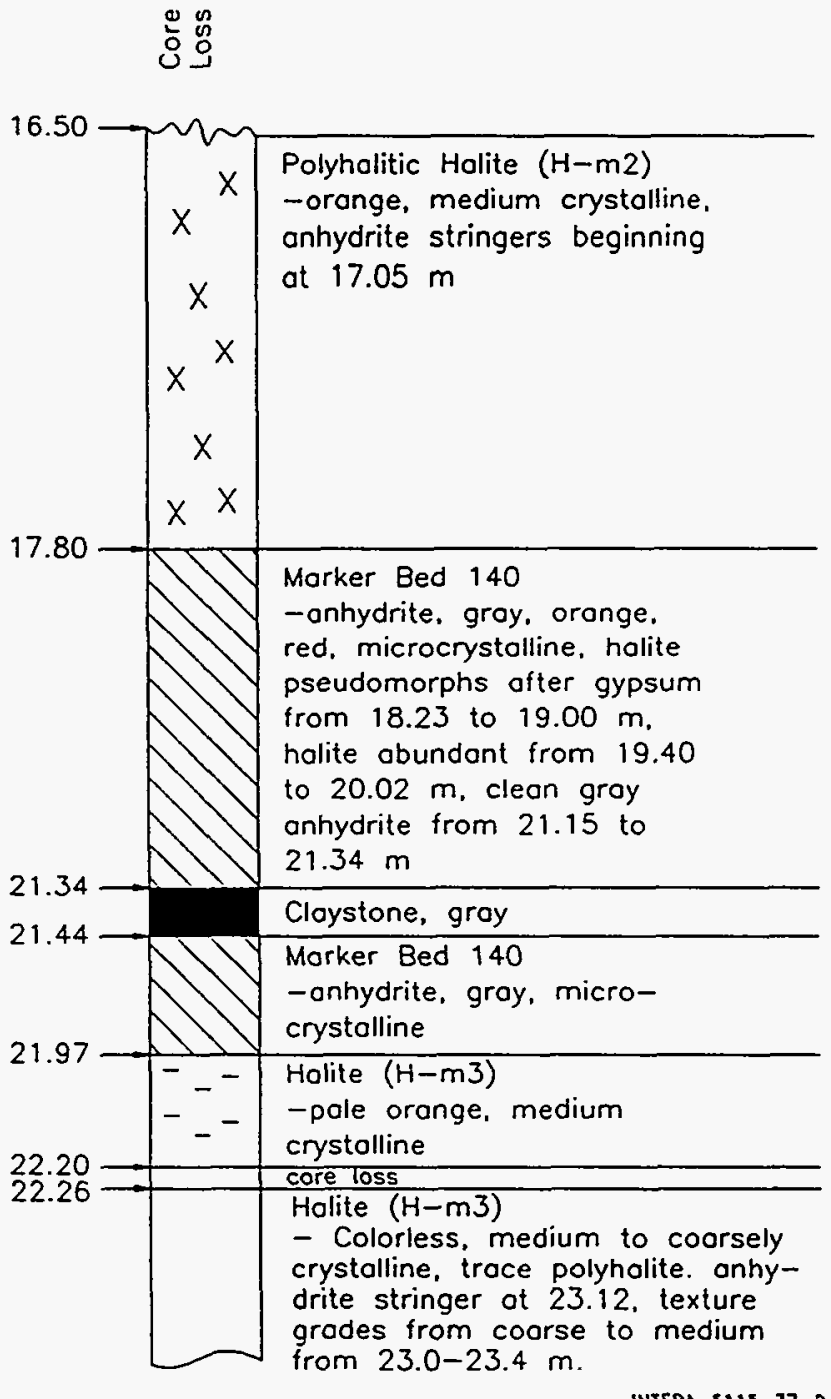

INTERA-6113-37-2

Figure 3-27a. Configuration \#3 of permeability test tool for sequence L4P51-D1. 
Borehole: L4P51

Test Tool: P51-DIB

Date: $10 / 27 / 94$

Hole depth: 30.48

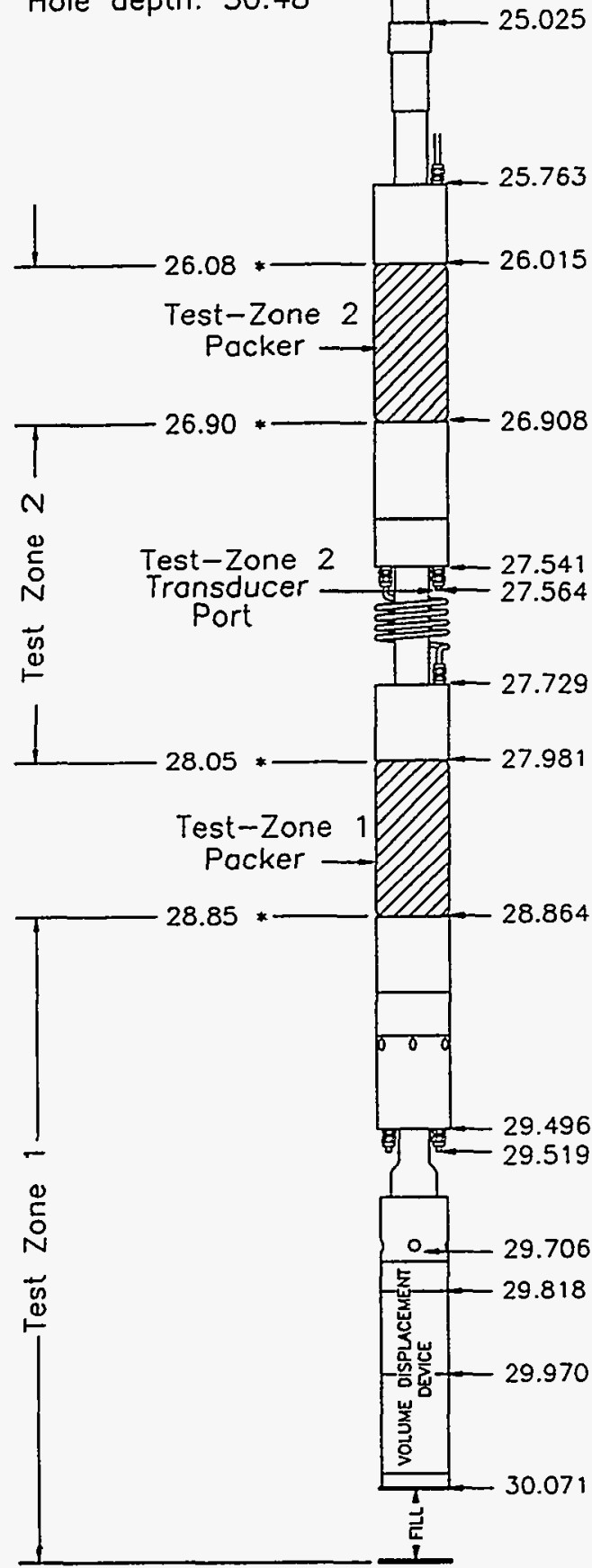

BOREHOLE STRATIGRAPHY

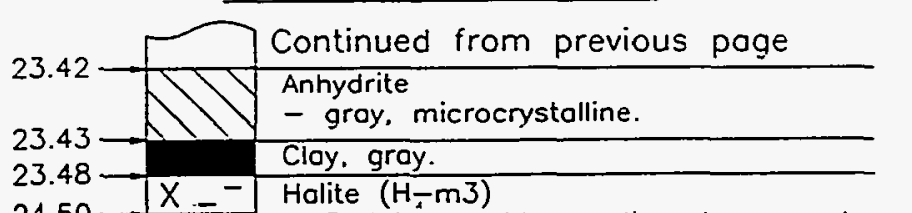

$25.34 \longrightarrow-1$ crystalline, minor polyholite, gray cloy.

Polyhalitic Holite ( $\mathrm{H}-\mathrm{m} 3)$

- Orange, coarsely crystalline.

$\begin{aligned} & - \\ & -\end{aligned}-\left[\begin{array}{l}\text { Holite }(H-m 3) \\ -\quad \text { Podulor muddy, brown clay. finely } \\ \text { to coorsely crystalline. }\end{array}\right.$

$\begin{gathered}- \\ -\end{gathered}-\begin{aligned} & \text { to coorsely crystalline. } \\ & -\quad \text { Colite (H-m3) } \\ & \text { finely to very texture grodes from }\end{aligned}$

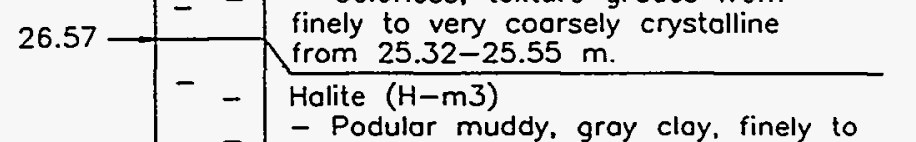

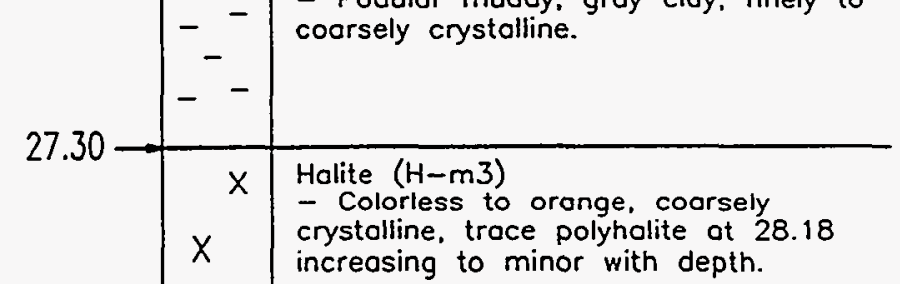

$X$ increosing to minor with depth.

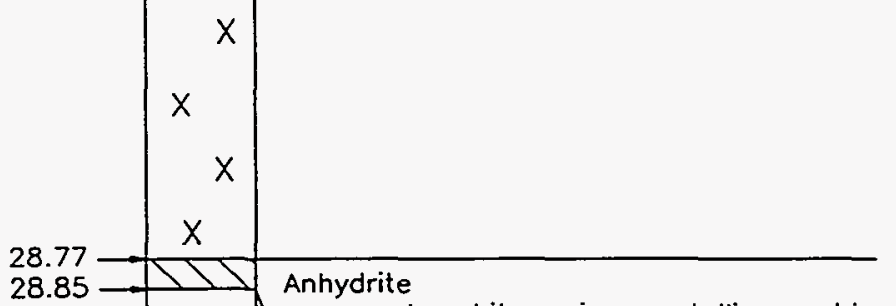

28.85

$X \quad$ - gray to white, microcrystalline, cubic
$\times \quad \begin{aligned} & \text { halite inclusion, } 1 \mathrm{~mm} \text { clay at base. } \\ & \text { Halite (H-m3) } \\ & - \text { Pole orange, coorsely crystalline, }\end{aligned}$
- Pole orange, coarsely crystalline, minor polyhalite.

$x$

NOTE: Meosurements in meters from floor before packer inflation.

- Estimated position after packer inflation.

INTERA-61 15-38-2

Figure 3-27b. Configuration \#3 of permeability test tool for sequence L4P51-D1 (continued). 


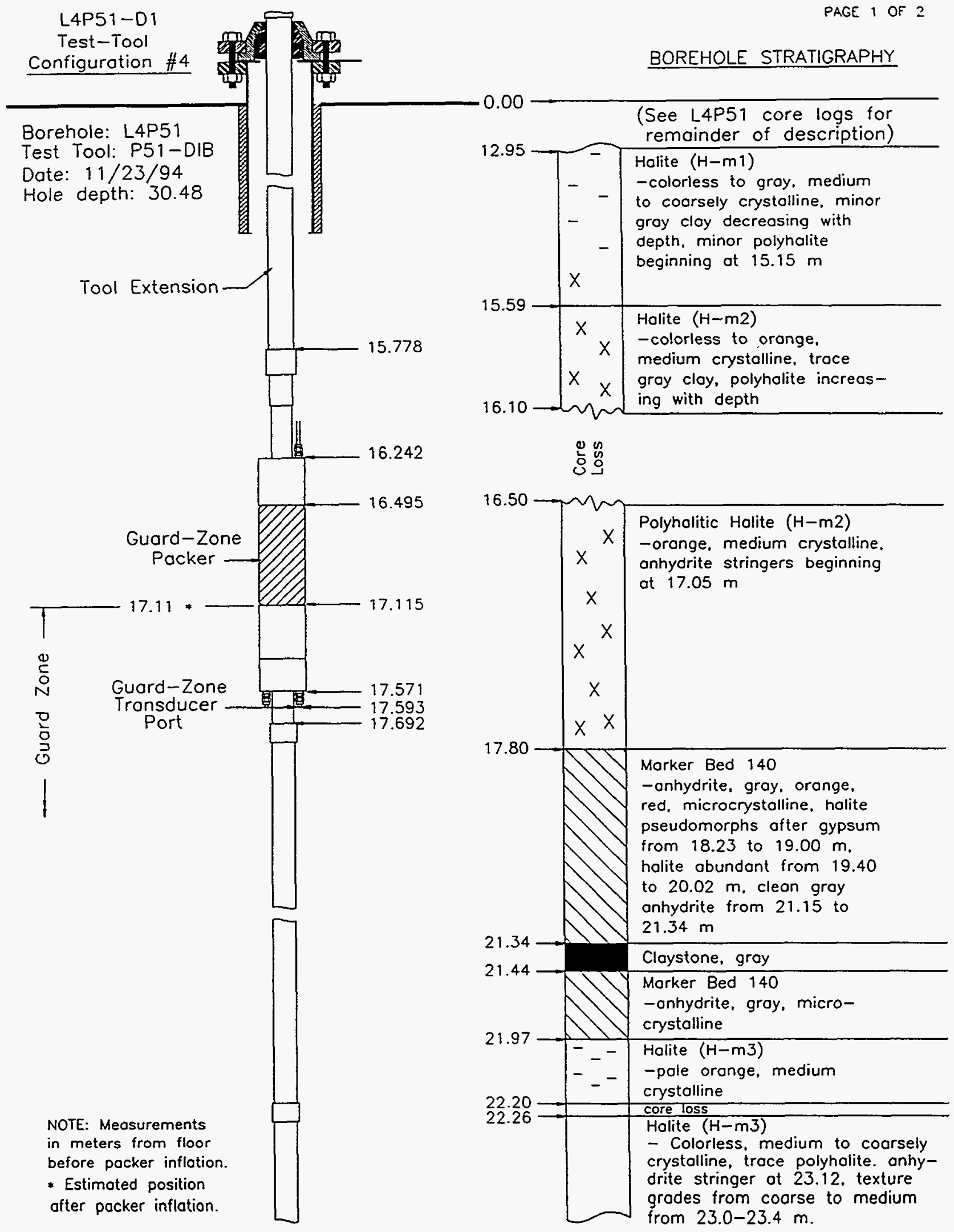

INTERA-6115-39-2

Figure 3-28a. Configuration \#4 of permeability test tool for sequence L4P51-D1. 
Borehole: L4P51

Test Tool: P51-DIB

Date: $11 / 23 / 94$

Hole depth: 30.48

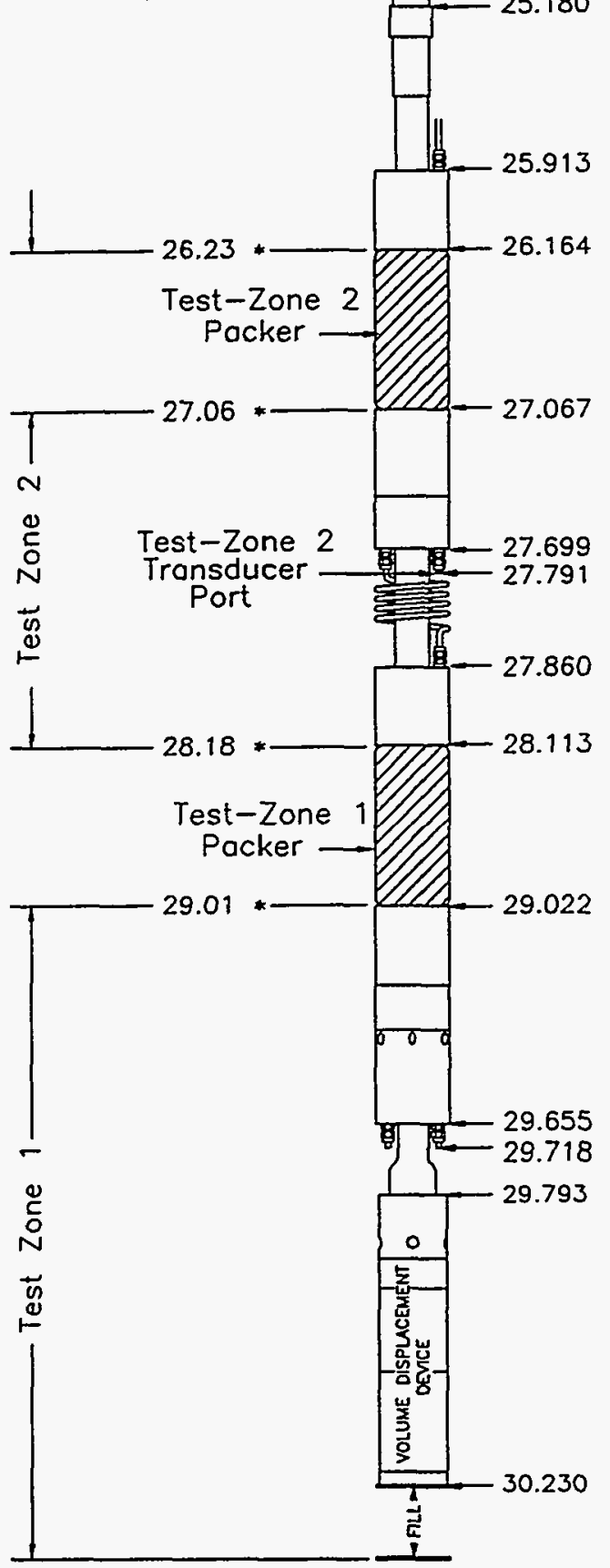

BOREHOLE STRATIGRAPHY

PAGE 2 OF 2

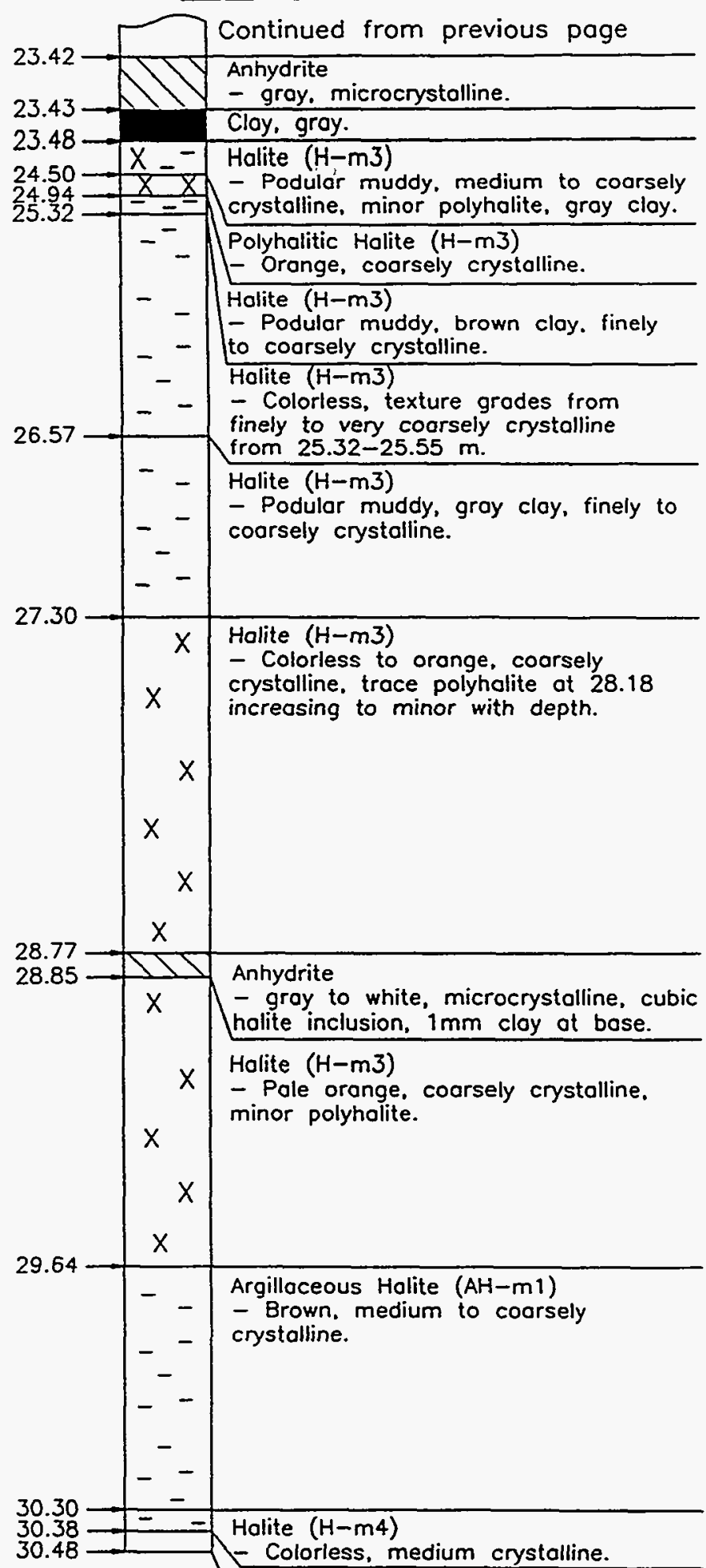

$30.48 \longrightarrow$ - Colorless, medium crystalline.

NOTE: Measurements in meters from floor before pocker inflation.

- Estimated position ofter pocker inflotion.

Figure 3-28b. Configuration \#4 of permeability test tool for sequence L4P51-D1 (continued). 


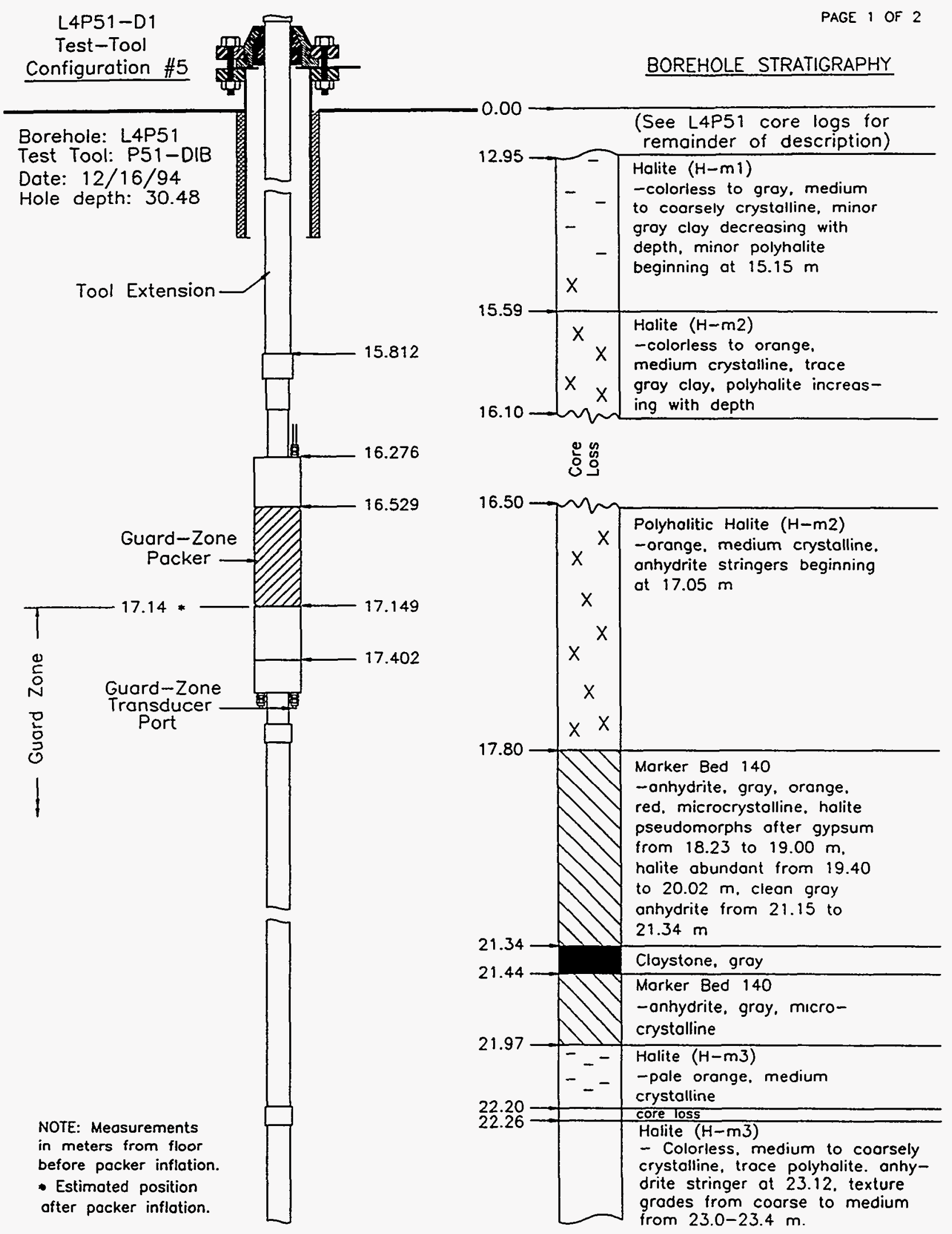

INTERA-61 15-41-1

Figure 3-29a. Configuration \#5 for permeability test tool for sequence L4P51-D1. 
Borehole: L4P51

Test Tool: P51-DIB

Date: $12 / 16 / 94$

Hole depth: 30.48

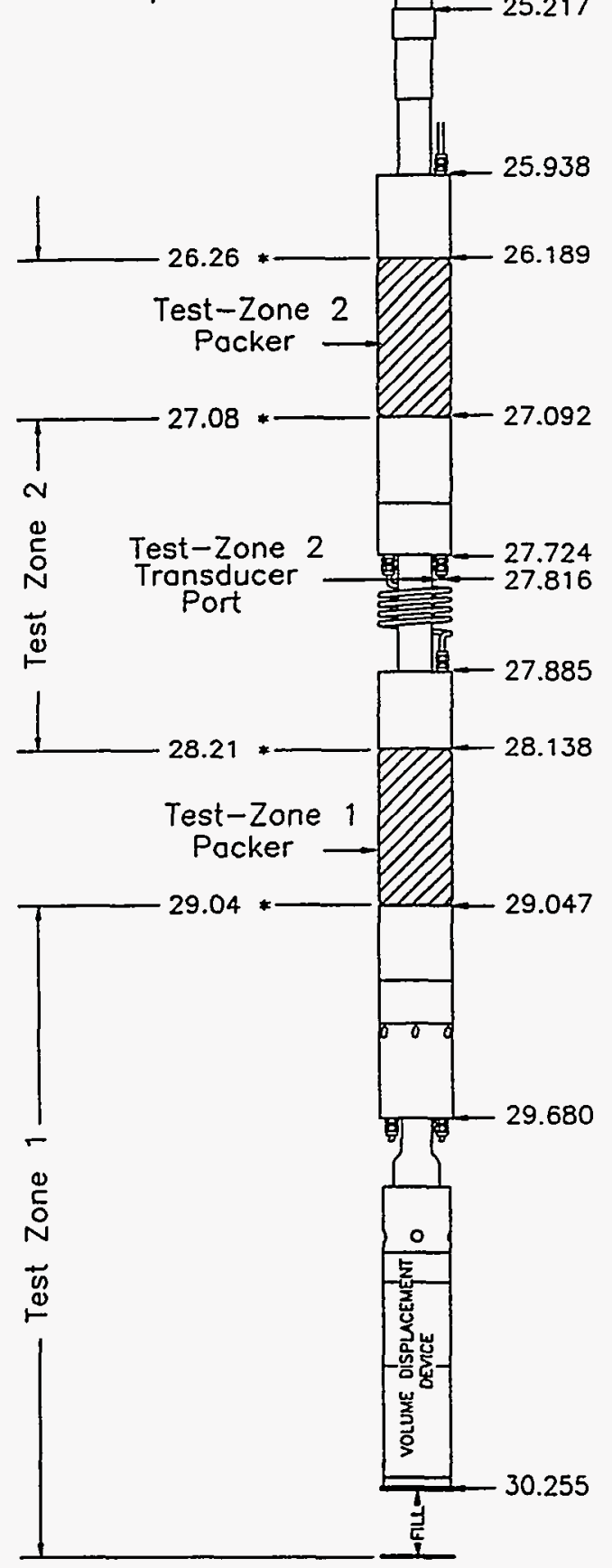

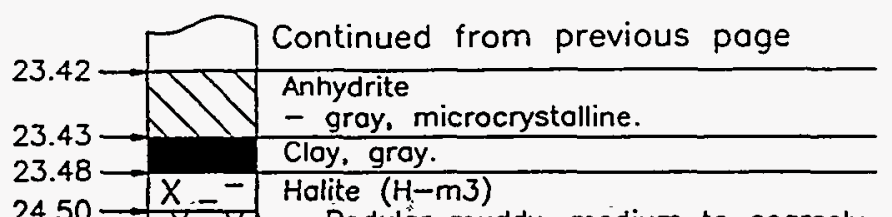

$24.50-\frac{X}{X}=-$ Podular muddy. medium to coorsely $25.32 \Rightarrow-2$ crystalline. minor polyhalite, groy clay.

$\begin{gathered}- \\ -\end{gathered}-\left\{\begin{array}{l}\text { Polyhalitic Holite }(\mathrm{H}-\mathrm{m} 3) \\ - \text { Oronge, coorsely crystalline. } \\ \text { Halite (H-m3) } \\ - \text { Podular muddy, brown clay, finely }\end{array}\right.$

- to coarsely crystalline.

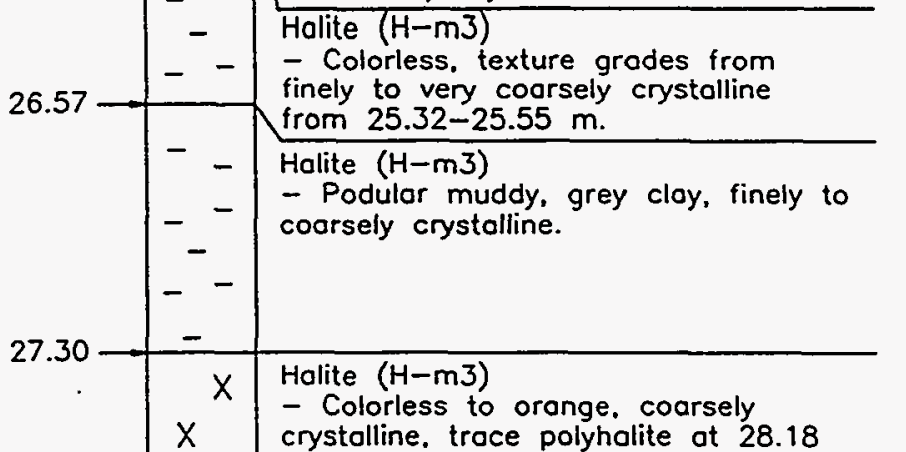
increasing to minor with depth.

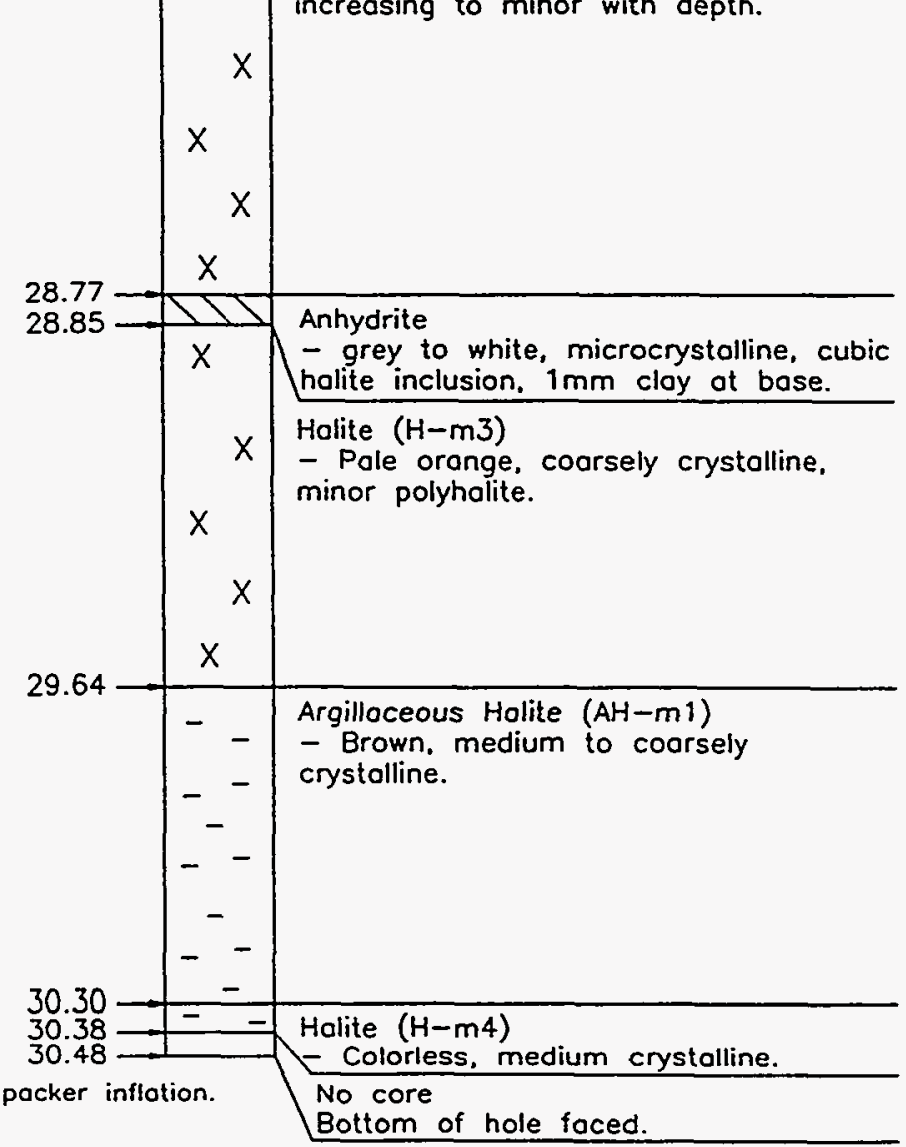

- Estimoted position ofter pocker inflation.

INTERA-6115-42-2

Figure 3-29b. Configuration \#5 for permeability test tool for sequence L4P51-D1 (continued). 
Borehole: L4P51

Test Tool: P51-D2

Date: 04/05/95

Hole depth: 30.48

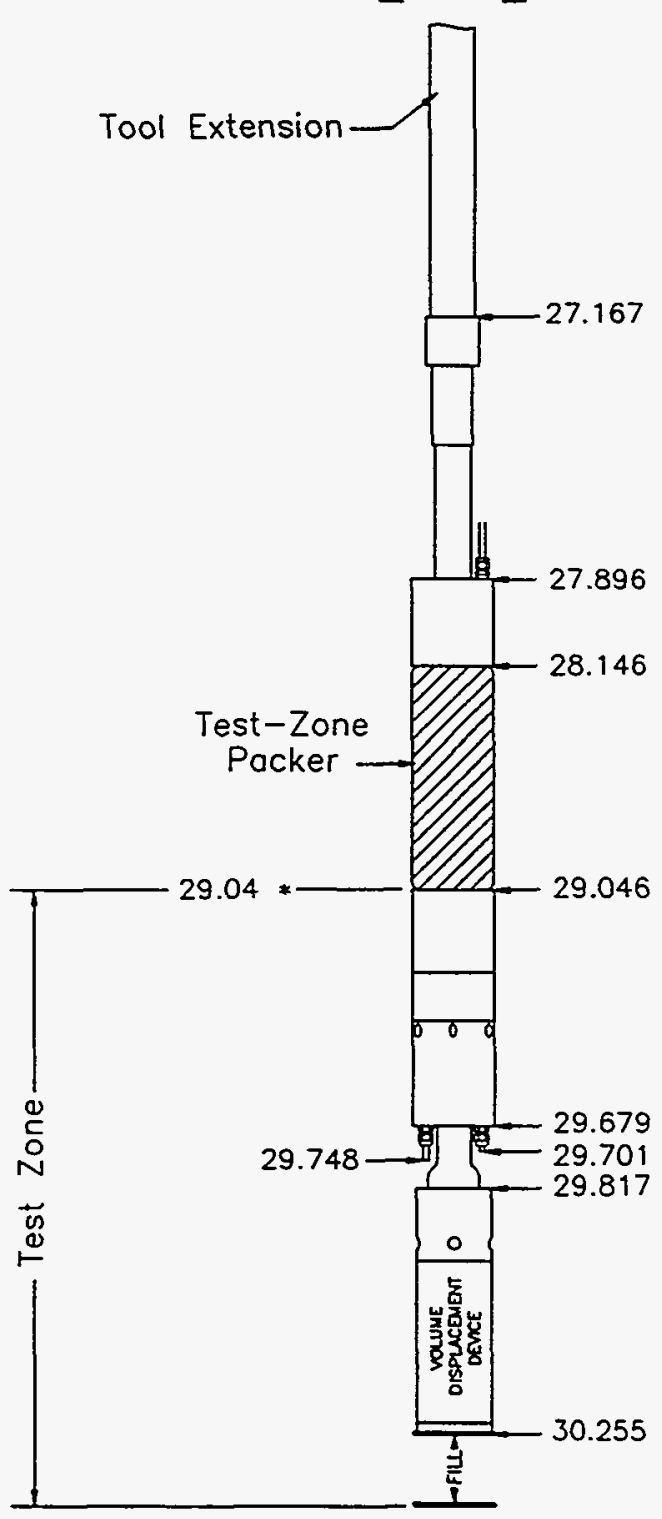

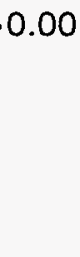

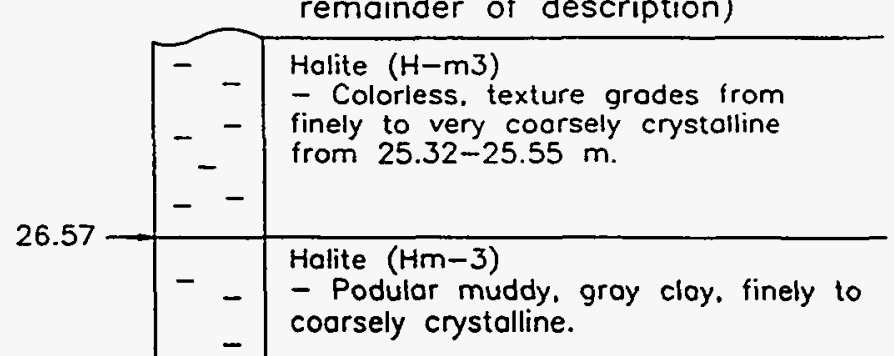

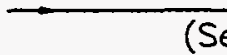

See L4P51 core logs for

remainder of description)

$-$

$(-$

$27.30-\frac{-}{-}-1$ Holite (H-m3)

- Colorless to orange. coorsely

crystalline, trace polyhalite at 28.18

increasing to minor with depth.

$x$

$x$

28.77

28.85

$x$

$x \quad$ - gray to white, microcrystalline, cubic halite inclusion. $1 \mathrm{~mm}$ cloy at base.

Halite $(\mathrm{H}-\mathrm{m} 3)$

$X$ - Pale orange. coorsely crystalline. minor polyhalite.

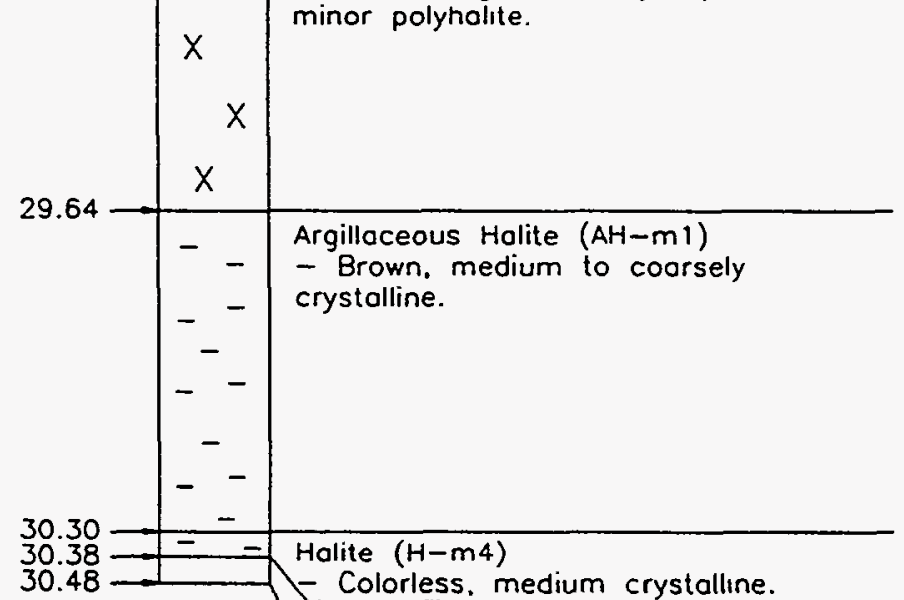

30.48

Colorless. medium crystalline.

No core

Bottom of hole foced.

NOTE: Measurements in meters from floor before packer inflation.

- Estimoted position ofter packer inflotion.

Figure 3-30. Configuration of permeability test tool for sequence L4P51-D2. 


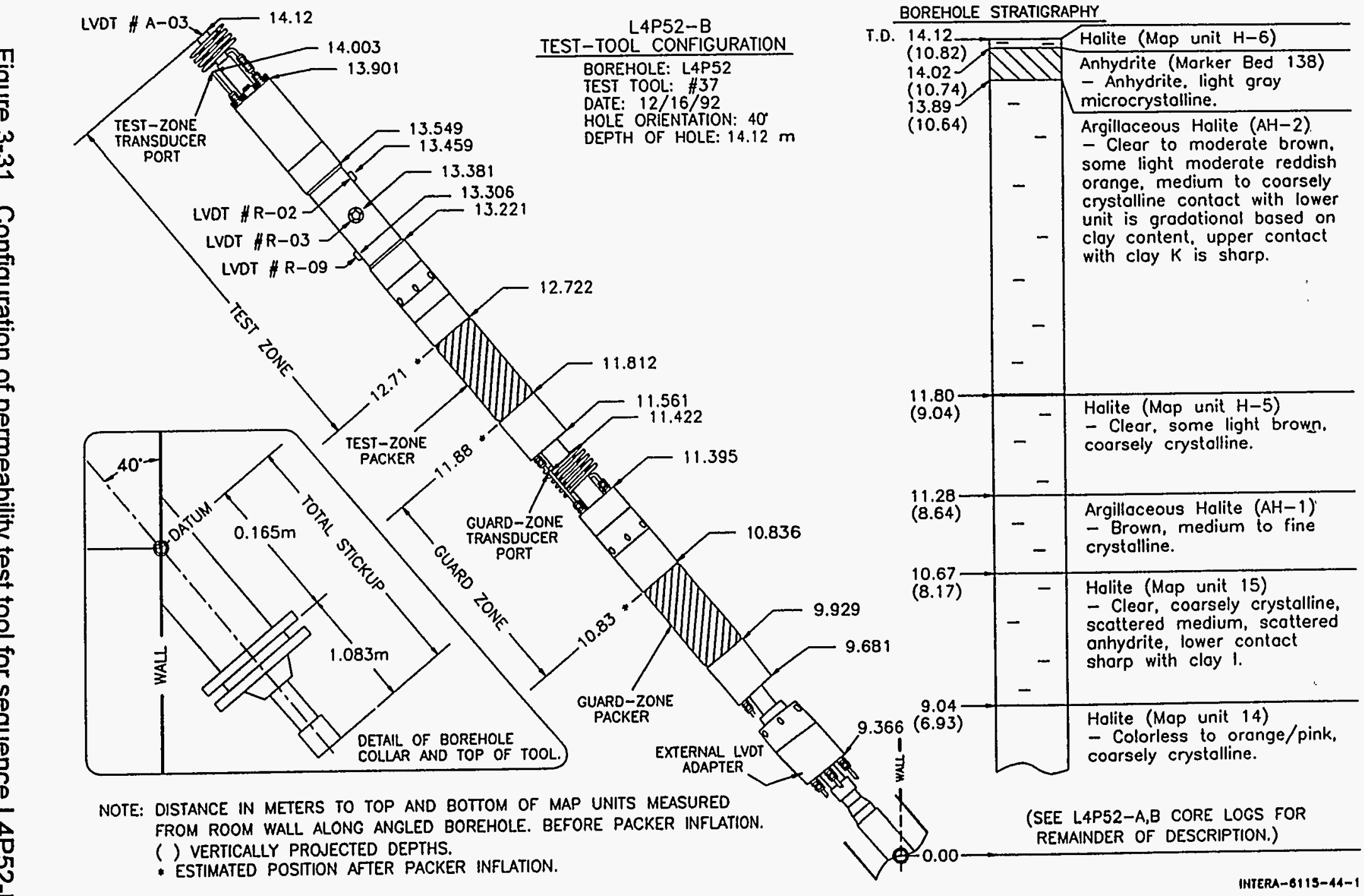

BOREHOLE STRATIGRAPHY 
LVDT \# A-02

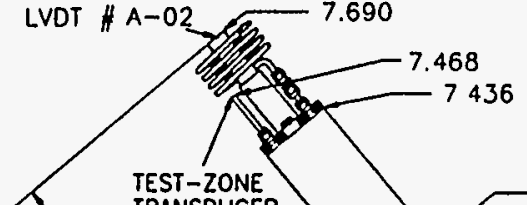

TEST-ZONE TRANSOUCER PORT

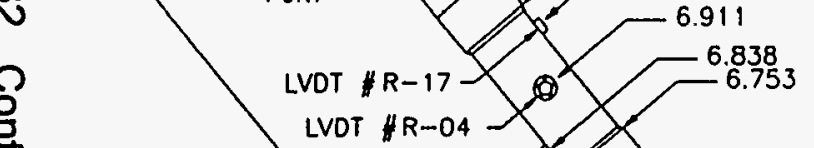

LVOT \#R-04

LVDT \# $R-16-$<smiles>[C]=C</smiles>
so
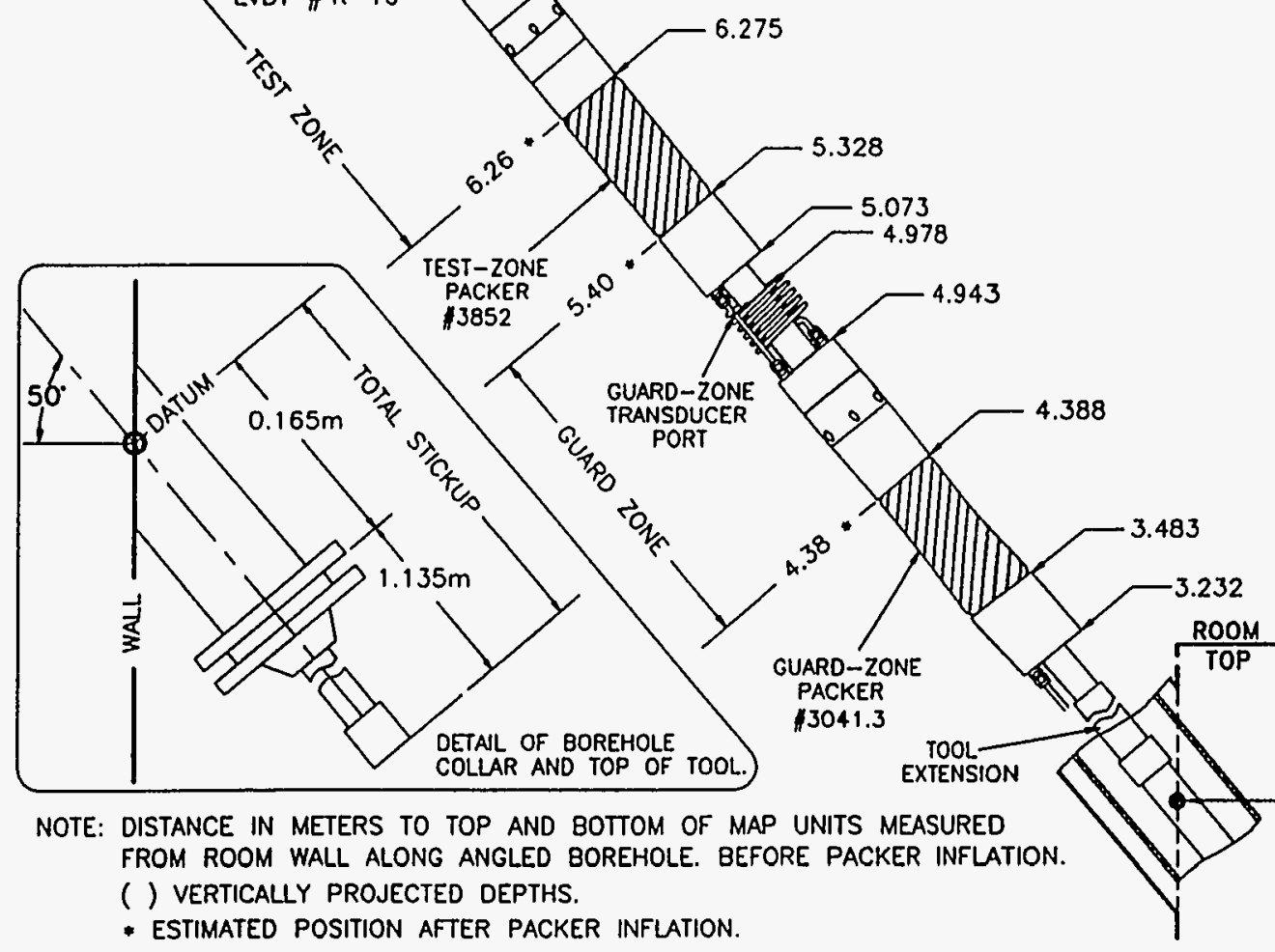

SIP74-A TEST-TOOL CONFIGURATION

BOREHOLE: SIP74

TEST TOOL. \#35

HOLE ORIENTATION: 50

DEPTH OF HOLE: $7.69 \mathrm{~m}$

BOREHOLE STRATIGRAPHY

T.D. $7.69-1-x \mid$ Map unit 12

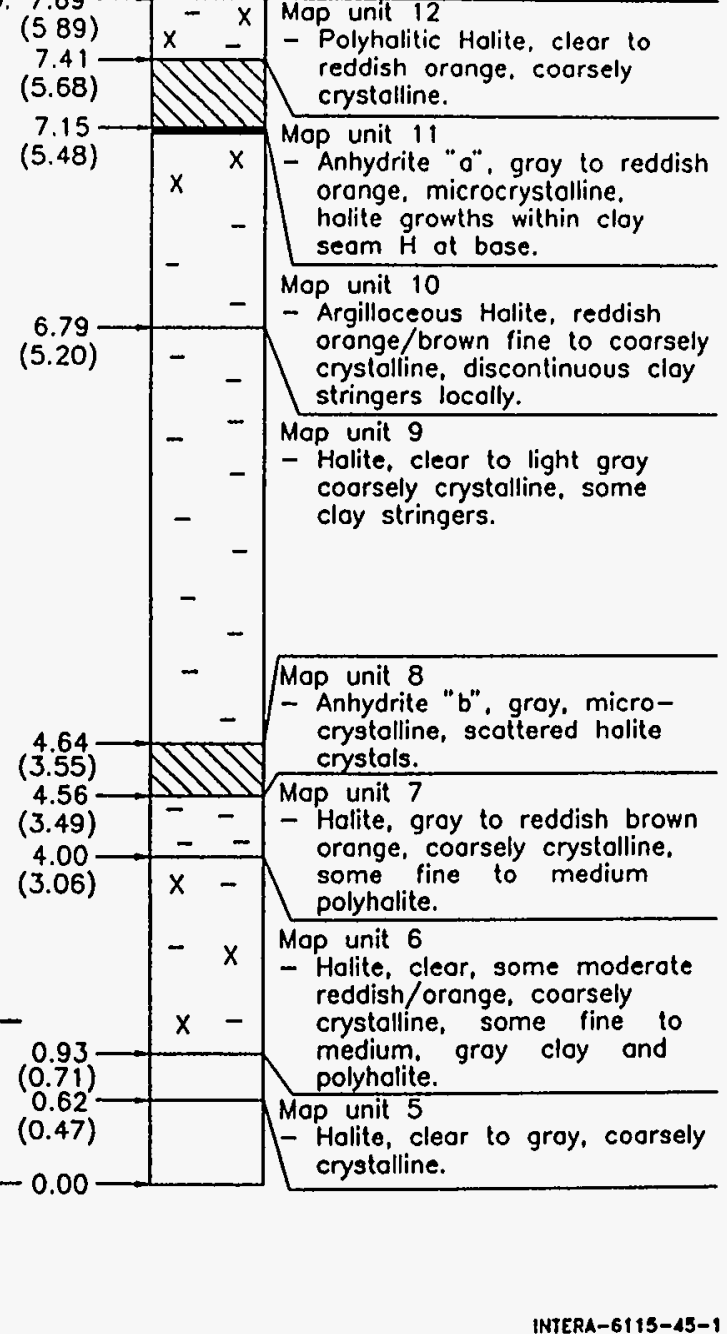


BOREHOLE STRATIGRAPHY

16.878
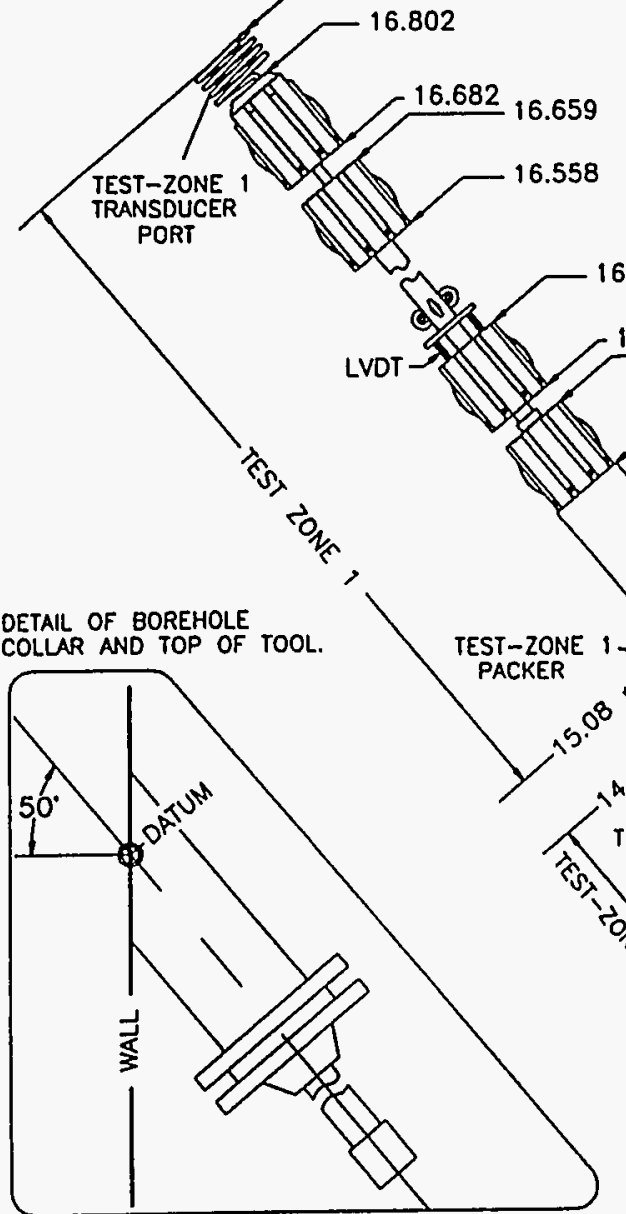

16.267

16.165

$-16.033$

NOTE: DISTANCE IN METERS TO TOP AND BOTTOM OF MAP UNITS MEASURED FROM ROOM WALL ALONG ANGLED BOREHOLE. BEFORE PACKER INFLATION.

() VERTICALLY PROJECTED DEPTHS.

- ESTIMATEO POSITION AFTER PACKER INFLATION.

$r^{13.931}$

$-13.198$
S1P74-B TEST-TOOL CONFIGURATION BOREHOLE: S1P74 TEST TOOL: P74-B HOLE ORIENTATION: DEPTH OF HOLE: $16.88 \mathrm{~m}$.
T.D.

\begin{tabular}{|c|c|c|}
\hline 12.93$)$ & & Borehole foced off. \\
\hline$\left(\begin{array}{l}6.82 \\
(12.88)\end{array}\right.$ & & $\begin{array}{l}\text { Halite (Mop unit } \mathrm{H}-6 \text { ) } \\
\text { Pole oronge, coorsely crystalline, } \\
\text { troce polyholite. }\end{array}$ \\
\hline & & $\begin{array}{l}\text { Anhydrite (MB 138) } \\
\text { - Grey, microcrystolline. }\end{array}$ \\
\hline
\end{tabular}

$(16.38 \rightarrow$

16.25
$(12.45)$

(1)

- Grey, microcrystalline.

Cloy (Clay K)

Helite (Mop und AH-2

- Podular mud AH-2) brown

clay, colorless, medium crystalline,

- clay lens ot $15.63,0.5 \mathrm{~cm}$ thick.

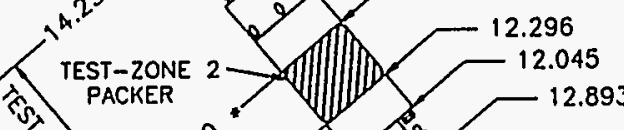

年, $10^{9}=11.340$

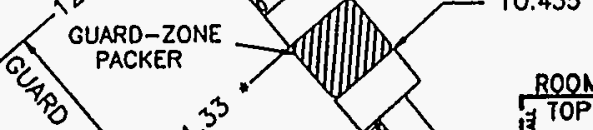

i $1: 15$

$-{ }^{-}-$

$-$

$(10.88)--$ Holite (Mop unit $\mathrm{H}-5)$

- troce of clay begins at 13.8

increosing with depth.

$(13.49-33)--\quad$ Holite (Map unit AH-1, Clay J)

$\begin{array}{lll}12.58-1 & - & \text { - Podular muddy, brown clay. } \\ \text { colorless, medium crystalline. }\end{array}$

-

- $\quad \begin{aligned} & \text { Halite (Map units } 14 \\ & \text { - Colorless, coorsely crystalline. }\end{aligned}$

- Anhydrite

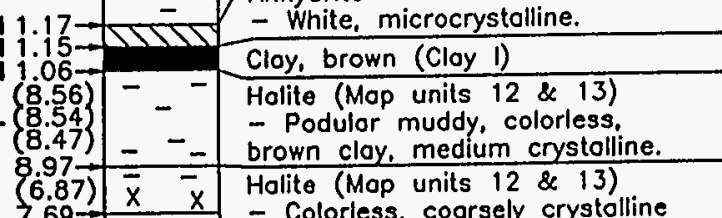

(5.89)

white onhydrite stringers from

7.725-7.88, trace polyhalite from 7.88-8.06, clay content gradually

increoses from 8.50-8.97.

See S1P74-A core description. 


\subsection{Permeability Test Data}

The following section presents the data from the permeability-testing sequences conducted. Four zone-compressibility tests (see Section 6.1), 7 pulse-withdrawal tests, 11 constant-pressure-injection tests, and 11 constant-pressure-withdrawal tests were performed.

Acronyms used in the tables presented in this section are as follows:

$T Z=$ Test Zone

TZP $=$ Test Zone Packer .

TZ1P = Test Zone 1 Packer (Triple-Packer Configuration)

TZ2P = Test Zone 2 Packer (Triple-Packer Configuration)

$G Z=$ Guard Zone

GZP = Guard Zone Packer

$\mathrm{CMP}=$ Compressibility Test

$\mathrm{CPI}=$ Constant-Pressure-Injection Test

CPW $=$ Constant-Pressure-Withdrawal Test

PW $=$ Pulse-Withdrawal Test

$\mathrm{TZ1}$ = Bottom-Hole Zone (Triple-Packer Configuration)

TZ2 = Middle Zone (Triple-Packer Configuration)

Zone Fluid Volume = Isolated Zone Volume+Tubing Volume-Tool Volume $\mathrm{AH}=$ Argillaceous Halite

Table 3-3 summarizes the information from these tests. Complete data files and abridged tabulations of the data are stored in the SWCF under WPO \#42269.

Table 3-3. Test Summary Associated with Permeability Testing

\begin{tabular}{|c|c|c|c|c|c|c|c|c|c|c|c|}
\hline $\begin{array}{c}\text { Permeability } \\
\text { Test } \\
\text { Sequence }\end{array}$ & Zone & $\begin{array}{c}\text { Date } \\
\text { Started } \\
\text { (mm-dd-yy) }\end{array}$ & $\begin{array}{l}\text { Test } \\
\text { Type }\end{array}$ & Unit & $\begin{array}{l}\text { Initial } \\
\text { Pressure } \\
\text { (MPa) }\end{array}$ & $\begin{array}{c}\text { Final } \\
\text { Pressure } \\
\text { (MPa) }\end{array}$ & $\begin{array}{c}\text { Fluid Volume } \\
\text { Injected (+) } \\
\text { Withdrawn (-) } \\
\text { (mL) }\end{array}$ & $\begin{array}{l}\text { Zone } \\
\text { Length } \\
\text { (cm) }\end{array}$ & $\begin{array}{l}\text { Zone } \\
\text { Radius } \\
\text { (cm) }\end{array}$ & $\begin{array}{c}\text { Tool } \\
\text { Volume } \\
\text { (mL) }\end{array}$ & $\begin{array}{l}\text { Zone Fluid } \\
\text { Volume } \\
\text { (mL) }\end{array}$ \\
\hline L4P51-C1 & GZ & $5-7-92$ & PW\#1 & Halite & 8.826 & 0.011 & -225 & 105 & 5.56 & 6586 & 3773 \\
\hline L4P51-C1 & $G Z$ & $5-26-92$ & CPW\#1 & Halite & 8.246 & 6.106 & -1750 & 104 & 5.56 & 6586 & 3773 \\
\hline L4P51-C1 & $T Z$ & $6-23-92$ & CPW\#1 & MB140 & 8.584 & 8.060 & -9834 & 477 & 5.165 & 32308 & 7999 \\
\hline L4P51-C1 & GZ & $7-29-92$ & PW\#2 & Halite & 7.567 & 0.073 & -96.5 & 104 & 5.56 & 6586 & 3773 \\
\hline L4P51-C1 & $\pi$ & $8-26-92$ & CPW\#2 & MB140 & 8.833 & 8407 & -5056 & 477 & 5.165 & 32308 & 7999 \\
\hline L4P51-C1 & $T Z$ & $10-13-92$ & CPW\#3 & MB140 & 8.952 & 8.462 & -13853 & 477 & 5.165 & 32308 & 7999 \\
\hline L4P51-C1 & $\pi$ & $10-26-92$ & CPW\#4 & MB140 & 8.650 & 7.943 & -22326 & 477 & 5.165 & 32308 & 7999 \\
\hline L4P51-C1 & $T Z$ & $2-11-93$ & CMP & MB140 & 7.578 & 0.344 & N/A & 477 & 5.165 & 32308 & 7999 \\
\hline L4P51-C1 & $\pi z$ & 4-15-93 & CPW\#5 & MB140 & 8.621 & 7.728 & -143869 & 477 & 5.165 & 32308 & 7999 \\
\hline L4P51-C2 & $T Z 2$ & $3-9-94$ & CPW\#1 & Halite & 7.849 & 7.255 & -1227 & 104 & 5.56 & 6466 & 3998 \\
\hline L4P51-C2 & TZ2 & $4-4-94$ & CPI \#1 & Halite & 7.963 & 8.136 & +3025 & 104 & 5.56 & 6466 & 3998 \\
\hline L4P51-C2 & TZ2 & $4-21-94$ & $\mathrm{CP} 1 \# 2$ & Halite & 8.080 & 8.885 & +4677 & 104 & 5.56 & 6466 & 3998 \\
\hline
\end{tabular}


Table 3-3 (Continued). Test Summary Associated with Permeability Testing

\begin{tabular}{|c|c|c|c|c|c|c|c|c|c|c|c|}
\hline $\begin{array}{c}\text { Permeability } \\
\text { Test } \\
\text { Sequence }\end{array}$ & Zone & $\begin{array}{c}\text { Date } \\
\text { Started } \\
\text { (mm-dd-yy) }\end{array}$ & $\begin{array}{l}\text { Test } \\
\text { Type }\end{array}$ & Unit & $\begin{array}{l}\text { Initial } \\
\text { Pressure } \\
\text { (MPa) }\end{array}$ & $\begin{array}{c}\text { Final } \\
\text { Pressure } \\
\text { (MPa) }\end{array}$ & $\begin{array}{l}\text { Fluid Volume } \\
\text { Injected (+) } \\
\text { Withdrawn (-) } \\
\text { (mL) }\end{array}$ & $\begin{array}{l}\text { Zone } \\
\text { Length } \\
\text { (cm) }\end{array}$ & $\begin{array}{c}\text { Zone } \\
\text { Radius } \\
\text { (cm) }\end{array}$ & $\begin{array}{c}\text { Tool } \\
\text { Volume } \\
\text { (mL) }\end{array}$ & $\begin{array}{l}\text { Zone Fluid } \\
\text { Volume } \\
\text { (mL) }\end{array}$ \\
\hline L4P51-C2 & TZ2 & $5-12-94$ & CPW\#2 & Halite & 8.346 & -7.8 & -466 & 104 & 5.56 & 6466 & 3998 \\
\hline L4P51-C2 & TZ2 & $5-17-94$ & PW & Halite & 8.126 & 4.158 & -38.2 & 104 & 5.56 & 6466 & 3998 \\
\hline L4P51-C2 & TZ2 & $6-14-94$ & CPW $\# 3$ & Halite & 8.133 & 4.206 & -3469 & 104 & 5.56 & 6466 & 3998 \\
\hline L4P51-D1 & TZ1 & $12-8-94$ & $\mathrm{CPI} \# 1$ & $\mathrm{AH}$ & 0.006 & 2.323 & +3389 & 147 & 5.165 & 7466 & 3692 \\
\hline L4P51-D1 & TZ1 & $12-21-94$ & CPI \#2 & $\mathrm{AH}$ & 0.004 & 2.325 & +2519 & 147 & 5.165 & 7466 & 3692 \\
\hline L4P51-D1 & TZ1 & $12-28-94$ & $\mathrm{CPI} \# 3$ & $\mathrm{AH}$ & 0.025 & 2.245 & +374 & 147 & 5.165 & 7466 & 3692 \\
\hline L4P51-D1 & TZ1 & $1-18-95$ & CPI \#4 & $\mathrm{AH}$ & 0.361 & 2.069 & +7 & 147 & 5.165 & 7466 & 3692 \\
\hline L4P51-D1 & $T Z 1$ & $1-19-95$ & CPI \#5 & $\mathrm{AH}$ & 2.013 & 4.404 & +279 & 147 & 5.165 & 7466 & 3692 \\
\hline L4P51-D1 & TZ1 & 1-23-95 & CPI\#6 & $\mathrm{AH}$ & 4.438 & 6.962 & +193 & 147 & 5.165 & 7466 & 3692 \\
\hline L4P51-D2 & \multicolumn{11}{|c|}{ Only initial pressure build-up data recorded } \\
\hline L4P52-B & $T Z$ & $2-11-93$ & CPW\#1 & MB138 & 9.084 & 7.971 & -435 & 141 & 5.08 & 8525 & 3114 \\
\hline L4P52-B & TZ & $6-7-93$ & $\mathrm{CPI} \# 1$ & MB138 & 9.155 & 9.598 & +219 & 141 & 5.08 & 8525 & 3114 \\
\hline L4P52-B & $T Z$ & $6-22-93$ & CPI \#2 & MB138 & 9.592 & 10.086 & +484 & 141 & 5.08 & 8525 & 3114 \\
\hline L4P52-B & TZ & $7-7-93$ & $\mathrm{CPI} \# 3$ & MB138 & 10.068 & 11.090 & +1241 & 141 & 5.08 & 8525 & 3114 \\
\hline S1P74-A & $T Z$ & 1-25-93 & CPW & $\begin{array}{l}\text { Anhydrite } \\
\text { "a" }\end{array}$ & 6.420 & 5.523 & -66 & 143 & 5.174 & 8399 & 3735 \\
\hline S1P74-A & $T Z$ & $8-3-93$ & CMP \#1 & $\begin{array}{l}\text { Anhydrite } \\
\text { "a" }\end{array}$ & 6.542 & -0.030 & N/A & 143 & 5.174 & 8399 & 3735 \\
\hline S1P74-A & $T Z$ & $8-3-93$ & CMP \#2 & $\begin{array}{l}\text { Anhydrite } \\
\text { "a" }\end{array}$ & -0.030 & -8.8 & N/A & 143 & 5.174 & 8399 & 3735 \\
\hline S1P74-A & $T Z$ & $8-3-93$ & PW & $\begin{array}{l}\text { Anhydrite } \\
\text { "a" }\end{array}$ & $\sim 8.8$ & 0.319 & -498 & 143 & 5.174 & 8399 & 3735 \\
\hline S1P74-A & GZ & $8-4-93$ & CMP \#1 & Halite & 4.045 & 0.024 & N/A & 102 & 5.174 & 6531 & 2079 \\
\hline S1P74-B & TZ1 & 4-10-95 & PW & MB138 & 9.273 & 8.860 & -8.9 & 180 & 5.174 & 5654 & 9284 \\
\hline S1P74-B & $T Z 2$ & $4-24-95$ & PW\#1 & $A H-1$ & 8.801 & 7.083 & -7.1 & 106 & 5.174 & 6587 & 2375 \\
\hline S1P74-B & $\mathrm{TZ2}$ & $5-13-95$ & PW\#2 & AH-1 & 8.814 & $4-977$ & -20.5 & 106 & 5.174 & 6587 & 2375 \\
\hline
\end{tabular}

\subsubsection{Permeability Testing in Room L4}

\subsubsection{BOREHOLE L4P51, PERMEABILITY-TESTING SEQUENCE L4P51-C1}

Permeability-testing sequence L4P51-C1 took place in Room L4 in borehole L4P51. This test sequence was designed to investigate the brine permeability of MB140. Table 3-4 gives a detailed description of the events that occurred during permeability-testing sequence L4P51-C1. 
Table 3-4. Permeability-Testing Sequence L4P51-C1 Events

\begin{tabular}{|c|c|c|c|c|}
\hline EVENT & DATE & $\begin{array}{l}\text { CALENDAR } \\
\text { DAY }\end{array}$ & $\begin{array}{c}1992 \\
\text { CALENDAR } \\
\text { DAY }\end{array}$ & $\begin{array}{c}\text { TIME } \\
\text { (HH:MM:SS) }\end{array}$ \\
\hline Ream borehole L4P51 to a diameter of 7.5 -inch $(19.05-\mathrm{cm})$ to a depth of 10 feet. & 3-30-92 & 90 & 90 & 12:00:00 \\
\hline Install and grout into place a 10-foot borehole flanged collar. & 3-30-92 & 90 & 90 & 14:00:00 \\
\hline Deepen borehole L4P51 with 4-inch $(10.16-\mathrm{cm})$ bit $(2-$ inch $(5.08-\mathrm{cm})$ core) to $11.10 \mathrm{~m}$. & 4-1-92 & 92 & 92 & 14:30:00 \\
\hline Deepen borehole L4P51 with 4-inch $(10.16-\mathrm{cm})$ bit $(2$-inch $(5.08-\mathrm{cm})$ core) to $12.62 \mathrm{~m}$. & $4-2-92$ & 93 & 93 & 12:45:00 \\
\hline Deepen borehole L4P51 with 4-inch $(10.16-\mathrm{cm})$ bit (2-inch $(5.08-\mathrm{cm})$ core) to $14.10 \mathrm{~m}$. & $4-2-92$ & 93 & 93 & 13:20:00 \\
\hline Deepen borehole L4P51 with 4-inch $(10.16-\mathrm{cm})$ bit $(2-$ inch $(5.08-\mathrm{cm})$ core) to $15.68 \mathrm{~m}$. & 4-2-92 & 93 & 93 & 14:20:00 \\
\hline Deepen borehole L4P51 with 4-inch (10.16-cm) bit (2-inch $(5.08-\mathrm{cm})$ core) to $17.18 \mathrm{~m}$. & 4-3-92 & 94 & 94 & 09:22:00 \\
\hline $\begin{array}{l}\text { Suspend drilling activities until a drilling fluid that is more fully saturated with respect } \\
\text { to } \mathrm{NaCl} \text { can be obtained. }\end{array}$ & $4-6-92$ & 97 & 97 & 08:00:00 \\
\hline Deepen borehole L4P51 with 4-inch (10.16-cm) bit (2-inch $(5.08-\mathrm{cm})$ core) to $18.10 \mathrm{~m}$. & 41492 & 105 & 105 & 13:40:00 \\
\hline Deepen borehole L4P51 with 4-inch (10.16-cm) bit (2-inch $(5.08-\mathrm{cm})$ core) to $18.61 \mathrm{~m}$. & 4-14-92 & 105 & 105 & 14:22:00 \\
\hline Deepen borehole L4P51 with 4-inch $(10.16-\mathrm{cm})$ bit $(2-$ inch $(5.08-\mathrm{cm})$ core) to $20.17 \mathrm{~m}$. & 4-15-92 & 106 & 106 & 09:45:00 \\
\hline Deepen borehole L4P51 with 4-inch (10.16- $\mathrm{cm})$ bit (2-inch $(5.08-\mathrm{cm})$ core) to $21.74 \mathrm{~m}$. & 4-15-92 & 106 & 106 & $13: 50: 00$ \\
\hline Deepen borehole L4P51 with 4-inch (10.16-cm) bit (2-inch $(5.08-\mathrm{cm})$ core) to $22.20 \mathrm{~m}$. & 4-15-92 & 106 & 106 & $14: 30: 00$ \\
\hline Perform video-log of borehole L4P51 (COLOG). & $4-22-92$ & $\$ 13$ & 113 & 12:00:00 \\
\hline $\begin{array}{l}\text { Install volume displacement device in borehole L4P51 as indicated in the test-tool } \\
\text { configuration diagram \#1 (Figure 3-22). }\end{array}$ & $4-23-92$ & 114 & 114 & 14:00:00 \\
\hline $\begin{array}{l}\text { Install multipacker test tool \#33A in borehole L4P51 for permeability testing sequence } \\
\text { L4P51-C1 as indicated in the test-tool configuration diagram \#1 (Figure 3-22). }\end{array}$ & 4-24-92 & 115 & 115 & 09:27:00 \\
\hline Begin data file L4P51C01. & $4-24-92$ & 115 & 115 & $09: 47: 37$ \\
\hline Inflate GZP to $\sim 10.3 \mathrm{MPa}$ & $4-24-92$ & 115 & 115 & 09:50:00 \\
\hline Inflate $T Z P$ to $\sim 10.3 \mathrm{MPa}$. & $4-24-92$ & 115 & 115 & 09:55:00 \\
\hline Increase GZP pressure. & $4-24-92$ & 115 & 115 & 09:58:00 \\
\hline Increase TZP pressure. & 4-24-92 & 115 & 115 & 10:00:00 \\
\hline Shut in $G Z$ and $T Z$. & $4-24.92$ & 115 & 115 & 10:07:00 \\
\hline Increase GZP pressure. & $4-27-92$ & 118 & 118 & 06:58:00 \\
\hline Increase TZP pressure. & $4-27-92$ & 118 & 118 & 07:00:00 \\
\hline Leaky fitting on GZ. & $4-28-92$ & 119 & 119 & 07:38:00 \\
\hline Leaky fitungs on $G Z$ and $T Z$. & $4-30-92$ & 121 & 121 & 09:00:00 \\
\hline LVDT \#1 has been disconnected since day 125. & $5-6-92$ & 127 & 127 & 08:03:00 \\
\hline End data file L4P51COI. & $5-7.92$ & 128 & 128 & 11:33:11 \\
\hline Power outage in Room L4. & $5-7-92$ & 128 & 128 & 11:45:00 \\
\hline Begin data file L4P51CO2. & $5-7-92$ & 128 & 128 & $12: 43: 40$ \\
\hline $\begin{array}{l}\text { Initiate pulse-withdrawal test \#1 in GZ from } 8.826 \text { to } 0.011 \mathrm{MPa} \text { removing } 225 \mathrm{~mL} \text { of } \\
\text { fuld. }\end{array}$ & $5-7-92$ & 128 & 128 & $12: 44: 00$ \\
\hline Shut in GZ. & $5-7-92$ & 128 & 128 & 12:46:10 \\
\hline Depressurze $T Z$ & 5-11-92 & 132 & 132 & $08: 32: 56$ \\
\hline Depressurize GZ ending pulse-withdrawal test \#1 in GZ. & $5-11-92$ & 132 & 132 & 08:34:24 \\
\hline Deflate TZP and GZP. & $5-11-92$ & 132 & 132 & 08:38:00 \\
\hline
\end{tabular}


Table 3-4 (Continued). Permeability-Testing Sequence L4P51-C1 Events

\begin{tabular}{|c|c|c|c|c|}
\hline EVENT & DATE & $\begin{array}{l}\text { CALENDAR } \\
\text { DAY }\end{array}$ & $\begin{array}{c}1992 \\
\text { CALENDAR } \\
\text { DAY }\end{array}$ & $\begin{array}{l}\text { TIME } \\
\text { (HH:MM:SS) }\end{array}$ \\
\hline End data file L4P51C02. & $5-11-92$ & 132 & 132 & 08:40:47 \\
\hline Remove multipacker test tool \#33A from borehole L4P51. & $5-11-92$ & 132 & 132 & $14: 00: 00$ \\
\hline $\begin{array}{l}\text { Install multipacker test tool \#33B in borehole L4P51 as indicated in the test-100l } \\
\text { configuration diagram \#2 (Figure 3-23). }\end{array}$ & 5-13-92 & 134 & 134 & 09:30:00 \\
\hline Begin data file L4P51 03. & 5-13-92 & 134 & 134 & 11:41:00 \\
\hline Inflate GZP to $\sim 10.3 \mathrm{MPa}$ & $5-13-92$ & 134 & 134 & 11:41:30 \\
\hline Inflate TZP to $-10.3 \mathrm{MPa}$ & 5-13-92 & 134 & 134 & $11: 43: 00$ \\
\hline Shut in $T Z$ and $G Z$ & 5-13-92 & 134 & 134 & $11: 45: 00$ \\
\hline Increase TZP pressure to $\sim 11 \mathrm{MPa}$. & $5-21-92$ & 142 & 142 & 10:15:43 \\
\hline End data file L4P51CO3. & $5-26-92$ & 147 & 147 & 07:53:40 \\
\hline Begin data file L4P5iCO4. & $5-26-92$ & 147 & 147 & 07:59:40 \\
\hline $\begin{array}{l}\text { Begin constant-pressure-withdrawal test in } \mathrm{GZ} \text { at } \sim 2 \mathrm{MPa} \text { below } \mathrm{GZ} \text { pressure } \\
\qquad(\sim 6.1 \mathrm{MPa}) .\end{array}$ & $5-26-92$ & 147 & 147 & 08:17:40 \\
\hline Increase back pressure on the DPT panel. & $5-28-92$ & 149 & 149 & 11:00:04 \\
\hline Leaky fitting on DPT panel. & $5-29-92$ & 150 & 150 & 09:34:00 \\
\hline Increase back pressure on the DPT panel. & $6-2-92$ & 154 & 154 & 09:36:00 \\
\hline Increase back pressure on the DPT panel. & $6-4-92$ & 156 & 156 & $09: 46: 00$ \\
\hline Increase back pressure on the DPT panel. & $6-5-92$ & 157 & 157 & 07:33:00 \\
\hline Shut in GZ from DPT panel, terminating the constant-pressure-withdrawal lest. & $6-8-92$ & 160 & 160 & 08:17:00 \\
\hline End data file L4P51CO4. & $6-10-92$ & 162 & 162 & 08:29:05 \\
\hline Begin data file L4P51C05. & $6-10-92$ & 162 & 162 & $08: 46: 24$ \\
\hline $\begin{array}{l}\text { Begin constant-pressure-withdrawal test \#1 in TZ at }-0.5 \mathrm{MPa} \text { below } \mathrm{TZ} \text { pressure } \\
\qquad(\sim 8.0 \mathrm{MPa}) .\end{array}$ & $6-23-92$ & 175 & 175 & 08:20:10 \\
\hline Shut in $\mathrm{TZ}$ from DPT panel terminating the constant-pressure-withdrawal test \#1 in $T Z$. & $6-26-92$ & 178 & 178 & 12:36:30 \\
\hline End data file L4P51C05. & $6-29-92$ & 181 & 181 & 12:16:13 \\
\hline Begin data file L4P51C06. & $6-29-92$ & 181 & 181 & 12:20:00 \\
\hline Open GZP to accumulator. & $7-8-92$ & 190 & 190 & 12:03:03 \\
\hline DAS nunning on unconditioned power since day 191. & 7-13-92 & 195 & 195 & 10:15:00 \\
\hline Increase TZP pressure. & $7-16-92$ & 198 & 198 & 12:15:37 \\
\hline Open TZP and GZP to same accumulator. & $7-16-92$ & 198 & 198 & 12:19:37 \\
\hline $\begin{array}{l}\text { Intiate pulso-withdrawal test \#2 in GZ from } 7.567 \text { to } 0.073 \mathrm{MPa} \text {, removing } 96.5 \mathrm{~mL} \text { of } \\
\text { fluid. }\end{array}$ & $7-29-92$ & 211 & 211 & 08:00:29 \\
\hline Deflate GZP. & 7-29-92 & 211 & 211 & 08:02:44 \\
\hline Inflate GZP to $10.898 \mathrm{MPa}$. & $7-29-92$ & 211 & 211 & 08:08:29 \\
\hline Increase GZP pressure. & $7-29-92$ & 211 & 211 & 08:19:14 \\
\hline Open GZP to accumulator. & $7-29-92$ & 211 & 211 & 08:25:14 \\
\hline Shut in GZ. & $7-29-92$ & 211 & 211 & 09:00:00 \\
\hline End data file L4P51C06 terminating pulse-withdrawal test \#2 in GZ & 8-14-92 & 227 & 227 & 07:57:06 \\
\hline
\end{tabular}




\section{Table 3-4 (Continued). Permeability-Testing Sequence L4P51-C1 Events}

\begin{tabular}{|c|c|c|c|c|}
\hline EVENT & DATE & $\begin{array}{l}\text { CALENDAR } \\
\text { DAY }\end{array}$ & $\begin{array}{c}1992 \\
\text { CALENDAR } \\
\text { DAY }\end{array}$ & $\begin{array}{c}\text { TIME } \\
\text { (HH:MM:SS) }\end{array}$ \\
\hline Begin data file L4P51CO7. & 8-14-92 & 227 & 227 & 08:31:00 \\
\hline End data file L4P51CO7. & 8-24-92 & 237 & 237 & 05:42:52 \\
\hline Begin data file L4P51CO8. & 8-24-92 & 237 & 237 & $12: 41: 28$ \\
\hline $\begin{array}{l}\text { Begin constant-pressure-withdrawal test } \# 2 \text { in } T Z \text { at } \sim 0.5 \mathrm{MPa} \text { below } \mathrm{TZ} \text { pressure } \\
\qquad(\sim 8.4 \mathrm{MPa}) .\end{array}$ & 8-26-92 & 239 & 239 & 08:41:37 \\
\hline Decrease back pressure on DPT panel. & 8-28-92 & 341 & 241 & 10:04:00 \\
\hline Decrease back pressure on DPT panel. & $8-31-92$ & 244 & 244 & 08:43:00 \\
\hline Leaky fitting on DPT panel. & 8-31-92 & 244 & 244 & $08: 45: 00$ \\
\hline Shut in $T Z$ from DPT panel terminating the constant-pressure withdrawal test $\# 2$ in $T Z$. & $9-1-92$ & 245 & 245 & $09: 54: 00$ \\
\hline End data file L4P51C08. & $9-8-92$ & 252 & 252 & 07:52:01 \\
\hline Begin data file L4P51C09. & 9-8-92 & 252 & 252 & $09: 51: 12$ \\
\hline End data file L4P51C09. & 9-21-92 & 265 & 265 & 07:42:34 \\
\hline Begin data file L4P51C10. & $10-2-92$ & 276 & 276 & 09:00:35 \\
\hline End data file L4P51C10. & 10-8-92 & 282 & 282 & 11:21:32 \\
\hline Begin data file L4P51C11. & 10-8-92 & 282 & 282 & 13:11:06 \\
\hline $\begin{array}{l}\text { Begin constant-pressure-withdrawal test } 3 \text { in } \mathrm{TZ} \text { at }-0.5 \mathrm{MPa} \text { below TZ pressure } \\
\qquad(-8.4 \mathrm{MPa}))\end{array}$ & 10-13-92 & 287 & 287 & 09:04:36 \\
\hline Increase back pressure on DPT panel. & $10-21-92$ & 295 & 295 & $12: 30: 00$ \\
\hline Decrease the rate of gas coming from the column. & $10-22-92$ & 296 & 296 & 12:21:30 \\
\hline Increase back pressure on DPT panel. & 10-23-92 & 297 & 297 & $11: 50: 00$ \\
\hline $\begin{array}{l}\text { Decrease back pressure on DPT panel to } \sim 7.4 \text { MPa terminating the constant- } \\
\text { pressure-withdrawal test } \# 3 \text { in } T Z \text { and beginning the constant-pressure-withdrawal test } \\
\qquad 4 \text { in } T Z .\end{array}$ & $10-26-92$ & 300 & 300 & 09:19:07 \\
\hline Shut in $T Z$ from DPT panel terminating the constant-pressure-withdrawal test $\# 4$ in TZ. & $11-6-92$ & 311 & 311 & 09:52:38 \\
\hline End data file L4P51C11. & $11-6-92$ & 311 & 311 & 14:25:28 \\
\hline Begin data file L4P51C12. & $11-6-92$ & 311 & 311 & $14: 29: 45$ \\
\hline End data file L4P51C12. & $1-8-93$ & 8 & 374 & $04: 21: 46$ \\
\hline Begin data file L4P51C13. & $1-8-93$ & 8 & 374 & $09: 44: 47$ \\
\hline Possible leaky fitting on DPT panel. & $1-13-93$ & 13 & 379 & $08: 00: 00$ \\
\hline $\begin{array}{l}\text { Pertorm compressibility test in TZ dropping the pressure in steps from } 7.578 \text { to } 0.344 \\
\qquad \begin{array}{l}\mathrm{MPa} \text {. }\end{array}\end{array}$ & $2-11-93$ & 42 & 408 & $12: 12: 00$ \\
\hline Depressurize $T Z$ terminating the $T Z$ compressibility test. & $2-11-93$ & 42 & 408 & 12:12:18 \\
\hline Depressurize GZ. & 2-11-93 & 42 & 408 & $12: 16: 48$ \\
\hline Defiate TZP. & 2-11-93 & 42 & 408 & 12:21:18 \\
\hline Deflate GZP. & $2-11-93$ & 42 & 408 & 12:23:18 \\
\hline Inflate TZP to $-10.3 \mathrm{MPa}$ & $2-11-93$ & 42 & 408 & 12:57:15 \\
\hline Inflate GZP to $-10.3 \mathrm{MPa}$ and increase TZP pressure. & $2-11-93$ & 42 & 408 & 12:59:15 \\
\hline Increase GZP pressure. & $2-11-93$ & 42 & 408 & 13:03:30 \\
\hline Open TZP to accumulator. & $2-11-93$ & 42 & 408 & 13:04:30 \\
\hline
\end{tabular}


Table 3-4 (Continued). Permeability-Testing Sequence L4P51-C1 Events

\begin{tabular}{|c|c|c|c|c|}
\hline EVENT & DATE & $\begin{array}{l}\text { CALENDAR } \\
\text { DAY }\end{array}$ & $\begin{array}{c}1992 \\
\text { CALENDAR } \\
\text { DAY }\end{array}$ & $\begin{array}{c}\text { TIME } \\
\text { (HH:MM:SS) }\end{array}$ \\
\hline Open GZP to accumulator. & $2-11-93$ & 42 & 408 & 13:04:45 \\
\hline Shut in TZ. & 2-11-93 & 42 & 408 & 13:10:46 \\
\hline Shut in GZ. & $2-11-93$ & 42 & 408 & 13:13:31 \\
\hline End data file L4P51C13. & $3-9-93$ & 68 & 434 & 11:24:11 \\
\hline Begin data file L4P51C14. & $3-9-93$ & 68 & 434 & $11: 32: 04$ \\
\hline End data file L4P51C14. & $4-1493$ & 104 & 470 & 07:08:13 \\
\hline Begin data file L4P51C15. & $4-14-93$ & 104 & 470 & $10: 16: 33$ \\
\hline 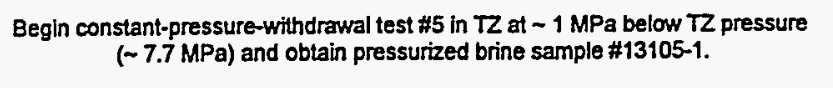 & 4-15-93 & 105 & 471 & 09:50:00 \\
\hline Shut in TZ from DPT panel due to overfull fluid reservoir. & $4-19-93$ & 109 & 475 & 10:02:22 \\
\hline Resume constant-pressure-withdrawal test \#5 in TZ & 4-19-93 & 109 & 475 & $11: 45: 00$ \\
\hline Shut in $T Z$ from DPT panel due to plugged flow line. & $4-21 \cdot 93$ & 111 & 477 & 12:52:14 \\
\hline Resume constant-pressure-withdrawal test $\# 5$ in $T Z$. & $4-21-93$ & 111 & 477 & 13:22:28 \\
\hline Shut in $T Z$ from DPT panel due to plugged flow line. & $4-27-93$ & 117 & 483 & 12:10:00 \\
\hline Resume constant-pressure-withdrawal test \#5 in $\mathrm{TZ}$. & $4-27-93$ & 117 & 483 & 12:16:56 \\
\hline Shut in TZ from DPT panel due to plugged flow line. & $5-7-93$ & 127 & 493 & $12: 00: 00$ \\
\hline End data file L4P5iC15. & $5-10-93$ & 130 & 496 & $11: 32: 21$ \\
\hline Begin data file L4P51C16. & $5-10-93$ & 130 & 496 & $11: 35: 40$ \\
\hline Resume constant-pressure-withdrawal test \#5 in TZ & $5-10-93$ & 130 & 496 & $11: 40: 00$ \\
\hline Decrease back pressure on DPT panel. & $5-10-93$ & 130 & 496 & $14: 26: 00$ \\
\hline Decroase back pressure on DPT panel. & 5-13-93 & 133 & 499 & 14:08:00 \\
\hline Decrease back pressure on DPT panel. & $5-17-93$ & 137 & 503 & $08: 20: 00$ \\
\hline Obtain pressurized brine sample \#13152-1 from TZ (cylinder flushed with argon). & $6-1-93$ & 152 & 518 & 08:58:00 \\
\hline Obtaln pressurized brine sample \#13167-1 from TZ (cylinder flushed with argon). & 6-25-93 & 176 & 542 & $11: 13: 53$ \\
\hline Obtain pressurized brine sample \#13176-1 from TZ (cylinder flushed with argon). & $7-2-93$ & 183 & 549 & 10:53:41 \\
\hline Oblain pressurized brine sample \#13183-1 from TZ (cylinder flushed with argon). & $7-9-93$ & 190 & 556 & $10: 31: 52$ \\
\hline Obtain pressurized brine sample \#13190-1 from TZ (cylinder flushed with argon). & $7-15-93$ & 196 & 562 & 13:22:44 \\
\hline End data file L4P51C16. & $8-6-93$ & 218 & 584 & 07:43:00 \\
\hline Begin data file L4P51C17. & $8-6-93$ & 218 & 584 & $07: 47: 24$ \\
\hline Obtain pressurized brine samplo \#13258-1 from TZ (cylinder flushed with argon). & $9-15-93$ & 258 & 624 & 08:05:33 \\
\hline Decrease back pressure on DPT panel. & $9-23-93$ & 266 & 632 & 07:37:47 \\
\hline End data file L4P51C17. & $9-23-93$ & 266 & 632 & $07: 46: 18$ \\
\hline Obtain pressurized brine sample \#13274-1 from TZ (cylinder flushed with argon). & $10-1-93$ & 274 & 640 & $08: 59: 30$ \\
\hline Decrease back pressure on DPT panel. & $10-6-93$ & 279 & 645 & 07:02:00 \\
\hline Obtain pressurized brine sample \#13281-1 from TZ (cylinder flushed with argon). & $10-8-93$ & 281 & 647 & 09:17:00 \\
\hline Decrease back pressure on DPT panel. & $10-11-93$ & 284 & 650 & $08: 45: 00$ \\
\hline Power outage in Room L4 since day 284. & $10-12-93$ & 285 & 651 & 13:52:00 \\
\hline
\end{tabular}


Table 3-4 (Continued). Permeability-Testing Sequence L4P51-C1 Events

\begin{tabular}{|c|c|c|c|c|}
\hline EVENT & DATE & $\begin{array}{l}\text { CALENDAR } \\
\text { DAY }\end{array}$ & $\begin{array}{c}1992 \\
\text { CALENDAR } \\
\text { DAY }\end{array}$ & $\begin{array}{c}\text { TIME } \\
\text { (HH:MM:SS) }\end{array}$ \\
\hline Decrease back pressure on DPT panel. & $10-15-93$ & 288 & 654 & 09:53:00 \\
\hline Decrease back pressure on DPT panel. & $10-18-93$ & 291 & 657 & 08:08:00 \\
\hline Obtain pressurized brine sample \#13294-1 from TZ (cylinder flushed with argon). & $10-21-93$ & 294 & 660 & 09:00:00 \\
\hline Begin data file L4P51C18. & $10-22-93$ & 295 & 661 & 08:38:02 \\
\hline Obtain pressurized brine sample \#13302-1 from TZ (Cylinder flushed with argon). & $10-29-93$ & 302 & 668 & 08:28:25 \\
\hline Increase back pressure on DPT panel. & $10-29-93$ & 302 & 668 & 08:38:07 \\
\hline Decrease back pressure on DPT panel. & $11-3-93$ & 307 & 673 & 10:15:32 \\
\hline Obtain pressurized brine sample \#13308-1 from TZ (cylinder flushed with argon). & $11-493$ & 308 & 674 & 09:36:44 \\
\hline Decrease back pressure on DPT panel. & 11-5-93 & 309 & 675 & 10:11:00 \\
\hline DAS powered by generator. & $11-10-93$ & 314 & 680 & $09: 27: 53$ \\
\hline Shut in $T Z$ from DPT panel terminating the constant-pressure-withdrawal test \#5 in TZ & $11-11-93$ & 315 & 681 & 08:58:00 \\
\hline Depressurize TZ. & 11-11-93 & 315 & 681 & 09:04:00 \\
\hline Depressurize $G Z$ ( $-100 \mathrm{~mL}$ of brine removed). & $11-11-93$ & 315 & 681 & 09:09:00 \\
\hline Deflate TZP. & $11-11-93$ & 315 & 681 & 09:15:00 \\
\hline Deflate GZP. & $11-11-93$ & 315 & 681 & 09:18:00 \\
\hline End data file L4P51C18. & $11-11-93$ & 315 & 681 & 09:26:00 \\
\hline $\begin{array}{l}\text { Remove multipacker test tool \#338 from borehole L4P51 and terminate permeability } \\
\text { testing sequence L4P51-C1. }\end{array}$ & $11-11-93$ & 315 & 681 & $12: 00: 00$ \\
\hline
\end{tabular}

Figures 3-34 through 3-40 illustrate the zone pressures, packer pressures, zone temperature, axial-LVDT displacement, radial-LVDT displacement, fluid production during constant-pressure-flow tests, and test-zone compressibility, respectively, for permeabilitytesting sequence L4P51-C1. It should be noted that Figure 3-39 (Fluid production during constant-pressure -flow tests in permeability testing sequence L4P51-C1) consists of two parts (Figures 3-39a and 3-39b). Copies of the video-log associated with testing sequence L4P51-C1 identified in Table 3-4 are provided in the SWCF under WPO \#45907. 


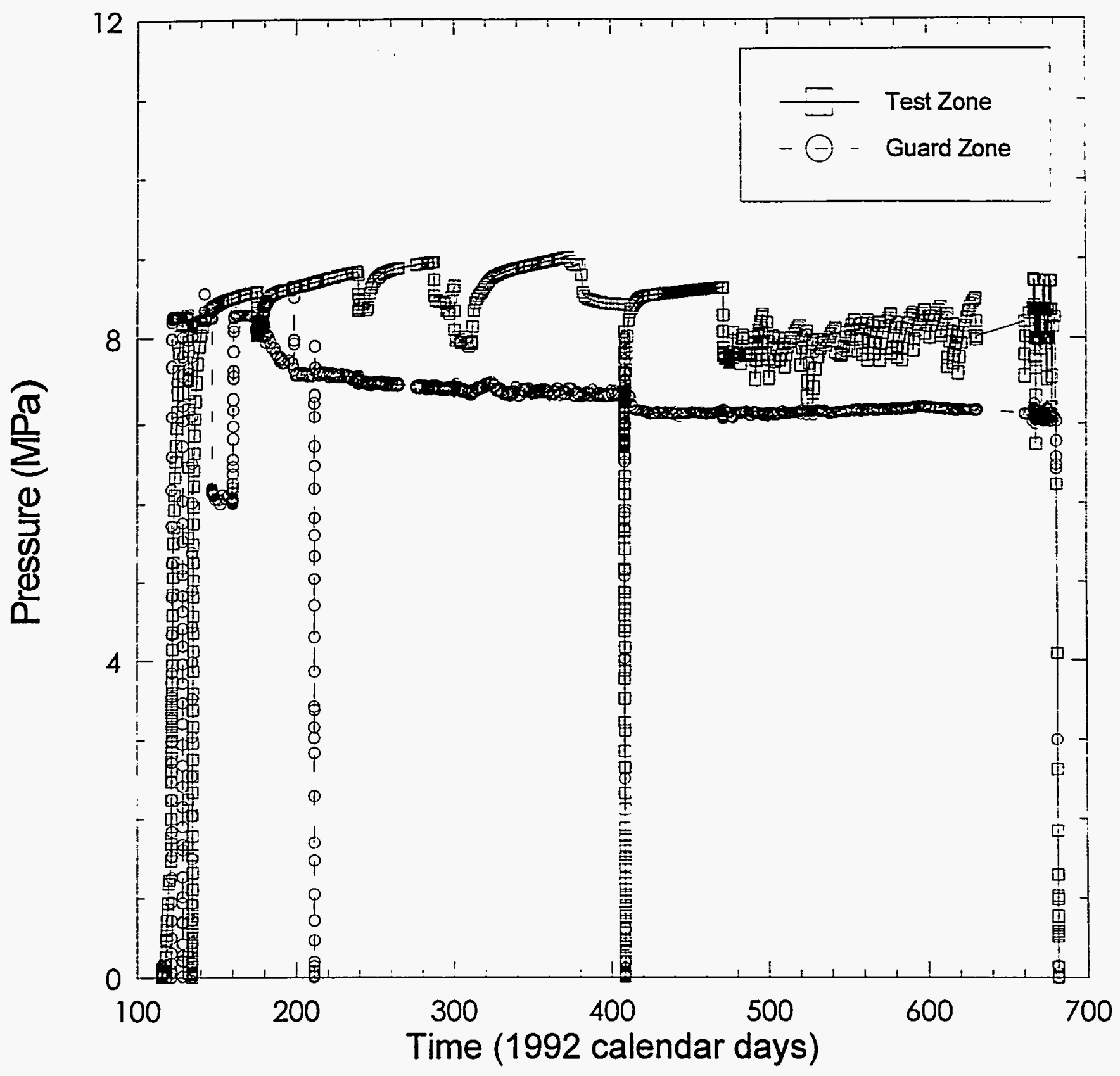

INTERA-6115-47-0

Figure 3-34. Zone pressures during permeability-testing sequence L4P51-C1. 


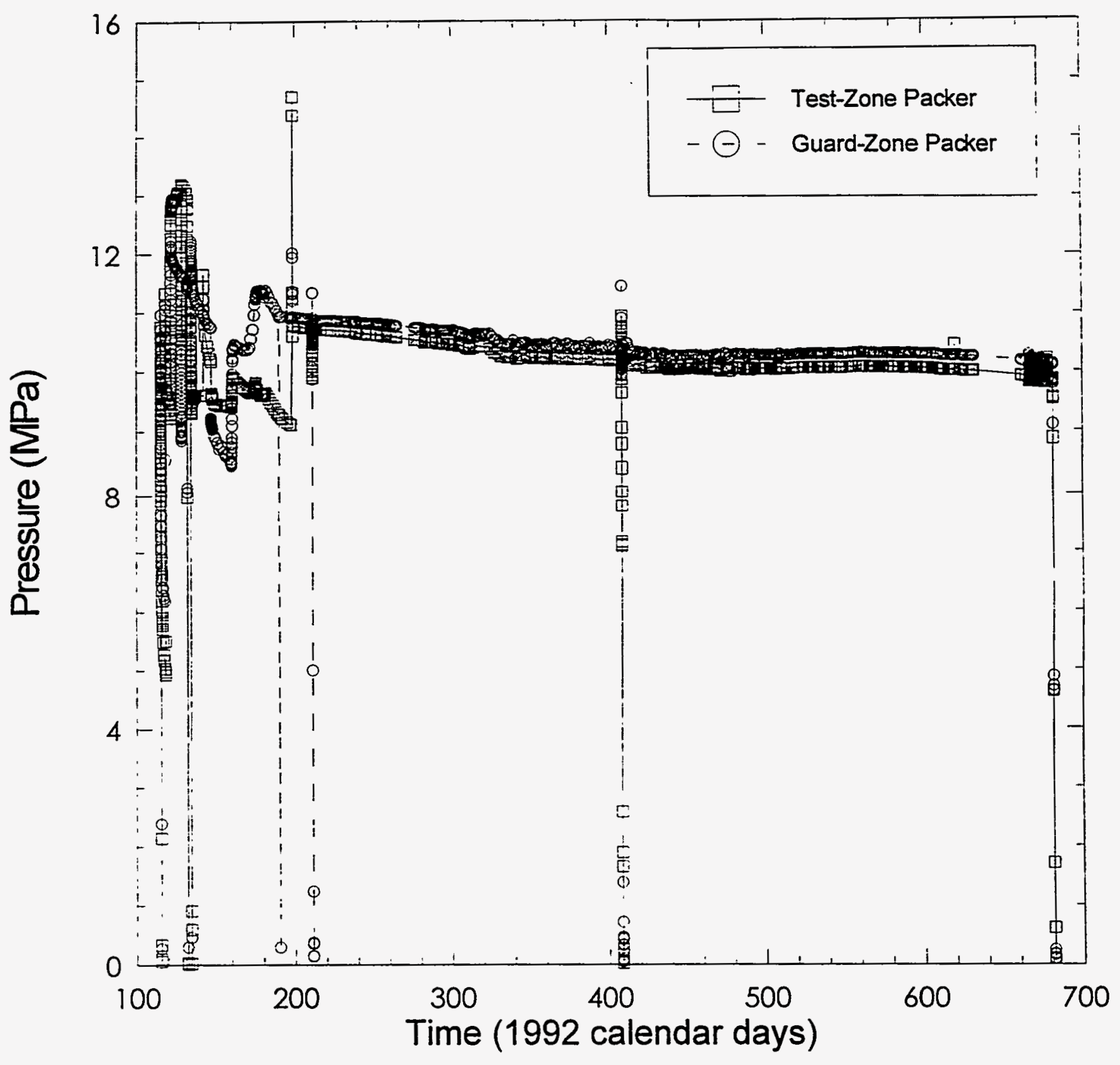

INTERABI15-48-O

Figure 3-35. Packer pressures during permeability-testing sequence L4P51-C1. 


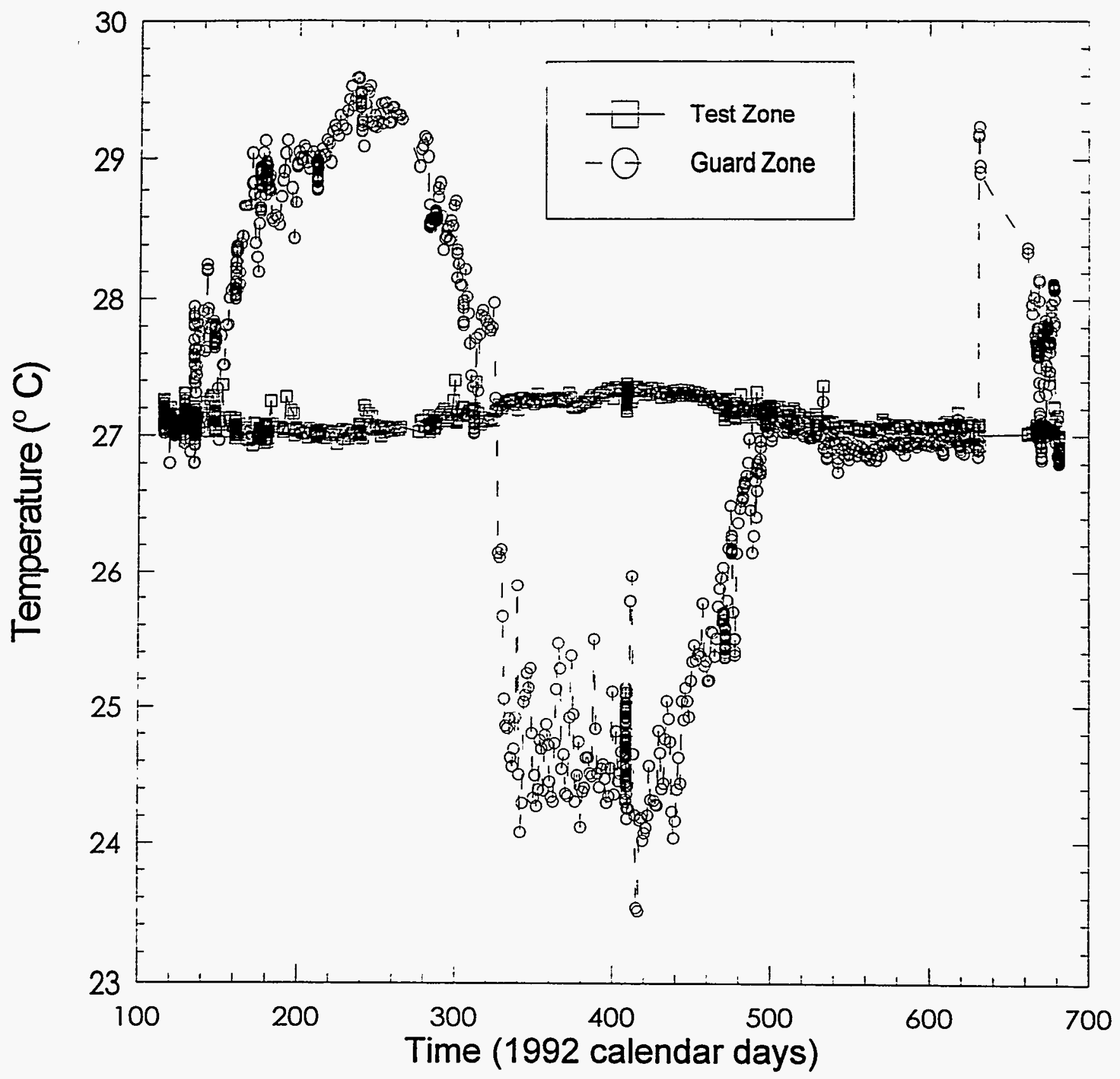

INTERA-ST15-490

Figure 3-36. Zone temperatures during permeability-testing sequenœ L4P51-C1. 


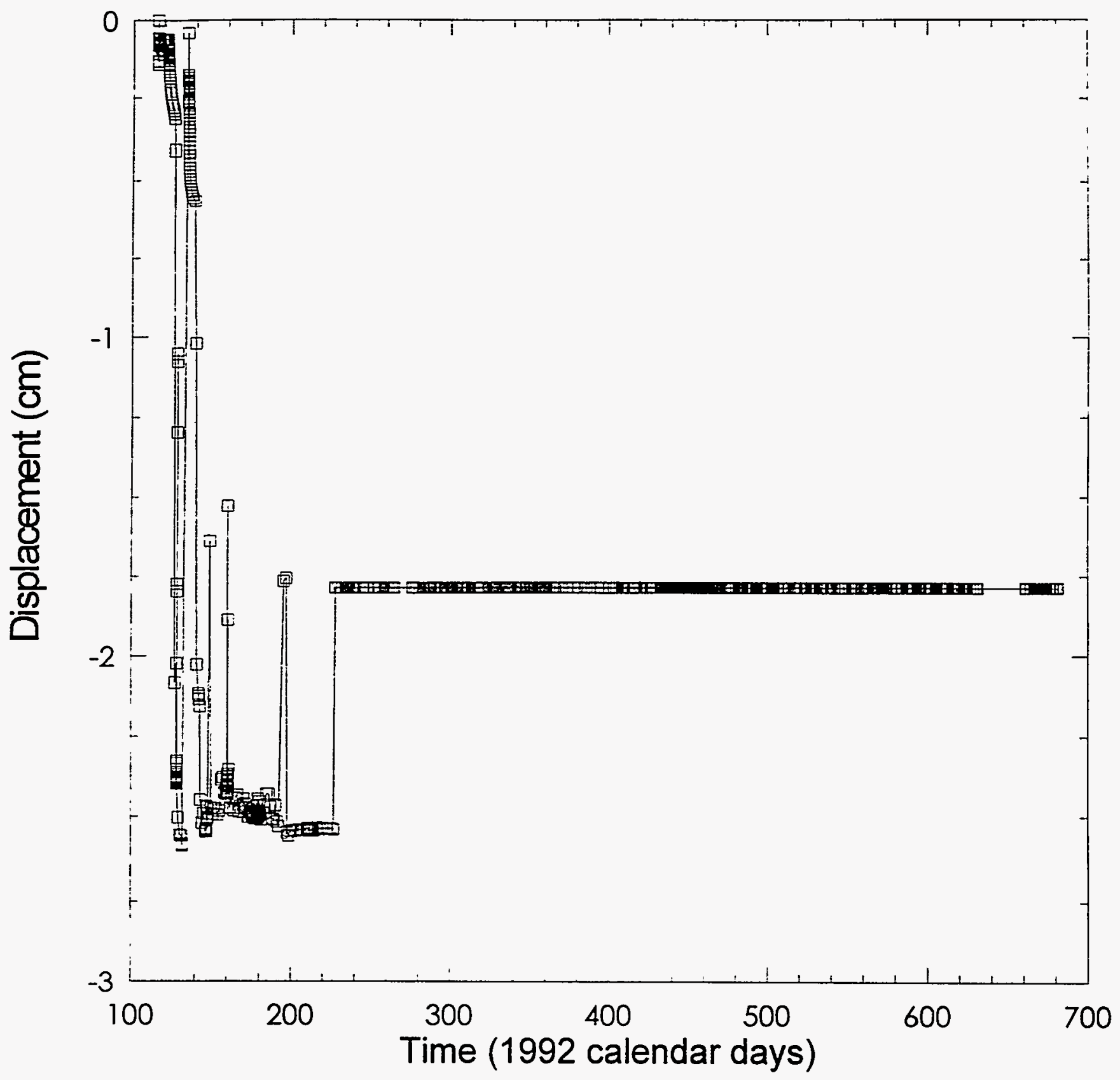

INTERA 6115-50-0

Figure 3-37. Axial-LVDT displacement during permeability-testing sequence L4P51-C1. 


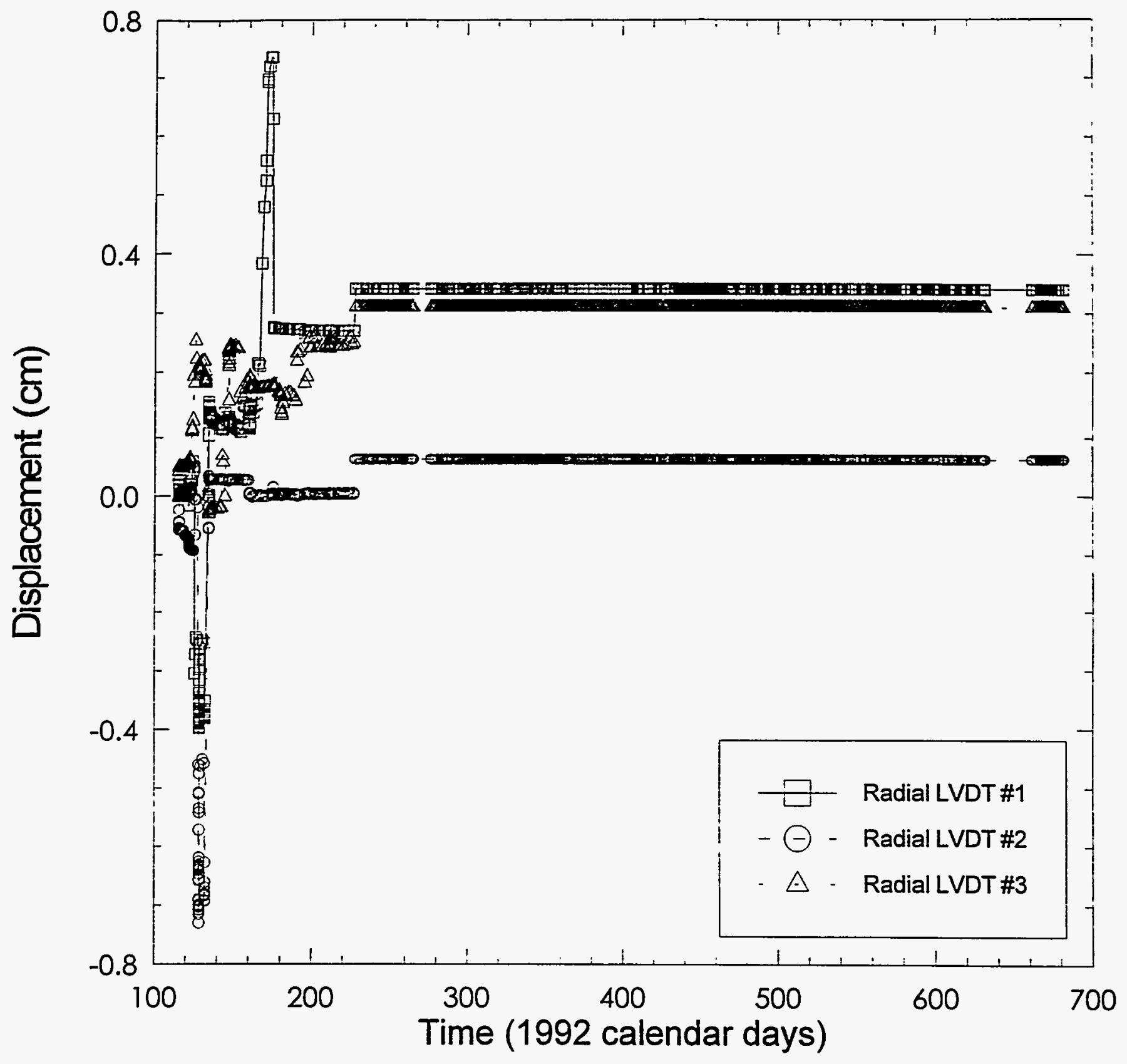

INTERA-6115-51-0

Figure 3-38. Radial-LVDT displacement during permeability-testing sequence L4P51-C1. 


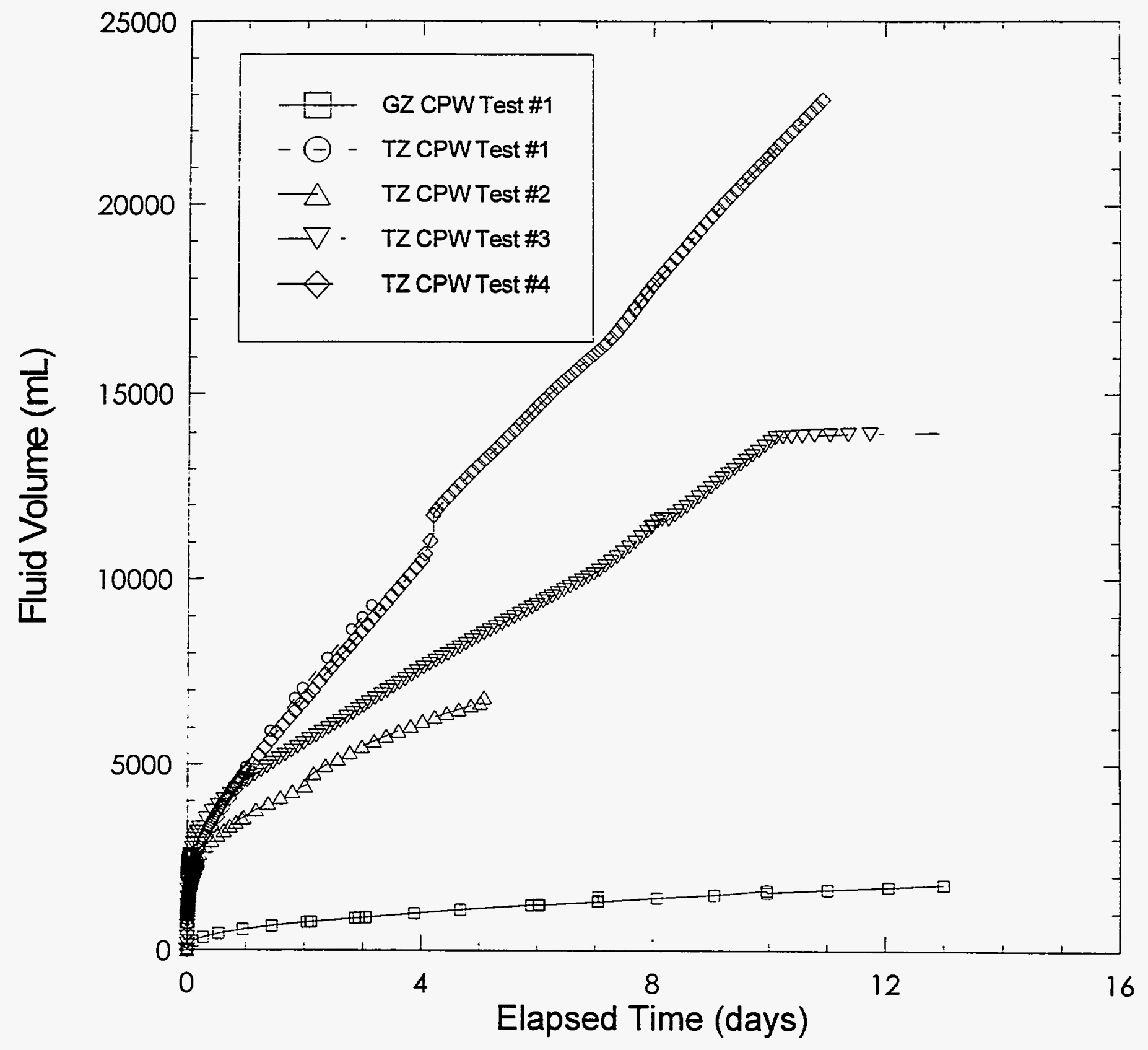

INTERA-6115-52-0

Figure 3-39a. Fluid production during constant-pressure-flow tests in permeabilitytesting sequence L4P51-C1. 


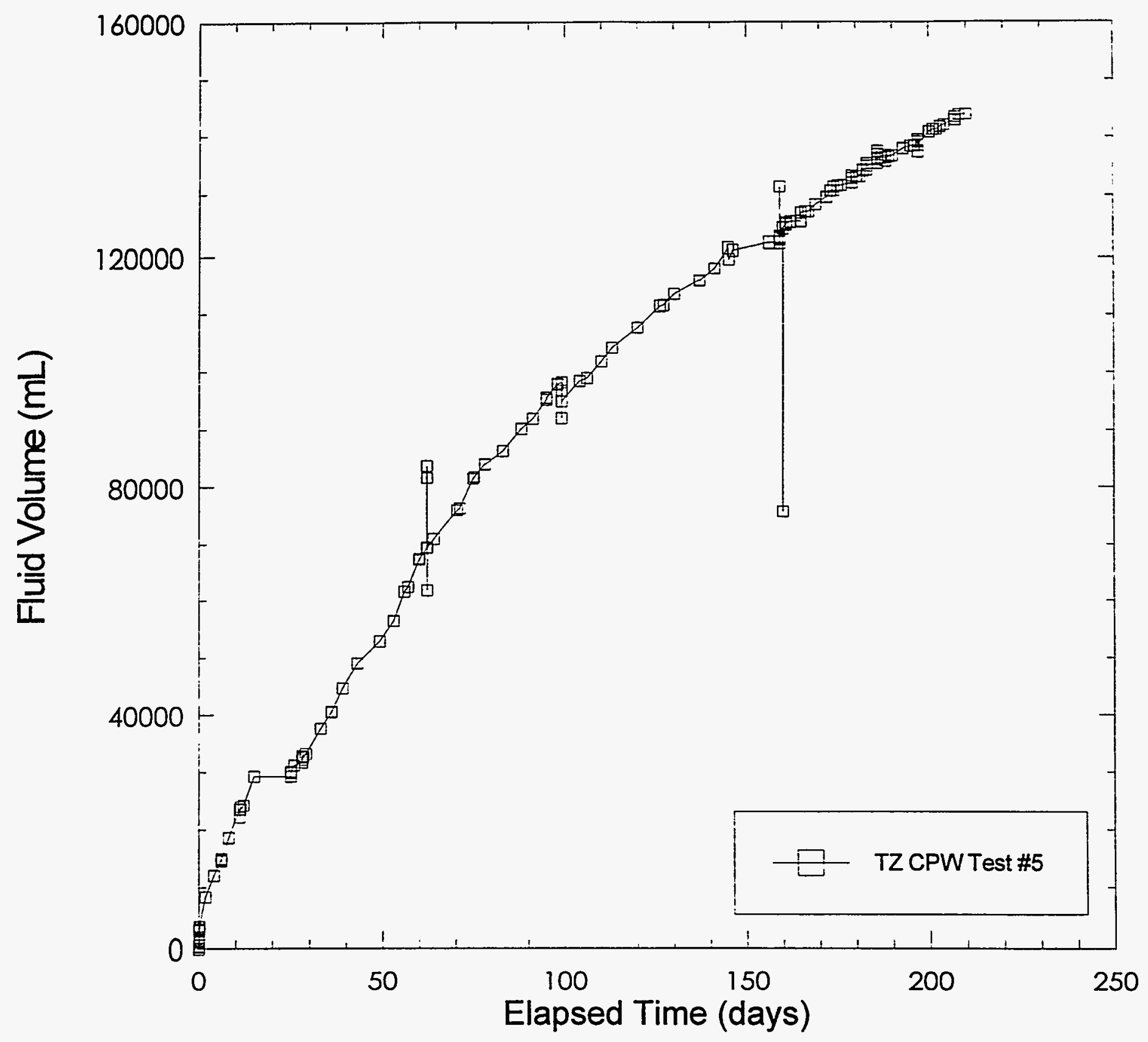

INTERA-6115-53-0

Figure 3-39b. Fluid production during constant-pressure-flow tests in permeabilitytesting sequence L4P51-C1 (continued). 


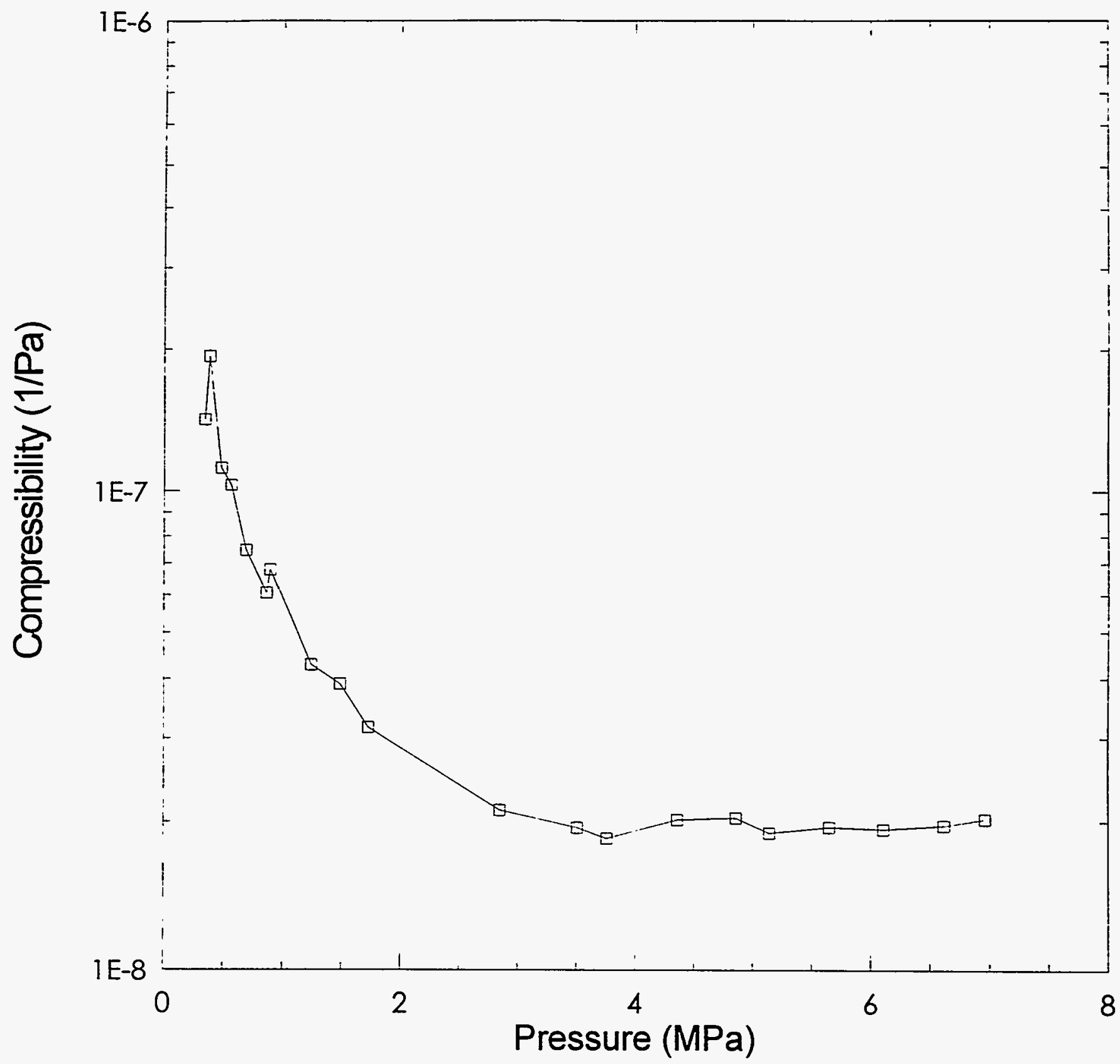

INTERA-6115-55-0

Figure 3-40. Test-zone compressibility as measured during permeability-testing sequence L4P51-C1. 
Table 3-5 indicates the equipment that was used and the duration that each instrument was used during permeability-testing sequence L4P51-C1.

Table 3-5. Permeability-Testing Sequence L4P51-C1 Equipment

\begin{tabular}{|c|c|c|c|c|}
\hline Equipment & Location & Serial \# & Installed & Removed \\
\hline DAS Software & N/A & PERM4F & 4-24-92 & $11-11-93$ \\
\hline DCU (HP3497A) & N/A & $2629 a 21996$ & $4-24-92$ & $10-2-92$ \\
\hline DCU (HP3497A) & N/A & $2629 a 21990$ & $10-2-92$ & $3-25-93$ \\
\hline DCU (HP3497A) & N/A & $2629 a 22040$ & $3-25-93$ & $9-6-93$ \\
\hline DCU (HP3497A) & N/A & $2514 a 17149$ & $9-6-93$ & $9-22-93$ \\
\hline DCU (HP3497A) & N/A & $2023 a 01688$ & $9-22-93$ & $11-11-93$ \\
\hline $\begin{array}{c}\text { Transducer } \\
\text { (Druck PDCR 830) }\end{array}$ & Guard Zone & 246914 & $4-24-92$ & $11-11-93$ \\
\hline $\begin{array}{c}\text { Transducer } \\
\text { (Druck PDCR 10/D) }\end{array}$ & $\begin{array}{l}\text { Test Zone } \\
\text { Packer }\end{array}$ & 211695 & $4-24-92$ & $11-11-93$ \\
\hline $\begin{array}{c}\text { Transducer } \\
\text { (Druck PDCR 830) }\end{array}$ & $\begin{array}{l}\text { Guard Zone } \\
\text { Packer }\end{array}$ & 246920 & $4-24-92$ & $11-11-93$ \\
\hline $\begin{array}{c}\text { Transducer } \\
\text { (Druck PDCR 10/D) }\end{array}$ & Test Zone & 211690 & $4-24-92$ & $11-11-93$ \\
\hline $\begin{array}{c}\text { Transducer } \\
\text { (Druck PDCR 830) }\end{array}$ & DPT Panel & 246910 & $5-26-92$ & $6-10-92$ \\
\hline $\begin{array}{c}\text { Transducer } \\
\text { (Druck PDCR 910) }\end{array}$ & DPT Panel & 322427 & $8-24-92$ & $9-8-92$ \\
\hline $\begin{array}{c}\text { Transducer } \\
\text { (Druck PDCR 910) }\end{array}$ & DPT Panel & 322427 & $10-8-92$ & $11-6-93$ \\
\hline $\begin{array}{c}\text { Transducer } \\
\text { (Druck PDCR 910) }\end{array}$ & DPT Panel & 322427 & $10-22-93$ & $11-11-93$ \\
\hline LVDT (Trans-Tek 241) & N/A & R04 & 4-24-92 & $5-11-92$ \\
\hline LVDT (Trans-Tek 241) & N/A & $\mathrm{R} 16$ & $4-24-92$ & $5-11-92$ \\
\hline LVDT Trans-Tek 241) & N/A & $\mathrm{R} 17$ & $4-24-92$ & $5-11-92$ \\
\hline LVDT (Trans-Tek 245) & N/A & $\mathrm{A} 02$ & 4-24-92 & $5-11-92$ \\
\hline LVDT (Trans-Tek 241) & N/A & R05 & $5-13-92$ & $10-22-93$ \\
\hline LVDT (Trans-Tek 241) & N/A & R11 & $5-13-92$ & $10-22-93$ \\
\hline LVDT (Trans-Tek 241) & N/A & $\mathrm{R} 12$ & $5-13-92$ & $10-22-93$ \\
\hline LVDT (Trans-Tek 245) & N/A & $\mathrm{A} 01$ & $5-13-92$ & $10-22-93$ \\
\hline $\begin{array}{c}\text { Thermocouple } \\
\text { (Type E) }\end{array}$ & Test Zone & 1 & 4-24-92 & $11-11-93$ \\
\hline
\end{tabular}


Table 3-5 (Continued). Permeability-Testing Sequence L4P51-C1 Equipment

\begin{tabular}{ccccc}
\hline Equipment & Location & Serial \# & Installed & Removed \\
\hline $\begin{array}{c}\text { Thermocouple } \\
\text { (Type E) }\end{array}$ & Guard Zone & 2 & $4-24-92$ & $11-11-93$ \\
\hline Injection Column & N/A & 76 & $6-23-92$ & $4-14-93$ \\
Injection Column & N/A & 77 & $5-26-92$ & $4-14-93$ \\
Injection Column & N/A & 38 & $4-14-93$ & $11-11-93$ \\
Injection Column & N/A & 88 & $4-14-93$ & $11-11-93$ \\
\hline DPT & N/A & 1409226 & $5-20-92$ & $10-12-92$ \\
(Rosemount 1151DP) & N/A & 1389938 & $10-12-92$ & $4-12-93$ \\
DPT & & 1140864 & $4-12-93$ & $11-11-93$ \\
(Rosemount 1151DP) & N/A & & & \\
DPT & & &
\end{tabular}

* Installed dates for injection columns refer to dates of initial use rather than date installed.

\subsubsection{BOREHOLE L4P51, PERMEABILITY-TESTING SEQUENCE L4P51-C2}

Permeability-testing sequence L4P51-C2 took place in Room L4 in borehole L4P51. This test sequence was designed to investigate the brine permeability of a halite directly above MB140. Table 3-6 gives a detailed description of the events that occurred during the permeability-testing sequence L4P51-C2.

Table 3-6. Permeability-Testing Sequence L4P51-C2 Events

\begin{tabular}{|c|c|c|c|c|}
\hline EVENT & DATE & $\begin{array}{c}\text { CALENDAR } \\
\text { DAY }\end{array}$ & $\begin{array}{c}1993 \\
\text { CALENDAR } \\
\text { DAY }\end{array}$ & $\begin{array}{c}\text { TIME } \\
\text { (HH:MM:SS) }\end{array}$ \\
\hline $\begin{array}{l}\text { Install } 4.63 \text { meter volume-displacement device in borehole L4P51 as indicated in the } \\
\text { test-tool configuration diagram (Figure 3-24). }\end{array}$ & $11-16-93$ & 320 & 320 & 13:18:00 \\
\hline $\begin{array}{l}\text { Install multipacker test tool \#41 in borehole L4P51 as indicated in the test-1001 } \\
\text { configuration diagram for permeability-testing sequence L4P51-C2 (Figure 3-24). }\end{array}$ & $19-17-93$ & 321 & 321 & 14:00:00 \\
\hline Begin data file L4P51C19. & $11-18-93$ & 322 & 322 & 12:58:00 \\
\hline Inflate TZIP to $-10.3 \mathrm{MPa}$ & $11-18-93$ & 322 & 322 & 13:08:20 \\
\hline Inflate TZ2P to $-10.3 \mathrm{MPa}$ & $11-18-93$ & 322 & 322 & 13:10:52 \\
\hline Inflate GZP to $-10.3 \mathrm{MPa}$ & $11-18-93$ & 322 & 322 & 13:13:20 \\
\hline Shut in TZ1. & $11-18-93$ & 322 & 322 & 13:16:01 \\
\hline Shut in $T Z 2$ and $G Z$ & $11-29-93$ & 333 & 333 & 08:58:00 \\
\hline Increase $G Z$ pressure to $\sim 4.1 \mathrm{MPa}$. & $12-1-93$ & 335 & 335 & 09:59:00 \\
\hline Increase $\mathrm{GZ}$ pressure to $5.888 \mathrm{MPa}$. & $12-2-93$ & 336 & 336 & 11:48:20 \\
\hline Increase GZP pressure to $11.209 \mathrm{MPa}$. & $12-2-93$ & 336 & 336 & 11:50:00 \\
\hline
\end{tabular}


Table 3-6 (Continued). Permeability-Testing Sequence L4P51-C2 Events

\begin{tabular}{|c|c|c|c|c|}
\hline EVENT & DATE & $\begin{array}{l}\text { CALENDAR } \\
\text { DAY }\end{array}$ & $\begin{array}{c}1993 \\
\text { CALENDAR } \\
\text { DAY }\end{array}$ & $\begin{array}{l}\text { TIME } \\
\text { (HH:MM:SS) }\end{array}$ \\
\hline Increase GZP pressure to $~ 12 \mathrm{MPa}$. & $12-8-93$ & 342 & 342 & 11:26:27 \\
\hline Open GZ to accumulator at $-6 \mathrm{MPa}$. & $12-8-93$ & 342 & 342 & $11: 30: 00$ \\
\hline Leaky fitting on GZP. & $12-15-93$ & 349 & 349 & 09:20:56 \\
\hline Replace gage on GZP. & $12-16-93$ & 350 & 350 & 09:31:49 \\
\hline Remove gage from GZP. & $12-17-93$ & 351 & 351 & 08:12:00 \\
\hline Increase GZP pressure. & $12-20-93$ & 354 & 354 & $09: 45: 00$ \\
\hline End data file L4P51C19. & 1-19-94 & 19 & 384 & $12: 23: 27$ \\
\hline Begin data file L4P51C20. & $1-19-94$ & 19 & 384 & $12: 27: 49$ \\
\hline Open TZ2P to accumulator at $11.175 \mathrm{MPa}$. & $2-8-94$ & 39 & 404 & 11:11:00 \\
\hline End data file L4P51C2O. & $2-24-94$ & 55 & 420 & 10:55:13 \\
\hline Begin data file L4P51C21. & $2-24-94$ & 55 & 420 & 11:07:33 \\
\hline End data file L4P51C21. & $3.7-94$ & 66 & 431 & 11:11:22 \\
\hline Begin data file L4P51C22. & 3.8 .94 & 67 & 432 & 12:11:46 \\
\hline Replace junction box on DAS to allow for the use of a DPT. & $3-8-94$ & 67 & 432 & 12:14:00 \\
\hline $\begin{array}{l}\text { Begin constant-pressure-withdrawal test \#1 in TZ2 at }-0.5 \mathrm{MPa} \text { below } 7 Z 2 \text { pressure } \\
\qquad(\sim 7.3 \mathrm{MPa}) .\end{array}$ & 3-9-94 & 68 & 433 & 11:03:45 \\
\hline Shut in TZ2 from DPT panel terminating constant-pressure-withdrawal test \#1 in TZ2. & 3-18-94 & 77 & 442 & 10:31:05 \\
\hline End data file L4P51C22. & $3-30-94$ & 89 & 454 & 08:21:50 \\
\hline Begin data file L4P51C23. & $3-30-94$ & 89 & 454 & 10:21:58 \\
\hline $\begin{array}{l}\text { Begin constant-pressure-injection test \#1 in } 7 Z 2 \text { at }-0.5 \mathrm{MPa} \text { above } \mathrm{TZ2} \text { pressure } \\
\qquad(-8.4 \mathrm{MPa}) .\end{array}$ & 4-4-94 & 94 & 459 & 09:17:00 \\
\hline Shut in GZP from accumulator. & 4-7.94 & 97 & 462 & 12:13:00 \\
\hline Open GZP to accumulator at $11.774 \mathrm{MPa}$. & 4-21-94 & 111 & 476 & 08:46:00 \\
\hline Terminate constant-pressure-injection test \#1 in TZ2 and end data file L4P51C23. & 4-21.94 & 111 & 476 & 12:56:09 \\
\hline Begin data file L4P51C24. & 4-21-94 & 111 & 476 & 12:59:10 \\
\hline $\begin{array}{l}\text { Begin constant-pressure-injection test \#2 in TZ2 at } \sim 1 \mathrm{MPa} \text { above TZ2 pressure } \\
\qquad(\sim 8.9 \mathrm{MPa}) .\end{array}$ & 4-21-94 & 111 & 476 & 13:00:33 \\
\hline Shut in TZ2 from DPT panel terminating constant-pressure-injection test \#2 in TZ2. & 4-27-94 & 117 & 482 & 10:58:35 \\
\hline DAS was not functioning property upon artival (problem occurred on day 117). & $4-29-94$ & 119 & 484 & $08: 31: 00$ \\
\hline End data file L4P51C24. & $429-94$ & 119 & 484 & 08:41:44 \\
\hline Begin data file L4P51C25. & 4-29-94 & 119 & 484 & 09:05:00 \\
\hline End data file L4P51C25. & $5-10-94$ & 130 & 495 & 11:38:35 \\
\hline Shut in all zones and packers to replace transducers. & $5-10-94$ & 930 & 495 & 11:52:00 \\
\hline Begin data file L4P51C26. & $5-90-94$ & 130 & 495 & 14:04:27 \\
\hline Open all zones and packers to new transducers. & $5-10.94$ & 130 & 495 & 14:06:00 \\
\hline Open TZ1P to accumulator at $11.985 \mathrm{MPa}$. & $5-12-94$ & 132 & 497 & $12: 40: 00$ \\
\hline $\begin{array}{l}\text { Begin constant-pressuro-withdrawal test } \# 2 \text { in } T 22 \text { at } ~ \\
\qquad(-7.8 \mathrm{MPa}) .5\end{array}$ & $5-12-94$ & 132 & 497 & 12:47:10 \\
\hline
\end{tabular}


Table 3-6 (Continued). Permeability-Testing Sequence L4P51-C2 Events

\begin{tabular}{|c|c|c|c|c|}
\hline EVENT & DATE & $\begin{array}{c}\text { CALENDAR } \\
\text { DAY }\end{array}$ & $\begin{array}{c}1993 \\
\text { CALENDAR } \\
\text { DAY }\end{array}$ & $\begin{array}{c}\text { TIME } \\
\text { (HH:MM:SS) }\end{array}$ \\
\hline Shut in $T Z 2$ from DPT panel terminating constant-pressure-withdrawal test $\# 2$ in $T Z 2$. & $5-12-94$ & 132 & 497 & 13:44:45 \\
\hline $\begin{array}{l}\text { Initiate pulse-withdrawal test in TZ2 dropping pressure from } 8.126 \text { to } 4.158 \mathrm{MPa} \\
\text { removing } 38.2 \mathrm{~mL} \text { of fluid. }\end{array}$ & 5-17-94 & 137 & 502 & 09:14:47 \\
\hline End data file L4P51C26. & 6-2-94 & 153 & 518 & $12: 08: 21$ \\
\hline Begin data file L4P51C27. & $6-2-94$ & 153 & 518 & $12: 42: 23$ \\
\hline Terminate pulse-withdrawal test in $\mathrm{TZ2}$ and end data file L4P51C27. & $6-6-94$ & 157 & 522 & 10:21:12 \\
\hline Begin data file L4P51C28. & $6-6-94$ & 157 & 522 & 10:49:54 \\
\hline $\begin{array}{l}\text { Begin constant-pressure-withdrawal test \#3 in TZ2 at } \sim 4 \mathrm{MPa} \text { below TZ2 pressure } \\
(-4.1 \mathrm{MPa}) \text { using a pressure controller. }\end{array}$ & $6-14-94$ & 165 & 530 & 11:48:15 \\
\hline $\begin{array}{l}\text { Shut down power to everything except the pressure controller and the reference } \\
\text { transducer in order to maintsin test conditions during a power outage. }\end{array}$ & 6-17-94 & 168 & 533 & 08:52:00 \\
\hline Power supplied to entire system. & $6-20-94$ & 171 & 536 & 09:22:00 \\
\hline Fluid reservoir level altered. & $6-23-94$ & 174 & 539 & $\sim$ 10:30:00 \\
\hline Retum fluid reservoir level to approximate original position. & $6-23-94$ & 174 & 539 & 13:10:00 \\
\hline End data file L4P51C28. & $6-27-94$ & 178 & 543 & 11:11:03 \\
\hline Begin data file L4P51C29. & $6-27-94$ & 178 & 543 & $11: 15: 46$ \\
\hline Shut in TZ2 from DPT panel terminating constant-pressure-withdrawal test $\$ 3$ in TZ2. & $7-6-94$ & 187 & 552 & 08:06:25 \\
\hline End data file L4P51C29. & $7-18-94$ & 199 & 564 & 10:14:19 \\
\hline Begin data file L4P51C30. & $7-18-94$ & 199 & 564 & 10:35:02 \\
\hline End data file $L 4 \mathrm{P} 51 \mathrm{C} 30$. & $7-19-94$ & 200 & 565 & 09:01:06 \\
\hline Depressurize all zones. & $7-25-94$ & 206 & 571 & 09:54:00 \\
\hline Deflate all packers. & $7-25-94$ & 206 & 571 & 10:03:00 \\
\hline $\begin{array}{l}\text { Remove multipacker test tool \#41 from borehole L4P51 terminating permeability } \\
\text { testing sequence L4P51-C2. }\end{array}$ & $7-25-94$ & 206 & 571 & $14: 00: 00$ \\
\hline Perform videa-log of borehole L4P51. & $7-26-94$ & 207 & 572 & 13:03:00 \\
\hline
\end{tabular}

Figures 3-41 through 3-44 illustrate the zone pressures, packer pressures, test-zone temperature, and fluid production during constant-pressure-flow tests, respectively, for permeability-testing sequence L4P51-C2. 


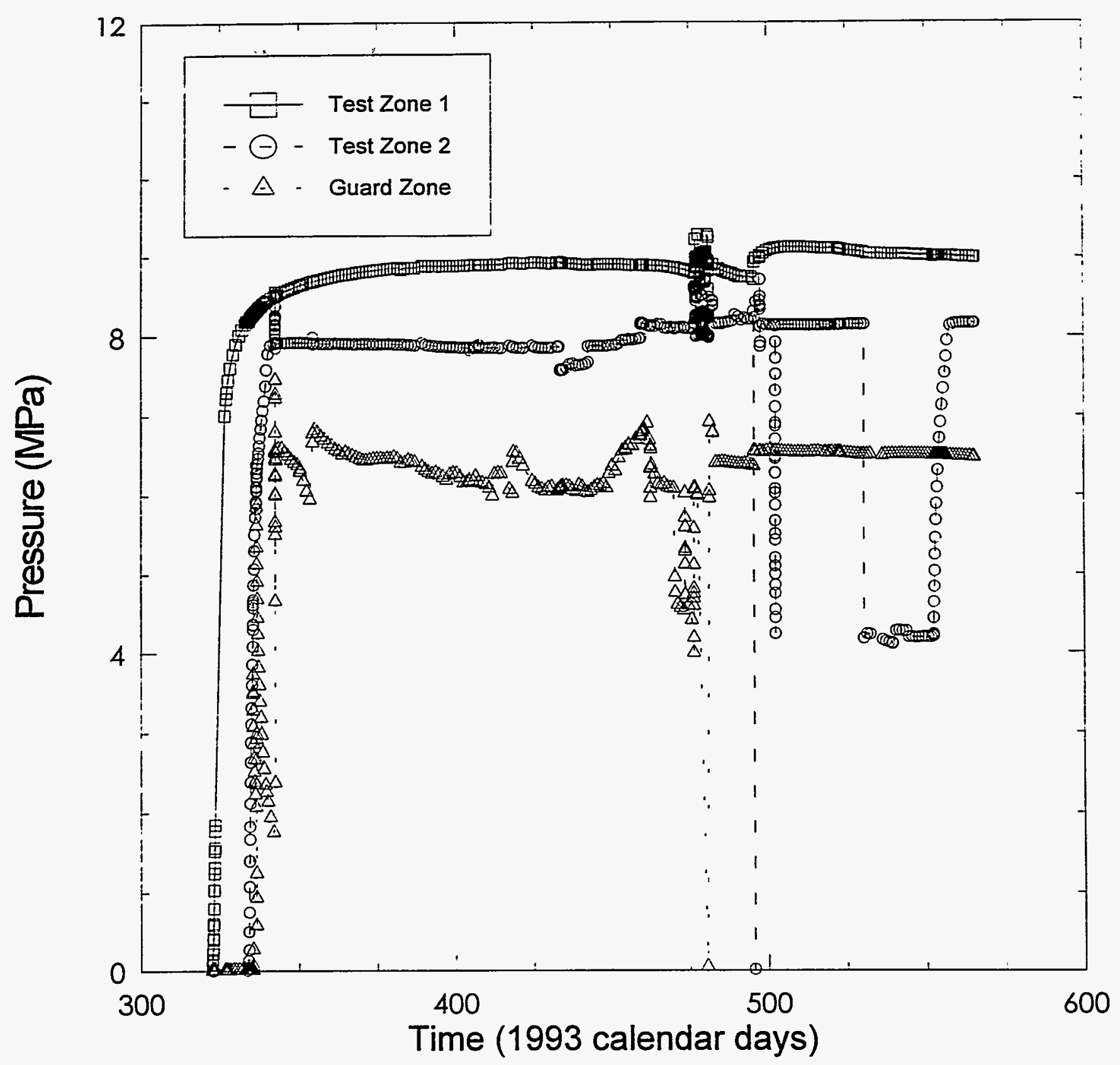

INTERA-6115-55-0

Figure 3-41. Zone pressures during permeability-testing sequence L4P51-C2. 


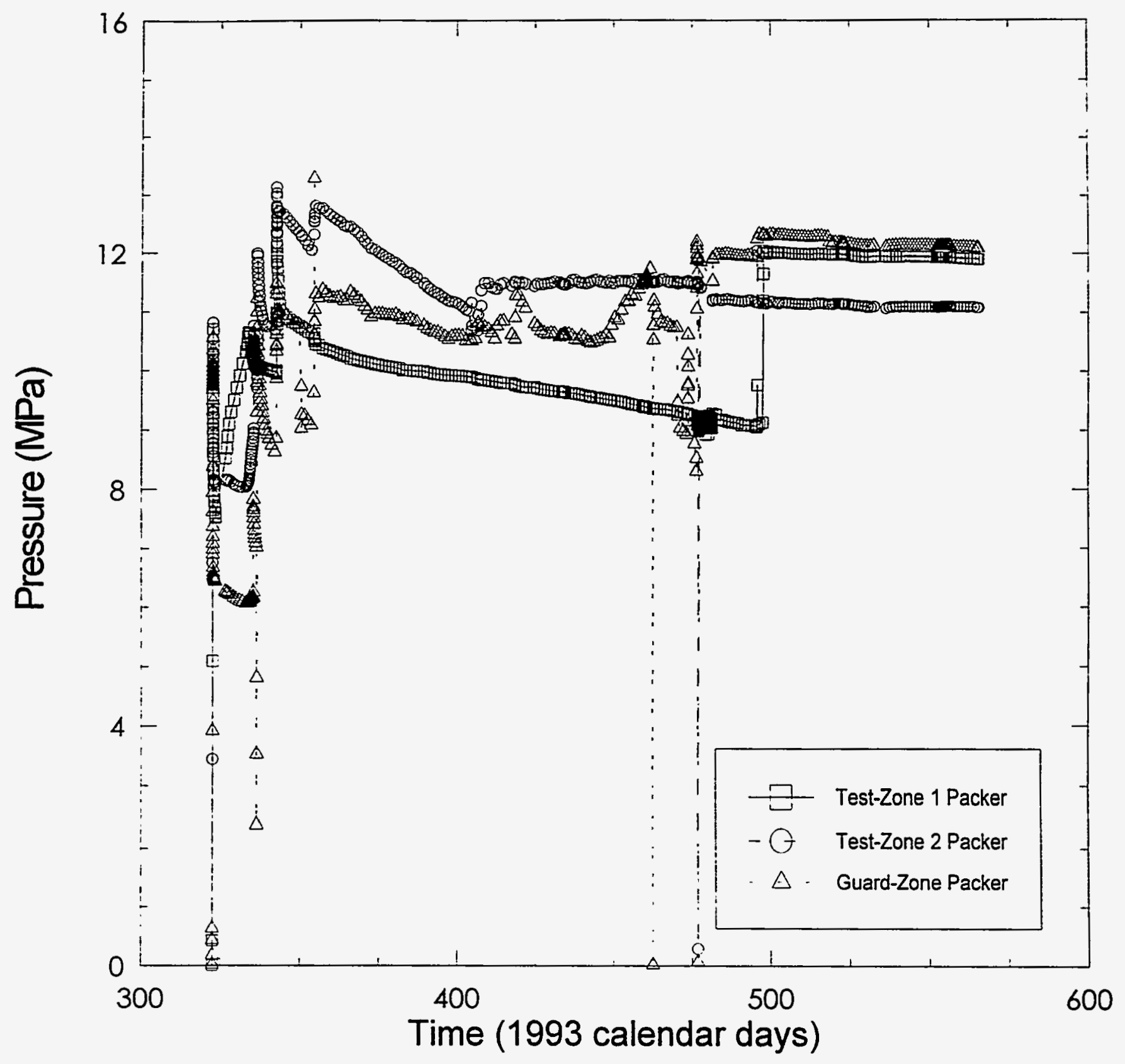

INTERA-6115-57-0

Figure 3-42. Packer pressures during permeability-testing sequence L4P51-C2. 


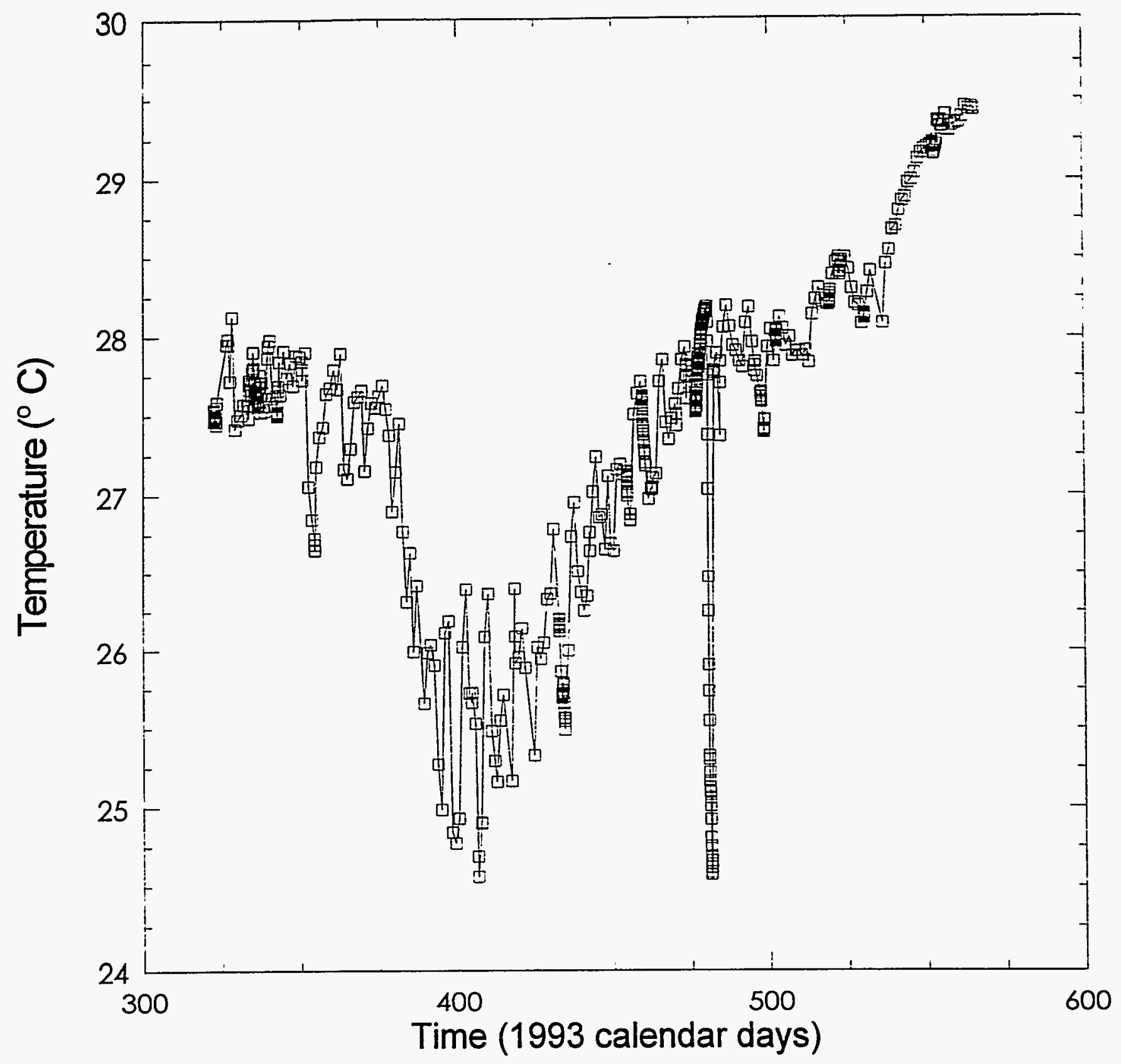

INTERAOI 15-SE-O

Figure 3-43. Test-zone 1 temperature during permeability-testing sequence L4P51-C2. 


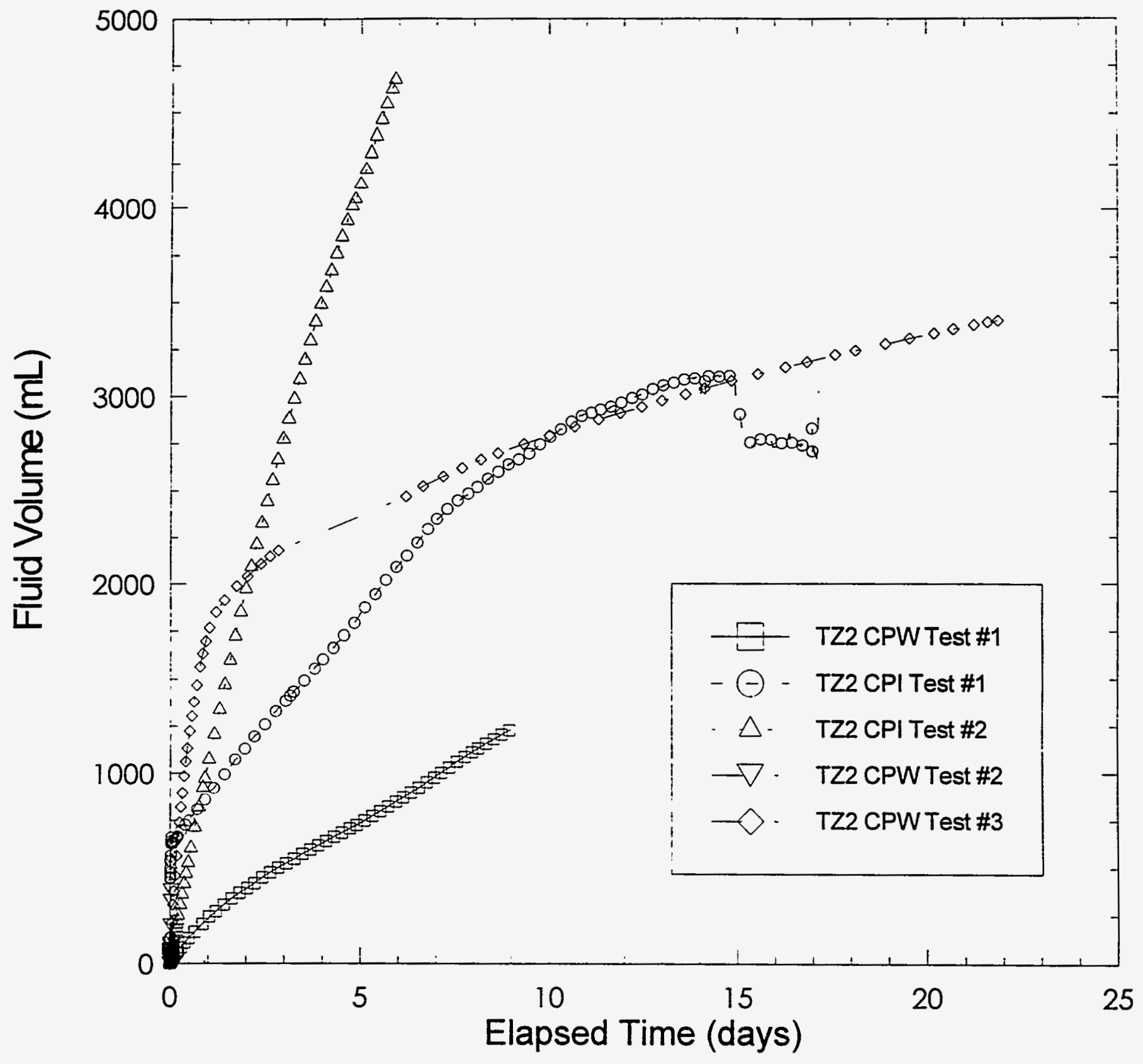

INTERA-6115-59-0

Figure 3-44. Fluid production during constant-pressure-flow tests in permeabilitytesting sequenœ L4P51-C2. 
Table 3-7 indicates the equipment that was used and the duration that each instrument was used during permeability-testing sequence L4P51-C2.

Table 3-7. Permeability-Testing Sequence L4P51-C2 Equipment

\begin{tabular}{|c|c|c|c|c|}
\hline Equipment & Location & Serial \# & Installed & Removed \\
\hline DAS Software & N/A & PERM4F & $11-18-93$ & $7-19-94$ \\
\hline DCU (HP3497A) & N/A & $2629 a 21990$ & $11-18-93$ & $3-7-94$ \\
\hline DCU (HP3497A) & N/A & $2023 a 01688$ & $3-7-94$ & $5-10-94$ \\
\hline DCU (HP3497A) & N/A & $2629 a 21996$ & $5-10-94$ & $7-19-94$ \\
\hline $\begin{array}{c}\text { Transducer } \\
\text { (Druck PDCR 910) }\end{array}$ & $\begin{array}{c}\text { Test Zone } 2 \\
\text { Packer }\end{array}$ & 308152 & $11-18-93$ & $7-19-94$ \\
\hline $\begin{array}{c}\text { Transducer } \\
\text { (Druck PDCR 830) }\end{array}$ & $\begin{array}{l}\text { Test Zone } 1 \\
\text { Packer }\end{array}$ & 246919 & $11-18-93$ & $7-19-94$ \\
\hline $\begin{array}{c}\text { Transducer } \\
\text { (Druck PDCR 830) }\end{array}$ & Test Zone 2 & 246910 & $11-18-93$ & $5-10-94$ \\
\hline $\begin{array}{c}\text { Transducer } \\
\text { (Druck PDCR 830) }\end{array}$ & Test Zone 1 & 246912 & $11-18-93$ & $5-10-94$ \\
\hline $\begin{array}{c}\text { Transducer } \\
\text { (Druck PDCR 910) }\end{array}$ & Guard Zone & 308143 & $11-18-93$ & $5-10-94$ \\
\hline $\begin{array}{c}\text { Transducer } \\
\text { (Druck PDCR 910) }\end{array}$ & $\begin{array}{c}\text { Guard Zone } \\
\text { Packer }\end{array}$ & 322423 & $11-18-93$ & $5-10-94$ \\
\hline $\begin{array}{c}\text { Transducer } \\
\text { (Druck PDCR 910) }\end{array}$ & DPT Panel & 321768 & $3-4-94$ & $3-30-94$ \\
\hline $\begin{array}{c}\text { Transducer } \\
\text { (Druck D930-18) }\end{array}$ & DPT Panel & 609364 & $3-30-94$ & $6-18-94$ \\
\hline $\begin{array}{c}\text { Transducer } \\
\text { (Druck D930-18) }\end{array}$ & Test Zone 1 & 609374 & $5-10-94$ & $7-19-94$ \\
\hline $\begin{array}{c}\text { Transducer } \\
\text { (Druck D930-18) }\end{array}$ & Test Zone 2 & 609367 & $5-10-94$ & $7-19-94$ \\
\hline $\begin{array}{c}\text { Transducer } \\
\text { (Druck D930-18) }\end{array}$ & Guard Zone & 609366 & $5-10-94$ & $7-19-94$ \\
\hline $\begin{array}{c}\text { Transducer } \\
\text { (Druck D930-18) }\end{array}$ & $\begin{array}{l}\text { Guard Zone } \\
\text { Packer }\end{array}$ & 609369 & $5-10-94$ & $7-19-94$ \\
\hline $\begin{array}{c}\text { Transducer } \\
\text { (Druck PDCR 910) } \\
\end{array}$ & $\begin{array}{l}\text { Pressure } \\
\text { Controller } \\
\end{array}$ & 322427 & $6-2-94$ & $6-18-94$ \\
\hline Thermocouple (Type E) & Test Zone 1 & 1 & $11-18-93$ & $7-19-94$ \\
\hline Injection Column & N/A & 264 & $3-4-94$ & $6-18-94$ \\
\hline Injection Column & N/A & 265 & $3-4-94$ & $6-3-94$ \\
\hline Injection Column & N/A & 88 & $6-3-94$ & $6-18-94$ \\
\hline
\end{tabular}


Table 3-7 (Continued). Permeability-Testing Sequence L4P51-C2 Equipment

\begin{tabular}{ccccc}
\hline Equipment & Location & Serial \# & Installed & Removed \\
\hline $\begin{array}{c}\text { DPT } \\
\text { (Rosemount 1151DP) }\end{array}$ & N/A & 1140864 & $3-4-94$ & $6-18-94$ \\
\hline
\end{tabular}

* Installed dates for injection columns refer to dates of initial use rather than date installed.

\subsubsection{BOREHOLE L4P51, PERMEABILITY-TESTING SEQUENCE L4P51-D1}

Permeability-testing sequence L4P51-D1 took place in Room L4 in borehole L4P51. This test sequence was designed to investigate the brine permeability of an argillaceous halite approximately $10 \mathrm{~m}$ below MB140. Unsuccessful attempts were made to perform permeability tests in the isolated test zone containing the argillaceous halite below MB140 in borehole L4P51 during permeability-testing sequence L4P51-D1. Testing was suspended for permeability-testing sequence L4P51-D1 when the test tool was determined to be unreliable. Table 3-8 gives a detailed description of the events that occurred during permeability-testing sequence L4P51-D1 and gives evidence of the unreliability of the test tool.

Table 3-8. Permeability-Testing Sequence L4P51-D1 Events

\begin{tabular}{|c|c|c|c|c|}
\hline EVENT & DATE & $\begin{array}{c}\text { CALENDAR } \\
\text { DAY }\end{array}$ & $\begin{array}{l}1994 \\
\text { CALENDAR } \\
\text { DAY }\end{array}$ & $\begin{array}{c}\text { TIME } \\
\text { (HH:MM:SS) }\end{array}$ \\
\hline Deepen borehole L4P5t with 4-inch $(10.16-\mathrm{cm})$ bit (2-inch $(5.08-\mathrm{cm})$ core) to $23.60 \mathrm{~m}$. & $9-20-94$ & 263 & 263 & 13:29:20 \\
\hline Deepen borehole L4P51 with 4-inch $(10.16-\mathrm{cm})$ bit (2-1nch $(5.08-\mathrm{cm})$ core) to $24.91 \mathrm{~m}$. & $9-21-94$ & 264 & 264 & 09:32:10 \\
\hline Deepen borehole L4P51 with 4-inch (10.16-cm) bit (2-inch $(5.08-\mathrm{cm})$ core) to $26.25 \mathrm{~m}$. & $9-21-94$ & 264 & 264 & 10:41:00 \\
\hline Deepen borehole L4P51 with 4-inch $(10.16-\mathrm{cm})$ bit (2-inch $(5.08-\mathrm{cm})$ core) to $27.62 \mathrm{~m}$. & $9-21-94$ & 264 & 264 & 13:01:00 \\
\hline Deepen borehole L4P51 with 4-inch (10.16-cm) bit (2-inch $(5.08-\mathrm{cm})$ core) to $28.85 \mathrm{~m}$. & $9-21-94$ & 264 & 264 & 13:46:15 \\
\hline Deepen borehole L4P51 with 4-inch $(10.16-\mathrm{cm})$ bit (2-inch $(5.08-\mathrm{cm})$ core) to $30.35 \mathrm{~m}$. & $9-21-94$ & 264 & 264 & $14: 39: 40$ \\
\hline Face off the bottom of the borehole with 4-inch (10.16-cm) plug brt to $30.45 \mathrm{~m}$. & $9-22-94$ & 265 & 265 & $10: 27: 30$ \\
\hline $\begin{array}{l}\text { Install muttipacker test tool \#P51-D1A in borehole L4P51 as indicated in the test-tool } \\
\text { configuration diagram \#1 to begin permeability-testing sequence L4P51-Di (Figure } 3 \text {. } \\
\qquad 25 \text { ). }\end{array}$ & $9-29-94$ & 272 & 272 & 12:00:00 \\
\hline Begin data file L4P51D01. & $9-30-94$ & 273 & 273 & 11:01:51 \\
\hline Circulate fluid through GZP to remove all possible gas. & $9-30-94$ & 273 & 273 & 11:42:00 \\
\hline Inflate GZP to $13.228 \mathrm{MPa}$. & $9-30-94$ & 273 & 273 & 11:45:00 \\
\hline Fill borehole with brine to remove all possible gas. & $9-30-94$ & 273 & 273 & 11:53:00 \\
\hline Inflate TZ1P to $13.514 \mathrm{MPa}$. & $9-30-94$ & 273 & 273 & 12:00:00 \\
\hline Inflate TZ2P to $13.743 \mathrm{MPa}$. & $9-30-94$ & 273 & 273 & $12: 04: 00$ \\
\hline Open all packers to accumulator at $\sim 13.9 \mathrm{MPa}$. & $9-30-94$ & 273 & 273 & 12:13:36 \\
\hline Shut in all zones. & $9-30-94$ & 273 & 273 & 12:15:17 \\
\hline Increase TZ2 pressure to $4.872 \mathrm{MPa}$. & $10-4-94$ & 277 & 277 & 09:45:00 \\
\hline End data file L4P51D01. & 10-6-94 & 279 & 279 & 11:08:01 \\
\hline
\end{tabular}


Table 3-8 (Continued). Permeability-Testing Sequence L4P51-D1 Events

\begin{tabular}{|c|c|c|c|c|}
\hline EVENT & DATE & $\begin{array}{l}\text { CALENDAR } \\
\text { DAY }\end{array}$ & $\begin{array}{c}1994 \\
\text { CALENDAR } \\
\text { DAY }\end{array}$ & $\begin{array}{c}\text { TIME } \\
\text { (HH:MM:SS) }\end{array}$ \\
\hline Begin data file L4P51D02. & $10-6-94$ & 279 & 279 & 11:12:59 \\
\hline Shut in all packer from accumulator. & $10-6-94$ & 279 & 279 & $11: 26: 00$ \\
\hline Depressurize TZ1. & $10-6-94$ & 279 & 279 & $11: 26: 54$ \\
\hline Depressurize TZ2. & $10-6-94$ & 279 & 279 & 11:27:25 \\
\hline Open GZ injection line to atmosphere. & $10-6-94$ & 279 & 279 & 11:27:44 \\
\hline Deflate TZ1P. & $10-6-94$ & 279 & 279 & 11:28:46 \\
\hline Deflate TZ2P. & $10-6-94$ & 279 & 279 & 11:29:56 \\
\hline Inflate TZ1P. & $10-6-94$ & 279 & 279 & 11:33:43 \\
\hline Inflate TZ2P. & $10-6-94$ & 279 & 279 & 11:34:48 \\
\hline Shut in TZ1. & $10-6-94$ & 279 & 279 & 11:39:19 \\
\hline Increase TZ2 pressure to $5.372 \mathrm{MPa}$. & $10-6-94$ & 279 & 279 & 11:42:02 \\
\hline DAS was not functioning property upon arrival. & $10-7.94$ & 280 & 280 & 10:38:00 \\
\hline End data file L4P51D02. & $10-10-94$ & 283 & 283 & 10:15:52 \\
\hline Begin data file L4P51D03. & $10-10-94$ & 283 & 283 & $10: 17: 14$ \\
\hline Depressurize all zones. & $10-10-94$ & 283 & 283 & $11: 38: 53$ \\
\hline Deflate TZ1P. & $10-10-94$ & 283 & 283 & $11: 43: 30$ \\
\hline Deflate TZ2P. & 10-10-94 & 283 & 283 & $11: 44: 21$ \\
\hline Circulate brine through the system. & $10-10-94$ & 283 & 283 & $11: 55: 00$ \\
\hline Inflate all packer to $~ 14 \mathrm{MPa}$. & $10-10-94$ & 283 & 283 & 12:12:35 \\
\hline Increase pressure in all packers to $-16.5 \mathrm{MPa}$. & $10-10-94$ & 283 & 283 & 12:13:55 \\
\hline TZ1P appears to be leaking into the TZ2. & $10-10-94$ & 283 & 283 & 12:20:00 \\
\hline Depressurize all zones. & $10-10-94$ & 283 & 283 & 13:20:00 \\
\hline Deflate all packers. & 10-10-94 & 283 & 283 & 13:28:00 \\
\hline Remove multipacker test -tool \#P51-D1A from borehole to fix leak. & 10-11-94 & 284 & 284 & 09:04:00 \\
\hline Remove LVDT housing from test tool and put on a volume-displacement device. & $10-11-94$ & 284 & 284 & $14: 21: 00$ \\
\hline Perform a leak check on test tool \# P51-D18. & $10-11-94$ & 284 & 284 & $15: 00: 00$ \\
\hline $\begin{array}{l}\text { Install multipacker test tool \#P51-D1B in borehole L4P51 as indicated in the test-tool } \\
\text { configuration diagram \#2 (Figure } 3-26) .\end{array}$ & 10-13-94 & 286 & 286 & 15:30:00 \\
\hline End data file L4P51D03. & 10-14-94 & 287 & 287 & 07:15:21 \\
\hline Begin data file L4P51D04. & $10-1494$ & 287 & 287 & 07:27:39 \\
\hline Inflate GZP to $13.357 \mathrm{MPa}$. & $10-14-94$ & 287 & 287 & 07:39:00 \\
\hline Circulate brine through the system to remove all possible gas. & 10-14-94 & 287 & 287 & 07:45:00 \\
\hline Inflate TZ1P to $13.880 \mathrm{MPa}$. & 10-14-94 & 287 & 287 & 07:49:00 \\
\hline Inflate TZ2P to $14.151 \mathrm{MPa}$. & $10-14-94$ & 287 & 287 & 07:54:00 \\
\hline Open all packers to sccumulator at $-13.8 \mathrm{MPa}$. & 10-14-94 & 287 & 287 & 08:07:00 \\
\hline Increase packer pressure to - $16.3 \mathrm{MPa}$. & 10-14-94 & 287 & 287 & 08:09:00 \\
\hline Increase TZ2 pressure to $5.338 \mathrm{MPa}$. & 10-14-94 & 287 & 287 & 08:12:25 \\
\hline
\end{tabular}


Table 3-8 (Continued). Permeability-Testing Sequence L4P51-D1 Events

\begin{tabular}{|c|c|c|c|c|}
\hline EVENT & DATE & $\begin{array}{l}\text { CALENDAR } \\
\text { DAY }\end{array}$ & $\begin{array}{c}1994 \\
\text { CALENDAR } \\
\text { DAY }\end{array}$ & $\begin{array}{c}\text { TIME } \\
\text { (HH:MM:SS) }\end{array}$ \\
\hline Shut in TZ1. & $10-14-94$ & 287 & 287 & 08:13:08 \\
\hline Switch $T Z 1$ inject and $T Z 1$ vent line due to plugging of $T Z 1$ inject line. & $10-14-94$ & 287 & 287 & $08: 34: 51$ \\
\hline Decrease TZ2 pressure. & $10-14-94$ & 287 & 287 & 08:41:00 \\
\hline DAS not functioning property upon arrival. & $10-17-94$ & 290 & 290 & 07:58:00 \\
\hline Depressurize TZ1. & $10-17-94$ & 290 & 290 & 08:07:29 \\
\hline Shut in TZ1. & $10-17-94$ & 290 & 290 & 08:08:30 \\
\hline End data file L4P51004. & $10-17-94$ & 290 & 290 & 09:54:00 \\
\hline Begin data file L4P51D05. & $10-17-94$ & 290 & 290 & 10:01:50 \\
\hline Depressurize TZ1. & $10-19-94$ & 292 & 292 & $09: 51: 55$ \\
\hline Depressurize TZ2. & $10-19-94$ & 292 & 292 & 09:52:51 \\
\hline Depressurize GZ. & $10-19-94$ & 292 & 292 & $09: 53: 20$ \\
\hline Deflate TZ1P and TZ2P. & $10-19-94$ & 292 & 292 & 09:59:20 \\
\hline Attempt to circulate brine through the system. & $10-19-94$ & 292 & 292 & 10:10:00 \\
\hline Inflate all packers via accumulator. & $10-19-94$ & 292 & 292 & 10:33:29 \\
\hline Shut in GZ. & $10-19-94$ & 292 & 292 & 10:34:05 \\
\hline Shut in $\mathrm{TZ2}$ and $\mathrm{TZ1}$. & $10-19-94$ & 292 & 292 & 10:36:44 \\
\hline Depressurize all zones. & $10-25-94$ & 298 & 298 & 07:30:09 \\
\hline Deflate all packers. & $10-25-94$ & 298 & 298 & 07:31:58 \\
\hline End data file L4P51D05. & $10-25-94$ & 298 & 298 & 07:33:36 \\
\hline Remove test tool \# P51-D1B from borehole L4P51 to unplug TZ1 inject line. & $10-25-94$ & 298 & 298 & 14:00:00 \\
\hline $\begin{array}{l}\text { Install multipacker test tool \#P51-D1B in borehole L4P51 as indicated in the test-tool } \\
\text { configuration diagram \#3 (Figure 3-27). }\end{array}$ & $10-27-94$ & 300 & 300 & 14:00:00 \\
\hline Begin data file L4P51D06. & $10-28-94$ & 301 & 301 & 07:33:53 \\
\hline Inflate GZP to $16.886 \mathrm{MPa}$. & $10-28-94$ & 301 & 301 & 08:02:59 \\
\hline Inflate TZ1P to $14.063 \mathrm{MPa}$. & $10-28-94$ & 301 & 301 & 08:15:00 \\
\hline Inflate TZ2P to $14.000 \mathrm{MPa}$ & $10-28-94$ & 301 & 301 & 08:20:0 \\
\hline Open all packers to accumulator at $-15.5 \mathrm{MPa}$. & $10-28-94$ & 301 & 301 & 08:21:41 \\
\hline Shut in all zones. & 10-28-94 & 301 & 301 & 09:41:53 \\
\hline TZ1 vent line is plugged again. & $10-28-94$ & 301 & 301 & 12:14:00 \\
\hline Depressurize all zones. & $10-28-94$ & 301 & 301 & $12: 16: 56$ \\
\hline Deflate all packers. & $10-28-94$ & 301 & 301 & 12:20:57 \\
\hline Remove multipacker test 1001 \#P51-D18 from borehole L4P51. & $11-1-94$ & 305 & 305 & 08:26:00 \\
\hline $\begin{array}{l}\text { Perform a video-log on borehole L4P51 to determine what is in the bottom of the } \\
\text { borehole. }\end{array}$ & $11-7-94$ & 311 & 311 & 10:33:00 \\
\hline $\begin{array}{l}\text { Circulate brine through the borehole using the Longyear } 0-38 \text { drilling rig in an attempt } \\
\text { to remove the sediment in the bottom of the borehole. }\end{array}$ & $11-22-94$ & 326 & 328 & $10: 44: 00$ \\
\hline $\begin{array}{l}\text { Perform a video-log on borehole L4P51 to make sure that the sediment has been } \\
\text { removed. }\end{array}$ & $11-22-94$ & 326 & 326 & $12: 04: 00$ \\
\hline End data file L4P51D06. & $11-23-94$ & 327 & 327 & 12:37:32 \\
\hline
\end{tabular}


Table 3-8 (Continued). Permeability-Testing Sequence L4P51-D1 Events

\begin{tabular}{|c|c|c|c|c|}
\hline EVENT & DATE & $\begin{array}{l}\text { CALENDAR } \\
\text { DAY }\end{array}$ & $\begin{array}{c}1994 \\
\text { CALENDAR } \\
\text { DAY }\end{array}$ & $\begin{array}{c}\text { TIME } \\
\text { (HH:MM:SS) }\end{array}$ \\
\hline $\begin{array}{l}\text { Install muttipacker test tool \#P51-D1B in borehole L4P51 as indicated in the test-tool } \\
\text { configuration diagram \#4 (Figure 3-28). }\end{array}$ & $11-23-94$ & 327 & 327 & 12:38:00 \\
\hline Begin data file L4P51D07. & $11-23-94$ & 327 & 327 & 12:39:30 \\
\hline Inflate all packers and put all packers on accumulator at $\sim 15.3 \mathrm{MPa}$. & $11-23-94$ & 327 & 327 & 13:55:44 \\
\hline Increase TZ2 pressure to $5.460 \mathrm{MPa}$. & $11-23-94$ & 327 & 327 & 13:57:35 \\
\hline Increase TZ2 pressure to $5.369 \mathrm{MPa}$. & $11-23-94$ & 327 & 327 & 14:01:00 \\
\hline Shut in $G Z$ and $T Z 1$. & $11-23-94$ & 327 & 327 & 14:05:12 \\
\hline Leaky fitting g on $T 22$. & $11-28-94$ & 332 & 332 & 11:30:00 \\
\hline Increase TZ2 pressure to $4.845 \mathrm{MPa}$. & $11-30-94$ & 334 & 334 & 08:10:20 \\
\hline End data file L4P51007. & $11-30-94$ & 334 & 334 & 08:13:23 \\
\hline Begin data file L4P51D08. & $12-6-94$ & 340 & 340 & 11:18:46 \\
\hline P7 and PB transducers are not reading correctly. & $12-6-94$ & 340 & 340 & 14:38:00 \\
\hline P7 and P8 transducers are reading correctly. & $12-7-94$ & 341 & 341 & 09:00:00 \\
\hline Begin constant-pressure-injection test \#1 in $\mathrm{TZ1}$ at $2 \mathrm{MPa}$. & $12-8-94$ & 342 & 342 & 12:39:20 \\
\hline Shut in TZ1 from DPT panel terminating constant-pressure-injection test \#1 in TZ1. & $12-9-94$ & 343 & 343 & $09: 42: 00$ \\
\hline Shut in all packers from accumulator. & $12-10-94$ & 344 & 344 & 10:04:00 \\
\hline Depressurize ali zones. & $12-10-94$ & 344 & 344 & 10:11:09 \\
\hline Deflate all packers. & $12-10-94$ & 344 & 344 & 10:13:00 \\
\hline Remove multipacker test tool \#P51-D1B from borehole L4P51 to fix suspected leak. & $12-10-94$ & 344 & 344 & 13:30:00 \\
\hline End data file L4P51D08. & $12-12-94$ & 346 & 346 & 09:15:26 \\
\hline Perform a leak check on TZiP. & $12-14-94$ & 348 & 348 & 12:59:00 \\
\hline $\begin{array}{l}\text { Install multupacker test tool \#P51-D1B in borehole L4P51 as indicated in the test-tool } \\
\text { configuration diagram \#5 (Figure 3-29). }\end{array}$ & $12-16-94$ & 350 & 350 & 14:00:00 \\
\hline Begin data file L4P51D09. & $12-20-94$ & 354 & 354 & 09:34:52 \\
\hline Circulate brine through TZ1 while inflating TZ1P to ensure all lines remain open. & $12-20-94$ & 354 & 354 & 10:02:00 \\
\hline Inflate all packers and put all packers on accumulator at $-14.5 \mathrm{MPa}$. & $12-20-94$ & 354 & 354 & 10:32:03 \\
\hline Shut in all zones. & $12-20-94$ & 354 & 354 & 10:32:55 \\
\hline Begin constant-pressure-injection test $\# 2$ in TZ1 at $2.325 \mathrm{MPa}$. & $12-21-94$ & 355 & 355 & 08:38:40 \\
\hline $\begin{array}{c}\text { Shut in TZ1 from DPT panel due to empty injection column terminating constant- } \\
\text { pressure-injection test } \# 2 \text { in } T Z 1 .\end{array}$ & $12-21-94$ & 355 & 355 & 08:47:47 \\
\hline Begin constant-pressure-injection test $\# 3$ in $\mathrm{TZ1}$ at $\sim 2.2 \mathrm{MPa}$. & $12-28-94$ & 362 & 362 & 12:14:05 \\
\hline Leaky fitting on accumulator. & 12-29-94 & 363 & 363 & 08:03:00 \\
\hline Shut in T21 trom DPT panel terminating constant-pressure-injection test $\# 3$ in TZ1. & $12-30-94$ & 364 & 364 & 08:10:17 \\
\hline Leaky valve on $\mathrm{GZ}$. & $12-30-94$ & 364 & 364 & 08:10:50 \\
\hline Replace bad gage on TZ2. & $12-30-94$ & 364 & 364 & 08:37:40 \\
\hline End data file L4P51D09. & $1-3-95$ & 3 & 368 & $08: 57: 35$ \\
\hline Begin data file L4P51D10. & 1-3-95 & 3 & 368 & 09:01:34 \\
\hline End data file L4P51D10. & $1.4-95$ & 4 & 369 & 10:03:16 \\
\hline
\end{tabular}


Table 3-8 (Continued). Permeability-Testing Sequence L4P51-D1 Events

\begin{tabular}{|c|c|c|c|c|}
\hline EVENT & DATE & $\begin{array}{c}\text { CALENDAR } \\
\text { DAY }\end{array}$ & $\begin{array}{c}1994 \\
\text { CALENDAR } \\
\text { DAY }\end{array}$ & $\begin{array}{c}\text { TIME } \\
\text { (HH:MM:SS) }\end{array}$ \\
\hline Begin data file L4P51D11. & $14-95$ & 4 & 369 & 10:06:10 \\
\hline Tubing string is completely filled with brine. & $1-16-95$ & 16 & 381 & 08:38:00 \\
\hline Begin constant-pressure-injection test \#4 in TZ1 at $-2 \mathrm{MPa}$. & 1-18-95 & 18 & 383 & 13:24:30 \\
\hline Shut in TZ1 from DPT panel terminating constant-pressure-injection test \#4 in TZ1. & $1-19-95$ & 19 & 384 & 08:32:10 \\
\hline Begin constant-pressure-injection test $\# 5$ in TZ1 at $-4.4 \mathrm{MPa}$. & 1-19.95 & 19 & 384 & 08:54:57 \\
\hline Shut in TZ1 from DPT panel terminating constant-pressure-injection test $\# 5$ in TZ1. & $1-23-95$ & 23 & 388 & 08:05:15 \\
\hline Begin constant-pressure-injection test \#6 in TZ1 at $\sim 7 \mathrm{MPa}$. & $1-23-95$ & 23 & 388 & 08:12:55 \\
\hline DAS was not functioning property upon artival. & $1-24-95$ & 24 & 389 & 09:16:00 \\
\hline End data file L4P51D11. & $1-30-95$ & 30 & 395 & 08:32:50 \\
\hline Begin data file L4P51D12. & $1-30-95$ & 30 & 395 & 08:35:56 \\
\hline Increase TZ2 pressure to $5.283 \mathrm{MPz}$. & 2-2-95 & 33 & 398 & 08:53:45 \\
\hline Open TZ2 to accumulator at $4.845 \mathrm{MPa}$. & $2-2-95$ & 33 & 398 & 13:08:20 \\
\hline Increase $T Z 2$ pressure via accumulator to $5.152 \mathrm{MPa}$. & 2-2-95 & 33 & 398 & 13:08:59 \\
\hline Shut in TZ1 from DPT panel terminating constant-pressure-injection test \#6 in TZ1. & $2-6-95$ & 37 & 402 & 08:35:52 \\
\hline End data file L4P51012. & 2-10-95 & 41 & 406 & 13:05:55 \\
\hline Begin data file L4P51D13. & 2-10-95 & 41 & 406 & 13:08:29 \\
\hline End data file L4P51D13. & 2-17-95 & 48 & 413 & 07:36:53 \\
\hline Shut in all packers from accumulator to eliminate temperature effects. & 2-20-95 & 51 & 416 & 12:19:00 \\
\hline Shut in $T Z 2$ from accumulator to eliminate temperature effects. & 2-20-95 & 51 & 416 & 12:21:00 \\
\hline Begin data file L4P51D14. & 2-20-95 & 51 & 416 & 12:43:24 \\
\hline End data file L4P51D14. & 3-3-95 & 62 & 427 & 07:57:22 \\
\hline Begin data file L4P51015. & 3-3-95 & 62 & 427 & 07:58:49 \\
\hline Depressurze all zones. & 3-20-95 & 79 & 444 & 11:27:00 \\
\hline Deflate all packers. & 3-20-95 & 79 & 444 & 11:28:30 \\
\hline End data file L4P51D15. & 3-21-95 & 80 & 445 & 08:53:25 \\
\hline Remove multipacker test tool \# P51-D1B from borehole L4P51. & 3-21-95 & 80 & 445 & 15:00:00 \\
\hline
\end{tabular}

Figures 3-45 through 3-49 illustrate the zone pressures, packer pressures, axial-LVDT displacement, radial-LVDT displacement, and fluid production during constant-pressureflow tests, respectively, for permeability-testing sequence L4P51-D1. It should be noted that Figure 3-49 (Fluid production during constant-pressure-flow tests in permeabilitytesting sequence L4P51-D1) consists of two parts (Figures 3-49a and 3-49b). Copies of the video-log associated with testing sequence L4P51-D1 identified in Table 3-8 are provided in the SWCF under WPO \#45907. 


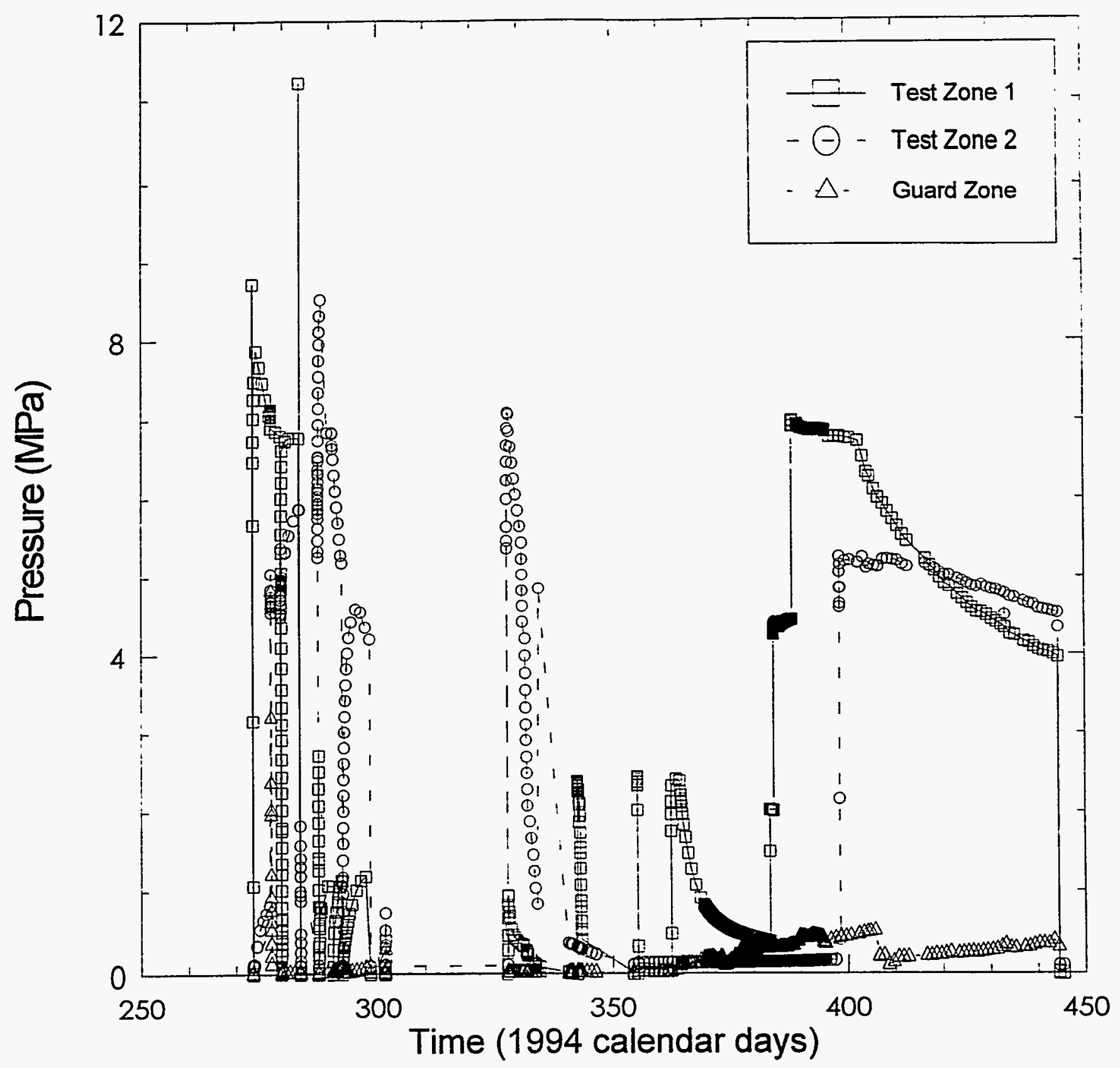

INTERABB115-81-0

Figure 3-45. Zone pressures during permeability-testing sequence L4P51-D1. 


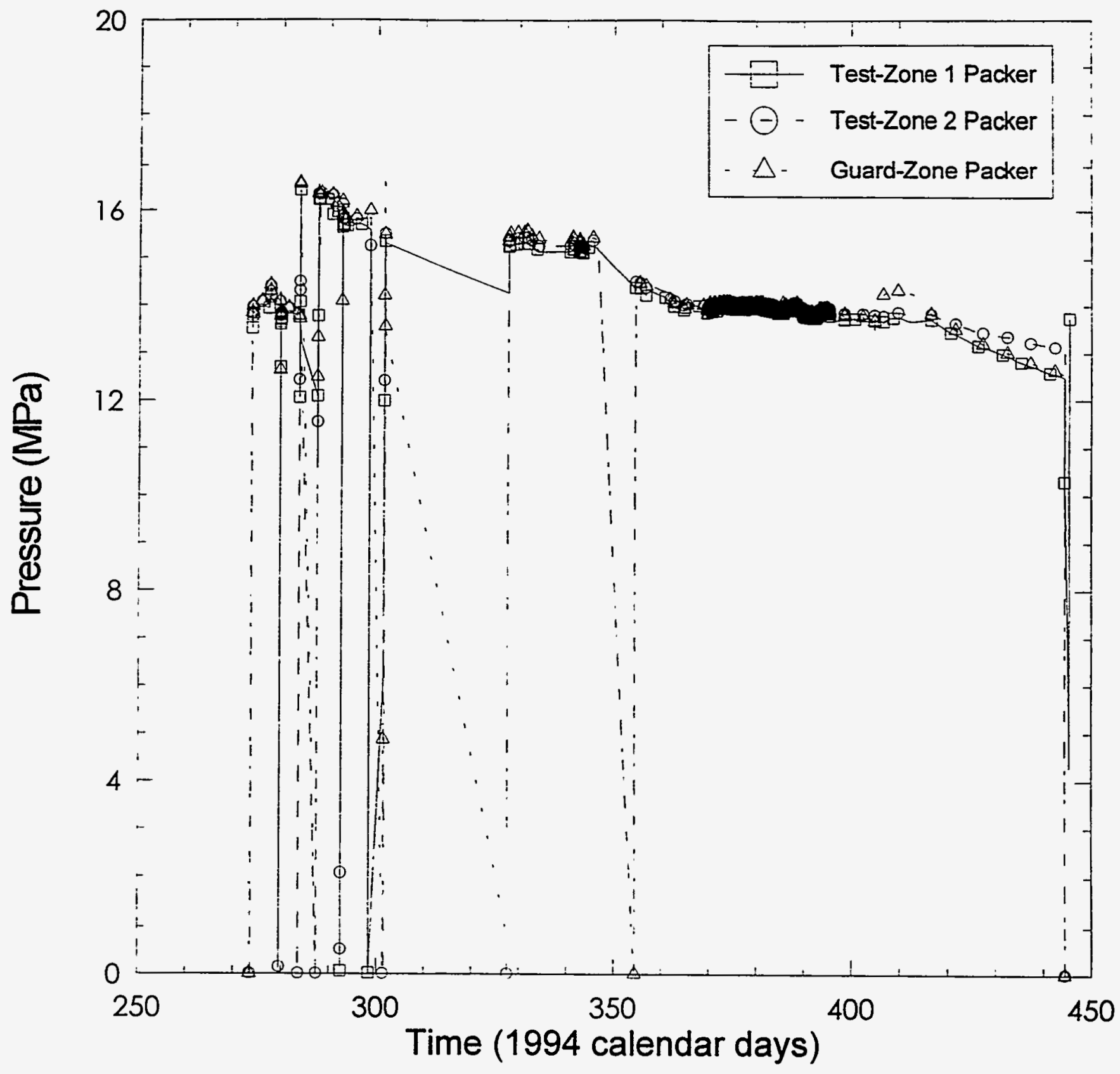

INTERA-T 15-62-0

Figure 3-46. Packer pressures during permeability-testing sequence L4P51-D1. 


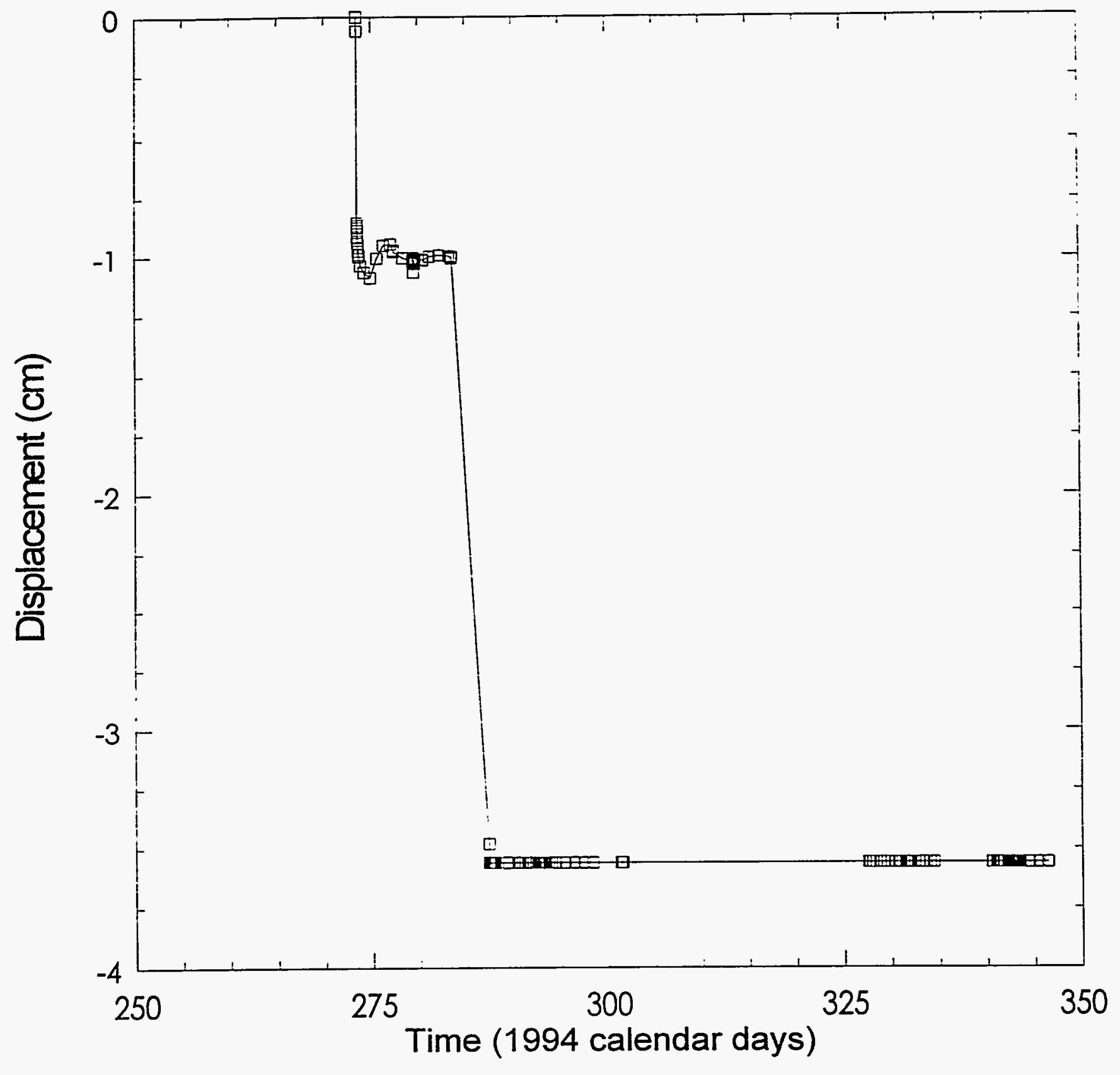

INTERA-6115-63-0

Figure 3-47. Axial-LVDT displacement during permeability-testing sequence L4P51-D1. 


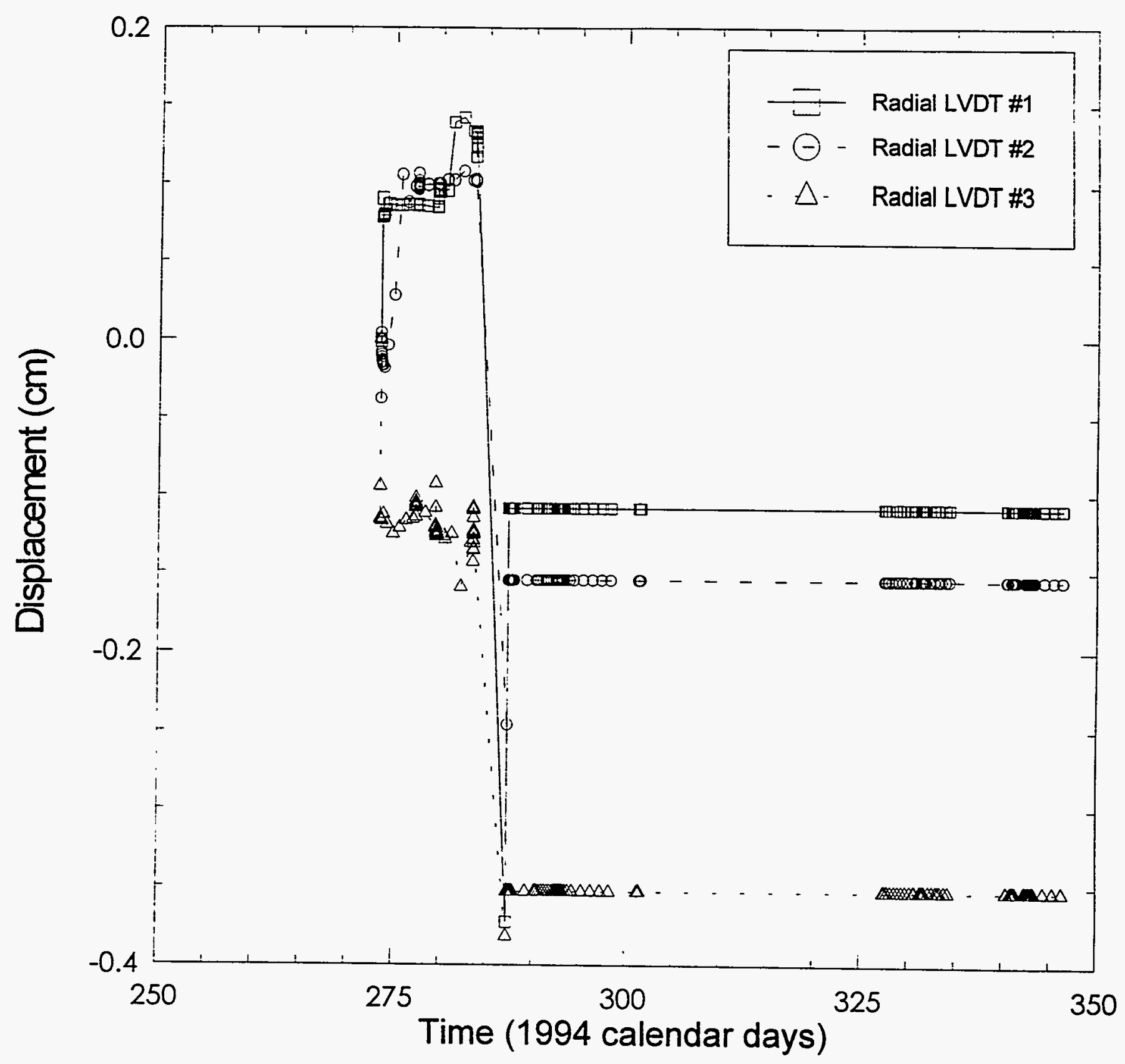

INTERA- $115-84-0$

Figure 3-48. Radial-LVDT displacement during permeability-testing sequence L4P51-D1. 


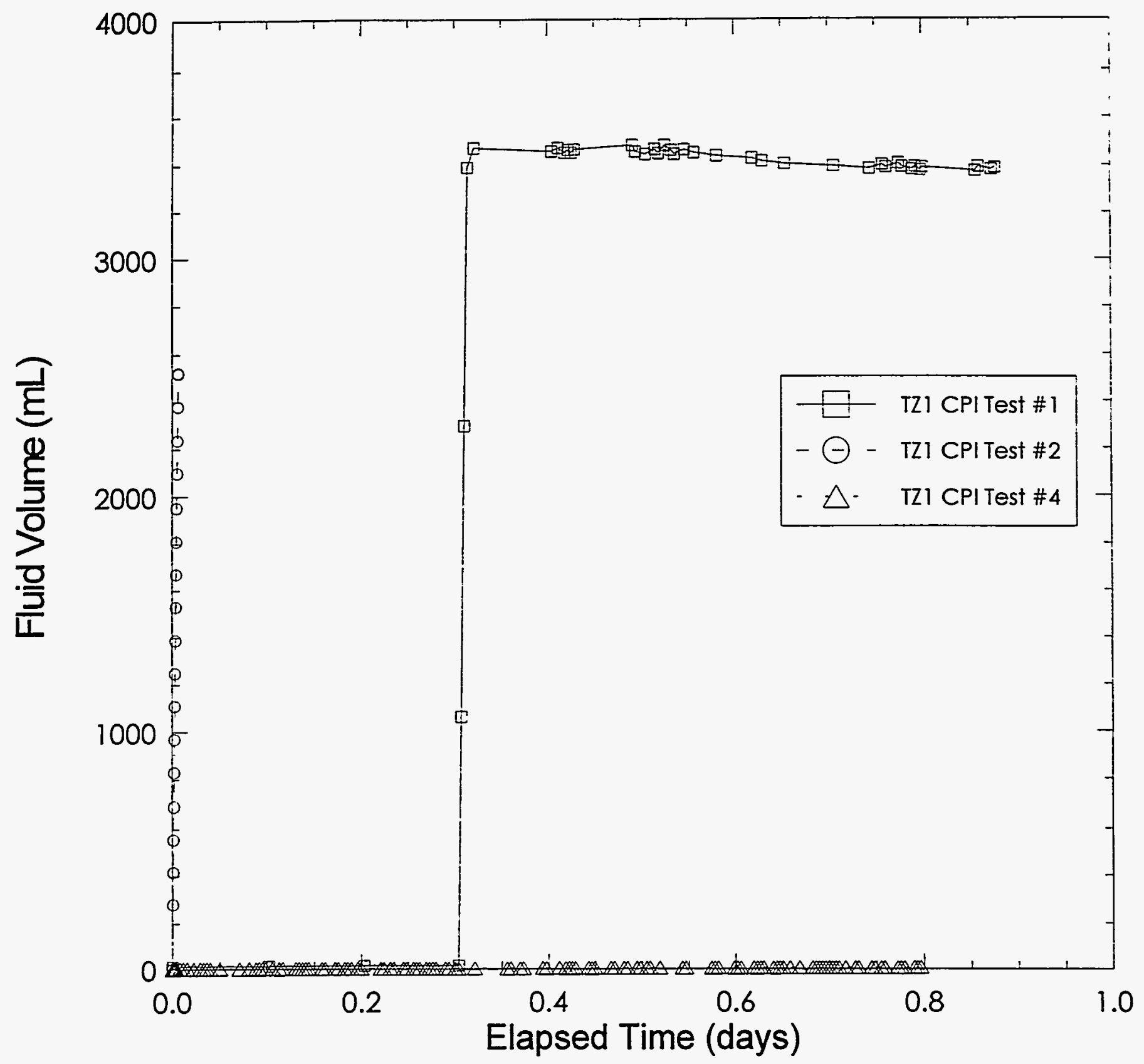

INTERABST15-85-0

Figure 3-49a. Fluid production during constant-pressure-flow tests in permeabilitytesting sequence L4P51-D1. 


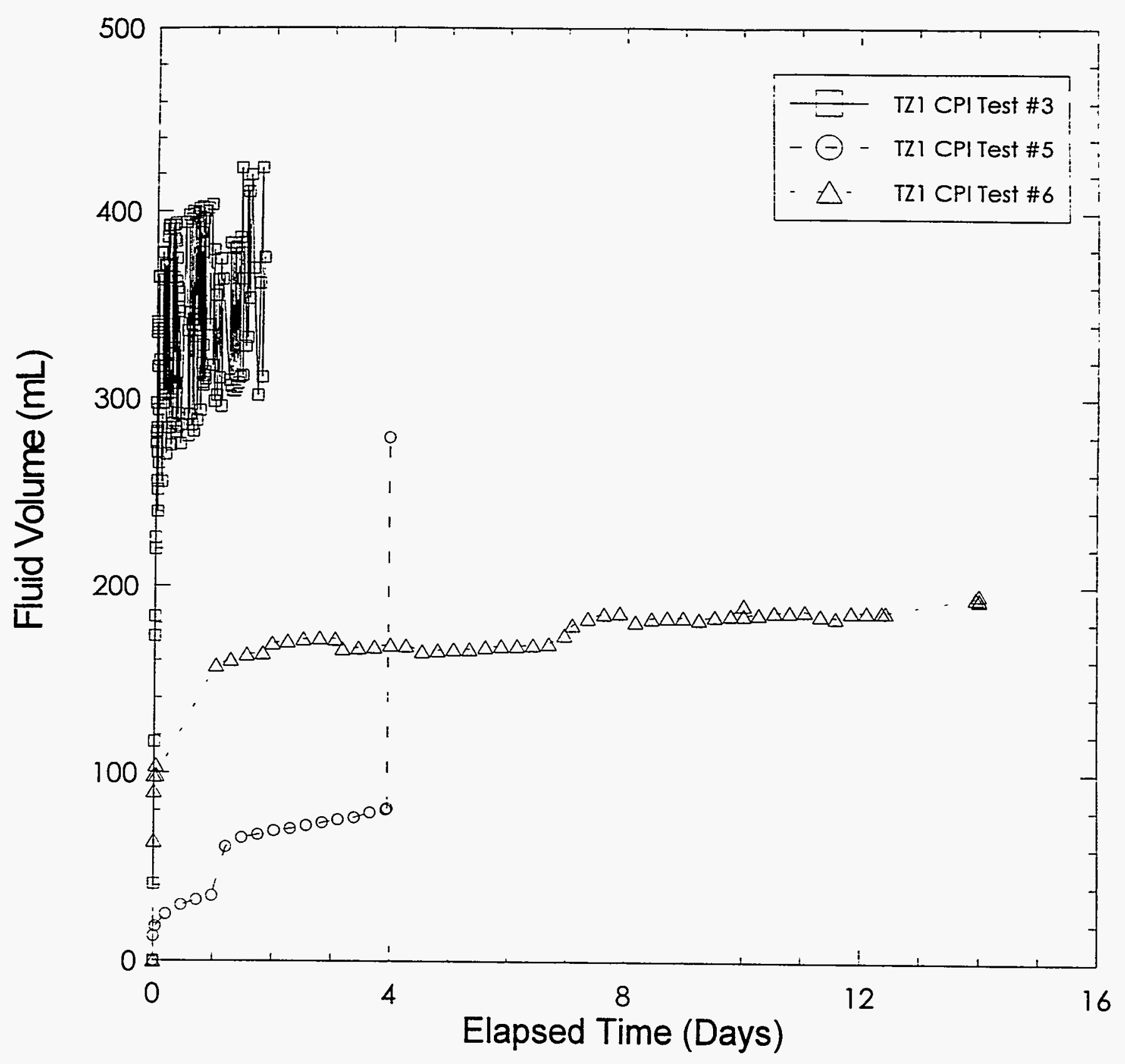

INTERA-811568-0

Figure $3-49 \mathrm{~b}$. Fluid production during constant-pressure-flow tests in permeabilitytesting sequence L4P51-D1 (continued). 
Table 3-9 indicates the equipment that was used and the duration that each instrument was used during permeability-testing sequence L4P51-D1.

Table 3-9. Permeability-Testing Sequence L4P51-D1 Equipment

\begin{tabular}{|c|c|c|c|c|}
\hline Equipment & Location & Serial \# & Installed & Removed \\
\hline DAS Software & N/A & PERM4F & $9-30-94$ & $3-20-95$ \\
\hline DCU (HP3497A) & N/A & $2629 a 22040$ & $9-30-94$ & $10-14-94$ \\
\hline DCU (HP3497A) & N/A & $2629 a 21996$ & $10-14-94$ & $3-3-95$ \\
\hline DCU (HP3497A) & N/A & $2629 a 22040$ & $3-3-95$ & $3-20-95$ \\
\hline $\begin{array}{c}\text { Transducer } \\
\text { (Druck D930-18) }\end{array}$ & Test Zone 1 & 609375 & $9-30-94$ & $3-20-95$ \\
\hline $\begin{array}{c}\text { Transducer } \\
\text { (Druck D930-18) }\end{array}$ & Test Zone 2 & 609368 & $9-30-94$ & $3-20-95$ \\
\hline $\begin{array}{c}\text { Transducer } \\
\text { (Druck D930-18) }\end{array}$ & $\begin{array}{l}\text { Test Zone } 1 \\
\text { Packer }\end{array}$ & 609371 & $9-30-94$ & $3-20-95$ \\
\hline $\begin{array}{c}\text { Transducer } \\
\text { (Druck D930-18) }\end{array}$ & $\begin{array}{l}\text { Test Zone } 2 \\
\text { Packer }\end{array}$ & 609374 & $9-30-94$ & $3-20-95$ \\
\hline $\begin{array}{c}\text { Transducer } \\
\text { (Druck D930-18) }\end{array}$ & Guard Zone & 609366 & $9-30-94$ & $3-20-95$ \\
\hline $\begin{array}{c}\text { Transducer } \\
\text { (Druck D930-18) }\end{array}$ & $\begin{array}{l}\text { Guard Zone } \\
\text { Packer }\end{array}$ & 609369 & $9-30-94$ & $3-20-95$ \\
\hline $\begin{array}{c}\text { Transducer } \\
\text { (Druck D930-18) }\end{array}$ & DPT Panel & 609372 & $12-6-94$ & $3-20-95$ \\
\hline $\begin{array}{c}\text { Transducer } \\
\text { (Druck D930-18) }\end{array}$ & $\begin{array}{l}\text { Pressure } \\
\text { Controller }\end{array}$ & 609370 & $12-6-94$ & $1-4-95$ \\
\hline LVDT (Trans-Tek 241) & N/A & R04 & $9-30-94$ & $10-11-94$ \\
\hline LVDT (Trans-Tek 241) & N/A & R16 & $9-30-94$ & $10-11-94$ \\
\hline LVDT (Trans-Tek 241) & N/A & $\mathrm{R} 17$ & $9-30-94$ & $10-11-94$ \\
\hline LVDT (Trans-Tek 245) & N/A & $\mathrm{AO} 2$ & $9-30-94$ & $10-11-94$ \\
\hline Injection Column & N/A & 265 & $12-6-94$ & $12-28-94$ \\
\hline Injection Column & N/A & 264 & $12-6-94$ & $12-28-94$ \\
\hline Injection Column & N/A & 88 & $12-28-94$ & $2-20-95$ \\
\hline $\begin{array}{c}\text { DPT } \\
\text { (Rosemount 1151DP) }\end{array}$ & N/A & 1140864 & $12-6-94$ & $1-4-95$ \\
\hline $\begin{array}{c}\text { DPT } \\
\text { (Rosemount 1151DP) }\end{array}$ & $N / A$ & 1389938 & $1-4-95$ & $2-20-95$ \\
\hline
\end{tabular}

* Installed dates for injection columns refer to dates of initial use rather than date installed. 
Permeability-testing sequence L4P51-D2 took place in Room L4 in borehole L4P51. This test sequence was designed to investigate the brine permeability of an argillaceous halite approximately $10 \mathrm{~m}$ below MB140. Table 3-10 gives a detailed description of the events that occurred during permeability-testing sequence L4P51-D2.

Table 3-10. Permeability-Testing Sequence L4P51-D2 Events

\begin{tabular}{|c|c|c|c|c|}
\hline EVENT & DATE & $\begin{array}{c}\text { CALENDAR } \\
\text { DAY }\end{array}$ & $\begin{array}{c}1995 \\
\text { CALENDAR } \\
\text { DAY }\end{array}$ & $\begin{array}{c}\text { TIME } \\
\text { (HH:MM:SS) }\end{array}$ \\
\hline Begin data file L4P51D16. & $4-6-95$ & 96 & 96 & 12:07:41 \\
\hline $\begin{array}{l}\text { Install single-packer test tool \#P51-D2 in borehole L4P51 as indicated in the test-tool } \\
\text { configuration diagram (Figure 3-30). }\end{array}$ & $4-6-95$ & 96 & 96 & 12:10:00 \\
\hline Inflate TZP to $-14 \mathrm{MPa}$ & 4-6-95 & 96 & 96 & 12:13:00 \\
\hline Open TZP to accumulator at $14.319 \mathrm{MPa}$. & $4-6-95$ & 96 & 96 & 12:17:00 \\
\hline Circulate brine through $T Z$ to remove all possible gas. & 4-6-95 & 96 & 96 & 12:18:00 \\
\hline Shut in $T Z$. & 4-6-95 & 96 & 96 & 12:18:34 \\
\hline End data file L4P51D16. & 5-17-95 & 137 & 137 & 07:50:35 \\
\hline Begin data file L4P51D17. & 5-17-95 & 137 & 137 & 07:51:59 \\
\hline End data file L4P51D17. & $9-14.95$ & 257 & 257 & 07:06:10 \\
\hline Begin data file L4P51D18. & $9-14-95$ & 257 & 257 & 07:31:59 \\
\hline Depressurize $R$. & $10-25-95$ & 298 & 298 & 08:00:00 \\
\hline Deflate TZP. & $10-25-95$ & 298 & 298 & 08:10:00 \\
\hline Remove test tool from borehole. & 10-25-95 & 298 & 298 & 11:00:00 \\
\hline End data file L4P51D18. & $10-25-95$ & 298 & 298 & $06: 49: 25$ \\
\hline
\end{tabular}

Figures 3-50 and 3-51 illustrate the zone pressure and packer pressure, respectively, for permeability-testing sequence L4P51-D2. 


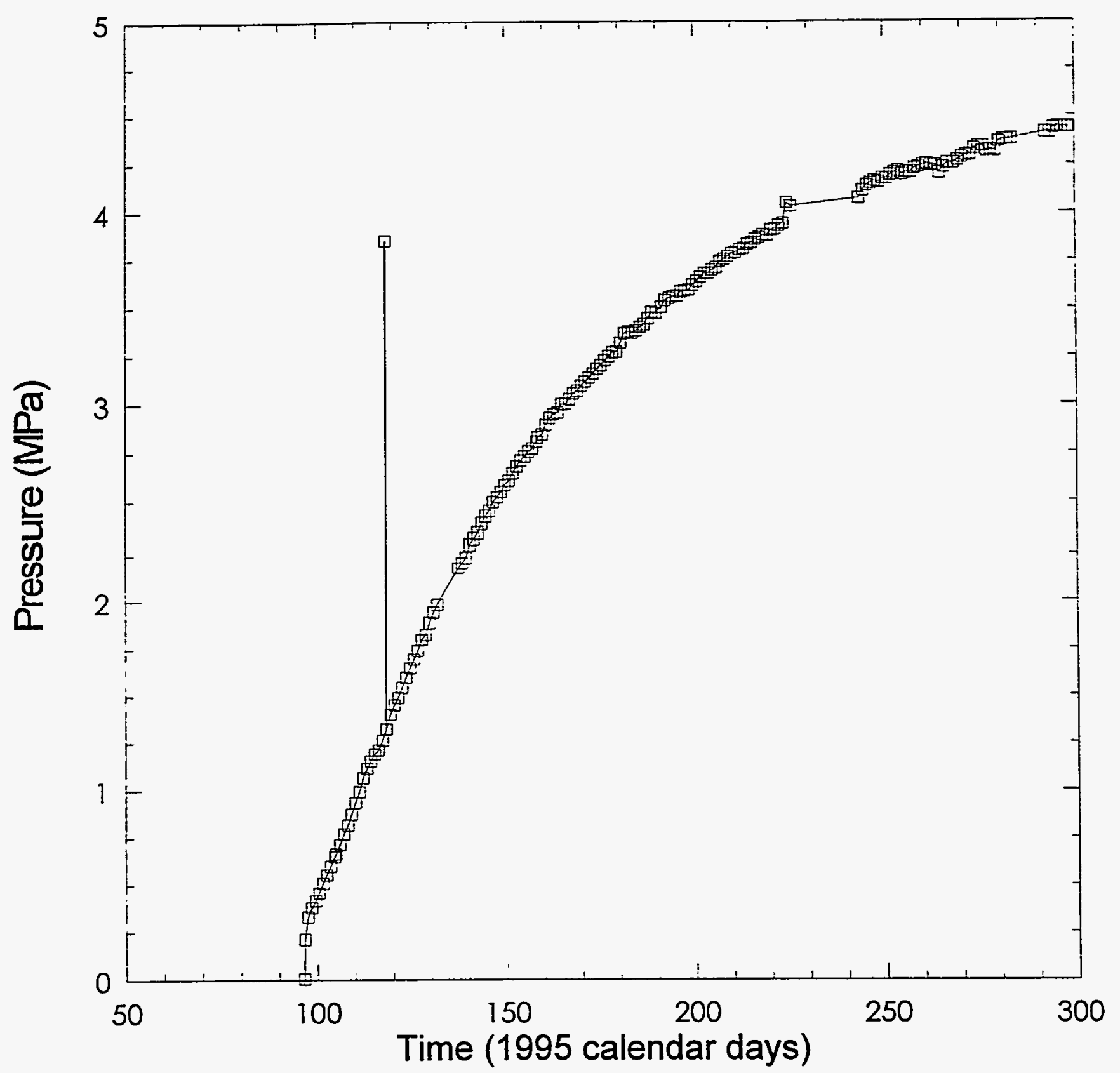

INTERA-6115-89-0

Figure 3-50. Zone pressure during permeability-testing sequence L4P51-D2. 


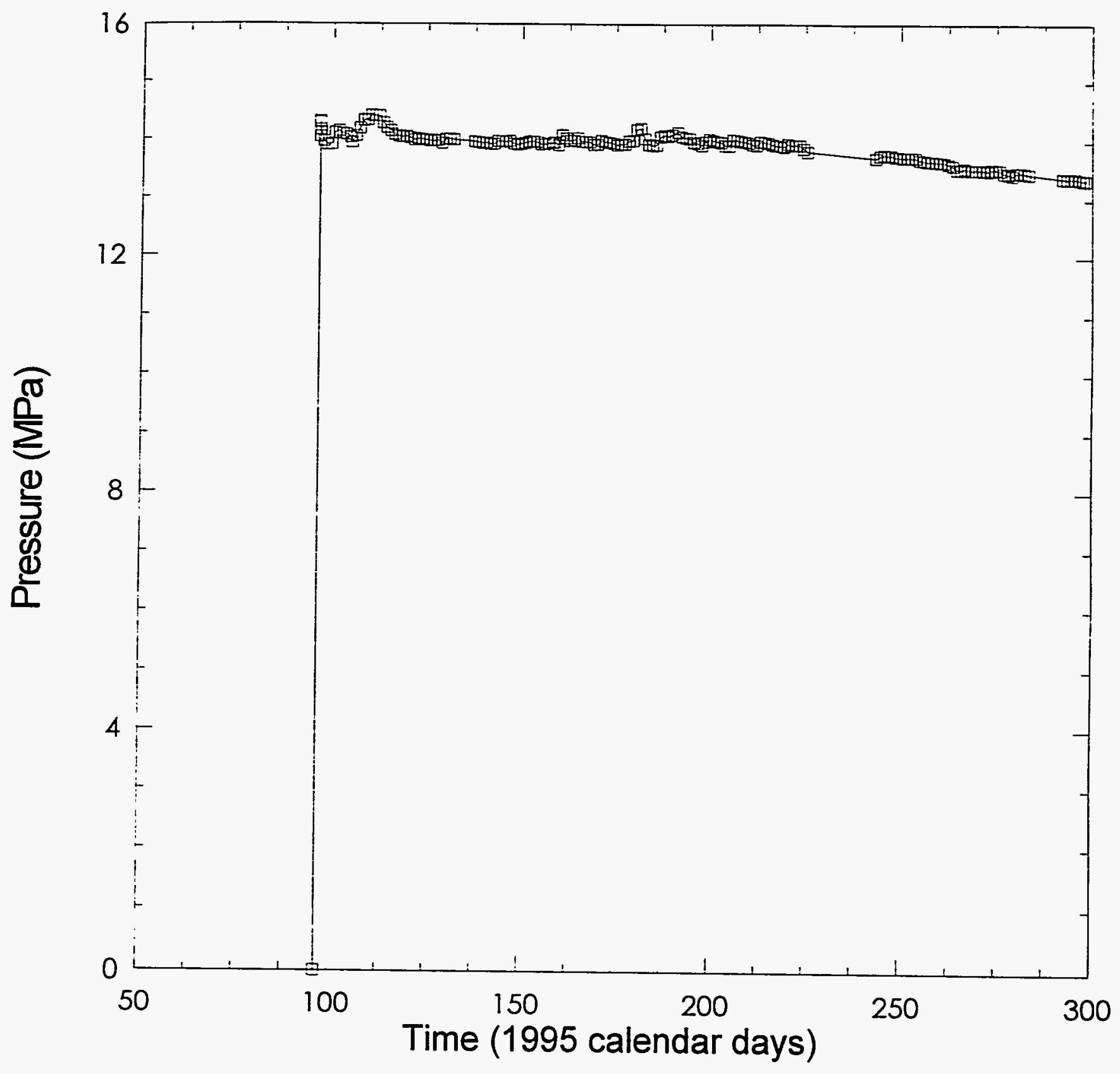

INTERABT15-70-0

Figure 3-51. Packer pressure during permeability-testing sequence L4P51-D2. 
Table 3-11 indicates the equipment that was used and the duration that each instrument was used during permeability-testing sequence L4P51-D2.

Table 3-11. Permeability-Testing Sequence L4P51-D2 Equipment

\begin{tabular}{ccccc}
\hline Equipment & Location & Serial \# & Installed & Removed \\
\hline DAS Software & N/A & PERM4F & $4-6-95$ & $10-25-95$ \\
\hline DCU (Hp3497A) & N/A & $2629 a 22040$ & $4-6-95$ & $10-25-95$ \\
\hline $\begin{array}{c}\text { Transducer } \\
\text { (Druck D930-18) }\end{array}$ & Test Zone & 609375 & $4-6-95$ & $10-25-95$ \\
\hline $\begin{array}{c}\text { Transducer } \\
\text { (Druck D930-18) }\end{array}$ & Test Zone Packer & 609368 & $4-6-95$ & $10-25-95$ \\
\hline
\end{tabular}

\subsubsection{BOREHOLE L4P52, PERMEABILITY-TESTING SEQUENCE L4P52-B}

Permeability-testing sequence L4P52-B took place in Room L4 in borehole L4P52. This test sequence was designed to investigate the brine permeability of MB138. Table 3-12 gives a detailed description of the events that occurred during permeability-testing sequence L4P52-B.

Table 3-12. Permeability-Testing Sequence L4P52-B Events

\begin{tabular}{|c|c|c|c|c|}
\hline EVENT & DATE & $\begin{array}{c}\text { CALENDAR } \\
\text { DAY }\end{array}$ & $\begin{array}{c}1992 \\
\text { CALENDAR } \\
\text { DAY }\end{array}$ & $\begin{array}{c}\text { TIME } \\
\text { (HH:MM:SS) }\end{array}$ \\
\hline Perform load test on the borehole flanged collar. & $12-10-92$ & 345 & 345 & 09:00:00 \\
\hline Deepen borehole with 4-inch $(10.16-\mathrm{cm})$ core barrel to 9.024 meters. & $12-10-92$ & 345 & 345 & 14:00:00 \\
\hline Deepen borehole with 4-inch $(10.16-\mathrm{cm})$ core barrel to 11.89 meters. & $12-11-92$ & 346 & 346 & $10: 20: 00$ \\
\hline Deepen borehole with 4-inch $(10.16-\mathrm{cm})$ core barrel to 14.18 meters. & $12-14-92$ & 349 & 349 & 11:00:00 \\
\hline $\begin{array}{l}\text { Install multipacker test tool \#37 in borehole L4P52 as indicated in the test- } \\
\text { tool configuration diagram (Figure 3-31). }\end{array}$ & $12-15-92$ & 350 & 350 & 12:00:00 \\
\hline Begin data file L4P52B01. & $12-17-92$ & 352 & 352 & 11:26:01 \\
\hline Inflate GZP to $-10.3 \mathrm{Mpa}$. & $12-17-92$ & 352 & 352 & 14:00:00 \\
\hline Deflate GZP. & $12 \_17-92$ & 352 & 352 & 14:05:00 \\
\hline Shut in GZ. & $12-17-92$ & 352 & 352 & $14: 20: 00$ \\
\hline Fill $T Z$ and $G Z$ with Brine. & $12-17-92$ & 352 & 352 & 14:38:00 \\
\hline GZP pressure decayed to $0.516 \mathrm{MPa}$ due to leaky fitting. & $12-18-92$ & 353 & 353 & 08:00:00 \\
\hline Inflate GZP to $\sim 10.3 \mathrm{Mpa}$ & $12-18-92$ & 353 & 353 & 08:56:00 \\
\hline Inflate TZP to $\sim 10.3 \mathrm{MPa}$ & 12-18-92 & 353 & 353 & 09:04:00 \\
\hline Decrease $\mathrm{TZ}$ and $\mathrm{GZ}$ pressure to $-0.2 \mathrm{MPa}$ and shut in. & $12-18-92$ & 353 & 353 & 09:12:00 \\
\hline Increase TZP pressure to $110.3 \mathrm{Mpa}$ & $12-18-92$ & 353 & 353 & 09:13:00 \\
\hline Decrease GZ pressure to $\sim 0.18 \mathrm{Mpa}$ and shut in. & $12-18-92$ & 353 & 353 & $09: 15: 00$ \\
\hline
\end{tabular}


Table 3-12 (Continued). Permeability-Testing Sequence L4P52-B Events

\begin{tabular}{|c|c|c|c|c|}
\hline EVENT & DATE & $\begin{array}{l}\text { CALENDAR } \\
\text { DAY }\end{array}$ & $\begin{array}{c}1992 \\
\text { CALENDAR } \\
\text { DAY }\end{array}$ & $\begin{array}{c}\text { TIME } \\
\text { (HH:MM:SS) }\end{array}$ \\
\hline No voltage from the power supply card to the transducers. & $1-21-93$ & 24 & 387 & 08:11:00 \\
\hline End data file L4P52B01. & $1-21-93$ & 21 & 387 & $11: 18: 57$ \\
\hline Begin data file L4P52B02. & $1-21-93$ & 21 & 387 & $11: 23: 00$ \\
\hline End data field L4P52B02. & 2-10-93 & 41 & 407 & $12: 00: 54$ \\
\hline Begin data file L4P52B03. & 2-10-93 & 41 & 407 & 12:02:44 \\
\hline $\begin{array}{c}\text { Begin constant-pressure-withdrawal test \#1 in TZ at } \sim 1 \text { Mpa below TZ } \\
\text { pressure }(\sim 8.05 \mathrm{Mpa}) .\end{array}$ & $2-11-93$ & 42 & 408 & $11: 23: 00$ \\
\hline Repair leaky fitting on the DPT panel. & 2-11-93 & 42 & 408 & $16: 15: 93$ \\
\hline Repair leak on the DPT panel. & 2-17-93 & 48 & 414 & $13: 49: 38$ \\
\hline $\begin{array}{l}\text { Shut in TZ from DPT panel terminating constant-pressure-withdrawal test } \\
\text { in } \mathrm{Z} \text {. }\end{array}$ & $2-24-93$ & 55 & 421 & $09: 13: 17$ \\
\hline DAS not functioning property upon arrival. & $3-19-93$ & 78 & 444 & $10: 14: 55$ \\
\hline End data file L4P52B03. & $3-23-93$ & 82 & 448 & $10: 18: 38$ \\
\hline Begin data file L4P52B04. & $-23-93$ & 82 & 448 & $11: 37: 18$ \\
\hline Potential problem with P4 transducer. & 3-25-93 & 84 & 450 & $09: 35: 00$ \\
\hline Open GZ to accumulator at $-7.1 \mathrm{Mpa}$. & 4-19-93 & 109 & 475 & $13: 30: 00$ \\
\hline End data file L4P52B04. & $5-10-93$ & 130 & 496 & 09:59:07 \\
\hline Begin data file L4P52B05. & $5-10-93$ & 130 & 496 & 10:01:58 \\
\hline Shut in GZ from accumulator. & $5-12-93$ & 132 & 498 & $09: 51: 58$ \\
\hline Open $G Z$ to full accumulator. & $5-12-93$ & 132 & 498 & 10:28:27 \\
\hline Increase GZP pressure via accumulator. & $5-19-93$ & 139 & 505 & $11: 06: 59$ \\
\hline Shut in GZP from accumulator. & $5-19-93$ & 139 & 505 & 11:10:59 \\
\hline Shut in GZ from accumulator. & 5-19-93 & 139 & 505 & $11: 12: 00$ \\
\hline Open GZ to full accumulator. & $5-19-93$ & 139 & 139 & $11: 21: 00$ \\
\hline Shut in GZ from accumulator. & 5-20-93 & 140 & 506 & $13: 14: 00$ \\
\hline $\begin{array}{c}\text { Begin constant-pressure-injection test \#1 in TZ at }-0.4 \mathrm{MPa} \text { above TZ } \\
\text { pressure }(9.598 \mathrm{MPa}) .\end{array}$ & $5.7-93$ & 158 & 524 & $09: 44: 24$ \\
\hline End data file L4P52B05. & $6-8-93$ & 159 & 525 & $12: 06: 46$ \\
\hline Begin data file L4P52B06. & $6-8-93$ & 159 & 525 & $12: 56: 20$ \\
\hline $\begin{array}{l}\text { Shut in } T Z \text { from DPT panel terminating constant-pressure-injection test \#1 } \\
\text { in } T Z \text {. }\end{array}$ & $6-22-93$ & 173 & 539 & 10:03:57 \\
\hline $\begin{array}{c}\text { Begin constant-pressure-injection test } \# 2 \text { in } T Z \text { at }-1 \mathrm{MPa} \text { above } T Z \\
\text { pressure }(\sim 10.1 \mathrm{MPa}) .\end{array}$ & $6-22-93$ & 173 & 539 & $10: 22: 49$ \\
\hline Increase TZP pressure to $\sim 13 \mathrm{MPa}$. & $7-7-93$ & 188 & 554 & 10:12:00 \\
\hline Increase GZP pressure to $-13 \mathrm{Mpa}$. & 7.7 .93 & 188 & 554 & 10:14:00 \\
\hline Decrease GZ pressure to $\sim 8.4 \mathrm{Mpa}$. & $7-7-93$ & 188 & 554 & $10: 15: 00$ \\
\hline $\begin{array}{l}\text { Shut in TZ from DPT panel terminating constant-pressure-injection test } \# 2 \\
\text { in } T 2 .\end{array}$ & $7-7-93$ & 188 & 554 & $10: 18: 00$ \\
\hline $\begin{array}{c}\text { Begin constant-pressure-injection test \#3 in TZ at }-2 \mathrm{MPa} \text { above } T Z \\
\text { pressure }(-11.1 \mathrm{Mpa}) .\end{array}$ & $7-7-93$ & 188 & 554 & $11: 44: 00$ \\
\hline $\begin{array}{l}\text { Shut in TZ from DPT panel teminating constant-pressure-injection test \#3 } \\
\text { in TZ. }\end{array}$ & $7-19-93$ & 200 & 566 & 07:57:00 \\
\hline
\end{tabular}


Table 3-12 (Continued). Permeability-Testing Sequence L4P52-B Events

\begin{tabular}{ccccc} 
EVENT & DATE & $\begin{array}{c}\text { CALENDAR } \\
\text { DAY }\end{array}$ & $\begin{array}{c}\text { 1992 } \\
\text { CALENDAR } \\
\text { DAY }\end{array}$ & $\begin{array}{c}\text { TIME } \\
\text { (HH:MM:SS) }\end{array}$ \\
\hline End data file L4P52B06. & $8-2-93$ & 214 & 580 & $05: 26: 34$ \\
Begin data file L4P52B07. & $8-2-93$ & 214 & 580 & $09: 13: 19$ \\
The P4 transducer is giving incorrect readings again. & $8-3-93$ & 215 & 581 & $09: 41: 00$ \\
DAS not functioning properly upon arrival. & $10-7-93$ & 280 & 646 & $08: 32: 00$ \\
DAS not functioning for the past 3 days. & $11-15-93$ & 319 & 685 & $09: 03: 00$ \\
End data file L4P52B07. & $12-29-93$ & 363 & 729 & $11: 48: 23$ \\
\hline
\end{tabular}

Figures 3-52 through 3-57 illustrate the zone pressures, packer pressures, zone temperatures, axial-LVDT displacement, radial-LVDT displacement, and fluid production during constant-pressure-flow tests, respectively, for permeability-testing sequence L4P52-B. 


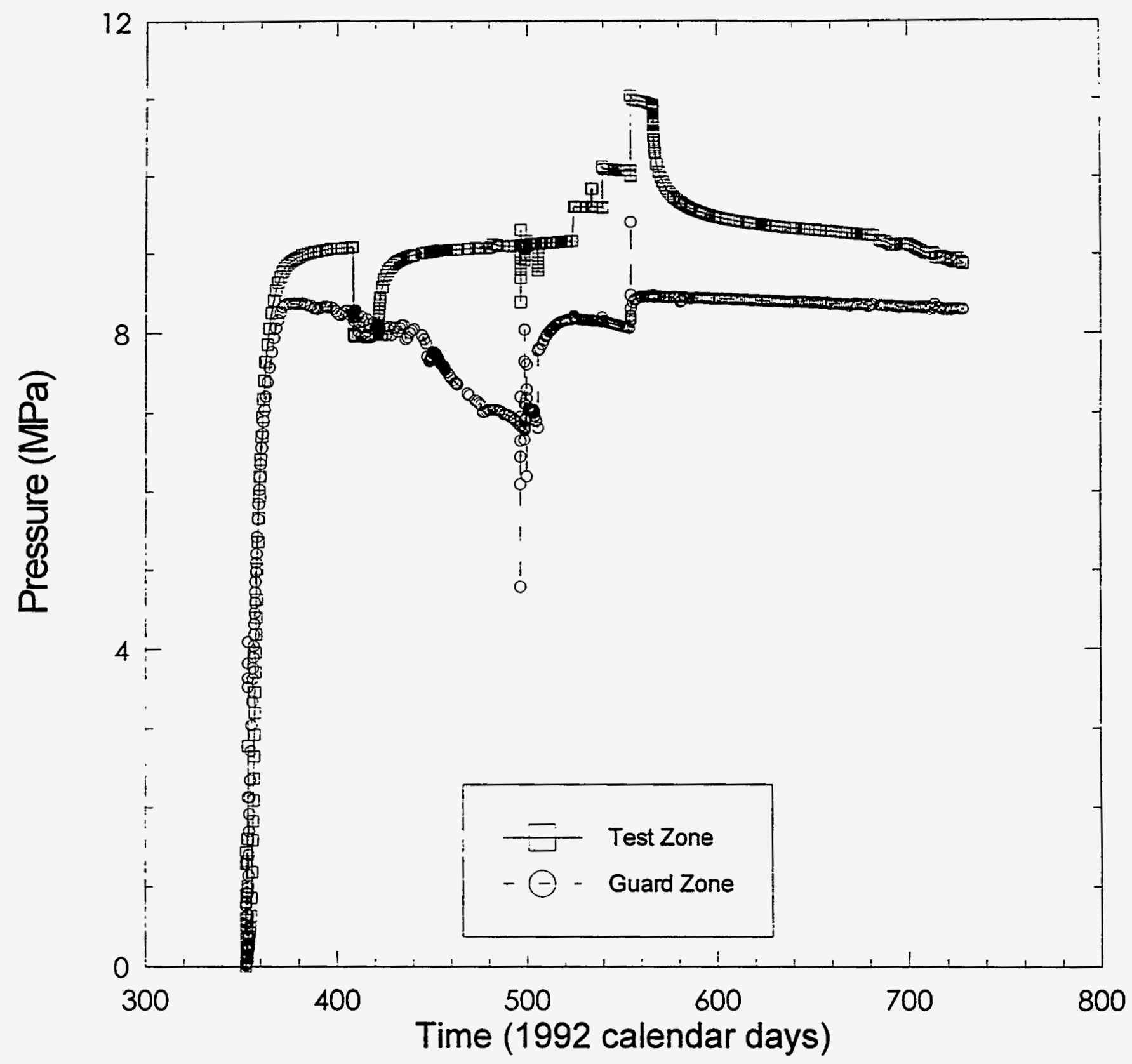

INTERA-61 15-71-0

Figure 3-52. Zone pressures during permeability-testing sequence L4P52-B. 


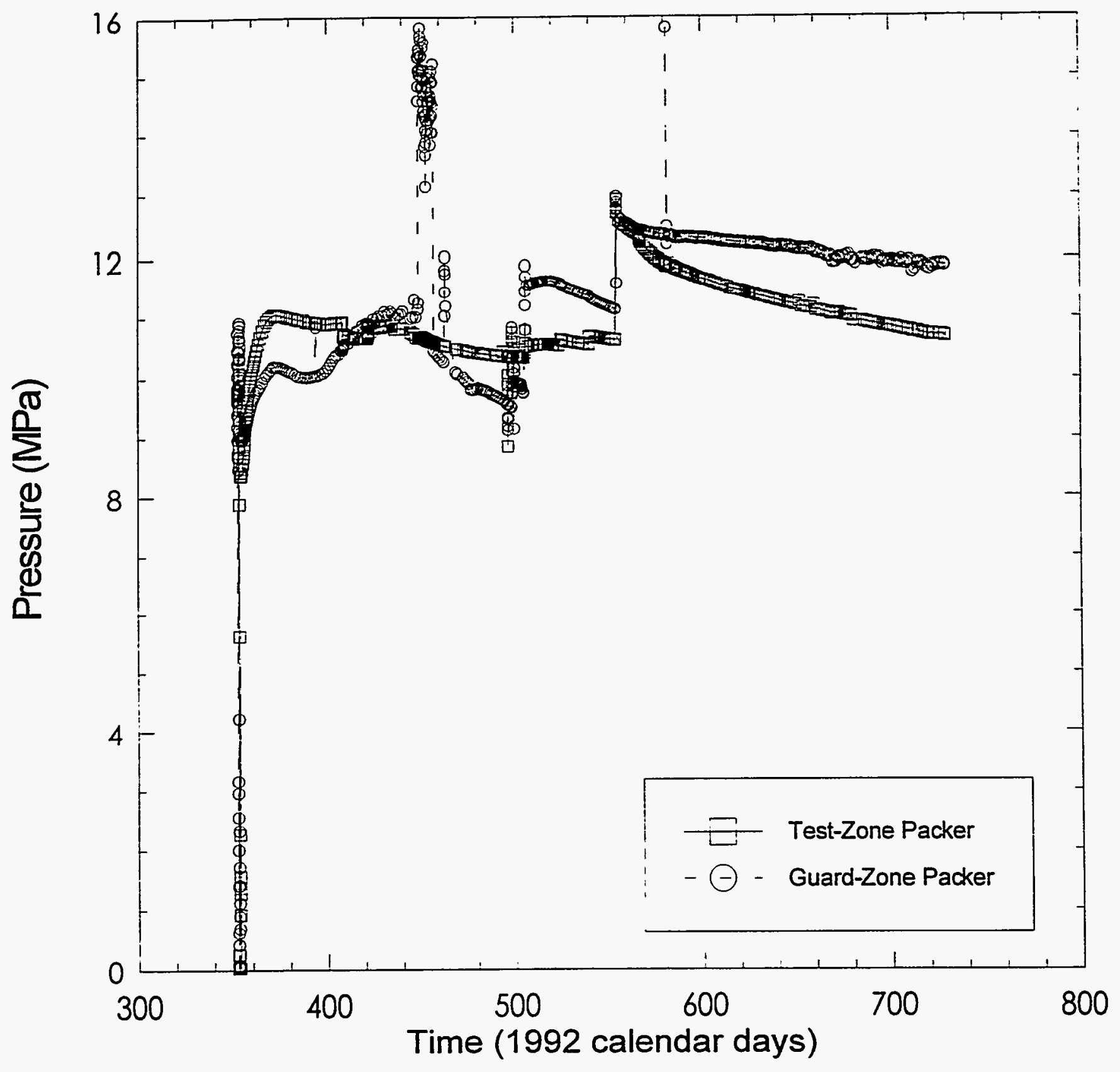

INTERA-6115-72-0

Figure 3-53. Packer pressures during permeability-testing sequence L4P52-B. 


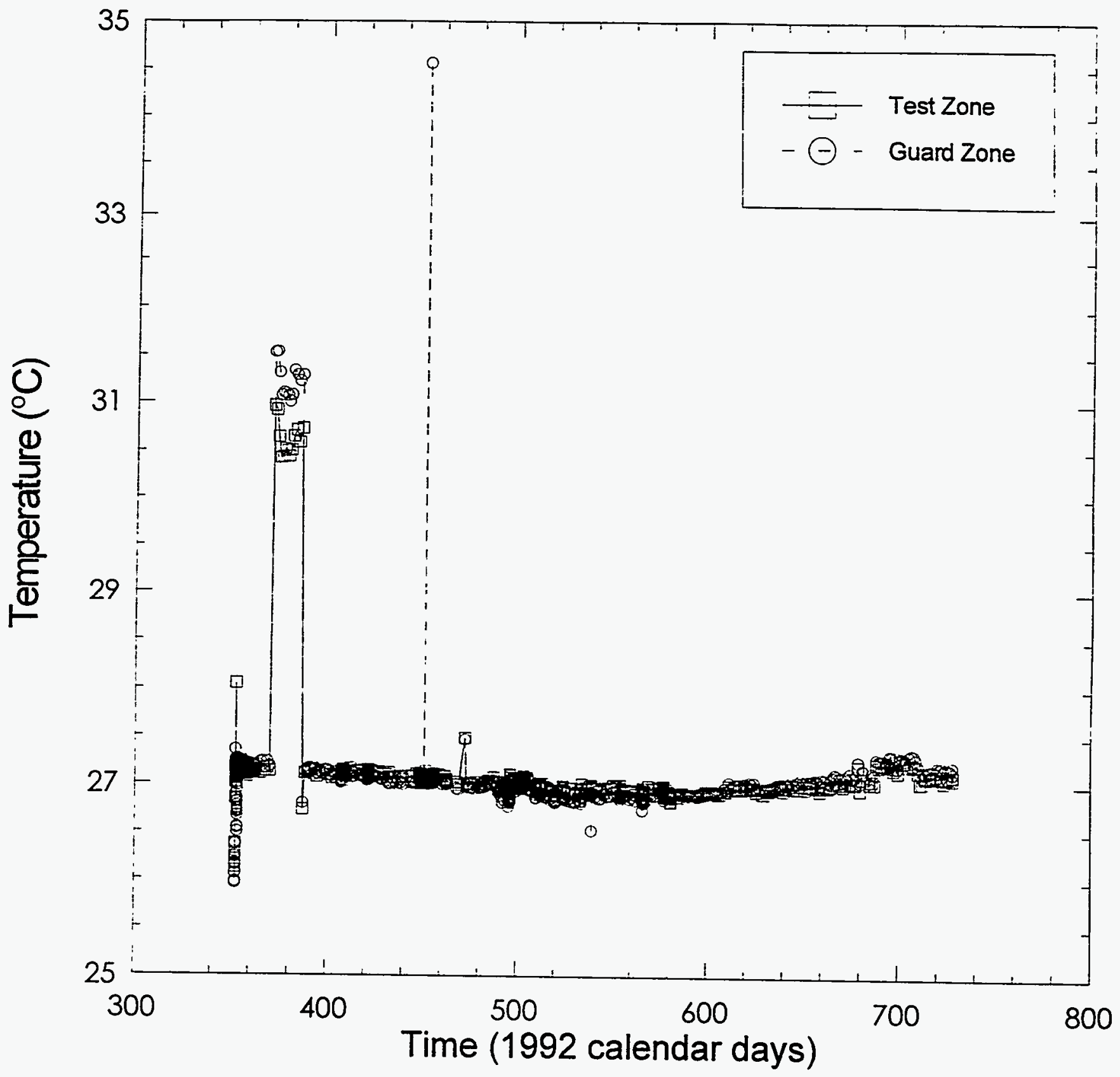

INTERA-61 15-73-0

Figure 3-54. Zone temperatures during permeability-testing sequence L4P52-B. 


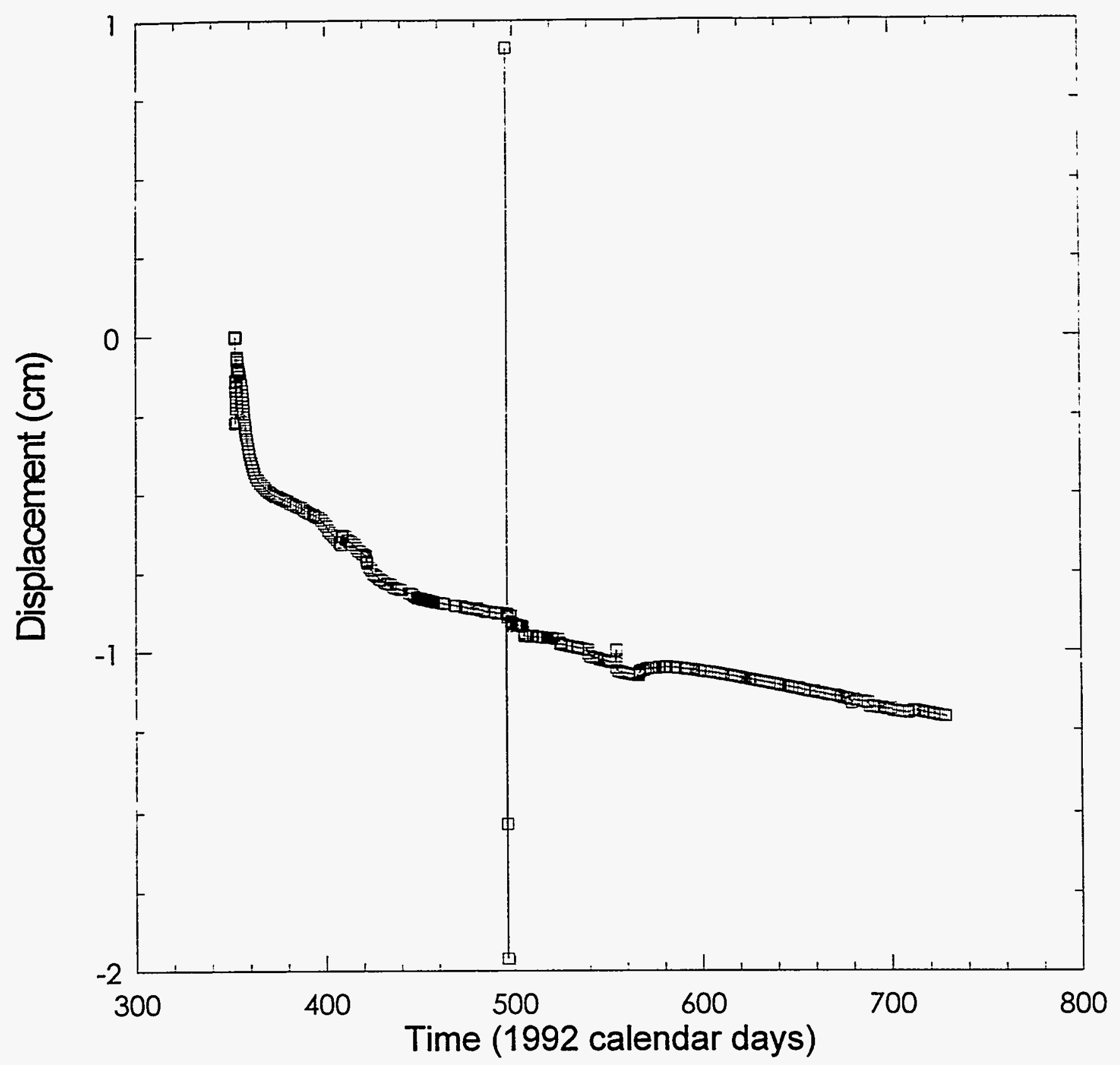

INTERABI15-740

Figure 3-55. Axial-LVDT displacement during permeability-testing sequence L4P52-B. 


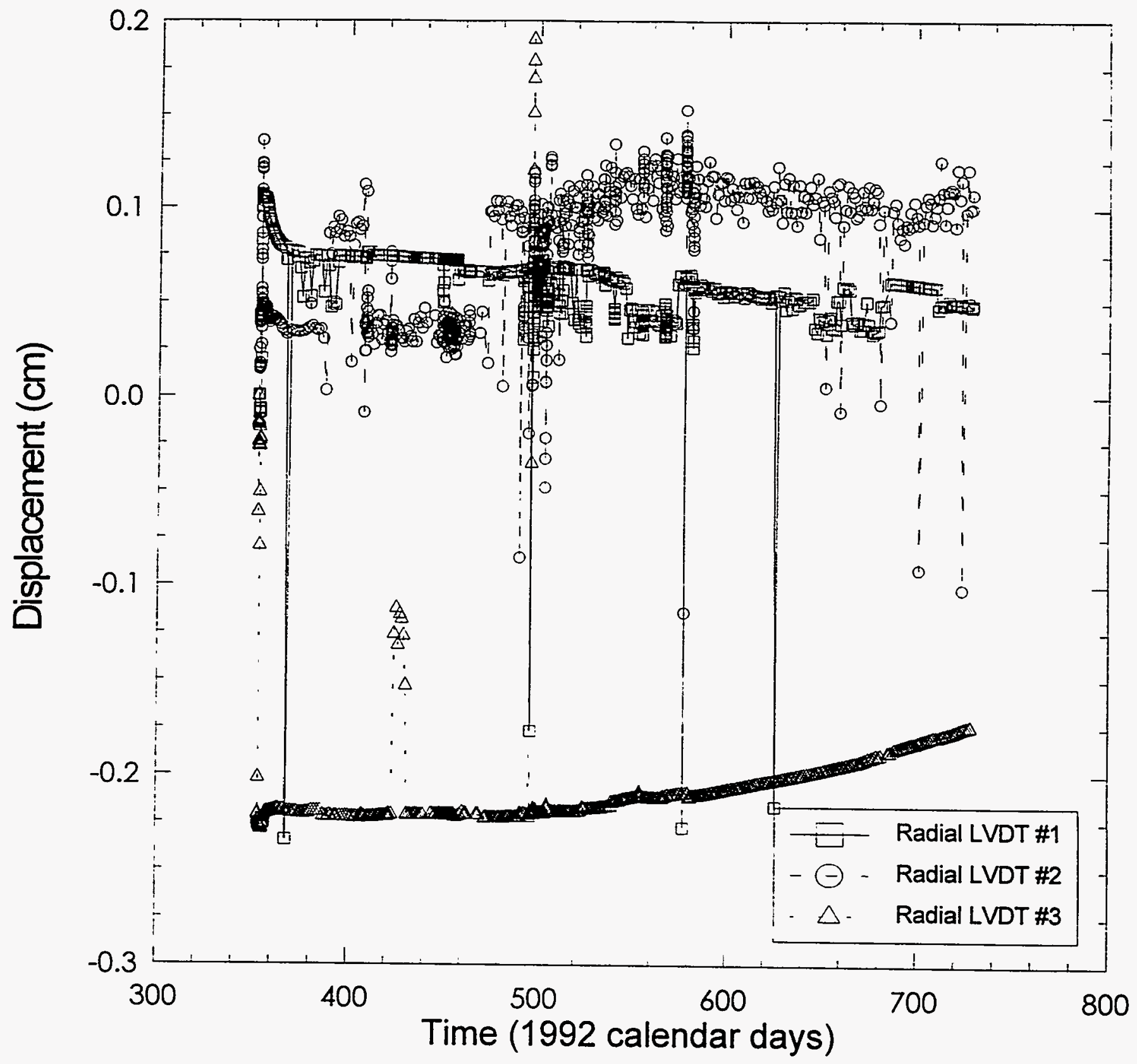

INTERA 8115-75-0

Figure 3-56. Radial-LVDT displacement during permeability-testing sequence L4P52-B. 


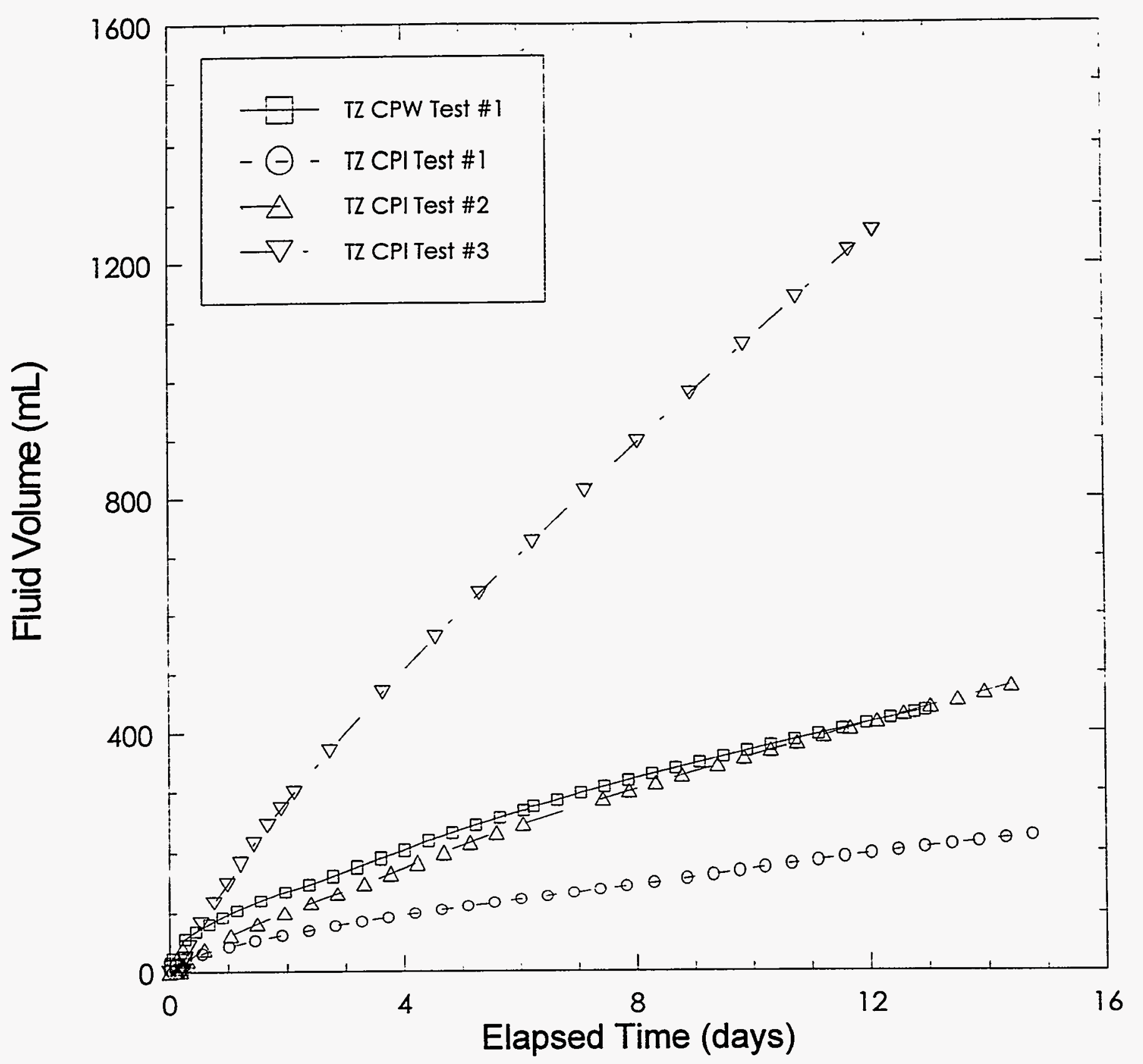

INTERA-6115-76-0

Figure 3-57. Fluid production during constant-pressure-flow tests in permeabilitytesting sequence L4P52-B. 
Table 3-13 indicates the equipment that was used and the duration that each instrument was used during permeability-testing sequence L4P52-B.

Table 3-13. Permeability-Testing Sequence L4P52-B Equipment

\begin{tabular}{|c|c|c|c|c|}
\hline Equipment & Location & Serial \# & Installed & Removed \\
\hline DAS Software & N/A & PERM4F & $12-17-92$ & $12-28-93$ \\
\hline DCU (HP3497A) & N/A & $2629 a 22040$ & $12-17-92$ & $1-21-93$ \\
\hline DCU (HP3497A) & N/A & $2629 a 21996$ & $4-16-93$ & $5-10-93$ \\
\hline DCU (HP3497A) & N/A & $2629 a 21990$ & $5-10-93$ & $9-22-93$ \\
\hline DCU (HP3497A) & N/A & $2629 a 21989$ & $9-22-93$ & $1-28-93$ \\
\hline $\begin{array}{c}\text { Transducer } \\
\text { (Druck PDCR 830) }\end{array}$ & Test Zone & 214048 & $12-17-92$ & $12-28-93$ \\
\hline $\begin{array}{c}\text { Transducer } \\
\text { (Druck PDCR 830) }\end{array}$ & Test Zone Packer & 214466 & $12-17-92$ & $12-28-93$ \\
\hline $\begin{array}{c}\text { Transducer } \\
\text { (Druck PDCR 830) }\end{array}$ & Guard Zone & 246913 & $12-17-92$ & $12-28-93$ \\
\hline $\begin{array}{c}\text { Transducer } \\
\text { (Druck PDCR 830) }\end{array}$ & $\begin{array}{l}\text { Guard Zone } \\
\text { Packer }\end{array}$ & 214470 & $12-17-92$ & $12-28-93$ \\
\hline $\begin{array}{c}\text { Transducer } \\
\text { (Druck PDCR 910) }\end{array}$ & DPT Panel & 322427 & $2-10-93$ & $3-23-93$ \\
\hline $\begin{array}{c}\text { Transducer } \\
\text { (Druck PDCR 830) }\end{array}$ & DPT Panel & 211694 & $3-23-93$ & $8-2-93$ \\
\hline LVDT (Trans-Tek 241) & N/A & Ro9 & $12-17-92$ & $12-28-93$ \\
\hline LVDT (Trans-Tek 241) & N/A & $\mathrm{R} 03$ & $12-17-92$ & $12-28-93$ \\
\hline LVDT (Trans-Tek 241) & N/A & $\mathrm{RO} 2$ & $12-17-92$ & $12-28-93$ \\
\hline LVDT (Trans-Tek 245) & N/A & $\mathrm{A} 03$ & $12-17-92$ & $12-28-93$ \\
\hline Thermocouple (Type E) & Test Zone & 1 & $12-17-92$ & $12-28-93$ \\
\hline Thermocouple (Type E) & Guard Zone & 2 & $12-17-92$ & $12-28-93$ \\
\hline Injection Column & N/A & 38 & $2-11-93$ & $2-24-93$ \\
\hline Injection Column & N/A & 77 & $6-7-93$ & $7-7-93$ \\
\hline Injection Column & N/A & 76 & $7-7-93$ & $7-19-93$ \\
\hline DPT (Rosemount 1151DP) & N/A & 1140864 & $2-10-93$ & $4-1-93$ \\
\hline DPT (Rosemount 1151DP) & N/A & 1140863 & $5-10-93$ & $9-1-93$ \\
\hline
\end{tabular}


*Installed dates for injection columns refer to dates of initial use rather than data installed.

\subsubsection{Permeability Testing in Waste Panel 1, Room 7}

\subsubsection{BOREHOLE S1P74, PERMEABILITY-TESTING SEQUENCE S1P74-A}

Permeability-testing sequence S1P74-A took place in Waste Panel 1, Room 7 in borehole S1P74. This test sequence was designed to investigate the brine permeability of anhydrite "a". Table 3-14 gives a detailed description of the events that occcurred during permeability-testing sequence S1P74-A.

\section{Table 3-14. Permeability-Testing Sequences S1P74-A Events}

\begin{tabular}{|c|c|c|c|c|}
\hline EVENT & DATE & $\begin{array}{l}\text { CALENDAR } \\
\text { DAY }\end{array}$ & $\begin{array}{c}1992 \\
\text { CALENDAR } \\
\text { DAY }\end{array}$ & $\begin{array}{c}\text { TIME } \\
\text { (HH:MM:SS) }\end{array}$ \\
\hline $\begin{array}{l}\text { Initiate borehole with } 7 \text {-inch }(17.78-\mathrm{cm}) \text { core barrel to } 0.62 \text { meters in order to } \\
\text { set a borehole collar. }\end{array}$ & $7-27-92$ & 209 & 209 & 11:15:00 \\
\hline Deepen borehole with 4-inch $(10.16-\mathrm{cm})$ core barrel to 1.65 meters. & 7-27-92 & 209 & 209 & 13:50:00 \\
\hline Deepen borehole with 4-inch $(10.16-\mathrm{cm})$ core barrel to 2.15 meters. & $7-27-92$ & 209 & 209 & $14: 30: 00$ \\
\hline Deepen borehole with 4-inch $(10.16-\mathrm{cm})$ core barrel to 3.07 meters. & 7-28-92 & 210 & 210 & 10:15:00 \\
\hline Deepen borehole with 4-inch $(10.16-\mathrm{cm})$ core barrel to 4.04 meters. & 7-28-92 & 210 & 210 & $11: 30: 00$ \\
\hline Deepen borehole with 4-inch $(10.16-\mathrm{cm})$ core barrel to 4.88 meters. & 7-28-92 & 210 & 210 & $13: 30: 00$ \\
\hline Deepen borehole with 4-inch $(10.16-\mathrm{cm})$ core barrel to 6.00 meters. & $7-28-92$ & 210 & 210 & 14:05:00 \\
\hline Deepen borehole with 4-inch $(10.16-\mathrm{cm})$ core barrel to 7.06 meters. & 7-28-92 & 210 & 210 & $14: 44: 00$ \\
\hline Deepen borehole with 4-inch $(10.16-\mathrm{cm})$ core barrel to 7.67 meters. & 7-29-92 & 211 & 211 & 09:35:00 \\
\hline $\begin{array}{l}\text { Face off the bottom of the borehole with } 4 \text {-inch }(10.16-\mathrm{cm}) \text { plug bit to } 7.69 \\
\text { meters. }\end{array}$ & 7-29-92 & 211 & 211 & 10:00:00 \\
\hline Perform videa-log of borehole S1P74. & $7-30-92$ & 212 & 212 & 13:15:00 \\
\hline Install borehole flanged collar. & $7-30-92$ & 212 & 212 & 14:00:00 \\
\hline Perform load test on the borehole flanged collar. & 7-31-92 & 213 & 213 & 12:00:00 \\
\hline $\begin{array}{l}\text { Install multipacker test tool \#35 in borehole S1P74 as indicated in the test-tool } \\
\text { configuration diagram (Figure 3-32). }\end{array}$ & 7-31-92 & 213 & 213 & 14:00:00 \\
\hline Power outage in Waste Panel 1, Room 7: cannot begin data collection. & $7-31-92$ & 213 & 213 & 14:30:00 \\
\hline $\begin{array}{l}\text { Remove test tool \#35 from borehole S1P74 in order to extend the TZ vent } \\
\text { line to the bottom of the borehole. }\end{array}$ & $8-3-92$ & 216 & 216 & 10:00:00 \\
\hline $\begin{array}{l}\text { Install multipacker test tool \#35 in borehole S1P74 as indicated in the test-tool } \\
\text { configuration diagram (Figure 3-32). }\end{array}$ & 8-3-92 & 216 & 216 & 12:00:00 \\
\hline Begin data file S1P741. & $8-5-92$ & 218 & 218 & $09: 27: 22$ \\
\hline Inflate GZP to-10.3 Mpa. & $8-5-92$ & 218 & 218 & 09:43:00 \\
\hline Fill $T Z$ and $G Z$ with brine. & 8-5-92 & 218 & 218 & 09:54:00 \\
\hline Diagnose leak in the $\mathbf{G Z}$ interval. & $8-5-92$ & 218 & 218 & 10:00:00 \\
\hline End data file S1P741. & $8-5-92$ & 218 & 218 & 10:18:31 \\
\hline Remove test tool $\# 35$ to find and repair leak in GZ interval. & $8-7-92$ & 220 & 220 & 08:00:00 \\
\hline
\end{tabular}


Table 3-14 (Continued). Permeability-Testing Sequence S1P74-A Events

\begin{tabular}{|c|c|c|c|c|}
\hline EVENT & DATE & $\begin{array}{l}\text { CALENDAR } \\
\text { DAY }\end{array}$ & $\begin{array}{c}1992 \\
\text { CALENDAR } \\
\text { DAY }\end{array}$ & $\begin{array}{c}\text { TIME } \\
\text { (HH:MM:SS) }\end{array}$ \\
\hline $\begin{array}{c}\text { Install multipacker test tool \#35 S1P74 as indicated in the test-tool configuration } \\
\text { diagram (Figure 3-32). }\end{array}$ & $8-7-92$ & 220 & 220 & $10: 30: 00$ \\
\hline Inflate GZP to $\sim 10.3 \mathrm{Mpa}$ & $8-7-92$ & 220 & 220 & 14:00:00 \\
\hline $\begin{array}{l}\text { Fill TZ and GZ with brine with } 0707 \mathrm{MPa} \text { check valves in place to maintain } \\
\text { pressure head. }\end{array}$ & $8-7-92$ & 220 & 220 & $14: 10: 00$ \\
\hline Begin data file S1P742. & $8-7-92$ & 220 & 220 & $14: 16: 37$ \\
\hline Inflate $\mathrm{RP}$ to $\sim 10.3 \mathrm{MPa}$ & $8-7-92$ & 220 & 220 & 14:20:00 \\
\hline Shut in GZ. & 8-7.92 & 220 & 220 & $14: 25: 00$ \\
\hline Shut in $T Z$. & $8-7-92$ & 220 & 220 & 14:29:00 \\
\hline Depressurize $G Z$ in order to repair leaky fitting. & $8-11-92$ & 224 & 224 & 09:22:00 \\
\hline Shut in GZ. & $8-11-92$ & 224 & 224 & 09:30:00 \\
\hline DAS not functioning property upon arrival. & 9-14-92 & 258 & 258 & 10:29:00 \\
\hline Increase $T Z$ pressure to $2.27 \mathrm{MPa}$. & $9-22-92$ & 266 & 266 & 14:36:00 \\
\hline DAS has not been functioning property for the past 3 days. & $9-28-92$ & 272 & 272 & $11: 35: 00$ \\
\hline End data file S1P742. & $10-1-92$ & 275 & 275 & 11:00:10 \\
\hline Replace DAS computer. & $10-1-92$ & 275 & 275 & 11:01:00 \\
\hline Begin data file S1P743. & $10-1-92$ & 256 & 275 & $12: 14: 00$ \\
\hline End data file S1P743. & $11-13-92$ & 318 & 318 & 10:46:35 \\
\hline Begin data file S1P744. & $11-13-92$ & 318 & 318 & $11: 26: 00$ \\
\hline End data file S1P744. & $1-25-93$ & 25 & 391 & 07:30:59 \\
\hline Begin data file S1P745. & 1-25-93 & 25 & 391 & 11:14:47 \\
\hline $\begin{array}{l}\text { Begin constant-pressure-withdrawal test in TZ at } 1 \mathrm{MPa} \text { below } \mathrm{TZ} \text { pressure } \\
\qquad(\sim 5.4 \mathrm{MPa}) .\end{array}$ & $1-25-93$ & 25 & 391 & 13:25:00 \\
\hline Decrease back-pressure on the system. & $1-25-93$ & 25 & 391 & 14:15:00 \\
\hline Shut in $T Z$ to change flow line from the bottom to the top of the fluid column. & $1-26-93$ & 26 & 392 & 10:54:00 \\
\hline Open $T Z$ to fluid column. & $1-26-93$ & 26 & 392 & 11:00:00 \\
\hline Shut in $T Z$ from fluid column terminating constant-pressure-withdrawal test in $T Z$. & 2-1-93 & 41 & 407 & $10: 30: 00$ \\
\hline End data file S1P745. & $2-10-93$ & 41 & 407 & 10:45:21 \\
\hline Begin data file S1P746. & $2-10-93$ & 41 & 407 & 10:45:35 \\
\hline Increase GZP pressure to $\sim 10.5 \mathrm{MPa}$. & $6-12-93$ & 193 & 559 & 13:04:17 \\
\hline Shut in GZP. & $6-12-93$ & 193 & 559 & 13:04:47 \\
\hline End data file S1P746 & 8-3-93 & 215 & 581 & $06: 28: 19$ \\
\hline Begin data file S1P747. & 8-3-93 & 215 & 581 & 11:15:14 \\
\hline Perform TZ-compressibility test \#1 from 6.542 to $0 \mathrm{MPa}$. & 8-3-93 & 215 & 581 & 13:14:e0 \\
\hline Shut in TZ terminating TZ-compressibility test \#1. & 8-3-93 & 215 & 581 & 13:53:00 \\
\hline Perform TZ-compressibility test \#2 from 0 to $\sim 8.8 \mathrm{MPa}$. & 8-3-83 & 215 & 581 & 13:58:41 \\
\hline Shut in $T Z$ terminating $T Z$ compressibility teset $\$ 2$. & $8-3-93$ & 215 & 581 & 14:02:00 \\
\hline $\begin{array}{l}\text { Initiate pulse-withdrawal test removing } 498 \mathrm{~mL} \text { of brine from the } T Z \text { in order to } \\
\text { decrease the } T Z \text { pressure. }\end{array}$ & 8-3-93 & 215 & 581 & 14:07:00 \\
\hline
\end{tabular}


Table 3-14 (Continued). Permeability-Testing Sequence S1P74-A Events

\begin{tabular}{ccccc}
\hline EVENT & DATE & $\begin{array}{c}\text { CALENDAR } \\
\text { DAY }\end{array}$ & $\begin{array}{c}\text { 1992 } \\
\text { CALENDAR } \\
\text { DAY }\end{array}$ & $\begin{array}{c}\text { TIME } \\
\text { (HH:MM:SS) }\end{array}$ \\
\hline Shut in TZ. & & 215 & 581 & $14: 07: 51$ \\
Terminate TZ pulse-withdrawal test & $8-3-93$ & 582 & $11: 25: 00$ \\
Perform GZ-compressibility test \#1 from 4.045 to 0 MPa. & $8-4-93$ & 216 & 582 & $11: 25: 06$ \\
Shut in GZ terminating GZ compressbility test. & $8-4-93$ & 216 & 582 & $12: 06: 56$ \\
End data file S1P747. & $8-4-93$ & 216 & 582 & $12: 07: 31$ \\
Begin data file S1P748. & $8-4-93$ & 216 & 582 & $12: 13: 53$ \\
End data file S1P748. & $8-4-93$ & 216 & 587 & $11: 05: 30$ \\
Depressurize TZ. & $8-9-93$ & 221 & 617 & $09: 00: 00$ \\
Depressurize GZ. & $9-8-93$ & 251 & 617 & $09: 05: 00$ \\
Deflate TZP. & $9-8-93$ & 251 & 617 & $09: 10: 00$ \\
Deflate GZP. & $9-8-93$ & 251 & 617 & $09: 15: 00$ \\
Remove multipacker test tool \#35 from borehole S1P74. & $9-8-93$ & 251 & 617 & $10: 30: 00$ \\
\hline
\end{tabular}

Figure 3-58 through 3-64 illustrate the zone pressures, packer pressures, zone temperatures, axial-LVDT displacement, radial-LVDT displacement, fluid production during a constant-pressure-withdrawal test, and test-zone compressibility as a function of pressure, respectively, for permeability-testing sequence S1P74-A. Copies of the video-log associated with testing sequence S1P74-A identified in Table 3-14 are provided in the SWCF under WPO \#45907. 


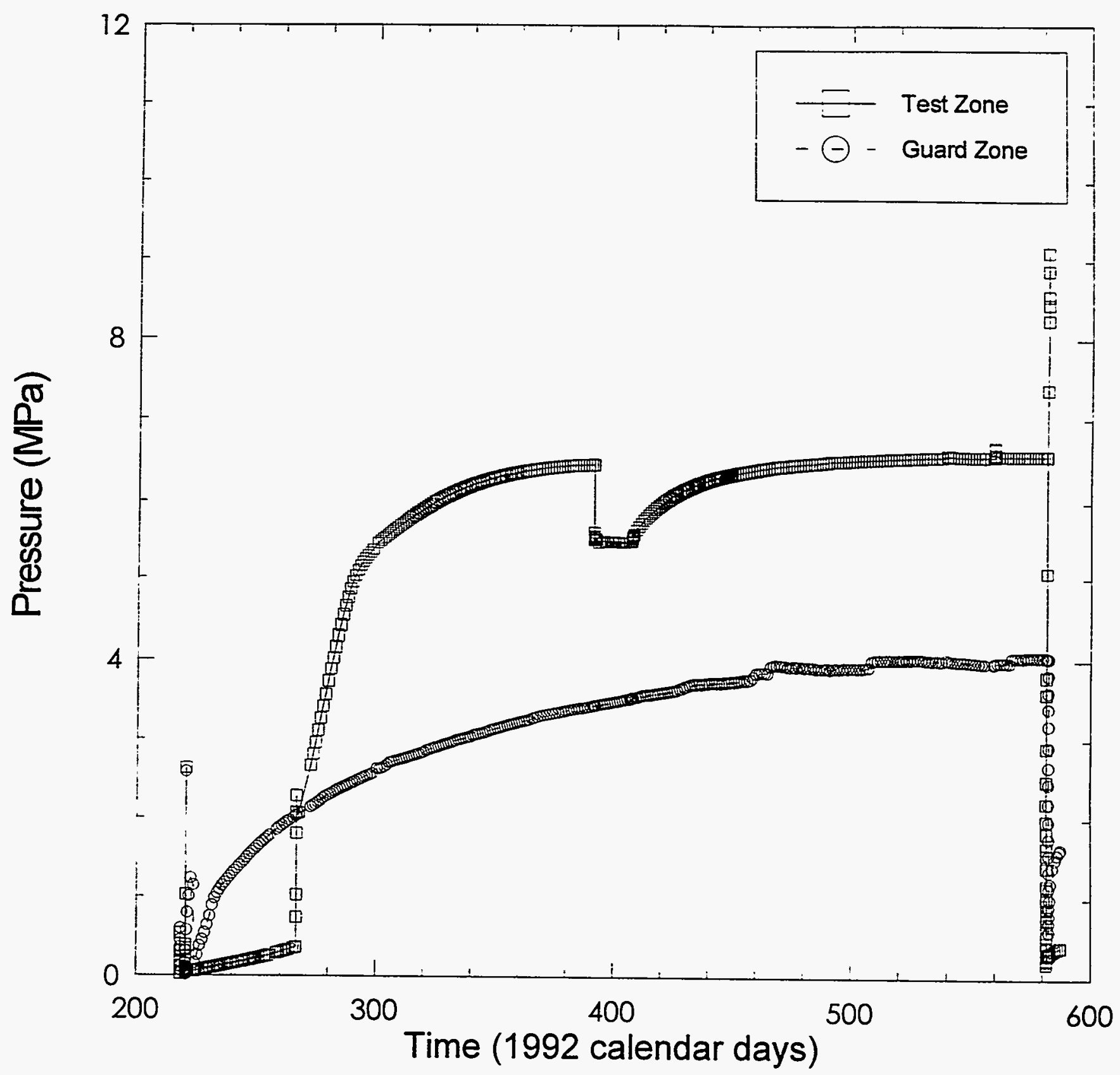

INTERA- 115-78-0

Figure 3-58. Zone pressures during permeability-testing sequence S1P74-A. 


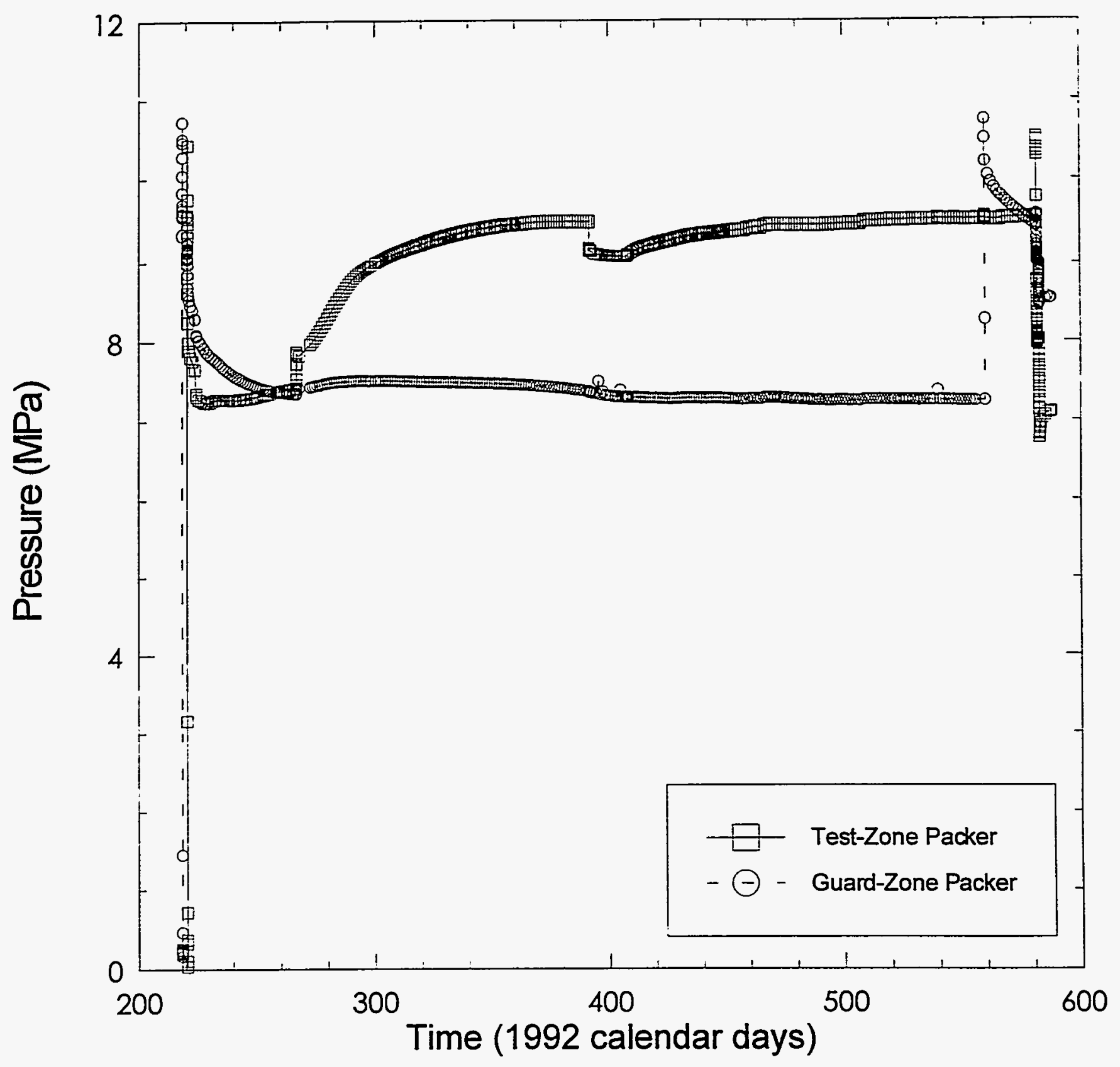

INTERABT15-700

Figure 3-59. Packer pressures during permeability-testing sequence S1P74A. 


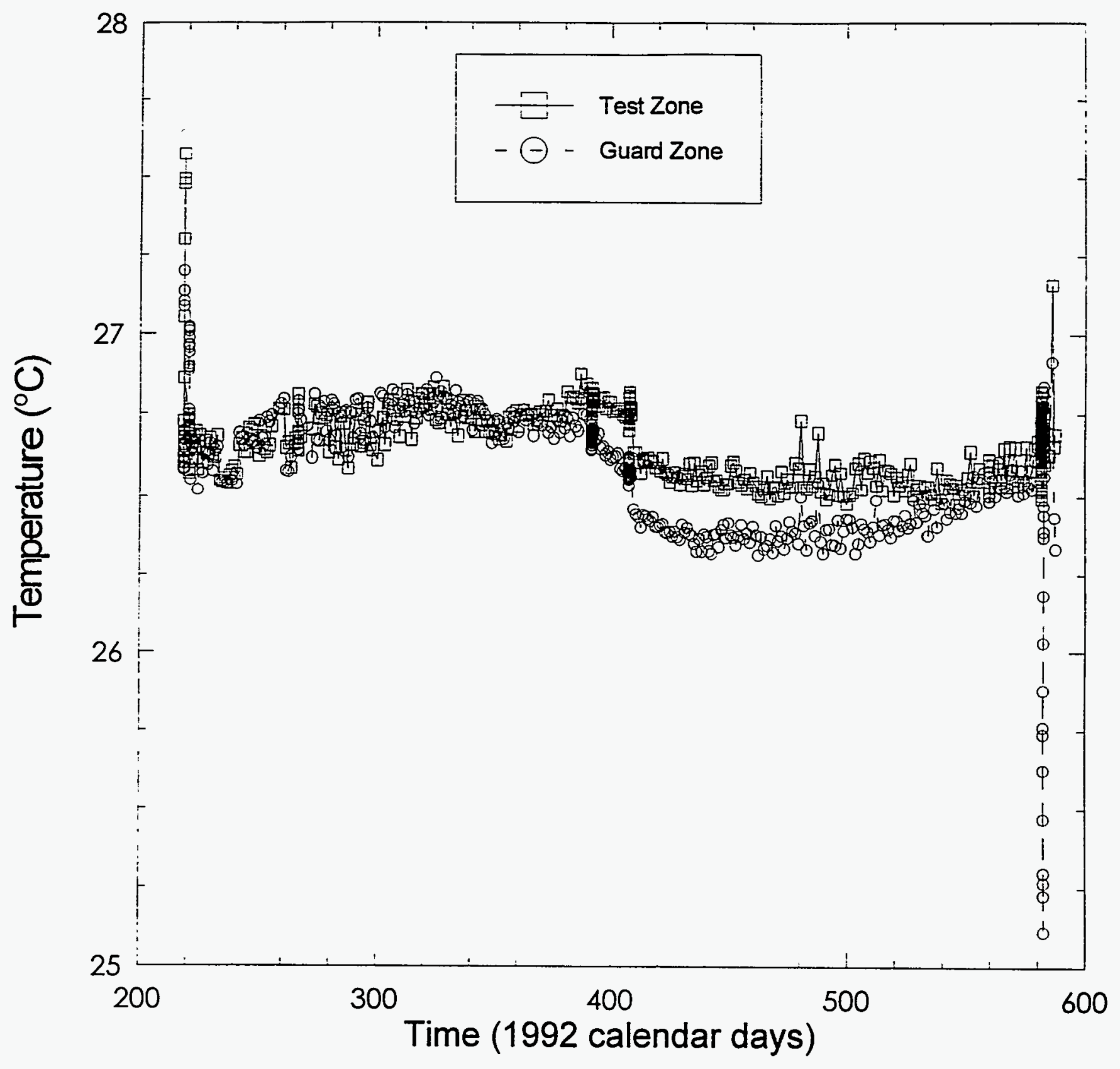

INTERA-BI15-80-0

Figure 3-60. Zone temperatures during permeability-testing sequence S1P74A. 


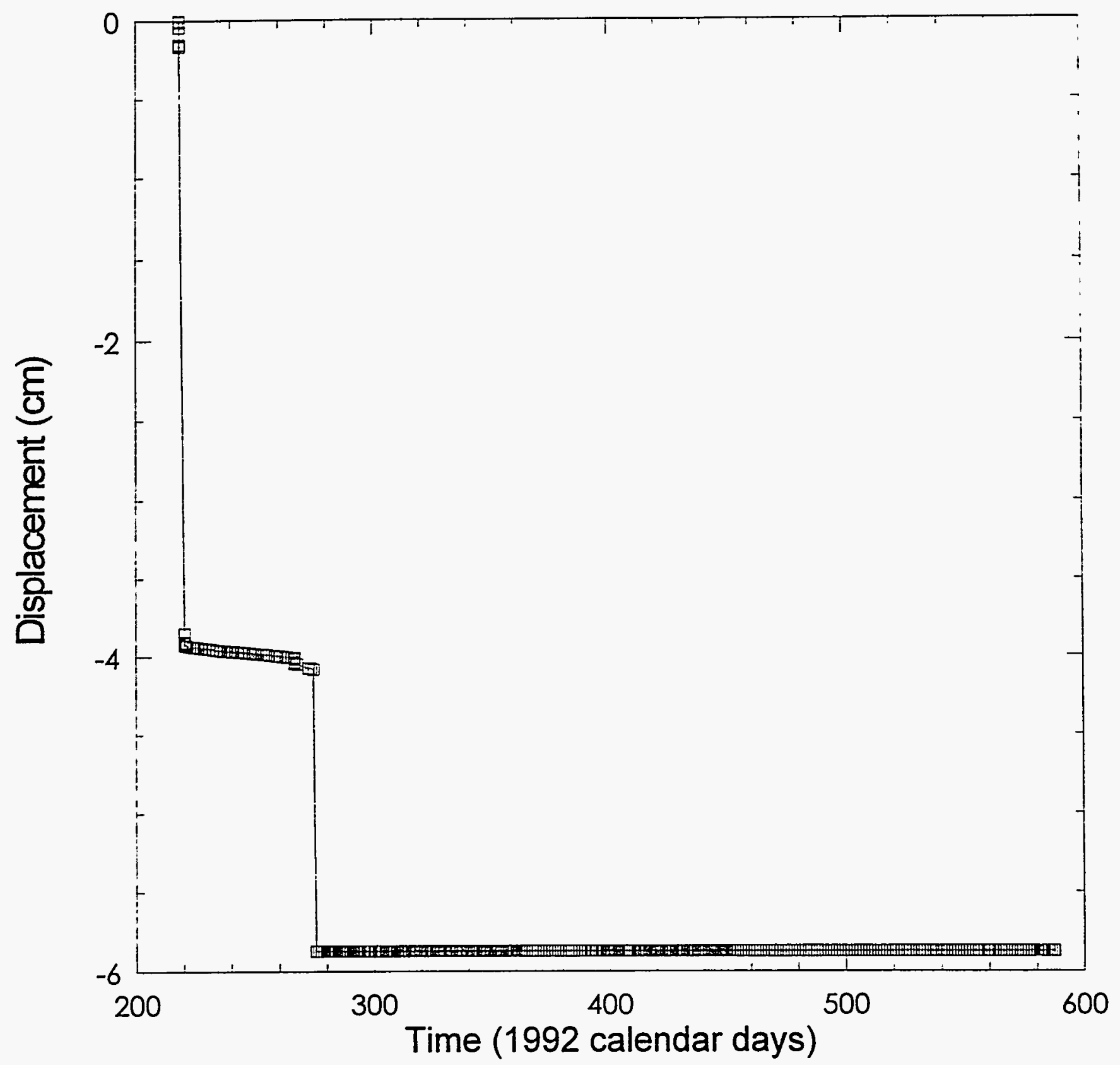

INTERA-115-81-0

Figure 3-61. Axial-LVDT displacement during permeability-testing sequence S1P74-A. 


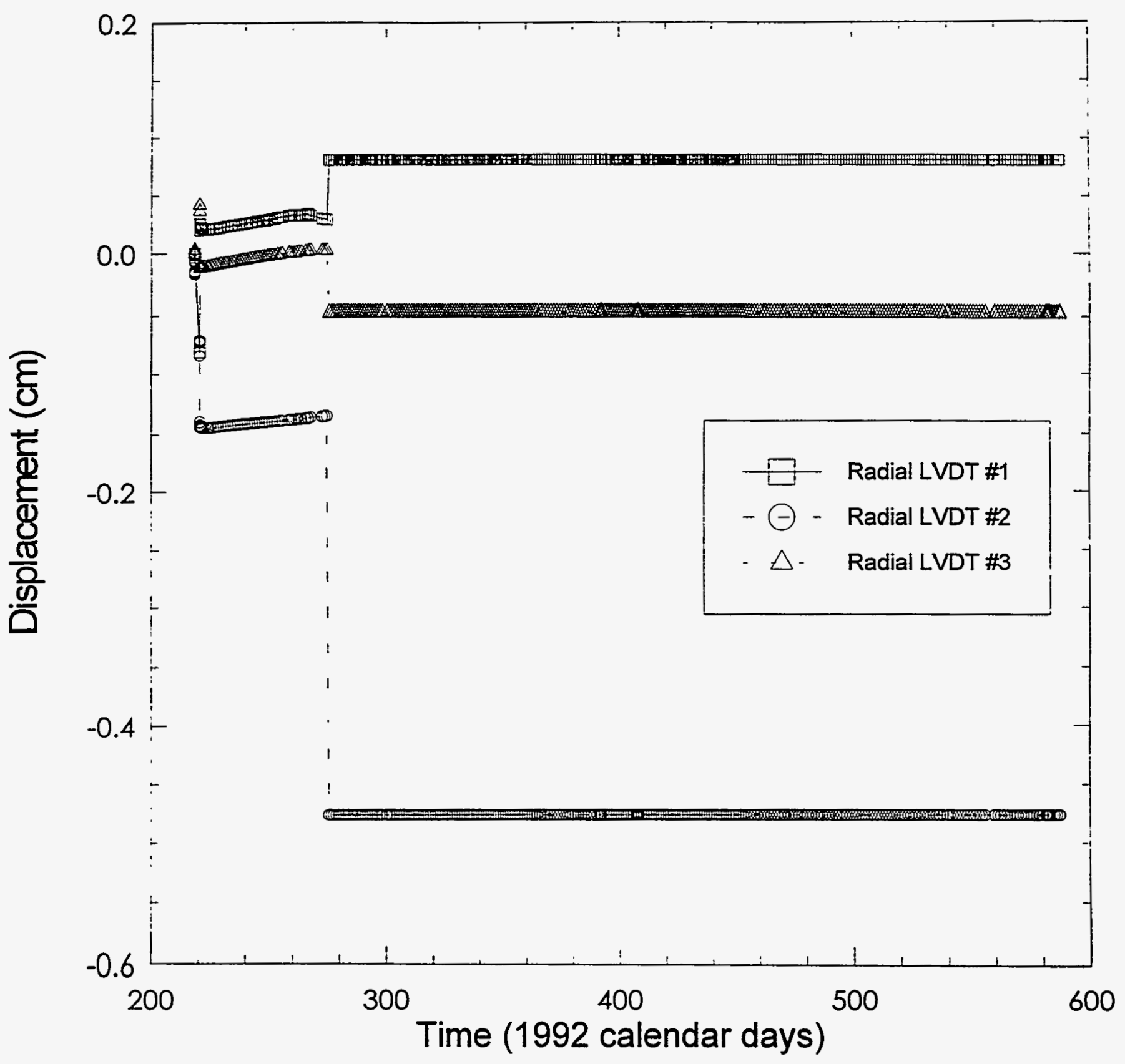

INTERA-6115-02-0

Figure 3-62. Radial-LVDT displacement during permeability-testing sequence S1P74-A. 


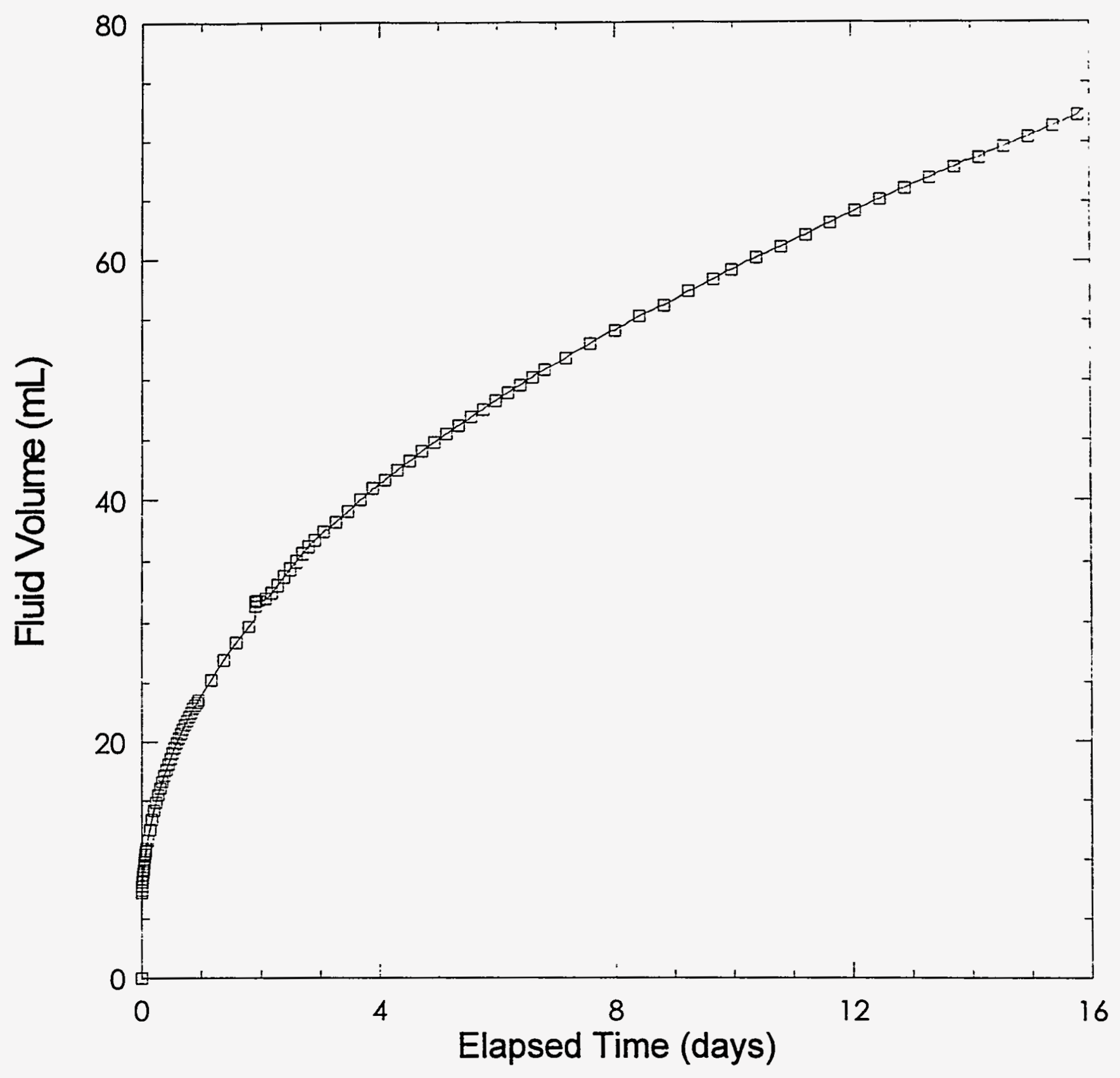

INTERA-6115-83-0

Figure 3-63. Fluid production during constant-pressure-flow tests in permeabilitytesting sequence S1P74-A. 


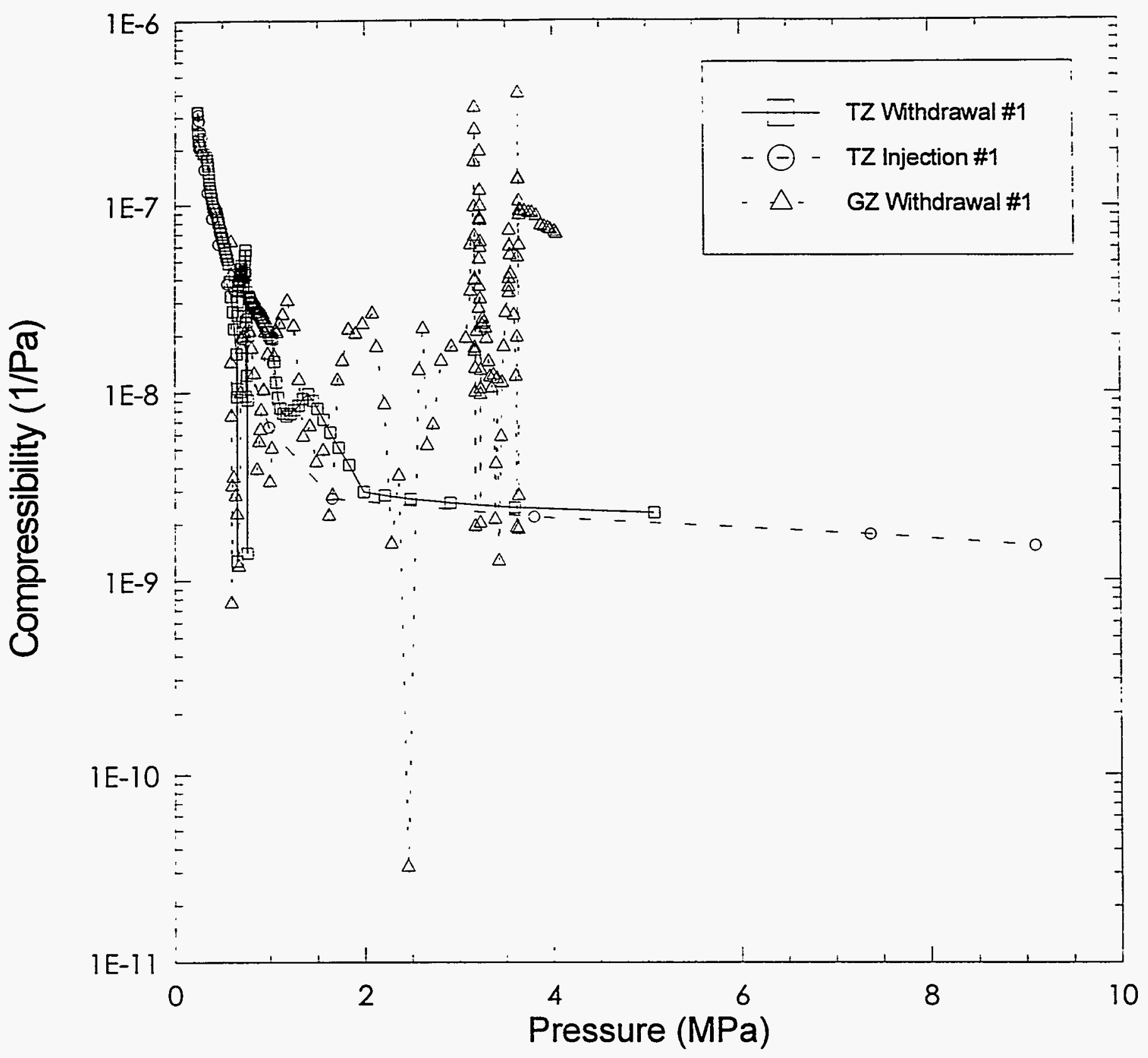

INTERA-6115-05-0

Figure 3-64. Zone compressibility as measured during permeability-testing sequence S1P74-A. 
Table 3-15 indicates the equipment that was used and the duration that each instrument was used during permeability-testing sequence S1P74-A.

Table 3-15. Permeability-Testing Sequence S1P74-A Equipment

\begin{tabular}{|c|c|c|c|c|}
\hline Equipment & Location & Serial \# & Installed & Removed \\
\hline DAS Software & N/A & PERM4C & $8-5-92$ & $10-1-92$ \\
\hline DAS Software & N/A & PERM4F & $10-1-92$ & $8-9-93$ \\
\hline DCU (HP3497A) & N/A & $2936 a 23831$ & $8-5-92$ & $11-13-92$ \\
\hline DCU (HP3497A) & N/A & $2023 a 01688$ & $11-13-92$ & $4-20-93$ \\
\hline DCU (HP3497A) & N/A & $2514 a 17149$ & $4-20-93$ & $8-9-93$ \\
\hline Transducer (Druck PDCR 910) & $\begin{array}{c}\text { Guard Zone } \\
\text { Packer }\end{array}$ & 322423 & $8-5-92$ & $8-9-93$ \\
\hline Tranducer (Druck PDCR 910) & $\begin{array}{l}\text { Test Zone } \\
\text { Packer }\end{array}$ & 308152 & $8-5-92$ & $8-9-93$ \\
\hline Transducer (Druck PDCR 830) & Guard Zone & 246910 & $8-5-92$ & $8-9-93$ \\
\hline Transducer (Druck PDCR 830) & Test Zone & 246912 & $8-5-92$ & $8-9-93$ \\
\hline Transducer (Druck PDCR 910) & DPT Panel & 322427 & $1-25-93$ & $2-10-93$ \\
\hline Transducer (Druck PDCR 10/D) & DPT Panel & 211694 & $8-3-93$ & $8-4-93$ \\
\hline LVDT (Trans-Tek 241) & N/A & $\mathrm{R} 04$ & $8-5-92$ & $8-9-93$ \\
\hline LVDT (Trans-Tek 241) & N/A & R16 & $8-5-92$ & $8-9-93$ \\
\hline LVDT (Trans-Tek 241) & N/A & R17 & $8-5-92$ & $8-9-93$ \\
\hline LVDT (Trans-Tek 245) & N/A & $\mathrm{A} 02$ & $8-5-92$ & $8-9-93$ \\
\hline Thermocouple (Type E) & Test Zone & 1 & $8-5-92$ & $8-9-93$ \\
\hline Thermocouple (Type E) & Guard Zone & 2 & $8-5-92$ & $8-9-93$ \\
\hline Injection Column & N/A & 39 & $1-25-93$ & $2-10-93$ \\
\hline Injection Column & N/A & 76 & $8-3-93$ & $8-4-93$ \\
\hline DPT (Rosemount 1151DP) & N/A & 1140864 & $1-25-93$ & $2-10-93$ \\
\hline DPT (Rosemount 1151DP) & N/A & 1140863 & $8-3-93$ & $8-4-93$ \\
\hline
\end{tabular}

*Installed dates for injection columns refers to dates of initial use rather than date installed. 


\subsubsection{BOREHOLE S1P74, PERMEABILITY-TESTING SEQUENCE S1P74-B}

Permeability-testing sequence S1P74-B took place in Waste Panel 1, Room 7 in borehole S1P74. This test sequence was designed to investigate the brine permeability of MB138. Table 3-16 gives a detailed description of the events that occurred during the permeabilitytesting sequence S1P74-B.

Table 3-16. Permeability-Testing Sequence S1P74-B Events

\begin{tabular}{|c|c|c|c|c|}
\hline EVENT & DATE & $\begin{array}{c}\text { CALENDAR } \\
\text { DAY }\end{array}$ & $\begin{array}{c}1995 \\
\text { CALENDAR } \\
\text { DAY }\end{array}$ & $\begin{array}{c}\text { TIME } \\
\text { (HH:MM:SS) }\end{array}$ \\
\hline Ream existing S1P74 borehole with 4-Inch (10.16-cm) bit prior to deepening. & $1-24-95$ & 24 & 24 & 12:31:00 \\
\hline Deepen borehole with 4-inch $(10.16-\mathrm{cm})$ core barrel to 8.55 meters. & 1-26-95 & 26 & 26 & 11:03:00 \\
\hline Deepen borehole with 4-inch (10.16-cm) core barrel to 9.50 meters. & $1-26-95$ & 26 & 26 & 13:05:00 \\
\hline Deepen borehole with 4-inch $(10.16-\mathrm{cm})$ core barrel to 10.36 meters. & 1-26-95 & 26 & 26 & 13:38:00 \\
\hline Deepen borehole with 4-inch $(10.16-\mathrm{cm})$ core barrel to 11.15 meters. & $1-26-95$ & 26 & 26 & 14:32:00 \\
\hline Deepen borehole with 4-inch $(10.16-\mathrm{cm})$ core barrel to 11.92 meters. & $1-27-95$ & 27 & 27 & 09:07:00 \\
\hline Deepen borehole with 4-inch $(10.16-\mathrm{cm})$ core barrel to 12.88 meters. & $1-27-95$ & 27 & 27 & $12: 25: 00$ \\
\hline Deepen borehole with 4-inch $(10.16-\mathrm{cm})$ core barrel to 13.80 meters. & $1-30-95$ & 30 & 30 & 09:56:00 \\
\hline Deepen borehole with 4 -inch $(10.16-\mathrm{cm})$ core barrel to 14.45 meters. & $1-30-95$ & 30 & 30 & $10: 42: 00$ \\
\hline Deepen borehole with 4-inch $(10.16-\mathrm{cm})$ core barrel to 15.32 meters. & $1-39-95$ & 31 & 31 & 09:43:00 \\
\hline Deepen borehole with 4 -inch $(10.16-\mathrm{cm})$ core barrel to 16.18 meters. & $1-31-95$ & 31 & 31 & 10:39:00 \\
\hline Deepen borehole with 4-inch $(10.16-\mathrm{cm})$ core barrel to 16.82 meters. & $1-31-95$ & 31 & 31 & 09:43:00 \\
\hline $\begin{array}{l}\text { Face off the bottom of the borehole with a } 4 \text {-inch }(10.16-\mathrm{cm}) \text { plug bit to } 16.88 \\
\text { meters. }\end{array}$ & $1-31-95$ & 31 & 31 & 13:30:00 \\
\hline Perform a videa-log of the borehole. & 2-1.95 & 32 & 32 & 09:06:00 \\
\hline $\begin{array}{l}\text { install the multipacker test tool \#P74-B until the bottom of the borehole is } \\
\text { reached. }\end{array}$ & $2-2-95$ & 33 & 33 & 12:30:00 \\
\hline $\begin{array}{l}\text { Remove the test tool \#P74-B to inspect the } T Z \text { vent line to ensure that the } \\
\text { vent line reaches the bottom of the borehole. }\end{array}$ & $2-6-95$ & 37 & 37 & 09:00:00 \\
\hline Tag borehole depth at 17.04 meters from the collar face. & $2-6-95$ & 37 & 37 & $10: 00: 00$ \\
\hline $\begin{array}{l}\text { Install the mulipacker test tool \#P74-B to a depth of } 16.960 \text { meters from the } \\
\text { collard face as indicated in the test-tool configuration diagram (Figure 3-33). }\end{array}$ & $2-6-95$ & 37 & 37 & 13:00:00 \\
\hline Begin data file S1P74B01. & $2-7-95$ & 38 & 38 & $08: 57: 47$ \\
\hline Pressurize GZP to $13.469 \mathrm{MPa}$. & $3-7-95$ & 38 & 38 & 10:03:00 \\
\hline Open GZP to accumulator at $13.419 \mathrm{MPa}$. & $2-7-95$ & 38 & 38 & 10:05:00 \\
\hline Fill borehole with brine. & 2-7.95 & 38 & 38 & 10:27:000 \\
\hline Pressurize TZ2P to $13.326 \mathrm{MPa}$. & 2-7-95 & 38 & 38 & $10: 58: 30$ \\
\hline Shut in GZ & $2-7-95$ & 38 & 38 & 10:58:45 \\
\hline Open TZ2P to accumulator at $13.747 \mathrm{MPa}$. & $2-7-95$ & 38 & 38 & 11:01:33 \\
\hline Pressurize TZ1P to $13395 \mathrm{MPa}$. & $2-7-95$ & 38 & 38 & 11:12:06 \\
\hline Shut in TZ2. & $2-7-95$ & 38 & 38 & $11: 12: 27$ \\
\hline Open TZ1P to accumulator at $13.435 \mathrm{MPa}$. & $2-7.95$ & 38 & 38 & 11:13:54 \\
\hline Shut in $\mathrm{Z1}$. & $2-7-95$ & 38 & 38 & $11: 17: 45$ \\
\hline
\end{tabular}


Table 3-16 (Continued). Permeability-Testing Sequence S1P74-B Events

\begin{tabular}{|c|c|c|c|c|}
\hline EVENT & DATE & $\begin{array}{l}\text { CALENDAR } \\
\text { DAY }\end{array}$ & $\begin{array}{c}1995 \\
\text { CALENDAR } \\
\text { DAY }\end{array}$ & $\begin{array}{c}\text { TIME } \\
\text { (HH:MM:SS) }\end{array}$ \\
\hline Leaky fitting on the $\mathrm{GZ}$ injection line. & $2-7-95$ & 38 & 38 & 08:10:00 \\
\hline End data file S1P74B01. & 2-10-95 & 41 & 41 & 09:17:04 \\
\hline Begin data file S1P74B02. & $2-10-95$ & 41 & 41 & $09: 28: 12$ \\
\hline Power outage and UPS failed to respond. & 2-10-95 & 41 & 41 & 09:45:00 \\
\hline Determine that the UPS is not functioning properly so it is replaced. & 2-10-95 & 41 & 41 & 12:00:00 \\
\hline DAS not functioning upon arrival. & 2-13-95 & 44 & 44 & 08:00:00 \\
\hline Shut in $G Z$ to replace leaky fitting. & $2-14-95$ & 45 & 45 & $08: 42: 00$ \\
\hline Open GZ to system. & 2-14-95 & 45 & 45 & 08:49:00 \\
\hline Tighten leaky fitting on the TZ1 inject line. & 3-2-95 & 61 & 61 & 08:00:00 \\
\hline Tighten the same leaky fitting on the TZ1 inject line. & $3-8-95$ & 67 & 67 & 08:00:00 \\
\hline End data file S1P74802. & $3-10-95$ & 69 & 69 & 08:47:33 \\
\hline Begin data file S1P74B03. & 3-10-95 & 69 & 69 & 08:52:33 \\
\hline End data file S1P74B03. & 3-14-95 & 73 & 73 & 09:04:50 \\
\hline Begin data file S1P74B04. & 3-14-95 & 73 & 73 & 09:10:64 \\
\hline $\begin{array}{l}\text { Shut in all packers from accumulators in order to try to eliminate the } \\
\text { temperature-dependent pressure fluctuations in the data. }\end{array}$ & 3-24-95 & 83 & -83 & $12: 10: 00$ \\
\hline End data file S1P74804. & 4-3-95 & 93 & 93 & 08:07:25 \\
\hline Begin data file S1P74B05. & 4-3-95 & 93 & 93 & $08: 21: 17$ \\
\hline $\begin{array}{l}\text { Open TZ1P and GZP to accumulator at } 113.3 \mathrm{MPa} \text { because of pressure } \\
\text { decay. }\end{array}$ & 4-3-95 & 93 & 93 & 08:48:00 \\
\hline Replace bad valve on the GZP infiation line. & 4-3-95 & 93 & 93 & 09:30:00 \\
\hline $\begin{array}{c}\text { Initiate pulse-withdrawal in TZ1 ( } 90.273 \text { to } 8,860 \mathrm{MPa} \text { ) removing } \sim 8.9 \\
\mathrm{~mL} \text { of brine. }\end{array}$ & 4-10-95 & 100 & 100 & 07:58:00 \\
\hline Open TZ2P to accumulator at $\sim 13.67 \mathrm{MPa}$. & 4-13-95 & 103 & 103 & 07:43:00 \\
\hline Terminate pulse-withdrawal test in TZ1. & $4-24-95$ & 114 & 114 & 08:23:00 \\
\hline $\begin{array}{l}\text { Initiate pulse-withdrawal test \#1 in TZ2 (8.801 to } 7.083 \mathrm{MPa} \text { ) removing } \\
\sim 7.1 \mathrm{~mL} \text { of binre. }\end{array}$ & 4-24-95 & 114 & 114 & 08:23:30 \\
\hline Begin several weeks of power outage. & $4-24-95$ & 114 & 114 & 09:28:00 \\
\hline Generator supplying power to the DAS broke down. & $4-25-95$ & 115 & 115 & 13:00:00 \\
\hline Upon arrival the generator was functioning again. & $4-26-95$ & 116 & 116 & 12:43:00 \\
\hline End data file S1P74B05. & 5.9 .95 & 131 & 131 & $16: 58: 52$ \\
\hline Begin data file S1P74B06. & $5-10-95$ & 132 & 132 & 14:20:17 \\
\hline Terminate puise-withdrawal test \#1 in $T 22$. & 5-13-95 & 135 & 135 & 07:50:00 \\
\hline $\begin{array}{l}\text { Initiate pulse-withdrawal test \#2 in TZ2 (8.814 to } 4.977 \mathrm{MPa}) \text { removing } \\
\qquad 20.5 \mathrm{~mL} \text { of brine. }\end{array}$ & $5-13-95$ & 135 & 135 & 07:52:42 \\
\hline Terminate pulse-withdrawal test $\# 2$ in $T Z 2$. & 5-18-95 & 138 & 138 & 07:49:00 \\
\hline Shut in all packers from accumulators. & 5-18-95 & 138 & 138 & 07:50:00 \\
\hline Depressurize TZ1. & $5-18-95$ & 138 & 138 & 08:05:00 \\
\hline Depressurize TZ2. & 5-18-95 & 138 & 138 & 08:06:00 \\
\hline Depresurnize GZ. & $5-18-95$ & 138 & 138 & 08:10:00 \\
\hline
\end{tabular}


Table 3-16 (Continued). Permeability-Testing Sequence S1P74-B Events

\begin{tabular}{|c|c|c|c|c|}
\hline EVENT & DATE & $\begin{array}{c}\text { CALENDAR } \\
\text { DAY }\end{array}$ & $\begin{array}{c}1995 \\
\text { CALENDAR } \\
\text { DAY }\end{array}$ & $\begin{array}{c}\text { TIME } \\
\text { (HH:MM:SS) }\end{array}$ \\
\hline Deflate TZ1P. & 5-18-95 & 138 & 138 & 08:20:00 \\
\hline Deflate TZ2P. & 5-18-95 & 138 & 138 & $08: 25: 00$ \\
\hline Deflate GZP. & 5-18-95 & 138 & 138 & 08:30:00 \\
\hline End data file S1P74B06. & 5-18-95 & 138 & 138 & $08: 02: 43$ \\
\hline Remove test tool \#P74-B from borehole S1P74. & $5-18-95$ & 138 & 138 & $14: 00: 00$ \\
\hline
\end{tabular}

Figures 3-65 through 3-67 illustrate the zone pressures, packer pressures, and axial-LVDT displacement, respectively, for permeability-testing sequence S1P74-B. Copies of the video-log associated with testing sequence S1P74-B identified in Table 3-16 are provided in the SWCF under WPO \#45907. 


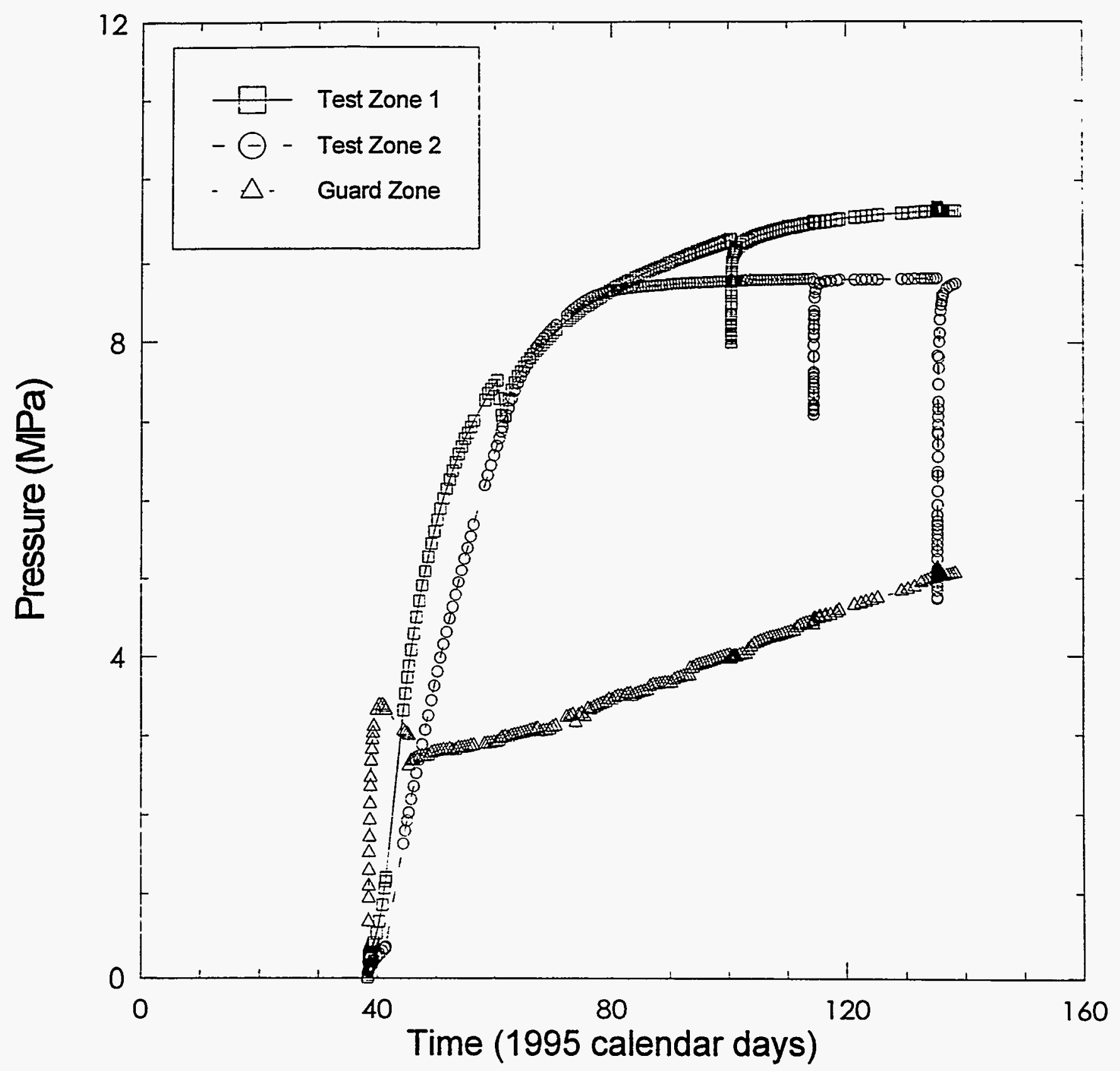

INTERAS115-86-0

Figure 3-65. Zone pressures during permeability-testing sequence S1P74-B. 


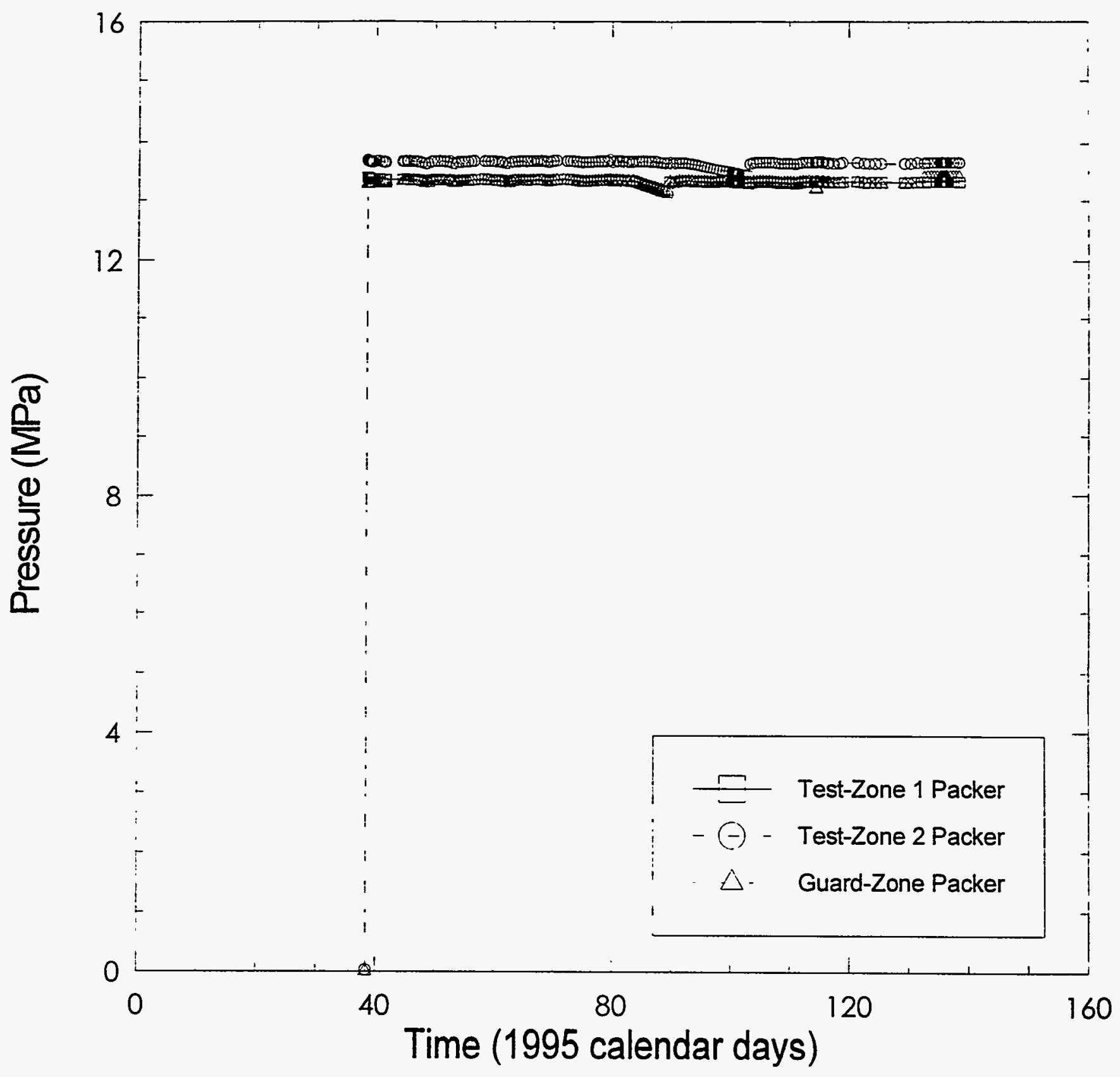

INTERA-6115-87-0

Figure 3-66. Packer pressures during permeability-testing sequence S1P74-B. 


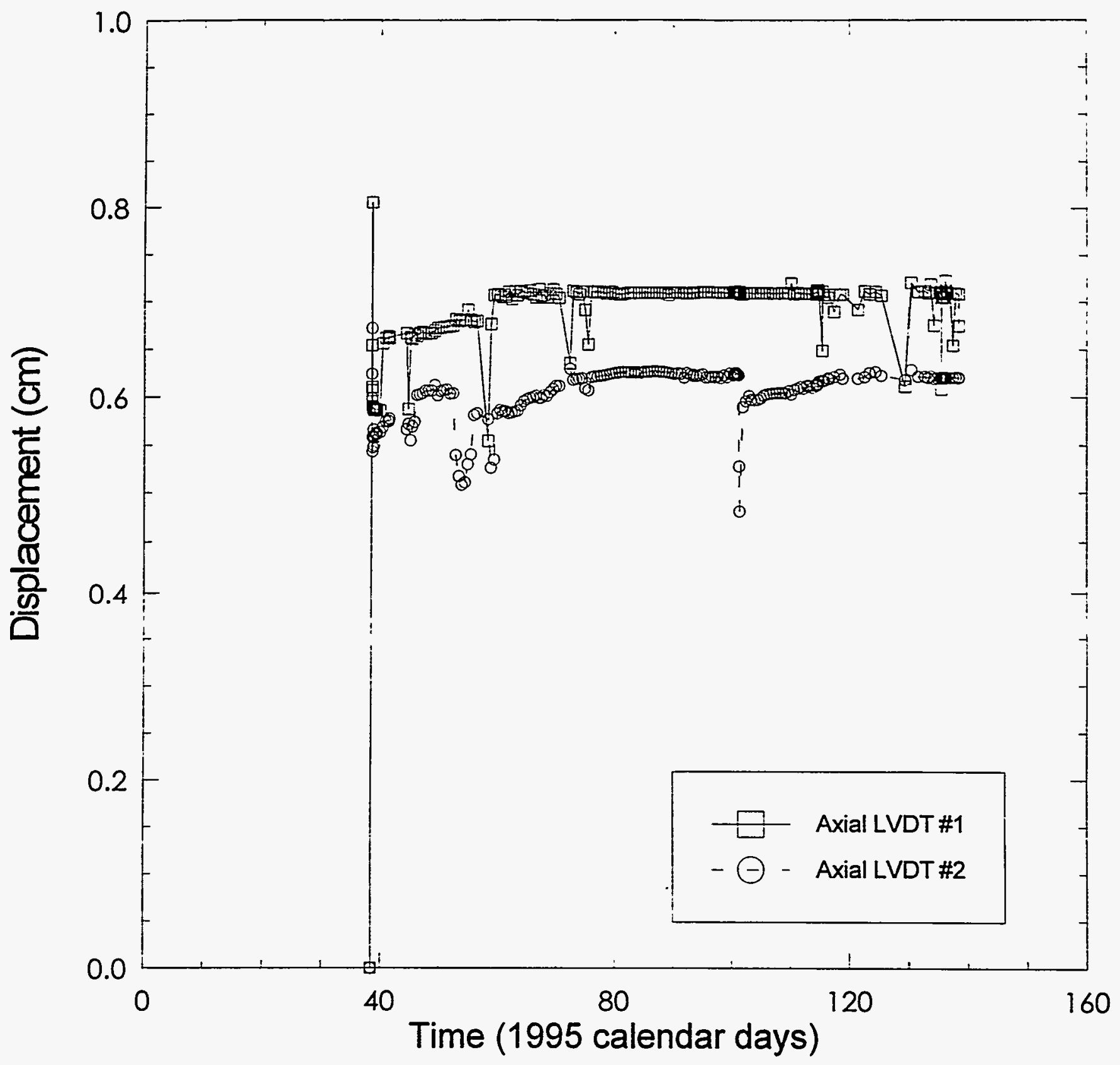

INTERA-8115-86-0

Figure 3-67. Axial-LVDT displacement during permeability-testing sequence S1P74-B. 


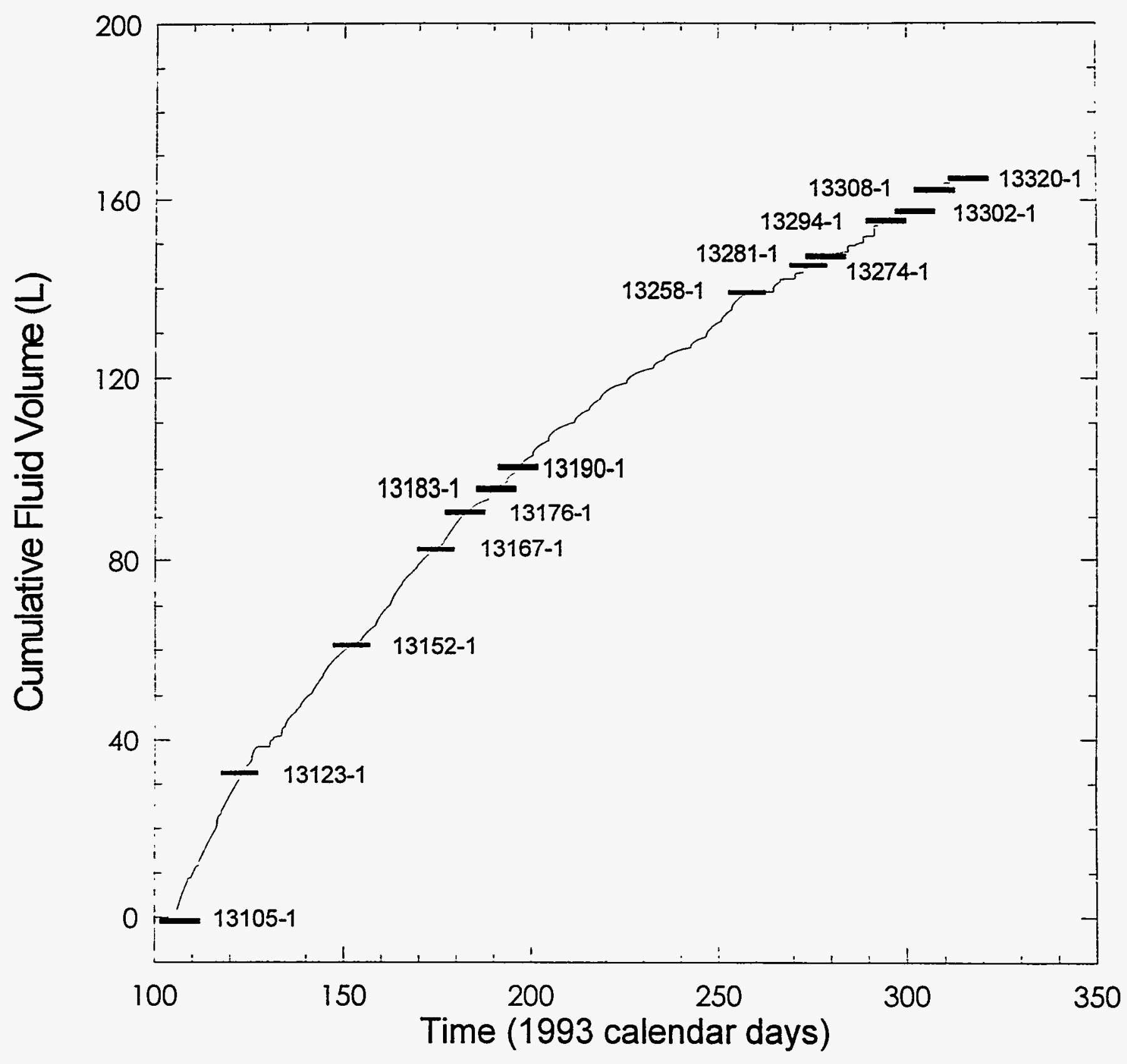

INTERA-6195-89-0

Figure 3-68. Cumulative production from the test zone of borehole L4P51 during the pressurized brine-sampling campaign (sample dates indicated). 


\section{COUPLED PERMEABILITY AND HYDROFRACTURE TESTING}

Complementary hydraulic fracturing and permeability tests were conducted in MB139 and in the deeper MB140 to address the consequences of gas generation in disposal rooms and, specifically, the experimental evaluation of analyses concerning the gas pressurization of anhydrite interbeds such as MB139 (Davies, 1991). Considerable variability was recorded in the measured formation (pore) pressures and permeabilities in MB139 one to two meters below the repository horizon and, to a lesser extent, some six meters below the floor of the experimental area in the WIPP. The observed variability suggests that the properties of MB139 have been altered by the influence of nearby excavations (Stormont et al., 1987; Stormont, 1990a; Beauheim et al., 1991). Because MB140 is located approximately $16 \mathrm{~m}$ deeper than MB139 (Holt and Powers, 1984), MB140 was expected to be sufficiently less disturbed to serve as a undisturbed analog of MB139 and other anhydrite interbeds with preexisting, partially healed fractures. Gas flow from the disposal rooms may not be restricted to MB139 immediately below the disposal areas. Therefore, hydraulic fracturing and hydrologic testing measurements in MB139 and MB140 were also intended to provide data useful for the evaluation of anhydrite interbeds above the WIPP horizon (Beauheim et al., 1993).

\subsection{Test Objectives}

This section presents data collected by the DAS used for permeability testing and to monitor responses to permeability and hydraulic fracturing tests in observation boreholes. A separate DAS was used to monitor and control the hydraulic fracturing tests and the data collected by that DAS are reported by Wawersik et al. (1997). The hydraulic fracturing program had six specific objectives. These objectives were:

- to determine the fluid pressures at which fracturing will occur in anhydrite interbeds, especially MB139, both in a potentially disturbed state and in its undisturbed state;

- to determine whether fracturing would take place by the opening and interconnection of preexisting, partially healed fractures or by the formation of new fractures;

- to determine at what induced pressure (liquid or gas) fracturing might be sustained;

- to determine if fracturing in MB139 involved the development of new fractures, would the fracturing be confined to the anhydrite interbed or would newly created fractures extend across the MB139 contacts;

- to determine if the total stress state (matrix stress plus pore pressure) in MB139 and MB140 is isotropic or not, and can near-field stress measurements around excavations be used to infer the undisturbed state of stress in the interbeds; and

- to determine the magnitude of the smallest principal stress in anhydrite interbeds, if the stress state is anisotropic. 
Complementary pre-and post-fracture hydrological measurements were intended to yield the following information:

- reliable values of formation (pore) fluid pressure;

- permeability values of the interbeds before and after hydraulic fracturing; and

- comparison of permeability measurements in MB139 relatively near existing excavations with equivalent data in MB140, which was expected to be essentially undisturbed.

The activities conducted as part of the hydraulic fracturing testing program were motivated foremost by the objectives of hydraulic fracturing tests and attendant studies of fracture formation and fracture growth in anhydrite interbeds. As a consequence, the test sections, i.e., the lengths of the pressurized intervals in all flow tests in the test boreholes, were considerably smaller than the thickness of the interbeds, and flow measurements may not provide good measures of average interbed permeabilities.

\subsection{Test Design}

The coupled permeability and hydrofracture testing sequences discussed in this report were conducted in two boreholes (C1X10 and C1X05) drilled in Room C1 in the WIPP underground facility. In addition to the test holes, the testing also utilized eight observation boreholes. Boreholes $\mathrm{C} 1 \mathrm{H} 05, \mathrm{C} 1 \mathrm{H} 06, \mathrm{C} 2 \mathrm{H} 01, \mathrm{DPD} 02$, and DPD03 were the observation boreholes associated with the $\mathrm{C} 1 \times 10$ (MB139) testing sequence. Boreholes $\mathrm{C} 1 \mathrm{X} 10$, $\mathrm{C} 2 \mathrm{H} 01, \mathrm{C} 1 \mathrm{H} 05, \mathrm{C} 1 \mathrm{H} 06, \mathrm{C} 1 \mathrm{H07}$, and $\mathrm{C} 1 \mathrm{XO} 6$ were the observation boreholes associated with the C1X05-A (MB139) testing sequence. Boreholes $\mathrm{C} 1 \mathrm{H} 07$ and $\mathrm{C} 1 \times 06$ were the observation boreholes associated with the C1X05-B (MB140) testing sequence. Note that during the C1X05-B testing sequence, a pulse-withdrawal test was initiated in borehole $\mathrm{C} 1 \mathrm{X} 06$ and monitored in boreholes $\mathrm{C} 1 \mathrm{X} 05$ and $\mathrm{C} 1 \mathrm{H} 07$. The stratigraphic positions and isolated zones of all of the coupled permeability and hydrofracture testing boreholes are shown in Figure 4-1.

Various combinations of single-, double-, and triple-packer test tools were used during the three coupled permeability and hydrofracture testing sequences. The zones of the test boreholes (C1X10 and C1X05) were filled with a mixture of Chevron Mineral Seal 38 oil and ZL-27A Zyglo penetrant. The zones of the observation boreholes were filled with 1.22 $\mathrm{kg} / \mathrm{L}$ sodium-chloride brine. After completing the pre-hydrofracture permeability tests, hydrofracturing of the anhydrite in the test zones was performed by pumping the ChevronMineral Seal 38 oil/ZL-27A Zyglo penetrant mixture into the test zone. Posthydrofracturing permeability tests were performed to determine how fracture development changed the anhydrite permeability and storage capacity and also to determine if postfracturing permeability and storage were constant or pressure dependent. 


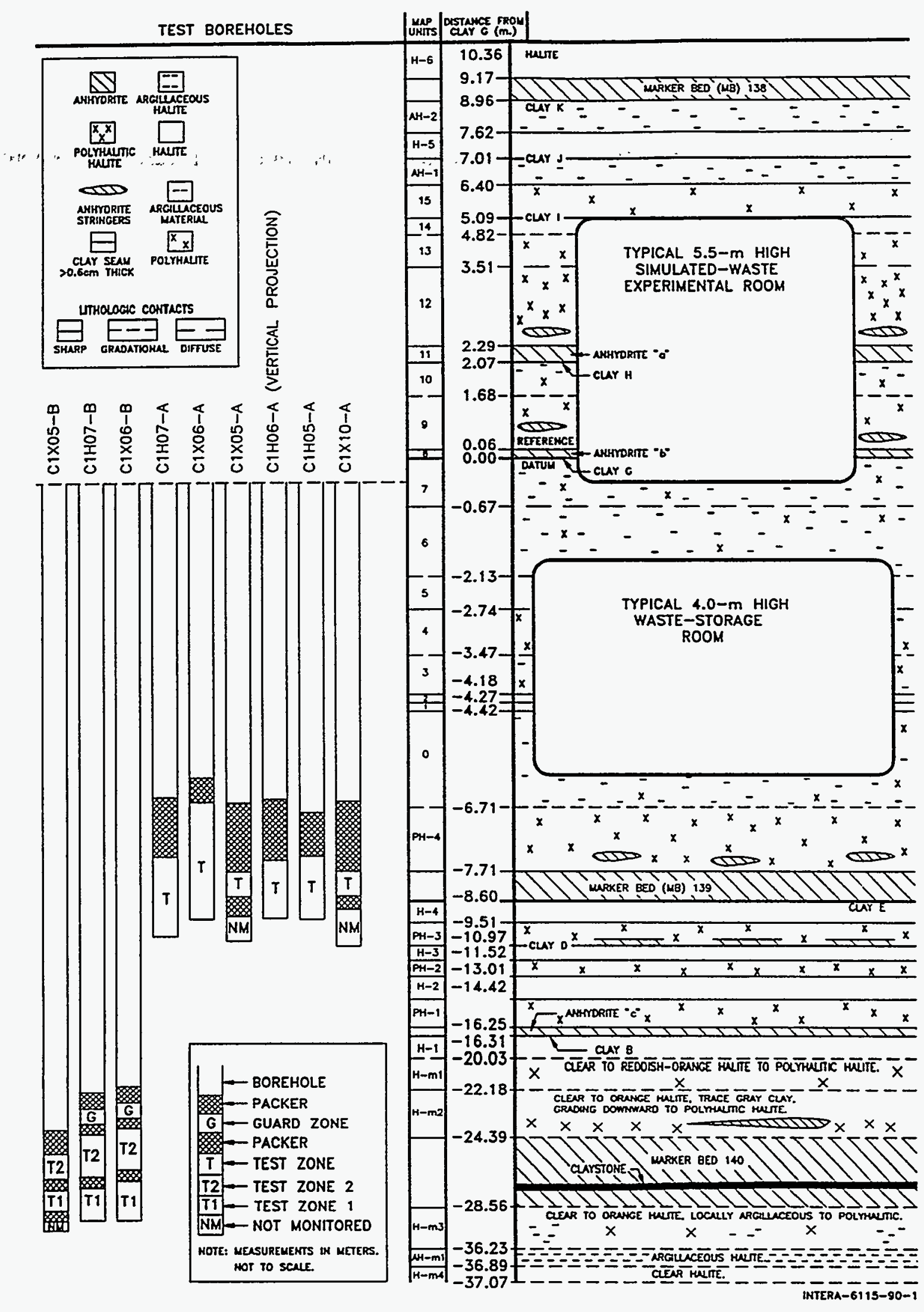

Figure 4-1. Stratigraphic positions of coupled permeability and hydrofracture test and observation boreholes with test zones indicated. 
Pulse-withdrawal testing and constant-pressure testing procedures are described in Section 3.4. Tables 4-1 and 4-2 summarize the locations and orientations of the boreholes in which hydrofracture and pre- and post-fracture permeability testing sequences were performed, the dates that the boreholes were drilled, the lengths of the boreholes, and the dates when the borehole locations were excavated.

\subsection{Test Equipment for Coupled Permeability and Hydrofracture Testing}

The following section (4.3.1) briefly describe the equipment used in the coupled permeability and hydrofracture testing program in the WIPP underground facility. The equipment includes single and multipacker test tools, data-acquisition systems (DAS), pressure transducers, thermocouples, linear variable-differential transformers (LVDT), and a differential-pressure-transmitter (DPT) panel. Equipment specific to individual testing sequences is provided in Section 4.6. More detailed descriptions of the testing equipment and the procedures are presented in Stensrud et al. (1992). Equipment calibration is discussed in Section 4.3.2.

\subsubsection{Description of Equipment}

\subsubsection{TEST TOOLS}

As described in Wawersik et al. (1997), the coupled permeability and hydrofracture test tools, shown in Figures 4-2 and 4-3, were variations of a conventional two-element straddle packer with stainless steel straddle rod(s) (Wawersik, 1991, 1992, 1993). The inflatable packer elements consisted of 40 -inch $(102-\mathrm{cm})$ long, 2-5/8-inch $(6.67-\mathrm{cm})$ diameter slattype elements acquired from Baker Service Tools with outer and inner packer tube designations \#808 and \#801, respectively. The length of the elastomer covers on all packers was $76 \mathrm{~cm}$. Figure 4-2 shows the assembly drawing of the tool that was fielded in two sets of combined fluid flow and hydrofracturing measurements in MB139. The straddle rod was machined of $2.5-\mathrm{cm}$ I.D. $\times 3.8-\mathrm{cm}$ O.D. $(1.0 \times 1.5$-inch) stainless steel pipe. The length of the straddle rod was selected so that each packer element would be centered over the upper and lower contacts of MB139 whose thickness in the test area varied between 0.80 and $0.95 \mathrm{~m}$. The packer ends on the interval side were free to move as the packer elements were pressurized and expanded. One adapter ('sub') of each packer was equipped with a 3000-psi (20.7 MPa) rupture disk. Additionally, the bottom sub of the tool contained a vent to purge the packer of air as it was filled with fluid. The top sub contained ports for separate fluid supply lines to the packers and to the coupled permeability and hydrofracturing interval between the packers. A third port permitted the installation of a pressure gage for downhole measurements of the interval pressure. A $0.953-\mathrm{cm}$ O.D. $\times 0.775-\mathrm{cm} \mathrm{I.D.} \mathrm{(0.375} \times 0.305$-inch) 304 stainless steel supply line to the test interval ensured that the interval volume remained nearly constant with time in order to obtain reliable measurements of the formation pressure before flow or coupled permeability and hydrofracturing tests were initiated. Early test simulations in a steel pipe indicated that this condition could not be maintained with a 23-m (75-foot)-long flexible 


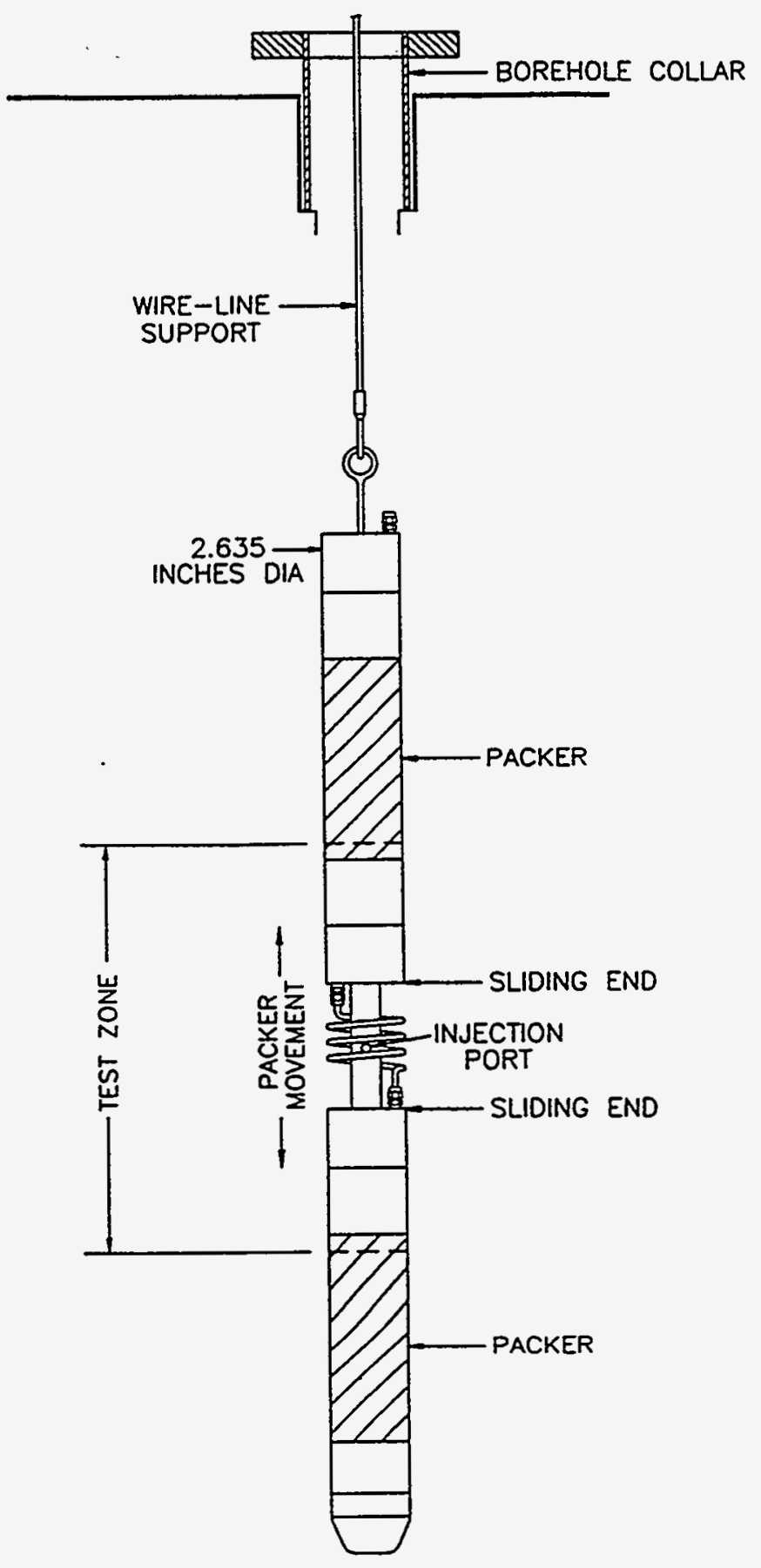

INTERA-6115-91-0

Figure 4-2. Generic double-packer test-tool configuration used for coupled permeability and hydrofracture testing. 


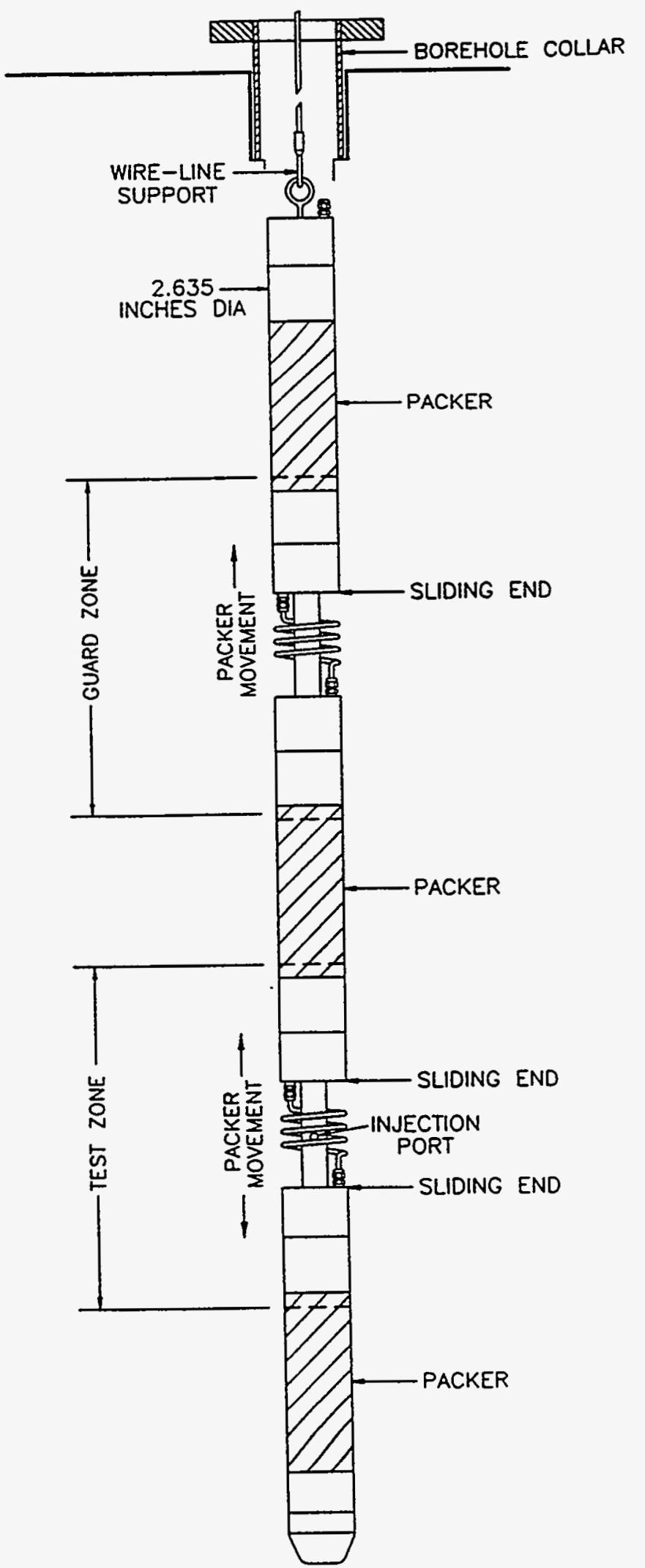

INTERA-61 15-92-0

Figure 4-3. Generic triple-packer test-tool configuration used for coupled permeability and hydrofracture testing. 
high-pressure hose (Aeroquip No. 2781-04, 0.63-cm/0.25-inch I.D.) whose internal diameter changed steadily with time. Therefore, a stainless Steel supply line was used. Figure 4-3 shows the assembly drawing of the straddle packer configuration that was used in MB140. To control pressure in two zones, the straddle packer in Figure 4-2 was modified in four ways. First, a new (middle) sub was built to incorporate a third packer element. Second, a manifold was mounted on top of the straddle packer, and third, a sliding sub was installed into the new (middle) sub in order to feed an additional, independent fluid supply line to the lower interval. Finally, fourth, the straddle rods between the top, middle, and bottom subs were segmented to facilitate the handling of the tool and the process of inserting and lowering it into boreholes with limited headroom.

The monitor tools, shown in Figures 3-2, 3-3, and 4-4, were designed and built specifically for the permeability-testing program by Baski Inc. in conjunction with SNL and INTERA Inc. Some of the test tools had two sliding-end, 3.75 -inch $(9.53-\mathrm{cm})$ O.D. packers mounted on a 1.9-inch $(4.83-\mathrm{cm})$ mandrel with the fixed ends oriented toward the bottom-hole end of the test tool (Figure 3-2). In other instances, the monitor tools had three sliding-end, 3.75inch $(9.53-\mathrm{cm})$ O.D. packers mounted on a 1.9-inch $(4.83-\mathrm{cm})$ O.D. mandrel with the fixed ends oriented toward the bottom-hole end of the monitor tool (Figure 3-3). The remaining monitor tools had one sliding-end, 3.75-inch (9.53-cm) O.D. packer mounted on a 1.9-inch $(4.83-\mathrm{cm})$ O.D. mandrel with the fixed end oriented toward the bottom-hole end of the monitor-tool (Figure 4-4). Figure 3-4 shows the nominal dimensions of the monitor tools' parts. Other information regarding the monitor tools is presented in Section 3.3.1.1.

\subsubsection{OTHER EQUIPMENT}

The DAS, pressure transducers, thermocouples, LVDTs, DPT panel, and pressuremaintenance system used for the permeability testing aspects of the coupled permeability and hydrofracture testing program are described in Sections 3.3.1.2 through 3.3.1.6 and 3.3.1.8. Wawersik et al. (1997) describes the fluid supply system used in the hydrofracture testing. The fluid and pressure source consisted of an air-driven Haskel pump (model GSF-60-6) in parallel with a 5-gallon (19-liter) Haskel accumulator (Haskel part no. 5-5100-1). Combinations of compact pressure transducers of Kulite Semiconductor Products (model HKM-365-5000A) and signal conditioners/readouts of Entran Devices Inc. and Beckman Instruments Co. were used throughout. In the MB140 experiments, the pressure in both the upper and lower straddle packer intervals were measured only at the console because the downhole pressure gage had been damaged during packer installation. Fluid flow during hydrofracturing tests was measured by means of a $0.4-11.4 \mathrm{~L} / \mathrm{min}(0.1-3 \mathrm{gpm})$ flow meter by Flow Technology, Inc. (model FT6-8-NEXB-LED-1). Fluid flow in the range of 1.9-11.4 L/min (0.5-3 gpm) was controlled by means of a Teledyne flow control valve (catalog series 664). Under all other conditions, fluid flow was regulated manually. Documentation associated with the equipment used for the actual hydrofracture tests can be found in the SWCF under WPO \#35174. 


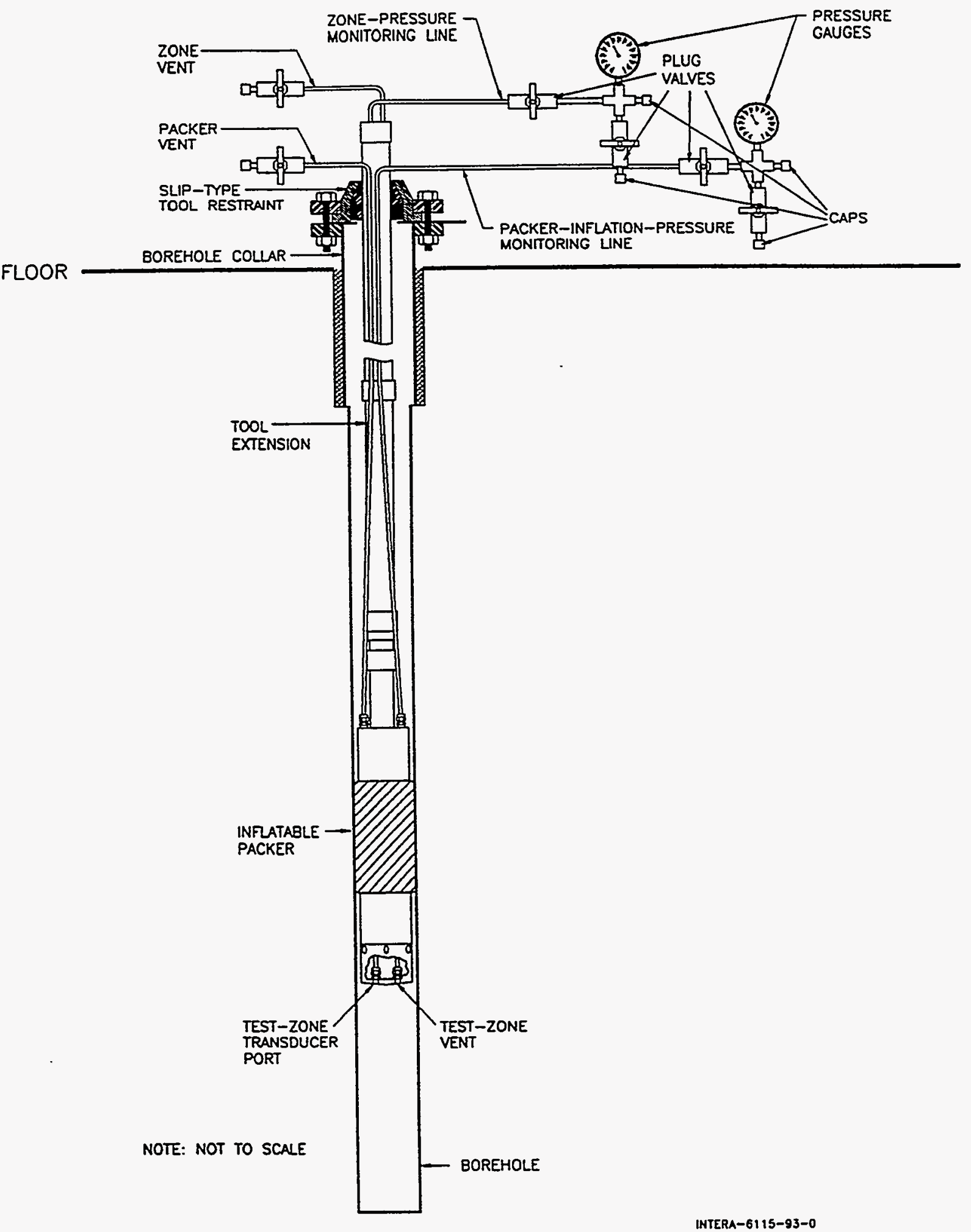

Figure 4-4. Generic single-packer monitor-tool configuration used for coupled permeability and hydrofracture testing. 


\subsubsection{Equipment Calibration}

Equipment calibration information is discussed in Section 3.3.2.

\subsection{Procedures for Permeability Testing Associated with Hydrofracture Experiments}

Test and monitor tools were installed as described in Section 3.4.1. Data associated with both test and observation boreholes were collected by the DAS associated with the test borehole. During the $\mathrm{C} 1 \mathrm{X} 05-\mathrm{B}$ testing sequence, borehole $\mathrm{C} 1 \mathrm{H} 07$ pressure was monitored for a brief period by a DAS associated with that observation borehole. This was necessary because borehole $\mathrm{C} 1 \mathrm{H} 07$ was deepened prior to deepening borehole $\mathrm{C} 1 \mathrm{X} 05$ (see Section 4.6.3.1).

During testing $\mathrm{C} 1 \mathrm{X} 05-\mathrm{B}$, borehole $\mathrm{C} 1 \mathrm{X} 05$ was primarily used as the testing borehole. In order to compare the directional pressure response between boreholes $\mathrm{C} 1 \mathrm{X} 05$ and $\mathrm{C} 1 \mathrm{X06}$, one pulse-withdrawal test was conducted in which borehole C1X06 was used as the testing borehole and borehole $\mathrm{C} 1 \mathrm{X05}$ and $\mathrm{C} 1 \mathrm{H} 07$ were used as the observation borehole. This pulse-withdrawal test is presented in Section 4.6.3.2 in Table 4-23.

One pulse-withdrawal test and multiple constant-pressure injection and withdrawal tests were conducted in association with the hydrofracture experiments. These tests followed the procedures outlined in Sections 3.4.2 and 3.4.3.

\subsection{Boreholes and Test-Tool Configurations for Coupled Permeability and Hydrofracture Testing}

Figure 2-1 shows the location of Room $\mathrm{C} 1$ in the WIPP facility where the coupled permeability and hydrofracture testing took place. Tables 4-1 and 4-2 provide information regarding the test and observation boreholes. To refer to a borehole which was deepened, a letter designation is added to the borehole number to indicate a different testing sequence.

Table 4-1. Coupled Permeability and Hydrofracture Test Borehole Information

\begin{tabular}{ccccccccc}
\hline $\begin{array}{c}\text { Test } \\
\text { Sequence }\end{array}$ & $\begin{array}{c}\text { Test } \\
\text { Horizon }\end{array}$ & $\begin{array}{c}\text { Time Test } \\
\text { Horizon } \\
\text { Penetrated }\end{array}$ & $\begin{array}{c}\text { Borehole } \\
\text { Diameter } \\
(\mathbf{c m})\end{array}$ & $\begin{array}{c}\text { Test Interval } \\
\text { Depth }(\mathbf{m})\end{array}$ & $\begin{array}{c}\text { Borehole } \\
\text { Depth }(\mathbf{m})\end{array}$ & $\begin{array}{c}\text { Sequence } \\
\text { Started }\end{array}$ & $\begin{array}{c}\text { Sequence } \\
\text { Terminated }\end{array}$ \\
\hline C1X10 & MB139 & $\begin{array}{c}11-11-91 \\
(13: 25)\end{array}$ & 7.62 & $7.50-8.10$ & 10.16 & $11-12-91$ & $6-26-92$ \\
C1X05-A & MB139 & $\begin{array}{c}6-29-92 \\
(10: 00)\end{array}$ & 7.62 & $6.72-7.60$ & 9.14 & $7-1-92$ & $4-12-93$ \\
C1X05-B & MB140 & $\begin{array}{l}6-14-93 \\
(10: 00)\end{array}$ & 7.62 & $23.09-27.14$ & 30.20 & $6-15-93$ & $4-11-94$ \\
\hline
\end{tabular}


Table 4-2 (Continued). Coupled Permeability and Hydrofracture Observation Borehole Information

\begin{tabular}{|c|c|c|c|c|c|c|}
\hline $\begin{array}{c}\text { Test } \\
\text { Sequence }\end{array}$ & $\begin{array}{l}\text { Observation } \\
\text { Borehole }\end{array}$ & $\begin{array}{c}\text { Test } \\
\text { Horizon }\end{array}$ & $\begin{array}{c}\text { Time Test } \\
\text { Horizon } \\
\text { Penetrated }\end{array}$ & $\begin{array}{c}\text { Borehole } \\
\text { Diameter } \\
\text { (cm) }\end{array}$ & $\begin{array}{l}\text { Test Interval } \\
\text { Depth (m) }\end{array}$ & $\begin{array}{l}\text { Borehole } \\
\text { Depth }(m)\end{array}$ \\
\hline C1X05-A & $\mathrm{C} 1 \mathrm{H} 05$ & MB139 & $\begin{array}{l}1-16-92 \\
(13: 30)\end{array}$ & 10.16 & $7.002-8.255$ & 8.26 \\
\hline C1X05-A & C1H06 & MB139 & $\begin{array}{l}1-21-92 \\
(13: 30)\end{array}$ & 10.16 & $8.200-9.398$ & 9.40 \\
\hline C1X05-A & $\mathrm{C} 1 \mathrm{HO} 07$ & MB139 & $\begin{array}{l}6-24-92 \\
(12: 00)\end{array}$ & 10.16 & $6.770-7.600$ & 8.18 \\
\hline C1X05-A & $\mathrm{C} 1 \times 06$ & MB139 & $\begin{array}{l}9-29-92 \\
(12: 30)\end{array}$ & 10.16 & $6.430-7.320$ & 7.63 \\
\hline C1X05-A & C1X10 & MB139 & $\begin{array}{c}11-11-91 \\
(13: 25)\end{array}$ & 10.16 & $7.50-8.10$ & 10.16 \\
\hline C1X05-A & $\mathrm{C} 2 \mathrm{H} 01$ & MB139 & $\begin{array}{c}12-13-89 \\
(12: 00)\end{array}$ & 10.16 & $6.80-7.76$ & 8.97 \\
\hline C1X05-B & $\mathrm{C} 1 \mathrm{H} 07$ & MB140 & $\begin{array}{c}5-25-93 \text { to } 5-26- \\
93\end{array}$ & 10.16 & $\begin{array}{l}22.688-23.657 \\
24.277-27.880\end{array}$ & 27.88 \\
\hline $\mathrm{C} 1 \times 05-\mathrm{B}$ & C1X06 & MB140 & $7-9-93 \& 8-12-93$ & 10.16 & $\begin{array}{l}22.973-23.945 \\
24.559-27.990\end{array}$ & 27.99 \\
\hline $\mathrm{C} 1 \times 10$ & $\mathrm{C} 1 \mathrm{H} 05$ & MB139 & $\begin{array}{l}1-16-92 \\
(13: 30)\end{array}$ & 10.16 & $7.002-8.255$ & 8.26 \\
\hline C1X10 & $\mathrm{C} 1 \mathrm{HOG}$ & MB139 & $\begin{array}{l}1-21-92 \\
(13: 30)\end{array}$ & 10.16 & $8.200-9.398$ & 9.40 \\
\hline $\mathrm{C} 1 \times 10$ & DPD02 & MB139 & $\begin{array}{l}4-86 \\
\text { (N/A) }\end{array}$ & 10.16 & $11.00-13.11$ & 13.11 \\
\hline $\mathrm{C} 1 \times 10$ & DPD03 & MB139 & $\begin{array}{l}4-86 \\
\text { (N/A) }\end{array}$ & 10.16 & $11.15-13.11$ & 13.11 \\
\hline $\mathrm{C} 1 \times 10$ & $\mathrm{C} 2 \mathrm{H} 01$ & MB139 & $\begin{array}{c}12-13-89 \\
(12: 00)\end{array}$ & 10.16 & $6.80-7.76$ & 8.97 \\
\hline
\end{tabular}

The drilling procedures describes in Section 3.5.2 were followed during the drilling of the boreholes associated with the coupled permeability and hydrofracture testing program. The air-rotary drilling method was used to drill all of the initial boreholes. The brine-rotary drilling method was used to deepen boreholes $\mathrm{C} 1 \mathrm{H} 07, \mathrm{C} 1 \mathrm{X} 05$, and $\mathrm{C} 1 \mathrm{X} 06$ prior to the C1X05-B coupled permeability and hydrofracture testing sequence.

Figures 4-5 and 4-6 schematically depict Room C1 in plan and cross-sectional views, and show the positions of all boreholes associated with the testing program. Figure 4-7 schematically depicts Room C2 in plan and cross-section showing the location of 


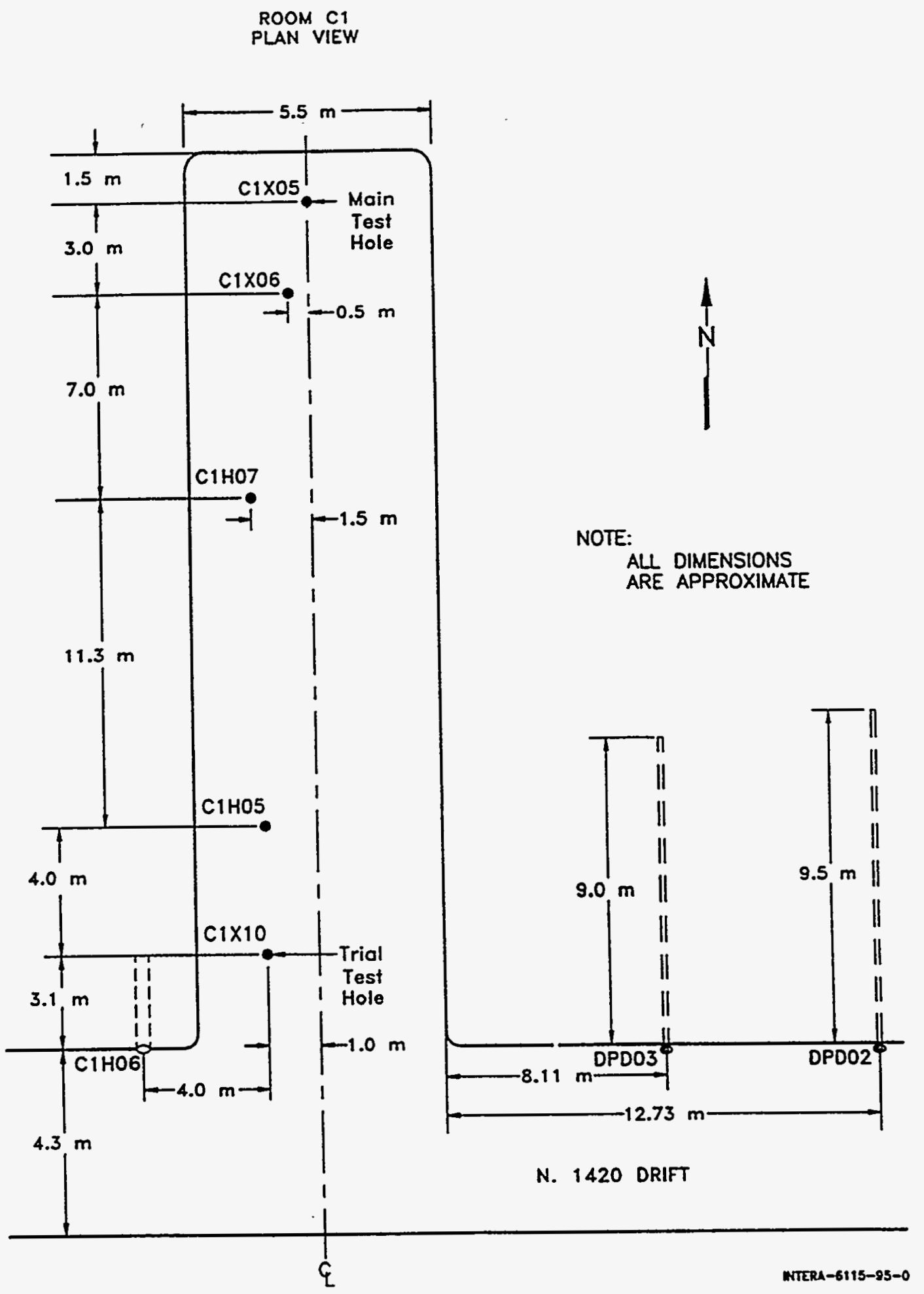

Figure 4-5. Spatial representation of Room C1 showing the locations of test and observation boreholes. 


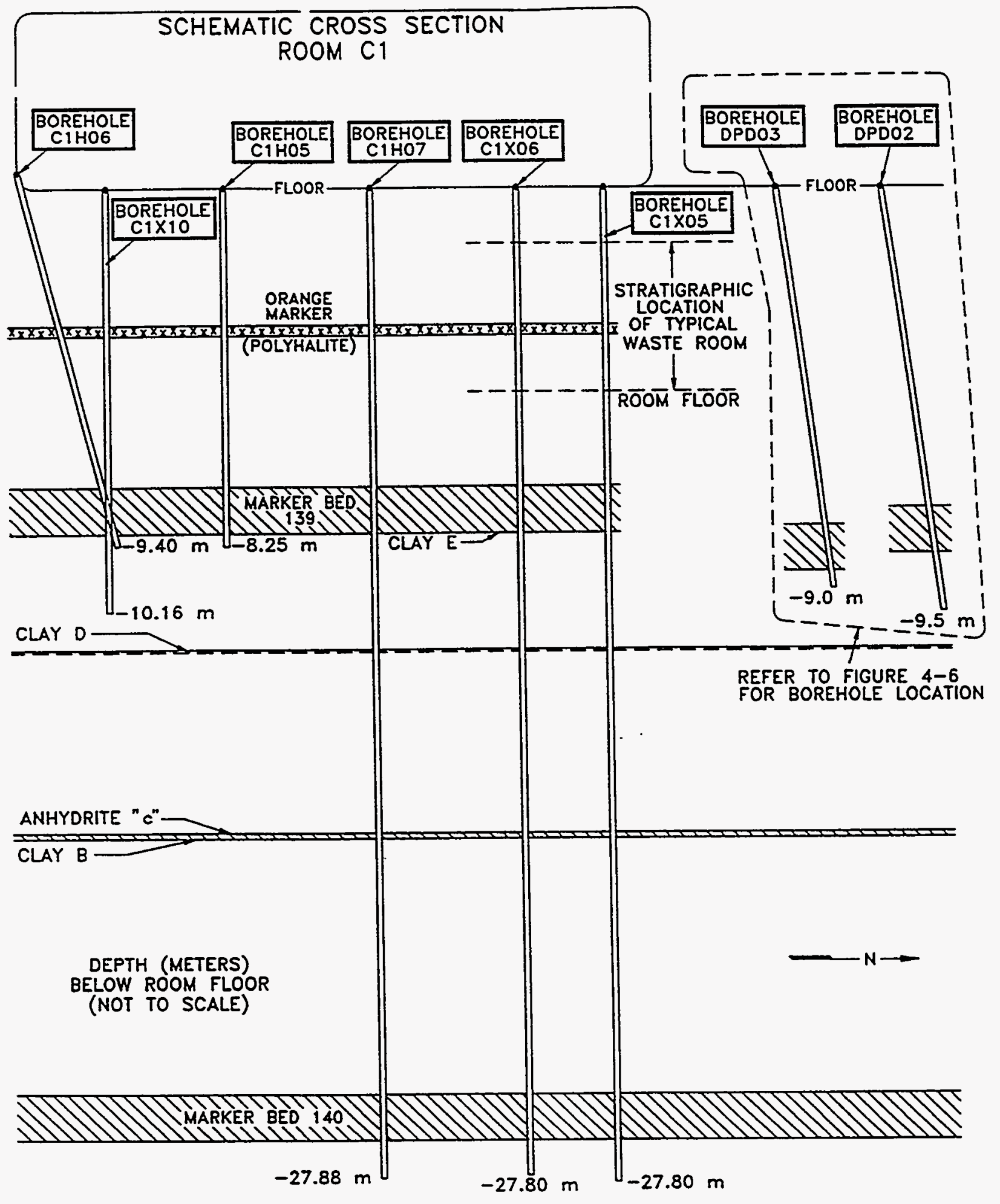

IMTERA-\$1 IS-16-0

Figure 4-6. Cross-sectional view of Room C1 showing the locations of test and observation boreholes. 


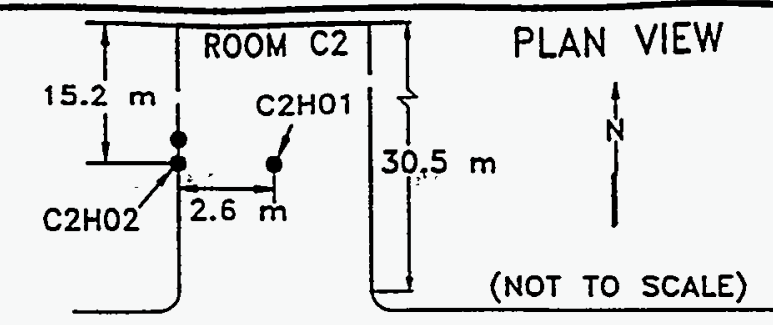

N. 1420 DRIFT

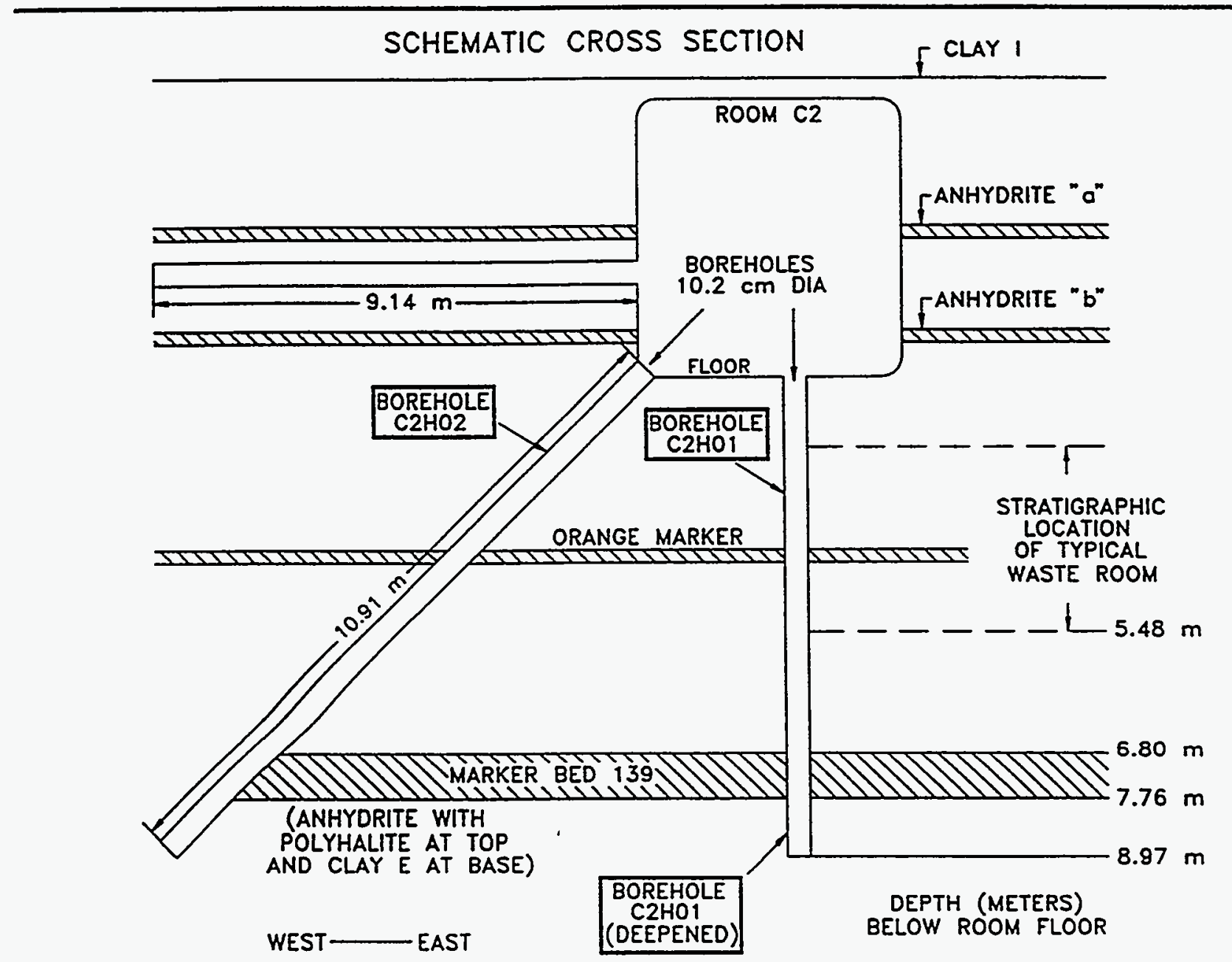

INTERA-61 15-180-0

Figure 4-7. Spatial representation of Room C2 showing the locations and orientations of test and observation boreholes $\mathrm{C} 2 \mathrm{H} 01$ and $\mathrm{C} 2 \mathrm{HO}$. 
observation borehole $\mathrm{C} 2 \mathrm{H} 01$. Figures 4-8 through 4-17 show the test- and observationtool configurations and associated installations that were used during the testing program. It should be noted that Figures 4-15, 4-16, and 4-17 each consist of two parts (ex. Fgiures 4-15a and 4-15b). Test-tool installation diagrams for boreholes $\mathrm{C} 2 \mathrm{H} 01, \mathrm{DPDO2}$, and DPD03 are contained in Section 7.5. Detailed desription of the core that was recovered from observation borehole $\mathrm{C} 2 \mathrm{HO} 1$ are provided in Saulnier et al., (1991). Observation boreholes DPD02 and DPD03 were constructed by S-CUBED under contract to SNL prior to 1988. Detailed descriptions of the core that was recovered from all of the other boreholes associated with the testing program are given in Appendix $A$ of this report. 


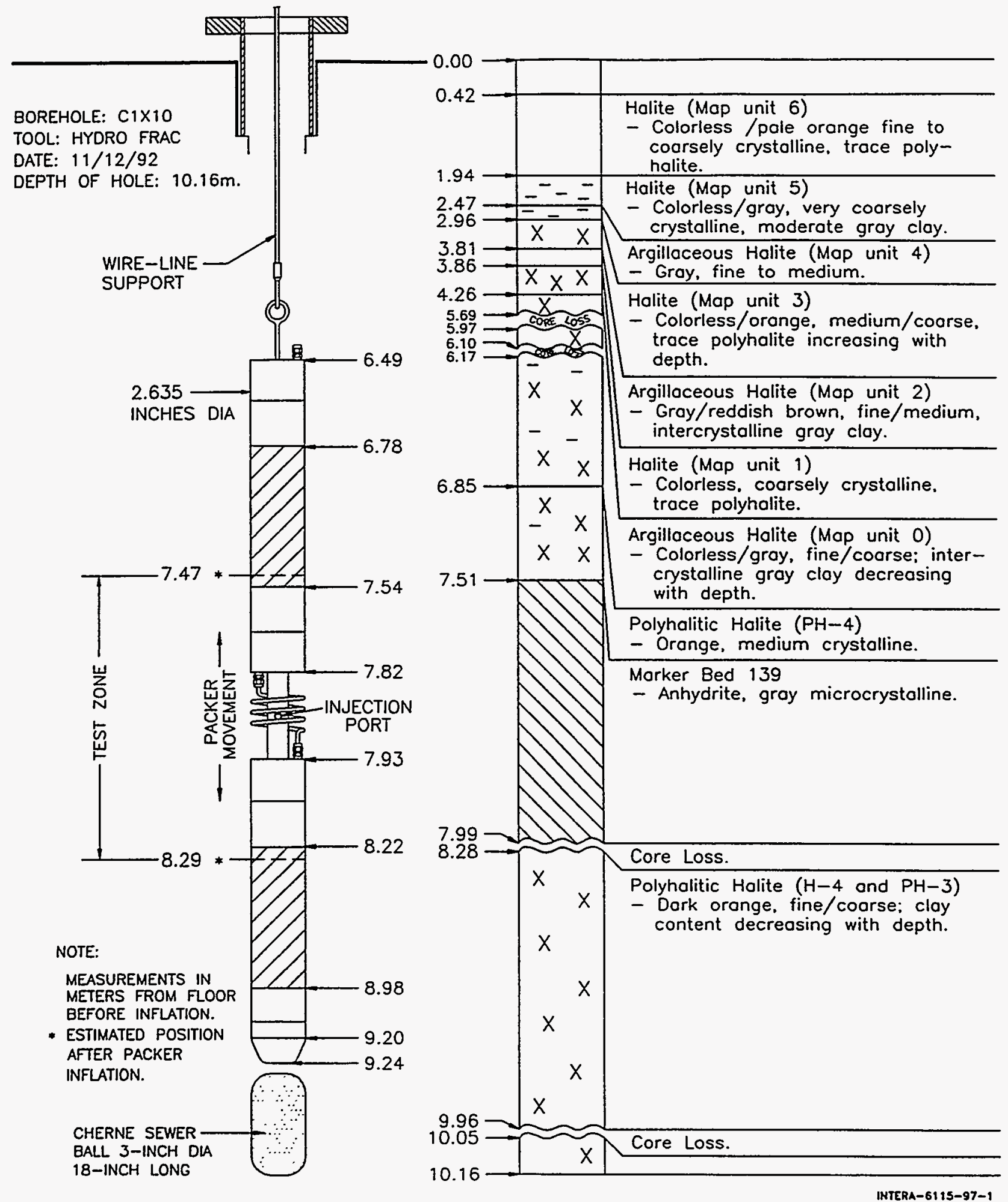

Figure 4-8. Configuration of the tool in test borehole $C 1 \times 10$ for testing sequences $C 1 \times 10$ and C1X05-A. 


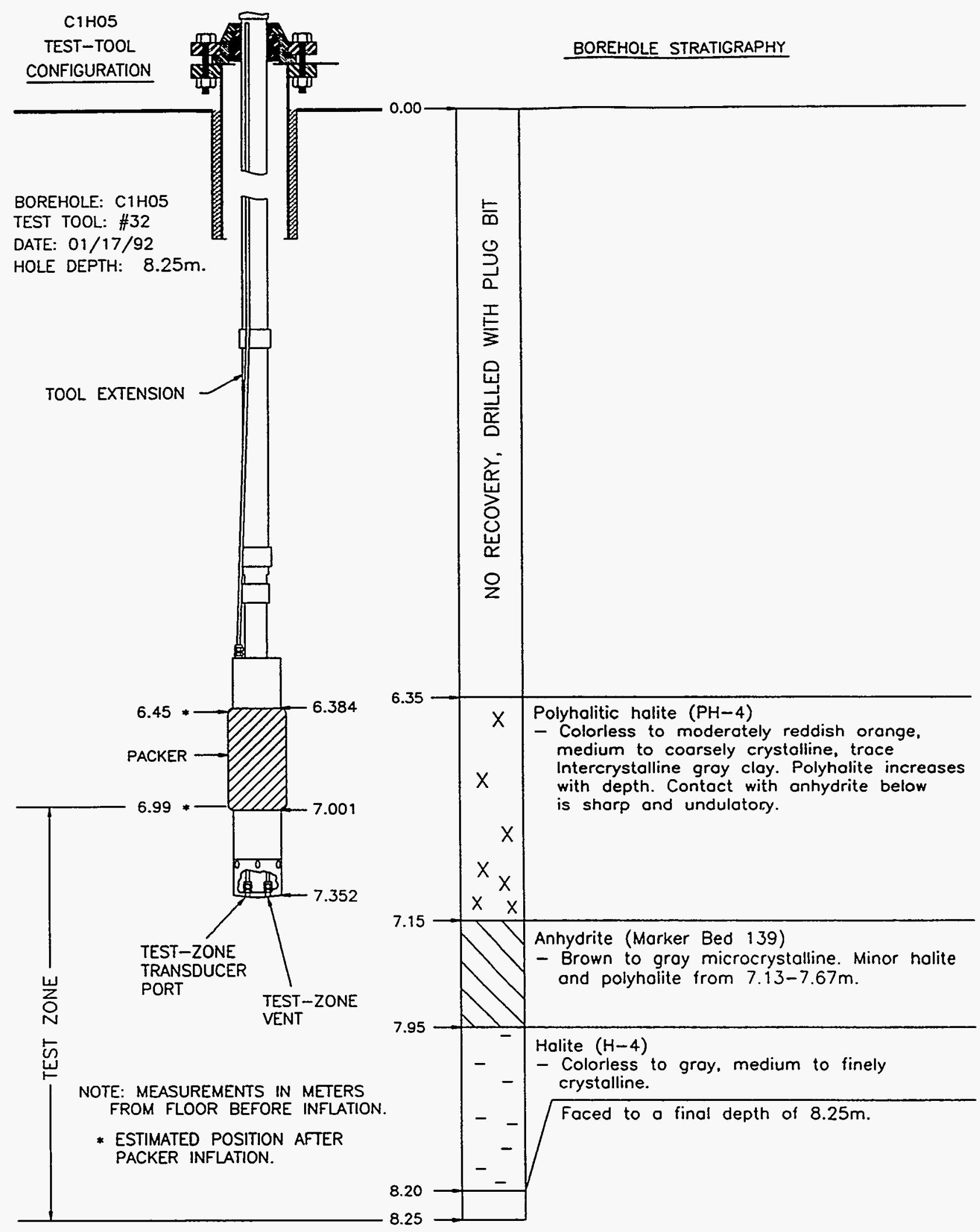

INTERA-6115-98-1

Figure 4-9. Configuration of the tool in observation borehole $\mathrm{C} 1 \mathrm{H} 05$ for testing sequences $\mathrm{C} 1 \times 10$ and $\mathrm{C} 1 \times 05-\mathrm{A}$. 


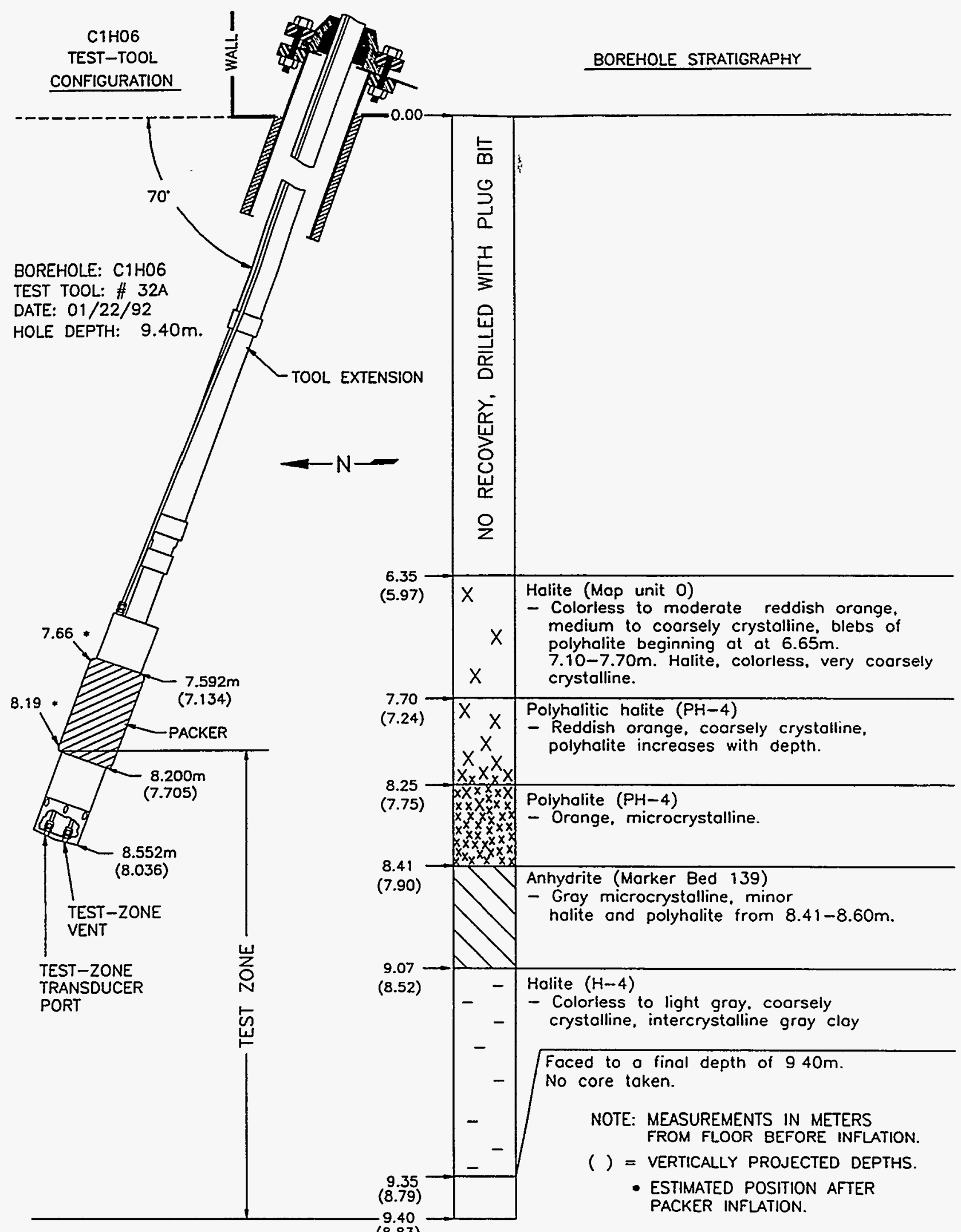

INTERA-61 15-99-1

Figure 4-10. Configuration of the tool in observation borehole $\mathrm{C} 1 \mathrm{H} 06$ for testing sequences $C 1 \times 10$ and $C 1 \times 05-A$. 
CIX05-A

BOREHOLE STRATIGRAPHY

TEST-TOOL CONFIGURATION

BOREHOLE: C1X05

TOOL: HYDRO FRAC

DATE: $07 / 02 / 92$

HOLE DEPTH: 9.14

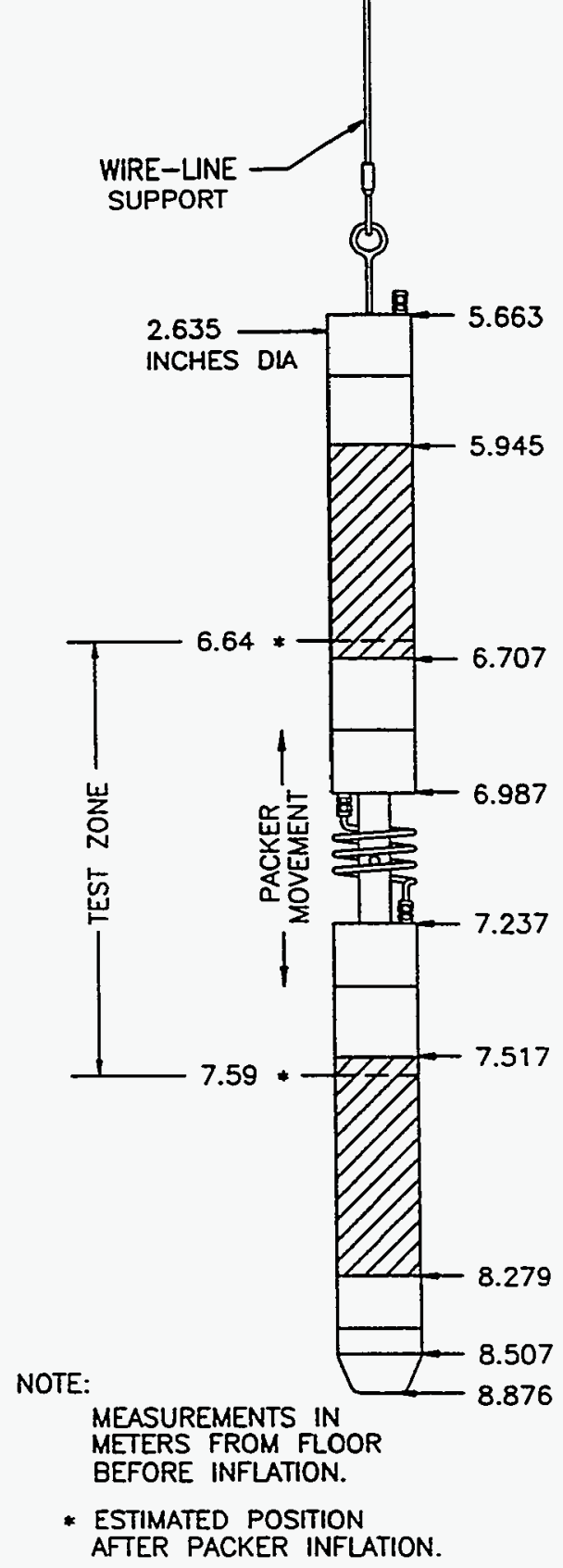

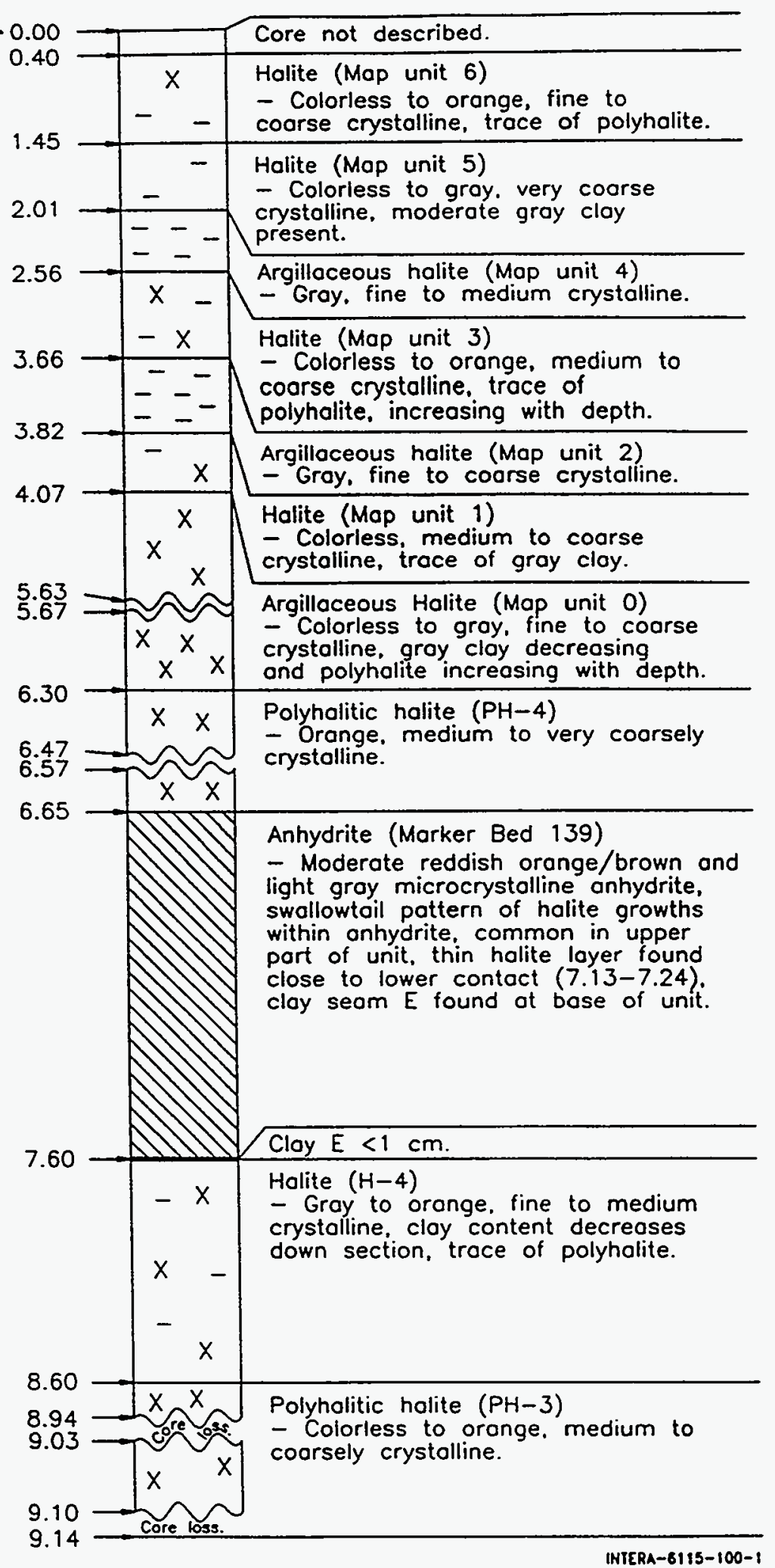

Figure 4-11. Configuration of the tool in test borehole $C 1 \times 05$ for testing sequence $C 1 \times 05-$ A. 


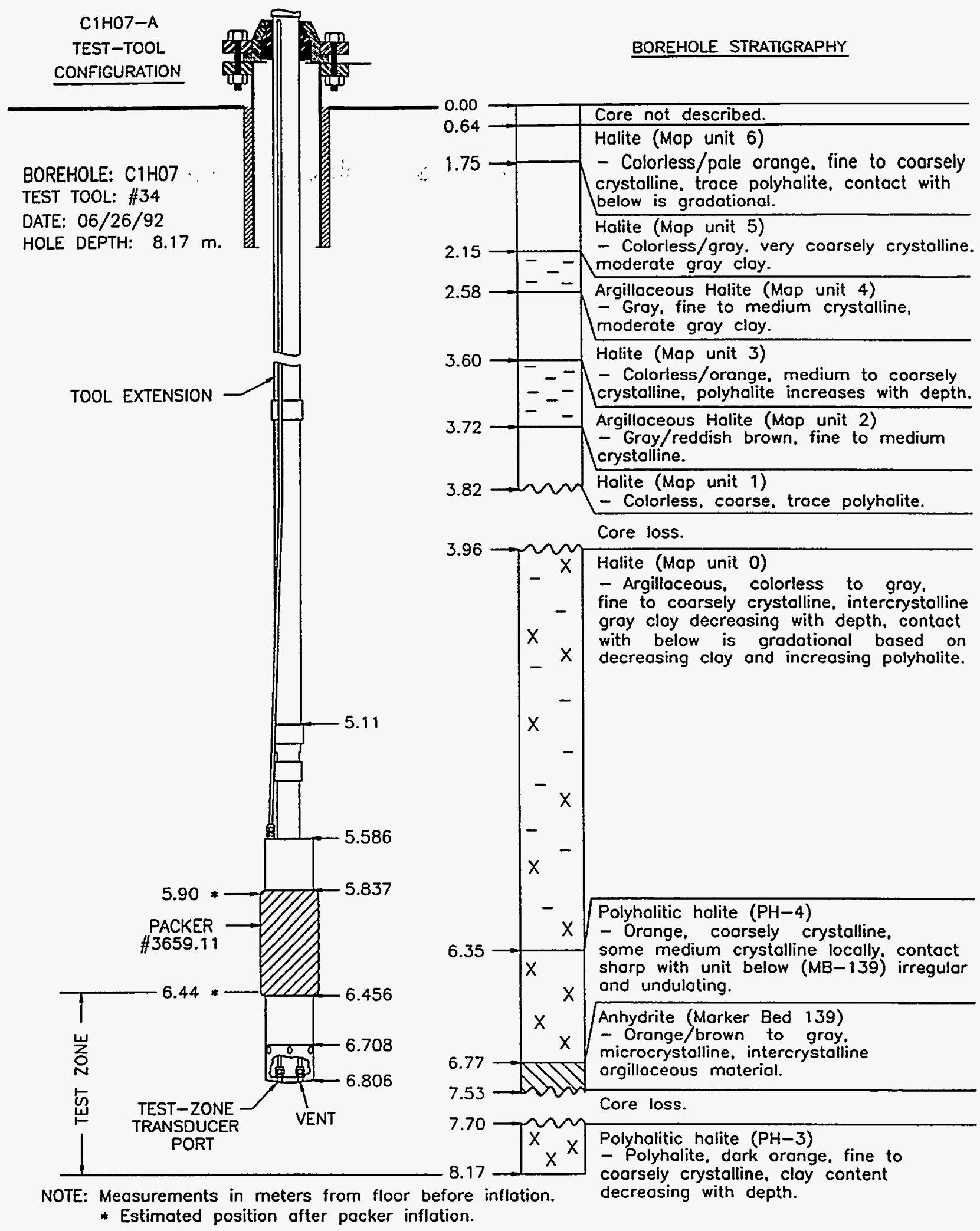

INTERA-61 15-101-2

Figure 4-12. Configuration of the tool in observation borehole $\mathrm{C} 1 \mathrm{H} 07$ for testing sequence C1X05-A. 


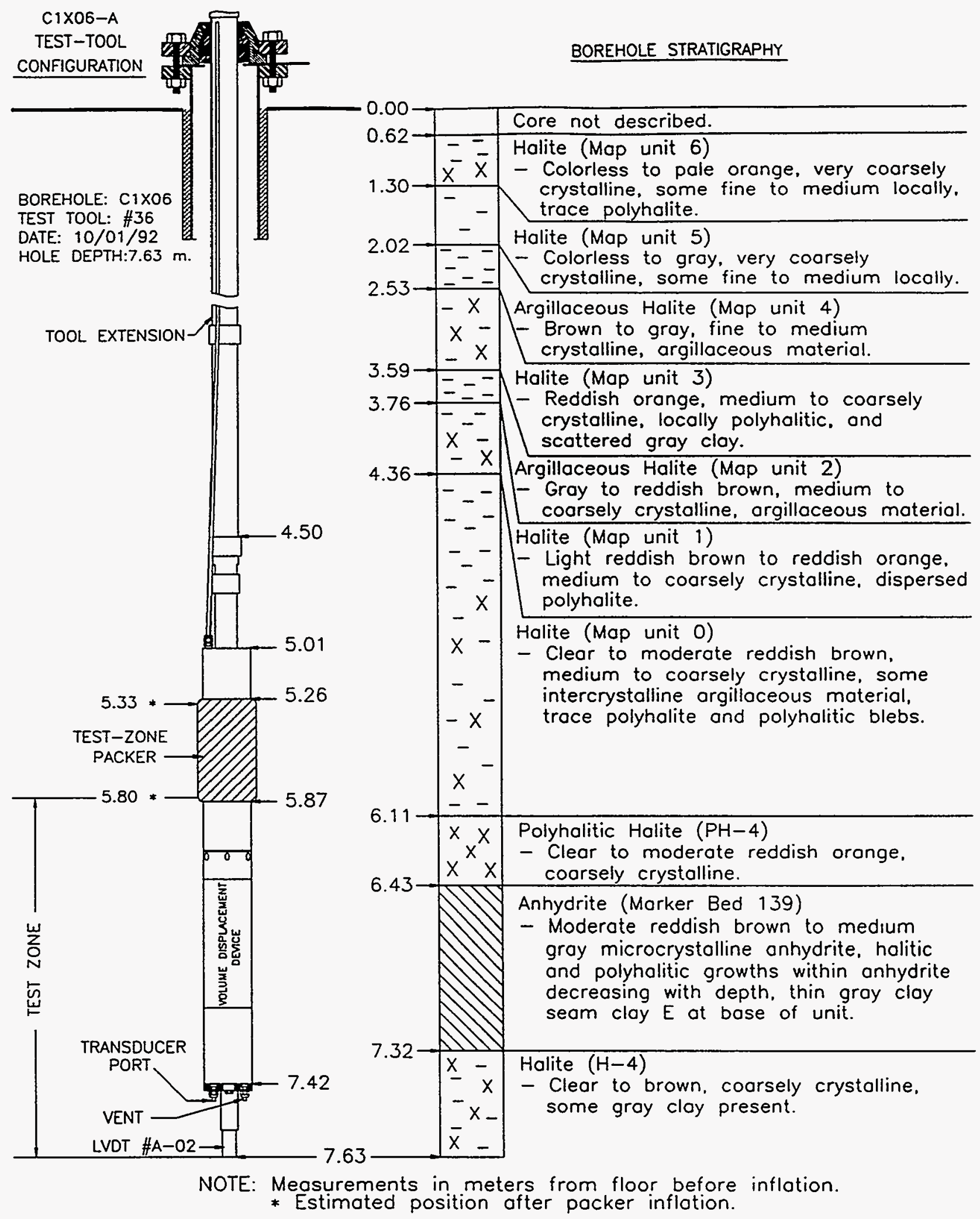

INTERA-6115-102-1

Figure 4-13. Configuration of the tool in observation borehole $\mathrm{C} 1 \mathrm{X} 06$ for testing sequence C1X05-A. 
C1X05-B

TEST-TOOL CONFIGURATION

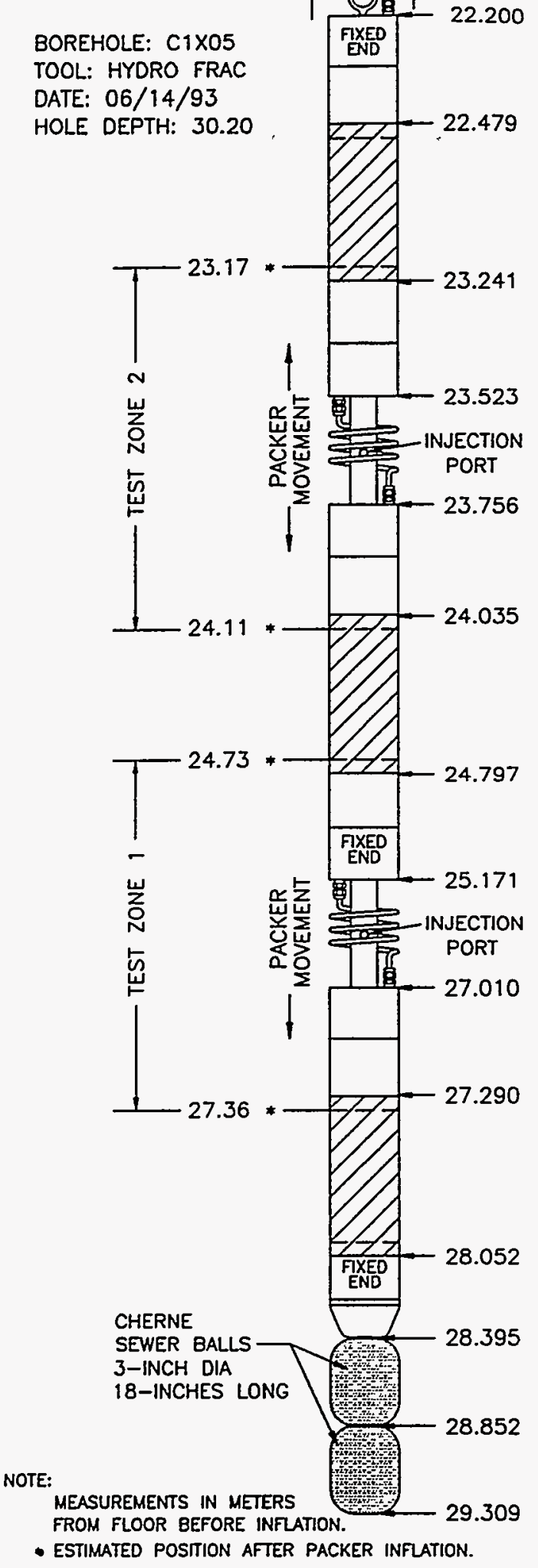

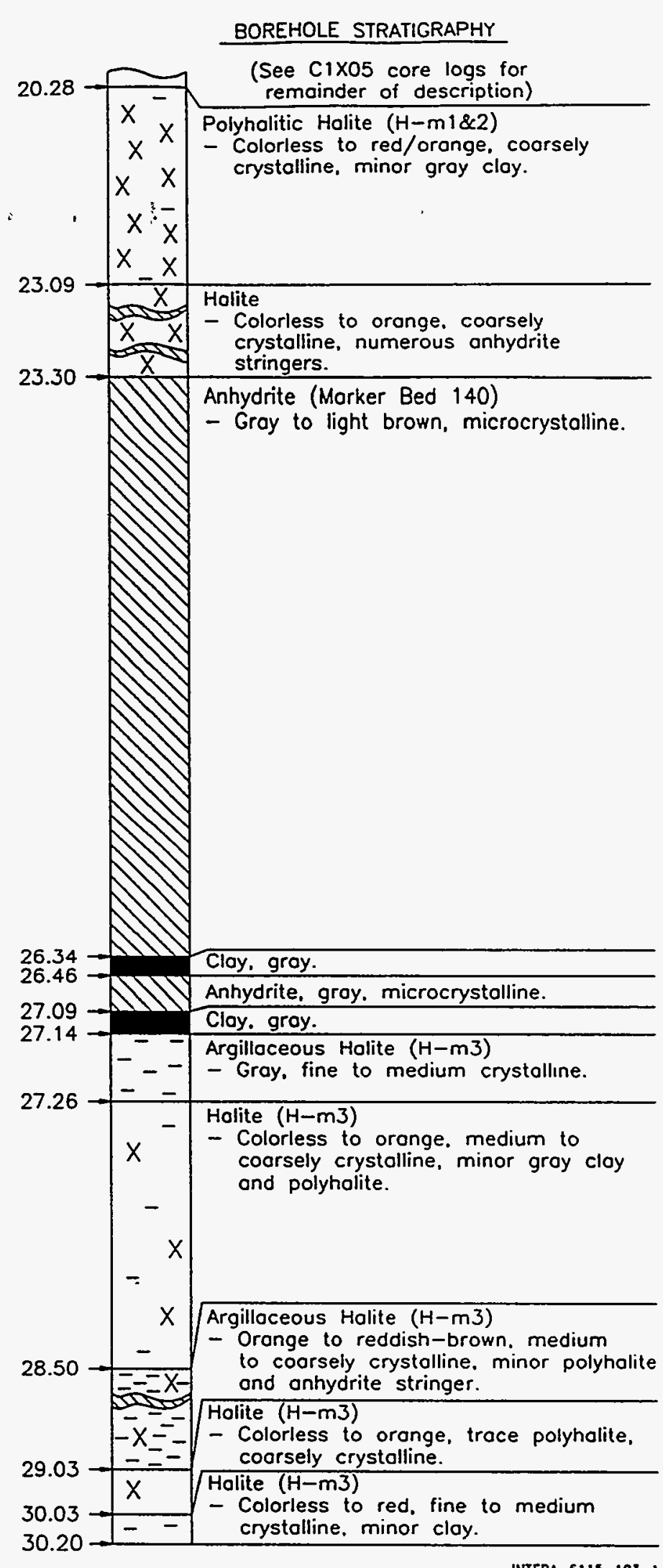

INTERA-6115-103-1

Figure 4-14. Configuration of the tool in test borehole $\mathrm{C} 1 \mathrm{X} 05$ for testing sequence $\mathrm{C} 1 \mathrm{X} 05$ B. 


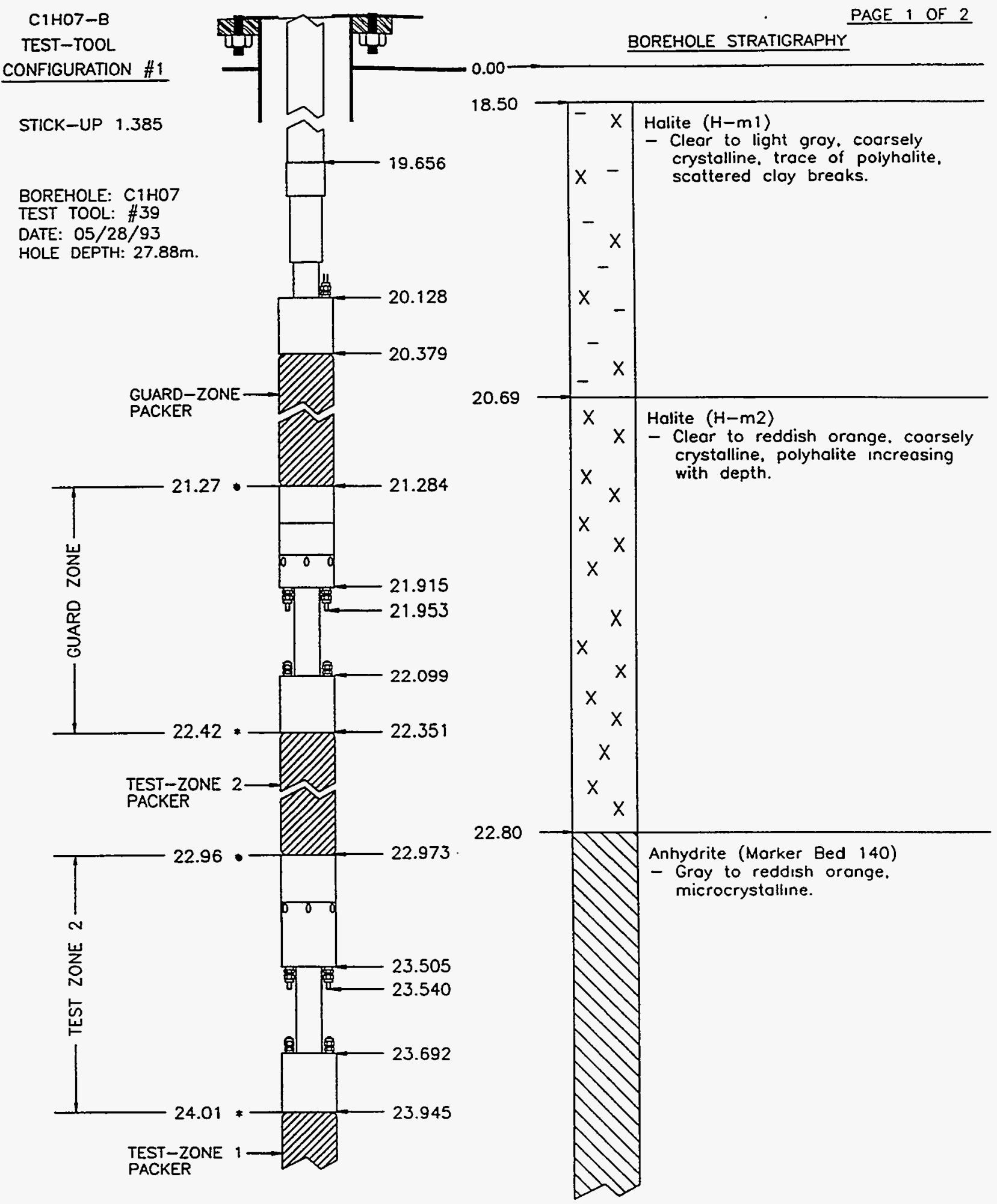

NOTE: Measurements in meters from floor before inflation.

* Estimated position ofter packer inflation.

INTERA-6115-104-1

Figure 4-15a. Configuration \#1 of the tool in observation borehole $\mathrm{C} 1 \mathrm{H} 07$ for testing sequence $\mathrm{C} 1 \mathrm{X} 05-\mathrm{B}$. 
BOREHOLE: C1HO7

TEST TOOL: \#39

DATE: $05 / 28 / 93$

HOLE DEPTH: $27.88 \mathrm{~m}$.
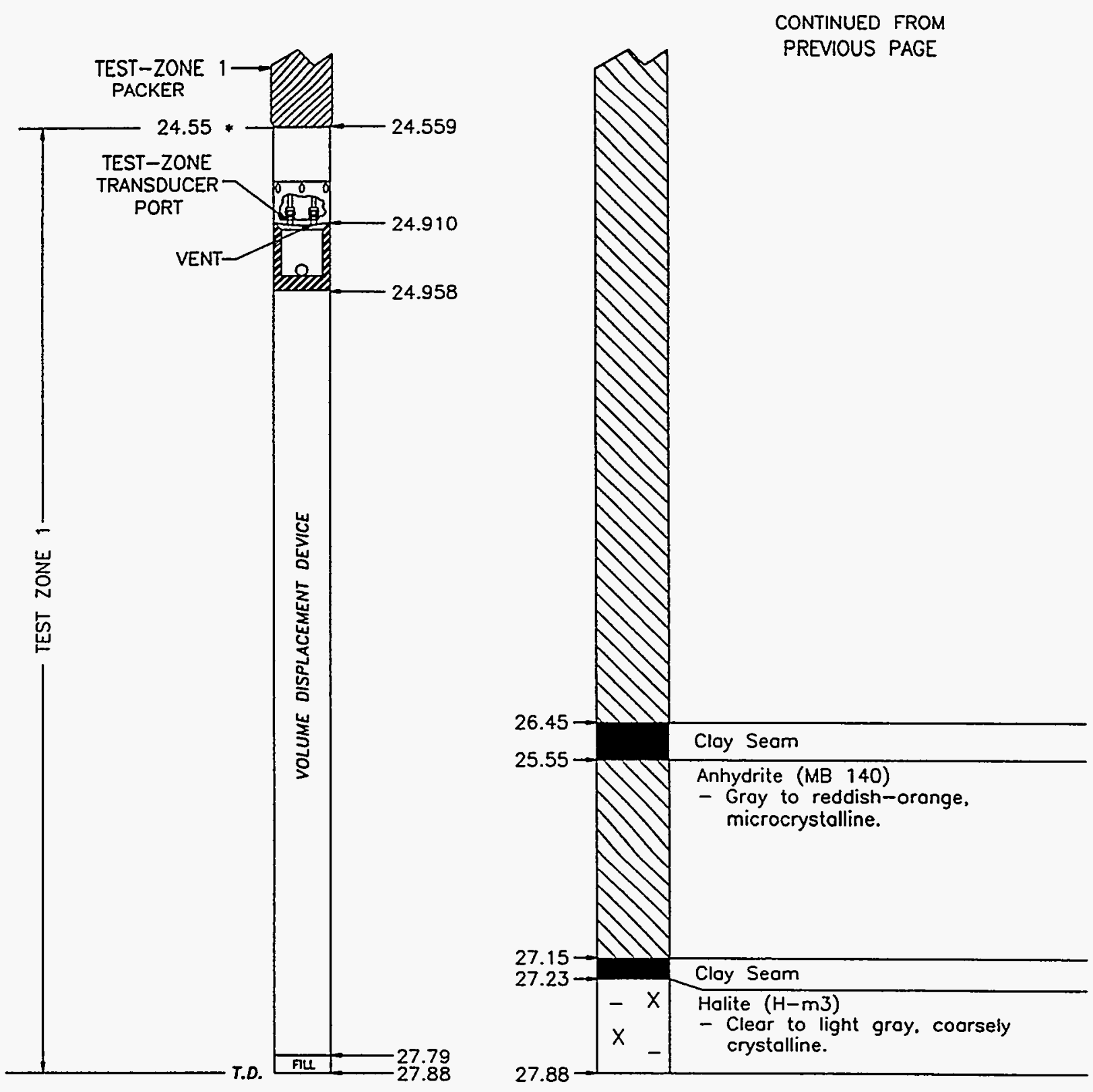

NOTE: Meosurements in meters from floor before inflation.

* Estimated position ofter packer inflation.

INTERA-6115-105-2

Figure 4-15b. Configuration \#1 of the tool in observation borehole $\mathrm{C} 1 \mathrm{H} 07$ for testing sequence $\mathrm{C} 1 \mathrm{X} 05-\mathrm{B}$ (continued). 


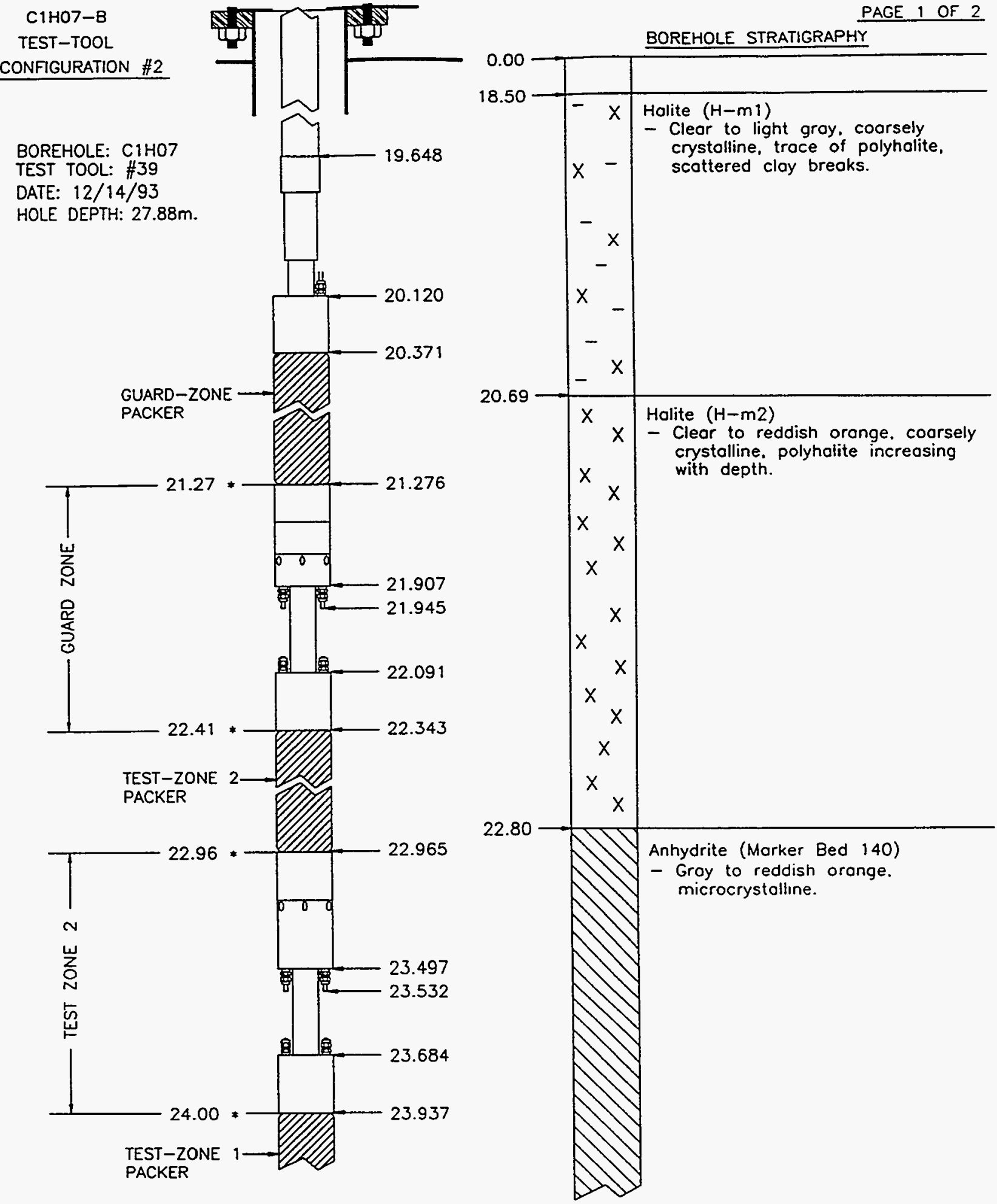

NOTE: Meosurements in meters from floor before inflation.

- Estimated position after pocker inflation.

INTERA-6I 15- 105-1

Figure 4-16a. Configuration \#2 of the tool in observation borehole $\mathrm{C} 1 \mathrm{H} 07$ for testing sequence $\mathrm{C} 1 \mathrm{X} 05-\mathrm{B}$. 
BOREHOLE: C1HO7

TEST TOOL: \#39

DATE: $12 / 14 / 93$

HOLE OEPTH: $27.88 \mathrm{~m}$.

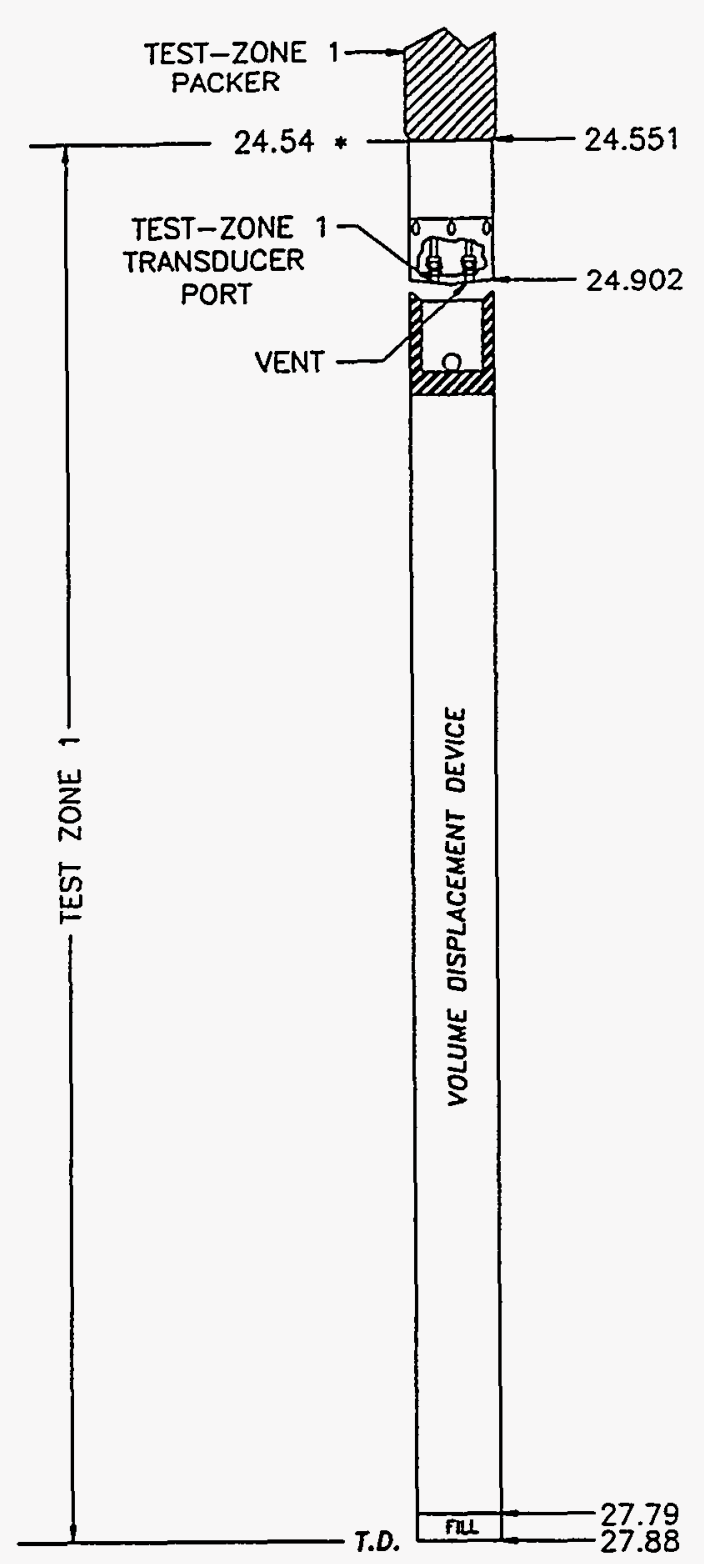

BOREHOLE STRATIGRAPHY 


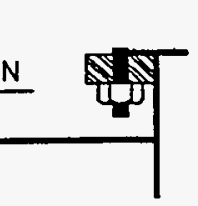

BOREHOLE: C1X06

TEST TOOL: \#40

DATE: $08 / 25 / 93$

HOLE DEPTH: $27.80 \mathrm{~m}$.
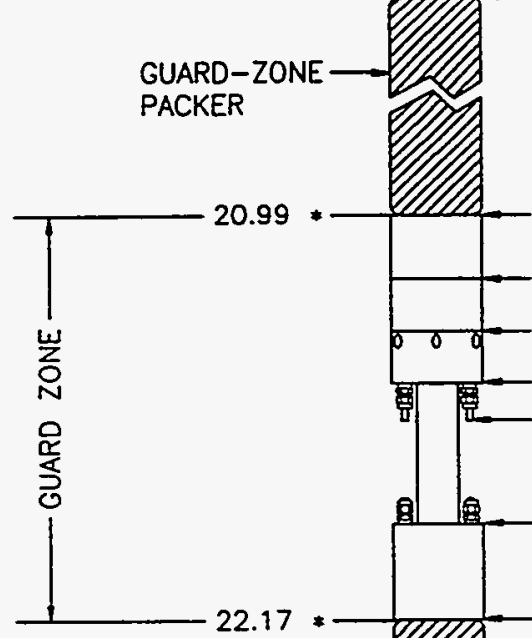

21.004

21.253

21.355

21.635

21.672

TEST-ZONE PACKER

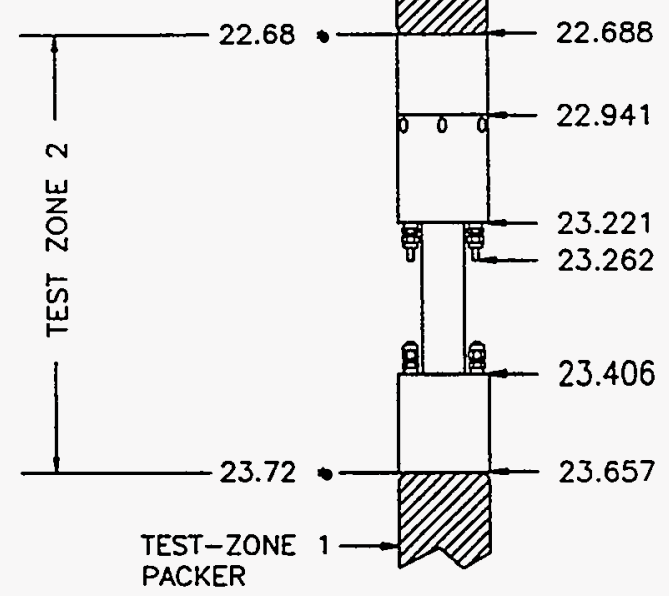

NOTE: Measurements in meters from floor before inflotion.

* Estimated position ofter pocker inflation. gypsum.

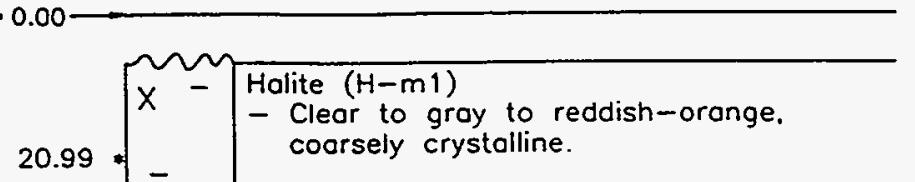
microcrystalline. Halite pseudomorphs ofter prismatic

Figure 4-17a. Configuration of the tool in observation borehole $\mathrm{C} 1 \times 06$ for testing sequence $\mathrm{C} 1 \mathrm{X} 05-\mathrm{B}$. 
BOREHOLE: C1X06

TEST TOOL: \#40

DATE: $08 / 25 / 93$

HOLE DEPTH: $27.80 \mathrm{~m}$.

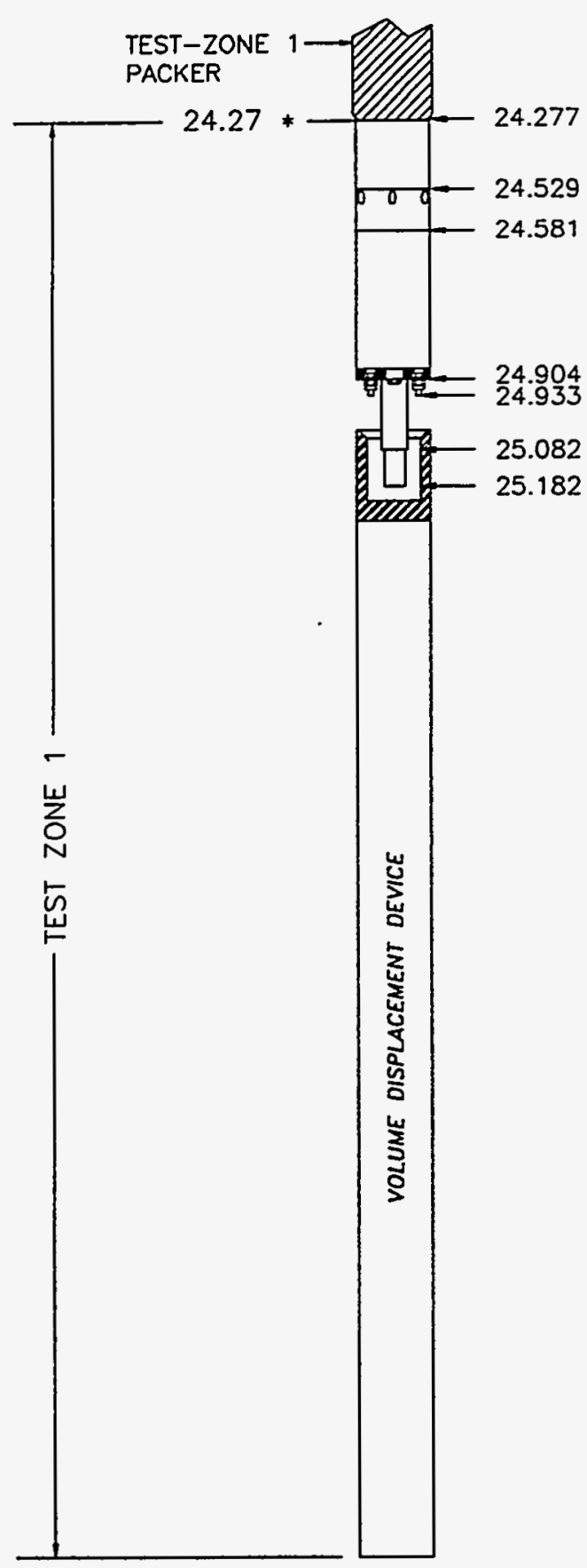

BOREHOLE STRATIGRAPHY

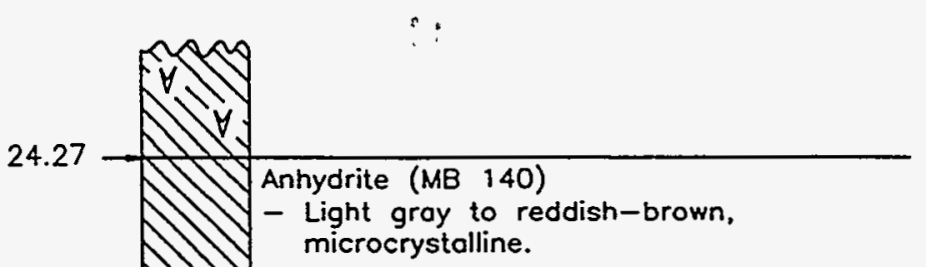

NOTE: Measurements in meters from floor before inflotion.

* Estimated position after packer inflation.

Figure 4-17b. Configuration of the tool in observation borehole $\mathrm{C} 1 \mathrm{X} 06$ for testing sequence $C 1 \times 05-B$ (continued). 


\subsection{Test Data}

This section presents data from the testing sequences in the two test boreholes that were investigated. One pulse-withdrawal test, 12 constant-pressure-injection tests, and 16 constant-pressure-withdrawal tests were performed.

Acronyms used in the tables presented in this section are as follows:

$\mathrm{CPI}=$ Constant-Pressure-Injection Test

$\mathrm{CPW}=$ Constant-Pressure-Withdrawal Test

PW $=$ Pulse-Withdrawal Test

$T Z=$ Test Zone

TZP Test Zone Packer

TZ1 = Test Zone 1 (triple-packer configuration)

TZ1P = Test Zone 1 Packer (triple-packer configuration)

TZ2 = Test Zone 2 (triple-packer configuration)

TZ2P = Test Zone 2 Packer (triple-packer configuration)

Zone Fluid Volume = Isolated Zone Volume+Tubing Volume-Tool Volume

* $=$ borehole in which the test was conducted

Table 4-3 summarizes information from these tests. Complete data files and abridged tabulations of the data are stored in the SWCF under WPO \#42269.

Table 4-3. Summary of Test Information from Test Boreholes

\begin{tabular}{|c|c|c|c|c|c|c|c|c|c|c|c|}
\hline $\begin{array}{l}\text { Hydro-Frac } \\
\text { Testing } \\
\text { Sequence }\end{array}$ & Zone & $\begin{array}{c}\text { Date } \\
\text { Started } \\
\text { (mm-dd-yy) }\end{array}$ & $\begin{array}{l}\text { Test } \\
\text { Type }\end{array}$ & Unit & $\begin{array}{l}\text { Initial } \\
\text { Pressure } \\
\text { (MPa) }\end{array}$ & $\begin{array}{c}\text { Final } \\
\text { Pressure } \\
\text { (MPa) }\end{array}$ & $\begin{array}{c}\text { Fluid Volume } \\
\text { Injected (+) } \\
\text { Withdrawm (-) } \\
\text { (mL) }\end{array}$ & $\begin{array}{c}\text { Zone } \\
\text { Length } \\
\text { (cm) }\end{array}$ & $\begin{array}{c}\text { Zone } \\
\text { Radius } \\
\text { (cm) }\end{array}$ & $\begin{array}{c}\text { Tool } \\
\text { Volume } \\
\text { (mL) }\end{array}$ & $\begin{array}{l}\text { Zone Fluid } \\
\text { Volume } \\
\text { (mL) }\end{array}$ \\
\hline C $1 \times 10$ & $T Z$ & $11-15-91$ & CPI\#1 & MB139 & 5.5 & 6.5 & +332 & 82 & 3.81 & 2454 & 2232 \\
\hline $\mathrm{C}_{1 \times 10}$ & $T Z$ & $11-19-91$ & $\mathrm{CPI} \# 2$ & MB139 & 6.1 & 6.4 & +809 & 82 & 3.81 & 2454 & 2232 \\
\hline C1X10 & $T Z$ & $11-26-91$ & CPI \#3 & MB139 & 6.5 & 9.0 & +7098 & 82 & 3.81 & 2454 & 2232 \\
\hline$C_{1 \times 10}$ & $T Z$ & $12-5-91$ & CPW\#1 & MB139 & 8.6 & 7.5 & -19089 & 82 & 3.81 & 2454 & 2232 \\
\hline $\operatorname{c} 1 \times 10$ & $T Z$ & $12-6-91$ & CPW\#2 & MB139 & 7.7 & 7.6 & -4393 & 82 & 3.81 & 2454 & 2232 \\
\hline C1X10 & $T Z$ & $12-11-91$ & CPW\#3 & MB139 & 7.7 & 7.4 & -94 & 82 & 3.81 & 2454 & 2232 \\
\hline $\mathrm{C} 1 \times 10$ & TZ & $2-6-92$ & $\mathrm{CPI} \# 4$ & MB139 & 7.5 & 9.0 & +874 & 82 & 3.81 & 2454 & 2232 \\
\hline C1X10 & $T Z$ & 3-18-92 & CPW\#4 & MB139 & 7.9 & 6.4 & -7831 & 82 & 3.81 & 2454 & 2232 \\
\hline Cix05-A & $T Z$ & $8-4-92$ & CPI \#1 & MB139 & 9.2 & 9.7 & +4 & 95 & 3.81 & 2270 & 2816 \\
\hline C1X05-A & $T Z$ & $8-4-92$ & $\mathrm{CPI} \# 2$ & MB139 & 9.7 & 10.2 & +34 & 95 & 3.81 & 2270 & 2816 \\
\hline C1X05-A & $T Z$ & $8-10-92$ & CPI \#3 & MB139 & 9.4 & 10.2 & +32 & 95 & 3.81 & 2270 & 2816 \\
\hline C1X05-A & $T Z$ & $9-16-92$ & CPW\#1 & MB139 & 11.7 & 9.5 & -241 & 95 & 3.81 & 2270 & 2816 \\
\hline$C_{1} \times 05-A$ & $T Z$ & $9-16-92$ & CPW\#2 & MB139 & 11.5 & 9.5 & -991 & 95 & 3.81 & 2270 & 2816 \\
\hline C1X05-A & $T Z$ & $9-16-92$ & CPW\#3 & MB139 & 11.3 & 9.5 & -1245 & 95 & 3.81 & 2270 & 2816 \\
\hline C1X05-A & $T Z$ & $9-16-92$ & CPW\#4 & MB139 & 10.8 & 9.2 & -3094 & 95 & 3.81 & 2270 & 2816 \\
\hline
\end{tabular}


Table 4-3 (Continued). Summary of Test Information from Test Boreholes

\begin{tabular}{|c|c|c|c|c|c|c|c|c|c|c|c|}
\hline $\begin{array}{l}\text { Hydro-Frac } \\
\text { Testing } \\
\text { Sequence }\end{array}$ & Zone & $\begin{array}{c}\text { Date } \\
\text { Started } \\
\text { (mm-dd-yy) }\end{array}$ & $\begin{array}{l}\text { Test } \\
\text { Type }\end{array}$ & Unit & $\begin{array}{l}\text { Initial } \\
\text { Pressure } \\
\text { (MPa) }\end{array}$ & $\begin{array}{l}\text { Final } \\
\text { Pressure } \\
\text { (MPa) }\end{array}$ & $\begin{array}{l}\text { Fluid Volume } \\
\text { Injected (+) } \\
\text { withdrawm (-) } \\
\text { (mL) }\end{array}$ & $\begin{array}{l}\text { Zone } \\
\text { Length } \\
\text { (cm) }\end{array}$ & $\begin{array}{c}\text { Zone } \\
\text { Radius } \\
\text { (cm) }\end{array}$ & $\begin{array}{c}\text { Tool } \\
\text { Volume } \\
\text { (mL) }\end{array}$ & $\begin{array}{l}\text { Zone Fluid } \\
\text { Volume } \\
\text { (mL) }\end{array}$ \\
\hline C1X05-A & $T Z$ & $9-17-92$ & CPW $\# 5$ & MB139 & 9.3 & 9.5 & -73 & 95 & 3.81 & 2270 & 2816 \\
\hline$C_{1} \times 05-A$ & $T Z$ & $11-17-92$ & $\mathrm{CPI} \# 4$ & MB139 & 9.4 & 10.3 & +6753 & 95 & 3.81 & 2270 & 2816 \\
\hline C1X05-A & $T Z$ & $12-1-92$ & CPI \#5 & MB139 & 10.4 & 11.5 & +21011 & 95 & 3.81 & 2270 & 2816 \\
\hline CiX05-A & $T Z$ & $1-13-93$ & CPW\#S & MB139 & 9.1 & 8.1 & -37 & 95 & 3.81 & 2270 & 2816 \\
\hline C1X05-A & TZ & 1-18-93 & CPW\#7 & MB139 & 9.1 & 8.1 & -112 & 95 & 3.81 & 2270 & 2816 \\
\hline C1X05-B & TZ2 & $10-21.93$ & CPI\#1 & MB140 & 9.4 & 10.6 & +11 & 94 & 3.81 & 2539 & 2771 \\
\hline C1X05-B & $T Z 2$ & $11-22-93$ & CPW\#1 & MB140 & 10.6 & 10.7 & -501 & 94 & 3.81 & 2539 & 2771 \\
\hline C1X05-B & $T Z 2$ & $11-22-93$ & CPW\#2 & MB140 & 12.5 & 10.0 & -4807 & 94 & 3.81 & 2539 & 2771 \\
\hline C1X05-B & $T Z 2$ & $11-22-93$ & CPW\#3 & MB140 & 20.1 & 12.2 & -1340 & 94 & 3.81 & 2539 & 2771 \\
\hline C1X05-B & $T Z 2$ & $11-23-93$ & CPW\#4 & MB140 & 14.0 & 12.2 & N/A & 94 & 3.81 & 2539 & 2771 \\
\hline C1X05-B & $T Z 2$ & $11-23-93$ & CPW\#5 & MB140 & 12.2 & 10.1 & $\sim 13210$ & 94 & 3.81 & 2539 & 2771 \\
\hline C1X05-B & TZ2 & $1-10-94$ & $\mathrm{CPI} \# 2$ & MB140 & 11.7 & 12.2 & +1085 & 94 & 3.81 & 2539 & 2771 \\
\hline C1X05-B & TZ2 & $1-20-94$ & $\mathrm{CPI} \# 3$ & MB140 & 11.9 & 12.7 & +27442 & 94 & 3.81 & 2539 & 2771 \\
\hline C1X05-8 & TZ2 & $2-15-94$ & CPW\#6 & MB140 & 11.7 & 11.2 & -3460 & 94 & 3.81 & 2539 & 2771 \\
\hline C1X05-8 & $T Z 2$ & $3-24-94$ & PW & MB140 & 11.8 & 0.2 & -254 & 94 & 3.81 & 2539 & 2771 \\
\hline $\begin{array}{c}\text { C1X05-B } \\
{ }^{*}(\mathrm{C} 1 \times 06-B)\end{array}$ & TZ1 & $4-8-94$ & PW & MB140 & 11.7 & 7.70 & -113 & 353 & 5.08 & 20376 & 8661 \\
\hline
\end{tabular}

\subsubsection{Borehole C1X10, Testing Sequence C1X10}

Testing sequence $\mathrm{C} 1 \times 10$ took place in Room $\mathrm{C} 1$ in borehole $\mathrm{C} 1 \times 10$ with associated observation boreholes $\mathrm{C} 1 \mathrm{H} 05, \mathrm{C} 1 \mathrm{HO6}, \mathrm{DPD} 02, \mathrm{DPD03}$, and $\mathrm{C} 2 \mathrm{H} 01$. This test sequence was designed to investigate the pre-and post-hydrofracture brine permeability of MB139. Table 4-4 gives a detailed description of the events that occurred during the testing sequence $\mathrm{C} 1 \mathrm{X} 10$ in borehole $\mathrm{C} 1 \mathrm{X} 10$.

Table 4-4. Events Associated with Test Borehole C1X10 During Testing Sequence C1X10

\begin{tabular}{|c|c|c|c|c|}
\hline EVENT & DATE & $\begin{array}{c}\text { CALENDAR } \\
\text { DAY }\end{array}$ & $\begin{array}{c}1991 \\
\text { CALENDAR } \\
\text { DAY }\end{array}$ & $\begin{array}{c}\text { TIME } \\
\text { (HH:MM:SS) }\end{array}$ \\
\hline Begin drilling borehole C1X10 with 3-inch $(7.62-\mathrm{cm})$ core barrel to 1.5 meters. & 8-16-91 & 228 & 228 & 10:33:00 \\
\hline Deepen borehole $\mathrm{C} 1 \times 10$ with 3 -inch $(7.62-\mathrm{cm})$ core barrel to 2.03 meters. & 8-16-91 & 228 & 228 & 11:07:00 \\
\hline Deepen borehole $\mathrm{C} 1 \mathrm{X} 10$ with 3-inch $(7.62-\mathrm{cm})$ core barrel to 2.87 meters. & $8-16-91$ & 228 & 228 & 12:45:00 \\
\hline Deepen borehole $\mathrm{C} 1 \mathrm{X} 10$ with 3 -inch $(7.62-\mathrm{cm})$ core barrel to 3.68 meters. & $8-16-91$ & 228 & 228 & 13:30:00 \\
\hline Deepen borehole $\mathrm{C} 1 \mathrm{X} 10$ with 3 -inch $(7.62-\mathrm{cm})$ core barrel to 4.55 meters. & 8-16-91 & 228 & 228 & 14:02:00 \\
\hline
\end{tabular}


Table 4-4 (Continued). Events Associated with Test Borehole C1X10 During Testing Sequence $\mathrm{C} 1 \times 10$

\begin{tabular}{|c|c|c|c|c|}
\hline EVENT & DATE & $\begin{array}{l}\text { CALENDAR } \\
\text { DAY }\end{array}$ & $\begin{array}{c}1991 \\
\text { CALENDAR } \\
\text { DAY }\end{array}$ & $\begin{array}{c}\text { TIME } \\
\text { (HH:MM:SS) }\end{array}$ \\
\hline Deepen borehole C1X10 with 3 -inch $(7.62-\mathrm{cm})$ core barrel to 5.46 meters. & $11-11-91$ & 315 & 315 & 10:18:00 \\
\hline Deepen borehole C1X10 with 3-inch $(7.62-\mathrm{cm})$ core barrel to 6.10 meters. & $11-11-91$ & 315 & 315 & 11:05:00 \\
\hline Deepen borehole $\mathrm{C} 1 \mathrm{X} 10$ with $3-$ inch $(7.62-\mathrm{cm})$ core barrel to 6.67 meters. & $11-11-91$ & 315 & 315 & $13: 16: 00$ \\
\hline Deepen borehole $\mathrm{C} 1 \times 10$ with 3 -inch $(7.62-\mathrm{cm})$ core barrel to 7.56 meters. & $11-11-91$ & 315 & 315 & $13: 35: 00$ \\
\hline Deepen borehole $\mathrm{C} 1 \mathrm{X} 10$ with 3-inch $(7.62-\mathrm{cm})$ core barrel to 8.44 meters. & $11-11-91$ & 315 & 315 & 13:57:00 \\
\hline Begin data file C1X1001. & $19-12-91$ & 316 & 316 & $09: 41: 32$ \\
\hline Deepen borehole $\mathrm{C} 1 \times 10$ with 3 -inch $(7.62-\mathrm{cm})$ core barrel to 9.30 meters. & $11-12-91$ & 316 & 316 & 10:15:00 \\
\hline Deepen borehole C1X10 with 3-inch $(7.62-\mathrm{cm})$ core barrel to 10.16 meters. & 11-12-91 & 316 & 396 & 12:15:00 \\
\hline Perform a video log of borehole C1X10. & $11-12-91$ & 316 & 316 & $14: 00: 00$ \\
\hline $\begin{array}{c}\text { Pour } 9 \text { liters of hydrofracture oil into borehole } \mathrm{C} 1 \times 10 \text { and install volume- } \\
\text { displacement device. }\end{array}$ & 11-12-91 & 316 & 316 & 15:00:00 \\
\hline $\begin{array}{l}\text { Install multipacker test tool into borehole } \mathrm{C} 1 \times 10 \text { as indieated in the test-tool } \\
\text { configuration diagram (Figure 4-8). }\end{array}$ & $11-12-91$ & 316 & 316 & $15: 30: 00$ \\
\hline Inflate TZP and GZP to $-12 \mathrm{MPa}$. & $11-12-91$ & 316 & 316 & 16:52:00 \\
\hline Shut in both zones. & $11-12-91$ & 316 & 316 & 17:00:00 \\
\hline End data file C1X1001. & $11-15-91$ & 319 & 319 & 11:34:42 \\
\hline Begin data file C1X1002. & $11-15-91$ & 319 & 319 & 12:35:13 \\
\hline $\begin{array}{l}\text { Begin constant-pressure-injection test \#1 in TZ at } \sim 1 \mathrm{MPa} \text { above } T Z \text { pressure } \\
\qquad(-6.5 \mathrm{MPa}) .\end{array}$ & $11-15-91$ & 319 & 319 & 13:44:00 \\
\hline Leaky fitting on $\pi z$. & $11-15-91$ & 319 & 319 & 13:46:00 \\
\hline Shut in $T Z$ from DPT panel terminating constant-pressure-injection test \#1 in $T Z$. & $19-15-91$ & 319 & 319 & 14:15:30 \\
\hline No power to DAS upon armval. & $11-18-91$ & 322 & 322 & 09:14:00 \\
\hline $\begin{array}{l}\text { Begin constant-pressure-injection test } \# 2 \text { in } T Z \text { at }-0.3 \mathrm{MPa} \text { above } T Z \text { pressure } \\
\qquad(-6.4 \mathrm{MPa}) .\end{array}$ & 11-19-91 & 323 & 323 & 10:56:30 \\
\hline Shut in TZ from DPT panel teminsting constant-pressure-injection test \#2 in TZ. & $11-19-91$ & 323 & 323 & $11: 47: 48$ \\
\hline $\begin{array}{l}\text { Begin constant-pressure-injection test } \$ 3 \mathrm{in} \mathrm{TZ} \text { at }-2.5 \mathrm{MPa} \text { above } \mathrm{TZ} \text { pressure } \\
\qquad(-9 \mathrm{MPa}) .\end{array}$ & $11-26-91$ & 330 & 330 & $08: 46: 40$ \\
\hline End data file $\mathrm{C} 1 \times 1002$ & $11-26-91$ & 330 & 330 & 14:46:08 \\
\hline Begin data file C1X1003. & $11-26-91$ & 330 & 330 & 14:54:00 \\
\hline Shut in TZ from DPT panel terminating constant-pressure-injection test \#3 in $T Z$. & $11-26-91$ & 330 & 330 & 14:54:40 \\
\hline Performed hydrofracture of MB139. & 12-5-91 & 339 & 339 & 12:27:00 \\
\hline Begin constant pressure withdrawal test \#1 in $7 \mathrm{Z}$ at $\sim 7.5 \mathrm{MPa}$. & $12-5-91$ & 339 & 339 & 14:32:20 \\
\hline Shut in $T Z$ from OPT panel terminating constant-pressure-withdrawal test $\# 1$ in $T Z$. & $12-6-91$ & 340 & 340 & 09:20:00 \\
\hline Begin constant-pressure-withdrawal test \#2 in $\pi$ at $\sim 7.6 \mathrm{MPa}$. & $12-6-91$ & 340 & 340 & 09:24:10 \\
\hline DAS not functioning property upon arival. & 12-9-91 & 343 & 343 & 10:40:00 \\
\hline Shut in $T Z$ from DPT panel terminating constant-pressure-withdrawal test \#2 in $T Z$. & $12-11-91$ & 345 & 345 & 10:47:00 \\
\hline Begin constant-pressure-withdrawal test 3 in $\mathrm{TZ}$ at $-7.4 \mathrm{MPa}$. & 12-11-91 & 345 & 345 & 11:03:20 \\
\hline Shut in $Z Z$ from DPT panel terminating constant-pressure-withdrawal test \#4 in TZ. & $12-13-91$ & 347 & 347 & $08: 49: 36$ \\
\hline
\end{tabular}


Table 4-4 (Continued). Events Associated with Test Borehole C1X10 During Testing Sequence $\mathrm{C} 1 \mathrm{X} 10$

\begin{tabular}{|c|c|c|c|c|}
\hline EVENT & DATE & $\begin{array}{c}\text { CALENDAR } \\
\text { DAY }\end{array}$ & $\begin{array}{c}1991 \\
\text { CALENDAR } \\
\text { DAY }\end{array}$ & $\begin{array}{c}\text { TIME } \\
\text { (HH:MM:SS) }\end{array}$ \\
\hline End data file Cix1003. & $1-17-92$ & 17 & 382 & 13:59:11 \\
\hline Begin monitoring fluid pressure in MB139 in borehole C1H05. & 1-17-92 & 17 & 382 & 14:00:00 \\
\hline Begin data file C $1 \times 1004$. & $1-17-92$ & 17 & 382 & 14:14:30 \\
\hline Begin monitoring fluid pressure in MB139 in borehole C1H06. & $1-22-92$ & 22 & 387 & 14:00:00 \\
\hline Begin monitoring fluld pressure in $\mathrm{MB139}$ in boreholes DPD02, DPD03, and C2H01. & 1-29-92 & 29 & 394 & 14:45:00 \\
\hline End data file $\mathrm{C} 1 \times 1004$. & 1-30-92 & 30 & 395 & 08:55:41 \\
\hline Begin data file $\mathrm{C} 1 \times 1005$. & 1-30-92 & 30 & 395 & 13:23:46 \\
\hline $\begin{array}{l}\text { Begin constant-pressure-injection test \#4 in TZ at } \sim 1.5 \mathrm{MPa} \text { above } \mathrm{TZ} \text { pressure } \\
\qquad(\sim 9 \mathrm{MPa}) .\end{array}$ & 2-6-92 & 37 & 402 & 10:48:17 \\
\hline End data file C1X1005. & $2-6-92$ & 37 & 402 & 14:46:29 \\
\hline Begin data file $C 1 \times 1006$ & $2-6-92$ & 37 & 402 & $14: 51: 00$ \\
\hline Shut in $T Z$ from DPT panel terminating constant-pressure-injection test \#4 in $T Z$ & 2-6-92 & 37 & 402 & 14:51:40 \\
\hline End data file $C 1 \times 1005$ & $3-18-92$ & 78 & 443 & $07: 37: 47$ \\
\hline Begin data file C1X1007. & $3-18-92$ & 78 & 443 & 08:19:00 \\
\hline $\begin{array}{l}\text { Begin constant-pressure-withdrawal test \#4 in TZ at }-1.5 \mathrm{MPa} \text { below } T Z \text { pressure } \\
\qquad(-6.4 \mathrm{MPa}) .\end{array}$ & 3-18-92 & 78 & 443 & 08:32:30 \\
\hline Shut in $T Z$ from DPT panel terminating constant-pressure-withdrawal test \#4 in $T Z$. & 3-18-92 & 78 & 443 & 14:31:09 \\
\hline Decrease TZ pressure (both oil and gas present). & $5-6-92$ & 127 & 492 & 09:36:00 \\
\hline Deflate TZP. & $5-6-92$ & 127 & 492 & 09:54:00 \\
\hline Inflate TZP and shut in TZ. & $5-6-92$ & 127 & 492 & 10:43:00 \\
\hline End data file C1X1007. & $5-7-92$ & 128 & 493 & $07: 48: 42$ \\
\hline Begin data file C1X1008. & $5-7-92$ & 128 & 493 & $10: 03: 45$ \\
\hline Calibrated transducers on P1, P5, and P7. & $5-26-92$ & 147 & 512 & $12: 56: 00$ \\
\hline End data file $C 1 \times 1008$. & $6-26-92$ & 178 & 543 & 08:18:34 \\
\hline
\end{tabular}

Figures 4-18 and 4-19 illustrate the zone pressures and fluid production during constantpressure-flow tests, respectively, in test borehole C1X10. It should be noted that Figure 4-19 (Fluid production during constant-pressure-flow tests during testing sequence $\mathrm{C} 1 \mathrm{X} 10$ ) consists of three parts (Figures 4-19a, 4-19b, and 4-19c). Copies of the video-log associated with testing sequence $\mathrm{C} 1 \times 10$ (borehole $\mathrm{C} 1 \times 10$ ) identified in Table 4-4 are provided in the SWCF under WPO \#45907

Table 4-5 indicates the equipment that was used and the duration that each instrument was used during testing sequence $\mathrm{C} 1 \mathrm{X} 10$ in test borehole $\mathrm{C} 1 \mathrm{X} 10$ and in the observation boreholes. 
Table 4-5. Testing Sequence C1X10 Equipment

\begin{tabular}{|c|c|c|c|c|}
\hline Equipment & Location & Serial \# & Installed & Removed \\
\hline DAS Software & N/A & PERM4F & $11-12-91$ & $6-26-92$ \\
\hline DCU (HP3497A) & N/A & $2629 a 21989$ & $11-12-91$ & $4-13-92$ \\
\hline DCU (HP3497A) & N/A & $2629 a 21990$ & $4-13-92$ & $6-26-92$ \\
\hline $\begin{array}{c}\text { Transducer } \\
\text { (Druck PDCR 830) }\end{array}$ & C1X10 TZ & 246909 & $11-12-91$ & $6-26-92$ \\
\hline $\begin{array}{c}\text { Transducer } \\
\text { (Druck PDCR 830) }\end{array}$ & DPT Panel & 246910 & $11-12-91$ & $5-7-92$ \\
\hline $\begin{array}{c}\text { Transducer } \\
\text { (Druck PDCR 830) }\end{array}$ & C1H05 TZ & 246917 & $1-17-92$ & $6-26-92$ \\
\hline $\begin{array}{c}\text { Transducer } \\
\text { (Druck PDCR 830) }\end{array}$ & C1H05 TZP & 246919 & $1-17-92$ & $6-26-92$ \\
\hline $\begin{array}{c}\text { Transducer } \\
\text { (Druck PDCR 830) }\end{array}$ & C1H06 TZ & 246916 & $1-17-92$ & $6-26-92$ \\
\hline $\begin{array}{c}\text { Transducer } \\
\text { (Druck PDCR 830) }\end{array}$ & C1H06 TZP & 246918 & $1-17-92$ & $6-26-92$ \\
\hline $\begin{array}{c}\text { Transducer } \\
\text { (Druck PDCR 10/D) }\end{array}$ & DPD03 TZ & 211694 & $1-30-92$ & $5-7-92$ \\
\hline $\begin{array}{c}\text { Transducer } \\
\text { (Druck PDCR 910) }\end{array}$ & DPD02 TZ & 316158 & $1-30-92$ & $5-7-92$ \\
\hline $\begin{array}{c}\text { Transducer } \\
\text { (Druck PDCR 910) }\end{array}$ & $\mathrm{C} 2 \mathrm{H} 01 \mathrm{TZ}$ & 321768 & $5-7-92$ & $6-26-92$ \\
\hline Injection Column & N/A & 76 & $11-15-91$ & $6-26-92$ \\
\hline Injection Column & N/A & 77 & $11-26-91$ & $6-26-92$ \\
\hline $\begin{array}{c}\text { DPT } \\
\text { (Rosemount 1151DP) }\end{array}$ & N/A & 1140863 & $11-15-91$ & $6-26-92$ \\
\hline
\end{tabular}

* Installed dates for injection columns refer to dates of initial use rather than date installed. 


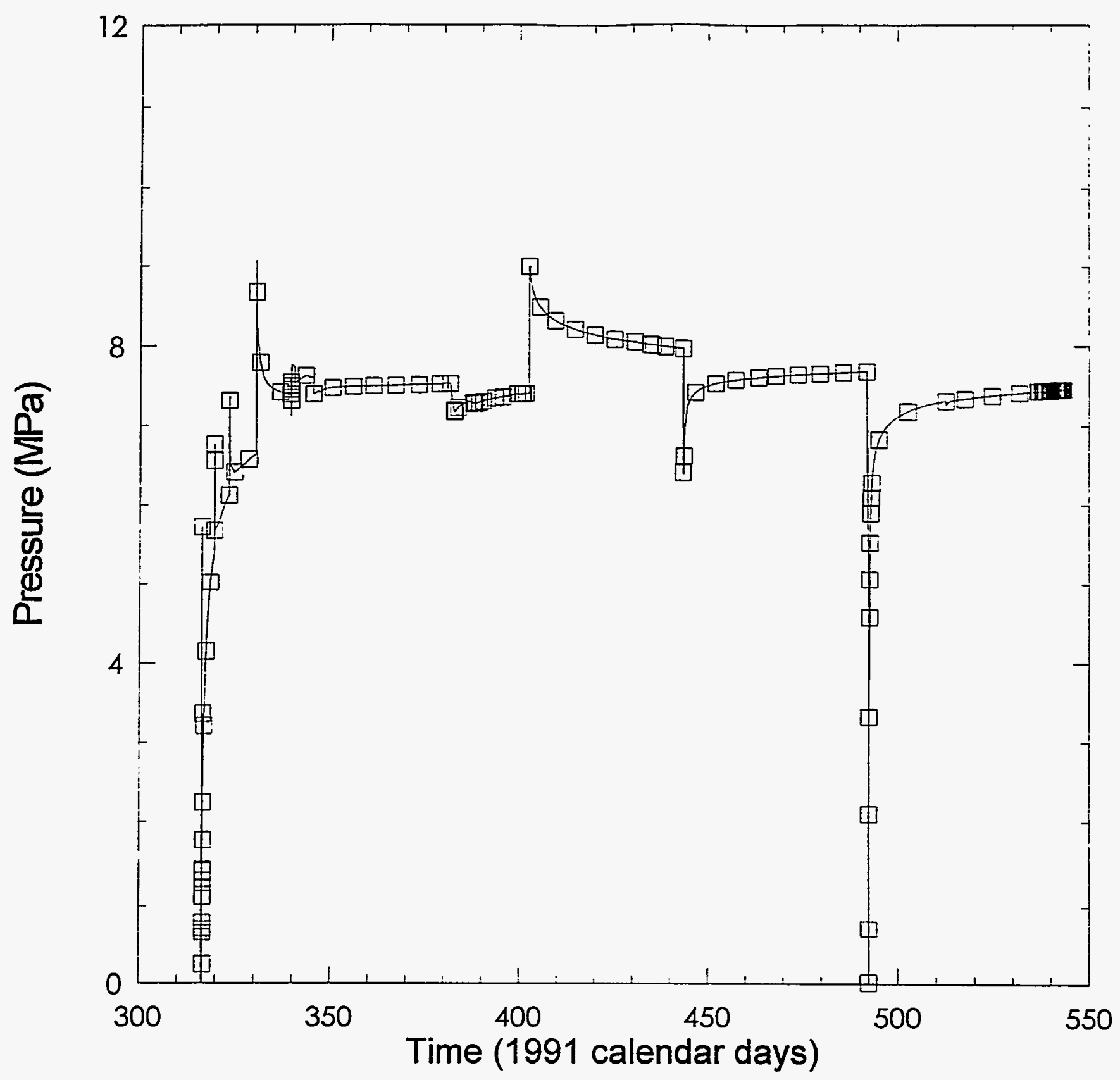

INTERA-6115-910-0

Figure 4-18. Test-zone pressure in test borehole $\mathrm{C} 1 \mathrm{X} 10$ during testing sequence $\mathrm{C} 1 \mathrm{X} 10$. 


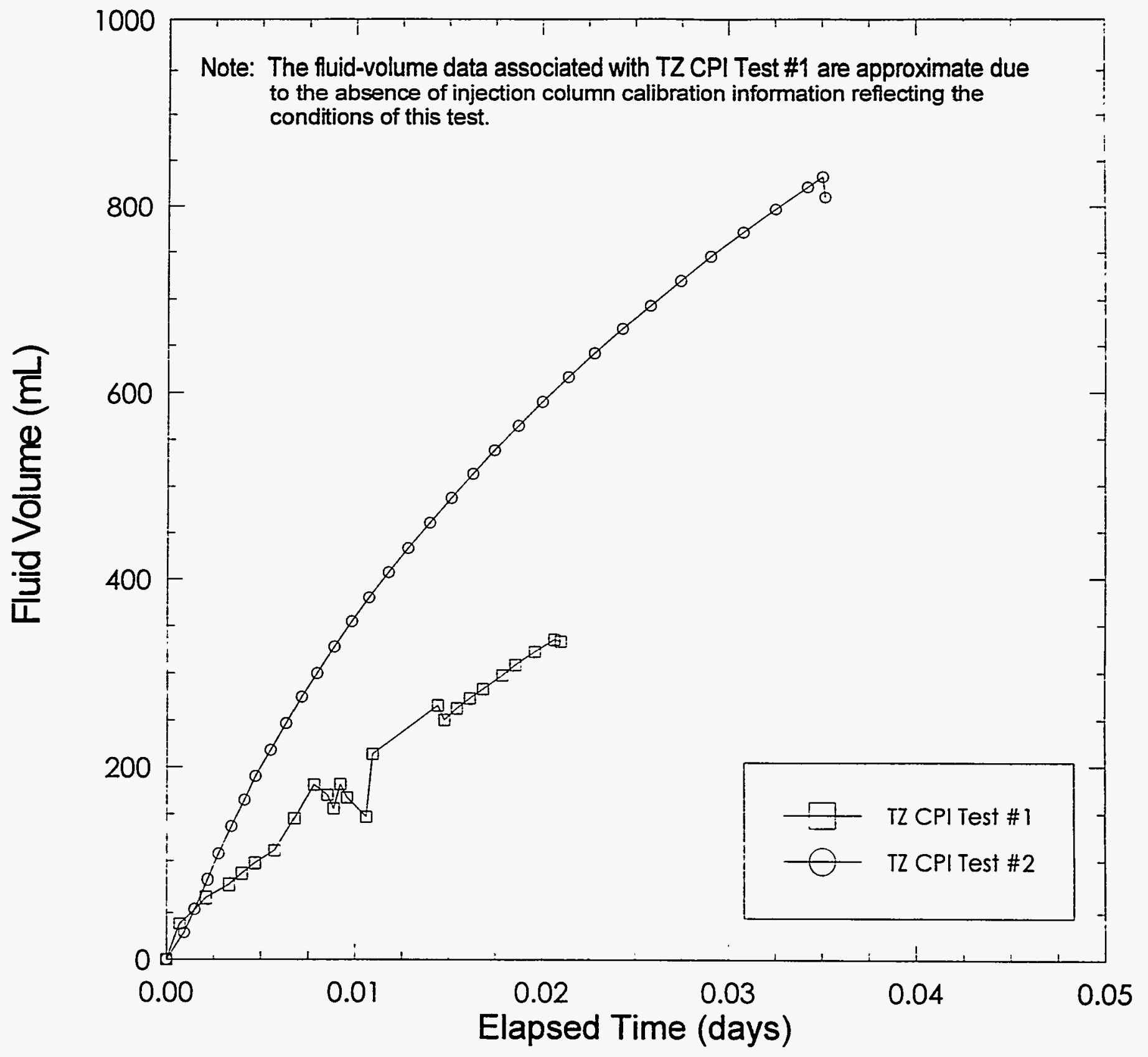

INTERA-S195-11200

Figure 4-19a. Fluid production during constant-pressure-flow tests during testing sequence $\mathrm{C} 1 \mathrm{X} 10$. 


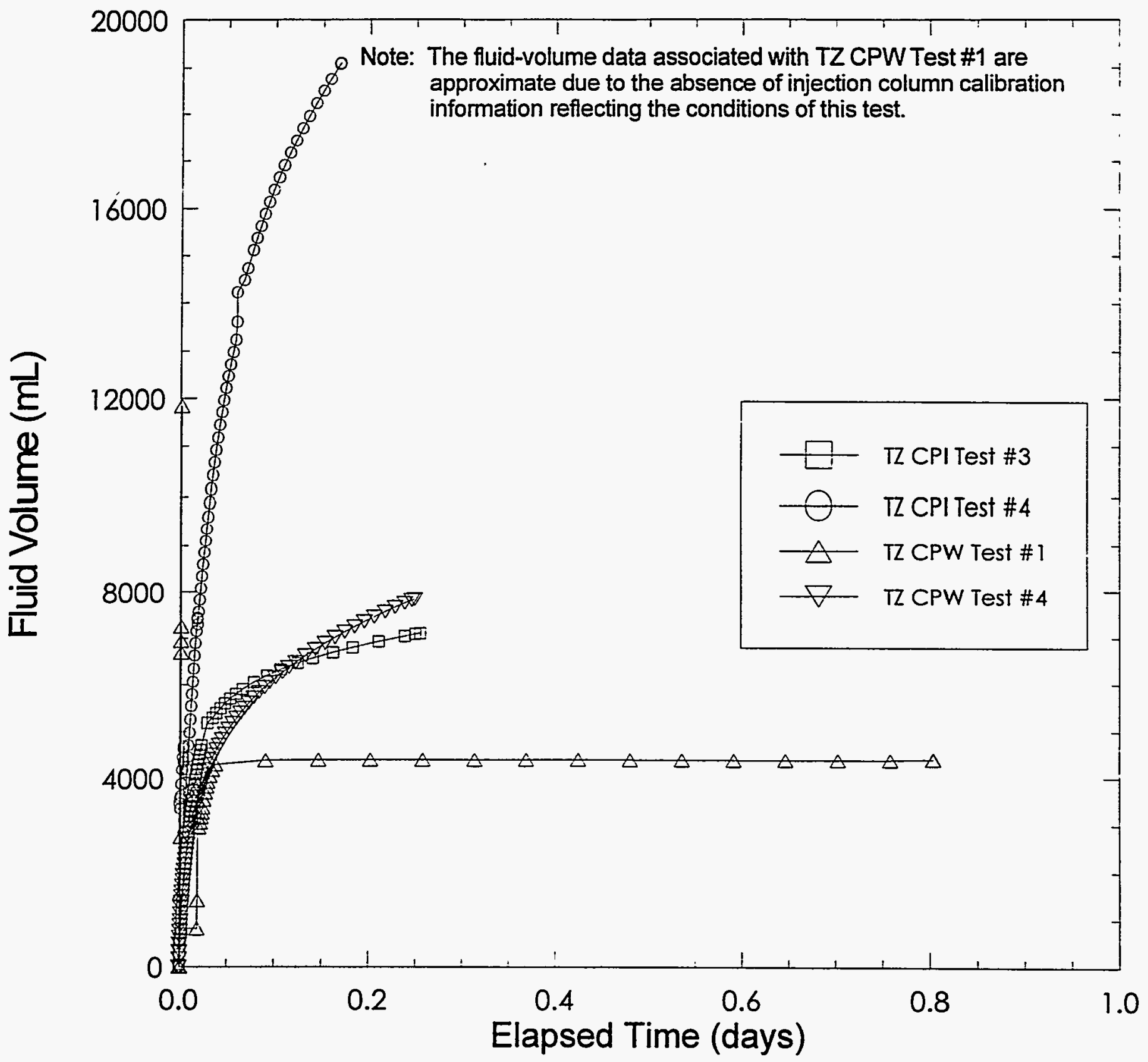

INTERA-6113-113-0

Figure 4-19b. Fluid production during constant-pressure-flow tests during testing sequence $\mathrm{C} 1 \mathrm{X} 10$ (continued). 


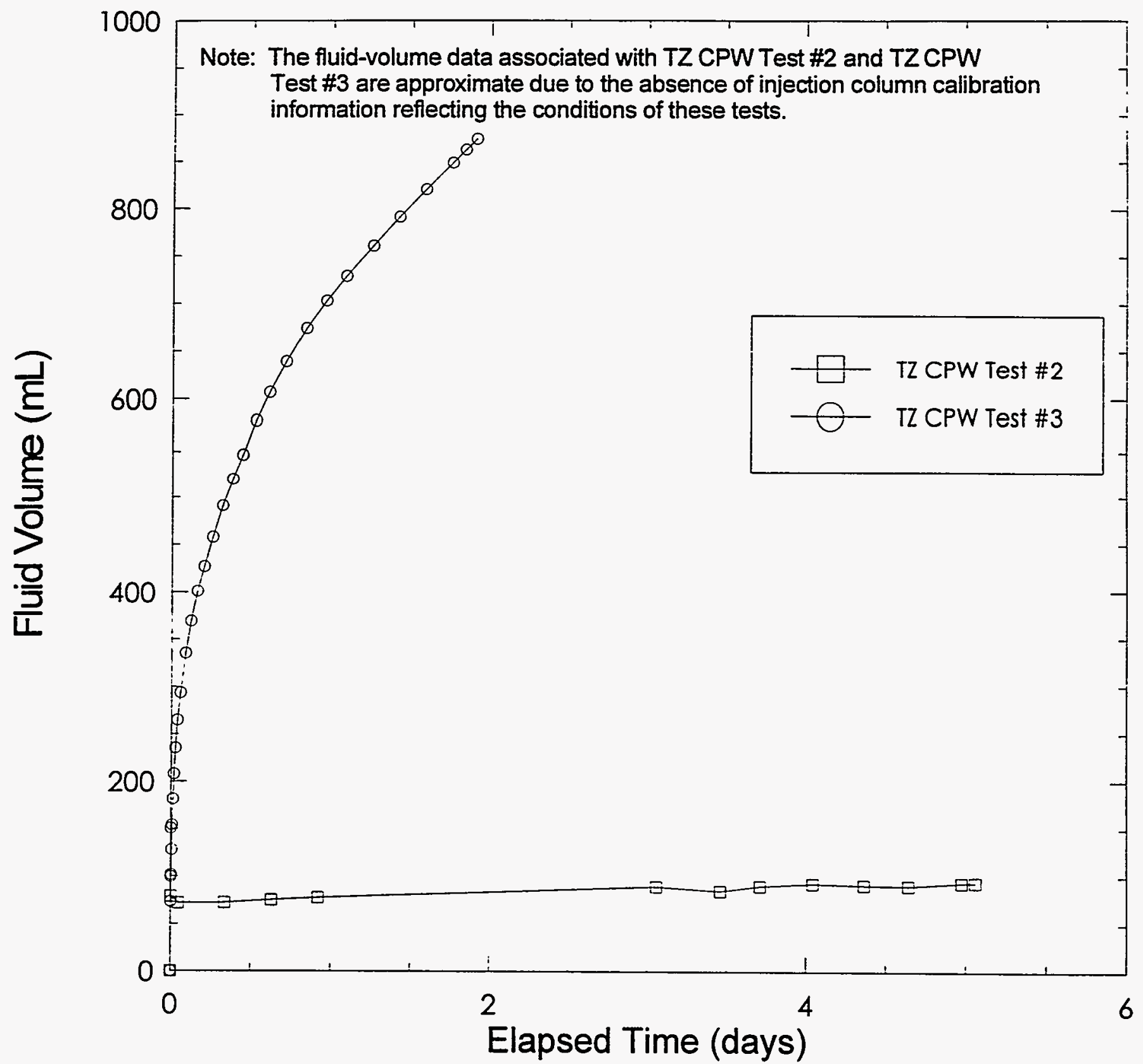

INTERA-6115-1140

Figure 4-19c. Fluid production during constant-pressure-flow tests during testing sequence $C 1 \times 10$ (continued). 


\subsubsection{OBSERVATION BOREHOLE C1H05, TESTING SEQUENCE C1X10}

Table 4-16 gives a detailed description of the events that occurred in observation borehole $\mathrm{C} 1 \mathrm{H} 05$ during testing sequence $\mathrm{C} 1 \mathrm{X} 10$.

Table 4-6. Events Associated with Observation Borehole C1H05 During Testing Sequence $\mathrm{C} 1 \times 10$

\begin{tabular}{|c|c|c|c|c|}
\hline EVENT & DATE & $\begin{array}{l}\text { CALENDAR } \\
\text { DAY }\end{array}$ & $\begin{array}{c}1991 \\
\text { CALENDAR } \\
\text { DAY }\end{array}$ & $\begin{array}{c}\text { TIME } \\
\text { (HH:MM:SS) }\end{array}$ \\
\hline Begin drilling borehole $\mathrm{C} 1 \mathrm{H} 05$ with 4 -inch $(10.16-\mathrm{cm})$ plug bit to 6.350 meters. & 1-15-92 & 15 & 380 & 10:15:00 \\
\hline Deepen borehole $\mathrm{C} 1 \mathrm{H} 05$ with 4 -inch $(10.16-\mathrm{cm})$ core barrel to 6.528 meters. & $1-16-92$ & 16 & 381 & 13:15:00 \\
\hline Deepen borehole $\mathrm{C} 1 \mathrm{H} 05$ with 4 inch $(10.16-\mathrm{cm})$ core barrel to 7.315 meters. & 1-16-92 & 16 & 381 & 13:45:00 \\
\hline $\begin{array}{c}\text { Deepen borehole C1H05 with 4-inch ( } 10.16-\mathrm{cm} \text { ) core barrel to } 8.204 \text { meters (noticed } \\
\text { hydrofracture oll in MB139 core). }\end{array}$ & 1-16-92 & 16 & 381 & 14:20:00 \\
\hline Pertorm video log of borehole $\mathrm{C} 1 \mathrm{H} 05$. & 1-17-92 & 17 & 382 & 10:00:00 \\
\hline Face bottom of borehole $\mathrm{C} 1 \mathrm{H} 05$ with 4 -inch $(10.16-\mathrm{cm})$ plug bit to 8.255 meters. & $1-17-92$ & 17 & 382 & 10:45:00 \\
\hline $\begin{array}{c}\text { Install single-packer monitor tool \#32A in borehole } \mathrm{C} 1 \mathrm{H} 05 \text { as indicated in the test-tool } \\
\text { configuration diagram (Figure 4-9). }\end{array}$ & 1-17-92 & 17 & 382 & 10:00:00 \\
\hline Inflate TZP to - $11 \mathrm{MPa}$. & $1-17-92$ & 17 & 382 & $14: 29: 00$ \\
\hline Shut in TZ. & $1-17-92$ & 17 & 382 & $14: 40: 30$ \\
\hline Terminate data collection. & $6-26-92$ & 178 & 543 & $08: 18: 34$ \\
\hline
\end{tabular}

Figures 4-20 and 4-21 illustrate the zone pressures and packer pressures, respectively, in observation borehole $\mathrm{C} 1 \mathrm{H} 05$ for testing sequence $\mathrm{C} 1 \mathrm{X} 10$. Copies of the video-log associated with testing sequence $\mathrm{C} 1 \mathrm{X} 10$ (borehole $\mathrm{C} 1 \mathrm{H} 05$ ) identified in Table 4-6 are provided in the SWCF under WPO \#45907 


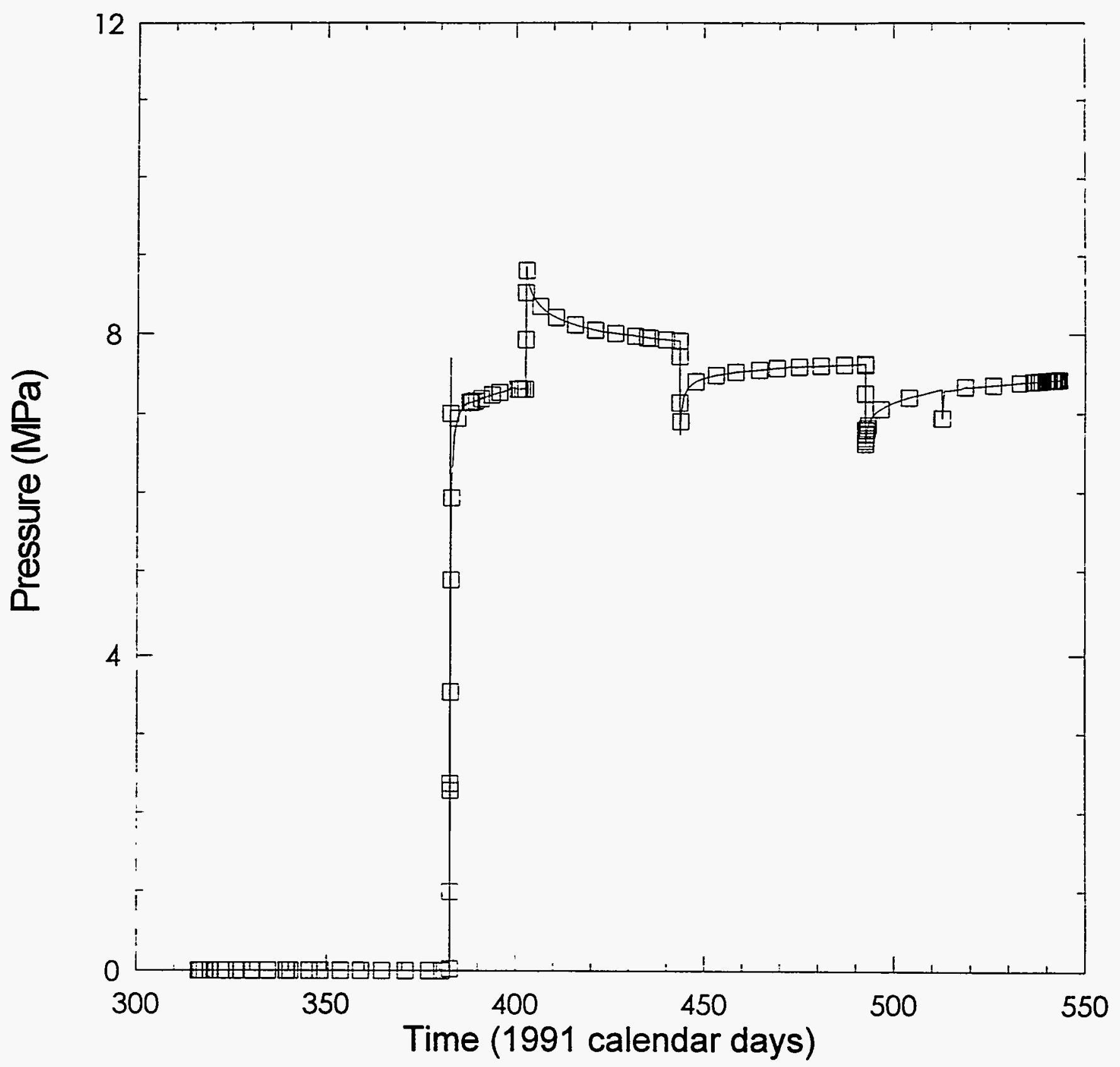

INTERAGI15-116-0

Figure 4-20. Test-zone pressure in observation borehole $\mathrm{C} 1 \mathrm{H} 05$ during testing sequence $\mathrm{C} 1 \mathrm{X} 10$. 


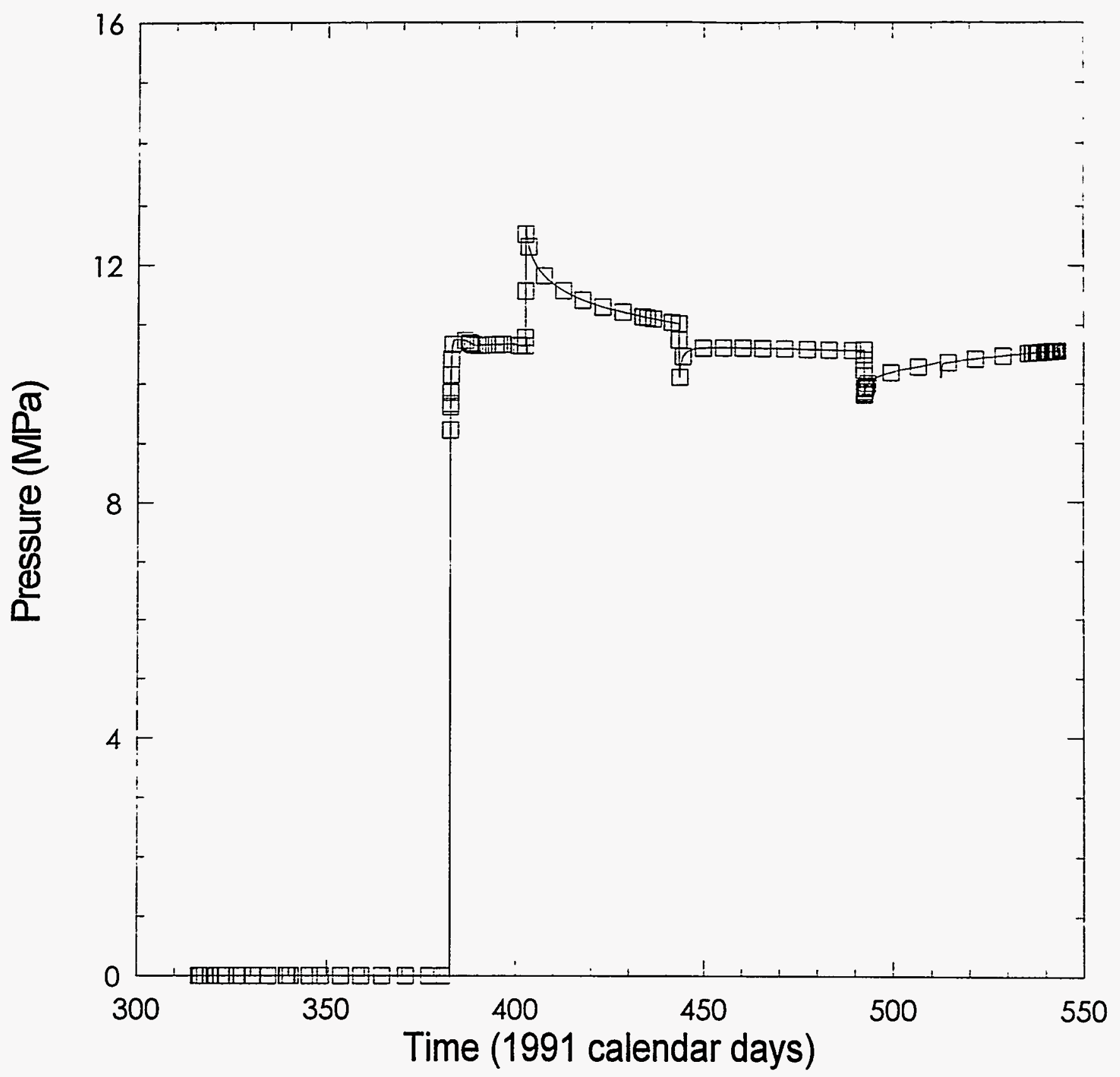

INTERA-6115-137-0

Figure 4-21. Test-zone packer pressure in observation borehole $\mathrm{C} 1 \mathrm{H} 05$ during testing sequence $\mathrm{C} 1 \mathrm{X} 10$. 
4.6.1.2 OBSERVATION BOREHOLE C1H06, TESTING SEQUENCE C1X10

Table 4-7 gives a detailed description of the events that occurred in observation borehole C1H06 during testing sequence $\mathrm{C} 1 \mathrm{X} 10$.

Table 4-7. Events Associated with Observation Borehole C1H06 During Testing Sequence $\mathrm{C} 1 \mathrm{X} 10$

\begin{tabular}{|c|c|c|c|c|}
\hline EVENT & DATE & $\begin{array}{c}\text { CALENDAR } \\
\text { DAY }\end{array}$ & $\begin{array}{c}1991 \\
\text { CALENDAR } \\
\text { DAY }\end{array}$ & $\begin{array}{c}\text { TIME } \\
\text { (HH:MM:SS) }\end{array}$ \\
\hline Begin drilting borehole $\mathrm{C} 1 \mathrm{H} 06$ with 4 -inch $(10.16-\mathrm{cm})$ plug bit to -6.4 meters. & 1-20.92 & 20 & 385 & 14:30:00 \\
\hline Deepen borehole C1HO6 with 4 inch $(10.16-\mathrm{cm})$ core barrel to 7.040 meters. & $1-21-92$ & 21 & 386 & 10:48:00 \\
\hline Deepen borehole C1H0S with 4-inch (10.16-cm) core barrel to 7.874 meters. & $1-21-92$ & 21 & 386 & 13:00:00 \\
\hline Deepen borehole C1H06 with 4-inch $(10.16-\mathrm{cm})$ core barrel to 8.611 meters. & $1-21-92$ & 21 & 386 & 13:29:00 \\
\hline Deepen borehole C1H06 with 4-inch (10.16-an) core barrel to 9.347 meters. & $1-21-92$ & 21 & 386 & 14:09:00 \\
\hline Face bottom of borehole C1H05 with 4 -inch $(10.16-\mathrm{cm})$ plug bit to 9.398 meters. & $1-21-92$ & 21 & 386 & 15:30:00 \\
\hline Perform video $\log$ of borehole CiHOs. & $9-22-92$ & 22 & 387 & $11: 30: 00$ \\
\hline $\begin{array}{l}\text { Install single-packer monitor tool \#32B in borehole C1HOS as indicated in the test-tool } \\
\text { configuration diagram (Figure } 4-10 \text { ). }\end{array}$ & $1-22-92$ & 22 & 387 & $10: 00: 00$ \\
\hline Inflate TZP to $-10 \mathrm{MPa}$ & $1-22-92$ & 22 & 387 & $14: 04: 00$ \\
\hline Shut in TZ. & $1-22-92$ & 22 & 387 & $14: 17: 00$ \\
\hline Terminate data collection. & $6-26-92$ & 178 & 543 & 08:18:34 \\
\hline
\end{tabular}

Figures 4-22 and 4-23 illustrate the zone pressures and packer pressures, respectively, in observation borehole $\mathrm{C} 1 \mathrm{H} 06$ for testing sequence $\mathrm{C} 1 \times 10$. Copies of the video-log associated with testing sequence $\mathrm{C} 1 \mathrm{X} 10$ (borehole $\mathrm{C} 1 \mathrm{H} 06$ ) identified in Table 4-7 are provided in the SWCF under WPO \#45907 


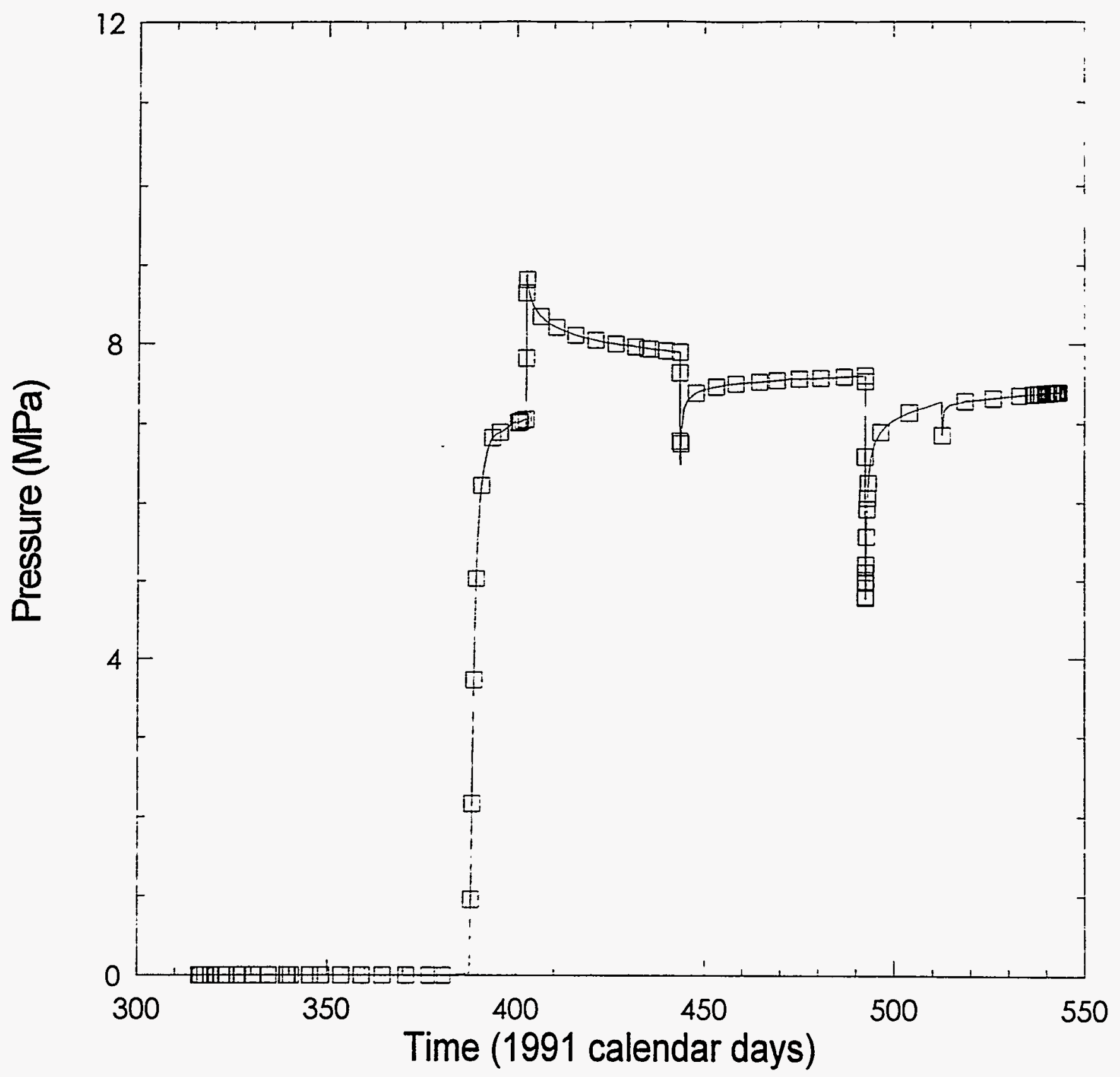

INTERA-6115-118-0

Figure 4-22. Test-zone pressure in observation borehole $\mathrm{C} 1 \mathrm{H} 06$ during testing sequence $\mathrm{C} 1 \times 10$. 


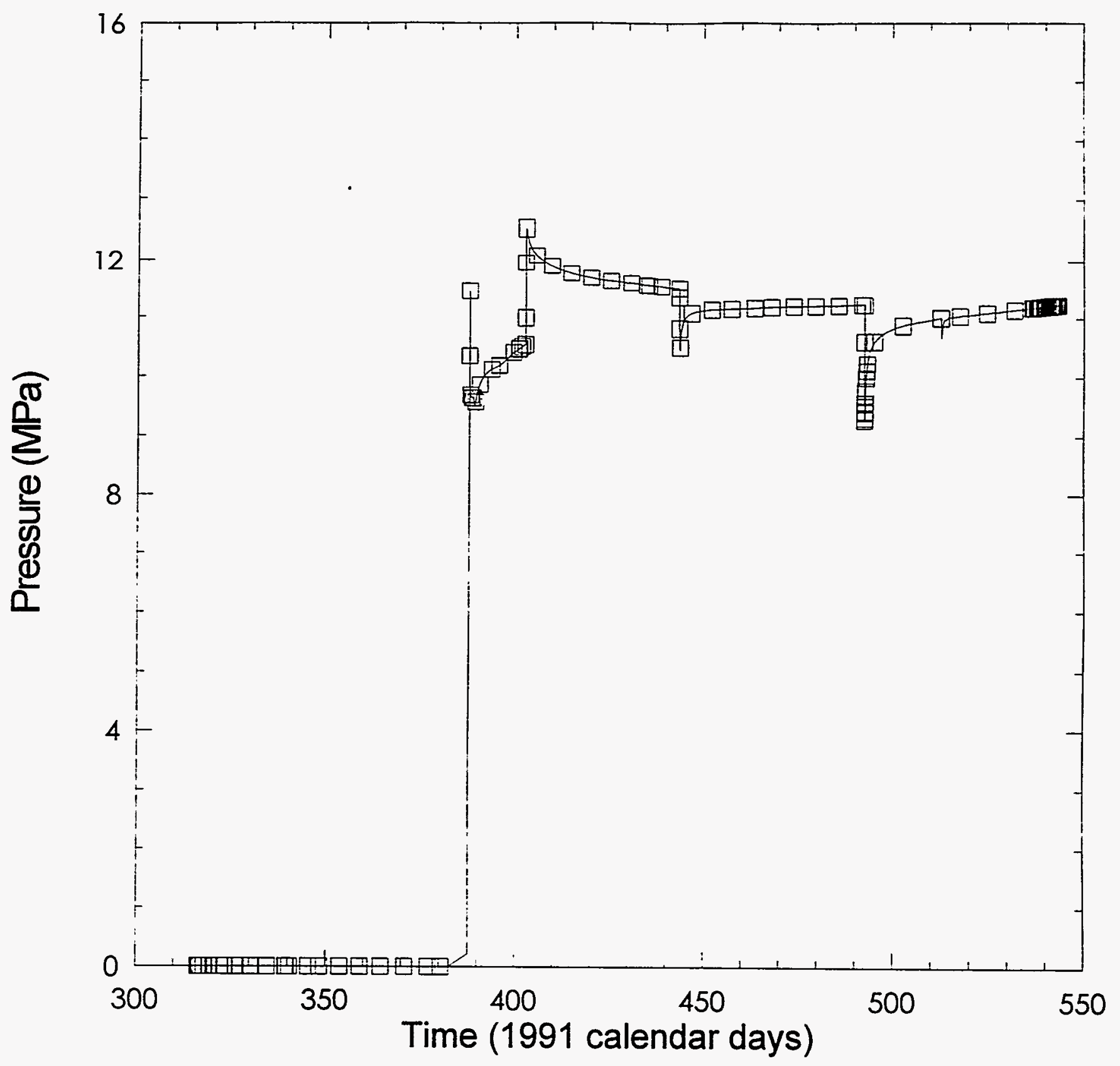

INTERAB115-1190

Figure 4-23. Test-zone packer pressure in observation borehole $\mathrm{C} 1 \mathrm{H} 06$ during testing sequence $\mathrm{C} 1 \mathrm{X} 10$. 


\subsubsection{OBSERVATION BOREHOLE DPD02, TESTING SEQUENCE C1X10}

Table 4-8 gives a detailed description of the events that occurred in observation borehole DPD02 during testing sequence $\mathrm{C} 1 \times 10$.

Table 4-8. Events Associated with Observation Borehole DPD02 During Testing Sequence $\mathrm{C} 1 \mathrm{X} 10$

\begin{tabular}{|c|c|c|c|c|}
\hline EVENT & DATE & $\begin{array}{l}\text { CALENDAR } \\
\text { DAY }\end{array}$ & $\begin{array}{c}1991 \\
\text { CALENDAR } \\
\text { DAY }\end{array}$ & $\begin{array}{c}\text { TIME } \\
\text { (HH:MM:SS) }\end{array}$ \\
\hline Borehole DPDO2 drilled to 13.11 meters. & $4-86$ & N/A & N/A & N/A \\
\hline Begin long-term fluid-pressure monitoring of MB139. & $5-3-89$ & 123 & N/A & NA \\
\hline $\begin{array}{l}\text { Install single-packer fluid-pressure monitoring tool as indicated in the test-tool } \\
\text { configuration diagram (Figure 7-20). }\end{array}$ & $12-4-91$ & 338 & 338 & N/A \\
\hline Begin nuid-pressure monitoring of MB139 associated with testing sequence $C 1 \times 10$. & 1-30-92 & 29 & 394 & 14:43:00 \\
\hline Terminate data collection. & $5-7.92$ & 128 & 493 & 14:02:00 \\
\hline
\end{tabular}

Figure 4-24 illustrates the zone pressure in observation borehole DPD02 for testing sequence $\mathrm{C} 1 \mathrm{X} 10$. 


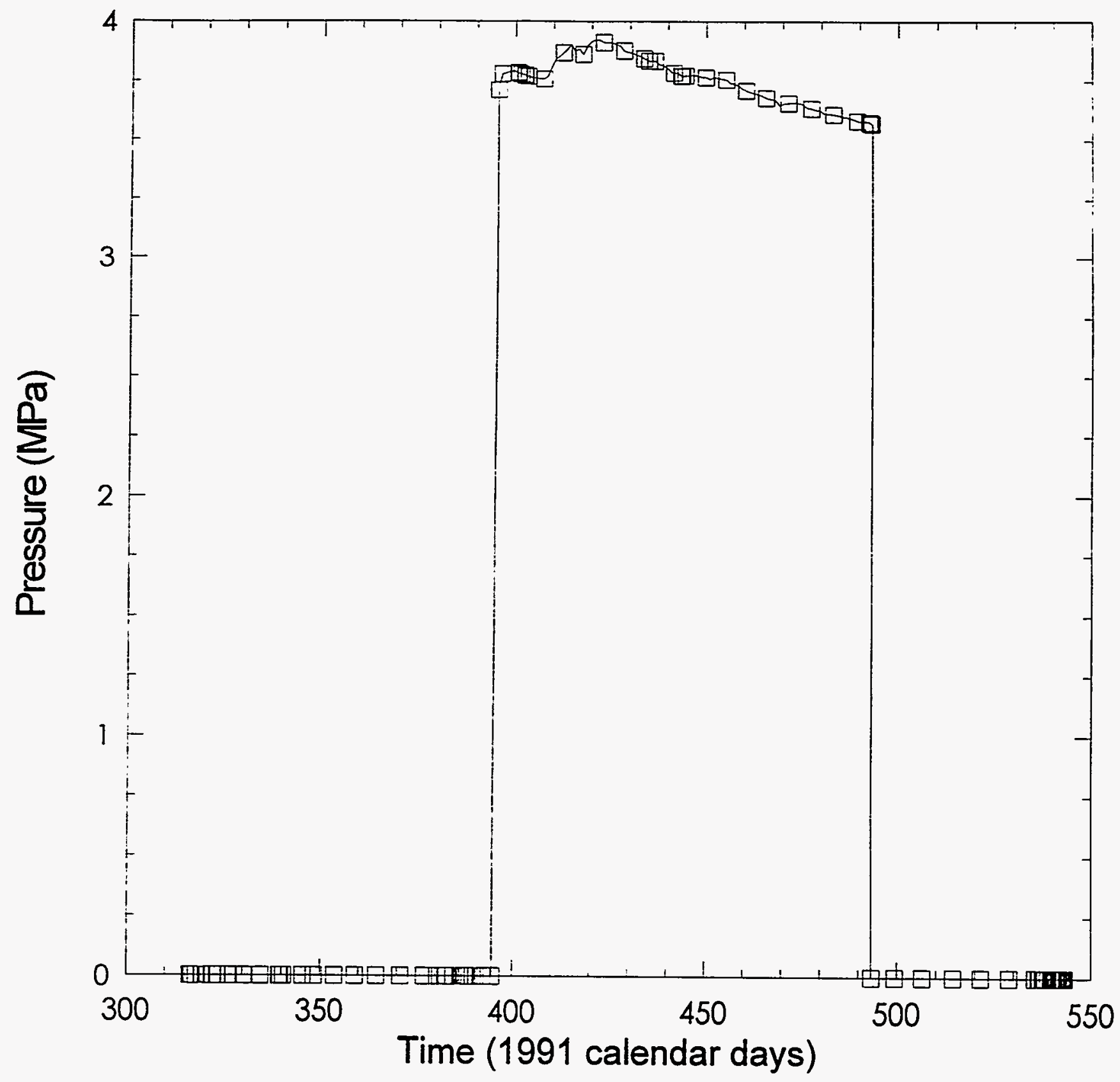

INTERA-6115-120-0

Figure 4-24. Test-zone pressure in observation borehole DPD02 during testing sequence C1X10. 


\subsubsection{OBSERVATION BOREHOLE DPD03, TESTING SEQUENCE C1X10.}

Table 4-9 gives a detailed description of the events that occurred in observation borehole DPD03 during testing sequence $\mathrm{C} 1 \mathrm{X} 10$.

Table 4-9. Events Associated with Observation Borehole DPD03 During Testing Sequence $\mathrm{C} 1 \mathrm{X} 10$

\begin{tabular}{|c|c|c|c|c|}
\hline EVENT & DATE & $\begin{array}{c}\text { CALENDAR } \\
\text { DAY }\end{array}$ & $\begin{array}{c}1991 \\
\text { CALENDAR } \\
\text { DAY }\end{array}$ & $\begin{array}{c}\text { TIME } \\
\text { (HH:MM:SS) }\end{array}$ \\
\hline Borehole DPD03 drilled to 13.11 meters. & 486 & N/A & NA & NA \\
\hline Begin long-term fluid-pressure monitoring of MB139. & 5-3-89 & 123 & N/A & N/A \\
\hline $\begin{array}{l}\text { Install single-packer fluid-pressure monitoring tool as indicated in the test-tool } \\
\text { configuration diagram (Figure 7-23). }\end{array}$ & $12-4-91$ & 338 & 338 & N/A \\
\hline Begin fluid-pressure monitoring of MB139 associated with testing sequence C1X10. & 1-30-92 & 29 & 394 & 14:43:00 \\
\hline Terminate data collection. & $5-7-92$ & 128 & 493 & 14:02:00 \\
\hline
\end{tabular}

Figure 4-25 illustrates the zone pressure in observation borehole DPD03 for testing sequence $\mathrm{C} 1 \mathrm{X} 10$. 


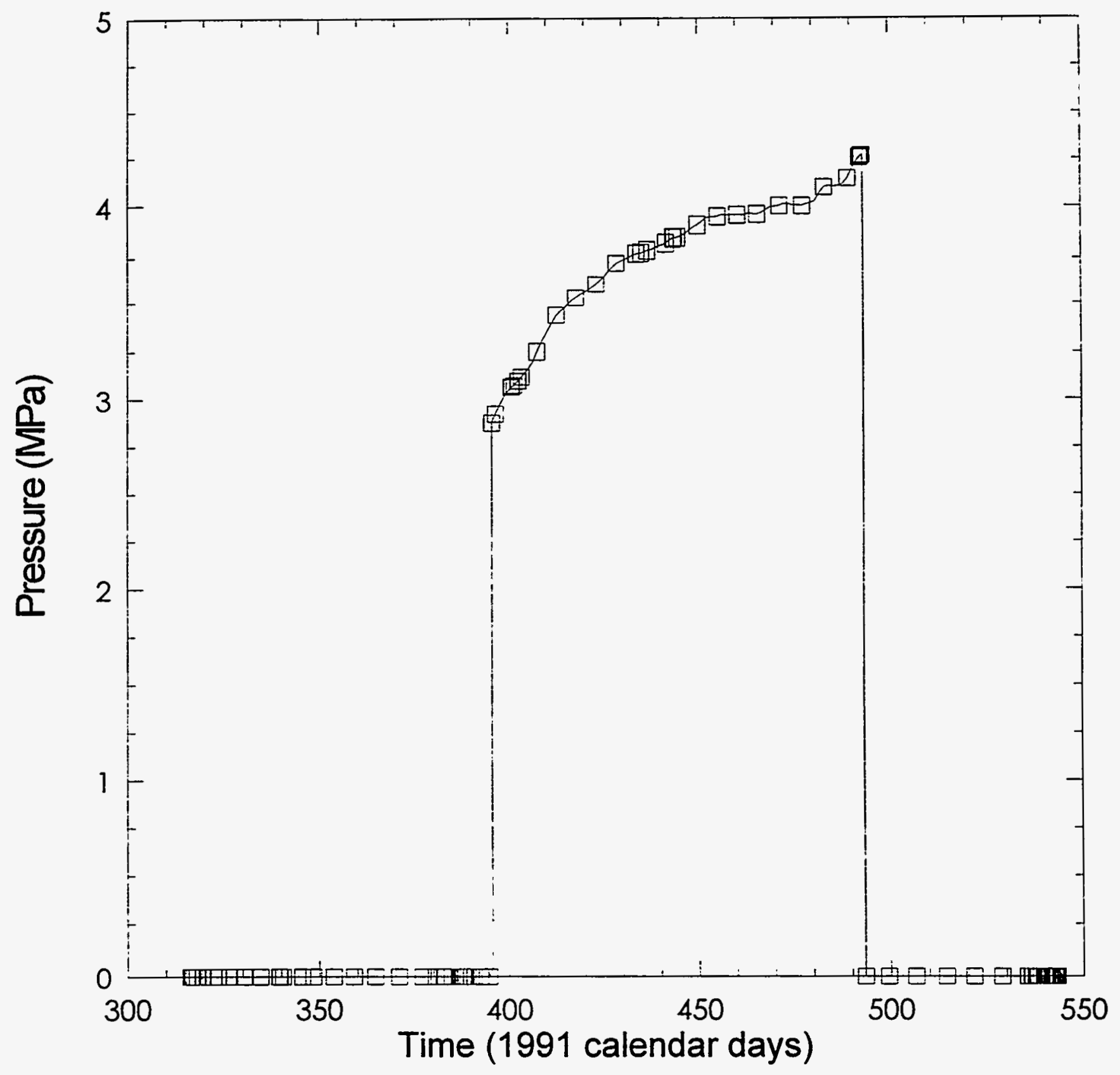

INTERA-6115-121-0

Figure 4-25. Test-zone pressure in observation borehole DPD03 during testing sequence C1X10. 
4.6.1.5 OBSERVATION BOREHOLE C2H01, TESTING SEQUENCE C1X10.

Table 4-10 gives a detailed description of the events that occurred in observation borehole $\mathrm{C} 2 \mathrm{H} 01$ during the testing sequence $\mathrm{C} 1 \times 10$.

Table 4-10. Events Associated with Observation Borehole C2H01 During Testing Sequence $\mathrm{C} 1 \mathrm{X} 10$

\begin{tabular}{|c|c|c|c|c|}
\hline EVENT & DATE & $\begin{array}{l}\text { CALENDAR } \\
\text { DAY }\end{array}$ & $\begin{array}{c}1991 \\
\text { CALENDAR } \\
\text { DAY }\end{array}$ & $\begin{array}{c}\text { TIME } \\
\text { (HH:MM:SS) }\end{array}$ \\
\hline Borehole $\mathrm{C} 2 \mathrm{HO} 1$ drilled to 5.58 meters. & $8-4-88$ & 217 & NA & NA \\
\hline Begin long-term fluid-pressure monitoring. & $8-22-89$ & 234 & N/A & N/A \\
\hline $\begin{array}{l}\text { Install single-packer fluid-pressure monitoring tool as indicated in the test-10ol } \\
\text { configuration diagram (Figure 5-9). }\end{array}$ & 3-13-91 & 72 & 72 & N/A \\
\hline Begin fluid-pressure monitoring of $M B 139$ associated with testing sequence $C 1 \times 10$. & $1-29-92$ & 29 & 394 & 14:43:00 \\
\hline Terminate data collection. & $6-26-92$ & $\uparrow 78$ & 548 & 08:18:34 \\
\hline
\end{tabular}

Figure 4-26 illustrates the zone pressure in observation borehole $\mathrm{C} 2 \mathrm{H} 01$ for testing sequence $\mathrm{C} 1 \times 10$. 


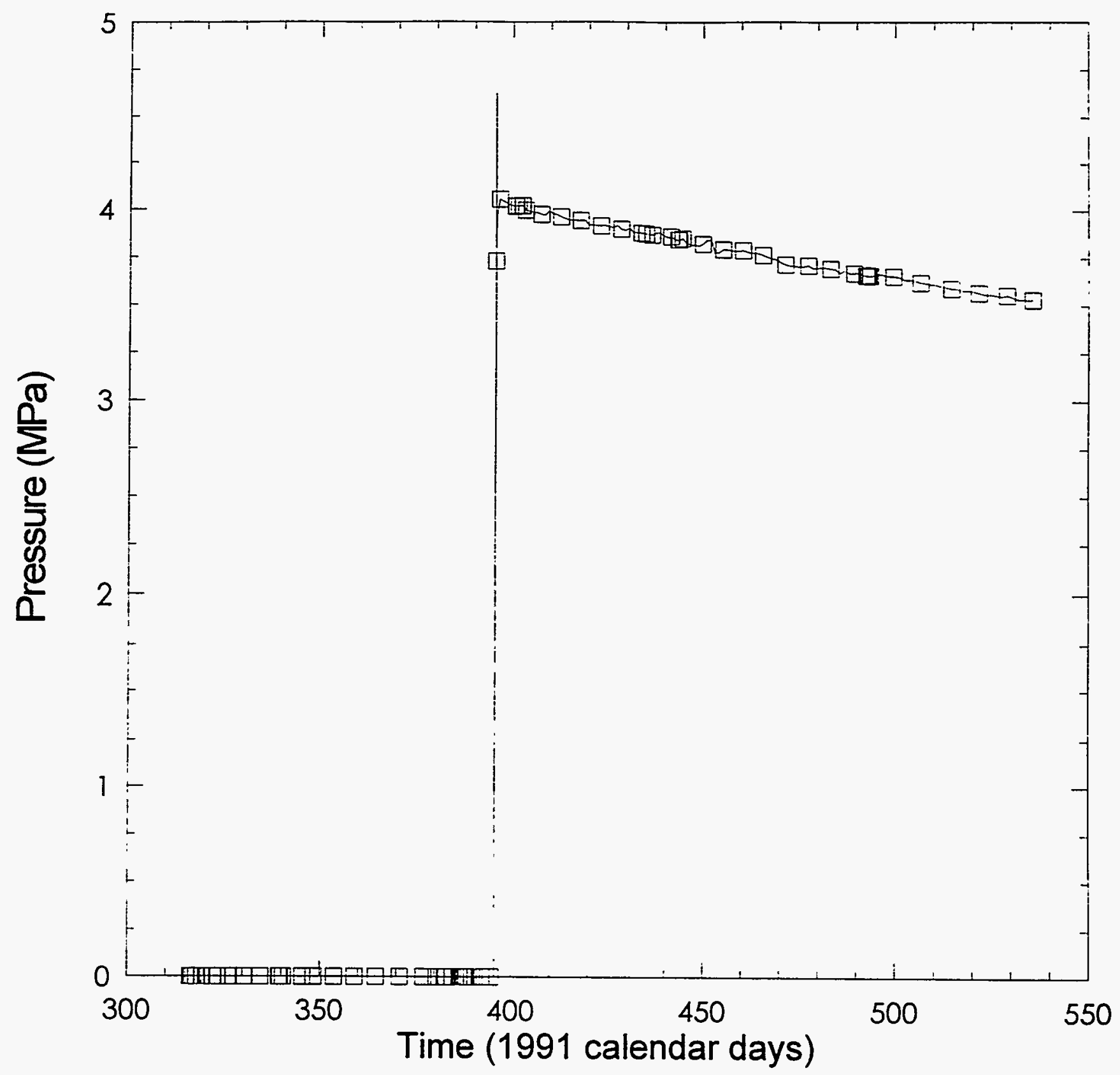

INTERAS115-122-0

Figure 4-26. Test-zone pressure in observation borehole $\mathrm{C} 2 \mathrm{H} 01$ during testing sequence $\mathrm{C} 1 \times 10$. 


\subsubsection{Borehole C1X05, Testing Sequence C1X05-A}

Testing sequence $\mathrm{C} 1 \mathrm{X} 05-\mathrm{A}$ took place in Room $\mathrm{C} 1$ in borehole $\mathrm{C} 1 \mathrm{X} 05$ with associated observation boreholes $\mathrm{C} 1 \mathrm{H} 05, \mathrm{C} 1 \mathrm{H} 06, \mathrm{C} 1 \mathrm{H} 07, \mathrm{C} 1 \mathrm{X06}, \mathrm{C} 1 \mathrm{X} 10$, and $\mathrm{C} 2 \mathrm{H} 01$. This test sequence was designed to investigate the pre- and post-hydrofracture brine permeability of MB139. Table 4-11 gives a detailed description of the events that occurred in test borehole $\mathrm{C} 1 \mathrm{X} 05$ during testing sequence $\mathrm{C} 1 \mathrm{X} 05-\mathrm{A}$.

Table 4-11. Events Associated with Test Borehole C1X05 During Testing Sequence C1X05-A

\begin{tabular}{|c|c|c|c|c|}
\hline EVENT & DATE & $\begin{array}{c}\text { CALENDAR } \\
\text { DAY }\end{array}$ & $\begin{array}{c}1992 \\
\text { CALENDAR } \\
\text { DAY }\end{array}$ & $\begin{array}{c}\text { TIME } \\
\text { (HH:MM:SS) }\end{array}$ \\
\hline Begin data file C1X0501. & $6-26-92$ & 178 & 178 & 12:03:32 \\
\hline Borehole C1X05 drilled with 3-inch $(7.62-\mathrm{cm})$ core barrel to 9.14 meters. & $6-29-92$ & 181 & 181 & 12:00:00 \\
\hline $\begin{array}{l}\text { Install multipacker test tool in borehole C1X05 as indicated in the test-tool } \\
\text { configuration diagram (Figure 4-11). }\end{array}$ & $6-30-92$ & 182 & 182 & 12:00:00 \\
\hline End data file C1X0501. & 6-30-92 & 182 & 182 & 14:46:37 \\
\hline Begin data file $\mathrm{C} 1 \times 0502$. & $6-30-92$ & 182 & 182 & 15:16:14 \\
\hline Begin data collection from borehole $\mathrm{C} 1 \times 05$. & $7-1-92$ & 183 & 183 & 08:28:00 \\
\hline End data file $\mathrm{C} 1 \times 0502$. & $7-6-92$ & 188 & 188 & 09:19:53 \\
\hline Begin data file $\mathrm{C}_{1 \times 0503 .}$ & $7-6-92$ & 188 & 188 & 09:48:15 \\
\hline DAS not functioning properly upon arrival. & $7-7-92$ & 189 & 189 & $13: 27: 00$ \\
\hline DAS not functioning properly upon arrival. & 7-20-92 & 202 & 202 & $13: 20: 00$ \\
\hline End data file C1X0503. & $7-30-92$ & 212 & 212 & 14:13:04 \\
\hline Begin data file $\mathrm{C} 1 \times 0504$. & $7-30-92$ & 212 & 212 & $14: 30: 08$ \\
\hline End data file C1X0504. & 8-3-92 & 216 & 216 & 13:14:19 \\
\hline Begin data file C1X0505. & 8-3-92 & 216 & 216 & 16:12:35 \\
\hline $\begin{array}{l}\text { Begin constant-pressure-injection test \#1 in TZ at } \sim 0.5 \mathrm{MPa} \text { above TZ pressure } \\
\qquad(\sim 9.7 \mathrm{MPa}) .\end{array}$ & $8-4-92$ & 217 & 217 & 10:24:00 \\
\hline Shut in $T Z$ from DPT panel terminating constant-pressure-injection test \#1 in $T Z$. & $8-4-92$ & 217 & 217 & 11:10:28 \\
\hline $\begin{array}{l}\text { Begin constant-pressure-injection test \#2 in TZ at } \sim 1 \mathrm{MPa} \text { above TZ pressure } \\
\qquad(-10.2 \mathrm{MPa}) .\end{array}$ & 8-4-92 & 217 & 217 & 11:22:00 \\
\hline Shut in TZ from DPT panel terminating constant-pressure-injection test \#2 in $T Z$. & 8-5-92 & 218 & 218 & $10: 47: 00$ \\
\hline End data file C1X0505. & 8-6-92 & 219 & 219 & 13:57:26 \\
\hline Begin data file C1X0506. & $8-6-92$ & 219 & 219 & 14:07:23 \\
\hline End data file $C_{1} \times 0506$. & $8-10-92$ & 223 & 223 & $10: 54: 48$ \\
\hline Begin data file C1X0507. & 8-10-92 & 223 & 223 & $11: 04: 38$ \\
\hline $\begin{array}{l}\text { Begin constant-pressure-injectlon test } \# 3 \text { in } T Z \text { at } \sim 1 \mathrm{MPa} \text { above } T Z \text { pressure } \\
\qquad(-10.2 \mathrm{MPa}) .\end{array}$ & $8-10-92$ & 223 & 223 & 11:12:37 \\
\hline End data file $\mathrm{C} 1 \times 0507$. & 8-18-92 & 231 & 231 & $09: 14: 13$ \\
\hline Begin data file C1X0508. & $8-20-92$ & 233 & 233 & 09:20:34 \\
\hline Shut in $T Z$ from DPT panel terminating constant-pressure-injection test \#3 in $T Z$. & 8-20-92 & 233 & 233 & 09:23:00 \\
\hline End data file C1X0508. & $9-10-92$ & 254 & 254 & 09:08:40 \\
\hline
\end{tabular}


Table 4-11 (Continued). Events Associated with Test Borehole C1X05 During Testing Sequence C1X05-A

\begin{tabular}{|c|c|c|c|c|}
\hline EVENT & DATE & $\begin{array}{l}\text { CALENDAR } \\
\text { DAY }\end{array}$ & $\begin{array}{c}1992 \\
\text { CALENDAR } \\
\text { DAY }\end{array}$ & $\begin{array}{c}\text { TIME } \\
\text { (HH:MM:SS) }\end{array}$ \\
\hline Begin data file C1X0509. & $9-10-92$ & 254 & 254 & $09: 43: 43$ \\
\hline End data file C1X0509. & $9-11-92$ & 255 & 255 & 09:16:30 \\
\hline Begin data file C1X0510. & $9-11-92$ & 255 & 255 & $09: 48: 28$ \\
\hline Increase TZP and GZP pressure. & $9-11-92$ & 255 & 255 & 13:26:00 \\
\hline Increase TZP and GZP pressure. & $9-16-92$ & 260 & 260 & 10:25:00 \\
\hline Perform hydrofracture of MB139 (injected $\sim 0.53 \mathrm{~L}$ of fluid into $T \mathrm{Z}$ ). & $9-16-92$ & 260 & 260 & 10:31:50 \\
\hline Begin constant-pressure-withdrawal test \#1 in $\mathrm{ZZ}$ at $\sim 9.5 \mathrm{MPa}$. & $9-16-92$ & 260 & 260 & 10:41:46 \\
\hline Shut in TZ from DPT panel terminating constant-pressure-withorawal test \#1 in $T Z$. & $9-16-92$ & 260 & 260 & 10:48:36 \\
\hline Decrease TZ pressure to $~ 9.6 \mathrm{MPa}$. & $9-16-92$ & 260 & 260 & 11:00:00 \\
\hline Pertorm hydrofracture of MB139 (injected $~-2.27 L$ of flutd). & $9-16-92$ & 260 & 260 & 11:02:30 \\
\hline Begin constant-pressure-withdrawal test $\# 2$ in $T Z$ at $\sim 9.5 \mathrm{MPa}$. & $9-16-92$ & 260 & 260 & 11:10:34 \\
\hline Shut in $T Z$ from DPT panel terminating constant-pressure-withdrawal test \#2 in $T Z$. & $9-16-92$ & 260 & 260 & 11:43:41 \\
\hline Perform hydrofracture of $M B 139$ (injected $\sim 5.98 \mathrm{~L}$ of fluid). & $9-16-92$ & 260 & 260 & 11:46:33 \\
\hline Begin constant-pressure-withdrawal test $\quad$ in TZ at $-9.5 \mathrm{MPa}$. & $9-16-92$ & 260 & 260 & 11:57:18 \\
\hline Shut in TZ from DPT panel terminating constant-pressure-withdrawal test $\# 3$ in $T Z$. & $9-16-92$ & 260 & 260 & $12: 11: 50$ \\
\hline Perform hydrotracture of MB139 (injected $~-8.55 \mathrm{~L}$ of fluid). & $9-16-92$ & 260 & 260 & 12:25:35 \\
\hline Begin constant-pressure-withdrawal test \#4 in $T Z$ at $\sim 9 \mathrm{MPa}$. & $9-16-92$ & 260 & 260 & 12:40:36 \\
\hline Decrease TZP pressure & $9-16-92$ & 260 & 260 & 13:19:52 \\
\hline Increase TZP pressure. & $9-16-92$ & 260 & 260 & 13:21:50 \\
\hline Shut in $T Z$ from DPT panel terminating constant-pressure-withdrawal test $\# 4$ in $T Z$. & $9-17-92$ & 261 & 261 & 10:14:54 \\
\hline Begin constant-pressure-withdrawal test $\# 5$ in $\mathrm{TZ}$ at $-9.5 \mathrm{MPa}$. & $9-17.92$ & 261 & 261 & 14:05:31 \\
\hline End data file C1X0sio. & $9-18-92$ & 262 & 262 & $09: 32: 16$ \\
\hline Begin data file C1X0511. & $9-18-92$ & 262 & 262 & 09:36:02 \\
\hline Shut in TZ from DPT panel terminating constant-pressure-withdrawal test \#5 in TZ. & $9-18-92$ & 262 & 262 & 09:37:46 \\
\hline End data file C1X0511. & 10-1-92 & 275 & 275 & 08:15:21 \\
\hline Begin data file C1X0512. & 10-1-92 & 275 & 275 & 10:54:03 \\
\hline End data file C1X0512. & 10-19-92 & 293 & 293 & 09:43:19 \\
\hline Begin data file C1X0513. & 10-19-92 & 293 & 293 & 11:11:28 \\
\hline End data file C1X0513. & $11-17-92$ & 322 & 322 & $09: 46: 07$ \\
\hline Begin data file C1X0514. & $11-17-92$ & 322 & 322 & 10:38:27 \\
\hline $\begin{array}{l}\text { Begin constant-pressure-injection test } 44 \text { in TZ at } \sim 1 \mathrm{MPa} \text { above TZ pressure } \\
\qquad(-10.3 \mathrm{MPa}) .\end{array}$ & $11-17-92$ & 322 & 322 & 10:47:52 \\
\hline Shut in TZ from DPT panel terminating constant-pressure-injection test \#4 in TZ. & 12-1-92 & 336 & 336 & $11: 09: 58$ \\
\hline $\begin{array}{l}\text { Begin constant-pressure-injection test } \# 5 \text { in } T Z \text { at } \sim 2.2 \mathrm{MPa} \text { above } T Z \text { pressure } \\
\qquad(-11.5 \mathrm{MPa}) .\end{array}$ & $12-1-92$ & 336 & 336 & 11:42:17 \\
\hline Shut in $T Z$ from DPT panel terminating constant-pressure-injection test $\# 5$ in TZ. & $12-1-92$ & 336 & 336 & 13:06:48 \\
\hline End data file C1X0514. & $1-8-93$ & 8 & 374 & 11:14:04 \\
\hline
\end{tabular}


Table 4-11 (Continued). Events Associated with Test Borehole C1X05 During Testing Sequence C1X05-A

\begin{tabular}{|c|c|c|c|c|}
\hline EVENT : & DATE & $\begin{array}{c}\text { CALENDAR } \\
\text { DAY }\end{array}$ & $\begin{array}{c}1992 \\
\text { CALENDAR } \\
\text { DAY }\end{array}$ & $\begin{array}{l}\text { TIME } \\
\text { (HH:MM:SS) }\end{array}$ \\
\hline Begin data file C1X0515. & $1-8-93$ & 8 & 374 & 12:38:36 \\
\hline $\begin{array}{l}\text { Begin constant-pressure-withdrawal test \#6 in TZ at } \sim 1 \mathrm{MPa} \text { below TZ pressure } \\
\qquad(\sim 8.1 \mathrm{MPa}) .\end{array}$ & 1-13-93 & 13 & 379 & 10:39:01 \\
\hline Leak in DPT panel caused TZ pressure decrease. & 1-18-93 & 18 & 384 & 10:12:00 \\
\hline Shut in TZ from DPT panel terminating constant-pressure-withdrawal test \#6 in TZ. & $1-18-93$ & 18 & 384 & 10:15:00 \\
\hline $\begin{array}{l}\text { Begin constant-pressure-withdrawal test \#7 in TZ at } \sim 1 \mathrm{MPa} \text { below TZ pressure } \\
\qquad(\sim 8.1 \mathrm{MPa}) .\end{array}$ & t-18-93 & 18 & 384 & 12:50:00 \\
\hline Shut in TZ from DPT panel terminating constant-pressure-withdrawal test \#7 in $T Z$. & $1-26-93$ & 26 & 392 & $15: 14: 02$ \\
\hline End data file C1X0515. & $3-11-93$ & 70 & 435 & $14: 41: 22$ \\
\hline Begin data file C1X0516. & $3-11-93$ & 70 & 435 & 14:52:50 \\
\hline End data file Cixo516. & 4-12-93 & 102 & 467 & 11:01:47 \\
\hline Remove multipacker test tool from borehole C1X05. & 4-16-93 & 106 & 472 & 09:44:00 \\
\hline
\end{tabular}

Table 4-12 indicates the equipment that was used and the duration that each instrument was used during testing sequence $\mathrm{C} 1 \mathrm{X} 05-\mathrm{A}$ in test borehole $\mathrm{C} 1 \mathrm{X} 05$ and in the observation boreholes. Figures 4-27 and 4-28 illustrate the zone pressures and fluid production during a constant-pressure-withdrawal tests, respectively, in test borehole C1X05 for testing sequence $\mathrm{C} 1 \mathrm{X} 05-\mathrm{A}$. It should be noted that Figure 4-28 (Fluid production during constantpressure-flow tests in test borehole $\mathrm{C} 1 \mathrm{X} 05$ during testing sequence $\mathrm{C} 1 \mathrm{X} 05-\mathrm{A}$ ) consists of five parts (Figures 4-28a, 4-28b, 4-28c, 4-28d, and 4-28e).

Table 4-12. Testing Sequence C1X05-A Equipment

\begin{tabular}{|c|c|c|c|c|}
\hline Equipment & Location & Serial \# & Installed & Removed \\
\hline DAS Software & N/A & PERM4F & $6-26-92$ & $4-12-93$ \\
\hline DCU (HP3497A) & N/A & $2629 a 21990$ & $6-26-92$ & $9-9-92$ \\
\hline DCU (HP3497A) & N/A & $2023 a 01688$ & $9-9-92$ & $10-19-92$ \\
\hline DCU (HP3497A) & N/A & $2629 a 21996$ & $10-19-92$ & $4-7-93$ \\
\hline $\begin{array}{c}\text { Transducer } \\
\text { (Druck PDCR 830) }\end{array}$ & $\mathrm{C} 1 \times 10 \mathrm{TZ}$ & 246909 & $6-26-92$ & $8-3-92$ \\
\hline $\begin{array}{c}\text { Transducer } \\
\text { (Druck PDCR 830) }\end{array}$ & C1X10 TZ & 246909 & $8-6-92$ & $3-11-93$ \\
\hline $\begin{array}{c}\text { Transducer } \\
\text { (Druck PDCR 830) }\end{array}$ & C1H05 TZ & 246917 & $6-26-92$ & $3-11-93$ \\
\hline $\begin{array}{c}\text { Transducer } \\
\text { (Druck PDCR 830) }\end{array}$ & C1H05 TZP & 246919 & $6-26-92$ & $10-1-92$ \\
\hline $\begin{array}{c}\text { Transducer } \\
\text { (Druck PDCR 910) }\end{array}$ & $\mathrm{C} 2 \mathrm{H} 01 \mathrm{TZ}$ & 321768 & $6-26-92$ & $8-3-92$ \\
\hline
\end{tabular}


Table 4-12 (Continued). Testing Sequence C1X05-A Equipment

\begin{tabular}{|c|c|c|c|c|}
\hline Equipment & Location & Serial \# & Installed & Removed \\
\hline $\begin{array}{c}\text { Transducer } \\
\text { (Druck PDCR 910) }\end{array}$ & C1H07 TZ & 322426 & $6-26-92$ & $4-12-93$ \\
\hline $\begin{array}{c}\text { Transducer } \\
\text { (Druck PDCR 10/D) }\end{array}$ & C1H07 TZP & 211691 & $6-26-92$ & $4-12-93$ \\
\hline $\begin{array}{c}\text { Transducer } \\
\text { (Druck PDCR 910) }\end{array}$ & $\mathrm{C} 1 \times 05 \mathrm{TZ}$ & 322424 & $6-30-92$ & $4-12-93$ \\
\hline $\begin{array}{c}\text { Transducer } \\
\text { (Druck PDCR 830) }\end{array}$ & C1H06 TZ & 246916 & $7-6-92$ & $3-11-93$ \\
\hline $\begin{array}{c}\text { Transducer } \\
\text { (Druck PDCR 830) }\end{array}$ & C1H06 TZP & 246918 & $7-6-92$ & $10-1-92$ \\
\hline $\begin{array}{c}\text { Transducer } \\
\text { (Druck PDCR 910) }\end{array}$ & DPT Panel & 322427 & $8-3-92$ & $8-18-92$ \\
\hline $\begin{array}{c}\text { Transducer } \\
\text { (Druck PDCR 910) }\end{array}$ & DPT Panel & 322427 & $9-10-92$ & $9-18-92$ \\
\hline $\begin{array}{c}\text { Transducer } \\
\text { (Druck PDCR 910) }\end{array}$ & DPT Panel & 322427 & $10-1-92$ & $11-17-92$ \\
\hline $\begin{array}{c}\text { Transducer } \\
\text { (Druck PDCR 910) }\end{array}$ & C1X06 TZ & 308143 & $10-1-92$ & $4-12-93$ \\
\hline $\begin{array}{c}\text { Transducer } \\
\text { (Druck PDCR 910) }\end{array}$ & C1X06 TZP & 308146 & 10-1-92 & $4-12-93$ \\
\hline $\begin{array}{c}\text { Transducer } \\
\text { (Druck PDCR 910) }\end{array}$ & DPT Panel & 316158 & $1-8-93$ & $4-12-93$ \\
\hline LVDT (Trans-Tek 245) & C1X06 & $\mathrm{A} 04$ & $10-1-92$ & $4-12-93$ \\
\hline $\begin{array}{c}\text { Thermocouple } \\
\text { (Type E) }\end{array}$ & $\mathrm{C} 1 \mathrm{HO}$ & 1 & $7-6-92$ & $4-12-93$ \\
\hline Injection Column & N/A & 77 & $8-4-92$ & $8-6-92$ \\
\hline Injection Column & N/A & 92 & $8-6-92$ & $8-24-92$ \\
\hline Injection Column & N/A & 77 & $9-16-92$ & $9-18-92$ \\
\hline Injection Column & N/A & 88 & $9-16-92$ & $9-18-92$ \\
\hline Injection Column & N/A & 77 & $11-17-92$ & $3-11-93$ \\
\hline Injection Column & N/A & 76 & $11-20-92$ & $3-11-93$ \\
\hline $\begin{array}{c}\text { DPT } \\
\text { (Rosemount 1151DP) }\end{array}$ & N/A & 1409226 & $8-3-92$ & $8-18-92$ \\
\hline $\begin{array}{c}\text { DPT } \\
\text { (Rosemount 1151DP) }\end{array}$ & N/A & 1389938 & $9-15-92$ & $3-11-93$ \\
\hline
\end{tabular}

* Installed dates for injection columns refer to dates of initial use rather than date installed. 


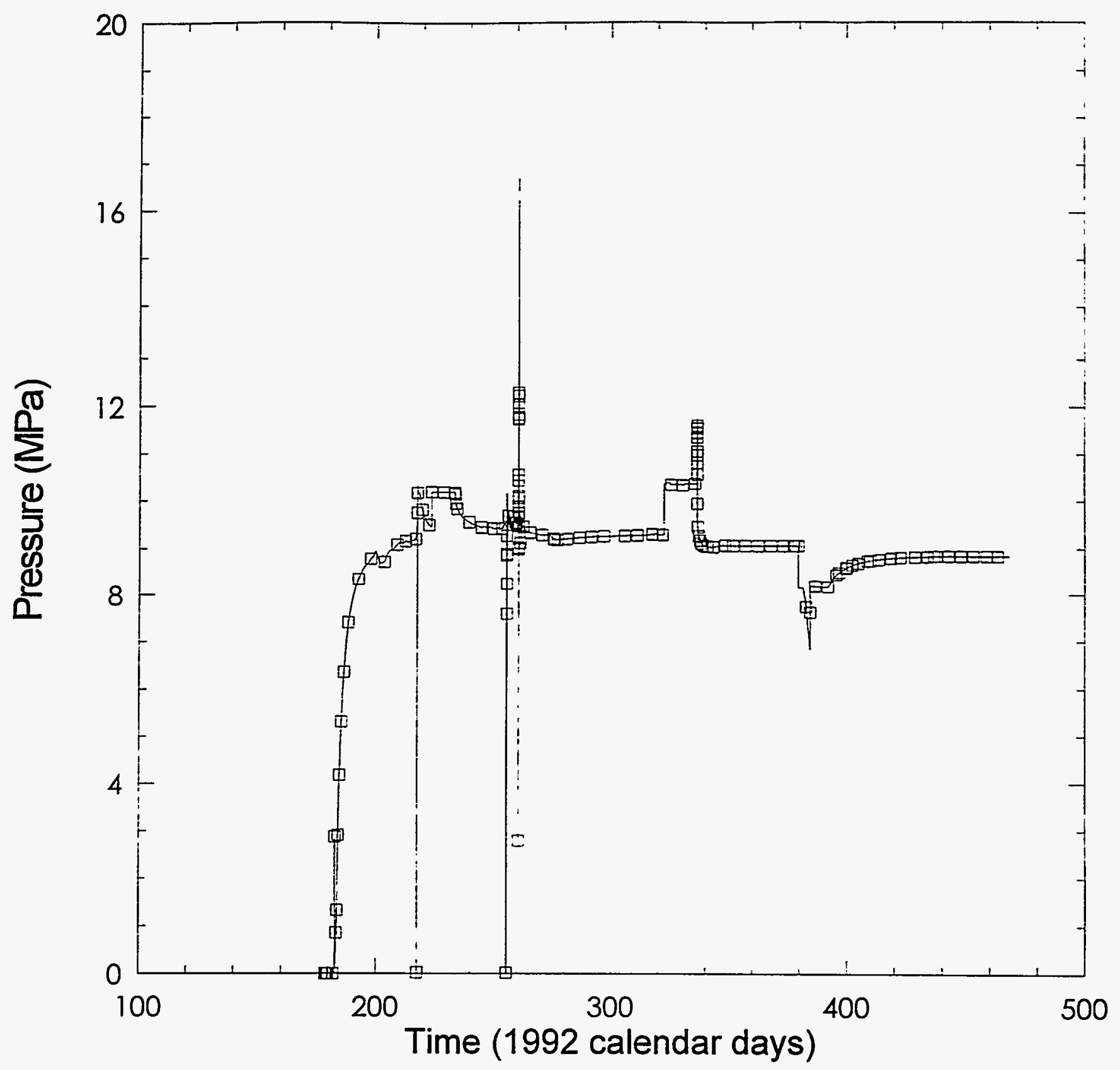

INTERA-6115-123-0

Figure 4-27. Test-zone pressure in test borehole $\mathrm{C} 1 \mathrm{X} 05$ during testing sequence $\mathrm{C} 1 \mathrm{X} 05-\mathrm{A}$. 


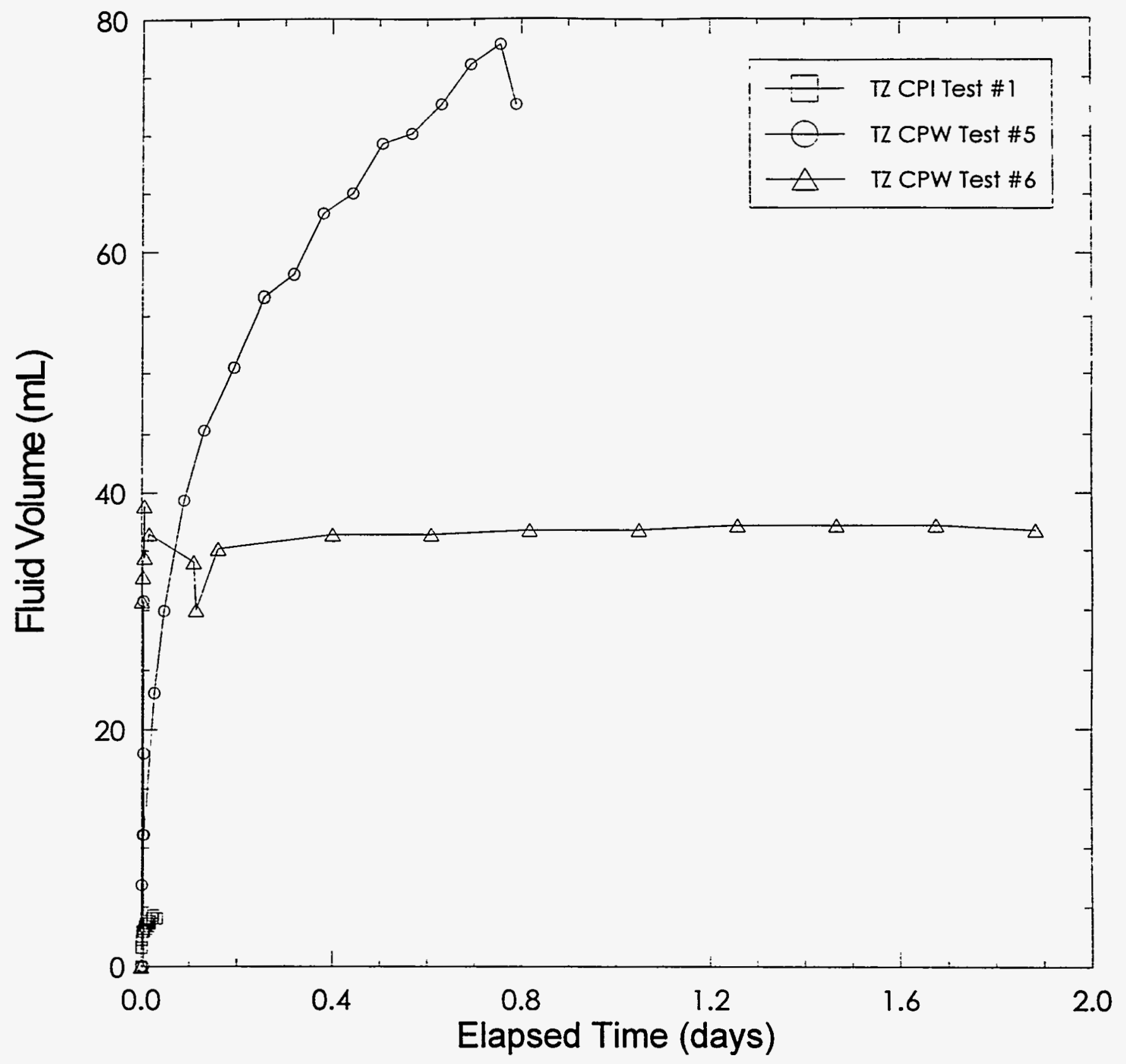

INTERA6115-125-0

Figure 4-28a. Fluid production during constant-pressure-flow tests in test borehole $\mathrm{C} 1 \mathrm{X} 05$ during testing sequence $\mathrm{C} 1 \times 05-\mathrm{A}$. 


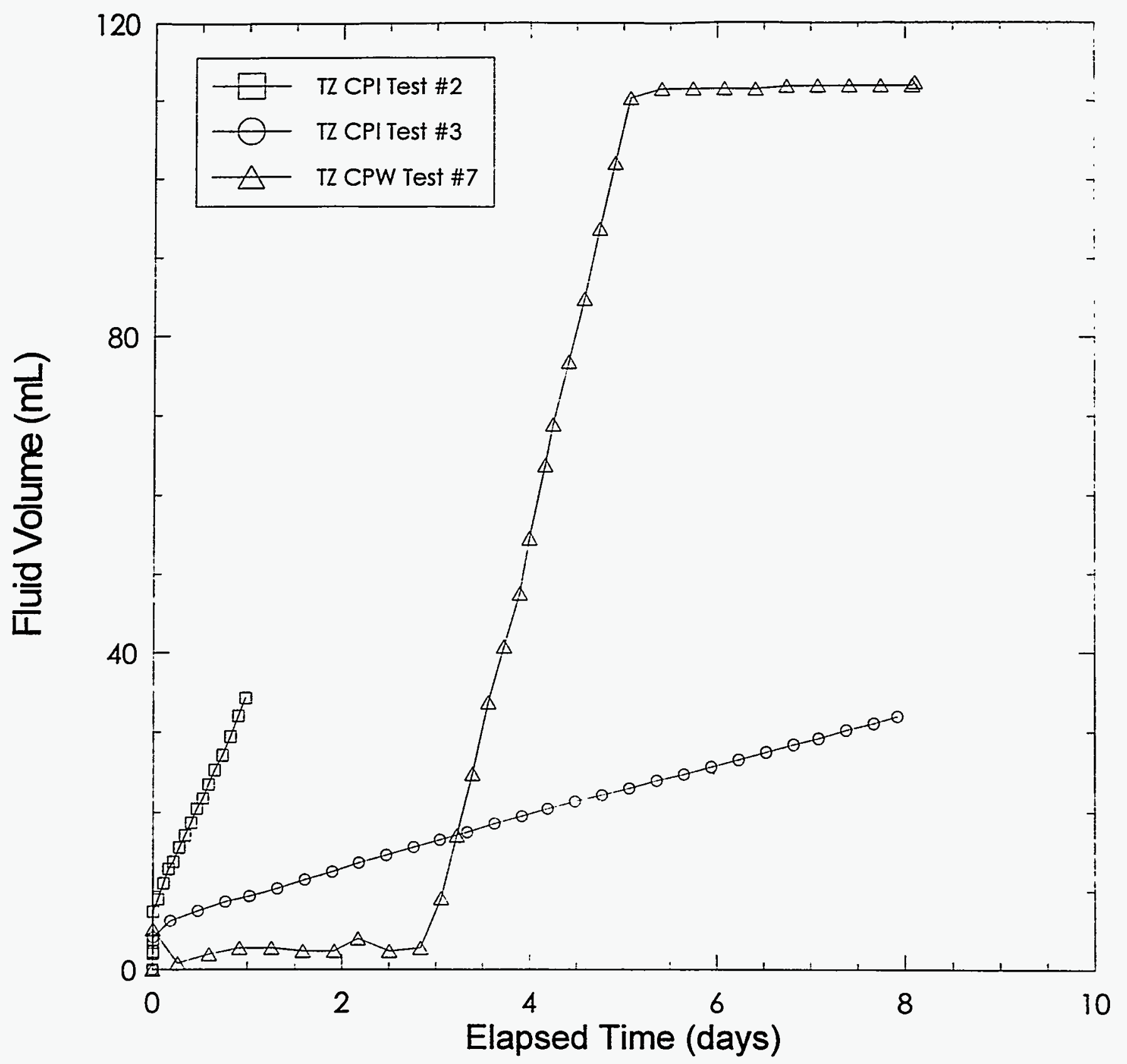

INTERA6115-12B-0

Figure 4-28b. Fluid production during constant-pressure-flow tests in test borehole $\mathrm{C} 1 \mathrm{X05}$ during testing sequence C1X05-A (continued). 


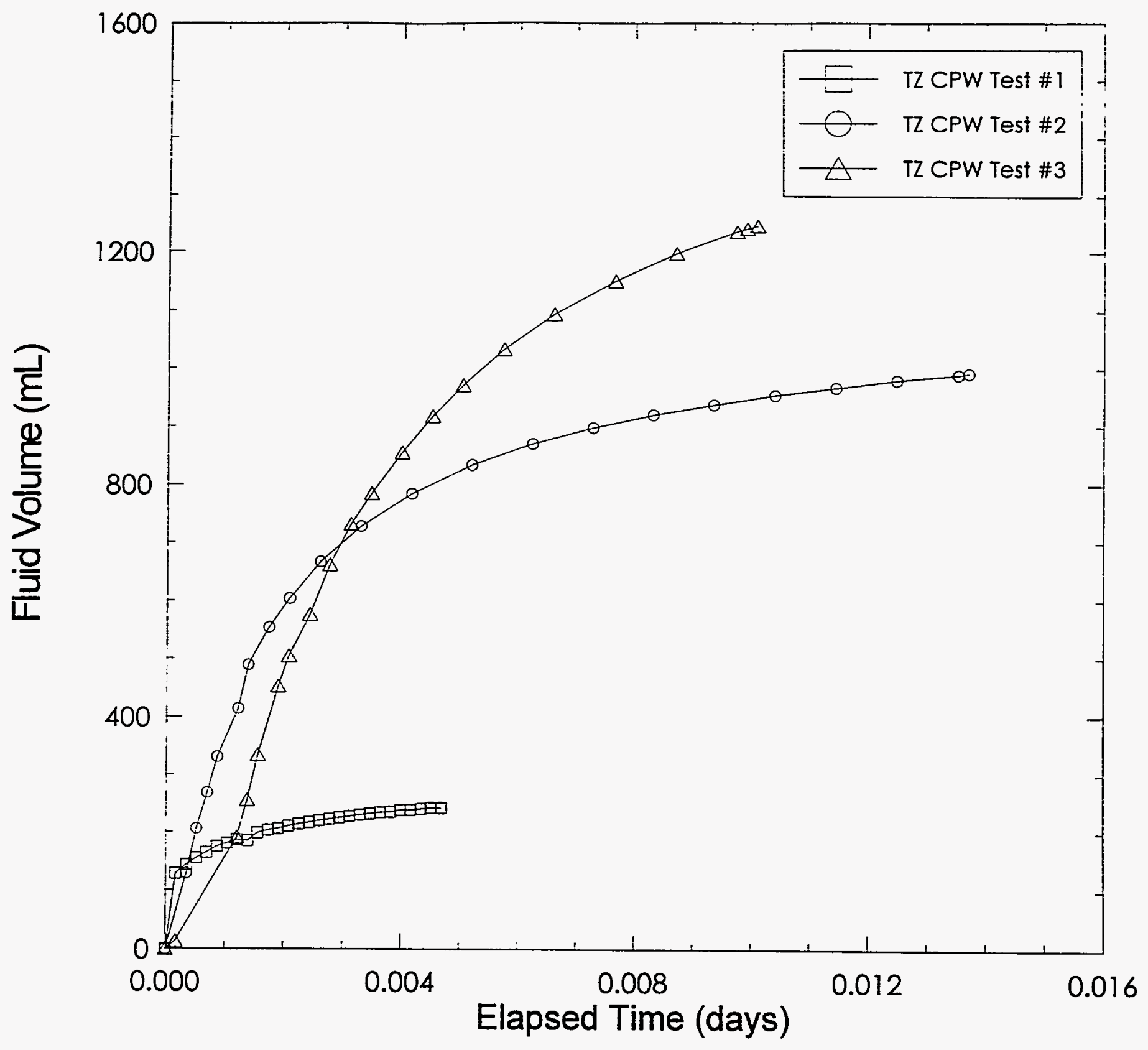

INTERA 6115-127-0

Figure 4-28c. Fluid production during constant-pressure-flow tests in test borehole C1X05 during testing sequence $\mathrm{C} 1 \mathrm{X} 05-\mathrm{A}$ (continued). 


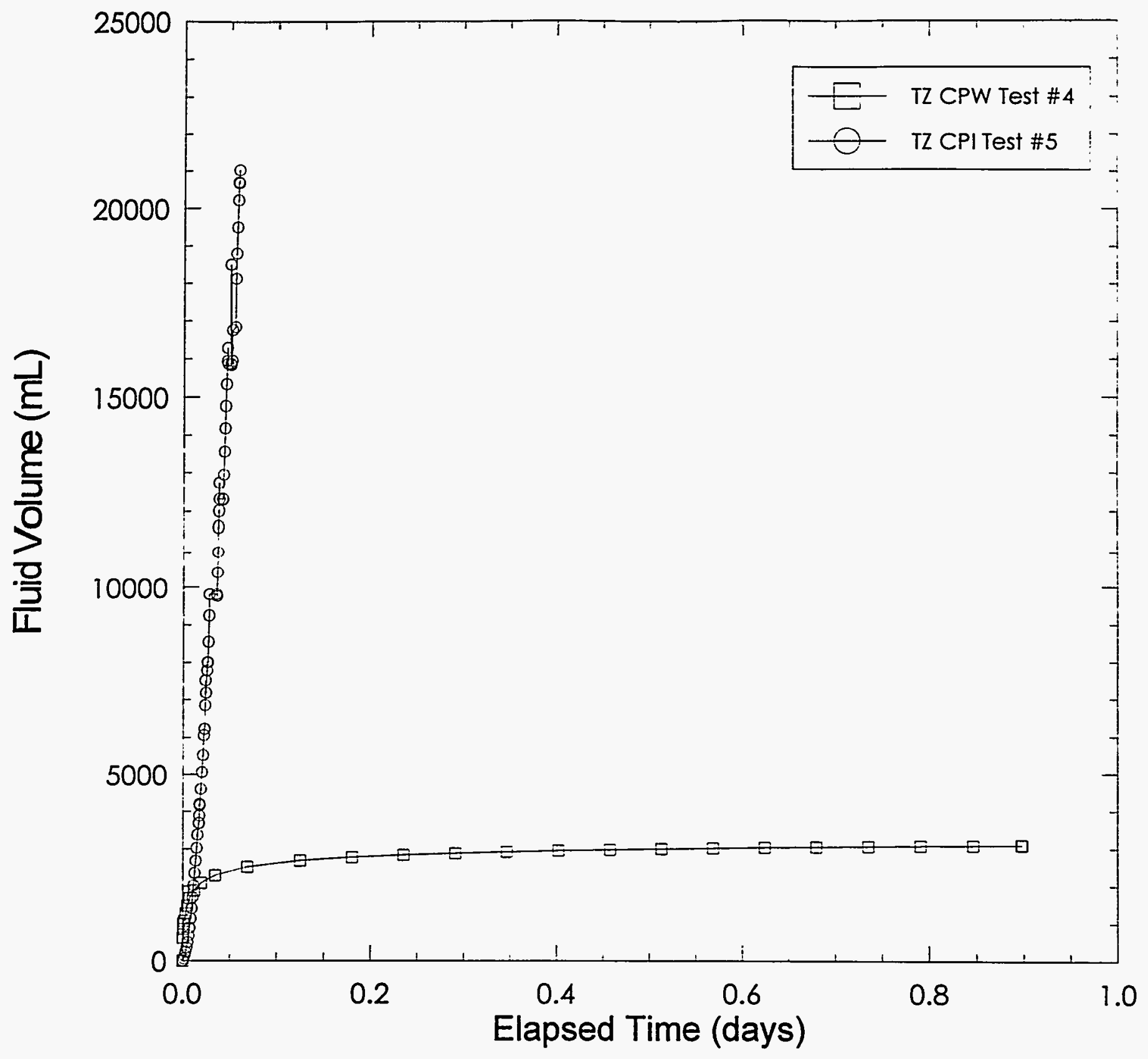

INTERA-8115-12800

Figure 4-28d. Fluid production during constant-pressure-flow tests in test borehole C1X05 during testing sequence C1X05-A (continued). 


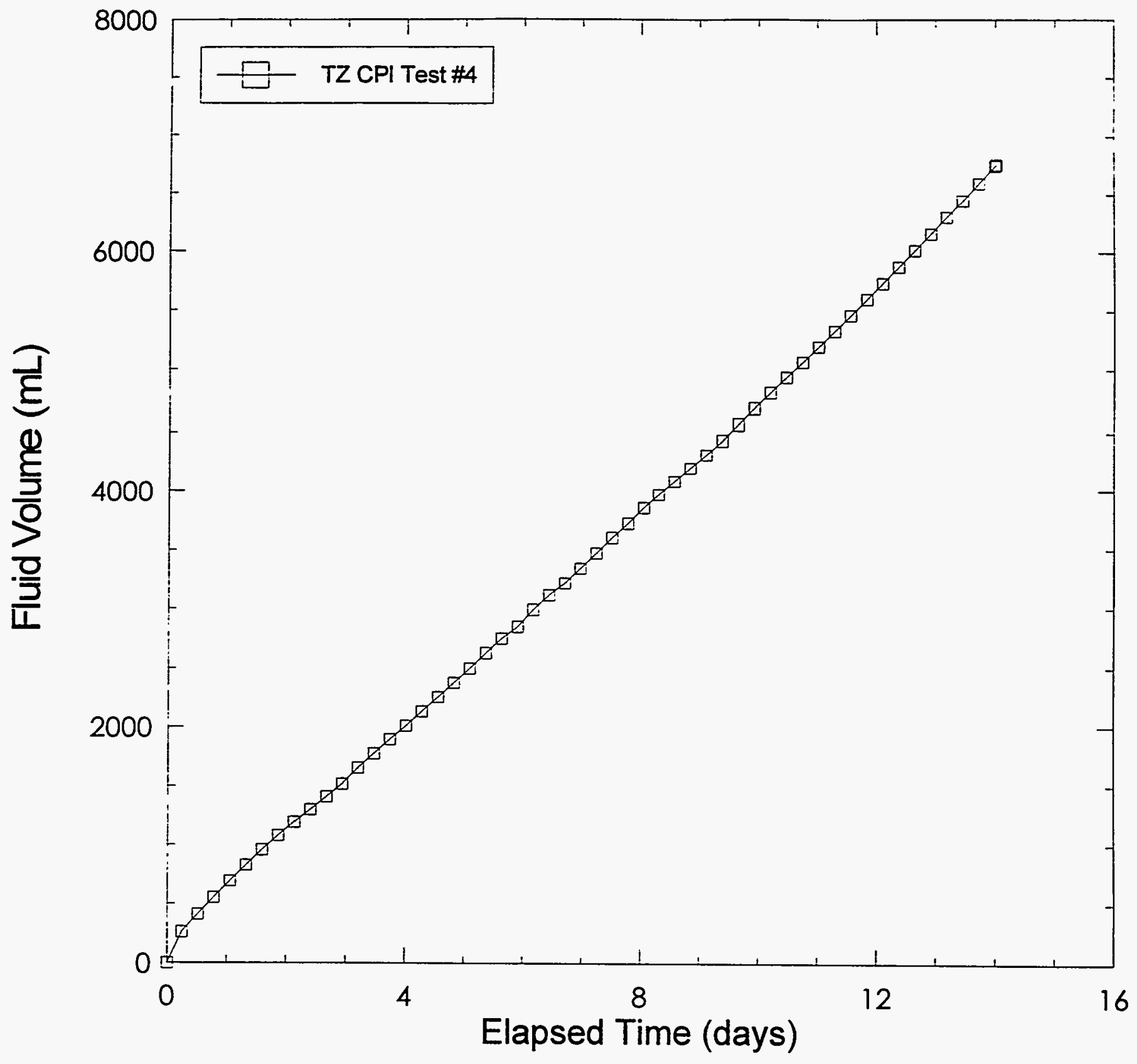

INTERA-115-1290

Figure 4-28e. Fluid production during constant-pressure-flow tests in test borehole $\mathrm{C} 1 \mathrm{X05}$ during testing sequence $\mathrm{C} 1 \times 05-\mathrm{A}$ (continued). 


\subsubsection{OBSERVATION BOREHOLE C1H05, TESTING SEQUENCE C1X05-A}

Table 4-13 gives a detailed description of the events that occurred in observation borehole C1H05 during testing sequence C1X05-A.

Table 4-13. Events Associated with Observation Borehole C1H05 During Testing Sequence C1X05-A

\begin{tabular}{|c|c|c|c|c|}
\hline EVENT & DATE & $\begin{array}{c}\text { CALENDAR } \\
\text { DAY }\end{array}$ & $\begin{array}{c}1992 \\
\text { CALENDAR } \\
\text { DAY }\end{array}$ & $\begin{array}{c}\text { TIME } \\
\text { (HH:MM:SS) }\end{array}$ \\
\hline Drill borehole C1H05 with 4-inch $(10.16-\mathrm{cm})$ core barrel to 8.20 meters. & 1-16-92 & 16 & 16 & 14:20:00 \\
\hline Perform video log of borehole $\mathrm{C} 1 \mathrm{H} 05$. & 1-17-92 & 17 & 17 & 10:00:00 \\
\hline Face off bottom of borehole with 4-inch $(10.16-\mathrm{cm})$ plug bit to 8.26 meters. & $1-17-92$ & 17 & 17 & 10:45:00 \\
\hline $\begin{array}{l}\text { Install single-packer monitor tool } 32 \mathrm{~A} \text { in borehole C1HO5 as indicated in the test-tool } \\
\text { configuration diagram (Figure 4-9). }\end{array}$ & $1-17-92$ & 17 & 17 & 10:00:00 \\
\hline Begin data collection associated with testing sequence $C 1 \times 10$. & 1-17-92 & 17 & 17 & $14: 30: 00$ \\
\hline Terminate data collection associated with testing sequence $\mathrm{C} 1 \times 10$. & 6-26-92 & 178 & 178 & 08:18:35 \\
\hline Begin data collection associated with testing sequence $\mathrm{C} 1 \times 05-\mathrm{A}$. & $6-26-92$ & 178 & 178 & 13:00:00 \\
\hline Terminate data collection associated with testing sequence $\mathrm{C} 1 \times 05-\mathrm{A}$. & 3-11-93 & 70 & 437 & 10:30:00 \\
\hline Remove monitor tool from borehole $\mathrm{C} 1 \mathrm{H} 05$. & 3-12-93 & 71 & 437 & 10:50:00 \\
\hline
\end{tabular}

Figures 4-29 and 4-30 illustrate the zone pressure and packer pressure, respectively, in observation borehole $\mathrm{C} 1 \mathrm{H} 05$ for testing sequence C1X05-A. Copies of the video-log associated with testing sequence $\mathrm{C} 1 \mathrm{X} 05-\mathrm{A}$ (borehole $\mathrm{C} 1 \mathrm{H} 05$ ) identified in Table 4-13 are provided in the SWCF under WPO \#45907 


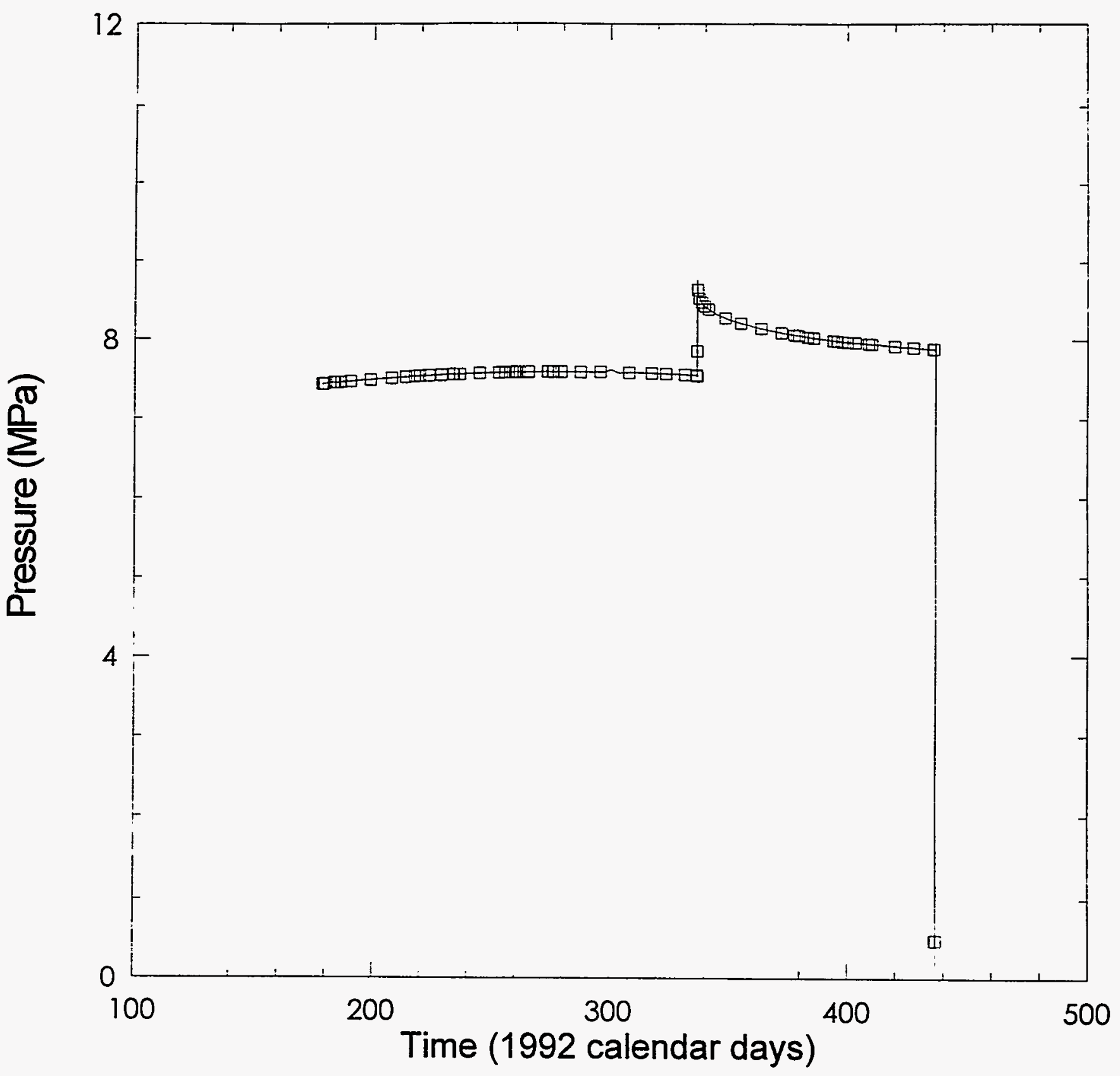

INTERA-61 15-132-0

Figure 4-29. Zone pressure in observation borehole $\mathrm{C} 1 \mathrm{H} 05$ during testing sequence $\mathrm{C} 1 \mathrm{X} 05-\mathrm{A}$. 


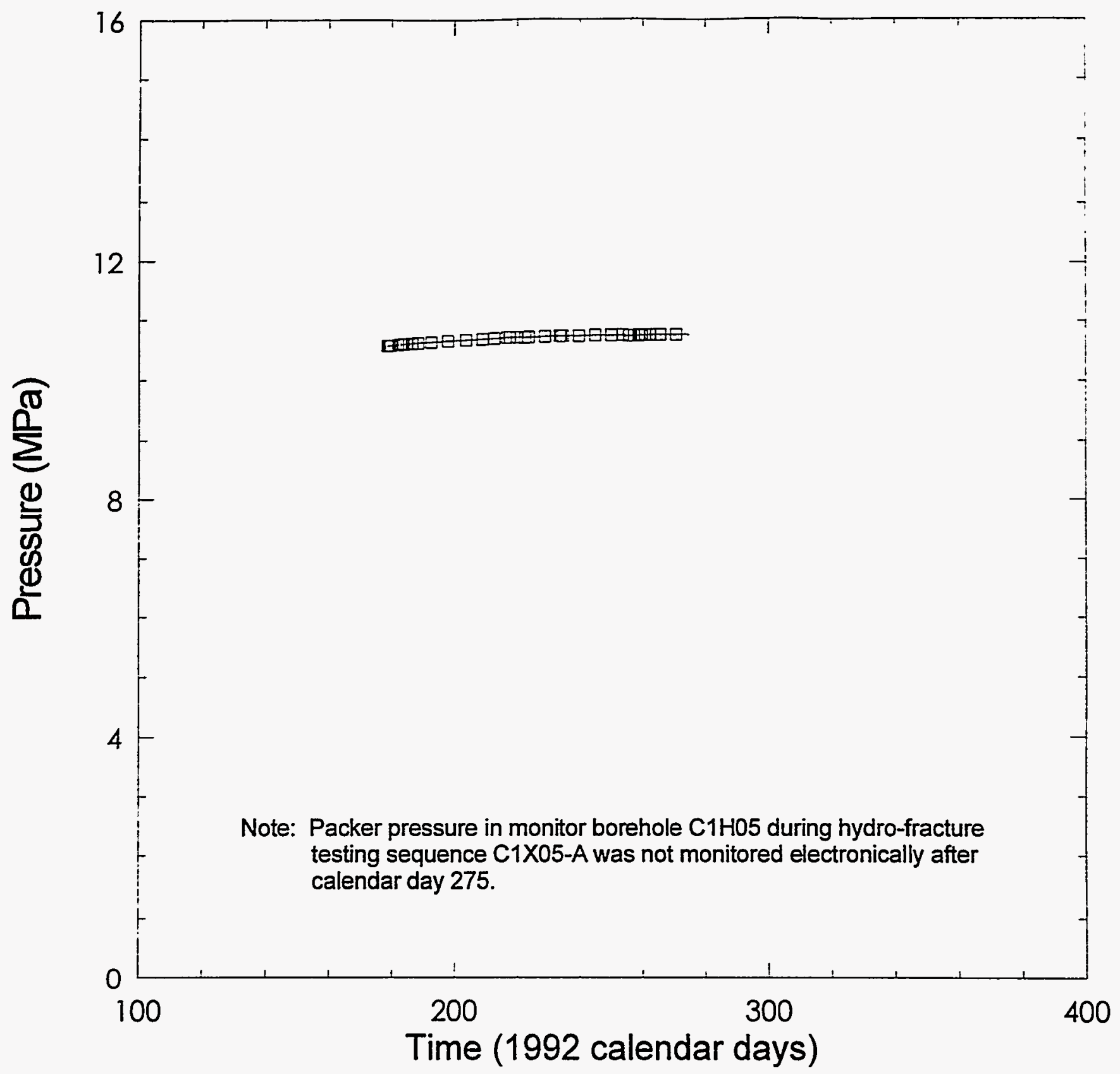

INTERA6115-133-0

Figure 4-30. Packer pressure in observation borehole $\mathrm{C} 1 \mathrm{H} 05$ during testing sequence $\mathrm{C} 1 \mathrm{X} 05-\mathrm{A}$. 


\subsubsection{OBSERVATION BOREHOLE C1H06, TESTING SEQUENCE C1X05-A}

Table 4-14 gives a detailed description the of events that occurred in observation borehole C1H06 during testing sequence C1X05-A.

Table 4-14. Events Associated with Observation Borehole C1H06 During Testing Sequence C1X05-A

\begin{tabular}{|c|c|c|c|c|}
\hline EVENT & DATE & $\begin{array}{c}\text { CALENDAR } \\
\text { DAY }\end{array}$ & $\begin{array}{c}1992 \\
\text { CALENDAR } \\
\text { DAY }\end{array}$ & $\begin{array}{c}\text { TIME } \\
\text { (HH:MM:SS) }\end{array}$ \\
\hline Drill borehole C1H06 with 4-inch $(10.16-\mathrm{cm})$ plug bit to 9.40 meters. & $1-21-92$ & 21 & 21 & 15:30:00 \\
\hline Perform video log of borehole C1HO6. & $1-22-92$ & 22 & 22 & 11:30:00 \\
\hline $\begin{array}{c}\text { Install single-packer monitor tool \#32B in borehole C1HOs as indicated in the test-10ol } \\
\text { configuration diagram (Figure } 4-10) \text {. }\end{array}$ & 1-22-92 & 22 & 22 & 10:00:00 \\
\hline Begin data collection associated with testing sequence $C 1 \times 10$. & $1-22-92$ & 22 & 22 & 14:04:00 \\
\hline Terminate data collection associated with testing sequence $\mathrm{C} 1 \times 10$. & $6-26-92$ & 178 & 178 & 08:18:35 \\
\hline Begin data collection associated with testing sequence $C 1 \times 05-A$. & $6-26-92$ & 178 & 178 & 13:00:00 \\
\hline Termmate data collection associated with testing sequence C1X05-A. & $3-11-93$ & 70 & 437 & 10:30:00 \\
\hline Remove monitor tool from borehole C1HO6. & 3-12-93 & 71 & 437 & 11:17:00 \\
\hline
\end{tabular}

Figures 4-31 and 4-32 illustrate the zone pressure and packer pressure, respectively, in observation borehole $\mathrm{C} 1 \mathrm{H} 06$ for testing sequence C1X05-A. Copies of the video-log associated with testing sequence $\mathrm{C} 1 \times 05-\mathrm{A}$ (borehole $\mathrm{C} 1 \mathrm{H06}$ ) identified in Table 4-14 are provided in the SWCF under WPO \#45907 


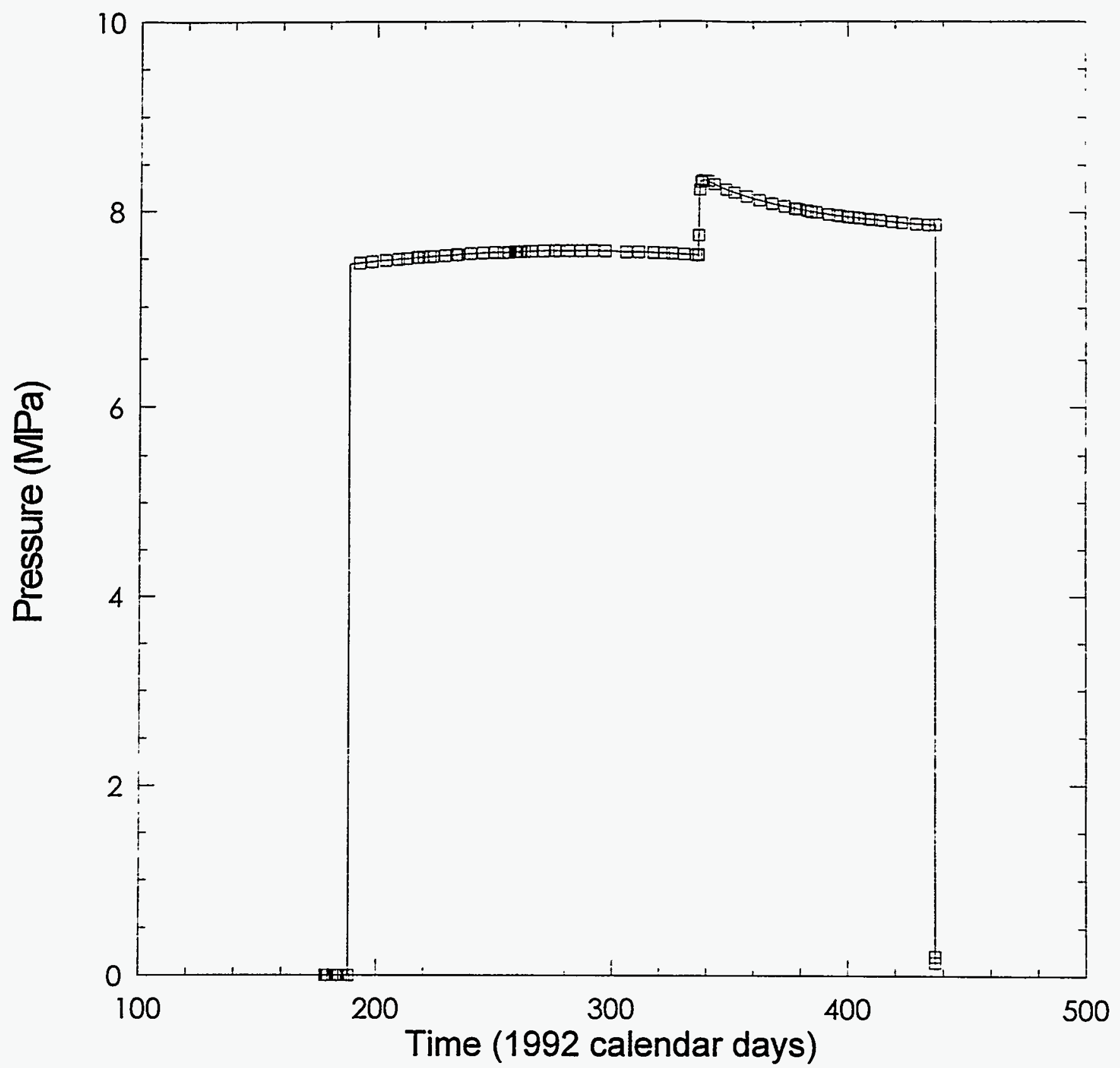

INTERABI15-1340

Figure 4-31. Zone pressure in observation borehole $\mathrm{C} 1 \mathrm{H} 06$ during testing sequence $\mathrm{C} 1 \mathrm{X} 05-\mathrm{A}$. 


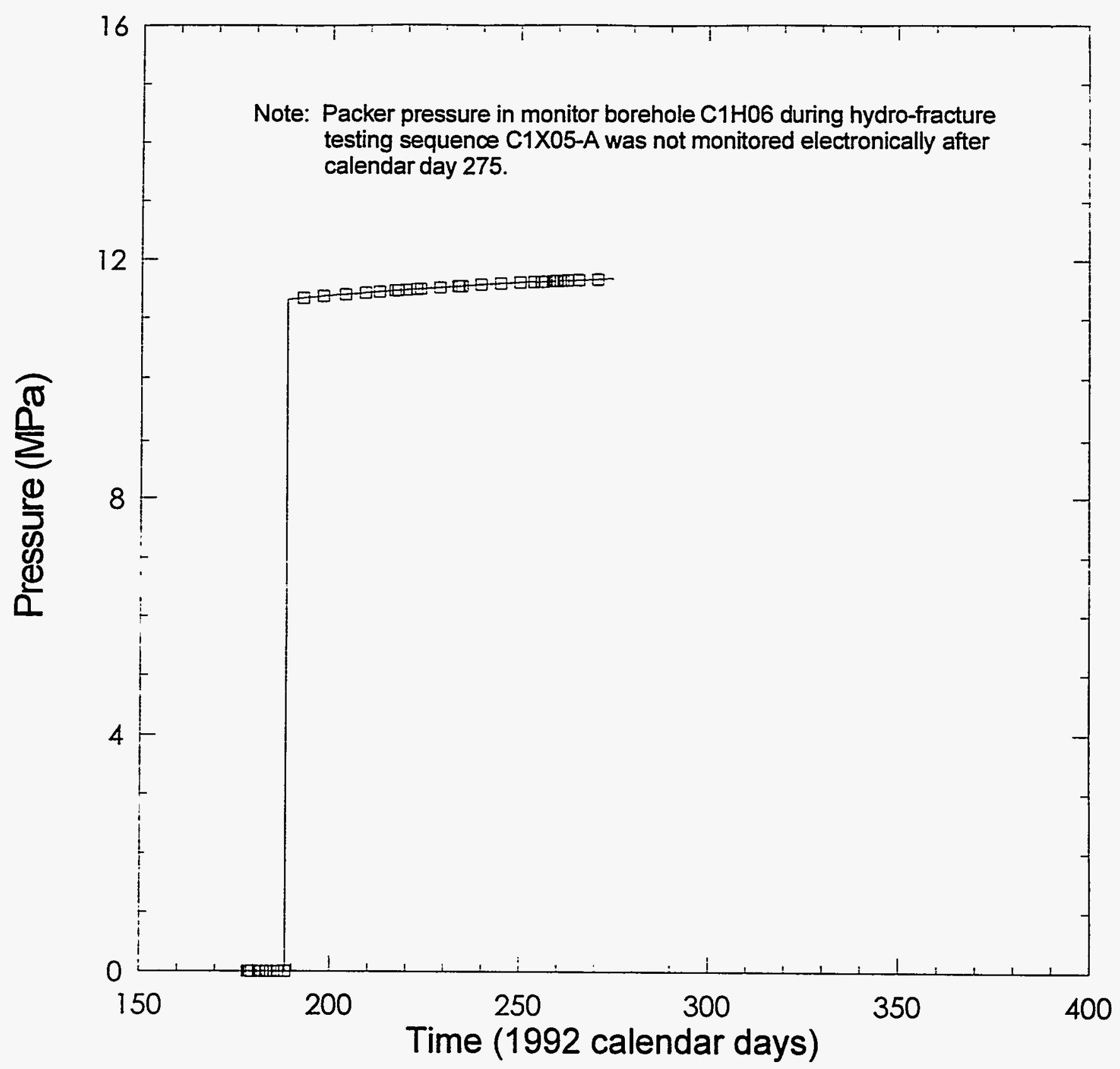

INTERA-6115-135-0

Figure 4-32. Packer pressure in observation borehole $\mathrm{C} 1 \mathrm{H} 06$ during testing sequence $\mathrm{C} 1 \mathrm{X} 05-\mathrm{A}$. 


\subsubsection{OBSERVATION BOREHOLE C1H07, TESTING SEQUENCE C1X05-A}

Table 4-15 gives a detailed description of the events that occurred in observation borehole C1H07 during testing sequence C1X05-A.

Table 4-15. Events Associated with Observation Borehole C1H07 During Testing Sequence C1X05-A

\begin{tabular}{|c|c|c|c|c|}
\hline EVENT & DATE & $\begin{array}{l}\text { CALENDAR } \\
\text { DAY }\end{array}$ & $\begin{array}{c}1992 \\
\text { CALENDAR } \\
\text { DAY) }\end{array}$ & $\begin{array}{l}\text { TIME } \\
\text { (HH:MM:SS) }\end{array}$ \\
\hline Start drilling borehole $\mathrm{C} 1 \mathrm{H} 07$ with 4-inch $(10.16-\mathrm{cm})$ core barrel to 1.47 meters. & $6-22-92$ & 174 & 174 & 10:30:00 \\
\hline Deepen borehole $\mathrm{C} 1 \mathrm{H} 07$ with 4 inch $(10.16-\mathrm{cm})$ core barrel to 2.47 meters. & $6-23-92$ & 175 & 175 & 09:18:00 \\
\hline Deepen bore hole $\mathrm{C} 1 \mathrm{H} 07$ with 4 inch $(10.16-\mathrm{cm})$ core barrel to 3.10 meters. & $6-23-92$ & 175 & 175 & 10:00:00 \\
\hline Deepen borehole C1H07 with 4-inch $(10.16-\mathrm{cm})$ core barrel to 3.82 meters. & $6-23-92$ & 175 & 175 & 10:30:00 \\
\hline Deepen borehole C1H07 with 4-inch $(10.16-\mathrm{cm})$ core barrel to 4.40 meters. & $6-23-92$ & 175 & 175 & 13:30:00 \\
\hline Deepen borehole $\mathrm{C} 1 \mathrm{H} 07$ with 4-inch $(10.16-\mathrm{cm})$ core barrel to 4.91 meters. & $6-23-92$ & 175 & 175 & 14:25:00 \\
\hline Deepen borehole $\mathrm{C} 1 \mathrm{H} 07$ with 4 inch $(10.16-\mathrm{cm})$ core barrel to 5.41 meters. & 6-24-92 & 176 & 176 & 10:00:00 \\
\hline Deepen borehole $\mathrm{C} 1 \mathrm{HO} 07$ with 4 -inch $(10.16-\mathrm{cm})$ core barrel to 6.18 meters. & $6-24-92$ & 176 & 176 & 10:30:00 \\
\hline Deepen borehole $\mathrm{C} 1 \mathrm{HO}$ with 4 -inch $(10.16-\mathrm{cm})$ core barrel to 6.88 meters. & $6-24-92$ & 176 & 176 & 11:10:00 \\
\hline Deepen borehole C1H07 with 4 inch $(10.16-\mathrm{cm})$ core barrel to 7.40 meters. & $6-24-92$ & 176 & 176 & 12:50:00 \\
\hline Deepen borehole $\mathrm{C} 1 \mathrm{H} 07$ with 4 -inch $(10.16-\mathrm{cm})$ core barrel to 8.18 meters. & 6-24-92 & 176 & 176 & 13:25:00 \\
\hline Perform video $\log$ of borehole C1HO7. & $6-26-92$ & 178 & 178 & 10:30:00 \\
\hline $\begin{array}{l}\text { Install single-packer monitor tool \#34 in borehole C1HO7-A as indicated in the test-100l } \\
\text { configuration diagram (Figure 4-12). }\end{array}$ & $6-26-92$ & 178 & 178 & 11:15:00 \\
\hline Begin data collection associated with testing sequence C1X05-A. & $6-26-92$ & 178 & 178 & 13:00:00 \\
\hline Inflate TZP to $\sim 10 \mathrm{MPa}$ & $6-26-92$ & 178 & 178 & 13:05:00 \\
\hline Shut in TZ. & $6-26-92$ & 178 & 178 & 13:05:00 \\
\hline Terminate data collection associated with testing sequence $C 1 \times 05-A$. & 4-7-93 & 97 & 463 & 09:30:43 \\
\hline Remove monitor tool \#34 from borehole $\mathrm{C} 1 \mathrm{H} 07$. & $4-16-93$ & 106 & 472 & 10:00:00 \\
\hline
\end{tabular}

Figures 4-33 through 4-35 illustrate the zone pressure, packer pressure, and zone temperature, respectively, in observation borehole $\mathrm{C} 1 \mathrm{H} 07$ for testing sequence $\mathrm{C} 1 \times 05-\mathrm{A}$.

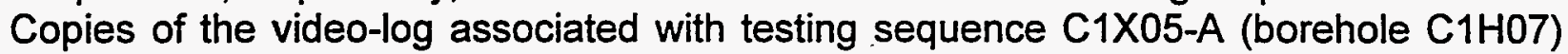
identified in Table 4-15 are provided in the SWCF under WPO \#45907 


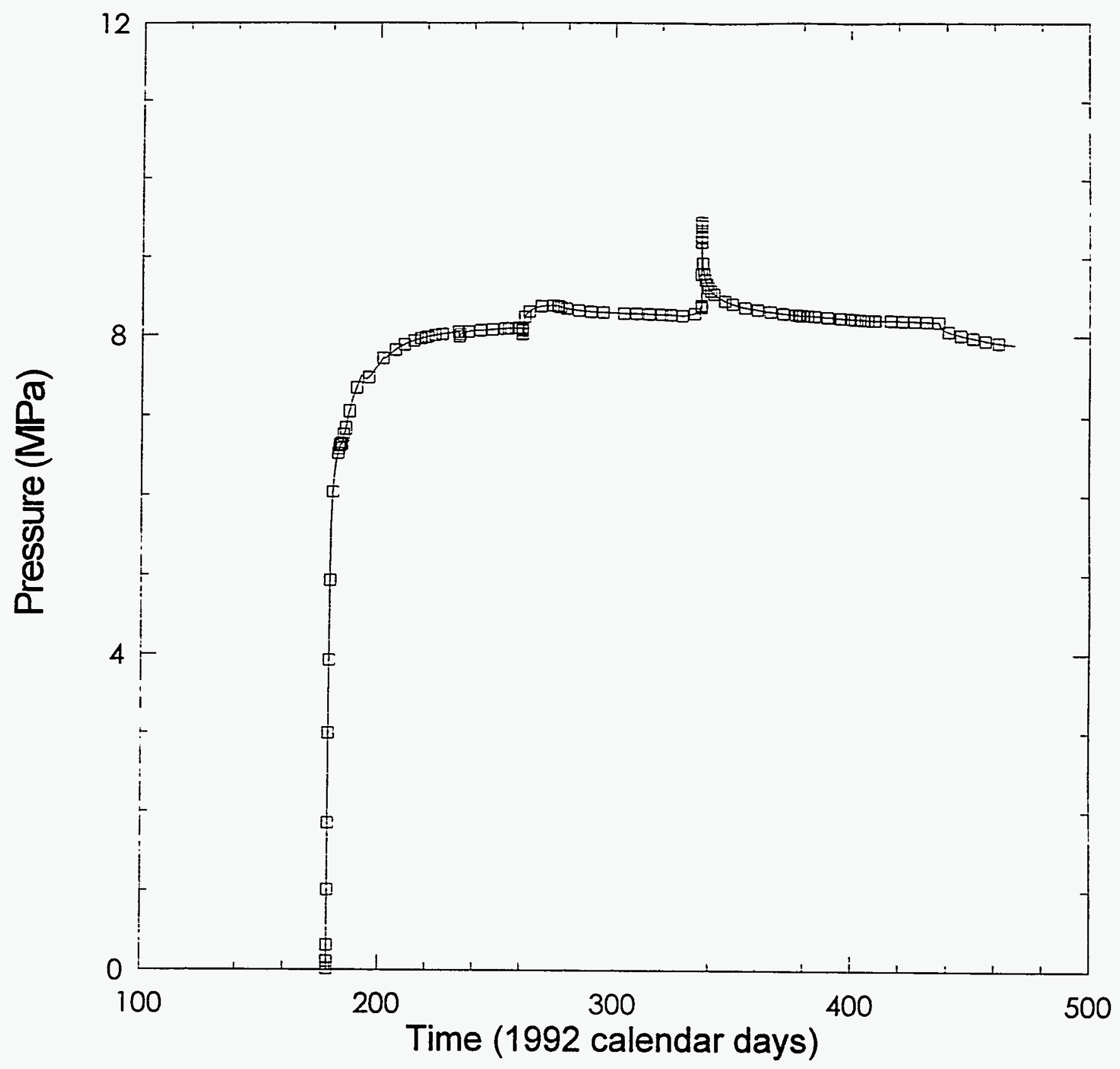

INTERU-B115-136-0

Figure 4-33. Zone pressure in observation borehole $\mathrm{C} 1 \mathrm{H} 07$ during testing sequence $\mathrm{C} 1 \mathrm{X} 05-\mathrm{A}$. 


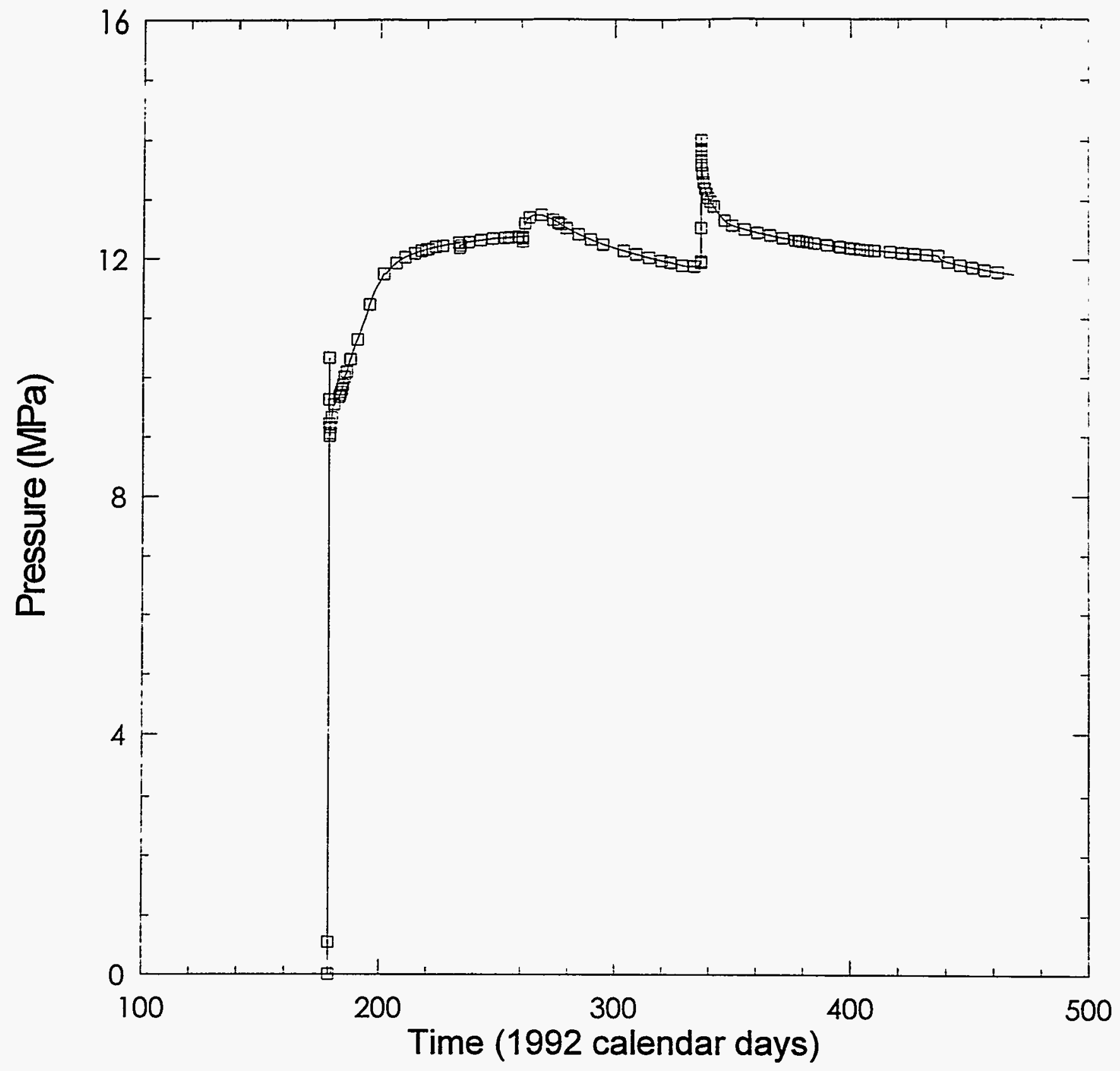

INTERA-6115-137-0

Figure 4-34. Packer pressure in observation borehole $\mathrm{C} 1 \mathrm{H} 07$ during testing sequence $\mathrm{C} 1 \mathrm{X} 05-\mathrm{A}$. 


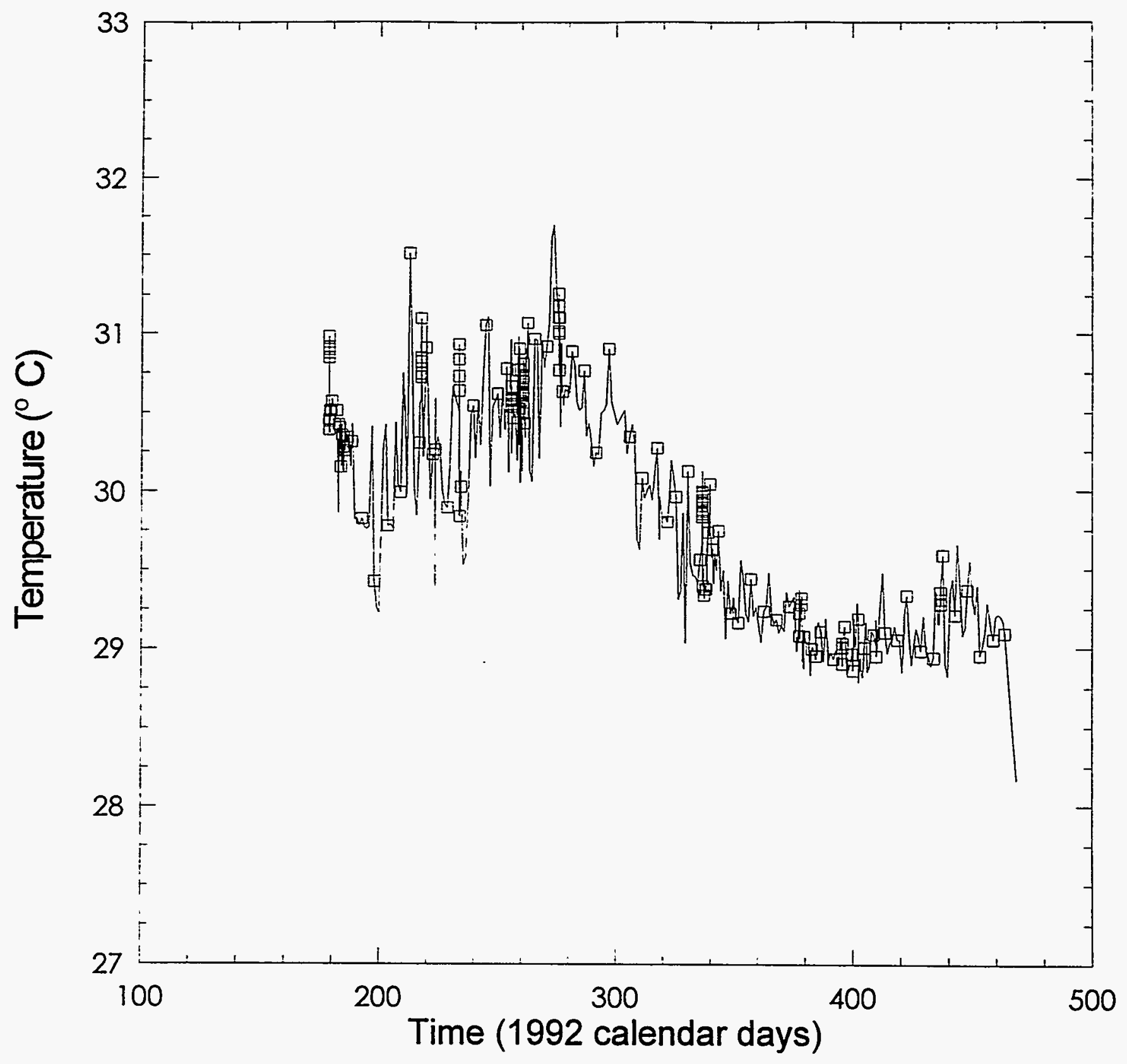

INTERA6t 15-138-0

Figure 4-35. Zone temperature in observation borehole $\mathrm{C} 1 \mathrm{HO}$ (uring testing sequence $\mathrm{C} 1 \mathrm{X} 05-\mathrm{A}$. 


\subsubsection{OBSERVATION BOREHOLE C1X06, TESTING SEQUENCE C1X05-A}

Table 4-16 gives a detailed description of the events that occurred in observation borehole C1X06 during testing sequence C1X05-A.

Table 4-16. Events Associated with Observation Borehole C1X06 During Testing Sequence C1X05-A

\begin{tabular}{|c|c|c|c|c|}
\hline EVENT & DATE & $\begin{array}{l}\text { CALENDAR } \\
\text { DAY }\end{array}$ & $\begin{array}{c}1992 \\
\text { CALENDAR } \\
\text { DAY }\end{array}$ & $\begin{array}{c}\text { TIME } \\
\text { (HH:MM:SS) }\end{array}$ \\
\hline Start drilling borehole $\mathrm{C} 1 \times 06$ with 4 inch $(10.16-\mathrm{cm})$ core barrel to 1.16 meters. & $9-28-92$ & 272 & 272 & 10:44:00 \\
\hline Deepen borehole C1X06 with 4-inch $(10.16-\mathrm{cm})$ core barrel to 2.01 meters. & $9-28-92$ & 272 & 272 & 11:20:00 \\
\hline Deepen borehole C1X06 with 4-inch $(10.16-\mathrm{cm})$ core barrel to 2.84 meters. & $9-28-92$ & 272 & 272 & 12:41:00 \\
\hline Deepen borehole C1X06 with 4-inch $(10.16-\mathrm{cm})$ core barrel to 3.60 meters. & $9-28-92$ & 272 & 272 & 13:09:00 \\
\hline Deepen borehole C1X06 with 4-inch $(10.16-\mathrm{cm})$ core barrel to 4.36 meters. & $9-28-92$ & 272 & 272 & 13:55:00 \\
\hline Deepen borehole C1X06 with 4-inch $(10.16-\mathrm{cm})$ core barrel to 5.16 meters. & 9-28-92 & 272 & 272 & 14:15:00 \\
\hline Deepen borehole C1X06 with 4-inch $(10.16-\mathrm{cm})$ core barrel to 6.05 meters. & $9-29-92$ & 273 & 273 & 09:56:00 \\
\hline Deepen borehole C1X06 with 4-inch $(10.16-\mathrm{cm})$ core barrel to 6.94 meters. & $9-29-92$ & 273 & 273 & 12:20:00 \\
\hline Deepen borehole C1X06 with 4-inch $(10.16-\mathrm{cm})$ core barrel to 7.37 meters. & $9-29-92$ & 273 & 273 & 13:05:00 \\
\hline Face off bottom of borehole $\mathrm{C} 1 \times 06$ with 4 -inch $(10.16-\mathrm{cm})$ plug bit to 7.63 meters. & $9-30-92$ & 274 & 274 & 10:00:00 \\
\hline $\begin{array}{l}\text { Install single-packer monitor tool \#36 in borehole } \mathrm{CixO6} \text { as indicated in the test-100l } \\
\text { configuration diagram (Figure 4-13). }\end{array}$ & $9-30-92$ & 274 & 274 & 12:00:00 \\
\hline Begin data collection associated with testing sequence C1X05-A. & $10-1-92$ & 275 & 275 & $11: 00: 00$ \\
\hline Inflate $T Z \mathrm{P}$ to $\sim 11 \mathrm{MPa}$ & $10-1-92$ & 275 & 275 & 11:19:05 \\
\hline Shut in $\mathrm{Z}$. & 10-1-92 & 275 & 275 & 11:49:37 \\
\hline Terminate data collection associated with testing sequence C1X05-A. & 4.7 .93 & 97 & 463 & 09:30:43 \\
\hline Remove monitor tool \#36 from borehole C $1 \times 06$. & 4-16-93 & 106 & 472 & 09:55:00 \\
\hline
\end{tabular}

Figures 4-36 through 4-38 illustrate the zone pressure, packer pressure, and axial-LVDT displacement, respectively, in observation borehole $\mathrm{C} 1 \mathrm{X06}$ for testing sequence $\mathrm{C} 1 \mathrm{X} 05-\mathrm{A}$. 


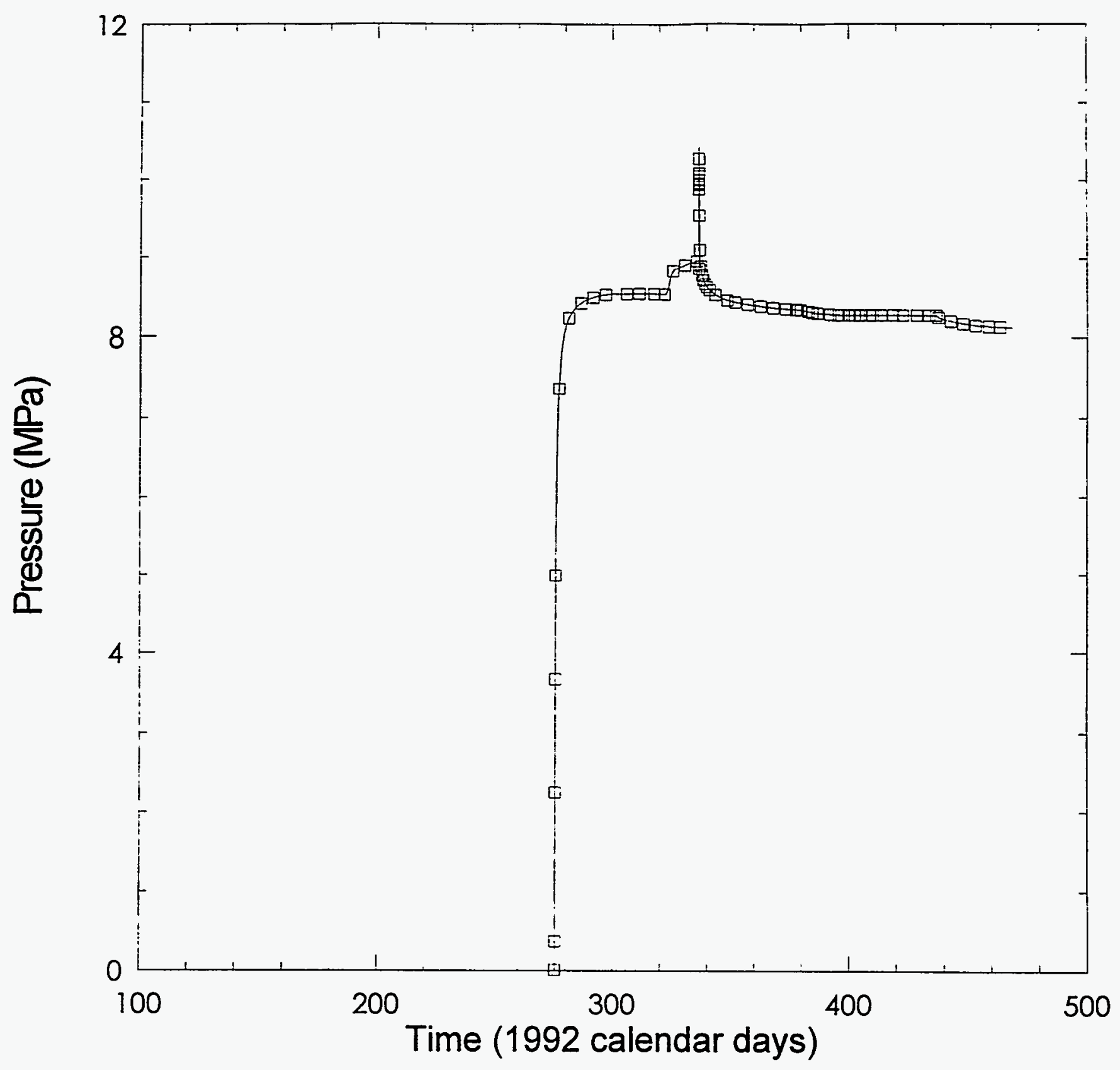

INTERA-6115-1320

Figure 4-36. Zone pressure in observation borehole $\mathrm{C} 1 \mathrm{X} 06$ during testing sequence $\mathrm{C} 1 \mathrm{X} 05-\mathrm{A}$. 


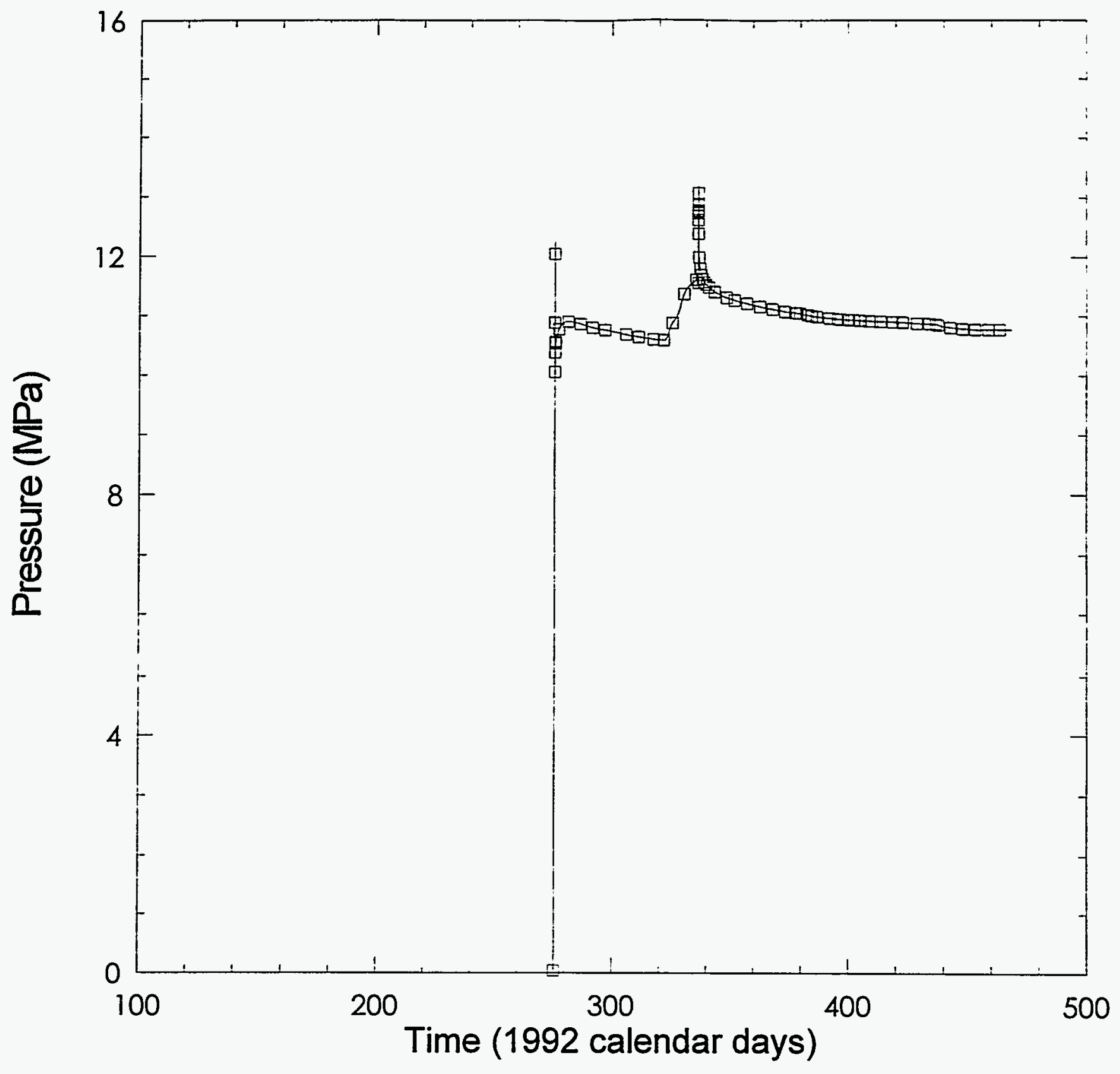

INTERA- $115-140-0$

Figure 4-37. Packer pressure in observation borehole $\mathrm{C} 1 \mathrm{X} 06$ during testing sequence $\mathrm{C} 1 \mathrm{X} 05-\mathrm{A}$. 


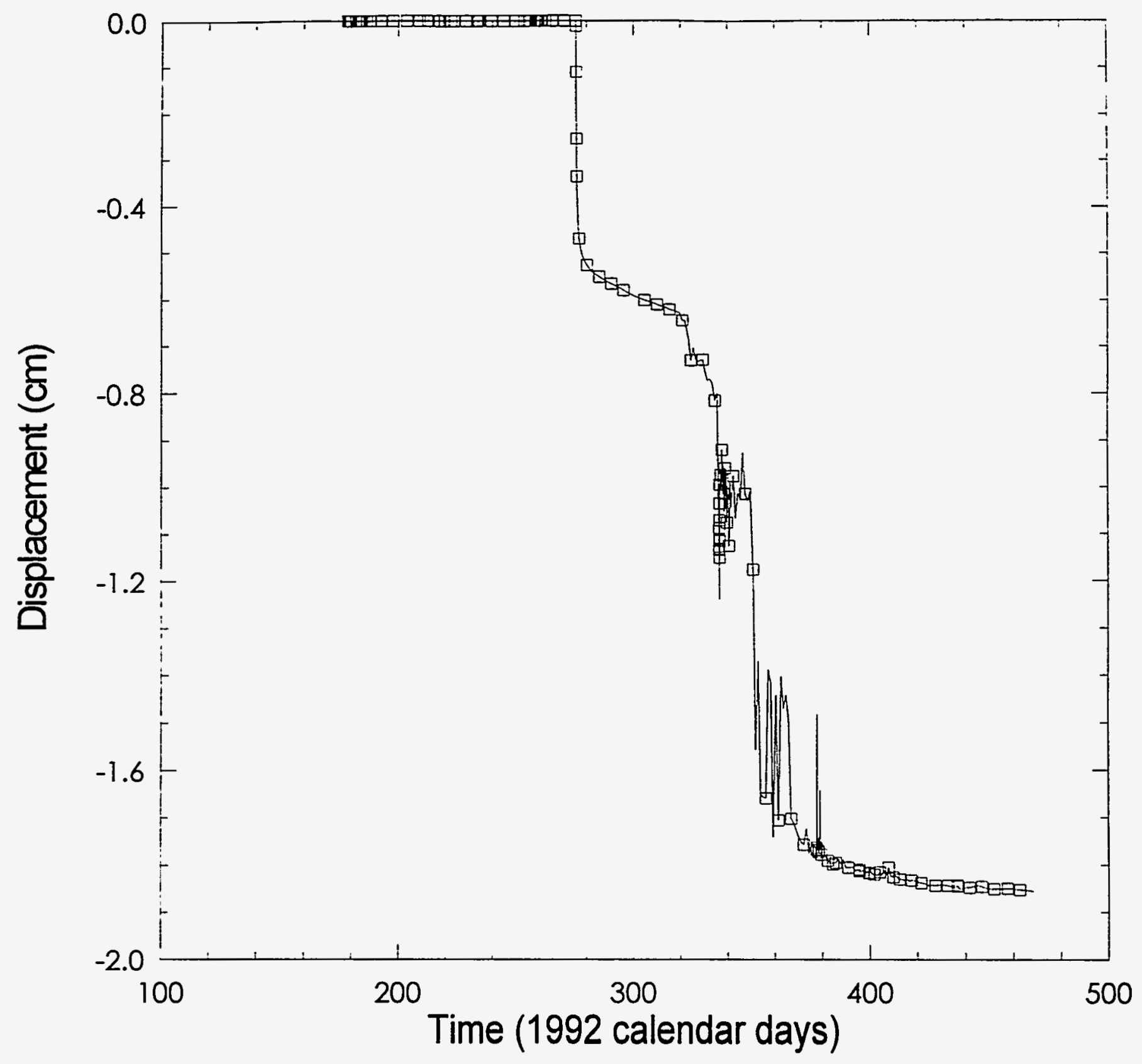

INTERA-6115-141-0

Figure 4-38. Axial-LVDT displacement in observation borehole C1X06 during testing sequence C1X05-A. 
4.6.2.5 OBSERVATION BOREHOLE C1X10, TESTING SEQUENCE C1X05-A

Table 4-17 gives a detailed description of the events that occurred in observation borehole C1X10 during testing sequence C1X05-A.

Table 4-17. Events Associated with Observation Borehole C1X10 During Testing Sequence C1X05-A

\begin{tabular}{|c|c|c|c|c|}
\hline EVENT & DATE & $\begin{array}{l}\text { CALENDAR } \\
\text { DAY }\end{array}$ & $\begin{array}{c}1992 \\
\text { CALENDAR } \\
\text { DAY }\end{array}$ & $\begin{array}{c}\text { TIME } \\
\text { (HH:MM:SS) }\end{array}$ \\
\hline Drill borehole C1X10 with 3-inch $(7.62-\mathrm{cm})$ core barrel to 10.16 meters. & $11-12-91$ & 316 & N/A & 14:00:00 \\
\hline Perform video log of borehole C1X10. & $11-12-91$ & 316 & NA & 15:00:00 \\
\hline $\begin{array}{l}\text { Install test tool in borehole } \mathrm{C} 1 \mathrm{X10} \text { as indicated in the test-tool configuration diagram } \\
\text { (Figure 4-8). }\end{array}$ & $11-12-91$ & 316 & NA & $15: 30: 00$ \\
\hline Begin testing sequence $\mathrm{C} 1 \times 10$. & $11-12-91$ & 316 & N/A & 16:52:00 \\
\hline Terminate testing sequence C1X10. & $6-26-92$ & 178 & 178 & 08:18:35 \\
\hline Begin data collection associated with testing sequence C1X05-A. & $6-26-92$ & 178 & 178 & 13:00:00 \\
\hline Terminate data collection associated with testing sequence $\mathrm{C} 1 \times 05-\mathrm{A}$. & 3-11-93 & 70 & 437 & 10:30:00 \\
\hline Remove monitor tool from borehole C1X10. & $3-12-93$ & 71 & 437 & 11:30:00 \\
\hline
\end{tabular}

Figure 4-39 illustrates the zone pressure in observation borehole $\mathrm{C} 1 \times 10$ for testing sequence $C 1 \times 05-A$. Copies of the video-log associated with testing sequence $C 1 \times 05-A$ (borehole C1X10) identified in Table 4-17 are provided in the SWCF under WPO \#45907. 


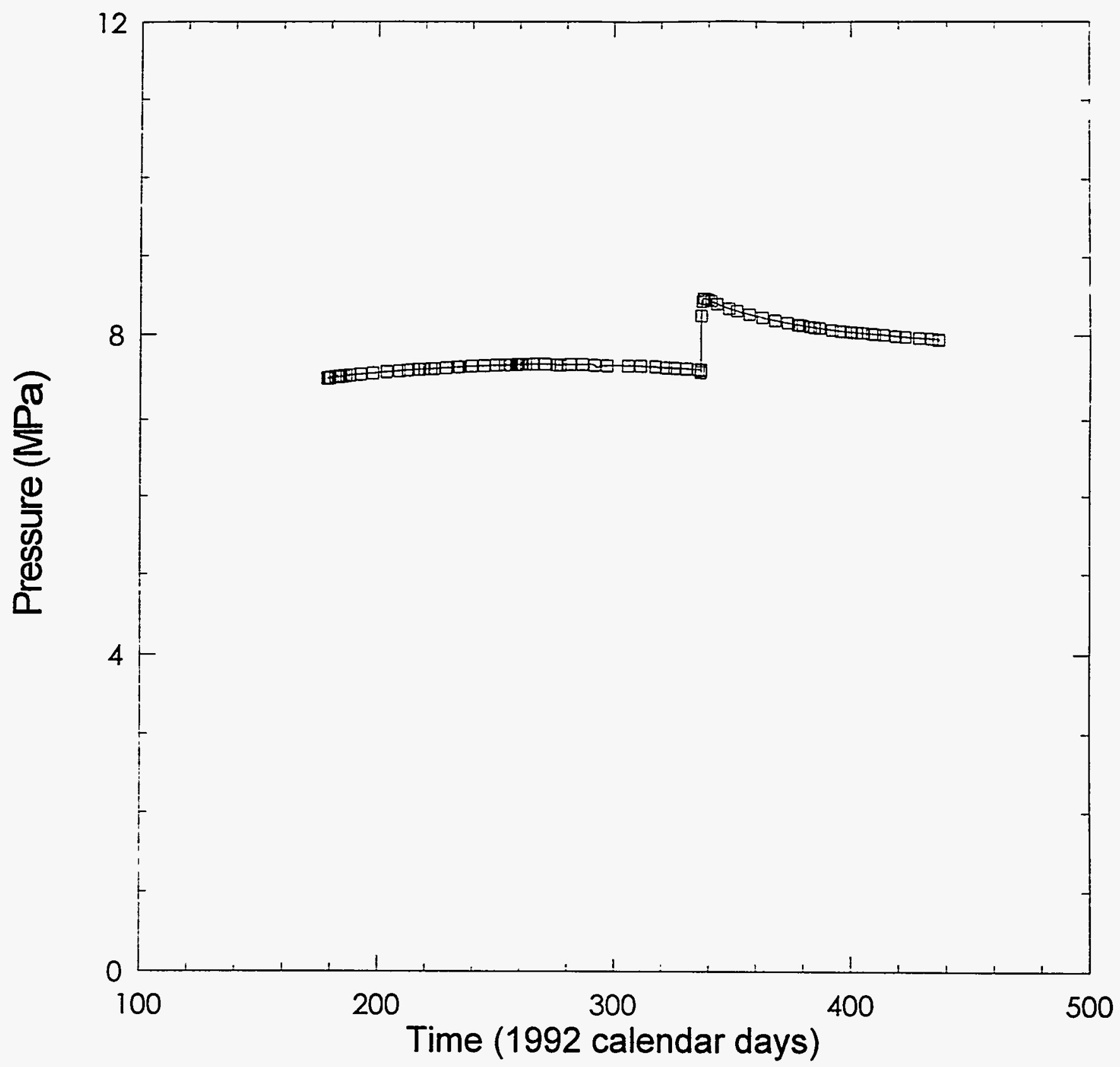

INTERA-6115-142-0

Figure 4-39. Test-zone pressure in observation borehole $\mathrm{C} 1 \times 10$ during testing sequence $\mathrm{C} 1 \mathrm{X} 05-\mathrm{A}$. 


\subsubsection{OBSERVATION BOREHOLE C2H01, TESTING SEQUENCE C1X05-A}

Table 4-18 gives a detailed description of the events that occurred in observation borehole $\mathrm{C} 2 \mathrm{H} 01$ during testing sequence $\mathrm{C} 1 \mathrm{X05}-\mathrm{A}$.

Table 4-18. Events Associated with Observation Borehole C2H01 During Testing Sequence C1X05-A

\begin{tabular}{|c|c|c|c|c|}
\hline EVENT & DATE & $\begin{array}{l}\text { CALENDAR } \\
\text { DAY }\end{array}$ & $\begin{array}{c}1992 \\
\text { CALENDAR } \\
\text { DAY }\end{array}$ & $\begin{array}{c}\text { TIME } \\
\text { (HH:MM:SS) }\end{array}$ \\
\hline Borehole С2HO1 drilled to 5.58 meters. & $8-4-88$ & 217 & NA & N/A \\
\hline Begin long-term fluid-pressure monitoring. & 8-22-89 & 234 & NA & N/A \\
\hline $\begin{array}{l}\text { Install single-packer fluid-pressure monitoring tool as indicated in the test-tool } \\
\text { configuration diagram (Figure 5-9). }\end{array}$ & 3-13-91 & 72 & N/A & N/A \\
\hline Begin data collection associated with testing sequence $\mathrm{C} 1 \times 10$. & 1-29-92 & 29 & 29 & 14:43:00 \\
\hline Terminate data collection associated with testing sequence $\mathrm{C} 1 \times 10$. & $6-26-92$ & 178 & 178 & 08:18:35 \\
\hline Begin data collection associated with testing sequence $\mathrm{C} 1 \times 05-\mathrm{A}$. & $6-26-92$ & 178 & 178 & 13:00:00 \\
\hline Terminate data collection associated with testing sequence $\mathrm{C} 1 \times 05-\mathrm{A}$. & 8-3-92 & 216 & 216 & $16: 06: 00$ \\
\hline
\end{tabular}

Figure 4-40 illustrates the zone pressure in observation borehole $\mathrm{C} 2 \mathrm{H} 01$ for testing sequence $\mathrm{C} 1 \mathrm{X} 05-\mathrm{A}$. 


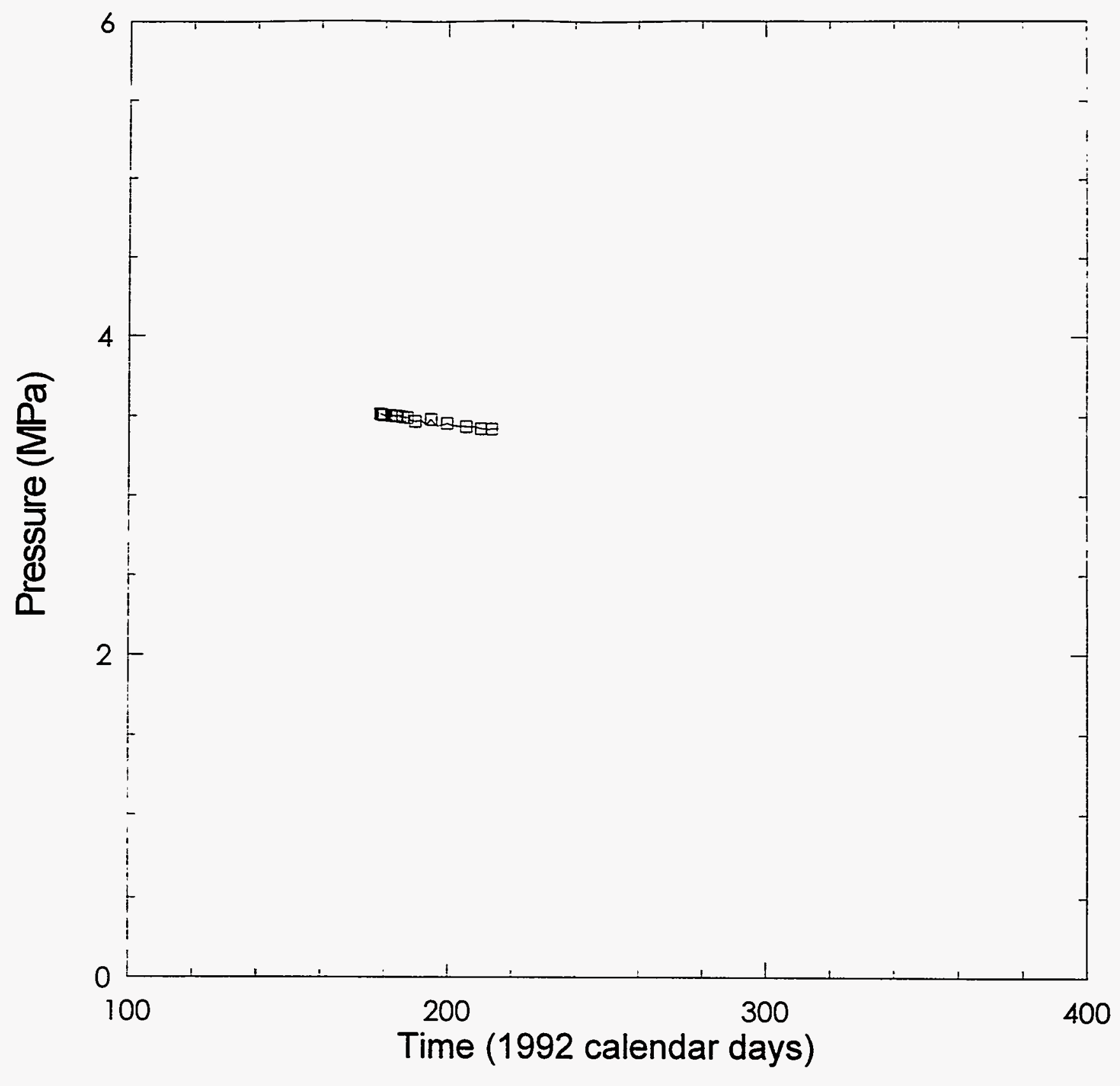

INTERA-6115-143-0

Figure 4-40. Zone pressure in observation borehole $\mathrm{C} 2 \mathrm{H} 01$ during testing sequence $\mathrm{C} 1 \times 05-\mathrm{A}$. 


\subsubsection{Borehole C1X05, Testing Sequence C1X05-B}

Testing sequence $\mathrm{C} 1 \mathrm{X} 05-\mathrm{B}$ took place in Room $\mathrm{C} 1$ in borehole $\mathrm{C} 1 \mathrm{X} 05-\mathrm{B}$ with associated observation boreholes $\mathrm{C} 1 \mathrm{H} 07$ and $\mathrm{C} 1 \mathrm{X} 06$. This test sequence was designed to investigate the pre- and post-hydrofracture brine permeability of MB140. Table 4-19 gives a detailed description of the events that occurred in test borehole $\mathrm{C} 1 \mathrm{X} 05$ during the testing sequence C1X05-B.

Table 4-19. Events Associated with Test Borehole C1X05 During Testing Sequence C1X05-B

\begin{tabular}{|c|c|c|c|c|}
\hline EVENT & DATE & $\begin{array}{c}\text { CALENDAR } \\
\text { DAY }\end{array}$ & $\begin{array}{c}1993 \\
\text { CALENDAR } \\
\text { DAY }\end{array}$ & $\begin{array}{c}\text { TIME } \\
\text { (HH:MM:SS) }\end{array}$ \\
\hline Borehole C1X05 drilled with 3-inch $(7.62-\mathrm{cm})$ core barrel to 9.14 meters. & $6-29-92$ & 181 & N/A & $12: 00: 00$ \\
\hline Begin testing sequence $C 1 \times 05-A$. & 6-30-92 & 182 & NA & 12:00:00 \\
\hline Terminate testing sequence $C 1 \times 05-A$. & 4-7-93 & 97 & 97 & $09: 30: 43$ \\
\hline Deepen borehole C1X05 with 3-inch $(7.62-\mathrm{cm})$ core barrel to 10.62 meters. & $6-7-93$ & 158 & 158 & 14:25:00 \\
\hline Deepen borehole C1X05 with 3-inch $(7.62-\mathrm{cm})$ core barrel to 12.04 meters. & $6-8-93$ & 159 & 159 & $09: 20: 00$ \\
\hline Deepen borehole C1X05 with 3-inch $(7.62-\mathrm{cm})$ core barrel to 13.51 meters. & $6-8-93$ & 159 & 159 & 09:50:00 \\
\hline Deepen borehole C1X05 with 3-inch $(7.62-\mathrm{cm})$ core barrel to 14.94 meters. & $6-8-93$ & 159 & 159 & 10:20:00 \\
\hline Deepen borehole C1X05 with 3-inch $(7.62-\mathrm{cm})$ core barrel to 16.33 meters. & 6-8-93 & 159 & 159 & 10:45:00 \\
\hline Deepen borehole C1X05 with 3-inch $(7.62-\mathrm{cm})$ core barrel to 17.78 meters. & $6-8-93$ & 159 & 159 & $12: 45: 00$ \\
\hline Deepen borehole C1X05 with 3-inch (7.62-cm) core barrel to 19.19 meters. & $6-8-93$ & 159 & 159 & 13:05:00 \\
\hline Deepen borehole $C_{1 X 05}$ with 3-inch $(7.62-\mathrm{cm})$ core barrel to 20.68 meters. & 6-11-93 & 162 & 162 & 12:30:00 \\
\hline Deepen borehole $C 1 \times 05$ with 3-inch $(7.62-\mathrm{cm})$ core barrel to 22.10 meters. & 6-14-93 & 165 & 165 & 08:25:00 \\
\hline Deepen borehole $C_{1 X 05}$ with 3 -inch $(7.62-\mathrm{cm})$ core bartel to 23.44 meters. & 6-14-93 & 165 & 165 & 08:51:00 \\
\hline Deepen borehole C1X05 with 3 -inch $(7.62-\mathrm{cm})$ core barrel to 24.92 meters. & 6-14-93 & 165 & 165 & 09:35:00 \\
\hline Deepen borehole C1X05 with 3-inch $(7.62-\mathrm{cm})$ core barrel to 26.34 meters. & 6-14-93 & 165 & 165 & $10: 30: 00$ \\
\hline Deepen borehole C1X05 with 3-inch $(7.62-\mathrm{cm})$ core barrel to 26.59 meters. & 6-14-93 & 165 & 165 & 11:20:00 \\
\hline Deepen borehole $\mathrm{C} 1 \mathrm{X} 05$ with 3-inch $(7.62-\mathrm{cm})$ core barrel to 28.09 meters. & 6-14-93 & 165 & 165 & 12:30:00 \\
\hline Deepen borehole $\mathrm{C} 1 \times 05$ with 3 -inch $(7.62-\mathrm{cm})$ core bartel to 29.54 melers. & 6-14-93 & 165 & 165 & 13:05:00 \\
\hline Deepen borehole $C_{1} \times 05$ with 3 -inch $(7.62-\mathrm{cm})$ core barrel to 30.20 meters. & 6-14-93 & 165 & 165 & 13:25:00 \\
\hline $\begin{array}{l}\text { Install multipacker test tool in borehole } C_{1} \times 05 \text { as indicated in the test-tool } \\
\text { configuration diagram (Figure 4-14). }\end{array}$ & 6-15-93 & 166 & 166 & 12:00:00 \\
\hline Begin data file C1X05B01. & 6-15.93 & 166 & 166 & 13:25:20 \\
\hline Increase GZ pressure via accumulator. & 6-15-93 & 166 & 166 & 13:28:15 \\
\hline Replace value on hydrofrac panel. & 7-7-93 & 188 & 188 & 11:07:37 \\
\hline Shut in GZ from accumulator. & 7-13-93 & 193 & 193 & 10:53:00 \\
\hline Increase TZ1P pressure to 13.8 MPa. & $7-15-93$ & 196 & 196 & 12:28:51 \\
\hline Increase TZ2P pressure to - 11.7 MPa. & $7-15-93$ & 196 & 196 & 12:32:15 \\
\hline Decreased TZ pressure. & $7-15-93$ & 196 & 196 & 12:35:25 \\
\hline Remove multipacker test tool for repairs. & $7-28-93$ & 209 & 209 & 08:23:00 \\
\hline
\end{tabular}




\section{Table 4-19 (Continued). Events Associated with Test Borehole C1X05 During Testing Sequence C1X05-B}

\begin{tabular}{|c|c|c|c|c|}
\hline EVENT & DATE & $\begin{array}{l}\text { CALENDAR } \\
\text { DAY }\end{array}$ & $\begin{array}{c}1993 \\
\text { CALENDAR } \\
\text { DAY }\end{array}$ & $\begin{array}{c}\text { TIME } \\
\text { (HH:MM:SS) }\end{array}$ \\
\hline Install multipacker test tool to original position. & $7-28-93$ & 209 & 209 & 14:00:00 \\
\hline End data file C1X05801. & $8-18-93$ & 230 & 230 & $12: 49: 30$ \\
\hline Replace gage on GZP. & 8-19-93 & 231 & 231 & $09: 48: 35$ \\
\hline Begin data file $\mathrm{C} 1 \times 05 \mathrm{~B} 02$. & 8-19-93 & 231 & 231 & 12:05:02 \\
\hline Remove gage from TZ1. & $9-1-93$ & 244 & 244 & 10:45:00 \\
\hline End data file C1X05B02. & $9-23-93$ & 266 & 266 & $04: 38: 21$ \\
\hline Begin data file C1 $\times 05 B 03$. & 9-23-93 & 266 & 266 & $08: 31: 50$ \\
\hline DAS not functioning property upon arrival. & $10-8-93$ & 281 & 281 & 08:08:00 \\
\hline End data file C1X05BO3. & $10-6-93$ & 292 & 292 & 12:23:35 \\
\hline Begin data file C1X05BO4. & $10-6-93$ & 292 & 292 & 12:43:17 \\
\hline $\begin{array}{l}\text { Begin constant-pressure-injection test \#1 in TZ2 at }-1 \mathrm{MPa} \text { above } \mathrm{TZ2} \text { pressure } \\
\qquad(-10.4 \mathrm{MPa}) .\end{array}$ & $10-21-93$ & 294 & 294 & 09:29:00 \\
\hline End data file C1X05B04. & $10-27-93$ & 300 & 300 & 07:38:44 \\
\hline Begin data file C1X05B05. & $10-27-93$ & 300 & 300 & 08:09:28 \\
\hline Shut in TZ2 from DPT panel terminating constant-pressure-injection test \#1 in TZ2. & $11-1-93$ & 305 & 305 & 09:02:00 \\
\hline DAS not functioning property upon arrival. & $11-8-93$ & 312 & 312 & 09:50:00 \\
\hline DAS power supplied by generator. & $11-10-93$ & 314 & 314 & 09:00:00 \\
\hline End data file C1X05805. & 11-19-93 & 323 & 323 & 12:44:37 \\
\hline Begin data file C1X05B05. & $11-19-93$ & 323 & 323 & 14:15:00 \\
\hline End data file C1X05B06. & $11-22-93$ & 326 & 326 & 09:32:21 \\
\hline Begin data file C1X05B07. & $11-22-93$ & 326 & 326 & 09:35:18 \\
\hline Attempt to perform hydrofracture of $\mathrm{MB140}$ at $22.8 \mathrm{MPa}$. & $11-22-93$ & 326 & 326 & $11: 20: 12$ \\
\hline Shut in $T Z 2$. & $11-22-93$ & 326 & 326 & $11: 25: 00$ \\
\hline Perform hydrofracture of MB140. & $11-22-93$ & 326 & 326 & 11:41:06 \\
\hline Shut in TZ2. & $11-22-93$ & 326 & 326 & 11:45:00 \\
\hline $\begin{array}{l}\text { Begin constant-pressure-withdrawal test \#1 in TZ2 at }-10.3 \mathrm{MPa} \\
\qquad(\sim 650 \mathrm{~mL} \text { of fluid recovered }) .\end{array}$ & $11-22-93$ & 326 & 326 & $11: 50: 46$ \\
\hline Shut in $T 22$ from DPT panel terminating constant-pressure-wthdrawal test \#1 in TZ2. & $11-22-93$ & 326 & 326 & 11:58:58 \\
\hline Perform hydrofracture of MB140 (injected - 5.7 liters of fluid in TZ2). & $11-22-93$ & 326 & 326 & 12:04:00 \\
\hline End data file C $1 \times 05 B 07$. & $11-22-93$ & 326 & 326 & 12:42:29 \\
\hline Begin data file C1X05B08. & $11-22-93$ & 326 & 326 & 14:06:44 \\
\hline $\begin{array}{l}\text { Begin constant-pressure-withdrawal test } \# 2 \text { in TZ2 at }-10.3 \mathrm{MPa} \\
\text { ( } \sim 5 \text { liters of fluid recovered). }\end{array}$ & $11-22-93$ & 326 & 326 & $14: 10: 40$ \\
\hline Shut in TZ2 from DPT panel terminating constant-pressure-withdrawal test $\# 2$ in $T Z 2$. & $11-22-93$ & 326 & 326 & 14:45:00 \\
\hline Perform hydrofracture of MB140. & $11-22-93$ & 326 & 326 & 15:13:00 \\
\hline Shut in TZ2. & $11-22-93$ & 326 & 326 & $16: 40: 00$ \\
\hline
\end{tabular}


Table 4-19 (Continued). Events Associated with Test Borehole C1X05 During Testing Sequence C1X05-B

\begin{tabular}{|c|c|c|c|c|}
\hline EVENT & DATE & $\begin{array}{l}\text { CALENDAR } \\
\text { DAY }\end{array}$ & $\begin{array}{c}1993 \\
\text { CALENDAR } \\
\text { DAY }\end{array}$ & $\begin{array}{c}\text { TIME } \\
\text { (HH:MM:SS) }\end{array}$ \\
\hline $\begin{array}{l}\text { Begin constant-pressure-withdrawal test } \# \text { in TZ2 at } \sim 10.3 \mathrm{MPa}(-1350 \mathrm{~mL} \text { of fluid } \\
\text { recovered). }\end{array}$ & $11-22-93$ & 326 & 326 & 16:55:00 \\
\hline Shut in TZ2 from DPT panel terminating constant-pressure-withdrawal test \#3 in TZ2. & $11-23-93$ & 327 & 327 & 08:41:00 \\
\hline Increase packer pressure to $18.3 \mathrm{MPa}$. & $11-23-93$ & 327 & 327 & 09:50:00 \\
\hline Perform hydrofracture of MB140 (injected $-8.3 \mathrm{~L}$ ). & $11-23-93$ & 327 & 327 & 10:12:35 \\
\hline Shut-in TZ2. & $11-23-93$ & 327 & 327 & 10:14:20 \\
\hline Drain fluid from $T 22$. & 11-23-93 & 327 & 327 & 10:21:00 \\
\hline Perform hydro-fracture of MB140 (injected 30.7). & $11-23-93$ & 327 & 327 & 10:41:30 \\
\hline Shut in TZ2. & $11-23-93$ & 327 & 327 & 10:47:00 \\
\hline Begin constant-pressure-withdrawal test \#4 in TZ2. & $11-23-93$ & 327 & 327 & 11:42:00 \\
\hline $\begin{array}{l}\text { Decrease packer pressure to } 15.4 \mathrm{MPa} \text { terminating constant-pressure-withdrawal test } \\
\qquad \# 4 \text { in TZ2. }\end{array}$ & $11-23-93$ & 327 & 327 & 13:30:00 \\
\hline $\begin{array}{l}\text { Begin constant-pressure-withdrawal test }+5 \text { in } 722 \text { at } \sim 10.0 \mathrm{MPa} \\
\qquad(\sim 13.7 \text { liters of fluid recovered). }\end{array}$ & 11-23-93 & 327 & 327 & 13:44:00 \\
\hline Shut in TZ2 from DPT panel terminating constant-pressure-withdrawal test \#5 in TZ2. & $11-24-93$ & 328 & 328 & 08:30:00 \\
\hline End data file C1X05B08. & $12-1-93$ & 335 & 335 & 08:26:15 \\
\hline Begin data file C1X05B09. & $12-1-93$ & 335 & 335 & 08:36:21 \\
\hline End data file C1X05809. & $12-30-93$ & 364 & 364 & 07:19:22 \\
\hline Begin data file C1X05B10. C1X05B11, and C1X05B12. & $12-30-93$ & 364 & 364 & 10:15:56 \\
\hline $\begin{array}{l}\text { Begin constant-pressure-injection test \#2 in TZ2 at } ~-0.5 \mathrm{MPa} \text { above } \mathrm{TZ2} \text { pressure } \\
\qquad(12.2 \mathrm{MPa}) .\end{array}$ & $1-10-94$ & 10 & 375 & 13:20:00 \\
\hline End data file C1X05B10, C1X05B11, and C1X05B12. & $1-13-94$ & 13 & 378 & 13:29:22 \\
\hline Begin data file C1X05B13. & $1-13-94$ & 13 & 378 & 13:32:37 \\
\hline Terminate constant-pressure-injection test $\# 2$ in TZ2. & $1-20-94$ & 20 & 385 & 09:48:00 \\
\hline $\begin{array}{l}\text { Begin constant-pressure-injection test } \$ 3 \text { in TZ2 at }-1 \mathrm{MPa} \text { above TZ2 pressure } \\
\qquad(-12.7 \mathrm{MPa}) .\end{array}$ & $1-20-94$ & 20 & 385 & 09:49:00 \\
\hline Shut in $T Z 2$ from DPT panel terminating constant-pressure-injection test \#3 in $7 Z 2$. & $1-20-94$ & 20 & 385 & 13:33:00 \\
\hline End data file C1X05B13. & $2-8-94$ & 39 & 404 & 11:34:25 \\
\hline Begin data file C1X05B14. & 2-8-94 & 39 & 404 & 13:36:36 \\
\hline DAS not functioning properly upon arrival. & 2-10-94 & 41 & 406 & 12:35:00 \\
\hline Increase packer pressure. & 2-11-94 & 42 & 407 & 12:50:00 \\
\hline $\begin{array}{l}\text { Begin constant-pressure-withdrawal test \#6 in TZ2 at } \sim 0.5 \mathrm{MPa} \text { below } \mathrm{TZ2} \text { pressure } \\
\qquad(\sim 11.2 \mathrm{MPa}) .\end{array}$ & 2-15-94 & 46 & 411 & 09:00:00 \\
\hline DAS not functioning property upon arrival. & 2-17-94 & 48 & 413 & 14:15:00 \\
\hline End data file C1X05B14. & 2-18-94 & 49 & 414 & 10:04:23 \\
\hline Begin data file C1X05B15. & 2-18-94 & 49 & 414 & 10:07:54 \\
\hline End data file C1X05B15. & 2-25-94 & 56 & 421 & 09:34:53 \\
\hline Begin data file C1X05B16. & 2-25-94 & 56 & 421 & 13:36:33 \\
\hline Shut in TZ2 from DPT panel terminating constant-pressure-withdrawal test \#6 in TZ2. & $3-1-94$ & 60 & 425 & 11:53:05 \\
\hline
\end{tabular}


Table 4-19 (Continued). Events Associated with Test Borehole C1X05 During Testing Sequence C1X05-B

\begin{tabular}{|c|c|c|c|c|}
\hline EVENT & DATE & $\begin{array}{l}\text { CALENDAR } \\
\text { DAY }\end{array}$ & $\begin{array}{c}1993 \\
\text { CALENDAR } \\
\text { DAY }\end{array}$ & $\begin{array}{c}\text { TIME } \\
\text { (HH:MM:SS) }\end{array}$ \\
\hline DAS not functioning property upon arrival. & $3-3-94$ & 62 & 427 & 10:33:00 \\
\hline End data file C1X05B16. & 3-15-94 & 74 & 439 & 11:48:09 \\
\hline Begin data file C1X05B17. & 3-15-94 & 74 & 439 & 11:53:13 \\
\hline Performed a 11.5 MPa pulse-withdrawal test on TZ2 removing $~ 254 \mathrm{~mL}$ of fluid. & $3-24-94$ & 83 & 448 & 13:04:28 \\
\hline Remove multipacker test tool from borehole $\mathrm{C} 1 \times 05$. & 4-11-94 & 101 & 466 & 12:00:00 \\
\hline Terminate pulse-withdrawal test in TZ2. & $6-7-94$ & 158 & 523 & 10:00:00 \\
\hline End data file C1X05B17. & $6-7-94$ & 158 & 523 & 10:04:17 \\
\hline
\end{tabular}

Figures 4-41 and 4-42 illustrate the zone pressures and fluid production during constantpressure tests, respectively, in test borehole $\mathrm{C} 1 \mathrm{X05}$ for testing sequence $\mathrm{C} 1 \mathrm{X05}-\mathrm{B}$. It should be noted that Figure 4-42 (Fluid production during constant-pressure-flow tests in test borehole $\mathrm{C} 1 \mathrm{X} 05$ during testing sequence $\mathrm{C} 1 \mathrm{X} 05-\mathrm{B}$ ) consists of three parts (Figures $4-42 a, 4-42 b$, and 4-42c). Constant-pressure-withdrawal test \#'s 3, 4, and 6 are not included due to the short duration of these tests.

Table 4-20 indicates the equipment that was used and the duration that each instrument was used during testing sequence $\mathrm{C} 1 \mathrm{X} 05-\mathrm{B}$ in test borehole $\mathrm{C} 1 \mathrm{X} 05$ and in the observation boreholes.

Table 4-20. Testing Sequence C1X05-B Equipment

\begin{tabular}{ccccc}
\hline Equipment & Location & Serial \# & Installed & Removed \\
\hline DAS Software & N/A & PERM4F & $6-15-93$ & $6-7-94$ \\
\hline DCU (HP3497A) & N/A & $2023 a 01688$ & $6-15-93$ & $9-16-93$ \\
DCU (HP3497A) & N/A & $2629 a 22040$ & $9-16-93$ & $2-25-94$ \\
DCU (HP3497A) & N/A & $2629 a 22040$ & $2-25-94$ & $6-7-94$ \\
\hline $\begin{array}{c}\text { Transducer } \\
\text { (Druck PDCR 830) }\end{array}$ & C1H07 TZ1 & 246909 & $6-15-93$ & $6-7-94$ \\
$\begin{array}{c}\text { Transducer } \\
\text { (Druck PDCR 910) }\end{array}$ & C1H07 TZ2 & 322426 & $6-15-93$ & $6-7-94$ \\
$\begin{array}{c}\text { Transducer } \\
\text { (Druck PDCR 910) }\end{array}$ & C1H07 GZ & 322424 & $6-15-93$ & $6-7-94$ \\
$\begin{array}{c}\text { Transducer } \\
\text { (Druck PDCR 830) }\end{array}$ & C1X05 TZ2 & 246917 & $6-15-93$ & $6-7-94$ \\
$\begin{array}{c}\text { Transducer } \\
\text { (Druck PDCR 830) }\end{array}$ & C1X06 TZ1 & 246918 & $8-19-93$ & $6-7-94$ \\
\hline
\end{tabular}


Table 4-20 (Continued). Testing Sequence C1X05-B Equipment

\begin{tabular}{|c|c|c|c|c|}
\hline Equipment & Location & Serial \# & Installed & Removed \\
\hline $\begin{array}{c}\text { Transducer } \\
\text { (Druck PDCR 830) }\end{array}$ & C1X06 TZ2 & 246916 & $8-19-93$ & $6-7-94$ \\
\hline $\begin{array}{c}\text { Transducer } \\
\text { (Druck PDCR 10/D) }\end{array}$ & $\mathrm{C} 1 \times 06 \mathrm{GZ}$ & 211691 & $8-19-93$ & $6-7-94$ \\
\hline $\begin{array}{c}\text { Transducer } \\
\text { (Druck PDCR 10/D) }\end{array}$ & DPT Panel & 211694 & $10-19-93$ & $3-15-94$ \\
\hline LVDT (Trans-Tek 245) & C1X06 & $\mathrm{A} 04$ & $8-19-93$ & $6-7-94$ \\
\hline Injection Column & N/A & 77 & $10-21-93$ & $10-22-93$ \\
\hline Injection Column & N/A & 94 & $10-22-93$ & $11-19-93$ \\
\hline Injection Column & N/A & 77 & $11-19-93$ & $11-22-93$ \\
\hline Injection Column & N/A & 76 & $11-19-93$ & $11-22-93$ \\
\hline Injection Column & N/A & 88 & $11-22-93$ & $12-8-93$ \\
\hline Injection Column & N/A & 76 & $1-10-94$ & $3-15-94$ \\
\hline Injection Column & N/A & 77 & $1-10-94$ & $3-15-94$ \\
\hline $\begin{array}{c}\text { DPT } \\
\text { (Rosemount 1151DP) }\end{array}$ & N/A & 1389938 & $10-19-93$ & $11-1-93$ \\
\hline $\begin{array}{c}\text { DPT } \\
\text { (Rosemount 1151DP) }\end{array}$ & N/A & 1140863 & $11-1-93$ & $3-15-94$ \\
\hline
\end{tabular}

* Installed dates for injection columns refer to dates of initial use rather than date installed. 


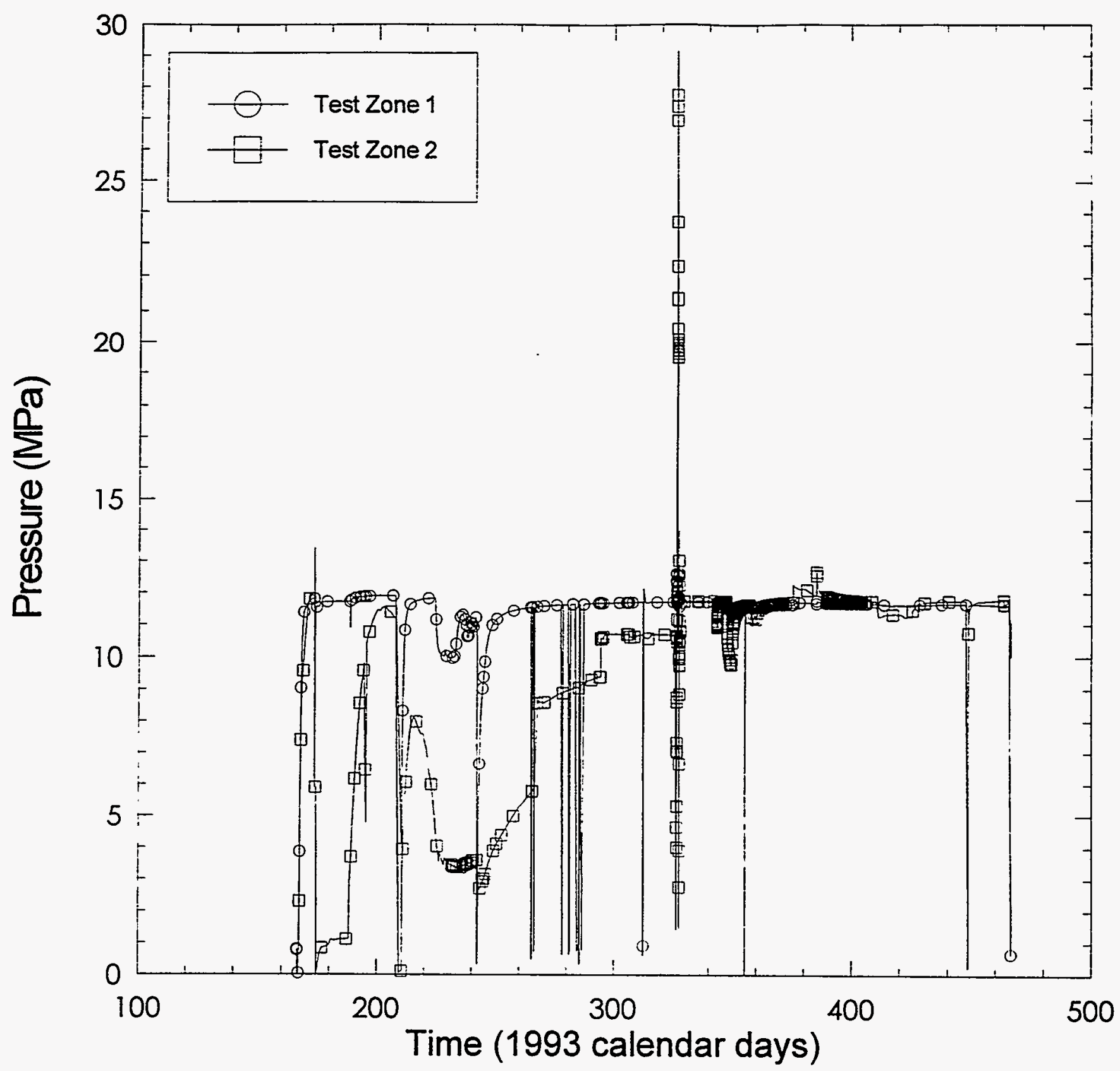

INTERAS115-1440

Figure 4-41. Zone pressures in test borehole C1X05 during testing sequence C1X05-B. 


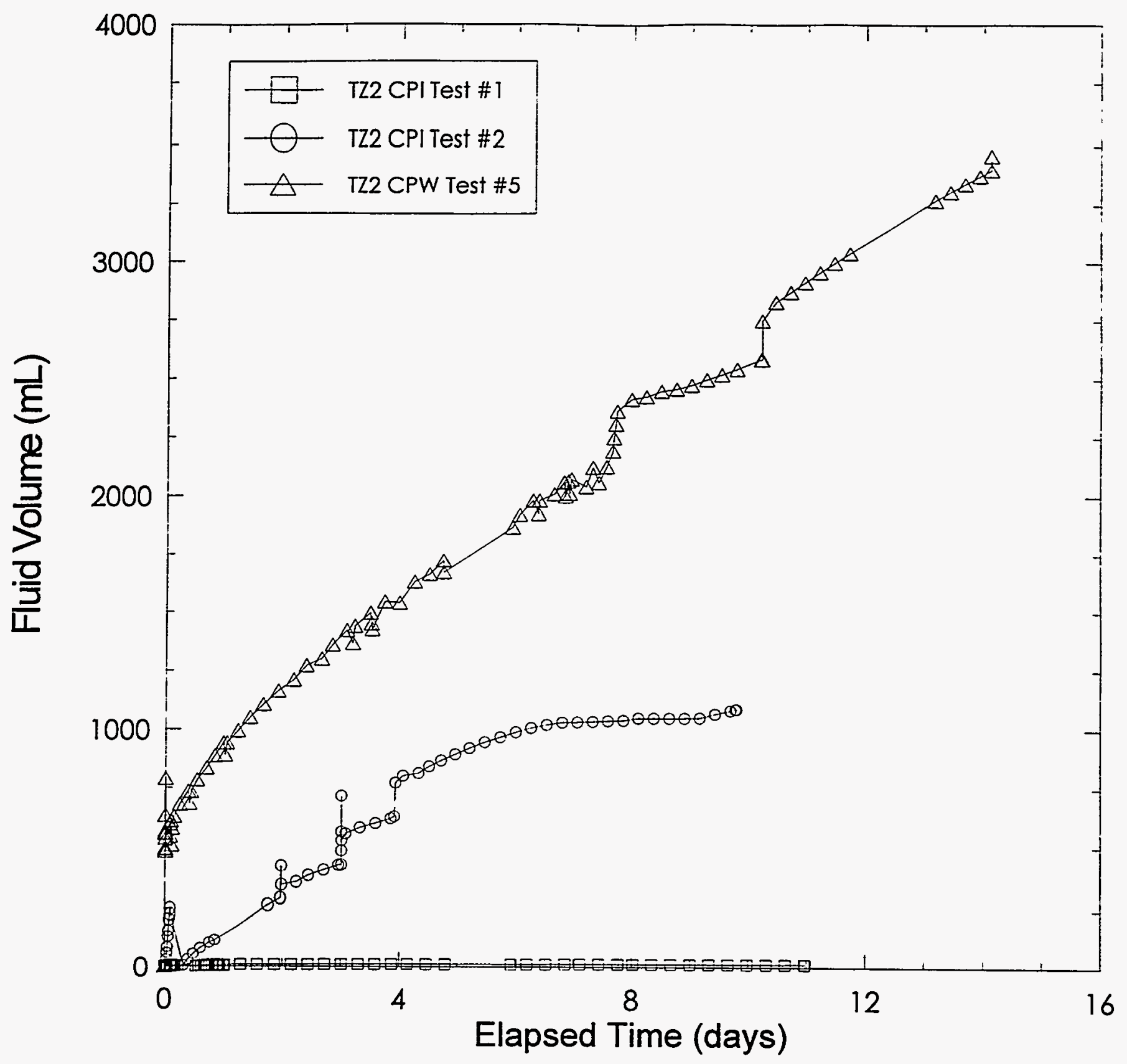

INTERA-8115-145-0

Figure 4-42a. Fluid production during constant-pressure-flow tests in test borehole $\mathrm{C}_{1 \mathrm{X} 05}$ during testing sequenœ C1X05-B. 


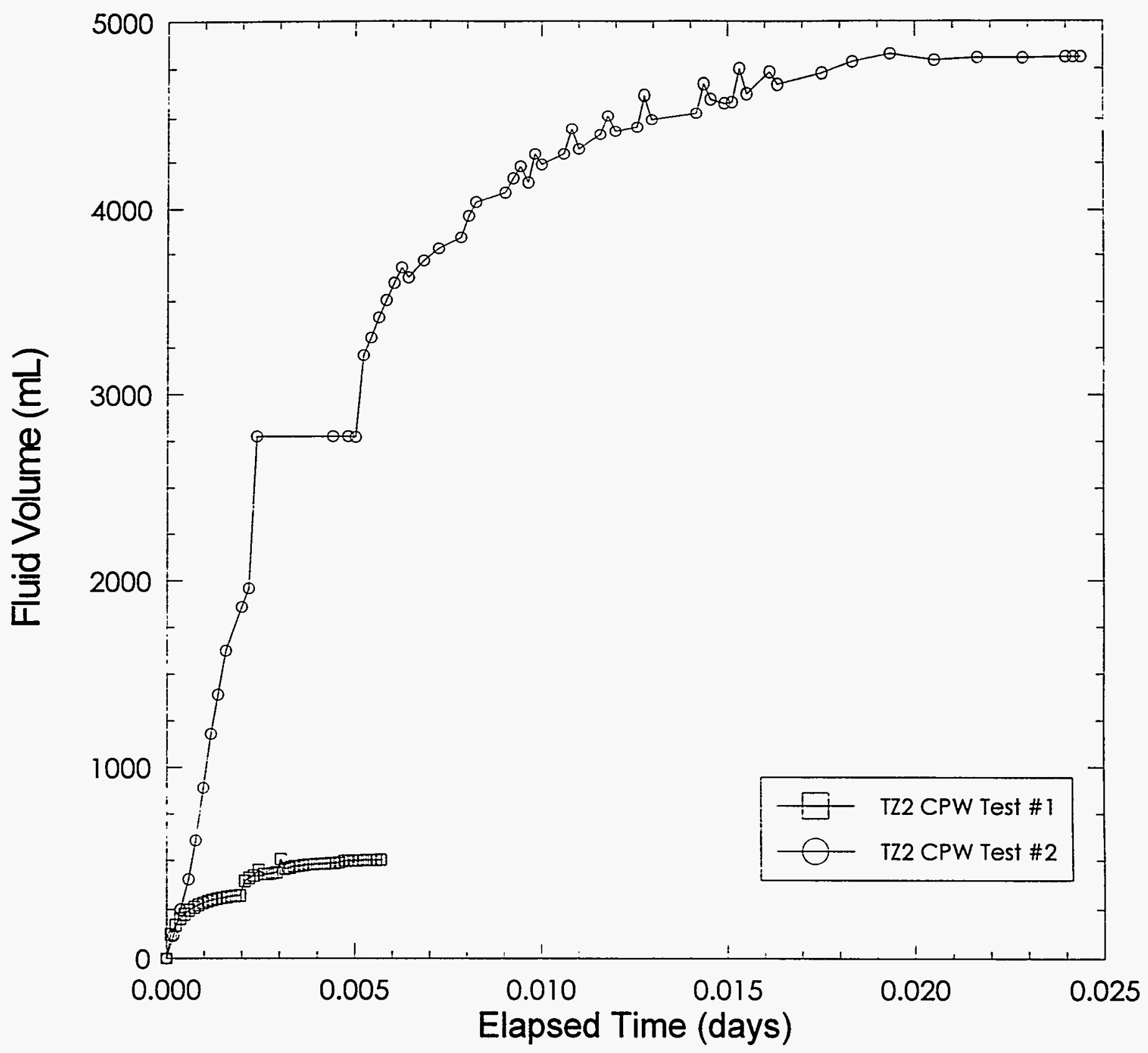

INTERA-SI15-146-0

Figure 4-42b. Fluid production during constant-pressure-flow tests in test borehole $\mathrm{C} 1 \mathrm{X05}$ during testing sequence $C 1 \times 05-B$ (continued). 


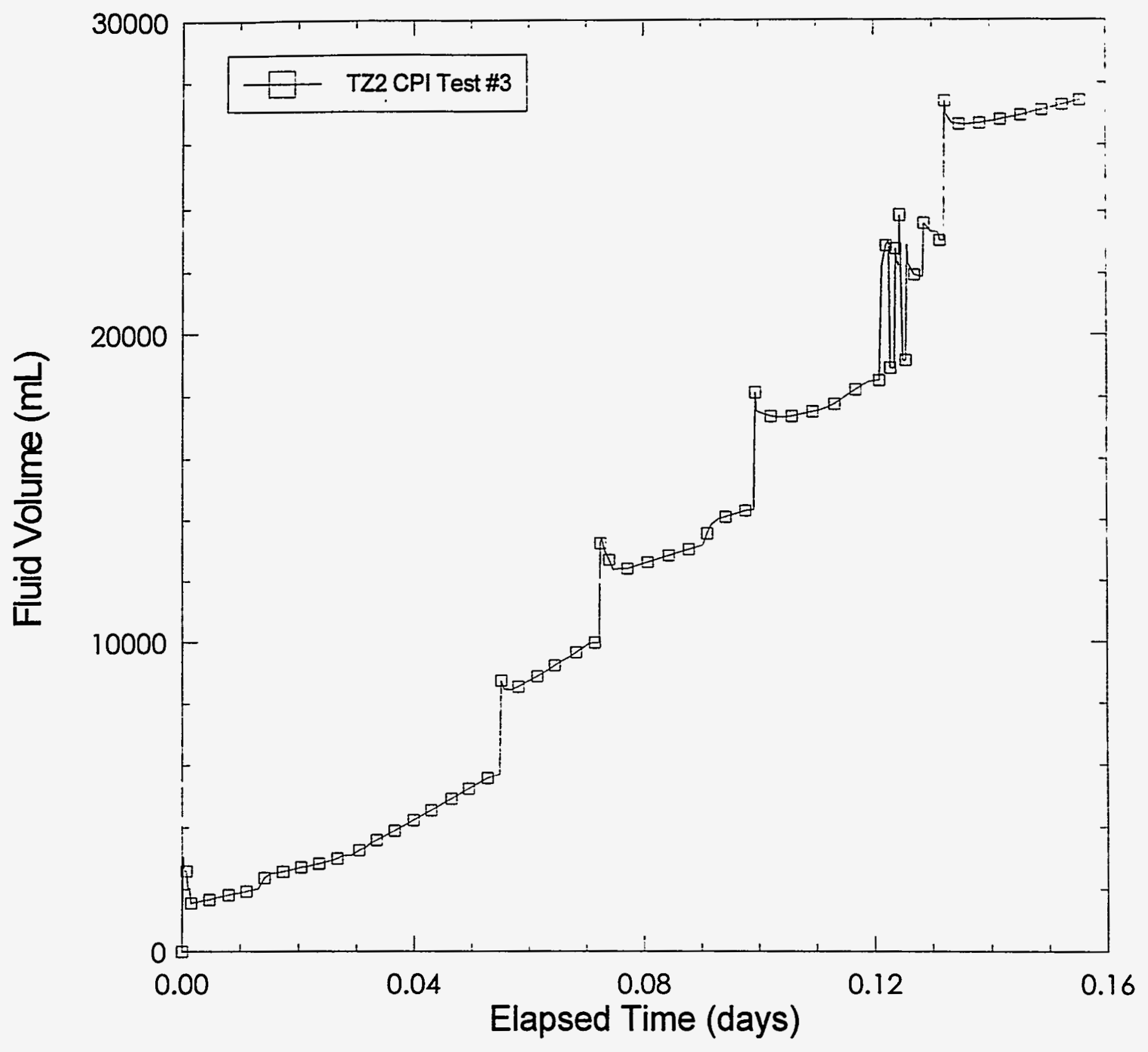

INTERA-6115-147-0

Figure 4-42c. Fluid production during constant-pressure-flow tests in test borehole C1X05 during testing sequence C1X05-B (continued). 


\subsubsection{OBSERVATION BOREHOLE C1H07, TESTING SEQUENCE C1X05-B}

Table 4-21 gives a detailed description of the events that occurred in observation borehole $\mathrm{C} 1 \mathrm{H} 07$ during testing sequence $\mathrm{C} 1 \mathrm{X} 05-\mathrm{B}$.

\section{Table 4-21. Events Associated with Observation Borehole C1H07 During Testing} Sequence C1X05-B

\begin{tabular}{|c|c|c|c|c|}
\hline EVENT & DATE & $\begin{array}{l}\text { CALENDAR } \\
\text { DAY }\end{array}$ & $\begin{array}{c}1993 \\
\text { CALENDAR } \\
\text { DAY }\end{array}$ & $\begin{array}{c}\text { TIME } \\
\text { (HH:MM:SS) }\end{array}$ \\
\hline Drill borehole C1H07-A with 4-inch $(10.16-\mathrm{cm})$ core barrel to 8.18 meters. & $6-24-92$ & 176 & N/A & 13:25:00 \\
\hline Begin fluid-pressure monitoring associated with testing sequence $\mathrm{C} 1 \times 05-\mathrm{A}$. & $6-26-92$ & 178 & N/A & 13:05:00 \\
\hline Teminate fluid-pressure monitoring associated with testing sequence C1X05-A. & 4-7-93 & 97 & 97 & 09:30:43 \\
\hline Deepen borehole $\mathrm{C}$ 1HO7 with 4-inch $(10.16-\mathrm{cm})$ core barrel to 9.37 meters. & $5-21-93$ & 141 & 141 & 11:05:00 \\
\hline Deepen borehole C1H07 with 4-inch $(10.16-\mathrm{cm})$ core barrel to - 10.58 meters. & $5-21-93$ & 141 & 141 & 12:45:00 \\
\hline Deepen borehole C1H07 with 4-inch $(10.16-\mathrm{cm})$ core barrel to 12.05 meters. & 5-21-93 & 141 & 141 & 13:25:00 \\
\hline Deepen borehole C1H07 with 4-inch $(10.16-\mathrm{cm})$ core barrel to -13.58 meters. & $5-21-93$ & 141 & 141 & 14:45:00 \\
\hline Deepen borehole $\mathrm{C} 1 \mathrm{H} 07$ with 4-inch $(10.16-\mathrm{cm})$ core barrel to -14.99 meters. & $5-24-93$ & 144 & 144 & 10:35:00 \\
\hline Deepen borehole $\mathrm{C} 1 \mathrm{H} 07$ with 4-inch $(10.16-\mathrm{cm})$ core barrel to $~ 16.52$ meters. & $5-24-93$ & 144 & 144 & 13:30:00 \\
\hline Deepen borehole C1H07 with 4inch $(10.16-\mathrm{cm})$ core barrel to 17.52 meters. & $5-24-93$ & 144 & 144 & 14:30:00 \\
\hline Deepen borehole C1H07 with 4-inch $(10.16-\mathrm{cm})$ core barrel to -18.73 meters. & $5-25-93$ & 145 & 145 & $09: 30: 00$ \\
\hline Deepen borehole $\mathrm{C} 1 \mathrm{HO} 07$ with 4 inch $(10.16-\mathrm{cm})$ core barrel to -20.51 meters. & $5-25-93$ & 145 & 145 & 10:05:00 \\
\hline Deepen borehole C1H07 with 4-inch (10.16-cm) core barrel to - 22.04 meters. & $5-25-93$ & 145 & 145 & 10:25:00 \\
\hline Deepen borehole C1HO7 with 4-inch $(10.16-\mathrm{cm})$ core barrel to -23.55 moters. & $5-25-93$ & 145 & 145 & 13:00:00 \\
\hline Deepen borehole $\mathrm{C} 1 \mathrm{H} 07$ with 4-inch $(10.16-\mathrm{cm})$ core barrel to -25.09 meters. & 5-25-93 & 145 & 145 & 14:00:00 \\
\hline Deepen borehole $\mathrm{C} 1 \mathrm{H} 07$ with 4 inch $(10.16-\mathrm{cm})$ core barrel to $\sim 26.05$ meters. & 5-25-93 & 145 & 145 & 14:40:00 \\
\hline Deepen borehole $\mathrm{C} 1 \mathrm{H} 107$ with 4 -inch $(10.16-\mathrm{cm})$ core bartel to $\sim 27.21$ melers. & $5-26-93$ & 146 & 146 & 09:25:00 \\
\hline Deepen borehole $\mathrm{C} 1 \mathrm{H} 07$ with 4 -inch $(10.16-\mathrm{cm})$ core barrel to -27.88 meters. & $5-26-93$ & 146 & 146 & 09:45:00 \\
\hline $\begin{array}{l}\text { Install multipacker test tool \#39 in borehole C1HO7 as indicated in the test-tool } \\
\text { configuration diagram \#1 (Figure 4-15). }\end{array}$ & $5-28-93$ & 148 & 148 & 14:14:00 \\
\hline Begin data file C1H07B01. & $5-28-93$ & 148 & 148 & 14:14:16 \\
\hline Add $\sim 8$ liters of brine to borehole $\mathrm{C} 1 \mathrm{H} 07$. & $5-28-93$ & 148 & 148 & 14:31:19 \\
\hline Inflate TZ1P to - $10 \mathrm{MPa}$ & 5-28-93 & 148 & 148 & 14:32:49 \\
\hline Inflate TZ2P to - $10 \mathrm{MPa}$ & $5-28-93$ & 148 & 148 & 14:36:49 \\
\hline Inflate GZP to - $10 \mathrm{MPa}$. & 5-28-93 & 148 & 148 & 14:38:19 \\
\hline Shut in all zones. & $5-28-93$ & 148 & 148 & $14: 40: 49$ \\
\hline DAS not functioning property upon arrival. & $6-11-93$ & 162 & 162 & 08:51:00 \\
\hline End data file C1HOTBO1. & $6-15-93$ & 166 & 166 & 11:16:48 \\
\hline Replace gage on GZP. & $9-7-93$ & 250 & 250 & 09:59:00 \\
\hline Inflate GZP to $-8.3 \mathrm{MPa}$ & $9-7-93$ & 250 & 250 & 10:15:00 \\
\hline Increase $\mathrm{GZ}$ pressure to $\sim 3.6 \mathrm{MPa}$. & $9-7-93$ & 250 & 250 & 10:22:00 \\
\hline
\end{tabular}


Table 4-21 (Continued). Events Associated with Observation Borehole C1H07 During Testing Sequence C1X05-B

\begin{tabular}{|c|c|c|c|c|}
\hline EVENT & DATE & $\begin{array}{l}\text { CALENDAR } \\
\text { DAY }\end{array}$ & $\begin{array}{c}1993 \\
\text { CALENDAR } \\
\text { DAY }\end{array}$ & $\begin{array}{l}\text { TIME } \\
\text { (HH:MM:SS) }\end{array}$ \\
\hline Increase GZP pressure to 8.3 MPa. & $9-22-93$ & 265 & 265 & 12:07:35 \\
\hline Increase TZ2P pressure to $\sim 11.7 \mathrm{MPa}$. & $9-22-93$ & 265 & 265 & 12:12:20 \\
\hline Increase TZ1P pressure to $\sim 14.5 \mathrm{MPa}$. & $9-22-93$ & 265 & 265 & 12:16:59 \\
\hline Increase TZ1P pressure to $~-15 \mathrm{MPa}$. & $11-2-93$ & 306 & 306 & $09: 45: 00$ \\
\hline Power outage. & $11-8-93$ & 312 & 312 & 09:34:00 \\
\hline DAS powered by generator. & $11-10-93$ & 314 & 314 & 08:47:00 \\
\hline Open TZ2P to accumulator at $\sim 10.3 \mathrm{MPa}$. & $12-1-93$ & 335 & 335 & 11:44:30 \\
\hline Shut in all zones from accumulators and depressurized. & $12-9-93$ & 343 & 343 & $09: 30: 00$ \\
\hline Deflate all packers. & $12-9-93$ & 343 & 343 & 09:50:00 \\
\hline $\begin{array}{l}\text { Partlally remove test tool \#39 from borehole C1H07 and repaired leaky fitting on TZ1 } \\
\text { and retumed test tool } \# 39 \text { to original position in borehole C1H07. }\end{array}$ & $12-9-93$ & 343 & 343 & 10:46:00 \\
\hline Replace gage on $T Z 2$. & $12-9-93$ & 343 & 343 & $11: 12: 00$ \\
\hline Inflate TZ1P to $\sim 15.9 \mathrm{MPa}$ & $12-9-93$ & 343 & 343 & 11:58:00 \\
\hline Inflate TZ2P to - $15.9 \mathrm{MPa}$. & $12-9-93$ & 343 & 343 & 12:19:00 \\
\hline Inflate GZP to $-15.9 \mathrm{MPa}$. & $12-9-93$ & 343 & 343 & 12:28:00 \\
\hline Increase $T Z 1$ pressure to $\sim 11.5 \mathrm{MPa}$. & $12-9-93$ & 343 & 343 & 12:40:00 \\
\hline Increase GZ pressure to $-8.5 \mathrm{MPa}$. & $12-9-93$ & 343 & 343 & 12:53:00 \\
\hline TZ2 will not hold pressure. & $12-9-93$ & 343 & 343 & 13:00:00 \\
\hline Increase TZ1 pressure to $11.660 \mathrm{MPa}$. & $12-9-93$ & 343 & 343 & $13: 20: 00$ \\
\hline Depressurze all zones. & $12-13-93$ & 347 & 347 & $09: 31: 30$ \\
\hline Deflate all packers. & $12-13-93$ & 347 & 347 & $09: 35: 00$ \\
\hline $\begin{array}{l}\text { Remove test tool \#39 from borehole } \mathrm{C}_{1 \mathrm{H}} \mathrm{O} 7 \text { in order to find and repair the leak in the } \\
\qquad \mathrm{TZ2} \text {. }\end{array}$ & $12-13-93$ & 347 & 347 & $12: 00: 00$ \\
\hline Test all lines for leaks. & $12-14-93$ & 348 & 348 & 10:05:00 \\
\hline $\begin{array}{c}\text { Install test tool \#39 in borehole } \mathrm{C}_{1 H} \mathrm{HO} \text { as indicated in the test tool configuration } \\
\text { diagram \#2 (Figure 4-16). }\end{array}$ & $12-15-93$ & 349 & 349 & 11:30:00 \\
\hline Inflate TZ1P to $-15 \mathrm{MPa}$. & $12-15-93$ & 349 & 349 & $11: 55: 00$ \\
\hline Inflate $T 22 \mathrm{P}$ to $-15 \mathrm{MPa}$ & $12-15-93$ & 349 & 349 & 11:57:00 \\
\hline Inflate GZP to - $15 \mathrm{MPa}$ & $12-15-93$ & 349 & 349 & 12:14:00 \\
\hline Increase TZ1 pressure to $\sim 11.5 \mathrm{MPa}$. & $12-15-93$ & 349 & 349 & 12:18:00 \\
\hline Increase TZ2 pressure to $\sim 8.5 \mathrm{MPa}$. & $12-15-93$ & 349 & 349 & 12:23:00 \\
\hline Increase TZ2 pressure to $-8.5 \mathrm{MPa}$. & 12-15-93 & 349 & 349 & 12:27:00 \\
\hline Increase $\mathrm{GZ}$ pressure to $\sim 8.5 \mathrm{MPa}$. & $12-15-93$ & 349 & 349 & 12:32:00 \\
\hline Leaky fitting on GZ gage. & $12-16-93$ & 350 & 350 & 08:42:00 \\
\hline Increase GZ pressure to $\sim 8.5 \mathrm{MPa}$ & $12-16-93$ & 350 & 350 & 08:48:00 \\
\hline Increase $T Z 2$ pressure to $8.5 \mathrm{MPa}$. & $12-16-93$ & 350 & 350 & 08:50:00 \\
\hline Increase TZ2P pressure to $\sim 15 \mathrm{MPa}$. & $12-16-93$ & 350 & 350 & $08: 56: 00$ \\
\hline
\end{tabular}


Table 4-21 (Continued). Events Associated with Observation Borehole C1H07 During Testing Sequence C1X05-B

\begin{tabular}{|c|c|c|c|c|}
\hline EVENT & DATE & $\begin{array}{c}\text { CALENDAR } \\
\text { DAY }\end{array}$ & $\begin{array}{c}1993 \\
\text { CALENDAR } \\
\text { DAY }\end{array}$ & $\begin{array}{c}\text { TIME } \\
\text { (HH:MM:SS) }\end{array}$ \\
\hline Open $T Z 2$ and $G Z$ to accumulator at $-8.5 \mathrm{MPa}$. & $12-20-93$ & 354 & 354 & 10:29:00 \\
\hline Leaky fitting on GZP gage. & $12-21-93$ & 355 & 355 & 08:51:00 \\
\hline Leaks on both TZ1, TZ2, and TZ2P. & $12-22-93$ & 356 & 356 & 09:17:00 \\
\hline Replace gage on TZ1. & $12-28-93$ & 362 & 362 & 08:56:00 \\
\hline Shut in $T Z 2$ and $G Z$ from accumulator. & $12-28-93$ & 362 & 362 & 09:07:00 \\
\hline Replace all zone gages with plugs to avoid any further leaks. & $12-31-93$ & 365 & 365 & 09:29:00 \\
\hline Open GZ to accumulator at $\sim 7.5 \mathrm{MPa}$. & $1-6-94$ & 6 & 371 & 12:26:00 \\
\hline DAS not functioning property upon arrival. & $2-10-94$ & 41 & 406 & 12:35:00 \\
\hline DAS not functioning property upon arrival. & $2-17-94$ & 48 & 413 & $14: 15: 00$ \\
\hline Terminate data collection associated with testing sequence C1X05-B. & $6-7-94$ & 158 & 523 & 10:00:00 \\
\hline Remove multipacker test tool \#39 from borehole C1H07. & $6-8-94$ & 159 & 524 & $13: 50: 00$ \\
\hline
\end{tabular}

Figure 4-43 illustrates the zone pressures in observation borehole $\mathrm{C} 1 \mathrm{HO}$ for testing sequence $\mathrm{C} 1 \mathrm{X} 05-\mathrm{B}$.

Table 4-22 indicates the equipment that was used and the duration that each instrument was used in observation borehole $\mathrm{C} 1 \mathrm{H} 07$ during testing sequence $\mathrm{C} 1 \mathrm{X} 05-\mathrm{B}$.

Table 4-22. Borehole C1H07 Equipment

\begin{tabular}{|c|c|c|c|c|}
\hline Equipment & Location & Serial \# & Installed & Removed \\
\hline DAS Software & N/A & PERM4F & $5-28-93$ & $6-15-93$ \\
\hline DCU (HP3497A) & N/A & $2023 a 01688$ & $5-28-93$ & $6-15-93$ \\
\hline $\begin{array}{c}\text { Transducer } \\
\text { (Druck PDCR 830) }\end{array}$ & Test Zone 1 & 246909 & $5-28-93$ & $6-15-93$ \\
\hline $\begin{array}{c}\text { Transducer } \\
\text { (Druck PDCR 830) }\end{array}$ & $\begin{array}{c}\text { Test Zone } 1 \\
\text { Packer }\end{array}$ & 246918 & $5-28-93$ & $6-15-93$ \\
\hline $\begin{array}{c}\text { Transducer } \\
\text { (Druck PDCR 910) }\end{array}$ & Test Zone 2 & 322426 & $5-28-93$ & $6-15-93$ \\
\hline $\begin{array}{c}\text { Transducer } \\
\text { (Druck PDCR 830) }\end{array}$ & $\begin{array}{c}\text { Test Zone } 2 \\
\text { Packer }\end{array}$ & 246917 & $5-28-93$ & $6-15-93$ \\
\hline $\begin{array}{c}\text { Transducer } \\
\text { (Druck PDCR 910) }\end{array}$ & Guard Zone & 322424 & $5-28-93$ & $6-15-93$ \\
\hline $\begin{array}{c}\text { Transducer } \\
\text { (Druck PDCR 10/D) }\end{array}$ & $\begin{array}{l}\text { Guard Zone } \\
\text { Packer }\end{array}$ & 211691 & $5-28-93$ & $6-15-93$ \\
\hline
\end{tabular}




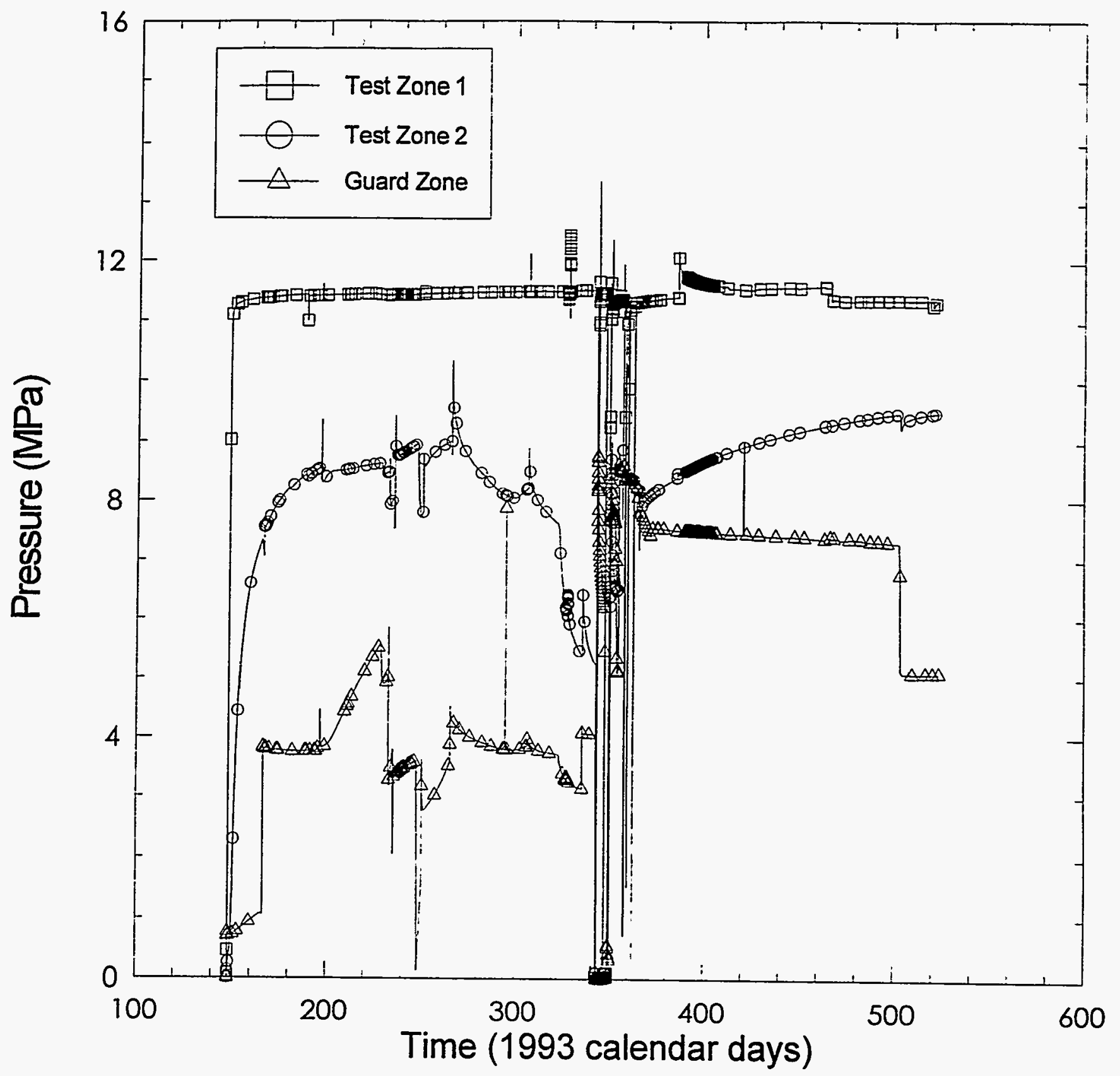

INTERA-6115-149-0

Figure 4-43. Zone pressures in observation borehole $\mathrm{C} 1 \mathrm{H} 07$ during testing sequence $\mathrm{C} 1 \mathrm{X} 05-\mathrm{B}$. 
Table 4-23 gives a detailed description of the events that occurred in observation borehole C1X06 during testing sequence C1X05-B.

\section{Table 4-23. Events Associated with Observation Borehole C1X06 During Testing} Sequence C1X05-B

\begin{tabular}{|c|c|c|c|c|}
\hline EVENT & DATE & $\begin{array}{l}\text { CALENDAR } \\
\text { DAY }\end{array}$ & $\begin{array}{c}1993 \\
\text { CALENDAR } \\
\text { DAY }\end{array}$ & $\begin{array}{c}\text { TIME } \\
\text { (HH:MM:SS) }\end{array}$ \\
\hline Drill borehole C1X06 with 4-inch core barrel to 7.63 meters. & $9-30-92$ & 274 & N/A & 10:00:00 \\
\hline Begin fluid-pressure monitoring associated with testing sequence C1X05-A. & 10-1-92 & 275 & N/A & $11: 00: 00$ \\
\hline Terminate fluid-pressure monitoring associated with testing sequence $\mathrm{C} 1 \times 05-\mathrm{A}$. & 4-7-93 & 97 & 97 & 09:30:43 \\
\hline Deepen borehole C1X06 with 4 inch $(10.16-\mathrm{cm})$ core barrel to 9.130 meters. & 7-7-93 & 188 & 188 & 13:07:00 \\
\hline Deepen borehole C1X06 with 4-inch $(10.16-\mathrm{cm})$ core barrel to 10.65 meters. & 7-7-93 & 188 & 188 & 14:22:00 \\
\hline Deepen borehole $\mathrm{C} 1 \times 06$ with 4 inch $(10.16-\mathrm{cm})$ core barrel to 12.15 meters. & $7-8-93$ & 189 & 189 & 09:35:00 \\
\hline Deepen borehole C1X06 with 4inch $(10.16-\mathrm{cm})$ core barrel to 13.65 meters. & $7-8-93$ & 189 & 189 & 10:15:00 \\
\hline Deepen borehole $\mathrm{C} 1 \times 06$ with 4 inch $(10.16-\mathrm{cm})$ core barrel to 15.17 meters. & $7-8-93$ & 189 & 189 & 10:45:00 \\
\hline Deepen borehole $\mathrm{C} 1 \mathrm{XOS}$ with 4 inch $(10.16-\mathrm{cm})$ core barrel to 16.67 meters. & $7-8-93$ & 189 & 189 & 12:45:00 \\
\hline Deepen borehole C1X06 with 4-inch $(10.16-\mathrm{cm})$ core barrel to 18.20 meters. & $7-8-93$ & 189 & 189 & 13:30:00 \\
\hline Deepen borehole C1X06 with 4-inch $(10.16-\mathrm{cm})$ core barrel to 19.94 meters. & 7-9-93 & 190 & 190 & 09:10:00 \\
\hline Deepen borehole C1X06 with 4-inch $(10.16-\mathrm{cm})$ core barrel to 21.21 meters. & $7-9-93$ & 190 & 190 & 10:00:00 \\
\hline Deepen borehole C1X06 with 4-inch $(10.16-\mathrm{cm})$ core barrel to 22.79 meters. & $7-9-93$ & 190 & 190 & 11:00:00 \\
\hline Deepen borehole C1X06 with 4-inch $(10.16-\mathrm{cm})$ core barrel to 24.27 meters. & 7-9-93 & 190 & 190 & 14:20:00 \\
\hline Deepen borehole $\mathrm{C} 1 \times 06$ with 4-inch $(10.16-\mathrm{cm})$ core barrel to 25.84 meters. & $8-12-93$ & 224 & 224 & 11:30:00 \\
\hline Deepen borehole $\mathrm{C} 1 \mathrm{X05}$ with 4-inch $(10.16-\mathrm{cm})$ core barrel to 27.34 meters. & $8-12-93$ & 224 & 224 & $12: 55: 00$ \\
\hline Deepen borehole $\mathrm{C} 1 \times 06$ with 4 -inch $(10.16-\mathrm{cm})$ core barel to 27.99 meters. & $8-12-93$ & 224 & 224 & 13:15:00 \\
\hline $\begin{array}{l}\text { Install multipacker test tool \#40 in borehole } C\{\times 06 \text { as indicated in the test-tool } \\
\text { configuration dragram (Figure } 417) \text {. }\end{array}$ & $8-19-93$ & 231 & 231 & 12:00:00 \\
\hline Begin data collection associated with testing sequence C $\mathrm{C} \times 05-\mathrm{B}$. & $8-19-93$ & 231 & 231 & 12:05:02 \\
\hline Inflate TZ1P to $\sim 12.4 \mathrm{MPa}$ & 8-19-93 & 231 & 231 & 12:15:56 \\
\hline Inflate TZ2P to $\sim 12.4 \mathrm{MPa}$ & 8-19-93 & 231 & 231 & 12:29:26 \\
\hline Inflate GZP to $-12.4 \mathrm{MPa}$ & 8-19-93 & 231 & 231 & 12:38:27 \\
\hline Shut in all zones. & 8-19-93 & 231 & 231 & $12: 40: 41$ \\
\hline Vented gas from all zones. & 8-20-93 & 232 & 232 & 12:26:37 \\
\hline Increase TZ2P pressure and shut in TZ2. & $8-20-93$ & 232 & 232 & 12:38:27 \\
\hline Increase GZP pressure. & 8-20-93 & 232 & 232 & 12:43:38 \\
\hline Replace gage on GZ. & $8-23-93$ & 235 & 235 & 08:57:13 \\
\hline Increase TZ1P pressure. & 8-23-93 & 235 & 235 & 09:09:32 \\
\hline Suspect TZ2P is leaking into TZ2. & $8-25-93$ & 237 & 237 & 08:18:00 \\
\hline Depressurize all zones. & 8-25-93 & 237 & 237 & 08:23:00 \\
\hline
\end{tabular}


Table 4-23 (Continued). Events Associated with Observation Borehole C1X06 During Testing Sequence C1X05-B

\begin{tabular}{|c|c|c|c|c|}
\hline$"$ EVENT $^{\circ}$ & DATE & $\begin{array}{c}\text { CALENDAR } \\
\text { DAY }\end{array}$ & $\begin{array}{c}\text { 1993 } \\
\text { CALENDAR } \\
\text { DAY }\end{array}$ & $\begin{array}{c}\text { TIME } \\
\text { (HH:MM:SS) }\end{array}$ \\
\hline Deflate all packers. & $8-25-93$ & 237 & 237 & 08:29:38 \\
\hline $\begin{array}{l}\text { Removed multipacker test tool \#40 from borehole C1X06 and repair leaky fitting and } \\
\text { re-install the test tool to original position with LVDT not connected. }\end{array}$ & $8-25-93$ & 237 & 237 & 13:22:00 \\
\hline Inflate GZP to $\sim 12.4 \mathrm{MPa}$. & $8-25-93$ & 237 & 237 & 13:26:55 \\
\hline Inflate TZ2P to $~ 12.4 \mathrm{MPa}$ & $8-25-93$ & 237 & 237 & $13: 30: 17$ \\
\hline Inflate TZ1P to $-12.4 \mathrm{MPa}$ & $8-25-93$ & 237 & 237 & 13:35:18 \\
\hline Shut in all zones. & $8-25-93$ & 237 & 237 & 13:38:30 \\
\hline Leak in $T Z 1$ injection line. & $8-27-93$ & 239 & 239 & 11:34:06 \\
\hline Leaky fitting on TZ2P. & $8-27-93$ & 239 & 239 & 11:44:47 \\
\hline Vent gas from TZ2. & $9-7-93$ & 250 & 250 & 09:50:00 \\
\hline Increase TZ2 pressure to $\sim 4 \mathrm{MPa}$. & $9-7.93$ & 250 & 250 & 09:58:00 \\
\hline Replace gage on $\mathrm{TZ2}$. & $9-14-93$ & 257 & 257 & 11:02:20 \\
\hline Increase TZ2 pressure to $\sim 3.4 \mathrm{MPa}$. & $9-1493$ & 257 & 257 & $11: 34: 00$ \\
\hline Opened GZ to accumulator at $4.233 \mathrm{MPa}$. & $9-22-93$ & 265 & 265 & 11:18:00 \\
\hline Increase TZ2 pressure via accumulator. & $9-24-93$ & 267 & 267 & 09:55:55 \\
\hline Shut in $\mathrm{TZ2}$ from accumulator. & $9-27-93$ & 270 & 270 & 08:50:00 \\
\hline Replace gage on $T Z 2$. & $9-28-93$ & 271 & 271 & $12: 46: 00$ \\
\hline Open GZ to accumulator. & $9-28-93$ & 271 & 271 & 12:50:00 \\
\hline Shut in GZ from accumulator. & $10-4-93$ & 277 & 277 & 09:21:00 \\
\hline Power outage. & $11-8-93$ & 312 & 312 & 09:34:00 \\
\hline DAS powered by generator. & $11-10-93$ & 314 & 314 & 08:47:00 \\
\hline No power supplied to transducers. & $1-7-94$ & 7 & 372 & 08:46:00 \\
\hline DAS not functioning properly upon arrival. & $2-10-94$ & 41 & 406 & 12:35:00 \\
\hline DAS not functioning property upon artival. & $2-17-94$ & 48 & 413 & 14:15:00 \\
\hline $\begin{array}{l}\text { Initiate pulse-withdrawal test on TZ1 dropping the pressure from } 11.747 \text { to } 7.680 \mathrm{MPa} \\
\text { and removing }-113 \mathrm{~mL} \text { of fluid. }\end{array}$ & $4-8-94$ & 98 & 463 & 12:29:26 \\
\hline Terminate data collection associated with testing sequence C1X05-B. & $6-7-94$ & 158 & 523 & 10:00:0 \\
\hline Remove multi-packer test tool \#40 from borehole C1X06. & $6-9-94$ & 160 & 525 & 14:00:00 \\
\hline
\end{tabular}

Figures 4-44 and 4-45 illustrate the zone pressures and axial-LVDT displacement, respectively, in observation borehole C1X06 for testing sequence C1X05-B. 


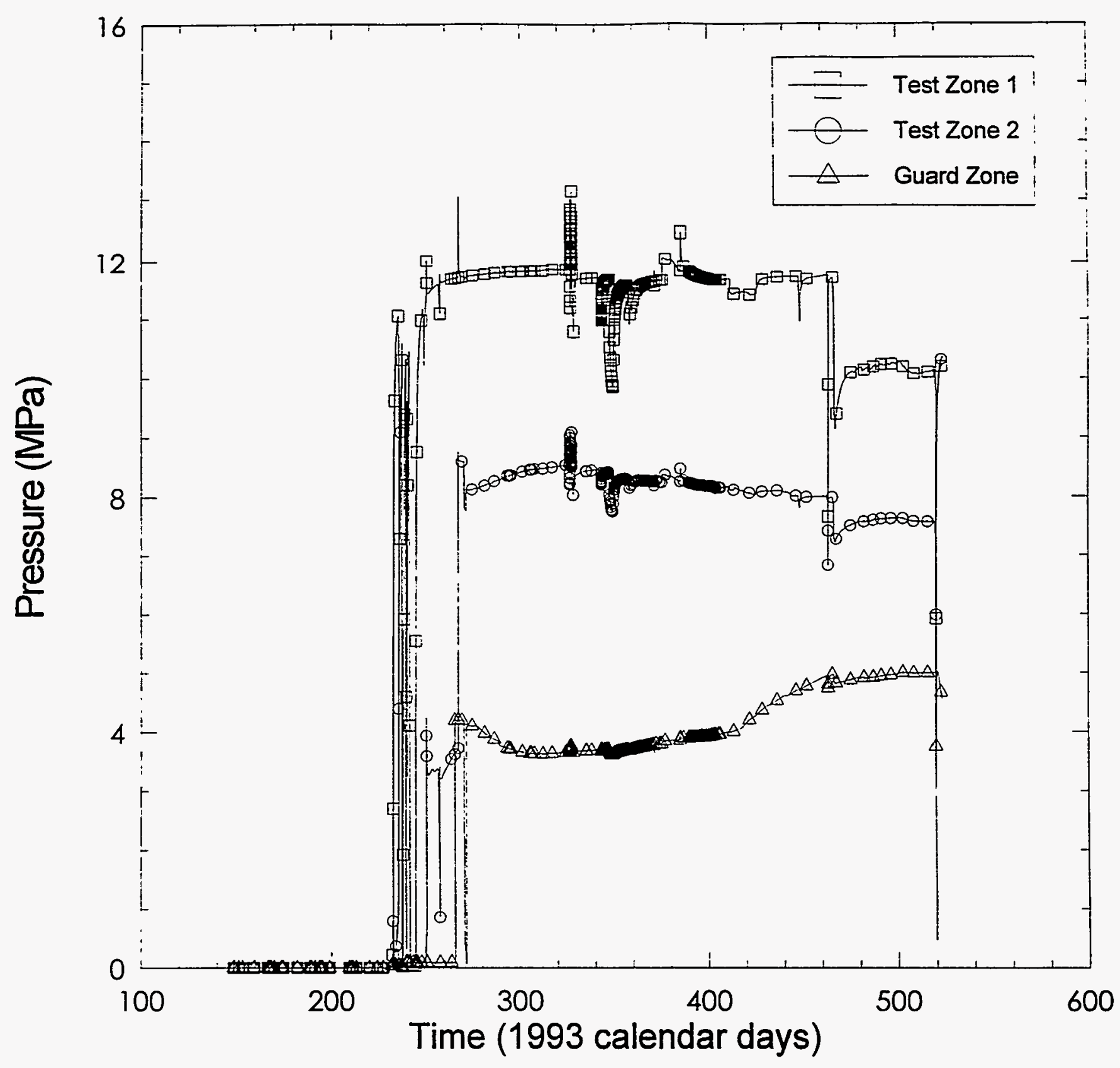

INTERA-6115-1500

Figure 4-44. Zone pressures in observation borehole C1X06 during testing sequence C1X05-B. 


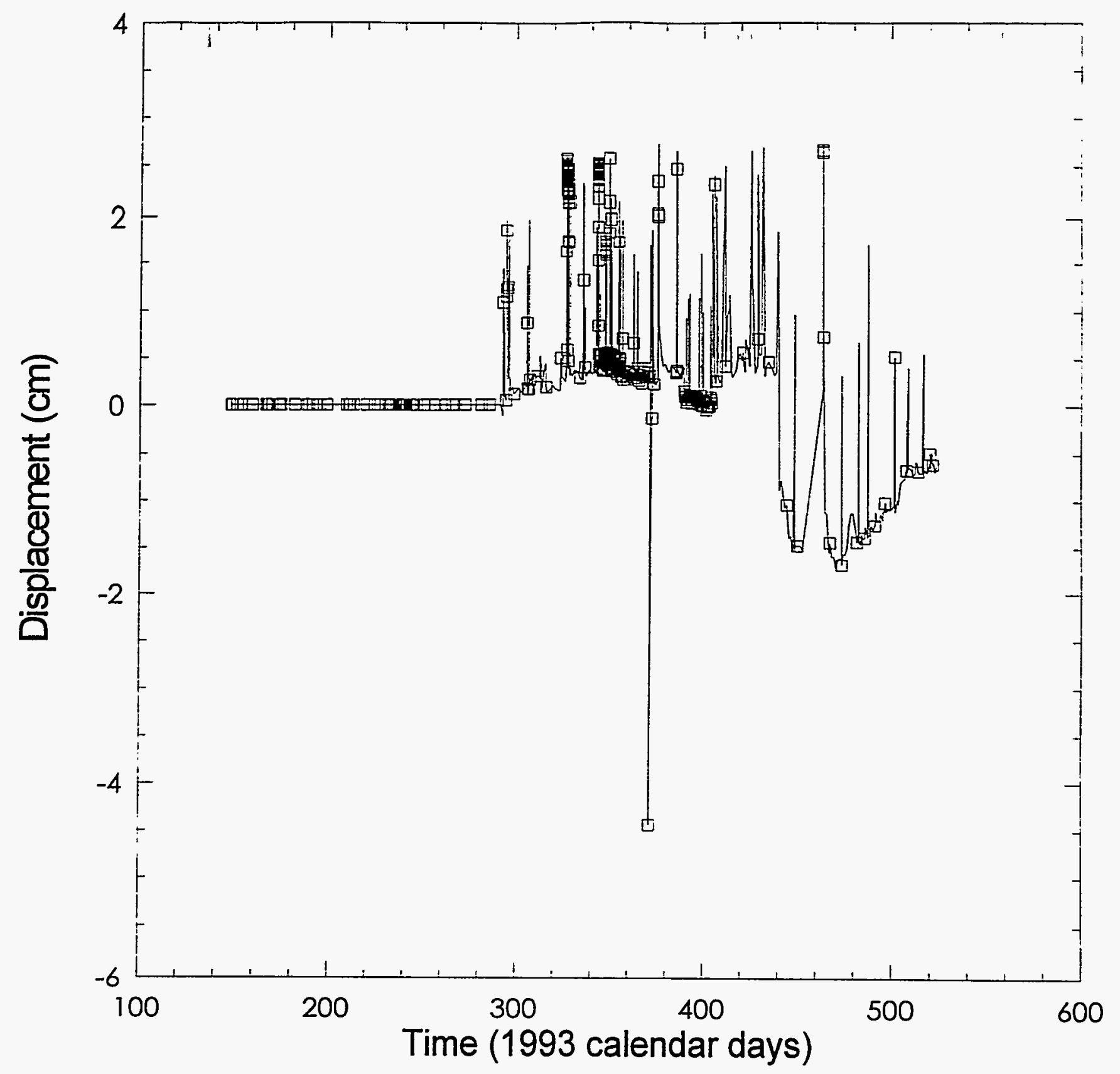

INTERA-S195-151-0

Figure 4-45. Axial-LVDT displacement in observation borehole C1X06 during testing sequence C1X05-B. 


\section{GAS-THRESHOLD-PRESSURE TESTING}

Post-closure degradation of the organic and metallic material included in the radioactive waste scheduled for burial in the WIPP underground repository is expected to generate gas in quantities potentially sufficient to pressurize the waste/backfill continuum in the underground rooms and drifts to lithostatic pressure (Lappin et al., 1989). Excessive gas pressure could cause fracturing of the formation surrounding the repository unless the generated gas can flow into the surrounding Salado Formation. Gas-threshold-pressure tests (GTPTs), were intended to provide estimates of the pressure at which Salado Formation anhydrite interbeds would allow initial penetration of the gas generated in the repository.

\subsection{Test Objectives}

The GTPT program in the WIPP underground facility was originally designed with the following objectives:

- to provide information about the far-field gas-threshold pressure in the Salado Formation anhydrite interbeds, particularly Marker Beds 138 and 139;

- to determine, if possible, the far-field gas-threshold pressure in both argillaceous and pure halite beds of the Salado Formation;

- to determine if the gas-threshold pressure varies with distance from the excavation in the WIPP underground facility;

- to determine if the gas-threshold pressure is related to formation permeability; and

- to determine if there will be sustained gas flow into the formation after gas-threshold pressure is reached, and at what rate and what pressure will the gas flow.

No tests of halite strata were ever performed to address the second objective because of concerns that the rock would fracture before accepting gas. The testing program was terminated before the third objective could be met.

\subsection{Test Design}

The GTPT sequences discussed in this data report were conducted in three boreholes drilled at three locations in the WIPP underground facility (Figure 2-1) and described in Section 5.5. The boreholes were drilled either angled downward or angled upward from the underground rooms. In the experimental area, borehole $\mathrm{C} 2 \mathrm{HO} 2$ was drilled in Room C2 and L4P52 was drilled in Room L4. In the operations area, borehole SCP01 was drilled in the Core Storage Library. The test boreholes were chosen to allow evaluation of gasthreshold pressures in the Salado Formation interbeds in locations (room pillars) where excavation induced damage was expected to be minimal. 
All of the boreholes tested in the GTPT program had previously undergone permeability testing. In boreholes SCP01 and $\mathrm{C} 2 \mathrm{HO2}$, long-term fluid-pressure monitoring tools were replaced by multipacker test tools configured for GTPT sequences. In borehole L4P52, the test tool used for permeability testing was used for the GTPT sequence. The multipacker test tools (see Section 5.3.1) were installed in each borehole in such a way that the packers isolated a bottom-hole test zone and a guard zone between the two packers. The zones were filled with $1.22 \mathrm{~kg} / \mathrm{L}$ sodium-chloride brine. The guard zones were used to detect pressure leakage from the bottom-hole test zone transmitted either through the test tool or through the formation and to reduce the pressure gradient across the test-zone packer. After packer inflation, the zones were shut in and the fluid-pressure buildup was monitored with pressure transducers and recorded on a computer controlled DAS. As fluid pressures stabilized, gas/brine exchanges were performed in the test zones (see Section 5.4). After completing the gas/brine exchange, the GTPT sequences were performed on the isolated, bottom-hole test zones as described in Section 5.4.

A pulse-withdrawal test was performed as part of the GTPT sequence in borehole $\mathrm{C} 2 \mathrm{HO} 2$ to provide data with which to estimate the compressibility of the zone being tested, formation hydraulic conductivity to brine, and brine pore pressure.

Figure 5-1 illustrates the stratigraphic position of each of the test and observation boreholes and shows which strata were included in the test intervals. Borehole $\mathrm{C} 2 \mathrm{H} 01$ was used as an observation borehole during testing sequence $\mathrm{C} 2 \mathrm{HO2}$.

\subsection{Test Equipment for Gas Threshold-Pressure Testing}

The following sections briefly describe the equipment used in the GTPT program in the WIPP underground facility. The equipment includes multipacker test tools, data-acquisition systems, pressure transducers, thermocouples, linear variable-differential transformers, a differential-pressure-transmitter panel, and a flow-control device. Section 5.3.2 discusses calibration of GTPT specific equipment. Calibration of other equipment is discussed in Section 3.3.2.

\subsubsection{Description of Equipment}

\subsubsection{MULTIPACKER TEST TOOLS}

Three types of multipacker test tools were used for this testing program. The first type of test tool, which was used in sequences SCP01-1, L4P52-B, and C2H02, was the doublepacker tool described in Section 3.3.1.1 and illustrated in Figure 3-2. The second type of test tool, which was also used in sequence SCP01-1, had the same overall configuration as the first type of test tool except that inflatable elements constructed of hard rubber by TAM international were used in place of the soft rubber elements manufactured by Baski, Inc. TAM elements were used to try to avoid pressure bypass around the packers as was suspected with the first type of test tool in sequence SCP01-1. The third type of test tool, which was used in sequence SCP01-2, had two sliding end, 3.5-inch (8.9-cm) O.D. 


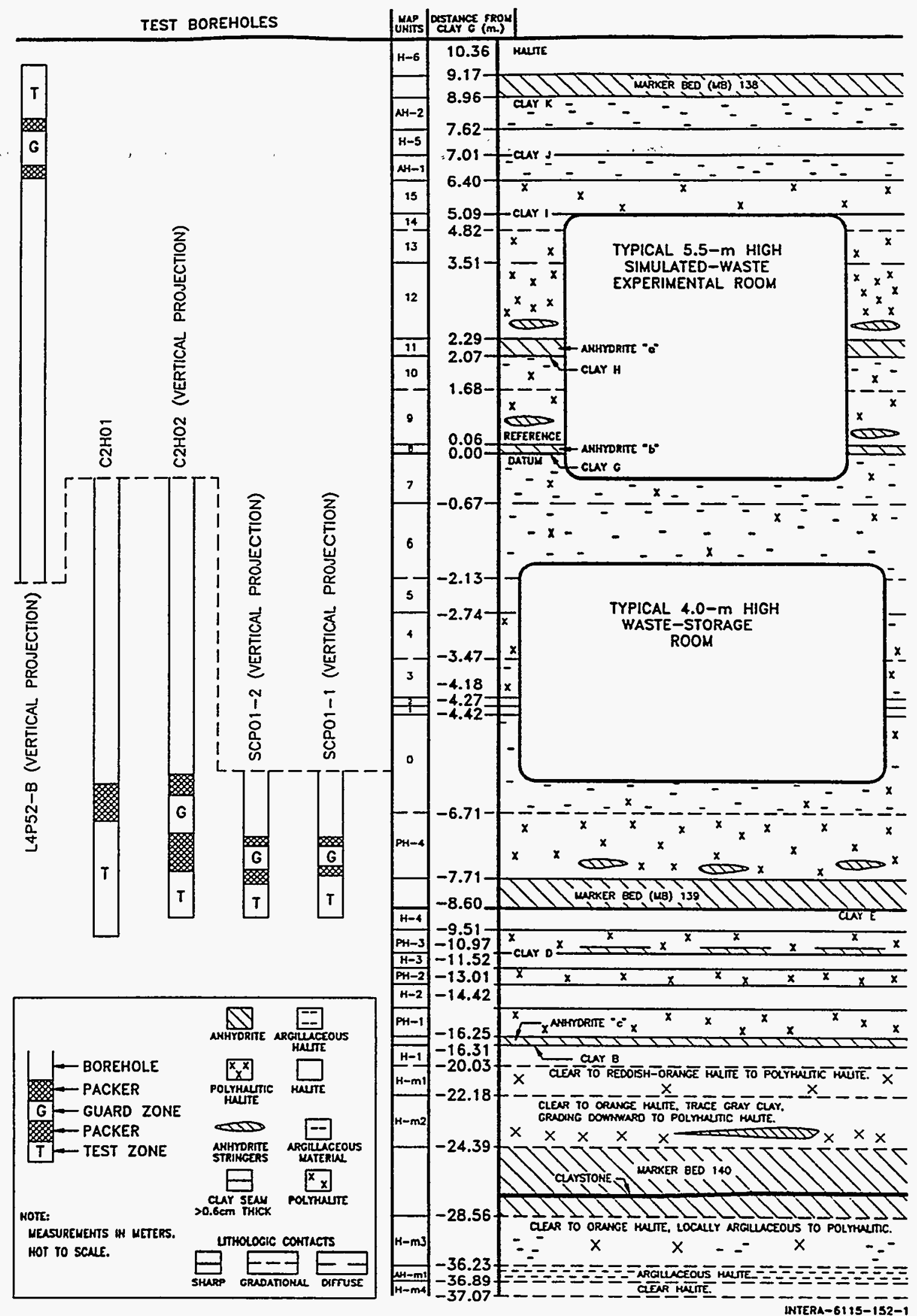

Figure 5-1. Stratigraphic positions of GTPT boreholes with test zones indicated. 
inflatable packers manufactured by Baker Oil Tools mounted on a 2.4-inch $(6.1-\mathrm{cm})$ O.D. mandrel oriented with the packers' fixed ends toward the bottom-hole end of the test-tool. The packers have 66 -inch $(1.7-\mathrm{m})$ long inflatable elastic elements also composed of natural rubber and synthetic materials. The packer elements have approximately 51 -inch $(1.3-\mathrm{m})$ seal lengths when inflated in 4-inch $(10.2-\mathrm{cm})$ diameter boreholes. In all cases, a set of radially oriented tapered jaws or slips that tighten on the test tool mandrel as the tool attempts to move out of the borehole in response to pressure buildup were used to restrain the test tool.

Each multipacker test tool was equipped with three sets of ports to the bottom-hole test zone and the guard zone between the packers. One set of ports was used to transmit pressures from the test and guard zones to the transducers, which were mounted outside of the boreholes. A second set of ports was used to dissipate "squeeze" pressures created during packer inflation and to vent fluid from the isolated intervals to decrease pressure. These two sets of ports were accessed by continuous lengths of 3/16-inch $(0.48-\mathrm{cm})$ O.D. stainless-steel tubing. In some cases (GTPT sequences SCP01-1, C2H02, and L4P52-B), the third set of ports provided access for $1 / 8$-inch $(0.32-\mathrm{cm})$ diameter Type $E$ thermocouples to measure temperatures in the test and guard zones. Packer-inflation pressures were monitored with transducers attached to the packer-inflation lines. Other information regarding the test tools is presented in Section 3.3.1.1.

\subsubsection{DATA-ACQUISITION SYSTEM (DAS)}

A computer-controlled data-acquisition system (DAS) was used to monitor the progress of each test and recorded pressure, in some cases temperature, and in some cases radial borehole closure and axial tool movement (Figure 3-8). Each DAS consisted of an IBM compatible desktop computer for system control and data storage, a Hewlett Packard (HP) 3497A and/or 75000 Data-Acquisition/Control Unit containing power supplies to excite the instruments associated with a given test (transducers, thermocouples, differential-pressure transmitters (DPTs, and LVDTs), a signal scanner to switch and read channels, a 5-1/2 digit voltmeter to measure the output from the instruments associated with a given test (transducers, thermocouples, DPTs, and LVDTs), and an Elgar Model 6000B uninteruptable power supply. The HP-75000 was used only with Labtech (version 4.1.0) data acquisition software. The HP-3497A was used on all other tests in which PERM data acquisition software was used (PERM4F). The data-acquisition software allowed sampling of the instrument output signals at user-specified time intervals ranging from approximately 10 seconds to several days. As data were acquired, they were stored both on the computer's hard disk and on a 3.5-inch diskette. Real-time listing of the data on an auxiliary printer and monitor and/or printer plots of the accumulated data were also possible. 


\subsubsection{MASS FLOW METER}

A Bronkhorst Model F-230C-FA-22-V mass flow meter with a high-pressure nitrogen reservoir as the gas source was used to provide constant gas-injection rates during the gas-threshold-pressure testing. This DAS-controlled mass flow meter allowed a constant gas-injection rate of 0 to $5 \mathrm{~mL} / \mathrm{min}$ to be maintained. The gas injection system used in all of the tests is shown in Figure 5-2. The DAS, pressure transducers, thermocouples, DPT panel, and pressure-maintenance system used for the GTPT program are described in Section 3.3.1.

\subsubsection{Equipment Calibration}

\subsubsection{DATA-ACQUISITION SYSTEM (DAS)}

The DAS equipment and associated software were tested prior to the start of the GTPT program to ensure proper operation. The procedure consisted of testing the response of the HP-3497A or HP-75000 Data-Acquisition/Control Units (DCU) and the DAS software using known input signals to ensure proper signal conversion. The PERM4F DAS software use in conjunction with the HP-3497A DCU was designed and tested by SNL and INTERA Inc. The Labtech 4.1.0 DAS software use in conjunction with the HP-75000 DCU was commercially available software. The DAS software was baselined and controlled in accordance with the SNL approved INTERA WIPP Quality Assurance Manual. In addition, the HP-3497A and HP-75000 DC voltmeter options were calibrated by SNL WIPP calibration laboratory using WIPP Procedure 182 and 427, respectively.

\subsubsection{MASS FLOW METER}

The Bronkhorst Model F-230C-FA-22-V mass flow meter was calibrated by the SNL calibration laboratory in Albuquerque, NM. No sensitivity values were generated for the instrument as it operated from an input voltage actuating a valve that allowed the desired flow rate to be produced. Flow rates were verified by the SNL calibration laboratory in Albuquerque, NM and the deviations from the input values were as much as $\pm 12.9 \%$ of full scale. Calibration information pertaining to the mass flow meter is contained in the SWCF under WPO \#42269. 


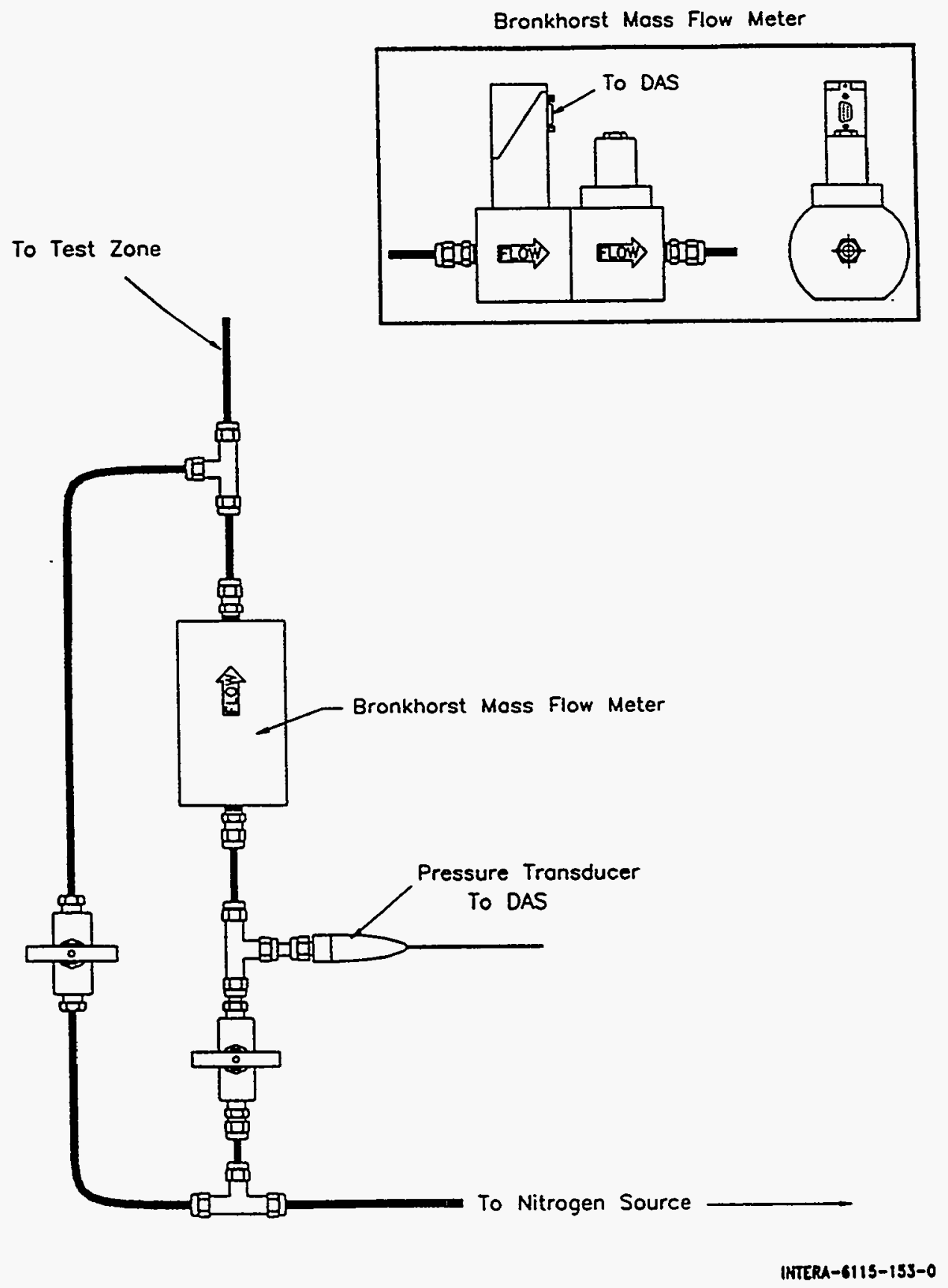

Figure 5-2. Schematic illustration of the constant-rate gas-injection system. 


\subsection{Procedures for Gas-Threshold-Pressure Testing}

Test tools were installed following the procedures described in Section 3.4.1. After the test-zone pressure had stabilized at the assumed formation pressure, the brine in the test zone had to be displaced by gas prior to beginning the constant-rate gas injection into the test zone for the gas-threshold pressure determination. In conducting the gas/brine exchange in a downward-angled borehole, the test-zone packer was deflated to decrease the zone pressure from its stabilization pressure. Nitrogen was injected into the test zone through the test-zone vent line, expelling brine from the test-zone injection line. During the exchange, care was taken to ensure that the gas pressure in the zone did not exceed the pre-exchange fluid pressure. This procedure was carried out until gas flowed from the testzone vent line, indicating that all fluid in the test zone had been displaced. The volume of fluid displaced from the test zone was measured and recorded. At this point, gas was injected through the test-zone vent line and an additional volume of fluid was removed from the guard zone in order to ensure that the test zone would be absent of fluid when the testzone packer was re-inflated. Again, the gas pressure was not allowed to exceed the initial fluid pressure in the zone during injection. In conducting the gas/brine exchange in an upward-angled borehole, the test-zone packer was deflated as previously described. However, in this case, nitrogen was injected into the test zone through the test-zone vent line and brine was expelled (and measured) out of the guard-zone injection line until the entire test-zone volume had been removed. Therefore, the test-zone volume had to be known prior to conducting this operation. Once the entire test-zone volume had been removed, the test-zone packer was re-inflated, taking the same precautions as described above.

After displacing the test-zone brine, nitrogen gas was injected at a constant rate into the test zone using a Bronkhorst Model F-230C-FA-22-V mass flow meter and the test-zone pressure buildup was monitored as the injected gas was compressed. The test-zone pressure was monitored closely until it was determined that the gas-threshold pressure was reached. The pressure was monitored closely in order to determine whether or not the test-zone pressure exceeded the hydrofracture pressure, thus creating an artificial pathway for gas entry into the formation. Once it was determined that gas-threshold pressure had been achieved, the gas injection was terminated and test-zone pressures were monitored. After the constant-rate gas injection in borehole $\mathrm{C} 2 \mathrm{H} 02$ was completed, the test-zone pressure was decreased by venting gas in a series of steps. After each step-decrease, the test-zone pressure was monitored to determine if it was decreasing, in which case the pressure was still above the threshold value and gas was flowing into the formation, or increasing, in which case gas was flowing from the formation back into the borehole. After the gas testing was completed in borehole $\mathrm{C} 2 \mathrm{HO} 2$, the gas was vented and the borehole was refilled with brine. After stable pressure conditions were reached, a pulse-withdrawal test was performed to determine if the brine permeability of MB139 had been altered by residual gas saturation. The test was performed following the procedures outlined in Section 3.4.2. 


\subsection{Boreholes and Test-Tool Configurations for Gas-Threshold-Pressure Testing}

Figure 2-1 shows the locations of the boreholes associated with the GTPT program. Tables 5-1 and 5-2 provide pertinent information on the boreholes and test zones associated with the testing program.

Table 5-1. Borehole Test-Zone Information

\begin{tabular}{ccccccccc}
\hline $\begin{array}{c}\text { Test } \\
\text { Sequence }\end{array}$ & $\begin{array}{c}\text { Test } \\
\text { Horizon }\end{array}$ & $\begin{array}{c}\text { Time Test } \\
\text { Horizon } \\
\text { Penetrated }\end{array}$ & $\begin{array}{c}\text { Borehole } \\
\text { Diameter } \\
(\mathbf{c m})\end{array}$ & $\begin{array}{c}\text { Test Interval } \\
\text { Depth }(\mathbf{m})\end{array}$ & $\begin{array}{c}\text { Borehole } \\
\text { Depth }(\mathbf{m})\end{array}$ & $\begin{array}{c}\text { Sequence } \\
\text { Started }\end{array}$ & $\begin{array}{c}\text { Sequence } \\
\text { Terminated }\end{array}$ \\
\hline SCP01-1 & MB139 & $\begin{array}{c}3-29-90 \\
(10: 30)\end{array}$ & 10.16 & $10.50-14.78$ & 15.48 & $3-30-93$ & $7-13-93$ \\
C2H02 & MB139 & $\begin{array}{c}4-14-89 \\
(09: 00)\end{array}$ & 10.16 & $9.20-10.68$ & 10.91 & $8-10-93$ & $3-21-94$ \\
L4P52-B & MB138 & $\begin{array}{c}12-14-92 \\
12: 00\end{array}$ & 10.16 & $13.89-14.02$ & 14.12 & $12-29-93$ & $3-31-94$ \\
SCP01-2 & MB139 & $\begin{array}{c}3-29-90 \\
(10: 30)\end{array}$ & 10.16 & $10.50-14.78$ & 15.48 & $4-15-94$ & $6-27-94$ \\
\hline
\end{tabular}

Table 5-2. Borehole Locations, Orientations, and Drilling Information

\begin{tabular}{|c|c|c|c|c|}
\hline $\begin{array}{l}\text { Test Sequence } \\
\text { (Orientation) }\end{array}$ & $\begin{array}{c}\text { Interval } \\
\text { Drilled/Cored } \\
\text { (m) }\end{array}$ & $\begin{array}{c}\text { Date } \\
\text { Drilled/Cored }\end{array}$ & Location & $\begin{array}{c}\text { Excavation Date of } \\
\text { Room }\end{array}$ \\
\hline $\begin{array}{c}\text { SCP01-1 } \\
\text { (downward } 77^{\circ} \text { ) } \\
\text { ( from vertical) }\end{array}$ & $0-15.48$ & $\begin{array}{l}3-26-90 \text { to } \\
3-30-90\end{array}$ & $\begin{array}{c}\text { Core Storage } \\
\text { Library }\end{array}$ & $\begin{array}{c}\text { April \& May } \\
1989\end{array}$ \\
\hline $\begin{array}{c}\mathrm{C} 2 \mathrm{HO} 2 \\
\text { (downward } 45^{\circ} \text { ) } \\
\text { (from vertical) }\end{array}$ & $0-10.91$ & $\begin{array}{l}4-12-89 \text { to } \\
4-17-89\end{array}$ & Room C2 & $\begin{array}{c}\text { March \& April } \\
1984\end{array}$ \\
\hline $\begin{array}{c}\text { L4P52-B } \\
\text { (upward 40 }) \\
\text { (from vertical) }\end{array}$ & $9.02-14.12$ & $\begin{array}{l}12-11-92 \text { to } \\
12-14-92\end{array}$ & Room L4 & $\begin{array}{c}\text { February } \\
1989\end{array}$ \\
\hline $\begin{array}{c}\text { SCP01-2 } \\
\text { (downward } 77^{\circ} \text { ) } \\
\text { ( from vertical) }\end{array}$ & $0-15.48$ & $\begin{array}{l}3-26-90 \text { to } \\
3-30-90\end{array}$ & $\begin{array}{l}\text { Core Storage } \\
\text { Library }\end{array}$ & $\begin{array}{l}\text { April \& May } \\
1989\end{array}$ \\
\hline
\end{tabular}

Figure 5-3 schematically depicts the Core Storage Library in plan view and in cross section, showing the location and orientation of borehole SCP01. The test-tool configurations, as they were installed for test sequences SCP01-1 and SCP01-2, are illustrated in Figures 5-4 through 5-8. 

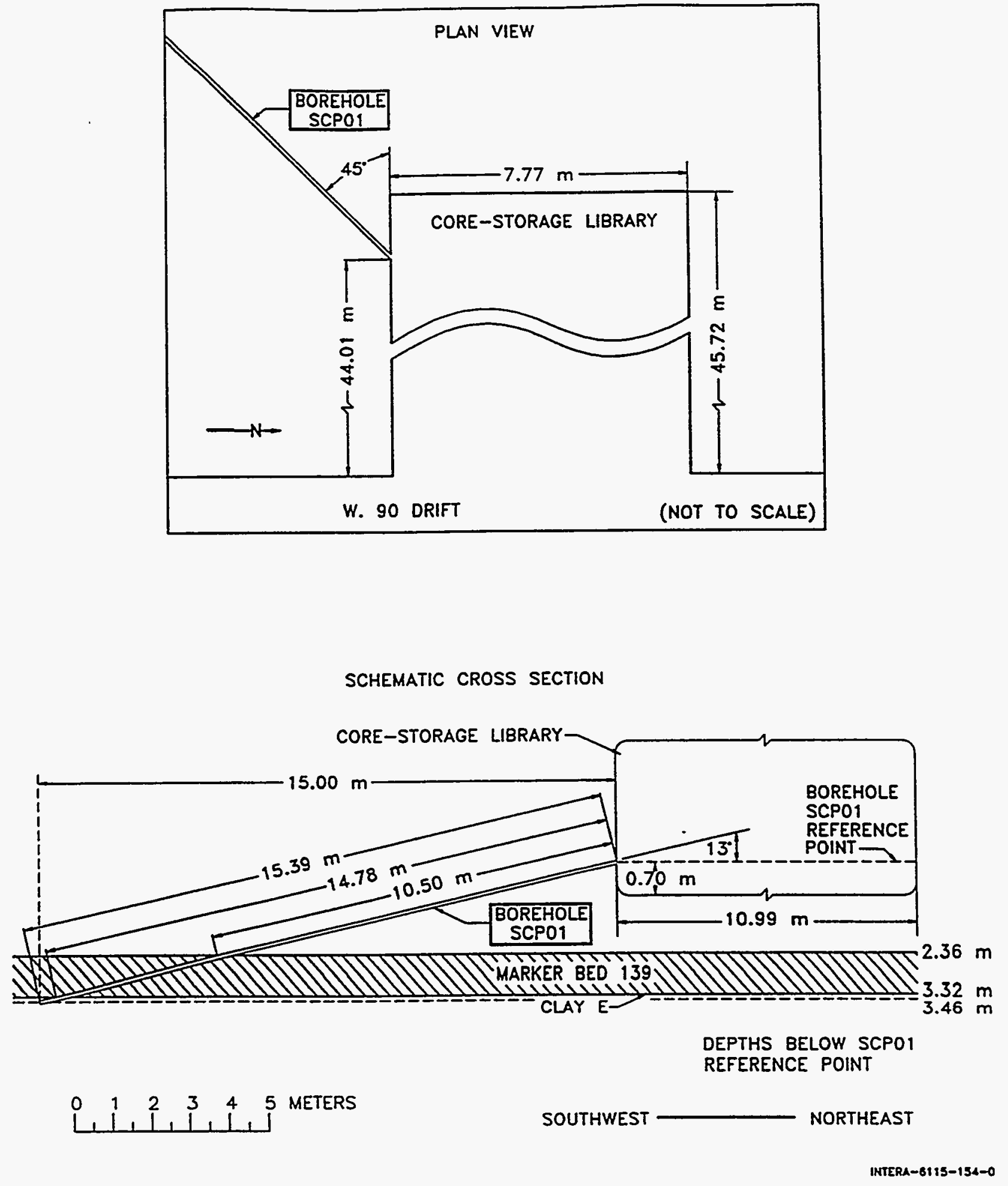

Figure 5-3. Spatial representation of the Core Storage Library showing the location and orientation of test borehole SCP01. 
SCPO1-1

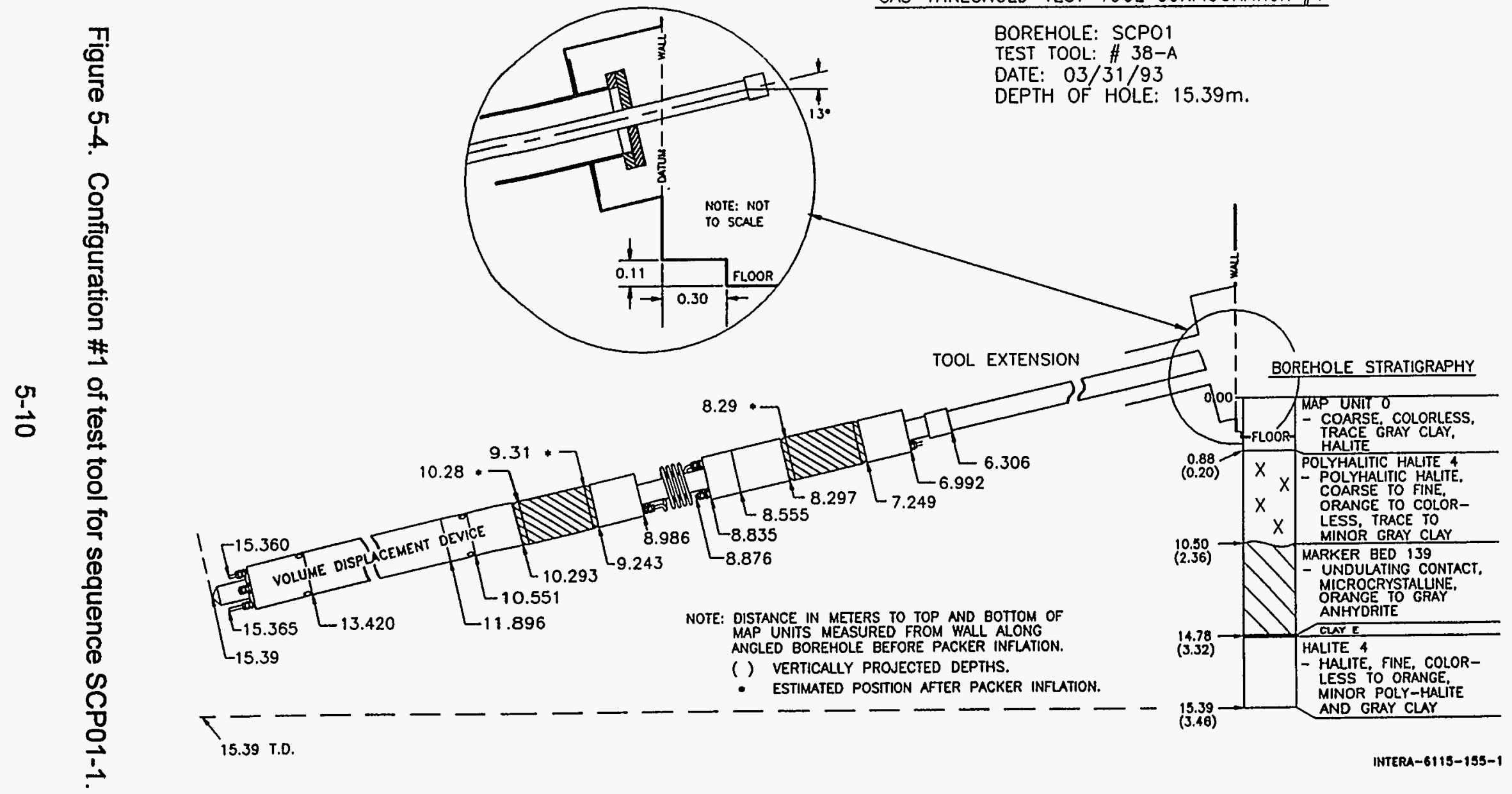


SCP01-1

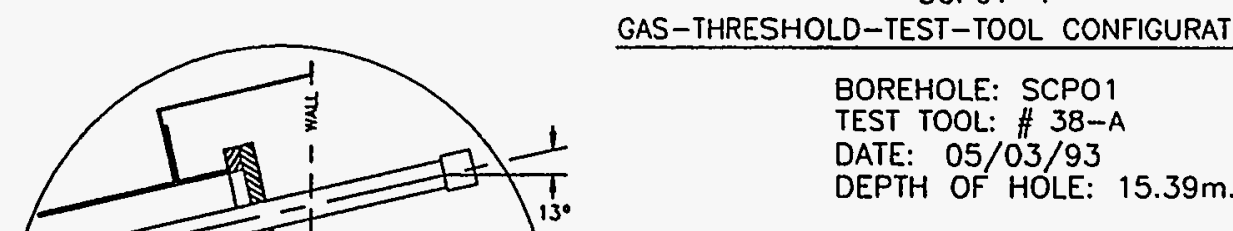

.

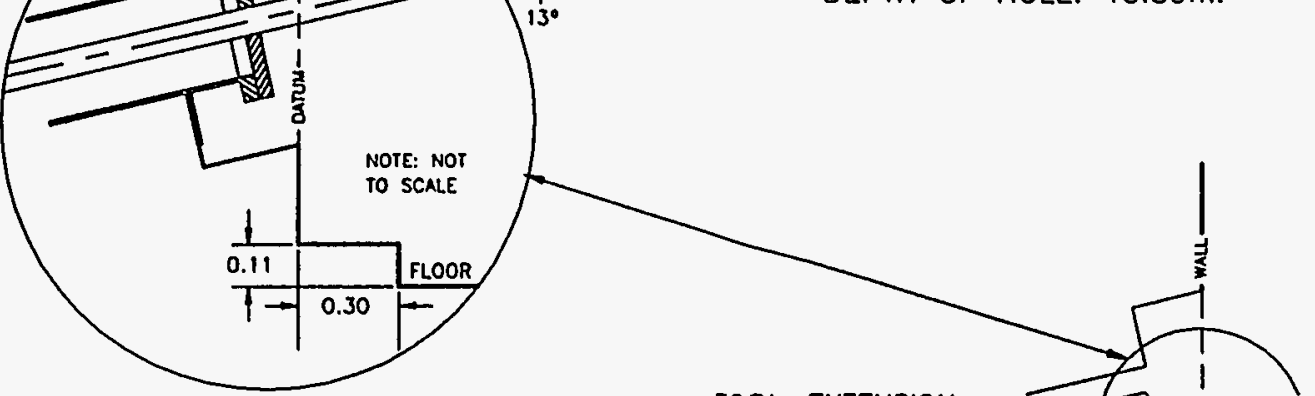

TOOL EXTENSION

ANGLEO BOREHOLE BEFORE PACKER INFLATION.

() VERTICALLY PROJECTED DEPTHS.

- ESTIMATED POSITION ATTER PACKER INFLATION.

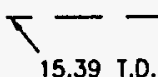


SCP01-1

GAS-THRESHOLD-TEST-TOOL CONFIGURATION \#3

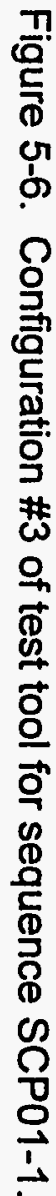

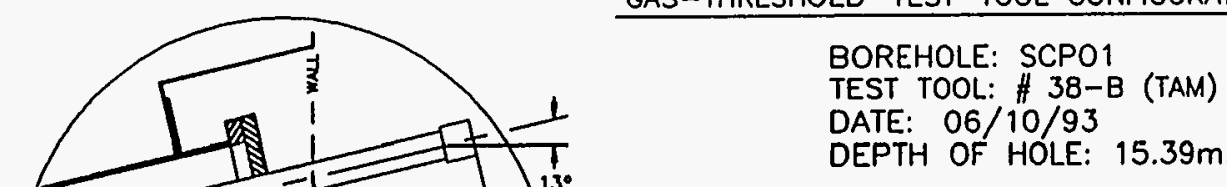

DEPTH OF HOLE: $15.39 \mathrm{~m}$.

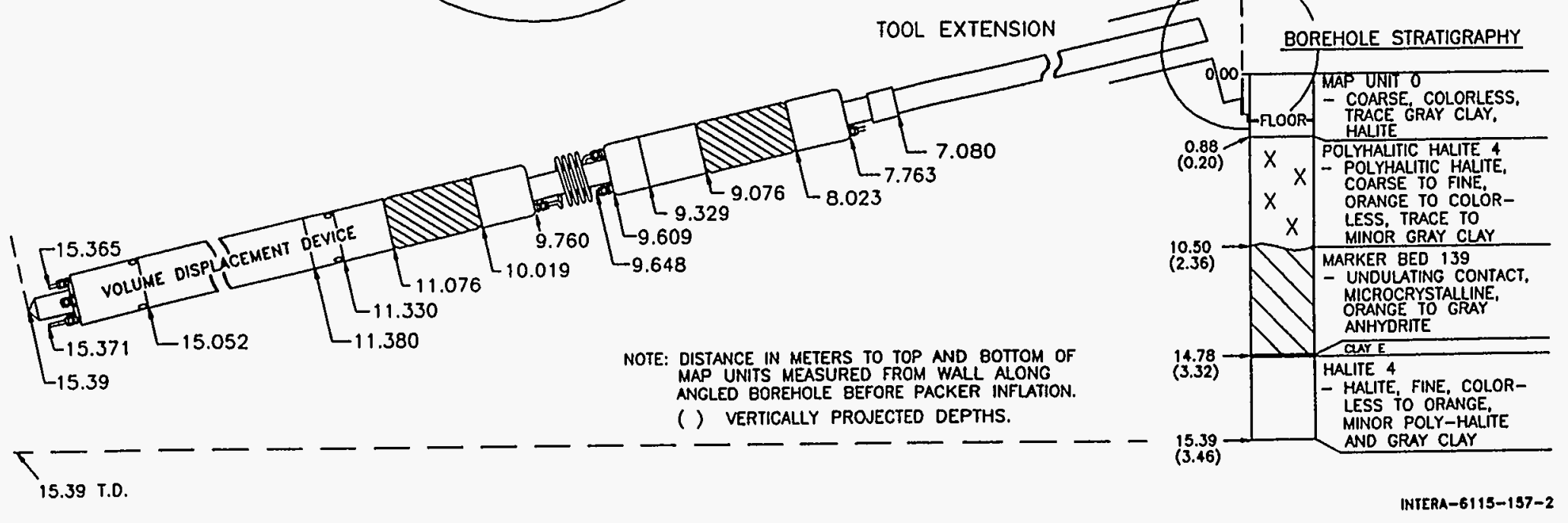




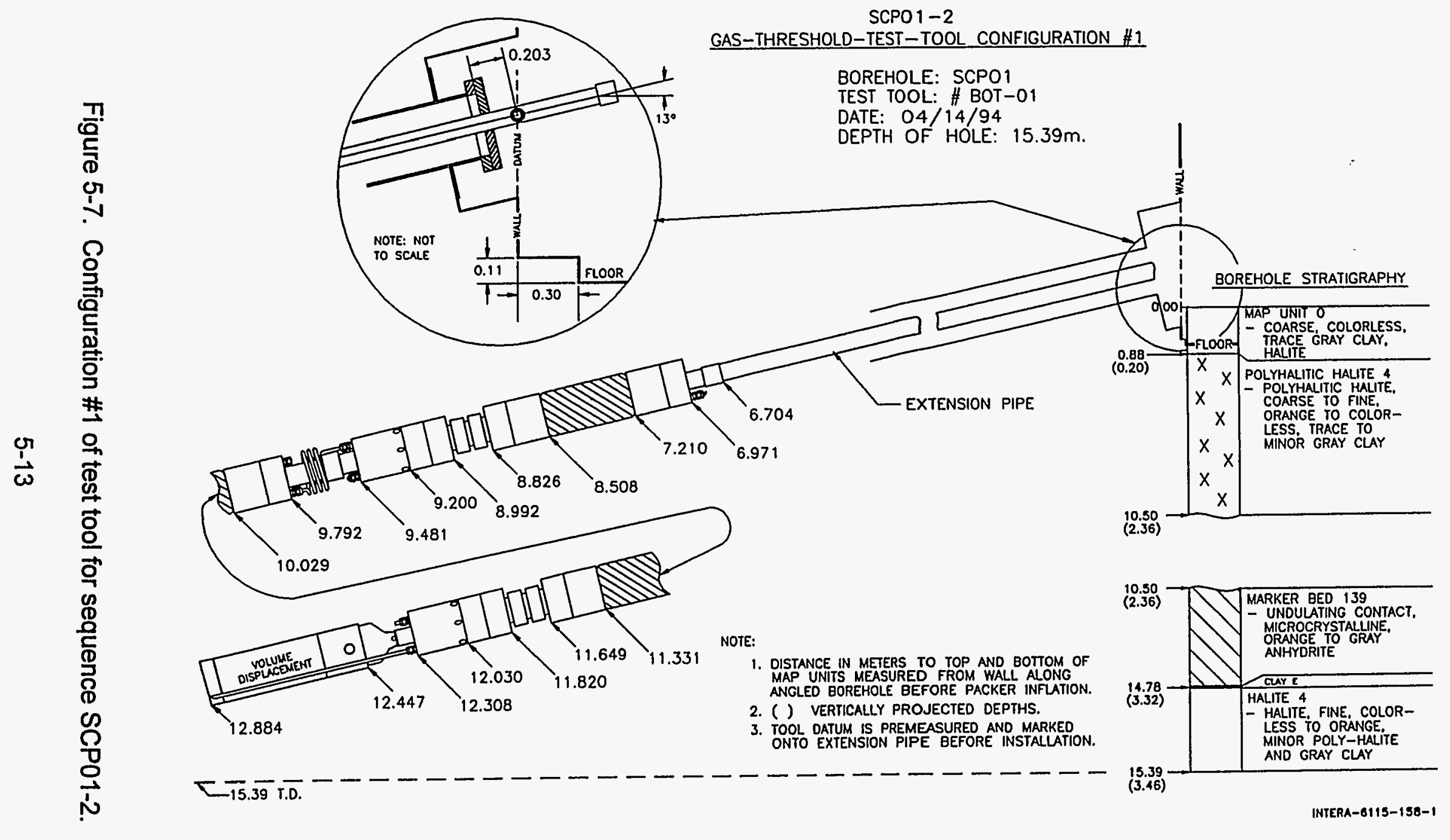




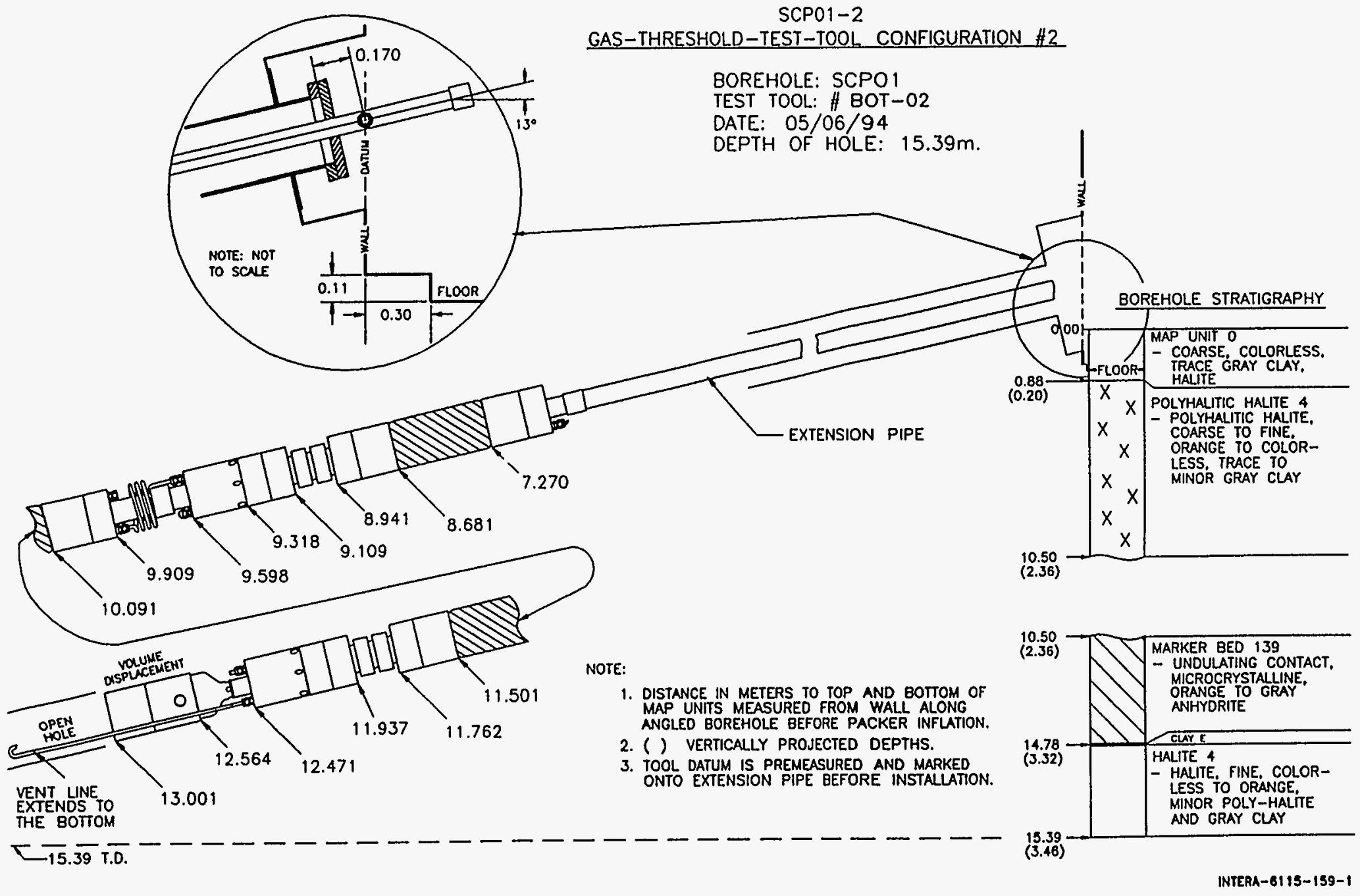


Figure 4-7 schematically depicts Room $\mathrm{C} 2$ in plan view and in cross section, showing the location and orientation of boreholes $\mathrm{C} 2 \mathrm{H} 01$ and $\mathrm{C} 2 \mathrm{HO}$. The test-tool configurations for test borehole $\mathrm{C} 2 \mathrm{H} 02$ and observation borehole $\mathrm{C} 2 \mathrm{HO1}$, as they were installed for test sequence $\mathrm{C} 2 \mathrm{H} 02$, are illustrated in Figures 5-9 through 5-11.

Figure 3-20 schematically depicts Room $L 4$ in plan view and in cross section, showing the location and orientation of borehole L4P52. The test-tool configuration, as it was installed for test sequence L4P52-B, is illustrated in Figure 3-31.

\subsubsection{Borehole Drilling}

The boreholes for the test program (i.e. SCP01, $\mathrm{C} 2 \mathrm{H} 02, \mathrm{C} 2 \mathrm{H} 01$, and L4P52) were drilled according to the procedures for compressed-air drilling outlined in Section 3.5.2. Core was not collected for test and observation boreholes $\mathrm{C} 2 \mathrm{H} 01, \mathrm{C} 2 \mathrm{H} 02$, and SCPO1 for GTPTs during the time period covered in this report. Core description for test borehole L4P52 (Bseries) are provided in Appendix A. Detailed descriptions of the core that was recovered from $\mathrm{C} 2 \mathrm{HO} 1$ and $\mathrm{C} 2 \mathrm{H} 02$ are given in Saulnier et al. (1991). Additional documentation for boreholes $\mathrm{C} 2 \mathrm{HO1}, \mathrm{C} 2 \mathrm{H02}$, L4P52, and SCP01 are provided in the SWCF under Group 5: Salado Testing Activities, (WPO \#23253). 
$\mathrm{C} 2 \mathrm{HO} 1$

SINGLE-PACKER TOOL CONFIGURATION \#2

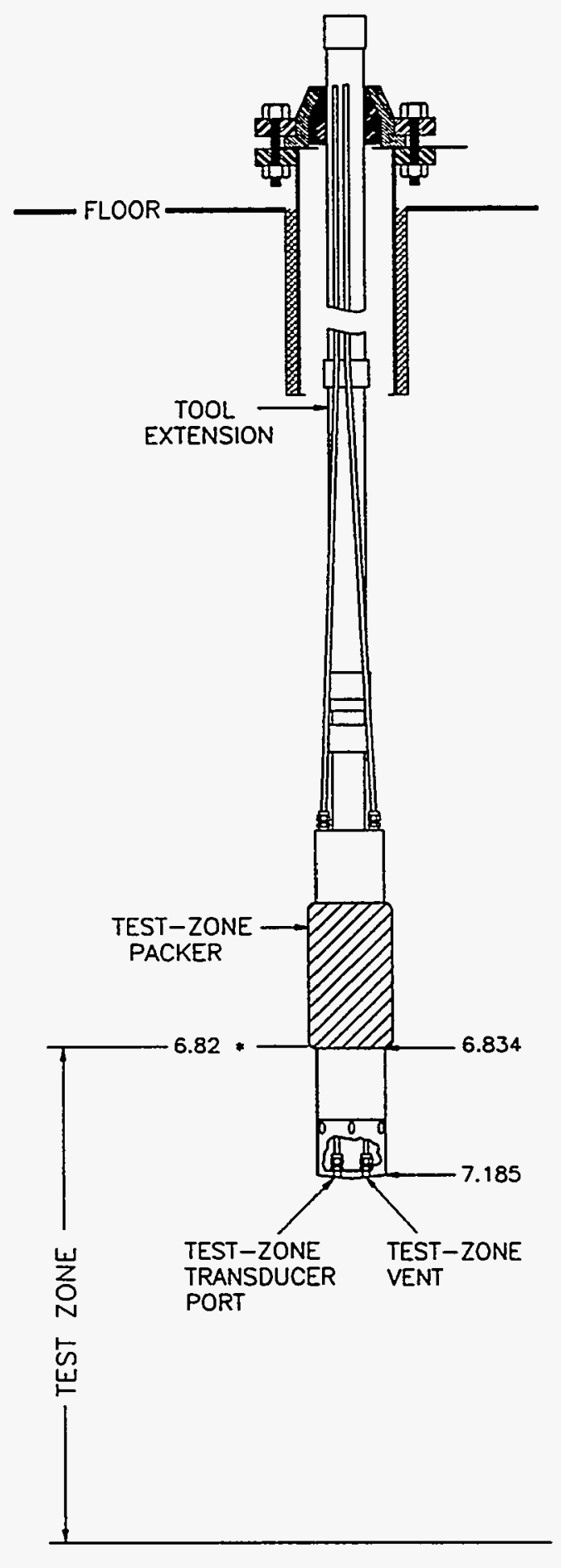

BOREHOLE: C2HO 1

DATE: $02 / 11 / 91$

DEPTH OF HOLE: $8.97 \mathrm{~m}$.

BOREHOLE STRATIGRAPHY

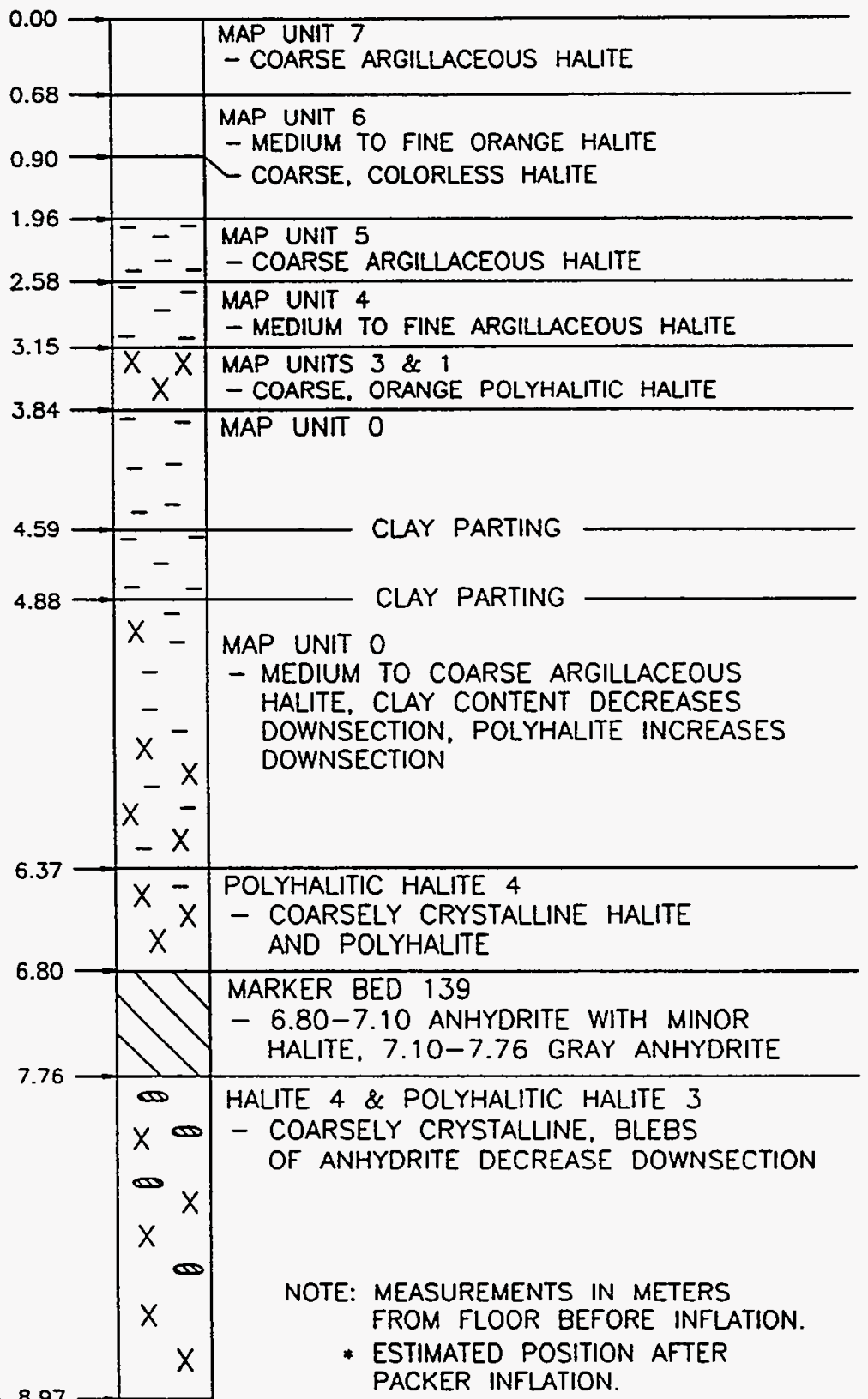

INTERA-6115-163-1

Figure 5-9. Configuration of monitor tool for sequence $\mathrm{C} 2 \mathrm{HO} 2$ in borehole $\mathrm{C} 2 \mathrm{H} 01$. 


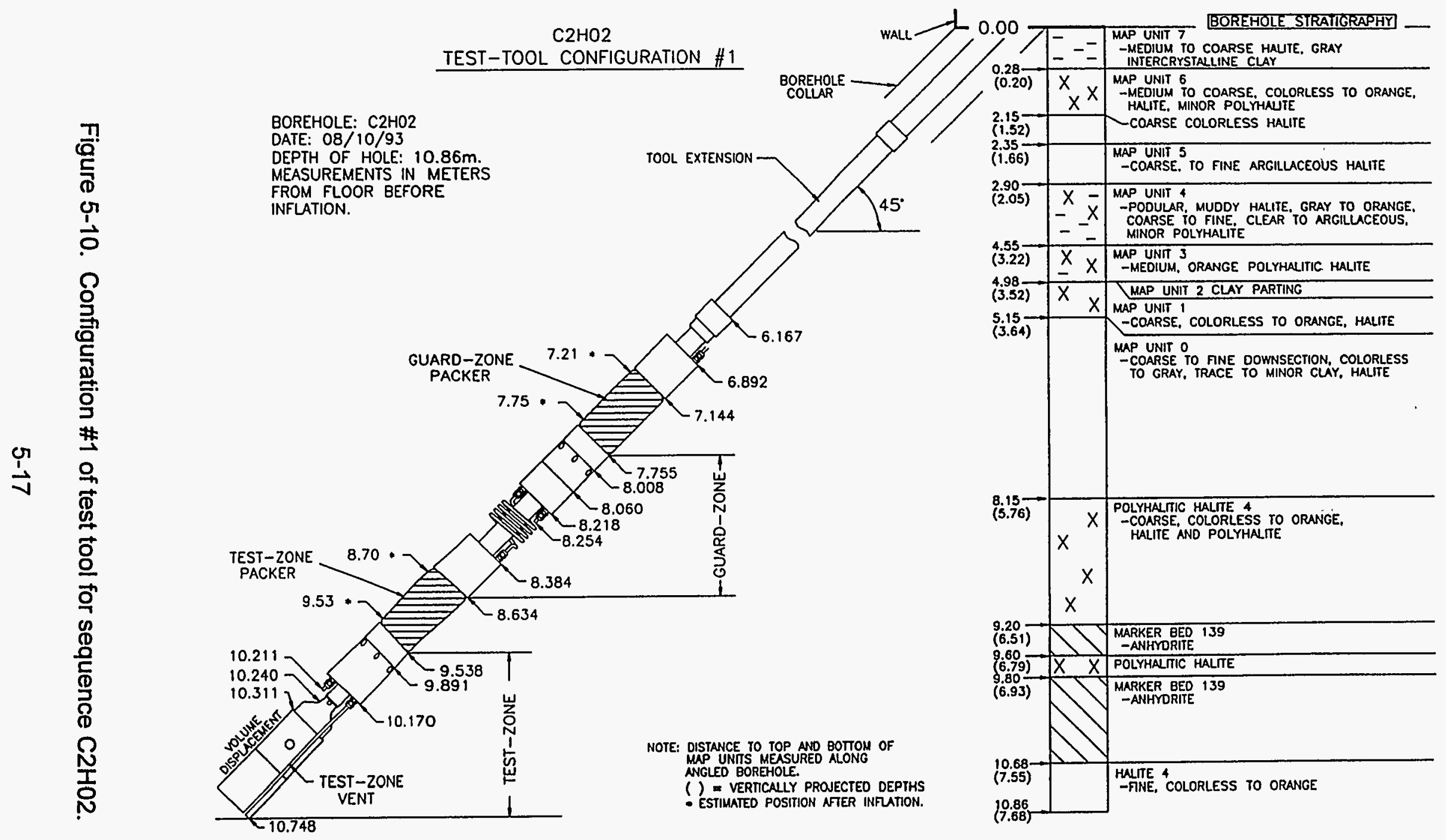

INTERA-B115-162-1 


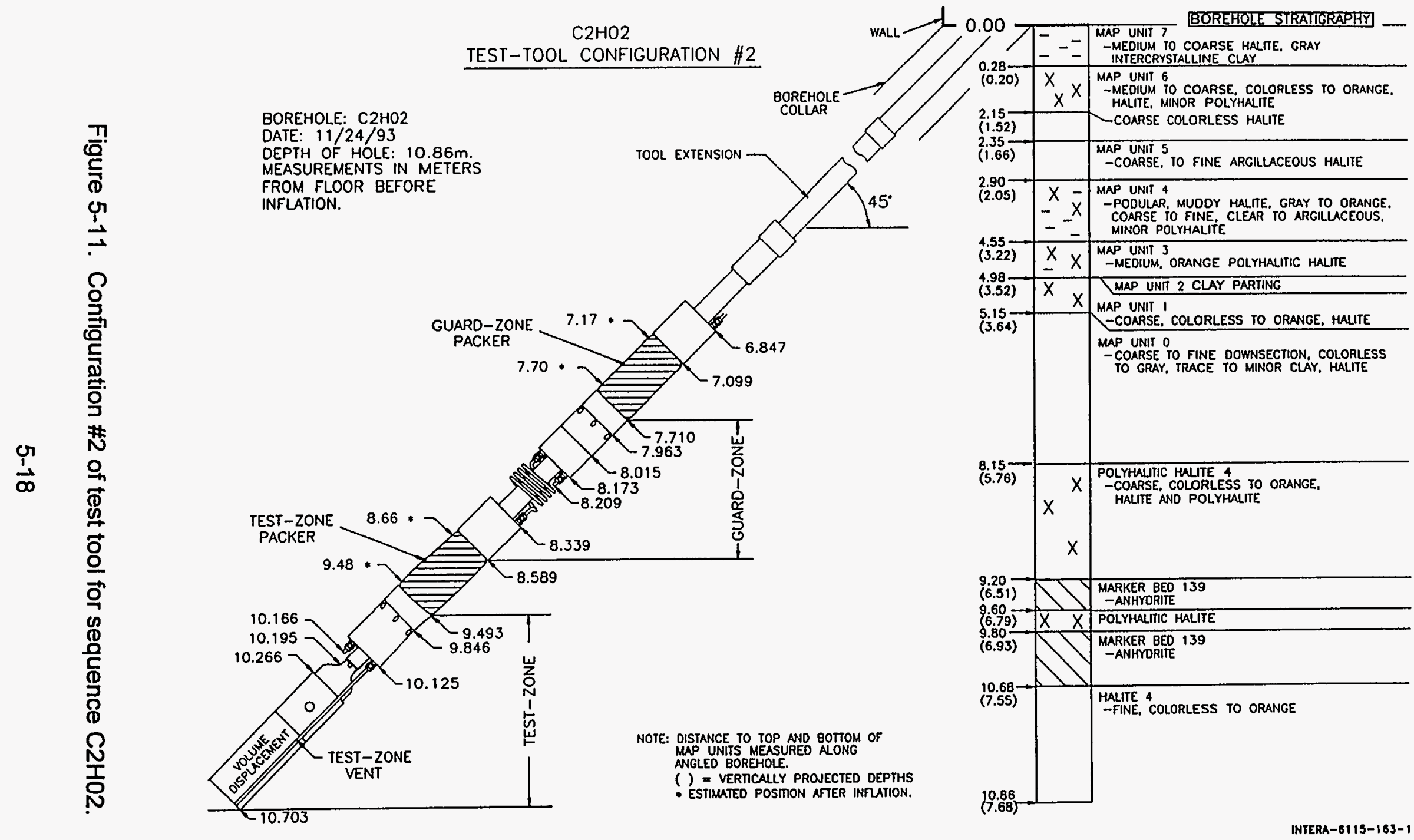




\subsection{Gas-Threshold-Pressure Test Data}

The following section presents the results of the tests in the three boreholes that were investigated: Ten constant-rate gas injection tests, two test-zone compressibility tests, seven gas/brine exchanges, eight depressurization steps, and four pulse-withdrawal tests were performed during the test program.

Acronyms used in the tables presented in this section are as follows:
CMP = Compressibility Test
$\mathrm{G} / \mathrm{BE}=\mathrm{Gas} /$ Brine Exchange
$\mathrm{GI}=$ Constant-Rate Gas-Injection Test
PW $=$ Pulse-Withdrawal Test
DPS = Depressurization Step
$T Z=$ Test Zone
TZP = Test Zone Packer
$G Z=$ Guard Zone
GZP = Guard Zone Packer
Zone Fluid Volume = Isolated Zone Volume+Tubing Volume-Tool Volume

Table 5-3 summarizes the results from all of these tests. Complete data files and abridged tabulations of the data are stored in the SWCF under WPO \#42269.

Table 5-3. Summary of Tests Associated with the GTPT Program

\begin{tabular}{|c|c|c|c|c|c|c|c|c|c|c|c|c|}
\hline $\begin{array}{c}\text { GTPT } \\
\text { Sequence }\end{array}$ & Zone & $\begin{array}{c}\text { Date } \\
\text { Started } \\
\text { (mm-dd-yy) }\end{array}$ & $\begin{array}{l}\text { Test } \\
\text { Type }\end{array}$ & Unit & $\begin{array}{c}\text { Initial } \\
\text { Pressure } \\
\text { (MPa) }\end{array}$ & $\begin{array}{c}\text { Final } \\
\text { Pressure } \\
\text { (MPa) }\end{array}$ & $\begin{array}{c}\text { Fluid Volume } \\
\text { Injected (+) } \\
\text { Withdrawn (-) } \\
\text { (mL) }\end{array}$ & $\begin{array}{c}\text { Flow } \\
\text { Rate } \\
\text { (mL/min) }\end{array}$ & $\begin{array}{l}\text { Zone } \\
\text { Length } \\
(\mathrm{cm})\end{array}$ & $\begin{array}{c}\text { Zone } \\
\text { Radius } \\
\text { (cm) }\end{array}$ & $\begin{array}{c}\text { Tool } \\
\text { Volume } \\
\text { (mL) }\end{array}$ & $\begin{array}{l}\text { Zone Fluid } \\
\text { Volume } \\
\text { (mL) }\end{array}$ \\
\hline SCP01-1 & $T Z$ & $3-31-93$ & CMP & MB139 & 10.700 & 0.000 & 230 & N/A & 511 & 13.831 & 35812 & 5853 \\
\hline SCP01-1 & $T Z$ & $4-26-93$ & G/BE & MB139 & 9.577 & 9.577 & -6500 & N/A & 511 & 13.831 & 35812 & 5853 \\
\hline SCP01-1 & $T Z$ & $4-26-93$ & $\mathrm{Gl} \# \mathbf{1}$ & MB139 & 9.577 & 9.577 & N/A & 1.0 & 511 & 13.831 & 35812 & 5853 \\
\hline SCP01-1 & $\pi$ & $4-27-93$ & GI \#2 & MB139 & 9.509 & 9.509 & N/A & 4.5 & 511 & 13.831 & 35812 & 5853 \\
\hline SCP01-1 & GZ & $5-26-93$ & PW & Halite & 7.950 & 7.713 & -18.5 & N/A & 102 & 13.831 & 6463 & 1915 \\
\hline $\mathrm{C} 2 \mathrm{HO}$ & $T Z$ & $9-10-93$ & G/BE \#1 & MB139 & 8.369 & 8.380 & -1760 & N/A & 133 & 6.259 & 7139 & 4318 \\
\hline $\mathrm{C} 2 \mathrm{HO}$ & TZ & $9-14-93$ & GI & MB139 & 8.359 & 8.361 & N/A & 3.0 & 133 & 6.259 & 7139 & 4318 \\
\hline $\mathrm{C} 2 \mathrm{HO}$ & $T Z$ & $10-11-93$ & DPS \#1 & MB139 & 10.475 & 9.970 & N/A & N/A & 133 & 6.259 & 7139 & 4318 \\
\hline $\mathrm{C} 2 \mathrm{HO}$ & $T Z$ & $10-15-93$ & DPS \#2 & MB139 & 9.836 & 9.587 & N/A & N/A & 133 & 6.259 & 7139 & 4318 \\
\hline $\mathrm{C} 2 \mathrm{HO} 2$ & $\mathrm{TZ}$ & $10-19-93$ & DPS \# & MB139 & 9.565 & 9.401 & N/A & N/A & 133 & 6.259 & 7139 & 4318 \\
\hline $\mathrm{C} 2 \mathrm{HO} 2$ & $T Z$ & $10-22-93$ & DPS \#4 & MB139 & 9.426 & 9.227 & N/A & N/A & 133 & 6.259 & 7139 & 4318 \\
\hline $\mathrm{C} 2 \mathrm{HO}$ & $T Z$ & $10-26-93$ & DPS \#5 & MB139 & 9.248 & 9.021 & N/A & N/A & 133 & 6.259 & 7139 & 4318 \\
\hline $\mathrm{C} 2 \mathrm{HO} 2$ & TZ & $11-1-93$ & DPS \#6 & MB139 & 9.073 & 8.950 & N/A & N/A & 133 & 6.259 & 7139 & 4318 \\
\hline $\mathrm{C} 2 \mathrm{HO} 2$ & $T Z$ & $11-11-93$ & DPS \#7 & MB139 & 8.975 & 8.856 & N/A & N/A & 133 & 6.259 & 7139 & 4318 \\
\hline $\mathrm{C}_{2} \mathrm{HO} 2$ & $T Z$ & $11-23-93$ & G/BE \#2 & MB139 & 8.891 & 8.154 & 4350 & N/A & 133 & 6.259 & 7139 & 4318 \\
\hline
\end{tabular}


Table 5-3 (Continued). Summary of Tests Associated with the GTPT Program

\begin{tabular}{|c|c|c|c|c|c|c|c|c|c|c|c|c|}
\hline $\begin{array}{c}\text { GTPT } \\
\text { Sequence }\end{array}$ & Zone & $\begin{array}{c}\text { Date } \\
\text { Started } \\
\text { (mm-dd-yy) }\end{array}$ & $\begin{array}{l}\text { Test } \\
\text { Type }\end{array}$ & Unit & $\begin{array}{l}\text { Initial } \\
\text { Pressure } \\
\text { (MPa) }\end{array}$ & $\begin{array}{c}\text { Final } \\
\text { Pressure } \\
\text { (MPa) }\end{array}$ & $\begin{array}{l}\text { Fluid Volume } \\
\text { Injected (+) } \\
\text { withdrawn (-) } \\
\text { (mL) }\end{array}$ & $\begin{array}{c}\text { Flow } \\
\text { Rate } \\
\text { (mL/min) }\end{array}$ & $\begin{array}{l}\text { Zone } \\
\text { Length } \\
\text { (cm) }\end{array}$ & $\begin{array}{c}\text { Zone } \\
\text { Radius } \\
\text { (cm) }\end{array}$ & $\begin{array}{c}\text { Tool } \\
\text { Volume } \\
\text { (mL) }\end{array}$ & $\begin{array}{l}\text { Zone Fluid } \\
\text { Volume } \\
\text { (mL) }\end{array}$ \\
\hline $\mathrm{C} 2 \mathrm{HO} 2$ & $T Z$ & $11-24-93$ & DPS \#8 & MB139 & 7.891 & 6.837 & N/A & N/A & 133 & 6.259 & 7139 & 4318 \\
\hline $\mathrm{C} 2 \mathrm{HO} 2$ & $\mathbf{G Z}$ & $11-29-93$ & PW & Halite & 6.413 & 0.000 & -1720 & N/A & 95 & 6.259 & 5908 & 2256 \\
\hline $\mathrm{C} 2 \mathrm{HO} 2$ & $T Z$ & $1-24-94$ & PW & MB139 & 9.159 & 6.743 & -10.4 & N/A & 138 & 6.259 & 7139 & 4699 \\
\hline L4P52-B & $T Z$ & $12-31-93$ & G/BE\#1 & N/A & 8.728 & 8.307 & -7330 & N/A & 141 & 5.850 & 8525 & 2718 \\
\hline L4P52-B & TZ & 1-17-94 & G/BE \#2 & N/A & 8.737 & 8.524 & -1990 & N/A & 141 & 5.850 & 8525 & 2718 \\
\hline L4P52-B & $T Z$ & $9-27-94$ & GI \#1 & MB138 & 8.602 & 8.602 & N/A & 2.0 & 141 & 5.850 & 8525 & 2718 \\
\hline L4P52-B & $\mathrm{TZ}$ & $1-28-94$ & G1 \#2 & MB138 & 10.543 & 10.543 & N/A & 0.8 & 141 & 5.850 & 8525 & 2718 \\
\hline L4P52-8 & $\mathrm{TZ}$ & $2-14-94$ & $\mathrm{GI} \# 3$ & MB138 & 9.391 & 9.391 & N/A & 1.1 & 141 & 5.850 & 8525 & 2718 \\
\hline L4P52-B & $T Z$ & $2-16-94$ & GI \#4 & MB138 & 10.455 & 10.455 & N/A & 1.1 & 141 & 5.850 & 8525 & 2718 \\
\hline L4P52-B & $\pi z$ & $3-9-94$ & GI \#5 & MB138 & 9.791 & 9.791 & N/A & 0.6 & 141 & 5.850 & 8525 & 2718 \\
\hline L4P52-B & $T z$ & $3-14-94$ & DPS & MB138 & 9.866 & 9.560 & N/A & N/A & 141 & 5.850 & 8525 & 2718 \\
\hline SCP01-2 & $T Z$ & 4-15-94 & CMP & MB139 & 5.500 & 5.446 & 330 & N/A & 406 & 13.831 & 4831 & 28384 \\
\hline SCP01-2 & TZ & $5-2-94$ & G/BE \#1 & MB139 & 9.514 & 9.514 & -2390 & N/A & 406 & 13.831 & 4831 & 28384 \\
\hline SCP01-2 & $T Z$ & $5-16-94$ & G/BE \#2 & MB139 & 9.512 & 9.344 & -24655 & N/A & 406 & 13.831 & 4831 & 28384 \\
\hline SCP01-2 & $T Z$ & $5-17-94$ & GI \#1 & MB139 & 9.261 & 9.261 & N/A & 2.0 & 406 & 13.831 & 4831 & 28384 \\
\hline SCP01-2 & $T Z$ & 5-26-94 & GI\#2 & MB139 & 8.708 & 8.659 & N/A & 0.2 & 406 & 13.831 & 4831 & 28384 \\
\hline
\end{tabular}

\subsubsection{Gas-Threshold-Pressure Testing in the Core Storage Library}

\subsubsection{GAS-THRESHOLD-PRESSURE TESTING SEQUENCE SCP01-1}

The first GTPT sequence to be conducted took place in the Core Storage Library in borehole SCP01. This test sequence was designed to investigate the gas threshold pressure of MB139 in an environment removed from the WIPP excavations. Problems during this testing sequence associated with the inability of the packer elements to form a reliable seal were encountered. These problems were not satisfactorily resolved while the testing sequence was in progress. Table 5-4 gives a detailed description of the events that occurred during test sequence SCP01-1. 
Table 5-4. Testing Sequence SCP01-1 Events

\begin{tabular}{|c|c|c|c|c|}
\hline EVENT & DATE & $\begin{array}{l}\text { CALENDAR } \\
\text { DAY }\end{array}$ & $\begin{array}{c}1993 \\
\text { CALENDAR } \\
\text { DAY }\end{array}$ & $\begin{array}{c}\text { TIME } \\
\text { (HH:MM:SS) }\end{array}$ \\
\hline Remove single-packer, long-term test tool from borehole. & $3-22-93$ & 81 & 81 & 13:19:00 \\
\hline Begin data file SCP01B01. & $3-30-93$ & 89 & 89 & $14: 19: 45$ \\
\hline End data file SCP01B01. & 3-30-93 & 89 & 89 & 14:30:16 \\
\hline $\begin{array}{l}\text { Install double-packer test tool \#38A in preparation for GTPT as indicated in tool } \\
\text { configuration diagram \#1 (Figure 5-4). }\end{array}$ & $3-30-93$ & 89 & 89 & $14: 33: 00$ \\
\hline Inflate GZP. & $3-30-93$ & 89 & 89 & 14:34:36 \\
\hline Shut in GZP. & 3-30-93 & 89 & 89 & $14: 37: 50$ \\
\hline Inflate TZP. & $3-30-93$ & 89 & 89 & $14: 40: 09$ \\
\hline Shut in TZP. & $3-30-93$ & 89 & 89 & $14: 44: 50$ \\
\hline Increase GZP pressure. & $3-30-93$ & 89 & 89 & $14: 47: 15$ \\
\hline Shut-in GZ and TZ. & $3-30-93$ & 89 & 89 & 14:48:14 \\
\hline Increase GZP pressure. & 3-30-93 & 89 & 89 & 14:55:11 \\
\hline Depressurize both zones and deflate both packers. & $3-31-93$ & 90 & 90 & 09:00:00 \\
\hline Pull tool out of borehole to the top of GZP. & $3-31-93$ & 90 & so & 11:00:00 \\
\hline Infate GZP to check for leaks. & 3-31-93 & 90 & 90 & 11:05:00 \\
\hline Install test tool to original position. & $3-31-93$ & 90 & 90 & $11: 40: 00$ \\
\hline Inflate GZP. & $3-31-93$ & 90 & 90 & 11:41:00 \\
\hline Shut in GZP. & $3-31-93$ & 90 & 90 & 11:43:00 \\
\hline Inflate TZP. & $3-31-93$ & 90 & 90 & 11:45:00 \\
\hline Shut in TZP. & $3-31-93$ & 90 & 90 & 11:47:00 \\
\hline Increase TZP pressure. & 3-31-93 & 90 & 90 & 12:03:00 \\
\hline Increase GZP pressure. & 3-31-93 & 90 & 90 & 12:06:00 \\
\hline Begin data file SCP01B02. & 3-31-93 & 90 & 90 & 15:01:14 \\
\hline Begin pressurization of $T Z$ via DPT panel to $-10 \mathrm{MPa}$ for $T Z$ compressibility test. & $3-31-93$ & 90 & 90 & 15:08:12 \\
\hline Increase TZP pressure to $-13.5 \mathrm{MPa}$ & $3.31-93$ & 90 & 90 & 16:04:03 \\
\hline Shut in $T Z$ terminating $T Z$ compressibility test. & $4-6-93$ & 96 & 96 & 11:44:02 \\
\hline End data file SCPO1BO2. & $4-12-93$ & 102 & 102 & 10:54:34 \\
\hline Begin data file SCP01803. & $4-12-93$ & 102 & 102 & 11:11:43 \\
\hline Open TZP to accumulator. & $4-12-93$ & 102 & 102 & 12:56:44 \\
\hline Increase TZP pressure via accumulator. & 4-12-93 & 102 & 102 & 12:57:29 \\
\hline Shut in TZP from accumulator due to leak. & $4-12-93$ & 102 & 102 & 13:07:51 \\
\hline Open GZP to accumulator and increase pressure. & $4-12-93$ & 102 & 102 & 13:13:42 \\
\hline Open TZP to accumulator. & 4-13-93 & 103 & 103 & 11:09:46 \\
\hline Increase TZP pressure via accumulator. & 4-13-93 & 103 & 103 & 11:16:30 \\
\hline Increase GZ pressure. & $4-13-93$ & 103 & 103 & 11:36:56 \\
\hline End data file SCPO1B03. & 4-19-93 & 109 & 109 & 12:11:52 \\
\hline Begin data file SCP01BO4. & 4-19-93 & 109 & 109 & 13:16:36 \\
\hline
\end{tabular}


Table 5-4 (Continued). Testing Sequence SCP01-1 Events

\begin{tabular}{|c|c|c|c|c|}
\hline EVENT & DATE & $\begin{array}{c}\text { CALENDAR } \\
\text { DAY }\end{array}$ & $\begin{array}{c}1993 \\
\text { CALENDAR } \\
\text { DAY }\end{array}$ & $\begin{array}{c}\text { TIME } \\
\text { (HH:MM:SS) }\end{array}$ \\
\hline Increase GZ pressure. & 4-22-93 & 112 & 112 & 11:10:51 \\
\hline Increase GZP pressure. & 4-22-93 & 112 & 112 & 11:28:23 \\
\hline Increase GZ pressure. & 4-22-93 & 112 & 112 & 11:36:24 \\
\hline Attempt to begin gas/brine exchange in $T Z$ but encountered a plugged line. & 4-26-93 & 116 & 116 & 13:07:00 \\
\hline Shut in zone. & 4-26-93 & 116 & 116 & 13:15:00 \\
\hline Remove $\sim 2$ liters of brine. & 4-26-93 & 116 & 116 & 13:35:00 \\
\hline Remove $\sim 1.7$ liters of brine. & 4-26-93 & 116 & 116 & 13:49:00 \\
\hline Remove -1.4 liters of brine. & 4-26-93 & 116 & 116 & 14:02:00 \\
\hline Remove $\sim 1.3$ fiters of brine. & $4-26-93$ & 116 & 116 & 14:08:00 \\
\hline Gas is coming from the vent line. & 4-26-93 & 116 & 116 & 14:11:00 \\
\hline Remove $-100 \mathrm{~mL}$ of brine while unplugging the original plugged line. & 4-26-93 & 116 & 116 & 15:12:00 \\
\hline Complete gas/brine exchange in $T Z$. & 4-26-93 & 116 & 116 & 15:13:00 \\
\hline Momentarily open $T Z$ to flow meter set at $1 \mathrm{~mL}$ min to test system. & 4-26-93 & 116 & 116 & 15:56:00 \\
\hline Begin $N_{2}$ injection \#1 into TZ at $1 \mathrm{~mL}$ min. & $4-26-93$ & 116 & 116 & 16:04:00 \\
\hline Shut in $T Z$ due to possible leak terminating $N_{2} \# 1$ in $T Z$. & $4-26-93$ & 196 & 116 & 16:16:00 \\
\hline Perform over night leak check. & 4-26-93 & 116 & 116 & 16:28:00 \\
\hline Begin $\mathrm{N}_{2}$ injection $\# 2$ into $\mathrm{TZ}$ at $4.5 \mathrm{~mL} \min$. & 4-27-93 & 117 & 117 & 11:07:00 \\
\hline Terminate $\mathrm{N}_{2}$ injection $\# 2$ due to incorrect flow meter reading. & 4-27-93 & 117 & 117 & 11:23:00 \\
\hline Resume $\mathrm{N}_{z}$ injection $\# 2$ into $T Z$ at $4.5 \mathrm{~mL} / \mathrm{min}$. & 4-27-93 & 117 & 117 & 11:33:00 \\
\hline Switch from MDT to MST for recording events in the log book. & 4-29-93 & 119 & 119 & N/A \\
\hline Bypass around TZP is suspected. & 4-29-93 & 119 & 119 & N/A \\
\hline Terminate $\mathrm{N}_{2}$ injection $\# 2$ into $\mathrm{TZ}$. & 4-29-93 & 119 & 119 & 11:22:00 \\
\hline Increase TZP pressure. & 4-29-93 & 119 & 119 & 12:31:00 \\
\hline Increase TZP pressure to $\sim 15 \mathrm{MPa}$. & 4-29-93 & 119 & 119 & 12:49:00 \\
\hline Decrease $G Z$ pressure. & $4-29-93$ & 119 & 119 & 13:18:00 \\
\hline Increase $\mathrm{GZ}$ pressure to $-10 \mathrm{MPa}$. & 4-30-93 & 120 & 120 & 11:39:37 \\
\hline End data file SCP01804. & $5-2-93$ & 122 & 122 & 11:40:16 \\
\hline Begin data file SCP01B05. & $5-2-93$ & 122 & 122 & 11:40:17 \\
\hline Test-zone thermocouple is no longer functioning. & $5-2-93$ & 122 & 122 & 13:38:30 \\
\hline Depressurize $T Z$ (25 to $50 \mathrm{~mL}$ of brine). & $5-3-93$ & 123 & 123 & $08: 45: 00$ \\
\hline Depressurize $G Z(-1480 \mathrm{~mL}$ of brine) & 5-3-93 & 123 & 123 & 08:52:00 \\
\hline Deflate GZP. & 5-3-93 & 123 & 123 & 09:01:00 \\
\hline Deflate TZP. & 5-3-93 & 123 & 123 & 09:06:00 \\
\hline Remove test tool for inspection (bubble on GZP, brine inside tool). & 5-3-93 & 123 & 123 & 11:34:00 \\
\hline $\begin{array}{l}\text { Reinstall tool so that TZP straddles the halite/anhydrite contact as indicated in tool } \\
\text { configuration diagram } \# 2 \text { (Figure 5-5). }\end{array}$ & 5-3-93 & 123 & 123 & 09:00:00 \\
\hline Inflate GZP to $\sim 12.4 \mathrm{MPz}$. & $5-6-93$ & 126 & 126 & 09:25:00 \\
\hline
\end{tabular}


Table 5-4 (Continued). Testing Sequence SCP01-1 Events

\begin{tabular}{|c|c|c|c|c|}
\hline EVENT & DATE & $\begin{array}{l}\text { CALENDAR } \\
\text { DAY }\end{array}$ & $\begin{array}{c}1993 \\
\text { CALENDAR } \\
\text { DAY }\end{array}$ & $\begin{array}{c}\text { TIME } \\
\text { (HH:MM:SS) }\end{array}$ \\
\hline Shut in GZP at $\sim 12.1 \mathrm{MPa}$. & $5-6-93$ & 126 & 126 & $09: 31: 00$ \\
\hline Inflate TZP to $-12.4 \mathrm{MPa}$ & $5-6-93$ & 126 & 126 & $09: 33: 00$ \\
\hline Shut in TZP at $\sim 11.7 \mathrm{MPa}$. & $5-6-93$ & 126 & 126 & 09:35:00 \\
\hline Open GZP to accumulator at $\sim 11.7 \mathrm{MPa}$. & $5-6-93$ & 126 & 126 & 09:40:00 \\
\hline Open TZP to accumulator at $\sim 12.4 \mathrm{MPa}$. & $5-6-93$ & 126 & 126 & 09:48:00 \\
\hline Circulate brine through $T Z$. & 5-6-93 & 126 & 126 & 09:56:00 \\
\hline Open $T Z$ to accumulator at $\sim 11.7 \mathrm{MPa}$ and observed pressure drop to $\sim 9.0 \mathrm{MPa}$. & $5-6-93$ & 126 & 126 & $11: 24: 00$ \\
\hline Increase $T Z$ pressure via accumulator to $-10.3 \mathrm{MPa}$. & $5-7-93$ & 127 & 127 & $11: 26: 00$ \\
\hline Shut in $T$ from accumulator. & $5-10-93$ & 130 & 130 & N/A \\
\hline Increase TZP pressure via accumulator to $13.134 \mathrm{MPa}$. & 5-17-93 & 137 & 137 & $10: 00: 36$ \\
\hline Increase GZP pressure via accumulator to $13.460 \mathrm{MPa}$. & 5-17-93 & 137 & 137 & 10:08:36 \\
\hline Shut in TZ from accumulator at $10.366 \mathrm{MPa}$. & $5-17-93$ & 137 & 137 & 10:14:06 \\
\hline Increase $\mathrm{GZ}$ pressure via accumulator to $11.257 \mathrm{MPa}$. & 5-17-93 & 137 & 137 & 10:34:06 \\
\hline Shut in TZP from accumulator. & 5-18-93 & 138 & 138 & 08:34:00 \\
\hline Open TZP to accumulator. & 5-18-93 & 138 & 138 & 08:38:00 \\
\hline Shut in GZ from accumulator to test for leaks from TZP. & $5-19-93$ & 139 & 139 & 09:47:00 \\
\hline Open GZ to accumulator. & $5-20-93$ & 140 & 140 & 09:21:00 \\
\hline Repair TZ thermocouple. & 5-20-93 & 140 & 140 & 11:44:00 \\
\hline Shut in $\mathrm{GZ}$ from accumutator. & $5-24-93$ & 144 & 144 & 09:03:00 \\
\hline Vent $G Z$ to expel gas that might have been introduced from the accumulator. & $5-24-93$ & 144 & 144 & 09:11:00 \\
\hline $\begin{array}{l}\text { Perform pulse-withdrawal test in } G Z \text { pressure to } 7.713 \mathrm{MPa} \text { removing } \sim 18.5 \mathrm{~mL} \text { of } \\
\text { brine (lesting for gas). }\end{array}$ & $5-26-93$ & 146 & 146 & 08:48:00 \\
\hline Shut in $T Z P$ from accumulator. & $5-26-93$ & 146 & 146 & $12: 42: 00$ \\
\hline End data file SCPO1B05. & $6-1-93$ & 152 & 152 & 07:29:25 \\
\hline Depressurize $T Z$ ( $-460 \mathrm{~mL}$ of fluid). & $6-1-93$ & 152 & 152 & 08:48:00 \\
\hline Depressurize GZ (large amount of gas). & 6.1 .93 & 152 & 152 & 08:55:00 \\
\hline Deflate GZP. & $6-1-93$ & 152 & 152 & 09:00:00 \\
\hline Deflate TZP. & $6-1-93$ & 152 & 152 & 10:00:00 \\
\hline Attempt to remove test tool to examine TZP for leaks but it got stuck in borehole. & $6-1-93$ & 152 & 152 & 11:00:00 \\
\hline $\begin{array}{l}\text { Reinstall test tool \#38B with new (hard nubber) TAM elements as indicated in tool } \\
\text { configuration diagram } \$ 3 \text { (Figure 5-6). }\end{array}$ & 6-10-93 & 161 & 161 & 12:00:00 \\
\hline Begin data file SCP01B06. & $6-10-93$ & 161 & 161 & 12:45:14 \\
\hline Inflate GZP via accumulator to $-13.5 \mathrm{MPa}$. & $6-10-93$ & 161 & 161 & 12:49:00 \\
\hline Circulate fluid through all of the lines. & $6-10-93$ & 161 & 161 & 14:10:00 \\
\hline Inflate TZP. & $6-10-93$ & 161 & 161 & $14: 20: 00$ \\
\hline Pressurize TZ via accumulator. & $6-90-93$ & 161 & 161 & 14:41:00 \\
\hline Shut in $\mathrm{GZ}$. & $6-10.93$ & 161 & 161 & 14:42:00 \\
\hline
\end{tabular}


Table 5-4 (Continued). Testing Sequence SCP01-1 Events

\begin{tabular}{|c|c|c|c|c|}
\hline EVENT & DATE & $\begin{array}{c}\text { CALENDAR } \\
\text { DAY }\end{array}$ & $\begin{array}{c}1993 \\
\text { CALENDAR } \\
\text { DAY }\end{array}$ & $\begin{array}{c}\text { TIME } \\
\text { (HH:MM:SS) }\end{array}$ \\
\hline Increase $T Z$ pressure via accumulator. & 6-11-93 & 162 & 162 & 10:16:00 \\
\hline Shut in $T Z$ from accumulator. & $6-11-93$ & 162 & 162 & 10:17:00 \\
\hline Open TZ to full accumulator. & 6-11-93 & 162 & 162 & 10:42:00 \\
\hline Shut in $\mathrm{TZ}$ from accumulator. & $6-14-93$ & 165 & 165 & 09:28:41 \\
\hline Shut in GZ from transducer to remove leaking gage. & $6-22-93$ & 173 & 173 & 10:58:00 \\
\hline Open $G Z$ to transducer and accumulator. & $6-22-93$ & 173 & 173 & 11:09:00 \\
\hline Increase GZP pressure via accumulator. & $6-28-93$ & 179 & 179 & 11:38:26 \\
\hline Increase $G Z$ pressure via accumulator. & $6-28-93$ & 179 & 179 & 12:36:38 \\
\hline Shut in $\mathrm{GZ}$ from accumulator. & $6-28-93$ & 179 & 179 & $12: 37: 30$ \\
\hline Increase TZP pressure due to bypass around the packer. & $6-29-93$ & 180 & 180 & 10:00:04 \\
\hline End data file SCP01BO6. & 7-1-93 & 183 & 183 & 08:38:57 \\
\hline Depressurize $T Z$ & $7-13-93$ & 194 & 194 & 08:55:00 \\
\hline Depressurize GZ. & 7-13-93 & 194 & 194 & 08:57:00 \\
\hline Deflate both packers. & $7-13-93$ & 194 & 194 & 09:02:00 \\
\hline Terminate data collection. & $7-13-93$ & 194 & 194 & 09:04:00 \\
\hline Remove test tool \#38B. & 7-13-93 & 194 & 194 & 13:00:00 \\
\hline
\end{tabular}

Figures 5-12 through 5-17 illustrate the zone pressures, packer pressures, nitrogeninjection rate, test-zone temperature, brine-injection volume during the test-zone compressibility test, and the test-zone compressibility as a function of pressure, respectively, during test sequence SCP01-1.

Table 5-5 indicates the equipment that was used and the duration that each instrument was used during testing sequence SCP01-1.

Table 5-5. Testing Sequence SCP01-1 Equipment

\begin{tabular}{|c|c|c|c|c|}
\hline Equipment & Location & Serial \# & Installed & Removed \\
\hline DAS Software & N/A & PERM4F & $3-30-93$ & $4-19-93$ \\
\hline DAS Software & N/A & LABTECH 4.1.0 & $4-19-93$ & $7-13-93$ \\
\hline DCU (HP3497A) & N/A & $2629 a 21989$ & $3-30-93$ & $4-19-93$ \\
\hline DCU (HP75000) & N/A & $3035 a 01445$ & 4-19-93 & $7-13-93$ \\
\hline $\begin{array}{c}\text { Transducer } \\
\text { (Druck PDCR 910) }\end{array}$ & Test Zone & 322422 & $3-30-93$ & $7-13-93$ \\
\hline $\begin{array}{c}\text { Transducer } \\
\text { (Druck PDCR 910) }\end{array}$ & $\begin{array}{l}\text { Test Zone } \\
\text { Packer }\end{array}$ & 308150 & $3-30-93$ & $7-13-93$ \\
\hline
\end{tabular}


Table 5-5 (Continued). Testing Sequence SCP01-1 Equipment

\begin{tabular}{|c|c|c|c|c|}
\hline Equipment & Location & Serial \# & Installed & Removed \\
\hline $\begin{array}{c}\text { Transducer } \\
\text { (Druck PDCR 910) }\end{array}$ & Guard Zone & 507864 & $3-30-93$ & $7-13-93$ \\
\hline $\begin{array}{c}\text { Transducer } \\
\text { (Druck PDCR 910) }\end{array}$ & $\begin{array}{l}\text { Guard Zone } \\
\text { Packer }\end{array}$ & 308145 & $3-30-93$ & $7-13-93$ \\
\hline $\begin{array}{c}\text { Transducer } \\
\text { (Druck PDCR 910) }\end{array}$ & $\begin{array}{l}\text { DPT Panel/Mass } \\
\text { Flow Meter }\end{array}$ & 322427 & $3-31-93$ & $4-12-93$ \\
\hline $\begin{array}{c}\text { DPT } \\
\text { (Rosemount 1151DP) }\end{array}$ & N/A & 1140864 & $3-31-93$ & $4-12-93$ \\
\hline Injection Column & N/A & 38 & $3-31-93$ & $3-31-93$ \\
\hline Thermocouple (Type E) & Test Zone & 1 & $3-30-93$ & $5-2-93$ \\
\hline $\begin{array}{c}\text { Flow Meter } \\
\text { (Bronkhorst F-230C-FA-22-V) }\end{array}$ & N/A & $921209 a$ & $4-19-93$ & $6-1-93$ \\
\hline
\end{tabular}

* Installed dates for injection columns refer to dates of initial use rather than date installed. 


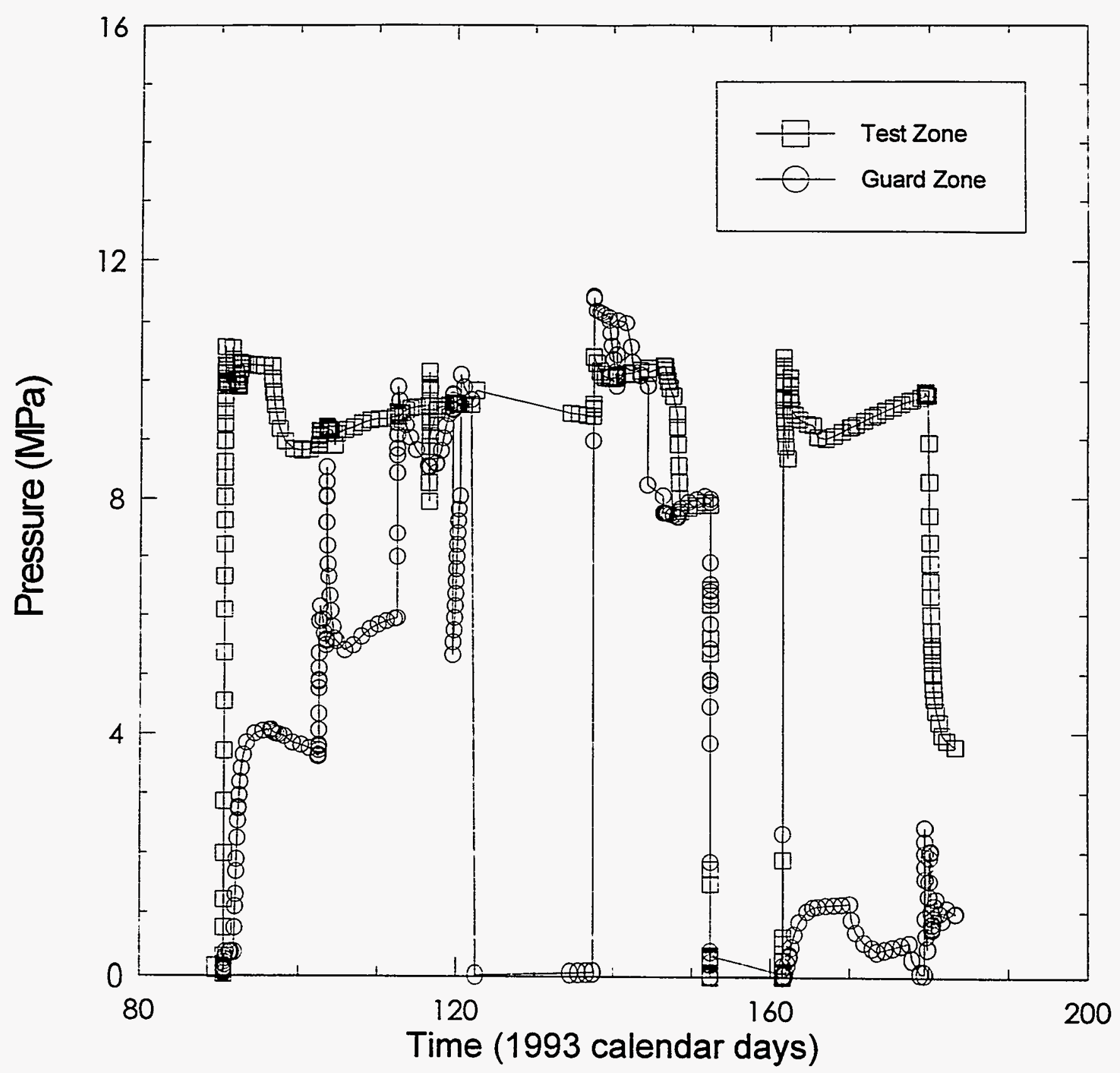

INTERA-6115-1040

Figure 5-12. Zone pressures during testing sequence SCP01-1. 


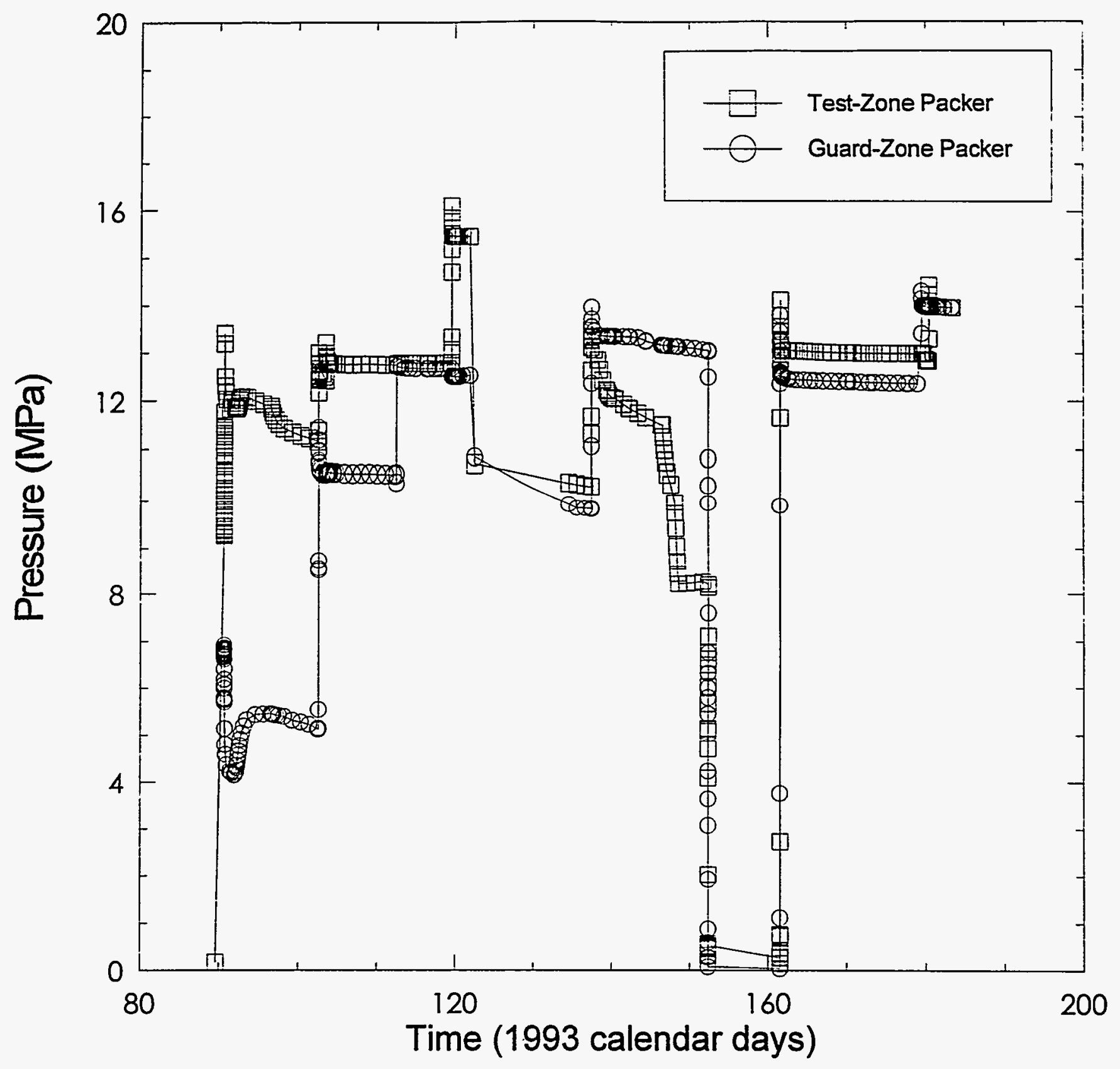

INTERA-6115-165-0

Figure 5-13. Packer pressures during testing sequence SCP01-1. 


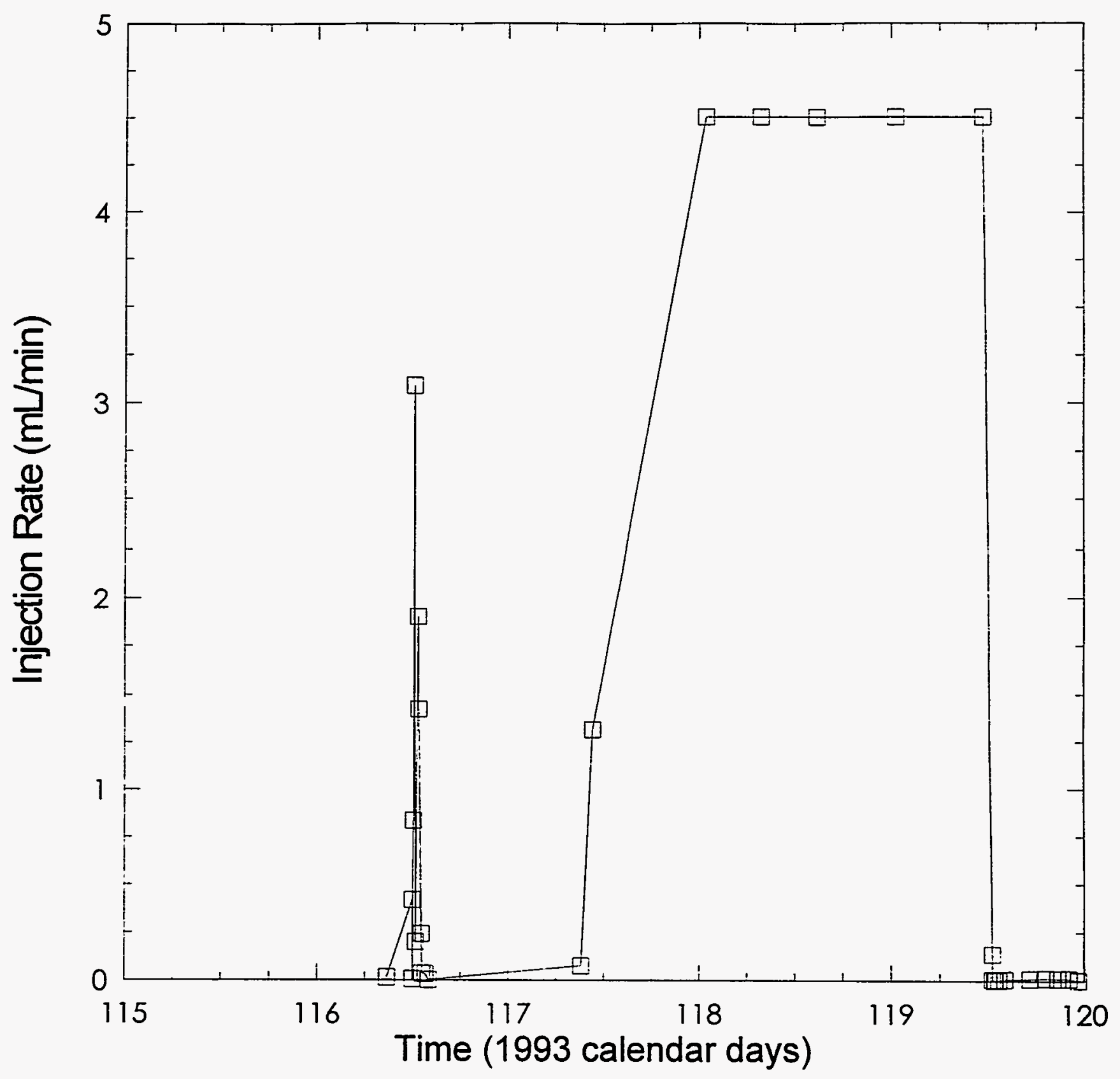

INTERA-SI15-186-0

Figure 5-14. Nitrogen-injection rate during testing sequence SCP01-1. 


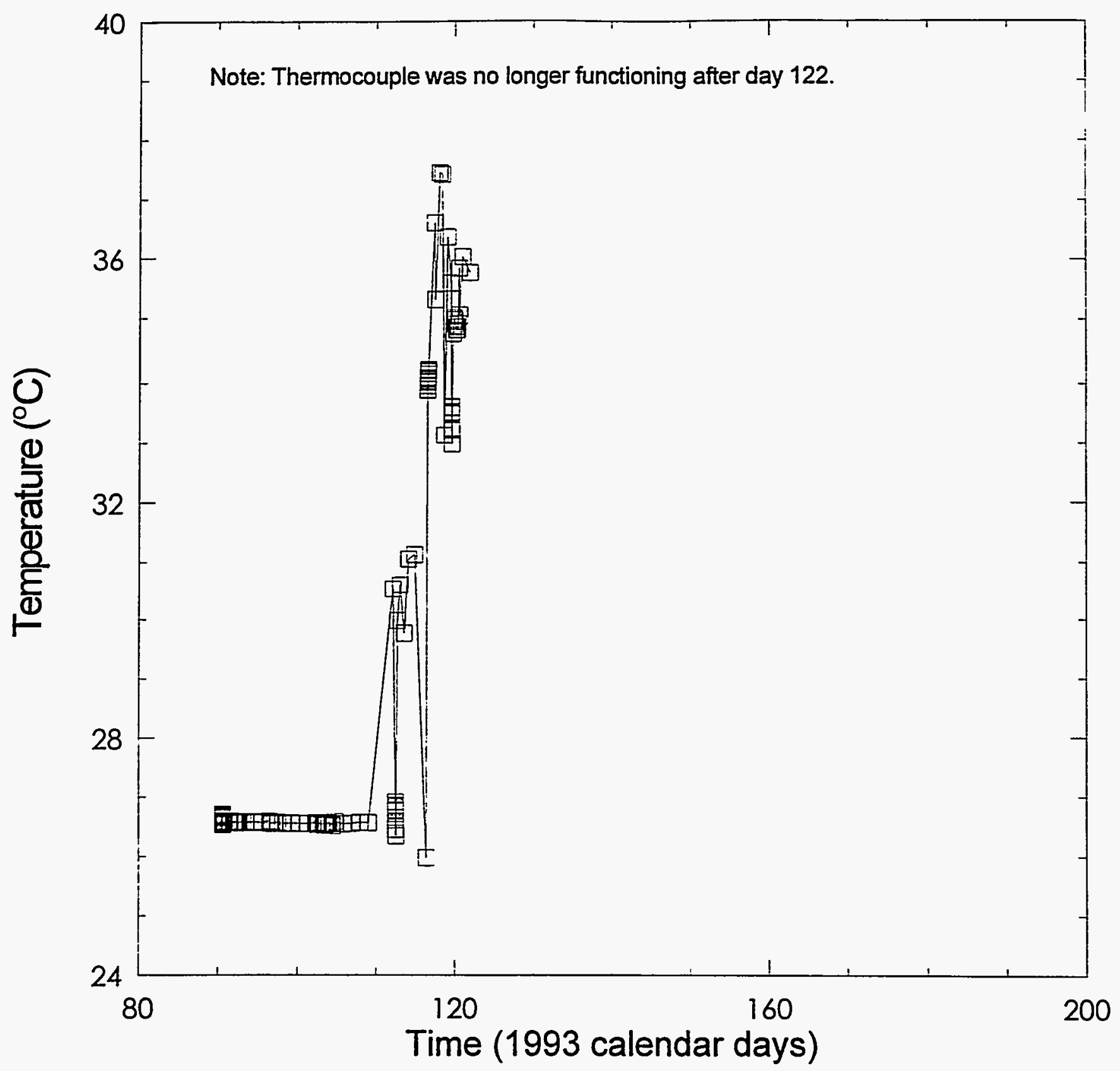

INTERA-6115-167-0

Figure 5-15. Test-zone temperature during testing sequence SCP01-1. 


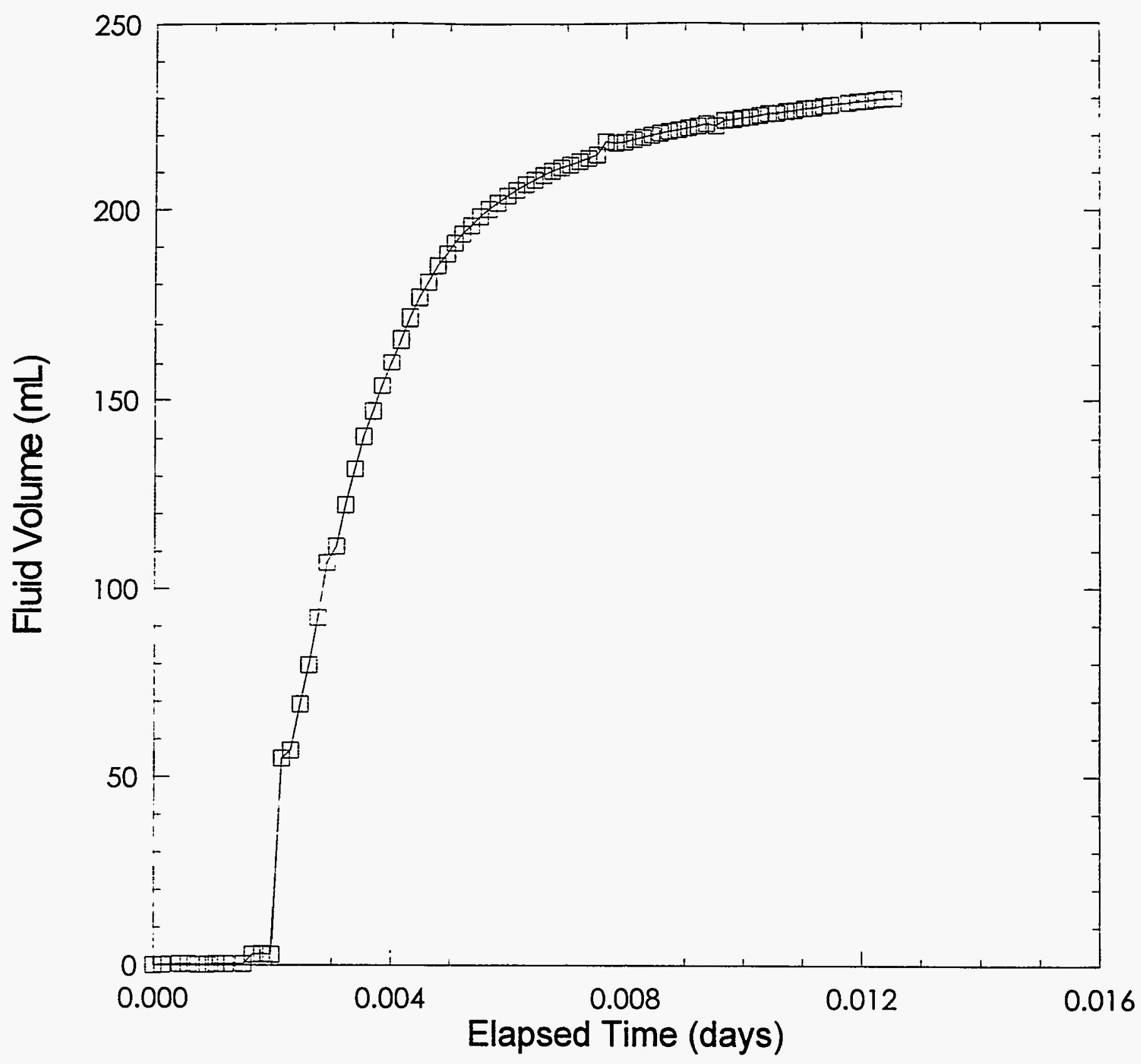

INTERA-6115-168-0

Figure 5-16. Brine-injection volume of test-zone compressibility test during testing sequence SCP01-1. 


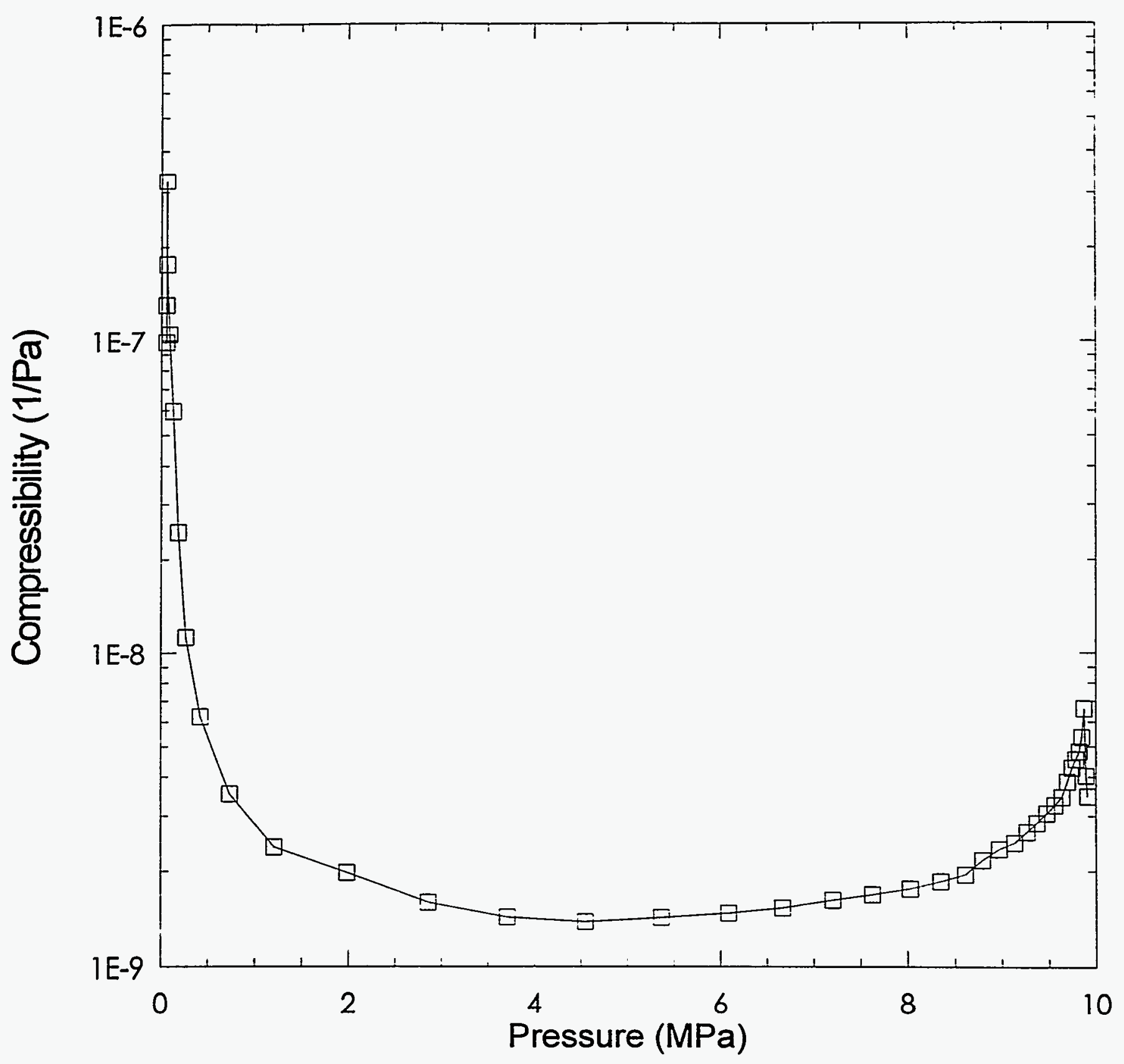

INTERA-8115-1620

Figure 5-17. Calculated test-zone compressibility as a function of pressure obtained from test-zone compressibility test during testing sequence SCP01-1. 
The last testing sequence was also conducted in the Core Storage Library in borehole SCP01. The objective and result of this testing sequence was to conduct a successful test sequence in MB139 in borehole SCP01 where equipment problems were encountered during the previous attempt. In test sequence SCP01-2, Baker packers were used to isolate MB139. It was decided to use these packers rather than Baski or TAM packers because of their longer seal length and on the basis of previous experiences in problematic boreholes. Table 5-6 gives a detailed description of the events that occurred during test sequence SCP01-2.

Table 5-6. Testing Sequence SCP01-2 Events

\begin{tabular}{|c|c|c|c|c|}
\hline EVENT & DATE & $\begin{array}{c}\text { CALENDAR } \\
\text { DAY }\end{array}$ & $\begin{array}{c}1994 \\
\text { CALENDAR } \\
\text { DAY }\end{array}$ & $\begin{array}{c}\text { TIME } \\
\text { (HH:MM:SS) }\end{array}$ \\
\hline Depressurize $T Z$ of the long-term test tool. & 4-13-94 & 103 & 103 & 10:22:00 \\
\hline Deflate TZP. & 4-13-94 & 103 & 103 & $10: 23: 00$ \\
\hline Remove single-packer, long-term test tool. & 4-13-94 & 103 & 103 & $11: 30: 00$ \\
\hline $\begin{array}{l}\text { Install double-packer test tool with Baker packers (TZ monitors MB139) as indicated in } \\
\text { tool configuration diagram \#1 (Figure 5-7). }\end{array}$ & $4-14-94$ & 104 & 104 & 14:30:00 \\
\hline Inflate TZP to $\sim 13.8 \mathrm{MPa}$ via accumulator. & $4-14-94$ & 104 & 104 & 15:00:00 \\
\hline Inflate GZP to $-13.8 \mathrm{MPa}$ via accumulator. & $4-14-94$ & 104 & 104 & 15:01:00 \\
\hline Begin data file SCP01B07. & 4-15-94 & 105 & 105 & 12:27:39 \\
\hline Pressurize $T Z$ via DPT panel to $-5.5 \mathrm{MPa}$ for $T Z$ compressibility test. & 4-15-94 & 105 & 105 & $13: 21: 26$ \\
\hline Shut in $T Z$ at $5.446 \mathrm{MPa}$ terminating $T Z$ compressibility test. & 4-15-94 & 105 & 105 & 13:44:30 \\
\hline Open TZ to accumulator. & 4-15-94 & 105 & 105 & $13: 47: 45$ \\
\hline Pressurize GZ to $5.077 \mathrm{MPa}$. & 4-15-94 & 105 & 105 & 13:55:00 \\
\hline Shut in $T Z$ from accumulator. & 4-18-94 & 108 & 108 & 15:04:00 \\
\hline End data file SCP01B07. & 4-19-94 & 109 & 109 & $09: 32: 36$ \\
\hline Switch DAS from PERMAF to LABTECH in preparation for GTPT. & $4-19-94$ & 109 & 109 & 09:34:00 \\
\hline Begin data file SCP01B08. & $4-21-94$ & 111 & 111 & 12:03:45 \\
\hline Incorrect flow meter readings. & 4-21-94 & 111 & 111 & 12:00:00 \\
\hline Shut in TZP and GZP from accumulator. & $4-27-94$ & 117 & 117 & 11:05:00 \\
\hline Increase $T Z$ pressure via accumulator to $-9.4 \mathrm{MPa}$. & 4-27-94 & 117 & 117 & 11:10:00 \\
\hline Shut in $\mathrm{TZ}$ from accumulator. & $4-28-94$ & 118 & 118 & 07:53:00 \\
\hline Flow meter is operating correctly and will be left over night at $1 \mathrm{mLmin}$ open. & 4.28.94 & 118 & 118 & 10:46:00 \\
\hline Shut in fiow meter and sot rate to $0 \mathrm{~mL} / \mathrm{min}$. & $4-29-94$ & 119 & 119 & 09:13:00 \\
\hline Pressurize $\mathrm{N}_{2}$ injection line to $\sim 9.5 \mathrm{MPa}$. & $5-2-94$ & 122 & 122 & 11:38:00 \\
\hline $\begin{array}{c}\text { Open } T Z \text { to } N_{2} \text { injection and open vent line to maintain constant } T Z \text { pressure durng } \\
\text { gas/brine exchange } \# 1 \text { in } T Z\end{array}$ & $5-2-94$ & 122 & 122 & $11: 49: 00$ \\
\hline Significant amount of gas flow with brine. & $5-2-94$ & 122 & 122 & 12:05:00 \\
\hline Shut in TZ ( $-1980 \mathrm{~mL}$ of brine withdrawn). & 5.2 .94 & 122 & 122 & 12:14:00 \\
\hline Open $T 2$ to continue gas/brine exchange. & $5-2-94$ & 122 & 122 & 12:16:00 \\
\hline
\end{tabular}


Table 5-6 (Continued). Testing Sequence SCP01-2 Events

\begin{tabular}{|c|c|c|c|c|}
\hline EVENT & DATE & $\begin{array}{l}\text { CALENDAR } \\
\text { DAY }\end{array}$ & $\begin{array}{c}1994 \\
\text { CALENDAR } \\
\text { DAY }\end{array}$ & $\begin{array}{c}\text { TIME } \\
\text { (HH:MM:SS) }\end{array}$ \\
\hline Shut in $T Z$ ( $-410 \mathrm{~mL}$ of brine withdrawn) terminating gas/brine exchange \#1 in $T Z$. & $5-2-94$ & 122 & 122 & 12:19:00 \\
\hline End data file SCP01B08. & $5-3-94$ & 123 & 123 & 08:39:58 \\
\hline Depressurize TZ & $5-3-94$ & 123 & 123 & 09:02:00 \\
\hline Depressurize GZ. & 5-3-94 & 123 & 123 & 09:04:00 \\
\hline Deflate GZP. & $5-3-94$ & 123 & 123 & 09:08:00 \\
\hline Deflate TZP. & $5-3-94$ & 123 & 123 & 09:10:00 \\
\hline Remove test tool to extend vent line to the bottom of the borehole. & 5-3-94 & 123 & 123 & 14:35:00 \\
\hline TZP was damaged during removal and cannot be used. & 5-3-94 & 123 & 123 & 15:00:00 \\
\hline Install single-packer test tool in borehole so that $T Z$ is not open to atmosphere. & $5-5-94$ & 125 & 125 & 14:32:00 \\
\hline Inflate TZP to $\sim 13.8 \mathrm{MPa}$. & $5-6-94$ & 126 & 126 & 10:22:00 \\
\hline Pressurize $T Z$ to $\sim 9.5 \mathrm{MPa}$. & $5-6-94$ & 126 & 126 & 10:30:00 \\
\hline Depressurize TZ. & $5-6-94$ & 126 & 126 & 08:29:00 \\
\hline Deflate TZP. & $5-6-94$ & 126 & 126 & 08:30:00 \\
\hline Remove single-packer test tool. & $5-6-94$ & 126 & 126 & 08:45:00 \\
\hline $\begin{array}{l}\text { Install double-packer test tool with new Baker elements to original position with a } \\
2.817 \mathrm{~m} \text { vent line extension to reach the bottom of the borehole as indicated in tool } \\
\text { configuration diagram } \# 2 \text { (Figure 5-8). }\end{array}$ & $5-9-94$ & 129 & 129 & 13:00:00 \\
\hline Begin data file SCP01B09. & $5-9-94$ & 129 & 129 & 13:01:12 \\
\hline Inflate $T Z P$ to $\sim 15.2 \mathrm{MPa}$ via accumulator. & $5-9-94$ & 129 & 129 & 13:07:00 \\
\hline Inflate GZP to $\sim 15.2 \mathrm{MPa}$ via accumulator. & $5-9-94$ & 129 & 129 & 13:29:00 \\
\hline Pressurize TZ to $-6.9 \mathrm{MPa}$. & $5-9-94$ & 129 & 129 & 13:45:00 \\
\hline Open TZ to accumulator at $\sim 9.5 \mathrm{MPa}$. & $5-9-94$ & 129 & 129 & 13:55:00 \\
\hline Pressurize GZ to $-5.5 \mathrm{MPa}$. & $5-9-94$ & 129 & 129 & 13:58:00 \\
\hline Open TZP to accumulator. & $5-9-94$ & 129 & 129 & 12:48:00 \\
\hline Shut in $T Z$ from accumulator. & 5-10-94 & 130 & 130 & 12:42:00 \\
\hline Shut in TZP and GZP from accumulator. & $5-11-94$ & 131 & 131 & 09:22:00 \\
\hline Decrease GZ pressure to $-5.5 \mathrm{MPa}$. & $5-12-94$ & 132 & 132 & $11: 39: 00$ \\
\hline Begin gas/brine exchange $\# 2$ in TZ (-24655 mL of brine withdrawn). & 5-16-94 & 136 & 136 & 12:33:00 \\
\hline Shut in $T Z$ terminating gas/brine exchange $\# 2$ in $T Z$ & 5-16-94 & 136 & 136 & 14:03:00 \\
\hline Open GZP to accumulator at $\sim B \mathrm{MPa}$. & 5-17-94 & 137 & 137 & 12:17:00 \\
\hline Pressurize $\mathrm{N}_{2}$ injection line to $\sim$ TZ pressure. & $5-17-94$ & 137 & 137 & 12:38:00 \\
\hline Open $T Z$ to flow meter set at $2 \mathrm{~mL} / \mathrm{min}$ beginning gas-injection test \#1 in $\mathrm{TZ}$. & 5-17-94 & 137 & 137 & 12:56:28 \\
\hline End data file SCPO1BO9. & 5-18-94 & 138 & 138 & 12:40:35 \\
\hline Begin data file SCP01B10. & $5-18-94$ & 138 & 138 & $12: 55: 26$ \\
\hline $\begin{array}{c}\text { Shut in } \mathrm{TZ} \text { from flow meter because } \mathrm{Z} \text { pressure had surpassed } N_{2} \text { pressure causing } \\
\text { anomalous flow readings terminating gas-injection test } \# 1 .\end{array}$ & $5-20-94$ & 140 & 140 & 11:50:00 \\
\hline End data file SCPO1B10. & $5-20-94$ & 140 & 140 & 12:11:57 \\
\hline Begin data file SCP01Bi1. & $5-20-94$ & 140 & 140 & 12:14:09 \\
\hline
\end{tabular}


Table 5-6 (Continued). Testing Sequence SCP01-2 Events

\begin{tabular}{|c|c|c|c|c|}
\hline EVENT & DATE & $\begin{array}{c}\text { CALENDAR } \\
\text { DAY }\end{array}$ & $\begin{array}{c}1994 \\
\text { CALENDAR } \\
\text { DAY }\end{array}$ & $\begin{array}{c}\text { TIME } \\
\text { (HH:MM:SS) }\end{array}$ \\
\hline End data file SCP01B11. & $5-23-94$ & 143 & 143 & $12: 40: 38$ \\
\hline Begin data file SCP01B12. & $5-23-94$ & 143 & 143 & 13:32:38 \\
\hline End data file SCP01B12. & $5-26-94$ & 146 & 146 & 11:04:35 \\
\hline Shut in GZP from accumulator. & $5-26-94$ & 146 & 146 & 11:11:00 \\
\hline Open GZP to full accumulator. & $5-26-94$ & 146 & 146 & 11:14:00 \\
\hline Pressurize N2 injection line to $\sim 8.7 \mathrm{MPa}$. & $5-26-94$ & 146 & 146 & $11: 16: 00$ \\
\hline Begin data file SCP01B13. & $5-26-94$ & 146 & 146 & 11:22:27 \\
\hline Open $T Z$ to flow meter set at $0.2 \mathrm{~mL}$ min beginning gas-injection test $\# 2$ in $T Z$ & $5-26-94$ & 146 & 146 & $11: 24: 46$ \\
\hline End data file SCP01B13. & $5-27-94$ & 147 & 147 & 12:27:25 \\
\hline Begin data file SCP01B14. & $5-27-94$ & 147 & 147 & 12:30:39 \\
\hline End data file SCP01B14. & $5-31-94$ & 151 & 151 & 11:59:20 \\
\hline Begin data file SCP01B15. & $5-31-94$ & 151 & 151 & 12:19:59 \\
\hline End data file SCP01B15. & $6-1-94$ & 152 & 152 & 10:42:02 \\
\hline Begin data file SCP01B16. & $6-1-94$ & 152 & 152 & 10:44:29 \\
\hline End data file SCP01B16. & $6-2-94$ & 153 & 153 & 13:38:54 \\
\hline Begin data file SCP01B17. & $6-2-94$ & 153 & 153 & 13:41:09 \\
\hline End data file SCP01B17. & $6-3-94$ & 154 & 154 & 11:23:42 \\
\hline Begin data file SCP01B18. & 6-3-94 & 154 & 154 & 11:26:49 \\
\hline Shut in $\mathrm{TZ}$ from flow meter terminating gas-injection test $\# 2$ in $T Z$. & $6-3-94$ & 154 & 154 & 11:58:00 \\
\hline End data file SCPO1B18. & $6-20-94$ & 171 & 171 & 12:05:04 \\
\hline Begin data file SCP01819. & $6-20-94$ & 171 & 171 & 12:25:40 \\
\hline End data file SCP01B19. & $6-27-94$ & 178 & 178 & 11:07:08 \\
\hline Shut in GZ from accumulator. & $8-2-94$ & 214 & 214 & 09:01:00 \\
\hline Depressurize TZ. & $8-2-94$ & 214 & 214 & 09:14:00 \\
\hline Depressurize GZ. & $8-2-94$ & 214 & 214 & 09:15:00 \\
\hline Deflate GZP. & $8-2-94$ & 214 & 214 & 09:21:00 \\
\hline Deflate TZP. & $8-2-94$ & 214 & 214 & 09:22:00 \\
\hline Remove GTPT tool \#8OT-02 from borehole & $8-2-94$ & 214 & 214 & 13:00:00 \\
\hline
\end{tabular}

Figures 5-18 through 5-22 illustrate the zone pressures, packer pressures, nitrogeninjection rate, fluid-injection volume during test-zone compressibility tests, and the testzone compressibility as a function of pressure, respectively, during testing sequence SCP01-2.

Table 5-7 indicates the equipment that was used and the duration that each instrument was used during testing sequence SCP01-2. 
Table 5-7. Testing Sequence SCP01-2 Equipment

\begin{tabular}{|c|c|c|c|c|}
\hline Equipment & Location & Serial \# & Installed & Removed \\
\hline DAS Software & N/A & PERM4F & $4-15-94$ & $4-18-94$ \\
\hline DAS Software & N/A & LABTECH 4.1.0 & 4-18-94 & 6-29-94 \\
\hline DCU (HP3497A) & N/A & $2629 a 21996$ & 4-15-94 & $4-18-94$ \\
\hline DCU (HP75000) & N/A & $3035 a 01445$ & 4-18-94 & 6-29-94 \\
\hline $\begin{array}{c}\text { Transducer } \\
\text { (Druck D930-18) }\end{array}$ & Test Zone & 609371 & $4-15-94$ & $6-29-94$ \\
\hline $\begin{array}{c}\text { Transducer } \\
\text { (Druck D930-18) }\end{array}$ & $\begin{array}{l}\text { Test Zone } \\
\text { Packer }\end{array}$ & 609370 & $4-15-94$ & $6-29-94$ \\
\hline $\begin{array}{c}\text { Transducer } \\
\text { (Druck D930-18) }\end{array}$ & Guard Zone & 609375 & $4-15-94$ & $6-29-94$ \\
\hline $\begin{array}{c}\text { Transducer } \\
\text { (Druck D930-18) }\end{array}$ & $\begin{array}{l}\text { Guard Zone } \\
\text { Packer }\end{array}$ & 609372 & $4-15-94$ & $6-29-94$ \\
\hline $\begin{array}{c}\text { Transducer } \\
\text { (Druck D930-18) } \\
\end{array}$ & $\begin{array}{l}\text { DPT Panel/Mass } \\
\text { Flow Meter }\end{array}$ & 609368 & $4-15-94$ & $6-29-94$ \\
\hline $\begin{array}{c}\text { DPT } \\
\text { (Rosemount 1151DP) } \\
\end{array}$ & N/A & 1140863 & $4-15-94$ & $4-18-94$ \\
\hline Injection Column & N/A & 76 & $4-15-94$ & $4-15-94$ \\
\hline $\begin{array}{l}\text { Flow Meter } \\
\text { (Bronkhorst F-230C-FA-22-V) }\end{array}$ & N/A & $921209 a$ & $4-18-94$ & $6-29-94$ \\
\hline
\end{tabular}

* Installed dates for injection columns refer to dates of initial use rather than date installed. 


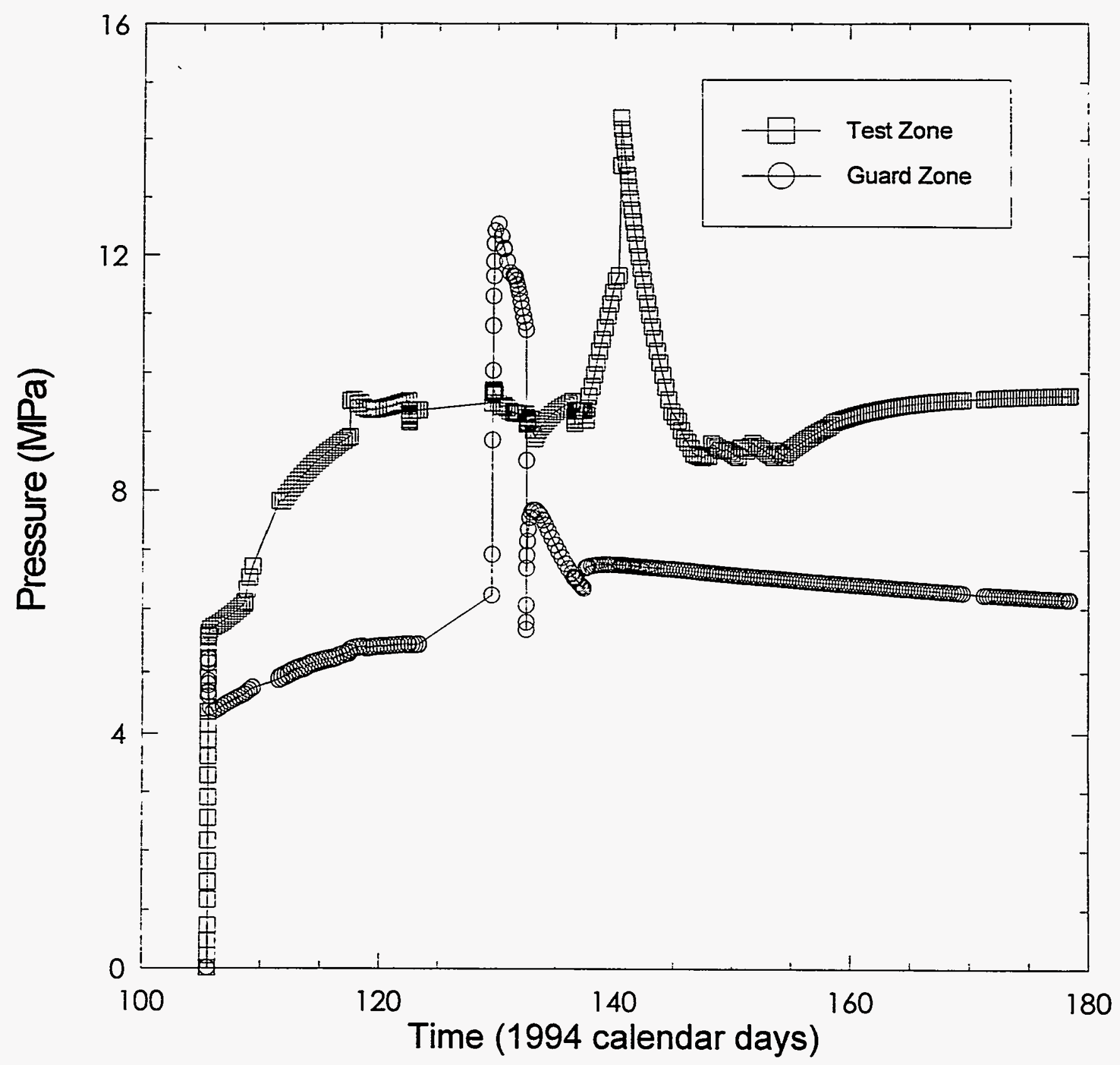

INTERA6115-170-0

Figure 5-18. Zone pressures during testing sequenœ SCP01-2. 


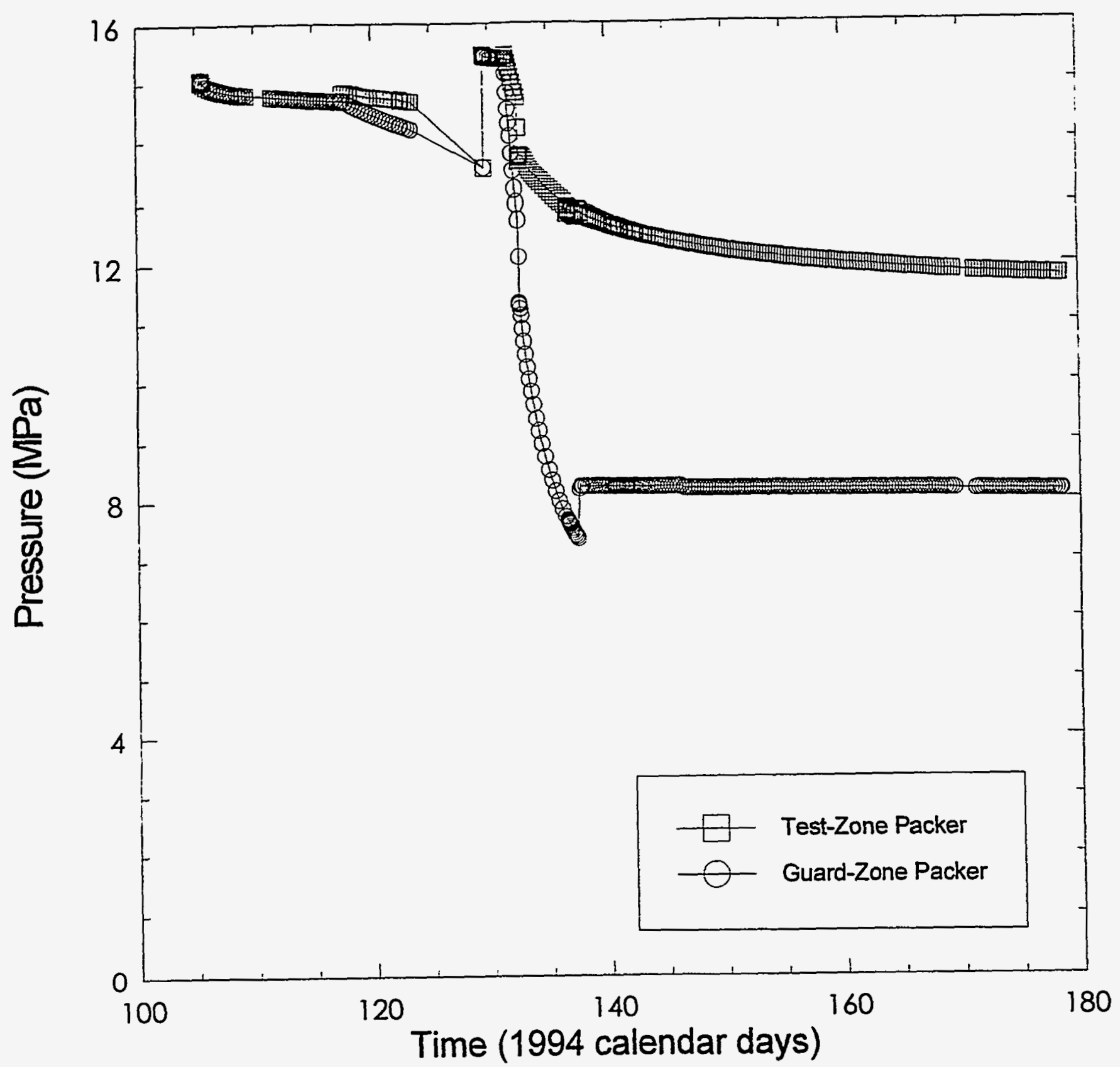

INTERA-6115-171-0

Figure 5-19. Packer pressures during testing sequence SCP01-2. 


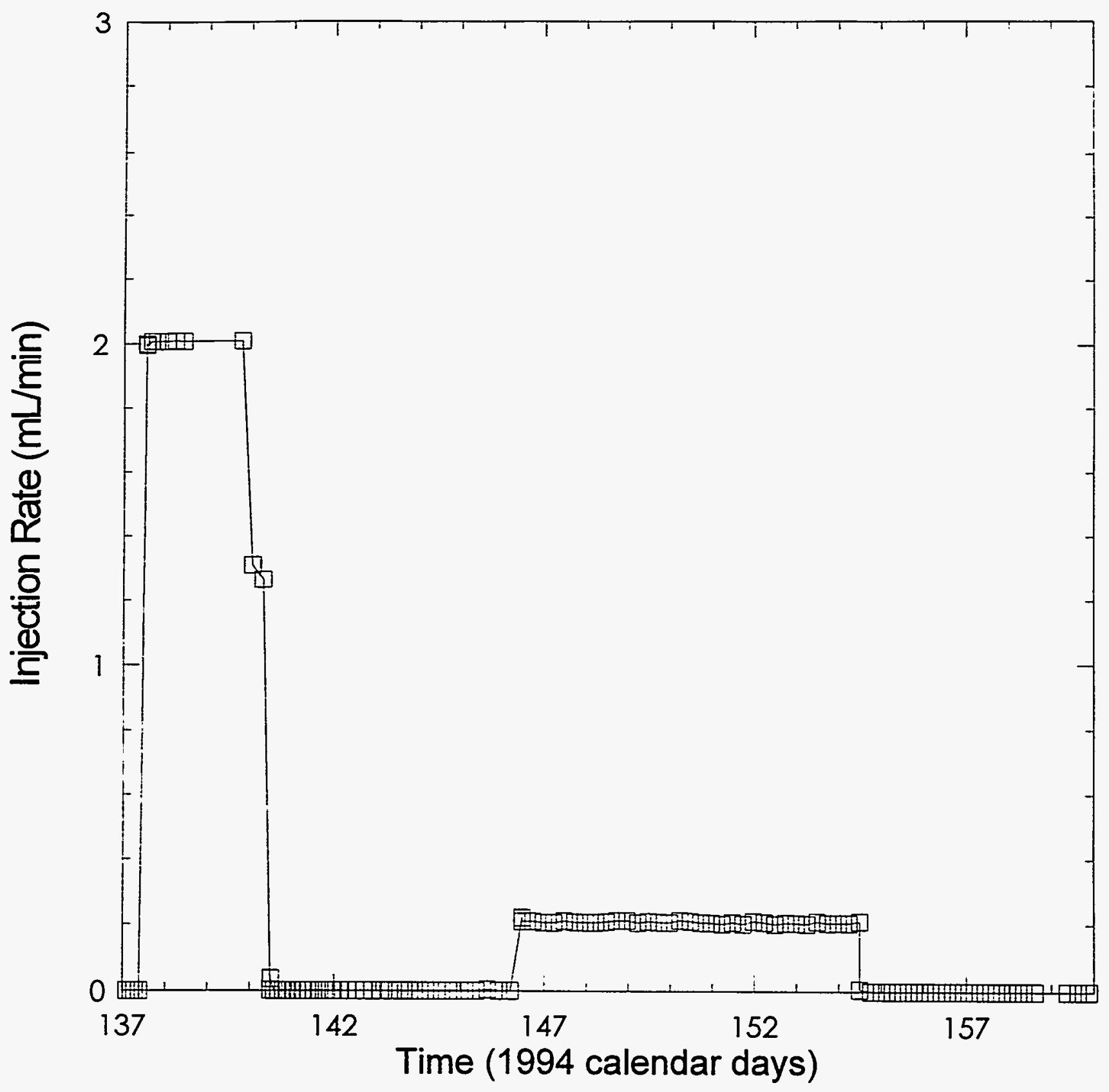

INTERA6 $115-172-0$

Figure 5-20. Nitrogen-injection rate during testing sequence SCP01-2. 


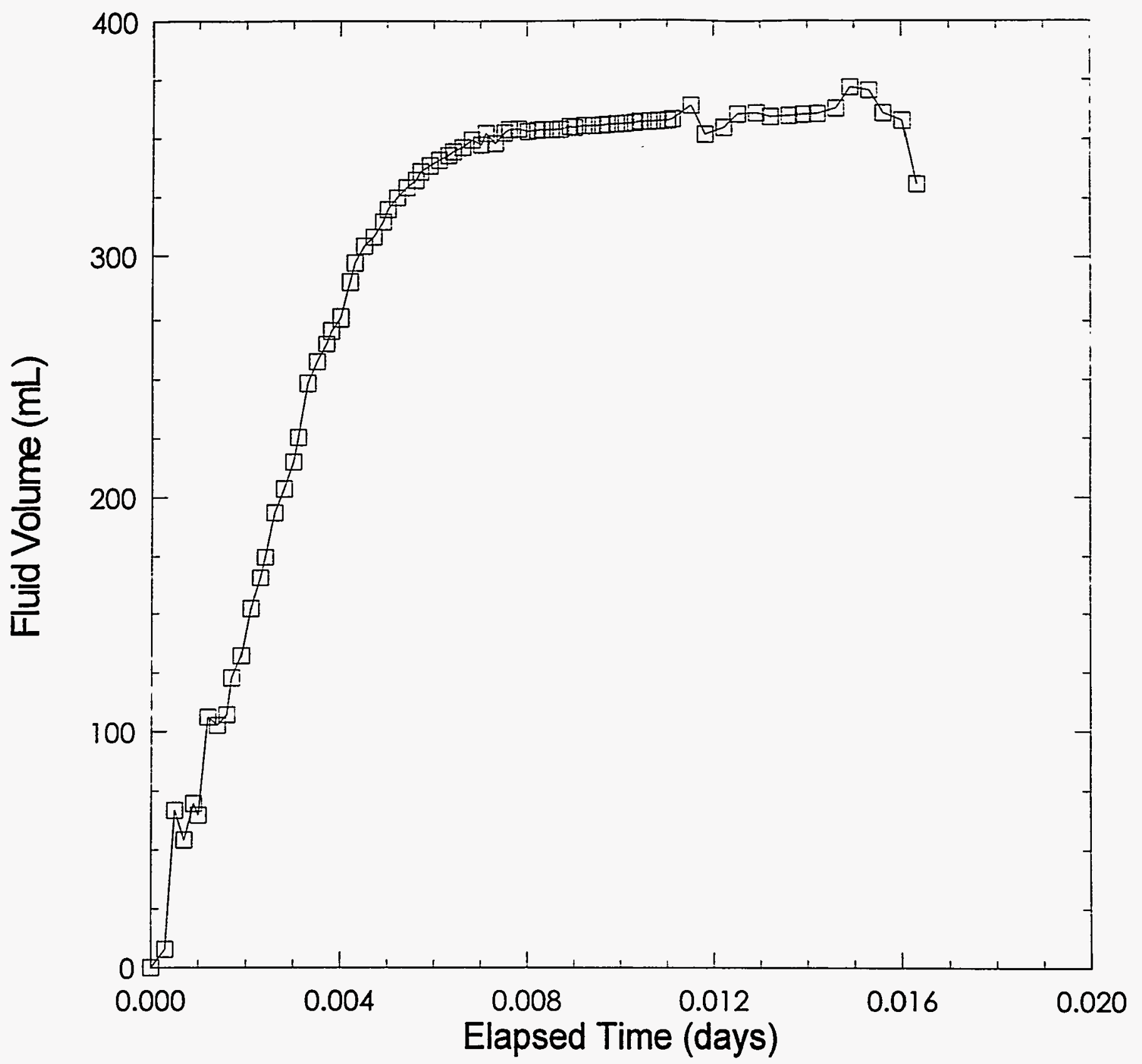

INTERA-115-173-0

Figure 5-21. Brine-injection volume of test-zone compressibility test during testing sequence SCP01-2. 


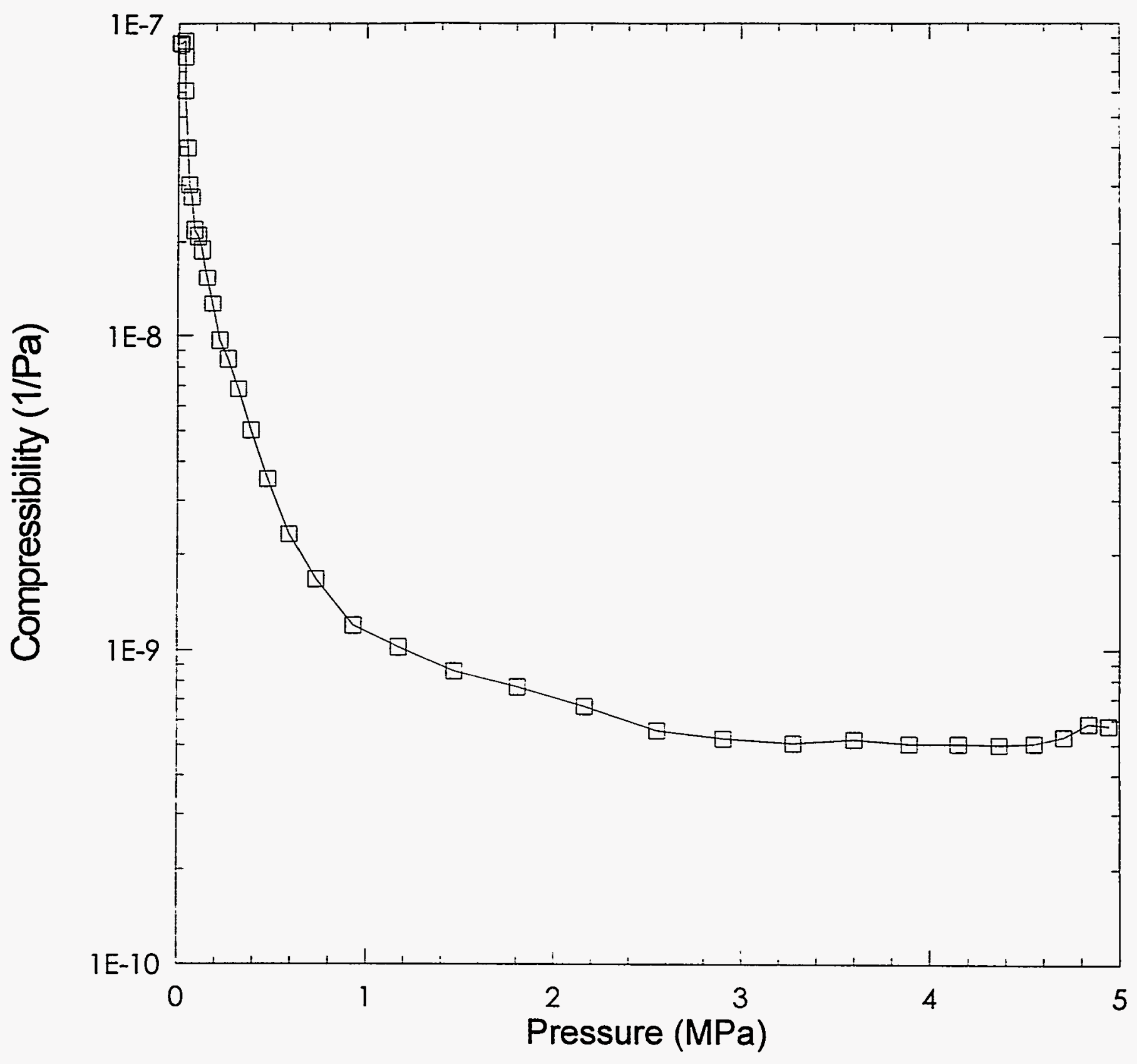

INTERAG115-1740

Figure 5-22. Calculated test-zone compressibility as a function of pressure obtained from test-zone compressibility test during testing sequence SCP01-2. 


\subsubsection{Gas-Threshold-Pressure Testing in Room C2}

Testing sequence $\mathrm{C} 2 \mathrm{H} 02$ was also designed to investigate the gas threshold pressure of MB139 in an environment removed from the WIPP excavations. Borehole $\mathrm{C} 2 \mathrm{H} 01$ was used as an observation borehole for a portion of testing sequence $\mathrm{C} 2 \mathrm{H} 02$. Table 5-8 gives a detailed description of the events that occurred during testing sequence $\mathrm{C} 2 \mathrm{HO} 2$.

Table 5-8. Testing Sequence C2H02 Events

\begin{tabular}{|c|c|c|c|c|}
\hline EVENT & DATE & $\begin{array}{c}\text { CALENDAR } \\
\text { DAY }\end{array}$ & $\begin{array}{c}1993 \\
\text { CALENDAR } \\
\text { DAY }\end{array}$ & $\begin{array}{c}\text { TIME } \\
\text { (HH:MM:SS) }\end{array}$ \\
\hline Remove long-term tool from borehole. & $8-9-93$ & 221 & 221 & 08:54:00 \\
\hline $\begin{array}{l}\text { Install double-packer test tool as indicated in test-tool configuration diagram \#1 } \\
\text { (Figure 5-10). }\end{array}$ & $8-10-93$ & 222 & 222 & $12: 00: 00$ \\
\hline Begin data file $\mathrm{C} 2 \mathrm{HO} 2 \mathrm{O}$. & $8-10-93$ & 222 & 222 & $12: 36: 11$ \\
\hline Inflate TZP. & 8-10-93 & 222 & 222 & $12: 36: 57$ \\
\hline Shut in TZP. & 8-10-93 & 222 & 222 & 12:38:57 \\
\hline Inflate GZP. & $8-10-93$ & 222 & 222 & 12:39:27 \\
\hline Shut in GZP. & 8-10-93 & 222 & 222 & 12:42:57 \\
\hline Open TZP to pressure accumulator. & 8-10-93 & 222 & 222 & 13:01:27 \\
\hline Open GZP to pressure accumulator. & 8-10-93 & 222 & 222 & $13: 06: 27$ \\
\hline Circulate brine through $T Z$ to eliminate all possible gas. & $8-10-93$ & 222 & 222 & 13:22:27 \\
\hline Pressurize TZ via accumulator to $-7.6 \mathrm{MPa}$. & 8-10-93 & 222 & 222 & $13: 40: 57$ \\
\hline Pressurize $\mathrm{GZ}$ to $\sim 5.2 \mathrm{MPa}$. & 8-10-93 & 222 & 222 & 13:46:57 \\
\hline Shut in GZ. & 8-10-93 & 222 & 222 & 13:53:57 \\
\hline Shut in TZ from accumulator. & $8-13-93$ & 225 & 225 & 09:33:00 \\
\hline Open GZ to accumulator at $\sim 5.3 \mathrm{MPa}$. & $8-17-93$ & 229 & 229 & 12:55:30 \\
\hline Shut in GZ from accumulator at $4.365 \mathrm{MPa}$. & $8-23-93$ & 235 & 235 & $08: 37: 25$ \\
\hline Open GZ to accumulator at $\sim 5.5 \mathrm{MPa}$ (power outage). & $8-27-93$ & 239 & 239 & 13:49:00 \\
\hline Increase GZP pressure via accumulator by $-1.5 \mathrm{MPa}$ to $\sim 10.8 \mathrm{MPa}$. & $9-6-93$ & 250 & 250 & 10:55:05 \\
\hline Shut in GZ from accumulator at $4.786 \mathrm{MPa}$. & $9-8-93$ & 252 & 252 & $11: 23: 35$ \\
\hline Withdraw $\sim 2 \mathrm{~mL}$ of brine trom $G Z$ to observe the pressure response. & $9-9-93$ & 253 & 253 & 08:11:19 \\
\hline End data file $\mathrm{C}_{2} \mathrm{HO} 2 \mathrm{O}$. & $9-9-93$ & 253 & 253 & 12:44:23 \\
\hline Begin data file $\mathrm{C}_{2} \mathrm{H} 02 \mathrm{O}$. & $9-9-93$ & 253 & 253 & 12:47:08 \\
\hline Shut in GZP from acoumulator at $10.588 \mathrm{MPa}$. & $9-10-93$ & 254 & 254 & 10:10:05 \\
\hline Open GZP to accumulator. & $9-10-93$ & 254 & 254 & 10:16:21 \\
\hline Open GZ to accumulator. & $9-10-93$ & 254 & 254 & 10:39:24 \\
\hline Shut in TZP from accumulator at $10.708 \mathrm{MPa}$. & $9-10-93$ & 254 & 254 & 10:43:54 \\
\hline Shut in GZ from accumulator at $4.783 \mathrm{MPa}$. & $9-10-93$ & 254 & 254 & 10:48:54 \\
\hline Perform leak check on the gas injection system at $12.629 \mathrm{MPa}$. & $9-10-93$ & 254 & 254 & $12: 31: 09$ \\
\hline Begin gas/brine exchange $\# 1$ in $T Z$. & $9-10-93$ & 254 & 254 & 13:12:00 \\
\hline Stop gas/brine exchange \#1 due to plugging of the flow valve. & $9-10-93$ & 254 & 254 & 13:16:00 \\
\hline
\end{tabular}


Table 5-8 (Continued). Testing Sequence $\mathrm{C} 2 \mathrm{HO} 2$ Events

\begin{tabular}{|c|c|c|c|c|}
\hline EVENT & DATE & $\begin{array}{c}\text { CALENDAR } \\
\text { DAY }\end{array}$ & $\begin{array}{c}1993 \\
\text { CALENDAR } \\
\text { DAY }\end{array}$ & $\begin{array}{c}\text { TIME } \\
\text { (HH:MM:SS) }\end{array}$ \\
\hline Resume gas/brine exchange \#1 removing $\sim 1760 \mathrm{~mL}$ of brine prior to gas flow. & 9-10-93 & 254 & 254 & 13:33:04 \\
\hline Shut in TZ at $8.380 \mathrm{MPa}$ terminating gas/brine exchange \#1 in TZ & $9-10-93$ & 254 & 254 & 13:40:04 \\
\hline Begin $\mathrm{N}_{2}$ injection at $3 \mathrm{mU} / \mathrm{min}$. & $9-13-93$ & 257 & 257 & 10:21:46 \\
\hline Flow meter is not responding property. & 9-13-93 & 257 & 257 & 10:24:26 \\
\hline Shut in TZ. & $9-13-93$ & 257 & 257 & 10:29:16 \\
\hline Open flow meter to $\mathrm{TZ}$ at $8.361 \mathrm{MPa}$. & $9-14-93$ & 258 & 258 & 08:52:30 \\
\hline Begin $\mathrm{N}_{2}$ injection at $3 \mathrm{~mL} / \mathrm{min}$. & $9-14-93$ & 258 & 258 & 08:59:45 \\
\hline Increase TZP pressure via accumulator and leave accumulator on line. & $9-20-93$ & 263 & 263 & 16:04:12 \\
\hline Shut in TZP from accumulator. & $9-21-93$ & 264 & 264 & $09: 39: 00$ \\
\hline Switch P5 (\#321768) from flow meter to DPT panel. & $9-22-93$ & 265 & 265 & $11: 51: 40$ \\
\hline End data file $\mathrm{C}_{2} \mathrm{H} 02 \mathrm{O} 2$. & $9-24-93$ & 267 & 267 & 12:47:09 \\
\hline Begin data file $\mathrm{C} 2 \mathrm{H} 0203$. & $9-24-93$ & 267 & 267 & 13:10:21 \\
\hline Increase TZP pressuro via accumulator and leave accumulator on line. & $9-24-93$ & 267 & 267 & 13:26:19 \\
\hline End data file $\mathrm{C} 2 \mathrm{HO} 203$. & $9-28-93$ & 271 & 271 & 11:15:05 \\
\hline Disconnect flow meter and P5 (\#321768) from the DAS. & $9-28-93$ & 271 & 271 & 12:25:00 \\
\hline Begin data file $\mathrm{C}_{2} \mathrm{HO} 02 \mathrm{O}$. & $9-28-93$ & 271 & 271 & $12: 52: 57$ \\
\hline Reconnect the flow meter to the DAS. & $9-28-93$ & 271 & 271 & 13:14:00 \\
\hline Switch P5 (\#321768) from the DPT panel to C2H01 TZ. & $9-28-93$ & 271 & 271 & 13:30:00 \\
\hline Shut in $T Z$ from the flow meter terminating gas-injection test in $R$. & $9-29-93$ & 272 & 272 & 13:37:00 \\
\hline Increase GZP pressure via accumulator to $13.500 \mathrm{MPa}$ and shut in. & $9-30-93$ & 273 & 273 & 10:26:00 \\
\hline Increase GZ pressure via accumulator to $10.120 \mathrm{MPa}$ and left accurmulator on line. & $9-30-93$ & 273 & 273 & 10:45:00 \\
\hline Shut in GZ from accumulator at $9.036 \mathrm{MPa}$. & 10-8-93 & 281 & 281 & 10:05:00 \\
\hline Perform DPS \#1 by decreasing $T Z$ pressure $-0.5 \mathrm{MPa}(\mathrm{P} 1=10.475 \mathrm{MPa})$. & $10-11-93$ & 284 & 284 & $14: 31: 00$ \\
\hline Shut in TZ at $9.970 \mathrm{MPa}$ terminating DPS \#1. & $10-11-93$ & 284 & 284 & 14:32:20 \\
\hline Shut in GZ from accumulator. & $10-1493$ & 287 & 287 & 07:25:00 \\
\hline Perform DPS \#2 by decreasing $T Z$ pressure $\sim 0.25 \mathrm{MPa}(P I=9.836 \mathrm{MPa})$. & $10-15-93$ & 288 & 288 & 08:58:00 \\
\hline Shut in $\mathrm{TZ}$ at $9.587 \mathrm{MPa}$ terminating DPS $\# 2$. & 10-15-93 & 288 & 288 & 08:59:20 \\
\hline Perform DPS \#3 by decreasing $T Z$ pressure $-0.15 \mathrm{MPa}(P 1=9.565 \mathrm{MPa})$. & $10-19-93$ & 292 & 292 & $08: 04: 00$ \\
\hline Shut in TZ at $9.401 \mathrm{MPa}$ terminating DPS $\# 3$. & $10-19-93$ & 292 & 292 & 08:04:50 \\
\hline Perform DPS \#4 by decreasing TZ pressure $-0.2 \mathrm{MPa}(\mathrm{PI}=9.426 \mathrm{MPa})$. & 10-22-93 & 295 & 295 & 09:22:00 \\
\hline Shut in TZ at $9.227 \mathrm{MPa}$ teminating DPS \#4. & $10-22-93$ & 295 & 295 & 09:23:05 \\
\hline End data file $\mathrm{C}_{2} \mathrm{HO} O 204$. & $10-26-93$ & 299 & 299 & 11:04:43 \\
\hline Begin data file $\mathrm{C} 2 \mathrm{H} 0205$. & 10-26-93 & 299 & 299 & 11:34:36 \\
\hline Perform DPS \#5 by decreasing TZ pressure $-0.2 \mathrm{MPa}(P 1=9.248 \mathrm{MPa})$. & 10-26-93 & 299 & 299 & 11:58:00 \\
\hline Shut in $\mathrm{TZ}$ at $9.021 \mathrm{MPa}$ termunating DPS $\# 5$. & 10-26-93 & 299 & 299 & 11:58:20 \\
\hline Perform DPS \#6 by decreasing TZ pressure $-0.12 \mathrm{MPa}(\mathrm{P} 1=9.073 \mathrm{MPa})$. & $11-1-93$ & 305 & 305 & 09:42:00 \\
\hline
\end{tabular}


Table 5-8 (Continued). Testing Sequence C2H02 Events

\begin{tabular}{|c|c|c|c|c|}
\hline EVENT & DATE & $\begin{array}{c}\text { CALENDAR } \\
\text { DAY }\end{array}$ & $\begin{array}{c}1993 \\
\text { CALENDAR } \\
\text { DAY }\end{array}$ & $\begin{array}{c}\text { TIME } \\
\text { (HH:MM:SS) }\end{array}$ \\
\hline Shut in $T Z$ at $8.950 \mathrm{MPa}$ terminating DPS \#6. & $11-1-93$ & 305 & 305 & $09: 42: 20$ \\
\hline Pertorm DPS \#7 by decreasing TZ pressure $\sim 0.1 \mathrm{MPa}(\mathrm{P1}=8.975 \mathrm{MPa})$. & $11-11-93$ & 315 & 315 & 11:41:00 \\
\hline Shut in $T Z$ at $8.856 \mathrm{MPa}$ terminating DPS $\# 7$. & $11-11-93$ & 315 & 315 & $11: 41: 20$ \\
\hline End data file $\mathrm{C} 2 \mathrm{H} 0205$. & 11-23-93 & 327 & 327 & 09:27:28 \\
\hline Begin data file $\mathrm{C} 2 \mathrm{H} 02 \mathrm{O}$. & 11-23-93 & 327 & 327 & 10:16:33 \\
\hline Deflate TZP in preparation for gas/brine exchange $(P 1=3.872 \mathrm{MPa})$. & $11-23-93$ & 327 & 327 & $12: 37: 45$ \\
\hline Begin gas/brine exchange $\$ 2$ in $T Z$ (injected $-4350 \mathrm{~mL}$ of brine into borehole). & $11-23-93$ & 327 & 327 & 12:54:32 \\
\hline Shut in TZ terminating gas/brine exchange $\# 2$. & $11-23-93$ & 327 & 327 & 13:00:00 \\
\hline Pertom DPS \#B by decreasing $T Z$ pressure to $P 1=6.837 \mathrm{MPa}$ (no gas in TZ). & 11-24-93 & 328 & 328 & 09:58:00 \\
\hline Shut in TZ at 6.837 MPa terminating DPS \#8. & 11-24-93 & 328 & 328 & 09:59:00 \\
\hline Inflate TZP to $-5.5 \mathrm{MPa}$. & 11-24-93 & 328 & 328 & 10:23:00 \\
\hline $\begin{array}{c}\text { Test tool moved out of the borehole }-4.5 \mathrm{~cm} \text { as indicated in tool configuration } \\
\text { diagram } \# 2 \text { (Figure 5-11). }\end{array}$ & 11-24-93 & 328 & 328 & 10:26:00 \\
\hline Deflate TZP. & $11-24-93$ & 328 & 328 & 11:01:00 \\
\hline Increase $T Z$ pressure to $7.984 \mathrm{MPa}$ by injecting $-760 \mathrm{~mL}$ of brine (no gas). & $11-24-93$ & 328 & 328 & 11:08:45 \\
\hline $\begin{array}{l}\text { Perform pulse-withdrawal in GZ to cheek for gas ( }-1020 \mathrm{~mL} \text { of gassy fluid then } \sim 700 \\
\text { mL of fluid). }\end{array}$ & $11-29-93$ & 333 & 333 & 09:51:00 \\
\hline Depressurize zone. & $11-29-93$ & 333 & 333 & 09:58:00 \\
\hline Inflate TZP to $-12 \mathrm{MPa}$ & 11-29-93 & 333 & 333 & 10:06:00 \\
\hline Open TZP to accumulator at $12.5 \mathrm{MPa}$. & $11-29-93$ & 333 & 333 & 10:10:00 \\
\hline Increase $\mathrm{GZ}$ pressure to $6.200 \mathrm{MPa}$. & 11-29-93 & 333 & 333 & 10:14:00 \\
\hline Increase TZ pressure to $9.140 \mathrm{MPa}$ (circulated brine to eliminate all possible gas). & $11-29-93$ & 333 & 333 & 10:23:00 \\
\hline Shut in both zones $(\mathrm{P1}=8.931, \mathrm{P3}=6.178 \mathrm{MPa})$. & $11-29-93$ & 333 & 333 & 10:25:35 \\
\hline End data file $\mathrm{C} 2 \mathrm{H}_{02} \mathrm{O}$. & $12-20-93$ & 354 & 354 & 08:23:13 \\
\hline Begin data file $\mathrm{C} 2 \mathrm{H} 02 \mathrm{OO}$. & 12-20-93 & 354 & 354 & 09:29:54 \\
\hline End data file C2H0207 (end PERM4F DAS software). & $12-28-93$ & 362 & 362 & $08: 56: 42$ \\
\hline Switch DAS from LABTECH to PERM4F (P5 is not responding properly). & $12-29-93$ & 363 & 363 & N/A \\
\hline Begin data file $\mathrm{C} 2 \mathrm{H} 02 \mathrm{OB}$. & $12-29-93$ & 363 & 363 & 13:25:06 \\
\hline End data file $\mathrm{C} 2 \mathrm{HO} 208$. & $1-10-94$ & 10 & 375 & 11:28:53 \\
\hline Begin data file $\mathrm{C}_{2} \mathrm{HO} O 209$. & $1-10-94$ & 10 & 375 & 11:29:31 \\
\hline End data file C2H0209. & 1-23-94 & 23 & 388 & 09:28:58 \\
\hline Begin data file C2HO21O. & $1-24-94$ & 24 & 389 & 12:17:06 \\
\hline $\begin{array}{l}\text { Perform pulse-withdrawal in TZ by decreasing TZ pressure to } 6.743 \mathrm{MPa}(-10.4 \mathrm{~mL} \text { of } \\
\text { brine removed). }\end{array}$ & 1-24-94 & 24 & 389 & $12: 31: 00$ \\
\hline Shut in $\pi$ & $1-24-94$ & 24 & 389 & $12: 33: 00$ \\
\hline End data file C2H0210. & $2-16-94$ & 46 & 411 & 09:20:23 \\
\hline Begin data file С2HO211. & $2-16-94$ & 46 & 411 & 09:23:44 \\
\hline End data file $\mathrm{C}_{2 \mathrm{HO}} \mathrm{H1}$. & $3-4-94$ & 63 & 434 & $14: 20: 41$ \\
\hline
\end{tabular}


Table 5-8 (Continued). Testing Sequence C2H02 Events

\begin{tabular}{ccccc}
\hline EVENT & DATE & $\begin{array}{c}\text { CALENDAR } \\
\text { DAY }\end{array}$ & $\begin{array}{c}\text { 1993 } \\
\text { CALNDAR } \\
\text { DAY }\end{array}$ & $\begin{array}{c}\text { TIME } \\
\text { (HH:MM:SS) }\end{array}$ \\
\hline Remove transducer 321768 (C2H01) from system. & $3-4-94$ & 63 & 434 & $14: 30: 00$ \\
Begin data file C2H0212. & $3-4-94$ & 63 & 434 & $14: 49: 44$ \\
End data file C2H0212. & $3-21-94$ & 80 & 448 & $11: 17: 50$ \\
Remove test tool from borehole C2H02. & $3-23-94$ & 82 & 450 & $14: 00: 00$ \\
\hline
\end{tabular}

Figures 5-23 through 5-27 illustrate the zone pressures, packer pressures, nitrogeninjection rate, and test-zone temperature, respectively, during testing sequence $\mathrm{C} 2 \mathrm{HO} 2$.

Table 5-9 indicates the equipment that was used and the duration that each instrument was used during testing sequence $\mathrm{C} 2 \mathrm{H} 02$.

Table 5-9. Testing Sequence $\mathrm{C} 2 \mathrm{H} 02$ Equipment

\begin{tabular}{|c|c|c|c|c|}
\hline Equipment & Location & Serial \# & Installed & Removed \\
\hline DAS Software & N/A & LABTECH 4.1.0 & $8-10-93$ & $12-28-93$ \\
\hline DAS Software & N/A & PERM4F & $12-29-93$ & $3-21-94$ \\
\hline DCU (HP75000) & N/A & $3035 a 01445$ & $8-10-93$ & $9-9-93$ \\
\hline DCU (HP75000) & N/A & $3035 a 01445$ & $9-9-93$ & $12-28-93$ \\
\hline DCU (HP3497A) & N/A & $2629 a 21989$ & $12-28-93$ & $3-21-94$ \\
\hline $\begin{array}{c}\text { Transducer } \\
\text { (Druck PDCR 910) }\end{array}$ & $\begin{array}{l}\text { C2H02 Guard } \\
\text { Zone Packer }\end{array}$ & 308145 & $8-10-93$ & $3-21-94$ \\
\hline $\begin{array}{c}\text { Transducer } \\
\text { (Druck PDCR 910) }\end{array}$ & $\begin{array}{l}\text { C2H02 Test } \\
\text { Zone Packer }\end{array}$ & 308150 & $8-10-93$ & $3-21-94$ \\
\hline $\begin{array}{c}\text { Transducer } \\
\text { (Druck PDCR 910) }\end{array}$ & $\begin{array}{l}\mathrm{C} 2 \mathrm{HO} 2 \text { Test } \\
\text { Zone }\end{array}$ & 322422 & $8-10-93$ & $3-21-94$ \\
\hline $\begin{array}{c}\text { Transducer } \\
\text { (Druck PDCR 910) }\end{array}$ & $\begin{array}{l}\text { C2H02 Guard } \\
\text { Zone }\end{array}$ & 507864 & $8-10-93$ & $3-21-94$ \\
\hline $\begin{array}{c}\text { Transducer } \\
\text { (Druck PDCR 910) } \\
\end{array}$ & $\begin{array}{c}\text { Mass Flow } \\
\text { Meter/C2H01 TZ } \\
\end{array}$ & 321768 & $9-10-93$ & $3-4-94$ \\
\hline Thermocouple (Type E) & $\mathrm{C} 2 \mathrm{H} 02 \mathrm{TZ}$ & 1 & $9-10-93$ & $3-4-94$ \\
\hline $\begin{array}{c}\text { Flow Meter } \\
\text { (Bronkhorst F-230C-FA-22-V) }\end{array}$ & N/A & $921209 a$ & $9-10-93$ & $9-28-93$ \\
\hline
\end{tabular}




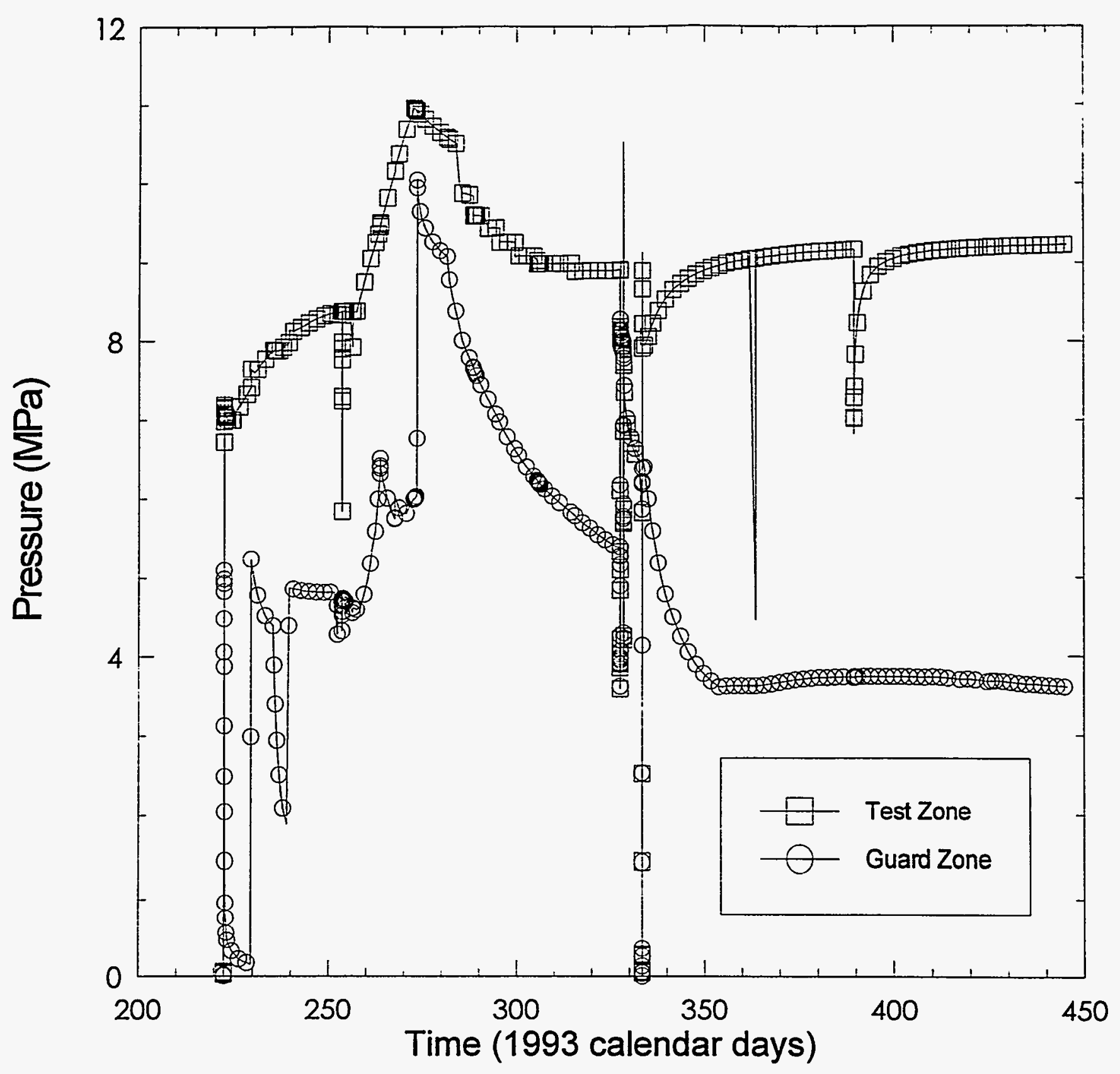

INTERA-115-175-0

Figure 5-23. Zone pressures during testing sequence $\mathrm{C} 2 \mathrm{HO} 2$ in test borehole $\mathrm{C} 2 \mathrm{HO}$. 


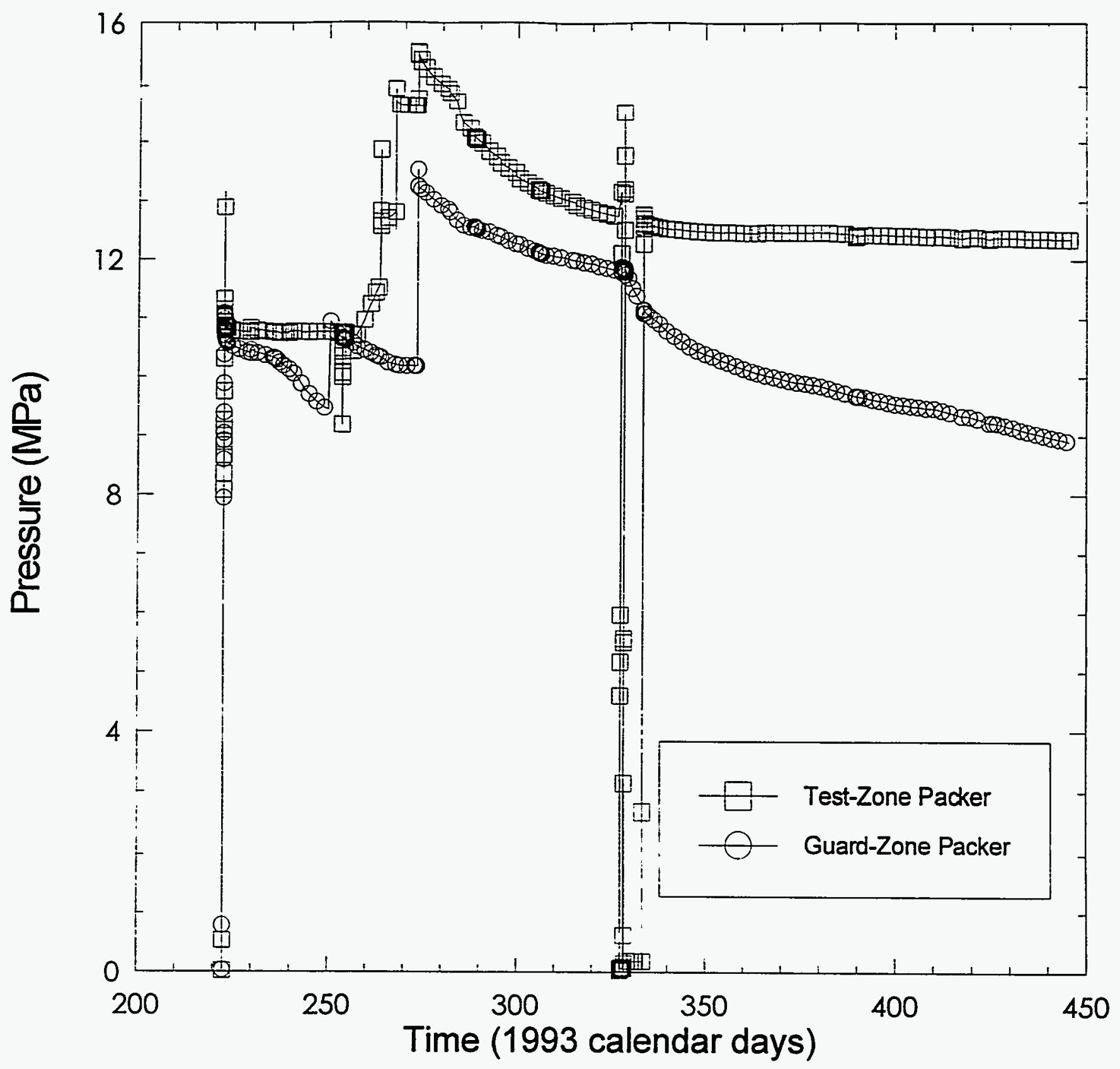

INTERA-115-176-0

Figure 5-24. Packer pressures during testing sequence $\mathrm{C} 2 \mathrm{H} O 2$ in test borehole $\mathrm{C} 2 \mathrm{H} 02$. 


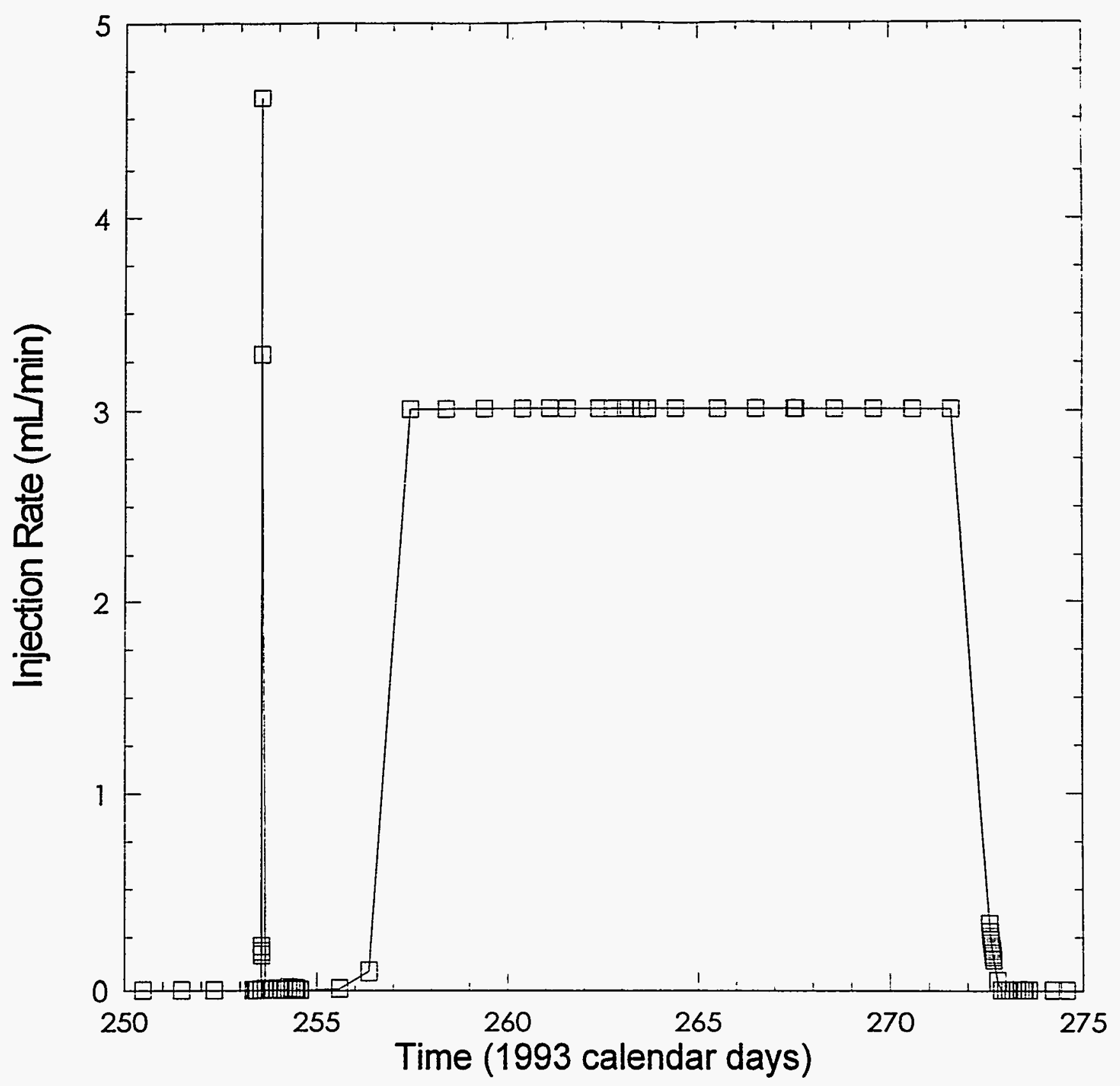

INTERA-ST15-177-0

Figure 5-25. Nitrogen-injection rate during testing sequence $\mathrm{C} 2 \mathrm{HO} 2$ in test borehole $\mathrm{C} 2 \mathrm{HO}$. 


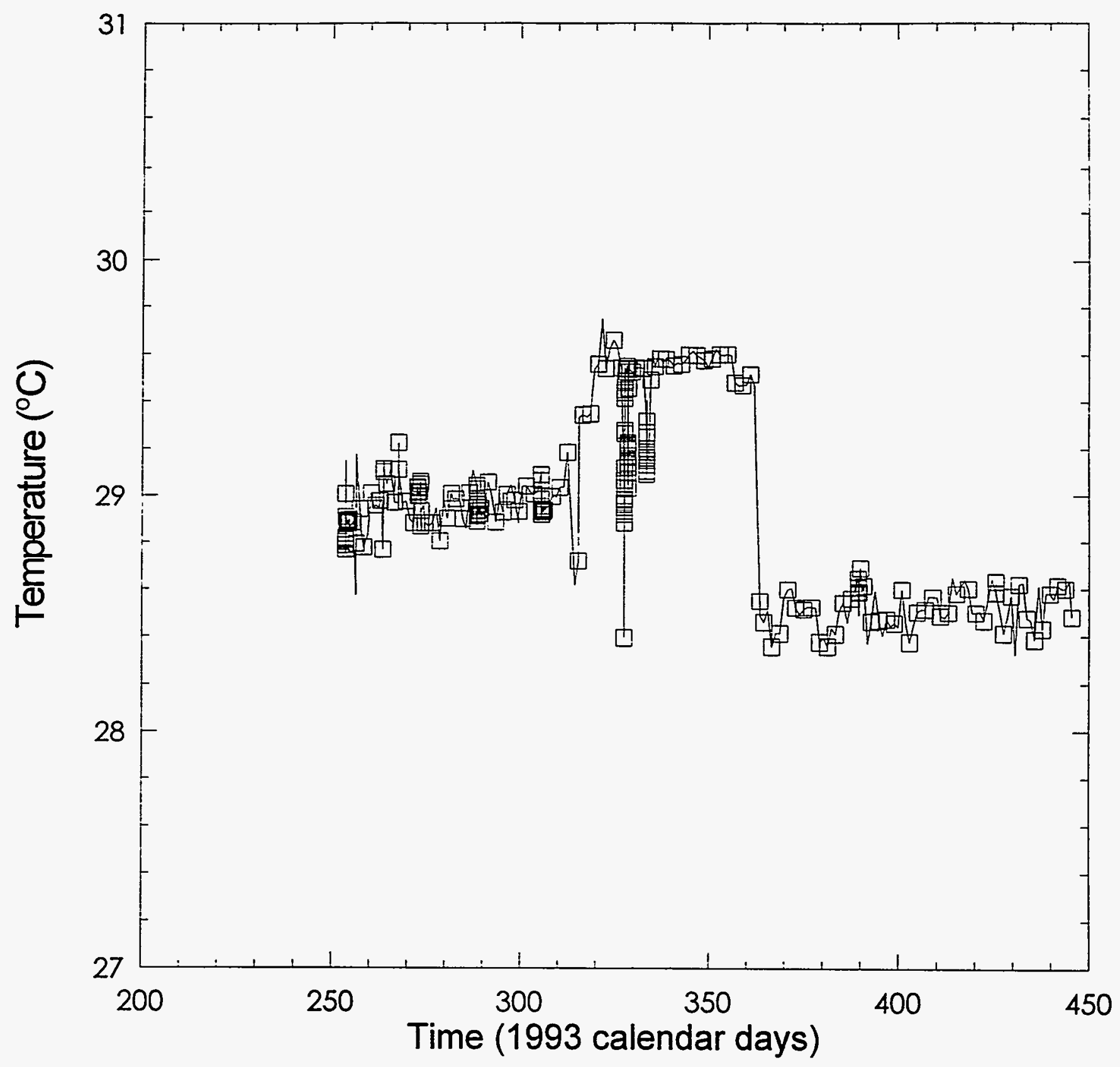

INTERAS115-178-0

Figure 5-26. Test-zone temperature during testing sequence $\mathrm{C} 2 \mathrm{HO}$ in test borehole $\mathrm{C} 2 \mathrm{HO}$. 


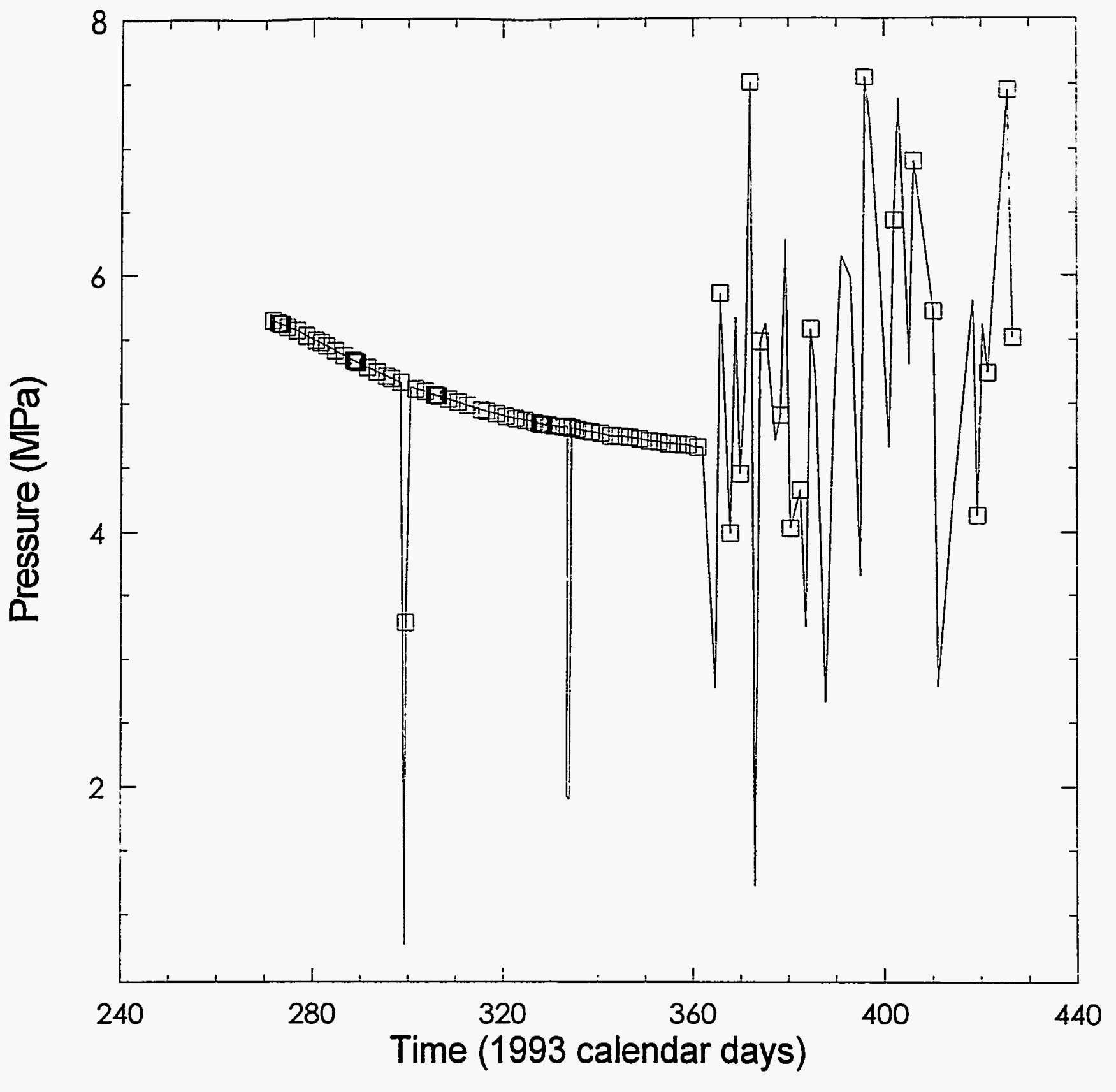

Figure 5-27. Zone pressure during testing sequence $\mathrm{C} 2 \mathrm{HO} 2$ in observation borehole $\mathrm{C} 2 \mathrm{H} 01$. 


\subsubsection{OBSERVATION BOREHOLE C2H01}

Table 5-10 gives a description of the events associated with observation borehole $\mathrm{C} 2 \mathrm{H} 01$ during testing sequence $\mathrm{C} 2 \mathrm{HO} 2$.

Table 5-10. Events in Borehole $\mathrm{C} 2 \mathrm{H} 01$ During Testing Sequence $\mathrm{C} 2 \mathrm{H} 02$

\begin{tabular}{ccccc}
\hline EVENT & DATE & $\begin{array}{c}\text { CALENDAR } \\
\text { DAY }\end{array}$ & $\begin{array}{c}\text { 1993 } \\
\text { CALENDAR } \\
\text { DAY }\end{array}$ & $\begin{array}{c}\text { TIME } \\
\text { (HH:MM:SS) }\end{array}$ \\
\hline $\begin{array}{c}\text { Install single-packer fluid-pressure monitoring tool as indicated in the test-tool } \\
\text { configuration diagram (Figure 5-9). }\end{array}$ & $3-13-91$ & 72 & N/A \\
Switch P5 (\$321768) from the DPT panel to C2H01 TZ & $9-28-93$ & 271 & 271 & $13: 30: 00$ \\
Remove transducer \#321768 trom system. & $3-4-94$ & 63 & 434 & $14: 30: 00$ \\
Terminate data collection. & $3-18-94$ & 77 & 442 & $11: 18: 00$ \\
Remove test tool from borehole C2H02. & $3-23-94$ & 82 & 447 & $14: 00: 00$ \\
\hline
\end{tabular}

MB139 in borehole $\mathrm{C} 2 \mathrm{H01}$ was monitored with Druck transducer \#321768 during testing sequence $\mathrm{C} 2 \mathrm{H} 02$. This transducer was incorporated into the $\mathrm{C} 2 \mathrm{HO} 2 \mathrm{DAS}$ and is listed in Table 5-9. Figure 5-27 illustrates the pressure in observation borehole $\mathrm{C} 2 \mathrm{H} 01$ during testing sequence $\mathrm{C} 2 \mathrm{HO} 2$.

\subsubsection{Gas-Threshold-Pressure Testing in Room L4}

Testing sequence L4P52-B was designed to investigate the gas-threshold pressure of MB138 in an environment removed from the WIPP excavations. Table 5-11 gives a detailed description of the events that occurred during testing sequence L4P52-B.

Table 5-11. Testing Sequence L4P52-B Events

\begin{tabular}{|c|c|c|c|c|}
\hline EVENT & DATE & $\begin{array}{c}\text { CALENDAR } \\
\text { DAY }\end{array}$ & $\begin{array}{c}1993 \\
\text { CALENDAR } \\
\text { DAY }\end{array}$ & $\begin{array}{c}\text { TIME } \\
\text { (HH:MM:SS) }\end{array}$ \\
\hline $\begin{array}{l}\text { Install double-packer test tool \#37 as indicated in test-1001 configuration diagram } \\
\text { (Figure 3-31). }\end{array}$ & $12-16-92$ & 350 & N/A & N/A \\
\hline Change DAS from PERM4F to LABTECH in preparation for GTPT. & $12-29-93$ & 363 & 363 & 13:01:00 \\
\hline Begin data file L4P52B08. & $12-29-93$ & 363 & 363 & 13:21:36 \\
\hline Repair a leaking fitting on $G Z$ inject line. & 12-30-93 & 364 & 364 & 09:41:00 \\
\hline Deflate TZP in preparation for gas/brine exchange. & 12-31-93 & 365 & 365 & 10:35:00 \\
\hline Pertorm gas/brine exchange \#1 by withdrawing $-180 \mathrm{~mL}$ of brine. & $12-31-93$ & 365 & 365 & 11:54:00 \\
\hline Continue gas/brine exchange \#1 withdrawing $6300 \mathrm{~mL}$ of brine to depressurze zone. & 12-31-93 & 365 & 365 & 11:59:00 \\
\hline Deflate GZP. & 12-31-93 & 365 & 365 & 13:11:00 \\
\hline Increase GZP pressure via accumulator to $-15 \mathrm{MPa}$. & 12-31-93 & 365 & 365 & 14:00:00 \\
\hline Fill zones with brine. & 12-31-93 & 365 & 365 & 14:46:00 \\
\hline Continue gas/brine exchange \#1 by withdrawing $-850 \mathrm{~mL}$ of brine to expose MB138. & $12-31-93$ & 365 & 365 & 14:56:00 \\
\hline
\end{tabular}


Table 5-11 (Continued). Testing Sequence L4P52-B Events

\begin{tabular}{|c|c|c|c|c|}
\hline EVENT & DATE & $\begin{array}{l}\text { CALENDAR } \\
\text { DAY }\end{array}$ & $\begin{array}{c}1993 \\
\text { CALENDAR } \\
\text { DAY }\end{array}$ & $\begin{array}{c}\text { TIME } \\
\text { (HH:MM:SS) }\end{array}$ \\
\hline Increase TZP pressure via accumulator to $\sim 15 \mathrm{MPa}$. & $12-31-93$ & 365 & 365 & 15:05:00 \\
\hline Increase TZ pressure to $-8 \mathrm{MPa}$. & 12-31-93 & 365 & 365 & 15:13:00 \\
\hline Increase $\mathrm{GZ}$ pressure to $-8 \mathrm{MPa}$. & $12-31-93$ & 365 & 365 & 15:15:00 \\
\hline Shut in both packers from accumulator. & 1.4 .94 & 4 & 369 & $09: 45: 00$ \\
\hline Open both packers to full accumulator. & $1-4-94$ & 4 & 369 & 10:34:00 \\
\hline Shut in both packers from accumulators, change $N_{2}$ bottle. & i-5-94 & 5 & 370 & $13: 20: 00$ \\
\hline Open both packers to accumulator at a higher pressure. & $1-5-94$ & 5 & 370 & 13:27:00 \\
\hline Increase TZ pressure to $-9.3 \mathrm{MPa}$. & $1-5-94$ & 5 & 370 & 13:37:00 \\
\hline Repair leaking fitting on $T Z$ inject line. & $1-6-94$ & 6 & 371 & 10:22:00 \\
\hline Shut in both packers from accumulator. & $1-17-94$ & 17 & 382 & 09:58:00 \\
\hline $\begin{array}{c}\text { Perform gas/brine exchange } \# 2 \text { by injecting } N_{2} \text { into } T Z \text { to remove }-990 \mathrm{~mL} \text { of brine } \\
\text { because MB138 is not fully exposed. }\end{array}$ & $1-17-94$ & 17 & 382 & 10:27:00 \\
\hline Deflate TZP. & 1-17-94 & 17 & 382 & 10:33:00 \\
\hline Continue gas/brine exchange $\# 2$ by withdrawing $-1000 \mathrm{~mL}$ of brine from the zone. & $1-17-94$ & 17 & 382 & $10: 40: 00$ \\
\hline Inflate TZP to $\sim 15 \mathrm{MPa}$. & 1-17-94 & 17 & 382 & 10:48:00 \\
\hline Pressurize TZ to $-8.7 \mathrm{MPa}$. & 1-17-94 & 17 & 382 & 10:53:00 \\
\hline Pressurize GZ to $\sim 8.3 \mathrm{MPa}$ & $1-17-94$ & 17 & 382 & 10:55:00 \\
\hline Open both packers to accumulator at $\sim 15 \mathrm{MPa}$. & 1-17-94 & 17 & 382 & 10:59:00 \\
\hline End data file L4P52B08. & $1-25-94$ & 25 & 390 & 11:06:19 \\
\hline Begin data file L4P52B09. & $1-25-94$ & 25 & 390 & 12:12:47 \\
\hline Open $T Z$ to the flow meter for a leak check of the system. & $1-25-94$ & 25 & 390 & 13:13:40 \\
\hline Open GZ to accumulator at $-10.2 \mathrm{MPa}$. & $1-26-94$ & 26 & 391 & 13:23:00 \\
\hline Increase $G Z$ pressure via accumulator to $-9.8 \mathrm{MPa}$ and left $N_{2}$ bottle in line. & 1-27-94 & 27 & 392 & 09:05:00 \\
\hline Open pressure valve to the flow meter. & $1.27-94$ & 27 & 392 & 09:16:00 \\
\hline Begin $\mathrm{N}_{2}$ injection \#1 into TZ at $2 \mathrm{~mL} / \mathrm{min}$. & $1-27-94$ & 27 & 392 & 09:18:00 \\
\hline $\begin{array}{l}\text { Change } \mathrm{N}_{2} \text { injection rate into } \mathrm{TZ} \text { to } 0.8 \mathrm{~mL} \mathrm{~min} \text { to avold possible fracture of } M B 138 \\
\text { terminating gas-injection test \#1 and beginning gas-injection test } \# 2 .\end{array}$ & $1-28-94$ & 28 & 393 & 14:30:00 \\
\hline Shut in TZ terminating gas-injection test $\# 2$. & 2-1-94 & 32 & 397 & 10:18:45 \\
\hline Change $\mathrm{N}_{2}$ injection rate $100 \mathrm{~mL} \mathrm{~min}$. & 2-1-94 & 32 & 397 & 10:19:00 \\
\hline End data file L4P52B09. & 2-3-94 & 34 & 399 & 10:46:35 \\
\hline Begin data file L4P52B10. & 2-3-94 & 34 & 399 & 11:03:58 \\
\hline Recharge GZ accumulator pressure. & $2-8-94$ & 39 & 404 & 11:14:00 \\
\hline Shut in GZ from accumulator. & 2-14-94 & 45 & 410 & 08:52:00 \\
\hline Open GZ to full accumulator at $-14.3 \mathrm{MPa}$. & 2-14-94 & 45 & 410 & 09:12:00 \\
\hline Open pressure valve to flow meter. & 2-14-94 & 45 & 410 & 10:53:00 \\
\hline Open flow meter to $T Z$. & 2-14-94 & 45 & 410 & 10:56:00 \\
\hline Begin $\mathrm{N}_{2}$ injection 43 into $T Z$ at $1.1 \mathrm{~mL} / \mathrm{min}$. & 2-14-94 & 45 & 410 & $11: 00: 00$ \\
\hline
\end{tabular}


Table 5-11 (Continued). Testing Sequence L4P52-B Events

\begin{tabular}{|c|c|c|c|c|}
\hline EVENT & DATE & $\begin{array}{c}\text { CALENDAR } \\
\text { DAY }\end{array}$ & $\begin{array}{l}1993 \\
\text { CALENDAR } \\
\text { DAY }\end{array}$ & $\begin{array}{c}\text { TIME } \\
\text { (HH:MM:SS) }\end{array}$ \\
\hline End data file L4P52B10. & $2-14-94$ & 45 & 410 & 11:19:06 \\
\hline Begin data file L4P52B11. & 2-14-94 & 45 & 410 & 11:42:59 \\
\hline $\begin{array}{l}\text { Decrease } \mathrm{N}_{2} \text { injection rate due to faulty regulator on } \mathrm{N}_{2} \text { source terminating gas- } \\
\text { injection test } \# 3 \text { in } T Z .\end{array}$ & 2-16-94 & 47 & 412 & $\sim$ 10:00:00 \\
\hline $\begin{array}{l}\text { Correct } \mathrm{N}_{2} \text { injection rate to } 1.1 \mathrm{mLmin} \text { by tapping on regulator beginning gas-injection } \\
\text { test } \# 4 \text { in } \mathrm{TZ} \text {. }\end{array}$ & 2-16-94 & 47 & 412 & 13:53:00 \\
\hline Shut in TZ terminating gas-injection test \#4. & $2-18-94$ & 49 & 414 & 09:04:00 \\
\hline Change $\mathrm{N}_{2}$ injection rate to $0 \mathrm{mU} \mathrm{min}$. & 2-18-94 & 49 & 414 & 09:04:20 \\
\hline Inadvertent depressurization of $G Z$ for -2 minutes. & 2-18-94 & 49 & 414 & $09: 26: 00$ \\
\hline Repair leaking fitting on $\mathrm{GZ}$ accumulator. & 2-18-94 & 49 & 414 & $09: 34: 00$ \\
\hline End data file L4P52B11. & $2-22-94$ & 53 & 418 & 09:52:11 \\
\hline Begin data file L4P52B12. & $2-22-94$ & 53 & 418 & 10:08:57 \\
\hline UPS malfunction (possible loss of data). & 3-3-94 & 62 & 427 & N/A \\
\hline Shut in GZ from accumulator. & $3-4-94$ & 63 & 428 & $12: 30: 00$ \\
\hline Open GZ to full accumulator. & $3-4-94$ & 63 & 428 & 13:02:00 \\
\hline End data file L4P52B12. & $3-4-94$ & 63 & 428 & 13:04:12 \\
\hline Begin data file L4P52B13. & 3-4-94 & 63 & 428 & 13:39:35 \\
\hline End data file L4P52B13. & 3-8-94 & 67 & 432 & 11:36:36 \\
\hline Temporary temination of data collection to calibrate DCU \#3035a01445. & 3-8-94 & 67 & 432 & $-12: 00: 00$ \\
\hline Replace DCU \#3035a01445. & 3.9 .94 & 68 & 433 & $19: 00: 00$ \\
\hline Begin data file L4P52B14. & $3-9.94$ & 68 & 433 & 11:53:01 \\
\hline Shut in GZ from accumulator. & 3-9.94 & 68 & 433 & 12:44:00 \\
\hline Open GZ to full accumulator. & $3-9-94$ & 68 & 433 & $13: 05: 00$ \\
\hline Open TZ to flow meter. & $3-9-94$ & 68 & 433 & $13: 09: 00$ \\
\hline Begin $\mathrm{N}_{2}$ injection $\# 5$ into TZ at $0.6 \mathrm{~mL} / \mathrm{min}$. & $3-9-94$ & 68 & 433 & 13:10:00 \\
\hline Shut in $\Pi Z$ terminating gas-injection test $\# 5$. & 3-11-94 & 70 & 435 & 13:03:00 \\
\hline Chango $\mathrm{N}_{2}$ injoction rate to $0 \mathrm{mUmin}$. & $3-11-94$ & 70 & 435 & 13:04:00 \\
\hline Shut in $G Z$ from accumulator. & 3-14-94 & 73 & 438 & 09:01:00 \\
\hline Open GZ to full accumulator. & 3-14-94 & 73 & 438 & $09: 33: 00$ \\
\hline End data file L4P52B14. & 3-14-94 & 73 & 438 & 11:11:06 \\
\hline Begin data file L4P52B15. & $3-14-94$ & 73 & 438 & $11: 21: 14$ \\
\hline Perform DPS by decreasing $T Z$ pressure from $9.866 \mathrm{MPa}$ to $-9.56 \mathrm{MPa}$. & 3-14-94 & 73 & 438 & $11: 25: 00$ \\
\hline Shut in $R$. & $3-14-94$ & 73 & 438 & $11: 25: 42$ \\
\hline Shut in $G Z$ from accumulator. & 3-18-94 & 77 & 442 & 12:09:00 \\
\hline Open GZ to full accumulator. & $3-18-94$ & 77 & 442 & 12:23:00 \\
\hline End data file L4P52B15. & $3-21-94$ & 80 & 445 & $11: 36: 10$ \\
\hline Begin data file L4P52B16. & $3-21-94$ & 80 & 445 & 12:17:10 \\
\hline
\end{tabular}


Table 5-11 (Continued). Testing Sequence L4P52-B Events

\begin{tabular}{|c|c|c|c|c|}
\hline EVENT & DATE & $\begin{array}{c}\text { CALENDAR } \\
\text { DAY }\end{array}$ & $\begin{array}{c}1993 \\
\text { CALENDAR } \\
\text { DAY }\end{array}$ & $\begin{array}{c}\text { TIME } \\
\text { (HH:MM:SS) }\end{array}$ \\
\hline Shut in GZ from accumulator. & $3-24-94$ & 83 & 448 & 10:18:00 \\
\hline Open $G Z$ to full accumulator. & $3-24-94$ & 83 & 448 & 11:02:00 \\
\hline Shut in $G Z$ from accumulator. & $3-25-94$ & 84 & 449 & 14:03:00 \\
\hline Shut in both packers from accumulator. & $3-28-94$ & 87 & 452 & 09:55:00 \\
\hline End data file L4P52B16. & $3-31-94$ & 90 & 455 & 11:27:05 \\
\hline Depressurize $T Z$ & $5-494$ & 124 & 489 & 10:46:00 \\
\hline Depressurize $\mathrm{GZ}$ & 5-4-94 & 124 & 489 & 10:49:00 \\
\hline Deflate GZP. & $5-4-94$ & 124 & 489 & 10:52:00 \\
\hline Deflate TZP. & $5-4-94$ & 124 & 489 & 10:54:00 \\
\hline Remove double-packer test tool \#37 from borehole L4P52. & $5-4-94$ & 124 & 489 & $14: 26: 00$ \\
\hline
\end{tabular}

Figures 5-28 through 5-31 illustrate the zone pressures, packer pressures, nitrogeninjection rate, and zone temperatures, respectively, during testing sequence L4P52-B.

Table 5-12 indicates the equipment that was used and the duration that each instrument was used during testing sequence L4P52-B.

Table 5-12. Testing Sequence L4P52-B Equipment

\begin{tabular}{|c|c|c|c|c|}
\hline Equipment & Location & Serial \# & Installed & Removed \\
\hline DAS Software & N/A & LABTECH 4.1 .0 & $12-29-93$ & $4-12-94$ \\
\hline DCU (HP75000) & N/A & $3035 a 01445$ & $12-29-93$ & $3-8-94$ \\
\hline DCU (HP75000) & N/A & $3035 a 01445$ & $3-9-94$ & 4-12-94 \\
\hline $\begin{array}{c}\text { Transducer } \\
\text { (Druck PDCR 830) }\end{array}$ & Test Zone & 214048 & $12-17-92$ & $4-12-94$ \\
\hline $\begin{array}{c}\text { Transducer } \\
\text { (Druck PDCR 830) }\end{array}$ & $\begin{array}{l}\text { Test Zone } \\
\text { Packer }\end{array}$ & 214466 & $12-17-92$ & $4-12-94$ \\
\hline $\begin{array}{c}\text { Transducer } \\
\text { (Druck PDCR 830) }\end{array}$ & $\begin{array}{l}\text { Guard Zone } \\
\text { Packer }\end{array}$ & 214470 & $12-17-92$ & $4-12-94$ \\
\hline $\begin{array}{c}\text { Transducer } \\
\text { (Druck PDCR 830) }\end{array}$ & Guard Zone & 246913 & $12-17-92$ & $4-12-94$ \\
\hline $\begin{array}{c}\text { Transducer } \\
\text { (Druck PDCR 910) } \\
\end{array}$ & $\begin{array}{c}\text { Mass Flow } \\
\text { Meter }\end{array}$ & 308148 & $1-20-94$ & $4-12-94$ \\
\hline Thermocouple (Type E) & Test Zone & 1 & $12-29-93$ & 4-12-94 \\
\hline $\begin{array}{c}\text { Flow Meter } \\
\text { (Bronkhorst F-230C-FA-22-V) }\end{array}$ & N/A & $921209 a$ & $1-20-94$ & $4-12-94$ \\
\hline
\end{tabular}




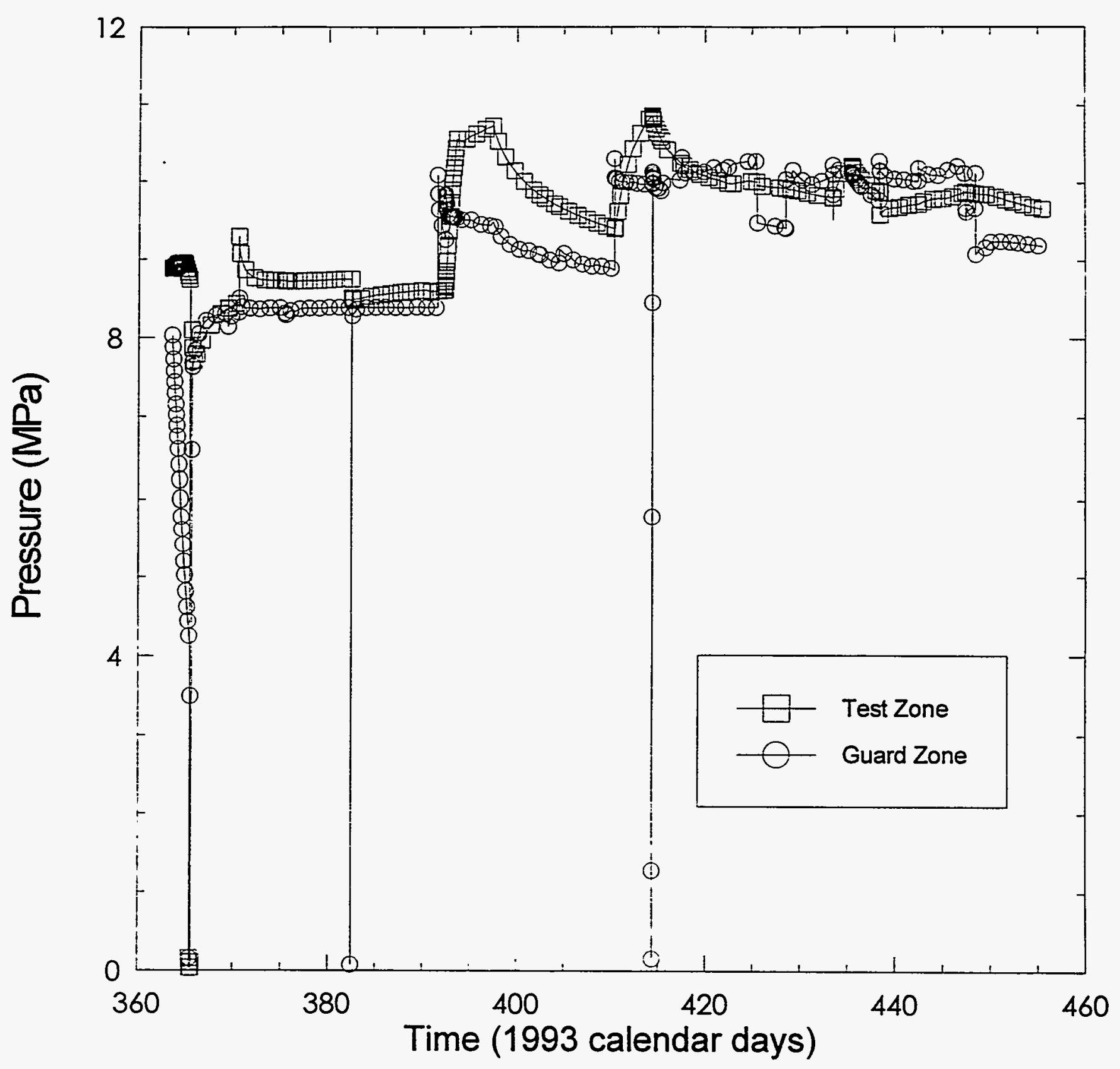

INTERAOI15-180-0

Figure 5-28. Zone pressures during testing sequence L4P52-B. 


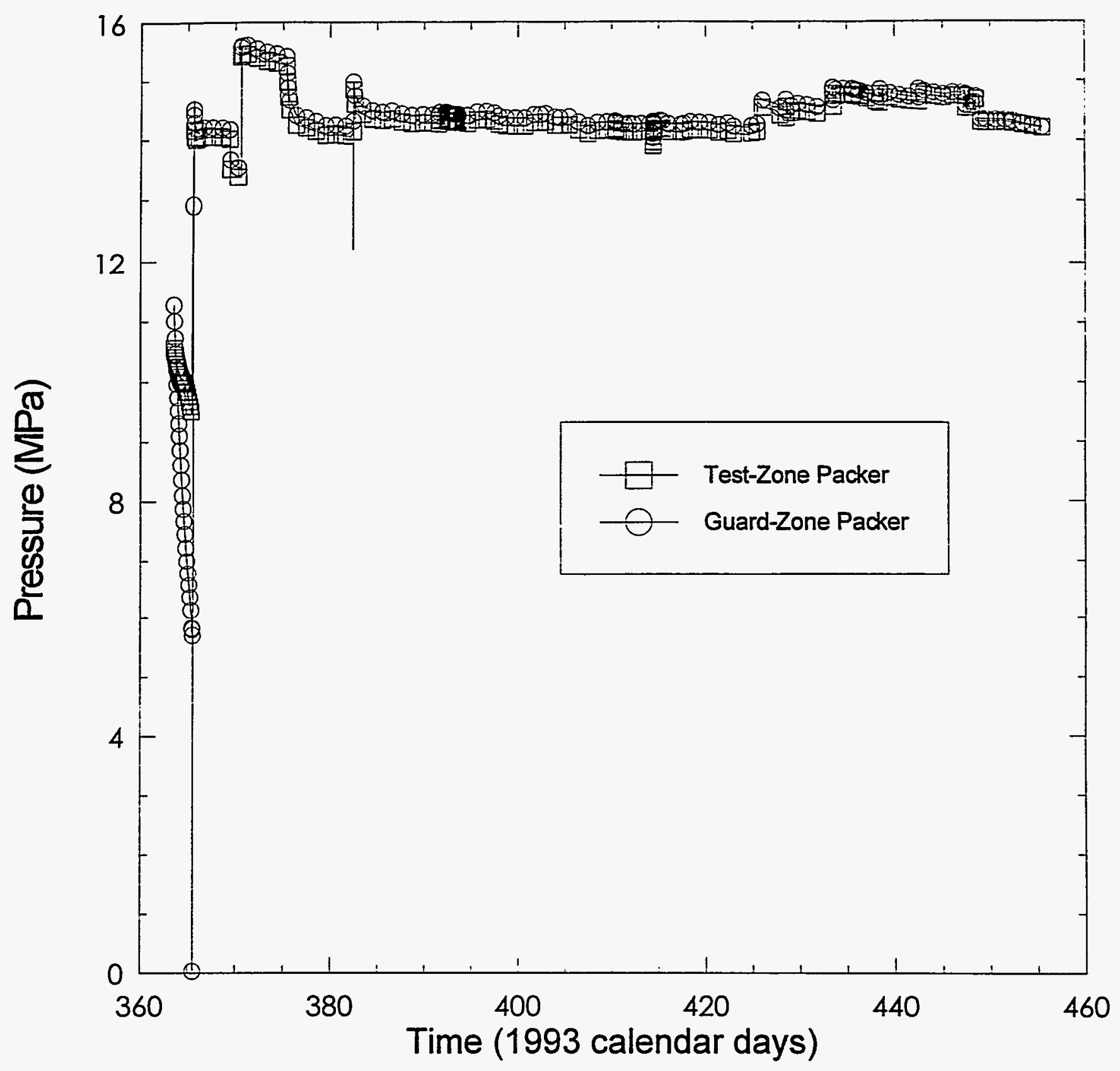

INTERA6115-181-0

Figure 5-29. Packer pressures during testing sequence L4P52-B. 


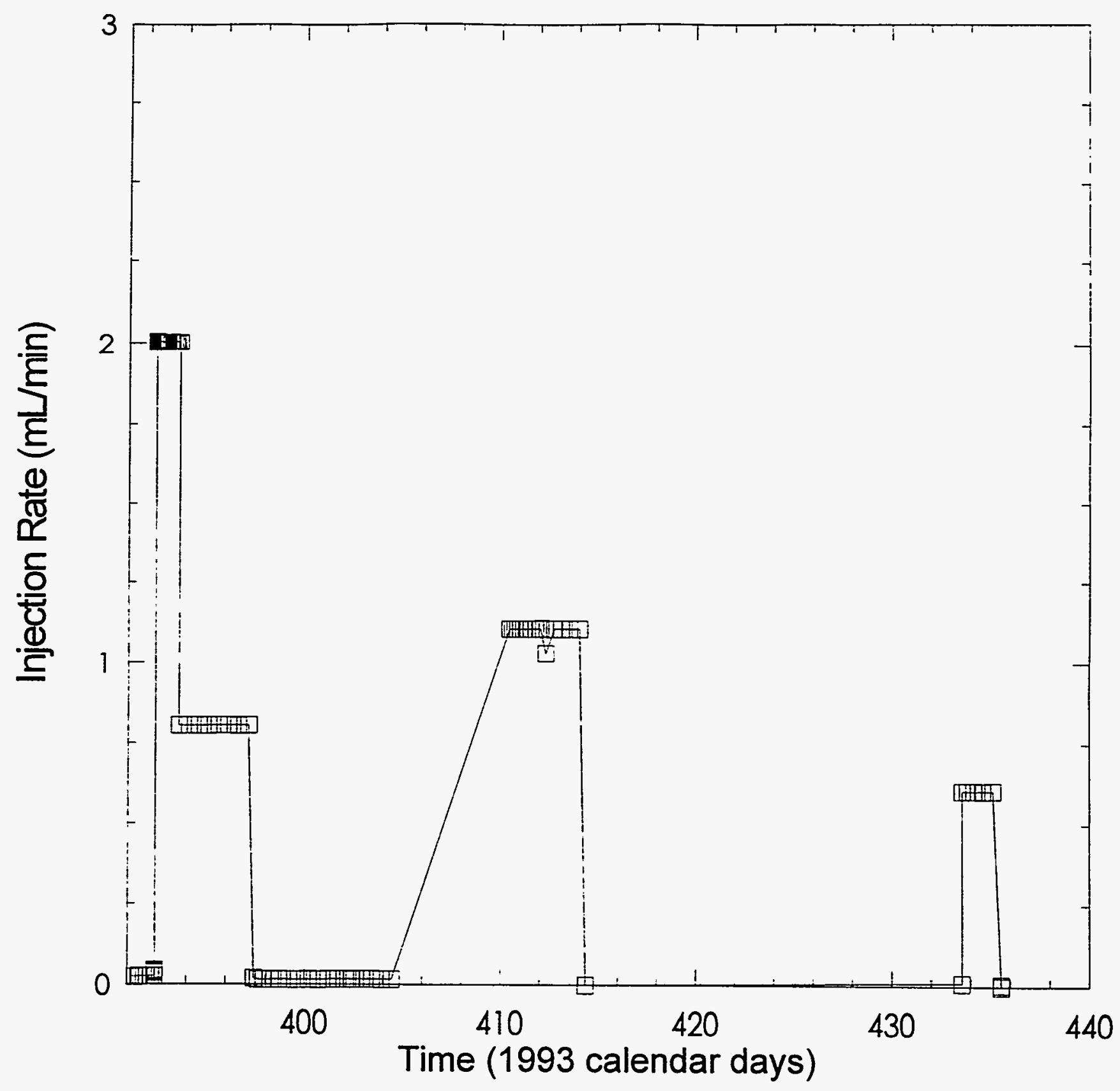

INTERA-6115-182-0

Figure 5-30. Nitrogen-injection rate during testing sequence L4P52-B. 


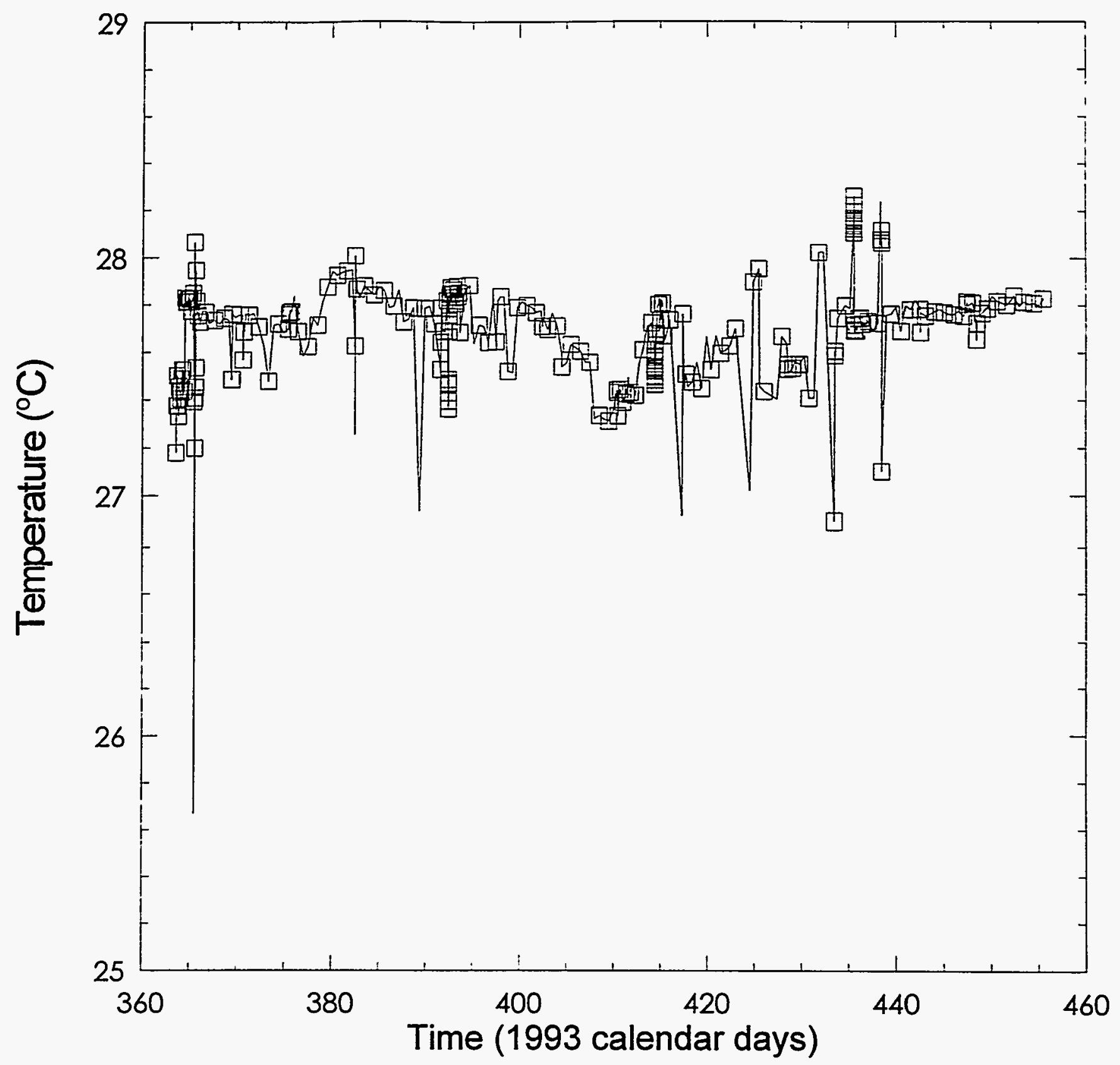

INTERA-6115-103-0

Figure 5-31. Test-zone temperature during testing sequence L4P52-B. 


\section{COMPLIANCE TESTING}

\subsection{Compliance-Testing Procedures}

Pickens et al. (1987) showed that test-tool movement in response to packer inflation and brine injection or withdrawal can affect fluid-pressure responses in isolated intervals in boreholes in low-permeability media. In particular, packer movement due to packer inflation can cause the packer element to displace brine in isolated intervals. In lowpermeability media, such displacement can cause changes in fluid pressure in the test zone. Changes in the shape, volume, or position of the test tool that affect fluid-pressure responses are referred to as compliance. Compliance may also affect the internal pressure of fluid-filled packers as the inflatable synthetic-rubber packer elements stretch or shrink in response to borehole or test-interval changes in pressure or temperature.

To evaluate the magnitude of compliance for the multipacker test tools, compliance tests were performed on each of the test tools prior to installation in test boreholes. The purpose of compliance testing was to (1) establish that the test tools were properly assembled and sealed, (2) to evaluate test-tool responses to packer inflation and applied pressure pulses, and (3) to quantify these test-tool responses by constructing test-zonecompressibility versus pressure relationships. The results of the compliance tests are presented below.

To begin compliance testing, a test tool or portion of a test tool was installed in a pressuretested stainless-steel chamber that was placed in a borehole in the underground WIPP facility (Figure 6-1). The compliance-testing chamber consisted of a 4.5-inch $(11.4-\mathrm{cm})$ O.D. and 5.1-meter long section of stainless-steel casing. The test chamber was sealed at one end with a welded cap and mated at the other end with a welded blind flange. The test chamber was pressure tested and certified to a maximum allowable working pressure of 1500 psig (10.3 MPa). The test chamber was placed in borehole ENP10 which was drilled in the East 140 drift between the North 1100 and North 1420 drifts for compliance testing (Figure 2-1). The borehole is a 5.5-inch $(13.97-\mathrm{cm})$ diameter, 15.83-meter long borehole, oriented $13^{\circ}$ downward from the horizontal. The compliance chamber contained enough brine to completely fill all zones after packer inflation. This testing configuration served to minimize temperature related effects that were previously found to create uncertainty in the compliance-testing results.

After a test tool was installed and before it was secured, all associated monitoring instruments (pressure transducers, linear variable-differential transformers (LVDTs), thermocouples, and differential-pressure transmitters (DPTs)) were connected to the data acquisition system (DAS). The DAS was then activated and the monitoring instruments were checked to verify that they were functioning properly. The test tool was then positioned in the compliance chamber by monitoring the axial-LVDT output signal until the axial LVDT was depressed approximately $5 \mathrm{~cm}$. If no axial LVDT was associated with a given test tool, the test tool was positioned in the compliance chamber to the desired level by test-tool measurements and tubing talleys. The test tool was then secured to the compliance chamber to prevent test-tool movement out of the compliance chamber. 


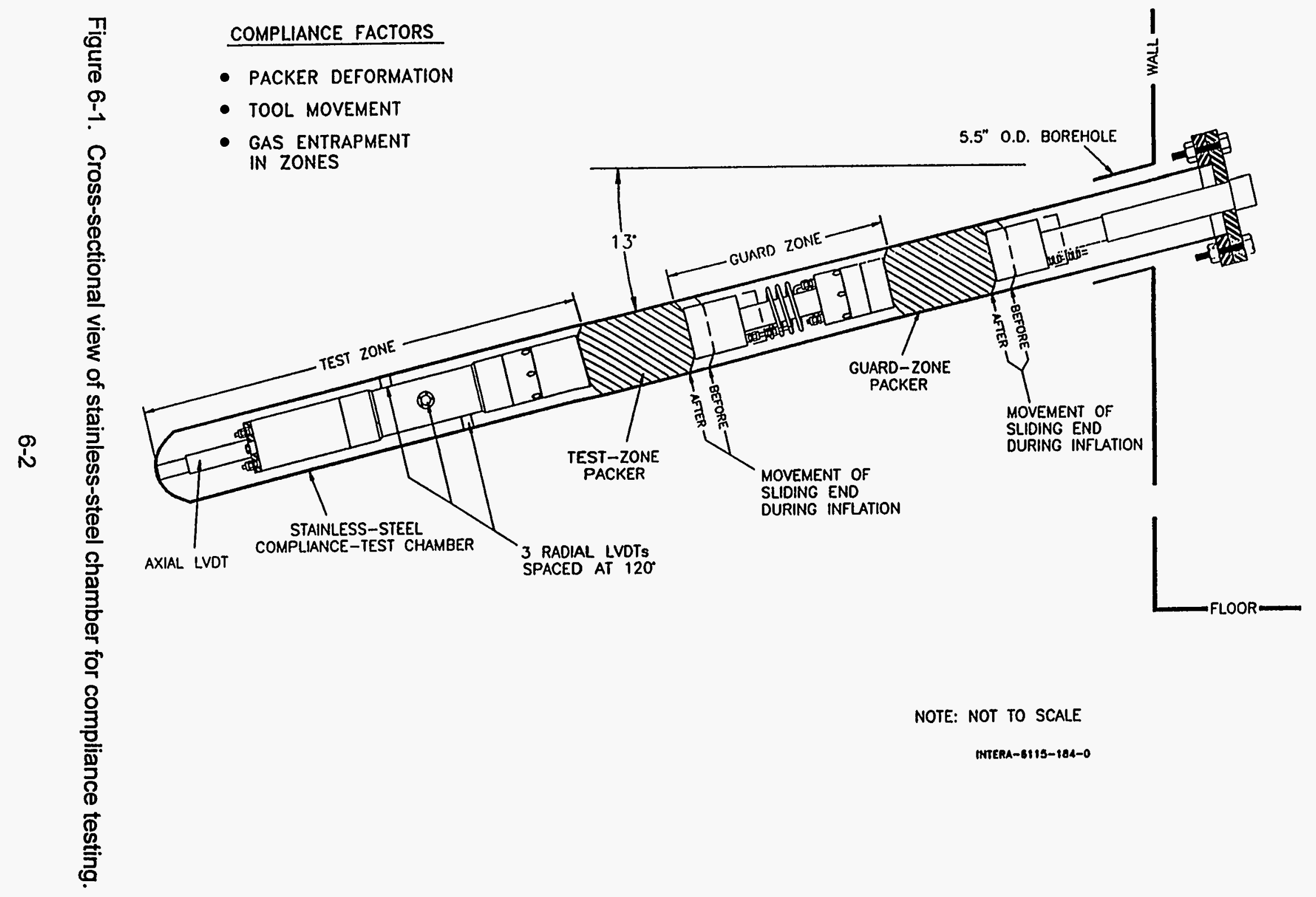


The test-tool's packers were sequentially inflated to pressures ranging from $10 \mathrm{MPa}$ to 20 $\mathrm{MPa}$ with fresh water, uppermost packer first, followed by the next downhole packer, until all packers were inflated. The packer-inflation pressures were monitored for 24 to 48 hours for evidence of leaks or improper performance. Packer-inflation pressures usually decrease during this period due to elasticity of the packer-element material. After monitoring this pressure decay for the 24- to 48-hour period, packer-inflation pressures were usually increased to the approximate original inflation pressure and monitored for an additional 24 to 48 hours.

After satisfactory completion of the leak check/packer-inflation pressure adjustment periods, the packers were usually attached to a pressure-maintenance system prior to applying any pressure to the associated zones. The zones were then subjected to pressure-injection pulses to the maximum pressure that would be expected in the borehole in which the test tool was to be installed (6 MPa to $14 \mathrm{MPa}$ range). The zones' fluid pressure responses were then monitored for evidence of leaks, and the associated packerinflation pressures were also monitored.

In most instances the test zone, and in some cases the second (guard) zone was subjected to compressibility tests. These tests involved increasing the zone pressure from $0 \mathrm{MPa}$ to maximum expected pressure (6 MPa to $14 \mathrm{MPa}$ range) in a continuous manner while monitoring the fluid volume necessary to provide such a pressure increase through the DPT panel. When maximum pressure was reached, the process was repeated in the reverse direction. Zone compressibility is an important factor in permeability testing performed under shut-in conditions because, given the volume of a zone, the zone compressibility governs the pressure change resulting from the flow of a given amount of fluid into or out of the zone. Beauheim et al., (1993) discusses zone compressibility calculations in detail. These series of tests were generally performed with the packers attached to pressure-maintenance systems. The information obtained from such a series of tests provided the test-zone-compressibility versus pressure relationships for the specific test-tool interval.

\subsection{Compliance-Test Data}

Table 6-1 provides information pertaining to compliance testing associated with the various testing programs. Complete data files and abridged tabulations of the data are stored in the SWCF under WPO \#42269.

Table 6-1. Compliance-Testing Information

\begin{tabular}{cccc}
\hline Test Tool \# & Testing Sequence & Borehole & Testing Performed \\
\hline none provided & $\mathrm{C} 1 \times 10$ & $\mathrm{C} 1 \times 10$ & No \\
$32 \mathrm{~A}$ & $\mathrm{C} 1 \times 10$ & $\mathrm{C} 1 \mathrm{H} 05$ & Yes \\
$32 \mathrm{~B}$ & $\mathrm{C} 1 \times 10$ & $\mathrm{C} 1 \mathrm{H} 06$ & Yes \\
none provided & $\mathrm{C} 1 \times 10 / \mathrm{C} 1 \times 05-\mathrm{A} C 2 \mathrm{H} 02$ & $\mathrm{C} 2 \mathrm{H} 01$ & No \\
\hline
\end{tabular}


Table 6-1 (Continued). Compliance-Testing Information

\begin{tabular}{|c|c|c|c|}
\hline Test Tool \# & Testing Sequence & Borehole & Testing Performed \\
\hline none provided & C1X10/C1X05-A & DPD02 & No \\
\hline none provided & C1X10/C1X05-A & DPD03 & No \\
\hline $33 A$ & L4P51-C1 & L4P51 & Yes \\
\hline $33 \mathrm{~B}$ & L4P51-C1 & L4P51 & Yes \\
\hline none provided & $C 1 \times 05-A$ & C1X05 & No \\
\hline 34 & C1X05-A & $\mathrm{C} 1 \mathrm{HO}$ & Yes \\
\hline 35 & S1P74-A & S1P74 & Yes \\
\hline 36 & C1X05-A & C1X06 & Yes \\
\hline 37 & L4P52-B & L4P52 & Yes \\
\hline $38 \mathrm{~A}$ & SCP01-1 & SCP01 & Yes \\
\hline $38 \mathrm{~B}$ & SCP01-1 & SCP01 & No \\
\hline none provided & C1X05-B & $\mathrm{C} 1 \times 05$ & No \\
\hline 39 & C1X05-B & $\mathrm{C} 1 \mathrm{HO} 07$ & Yes \\
\hline 40 & C1X05-B & C1X06 & Yes \\
\hline 41 & L4P51-C2 & L4P51 & Yes \\
\hline BOT-01 & SCP01-2 & SCP01 & Yes \\
\hline BOT-02 & SCP01-2 & SCP01 & No \\
\hline P51-D1A & L4P51-D1 & L4P51 & Yes \\
\hline P51-D1B & L4P51-D1 & L4P51 & No \\
\hline P74-B & S1P74-B & S1P74 & Yes \\
\hline P51-D2 & L4P51-D2 & L4P51 & No \\
\hline
\end{tabular}

Acronyms used in the tables presented in this section are as follows:

$\mathrm{TZ}=$ Test Zone

TZ1 $=$ Test Zone 1

$T Z 2=$ Test Zone 2

$\mathrm{GZ}=$ Guard Zone 


\subsubsection{Test Tool \#32A (Borehole C1H05, Coupled Permeability and Hydrofracture- Testing Sequences $\mathrm{C} 1 \mathrm{X} 10$ and $\mathrm{C} 1 \mathrm{X05}-\mathrm{A})$}

Table 6-2 gives a detailed description of the events that occurred during compliance testing of test tool \#32A. Figures 6-2 through 6-4 illustrate the zone pressure, packer pressure, and zone temperature, respectively, for test tool \#32A. Figure 4-9 illustrates the configuration of test tool \#32A as assembled for compliance testing.

Table 6-2. Events Associated with Compliance Testing of Test Tool \#32A; Borehole C1H05; Sequence C1X10

\begin{tabular}{|c|c|c|c|c|}
\hline EVENT & DATE & $\begin{array}{l}\text { CALENDAR } \\
\text { DAY }\end{array}$ & $\begin{array}{c}1992 \\
\text { CALENDAR } \\
\text { DAY }\end{array}$ & $\begin{array}{l}\text { TIME } \\
\text { (HH:MM:SS) }\end{array}$ \\
\hline $\begin{array}{l}\text { Assemble single-packer test } 1001 \text { \#32A to be used in borehole } \mathrm{C} 1 \mathrm{H} 05 \text { during testing } \\
\text { sequence } \mathrm{C} 1 \times 10 .\end{array}$ & $1-10-92$ & 10 & 10 & 13:43:00 \\
\hline FIII $T Z$ with fresh water. & $1-10-92$ & 10 & 10 & 14:15:00 \\
\hline Inflate packer to $-10.3 \mathrm{MPa}$. & $1-10-92$ & 10 & 10 & $14: 30: 00$ \\
\hline Begin data file COMP32. & 1-10-92 & 10 & 10 & 14:32:36 \\
\hline Increase $T Z$ pressure $-6.9 \mathrm{MPa}$. & $1-10-2$ & 10 & 10 & 14:35:00 \\
\hline DAS not functioning upon arrival. & $1-13-92$ & 13 & 13 & 08:55:00 \\
\hline End data file COMP32. & 1-13-92 & 13 & 13 & 11:15:20 \\
\hline Begin data file COMP32A. & $1-13-92$ & 13 & 13 & 11:18:20 \\
\hline End data file COMP32A. & $1-15-92$ & 15 & 15 & 09:23:01 \\
\hline Remove test tool from compliance chamber and move to borehole $\mathrm{C} 1 \mathrm{H} 05$. & 1-15-92 & 15 & 15 & $09: 25: 00$ \\
\hline
\end{tabular}

Table 6-3 indicates the equipment that was used and the duration that each instrument was used during compliance testing of test tool \#32A. Test tool \#32A was used in observation borehole $\mathrm{C} 1 \mathrm{H} 05$ during testing sequences $\mathrm{C} 1 \mathrm{X} 10$ and $\mathrm{C} 1 \mathrm{X} 05-\mathrm{A}$.

Table 6-3. Compliance Equipment Associated with Test Tool \#32A; Borehole C1H05; Sequences $\mathrm{C} 1 \mathrm{X} 10$ and $\mathrm{C} 1 \mathrm{X} 05-\mathrm{A}$

\begin{tabular}{ccccc}
\hline Equipment & Location & Serial \# & Installed & Removed \\
\hline DAS Software & N/A & PERM4F & 1-10-92 & $1-15-92$ \\
\hline DCU (HP3497A) & N/A & $2514 a 17149$ & $1-10-92$ & $1-15-92$ \\
\hline $\begin{array}{c}\text { Transducer } \\
\text { (Druck PDCR 830) } \\
\begin{array}{c}\text { Transducer } \\
\text { (Druck PDCR 830) }\end{array}\end{array}$ & $\begin{array}{c}\text { Test Zone } \\
\text { Packer }\end{array}$ & 246917 & $1-10-92$ & $1-15-92$ \\
\hline Thermocouple (Type E) & Test Zone & 246919 & $1-10-92$ & $1-15-92$ \\
\hline
\end{tabular}




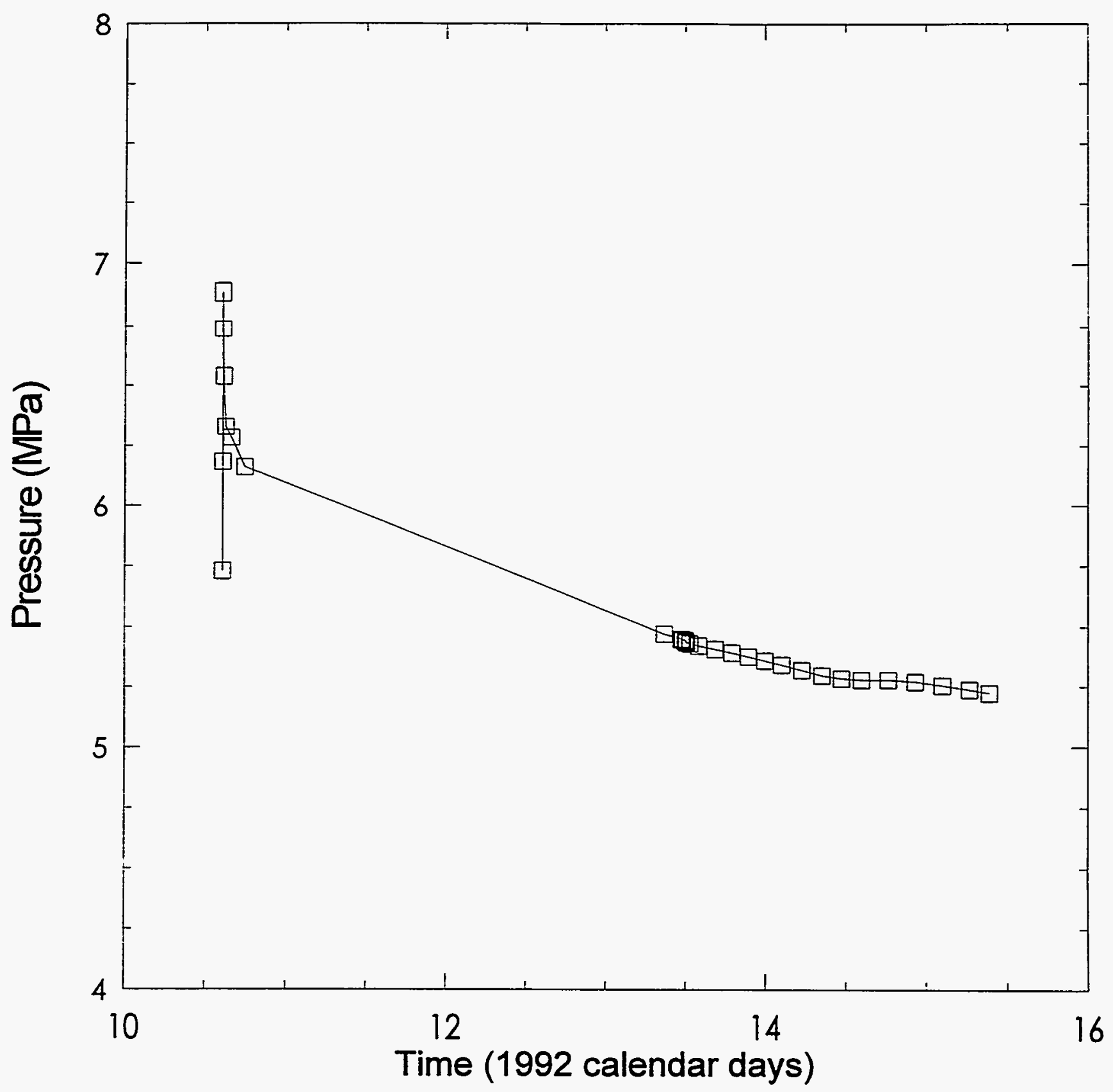

INTERA- 118-185-0

Figure 6-2. Zone pressure during compliance testing of test tool \#32A. 


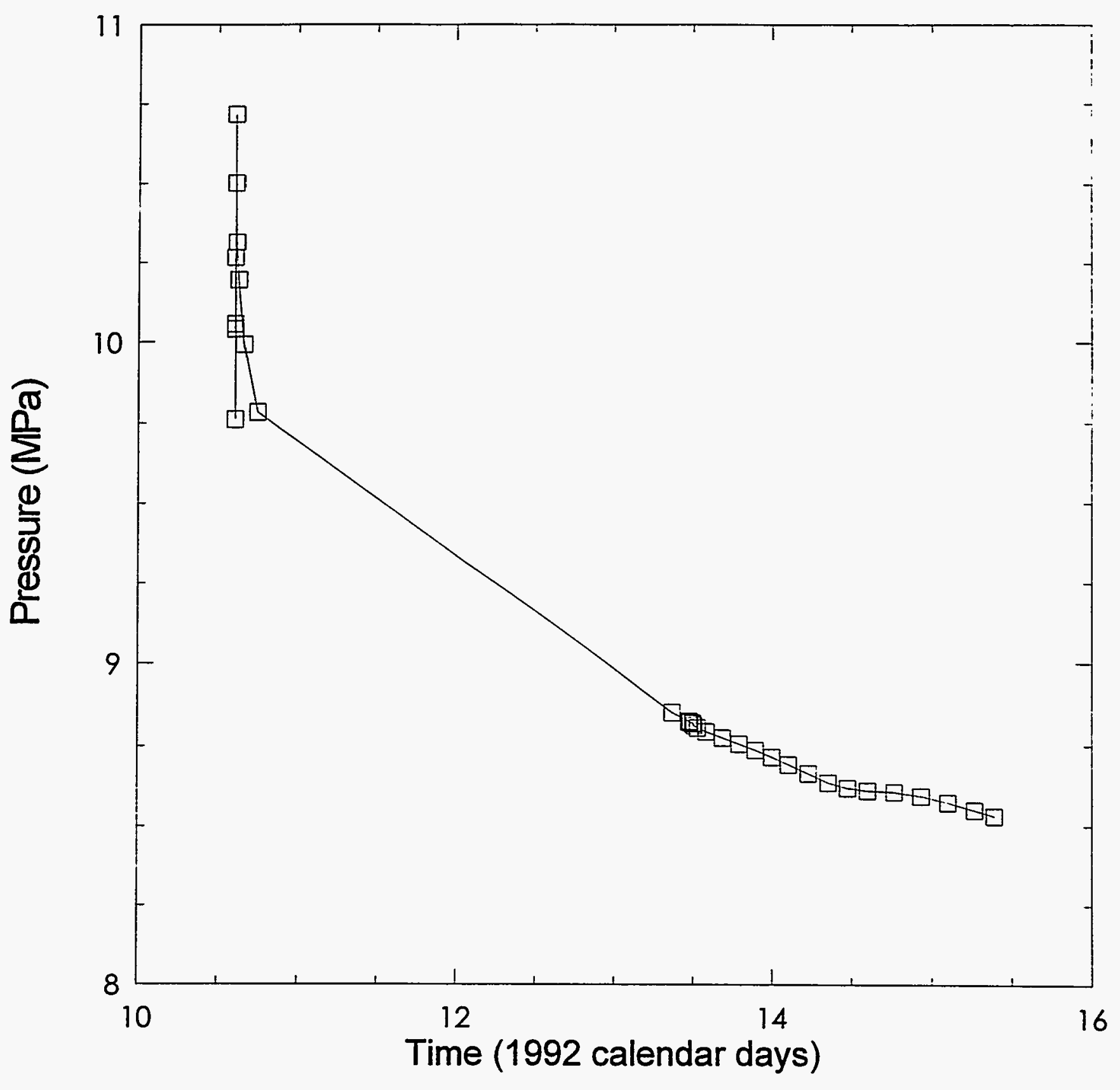

INTERA-6115-186-0

Figure 6-3. Packer pressure during compliance testing of test tool \#32A. 


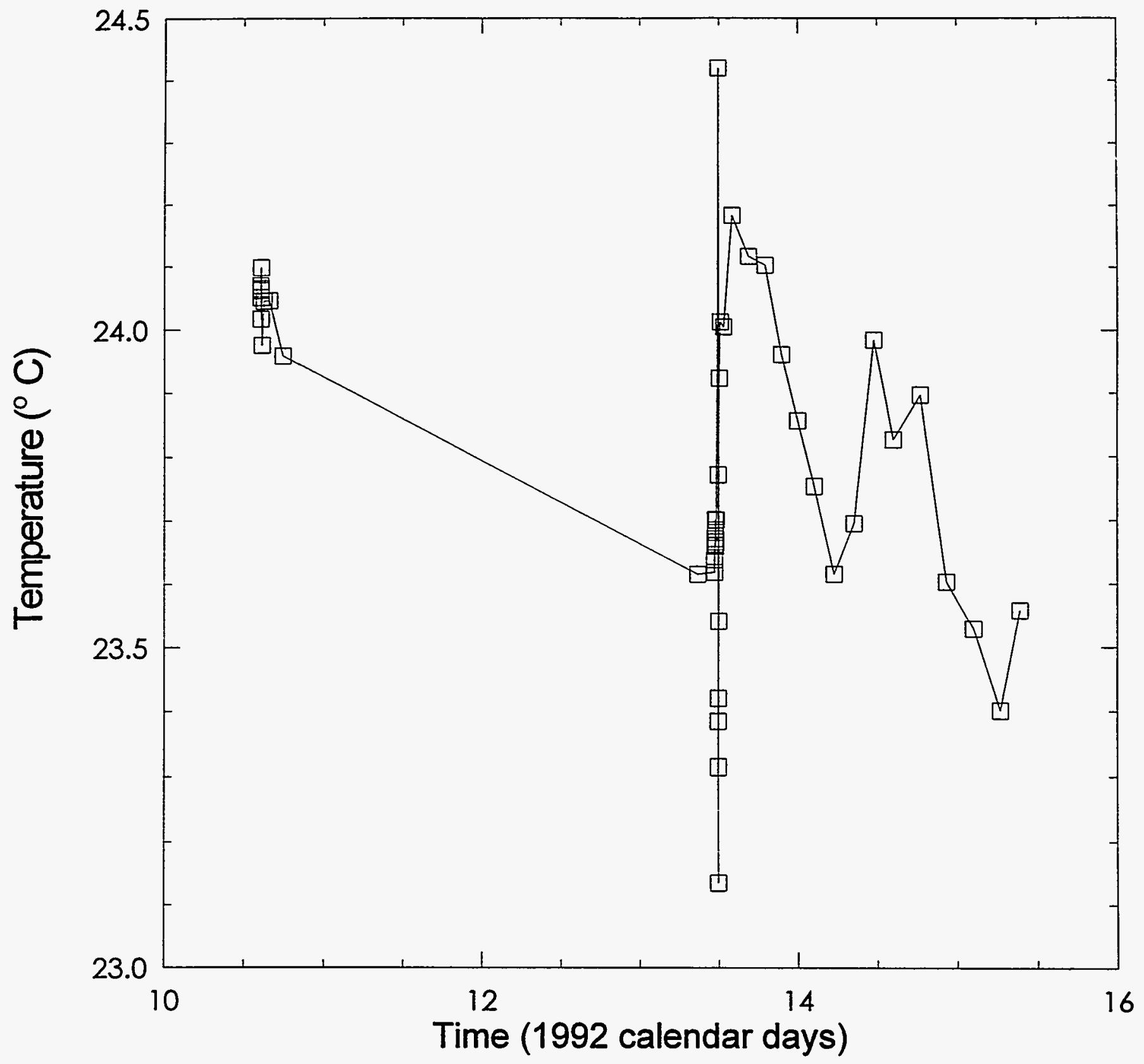

INTERA-6118-187-0

Figure 6-4. Zone temperature during compliance testing of test tool \#32A. 


\subsubsection{Test Tool \#32B (Borehole C1H06, Coupled Permeability and Hydrofracture- Testing Sequences C1X10 and C1X05-A)}

Table 6-4 gives a detailed description of the events that occurred during compliance testing of test tool \#32B. Figures 6-5 and 6-6 illustrate the zone and packer pressures, respectively, for test tool \#32B. Figure 4-10 illustrates the configuration of test tool \#32B as assembled for compliance testing.

Table 6-4. Events Associated with Compliance Testing of Test Tool \#32B; Borehole C1H06; Sequences C1X10

\begin{tabular}{|c|c|c|c|c|}
\hline EVENT & DATE & $\begin{array}{c}\text { CALENDAR } \\
\text { DAY }\end{array}$ & $\begin{array}{c}1992 \\
\text { CALENDAR } \\
\text { DAY }\end{array}$ & $\begin{array}{c}\text { TIME } \\
\text { (HH:MM:SS) }\end{array}$ \\
\hline $\begin{array}{l}\text { Assemble single-packer test tool \#32B to be used in borehole } \mathrm{C} 1 \mathrm{H} 0 \mathrm{C} \text { during testing } \\
\text { sequence } \mathrm{C} 1 \mathrm{X} 10 .\end{array}$ & $1-13-92$ & 13 & 13 & 11:18:00 \\
\hline Begin data file COMP32A. & 1-13-92 & 13 & 13 & 11:18:20 \\
\hline Inflate packer. & $1-13-92$ & 13 & 13 & $11: 22: 00$ \\
\hline Increase TZ pressure. & 1-\$3-92 & 13 & 13 & 11:27:00 \\
\hline Depressurize $T Z$ and deflate packer. & $1-13-92$ & 13 & 13 & 11:38:00 \\
\hline Inflate packer. & i-13-92 & 13 & 13 & 11:53:00 \\
\hline Increase TZ pressure. & $1-13-92$ & 13 & 13 & 11:58:00 \\
\hline End data file COMP32A. & $1-15-92$ & 15 & 15 & 09:23:01 \\
\hline Remove test tool from compliance chamber and move to borehole C1H06. & $1-15-92$ & 15 & 15 & $09: 25: 00$ \\
\hline
\end{tabular}

Table 6-5 indicates the equipment that was used and the duration that each instrument was used during compliance testing of test tool \#32B. Test tool \#32B was used in observation borehole $\mathrm{C} 1 \mathrm{H} 06$ during testing sequences $\mathrm{C} 1 \mathrm{X} 10$ and $\mathrm{C} 1 \mathrm{X} 05-\mathrm{A}$.

Table 6-5. Compliance Equipment Associated with Test Tool \#32B; Borehole C1H06; Sequences $\mathrm{C} 1 \mathrm{X} 10$ and $\mathrm{C} 1 \mathrm{X05-A}$

\begin{tabular}{ccccc}
\hline Equipment & Location & Serial \# & Installed & Removed \\
\hline DAS Software & N/A & PERM4F & $1-13-92$ & $1-15-92$ \\
\hline DCU (HP3497A) & N/A & 2514a17149 & $1-13-92$ & $1-15-92$ \\
\hline $\begin{array}{c}\text { Transducer } \\
\text { (Druck PDCR 830) } \\
\begin{array}{c}\text { Transducer } \\
\text { (Druck PDCR 830) }\end{array}\end{array}$ & $\begin{array}{c}\text { Test Zone } \\
\text { Pest Zone }\end{array}$ & 246916 & $1-13-92$ & $1-15-92$ \\
\hline
\end{tabular}




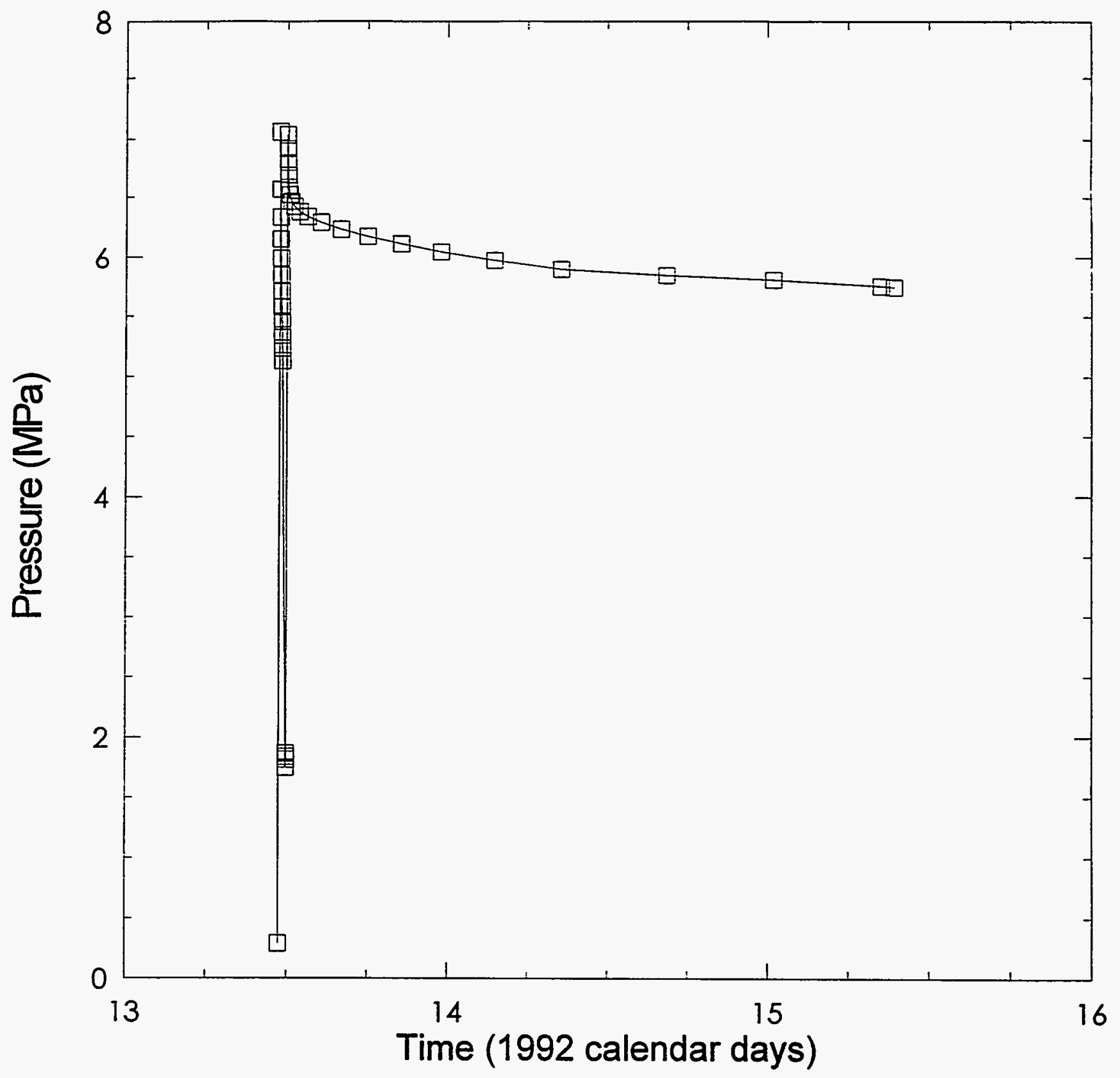

INTERA-6116-188-0

Figure 6-5. Zone pressure during compliance testing of test tool \#32B. 


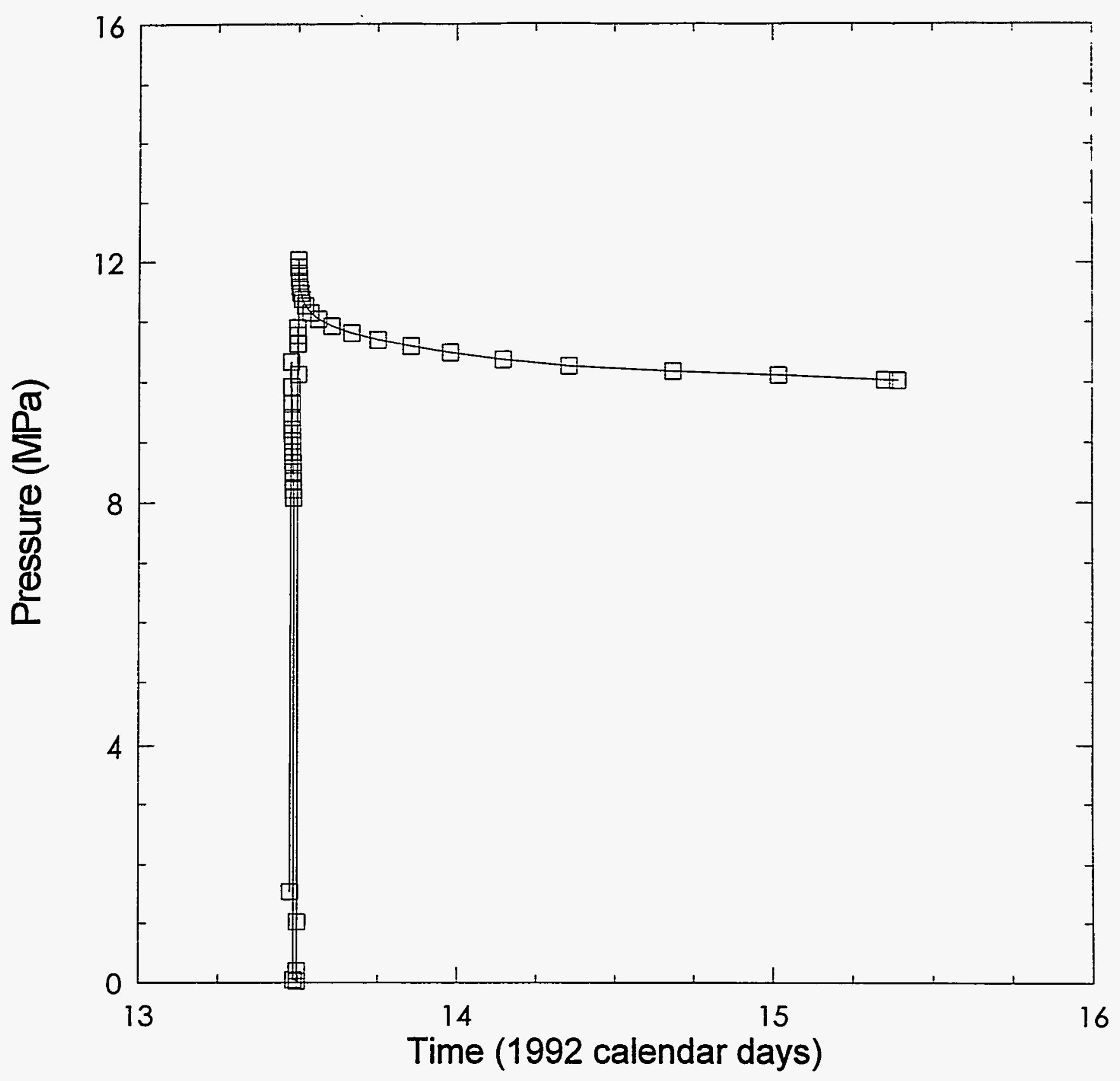

INTERABI15-189-0

Figure 6-6. Packer pressure during compliance testing of test tool \#32B. 


\subsubsection{Test Tool \#33A (Permeability-Testing Sequence L4P51-C1)}

Table 6-6 gives a detailed description of the events that occurred during compliance testing of test tool \#33A. Figures 6-7 through 6-13 illustrate the zone pressures, packer pressures, zone temperatures, axial-LVDT displacement, radial-LVDT displacement, fluid-injection volumes during compressibility tests, and test-zone compressibility as a function of pressure, respectively, for multipacker test tool \#33A. It should be noted that Figures 6-12 and 6-13 each consist of two parts (ex. Figures 6-12a and 6-12b). Figure 3-22 illustrates the configuration of test tool \#33A as assembled for compliance testing.

Table 6-6. Events Associated with Compliance Testing of Test Tool \#33A; Borehole L4P51; Sequence L4P51-C1

\begin{tabular}{|c|c|c|c|c|}
\hline EVENT & DATE & $\begin{array}{l}\text { CALENDAR } \\
\text { DAY }\end{array}$ & $\begin{array}{c}1992 \\
\text { CALENDAR } \\
\text { DAY }\end{array}$ & $\begin{array}{c}\text { TIME } \\
\text { (HH:MM:SS) }\end{array}$ \\
\hline $\begin{array}{l}\text { Assemble multipacker test tool I33A to be used in borehole L4P5i during testing } \\
\text { sequence L4P51-C1. }\end{array}$ & $2-24-92$ & 55 & 55 & 12:00:00 \\
\hline Begin data file COMP33A. & $2-25-92$ & 56 & 56 & 13:46:11 \\
\hline Inflate GZ packer. & $2-25-92$ & 56 & 56 & 14:12:00 \\
\hline Inllate TZ packer. & $2-25-92$ & 56 & 56 & 14:15:00 \\
\hline Open GZ packer to accumulator. & $2-26-92$ & 57 & 57 & 10:24:30 \\
\hline Open TZ packer to accumulator. & $2-26-92$ & 57 & 57 & 10:25:15 \\
\hline End data file COMP33A. & $2-26-92$ & 57 & 57 & 11:51:18 \\
\hline Begin data file COMP33B. & $2-26-92$ & 57 & 57 & 11:59:44 \\
\hline Increase GZ pressure via DPT panel. & $2-26-92$ & 57 & 57 & 13:17:00 \\
\hline Depressurize GZ. & $2-26-92$ & 57 & 57 & 13:24:00 \\
\hline Increase GZ pressure via DPT panel. & $2-26-92$ & 57 & 57 & 13:27:00 \\
\hline Shut in GZ. & $2-26-92$ & 57 & 57 & 13:35:45 \\
\hline Increase TZ pressure via DPT panel. & $2-26-92$ & 57 & 57 & 13:46:13 \\
\hline Shut in TZ. & $2-26-92$ & 57 & 57 & 14:10:44 \\
\hline Depressurze GZ via DPT panel. & $3-3-92$ & 63 & 63 & 10:37:00 \\
\hline Increase GZ pressure via DPT panel. & $3-3-92$ & 63 & 63 & 10:44:52 \\
\hline Shut in $\mathrm{GZ}$. & 3-3-92 & 63 & 63 & 10:51:08 \\
\hline Shut in packers from accumulators. & 3-3-92 & 63 & 63 & 10:52:53 \\
\hline Increase TZ pressure via DPT panel. & 3-3-92 & 63 & 63 & 11:00:00 \\
\hline Decrease TZ packer pressure via DPT panel. & 3-3-92 & 63 & 63 & 11:52:39 \\
\hline Shut in TZ trom DPT panel. & 3-9.92 & 69 & 69 & 09:35:30 \\
\hline End data filo COMP33B. & $3-24-92$ & 84 & 84 & 08:43:52 \\
\hline Begin data file COMP33C. & $3-24-92$ & 84 & 84 & 09:51:25 \\
\hline Begin TZ compressiblitity test. & $3-24-92$ & 84 & 84 & 10:02:00 \\
\hline Shut in $T z$ & $3-24-92$ & 84 & 84 & 12:14:05 \\
\hline Open packers to accumulator. & $3-24-92$ & 84 & 84 & 12:31:06 \\
\hline Removed $-10 \mathrm{~mL}$ of fluld from $T Z$ & $3-26-92$ & 86 & 86 & 09:11:00 \\
\hline
\end{tabular}


Table 6-6 (Continued). Events Associated with Compliance Testing of Test Tool \#33A; Borehole L4P51; Sequence L4P51-C1

\begin{tabular}{|c|c|c|c|c|}
\hline EVENT & DATE & $\begin{array}{c}\text { CALENDAR } \\
\text { DAY }\end{array}$ & $\begin{array}{c}1992 \\
\text { CALENDAR } \\
\text { DAY }\end{array}$ & $\begin{array}{c}\text { TIME } \\
\text { (HH:MM:SS) }\end{array}$ \\
\hline Increase $T Z$ pressure via DPT panel. & $3-31.92$ & 91 & 91 & 14:02:52 \\
\hline Depressurize GZ via DPT panel. & $4-8-92$ & 99 & 99 & $08: 42: 35$ \\
\hline Increase GZ pressure via DPT panel. & 4-8-92 & 99 & 99 & $09: 18: 10$ \\
\hline Shut in GZ. & 4-8-92 & 99 & 99 & $10: 45: 36$ \\
\hline Depressurize $T Z$ via DPT panel. & $4-22-92$ & 113 & 113 & $09: 51: 33$ \\
\hline Depressurize $G Z$ via DPT panel. & 4-22-92 & 113 & 113 & 10:15:33 \\
\hline Deflate GZ packer. & 4-22-92 & 113 & 113 & 10:38:03 \\
\hline Deflate TZ packer. & $4-22-92$ & 113 & 113 & $10: 40: 33$ \\
\hline End data file COMP33C. & $4-22-92$ & 113 & 113 & $11: 13: 49$ \\
\hline Remove test tool \#33A from compliance chamber and move to borehole L4PS1. & 4-22-92 & 113 & 113 & 12:00:00 \\
\hline
\end{tabular}

Table 6-7 indicates the equipment that was used and the duration that each instrument was used during compliance testing of test-tool \#33A. Test tool \#33A was used in permeability-testing sequence L4P51-C1.

Table 6-7. Compliance Equipment Associated with Test Tool \#33A; Borehole L4P51; Sequence L4P51-C1

\begin{tabular}{|c|c|c|c|c|}
\hline Equipment & Location & Serial \# & Installed & Removed \\
\hline DAS Software & N/A & PERM4F & $2-25-92$ & $4-22-92$ \\
\hline DCU (HP3497A) & N/A & $2514 a 17149$ & $2-25-92$ & $4-22-92$ \\
\hline $\begin{array}{c}\text { Transducer } \\
\text { (Druck PDCR 10/D) }\end{array}$ & Test Zone & 211690 & $2-25-92$ & $4-22-92$ \\
\hline $\begin{array}{c}\text { Transducer } \\
\text { (Druck PDCR 10/D) }\end{array}$ & $\begin{array}{l}\text { Test Zone } \\
\text { Packer }\end{array}$ & 211695 & $2-25-92$ & $4-22-92$ \\
\hline $\begin{array}{c}\text { Transducer } \\
\text { (Druck PDCR 830) }\end{array}$ & Guard Zone & 246914 & $2-25-92$ & $4-22-92$ \\
\hline $\begin{array}{c}\text { Transducer } \\
\text { (Druck PDCR 830) }\end{array}$ & $\begin{array}{l}\text { Guard Zone } \\
\text { Packer }\end{array}$ & 246920 & $2-25-92$ & $4-22-92$ \\
\hline $\begin{array}{c}\text { Transducer } \\
\text { (Druck PDCR 830) } \\
\end{array}$ & DPT Panel & 246912 & $2-25-92$ & $4-22-92$ \\
\hline LVDT (Trans-Tek 241) & N/A & R04 & $2-25-92$ & $4-22-92$ \\
\hline LVDT (Trans-Tek 241) & N/A & R16 & $2-25-92$ & $4-22-92$ \\
\hline LVDT (Trans-Tek 241) & N/A & R17 & $2-25-92$ & $4-22-92$ \\
\hline LVDT (Trans-Tek 245) & N/A & $\mathrm{A} 02$ & $2-25-92$ & $4-22-92$ \\
\hline
\end{tabular}


Table 6-7 (Continued). Compliance Equipment Associated with Test Tool \#33A; Borehole L4P51; Sequence L4P51-C1

\begin{tabular}{ccccc}
\hline Equipment & Location & Serial \# & Installed & Removed \\
\hline Thermocouple (Type E) & Test Zone & 1 & $2-25-92$ & $4-22-92$ \\
Thermocouple (Type E) & Guard Zone & 2 & $2-25-92$ & $4-22-92$ \\
\hline Injection Column & N/A & 38 & $2-25-92$ & $4-22-92$ \\
Injection Column & N/A & 39 & $2-25-92$ & $4-22-92$ \\
\hline DPT & N/A & 1389938 & $2-25-92$ & $4-22-92$ \\
\hline
\end{tabular}




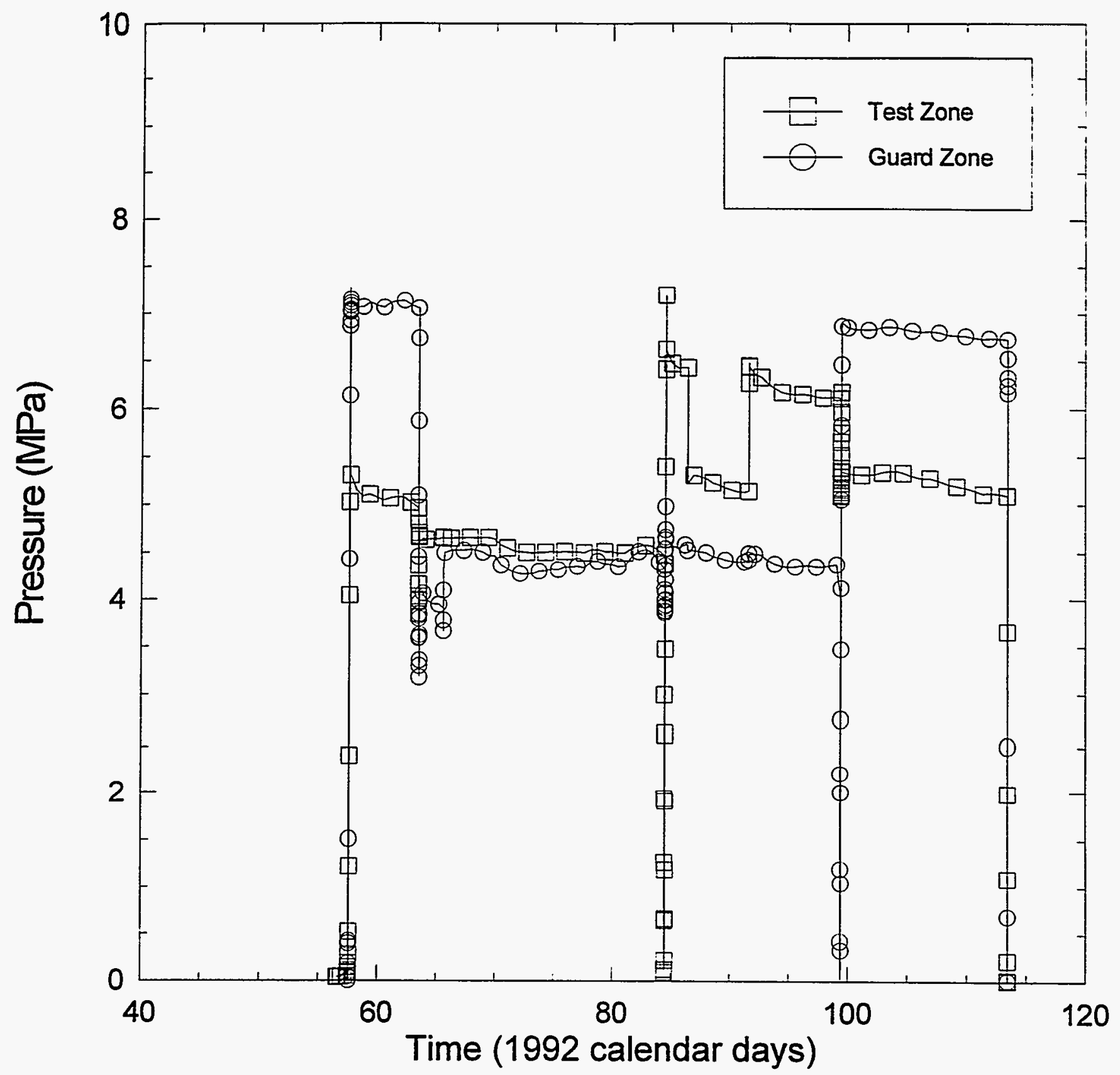

INTERA-6115-180-0

Figure 6-7. Zone pressures during compliance testing of test tool \#33A. 


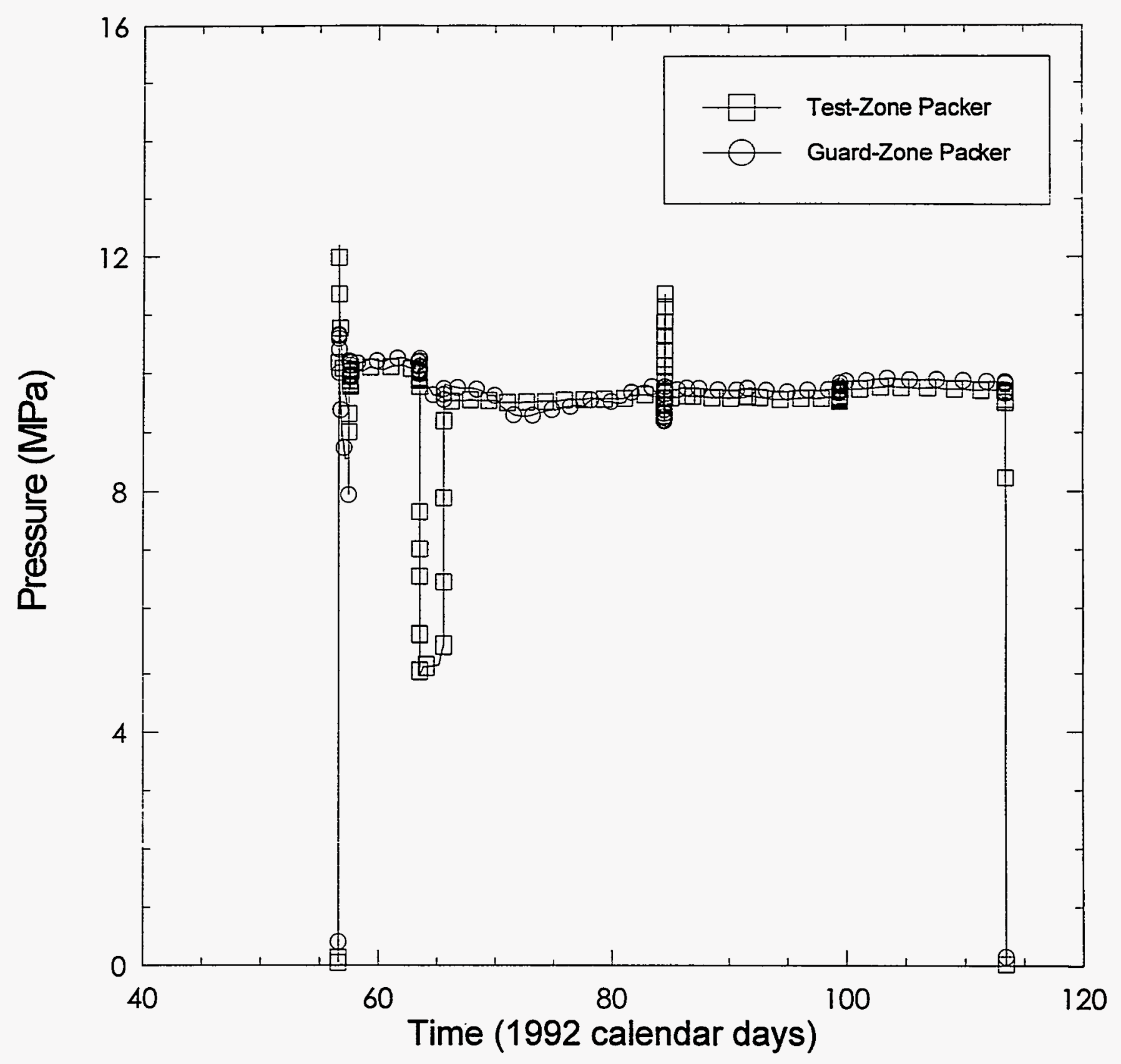

INTERA6T15-191-0

Figure 6-8. Packer pressures during compliance testing of test tool \#33A. 


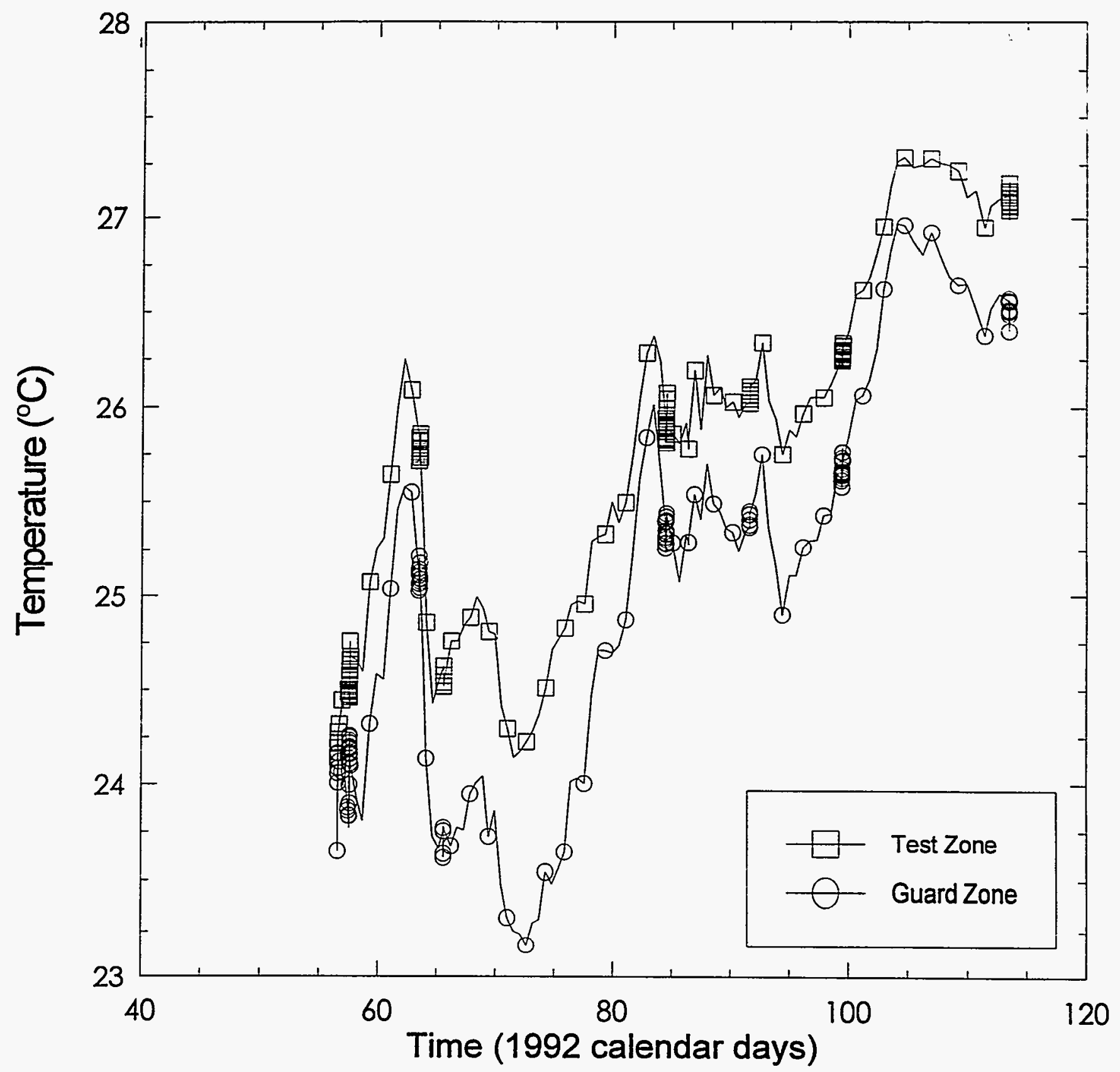

INTERABI15-182-0

Figure 6-9. Zone temperatures during compliance testing of test tool \#33A. 


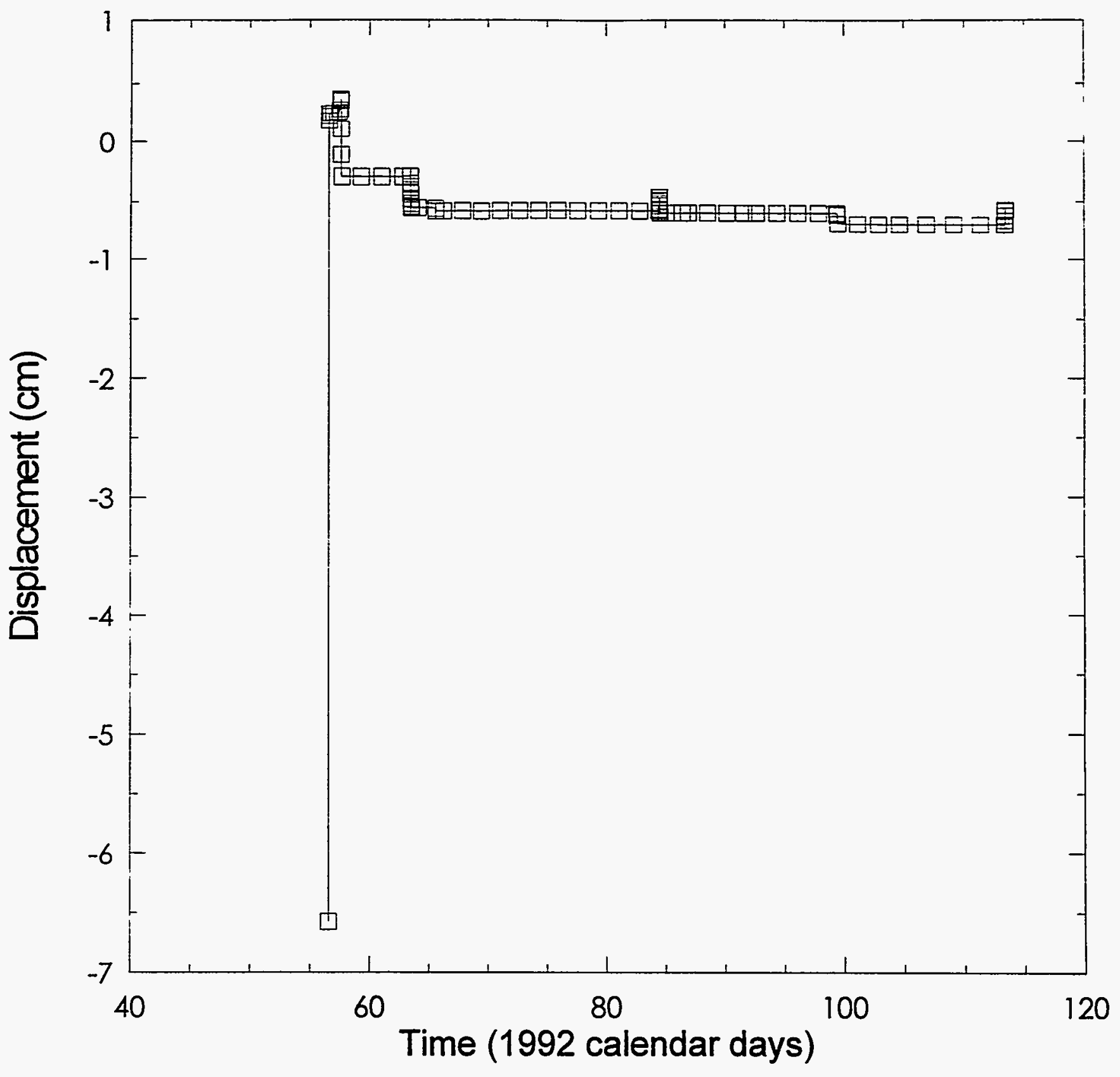

INTERA6115-193-0

Figure 6-10. Axial-LVDT displacement during compliance testing of test tool \#33A. 


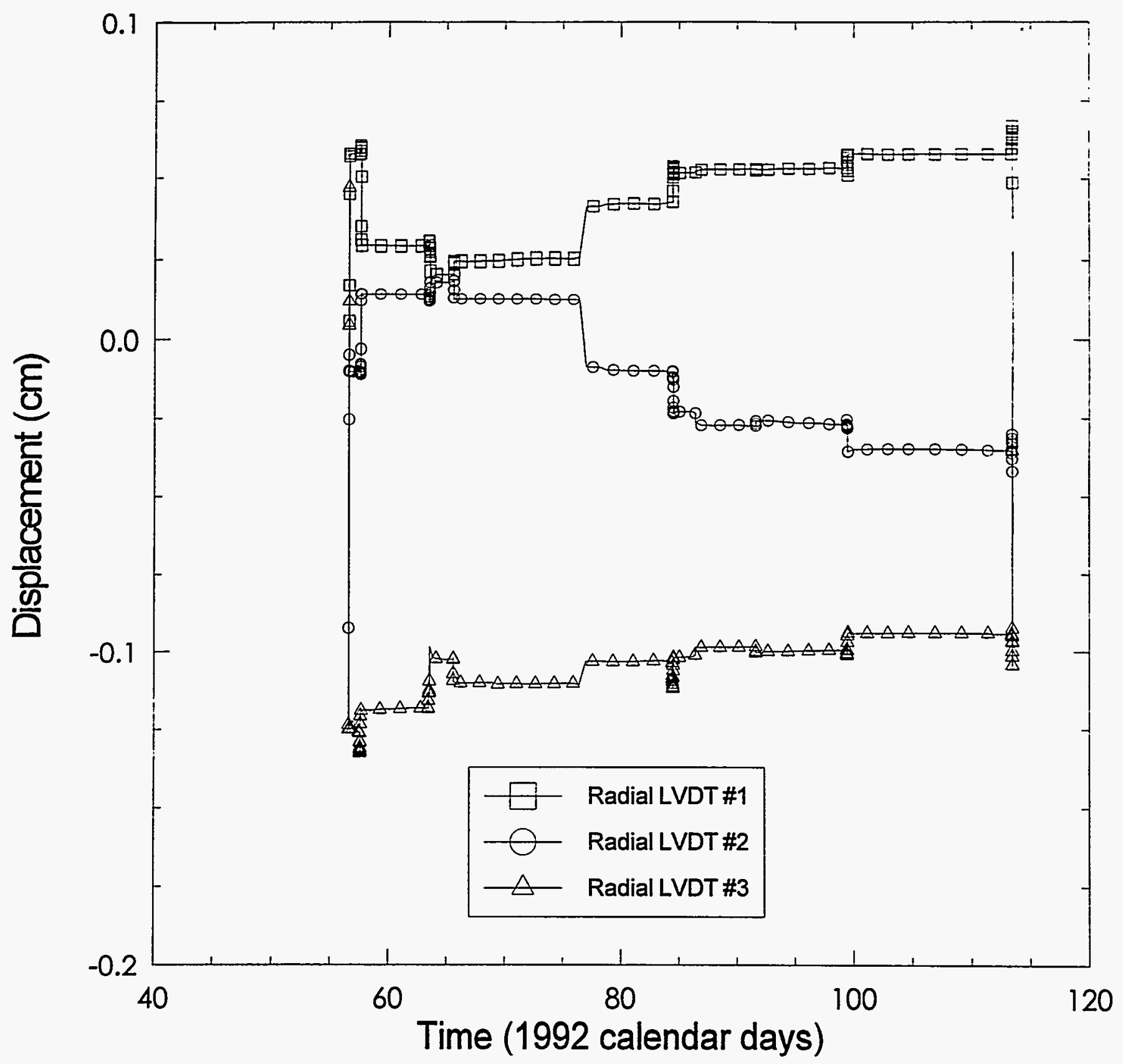

INTERA-61 15-194-O

Figure 6-11. Radial-LVDT displacement during compliance testing of test tool \#33A. 


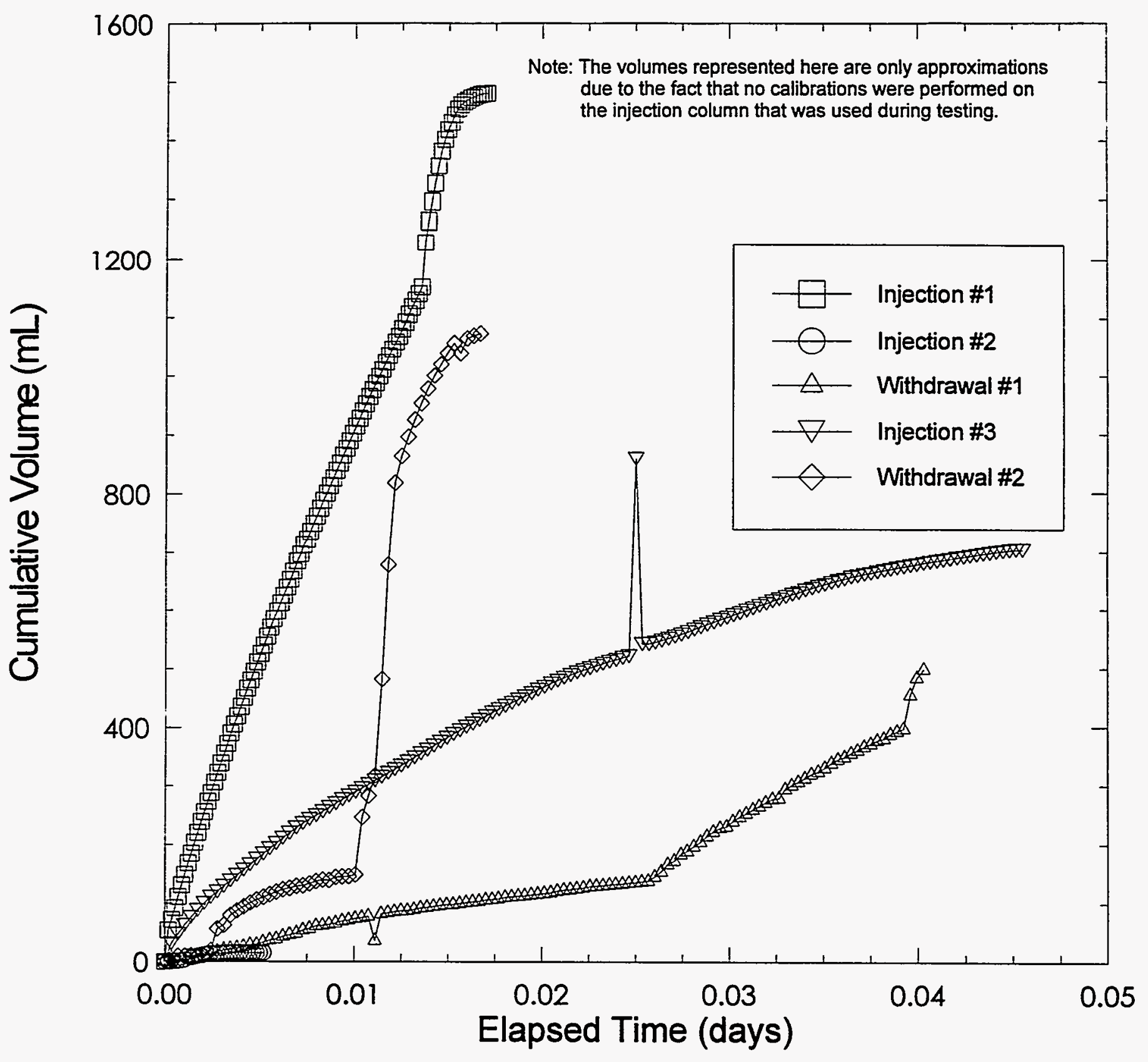

INTERA-115-105-0

Figure 6-12a. Test-zone fluid-injection volumes during compliance testing of test tool \#33A. 


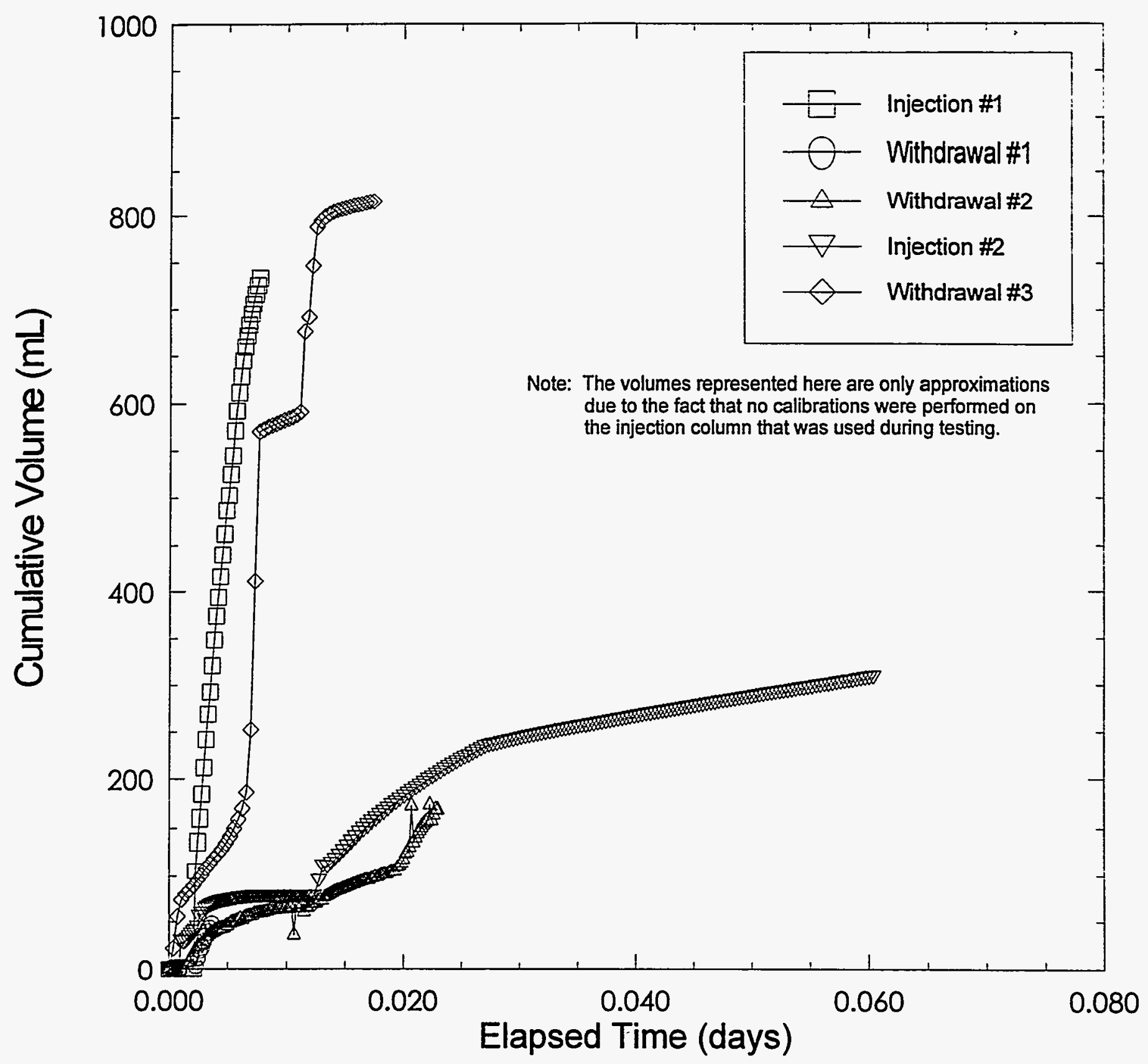

INTERA-115-108-0

Figure 6-12b. Guard-zone fluid-injection volumes during compliance testing of test tool \#33A. 


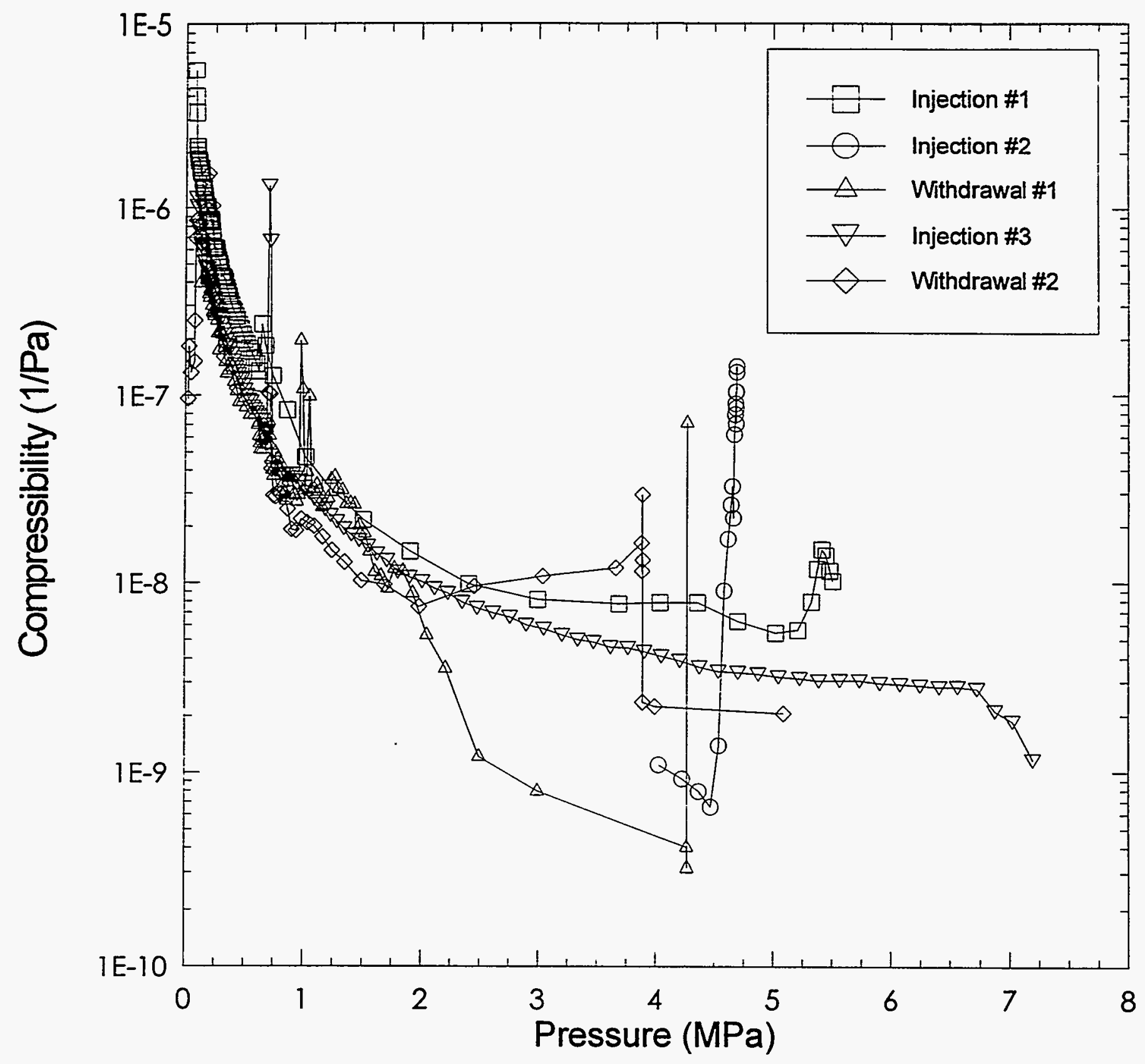

INTERA-6115-197-0

Figure 6-13a. Test-zone compressibility as measured during compliance testing of test tool \#33A. 


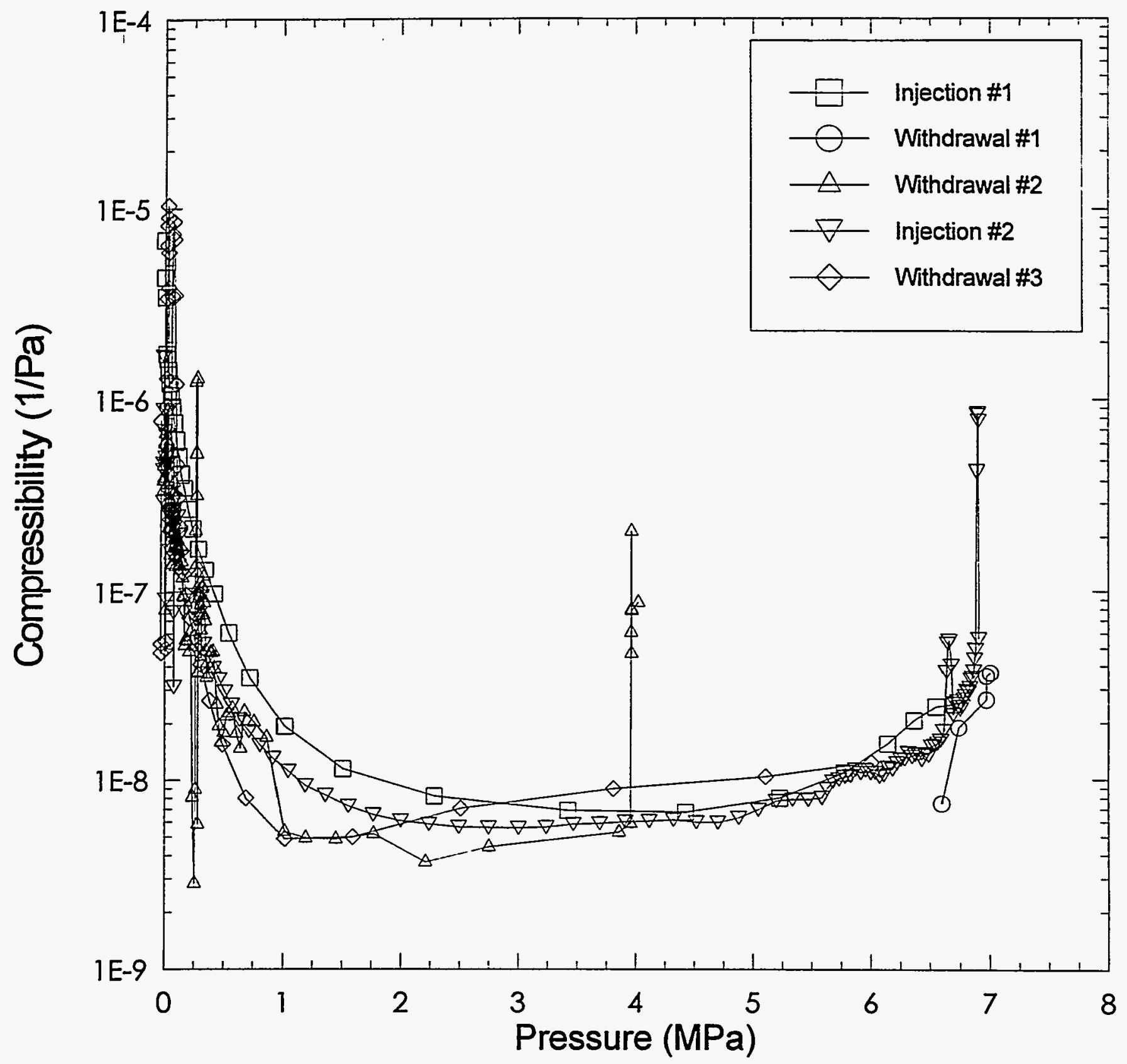

INTERA-6115-188-0

Figure 6-13b. Guard-zone compressibility as measured during compliance testing of test tool \#33A. 


\subsubsection{Test Tool \#34 (Borehole C1H07, Coupled Permeability and Hydrofracture- Testing Sequence C1X05-A)}

Table 6-8 gives a detailed description of the events that occurred during compliance testing of test tool \#34. Figures 6-14 and 6-15 illustrate the zone and packer pressures, respectively, for test tool \#34. Figure 4-12 illustrates the configuration of test tool \#34 as assembled for compliance testing.

Table 6-8. Events Associated with Compliance Testing of Test Tool \#34; Borehole C1H07; Sequence C1X05-A

\begin{tabular}{|c|c|c|c|c|}
\hline EVENT & DATE & $\begin{array}{c}\text { CALENDAR } \\
\text { DAY }\end{array}$ & $\begin{array}{c}1992 \\
\text { CALENDAR } \\
\text { DAY }\end{array}$ & $\begin{array}{c}\text { TIME } \\
\text { (HH:MM:SS) }\end{array}$ \\
\hline $\begin{array}{l}\text { Assemble single-packer test tool \#34 to be used in borehole C1H07 during testing } \\
\text { sequence C1X05-A. }\end{array}$ & $6-2 \cdot 92$ & 154 & 154 & 09:30:00 \\
\hline Begin data file COMP34A. & $6-2-92$ & 154 & 154 & 09:58:03 \\
\hline Inflate packer to -10 MPa. & $6-2-92$ & 154 & 154 & $09: 59: 13$ \\
\hline Shut in packer. & $6-2-92$ & 154 & 154 & $10: 00: 43$ \\
\hline Increase $T Z$ pressure to $-6 \mathrm{MPa}$. & $6-2-92$ & 154 & 154 & 10:06:13 \\
\hline Shut in TZ. & $6-2-92$ & 154 & 154 & $10: 06: 43$ \\
\hline Increase $T Z$ pressure to $-4.1 \mathrm{MPa}$ & $6-4-92$ & 156 & 156 & 10:23:00 \\
\hline Shut in TZ. & $6-4-92$ & 156 & 156 & 10:24:00 \\
\hline End data file COMP34A. & $6-17-92$ & 169 & 169 & $09: 43: 42$ \\
\hline Remove test tool $\$ 34$ from compliance chamber and move to borehole C1HO7. & $6-17-92$ & 169 & 169 & 12:00:00 \\
\hline
\end{tabular}

Table 6-9 indicates the equipment that was used and the duration that each instrument was used during compliance testing of test tool \#34. Test tool \#34 was used in observation borehole $\mathrm{C} 1 \mathrm{HO} 7$ during testing sequence $\mathrm{C} 1 \mathrm{X} 05-\mathrm{A}$.

Table 6-9. Compliance Equipment Associated with Test Tool \#34; Borehole C1H07; Sequence C1X05-A

\begin{tabular}{ccccc}
\hline Equipment & Location & Serial \# & Installed & Removed \\
\hline DAS Software & N/A & PERM4F & $6-2-92$ & $6-17-92$ \\
\hline DCU (HP3497A) & N/A & 2629a22040 & $6-2-92$ & $6-17-92$ \\
\hline $\begin{array}{c}\text { Transducer } \\
\text { (Druck PDCR 910) }\end{array}$ & Test Zone & 322424 & $6-2-92$ & $6-17-92$ \\
$\begin{array}{c}\text { Transducer } \\
\text { (Druck PDCR 910) }\end{array}$ & $\begin{array}{c}\text { Test Zone } \\
\text { Packer }\end{array}$ & 322426 & $6-2-92$ & $6-17-92$ \\
\hline
\end{tabular}




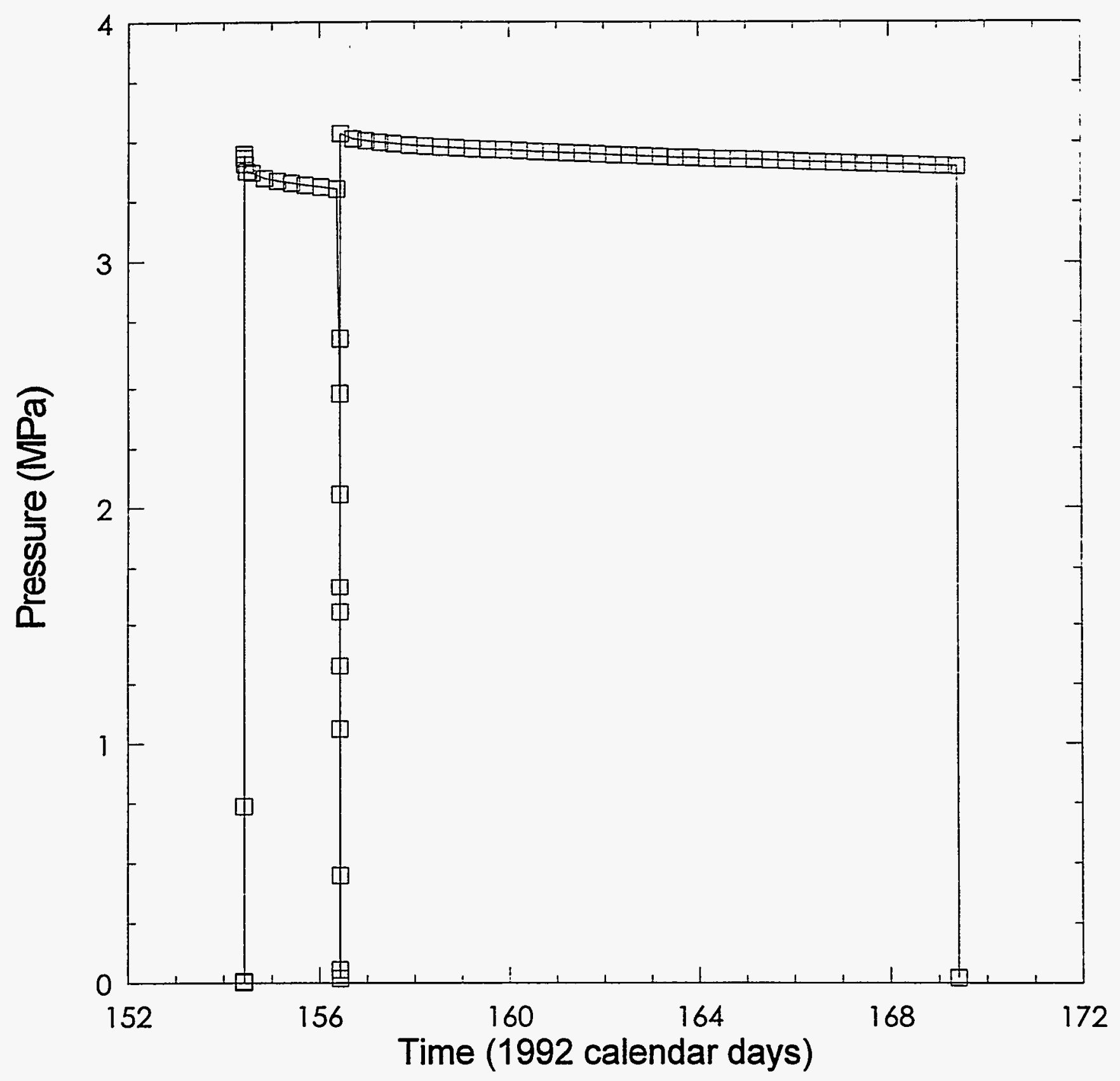

INTERA-6115-1990

Figure 6-14. Zone pressure during compliance testing of test tool \#34. 


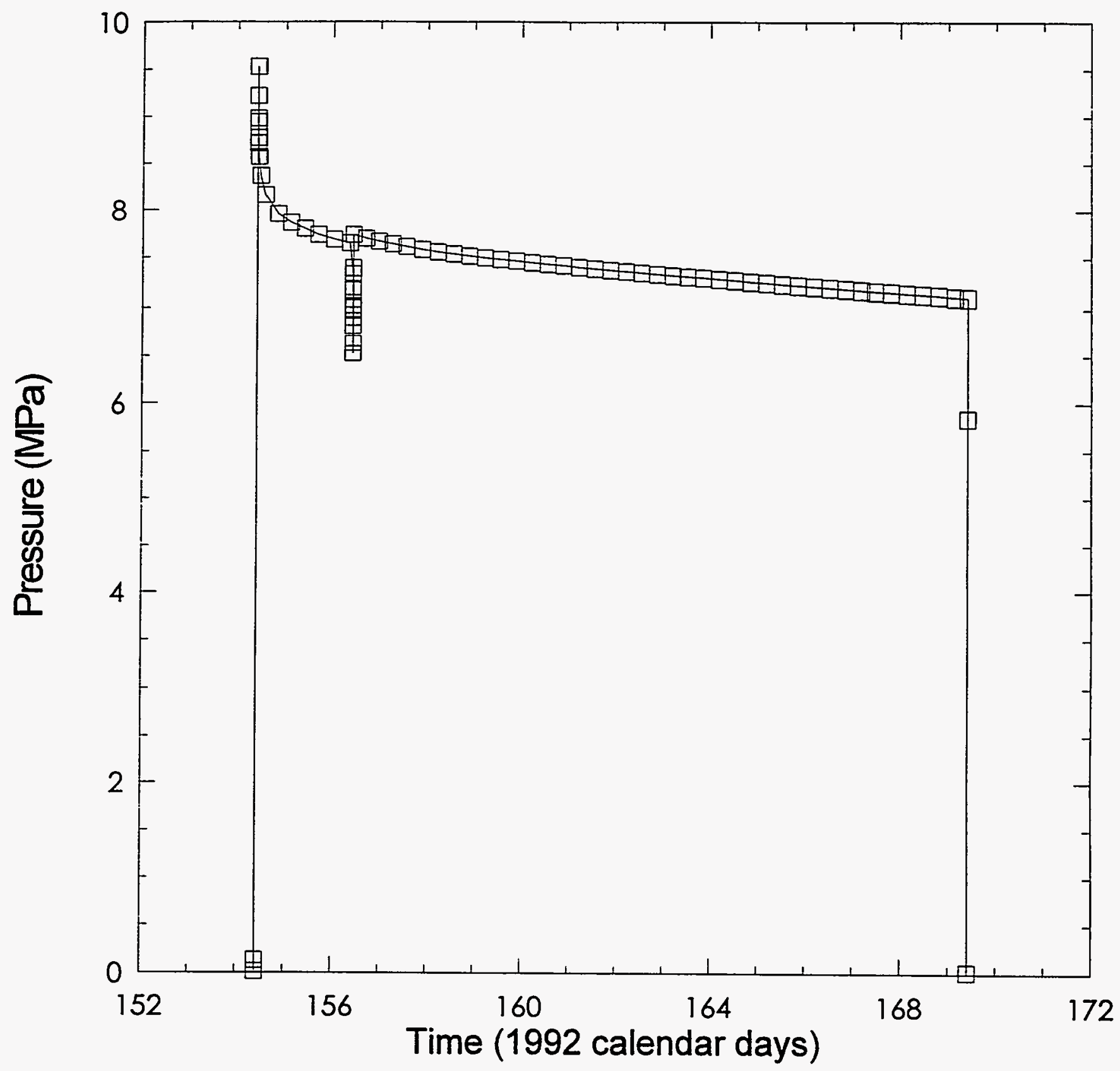

WTERAB1 15-200-0

Figure 6-15. Packer pressure during compliance testing of test tool \#34. 


\subsubsection{Test Tool \#35 (Permeability-Testing Sequence S1P74-A)}

Table 6-10 gives a detailed description of the events that occurred during compliance testing of test tool \#35. Figures 6-16 through 6-22 illustrate the zone pressures, packer pressures, zone temperatures, axial-LVDT displacement, radial-LVDT displacement, fluidinjection volumes during compressibility tests, and test-zone compressibility as a function of pressure, respectively, for multipacker test tool \#35. It should be noted that Figures 6-21 and 6-22 each consist of two parts (ex. Figures 6-21a and 6-21b). Figure 3-32 illustrates the configuration of test tool \#35 as assembled for compliance testing.

Table 6-10. Events Associated with Compliance Testing of Test Tool \#35; Borehole S1P74; Sequence S1P74-A

\begin{tabular}{|c|c|c|c|c|}
\hline EVENT & DATE & $\begin{array}{c}\text { CALENDAR } \\
\text { DAY }\end{array}$ & $\begin{array}{c}1992 \\
\text { CALENDAR } \\
\text { DAY }\end{array}$ & $\begin{array}{c}\text { TIME } \\
\text { (HH:MM:SS) }\end{array}$ \\
\hline $\begin{array}{l}\text { Assembled multipacker test tool \#35 to be used in borehole S1P74 during testing } \\
\text { sequence S1P74A. }\end{array}$ & $6-17-92$ & 169 & 169 & 12:00:00 \\
\hline Begin data file COMP35A. & $6-18-92$ & 170 & 170 & 11:02:51 \\
\hline Inflate $G Z$ packer to $\sim 11.0 \mathrm{MPa}$ and shut in. & $6-18-92$ & 170 & 170 & $11: 05: 00$ \\
\hline Inflate $T Z$ packer. & $6-18-92$ & 170 & 170 & 11:06:00 \\
\hline Shut in TZ packer. & $6-18-92$ & 170 & 170 & 11:08:00 \\
\hline DAS not functioning upon arrival. & 6-19-92 & 171 & 171 & 08:30:00 \\
\hline Increase GZ pressure to $-4.1 \mathrm{MPa}$. & $6-19-92$ & 171 & 171 & 09:01:00 \\
\hline Shut in GZ. & 6-19-92 & 171 & 171 & 09:04:00 \\
\hline Increase TZ pressure to $\sim 4.1 \mathrm{MPa}$ and shut in. & 6-19-92 & 171 & 171 & 09:05:00 \\
\hline Appears to be a leak in $\mathrm{GZ}$. & $6-22-92$ & 174 & 174 & 09:00:00 \\
\hline End data file COMP35A. & $6-22-92$ & 174 & 174 & 11:08:44 \\
\hline $\begin{array}{l}\text { Remove test tool from compliance chamber, diagnose and eliminate leak in } G Z \text {, and } \\
\text { reinstall test tool in compliance chamber. }\end{array}$ & $6-22-92$ & 174 & 174 & 11:30:00 \\
\hline Begin data file COMP358. & $6-22-92$ & 174 & 174 & 11:42:16 \\
\hline Inflate $G Z$ and $T Z$ packers. & 6-22-92 & 174 & 174 & 11:45:00 \\
\hline Increase $T Z$ pressure to $-4.1 \mathrm{MPa}$. & $6-22-92$ & 174 & 174 & $12: 00: 00$ \\
\hline Leak on $T Z$ packer caused packer deflation and depressurization of $T Z$ and $G Z$. & $6-26-92$ & 178 & 178 & $12: 00: 00$ \\
\hline End data file COMP35B. & 6-26-92 & 178 & 178 & 12:30:00 \\
\hline Begin data file COMP35C. & $6-29-92$ & 181 & 181 & 09:25:15 \\
\hline Inflate $T Z$ and $G Z$ packers. & $6-29-92$ & 181 & 181 & 09:53:25 \\
\hline Shut in $T Z$ and $G Z$ packers at $\sim 11.0 \mathrm{MPa}$. & $6-29-92$ & 181 & 181 & $09: 56: 50$ \\
\hline End data file COMP35C. & $6-30-92$ & 182 & 182 & $11: 25: 53$ \\
\hline Remove transducers from P1 and P3. & 6-30-92 & 182 & 182 & $11: 30: 00$ \\
\hline Install transducers 246912 and 246910 , respectively on P1 and P3. & $6-30-92$ & 182 & 182 & 11:45:00 \\
\hline Begin data file COMP35D. & $6-30-92$ & 182 & 182 & 13:19:00 \\
\hline Increase GZ pressure to $-4.1 \mathrm{MPa}$. & $7-9-92$ & 183 & 183 & 08:22:00 \\
\hline Decrease $T Z$ packer pressure from $12.4 \mathrm{MPa}$ to $10.7 \mathrm{MPa}$. & $7-1-92$ & 183 & 183 & 08:25:00 \\
\hline
\end{tabular}


Table 6-10 (Continued). Events Associated with Compliance Testing of Test Tool \#35; Borehole S1P74; Sequence S1P74-A

\begin{tabular}{|c|c|c|c|c|}
\hline EVENT & DATE & $\begin{array}{c}\text { CALENDAR } \\
\text { DAY }\end{array}$ & $\begin{array}{c}1992 \\
\text { CALENDAR } \\
\text { DAY }\end{array}$ & $\begin{array}{c}\text { TIME } \\
\text { (HH:MM:SS) }\end{array}$ \\
\hline Increase $T Z$ pressure to $-4.1 \mathrm{MPa}$. & $7-1-92$ & 183 & 183 & 09:11:00 \\
\hline Decrease $T Z$ pressure by removing $20 \mathrm{~mL}$ of fluid. & $7-8-92$ & 190 & 190 & 09:06:00 \\
\hline Decrease GZ pressure by removing $19 \mathrm{~mL}$ of fluid. & $7-8-92$ & 190 & 190 & 09:07:00 \\
\hline End data file COMP35D. & $7-8-92$ & 190 & 190 & 10:34:44 \\
\hline Begin data file COMP35E. & $7-8-92$ & 190 & 190 & $11: 17: 34$ \\
\hline Begin TZ compressibility test. & $7-8-92$ & 190 & 190 & 11:19:05 \\
\hline Shut in $T Z$ & $7-8-92$ & 190 & 190 & $11: 20: 21$ \\
\hline Depressurize $T Z$ & $7-8-92$ & 190 & 190 & $11: 24: 43$ \\
\hline Begin TZ compressibility test. & $7-8-92$ & 190 & 190 & 11:28:18 \\
\hline Shut in $T Z$ & $7-8-92$ & 190 & 190 & 11:46:52 \\
\hline Begin GZ compressibility test. & $7-8-92$ & 190 & 190 & 12:29:11 \\
\hline Shut in GZ. & $7-8-92$ & 190 & 490 & 12:31:00 \\
\hline Depressurize GZ & $7-8-92$ & 190 & 190 & 12:34:00 \\
\hline Begin $G Z$ compressibility test. & $7-8-92$ & 190 & 190 & 12:39:10 \\
\hline Shut in $G Z$ & $7-8-92$ & 190 & 190 & 12:51:26 \\
\hline Begin constant pressure withdrawal from TZ at $6.4 \mathrm{MPa}$. & 7-13-92 & 195 & 195 & 10:48:04 \\
\hline Shut in $\mathrm{Z}$. & $7-16-92$ & 198 & 198 & 12:59:54 \\
\hline End data file COMP35E. & $7-20-92$ & 202 & 202 & 13:35:49 \\
\hline Remove test tool 35 from compliance chamber and move to borehole S1P74. & $7-21-92$ & 203 & 203 & 12:00:00 \\
\hline
\end{tabular}

Table 6-11 indicates the equipment that was used and the duration that each instrument was used during compliance testing of test tool \#35. Test tool \#35 was used in permeability-testing sequence S1P74-A.

Table 6-11. Compliance Equipment Associated with Test Tool \#35; Borehole S1P74; Sequence S1P74-A

\begin{tabular}{|c|c|c|c|c|}
\hline Equipment & Location & Serial \# & Installed & Removed \\
\hline DAS Software & N/A & PERM4F & $6-18-92$ & $7-21-92$ \\
\hline DCU (HP3497A) & N/A & $2629 a 22040$ & $6-18-92$ & $7-21-92$ \\
\hline $\begin{array}{c}\text { Transducer } \\
\text { (Druck PDCR 910) }\end{array}$ & Test Zone & 322424 & $6-18-92$ & $6-30-92$ \\
\hline $\begin{array}{c}\text { Transducer } \\
\text { (Druck PDCR 910) }\end{array}$ & $\begin{array}{l}\text { Test Zone } \\
\text { Packer }\end{array}$ & 308152 & $6-18-92$ & $7-21-92$ \\
\hline $\begin{array}{c}\text { Transducer } \\
\text { (Druck PDCR 910) }\end{array}$ & Guard Zone & 322427 & $6-18-92$ & $6-30-92$ \\
\hline
\end{tabular}


Table 6-11 (Continued). Compliance Equipment Associated with Test Tool \#35; Borehole S1P74; Sequence S1P74-A

\begin{tabular}{|c|c|c|c|c|}
\hline Equipment & Location & Serial \# & Installed & Removed \\
\hline $\begin{array}{c}\text { Transducer } \\
\text { (Druck PDCR 910) }\end{array}$ & DPT Panel & 322427 & $7-8-92$ & $7-21-92$ \\
\hline $\begin{array}{c}\text { Transducer } \\
\text { (Druck PDCR 910) }\end{array}$ & $\begin{array}{l}\text { Guard Zone } \\
\text { Packer }\end{array}$ & 322423 & $6-18-92$ & $7-21-92$ \\
\hline $\begin{array}{c}\text { Transducer } \\
\text { (Druck PDCR 830) }\end{array}$ & Test Zone & 246912 & $6-30-92$ & $7-21-92$ \\
\hline $\begin{array}{c}\text { Transducer } \\
\text { (Druck PDCR 830) }\end{array}$ & Guard Zone & 246910 & $6-30-92$ & $7-21-92$ \\
\hline LVDT (Trans-Tek 241) & N/A & R16 & $6-18-92$ & $7-21-92$ \\
\hline LVDT (Trans-Tek 241) & N/A & $\mathrm{RO4}$ & $6-18-92$ & $7-21-92$ \\
\hline LVDT (Trans-Tek 241) & N/A & R17 & $6-18-92$ & $7-21-92$ \\
\hline LVDT (Trans-Tek 245) & N/A & $\mathrm{A} 02$ & $6-18-92$ & $7-21-92$ \\
\hline Thermocouple (Type E) & Test Zone & 1 & $6-18-92$ & $7-21-92$ \\
\hline Thermocouple (Type E) & Guard Zone & 2 & $6-18-92$ & $7-21-92$ \\
\hline Injection Column & N/A & 38 & $7-8-92$ & $7-21-92$ \\
\hline Injection Column & N/A & 39 & $7-8-92$ & $7-21-92$ \\
\hline $\begin{array}{c}\text { DPT } \\
\text { (Rosemount 1151DP) }\end{array}$ & N/A & 1140863 & $7-8-92$ & $7-21-92$ \\
\hline
\end{tabular}




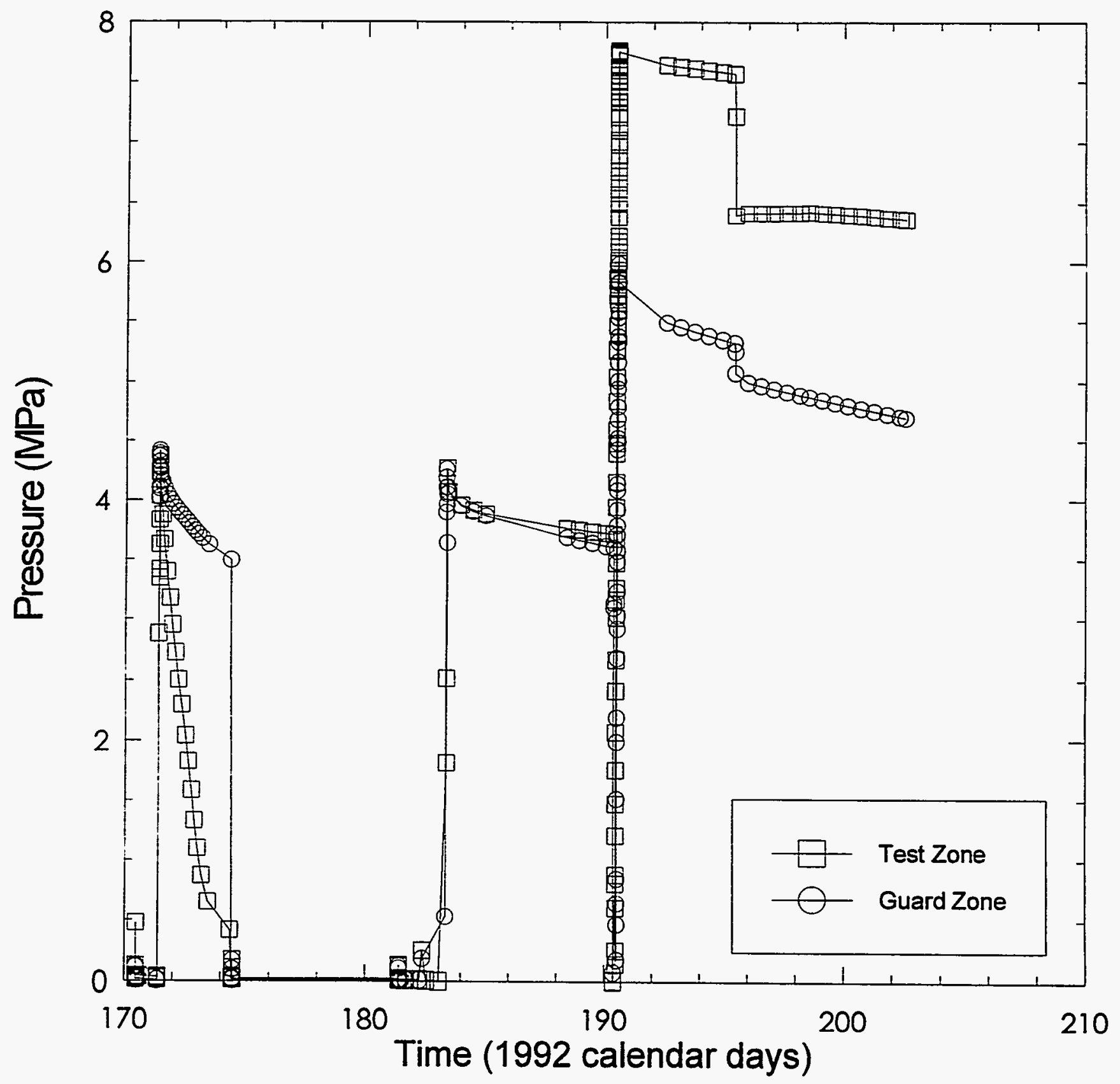

INTERA-115-201-0

Figure 6-16. Zone pressures during compliance testing of test tool \#35. 


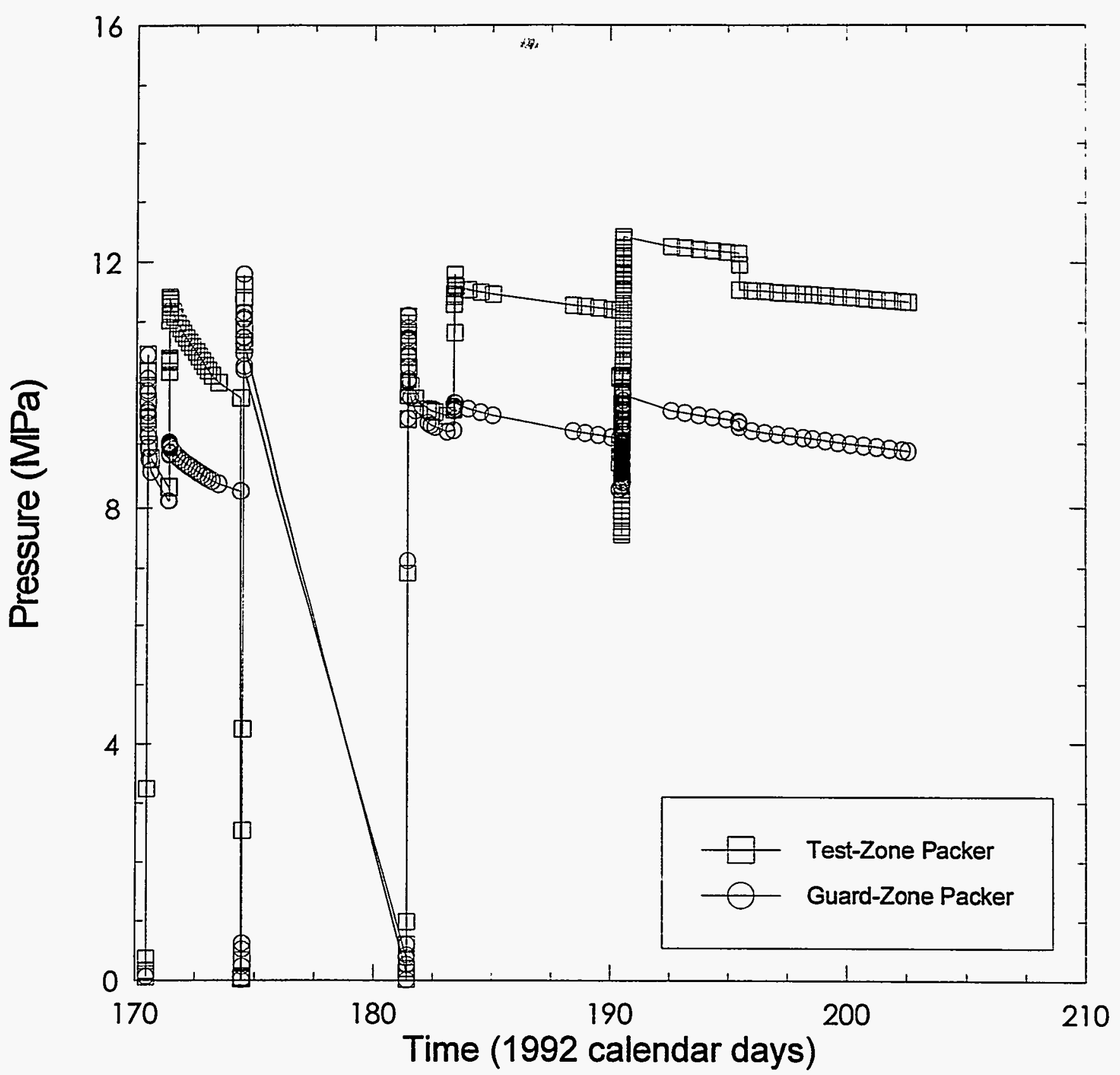

INTERA-115-202-0

Figure 6-17. Packer pressures during compliance testing of test tool \#35. 


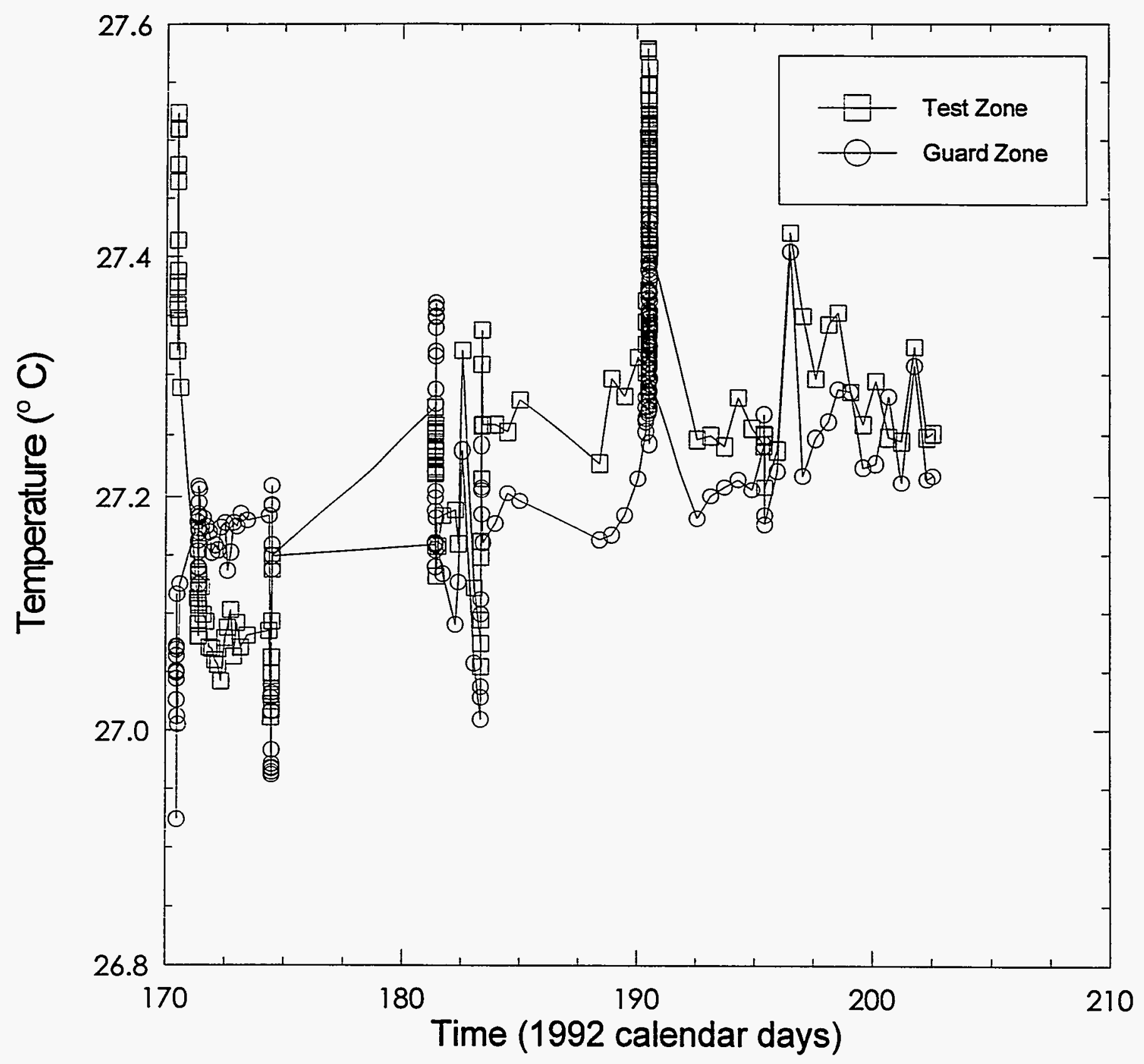

INTERA-115-203-0

Figure 6-18. Zone temperatures during compliance testing of test tool \#35. 


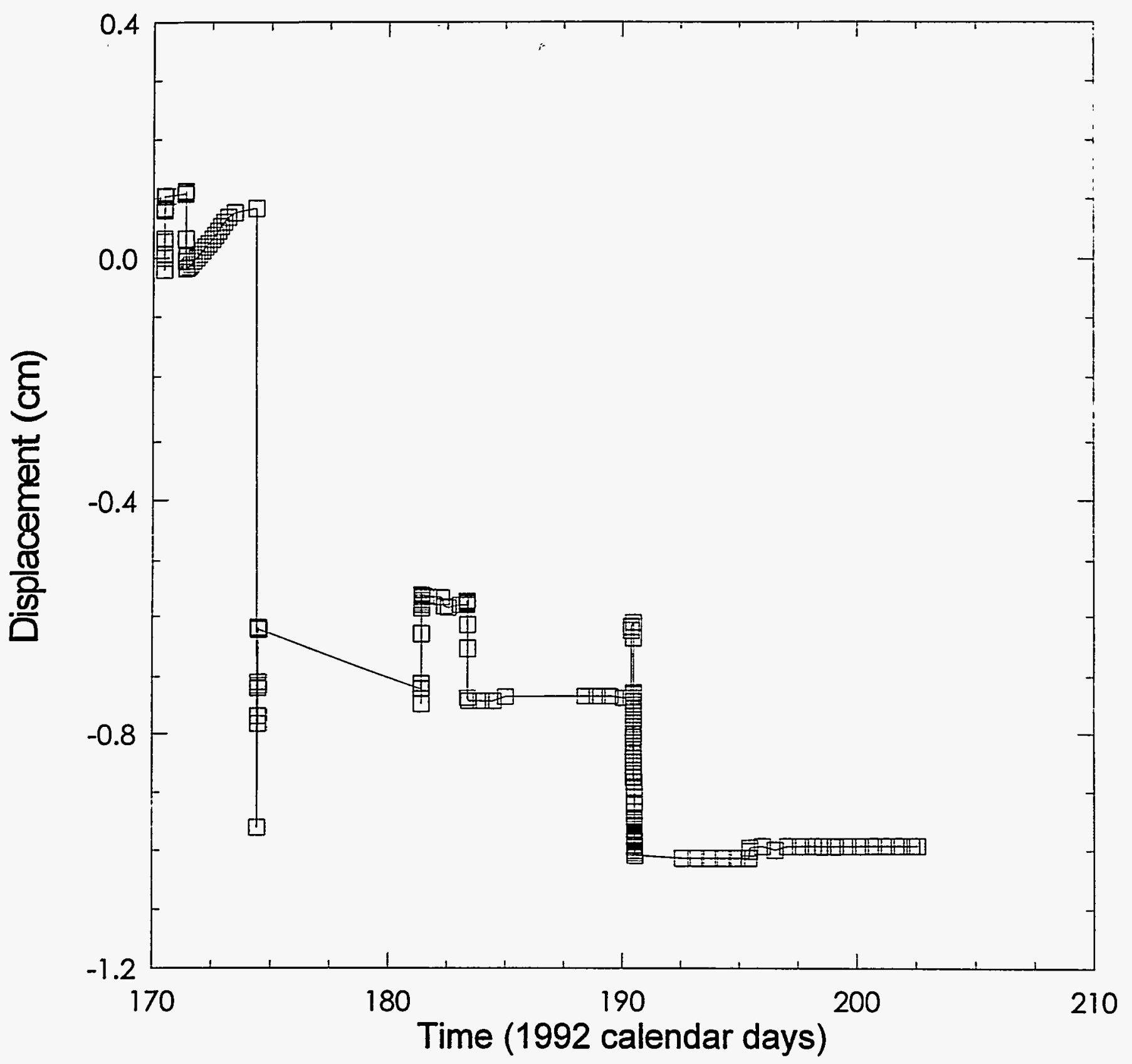

INTERA-6115-2040

Figure 6-19. Axial-LVDT displacement during compliance testing of test tool \#35. 


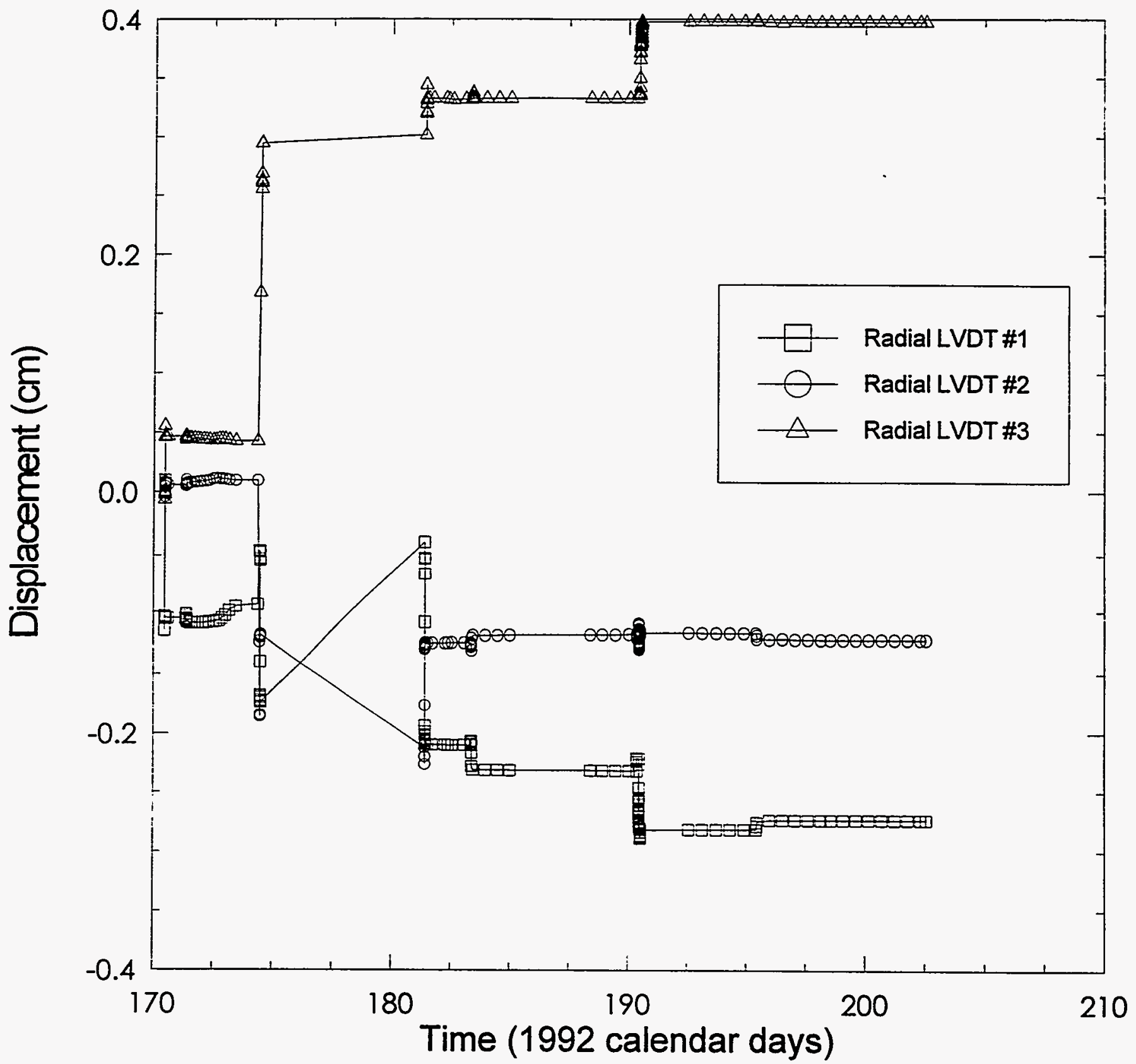

INTERA-O115-205-0

Figure 6-20. Radial-LVDT displacement during compliance testing of test tool \#35. 


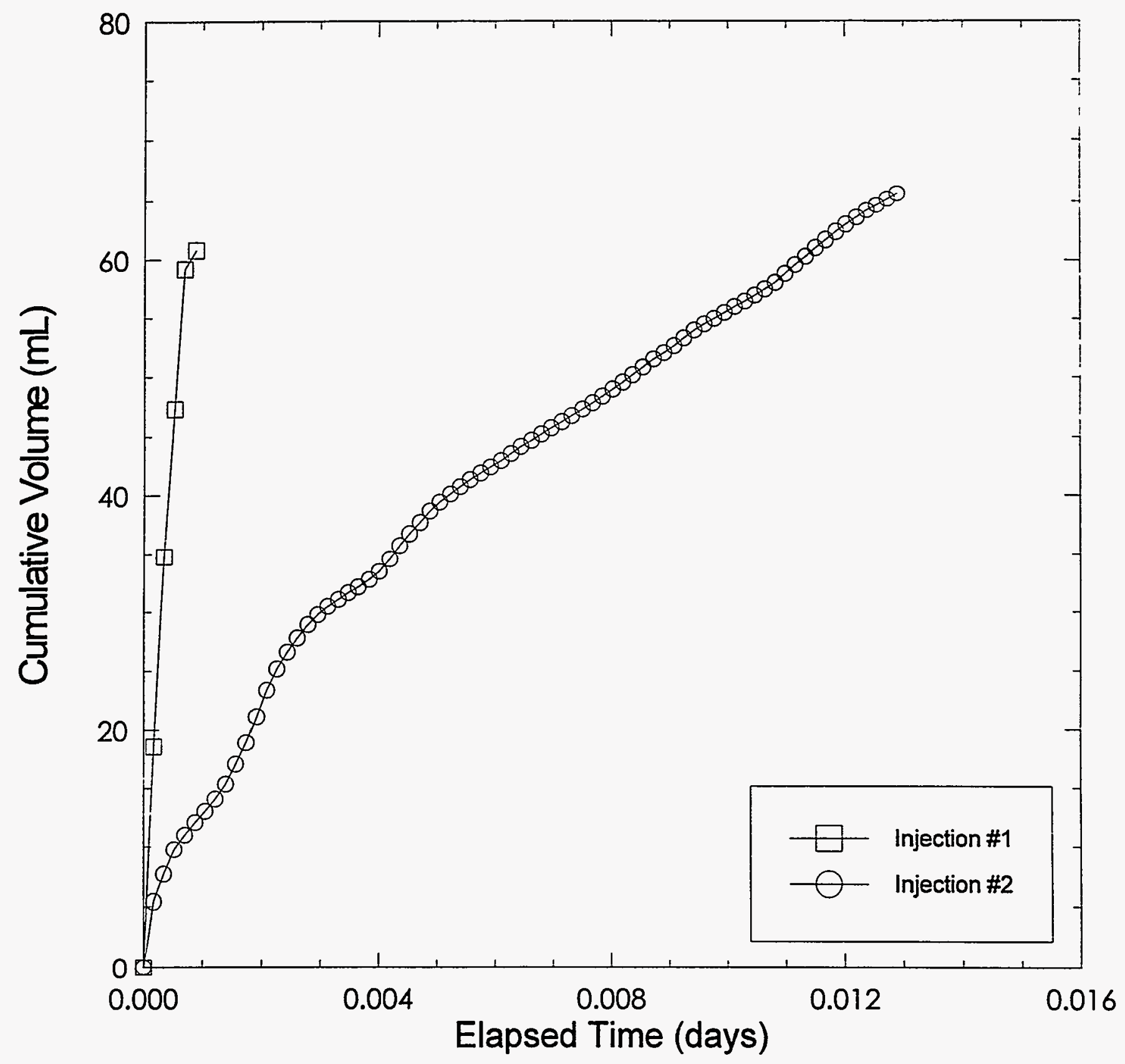

INTERA-6115-208-0

Figure 6-21a. Test-zone fluid-injection volumes during compliance testing of test tool \#35. 


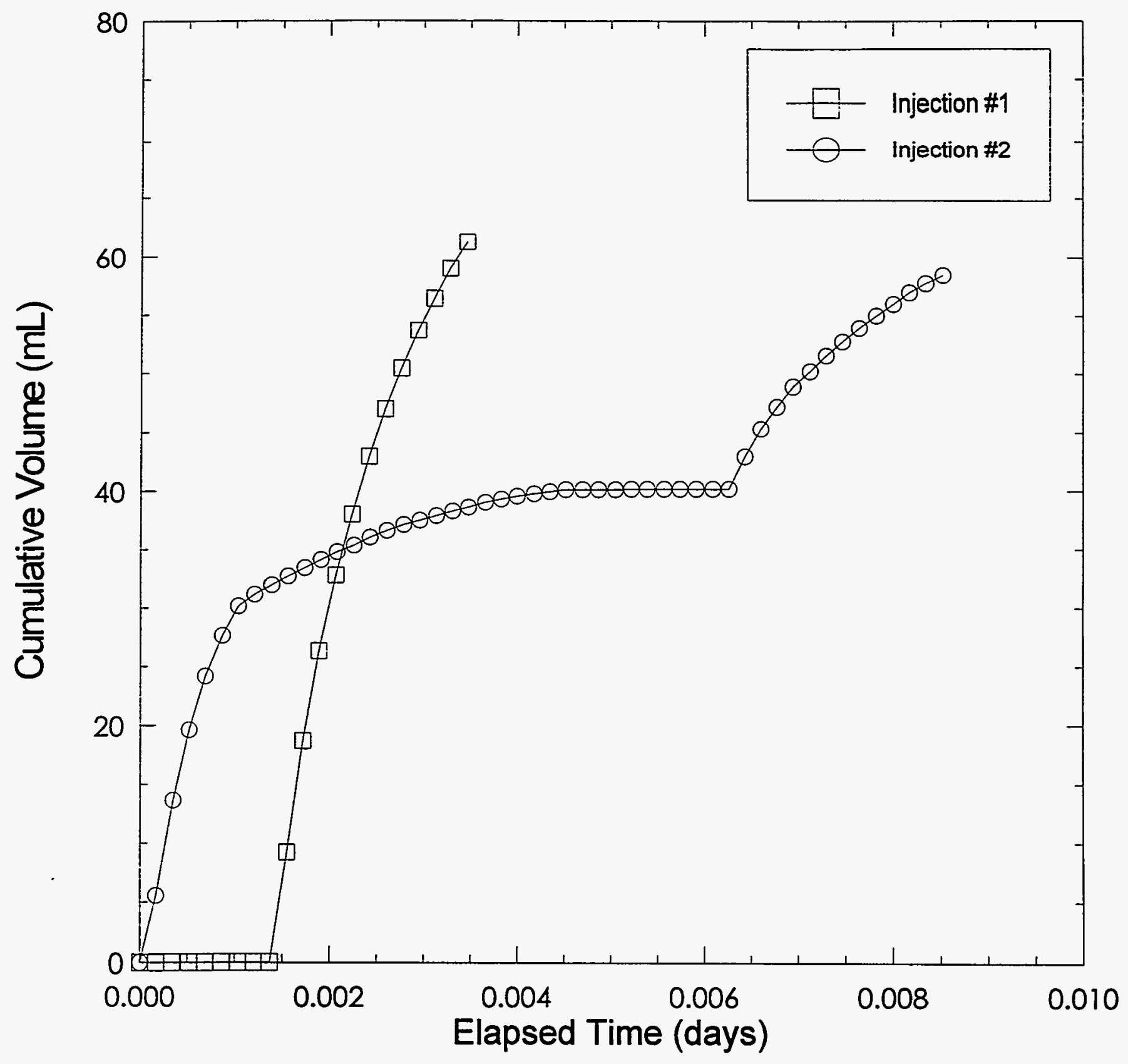

INTERA-8115-207-0

Figure 6-21b. Guard-zone fluid-injection volumes during compliance testing of test tool \#35. 


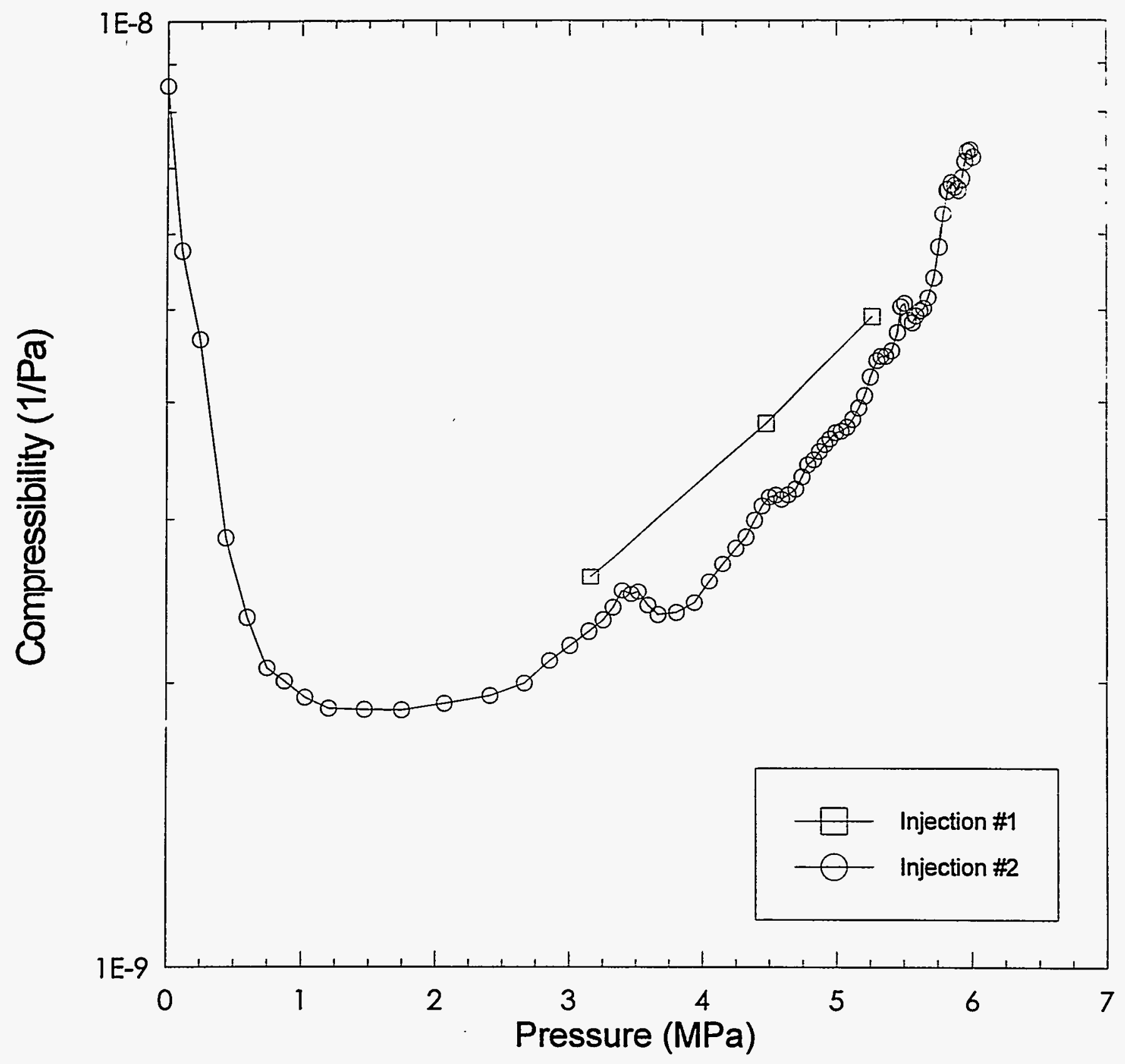

INTERA-6115-200-0

Figure 6-22a. Test-zone compressibility as measured during compliance testing of test tool \#35. 


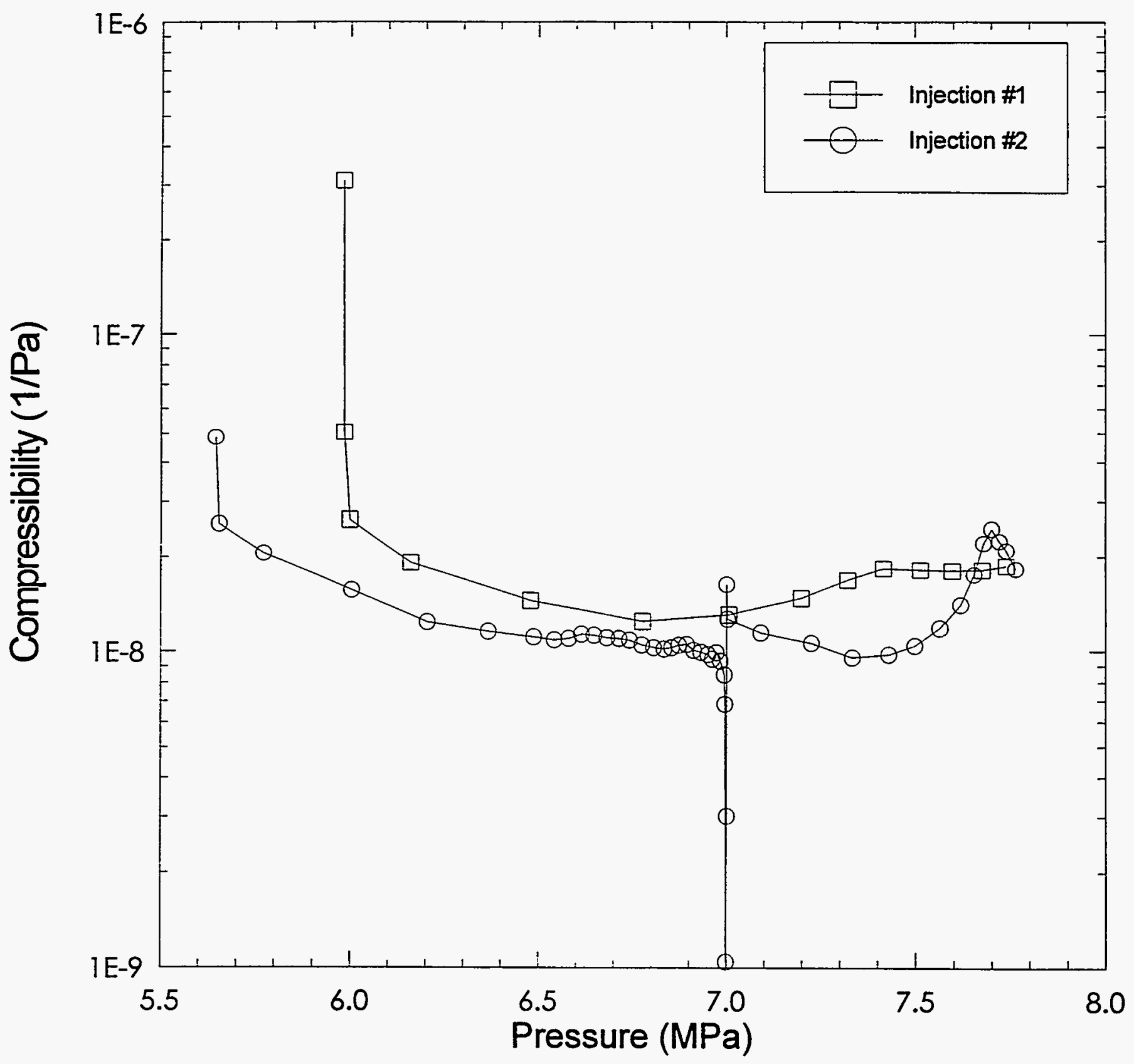

INTERAB115-2090

Figure 6-22b. Guard-zone compressibility as measured during compliance testing of test tool \#35. 


\subsubsection{Test Tool \#36 (Borehole C1X06, Coupled Permeability and Hydrofracture- Testing Sequence C1X05-A)}

Table 6-12 gives a detailed description of the events that occurred during compliance testing of test tool \#36. Figures 6-23 and 6-24 illustrate the zone and packer pressures, respectively, for test tool \#36. Figure 4-13 illustrates the configuration of test tool \#36 as assembled for compliance testing.

Tabie 6-12. Events Associated with Compliance Testing of Test Tool \#36; Borehole C1X06; Sequence C1X05-A

\begin{tabular}{|c|c|c|c|c|}
\hline EVENT & DATE & $\begin{array}{c}\text { CALENDAR } \\
\text { DAY }\end{array}$ & $\begin{array}{c}1992 \\
\text { CALENDAR } \\
\text { DAY }\end{array}$ & $\begin{array}{c}\text { TIME } \\
\text { (HH:MM:SS) }\end{array}$ \\
\hline $\begin{array}{l}\text { Assemble multipacker test tool \#36 to be used in borehole C1X06 during testing } \\
\text { sequence C1X05-A. }\end{array}$ & $9-24-92$ & 268 & 268 & 09:00:00 \\
\hline Begin data file COMP36. & $9-24-92$ & 268 & 268 & 11:58:47 \\
\hline Inflate packer. & $9-24-92$ & 268 & 268 & 12:08:56 \\
\hline Shut in packer. & $9-24-92$ & 268 & 268 & $12: 11: 26$ \\
\hline Increase TZ pressure. & $9-24-92$ & 268 & 268 & 12:17:56 \\
\hline Shut in $T Z$. & $9-24-92$ & 268 & 268 & $12: 20: 26$ \\
\hline End data file COMP36. & $9-28-92$ & 272 & 272 & 08:27:37 \\
\hline $\begin{array}{l}\text { Depressurize TZ, deflate packer, remove test tool from compliance chamber, install } \\
\text { axiafLVDT, and reinstall test tool in compliance chamber. }\end{array}$ & $9-28-92$ & 272 & 272 & 08:30:00 \\
\hline Begin data file COMP36A. & $9-28-92$ & 272 & 272 & $14: 10: 12$ \\
\hline End data file COMP36A. & $9-30-92$ & 274 & 274 & 13:33:04 \\
\hline $\begin{array}{l}\text { Depressurize TZ, deflate packer, and remove test tool from compliance chamber and } \\
\text { move to borehole C1X06. }\end{array}$ & $9-30-92$ & 274 & 274 & 14:00:00 \\
\hline
\end{tabular}

Table 6-13 indicates the equipment that was used and the duration that each instrument was used during compliance testing of test tool \#36. Test tool \#36 was used in observation borehole $\mathrm{C} 1 \mathrm{X06}$ during testing sequence $\mathrm{C} 1 \mathrm{X} 05-\mathrm{A}$.

Table 6-13. Compliance Equipment Associated with Test Tool \#36; Borehole C1X06; Sequence C1X05-A

\begin{tabular}{ccccc}
\hline Equipment & Location & Serial \# & Installed & Removed \\
\hline DAS Software & N/A & PERM4F & $9-24-92$ & $9-30-92$ \\
\hline DCU (HP3497A) & N/A & 2629a21990 & $9-24-92$ & $9-30-92$ \\
\hline $\begin{array}{c}\text { Transducer } \\
\text { (Druck PDCR 910) }\end{array}$ & $\begin{array}{c}\text { Test Zone } \\
\text { Packer }\end{array}$ & 308143 & $9-24-92$ & $9-30-92$ \\
$\begin{array}{c}\text { Transducer } \\
\text { (Druck PDCR 910) }\end{array}$ & Test Zone & 308146 & $9-24-92$ & $9-30-92$ \\
\hline
\end{tabular}




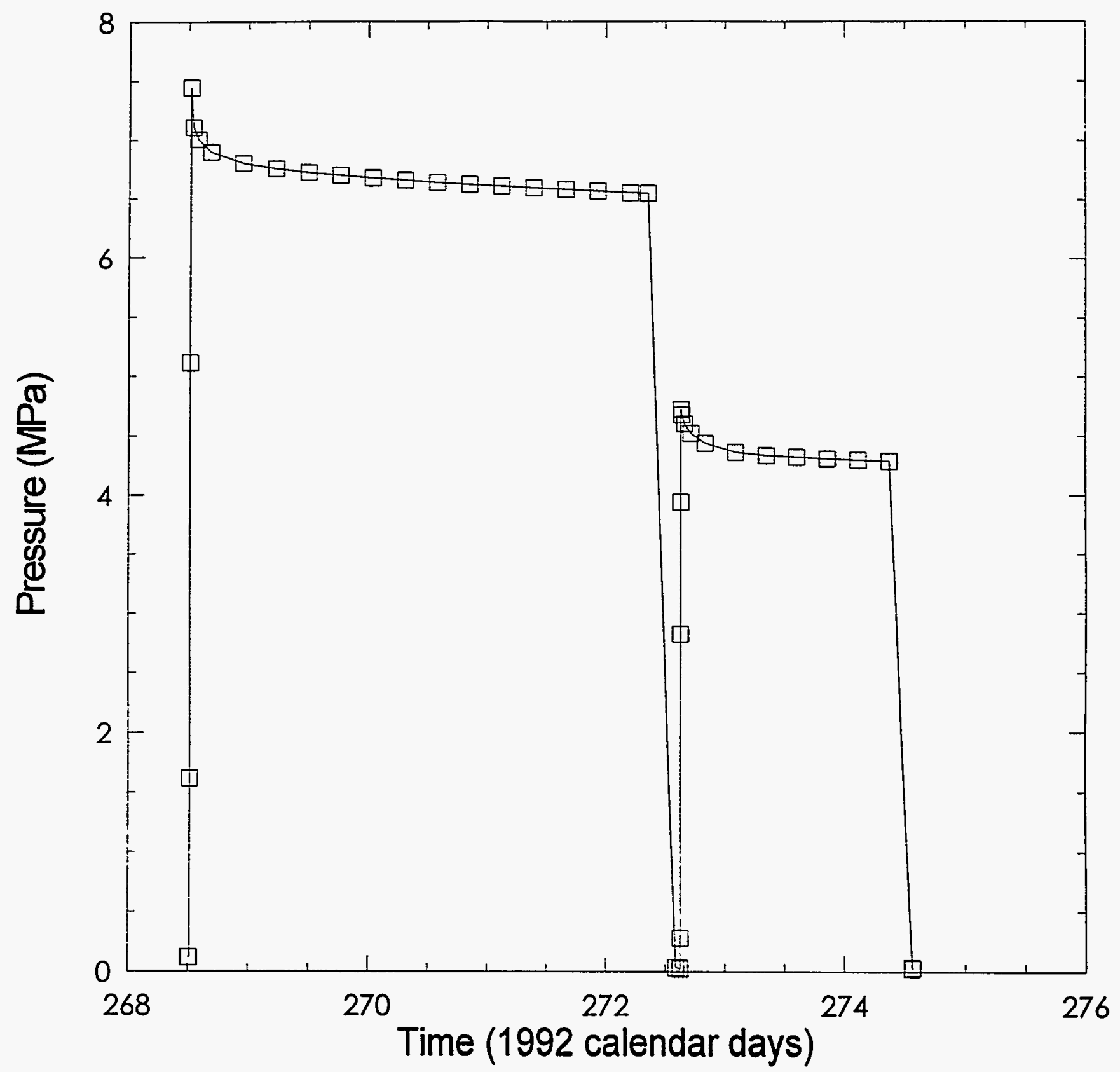

INTERAC115-2100

Figure 6-23. Zone pressure during compliance testing of test tool \#36. 


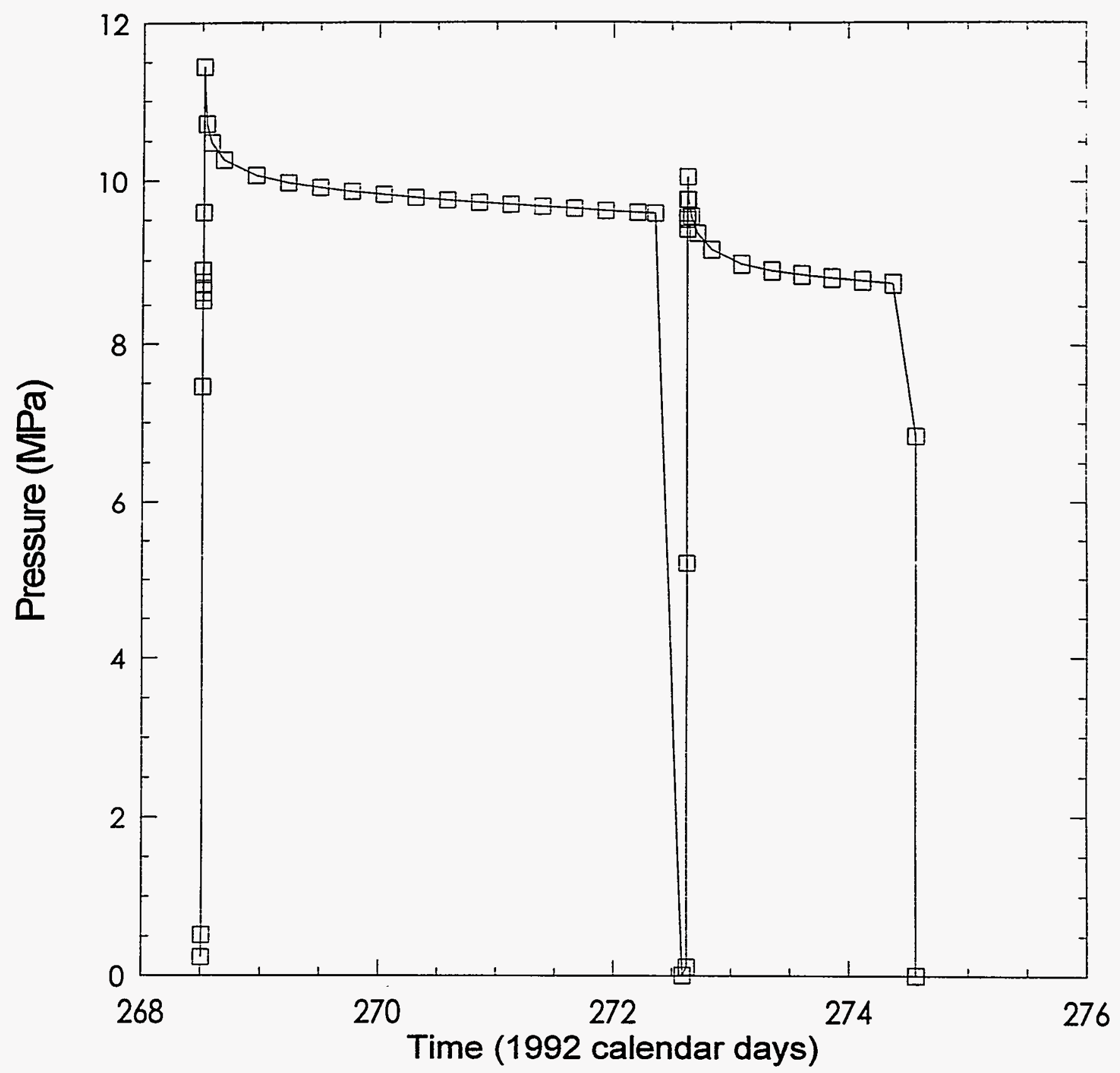

INTERAGI15-211-0

Figure 6-24. Packer pressure during compliance testing of test tool $\# 36$. 


\subsubsection{Test Tool \#37 (Permeability-Testing Sequence L4P52-B)}

Table 6-14 gives a detailed description of the events that occurred during compliance testing of test tool \#37. Figures 6-25 through 6-31 illustrate the zone pressures, packer pressures, zone temperatures, axial-LVDT displacement, radial-LVDT displacement, fluidinjection volumes during compressibility tests, and test-zone compressibility as a function of pressure, respectively, for multipacker test tool \#37. Figure 3-31 illustrates the configuration of test tool \#37 as assembled for compliance testing.

Table 6-14. Events Associated with Compliance Testing of Test Tool \#37; Borehole L4P52; Sequence L4P52-B

\begin{tabular}{|c|c|c|c|c|}
\hline EVENT & DATE & $\begin{array}{l}\text { CALENDAR } \\
\text { DAY }\end{array}$ & $\begin{array}{c}1992 \\
\text { CALENDAR } \\
\text { DAY }\end{array}$ & $\begin{array}{c}\text { TIME } \\
\text { (HH:MM:SS) }\end{array}$ \\
\hline $\begin{array}{l}\text { Assemble multipacker test tool \#37 to be used in borehole L4P52 during testing } \\
\text { sequence L4P52-8. }\end{array}$ & $10-30-92$ & 304 & 304 & 12:00:00 \\
\hline Inflate $T Z$ packer. & $10-31-92$ & 305 & 305 & 12:10:06 \\
\hline Shut in $Z$ packer. & $10-31-92$ & 305 & 305 & 12:11:07 \\
\hline Inflate GZ packer. & $10-31-92$ & 305 & 305 & 12:12:37 \\
\hline Shut in $G Z$ packer. & $10-31-92$ & 305 & 305 & 12:13:37 \\
\hline Increase $T Z$ packer and $G Z$ packer pressure. & $10-31-92$ & 305 & 305 & 12:19:07 \\
\hline Begin data file COMP3701. & $11-2-92$ & 307 & 307 & 12:04:57 \\
\hline Increase $T Z$ and $G Z$ pressure to $-4.8 \mathrm{MPa}$. & $11-9-92$ & 314 & 314 & 10:11:00 \\
\hline Depressurize $T Z$ and $G Z$ to fix leaky fitting. & $11-10-92$ & 315 & 315 & 12:38:00 \\
\hline End data file COMP3701. & $11-10-92$ & 315 & 315 & 14:17:58 \\
\hline Begin data file COMP3702. & 11-11-92 & 316 & 316 & 08:57:15 \\
\hline Begin $T Z$ compressibility tost. & 11-11-92 & 316 & 316 & 10:31:00 \\
\hline Shut in $T Z$ & $11-11-92$ & 316 & 316 & 10:52:00 \\
\hline Begin GZ compressiblility test. & $11-11-92$ & 316 & 316 & 11:00:00 \\
\hline Shut in $G Z$. & $11-11-92$ & 316 & 316 & 11:18:00 \\
\hline End data file COMP3702. & $11-13-92$ & 318 & 318 & 10:39:40 \\
\hline Begin data file COMP3703. & $11-13-92$ & 398 & 318 & $10: 43: 53$ \\
\hline End data file COMP3703. & $11-17-92$ & 322 & 322 & 07:55:46 \\
\hline Replace valves on $T Z$ and $G Z$ & $11-23-92$ & 328 & 328 & 10:19:00 \\
\hline DAS not functioning upon arrival. & $11-30-92$ & 335 & 335 & 12:00:00 \\
\hline Begin data file COMP3704. & $11-30-92$ & 335 & 335 & 13:29:08 \\
\hline Incroase $T Z$ and $G Z$ pressure. & $11-30-92$ & 335 & 335 & $13: 49: 00$ \\
\hline Depressurize zones and deflate packers. & 12-2-92 & 337 & 337 & 12:00:00 \\
\hline End data file COMP3704. & 12-2-92 & 337 & 337 & 12:20:04 \\
\hline Begin data file COMP3705. & 12-3-92 & 338 & 338 & 09:55:27 \\
\hline Inflate $T Z$ packer and $G Z$ packer. & $12-3-92$ & 338 & 338 & 10:12:00 \\
\hline Increase $T Z$ and $G Z$ pressure. & $12-4-92$ & 339 & 339 & $12: 00: 00$ \\
\hline
\end{tabular}


Table 6-14 (Continued). Events Associated with Compliance Testing of Test Tool \#37; Borehole L4P52; Sequence L4P52-B

\begin{tabular}{|c|c|c|c|c|}
\hline : EVENT $:$ & DATE & $\begin{array}{l}\text { CALENDAR } \\
\text { DAY }\end{array}$ & $\begin{array}{c}1992 \\
\text { CALENDAR } \\
\text { DAY }\end{array}$ & $\begin{array}{c}\text { TIME } \\
\text { (HH:MM:SS) }\end{array}$ \\
\hline Depressurize TZ and GZ. & $12-4-92$ & 339 & 339 & 12:01:00 \\
\hline Increase TZ pressure to $\sim 4.5 \mathrm{MPa}$. & $12-492$ & 339 & 339 & $13: 23: 00$ \\
\hline Increase GZ pressure to $\sim 4.5 \mathrm{MPa}$. & $12-4-92$ & 339 & 339 & 13:25:00 \\
\hline End data file COMP370S. & $12-7-92$ & 342 & 342 & $11: 16: 27$ \\
\hline Remove test tool \#37 from compliance chamber and move to borehole L4P52. & $12-14-92$ & 349 & 349 & $09: 40: 28$ \\
\hline
\end{tabular}

Table 6-15 indicates the equipment that was used and the duration that each instrument was used during compliance testing of test tool \#37. Test tool \#37 was used in permeability-testing sequence L4P52-B.

Table 6-15. Compliance Equipment Associated with Test Tool \#37; Borehole L4P52; Sequence L4P52-B

\begin{tabular}{|c|c|c|c|c|}
\hline Equipment & Location & Serial \# & Installed & Removed \\
\hline DAS Software & N/A & PERM4F & $10-31-92$ & $12-7-92$ \\
\hline DCU (HP3497A) & N/A & $2629 a 22040$ & $10-31-92$ & $12-7-92$ \\
\hline $\begin{array}{c}\text { Transducer } \\
\text { (Druck PDCR 830) }\end{array}$ & Test Zone & 214048 & $10-31-92$ & $12-7-92$ \\
\hline $\begin{array}{c}\text { Transducer } \\
\text { (Druck PDCR 830) }\end{array}$ & $\begin{array}{l}\text { Test Zone } \\
\text { Packer }\end{array}$ & 214466 & $10-31-92$ & $12-7-92$ \\
\hline $\begin{array}{c}\text { Transducer } \\
\text { (Druck PDCR 830) }\end{array}$ & Guard Zone & 246913 & $10-31-92$ & $12-7-92$ \\
\hline $\begin{array}{c}\text { Transducer } \\
\text { (Druck PDCR 830) }\end{array}$ & $\begin{array}{l}\text { Guard Zone } \\
\text { Packer }\end{array}$ & 214470 & $10-31-92$ & $12-7-92$ \\
\hline $\begin{array}{c}\text { Transducer } \\
\text { (Druck PDCR 910) }\end{array}$ & DPT Panel & 322427 & $11-11-92$ & $11-13-92$ \\
\hline LVDT (Trans-Tek 241) & N/A & R09 & $10-31-92$ & $12-7-92$ \\
\hline LVDT (Trans-Tek 241) & N/A & $\mathrm{R} 03$ & $10-31-92$ & $12-7-92$ \\
\hline LVDT (Trans-Tek 241) & N/A & $\mathrm{R} 02$ & $10-31-92$ & $12-7-92$ \\
\hline LVDT (Trans-Tek 245) & N/A & $\mathrm{AO3}$ & $10-31-92$ & $12-7-92$ \\
\hline Thermocouple (Type E) & Test Zone & 1 & $10-31-92$ & $12-7-92$ \\
\hline Thermocouple (Type E) & Guard Zone & 2 & $10-31-92$ & $12-7-92$ \\
\hline Injection Column & N/A & 39 & $11-11-92$ & $11-11-92$ \\
\hline $\begin{array}{c}\text { DPT } \\
\text { (Rosemount 1151DP) }\end{array}$ & N/A & 1140863 & $11-11-92$ & $11-13-92$ \\
\hline
\end{tabular}




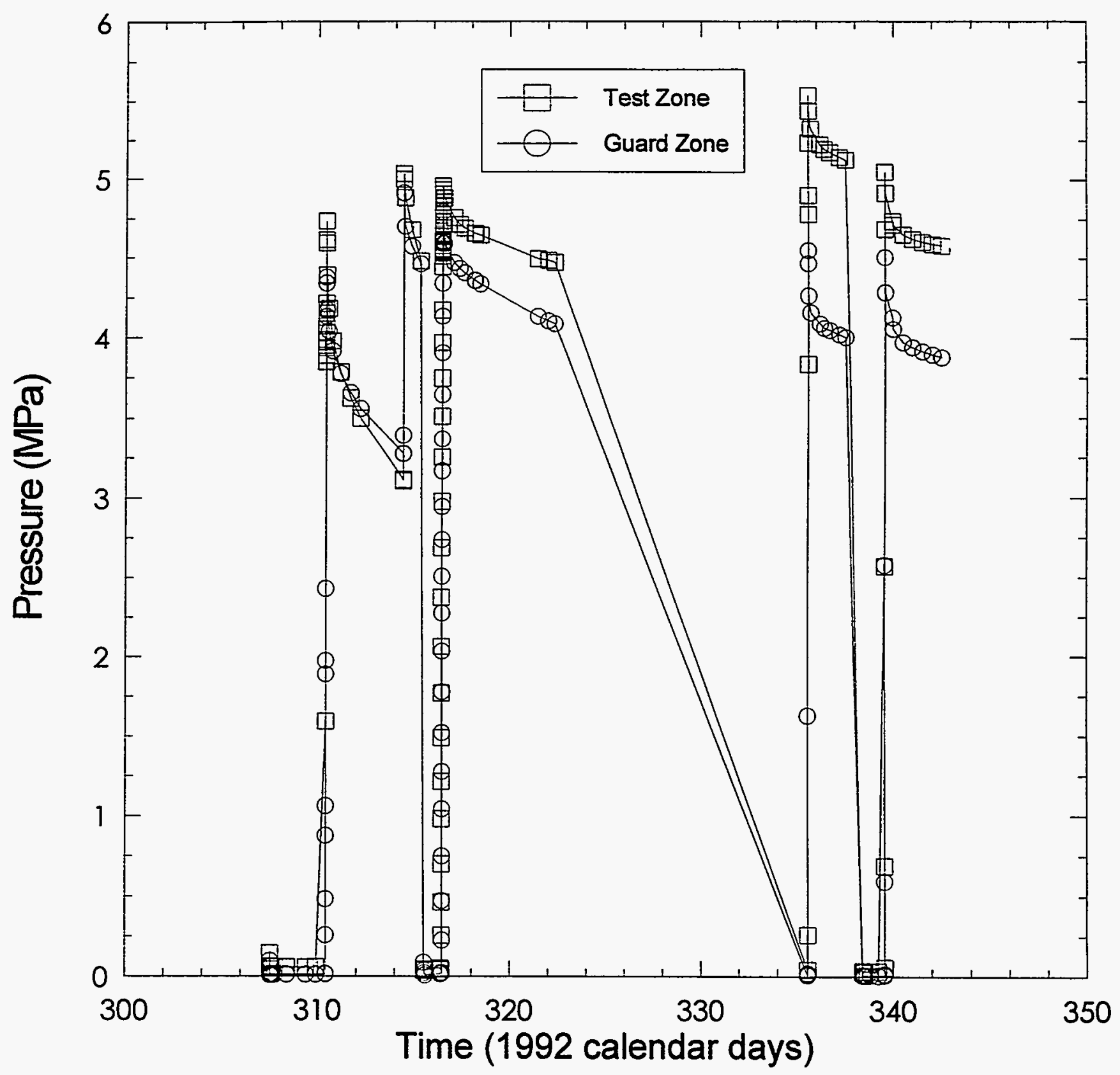

INTERA-115-212-0

Figure 6-25. Zone pressures during compliance testing of test tool \#37. 


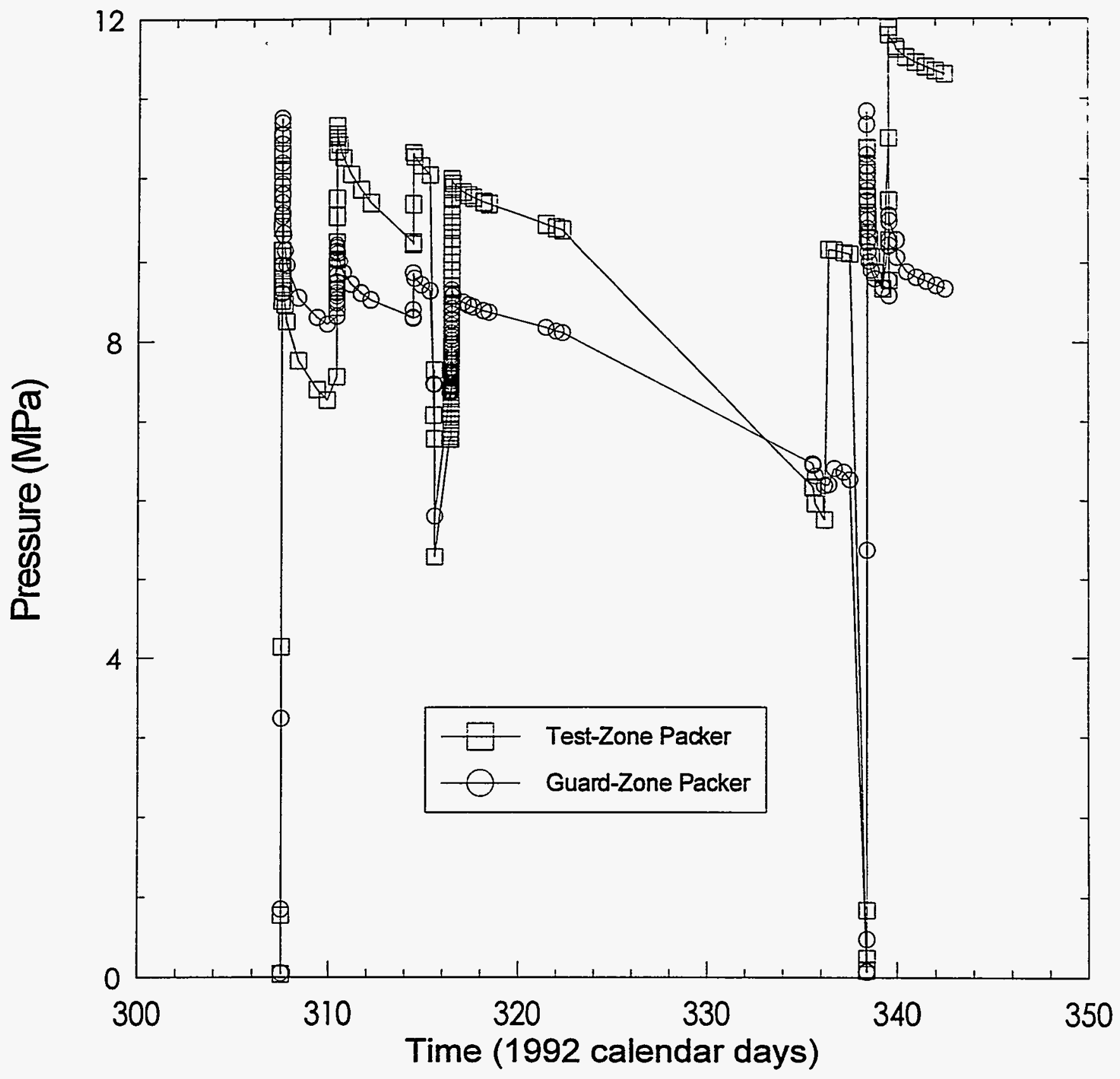

INTERAS115-213-0

Figure 6-26. Packer presures during compliance testing of test tool \#37. 


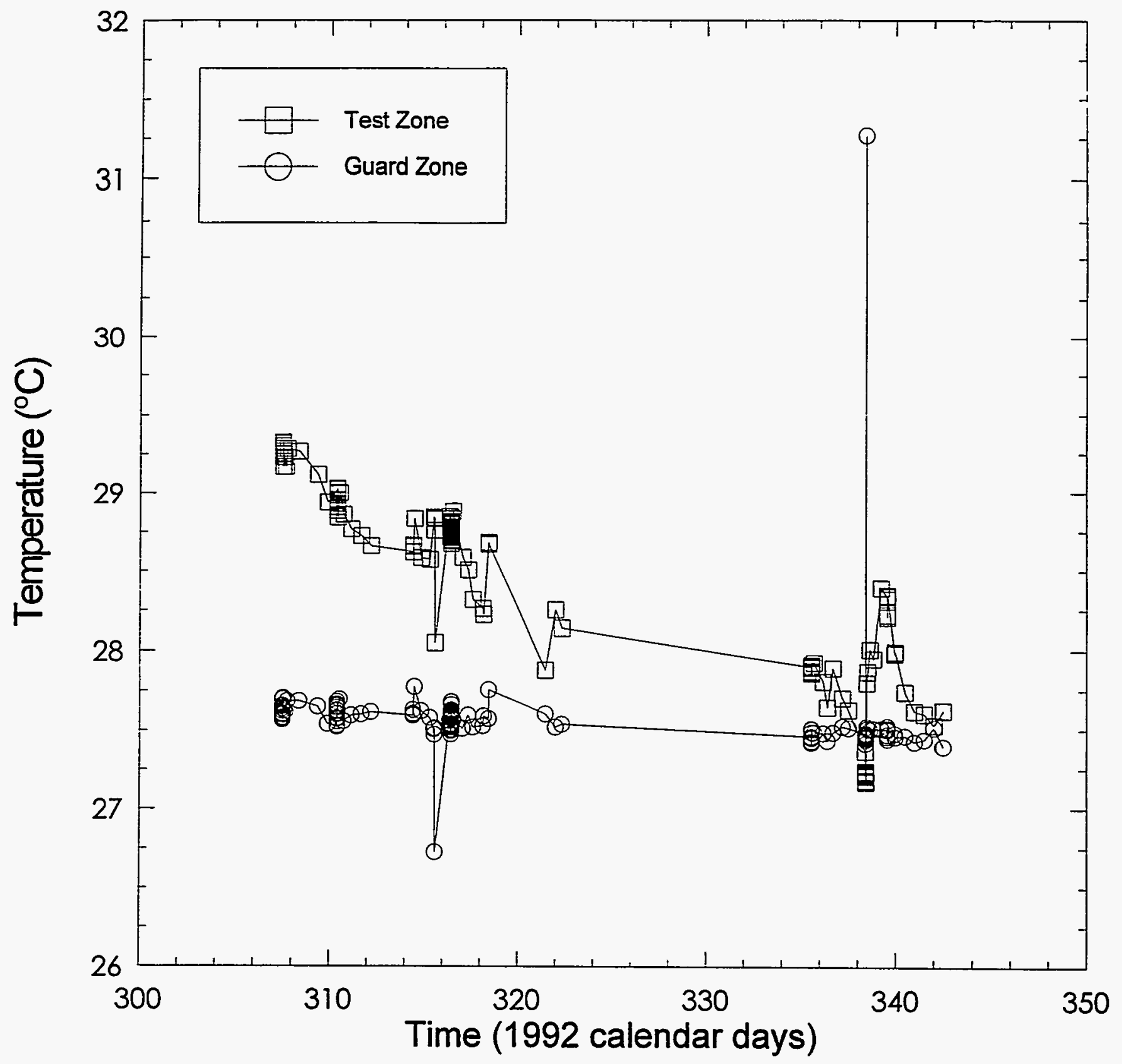

INTERA-6115-2140

Figure 6-27. Zone temperatures during compliance testing of test tool \#37. 


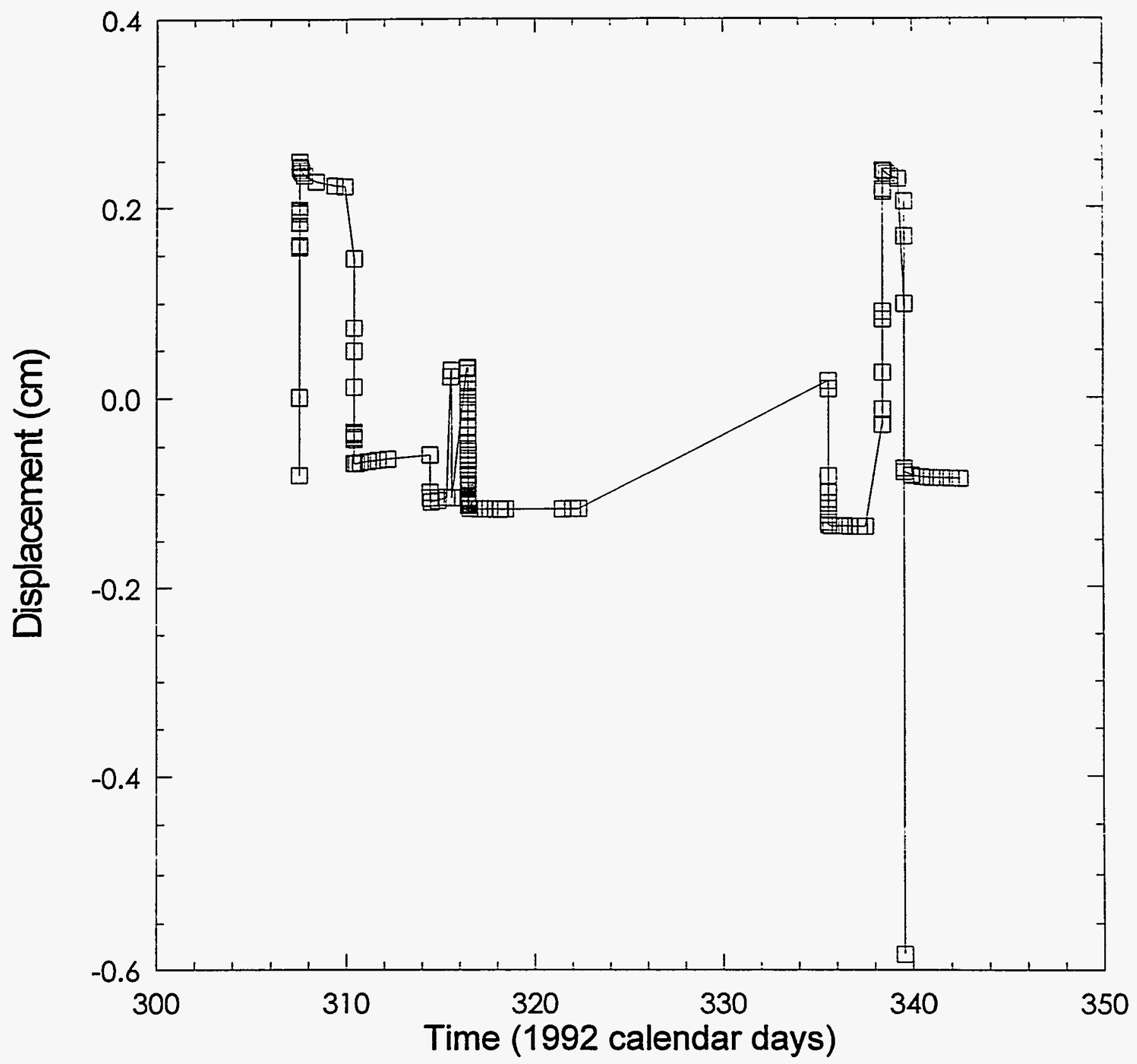

INTERA-6115-215-0

Figure 6-28. Axial-LVDT displacement during compliance testing of test tool \#37. 


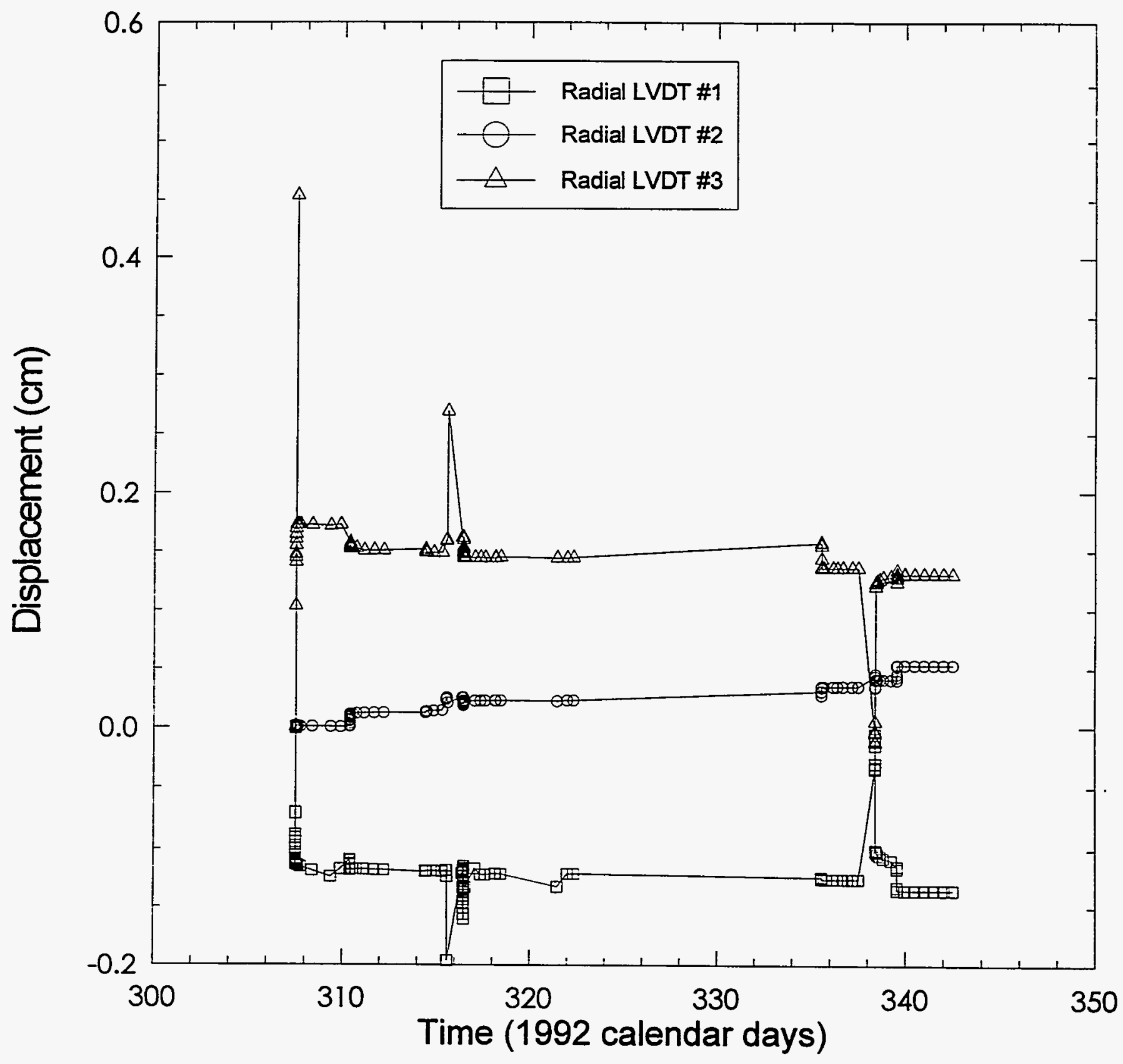

INTER46115-218-0

Figure 6-29. Radial-LVDT displacement during compliance testing of test tool \#37. 


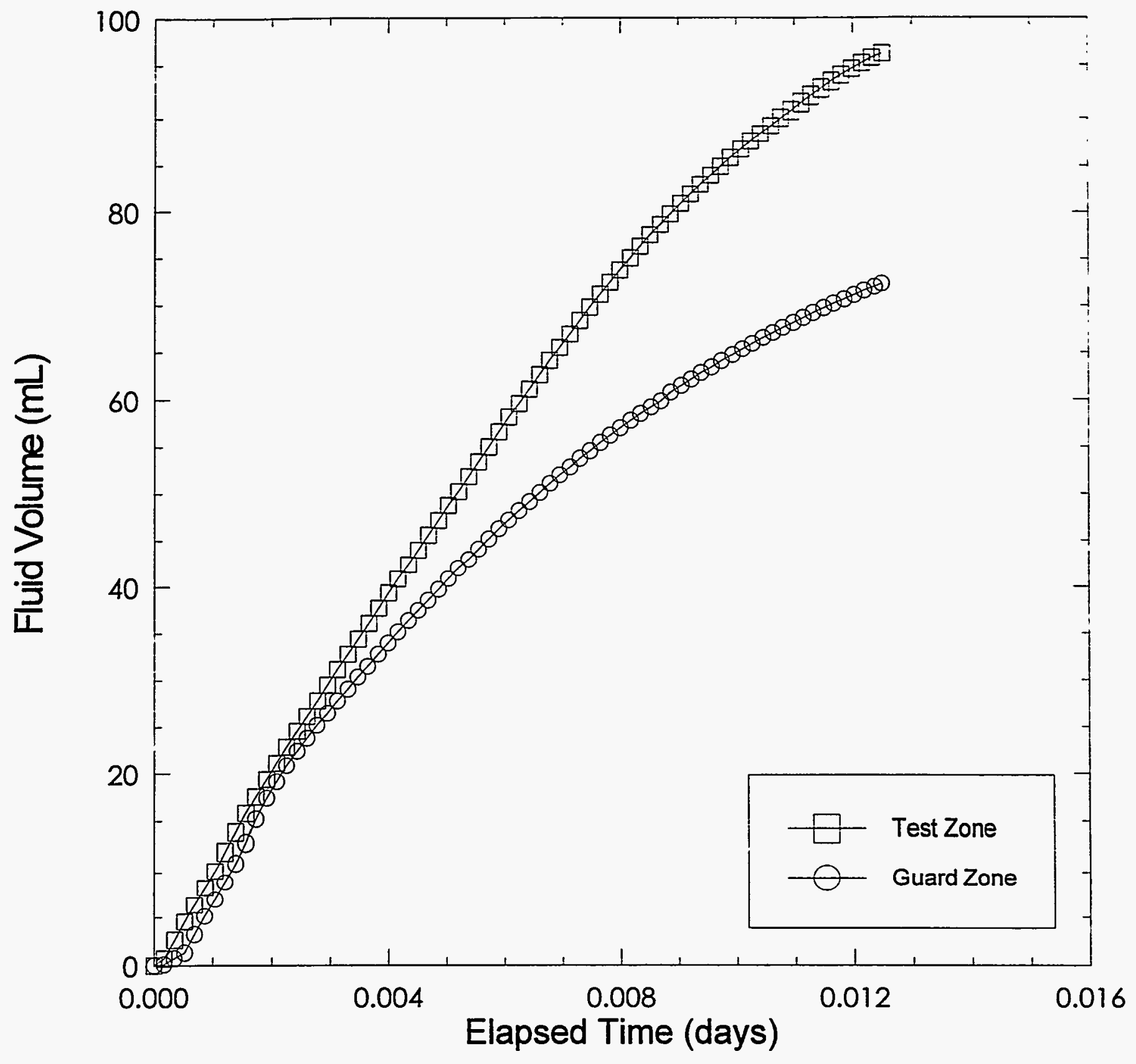

INTERA-BI15-217-0

Figure 6-30. Test-zone fluid-injection volumes during compliance testing of test tool \#37. 


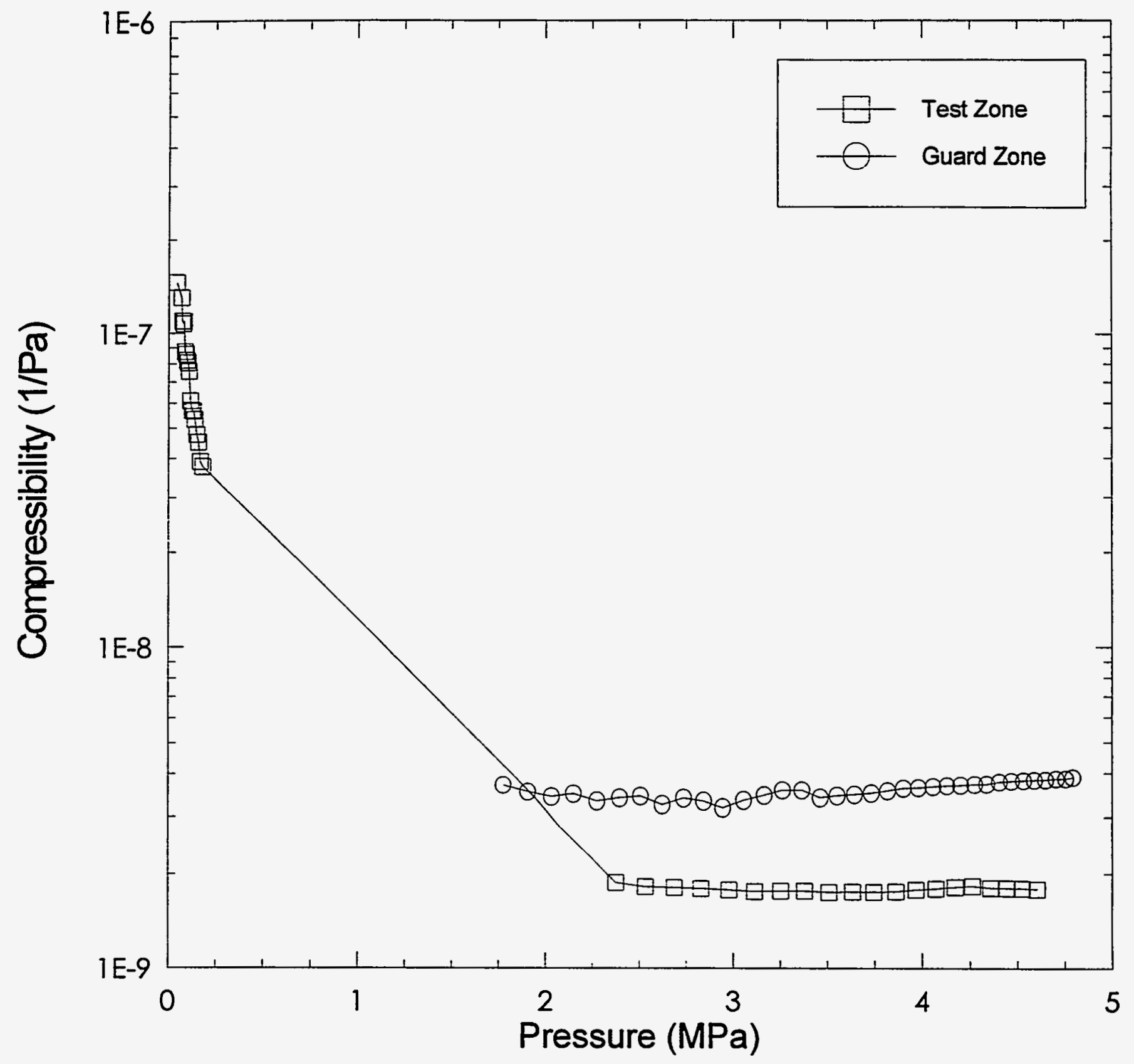

INTERAOI18-218-0

Figure 6-31. Test-zone compressibility as measured during compliance testing of test tool \#37. 


\subsubsection{Test Tool \#38A (Gas-Threshold-Pressure Testing Sequence SCP01-1)}

Table 6-16 gives a detailed description of the events that occurred during compliance testing of test tool \#38A. Figures 6-32 through 6-36 illustrate the zone pressures, packer pressures, zone temperatures, fluid-injection volumes during test-tool compressibility tests, and test-tool compressibility as a function of pressure, respectively, for multipacker test tool \#38A. Figure 5-4 illustrates the configuration of test tool \#38A as assembled for compliance testing.

Table 6-16. Events Associated with Compliance Testing of Test Tool \#38A; Borehole SCP01; Sequence SCP01-1

\begin{tabular}{|c|c|c|c|c|}
\hline EVENT & DATE & $\begin{array}{l}\text { CALENDAR } \\
\text { DAY }\end{array}$ & $\begin{array}{c}1993 \\
\text { CALENDAR } \\
\text { DAY }\end{array}$ & $\begin{array}{c}\text { TIME } \\
\text { (HH:MM:SS) }\end{array}$ \\
\hline $\begin{array}{l}\text { Assemble multipacker test tool \#38A to be used in borehole SCP01 during testing } \\
\text { sequence SCP01-1. }\end{array}$ & $1-4-93$ & 4 & 4 & 12:00:00 \\
\hline Begin data file COMP3801. & $1-5-93$ & 5 & 5 & $11: 45: 21$ \\
\hline Inflate $T Z$ packer. & $1-5-93$ & 5 & 5 & 12:38:40 \\
\hline Fix leak at TZ packer transducer. & $1-5-93$ & 5 & 5 & $12: 41: 21$ \\
\hline Inflate TZ packer. & $1-5-93$ & 5 & 5 & 12:50:19 \\
\hline Shut in TZ packer. & i-5-93 & 5 & 5 & 12:53:53 \\
\hline Inflate GZ packer. & $1.5-93$ & 5 & 5 & 12:55:42 \\
\hline Shut in $G Z$ packer. & $1-5-93$ & 5 & 5 & 12:58:23 \\
\hline Deflate $T Z$ packer and $G Z$ packer. & $1-6-93$ & 6 & 6 & 10:18:24 \\
\hline Repair $T Z$ packer. & $1-6-93$ & 6 & 6 & 11:15:45 \\
\hline Inflate TZ packer. & $1-6-93$ & 6 & 6 & 11:18:25 \\
\hline Shut in $T Z$ packer at $11.207 \mathrm{MPa}$. & $1-6-93$ & 6 & 6 & 11:20:11 \\
\hline Inflate GZ packer. & $1-6-93$ & 6 & 6 & 11:21:04 \\
\hline Shut in GZ packer at $10.073 \mathrm{MPa}$. & $1-6-93$ & 6 & 6 & 11:22:51 \\
\hline Increase TZ packer pressure. & $1-6-93$ & 6 & 6 & 12:30:53 \\
\hline Increase GZ packer pressure. & $1-6-93$ & 6 & 6 & 12:31:42 \\
\hline End data file COMP3801. & $9-7-93$ & 7 & 7 & 10:16:05 \\
\hline Begin data file COMP3802. & $1.7-93$ & 7 & 7 & 10:49:12 \\
\hline Inject nuld into TZ via DPT panel (no response). & 1.7 .93 & 7 & 7 & 12:54:25 \\
\hline Inject fuld into TZ via DPT panel (no response). & $1-7.93$ & 7 & 7 & 13:35:24 \\
\hline Dentete packers. & $9-7-93$ & 7 & 7 & 14:03:56 \\
\hline Inflate $7 Z$ packer. & $1-7-93$ & 7 & 7 & 14:07:56 \\
\hline Inllate GZ packer. & $1.7-93$ & 7 & 7 & 14:09:11 \\
\hline Increase $T Z$ pressure via DPT panel. & $1-8-93$ & 8 & 8 & 10:06:48 \\
\hline Shut in $\mathrm{TZ}$ & $1-8-93$ & 8 & 8 & 10:07:33 \\
\hline Depressurize TZ. & $1-8-93$ & 8 & 8 & 10:10:19 \\
\hline Increase $T Z$ pressure via DPT panel. & $1-8-93$ & 8 & 8 & 10:16:44 \\
\hline
\end{tabular}


Table 6-16 (Continued). Events Associated with Compliance Testing of Test Tool \#38A; Borehole SCP01; Sequence SCP01-1

\begin{tabular}{|c|c|c|c|c|}
\hline EVENT & DATE & $\begin{array}{l}\text { CALENDAR } \\
\text { DAY }\end{array}$ & $\begin{array}{c}1993 \\
\text { CALENDAR } \\
\text { DAY }\end{array}$ & $\begin{array}{c}\text { TIME } \\
\text { (HH:MM:SS) }\end{array}$ \\
\hline Shut in $T Z$ & $1-8-93$ & 8 & 8 & 10:21:15 \\
\hline Depressurize TZ & $1-8-93$ & $\mathbf{B}$ & 8 & 10:26:16 \\
\hline Increase TZ pressure via DPT panel. & $1-8-93$ & 8 & 8 & 10:31:16 \\
\hline Shut in $T Z$ & $1-8-93$ & 8 & 8 & 10:40:46 \\
\hline Increase GZ pressure via DPT panel. & $1-8-93$ & 8 & 8 & 11:00:02 \\
\hline Shut in $\mathrm{GZ}$ & $1-8-93$ & 8 & 8 & 11:08:13 \\
\hline Depressurize $T Z$ and $G Z$ & $1-11-93$ & 11 & 19 & 10:40:39 \\
\hline Increase $G Z$ packer pressure to $\sim 11.0 \mathrm{MPa}$. & 1-11-93 & 11 & 11 & $10: 43: 40$ \\
\hline Increase TZ packer pressure to $-11.0 \mathrm{MPa}$. & $1-11-93$ & 11 & 11 & 10:45:25 \\
\hline DAS not functioning upon arrival. & $1-12-93$ & 12 & 12 & 10:34:23 \\
\hline Increase TZ packer pressure. & $1-15-93$ & 15 & 15 & 10:36:32 \\
\hline Shut in TZ packer. & $1-15-93$ & 15 & 15 & $10: 38: 56$ \\
\hline Begin $T Z$ compressibility test. & $1-18-93$ & 18 & 18 & $12: 40: 00$ \\
\hline Shut in $T Z$. & $1-18-93$ & 18 & 18 & 12:53:00 \\
\hline End data file COMP3802. & $1-19-93$ & 19 & 19 & 08:51:50 \\
\hline Begin data file COMP3803. & $1-20-93$ & 20 & 20 & 10:47:01 \\
\hline Decrease TZ pressure. & $1-20-93$ & 20 & 20 & 11:00:00 \\
\hline Depressurize TZ. & $1-22-93$ & 22 & 22 & $09: 53: 18$ \\
\hline Deflate $T Z$ packer and $G Z$ packer. & $1-22-93$ & 22 & 22 & $09: 54: 30$ \\
\hline Inflate $T Z$ packer. & $1-22-93$ & 22 & 22 & 11:10:11 \\
\hline Shut in $T Z$ packer. & $1-22-93$ & 22 & 22 & $11: 12: 53$ \\
\hline Inflate GZ packer. & $1-22-93$ & 22 & 22 & $11: 13: 47$ \\
\hline Shut in $G Z$ packer. & $1-22-93$ & 22 & 22 & $11: 16: 27$ \\
\hline Increase TZ pressure. & $1-22-93$ & 22 & 22 & $13: 17: 18$ \\
\hline Increase GZ pressure. & $1-22-93$ & 22 & 22 & $13: 20: 30$ \\
\hline$T Z, T Z$ packer, and $G Z$ lost pressure due to leaks. & $1-25-93$ & 25 & 25 & 11:12:48 \\
\hline Inflate $T Z$ packer. & $1-25-93$ & 25 & 25 & $11: 22: 52$ \\
\hline Shut in $T Z$ packer. & $1-25-93$ & 25 & 25 & 11:25:16 \\
\hline Perform gas/brine exchange. & $1-25-93$ & 25 & 25 & 13:33:54 \\
\hline End data file COMP3803. & 1-28-93 & 28 & 28 & 13:04:39 \\
\hline Remove test tool from compliance chamber and move to borehole SCP01. & $2-6-93$ & 37 & 37 & $12: 00: 00$ \\
\hline
\end{tabular}

Table 6-17 indicates the equipment that was used and the duration that each instrument was used during compliance testing of test tool \#38A which was used in testing sequence SCP01-1. 
Table 6-17. Compliance Equipment Associated with Test Tool \#38A; Borehole SCP01; Sequence SCP01-1

\begin{tabular}{|c|c|c|c|c|}
\hline Equipment & Location & Serial \# & Installed & Removed \\
\hline DAS Software & N/A & PERM4F & $1-5-93$ & $2-5-93$ \\
\hline DCU (HP3497A) & N/A & $2629 a 21989$ & $1-5-93$ & $2-5-93$ \\
\hline $\begin{array}{c}\text { Transducer } \\
\text { (Druck PDCR 910) }\end{array}$ & Test Zone & 322422 & $1-5-93$ & $2-5-93$ \\
\hline $\begin{array}{c}\text { Transducer } \\
\text { (Druck PDCR 910) }\end{array}$ & $\begin{array}{l}\text { Test Zone } \\
\text { Packer }\end{array}$ & 308150 & $1-5-93$ & $2-5-93$ \\
\hline $\begin{array}{c}\text { Transducer } \\
\text { (Druck PDCR 910) }\end{array}$ & Guard Zone & 507864 & $1-5-93$ & $2-5-93$ \\
\hline $\begin{array}{c}\text { Transducer } \\
\text { (Druck PDCR 910) }\end{array}$ & $\begin{array}{l}\text { Guard Zone } \\
\text { Packer }\end{array}$ & 308145 & $1-5-93$ & $2-5-93$ \\
\hline $\begin{array}{c}\text { Transducer } \\
\text { (Druck PDCR 910) } \\
\end{array}$ & DPT Panel & 322427 & $1-5-93$ & $2-5-93$ \\
\hline Thermocouple (Type E) & Test Zone & 1 & $1-5-93$ & $2-5-93$ \\
\hline Thermocouple (Type E) & Guard Zone & 2 & $1-5-93$ & $2-5-93$ \\
\hline Injection Column & N/A & 38 & $1-7-93$ & $1-18-93$ \\
\hline Injection Column & N/A & 39 & $1-18-93$ & $1-20-93$ \\
\hline $\begin{array}{c}\text { DPT } \\
\text { (Rosemount 1151DP) }\end{array}$ & N/A & 1140864 & $1-7-93$ & $1-20-93$ \\
\hline
\end{tabular}




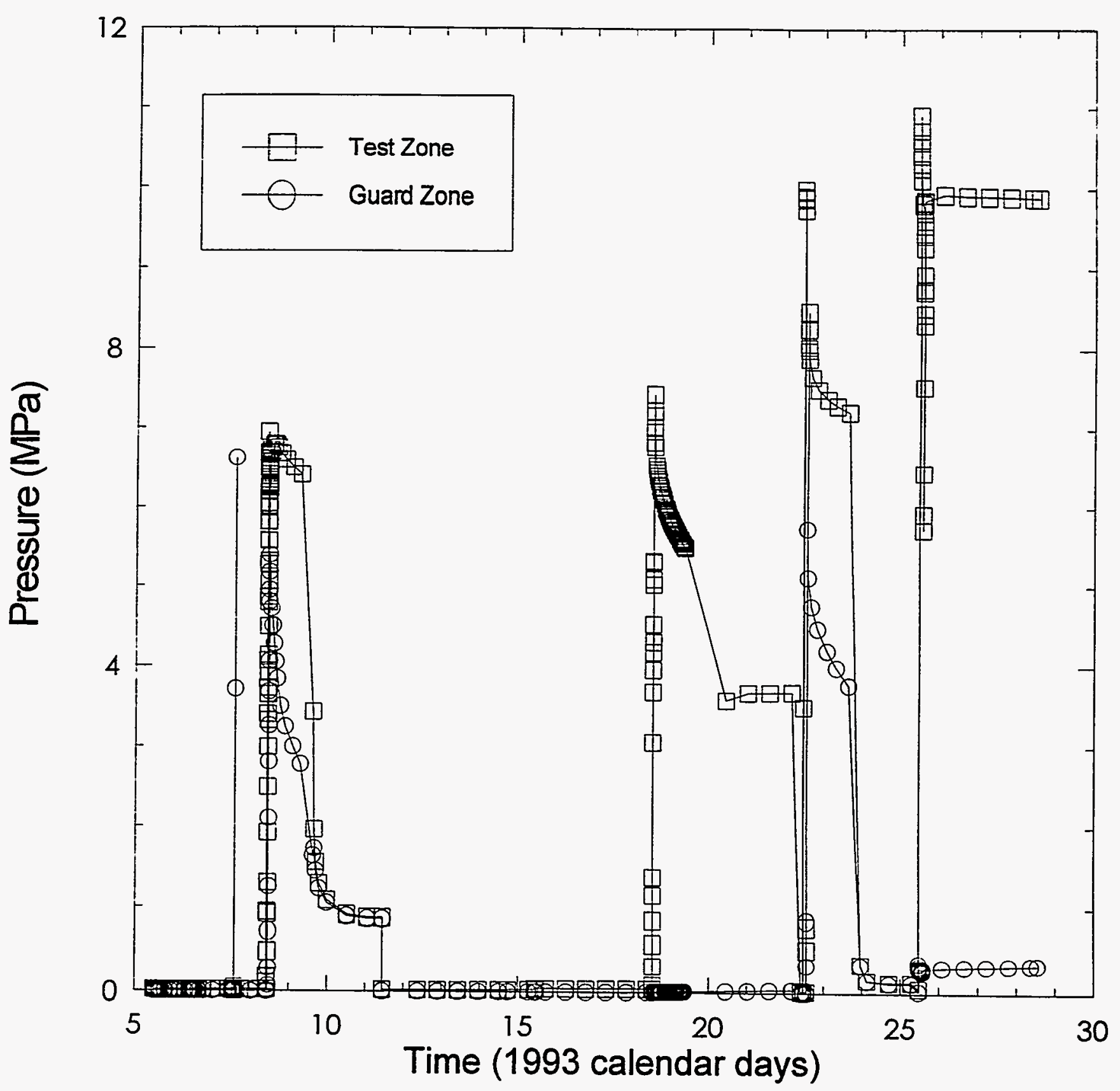

INTERA-4115-2190

Figure 6-32. Zone pressures during compliance testing of test tool \#38A. 


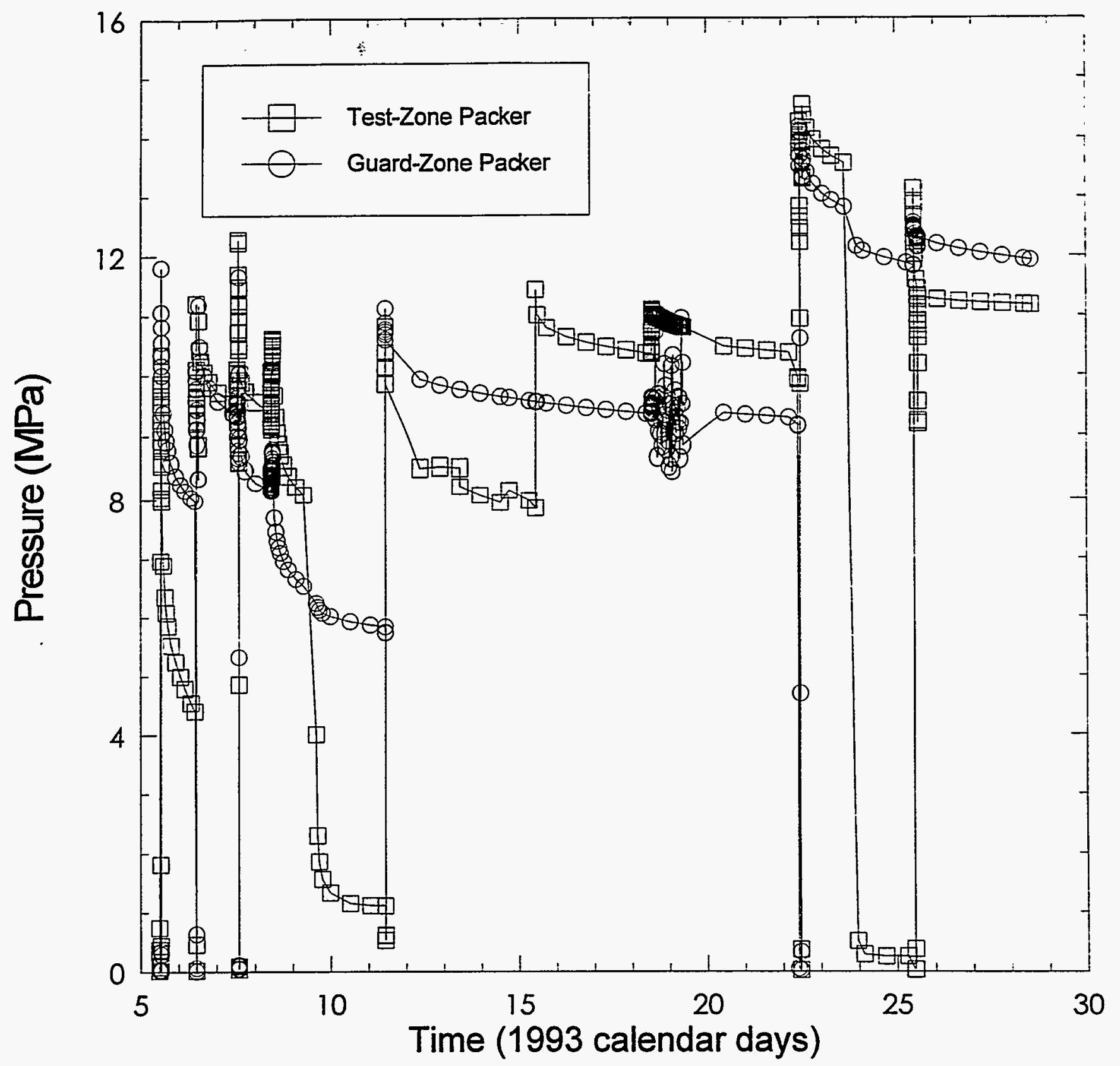

INTERA-61 15-220-0

Figure 6-33. Packer pressures during compliance testing of test tool \#38A. 


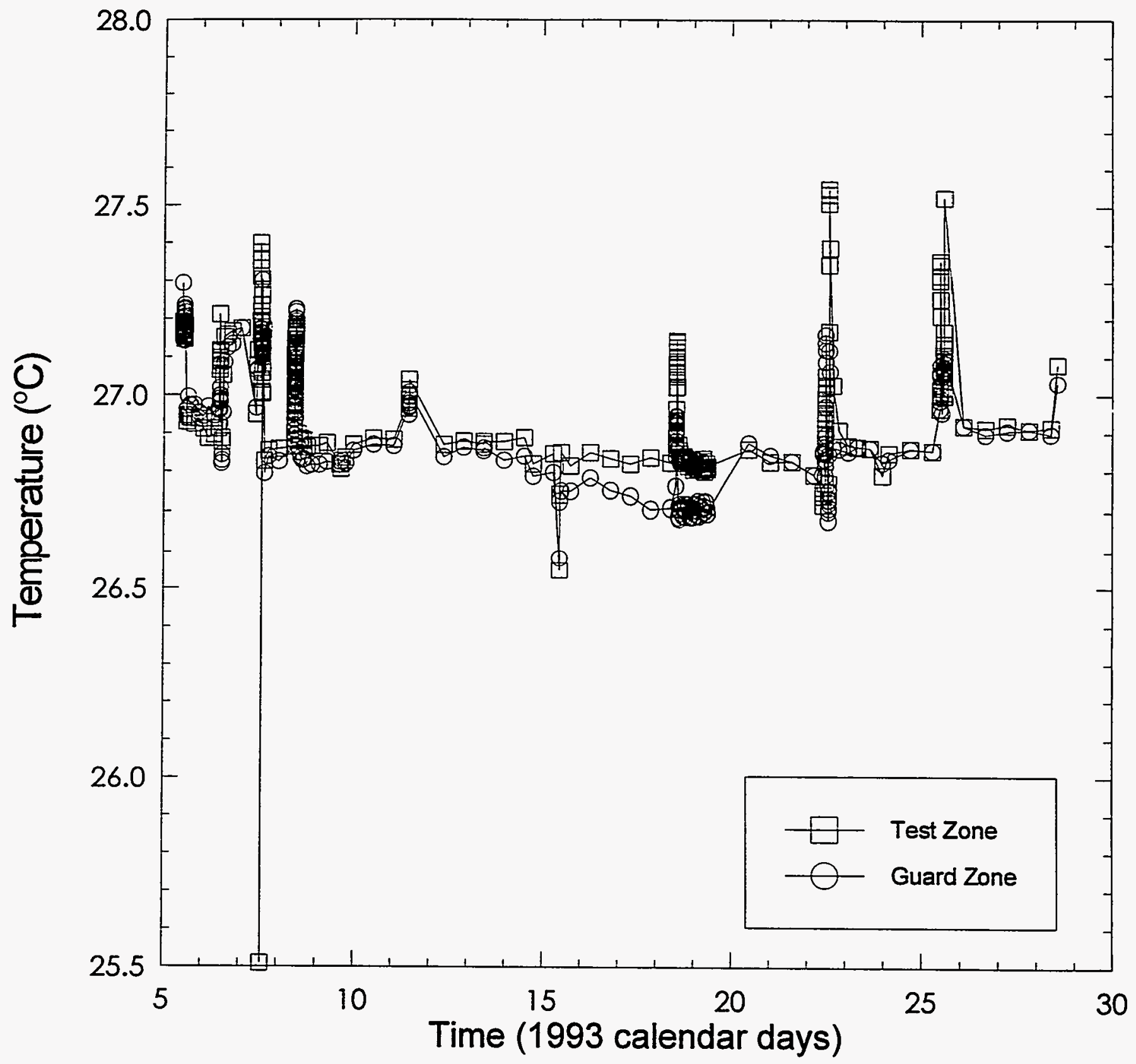

INTERAS115-221-0

Figure 6-34. Zone temperatures during compliance testing of test tool \#38A. 


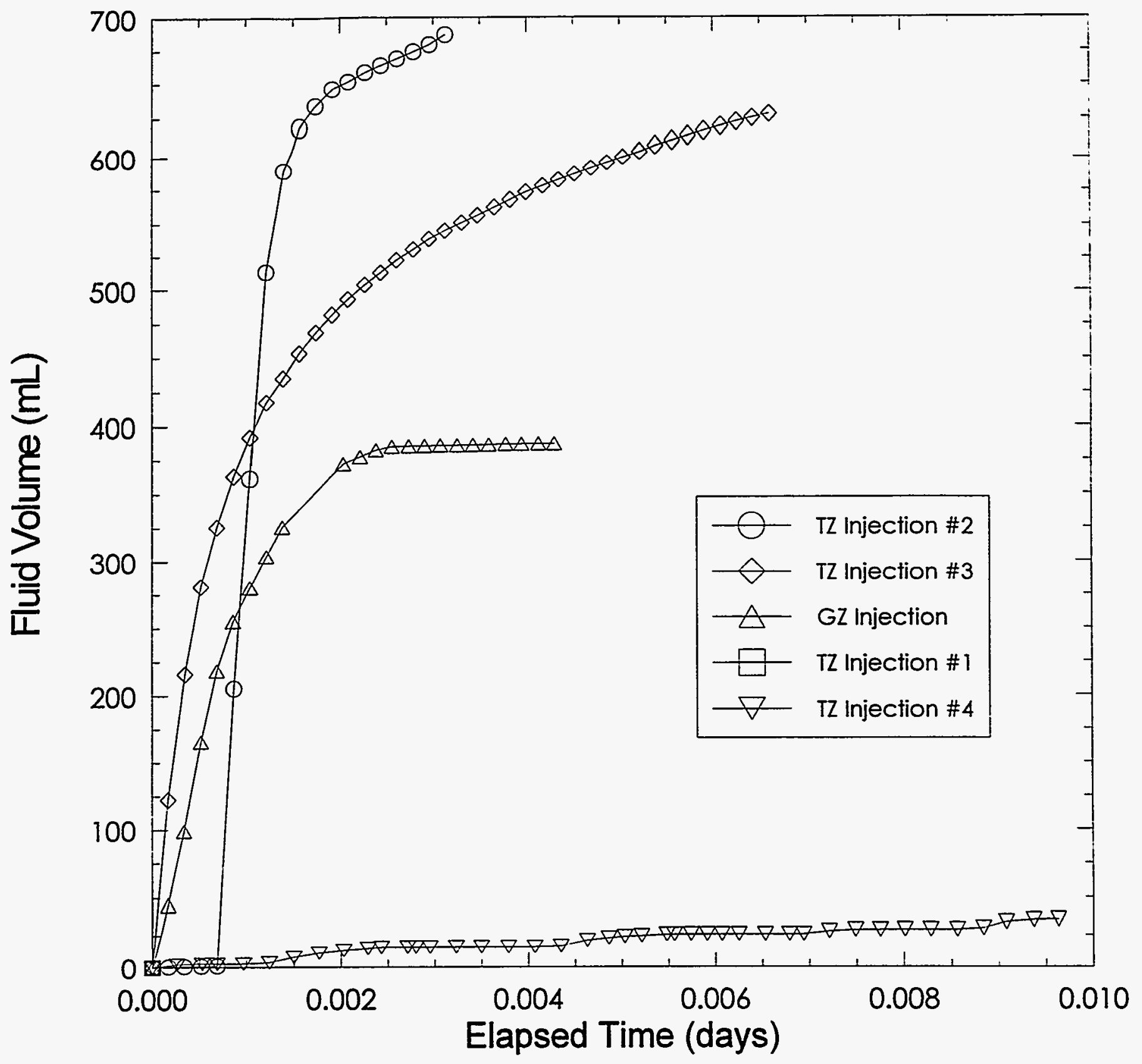

INTERA-6115-222-0

Figure 6-35. Test-zone fluid-injection volumes during compliance testing of test tool \#38A. 


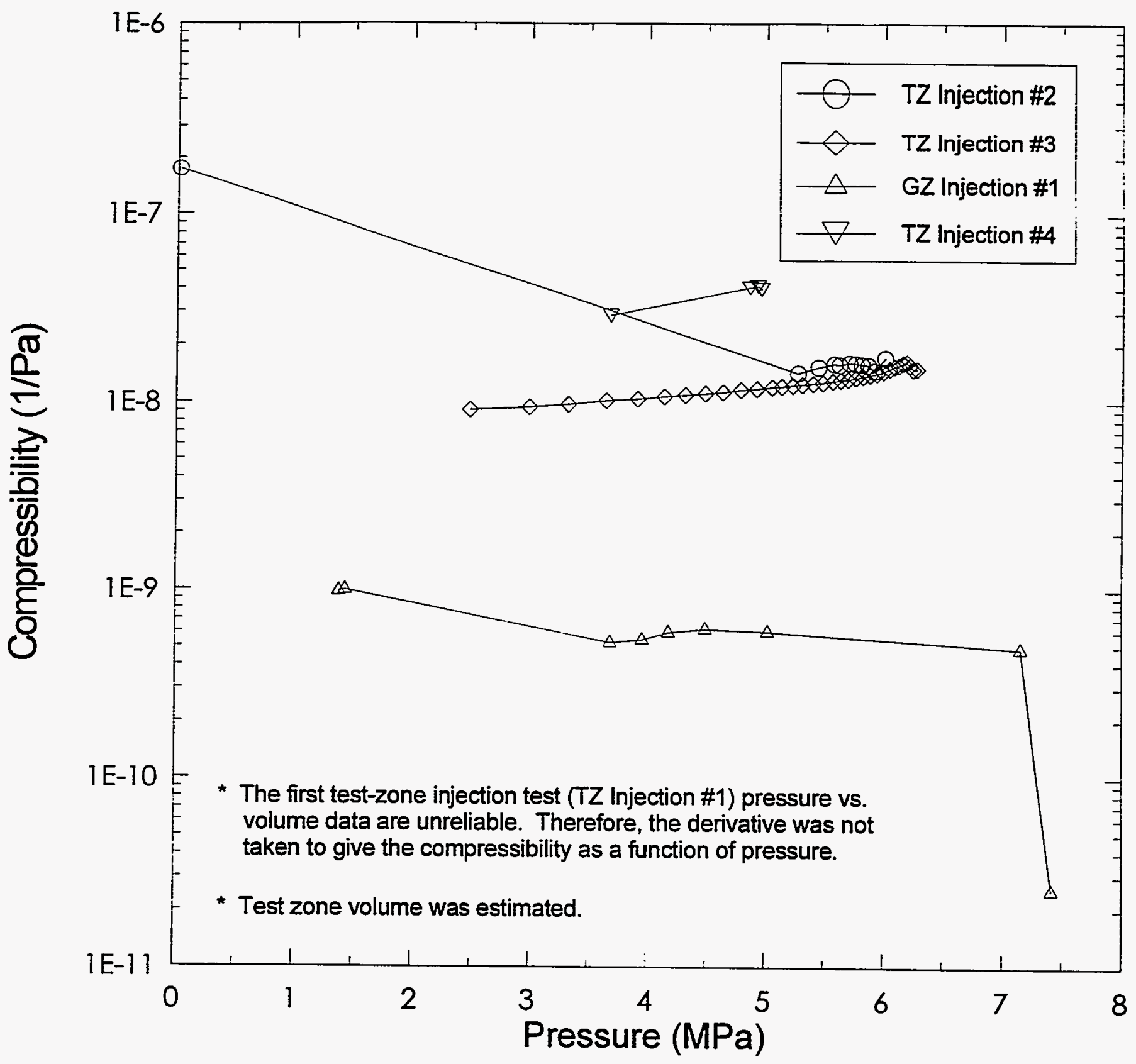

INTERA-6115-223-0

Figure 6-36. Test-zone compressibility as measured during compliance testing of test tool \#38A. 


\subsubsection{Test Tool \#39 (Borehole C1H07, Coupled Permeability and Hydrofracture- Testing Sequence C1X05-B)}

Table 6-18 gives a detailed description of the events that occurred during compliance testing of test tool \#39. Figures 6-37 and 6-38 illustrate the zone and packer pressures, respectively, for test tool \#39. Figure 4-15 illustrates the configuration of test tool \#39 as assembled for compliance testing.

Table 6-18. Events Associated with Compliance Testing of Test Tool \#39; Borehole C1H07; Sequence C1X05-B

\begin{tabular}{|c|c|c|c|c|}
\hline EVENT & DATE & $\begin{array}{l}\text { CALENDAR } \\
\text { DAY }\end{array}$ & $\begin{array}{c}1993 \\
\text { CALENDAR } \\
\text { DAY }\end{array}$ & $\begin{array}{c}\text { TIME } \\
\text { (HH:MM:SS) }\end{array}$ \\
\hline $\begin{array}{l}\text { Assemble multipacker test tool \#39 to be used in borehole } \mathrm{C} 1 \mathrm{H} 07 \text { during testing } \\
\text { sequence } \mathrm{C} 1 \times 05 \mathrm{~B} \text {. }\end{array}$ & $5-15-93$ & 135 & 135 & 12:00:00 \\
\hline Begin data file COMP3901. & $5-18-93$ & 138 & 138 & 09:32:59 \\
\hline Inflate packers. & 5-18-93 & 138 & 138 & 12:34:00 \\
\hline TZ1 packer appears to bo leaking; remove test tool and fix leak. & 5-18-93 & 138 & 138 & 13:00:00 \\
\hline Inflate TZ1 packer and shut in. & 5-19-93 & 139 & 139 & 11:14:00 \\
\hline Inflate $T Z 2$ packer and shut in. & 5-19-93 & 139 & 139 & 11:16:00 \\
\hline Inflate $G Z$ packer and shut in. & 5-19-93 & 139 & 139 & 11:18:00 \\
\hline Increase $T Z 1$ pressure. & $5-20-93$ & 140 & 140 & 11:15:00 \\
\hline Increase TZ2 pressure (no response on transducer). & 5-20-93 & 140 & 140 & 12:15:93 \\
\hline End data file COMP3901. & $5-20-93$ & 140 & 140 & 12:32:14 \\
\hline Remove transducer 308146 and install transducer 322424. & $5-20-93$ & 140 & 140 & 12:35:00 \\
\hline Begin data file COMP3902. & 5-20-93 & 140 & 140 & 12:35:41 \\
\hline Increase $G Z$ pressure. & $5-20-93$ & 140 & 140 & 13:33:00 \\
\hline Repaired leaky fitting on $T Z 2$. & $5-21-93$ & 141 & 141 & 10:14:00 \\
\hline Increase TZ2 pressure to $4.4 \mathrm{MPa}$. & 5-21-93 & 141 & 141 & 10:18:00 \\
\hline Increase GZ pressure to $4.3 \mathrm{MPa}$. & 5-21-93 & 141 & 141 & 10:20:00 \\
\hline End data file COMP3902. & $5-28-93$ & 148 & 148 & 04:07:08 \\
\hline Remove test tool from compliance chamber and move to borehole $\mathrm{C} 1 \mathrm{HO}$. & $5-28-93$ & 148 & 148 & 12:00:00 \\
\hline
\end{tabular}

Table 6-19 indicates the equipment that was used and the duration that each instrument was used during compliance testing of test tool \#39. Test tool \#39 was used in observation borehole $\mathrm{C} 1 \mathrm{H} 07$ during testing sequence $\mathrm{C} 1 \mathrm{X} 05-\mathrm{B}$. 
Table 6-19. Compliance Equipment Associated with Test Tool \#39; Borehole C1H07; Sequence C1X05-B

\begin{tabular}{|c|c|c|c|c|}
\hline Equipment & Location & Serial \# & Installed & Removed \\
\hline DAS Software & N/A & PERM4F & 5-18-93 & $5-28-93$ \\
\hline DCU (HP3497A) & N/A & $2023 a 01688$ & 5-18-93 & $5-28-93$ \\
\hline $\begin{array}{c}\text { Transducer } \\
\text { (Druck PDCR 830) }\end{array}$ & Test Zone 1 & 246909 & $5-18-93$ & $5-28-93$ \\
\hline $\begin{array}{c}\text { Transducer } \\
\text { (Druck PDCR 830) }\end{array}$ & $\begin{array}{c}\text { Test Zone } 1 \\
\text { Packer }\end{array}$ & 246918 & $5-18-93$ & $5-28-93$ \\
\hline $\begin{array}{c}\text { Transducer } \\
\text { (Druck PDCR 910) }\end{array}$ & Test Zone 2 & 308146 & $5-18-93$ & $5-20-93$ \\
\hline $\begin{array}{c}\text { Transducer } \\
\text { (Druck PDCR 830) }\end{array}$ & $\begin{array}{l}\text { Test Zone } 2 \\
\text { Packer }\end{array}$ & 246917 & $5-18-93$ & $5-28-93$ \\
\hline $\begin{array}{c}\text { Transducer } \\
\text { (Druck PDCR 910) }\end{array}$ & Test Zone 2 & 322426 & $5-20-93$ & $5-28-93$ \\
\hline $\begin{array}{c}\text { Transducer } \\
\text { (Druck PDCR 910) }\end{array}$ & Guard Zone & 322424 & $5-18-93$ & $5-28-93$ \\
\hline $\begin{array}{c}\text { Transducer } \\
\text { (Druck PDCR 10/D) }\end{array}$ & $\begin{array}{l}\text { Guard Zone } \\
\text { Packer }\end{array}$ & 211691 & $5-18-93$ & $5-28-93$ \\
\hline
\end{tabular}




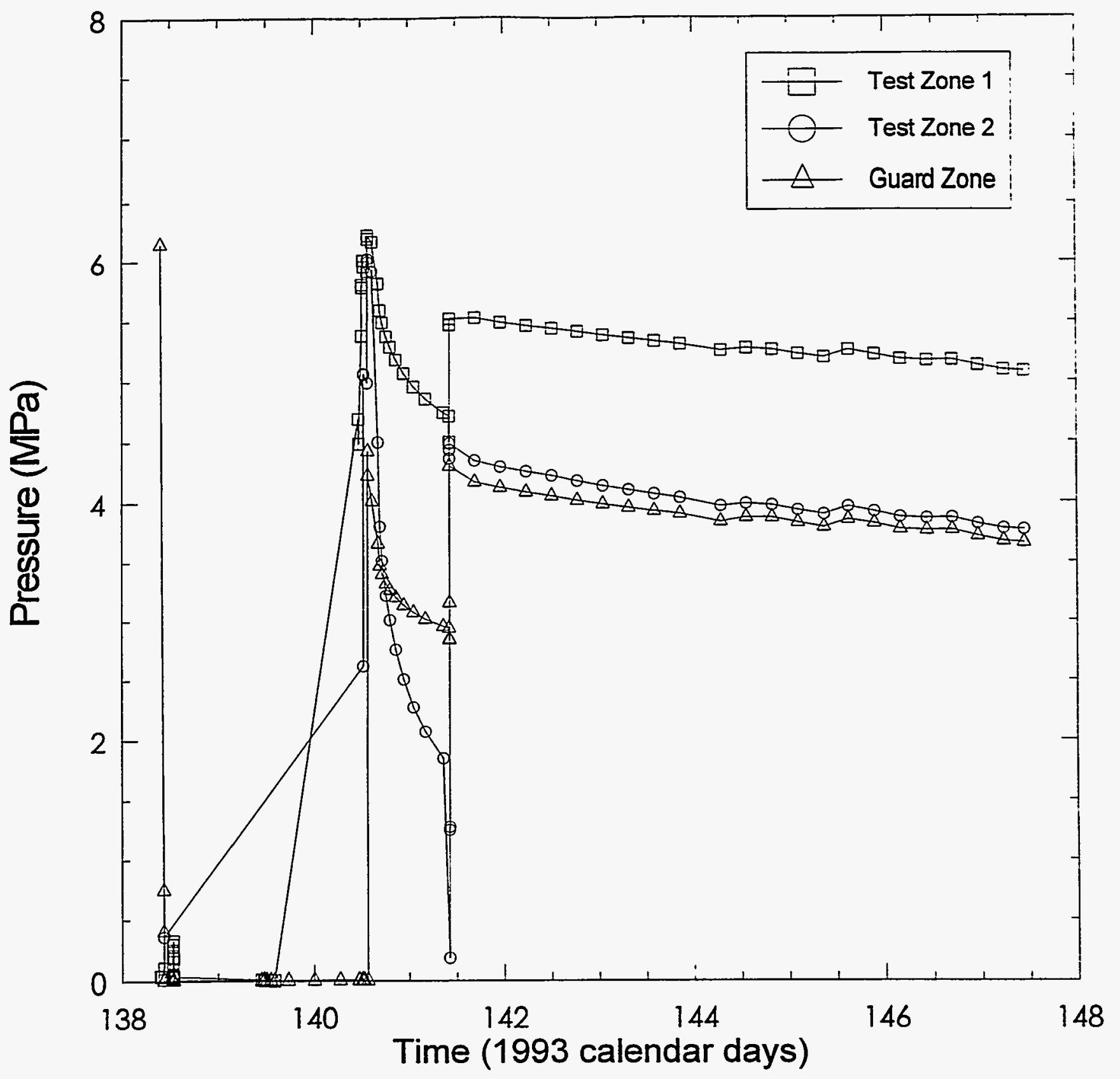

INTERA-6115-224-0

Figure 6-37. Zone pressures during compliance testing of test tool \#39. 


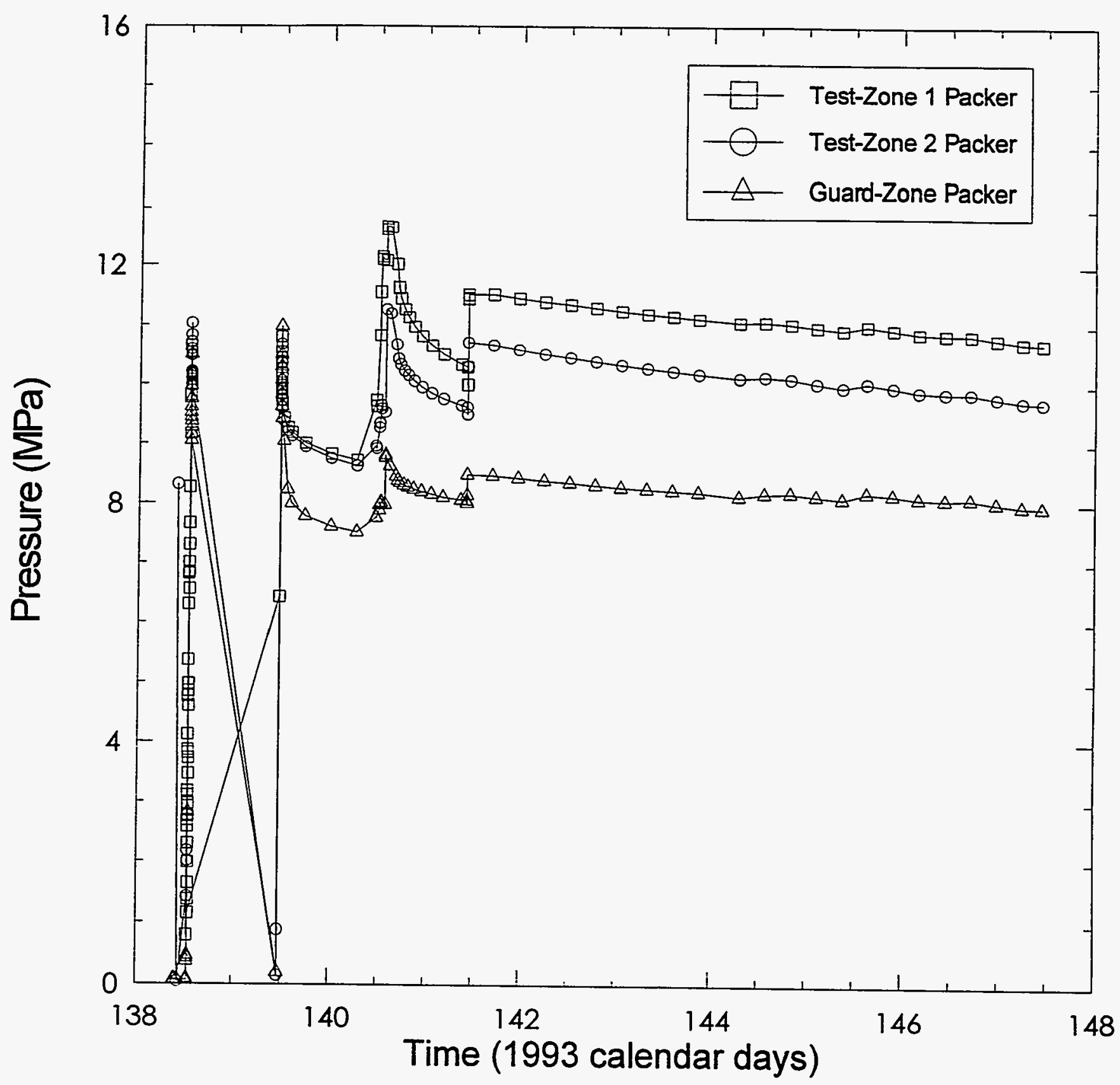

INTERA-115-225-0

Figure 6-38. Packer pressures during compliance testing of test tool \#39. 


\subsubsection{Test Tool \#40 (Borehole C1X06, Coupled Permeability and Hydrofracture- Testing Sequence C1X05-B)}

Table 6-20 gives a detailed description of the events that occurred during compliance testing of test tool \#40. Figures 6-39 and 6-40 illustrate the zone and packer pressures, respectively, for test tool \#40. Figure 4-17 illustrates the configuration of test tool \#40 as assembled for compliance testing.

Table 6-20. Events Associated with Compliance Testing of Test Tool \#40; Borehole C1X06; Sequence C1X05-B

\begin{tabular}{|c|c|c|c|c|}
\hline EVENT & DATE & $\begin{array}{c}\text { CALENDAR } \\
\text { DAY }\end{array}$ & $\begin{array}{c}1993 \\
\text { CALENDAR } \\
\text { DAY }\end{array}$ & $\begin{array}{c}\text { TIME } \\
\text { (HH:MM:SS) }\end{array}$ \\
\hline $\begin{array}{l}\text { Assemble multipacker test tool \#30 to be used in borehole C1X06 during testing } \\
\text { sequence C1X05-B. }\end{array}$ & 7-1-93 & 182 & 182 & $12: 00: 00$ \\
\hline Begin data file COMP4002. & $7-2-93$ & 183 & 183 & $12: 45: 22$ \\
\hline Inflate TZ2 packer. & $7-2-93$ & 183 & 183 & $13: 13: 33$ \\
\hline Shut in TZ2 packer. & $7-2-93$ & 183 & 183 & 13:15:10 \\
\hline Inflate TZ1 packer. & $7-2-93$ & 183 & 183 & 13:18:02 \\
\hline Shut in $7 Z 1$ packer. & $7-2-93$ & 183 & 183 & $13: 19: 20$ \\
\hline Inflate GZ packer. & $7-2-93$ & 183 & 183 & 13:19:45 \\
\hline Shut in GZ packer. & $7-2-93$ & 183 & 183 & 13:21:21 \\
\hline Increase TZ2 pressure. & $7-2-93$ & 183 & 183 & 13:25:21 \\
\hline Increase TZ1 pressure. & $7-2-93$ & 183 & 183 & $13: 40: 30$ \\
\hline Shut in $T Z 2$ and $T Z 1$. & $7-2-93$ & 183 & 183 & 13:40:58 \\
\hline increase $\mathrm{GZ}$ pressure. & $7-2-93$ & 183 & 183 & 13:44:01 \\
\hline Shut in $\mathbf{G Z}$. & $7-2-93$ & 183 & 183 & 13:46:00 \\
\hline Increase $T Z 1$ and $G Z$ pressure. & $7-30-93$ & 211 & 211 & 12:07:14 \\
\hline End data file COMP4002. & $8-2-93$ & 214 & 214 & 06:59:22 \\
\hline Remove test tool \#40 form compliance chamber and move to borehole $\mathrm{C} 1 \times 06$. & 8-4-93 & 216 & 216 & 12:00:00 \\
\hline
\end{tabular}

Table 6-21 indicates the equipment that was used and the duration that each instrument was used during compliance testing of test tool \#40. Test tool \#40 was used in observation borehole $\mathrm{C} 1 \mathrm{X06}$ during coupled permeability and hydrofracture-testing sequence $\mathrm{C} 1 \mathrm{X} 05-\mathrm{B}$. 
Table 6-21. Compliance Equipment Associated with Test Tool \#40; Borehole C1X06; Sequence C1X05-B

\begin{tabular}{|c|c|c|c|c|}
\hline Equipment & Location & Serial \# & Installed & Removed \\
\hline DAS Software & N/A & PERM4F & $7-2-93$ & $8-4-93$ \\
\hline DCU (HP3497A) & N/A & $2629 a 21989$ & $7-2-93$ & $8-4-93$ \\
\hline $\begin{array}{c}\text { Transducer } \\
\text { (Druck PDCR 830) }\end{array}$ & Test Zone 1 & 246918 & $7-2-93$ & $8-4-93$ \\
\hline $\begin{array}{c}\text { Transducer } \\
\text { (Druck PDCR 910) }\end{array}$ & $\begin{array}{l}\text { Test Zone } 1 \\
\text { Packer }\end{array}$ & 308143 & $7-2-93$ & $8-4-93$ \\
\hline $\begin{array}{c}\text { Transducer } \\
\text { (Druck PDCR 830) }\end{array}$ & Test Zone 2 & 246916 & $7-2-93$ & $8-4-93$ \\
\hline $\begin{array}{c}\text { Transducer } \\
\text { (Druck PDCR 910) }\end{array}$ & $\begin{array}{c}\text { Test Zone } 2 \text { \& } \\
\text { Guard Zone } \\
\text { Packer }\end{array}$ & 308148 & $7-2-93$ & $8-4-93$ \\
\hline $\begin{array}{c}\text { Transducer } \\
\text { (Druck PDCR 10/D) }\end{array}$ & Guard Zone & 211691 & $7-2-93$ & $8-4-93$ \\
\hline
\end{tabular}




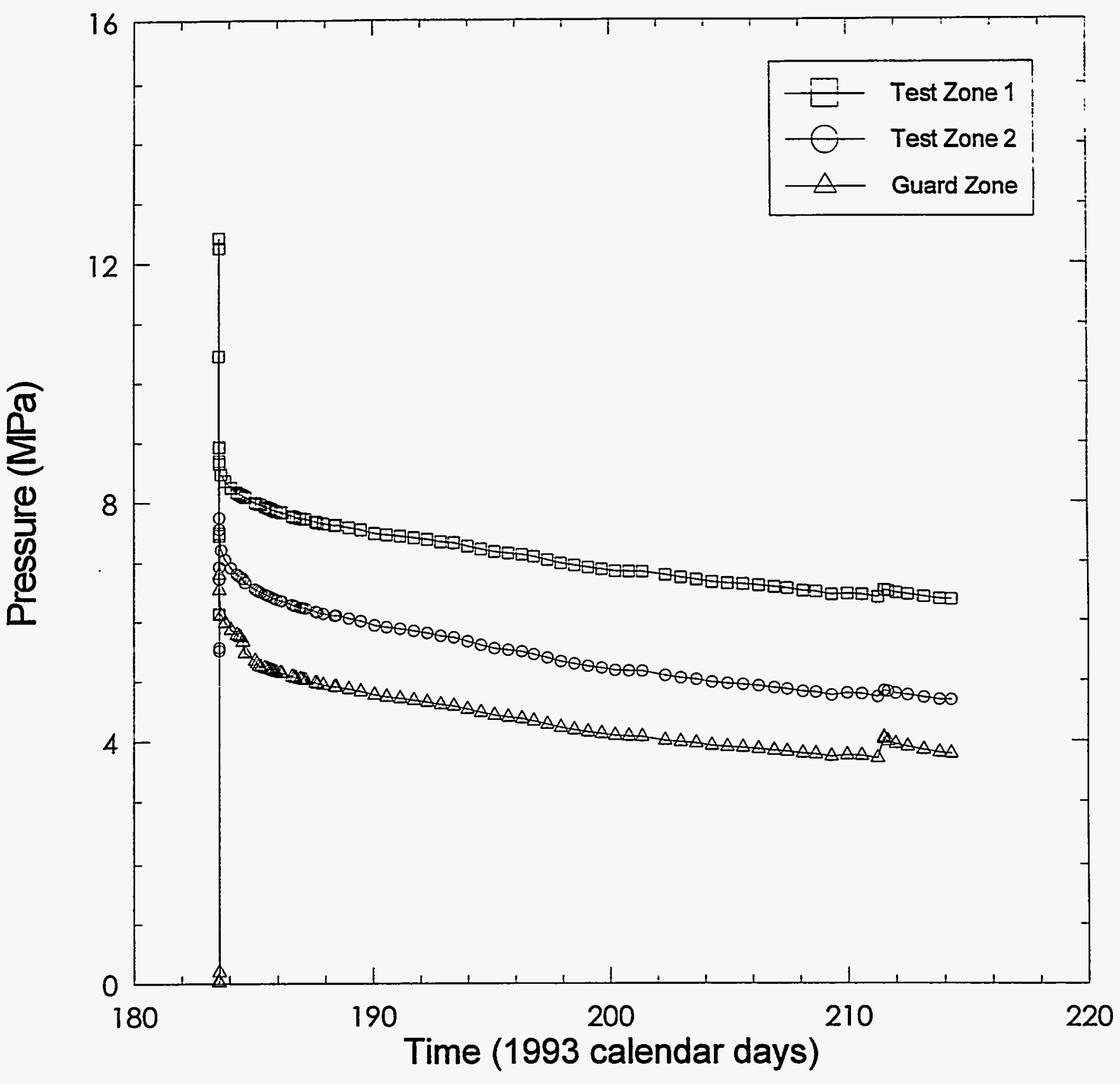

INTERA-6115-228-0

Figure 6-39. Zone pressures during compliance testing of test tool \#40. 


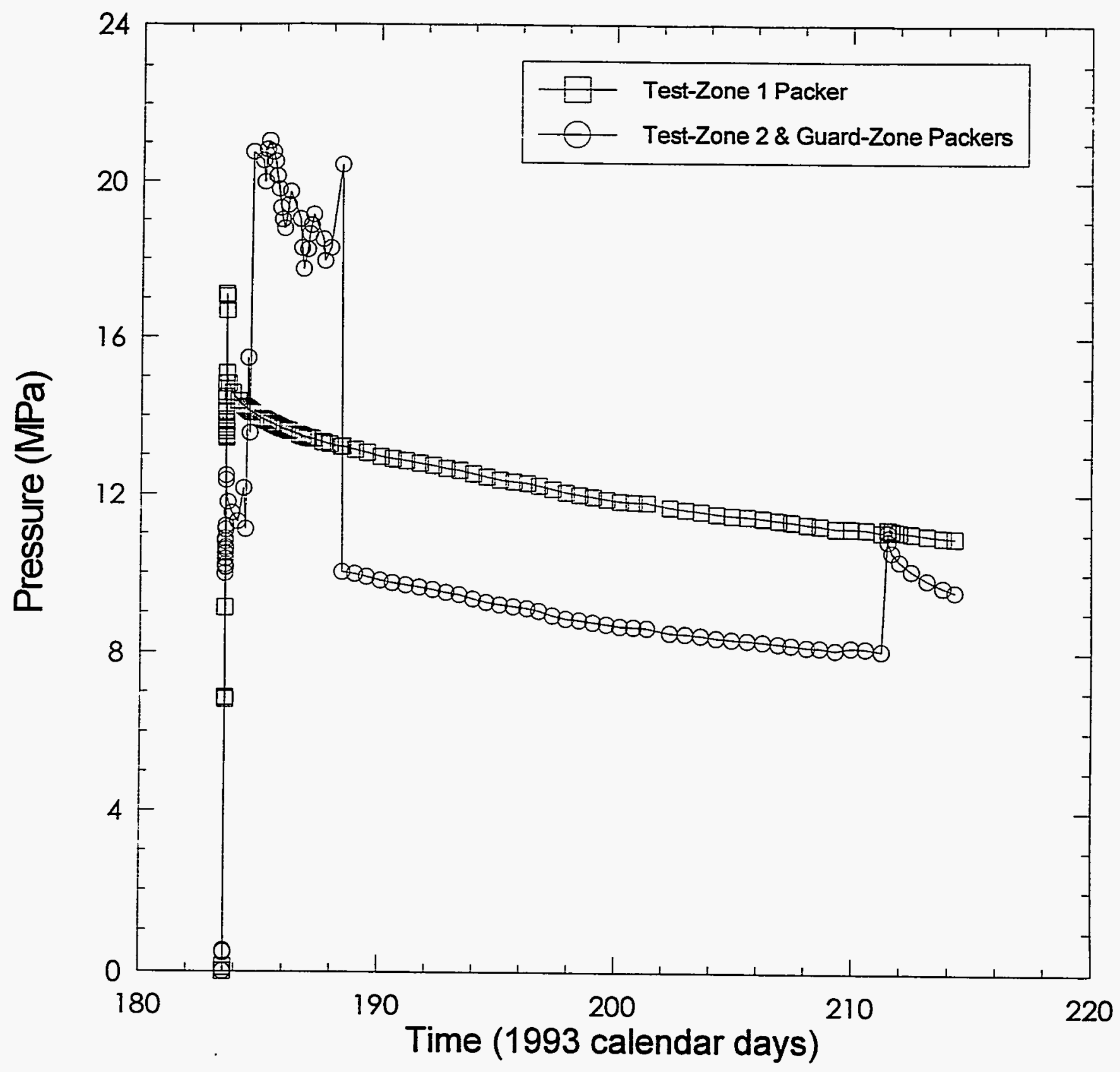

INTERA- 115-227-0

Figure 6-40. Packer pressures during compliance testing of test tool $\# 40$. 


\subsubsection{Test Tool \#41 (Permeability-Testing Sequence L4P51-C2)}

Table 6-22 gives a detailed description of the events that occurred during compliance testing of test tool \#41. Figures 6-41 through 6-43 illustrate the zone pressures, packer pressures, and test zone temperature, respectively, for multipacker test tool \#41. Figure 3-24 illustrates the configuration of test tool \#41 as assembled for compliance testing.

Table 6-22. Events Associated with Compliance Testing of Test Tool \#41; Borehole L4P51; Sequence L4P51-C2

\begin{tabular}{|c|c|c|c|c|}
\hline EVENT & DATE & $\begin{array}{l}\text { CALENDAR } \\
\text { DAY }\end{array}$ & $\begin{array}{c}1993 \\
\text { CALENDAR } \\
\text { DAY }\end{array}$ & $\begin{array}{c}\text { TIME } \\
\text { (HH:MM:SS) }\end{array}$ \\
\hline $\begin{array}{l}\text { Assemble multipacker test tool \#41 to be used in borehole L4P51 during testing } \\
\text { sequence L4P51-C2. }\end{array}$ & $10-2493$ & 297 & 297 & 12:00:00 \\
\hline Inflate TZ1 packer. & 10-26-93 & 299 & 299 & 09:27:29 \\
\hline Deflate TZ1 packer. & 10-26-93 & 299 & 299 & 09:28:00 \\
\hline Inflate TZ1 packer. & $10-26-93$ & 299 & 299 & 09:30:00 \\
\hline Deflate TZ1 packer. & 10-26-93 & 299 & 299 & 09:31:00 \\
\hline Inflate TZ1 packer. & 10-26-93 & 299 & 299 & 09:37:08 \\
\hline Shut in $T Z 1$ packer. & 10-26-93 & 299 & 299 & 09:38:46 \\
\hline Inflate TZ2 packer (incorrect response). & $10-26-93$ & 299 & 299 & 09:43:25 \\
\hline Removed test tool to find mislabeled inflation lines. & $10-26-93$ & 299 & 299 & 13:00:00 \\
\hline Begin data file COMP4101. & 10-26-93 & 299 & 299 & 13:37:03 \\
\hline Inflate TZ1 packer. & $10-27-93$ & 300 & 300 & 08:10:00 \\
\hline Inflate $T Z 2$ packer. & $10-27-93$ & 300 & 300 & 08:25:00 \\
\hline Decrease TZ1 packer pressure. & $10-27-93$ & 300 & 300 & 08:25:30 \\
\hline Innlete GZ packer. & $10-27-93$ & 300 & 300 & 08:35:00 \\
\hline Increase $T Z 1$ pressure. & 10-28-93 & 301 & 301 & 07:53:00 \\
\hline Increase $T Z 1$ pressure. & 10-28-93 & 301 & 301 & 07:55:00 \\
\hline Increase $T Z 2$ packer pressure to $\sim 10.3 \mathrm{MPa}$. & 10-28-93 & 301 & 301 & 08:15:00 \\
\hline TZ1 will not maintain pressure. & 10-28-93 & 301 & 301 & 09:00:00 \\
\hline Deflate packers. & $10-28-93$ & 301 & 301 & 09:02:00 \\
\hline Remove test tool and fix leaky fitting. & $10-28-93$ & 301 & 301 & 09:30:00 \\
\hline Reinstall test tool in compliance chamber. & 10-28-93 & 301 & 301 & 10:02:00 \\
\hline Inflate TZ1 packer. & $10-28-93$ & 301 & 301 & 11:09:00 \\
\hline Inflate TZ2 packer. & 10-28-93 & 301 & 301 & 11:12:00 \\
\hline Inflate GZ packer. & $10-28-93$ & 301 & 301 & 11:15:00 \\
\hline Increase TZ1 pressure. & $10-28-93$ & 301 & 301 & 12:22:00 \\
\hline Increase TZ2 pressure. & 10-28-93 & 301 & 301 & 12:23:00 \\
\hline Increase GZ pressure. & $10-28-93$ & 301 & 301 & 12:25:00 \\
\hline End data file COMP4101. & $10-28.93$ & 301 & 301 & 12:32:39 \\
\hline Begin data file COMP4102. & 10-28-93 & 301 & 301 & 12:36:51 \\
\hline
\end{tabular}


Table 6-22 (Continued). Events Associated with Compliance Testing of Test Tool \#41; Borehole L4P51; Sequence L4P51-C2

\begin{tabular}{ccccc}
\hline EVENT & DATE & $\begin{array}{c}\text { CALENDAR } \\
\text { DAY }\end{array}$ & $\begin{array}{c}\text { 1993 } \\
\text { CALENDAR } \\
\text { DAY }\end{array}$ & $\begin{array}{c}\text { TIME } \\
\text { (HH:MM:SS) }\end{array}$ \\
\hline P5 gage is reading incorrectly (high) as determined by Heise gage. & $11-2-93$ & 306 & 306 & $10: 42: 15$ \\
End data fite coMP4102. & $11-11-93$ & 315 & 315 & $12: 31: 36$ \\
Remove test tool \#41 from compliance chamber and move to borehole L4P51. & $11-16-93$ & 320 & 320 & $12: 00: 00$ \\
\hline
\end{tabular}

Table 6-23 indicates the equipment that was used and the duration that each instrument was used during compliance testing of test tool \#41. Test tool \#41 was used in permeability-testing sequence L4P51-C2.

Table 6-23. Compliance Equipment Associated with Test Tool \#41; Borehole L4P51; Sequence L4P51-C2

\begin{tabular}{|c|c|c|c|c|}
\hline Equipment & Location & Serial \# & Installed & Removed \\
\hline DAS Software & N/A & PERM4F & $10-26-93$ & $11-16-93$ \\
\hline DCU (HP3497A) & N/A & $2629 a 21990$ & $10-26-93$ & $11-16-93$ \\
\hline $\begin{array}{c}\text { Transducer } \\
\text { (Druck PDCR 830) }\end{array}$ & Test Zone 1 & 246912 & $10-26-93$ & $11-16-93$ \\
\hline $\begin{array}{c}\text { Transducer } \\
\text { (Druck PDCR 830) }\end{array}$ & $\begin{array}{l}\text { Test Zone } 1 \\
\text { Packer }\end{array}$ & 246919 & $10-26-93$ & $11-16-93$ \\
\hline $\begin{array}{c}\text { Transducer } \\
\text { (Druck PDCR 830) }\end{array}$ & Test Zone 2 & 246910 & $10-26-93$ & $11-16-93$ \\
\hline $\begin{array}{c}\text { Transducer } \\
\text { (Druck PDCR 910) }\end{array}$ & $\begin{array}{l}\text { Test Zone } 2 \\
\text { Packer }\end{array}$ & 308152 & $10-26-93$ & $11-16-93$ \\
\hline $\begin{array}{c}\text { Transducer } \\
\text { (Druck PDCR 910) }\end{array}$ & Guard Zone & 308143 & $10-26-93$ & $11-16-93$ \\
\hline $\begin{array}{c}\text { Transducer } \\
\text { (Druck PDCR 910) } \\
\end{array}$ & $\begin{array}{c}\text { Guard Zone } \\
\text { Packer }\end{array}$ & 322423 & $10-26-93$ & $11-16-93$ \\
\hline Thermocouple (Type E) & Test Zone 1 & 1 & $10-26-93$ & $11-16-93$ \\
\hline
\end{tabular}




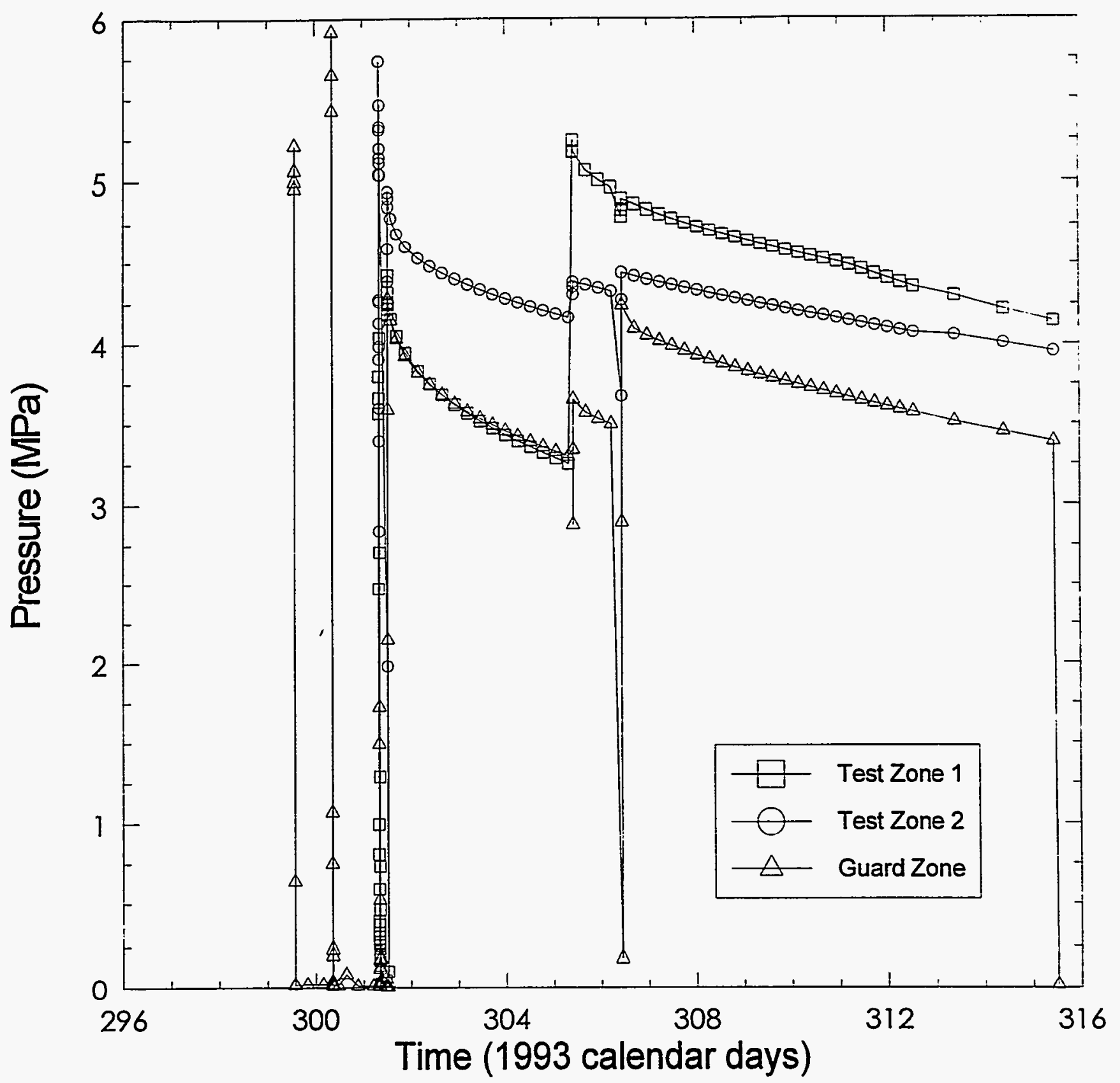

INTERA-6115-228-0

Figure 6-41. Zone pressures during compliance testing of test tool \#41. 


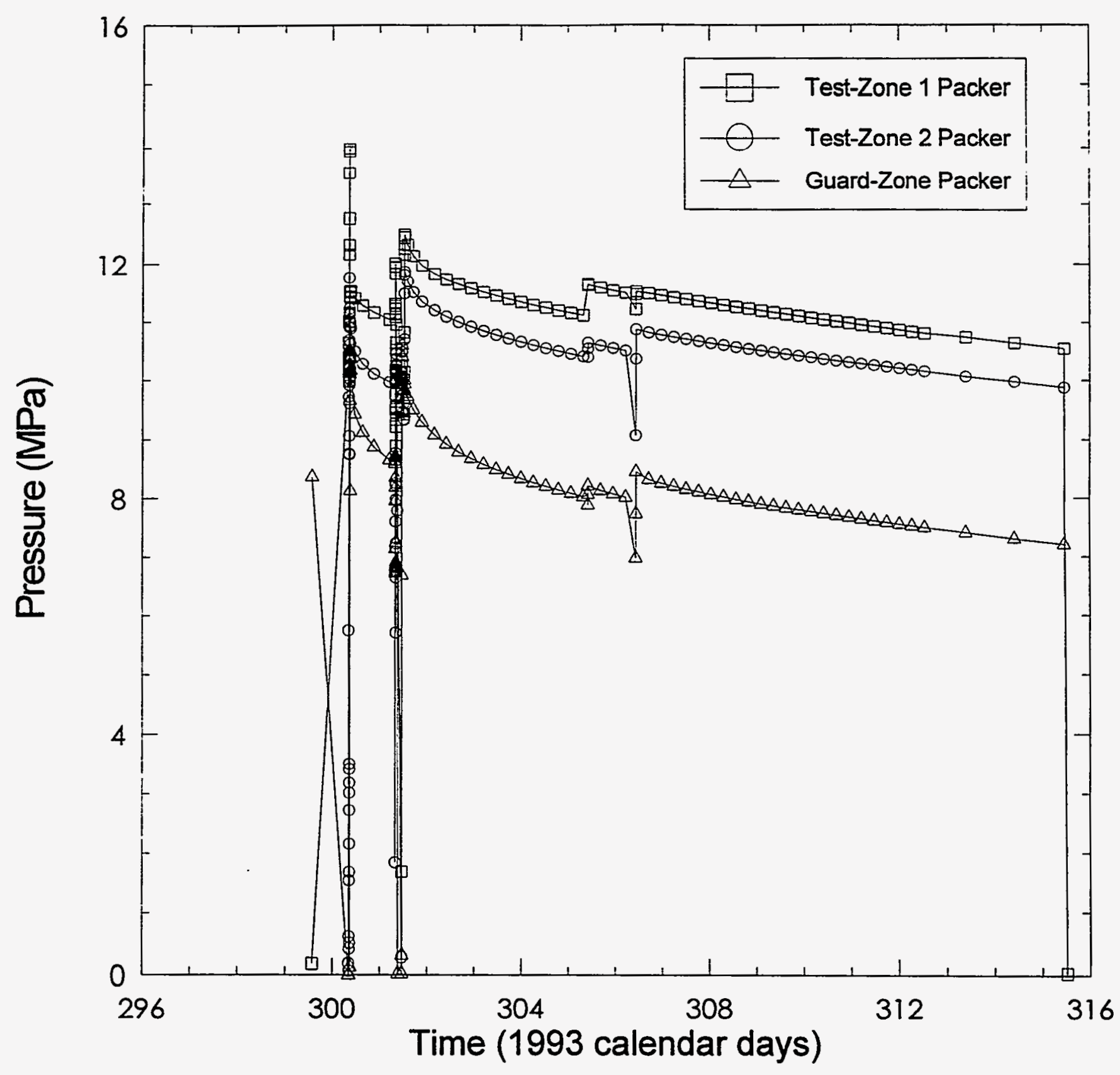

INTERA-6115-229-0

Figure 6-42. Packer pressures during compliance testing of test tool \#41. 


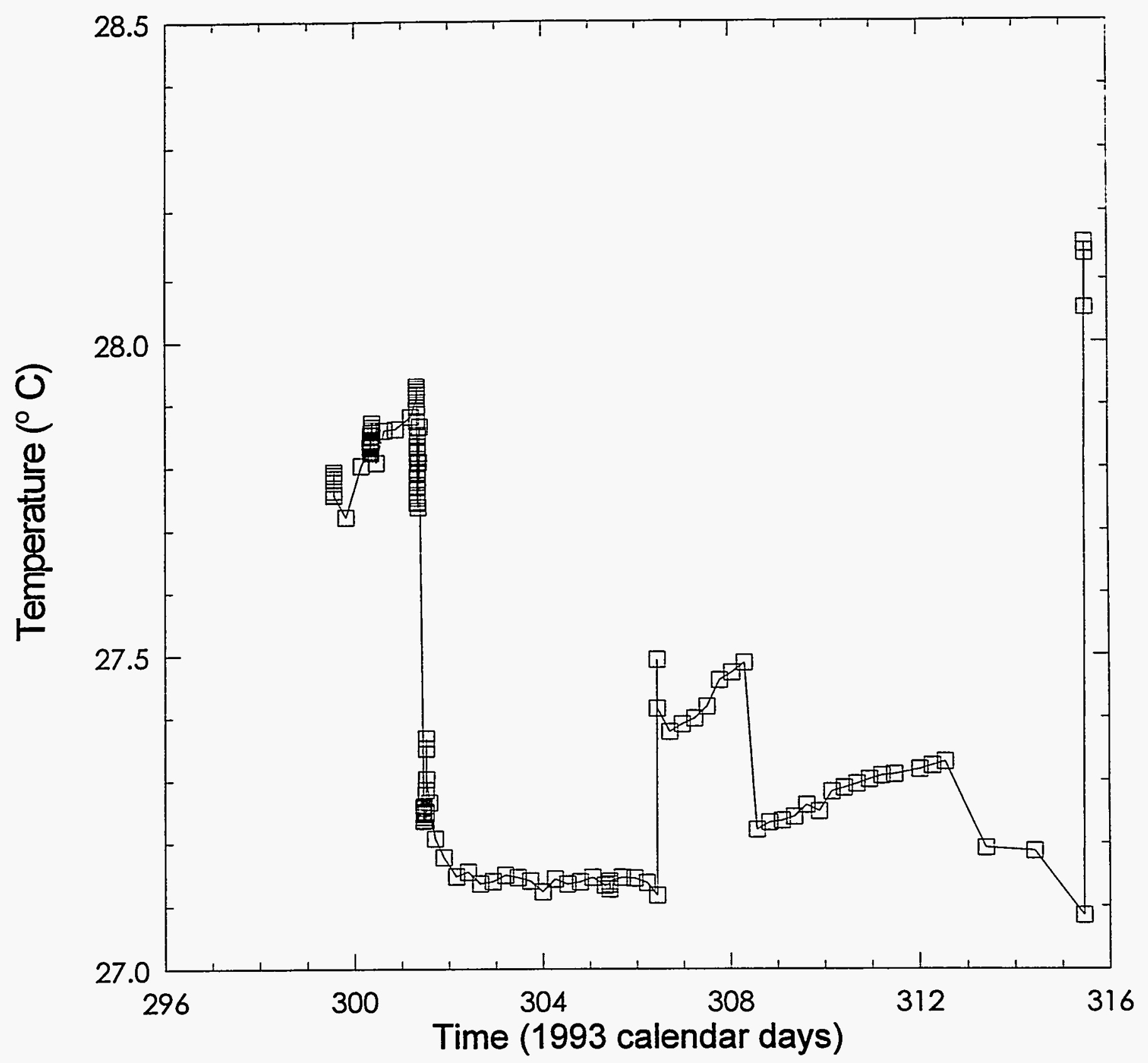

INTERA-6115-230-0

Figure 6-43. Test-zone 1 temperature during compliance testing of test tool \#41. 


\subsubsection{Test Tool \#BOT- 01 (Gas-Threshold Pressure-Testing Sequence SCP01-2)}

Table 6-24 gives a detailed description of the events that occurred during compliance testing of test tool \#BOT-01. Figures 6-44 through 6-47 illustrate the zone pressures, packer pressures, fluid-injection volumes during test-zone compressibility tests, and testzone compressibility as a function of pressure, respectively, for multipacker test tool \#BOT01. Figure 5-7 illustrates the configuration of test tool \#BOT-01 as assembled for compliance testing.

Table 6-24. Events Associated with Compliance Testing of Test Tool \#BOT-01; Borehole SCP01; Sequence SCP01-2

\begin{tabular}{|c|c|c|c|c|}
\hline EVENT & DATE & $\begin{array}{c}\text { CALENDAR } \\
\text { DAY }\end{array}$ & $\begin{array}{c}1994 \\
\text { CALENDAR } \\
\text { DAY }\end{array}$ & $\begin{array}{c}\text { TIME } \\
\text { (HH:MM:SS) }\end{array}$ \\
\hline $\begin{array}{l}\text { Assemble multipacker test tool \#BOT-01 to be used in borehole SCP01 during testing } \\
\text { sequence SCP01-2. }\end{array}$ & $3-16-94$ & 75 & 75 & $12: 00: 00$ \\
\hline Begin data file BAKER1. & $3-17-94$ & 76 & 76 & 15:05:15 \\
\hline Inflate TZ packer to $17.2 \mathrm{MPa}$. & $3-17-94$ & 76 & 76 & 15:15:00 \\
\hline Inflate GZ packer to $17.7 \mathrm{MPa}$. & 3-17-94 & 76 & 76 & $15: 25: 00$ \\
\hline Increase $T Z$ pressuro to $13.6 \mathrm{MPa}$. & $3-17-94$ & 76 & 76 & $15: 28: 00$ \\
\hline Increase GZ pressure to $-11.0 \mathrm{MPa}$. & $3-17-94$ & 76 & 76 & 15:30:00 \\
\hline Depressurize GZ. & $3-17-94$ & 76 & 76 & $15: 35: 00$ \\
\hline Increase $\mathrm{GZ}$ pressure to $10.8 \mathrm{MPa}$. & 3-17-94 & 76 & 76 & $15: 42: 00$ \\
\hline Depressurize GZ. & 3-17-94 & 76 & 76 & 15:52:00 \\
\hline Increase $\mathrm{GZ}$ pressure to $10.525 \mathrm{MPa}$. & 3-17-94 & 76 & 76 & 15:54:00 \\
\hline DAS not functioning upon arrival. & 3-18-94 & $\pi 7$ & 77 & 09:47:00 \\
\hline Depressurize GZ. & 3-18-94 & 77 & 77 & 09:49:00 \\
\hline Depressurize TZ. & 3-18-94 & 77 & 77 & 09:50:00 \\
\hline Deflate GZ packer. & $3-18-94$ & 77 & 77 & 09:54:00 \\
\hline Deflate TZ packer. & 3-18-94 & 77 & 77 & 10:05:00 \\
\hline Remove test tool from compliance chamber to fix leak. & 3-18-94 & 77 & 77 & 10:10:00 \\
\hline Reinstall test tool in compliance chamber. & 3-18-94 & 77 & 77 & 10:40:00 \\
\hline Inflate packers and increase $T Z$ and $G Z$ pressure. & 3-18-94 & 77 & 77 & 12:15:00 \\
\hline $\begin{array}{l}\text { Increase } T Z \text { packer and } G Z \text { packer pressure to }-17 \mathrm{MPa} \text {, increase } T Z \text { pressure to } \\
-13.56 \mathrm{MPa} \text {, and thcrease } G Z \text { pressure to }-10.2 \mathrm{MPa} \text {. }\end{array}$ & $3-21-94$ & 80 & 80 & 13:51:00 \\
\hline Decrease TZ pressure. & $3-23-94$ & 82 & 82 & 11:42:00 \\
\hline Decrease TZ packer pressure. & $3-23-94$ & 82 & 82 & 11:44:00 \\
\hline Decrease GZ pressure. & $3-25-94$ & 84 & 84 & $12: 50: 40$ \\
\hline End data file BAKER1. & $3-25-94$ & 84 & 84 & 13:15:10 \\
\hline Remove transducer 609364. & $3-25-94$ & 84 & 84 & 13:16:00 \\
\hline Begin data file BAKER2. & $3-25-94$ & 84 & 84 & 13:24:06 \\
\hline Increase $T Z, G Z$, and packer pressures. & 3-31-94 & 90 & 90 & 14:04:00 \\
\hline End data file BAKER2. & 3-31-94 & 90 & 90 & 14:08:54 \\
\hline
\end{tabular}


Table 6-24 (Continued). Events Associated with Compliance Testing of Test Tool \#BOT-01; Borehole SCP01; Sequence SCP01-2

\begin{tabular}{|c|c|c|c|c|}
\hline EVENT & DATE & $\begin{array}{c}\text { CALENDAR } \\
\text { DAY }\end{array}$ & $\begin{array}{c}1994 \\
\text { CALENDAR } \\
\text { DAY }\end{array}$ & $\begin{array}{c}\text { TIME } \\
\text { (HH:MM:SS) }\end{array}$ \\
\hline Install transducer 609370 to monitor $T Z$ packer. & 3-31-94 & 90 & 90 & 14:10:00 \\
\hline Begin data file BAKER3. & 3-31-94 & so & 90 & 14:17:08 \\
\hline End data file BAKER3. & $4-7-94$ & 97 & 97 & 14:59:36 \\
\hline Incorporate DPT panel with new transducer into system. & 4-8-94 & 98 & 98 & 13:11:00 \\
\hline Begin data file BAKER4. & $4-8-94$ & 98 & 98 & 13:17:59 \\
\hline Depressurizo $T Z$ by removing $-65 \mathrm{~mL}$ of fluid. & 4-11-94 & 101 & 101 & 13:28:00 \\
\hline Increase $T Z$ pressure. & $4-11-94$ & 101 & 101 & 13:36:00 \\
\hline Begin $T Z$ compressiblity test. & 4-12-94 & 102 & 102 & 11:46:17 \\
\hline Shut in TZ. & $4-12-94$ & 102 & 102 & 12:42:44 \\
\hline Begin TZ compressibility test. & 4-12-94 & 102 & 102 & 12:45:09 \\
\hline Shut in $T Z$ & 4-12-94 & 102 & 102 & 13:04:08 \\
\hline Begin $T Z$ compressibility test. & 4-12-94 & 102 & 102 & 13:05:09 \\
\hline Shut in $T$. & 4-12-94 & 102 & 102 & 13:17:18 \\
\hline Depressurize TZ and GZ. & 4-13-94 & 103 & 103 & 12:22:00 \\
\hline Deflate packers. & 4-13-94 & 103 & 103 & 12:23:00 \\
\hline End data file BAKER4. & 4-13-94 & 103 & 103 & 13:18:02 \\
\hline Remove test tool \#BOT-01 from compliance chamber and move to borehole SCP01. & 4-13-94 & 103 & 103 & 15:00:00 \\
\hline
\end{tabular}

Table 6-25 indicates the equipment that was used and the duration that each instrument was used during compliance testing of test tool \#BOT-01. Test tool \#BOT-01 was used in testing sequence SCP01-2.

Table 6-25. Compliance Equipment Associated with Test Tool \#BOT-01; Borehole SCP01; Sequence SCP01-2

\begin{tabular}{|c|c|c|c|c|}
\hline Equipment & Location & Serial \# & Installed & Removed \\
\hline DAS Software & N/A & PERM4F & $3-17-94$ & $4-13-94$ \\
\hline DCU (HP3497A) & N/A & $2629 a 21996$ & $3-17-94$ & 4-13-94 \\
\hline $\begin{array}{c}\text { Transducer } \\
\text { (Druck D930-18) }\end{array}$ & Test Zone & 609371 & $3-17-94$ & $4-13-94$ \\
\hline $\begin{array}{c}\text { Transducer } \\
\text { (Druck D930-18) }\end{array}$ & $\begin{array}{l}\text { Test Zone } \\
\text { Packer }\end{array}$ & 609364 & $3-17-94$ & $3-25-94$ \\
\hline $\begin{array}{c}\text { Transducer } \\
\text { (Druck D930-18) }\end{array}$ & $\begin{array}{l}\text { Test Zone } \\
\text { Packer }\end{array}$ & 609370 & $3-31-94$ & $4-13-94$ \\
\hline $\begin{array}{c}\text { Transducer } \\
\text { (Druck D930-18) }\end{array}$ & Guard Zone & 609375 & $3-17-94$ & 4-13-94 \\
\hline
\end{tabular}


Table 6-25 (Continued). Compliance Equipment Associated with Test Tool \#BOT-01; Borehole SCPO1; Sequence SCP01-2

\begin{tabular}{ccccc}
\hline Equipment & Location & Serial \# & Installed & Removed \\
\hline $\begin{array}{c}\text { Transducer } \\
\text { (Druck D930-18) } \\
\begin{array}{c}\text { Transducer } \\
\text { (Druck D930-18) }\end{array}\end{array}$ & $\begin{array}{c}\text { Guard Zone } \\
\text { Packer }\end{array}$ & 609372 & $3-17-94$ & $4-13-94$ \\
\hline Injection Column & DPT Panel & 609368 & $4-8-94$ & $4-13-94$ \\
\hline $\begin{array}{c}\text { DPT } \\
\text { (Rosemount 1151DP) }\end{array}$ & N/A & 94 & $4-8-94$ & $4-13-94$ \\
\hline
\end{tabular}




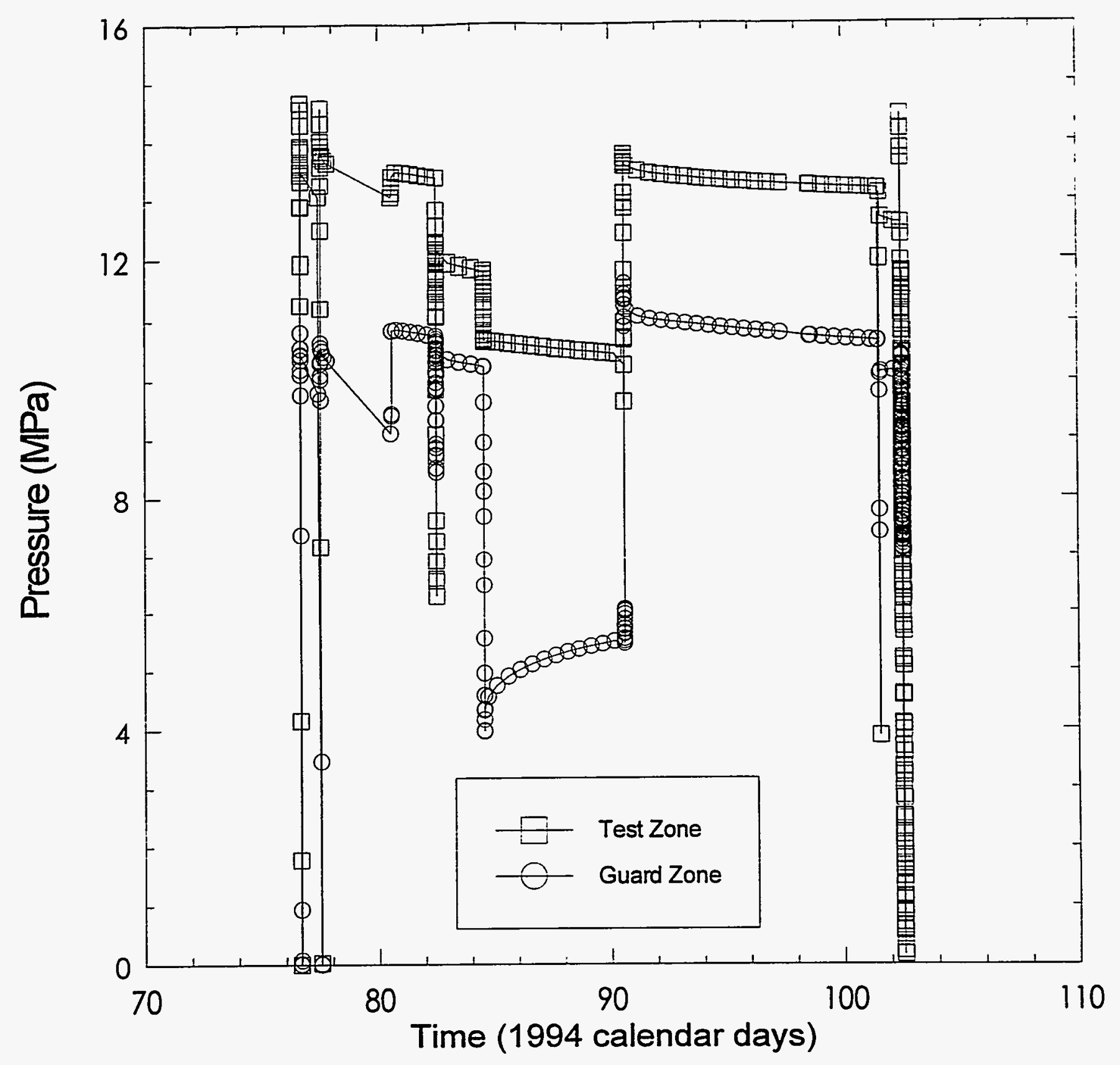

Figure 6-44. Zone pressures during compliance testing of test tool \#BOT-01. 


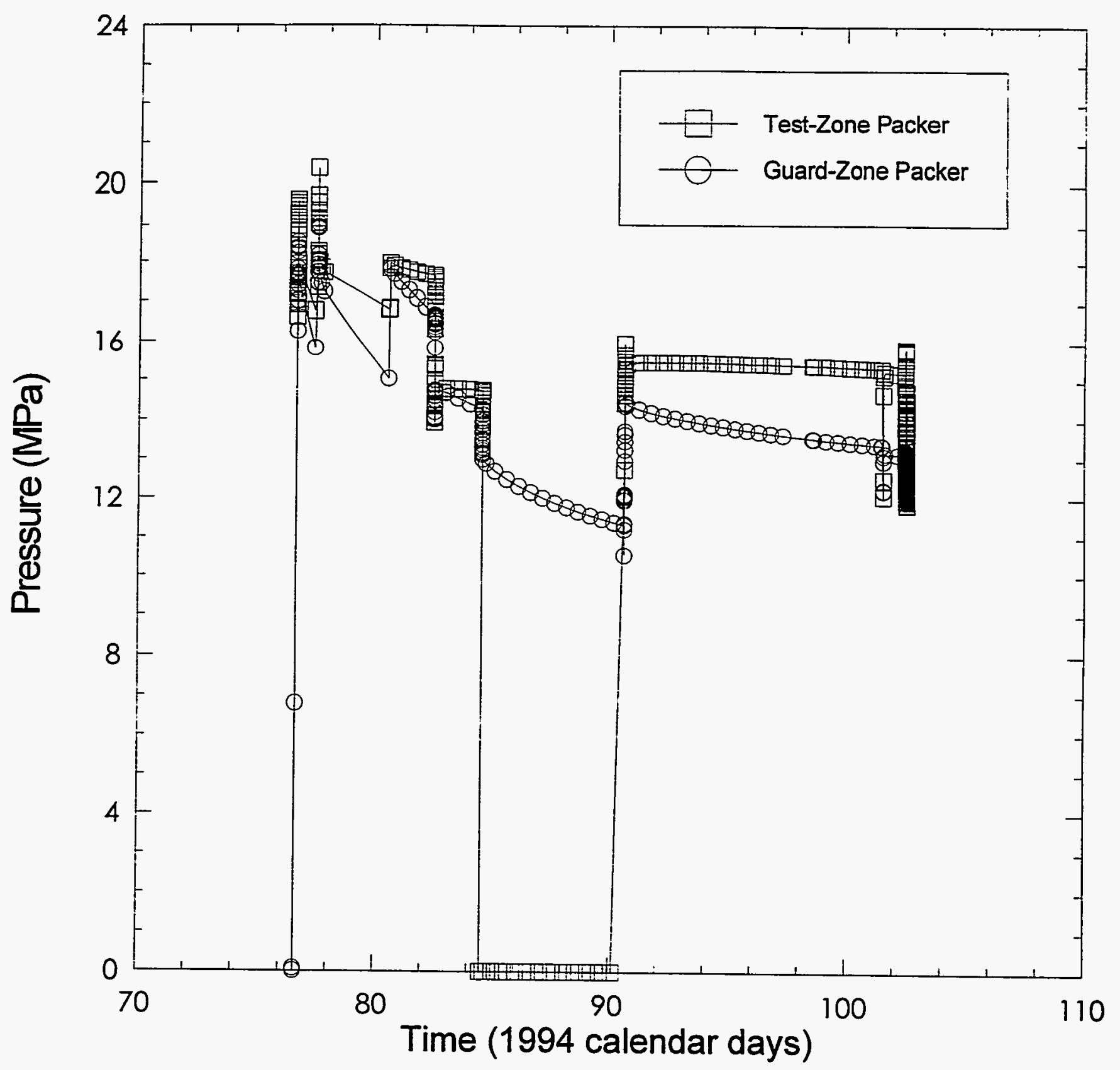

INTERA-115-232-0

Figure 6-45. Packer pressures during compliance testing of test tool \#BOT-01. 


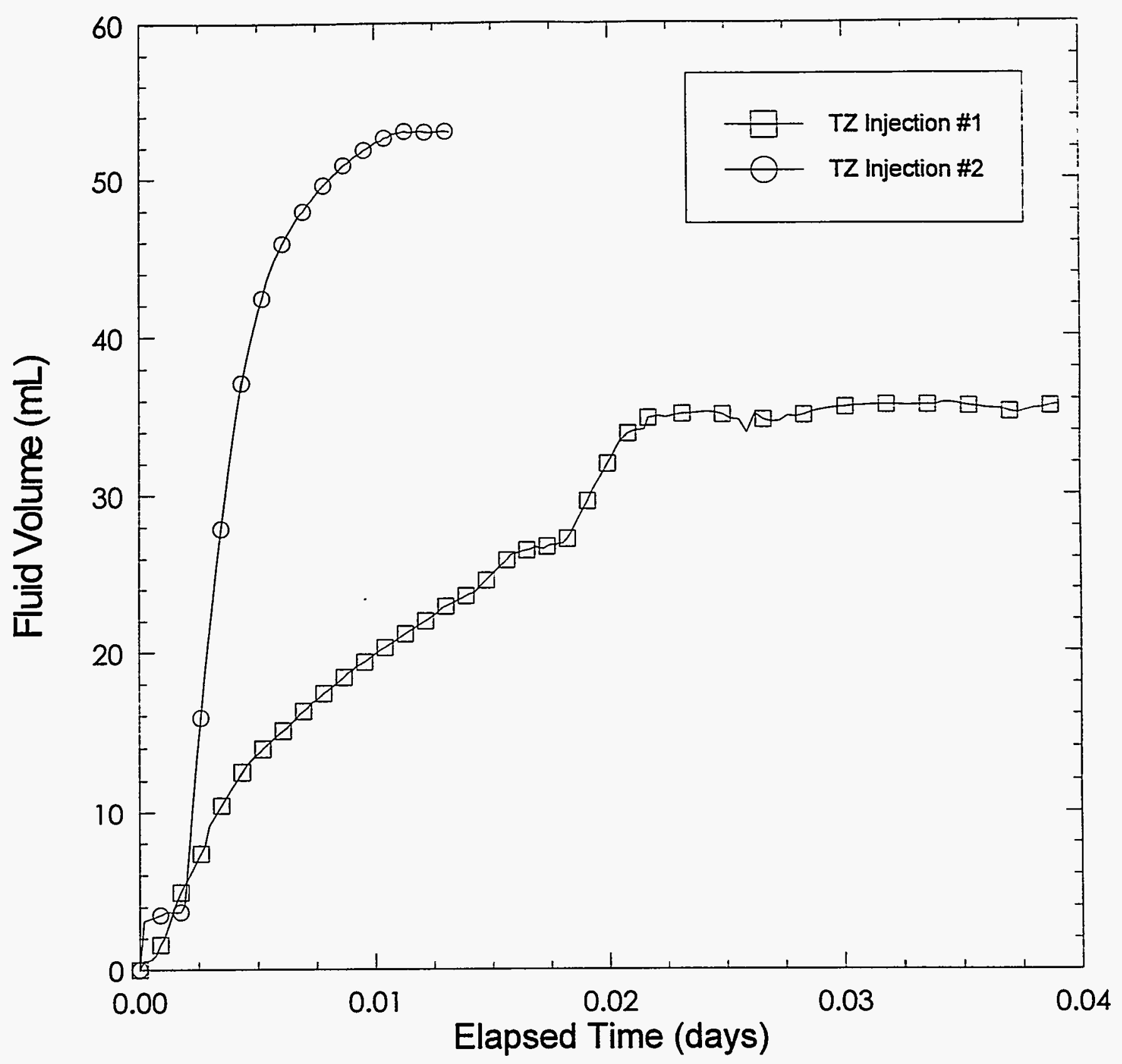

INTERA-6115-233-0

Figure 6-46. Test-zone fluid-injection volumes during compliance testing of test tool \#BOT-01. 


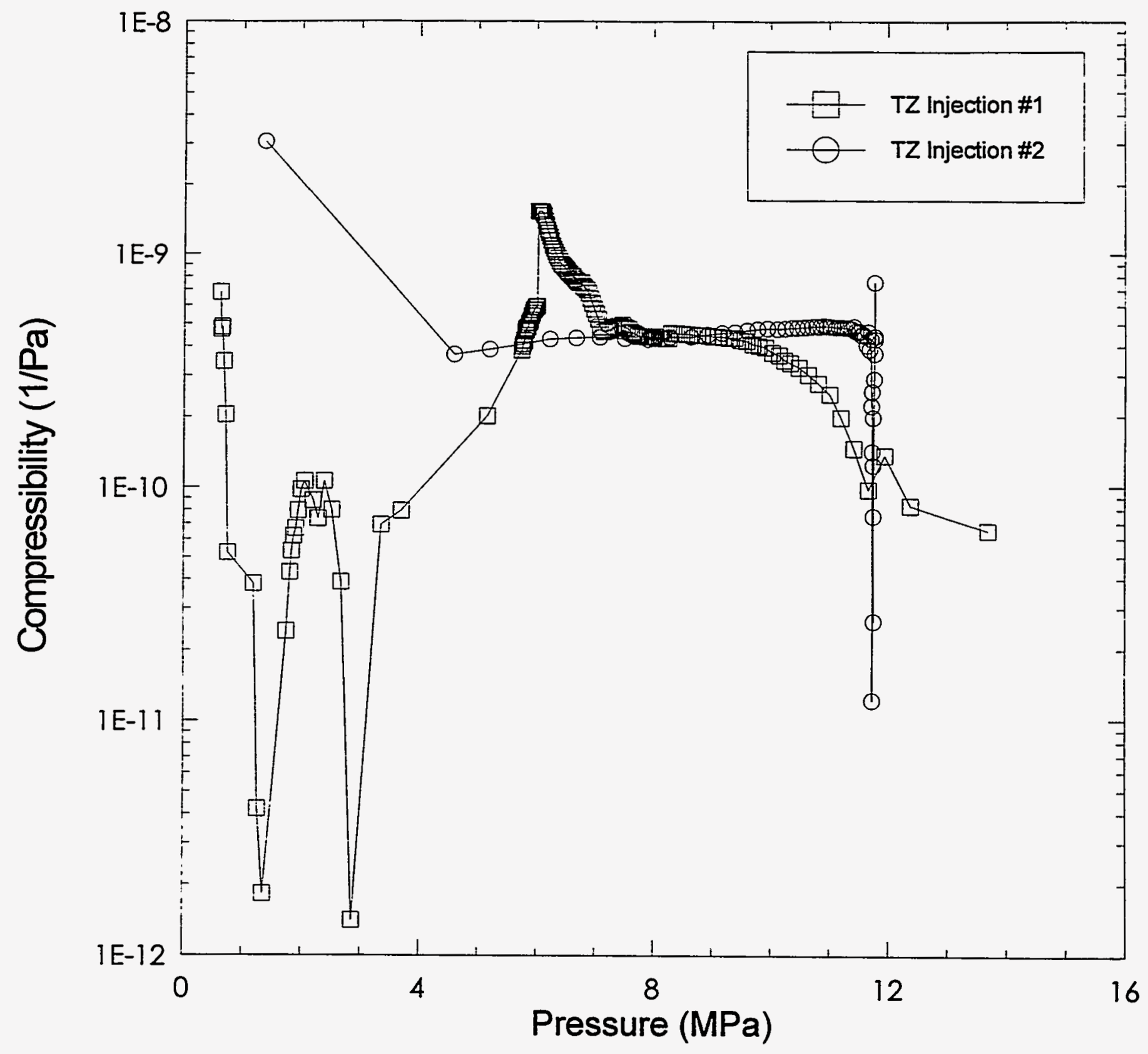

INTERA-6115-2340

Figure 6-47. Test-zone compressibility as measured during compliance testing of test tool \#BOT-01. 


\subsubsection{Test Tool \#P51-D1A (Permeability-Testing Sequence L4P51-D1)}

Table 6-26 gives a detailed description of the events that occurred during compliance testing of test tool \#P51-D1A. Figures 6-48 through 6-53 illustrate the zone pressures, packer pressures, axial-LVDT displacement, radial-LVDT displacement, fluid-injection volumes during compressibility tests, and test-zone compressibility as a function of pressure, respectively, for multipacker test tool \#P51-D1A. Figure 3-25 illustrates the configuration of test tool \#P51-D1A as assembled for compliance testing.

Table 6-26. Events Associated with Compliance Testing of Test Tool \#P51-D1A; Borehole L4P51; Sequence L4P51-D1

\begin{tabular}{|c|c|c|c|c|}
\hline EVENT & DATE & $\begin{array}{c}\text { CALENDAR } \\
\text { DAY }\end{array}$ & $\begin{array}{c}1994 \\
\text { CALENDAR } \\
\text { DAY }\end{array}$ & $\begin{array}{c}\text { TIME } \\
\text { (HH:MM:SS) }\end{array}$ \\
\hline $\begin{array}{l}\text { Assemble multipacker test tool \#P51-D1A to be used in borehole L4P51 during testing } \\
\text { sequence L4P51-D1. }\end{array}$ & $8-26-94$ & 238 & 238 & $12: 29: 00$ \\
\hline Install test tool in compliance chamber. & 8-30-94 & 242 & 242 & $11: 50: 00$ \\
\hline Inflate $T Z 1$ packer to $-15.2 \mathrm{MPa}$ & $8-30-94$ & 242 & 242 & 12:35:00 \\
\hline Inflate $T 22$ packer to $\sim 14.5 \mathrm{MPa}$. & $8-30-94$ & 242 & 242 & 12:41:00 \\
\hline Begin data file CMPP51. & $8-30-94$ & 242 & 242 & $12: 59: 42$ \\
\hline Increase TZ2 packer pressure to $15.519 \mathrm{MPa}$. & 8-30-94 & 242 & 242 & 13:02:00 \\
\hline Increase TZ1 packer pressure to $15.960 \mathrm{MPa}$. & $8-30-94$ & 242 & 242 & 13:04:00 \\
\hline Deflate packers. & $8-31-94$ & 243 & 243 & $11: 29: 00$ \\
\hline Inflate TZ1 packer to $15.640 \mathrm{MPa}$ & $8-31-94$ & 243 & 243 & $11: 36: 00$ \\
\hline Inflate TZ2 packer to $15.480 \mathrm{MPa}$. & $8-31-94$ & 243 & 243 & $11: 40: 00$ \\
\hline Increase TZ1 pressure to $11.881 \mathrm{MPa}$. & 8-31.94 & 243 & 243 & 12:15:00 \\
\hline Decrease $T Z 1$ packer pressure. & 8-31-94 & 243 & 243 & 12:19:00 \\
\hline Increase TZ2 pressure to $8.080 \mathrm{MPa}$. & 8-31-94 & 243 & 243 & 12:30:00 \\
\hline Depressurize TZ1 and TZ2. & $9-1-94$ & 244 & 244 & 07:56:00 \\
\hline Deflate $T Z 1$ packer. & $9-1-94$ & 244 & 244 & 07:57:00 \\
\hline Deflate TZ2 packer. & $9-1-94$ & 244 & 244 & 07:57:00 \\
\hline Remove test tool from complianco chamber to inspect for leaks. & $9-1-94$ & 244 & 244 & 08:30:00 \\
\hline Reinstall test tool in complianca chamber. & 2-1-94 & 244 & 244 & 13:19:00 \\
\hline Inflate TZ1 packer to $15.450 \mathrm{MPa}$. & $9-6-94$ & 249 & 249 & 08:36:00 \\
\hline Inflate TZ2 packer to $15.710 \mathrm{MPa}$. & $9-6-94$ & 249 & 249 & 08:40:00 \\
\hline Doflate packers. & $9-6-94$ & 249 & 249 & 10:19:00 \\
\hline Inflate TZ1 packer to $15.532 \mathrm{MPa}$. & $9-6-94$ & 249 & 249 & 10:21:00 \\
\hline Inflate TZ2 packer to $15.823 \mathrm{MPa}$. & $9-6-94$ & 249 & 249 & 10:24:00 \\
\hline Open TZ1 packer to accumulator at $13.992 \mathrm{MPa}$. & $9-6-94$ & 249 & 249 & $11: 30: 00$ \\
\hline Open TZ2 packer to accumulator at $14.062 \mathrm{MPa}$. & $9-6-94$ & 249 & 249 & 11:31:00 \\
\hline Increase TZ2 pressure to $8.605 \mathrm{MPa}$. & $9-6-94$ & 249 & 249 & 12:14:00 \\
\hline Increase TZ2 pressure to $8.744 \mathrm{MPa}$. & $9-6-94$ & 249 & 249 & 12:38:00 \\
\hline
\end{tabular}


Table 6-26 (Continued). Events Associated with Compliance Testing of Test Tool \#P51-D1A; Borehole L4P51; Sequence L4P51-D1

\begin{tabular}{|c|c|c|c|c|}
\hline EVENT & DATE & $\begin{array}{c}\text { CALENDAR } \\
\text { DAY }\end{array}$ & $\begin{array}{c}1994 \\
\text { CALENDAR } \\
\text { DAY }\end{array}$ & $\begin{array}{c}\text { TIME } \\
\text { (HH:MM:SS) }\end{array}$ \\
\hline Shut in packers from accumulator. & $9-7-94$ & 250 & 250 & 07:28:00 \\
\hline Depressurize TZ2. & $9-7.94$ & 250 & 250 & 07:30:00 \\
\hline Deflate TZ1 packer. & $9-7-94$ & 250 & 250 & 07:32:00 \\
\hline Circulate fluid from the TZ1 to TZ2. & $9-7-94$ & 250 & 250 & 07:38:00 \\
\hline Inflate TZ1 packer and open both packers to accumulator. & $9-7.94$ & 250 & 250 & 07:48:00 \\
\hline Increase TZ2 pressure to $8.683 \mathrm{MPa}$. & $9-7-94$ & 250 & 250 & 07:54:00 \\
\hline Depressurize TZ2. & $97-94$ & 250 & 250 & $09: 41: 00$ \\
\hline Increase T22 pressure to $8.635 \mathrm{MPa}$. & $97-94$ & 250 & 250 & 09:51:00 \\
\hline Shut in both packers from accumulator. & $97-94$ & 250 & 250 & 10:13:00 \\
\hline Disconnect all LVDTs from DAS. & $9-7-94$ & 250 & 250 & $11: 20: 00$ \\
\hline Connect all LVDTs to DAS. & $9-84$ & 251 & 251 & 07:56:00 \\
\hline Increase TZ1 pressure. & $9-8-94$ & 251 & 251 & $08: 06: 00$ \\
\hline Depressurize TZ1. & $9-8-94$ & 251 & 251 & $08: 08: 00$ \\
\hline Increase TZ1 pressure to $13.210 \mathrm{MPa}$. & $9-8-94$ & 251 & 251 & 08:15:00 \\
\hline Depressurize TZ1. & $9-8-94$ & 251 & 251 & 11:28:00 \\
\hline Depressurize TZ2. & $9-8-94$ & $25 t$ & 251 & 11:30:00 \\
\hline Open both packers to accumulator. & $9-8-94$ & 251 & 251 & $11: 43: 00$ \\
\hline End data file CMPP51. & $9-12-94$ & 255 & 255 & 07:23:34 \\
\hline Begin data file CMPRES1. & $9-12-94$ & 255 & 255 & 07:46:19 \\
\hline Increase TZ2 pressure to $8.571 \mathrm{MPa}$. & $9-12-94$ & 255 & 255 & 07:49:00 \\
\hline Begin $T Z 1$ compressibility test. & $9-12-94$ & 255 & 255 & 08:05:00 \\
\hline Shut in TZ1. & $9-12-94$ & 255 & 255 & 09:12:00 \\
\hline Begin $T Z 1$ compressibility test. & $9-12-94$ & 255 & 255 & $10: 49: 00$ \\
\hline Shut in TZ1. & $9-12-94$ & 255 & 255 & 11:22:00 \\
\hline Begin TZ1 compressibility lest. & $9-12-94$ & 255 & 255 & $11: 30: 00$ \\
\hline Shut in TZ1. & $9-12-94$ & 255 & 255 & 12:13:00 \\
\hline increase TZ2 pressure to $8.088 \mathrm{MPa}$. & $9-12-94$ & 255 & 255 & 12:16:00 \\
\hline Depressurize TZ1. & $9-13-94$ & 256 & 256 & 08:40:00 \\
\hline Increase TZ2 pressure to $8.165 \mathrm{MPa}$. & $9-13-94$ & 256 & 256 & 08:48:00 \\
\hline Begin TZ1 compresslbility test. & $9-13-94$ & 256 & 256 & 09:06:00 \\
\hline Shut in TZ1. & $9-13-94$ & 256 & 256 & 10:18:00 \\
\hline Depressurize TZ1. & $9-13-94$ & 256 & 256 & 10:20:00 \\
\hline Begin TZ1 compressibility test. & $9-13.94$ & 256 & 256 & 10:36:00 \\
\hline Shut in TZ1. & $9-13-94$ & 256 & 256 & 12:08:00 \\
\hline Increase TZ1 pressure to $12.094 \mathrm{MPa}$. & $9-19-94$ & 262 & 262 & 07:07:00 \\
\hline
\end{tabular}


Table 6-26 (Continued). Events Associated with Compliance Testing of Test Tool \#P51-D1A; Borehole L4P51; Sequence L4P51-D1

\begin{tabular}{|c|c|c|c|c|}
\hline EVENT & DATE & $\begin{array}{c}\text { CALENDAR } \\
\text { DAY }\end{array}$ & $\begin{array}{c}1994 \\
\text { CALENDAR } \\
\text { DAY }\end{array}$ & $\begin{array}{c}\text { TIME } \\
\text { (HH:MM:SS) }\end{array}$ \\
\hline TZ2 gage busted out which explains the TZ2 pressure. & $9-26-94$ & 269 & 269 & 08:07:00 \\
\hline Depressurize all zones and deflate all packers. & $9-26-94$ & 262 & 262 & 08:09:00 \\
\hline End data file CMPRES1. & $9-26-94$ & 262 & 262 & 08:09:54 \\
\hline Remove test tool \#P51-D1A from compliance chamber and move to borehole L4P51. & $9-26-94$ & 262 & 262 & 12:00:00 \\
\hline
\end{tabular}

Table 6-27 indicates the equipment that was used and the duration that each instrument was used during compliance testing of test tool \#P51-D1A which was used in permeabilitytesting sequence L4P51-D1.

Table 6-27. Compliance Equipment Associated with Test Tool \#P51-D1A; Borehole L4P51; Sequence L4P51-D1

\begin{tabular}{|c|c|c|c|c|}
\hline Equipment & Location & Serial \# & Installed & Removed \\
\hline DAS Software & N/A & PERM4F & $8-22-94$ & $9-26-94$ \\
\hline DCU (HP3497A) & N/A & $2629 a 22040$ & $8-22-94$ & $9-26-94$ \\
\hline $\begin{array}{c}\text { Transducer } \\
\text { (Druck D930-18) }\end{array}$ & Test Zone 1 & 609375 & $8-22-94$ & $9-26-94$ \\
\hline $\begin{array}{c}\text { Transducer } \\
\text { (Druck D930-18) }\end{array}$ & $\begin{array}{l}\text { Test Zone } 1 \\
\text { Packer }\end{array}$ & 609371 & $8-22-94$ & $9-26-94$ \\
\hline $\begin{array}{c}\text { Transducer } \\
\text { (Druck D930-18) }\end{array}$ & Test Zone 2 & 609368 & $8-22-94$ & $9-26-94$ \\
\hline $\begin{array}{c}\text { Transducer } \\
\text { (Druck D930-18) }\end{array}$ & $\begin{array}{c}\text { Test Zone } 2 \\
\text { Packer }\end{array}$ & 609374 & $8-22-94$ & $9-26-94$ \\
\hline $\begin{array}{c}\text { Transducer } \\
\text { (Druck D930-18) }\end{array}$ & DPT Panel & 609372 & $8-22-94$ & $9-26-94$ \\
\hline $\begin{array}{c}\text { Transducer } \\
\text { (Druck D930-18) }\end{array}$ & $\begin{array}{l}\text { Pressure } \\
\text { Controller }\end{array}$ & 609370 & $8-22-94$ & $9-26-94$ \\
\hline LVDT (Trans-Tek 241) & N/A & R17 & $8-22-94$ & $9-26-94$ \\
\hline LVDT (Trans-Tek 241) & N/A & $\mathrm{RO4}$ & $8-22-94$ & $9-26-94$ \\
\hline LVDT (Trans-Tek 241) & N/A & R16 & $8-22-94$ & $9-26-94$ \\
\hline LVDT (Trans-Tek 245) & N/A & $\mathrm{AO} 2$ & $8-22-94$ & $9-26-94$ \\
\hline Injection Column & N/A & 92 & $8-22-94$ & $9-26-94$ \\
\hline $\begin{array}{c}\text { DPT } \\
\text { (Rosemount 1151DP) }\end{array}$ & N/A & 1140864 & $8-22-94$ & $9-26-94$ \\
\hline
\end{tabular}




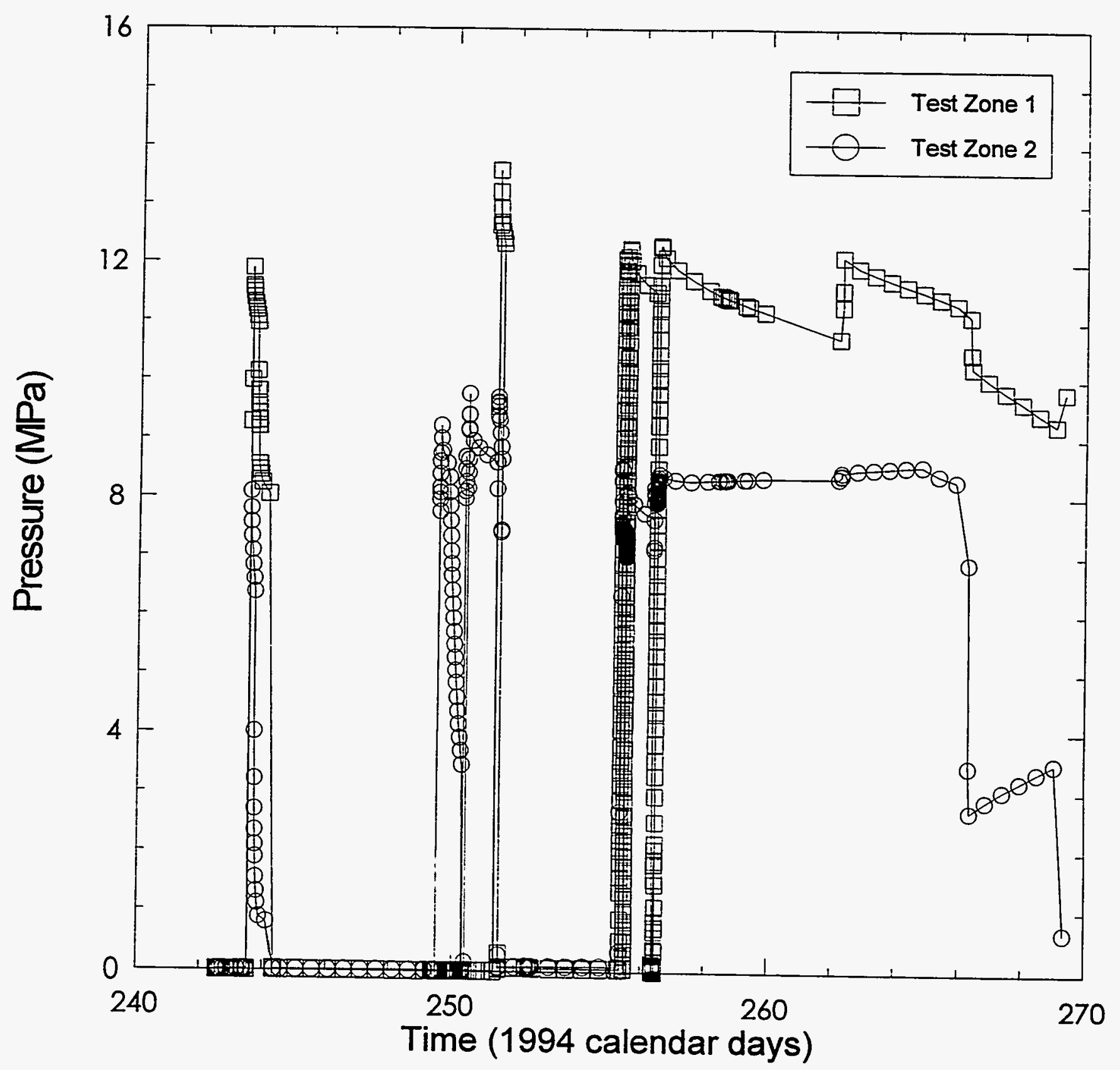

INTERA-6115-235-O

Figure 6-48. Zone pressures during compliance testing of test tool \#P51-D1A. 


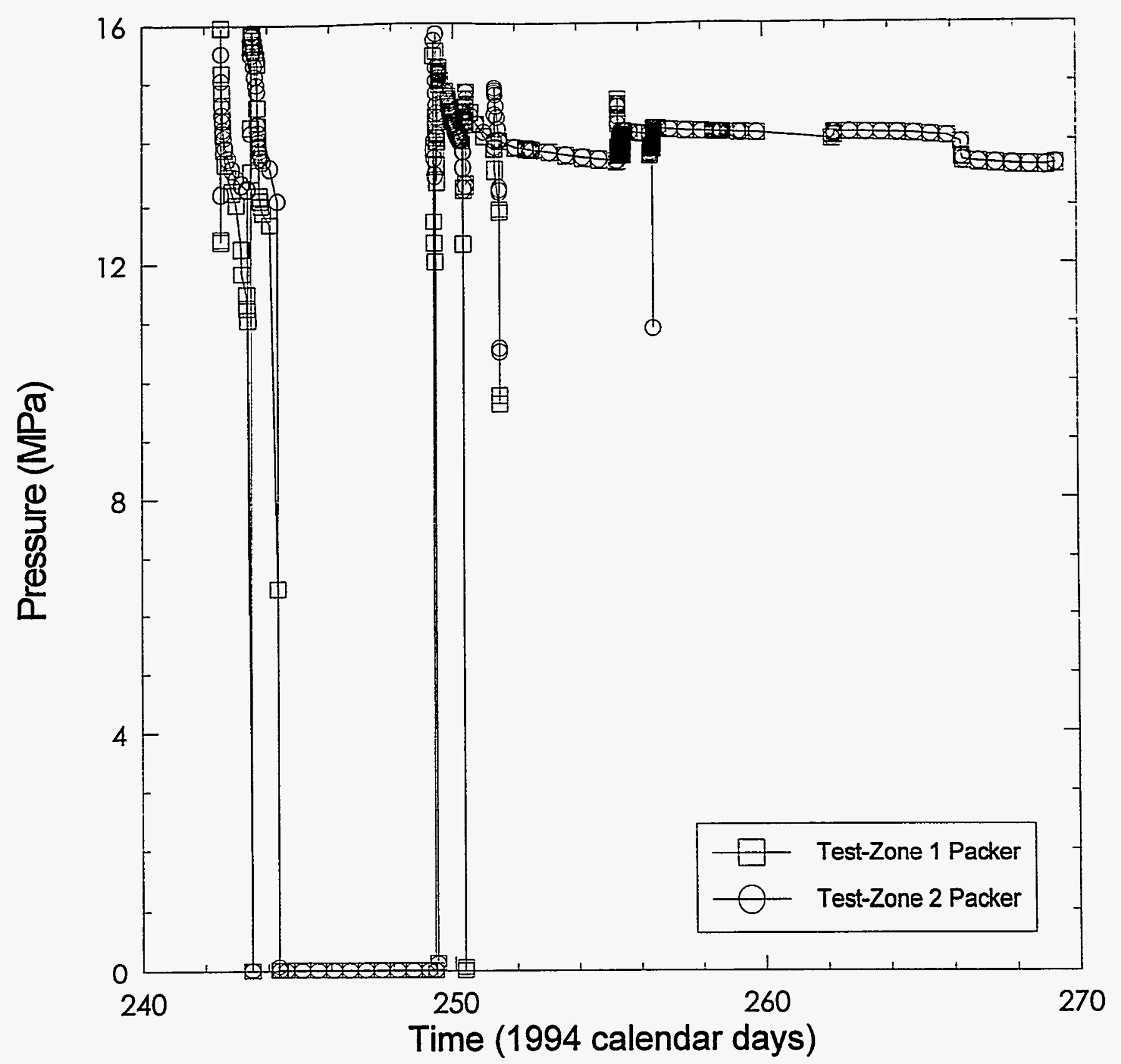

INTERA6115-238-0

Figure 6-49. Packer pressures during compliance testing of test tool \#P51-D1A. 


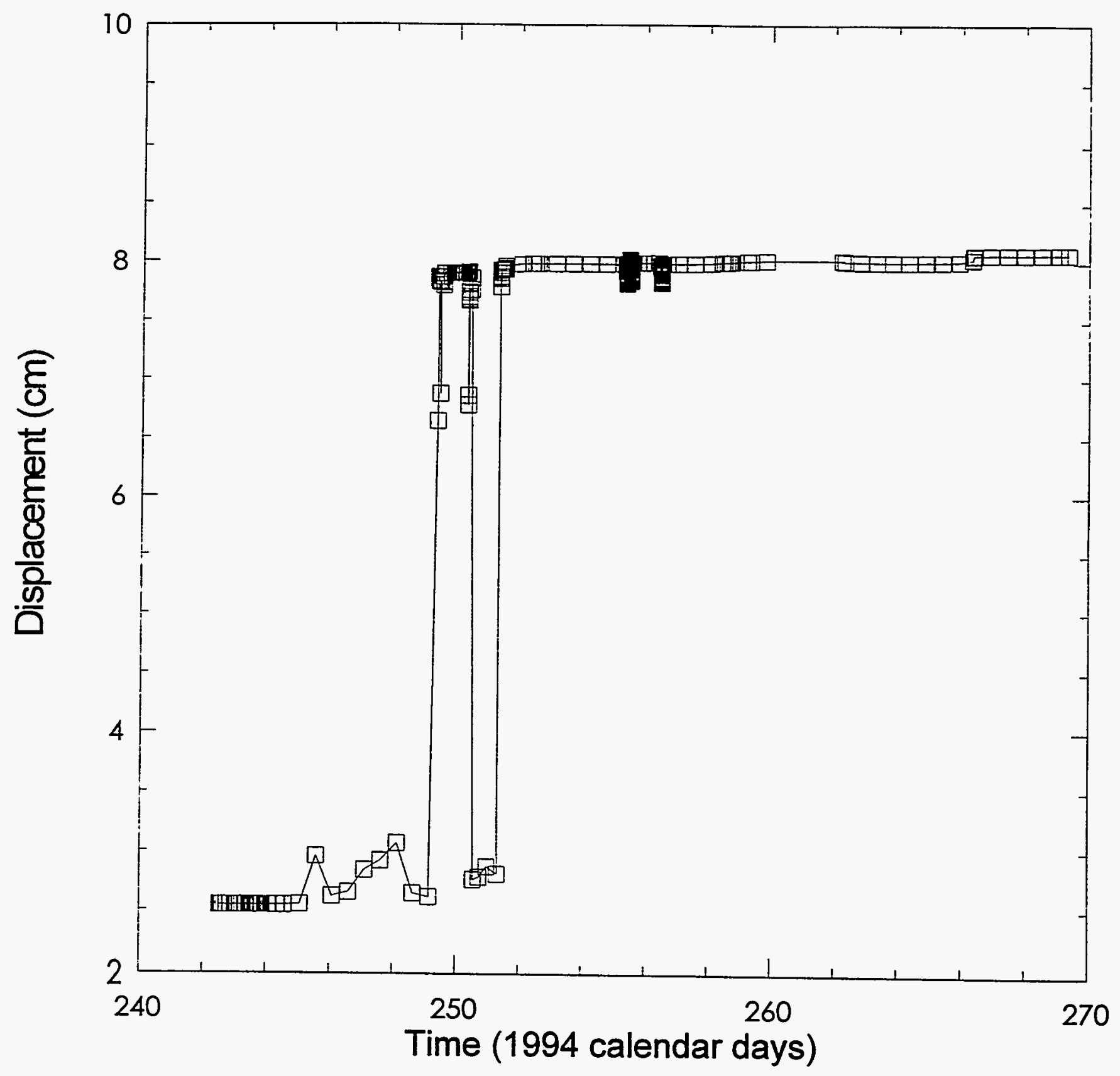

INTERA-115-237-0

Figure 6-50. Axial-LVDT displacement during compliance testing of test tool \#P51-D1A. 


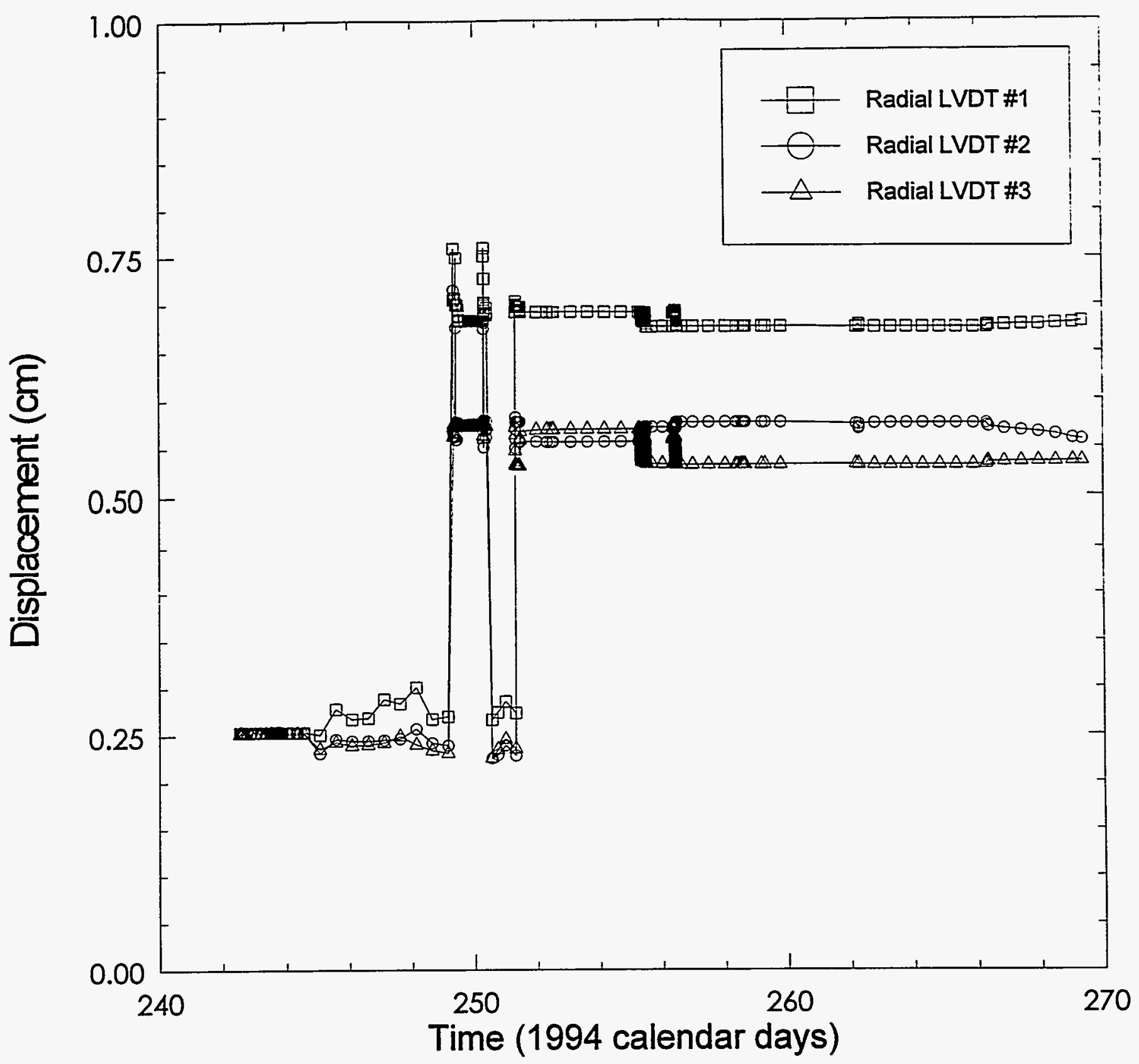

INTERAB115-238-0

Figure 6-51. Radial-LVDT displacement during compliance testing of test tool \#P51-D1A. 


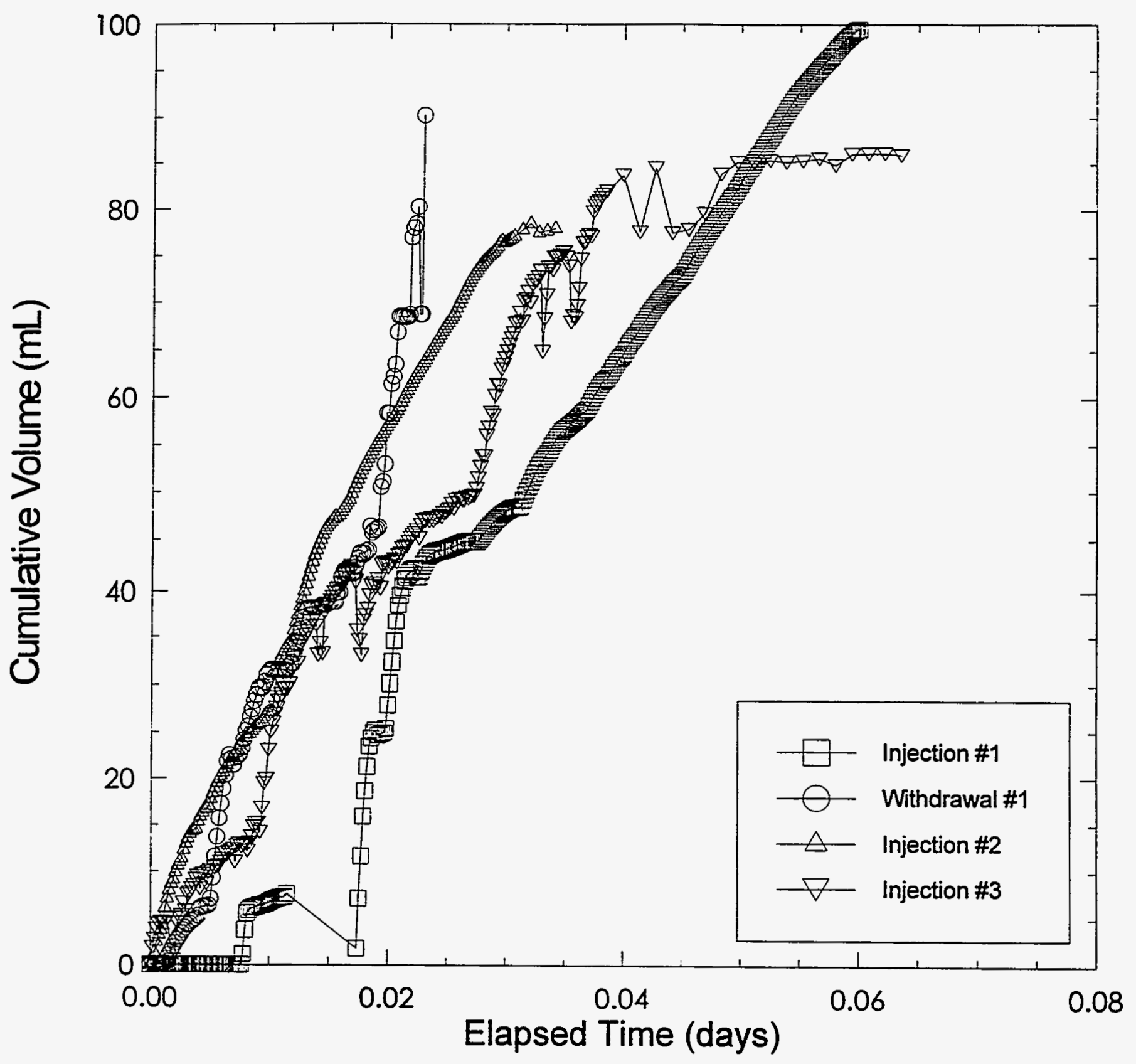

INTERA6115-2390

Figure 6-52. Fluid-injection volumes during compliance testing of test tool \#P51-D1A. 


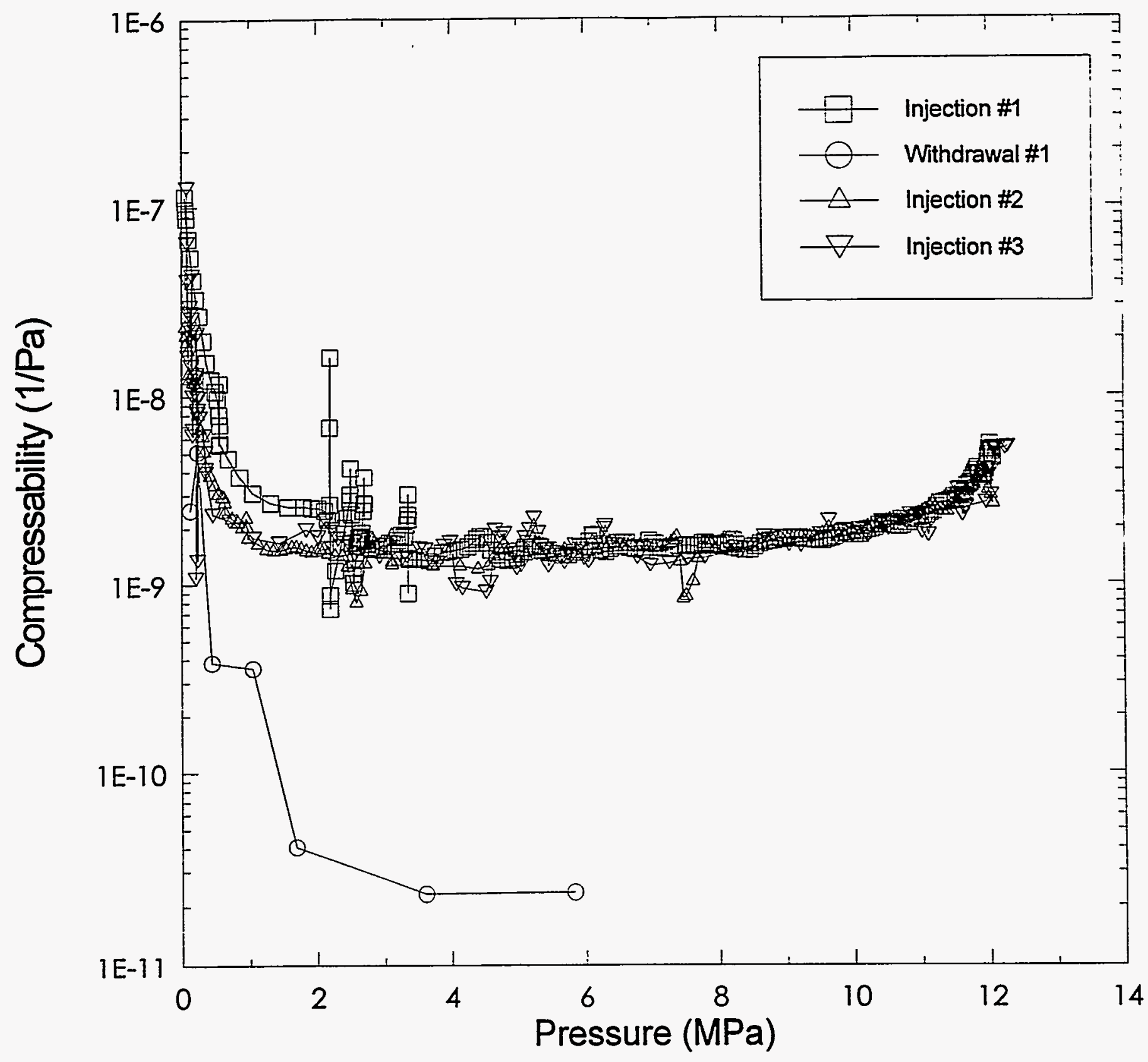

INTERA-6115-240-0

Figure 6-53. Test-zone compressibility as measured during compliance testing of test tool \#P51-D1A. 


\subsubsection{Test Tool \#P74-B (Permeability-Testing Sequence S1P74-B)}

Table 6-28 gives a detailed description of the events that occurred during compliance testing of test tool \#P74-B. Figures 6-54 through 6-58 illustrate the zone pressures, packer pressures, axial-LVDT displacement, fluid-injection volumes during compressibility tests, and test-zone compressibility as a function of pressure, respectively, for multipacker test tool \#P74-B. Figure 3-33 illustrates the configuration of test tool \#P74-B as assembled for compliance testing.

Table 6-28. Events Associated with Compliance Testing of Test Tool \#P74-B; Borehole S1P74; Sequence S1P74-B

\begin{tabular}{|c|c|c|c|c|}
\hline EVENT & DATE & $\begin{array}{c}\text { CALENDAR } \\
\text { DAY }\end{array}$ & $\begin{array}{c}1995 \\
\text { CALENDAR } \\
\text { DAY }\end{array}$ & $\begin{array}{c}\text { TIME } \\
\text { (HH:MM:SS) }\end{array}$ \\
\hline $\begin{array}{l}\text { Assemble multipacker test tool \#P74-B to be used in borehole S1P74 during testing } \\
\text { sequence S1P74-B. }\end{array}$ & $1-18-95$ & 18 & 18 & $12: 00: 00$ \\
\hline Begin data file COMPP74. & 1-19-95 & 19 & 19 & 14:18:33 \\
\hline Inflate TZ1 packer to $-13.8 \mathrm{MPa}$. & $1-19-95$ & 19 & 19 & $14: 20: 00$ \\
\hline Open TZ1 packer to accumulator at $13.833 \mathrm{MPa}$. & $9-19-95$ & 19 & 19 & 14:24:00 \\
\hline Infiate TZ2 packer to $\sim 13.8 \mathrm{MPa}$. & $1-19-95$ & 19 & 19 & $14: 33: 00$ \\
\hline Open TZ2 packer to accumulator at $14.058 \mathrm{MPa}$. & 1-19-95 & 19 & 19 & 14:37:00 \\
\hline Inflate $G Z$ packer to $\sim 13.8 \mathrm{MPa}$. & $1-19-95$ & 19 & 19 & $14: 42: 00$ \\
\hline Open GZ packer to accumulator at $13.712 \mathrm{MPa}$. & $1-19-95$ & 19 & 19 & 14:44:00 \\
\hline End data file COMPP74. & $9-20-95$ & 20 & 20 & 10:16:46 \\
\hline Begin data file COMPP742. & $1-20-95$ & 20 & 20 & 11:16:19 \\
\hline Increase TZ1 pressure to $9.909 \mathrm{MPa}$. & $1-20-95$ & 20 & 20 & 12:31:00 \\
\hline Increase TZ2 pressure to $10.031 \mathrm{MPa}$. & $1-20-95$ & 20 & 20 & $12: 35: 00$ \\
\hline Increase $\mathrm{GZ}$ pressure to $9.257 \mathrm{MPa}$. & $1-20-95$ & 20 & 20 & 12:39:00 \\
\hline Significant pressure decay in GZ. & 1-20-95 & 20 & 20 & $12: 46: 00$ \\
\hline Remove $G Z$ portion of test tool from compliance chamber to find leak. & $1-20-95$ & 20 & 20 & 14:00:00 \\
\hline Leak caused by broken $1 / 8$-inch fitting with no replacement. & 1-20-95 & 20 & 20 & 14:15:00 \\
\hline Reinstall test tool in compliance chamber to test TZ2. & $1-20-95$ & 20 & 20 & 14:20:00 \\
\hline Inflate TZ2 packer to $12.834 \mathrm{MPa}$. & 1-20-95 & 20 & 20 & 14:30:00 \\
\hline Open TZ2 packer to accumulator at $14.047 \mathrm{MPa}$. & 1-20-95 & 20 & 20 & 14:33:00 \\
\hline Inflate GZ packer to $13.8 \mathrm{MPa}$. & $1-20-95$ & 20 & 20 & 14:37:00 \\
\hline Open GZ packer to accumulator at $13.691 \mathrm{MPa}$. & $1-20-95$ & 20 & 20 & 14:39:00 \\
\hline Increase TZ2 pressure to $9.822 \mathrm{MPa}$. & 1-20-95 & 20 & 20 & 14:44:00 \\
\hline Depressurize TZ2. & $1-23-95$ & 23 & 23 & 09:25:00 \\
\hline Deflate TZ2 packer and GZ packer. & $1-23-95$ & 23 & 23 & 09:29:00 \\
\hline Replace broken 1/8-inch fitting. & $1-23-95$ & 23 & 23 & 09:48:00 \\
\hline Inflate TZ2 packer to $\sim 13.8 \mathrm{MPa}$. & $1-23-95$ & 23 & 23 & 10:14:00 \\
\hline Open TZ2 packer to accumulator at $14.036 \mathrm{MPa}$. & $1-23-95$ & 23 & 23 & 10:15:00 \\
\hline
\end{tabular}


Table 6-28 (Continued). Events Associated with Compliance Testing of Test Tool \#P74-B; Borehole S1P74; Sequence S1P74-B

\begin{tabular}{|c|c|c|c|c|}
\hline EVENT & DATE & $\begin{array}{l}\text { CALENDAR } \\
\text { DAY }\end{array}$ & $\begin{array}{c}1995 \\
\text { CALENDAR } \\
\text { DAY }\end{array}$ & $\begin{array}{c}\text { TIME } \\
\text { (HH:MM:SS) }\end{array}$ \\
\hline Inflate $\mathrm{GZ}$ packer to $\sim 13.8 \mathrm{MPa}$. & 1-23-95 & 23 & 23 & 10:20:00 \\
\hline Open GZ packer to accumulator at $13.683 \mathrm{MPa}$. & $1-23-95$ & 23 & 23 & 10:22:00 \\
\hline Increase GZ pressure to $4.687 \mathrm{MPa}$. & $1-23-95$ & 23 & 23 & 10:27:00 \\
\hline Decrease GZ pressure to $\sim 2.7 \mathrm{MPa}$. & $1-23-95$ & 23 & 23 & 10:37:00 \\
\hline Increase $\mathrm{GZ}$ pressure to $4.459 \mathrm{MPa}$. & $1-23-95$ & 23 & 23 & 10:38:00 \\
\hline End data file COMPP742. & $1-23-95$ & 23 & 23 & 12:42:07 \\
\hline - Begin data file COMPP743. & $1-23-95$ & 23 & 23 & $14: 31: 39$ \\
\hline Increase GZ pressure after replacing leaky fitting. & $1-24-95$ & 24 & 24 & $10: 24: 23$ \\
\hline Depressurize TZ1. & $1-24-95$ & 24 & 24 & $10: 27: 47$ \\
\hline Begin TZ1 compressibility test. & $1-24-95$ & 24 & 24 & 10:50:00 \\
\hline Shut in TZ1. & $1-24-95$ & 24 & 24 & 11:12:00 \\
\hline Begin TZ1 compressibility test. & $1-24-95$ & 24 & 24 & 11:17:00 \\
\hline Shut in TZ1. & $1-24-95$ & 24 & 24 & $11: 45: 00$ \\
\hline Begin $T Z 1$ compressibility test. & $1-24-95$ & 24 & 24 & $11: 52: 00$ \\
\hline Shut in TZ1. & $1-24-95$ & 24 & 24 & $12: 32: 00$ \\
\hline Begin $T Z 1$ compressibility test. & $1-24-95$ & 24 & 24 & $12: 35: 00$ \\
\hline Shut in $T Z 1$. & $1-24-95$ & 24 & 24 & $12: 58: 00$ \\
\hline End data file COMPP743. & $1-2495$ & 24 & 24 & 12:58:51 \\
\hline Remove test tool \#P74-B from compliance chamber and move to borehole S1P74. & $2-1-95$ & 32 & 32 & 12:00:00 \\
\hline
\end{tabular}

Table 6-29 indicates the equipment that was used and the duration that each instrument was used during compliance testing of test tool \#P74-B. Test tool \#P74-B was used in permeability-testing sequence S1P74-B.

Table 6-29. Compliance Equipment Associated with Test Tool \#P74-B; Borehole S1P74; Sequence S1P74-B

\begin{tabular}{|c|c|c|c|c|}
\hline Equipment & Location & Serial \# & Installed & Removed \\
\hline DAS Software & N/A & PERM4F & 1-19-95 & 2-1-95 \\
\hline $\mathrm{DCU}(\mathrm{HP} 3497 \mathrm{~A})$ & N/A & $2629 a 21990$ & $1-19-95$ & 2-1-95 \\
\hline $\begin{array}{c}\text { Transducer } \\
\text { (Druck PDCR 910) }\end{array}$ & Test Zone 1 & 321768 & $1-19-95$ & $2-1-95$ \\
\hline $\begin{array}{c}\text { Transducer } \\
\text { (Druck PDCR 10/D) }\end{array}$ & $\begin{array}{l}\text { Test Zone } 1 \\
\text { Packer }\end{array}$ & 211695 & $1-19-95$ & $2-1-95$ \\
\hline
\end{tabular}


Table 6-29 (Continued). Compliance Equipment Associated with Test Tool \#P74-B; Borehole S1P74; Sequence S1P74-B

\begin{tabular}{|c|c|c|c|c|}
\hline Equipment & Location & Serial \# & Installed & Removed \\
\hline $\begin{array}{c}\text { Transducer } \\
\text { (Druck D930-18) }\end{array}$ & Test Zone 2 & 609364 & $1-19-95$ & $2-1-95$ \\
\hline $\begin{array}{c}\text { Transducer } \\
\text { (Druck PDCR 910) }\end{array}$ & $\begin{array}{c}\text { Test Zone } 2 \\
\text { Packer }\end{array}$ & 322422 & $1-19-95$ & $2-1-95$ \\
\hline $\begin{array}{c}\text { Transducer } \\
\text { (Druck PDCR 910) }\end{array}$ & Guard Zone & 507864 & $1-19-95$ & $2-1-95$ \\
\hline $\begin{array}{c}\text { Transducer } \\
\text { (Druck PDCR 910) }\end{array}$ & $\begin{array}{l}\text { Guard Zone } \\
\text { Packer }\end{array}$ & 322426 & $1-19-95$ & $2-1-95$ \\
\hline $\begin{array}{c}\text { Transducer } \\
\text { (Druck PDCR 910) } \\
\end{array}$ & DPT Panel & 308152 & $1-19-95$ & $2-1-95$ \\
\hline LVDT (Trans-Tek 242) & N/A & 1 & $1-19-95$ & $2-1-95$ \\
\hline LVDT (Trans-Tek 242) & N/A & 2 & $1-19-95$ & $2-1-95$ \\
\hline Injection Column & N/A & 92 & 1-19-95 & $2-1-95$ \\
\hline $\begin{array}{c}\text { DPT } \\
\text { (Rosemount 1151DP) }\end{array}$ & N/A & 1689030 & $1-19-95$ & $2-1-95$ \\
\hline
\end{tabular}




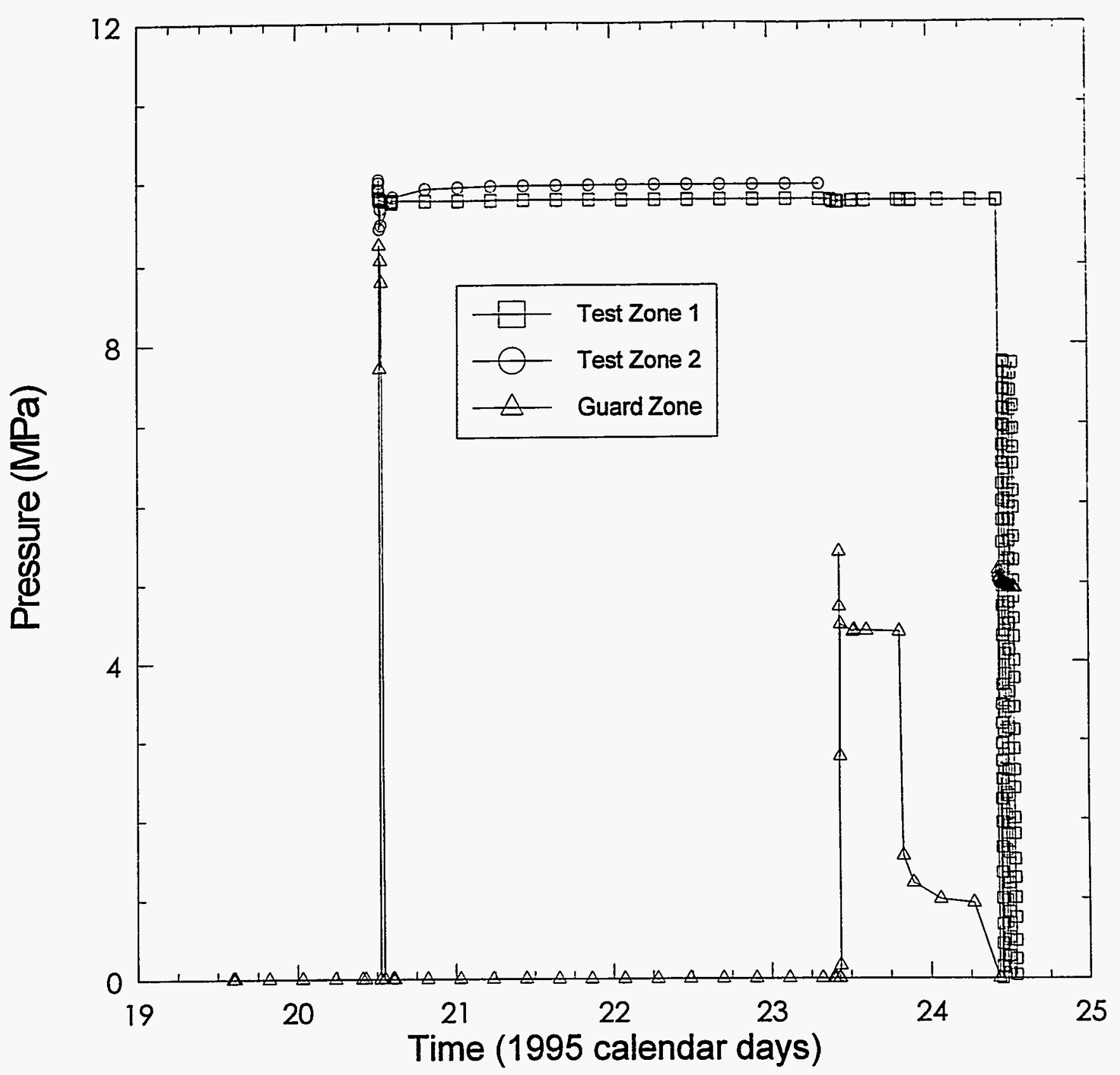

INTERA6115-241-0

Figure 6-54. Zone pressures during compliance testing of test tool \#P74-B. 


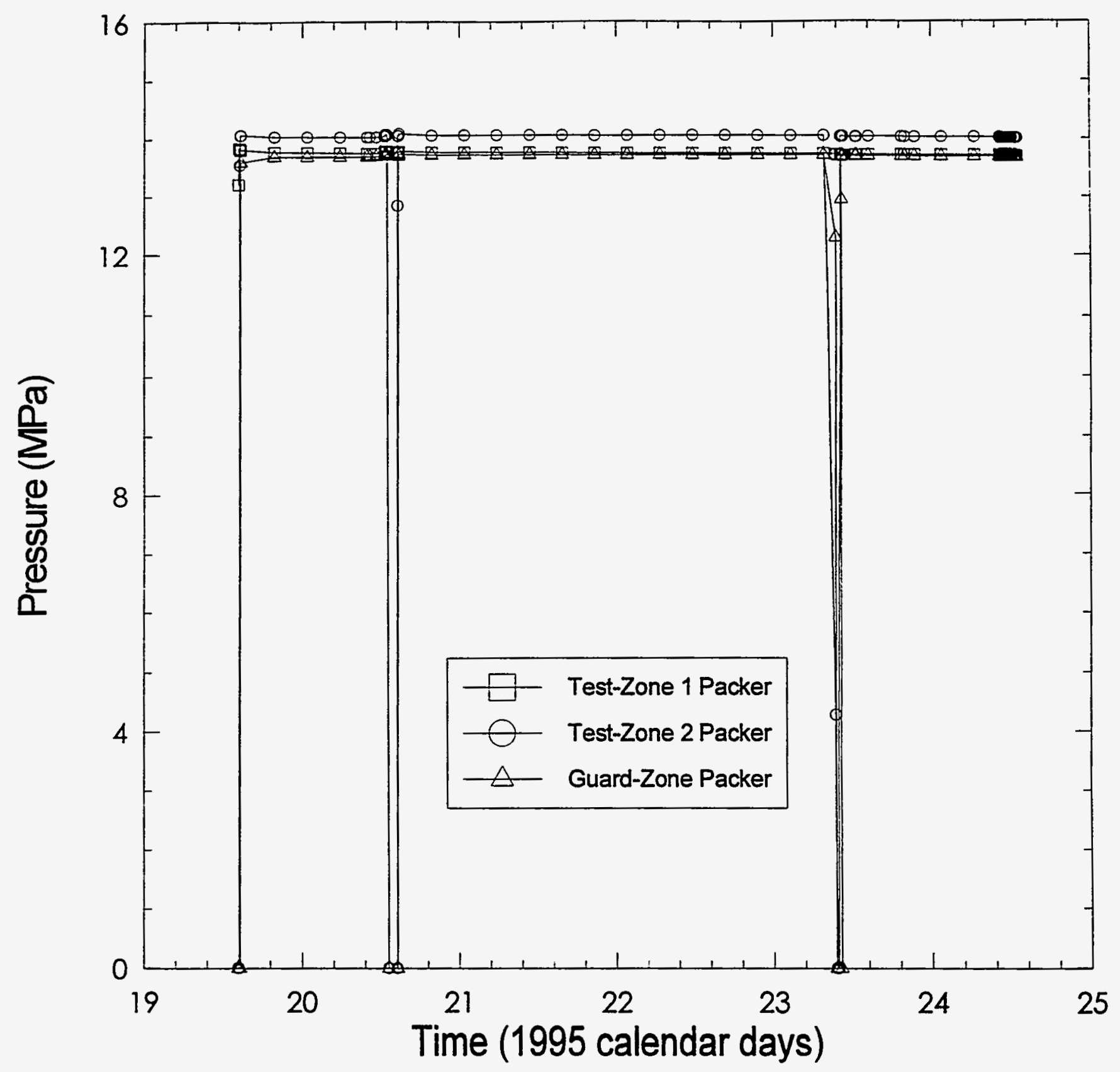

INTERA-115-242-0

Figure 6-55. Packer pressures during compliance testing of test tool \#P74-B. 


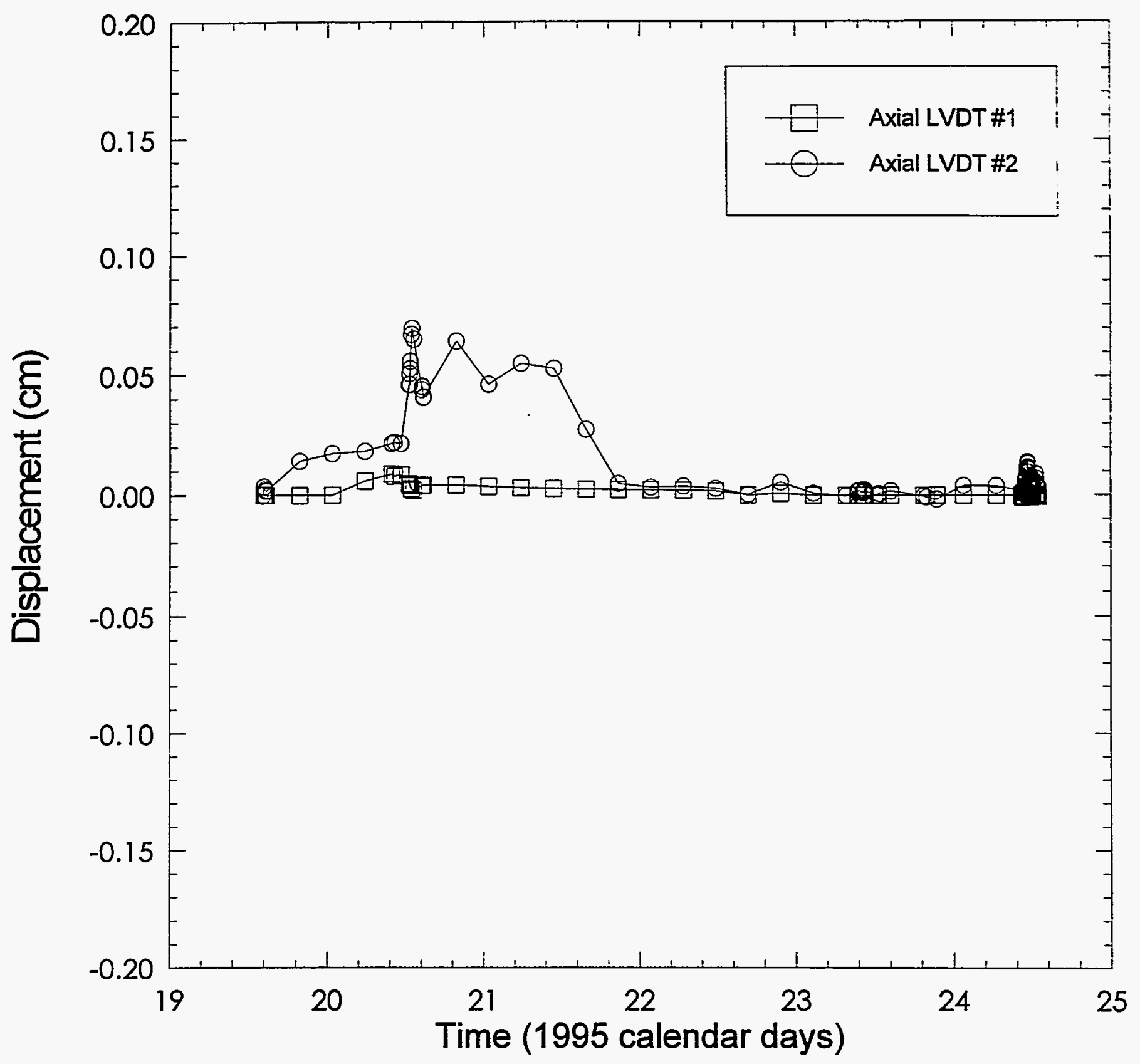

INTERA-6115-243-0

Figure 6-56. Axial-LVDT displacement during compliance testing of test tool \#P74-B. 


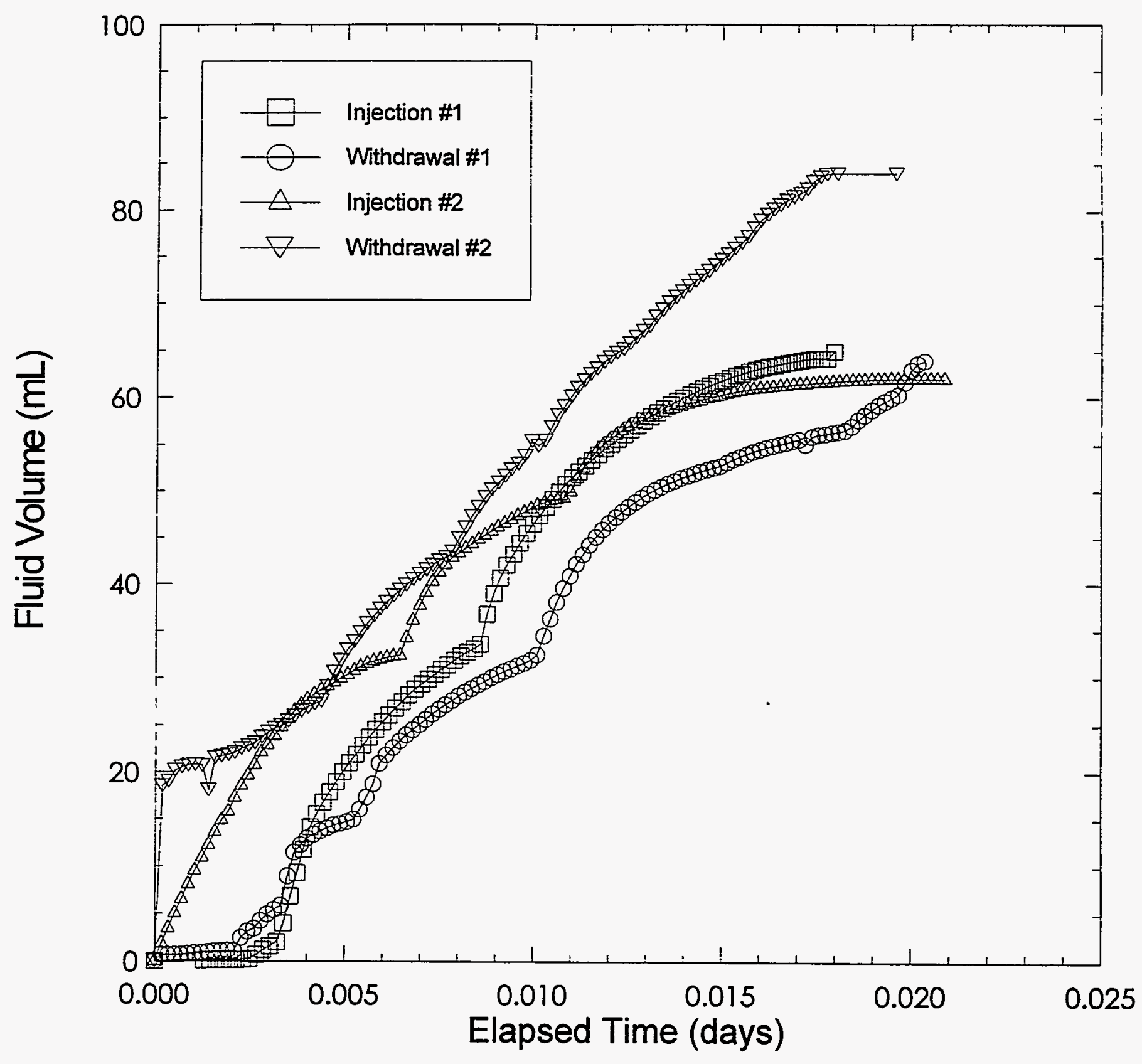

INTERABI15-2440

Figure 6-57. Fluid-injection volumes during compliance testing of test tool \#P74-B. 


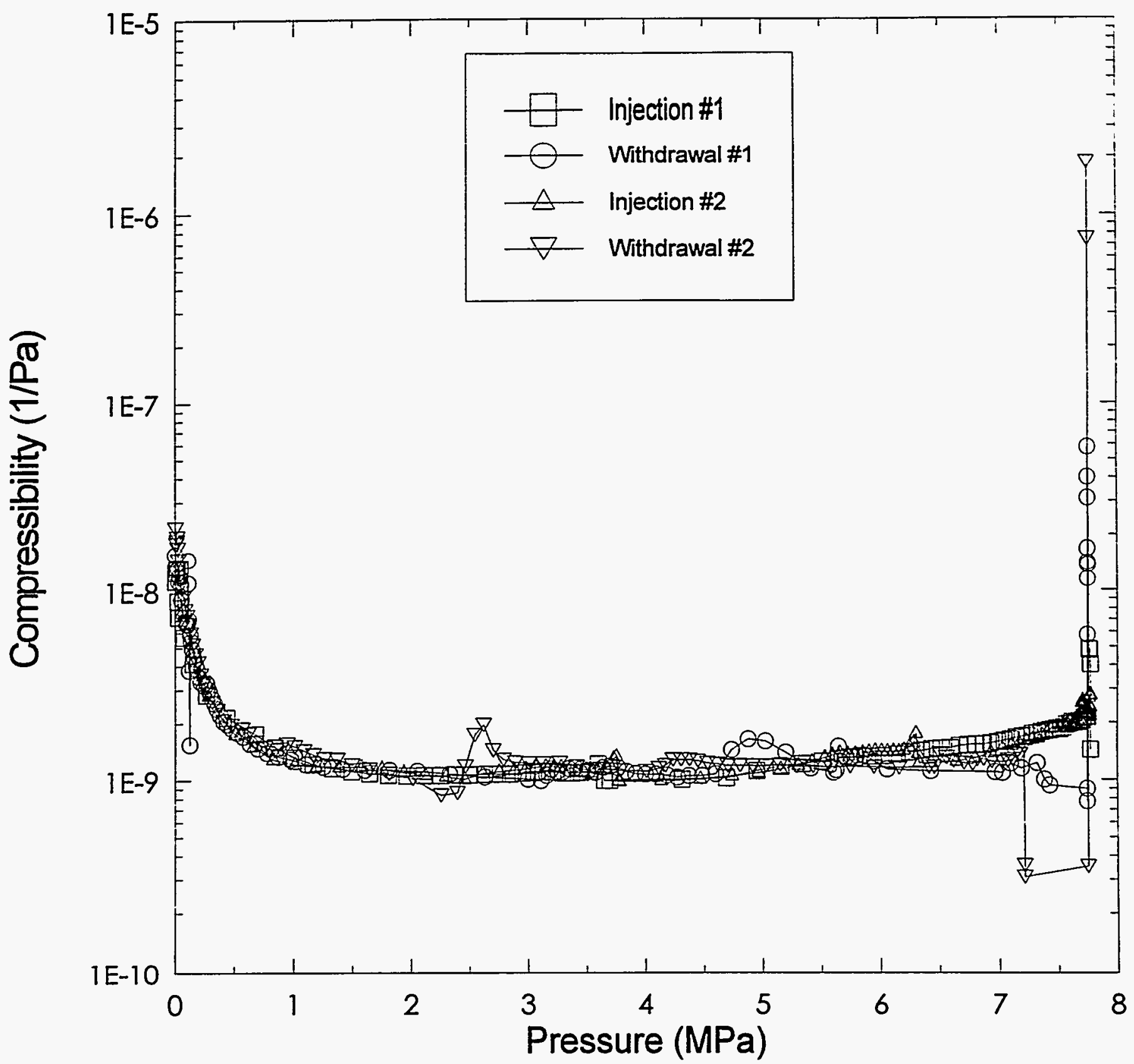

INTERA-B115-245-0

Figure 6-58. Test-zone compressibility as measured during compliance testing of test tool \#P74-B. 


\section{LONG-TERM FLUID-PRESSURE MONITORING}

The fluid-pressure data obtained during permeability testing, coupled permeability and hydrofracture testing, and gas-threshold pressure testing (GTPT) were supplemented with fluid-pressure data from packer-isolated sections of selected boreholes. The specific objectives of the long-term fluid-pressure monitoring were:

- to determine if pore pressures in the various Salado Formation interbeds change as a function of time due to the underground excavation; and

- to determine the relationship between proximity to an excavation and change in formation pore pressure as a function of time.

Single-packer fluid-pressure monitoring tools as described in Section 7.2 were installed in boreholes C2H01, C2H02, DPD01, DPD02, DPD03, L4P52, SCP01, S1P71, and S1P72. Permeability testing was conducted in boreholes $\mathrm{C} 2 \mathrm{H01}, \mathrm{C} 2 \mathrm{H} 02$, L4P52 (permeabilitytesting sequences L4P52-A and L4P52-B), SCP01, S1P71 (permeability-testing sequences S1P71-A and S1P71-B), and S1P72. GTPT was conducted in boreholes SCP01 (GTPT sequences SCP01-1 and SCP01-2), C2H02, and L4P52 (GTPT sequence L4P52-B). Permeability-testing sequences $\mathrm{C} 2 \mathrm{H} 01, \mathrm{C} 2 \mathrm{H} 02$, and S1P71-A are described in Saulnier et al. (1991). Permeability-testing sequences L4P52-A, SCP01, S1P71-B, and S1P72 are described in Stensrud et al. (1992). Permeability-testing sequence L4P52-B is described in Section 3.6.1.5 of this report. GTPT sequences C2H02, L4P52-B, SCP01-1, and SCP01-2 are described in Section 5.6 of this report. The dates of permeability testing and GTPT in these boreholes are given in Table 7-1. Figure 2-1 shows the locations of the boreholes involved in the long-term fluid-pressure monitoring.

Note: Fluid pressures associated with the long-term fluid-pressure monitoring were recorded in units of pounds per square inch (psig) and are reported as such.

Table 7-1. Locations and Dates of Testing Performed in Long-Term Fluid-Pressure Monitoring Boreholes

\begin{tabular}{ccccc}
\hline $\begin{array}{c}\text { Borehole } \\
\text { (Orientation) }\end{array}$ & Location & Dates Tested & $\begin{array}{c}\text { Excavation } \\
\text { Date of Room }\end{array}$ & Test Type \\
\hline $\begin{array}{c}\text { C2H01 } \\
\text { (vertical down) }\end{array}$ & Room C2 & $8-88$ to 2-89 & $\begin{array}{c}\text { March \& April } \\
1984\end{array}$ & Permeability \\
$\begin{array}{c}\text { C2H01 (Deepened) } \\
\text { (vertical down) }\end{array}$ & Room C2 & $2-89$ to 5-89 & $\begin{array}{c}\text { March \& April } \\
1984\end{array}$ & Permeability \\
$\begin{array}{c}\text { C2H02 } \\
\text { (downward 45 } \\
\text { from vertical) }\end{array}$ & Room C2 & $4-89$ to 12-89 & $\begin{array}{c}\text { March \& April } \\
1984\end{array}$ & Permeability \\
$\begin{array}{c}\text { C2H02 } \\
\text { (downward 45 } \\
\text { from vertical) }\end{array}$ & Room C2 & $8-93$ to 3-94 & March \& April & Gas-Threshold \\
\hline
\end{tabular}


Table 7-1 (Continued). Locations and Dates of Testing Performed in Long-Term Fluid-Pressure Monitoring Boreholes

\begin{tabular}{|c|c|c|c|c|}
\hline $\begin{array}{c}\text { Borehole } \\
\text { (Orientation) }\end{array}$ & Location & Dates Tested & $\begin{array}{c}\text { Excavation } \\
\text { Date of Room }\end{array}$ & Test Type \\
\hline $\begin{array}{c}\text { DPD01 } \\
\text { (downward } 46^{\circ} \\
\text { from vertical) }\end{array}$ & North 1400 Drift & N/A & March 1983 & N/A \\
\hline $\begin{array}{l}\text { DPD02 } \\
\text { (downward } 47^{\circ} \\
\text { from vertical) }\end{array}$ & North 1400 Drift & N/A & March 1983 & N/A \\
\hline $\begin{array}{c}\text { DPD03 } \\
\text { (downward } 46^{\circ} \\
\text { from vertical) }\end{array}$ & North 1400 Drift & N/A & March 1983 & N/A \\
\hline $\begin{array}{c}\text { L4P52 } \\
\text { (upward } 40^{\circ} \\
\text { from vertical) }\end{array}$ & Room L4 & $12-92$ to $12-93$ & February 1989 & Permeability \\
\hline $\begin{array}{c}\text { L4P52 } \\
\text { (upward } 40^{\circ} \\
\text { from vertical) }\end{array}$ & Room L4 & $12-93$ to $3-94$ & February 1989 & $\begin{array}{l}\text { Gas-Threshold } \\
\text { Pressure }\end{array}$ \\
\hline $\begin{array}{c}\text { SCP01 } \\
\text { (downward } 77^{\circ} \\
\text { from vertical) }\end{array}$ & Core Storage Library & $4-90$ to $10-90$ & May 1989 & Permeability \\
\hline $\begin{array}{c}\text { SCP01 } \\
\text { (downward } 77^{\circ} \\
\text { from vertical) }\end{array}$ & Core Storage Library & $4-94$ to $6-94$ & May 1989 & $\begin{array}{c}\text { Gas-Threshold } \\
\text { Pressure }\end{array}$ \\
\hline $\begin{array}{c}\text { \$1P71 } \\
\text { (vertical down) }\end{array}$ & $\begin{array}{c}\text { Waste Panel 1, Room } \\
7\end{array}$ & $11-88$ to $7-89$ & March 1988 & Permeability \\
\hline $\begin{array}{l}\text { S1P71 } \\
\text { (vertical down) }\end{array}$ & $\begin{array}{c}\text { Waste Panel } 1, \text { Room } \\
7\end{array}$ & $8-89$ to $5-90$ & March 1988 & Permeability \\
\hline $\begin{array}{c}\text { S1P72 } \\
\text { (downward 58 } \\
\text { from vertical) }\end{array}$ & $\begin{array}{c}\text { Waste Panel 1, Room } \\
7\end{array}$ & $12-90$ to $2-91$ & March 1988 & Permeability \\
\hline
\end{tabular}

\subsection{Implementation}

The long-term fluid-pressure monitoring discussed in this data report was conducted in nine boreholes drilled at five locations in the WIPP underground facility shown in Figure 2-1 and described in detail in Sections 7.4 and 7.5. The boreholes were drilled vertically downward, angled downward, and angled upward. In the experimental area, boreholes $\mathrm{C} 2 \mathrm{H} 01$ and $\mathrm{C} 2 \mathrm{H} 02$ were drilled in Room C2, L4P52 was drilled in Room L4, and boreholes DPD01, DPD02, and DPD03 were drilled on the north rib of the north 1400 drift between Room C1 and Room M. In the operations area, borehole SCP01 was drilled in the Core Storage Library. In the waste storage area, boreholes S1P71 and S1P72 were 
drilled in Waste Panel 1, Room 7. The monitoring locations were chosen to provide an adequate representation of long-term fluid pressures in the Salado Formation and its interbeds at and near the repository horizon. Figure 7-1 illustrates the stratigraphic positions of each of the monitoring boreholes in relation to the typical WIPP excavation.

The borehole zones isolated by the single-packer tools were MB138 and MB139. Table 7-2 lists the pertinent information for the boreholes and zones.

Table 7-2. Fluid-Pressure Monitoring Boreholes; Test-Zone Information

\begin{tabular}{cccccccc}
\hline Borehole & $\begin{array}{c}\text { Test } \\
\text { Horizon }\end{array}$ & $\begin{array}{c}\text { Test Horizon } \\
\text { Penetrated }\end{array}$ & $\begin{array}{c}\text { Borehole } \\
\text { Diameter }(\mathrm{cm})\end{array}$ & $\begin{array}{c}\text { Test Interval } \\
\text { Depth }(\mathrm{m})\end{array}$ & $\begin{array}{c}\text { Borehole } \\
\text { Depth }(\mathrm{m})\end{array}$ & $\begin{array}{c}\text { Monitoring } \\
\text { Started }\end{array}$ & $\begin{array}{c}\text { Monitoring } \\
\text { Terminated }\end{array}$ \\
\hline C2H01 & MB139 & $2-13-89$ & 10.16 & $6.80-7.76$ & 8.97 & $8-89$ & $4-95$ \\
C2H02 & MB139 & $4-14-89$ & 10.16 & $9.80-10.68$ & 10.91 & $2-91$ & $4-95$ \\
L4P52-B & MB138 & $12-14-92$ & 10.16 & $13.89-14.02$ & 14.12 & $5-94$ & $5-95$ \\
S1P71-B & anhydrite "C" & $7-24-89$ & 10.16 & $9.75-9.80$ & 10.15 & $2-91$ & $5-95$ \\
S1P72 & MB139 & $12-13-89$ & 10.16 & $4.40-6.00$ & 6.05 & $2-91$ & $5-95$ \\
SCP01 & MB139 & $3-29-90$ & 10.16 & $10.50-14.78$ & 15.48 & $10-90$ & $5-95$ \\
\hline
\end{tabular}

After drilling or after the completion of another testing sequence, a single-packer tool (see Section 7.2) was installed in each of the boreholes. The packers were used to isolate a selected zone below the packers. The zones of the long-term fluid-pressure monitoring boreholes were filled with $1.22 \mathrm{~kg} / \mathrm{L}$ sodium-chloride brine. After packer inflation, the zone of each tool was shut in and the fluid-pressure buildup was monitored with manually read Bourdon tube pressure gages (Ashcroft or Wika) and recorded on appropriate data collection forms. No additional testing was conducted in the boreholes after the tools were installed.

\subsection{Equipment for Long-Term Fluid-Pressure Monitoring}

The following sections briefly describe the equipment used in the long-term fluid-pressure monitoring in the WIPP underground facility. The equipment includes single-packer tools and Bourdon tube pressure gages.

\subsubsection{Single-Packer Tool}

The single-packer tools installed for this monitoring are shown in Sections 7.4 and 7.5. The tools consist of a sliding-end, 9.5-cm outside diameter (O.D.) inflatable packer mounted on a 4.83-cm O.D. mandrel oriented with the packers' fixed end toward the bottom-hole end of the test tool. The packers have $0.92-\mathrm{m}$-long inflatable elastic elements composed of natural rubber and synthetic materials. The packer elements have 


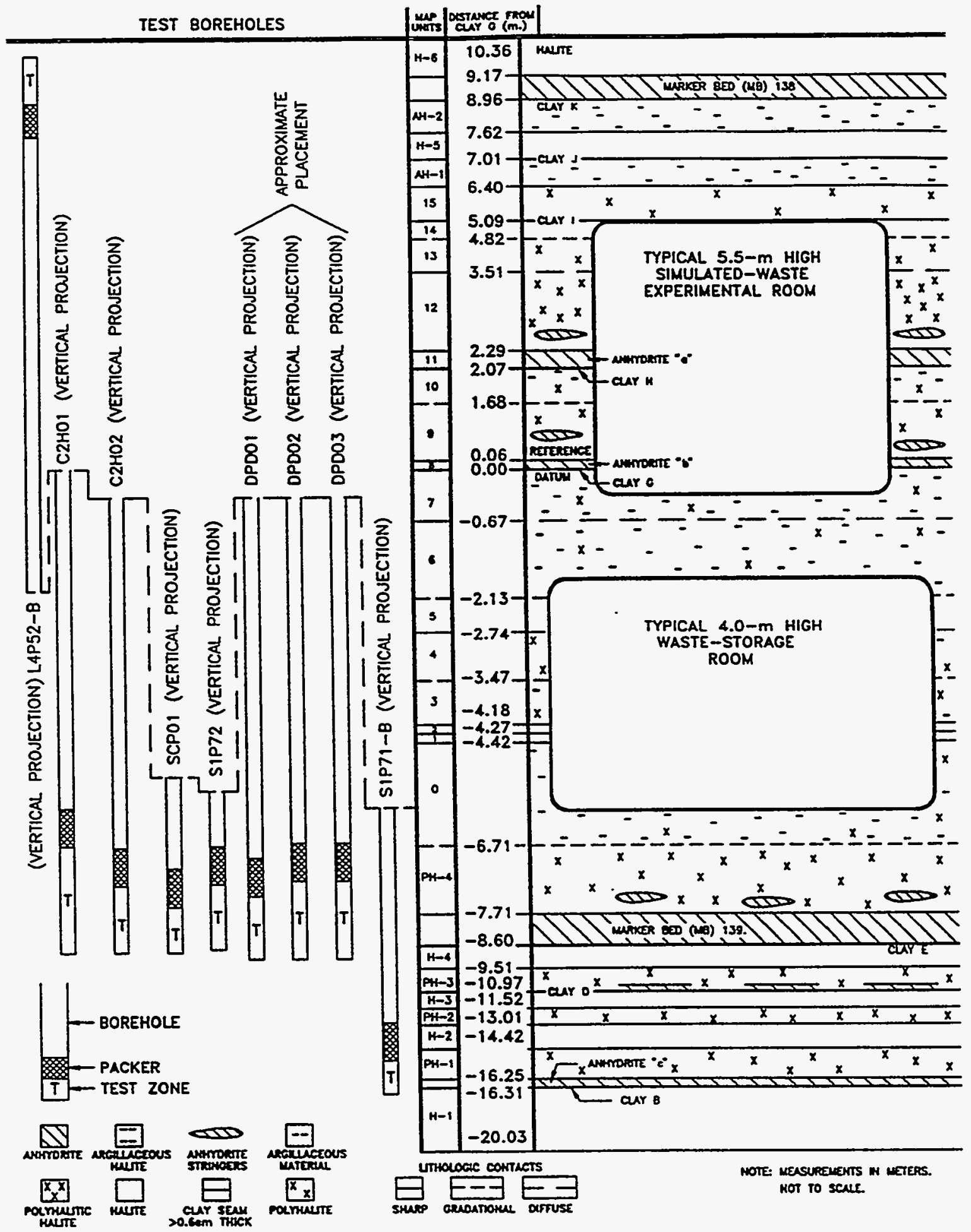

INTERA-61 15-246-0

Figure 7-1. Stratigraphic positions of long-term fluid-pressure monitoring boreholes with test zones indicated. 
approximately $0.9-\mathrm{m}$ seal lengths when inflated in 4-inch $(10.2-\mathrm{cm})$ diameter boreholes. In all cases, a set of radially oriented tapered jaws or slips that tighten on the tool mandrel as the tool attempted to move out of the borehole, in response to pressure buildup, was used to restrain the tool.

Each single-packer tool was equipped with two ports to the bottom-hole test zone below the packers. One port was used to transmit pressures from the test zones to the Bourdon tube gages, which were mounted outside of the boreholes. The second port was used to dissipate "squeeze" pressures created during packer inflation. These two ports were accessed by continuous lengths of $3 / 16$-inch $(0.48-\mathrm{cm})$ O.D. stainless-steel tubing.

\subsubsection{Bourdon Tube Gages}

Bourdon tube pressure gages of varying ranges (WIKA and Ashcroft brand) were used for all of the long term fluid-pressure measurements. The pressure range of each gage used was appropriate for the borehole pressure being monitored. Both aone and packer pressures were monitored and manually recorded. The gages were not calibrated and were used for indication purposes only. As such, the pressure measurements obtained from these gages should not be used for data interpretation.

\subsection{Procedures for Long-Term Fluid-Pressure Monitoring}

\subsubsection{Tool Installation Procedure}

The following describes a typical tool-installation sequence. Single-packer tools were installed in each of the boreholes as soon after the completion of a permeability-testing sequence or a GTPT sequence as possible in order to minimize depressurization of the formation surrounding the borehole. Prior to initiating long-term fluid-pressure monitoring, a multipacker test tool configured appropriately for either permeability testing or GTPT sequences was replaced by a single-packer tool. All single-packer tools were installed following tool-installation procedures presented in SOP INT-5 (INTERA, 1992) and/or WIPP Procedures for test-tool installation (479, 480, and 481) (see Figures 3-15 through 3-17). Before tool installation in vertically down or angled downward borehole orientations, enough brine was added to the borehole to fill the zone to be monitored, helping to minimize air entrapment. After the single-packer tool was installed, the packer was inflated to an appropriate pressure as indicated on the Bourdon tube pressure gage and the zone was shut in. Both zone and packer pressures were monitored and recorded.

For boreholes oriented vertically or angled upward, the single-packer tools were installed and the packer inflated to an appropriate pressure. The isolated borehole was then filled with brine while a vacuum pump attached to the zone vent line removed entrapped air. In some cases, 10 -psi $(68.95 \mathrm{kPa})$ check valves were attached to the vent lines to prevent 
brine from draining from the isolated borehole interval, assuring fluid-filled zones (Figure 3-18).

\subsection{Fluid-Pressure Monitoring}

Information and plots of the fluid-pressure monitoring data are presented below. Complete tabulations of the manually recorded data are stored in the SWCF under WPO \#42269.

\subsubsection{Borehole C2HO1}

Table 7-3 lists the events associated with borehole $\mathrm{C} 2 \mathrm{H} 01$ from the time the borehole was drilled to the termination of all testing and/or monitoring in the borehole with the events associated with the fluid-pressure monitoring emphasized. Descriptions of events that occurred during permeability testing in this borehole and of the core recovered from the borehole can be found in Saulnier et al. (1991).

Table 7-3. Long-Term Fluid-Pressure Monitoring C2H01 Events

\begin{tabular}{|c|c|c|c|}
\hline EVENT & DATE & $\begin{array}{l}\text { CALENDAR } \\
\text { DAY }\end{array}$ & $\begin{array}{c}1989 \\
\text { CALENDAR DAY }\end{array}$ \\
\hline Drill borehole $\mathrm{C} 2 \mathrm{H} 01$ to 5.58 meters. & $8-4-88$ & 217 & N/A \\
\hline Begin permeability-testing sequence $\mathrm{C} 2 \mathrm{H} 01-\mathrm{A}$. & $8-6-88$ & 219 & N/A \\
\hline Terminate permeability-testing sequence $\mathrm{C} 2 \mathrm{H} 01-\mathrm{A}$. & $9-1-88$ & 245 & N/A \\
\hline Begin permeability-testing sequence $\mathrm{C} 2 \mathrm{H} 01-\mathrm{B}$. & $9-2-88$ & 246 & N/A \\
\hline Terminate permeability-testing sequence $\mathrm{C} 2 \mathrm{H} 01-\mathrm{B}$. & $2-9-89$ & 40 & 40 \\
\hline Borehole $\mathrm{C} 2 \mathrm{H} 01$ deepened to 8.97 meters & $2-15-89$ & 46 & 46 \\
\hline Begin permeability-testing sequence $\mathrm{C} 2 \mathrm{H} 01-\mathrm{C}$. & $2-16-89$ & 47 & 47 \\
\hline Terminate permeability-testing sequence $\mathrm{C} 2 \mathrm{H} 01-\mathrm{C}$. & $5-22-89$ & 142 & 142 \\
\hline $\begin{array}{c}\text { Install single-packer long-term fluid-pressure monitor tool } \\
\text { to monitor fluid pressure in MB139 as indicated in } \\
\text { Figure } 7-2 .\end{array}$ & $8-22-89$ & 234 & 234 \\
\hline Inflate packer to $\sim 1500$ psig. & $8-22-89$ & 234 & 234 \\
\hline Increase packer pressure to $\sim 1500$ psig. & $8-24-89$ & 236 & 236 \\
\hline $\begin{array}{c}\text { Diagnose that the monitor tool was moving out of the } \\
\text { borehole. }\end{array}$ & $10-30-89$ & 303 & 303 \\
\hline Depressurize test zone. & $7-5-90$ & 186 & 551 \\
\hline Deflate packer. & $7-5-90$ & 186 & 551 \\
\hline Remove long-term fluid-pressure monitoring tool. & $7-5-90$ & 186 & 551 \\
\hline
\end{tabular}


Table 7-3 (Continued). Long-Term Fluid-Pressure Monitoring C2H01 Events

\begin{tabular}{|c|c|c|c|}
\hline EVENT & DATE & $\begin{array}{l}\text { CALENDAR } \\
\text { DAY }\end{array}$ & $\begin{array}{c}1989 \\
\text { CALENDAR DAY }\end{array}$ \\
\hline $\begin{array}{l}\text { Ream borehole } \mathrm{C} 2 \mathrm{HO} 1 \text { to } 4-\text { inch }(10.16-\mathrm{cm}) \text { to bottom of } \\
\text { borehole. }\end{array}$ & $1-22-91$ & 22 & 752 \\
\hline Video-log borehole. & 2-7-91 & 38 & 768 \\
\hline $\begin{array}{c}\text { Install single-packer long-term fluid-pressure monitor tool } \\
\text { to monitor fluid pressure in MB139 as indicated in } \\
\text { Figure } 5-9 \text {. }\end{array}$ & $2-11-91$ & 42 & 772 \\
\hline Inflate packer to $\sim 1500 \mathrm{psig}$ & $2-12-91$ & 43 & 773 \\
\hline Shut in test zone. & $2-12-91$ & 43 & 773 \\
\hline Depressurize test zone. & 3-13-91 & 72 & 802 \\
\hline Deflate packer. & 3-13-91 & 72 & 802 \\
\hline Remove long-term fluid-pressure monitoring tool. & 3-13-91 & 72 & 802 \\
\hline $\begin{array}{c}\text { Re-install single-packer long-term fluid-pressure monitor } \\
\text { tool to monitor fluid pressure in MB139 as indicated in } \\
\text { Figure 5-9. }\end{array}$ & $3-13-91$ & 72 & 802 \\
\hline Inflate packer to $\sim 1500$ psig. & $3-13-91$ & 72 & 802 \\
\hline Increase test zone pressure to $\sim 275$ psig. & $3-14-91$ & 73 & 803 \\
\hline $\begin{array}{l}\text { Install transducer \#321768 to monitor pressure during } \\
\text { coupled permeability and hydrofracture-testing sequence } \\
\text { C } 1 \times 10 .\end{array}$ & $1-29-92$ & 29 & 1124 \\
\hline $\begin{array}{l}\text { Begin fluid-pressure monitoring of MB139 associated with } \\
\text { testing sequence } C 1 \times 10 .\end{array}$ & $1-29-92$ & 29 & 1124 \\
\hline $\begin{array}{c}\text { Terminate fluid-pressure monitoring associated with } \\
\text { testing sequence } \mathrm{C} 1 \times 10 .\end{array}$ & $6-26-92$ & 178 & 1273 \\
\hline $\begin{array}{l}\text { Begin fluid-pressure monitoring of MB139 associated with } \\
\text { testing sequence } \mathrm{C} 1 \times 05-\mathrm{A} \text {. }\end{array}$ & $6-26-92$ & 178 & 1273 \\
\hline $\begin{array}{l}\text { Terminate fluid-pressure monitoring associated with } \\
\text { testing sequence } C 1 \times 05-A \text {. }\end{array}$ & $8-3-92$ & 216 & 1311 \\
\hline $\begin{array}{l}\text { Resume long-term fluid-pressure monitoring of fluid } \\
\text { pressure in MB139. }\end{array}$ & $8-3-92$ & 216 & 1311 \\
\hline Increase packer pressure. & $7-12-93$ & 193 & 1653 \\
\hline $\begin{array}{l}\text { Begin fluid-pressure monitoring of } \mathrm{MB139} \text { associated with } \\
\text { testing sequence } \mathrm{C} 2 \mathrm{H} 02 \text {. }\end{array}$ & $9-28-93$ & 271 & 1731 \\
\hline $\begin{array}{c}\text { Terminate fluid-pressure monitoring associated with } \\
\text { testing sequence } \mathrm{C} 2 \mathrm{H} 02 \text {. }\end{array}$ & $3-4-94$ & 77 & 1894 \\
\hline $\begin{array}{l}\text { Resume long-term fluid-pressure monitoring of fluid } \\
\text { pressure in MB139. }\end{array}$ & $3-4-94$ & 77 & 1894 \\
\hline
\end{tabular}


Table 7-3 (Continued). Long-Term Fluid-Pressure Monitoring C2H01 Events

\begin{tabular}{cccc}
\hline EVENT & DATE & $\begin{array}{c}\text { CALENDAR } \\
\text { DAY }\end{array}$ & $\begin{array}{c}1989 \\
\text { CALENDAR DAY }\end{array}$ \\
\hline Depressurize test zone. & $4-20-95$ & 110 & 1935 \\
Deflate packer. & $4-20-95$ & 110 & 1935 \\
Remove long-term fluid-pressure monitoring tool. & $4-20-95$ & 110 & 1935 \\
Terminate long-term fluid-pressure monitoring. & $4-20-95$ & 110 & 1935 \\
\hline
\end{tabular}

Figure 4-7 schematically depicts Room $\mathrm{C} 2$ in plan view and in cross section, showing the locations and orientations of boreholes $\mathrm{C} 2 \mathrm{H} 01$ and $\mathrm{C} 2 \mathrm{H} 02$. Figures 7-2 and 5-9 illustrate both of the tool configurations and associated installations that were used during the fluidpressure monitoring in borehole $\mathrm{C} 2 \mathrm{H} 01$. Figure $7-3$ is a plot of the fluid pressure observed in borehole $\mathrm{C} 2 \mathrm{H} 01$. 
$\mathrm{C} 2 \mathrm{HO} 1$

SINGLE-PACKER TOOL CONFIGURATION \#1
BOREHOLE: C2HO1

DATE: $08 / 22 / 89$

DEPTH OF HOLE: $8.97 \mathrm{~m}$.

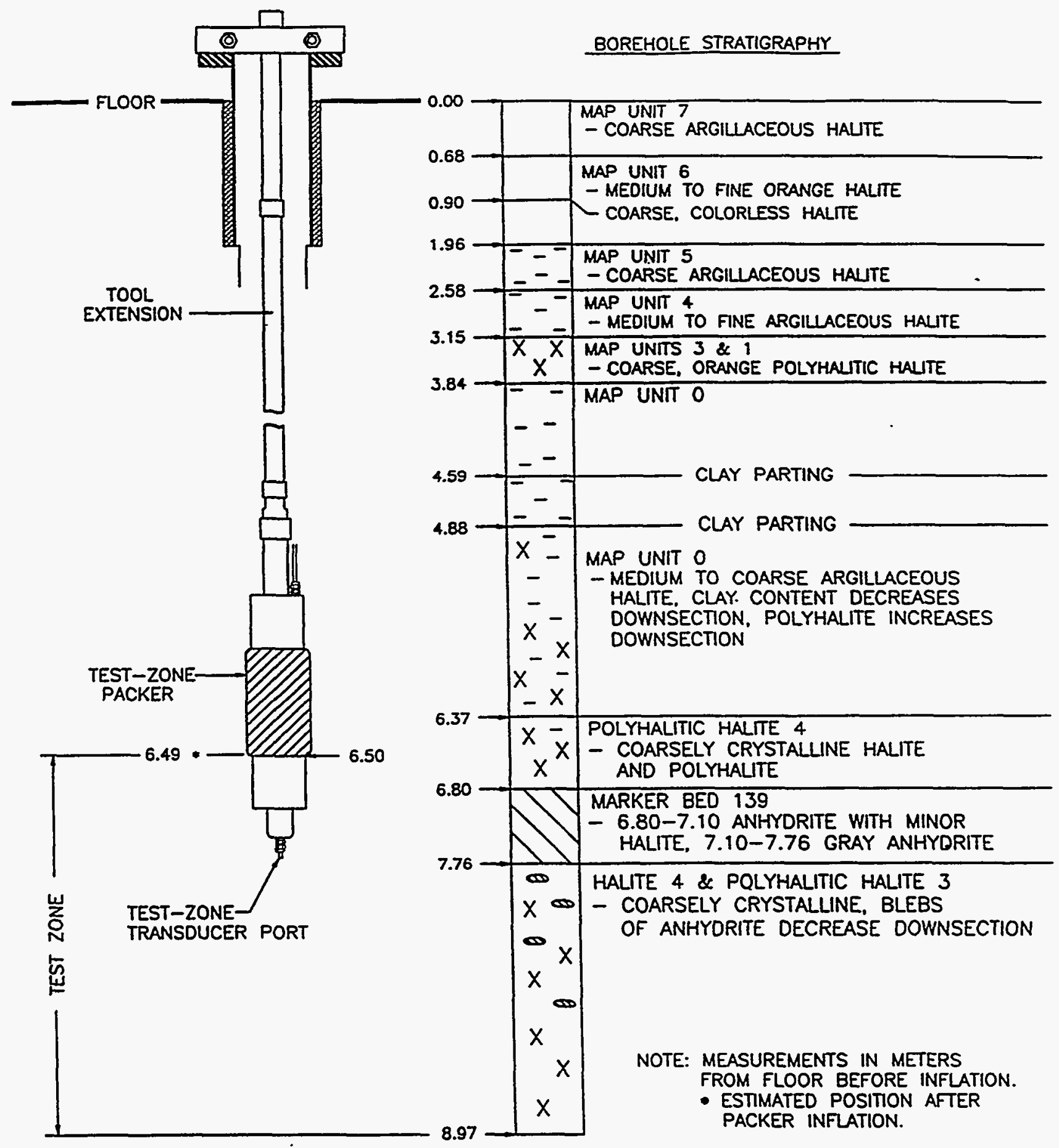

IKTERA-8115-247-0

Figure 7-2. Configuration \#1 of long-term fluid-pressure monitoring tool in borehole $\mathrm{C} 2 \mathrm{H} 01$. 


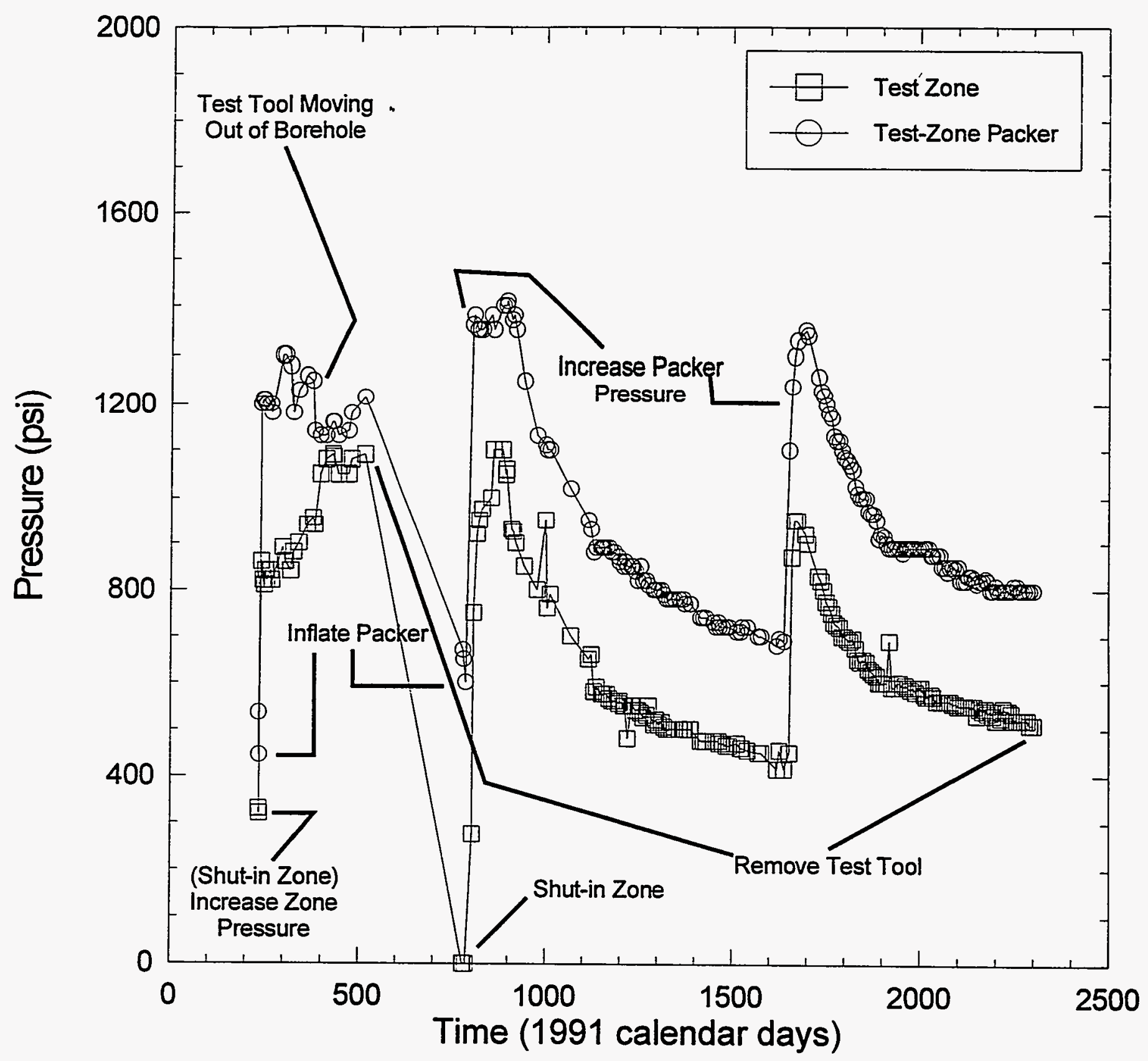

INTERA-6115-249-0

Figure 7-3. Long-term fluid pressure in MB139 in borehole $\mathrm{C} 2 \mathrm{H} 01$. 


\subsubsection{Borehole $\mathrm{C} 2 \mathrm{HO}$}

Table 7-4 lists the events associated with borehole $\mathrm{C} 2 \mathrm{H} 02$ from the time the borehole was drilled to the termination of all testing and/or monitoring in the borehole with the events associated with the fluid-pressure monitoring emphasized. Descriptions of events that occurred during permeability testing in this borehole and the core recovered from the borehole can be found in Saulnier et al. (1991). Descriptions of events that occurred during the GTPT in this borehole can be found in Section 5.6.2 of this report.

Table 7-4. Long-Term Fluid-Pressure Monitoring $\mathrm{C} 2 \mathrm{H} 02$ Events

\begin{tabular}{|c|c|c|c|}
\hline EVENT & DATE & $\begin{array}{c}\text { CALENDAR } \\
\text { DAY }\end{array}$ & $\begin{array}{l}1991 \\
\text { CALENDAR DAY }\end{array}$ \\
\hline Drill borehole $\mathrm{C} 2 \mathrm{HO} 2$ to 10.86 meters. & $4-17-89$ & 107 & N/A \\
\hline Begin permeability-testing sequence $\mathrm{C} 2 \mathrm{HO}$. & 4-19-89 & 109 & N/A \\
\hline Terminate permeability-testing sequence $\mathrm{C} 2 \mathrm{H} 02$. & $12-12-89$ & 346 & N/A \\
\hline $\begin{array}{c}\text { Ream borehole } \mathrm{C} 2 \mathrm{H} 02 \text { 4-inch }(10.16-\mathrm{cm}) \text { to bottom of } \\
\text { borehole. }\end{array}$ & $1-22-91$ & 22 & 22 \\
\hline Video log borehole. & 2-7-91 & 38 & 38 \\
\hline $\begin{array}{c}\text { Install single-packer long-term fluid-pressure monitor tool } \\
\text { to monitor fluid pressure in MB139 as indicated in } \\
\text { Figure } 7-4 .\end{array}$ & $2-11-91$ & 42 & 42 \\
\hline Inflate packer to $~ 1500$ psig. & $2-12-91$ & 43 & 43 \\
\hline Shut in test zone. & $2-12-91$ & 43 & 43 \\
\hline Diagnose and fix leak on test zone. & $10-9-91$ & 282 & 282 \\
\hline Replace bad gage on test zone. & $1-7-92$ & 7 & 372 \\
\hline Depressurize test zone. & $8-9-93$ & 221 & 951 \\
\hline Deflate packer. & $8-9-93$ & 221 & 951 \\
\hline Remove long-term fluid-pressure monitoring tool. & $8-9-93$ & 221 & 951 \\
\hline $\begin{array}{l}\text { Install GTPT test tool as indicated in Figure } 5-10 \text { and } \\
\text { begin GTPT sequence } \mathrm{C} 2 \mathrm{HO2} \text {. }\end{array}$ & $8-10-93$ & 222 & 952 \\
\hline $\begin{array}{l}\text { Remove GTPT test tool and terminate GTPT sequence } \\
\qquad \mathrm{C} 2 \mathrm{H} 02 .\end{array}$ & $3-23-94$ & 82 & 1177 \\
\hline $\begin{array}{l}\text { Install single-packer long-term fluid-pressure monitor tool } \\
\text { to monitor fluid pressure in MB139 as indicated in } \\
\text { Figure 7-5. }\end{array}$ & $3-24-94$ & 83 & 1178 \\
\hline Add approximately 10 liters of brine to borehole. & $3-25-94$ & 84 & 1179 \\
\hline Inflate packer to -1750 psig. & $3-25-94$ & 84 & 1179 \\
\hline Increase test zone pressure to $\sim 1080$ psig. & $3-25-94$ & 84 & 1179 \\
\hline
\end{tabular}


Table 7-4 (Continued). Long-Term Fluid-Pressure Monitoring C2H02 Events

\begin{tabular}{cccc}
\hline EVENT & DATE & $\begin{array}{c}\text { CALENDAR } \\
\text { DAY }\end{array}$ & $\begin{array}{c}\text { 1991 } \\
\text { CALENDAR DAY }\end{array}$ \\
\hline Depressurize test zone. & $4-20-95$ & 110 & 1570 \\
Deflate packer. & $4-20-95$ & 110 & 1570 \\
Remove long-term fluid-pressure monitoring tool. & $4-20-95$ & 110 & 1570 \\
Terminate long-term fluid-pressure monitoring. & $4-20-95$ & 110 & 1570 \\
\hline
\end{tabular}

Figures $7-4$ and $7-5$ illustrate the tool configurations and associated installations that were used during the fluid-pressure monitoring in borehole $\mathrm{C} 2 \mathrm{H} 02$. Figure 7-6 is a plot of the fluid pressure observed in borehole $\mathrm{C} 2 \mathrm{HO} 2$. 


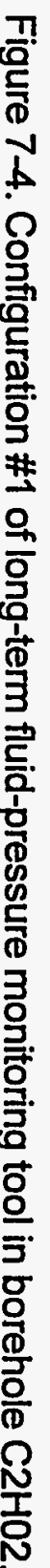

$\mathrm{C} 2 \mathrm{HO} 2$ SINGLE-PACKER TOOL CONFIGURATION \#1

BOREHOLE: $\mathrm{C} 2 \mathrm{HO} 2$

DATE: 01/22/91

DEPTH OF HOLE: $10.86 \mathrm{~m}$
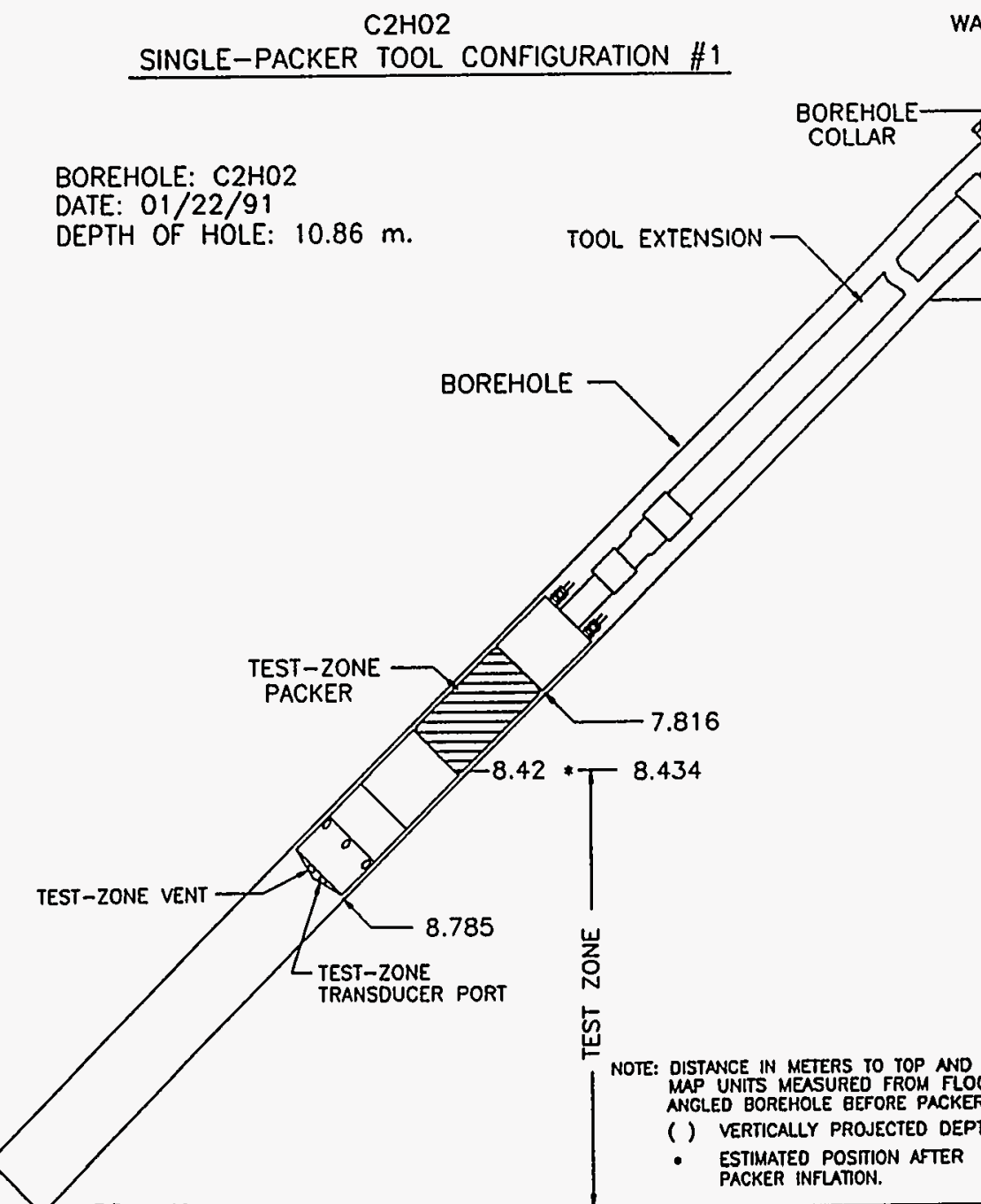

TOOL EXTENSION
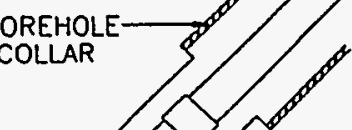

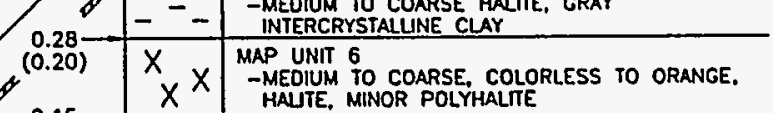

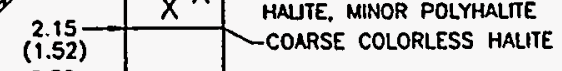

\begin{tabular}{c|c}
2.35 \\
$(1.66)$
\end{tabular}$\quad \begin{gathered}\text { MAP UNII } 5 \\
\text {-COARE, TO FINE ARGILACEOUS HALITE }\end{gathered}$

(2.05) MAP UNT

$(3.92$

\begin{tabular}{|c|c|c|}
\hline $\begin{array}{l}4.55-- \\
(3.22)\end{array}$ & \begin{tabular}{|c|}
$x-\bar{x}$ \\
$-\bar{x}$
\end{tabular} & $\begin{array}{l}\text { MAP UNIT }{ }^{3} \text { - MEDUM, ORANGE POLYHALITC HNLITE } \\
\text { - }\end{array}$ \\
\hline & $\mid \begin{array}{ll}- & 1 \\
x & x\end{array}$ & $\begin{array}{l}\text { MAP UNT } 2 \text { CLAY PARTING } \\
\text { MAP UNT } 1 \text { CLPLST }\end{array}$ \\
\hline & & 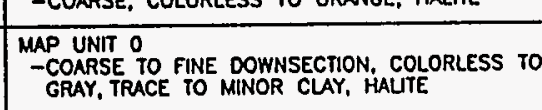 \\
\hline
\end{tabular}

(5.)

\begin{tabular}{rl|l}
$\times$ & $\times$ & $\begin{array}{c}\text { POLYHALLTIC HALITE } 4 \\
\text {-COARSE, COLORLESS TO ORANGE, } \\
\text { HALITE AND POLYHALITE }\end{array}$ \\
$\times$ &
\end{tabular}

DTE: DISTANCE IN METERS TO TOP NND BOTTOM OF MAP UEITS MEASURED FROM FLOOR ALONG ANGLED BOREHOLE BEFORE PACKER INR - estimateo postion after
packer inflation.

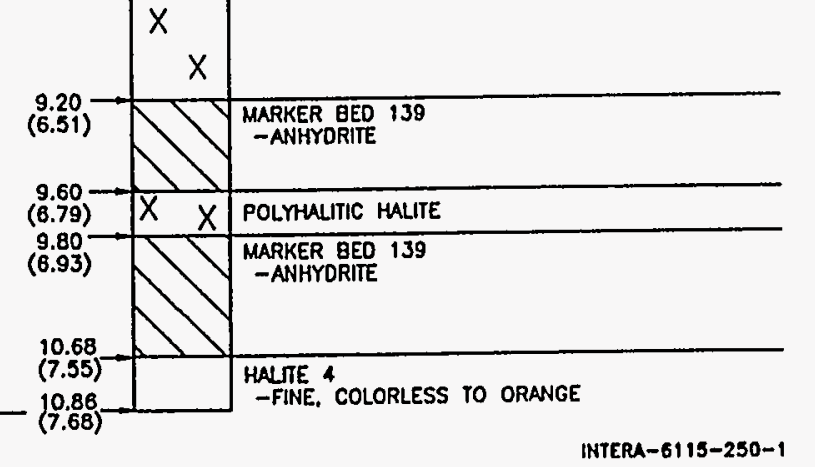




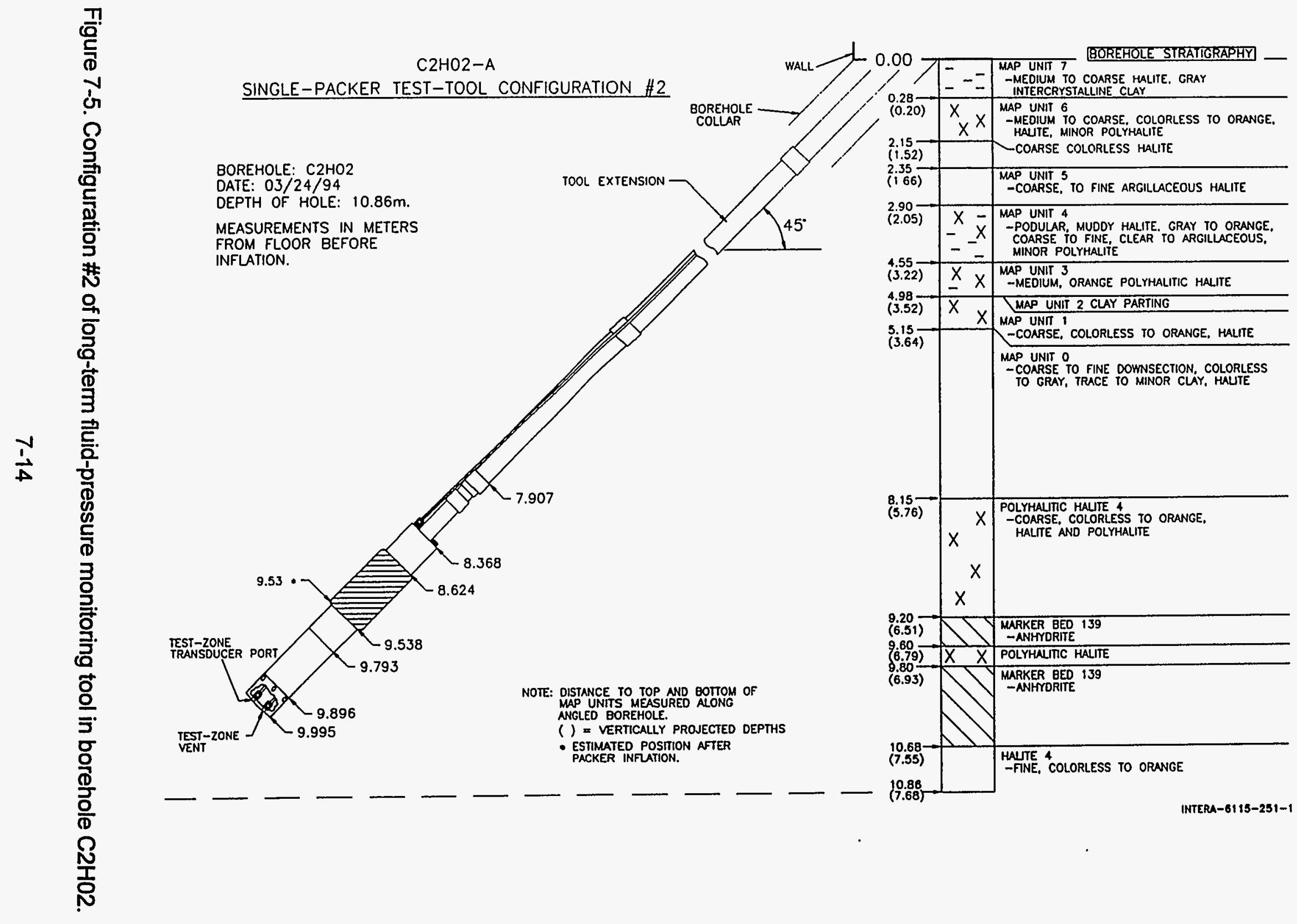




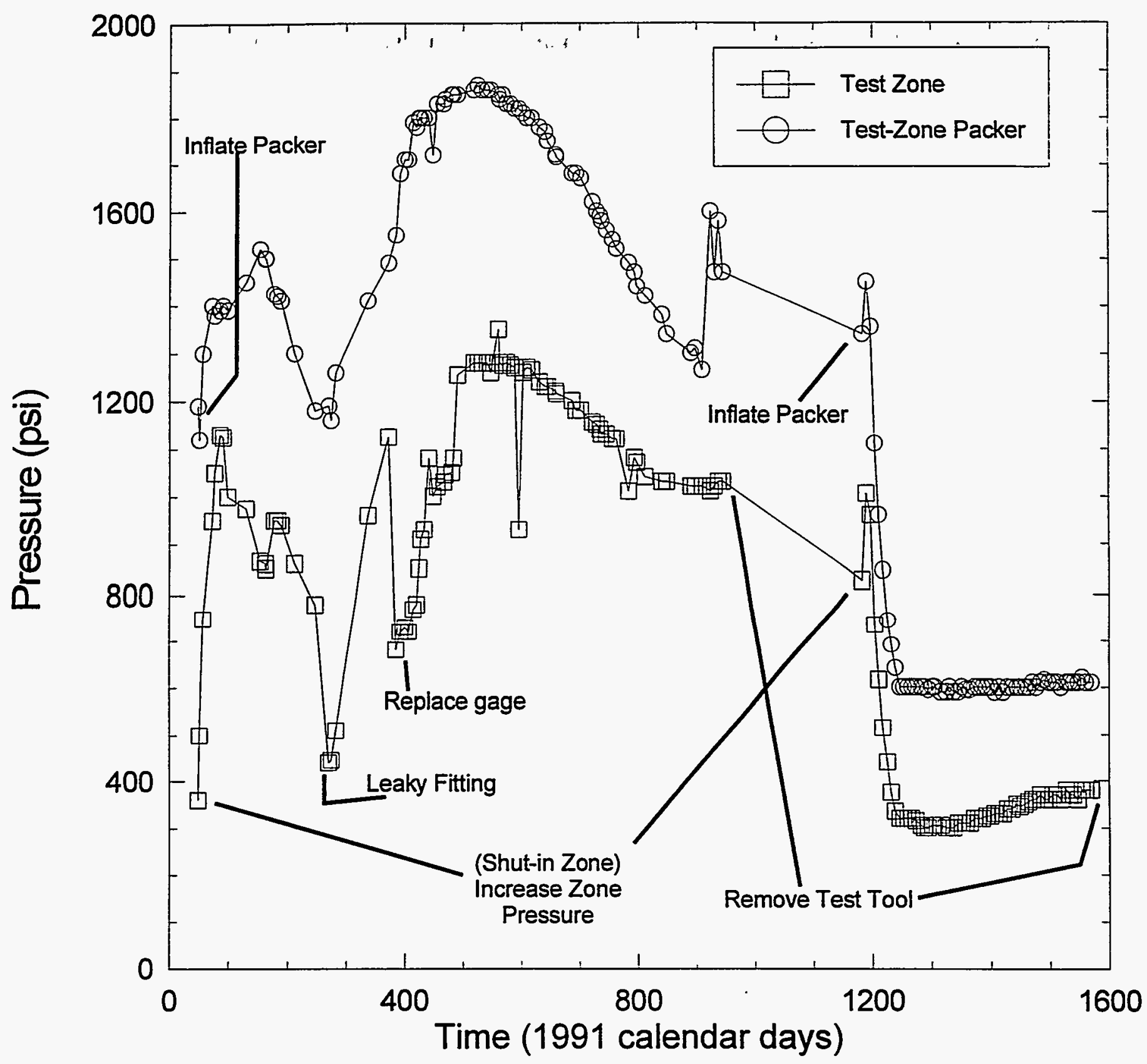

INTERA-8115-252-0

Figure 7-6. Long-term fluid pressure in MB139 in borehole $\mathrm{C} 2 \mathrm{H} 02$. 


\subsubsection{Borehole L4P52}

Table 7-5 lists the events associated with borehole L4P52 from the time the borehole was drilled to the termination of all testing and/or monitoring in the borehole with the events associated with the fluid-pressure monitoring emphasized. Descriptions of events that occurred during both the permeability testing and the GTPT in this borehole can be found in Sections 3.6.1.5 and 5.6.3 of this report. A description of the core recovered from borehole L4P52 can be found in Appendix A of this report.

Table 7-5. Long-Term Fluid-Pressure Monitoring L4P52 Events

\begin{tabular}{|c|c|c|c|}
\hline EVENT & DATE & $\begin{array}{l}\text { CALENDAR } \\
\text { DAY }\end{array}$ & $\begin{array}{c}1994 \\
\text { CALENDAR DAY }\end{array}$ \\
\hline Borehole L4P52 drilled to 5.56 meters. & $4-2-91$ & 92 & N/A \\
\hline Video-log borehole. & 4-8-91 & 98 & N/A \\
\hline Begin permeability-testing sequence L4P52-A. & 4-12-91 & 102 & N/A \\
\hline Terminate permeability-testing sequence L4P52-A. & $8-11-92$ & 224 & N/A \\
\hline Deepen borehole L4P52 to 14.18 meters. & $12-14-92$ & 349 & N/A \\
\hline Install test tool as indicated in Figure 3-31. & $12-16-93$ & 351 & N/A \\
\hline Begin permeability-testing sequence L4P52-B. & $12-17-92$ & 352 & N/A \\
\hline Terminate permeability-testing sequence L4P52-B. & $12-28-93$ & 362 & N/A \\
\hline Begin GTPT sequence L4P52-B. & $12-29-93$ & 363 & N/A \\
\hline Remove test tool and terminate GTPT sequence L4P52-B. & $5-4-94$ & 124 & 124 \\
\hline $\begin{array}{c}\text { Install single-packer long-term fluid-pressure monitor tool } \\
\text { to monitor fluid pressure in MB138 as indicated in } \\
\text { Figure } 7-7 .\end{array}$ & $5-4-94$ & 124 & 124 \\
\hline Inflate packer to $\sim 1500$ psig. & $5-5-94$ & 125 & 125 \\
\hline Shut in test zone. & $5-5-94$ & 125 & 125 \\
\hline Depressurize test zone. & $5-16-95$ & 136 & 501 \\
\hline Deflate packer. & $5-16-95$ & 136 & 501 \\
\hline Remove long-term fluid-pressure monitoring tool. & $5-16-95$ & 136 & 501 \\
\hline Terminate long-term fluid-pressure monitoring. & $5-16-95$ & 136 & 501 \\
\hline
\end{tabular}

Figure 3-20 schematically depicts Room L4 in plan view and in cross section, showing the location and orientation of borehole L4P52. Figure 7-7 illustrates the test-tool configuration and associated installation that was used during the fluid-pressure monitoring in borehole L4P52. Figure 7-8 is a plot of the fluid pressure observed in borehole L4P52-B. 
L4P52-B

SINGLE-PACKER TEST-TOOL CONFIGURATION

BOREHOLE: L4P52

DATE: OS: 44942

DEPTH OF HOLE: $14.12 \mathrm{~m}$
BOREHOLE STRATGRAPHY

T.D. 14.12

(10.82) A1V Anhydrite (Marker Bed 138)
- Anhydrite, light gray

- microcrystalline.

(10.64) Argillaceous Halite (AH-2)

- Clear to moderate brown. some light moderate reddish

- orange. medium to coorsely crystalline contact with lower unit is gradational based on clay content, upper contact

TEST-ZONE TEST-

TRANSDUCER PORT

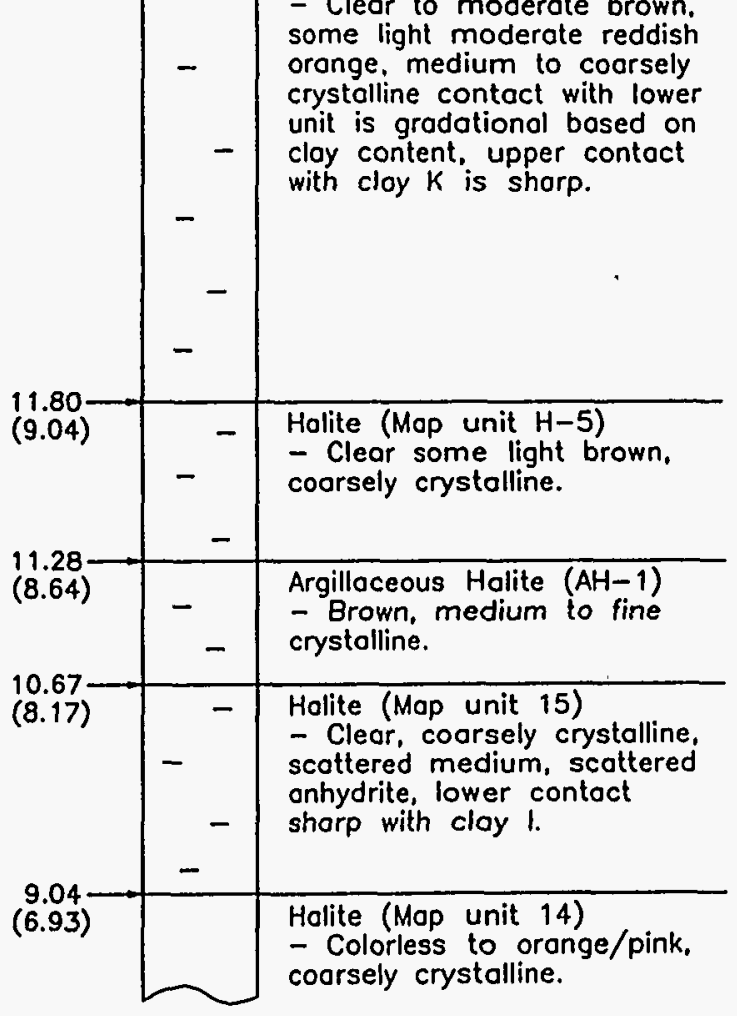

NOTE: DISTANCE IN METERS TO TOP ANO BOTTOM OF MAP UNITS MEASURED FROM ROOM WALL ALONG ANGLED BOREHOLE. BEFORE PACKER INFLATION. ( ) VERTICALLYY PROJECTED OEPTHS.

- ESTIMATEO POSITION AFTER PACKER INFLATION.
(SEE LAP52-A,B CORE LOGS FOR
$0.00+$ REMAINDER OF DESCRIPTION.) 


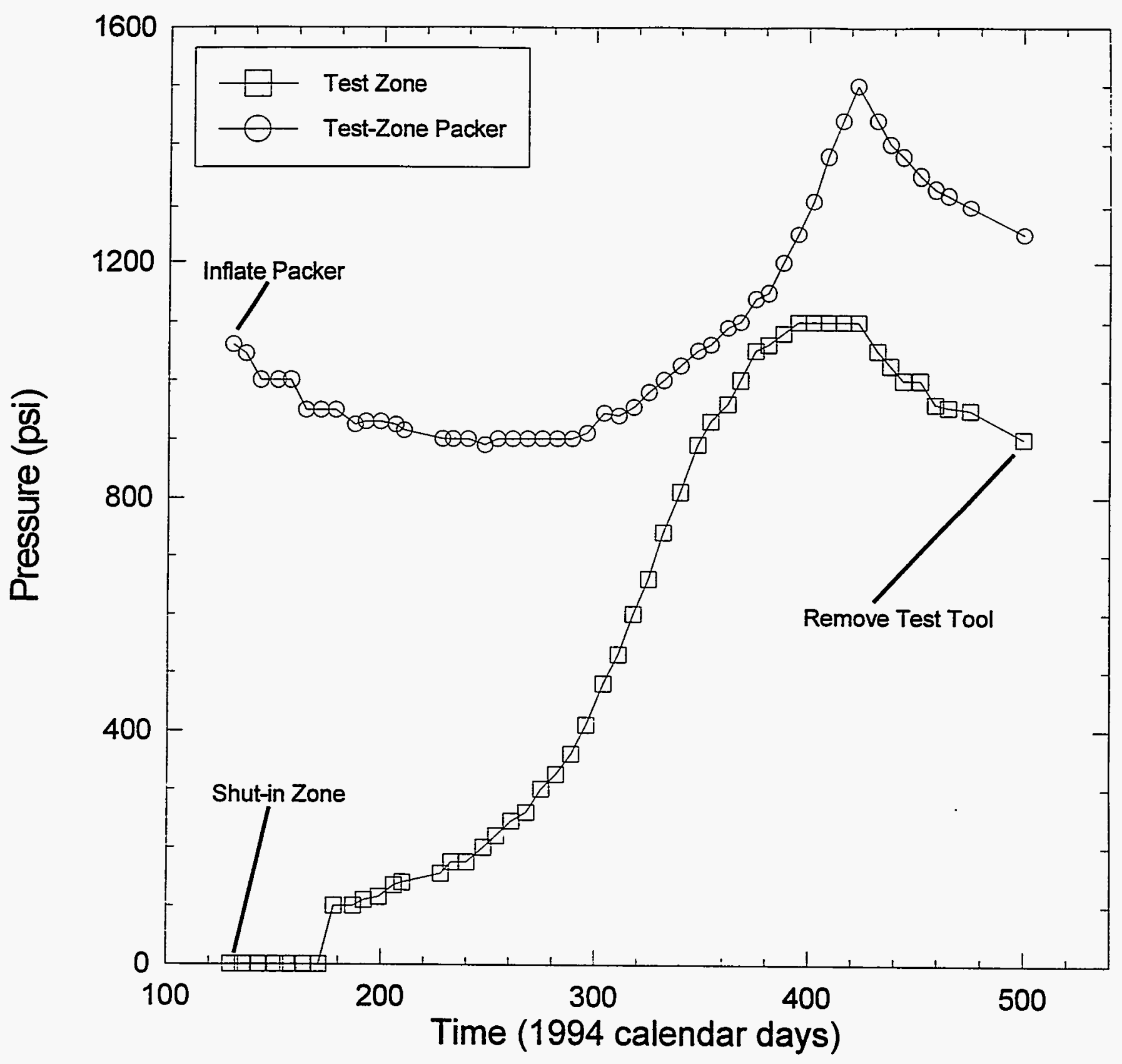

INTERA6115-2540

Figure 7-8. Long-term fluid pressure in MB138 in borehole L4P52. 


\subsubsection{Borehole SCP01}

Table 7-6 lists the events associated with borehole SCP01 from the time the borehole was drilled to the termination of all testing and/or monitoring in the borehole with the events associated with the fluid-pressure monitoring emphasized. Descriptions of events that occurred during permeability testing in this borehole and of the core recovered from the borehole can be found in Stensrud et al. (1992). A description of events that occurred during GTPT in this borehole can be found in Sections 5.6.1.1 and 5.6.1.2 of this report.

Table 7-6. Long Term Fluid-Pressure Monitoring SCP01 Events

\begin{tabular}{|c|c|c|c|}
\hline EVENT & DATE & $\begin{array}{c}\text { CALENDAR } \\
\text { DAY }\end{array}$ & $\begin{array}{c}1992 \\
\text { CALENDAR DAY }\end{array}$ \\
\hline Drill borehole SCPO1 to 15.39 meters. & $3-30-90$ & 89 & 89 \\
\hline Begin permeability-testing sequence SCP01. & $4-10-90$ & 100 & 100 \\
\hline Terminate permeability-testing sequence SCPO1. & $10-11-90$ & 284 & 284 \\
\hline $\begin{array}{c}\text { Install single-packer long-term fluid-pressure monitor tool } \\
\text { to monitor fluid pressure in MB139 as indicated in } \\
\text { Figure } 7-9 .\end{array}$ & $10-12-90$ & 285 & 285 \\
\hline Add approximately $30.3 \mathrm{~L}$ of brine to borehole. & $10-15-90$ & 288 & 288 \\
\hline Inflate packer to $1500 \mathrm{psi}$. & $10-15-90$ & 288 & 288 \\
\hline Re-inflate packer to 1500 psi. & $10-15-90$ & 288 & 288 \\
\hline Re-inflate packer to $1540 \mathrm{psi}$. & $10-16-90$ & 289 & 289 \\
\hline Shut in test zone. & $10-16-90$ & 289 & 289 \\
\hline Monitor tool moved out of borehole $1.2-\mathrm{cm}$ total distance. & $11-6-90$ & 310 & 310 \\
\hline Monitor tool moved out of borehole $1.3-\mathrm{cm}$ total distance. & $11-8-90$ & 312 & 312 \\
\hline Monitor tool moved out of borehole $1.5-\mathrm{cm}$ total distance. & $11-9-90$ & 313 & 313 \\
\hline Monitor tool moved out of borehole $3.0-\mathrm{cm}$ total distance. & $11-12-90$ & 316 & 316 \\
\hline Monitor tool moved out of borehole $4.3-\mathrm{cm}$ total distance. & $11-14-90$ & 318 & 318 \\
\hline $\begin{array}{l}\text { Replace restraining device with slip-type to eliminate } \\
\text { monitor tool movement. }\end{array}$ & $11-19-90$ & 323 & 323 \\
\hline Replace bad gages on both test zone and packer. & $12-6-90$ & 340 & 340 \\
\hline Inflate packer to $\sim 1475$ psi. & $12-6-90$ & 340 & 340 \\
\hline Depressurize test zone. & $12-10-92$ & 344 & 1075 \\
\hline Deflate packer. & $12-10-92$ & 344 & 1075 \\
\hline Rotate packer $90^{\circ}$ clockwise. & $12-10-92$ & 344 & 1075 \\
\hline Inflate packer to 1500 psi. & $12-10-92$ & 344 & 1075 \\
\hline Shut in test zone. & $12-10-92$ & 344 & 1075 \\
\hline
\end{tabular}


Table 7-6 (Continued). Long Term Fluid-Pressure Monitoring SCP01 Events

\begin{tabular}{|c|c|c|c|}
\hline EVENT & DATE & $\begin{array}{l}\text { CALENDAR } \\
\text { DAY }\end{array}$ & $\begin{array}{l}1992 \\
\text { CALENDAR DAY }\end{array}$ \\
\hline Depressurize test zone. & $3-22-93$ & 81 & 1176 \\
\hline Deflate packer. & $3-22-93$ & 81 & 1176 \\
\hline Remove long-term fluid-pressure monitor tool. & $3-22-93$ & 81 & 1176 \\
\hline $\begin{array}{l}\text { Install GTPT test tool as indicated in Figure } 5-4 \text { and begin } \\
\text { GTPT sequence SCPO1-1. }\end{array}$ & 3-30-93 & 89 & 1184 \\
\hline $\begin{array}{l}\text { Remove GTPT test tool and terminate GTPT sequence } \\
\text { SCP01-1. }\end{array}$ & $7-13-93$ & 194 & 1289 \\
\hline $\begin{array}{l}\text { Install long-term fluid-pressure monitoring tool to monitor } \\
\text { fluid pressure in MB139. }\end{array}$ & $7-14-93$ & 195 & 1290 \\
\hline Inflate packer to $\sim 1500 \mathrm{psi}$. & 7-14-93 & 195 & 1290 \\
\hline Shut in test zone. & $7-14-93$ & 195 & 1290 \\
\hline Depressurize test zone. & $4-13-94$ & 103 & 1563 \\
\hline Deflate packer. & 4-13-94 & 103 & 1563 \\
\hline Remove long-term fluid-pressure monitoring tool. & 4-13-94 & 103 & 1563 \\
\hline $\begin{array}{l}\text { Install GTPT test tool as indicated in Figure 5-7 and begin } \\
\text { GTPT sequence SCP01-2. }\end{array}$ & $4-14-94$ & 104 & 1564 \\
\hline $\begin{array}{l}\text { Remove GTPT test tool and terminate GTPT sequence } \\
\qquad \text { SCP01-2. }\end{array}$ & $8-2-94$ & 214 & 1674 \\
\hline $\begin{array}{c}\text { Install single-packer long-term fiuid-pressure monitor tool } \\
\text { to monitor fluid pressure in MB139 as indicated in } \\
\text { Figure } 7-10 \text {. }\end{array}$ & $8-2-94$ & 214 & 1674 \\
\hline Inflate packer to $\sim 1500 \mathrm{psi}$. & $8-2-94$ & 214 & 1674 \\
\hline Shut in test zone. & $8-2-94$ & 214 & 1674 \\
\hline Leak in packer. & $8-3-94$ & 215 & 1675 \\
\hline Depressurize test zone. & $5-2-95$ & 122 & 1947 \\
\hline Deflate packer. & $5-2-95$ & 122 & 1947 \\
\hline Remove long-term fluid-pressure monitoring tool. & $5-2-95$ & 122 & 1947 \\
\hline Terminate long-term fluid-pressure monitoring. & $5-2-95$ & 122 & 1947 \\
\hline
\end{tabular}

Figure 5-3 schematically depicts the Core Storage Library in plan view and in cross section, showing the location and orientation of borehole SCP01. Figures 7-9 and 7-10 illustrate all of the test-tool configurations and associated installations that were used during the fluid-pressure monitoring in borehole SCPO1 (information regarding configuration \#2 is not available and, therefore, there is no associated installation diagram). Figure $7-11$ is a plot of the fluid pressure observed in borehole SCP01. 


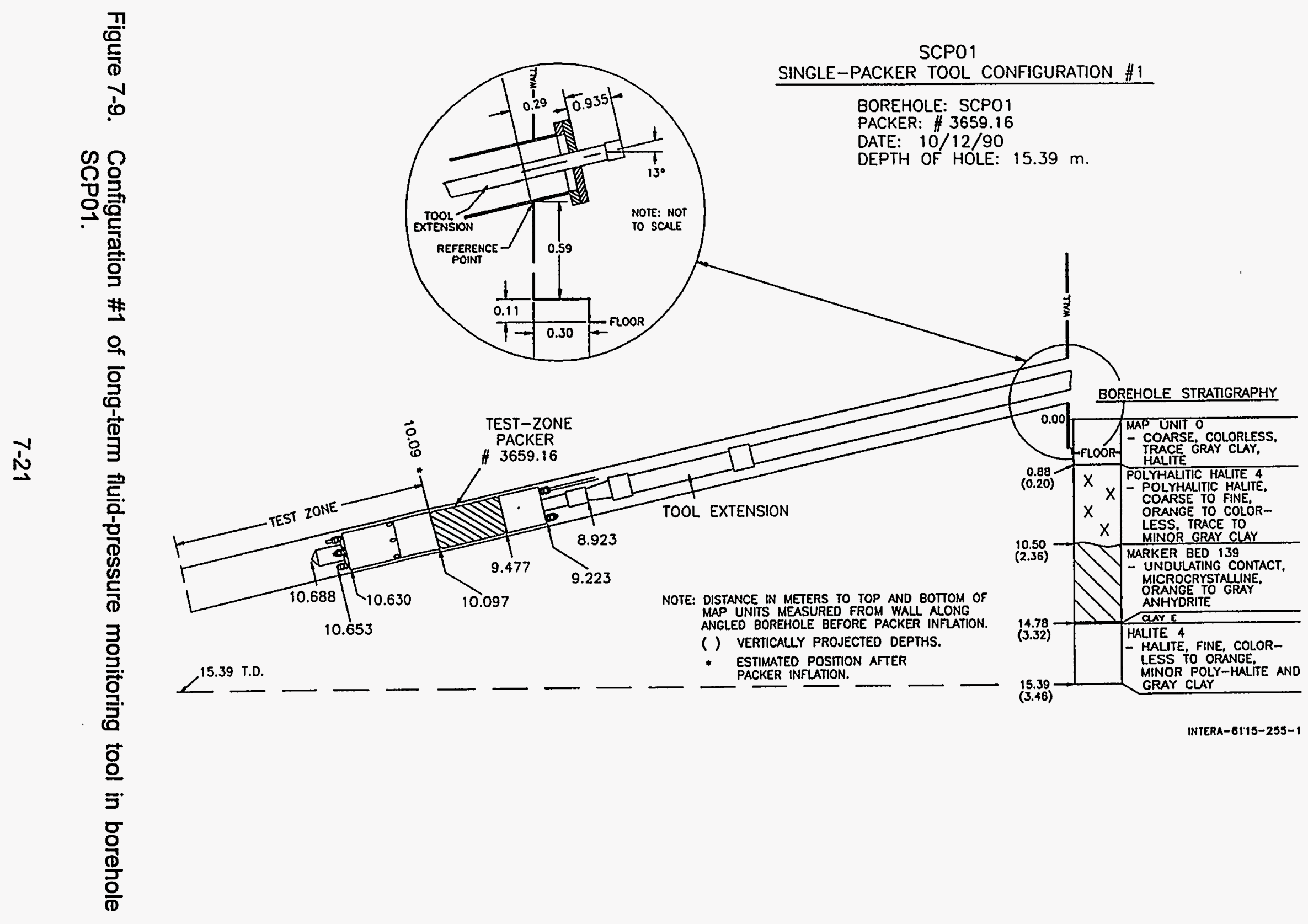




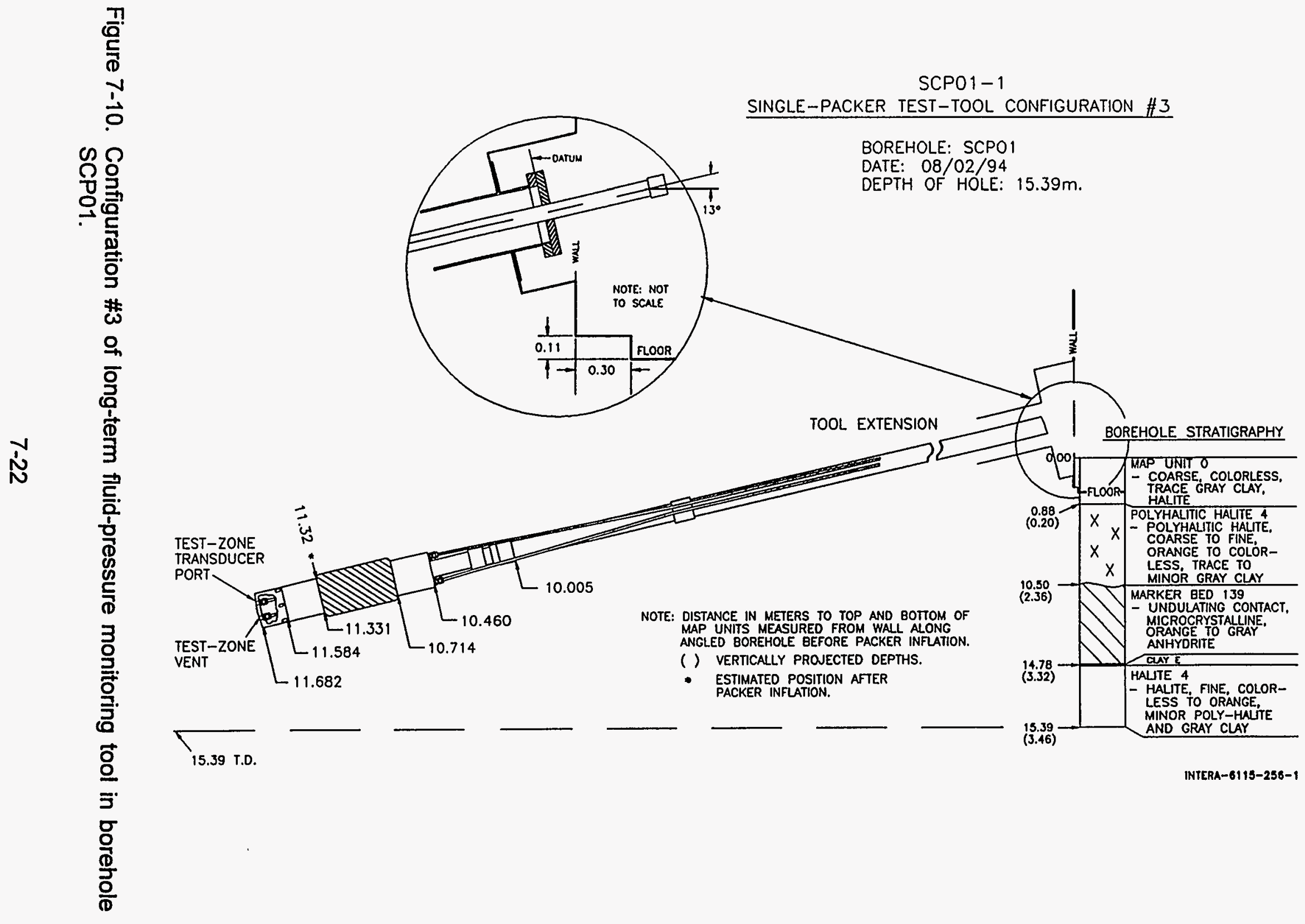




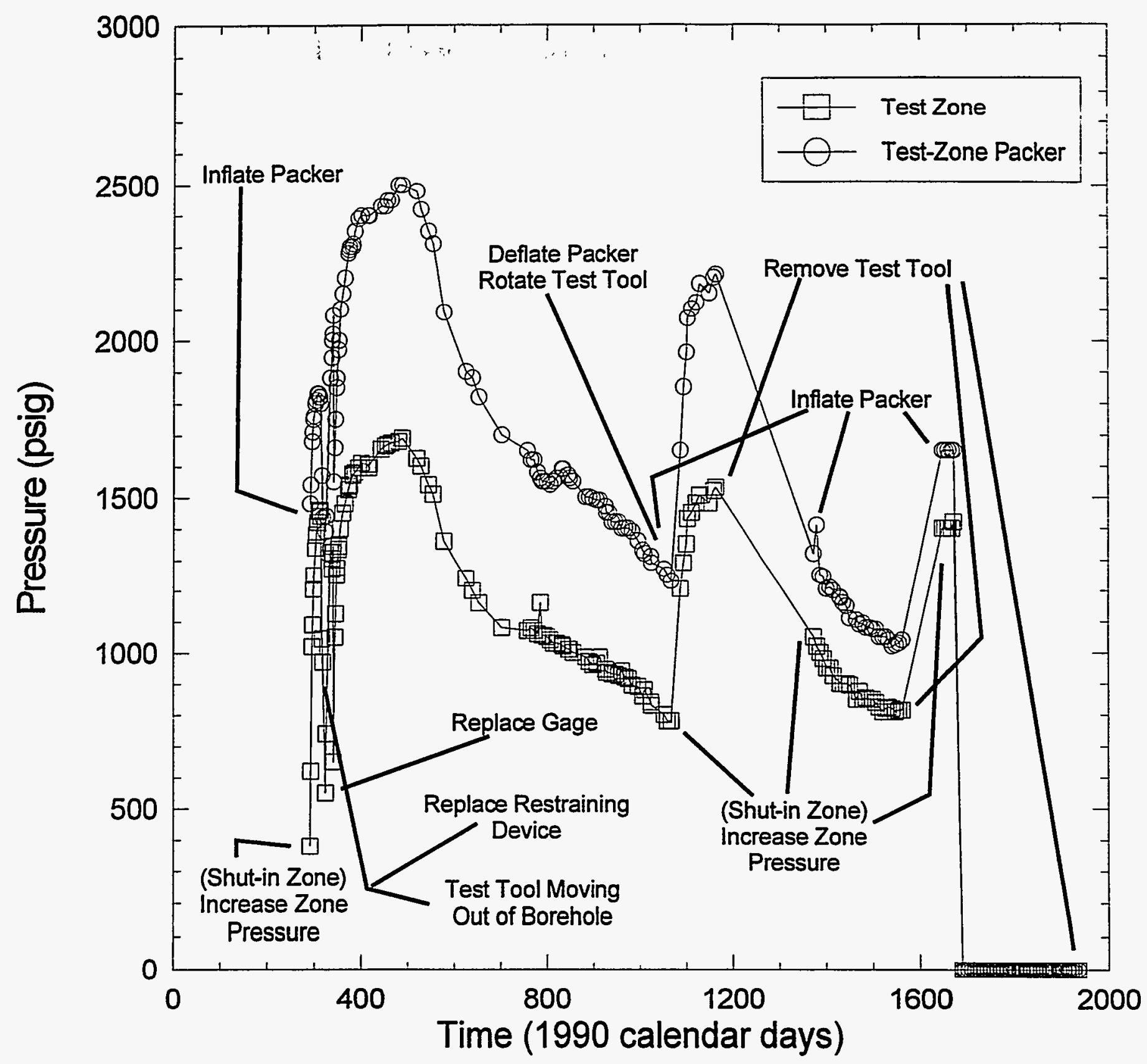

INTERA-B115-257-0

Figure 7-11. Long-term fluid pressure in MB139 in borehole SCP01. 


\subsubsection{Borehole S1P71}

Table 7-7 lists the events associated with borehole S1P71 from the time the borehole was drilled to the termination of all testing and/or monitoring in the borehole with the events associated with the fluid-pressure monitoring emphasized. A description of events that occurred during permeability-testing sequence S1P71-A can be found in Saulnier et al. (1991). Events that occurred during permeability-testing sequence S1P71-B, as well as core recovered from the borehole, are described in Stensrud et al. (1992).

Table 7-7. Long-Term Fluid-Pressure Monitoring S1P71 Events

\begin{tabular}{|c|c|c|c|}
\hline EVENT & DATE & $\begin{array}{l}\text { CALENDAR } \\
\text { DAY }\end{array}$ & $\begin{array}{l}1991 \\
\text { CALENDAR DAY }\end{array}$ \\
\hline Drill borehole S1P71 to 4.56 meters. & $11-10-88$ & 316 & N/A \\
\hline Begin permeability-testing sequence S1P71-A. & $11-11-88$ & 317 & N/A \\
\hline Terminate permeability-testing sequence S1P71-A. & $7-10-89$ & 191 & N/A \\
\hline Deepen borehole S1P71 to 10.15 meters. & $7-24-89$ & 205 & N/A \\
\hline Begin permeability-testing sequence S1P71-B. & $7-25-89$ & 206 & N/A \\
\hline Terminate permeability-testing sequence S1P71-B. & $5-24-90$ & 144 & N/A \\
\hline $\begin{array}{l}\text { Ream borehole S1P71 to 4-inch }(10.16-\mathrm{cm}) \text { to bottom of } \\
\text { borehole. }\end{array}$ & $6-7-91$ & 158 & 158 \\
\hline Set and grout new collar. & $6-7-91$ & 158 & 158 \\
\hline Load test new collar. & $6-10-91$ & 161 & 161 \\
\hline Video-log borehole. & $6-11-91$ & 162 & 162 \\
\hline $\begin{array}{l}\text { Install long-term fluid-pressure monitoring tool to monitor } \\
\text { fluid pressure in anhydrite "c" as indicated in Figure 7-12. }\end{array}$ & $6-11-91$ & 162 & 162 \\
\hline Inflate packer to $\sim 1490 \mathrm{psi}$ & $6-13-91$ & 164 & 164 \\
\hline Deflate packer. & $6-13-91$ & 164 & 164 \\
\hline Re-inflate packer to $\sim 1490$ psi. & $6-13-91$ & 164 & 164 \\
\hline Increase packer pressure to $\sim 1580 \mathrm{psi}$. & $6-13-91$ & 164 & 164 \\
\hline Shut in test zone. & $6-13-91$ & 164 & 164 \\
\hline Increase packer pressure. & $7-12-93$ & 193 & 923 \\
\hline Depressurize test zone. & $5-16-95$ & 136 & 1596 \\
\hline Deflate packer. & $5-16-95$ & 136 & 1596 \\
\hline Remove long-term fluid-pressure monitoring tool. & $5-16-95$ & 136 & 1596 \\
\hline Terminate long-term fluid-pressure monitoring. & $5-16-95$ & 136 & 1596 \\
\hline
\end{tabular}


Figure 3-21 schematically depicts Waste Panel 1, Room 7 in plan view and in cross section, showing the location and orientation of boreholes S1P71 and S1P72. Figure 7-12 illustrates the test-tool configuration and associated installation that was used during the fluid-pressure monitoring in borehole S1P71. Figure 7-13 is a plot of the fluid pressure observed in borehole S1P71. 


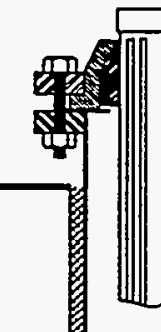

BOREHOLE: S1P71

TEST TOOL: \#3659.20

DATE: $06 / 11 / 91$

DEPTH OF HOLE: $10.15 \mathrm{~m}$.
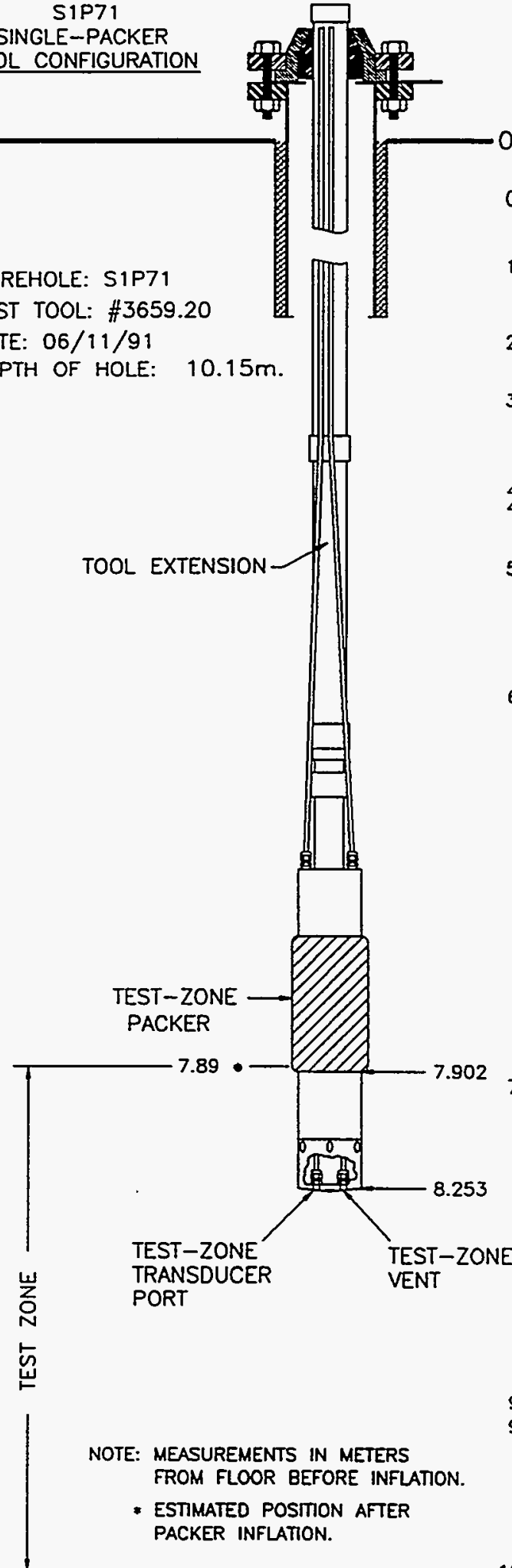

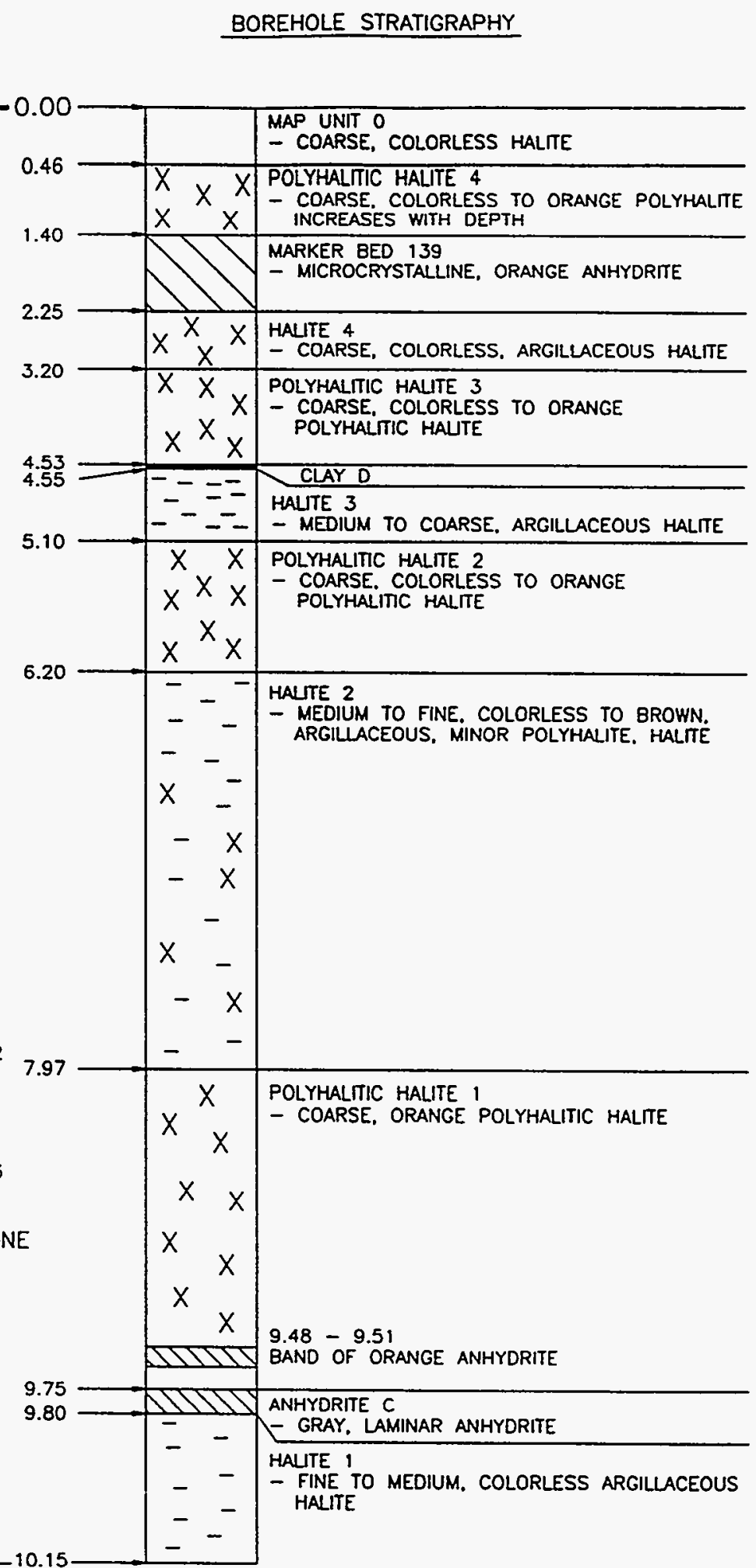

INTERA-6115-258-1

Figure 7-12. Configuration of long-term fluid-pressure monitoring tool in borehole S1P71. 


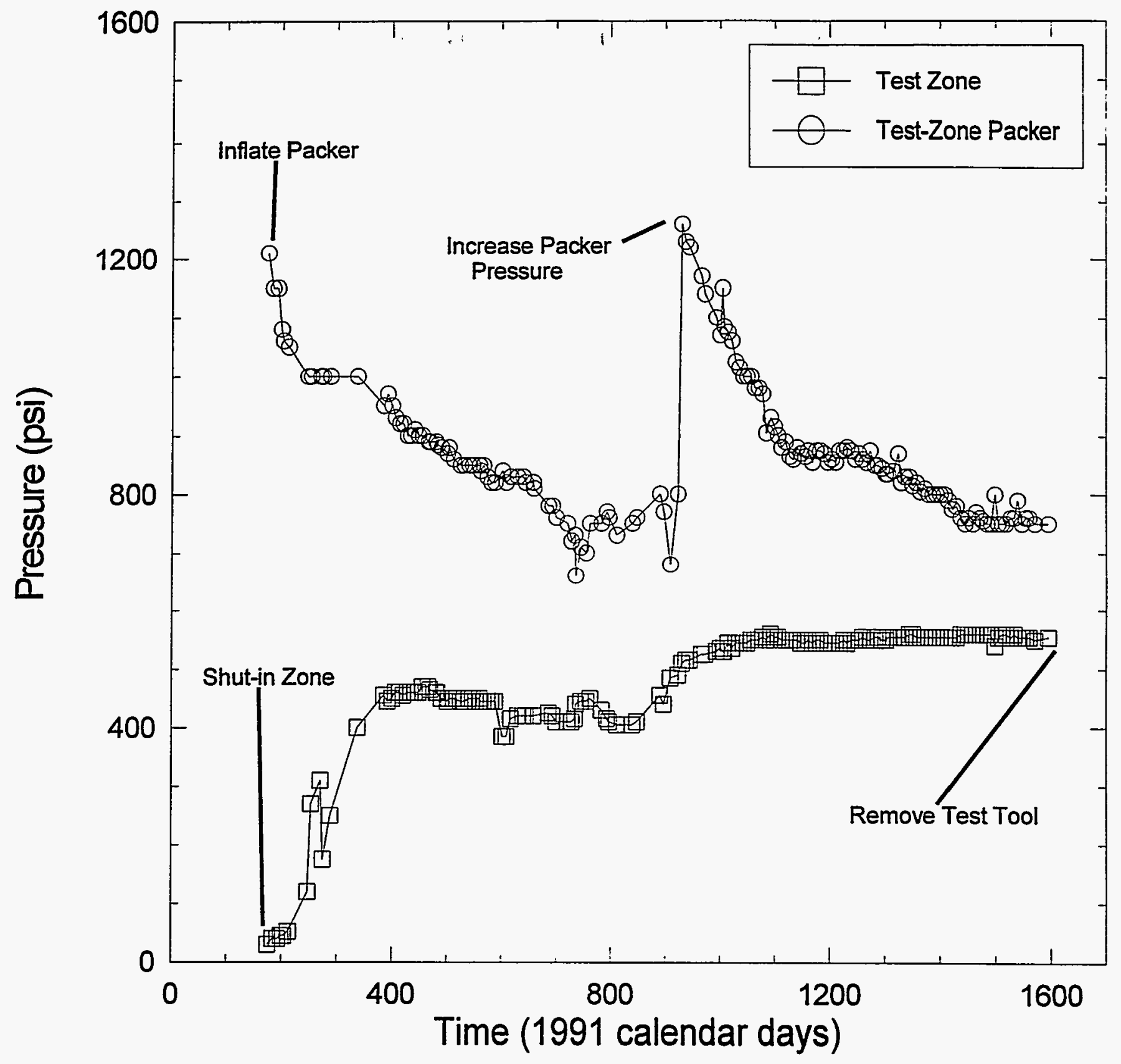

INTERA-O1 15-252-0

Figure 7-13. Long-term fluid presure in anhydrite "C" in borehole S1P71. 


\subsubsection{Borehole S1P72}

Table 7-8 lists the events associated with borehole S1P72 from the time the borehole was drilled to the termination of all testing and/or monitoring in the borehole with the events associated with the fluid-pressure monitoring emphasized. Descriptions of events that occurred during permeability testing and of core recovered from this borehole can be found in Stensrud et al. (1992).

Table 7-8. Long-Term Fluid-Pressure Monitoring S1P72 Events

\begin{tabular}{|c|c|c|c|}
\hline EVENT & DATE & $\begin{array}{l}\text { CALENDAR } \\
\text { DAY }\end{array}$ & $\begin{array}{l}1991 \\
\text { CALENDAR DAY }\end{array}$ \\
\hline Drill borehole S1P72 to 6.05 meters. & $12-13-89$ & 347 & N/A \\
\hline Begin permeability-testing sequence S1P72. & $12-18-89$ & 352 & N/A \\
\hline Terminate permeability-testing sequence S1P72. & $1-30-91$ & 30 & 30 \\
\hline $\begin{array}{l}\text { Install single-packer long-term fluid-pressure monitoring } \\
\text { tool to monitor fluid pressure in MB139 as indicated in } \\
\text { Figure } 7-14 .\end{array}$ & $1-31-91$ & 31 & 31 \\
\hline Inflate packer to $\sim 1500 \mathrm{psi}$. & $1-31-91$ & 31 & 31 \\
\hline Shut in test zone. & $1-31-91$ & 31 & 31 \\
\hline Leak in packer. & $2-1-91$ & 32 & 32 \\
\hline $\begin{array}{c}\text { Remove long-term fluid-pressure monitoring tool form } \\
\text { borehole. }\end{array}$ & $2-1-91$ & 32 & 32 \\
\hline Fix leak in the packer inflation line. & $2-1-91$ & 32 & 32 \\
\hline $\begin{array}{l}\text { Re-install single-packer long-term fluid-pressure } \\
\text { monitoring tool to monitor fluid pressure in MB139 as } \\
\text { indicated in Figure } 7-14 \text {. }\end{array}$ & $2-1-91$ & 32 & 32 \\
\hline Inflate packer to $~ 1650$ psi. & $2-1-91$ & 32 & 32 \\
\hline Shut in test zone. & $2-1-91$ & 32 & 32 \\
\hline Replace bad gage on packer. & $10-19-92$ & 294 & 658 \\
\hline Depressurize test zone. & $5-16-95$ & 136 & 1596 \\
\hline Deflate packer. & $5-16-95$ & 136 & 1596 \\
\hline $\begin{array}{l}\text { Attempt to remove long-term fiuid-pressure monitoring } \\
\text { tool. }\end{array}$ & 5-16-95 & 136 & 1596 \\
\hline Terminate long-term fluid-pressure monitoring. & $5-16-95$ & 136 & 1596 \\
\hline
\end{tabular}

Figure 7-14 illustrates the test-tool configuration and associated installation that was used during the fluid-pressure monitoring in borehole S1P72. Figure 7-15 is a plot of the fluid pressure observed in borehole S1P72. 


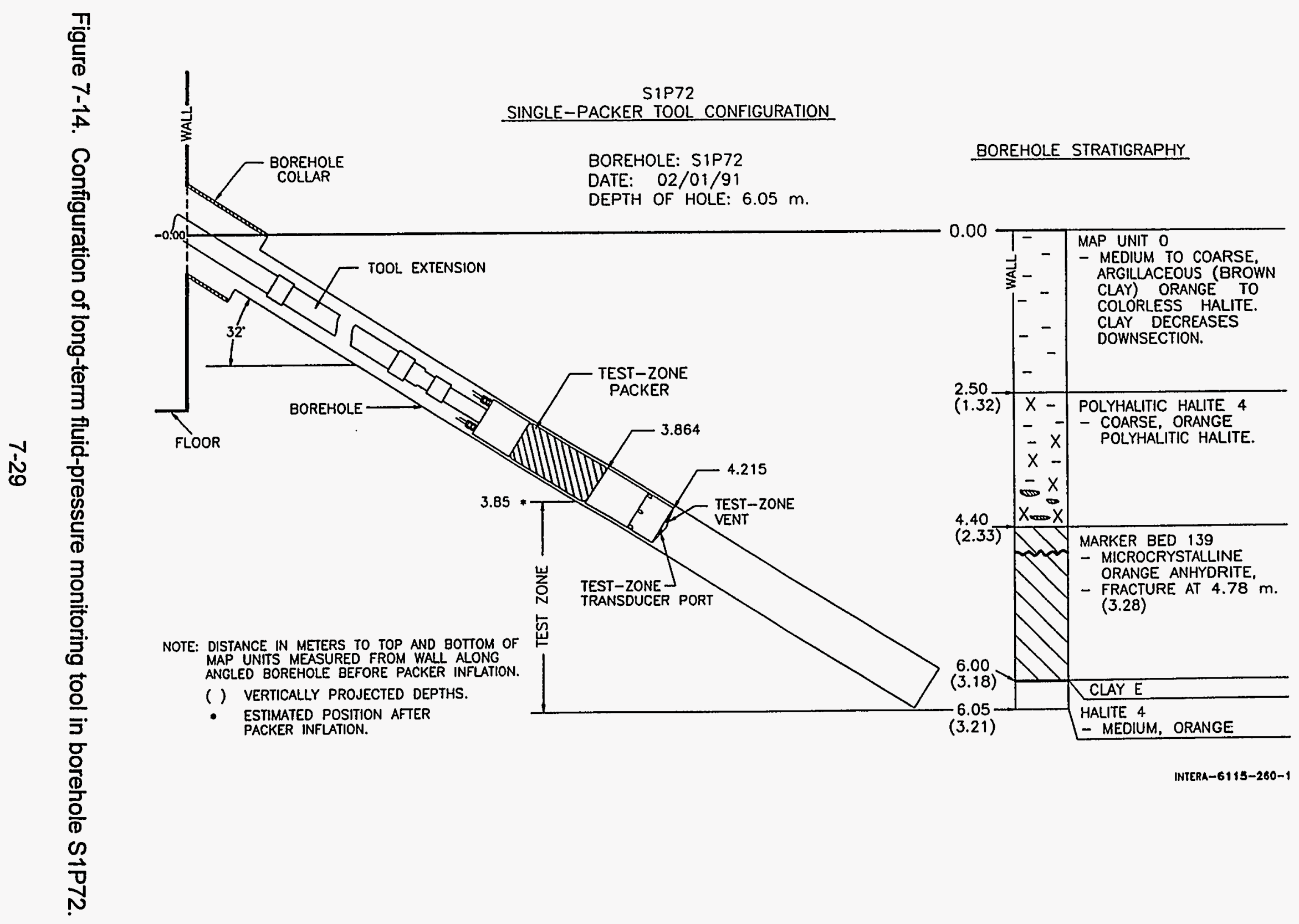




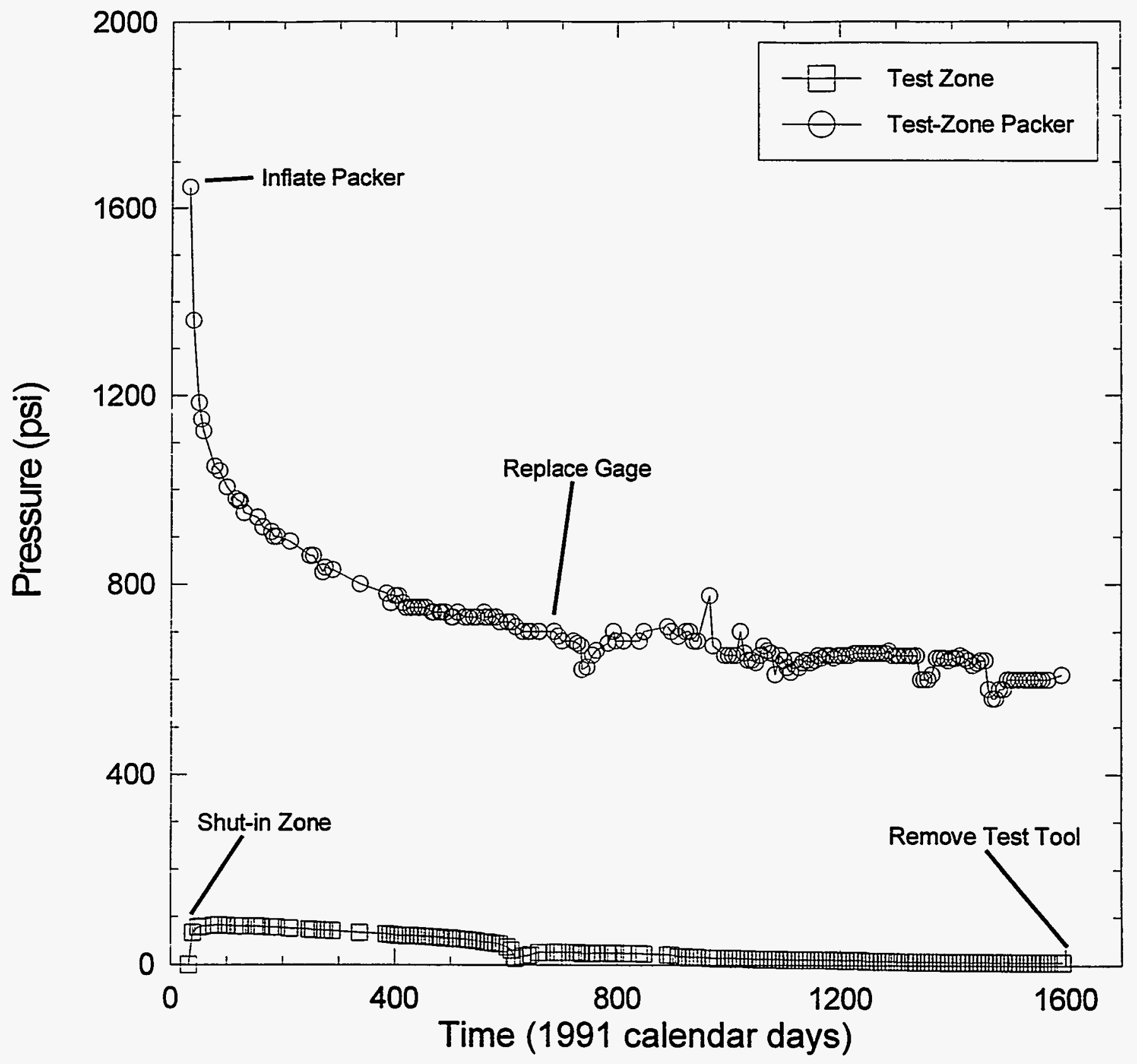

INTERA-T1 15-2B1-0

Figure 7-15. Long-term fluid pressure in MB139 in borehole S1P72. 


\subsection{Fluid-Pressure Monitoring in Other Boreholes}

The long-term fluid-pressure monitoring tools that were used in boreholes DPD01, DPD02, and DPD03 were similar in design to those described in Section 7.2. These monitor tools consisted of fluid-inflatable, 3.5-inch $(8.9-\mathrm{cm})$ diameter packers mounted on a 1.9-inch $(4.83-\mathrm{cm})$ mandrel. $3 / 16$-inch $(0.48-\mathrm{cm})$ stainless-steel tubing was used to access the associated zone and to transmit pressure to pressure gages located outside of the borehole. Prior to installation of the long-term fluid-pressure monitoring tools, the boreholes were filled with WIPP-facility brine $(1.22 \mathrm{~kg} / \mathrm{L})$ to a level such that the monitor tool was completely submerged. The monitor tools in boreholes DPD01, DPD02, and DPD03 were not restrained from moving out of the boreholes in response to pressure buildup. Table 7-9 lists the pertinent information for the boreholes and test zones monitored in the fluid-pressure monitoring program which had not been previously tested under the permeability and/or the GTPT program. Figure 2-1 shows the location of these boreholes within the WIPP underground facility.

Table 7-9. Fluid-Pressure Monitoring in Other Boreholes; Test-Zone Information

\begin{tabular}{cccccccc}
\hline Borehole & $\begin{array}{c}\text { Test } \\
\text { Horizon }\end{array}$ & $\begin{array}{c}\text { Test Horizon } \\
\text { Penetrated }\end{array}$ & $\begin{array}{c}\text { Borehole } \\
\text { Diameter }(\mathrm{cm})\end{array}$ & $\begin{array}{c}\text { Test Interval } \\
\text { Depth }(\mathbf{m})\end{array}$ & $\begin{array}{c}\text { Borehole } \\
\text { Depth }(\mathbf{m})\end{array}$ & $\begin{array}{c}\text { Monitoring } \\
\text { Started }\end{array}$ & $\begin{array}{c}\text { Monitoring } \\
\text { Terminated }\end{array}$ \\
\hline DPD01 & MB139 & $11-84$ & 12.7 & 10.39 & 12.34 & $5-89$ & $8-89$ \\
DPD02 & MB139 & $4-86$ & 10.8 & 11.00 & 13.11 & $5-89$ & $4-95$ \\
DPD03 & MB139 & $4-86$ & 10.8 & 11.15 & 13.11 & $5-89$ & $4-95$ \\
\hline
\end{tabular}

Table 7-10 summarizes the location and orientation of each of the boreholes associated with the long-term fluid-pressure monitoring in which brine permeability and/or GTPT sequences had not been performed. Borehole orientations at an underground location attempt to accomplish two objectives:

- to characterize the interbeds in close areal proximity; and

- to characterize the interbeds at a maximum distance from the excavation.

Table 7-10. Summary of Long-Term Fluid-Pressure Monitoring Borehole Locations and Orientations not Previously Tested

\begin{tabular}{ccccc}
\hline $\begin{array}{c}\text { Borehole } \\
\text { (Orientation) }\end{array}$ & $\begin{array}{c}\text { Interval } \\
\text { Drilled/ } \\
\text { Cored }(\mathbf{m})\end{array}$ & $\begin{array}{c}\text { Date } \\
\text { Drilled/ } \\
\text { Cored }\end{array}$ & Location & $\begin{array}{c}\text { Excavation Date } \\
\text { of Drift }\end{array}$ \\
\hline $\begin{array}{c}\text { DPD01 } \\
\text { (downward 46 }) \\
\text { (from vertical) } \\
\begin{array}{c}\text { DPD02 } \\
\text { (downward 47 }) \\
\text { (from vertical) }\end{array}\end{array} \quad 0-12.34$ & $\begin{array}{c}\text { November } \\
1984\end{array}$ & $\begin{array}{c}\text { North 1400 Drift } \\
\text { East of Room C1 }\end{array}$ & March 1983 \\
\hline
\end{tabular}


Table 7-10 (Continued). Summary of Long-Term Fluid-Pressure Monitoring Borehole Locations and Orientations not Previously Tested

\begin{tabular}{ccccc}
\hline $\begin{array}{c}\text { Borehole } \\
\text { (Orientation) }\end{array}$ & $\begin{array}{c}\text { Interval } \\
\text { Drilled/ } \\
\text { Cored }(\mathbf{m})\end{array}$ & $\begin{array}{c}\text { Date } \\
\text { Drilled/ } \\
\text { Cored }\end{array}$ & Location & $\begin{array}{c}\text { Excavation Date } \\
\text { of Drift }\end{array}$ \\
\hline $\begin{array}{c}\text { DPD03 } \\
\text { (downward 46 }) \\
\text { (from vertical) }\end{array}$ & $0-13.11$ & April 1986 & $\begin{array}{c}\text { North 1400 Drift } \\
\text { East of Room C1 }\end{array}$ & March 1983 \\
\hline
\end{tabular}

Figure 7-16 illustrates the locations and orientations of boreholes DPD01, DPD02, and DPD03 relative to the surrounding area of the WIPP underground facility. 

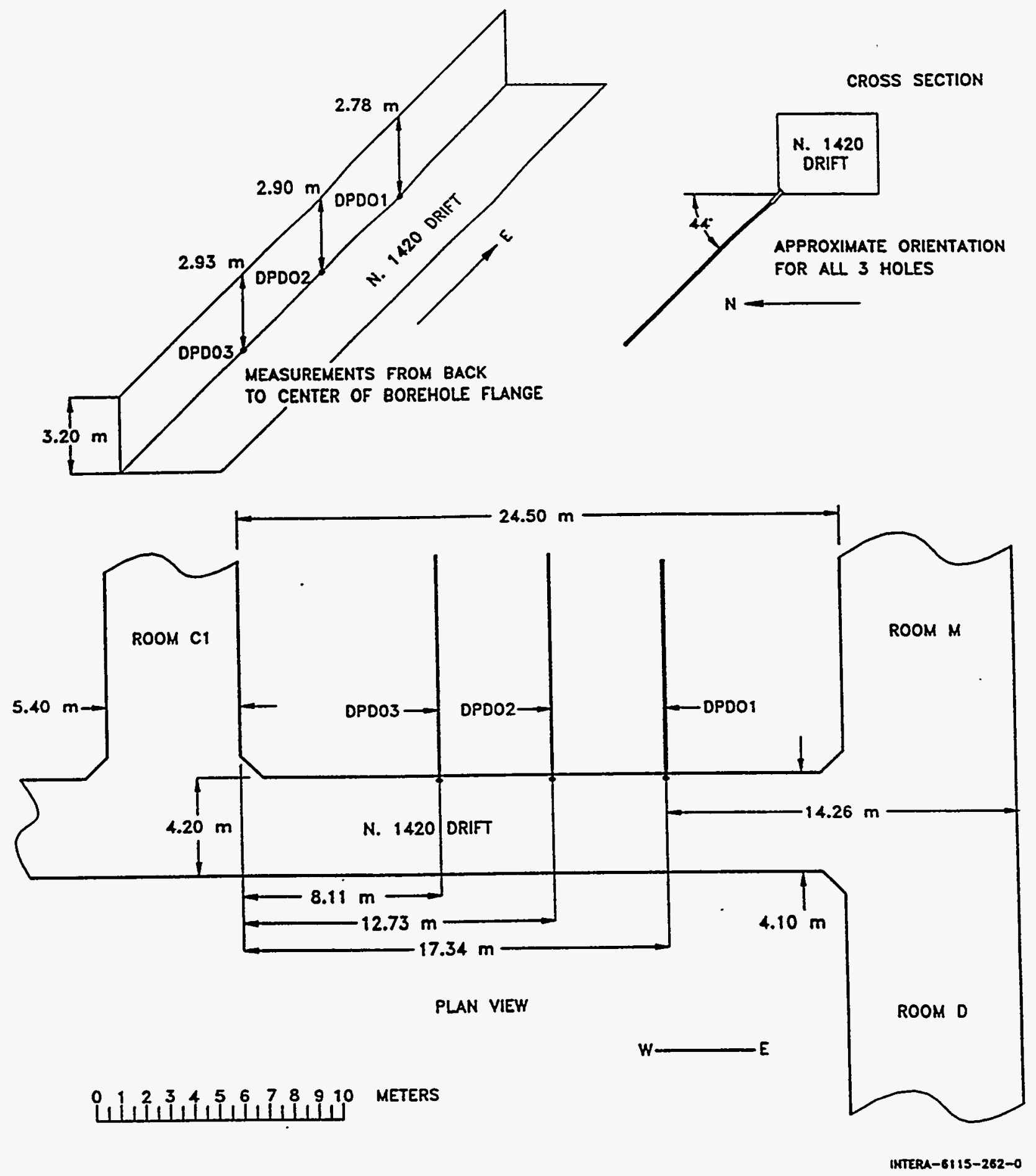

Figure 7-16. Locations and orientations of long-term fluid-pressure monitoring boreholes DPD01, DPD02, and DPD03. 


\subsubsection{Borehole DPD01}

Table 7-11 lists the events associated with the fluid-pressure monitoring in borehole DPD01.

Table 7-11. Long-Term Fluid-Pressure Monitoring DPD01 Events

\begin{tabular}{|c|c|c|c|}
\hline EVENT & DATE & $\begin{array}{l}\text { CALENDAR } \\
\text { DAY }\end{array}$ & $\begin{array}{c}1989 \\
\text { CALENDAR DAY }\end{array}$ \\
\hline Drill borehole DPD01 to 12.34 meters. & $11-84$ & N/A & N/A \\
\hline Begin fluid level measurements. & 4-27-89 & 117 & 117 \\
\hline Video-log borehole DPD01 to determine MB139 position. & $4-28-89$ & 118 & 118 \\
\hline $\begin{array}{l}\text { Install single-packer long-term fluid-pressure monitoring } \\
\text { tool to monitor fluid pressure in MB139 as indicated in } \\
\text { Figure } 7-17 .\end{array}$ & 4-28-89 & 118 & 118 \\
\hline Survey borehole DPD01 to determine inclination. & 4-28-89 & 118 & 118 \\
\hline Inflate packer to $\sim 1500$ psi. & $5-2-89$ & 122 & 122 \\
\hline Deflate packer. & $6-5-89$ & 156 & 156 \\
\hline Add approximately $18.9 \mathrm{~L}$ of brine to the borehole. & $6-5-89$ & 156 & 156 \\
\hline Inflate packer to 1380 psi. & $6-5-89$ & 156 & 156 \\
\hline Shut in test zone. & $6-5-89$ & 156 & 156 \\
\hline Increase packer pressure to $\sim 1500 \mathrm{psi}$. & $7-18-89$ & 199 & 199 \\
\hline Remove long-term fluid-pressure monitoring tool. & $8-22-89$ & 234 & 234 \\
\hline Long-term fluid-pressure monitoring terminated. & $8-22-89$ & 234 & 234 \\
\hline Ream borehole DPD01 to 5-inches $(12.7-\mathrm{cm})$. & $7-30-91$ & 211 & 941 \\
\hline Install and grout in new collar. & $7-30-91$ & 211 & 941 \\
\hline Load test new collar. & $8-6-91$ & 218 & 948 \\
\hline Add approximately $18.9 \mathrm{~L}$ of brine to borehole. & $12-4-91$ & 338 & 1068 \\
\hline Install new long-term fluid-pressure monitor tool. & $12-4-91$ & 338 & 1068 \\
\hline Inflate packer to $\sim 500 \mathrm{psi}$. & $12-4-91$ & 338 & 1068 \\
\hline Packer failed. & $12-4-91$ & 338 & 1068 \\
\hline Remove monitor tool from borehole. & $12-4-91$ & 338 & 1068 \\
\hline
\end{tabular}

Figure 7-17 illustrates the tool configuration and associated installation that was used during the fluid-pressure monitoring in borehole DPD01. Figure 7-18 shows that no fluidpressure buildup was observed in borehole DPD01. Apparently, the packer diameter was too small to seal the observation zone. 


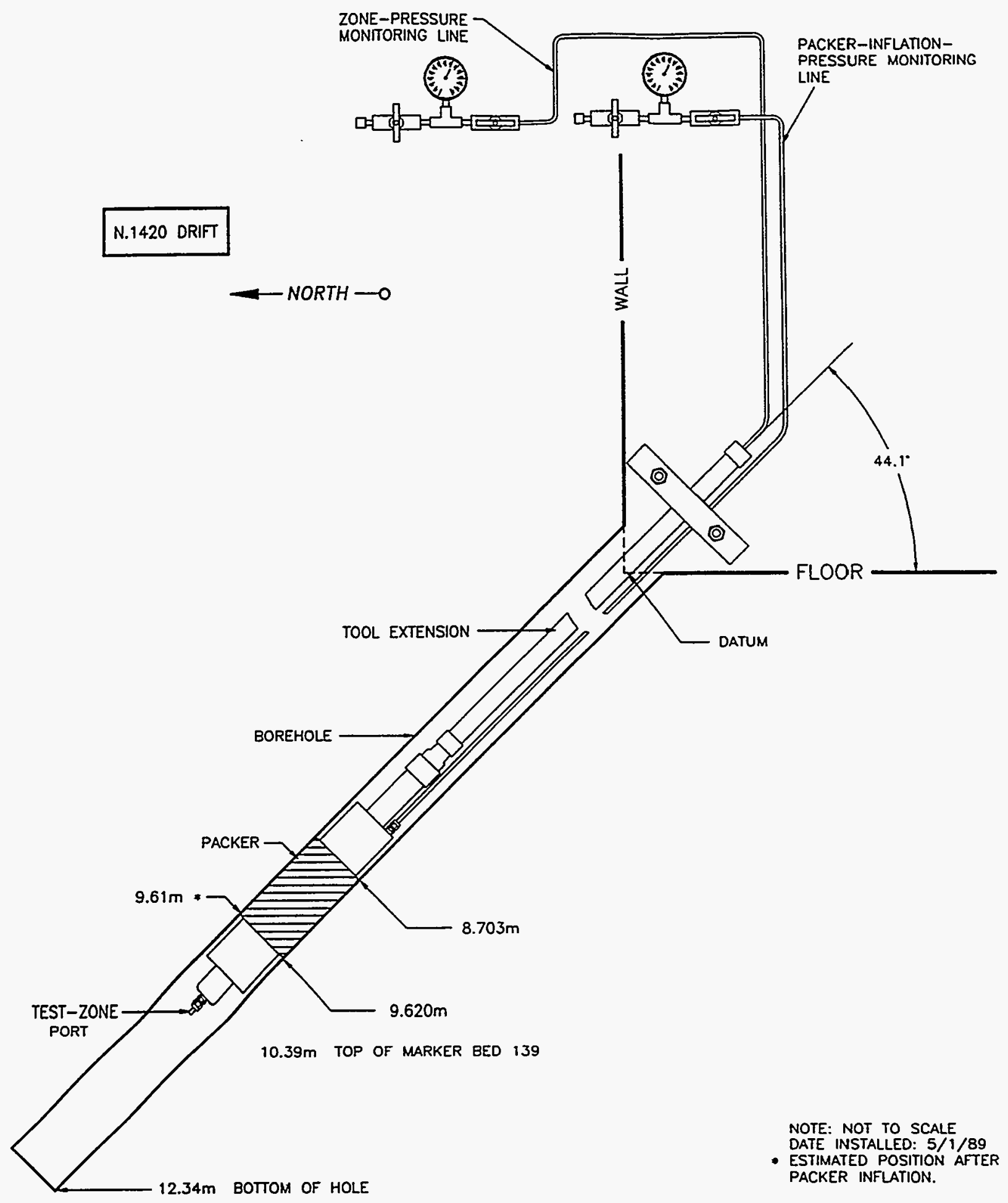

INTERA-61 15-263-1

Figure 7-17. Configuration of long-term fluid-pressure monitoring tool in borehole DPD01. 


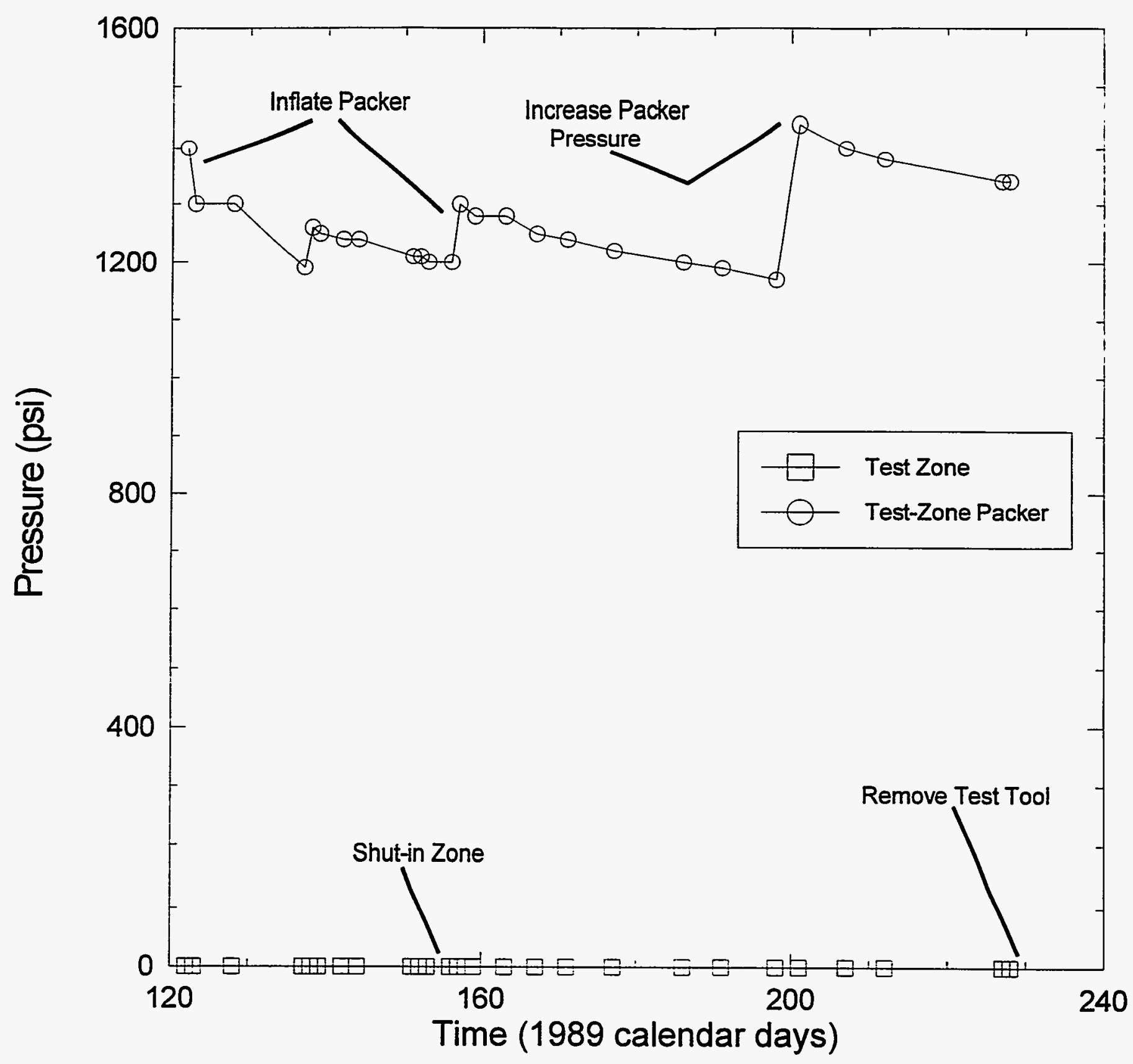

WNERA6115-2640

Figure 7-18. Long-term fluid pressure in MB139 in borehole DPD01. 


\subsubsection{Borehole DPD02}

Table 7-12 lists the events associated with the fluid-pressure monitoring in borehole DPD02.

Table 7-12. Long-Term Fluid-Pressure Monitoring DPD02 Events

\begin{tabular}{|c|c|c|c|}
\hline EVENT & DATE & $\begin{array}{c}\text { CALENDAR } \\
\text { DAY }\end{array}$ & $\begin{array}{c}1989 \\
\text { CALENDAR DAY }\end{array}$ \\
\hline Drill borehole DPD02 to 13.11 meters. & 4-86 & N/A & N/A \\
\hline Begin fluid level measurements. & 4-27-89 & 117 & 117 \\
\hline Video-log borehole DPD02 to determine MB139 position. & $4-28-89$ & 118 & 118 \\
\hline $\begin{array}{l}\text { Install single-packer long-term fluid-pressure monitoring } \\
\text { tool to monitor fluid pressure in MB139 as indicated in } \\
\text { Figure } 7-19 .\end{array}$ & 4-28-89 & 118 & 118 \\
\hline Survey borehole DPD02 to determine inclination. & 4-28-89 & 118 & 118 \\
\hline Inflate packer to $\sim 1500 \mathrm{psi}$. & $5-2-89$ & 122 & 122 \\
\hline Begin long-term fluid-pressure monitoring in MB139. & $5-3-89$ & 123 & 123 \\
\hline Increase packer pressure to $\sim 1500 \mathrm{psi}$. & $7-18-89$ & 199 & 199 \\
\hline Diagnose and attempt to fixed a leak in the test zone. & $7-26-89$ & 207 & 207 \\
\hline Test zone continues to leak. & $7-31-89$ & 212 & 212 \\
\hline Replace bad valve on test zone. & $8-15-89$ & 227 & 227 \\
\hline Remove monitor tool from borehole. & $7-16-91$ & 197 & 927 \\
\hline Ream borehole DPD02 to 4-inch $(10.16-\mathrm{cm})$. & $8-2-91$ & 214 & 944 \\
\hline Install and grout in new collar. & $8-5-91$ & 217 & 947 \\
\hline Load test new collar. & 8-6-91 & 218 & 948 \\
\hline Add approximately $18.9 \mathrm{~L}$ of brine to borehole. & $12-4-91$ & 338 & 1068 \\
\hline $\begin{array}{l}\text { Install new single-packer long-term fluid-pressure monitor } \\
\text { tool to monitor fluid pressure in MB139 as indicated in } \\
\text { Figure } 7-20 .\end{array}$ & $12-4-91$ & 338 & 1068 \\
\hline Inflate packer to 1500 psi. & $12-4-91$ & 338 & 1068 \\
\hline Increase test zone pressure to $\sim 500 \mathrm{psi}$. & $12-4-91$ & 338 & 1068 \\
\hline Replace pressure gage on test zone. & $12-16-91$ & 350 & 1080 \\
\hline $\begin{array}{l}\text { Install transducer \#316158 to monitor pressure during } \\
\text { C1X10 coupled permeability and hydrofracture-testing } \\
\text { sequence. }\end{array}$ & $1-29-92$ & 29 & 1124 \\
\hline $\begin{array}{c}\text { Begin fluid-pressure monitoring of MB139 associated with } \\
\text { testing sequence } C 1 \times 10 \text {. }\end{array}$ & $1-30-92$ & 30 & 1125 \\
\hline
\end{tabular}


Table 7-12 (Continued). Long-Term Fluid-Pressure Monitoring DPD02 Events

\begin{tabular}{cccc}
\hline EVENT & DATE & $\begin{array}{c}\text { CALENDAR } \\
\text { DAY }\end{array}$ & $\begin{array}{c}1989 \\
\text { CALENDAR DAY }\end{array}$ \\
\hline $\begin{array}{c}\text { Terminate fluid-pressure monitoring associated with } \\
\text { testing sequence C1X10. }\end{array}$ & $6-26-92$ & 178 & 1273 \\
$\begin{array}{c}\text { Begin fluid-pressure monitoring of MB139 associated with } \\
\text { testing sequence C1X05-A. }\end{array}$ & $6-26-92$ & 178 & 1273 \\
$\begin{array}{c}\text { Terminate fluid-pressure monitoring associated with } \\
\text { testing sequence C1X05-A. }\end{array}$ & $8-3-92$ & 216 & 1311 \\
$\begin{array}{c}\text { Resume long-term fluid-pressure monitoring of fluid } \\
\text { pressure in MB139. }\end{array}$ & $8-3-92$ & 216 & 1311 \\
Replace bad gage on test zone. & $9-8-92$ & 252 & 1347 \\
$\begin{array}{c}\text { Increase packer pressure. } \\
\text { Repiace bad gage on test zone. }\end{array}$ & $7-12-93$ & 193 & 1653 \\
Replace bad gage on test zone. & $11-29-93$ & 333 & 1793 \\
Depressurize test zone. & $3-1-94$ & 60 & 1885 \\
Deflate packer. & $4-21-95$ & 110 & 2300 \\
\hline Remove long-term monitoring tool from borehole. & $4-21-95$ & 110 & 2300 \\
Terminate long-term fluid-pressure monitoring. & $4-21-95$ & 110 & 2300 \\
\hline
\end{tabular}

Figures 7-19 and 7-20 illustrate both of the tool configurations and associated installations that were used during the fluid-pressure monitoring in borehole DPD02. Figure 7-21 is a plot of the fluid pressure observed in borehole DPD02 for the entire duration of the fluidpressure monitoring. 


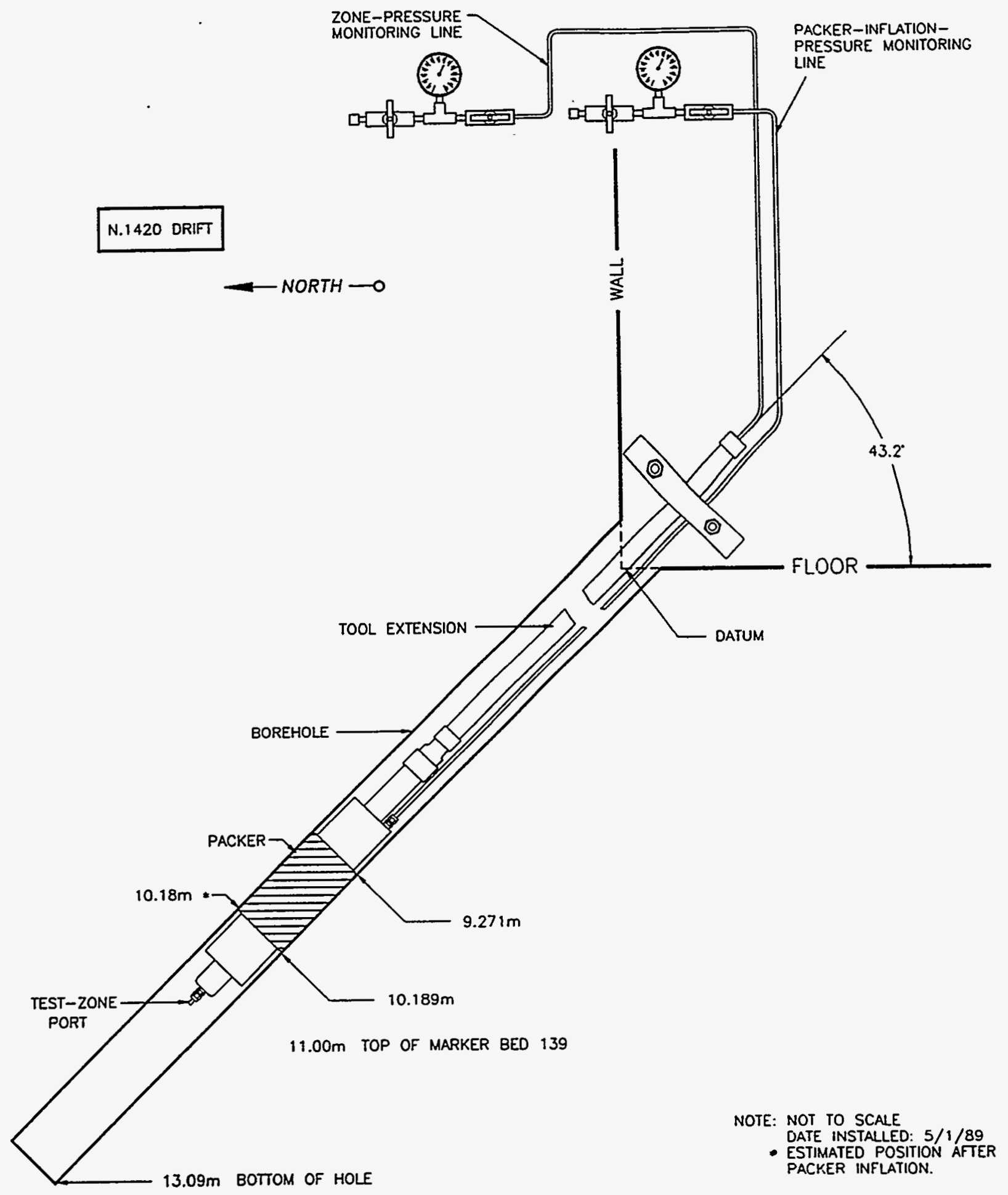

INTERA-61 15-265-1

Figure 7-19. Configuration \#1 of long-term fluid-pressure monitoring tool in borehole DPD02. 


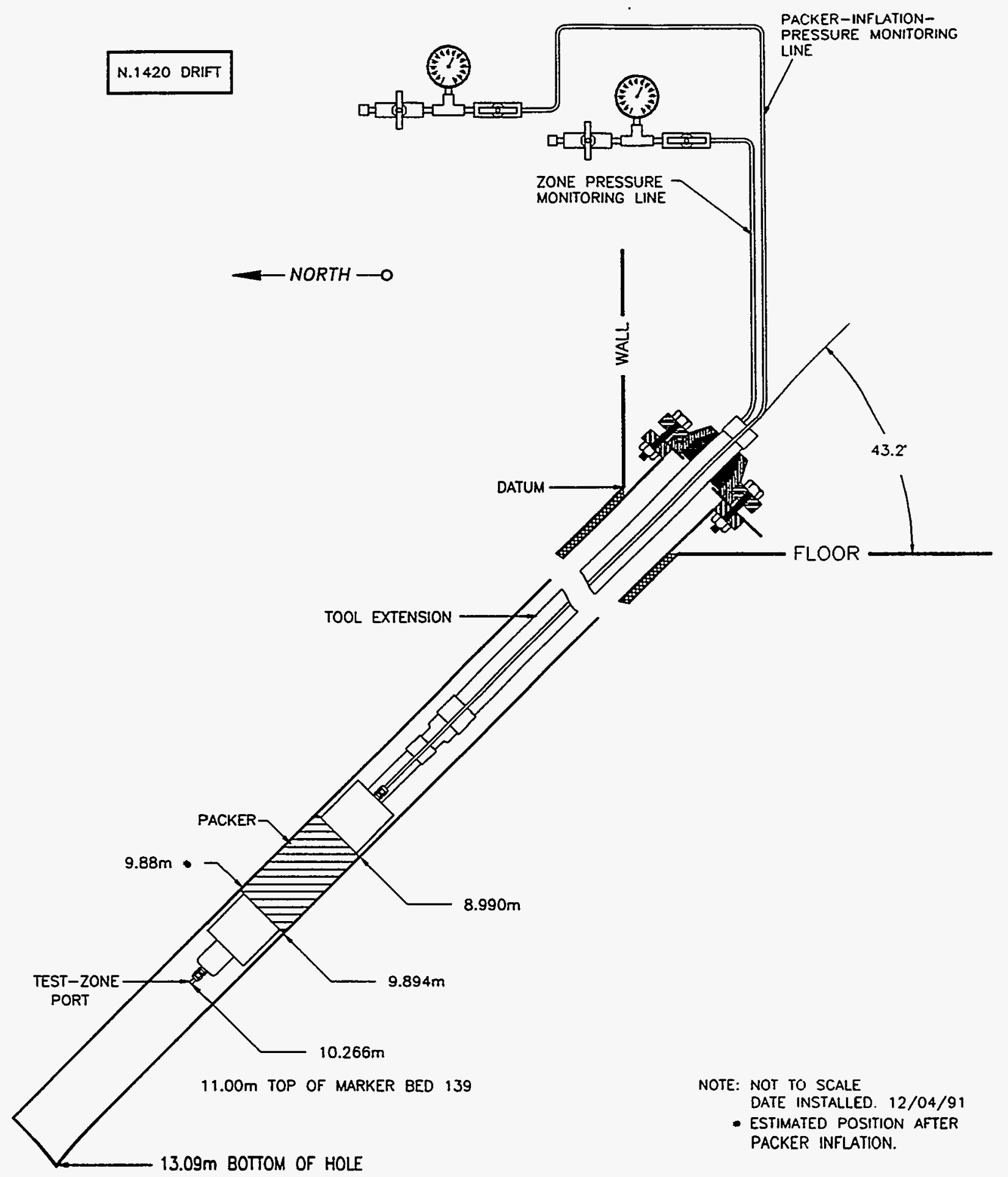

INTERA-6115-265-1

Figure 7-20. Configuration \#2 of long-term fluid-pressure monitoring tool in borehole DPD02. 


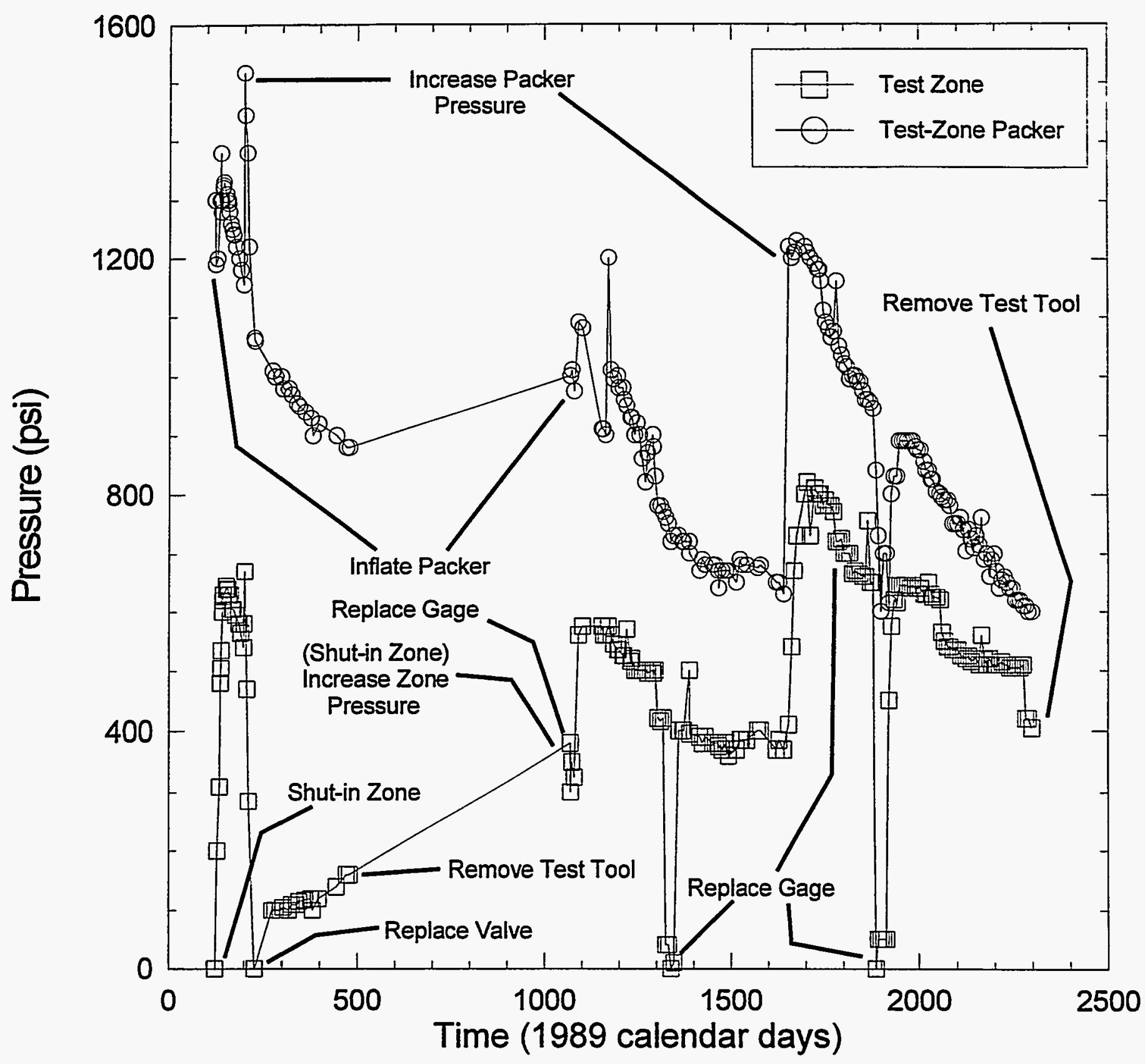

INTERA6115-257-0

Figure 7-21. Long-term fluid pressure in MB139 in borehole DPD02. 


\subsubsection{Borehole DPD03}

Table 7-13 lists the events associated with the fluid-pressure monitoring in borehole DPD03.

Table 7-13. Long Term Fluid-Pressure Monitoring DPD03 Events

\begin{tabular}{|c|c|c|c|}
\hline EVENT & DATE & $\begin{array}{l}\text { CALENDAR } \\
\text { DAY }\end{array}$ & $\begin{array}{c}1989 \\
\text { CALENDAR DAY }\end{array}$ \\
\hline Drill borehole DPD03 to 13.11 meters. & $4-86$ & N/A & N/A \\
\hline Begin fiuid level measurements. & $4-27-89$ & 117 & 117 \\
\hline Video-log borehole DPD03 to determine MB139 position. & 4-28-89 & 118 & 118 \\
\hline $\begin{array}{c}\text { Install single-packer long-term fluid-pressure monitoring } \\
\text { tool to monitor fluid pressure in MB139 as indicated in } \\
\text { Figure 7-22. }\end{array}$ & $4-28-89$ & 118 & 118 \\
\hline Survey borehole DPD03 to determine inclination. & $4-28-89$ & 118 & 118 \\
\hline Infiate packer to $\sim 1500 \mathrm{psi}$. & $5-2-89$ & 122 & 122 \\
\hline Shut in test zone. & $5-3-89$ & 123 & 123 \\
\hline Increase packer pressure to $\sim 1500$ psi. & $7-18-89$ & 199 & 199 \\
\hline Attempt to remove monitor tool from borehole. & $7-16-91$ & 197 & 927 \\
\hline Push monitor tool to the bottom of the borehole. & $8-2-91$ & 214 & 944 \\
\hline $\begin{array}{l}\text { Ream borehole DPDO3 to } 4 \text {-inch }(10.16-\mathrm{cm}) \text { to the top of } \\
\text { the tool. }\end{array}$ & $8-2-91$ & 214 & 944 \\
\hline Remove monitor tool from borehole. & $8-5-91$ & 217 & 947 \\
\hline Install and grout in new collar. & $8-5-91$ & 217 & 947 \\
\hline Load test new collar. & $8-6-91$ & 218 & 948 \\
\hline Add approximately $18.9 \mathrm{~L}$ of brine to borehole. & $12-4-91$ & 338 & 1068 \\
\hline $\begin{array}{l}\text { Install new single-packer long-term fluid-pressure monitor } \\
\text { tool to monitor fluid pressure in MB139 as indicated in } \\
\text { Figure } 7-23 \text {. }\end{array}$ & $12-4-91$ & 338 & 1068 \\
\hline Inflate packer to $\sim 1500 \mathrm{psi}$. & $12-4-91$ & 338 & 1068 \\
\hline Increase test zone pressure to $\sim 500 \mathrm{psi}$. & $12-4-91$ & 338 & 1068 \\
\hline $\begin{array}{l}\text { Install transducer \#211694 to monitor pressure during } \\
\text { C1X10 coupled permeability and hydrofracture-testing } \\
\text { sequence. }\end{array}$ & $1-29-92$ & 29 & 1124 \\
\hline $\begin{array}{l}\text { Begin fluid-pressure monitoring of MB139 associated with } \\
\text { testing sequence } \mathrm{C} 1 \times 10 .\end{array}$ & $1-30-92$ & 30 & 1125 \\
\hline Diagnose and fix leak in test zone & $2-10-92$ & 41 & 1136 \\
\hline
\end{tabular}


Table 7-13 (Continued). Long Term Fluid-Pressure Monitoring DPD03 Events

\begin{tabular}{cccc}
\hline EVENT & DATE & $\begin{array}{c}\text { CALENDAR } \\
\text { DAY }\end{array}$ & $\begin{array}{c}1989 \\
\text { CALENDAR DAY }\end{array}$ \\
\hline $\begin{array}{c}\text { Terminate fluid-pressure monitoring associated with } \\
\text { testing sequence C1X10. }\end{array}$ & $6-26-92$ & 178 & 1273 \\
$\begin{array}{c}\text { Begin fluid-pressure monitoring of MB139 associated with } \\
\text { testing sequence C1X05-A. }\end{array}$ & $6-26-92$ & 178 & 1273 \\
$\begin{array}{c}\text { Terminate fluid-pressure monitoring associated with } \\
\text { testing sequence C1X05-A. }\end{array}$ & $8-3-92$ & 216 & 1311 \\
$\begin{array}{c}\text { Resume long-term fluid-pressure monitoring of fluid } \\
\text { pressure in MB139. }\end{array}$ & $8-3-92$ & 216 & 1311 \\
$\begin{array}{c}\text { Replace bad gage on test zone. } \\
\text { Increase packer pressure. }\end{array}$ & $10-19-92$ & 293 & 1388 \\
Depressurize test zone. & $7-12-93$ & 193 & 1653 \\
Deflate packer. & $4-21-95$ & 110 & 2300 \\
\hline $\begin{array}{c}\text { Remove long-term monitoring tool from borehole. } \\
\text { Terminate long-term fluid-pressure monitoring. }\end{array}$ & $4-21-95$ & 110 & 2300 \\
\hline
\end{tabular}

Figures 7-22 and 7-23 illustrate both of the tool configurations and associated installations that were used during the fluid-pressure monitoring in borehole DPD03. Figure 7-24 is a plot of the fluid pressure observed in borehole DPD03 for the entire duration of the fluidpressure monitoring. 


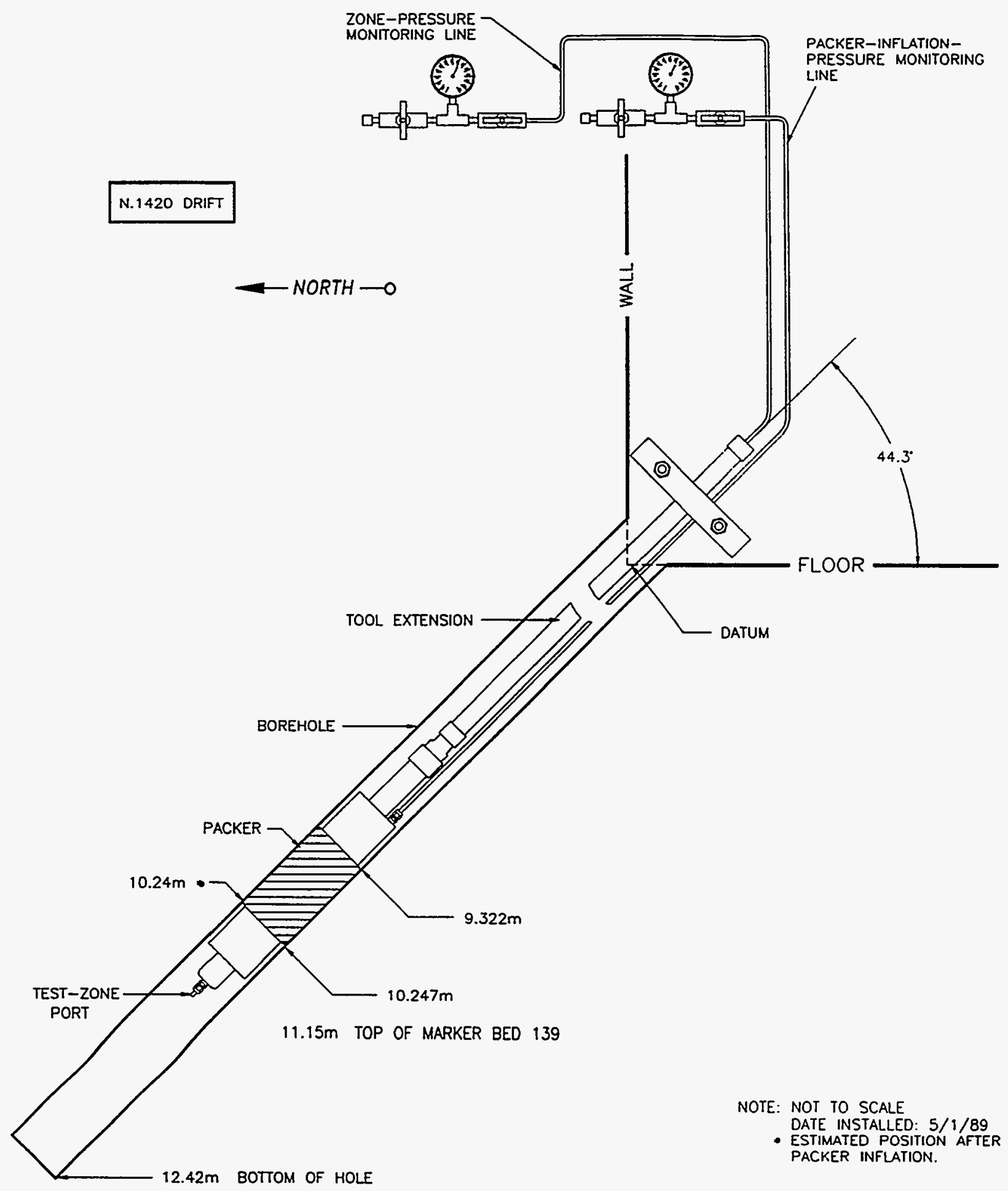

INTCRA-6115-268-1

Figure 7-22. Configuration \#1 of long-term fluid-pressure monitoring tool in borehole DPD03. 


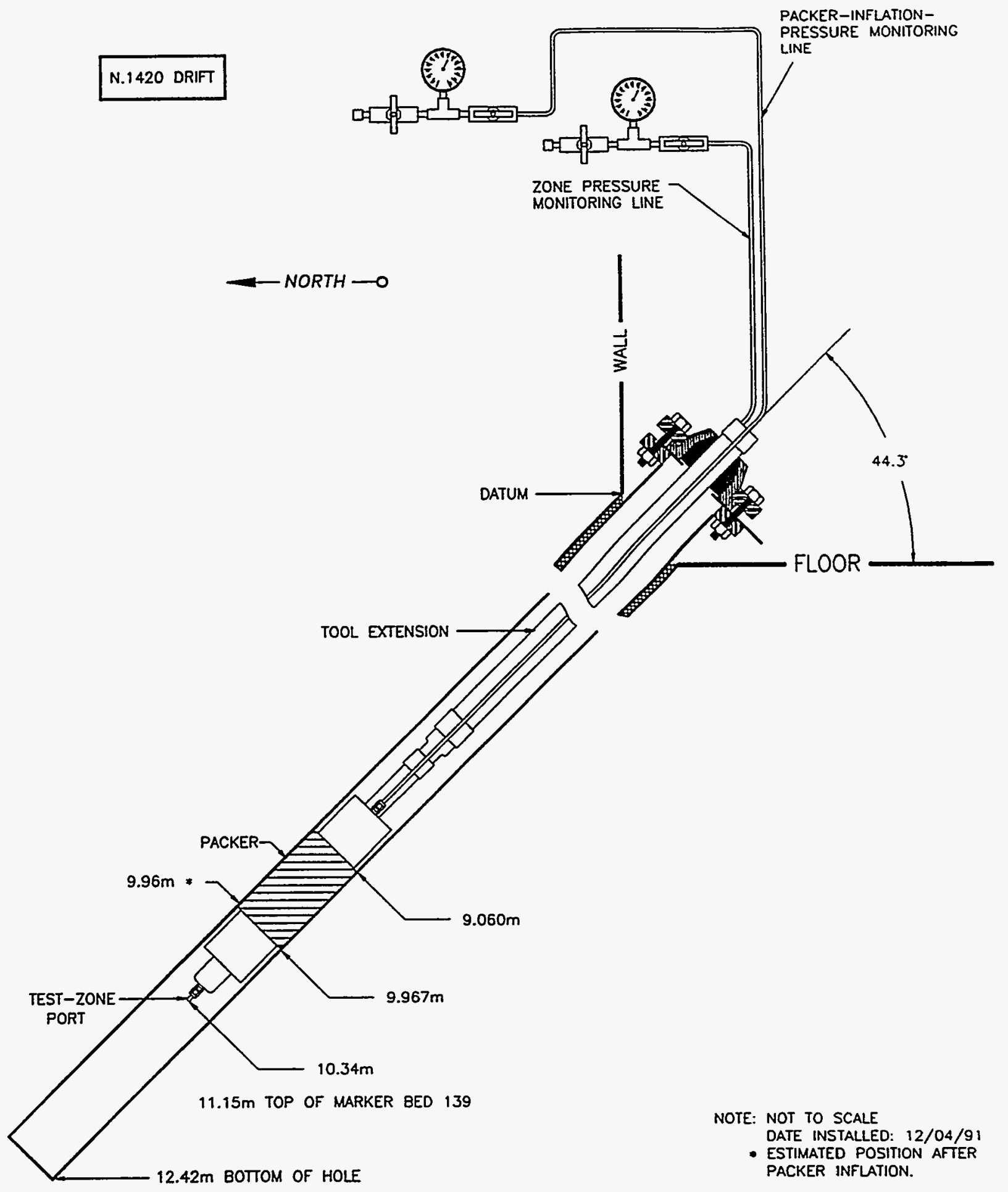

INTERA-61 15-269-1

Figure 7-23. Configuration \#2 of long-term fluid-pressure monitoring tool in borehole DPD03. 


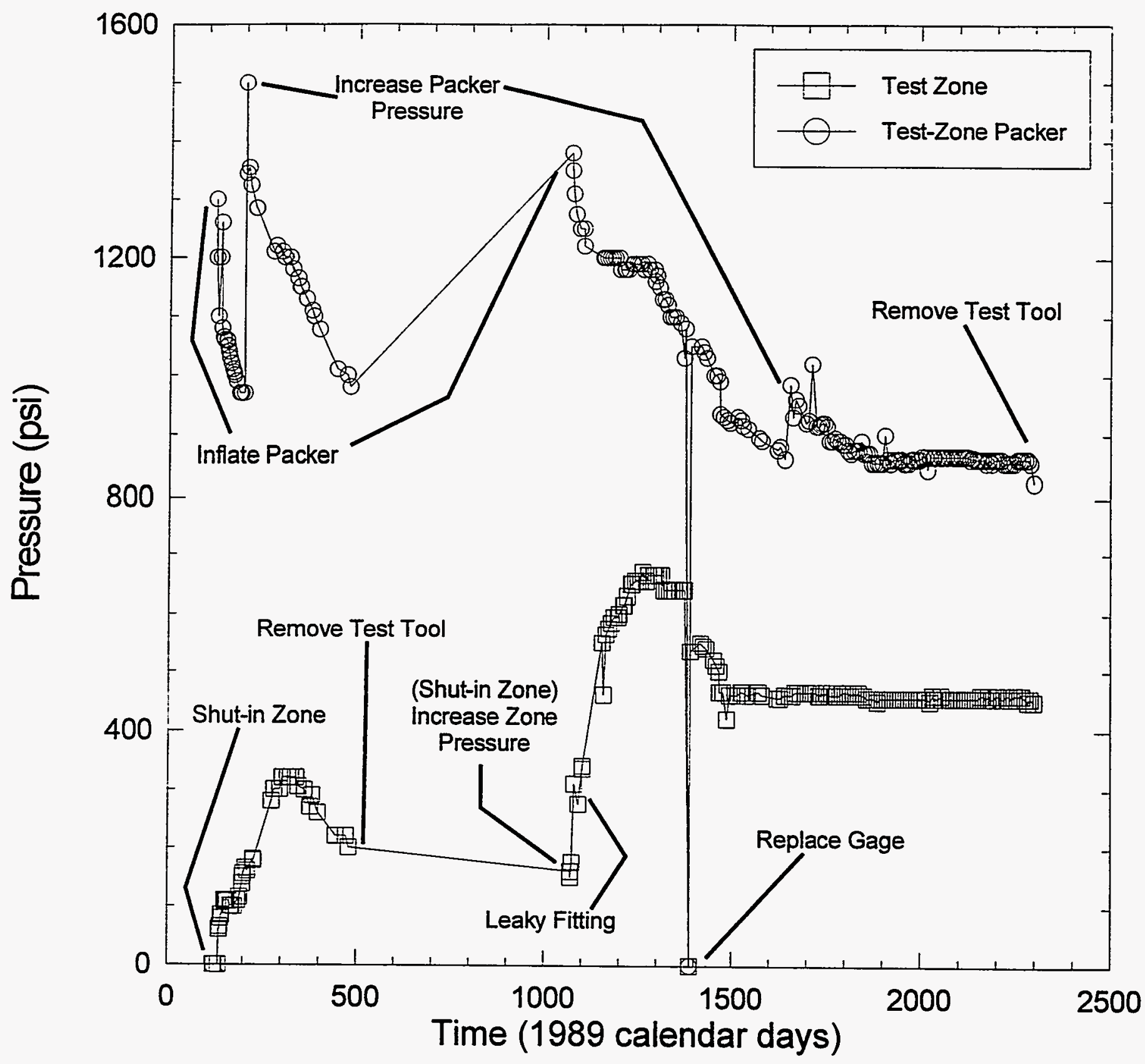

INTERA-6115-270-0

Figure 7-24. Long-term fluid pressure in MB139 in borehole DPD03. 


\section{REFERENCES}

Beauheim, R.L., G.J. Saulnier Jr., and J.D. Avis. 1991. Interpretation of BrinePermeability Tests of the Salado Formation at the Waste Isolation Pilot Plant Site: First Interim Report. SAND90-0083. Albuquerque, NM: Sandia National Laboratories (SNL). (Available from the National Technical Information Service (NTIS), Springfield, VA as DE92001853/XAB and is on file in the Sandia WIPP Central Files (SWCF), SNL, Albuquerque, NM under WPO \#26003.)

Beauheim, R.L., R.M. Roberts, T.F. Dale, M.D. Fort, and W.A. Stensrud. 1993. Hydraulic Testing of Salado Formation Evaporites at the Waste Isolation Pilot Plant Site: Second Interpretive Report. SAND92-0533. Albuquerque, NM: Sandia National Laboratories.

Bechtel National, Inc. 1986. Interim Geotechnical Field Data Report, Fall 1986. DOEWIPP 86-012. Carlsbad, NM: U.S. Department of Energy, Waste Isolation Pilot Plant Project Office. (Available from the NTIS, Springfield, VA as DE87003701/XAB.)

Davies, P.B. 1991. Evaluation of the Role of Threshold Pressure in Controlling Flow of Waste-Generated Gas into Bedded Salt at the Waste Isolation Pilot Plant. SAND90-3246. Albuquerque, NM: Sandia National Laboratories. (Available from the NTIS, Springfield, VA as DE91016498/XAB and is on file in the SWCF under WPO \#26169.)

Deal, D.E., R.J. Abitz, D.S. Belski, J.B. Case, M.E. Crawley, R.M. Deshler, P.E. Drez, C.A. Givens, R.B. King, B.A. Lauctes, J. Myers, S. Niou, J.M. Pietz, W.M. Roggenthen, J.R. Tyburski, and M.G. Wallace. 1989. Brine Sampling and Evaluation Program 1988 Report. DOE-WIPP- 89-015. Carlsbad, NM: Westinghouse Electric Corporation: Waste Isolation Division (WID). (Available from the NTIS, Springfield, VA as DE91008821/XAB.)

Holt, R.M. and D.W. Powers. 1984. Geotechnical Activities in the Waste Handling Shaft, Waste Isolation Pilot Plant (WIPP) Project, Southeastern New Mexico. WTSD-TME-038. Carlsbad, NM: U.S. Department of Energy, Waste Isolation Pilot Plant. (Available from the NTIS, Springfield, VA as DE86004328/XAB.)

INTERA, Inc. 1992. "SOP INT-5, Safe Operating Procedures for Permeability Testing in the WIPP-Site Underground Facility." SP-471061, Issue B. Austin, TX: INTERA, Inc.; Albuquerque, NM: Sandia National Laboratories. (Copy on file in the SWCF, SNL, Albuquerque, NM under WPO \#6783.)

Jensen, A.L. 1990. Borehole Closure and Test Zone Volume Determination Program for Brine-Permeability Test Results Within the Waste Isolation Pilot Plant Underground Facility. SAND90-0228. Albuquerque, NM: Sandia National Laboratories. (Available from the NTIS, Spingfield, VA as DE90013629/XAB and is on file in the SWCF under WPO \#26007.) 
Lappin, A.R., R.L. Hunter, D.P. Garber, and P.B. Davies, eds. 1989. Systems Analysis, Long-Term Radionuclide Transport, and Dose Assessments, Waste Isolation Pilot Plant (WIPP), Southeastem New Mexico; March 1989. SAND89-0462. Albuquerque, NM: Sandia National Laboratories. (Available from the NTIS, Springfield, VA as DE89014586/XAB and is on file in the SWCF under WPO \#24125.)

Pickens, J.F., G.E. Grisak, J.D. Avis, D.W. Belanger, and M. Thury. 1987. "Analysis and Interpretation of Borehole Hydraulic Tests in Deep Boreholes: Principles, Model Development, and Applications," Water Resources Research. Vol. 23, no. $7,1341-1375$. (This journal is in the NWM Library collection.)

Saulnier, G.J., Jr., and J.D. Avis. 1988. Interpretation of Hydraulic Tests Conducted in the Waste-Handling Shaft at the Waste Isolation Pilot Plant (WIPP) Site. SAND887001. Albuquerque, NM: Sandia National Laboratories. (Available from the NTIS, Springfield, VA as DE88014193/XAB and is on file in the SWCF under WPO \#24164.)

Saulnier, G.J., Jr., P.S. Domski, J.B. Palmer, R.M. Roberts, W.A. Stensrud, and A.L. Jensen. 1991. WIPP Salado Hydrology Program Data Report \#1. SAND907000. Albuquerque, NM: Sandia National Laboratories. (Available from the NTIS as DE91014564/XAB and is on file in the SWCF under WPO \#25746.)

Stensrud, W.A., M.A. Bame, K.D. Lantz, T.L. Cauffman, J.B. Palmer, and G.J. Saulnier, Jr. 1988. WIPP Hydrology Program, Waste Isolation Pilot Plant, Southeastern New Mexico, Hydrologic Data Report \#6. SAND87-7166. Albuquerque, NM: Sandia National Laboratories. (Available from the NTIS, Springfield, VA as DE88013135/XAB and is on file in the SWCF under WPO \#29674.)

Stensrud, W.A., T.F. Dale, P.S. Domski, J.B. Palmer, R.M. Roberts, M.D. Fort, G.J. Saulnier, Jr., and A.L. Jensen. 1992. Waste Isolation Pilot Plant Salado Hydrology Program Data Report \#2. SAND92-7072. Albuquerque, NM: Sandia National Laboratories. (Available from the NTIS, Springfield, VA as DE93012398/XAB and is on file in the SWCF under WPO \#26432.)

Stormont, J.C. 1990. Summary of 1988 WIPP Facility Horizon Gas Flow Measurements. SAND89-2497. Albuquerque, NM: Sandia National Laboratories. (Available from the NTIS, Springfield, VA as DE91006099/XAB and is on file in the SWCF under WPO \#24044.)

Stormont, J.C., E.W. Peterson, and P.L. Lagus. 1987. Summary of and Observations About WIPP Facility Horizon Flow Measurements Through 1986. SAND870176. Albuquerque, NM: Sandia National Laboratories. (Available from the NTIS, Springfield, VA as DE87011611/XAB and is on file in the SWCF under WPO \#27053.) 
Wawersik, W.R. 1991-1993. "Activity-Specific ES\&H Standard Operating Procedure Instructions for Hydraulic Fracturing Tests in MB139 and MB140." SP471962, Issue A-C. Albuquerque, NM: SNL. (Copy on file in the SWCF under WPO \#'s 38248 , \#38249, and \#38251.)

Wawersik, W.R., L.W. Carlson, J.A. Henfling, D.J. Borns, R.L. Beauheim, C.L. Howard, and R.M. Roberts. 1997. Hydraulic Fracturing Tests in Anhydrite Interbeds in the WIPP, Marker Beds 139 and 140. SAND95-0596. Albuquerque, NM: Sandia National Laboratories. (Copy on file in the SWCF under WPO \#45491.)

Westinghouse Electric Corporation. 1990. Geotechnical Field Data and Analysis Report, July 1988-June 1989. DOEMIPP 90-006, Vol. 2. Carisbad, NM: U.S. Department of Energy. (Available from the NTIS, Springfield, VA as DE91004495/XAB.)

WIPP Technical Operating Procedure 054. 1992. "Hydraulic Pressure Gage and Transducer Repair and Calibration." TOP 054, Rev. 2. Albuquerque, NM: SNL. (Copy on file in the SWCF under WPO \#37062.)

WIPP Technical Operating Procedure 125. 1992. "SNL Experimental Program Work Requests." TOP 125, Rev. 5. Albuquerque, NM: SNL. (Copy on file in the SWCF under WPO \#37996.)

WIPP Technical Operating Procedure 140. 1991. "Underground Drilling Request and Hole Identification System." TOP 140, Rev. 4. Albuquerque, NM: SNL. (Copy on file in the SWCF under WPO \#38011.)

WIPP Technical Operating Procedure 182. 1991. "Calibration of HP-3497A DC Voltmeter Option." TOP 182, Rev. 3. Albuquerque, NM: SNL. (Copy on file in the SWCF under WPO \#38069.)

WIPP Technical Operating Procedure 204. 1988. "Radial Closure Detector Setup and Calibration (Trans-Tek Model 0241-0000LVDT)." Top 204, Rev. 0. Albuquerque, NM: SNL. (Copy on file in the SWCF under WPO \#38112.)

WIPP Technical Operating Procedure 205. 1992. "Radial-Closure Detector Calibration." (Note: the title of this TOP is in error. The correct tile should be "Axial Motion Detector Setup and Calibration" as indicated in step I.A. of the TOP) TOP 205, Rev. 1. Albuquerque, NM: SNL. (Copy on file in the SWCF under WPO \#38113.)

WIPP Technical Operating Procedure 353. 1992. "Manual Calibration of Pressure Transducers." TOP 353, Rev. 2. Albuquerque, NM: SNL. (Copy on file in the SWCF in the Controlled Document Center.)

WIPP Technical Operating Procedure 427. 1993. "HP-75000 Series B 5 1/2 Digit HP (E1326B) DVM Calibration." TOP 427, Rev. 0. Albuquerque, NM: SNL. (Copy on file in the SWCF in the Controlled Document Center.) 
WIPP Technical Operating Procedure 467. 1993. "Installation and Retrieval of Pressurized Brine Sample Cylinders." TOP 467, Rev. 0. Albuquerque, NM: SNL. (Copy on file in the SWCF under WPO \#39278.)

WIPP Technical Operating Procedure 470. 1994. "Operation and Use of Pressure Accumulators." TOP 470, Rev. 0. Albuquerque, NM: SNL. (Copy on file in the SWCF under WPO \#39281.)

WIPP Technical Operating Procedure 473. 1994. "Radial LVDT Calibration Measurement." TOP 473, Rev. 0. Albuquerque, NM: SNL. (Copy on file in the SWCF under WPO \#39284.)

WIPP Technical Operating Procedure 476. 1995. "Constant-Pressure InjectionMithdrawal System." TOP 476, Rev. 0. Albuquerque, NM: SNL. (Copy on file in the SWCF under WPO \#39282.)

WIPP Technical Operating Procedure 479. 1995. "Multipacker-Test-Tool Installation for Borehole Drilled Vertically Downward." TOP 479, Rev. 1. Albuquerque, NM: SNL. (Copy on file in the SWCF under WPO \#39289.)

WIPP Technical Operating Procedure 480. 1995. "Multipacker-Test-Tool Installation for Borehole Drilled at an Upward Angle into the Rib or Back." TOP 480, Rev. 2. Albuquerque, NM: SNL. (Copy on file in the SWCF under WPO \#39285.)

WIPP Technical Operating Procedure 481. 1995. "Multipacker-Test-Tool Installation for Borehole Drilled Vertically Upward." TOP 481, Rev. 1. Albuquerque, NM: SNL. (Copy on file in the SWCF under WPO \#39288.)

WIPP Technical Operating Procedure 483. 1995. "Permeability Testing." TOP 483, Rev. 0. Albuquerque, NM: SNL. (Copy on file in the SWCF under WPO \#39292.) 


\section{APPENDIX A: CORE DESCRIPTION OF TEST AND MONITOR BOREHOLES}


TABLE A.1

CORE DESCRIPTION OF BOREHOLE S1P74-A

A - 2 
TABLE A.1

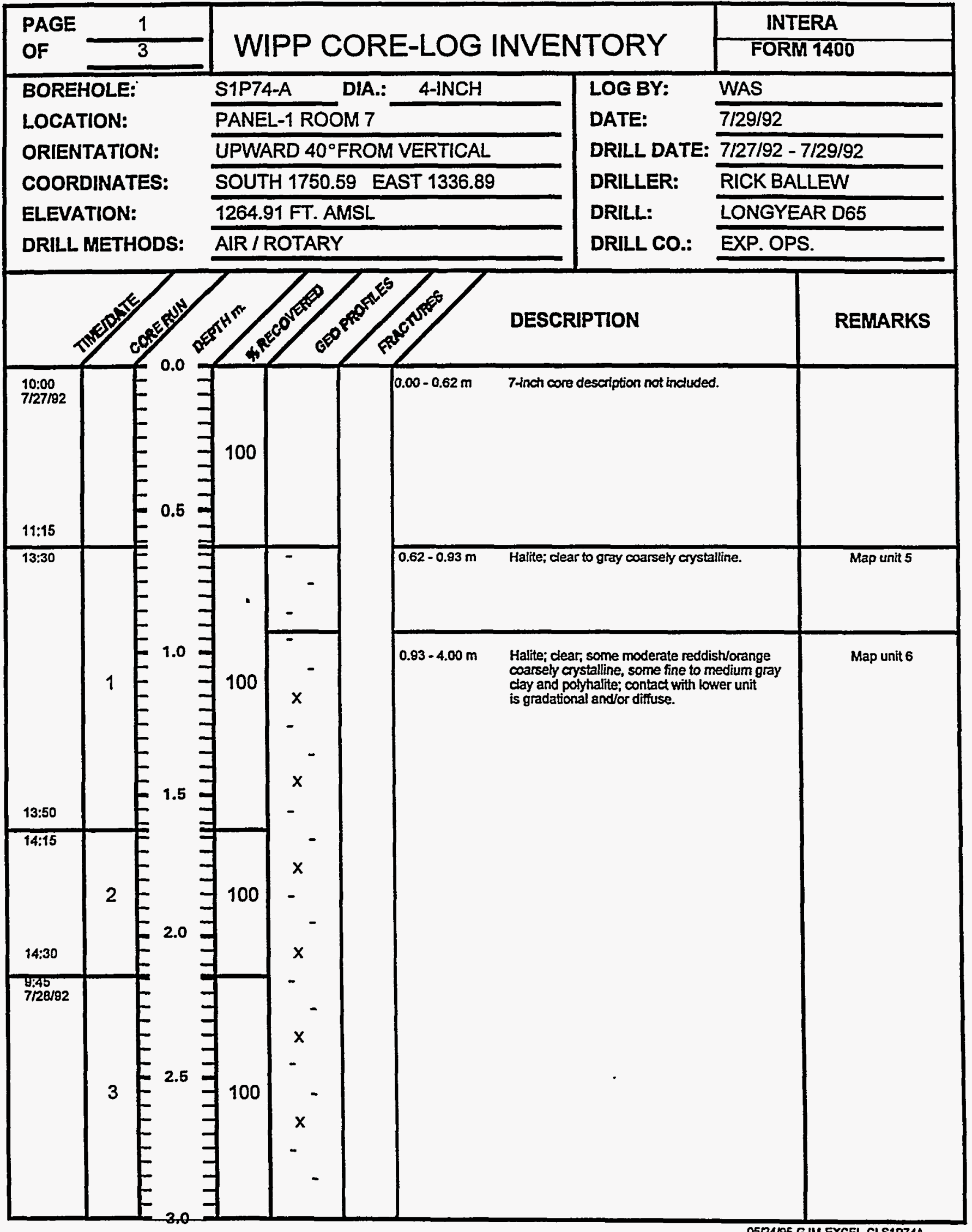


TABLE A.1 (Continued)

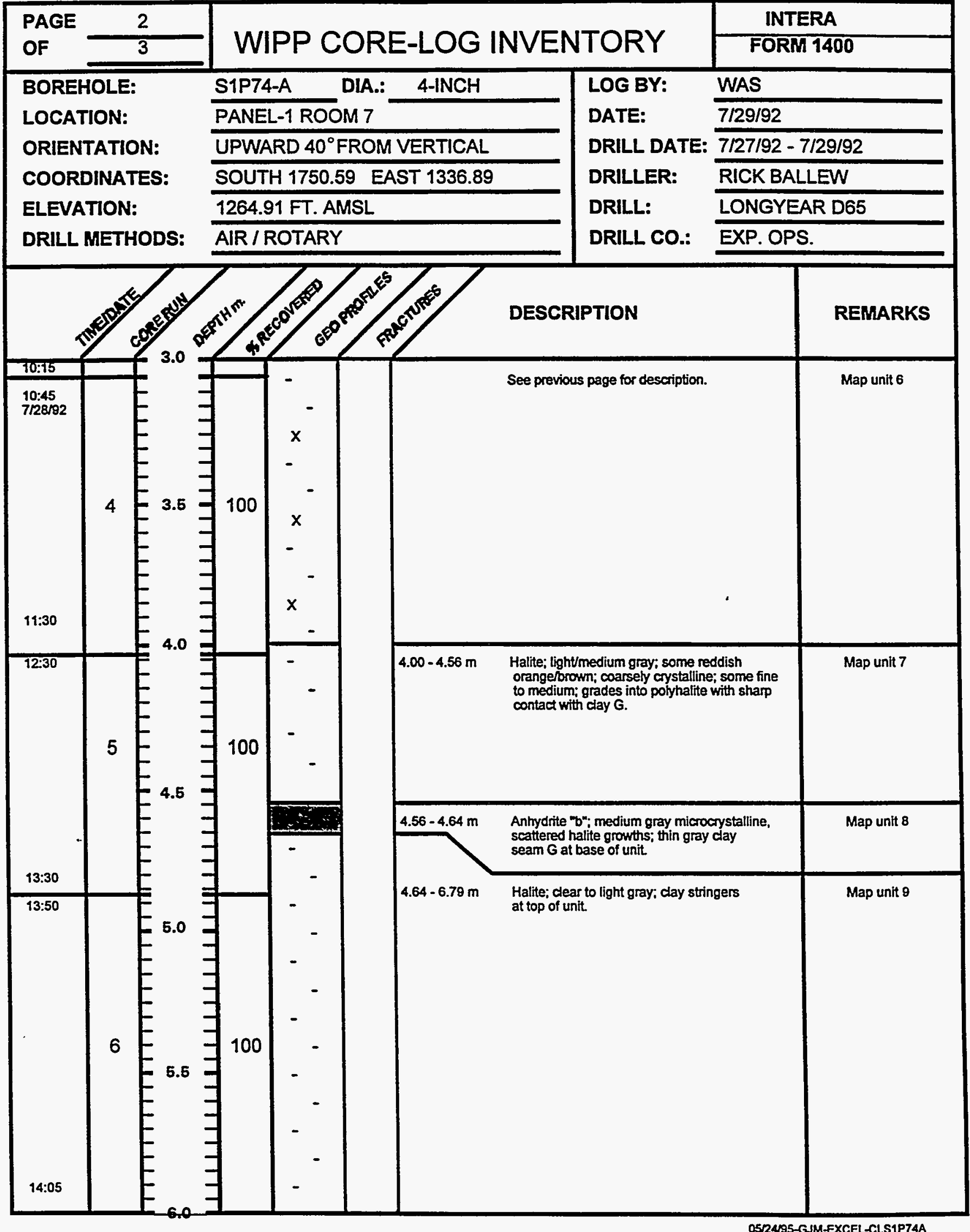


TABLE A.1 (Continued)

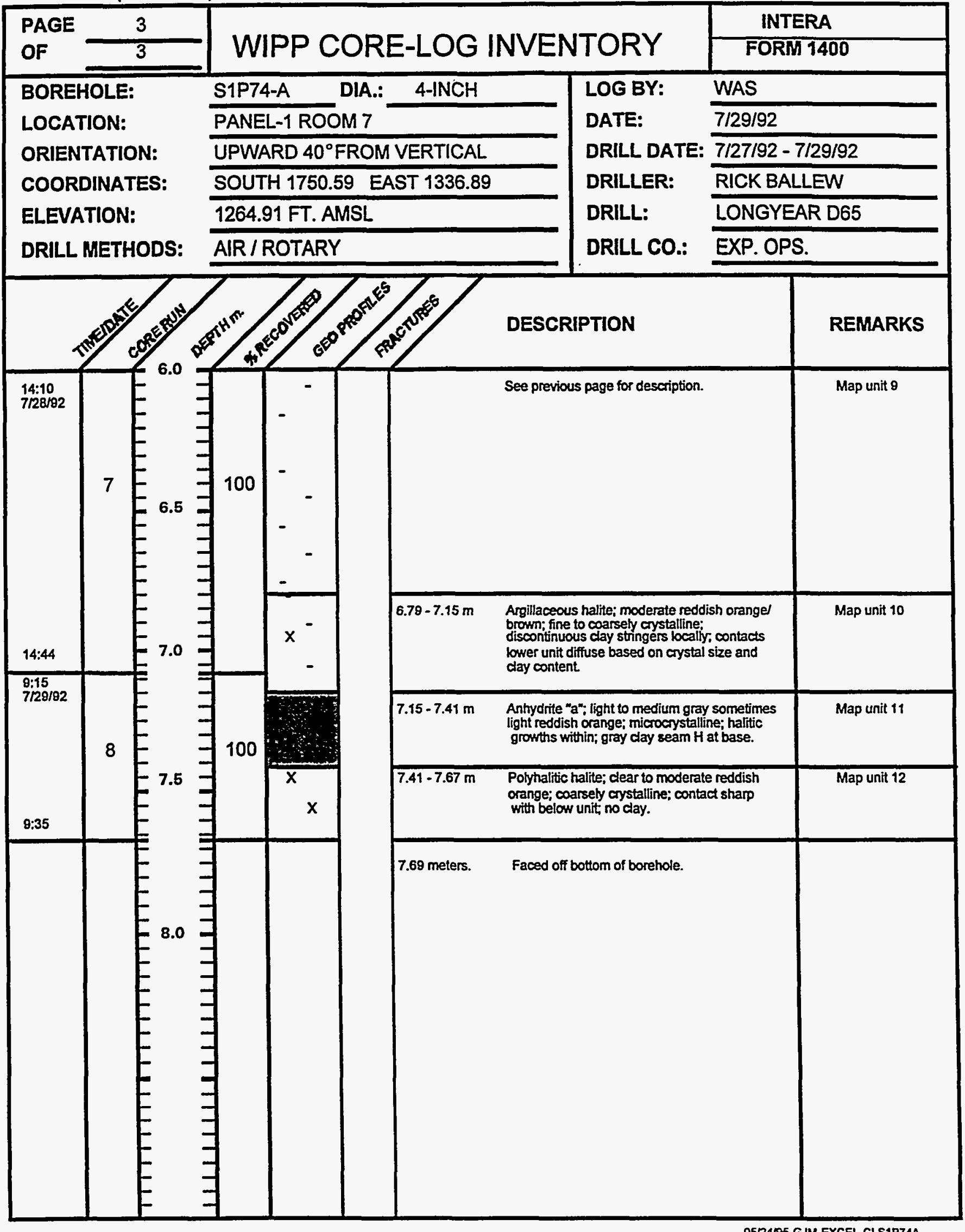


TABLE A.2

CORE DESCRIPTION OF BOREHOLE S1P74-B

A -6 
TABLE A.2

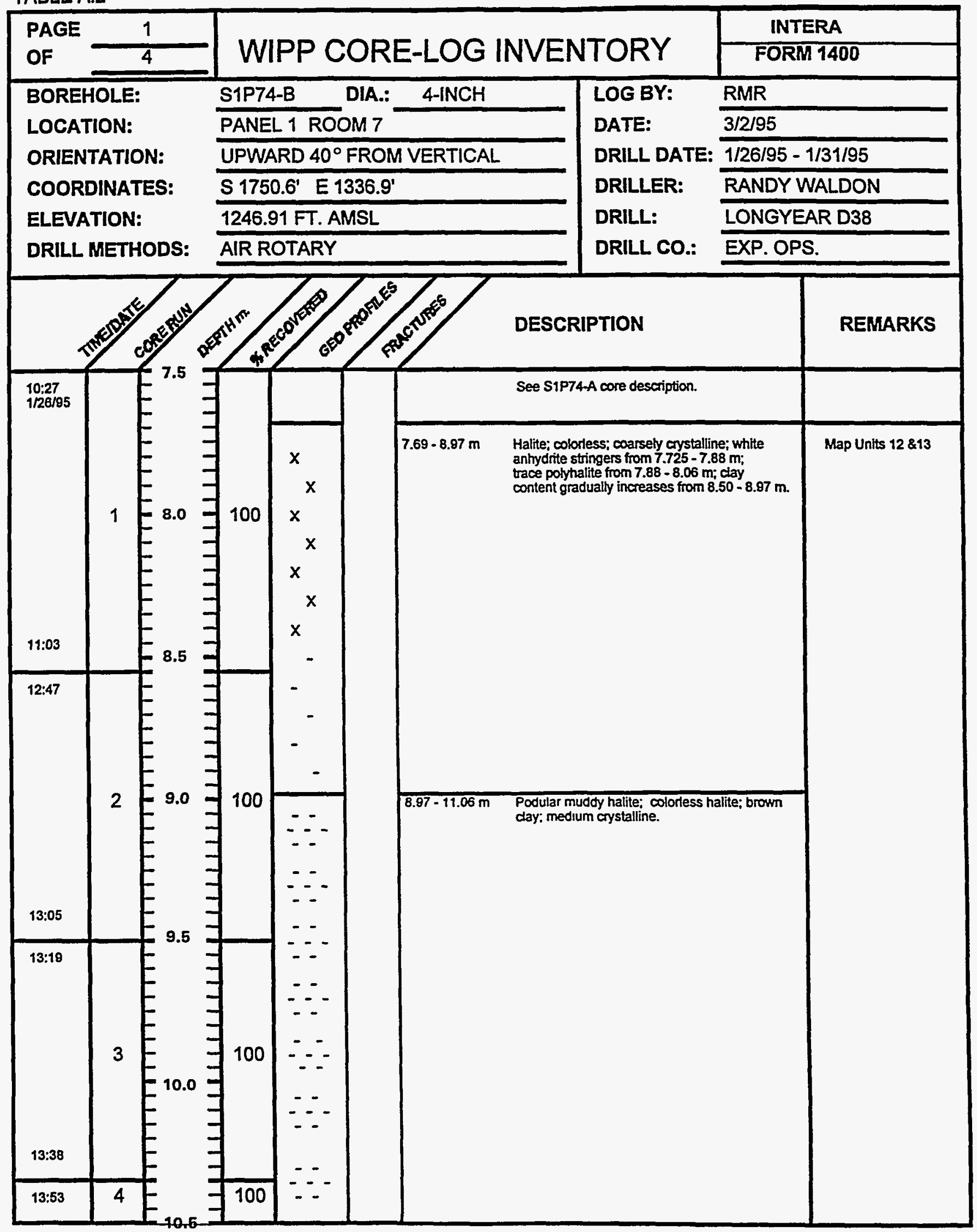


TABLE A.2 (Continued)

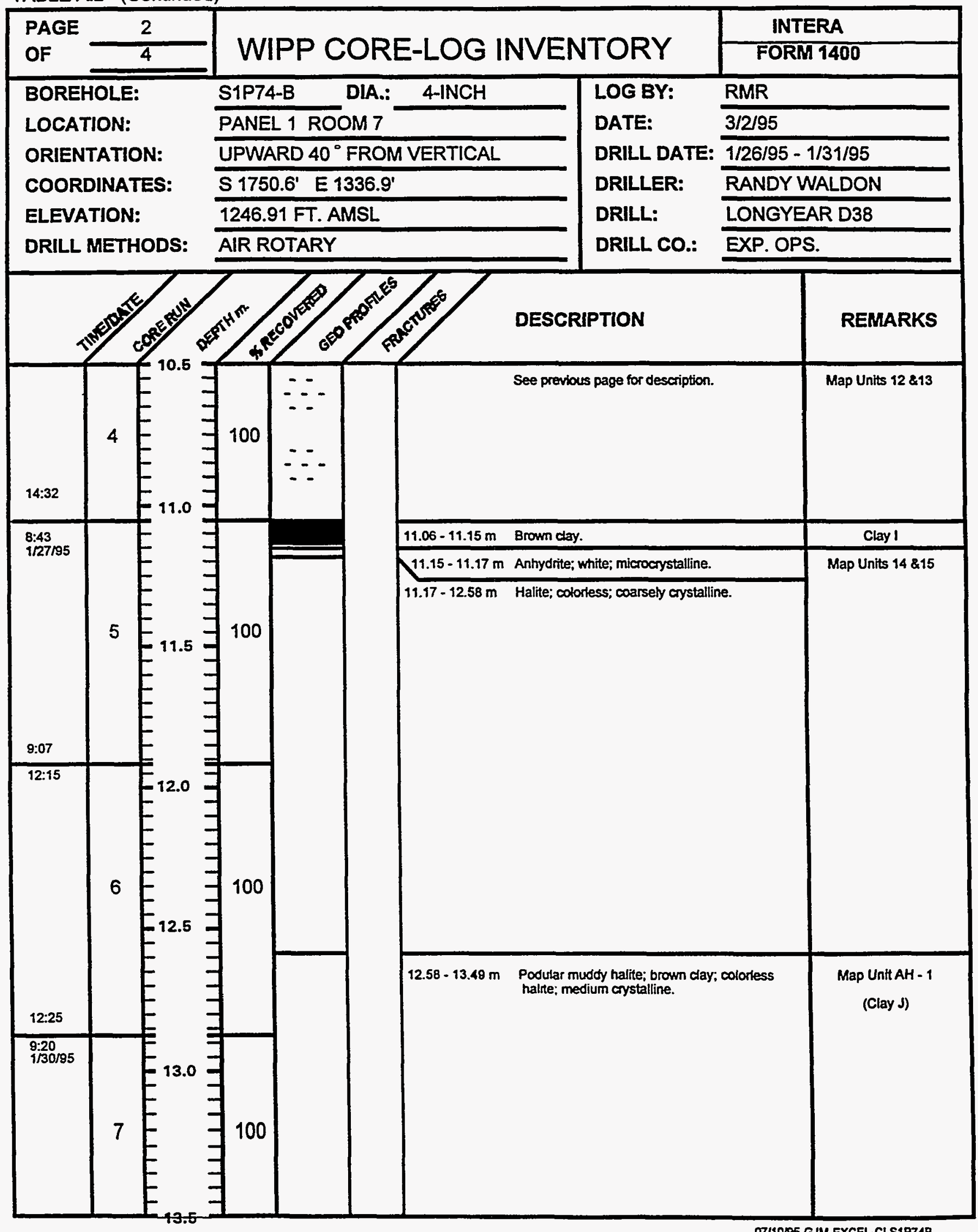


TABLE A.2 (Continued)

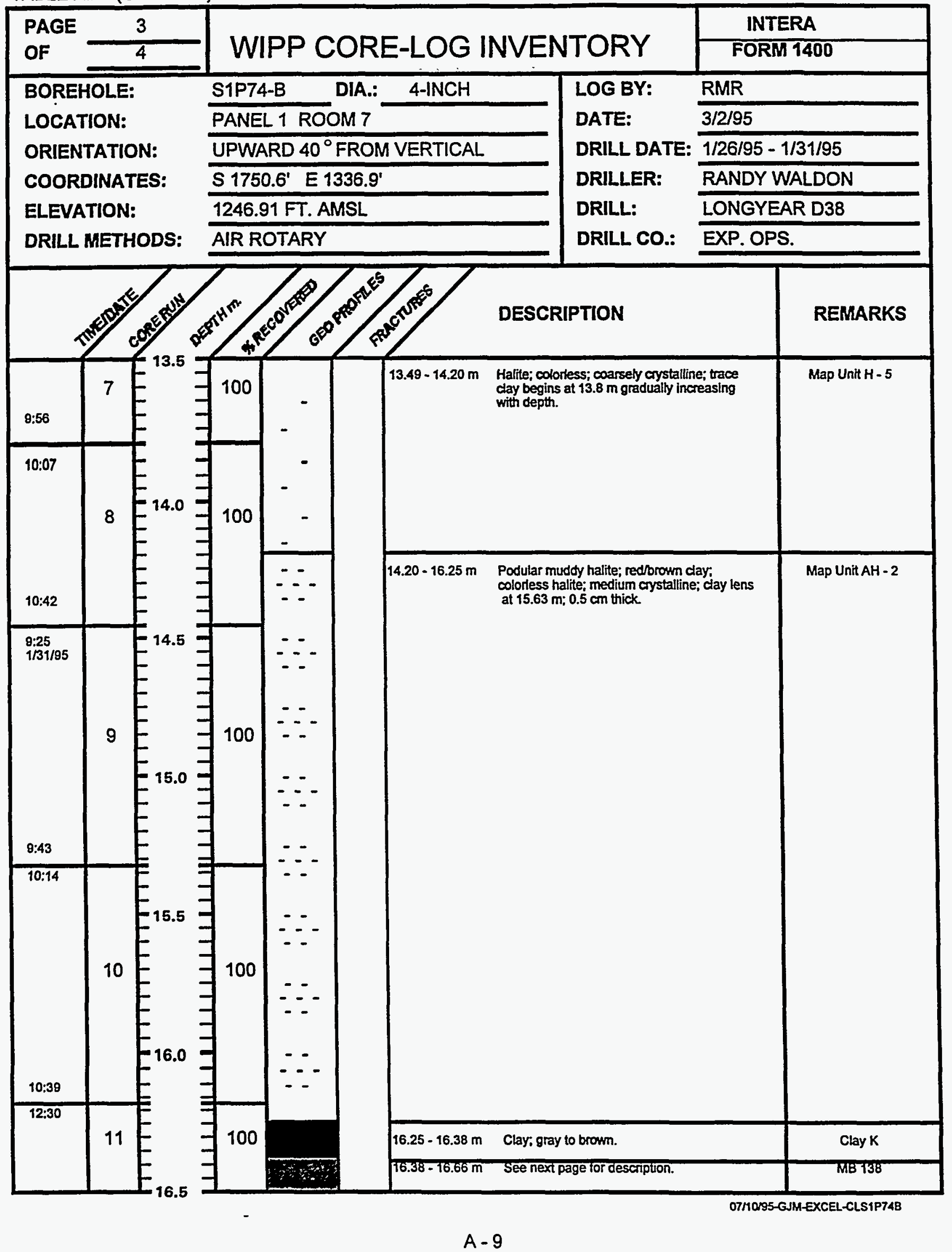


TABLE A.2 (Continued)

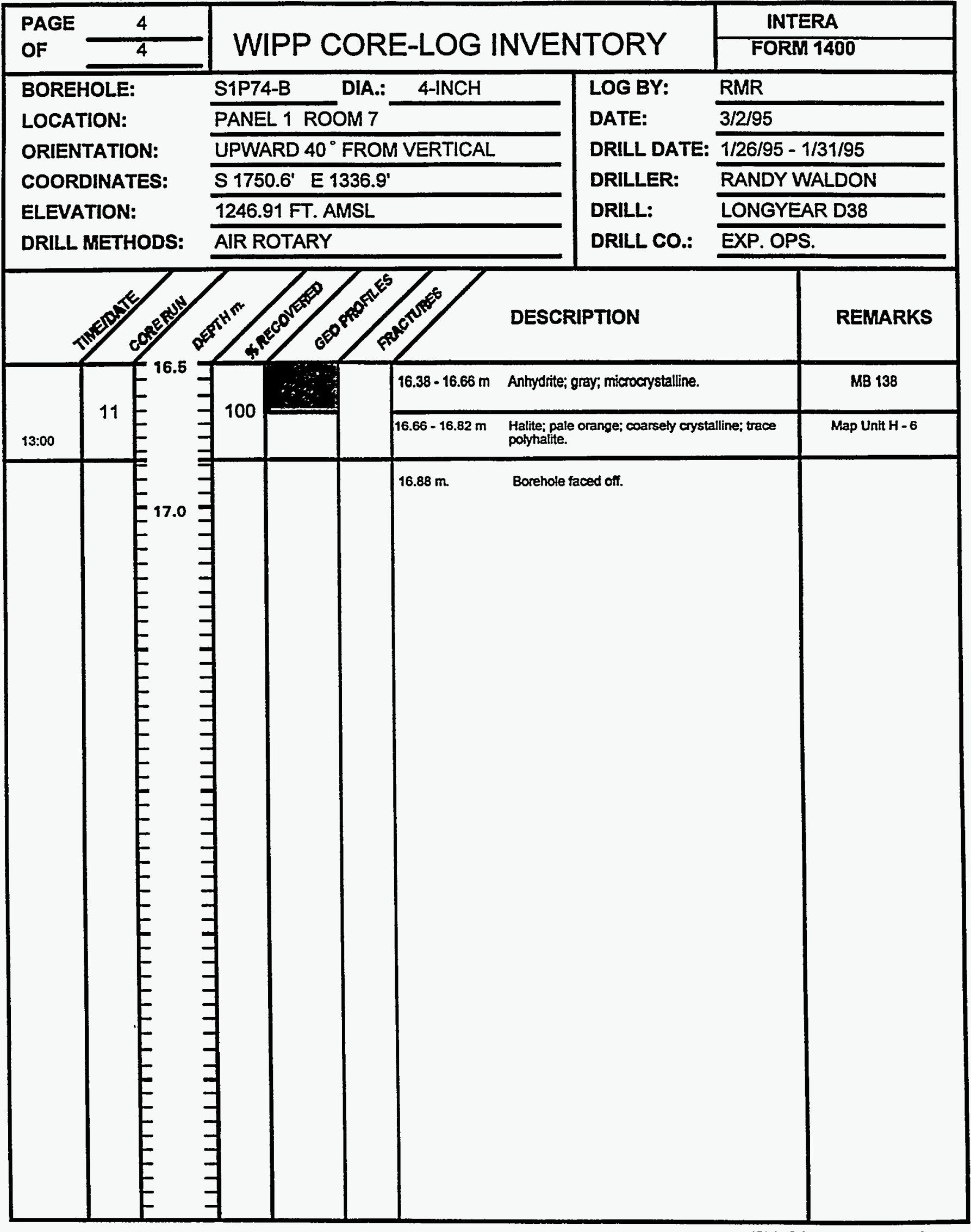


TABLE A.3

CORE DESCRIPTION OF BOREHOLE C1H05

A - 11 
TABLE A.3

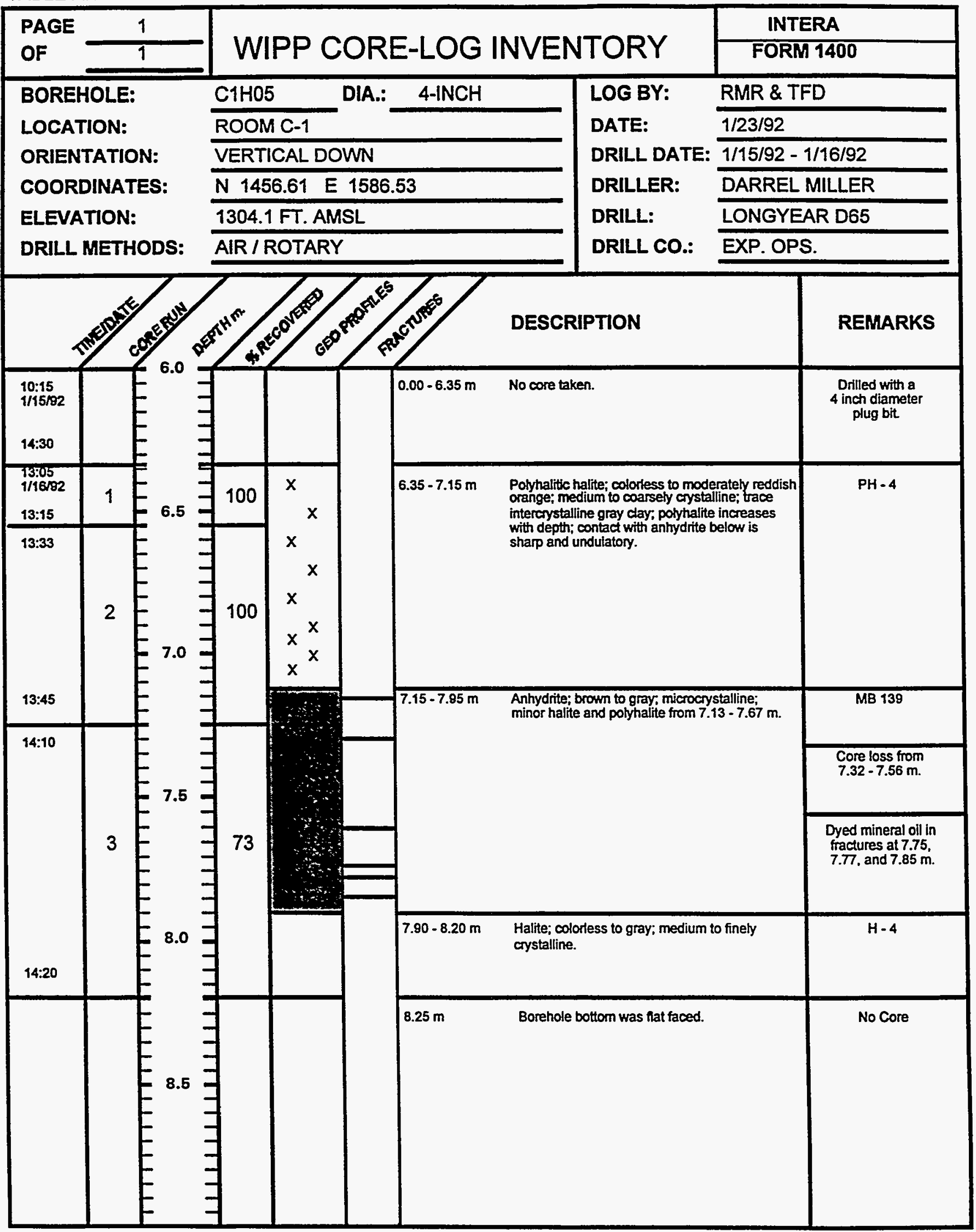


CORE DESCRIPTION OF BOREHOLE C1H07-A 
TABLE A.4

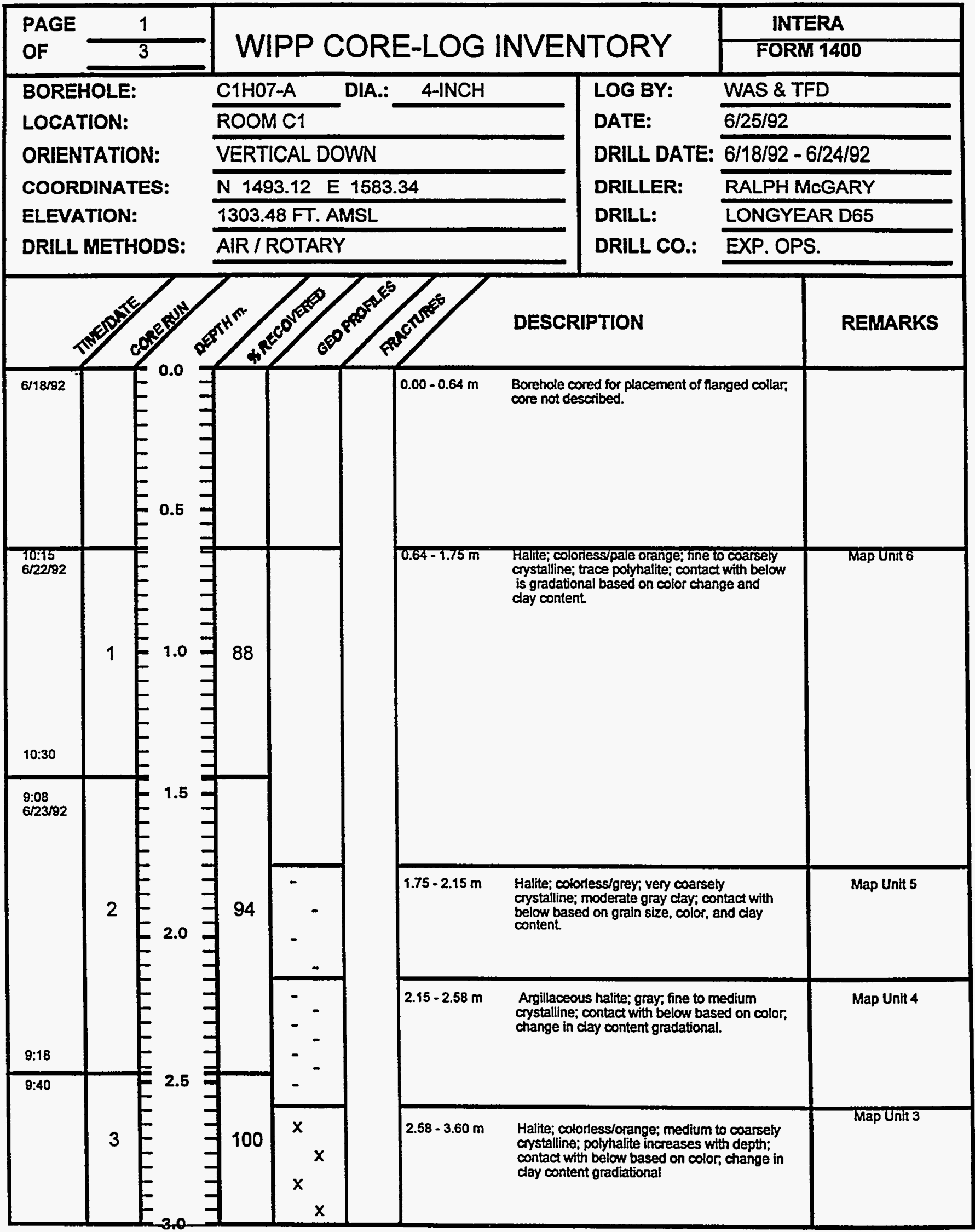


TABLE A.4 (Continued)

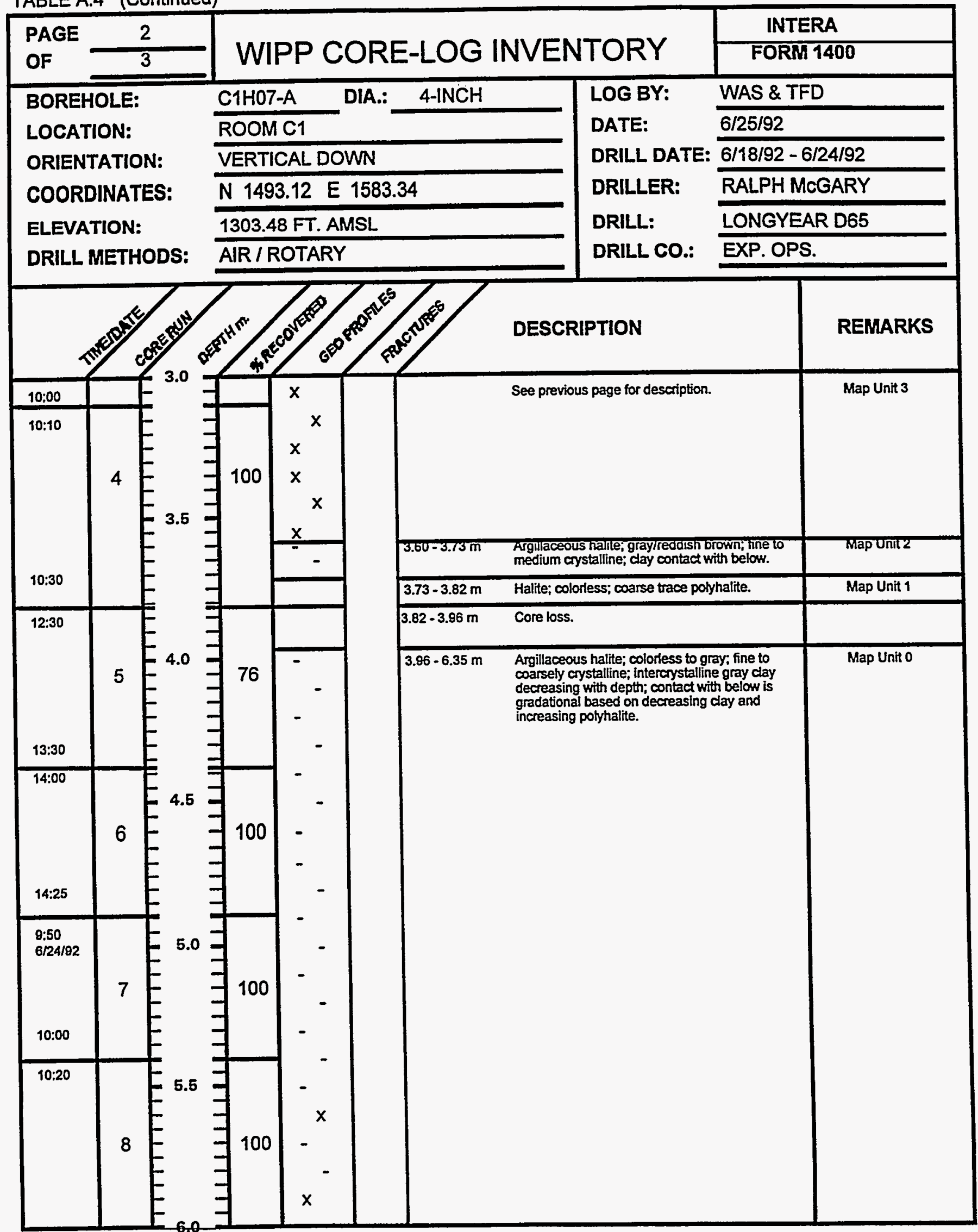


TABLE A.4 (Continued)

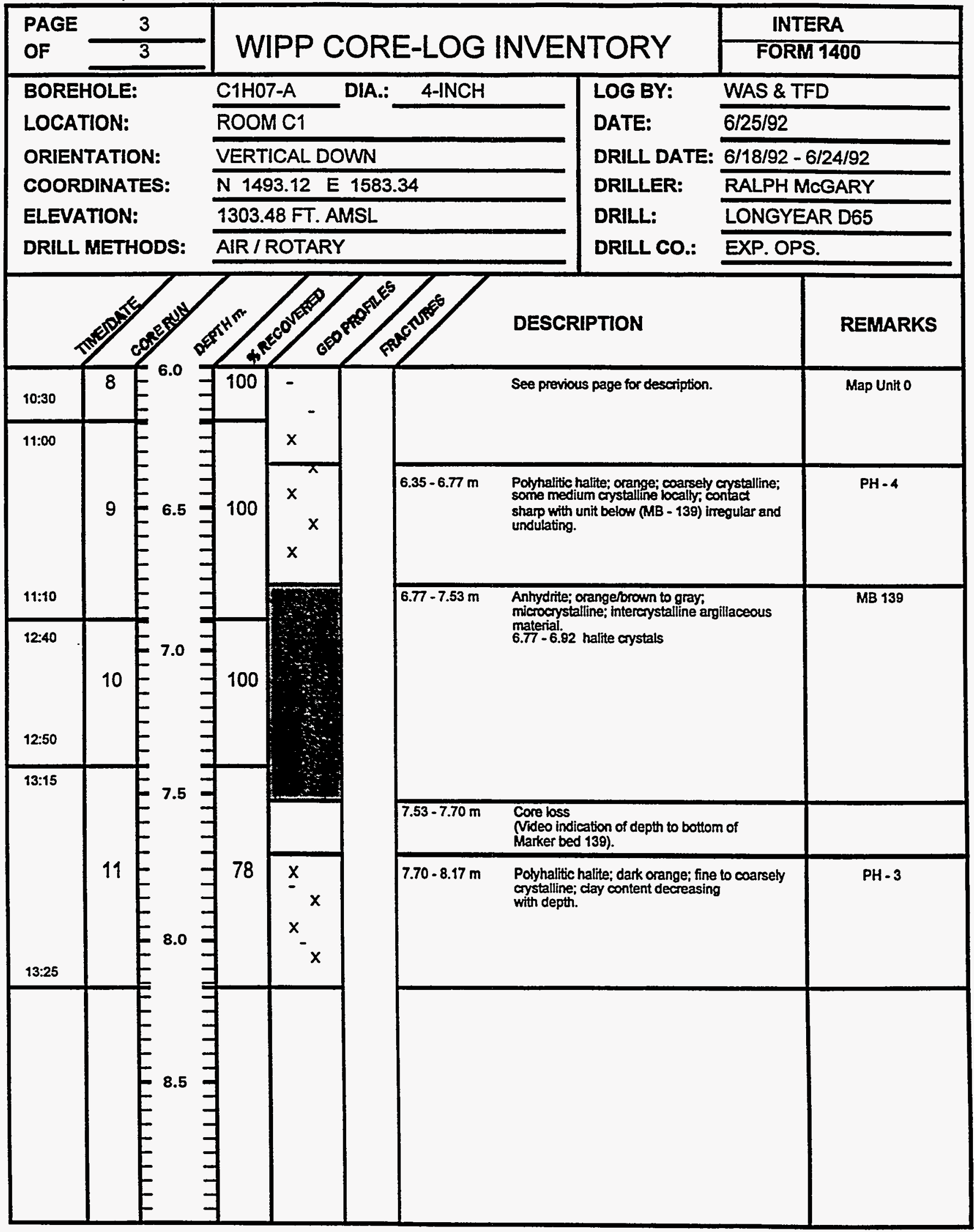


TABLE A.5

CORE DESCRIPTION OF BOREHOLE C1H07-B

A -17 
TABLE A.5

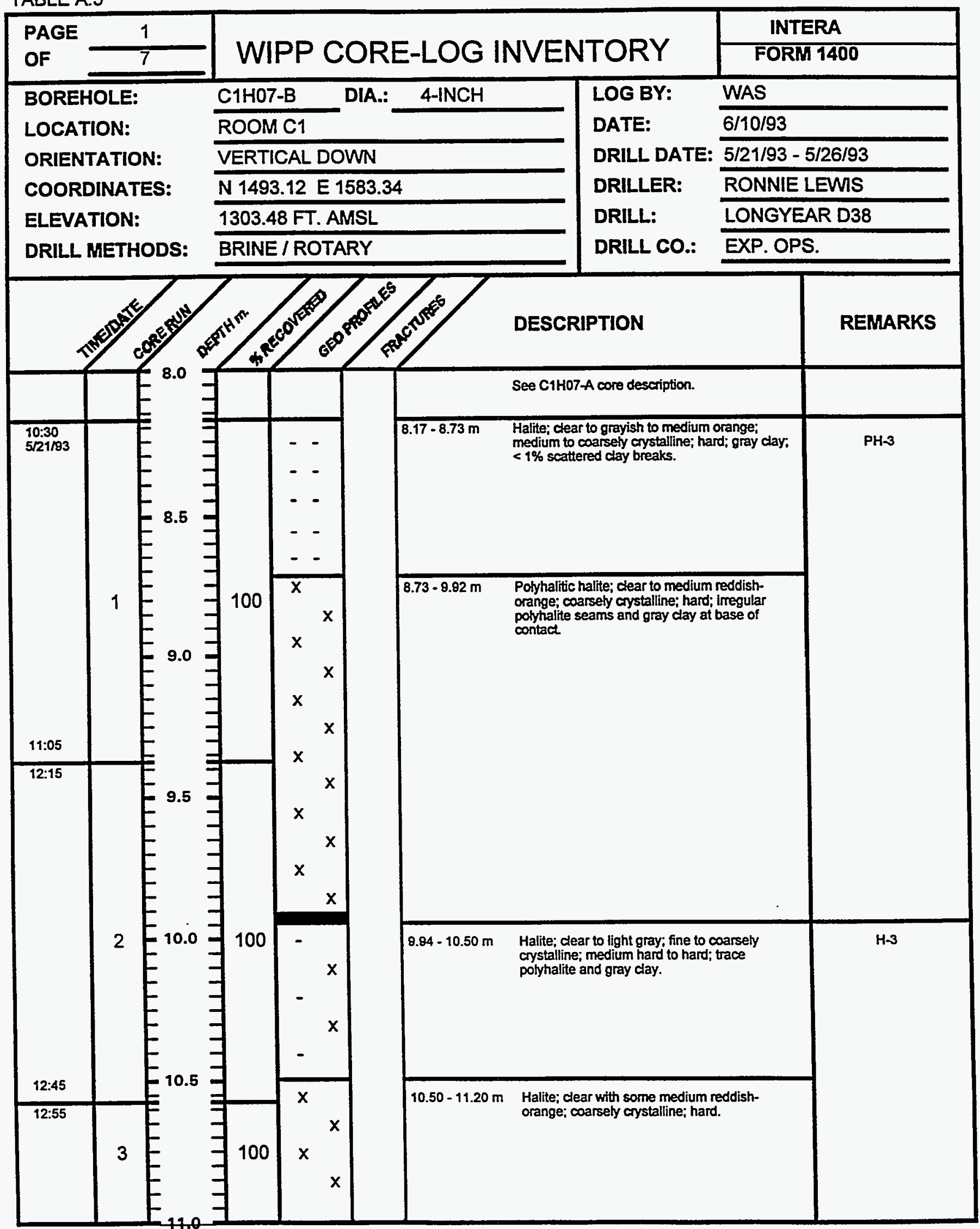


TABLE A.5 (Continued)

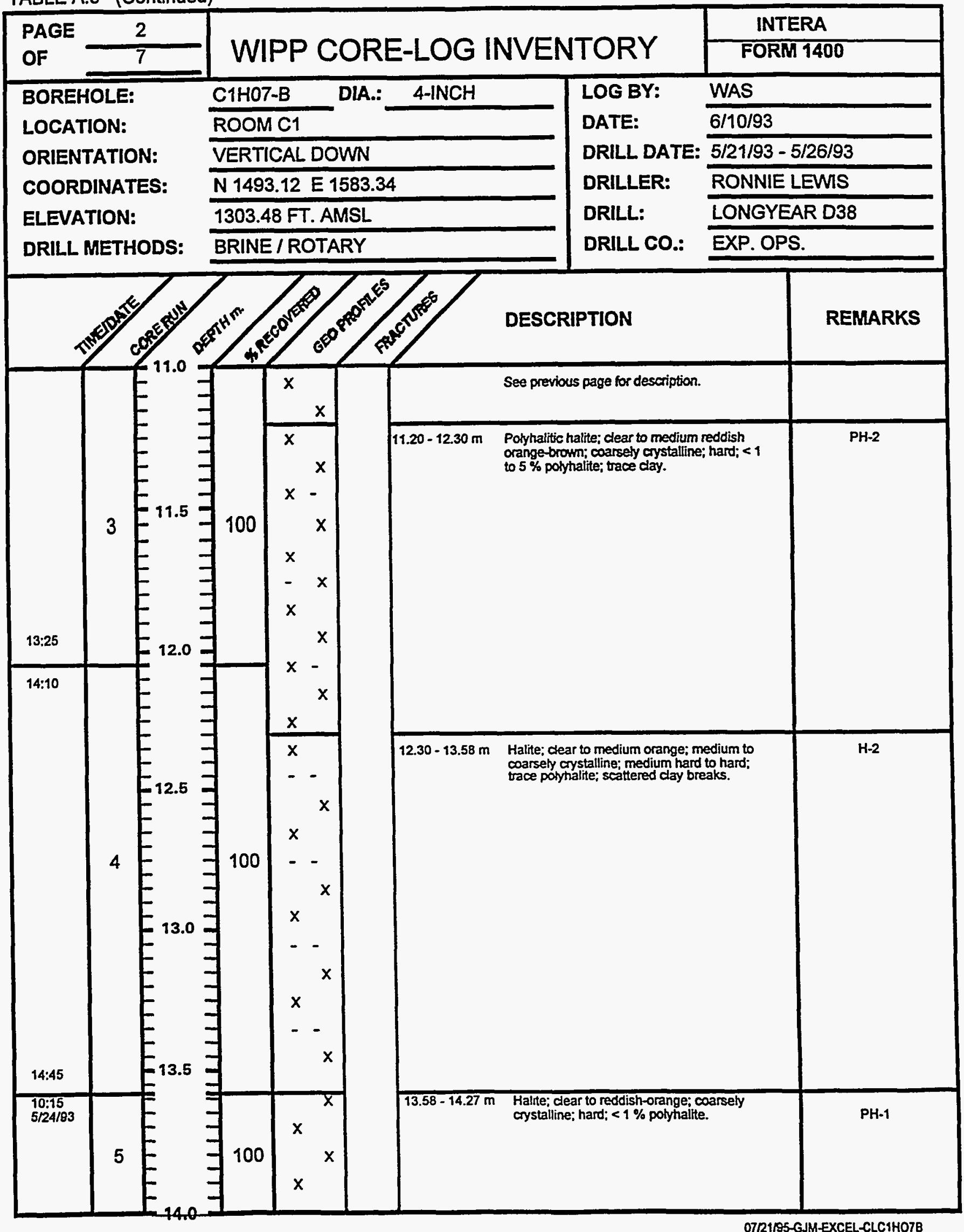


TABLE A.5 (Continued)

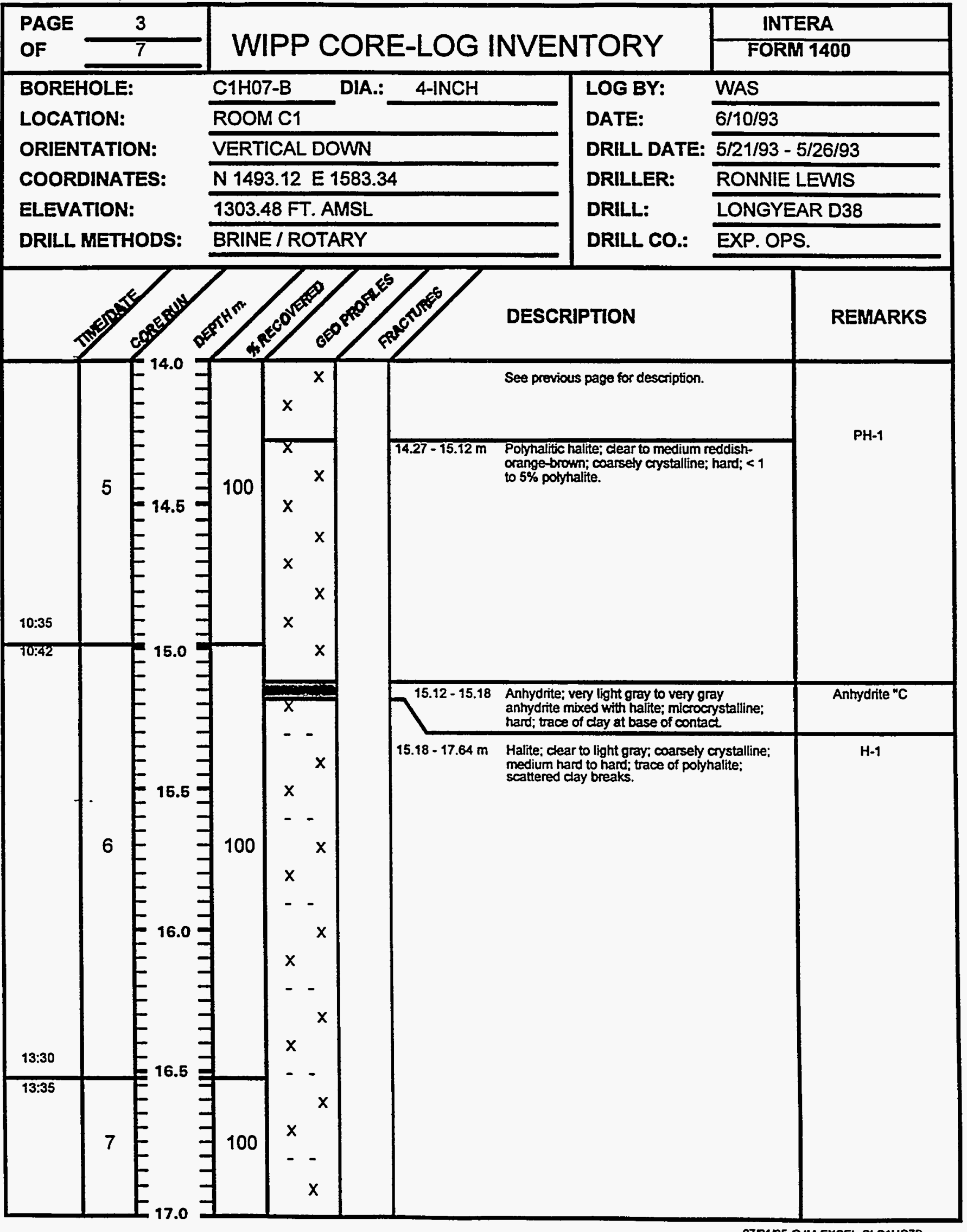


TABLEA.5 (Continued)

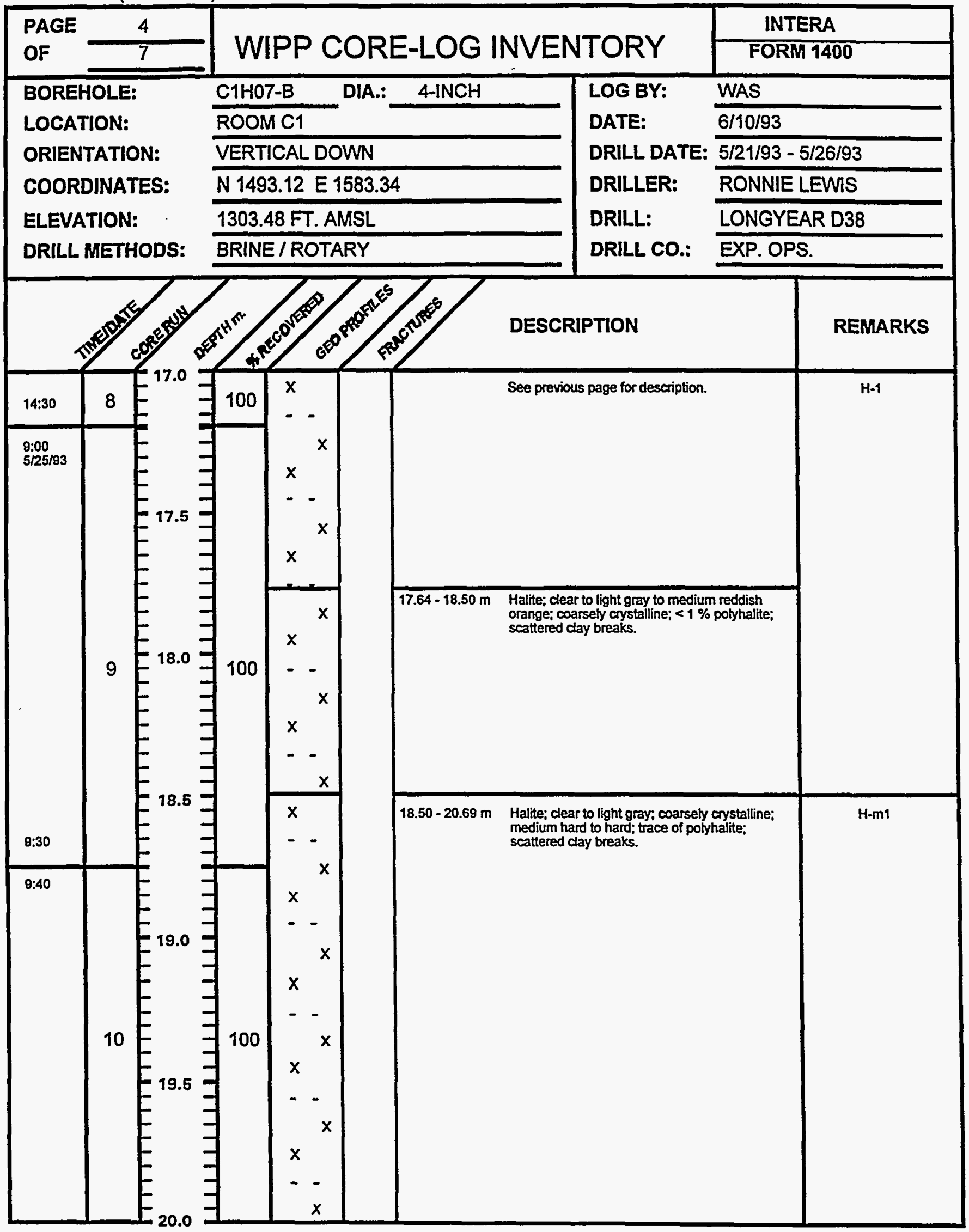


TABLE A.5 (Continued)

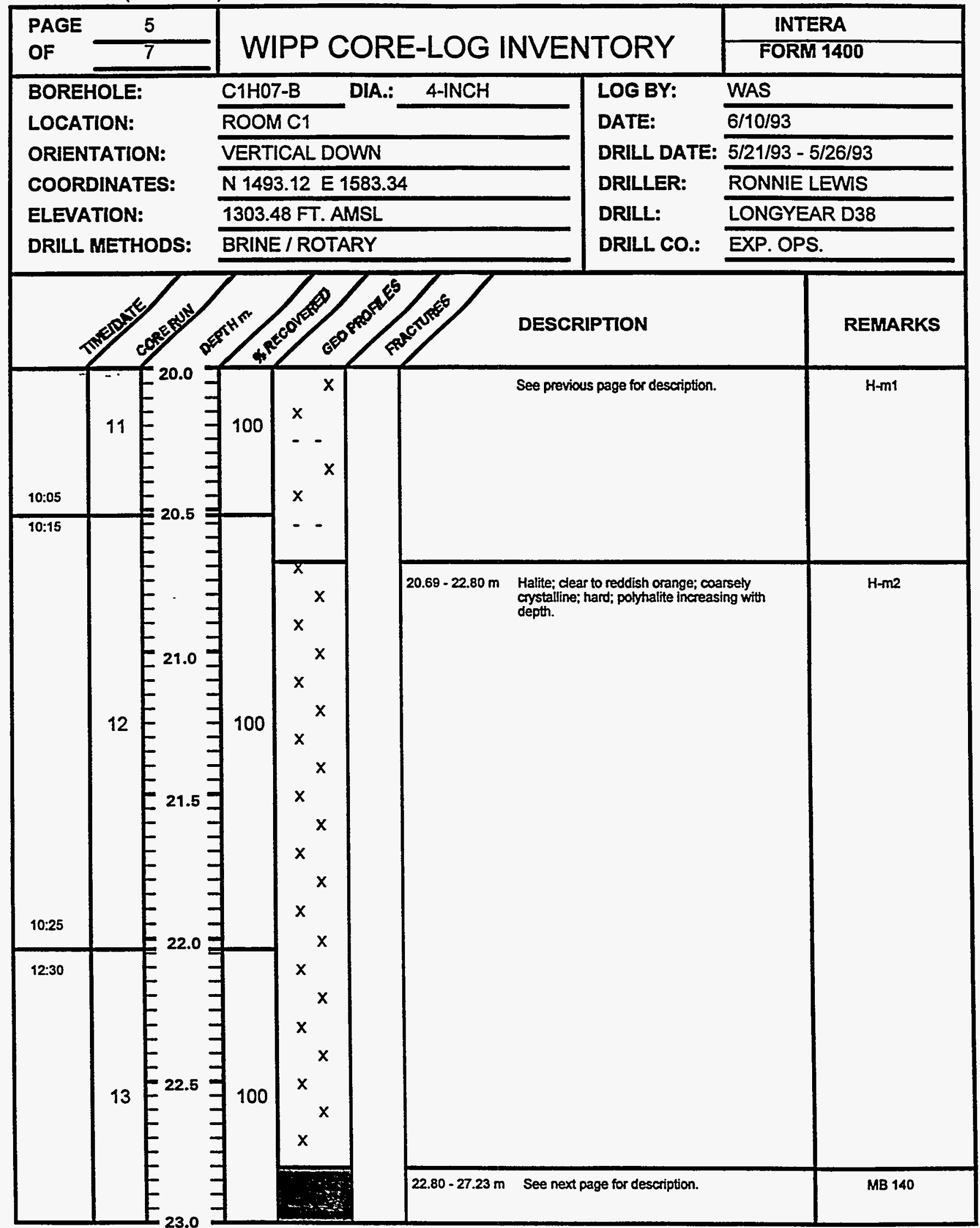

07/21/95-GJM-EXCEL-CLC1HO7B 
TABLE A.5 (Continued)

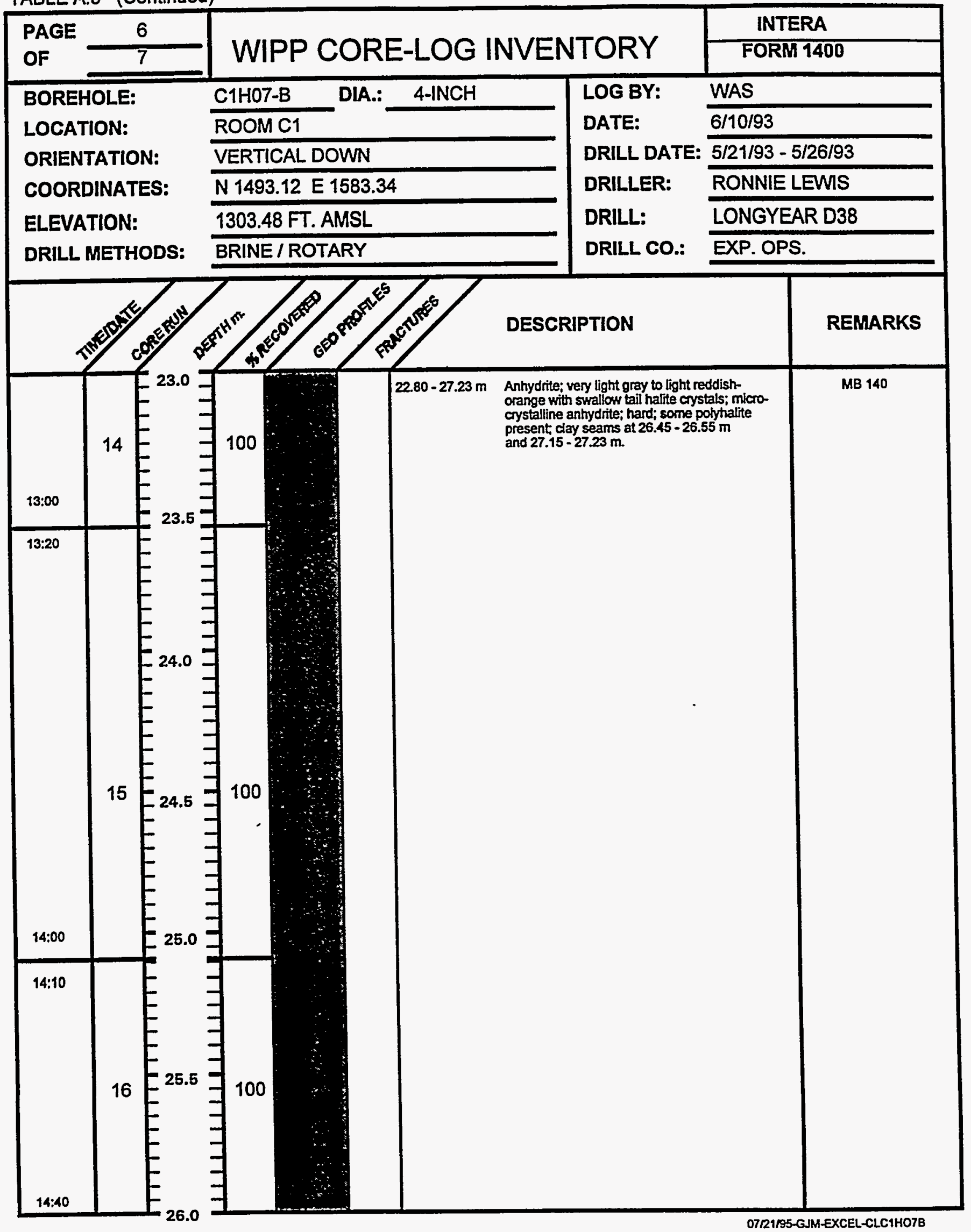


TABLE A.5 (Continued)

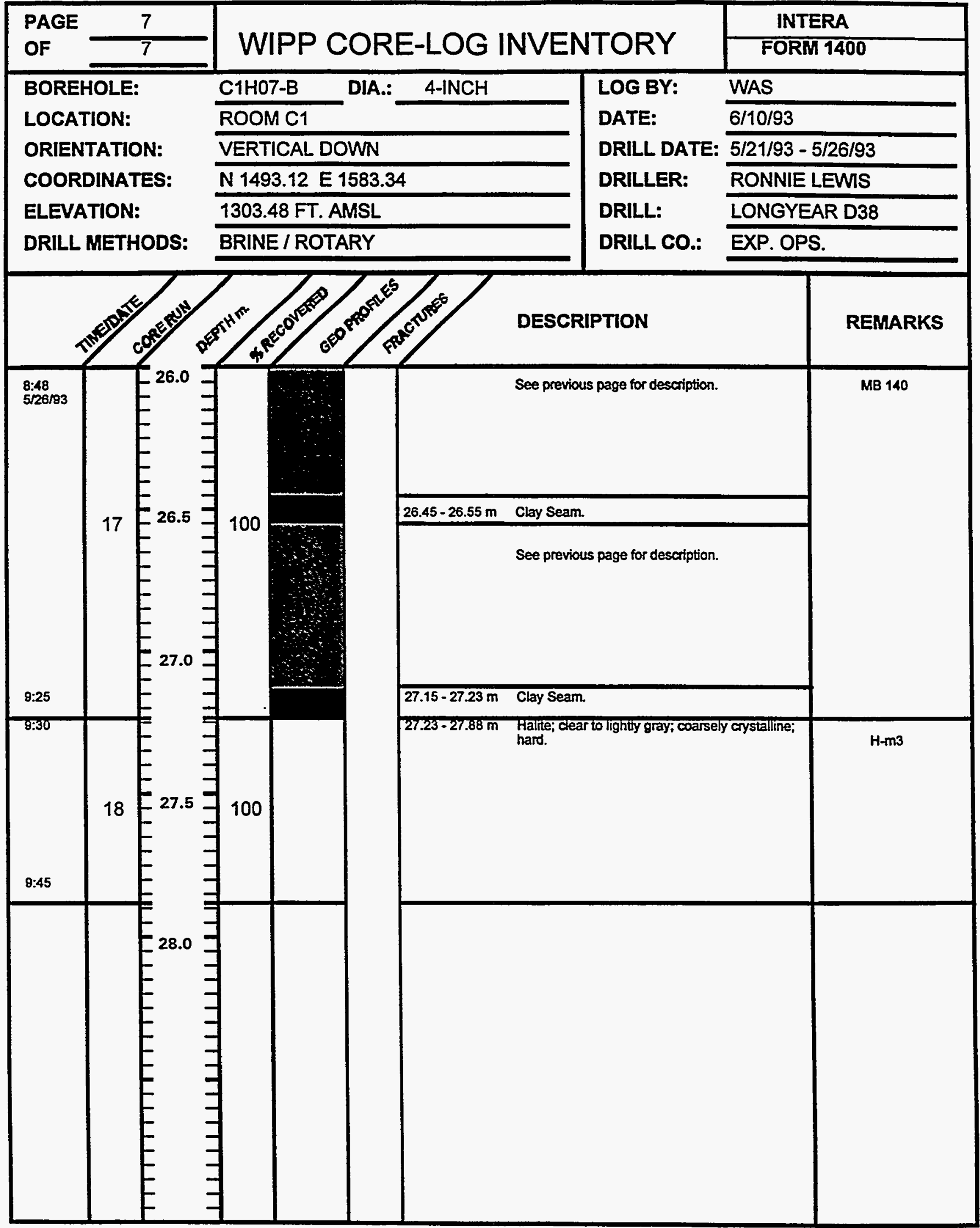


CORE DESCRIPTION OF BOREHOLE C1HO6 
TABLE A.6

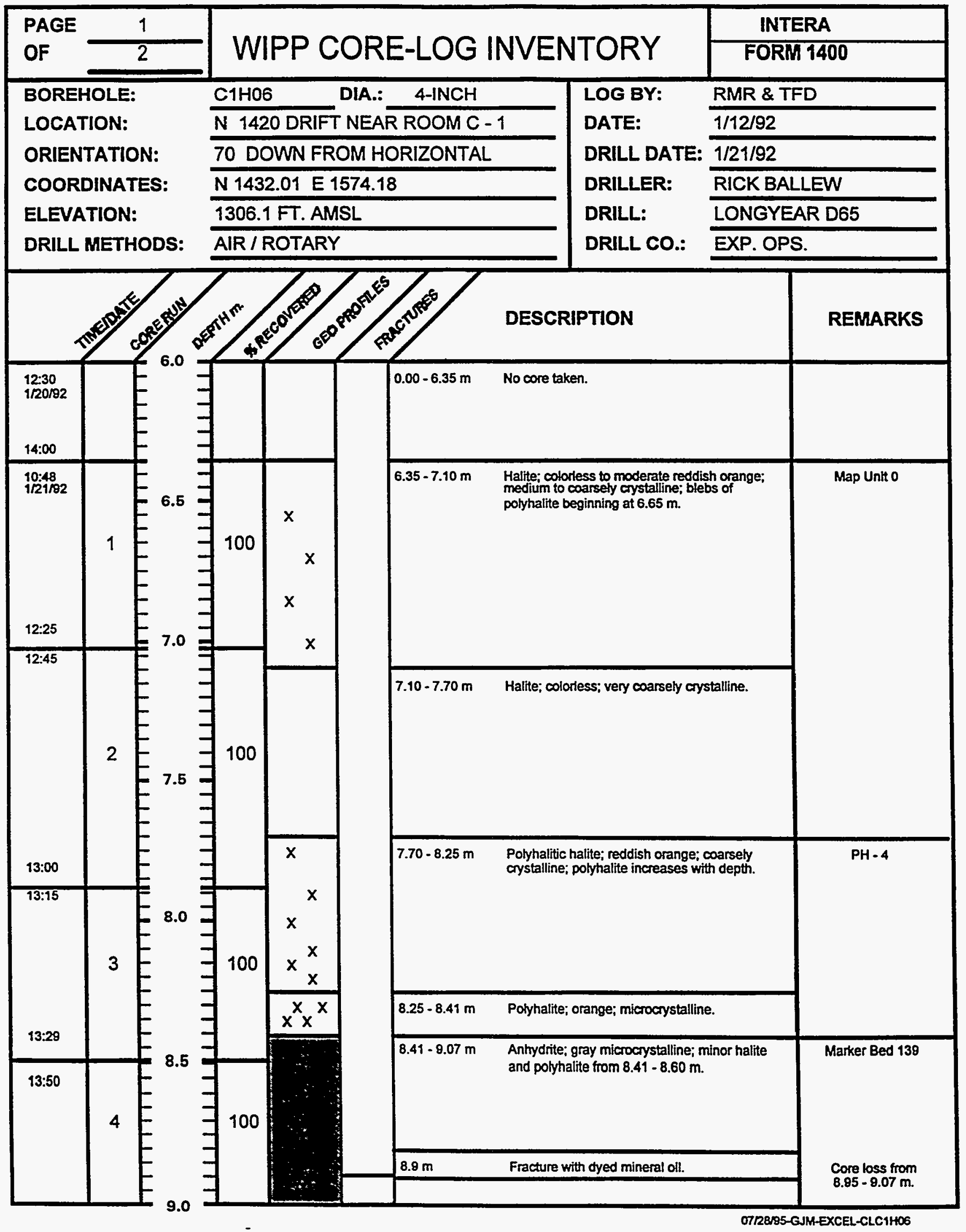


TABLE A.6 (Continued)

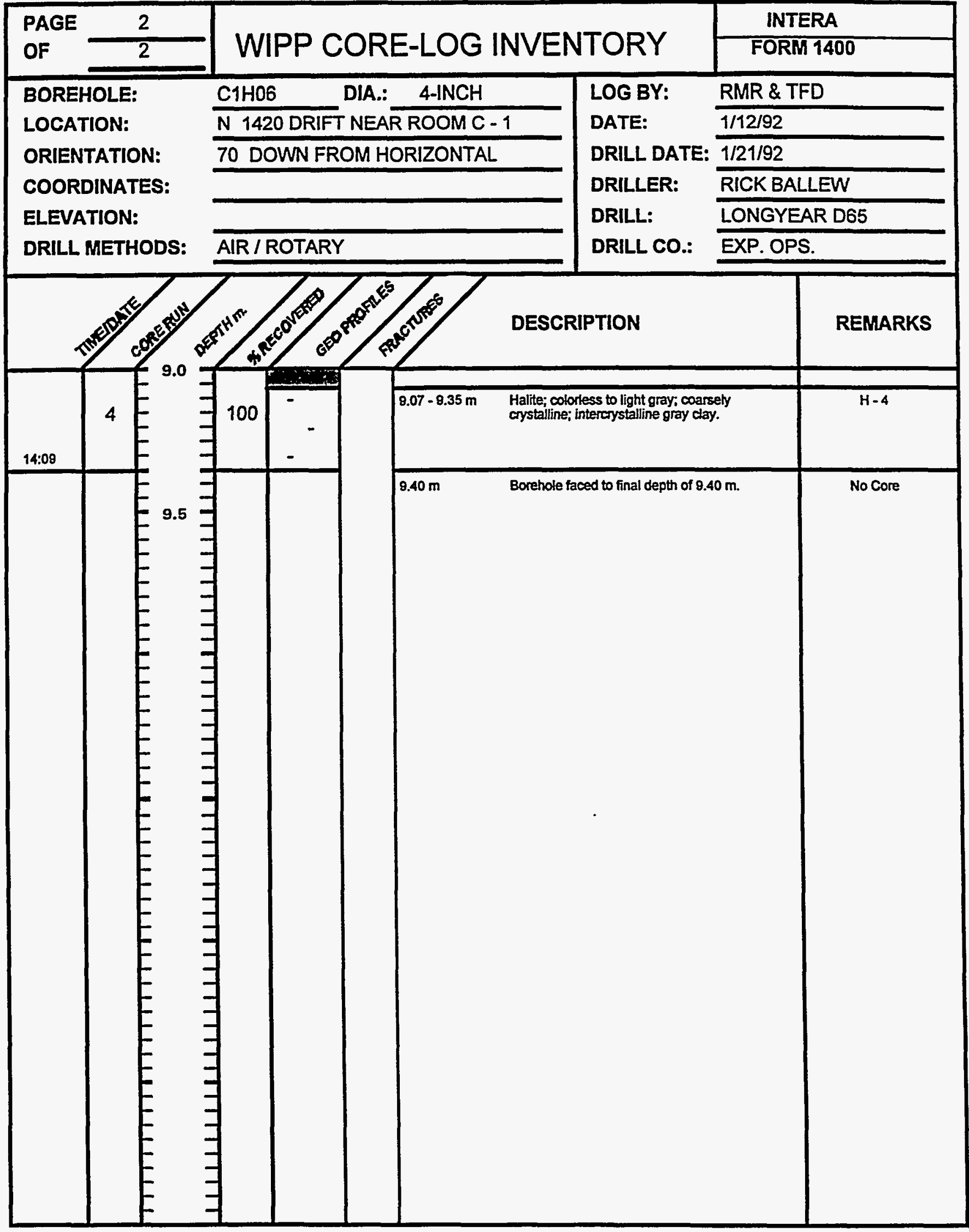


TABLE A.7

CORE DESCRIPTION OF BOREHOLE C1X05-A

A - 28 
TABLE A.7

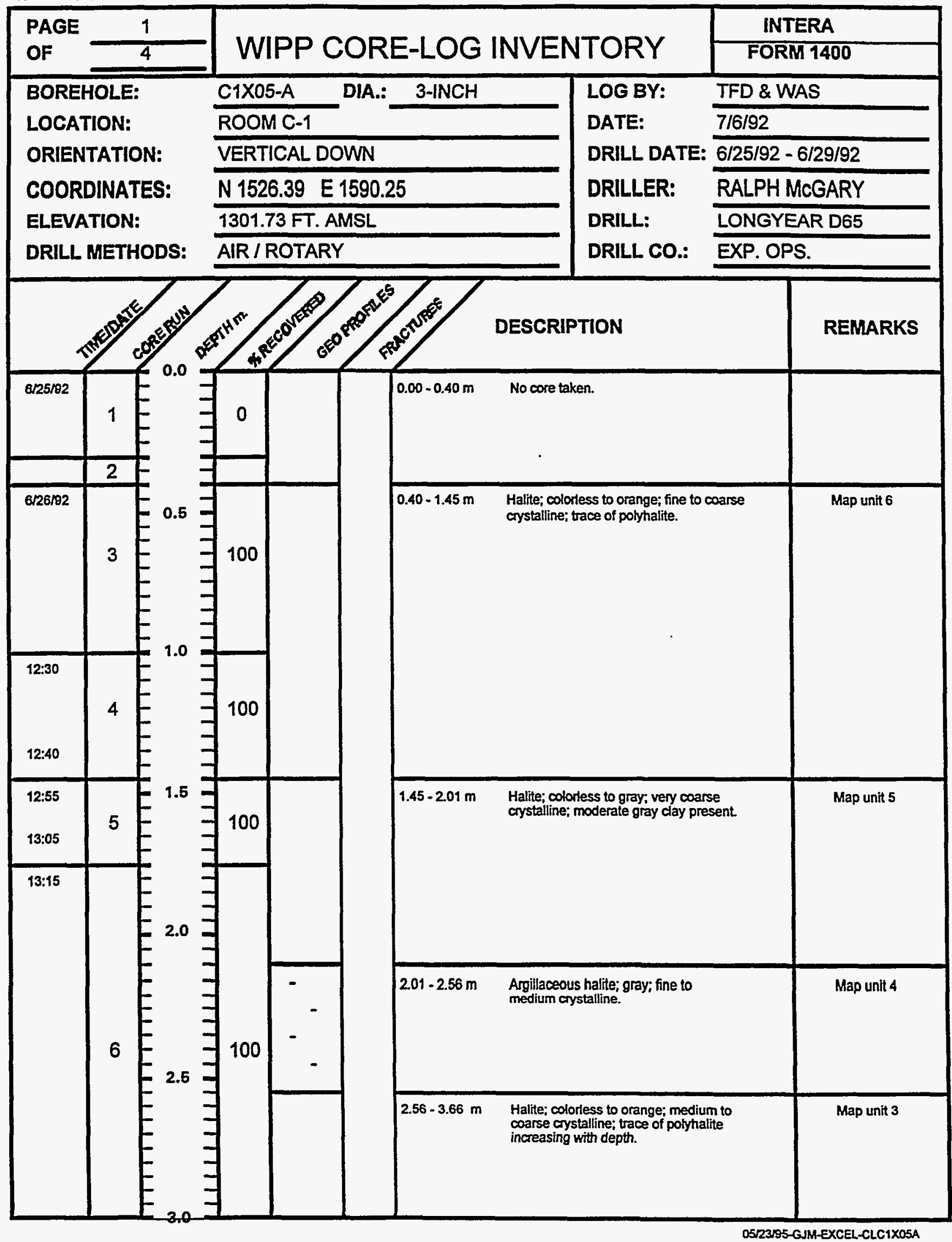


TABLE A.7 (Continued)

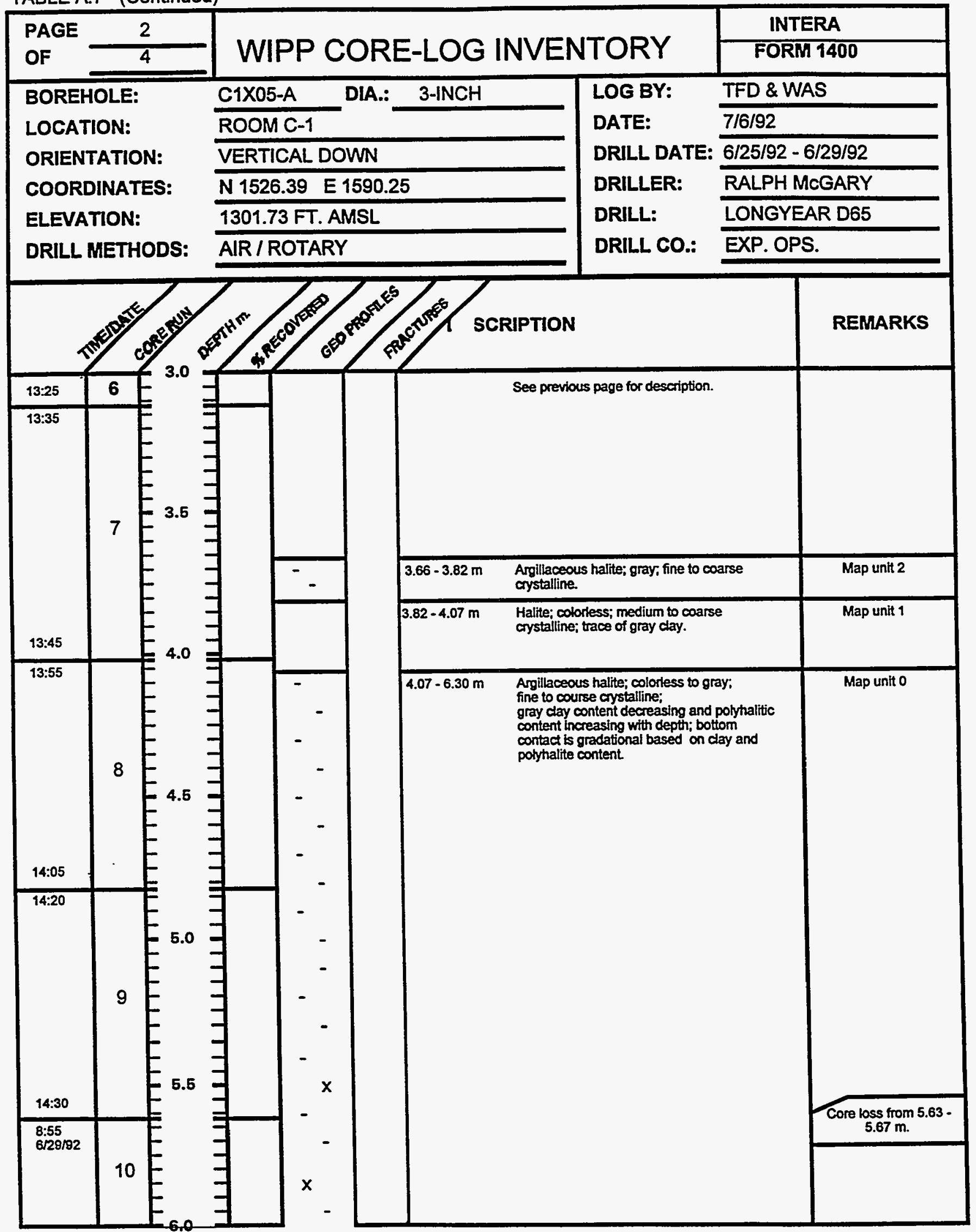


TABLE A.7 (Continued)

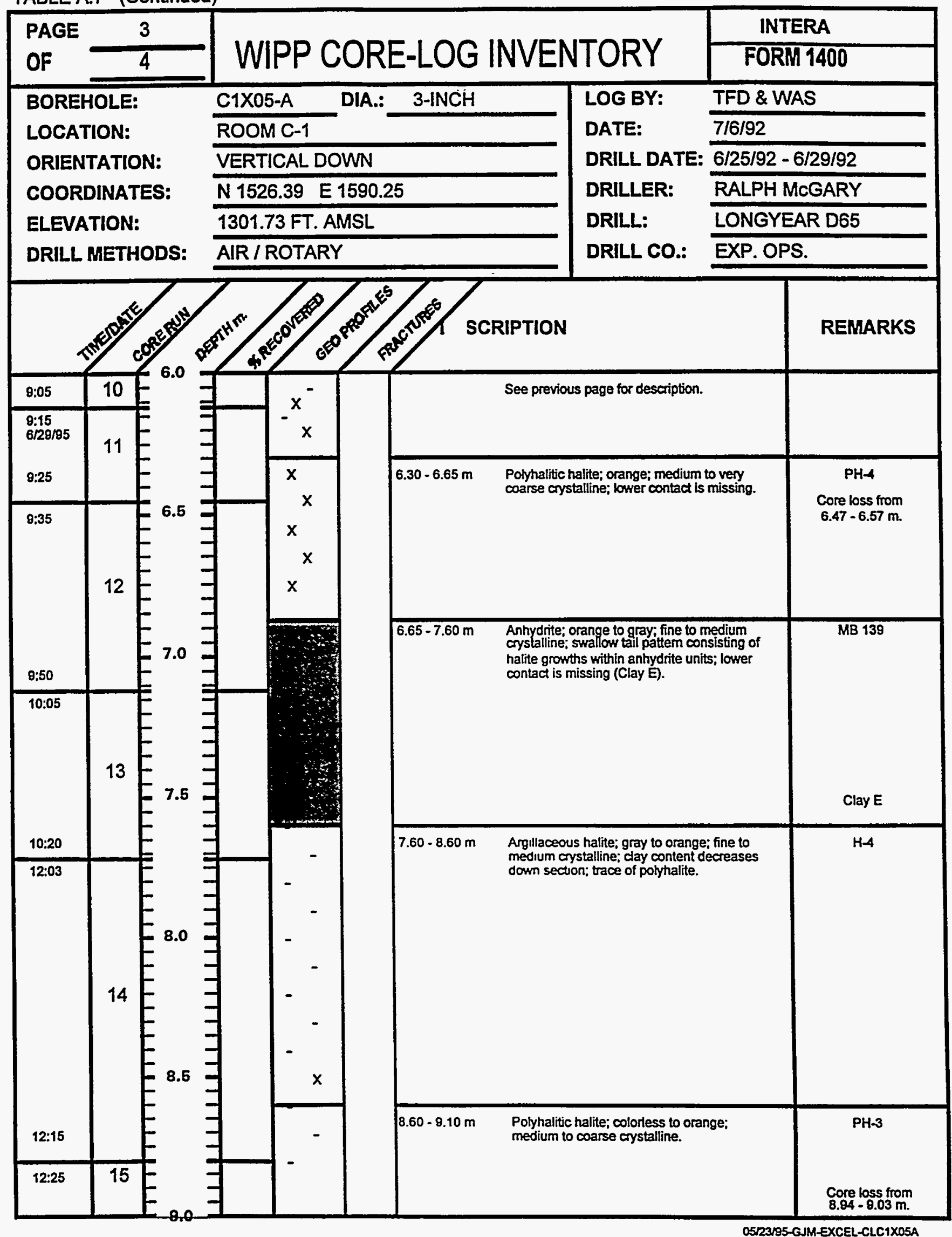


TABLE A.7 (Continued)

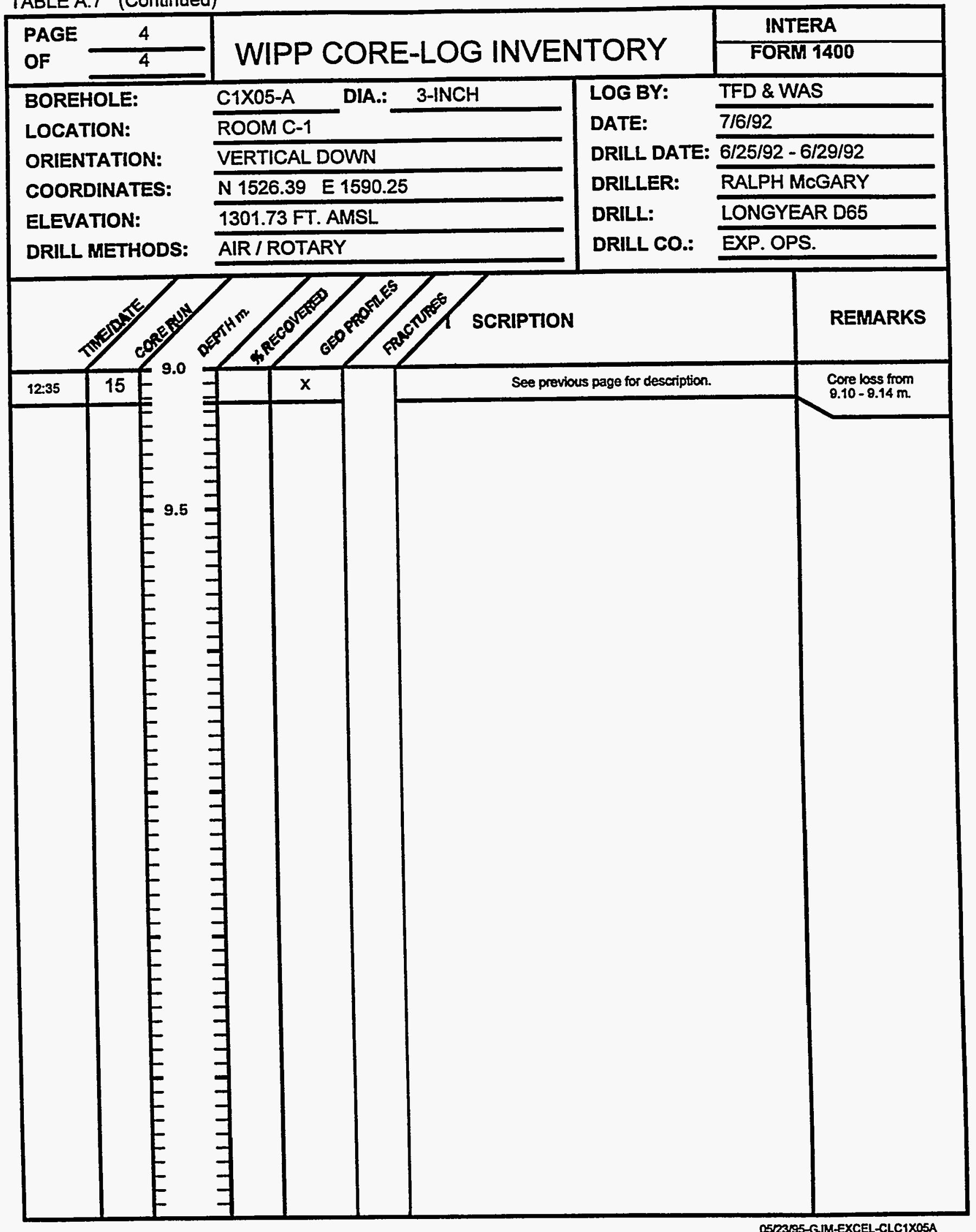


TABLE A.8

CORE DESCRIPTION OF BOREHOLE C1X05-B 
TABLE A.8

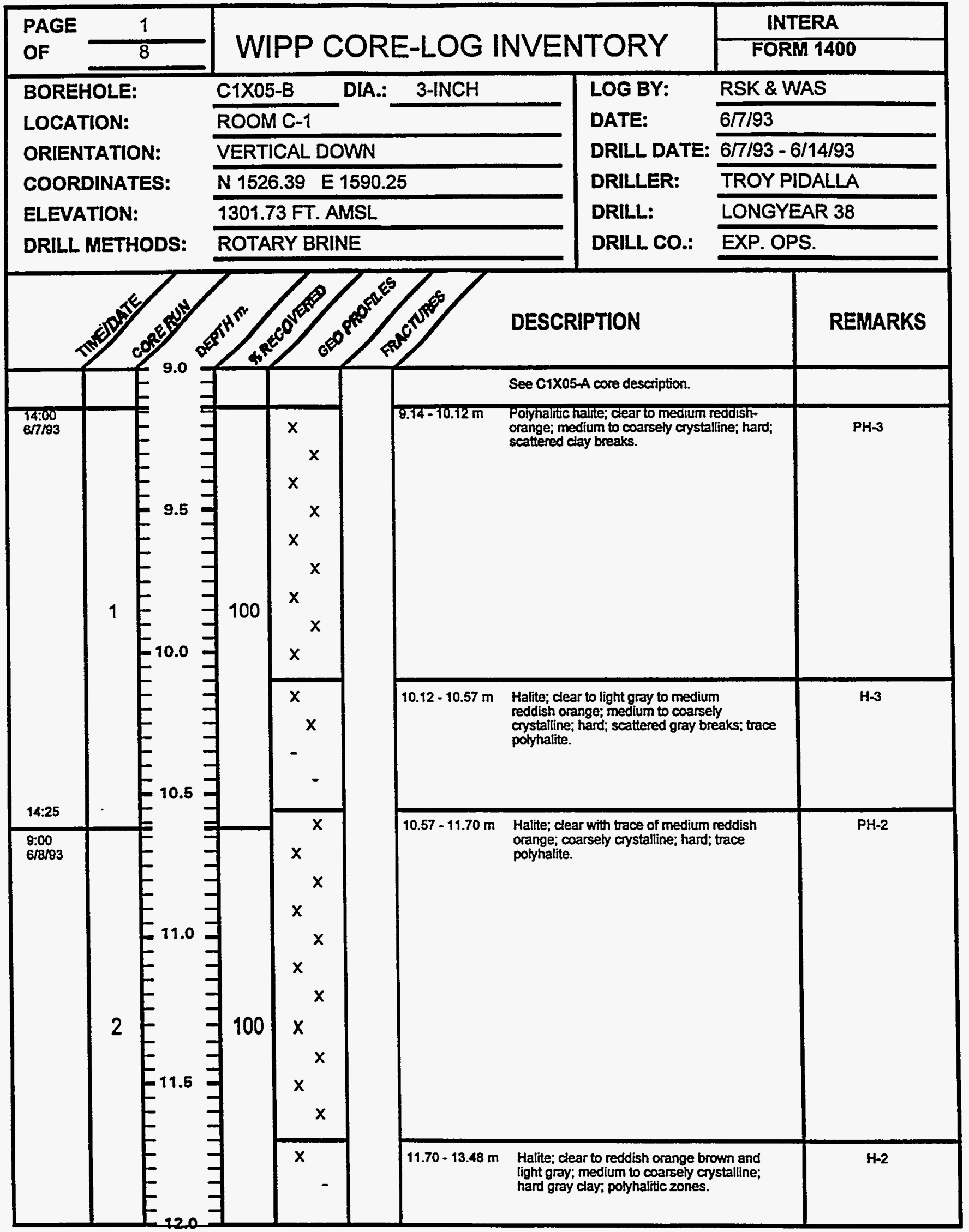


TABLE A.8 (Continued)

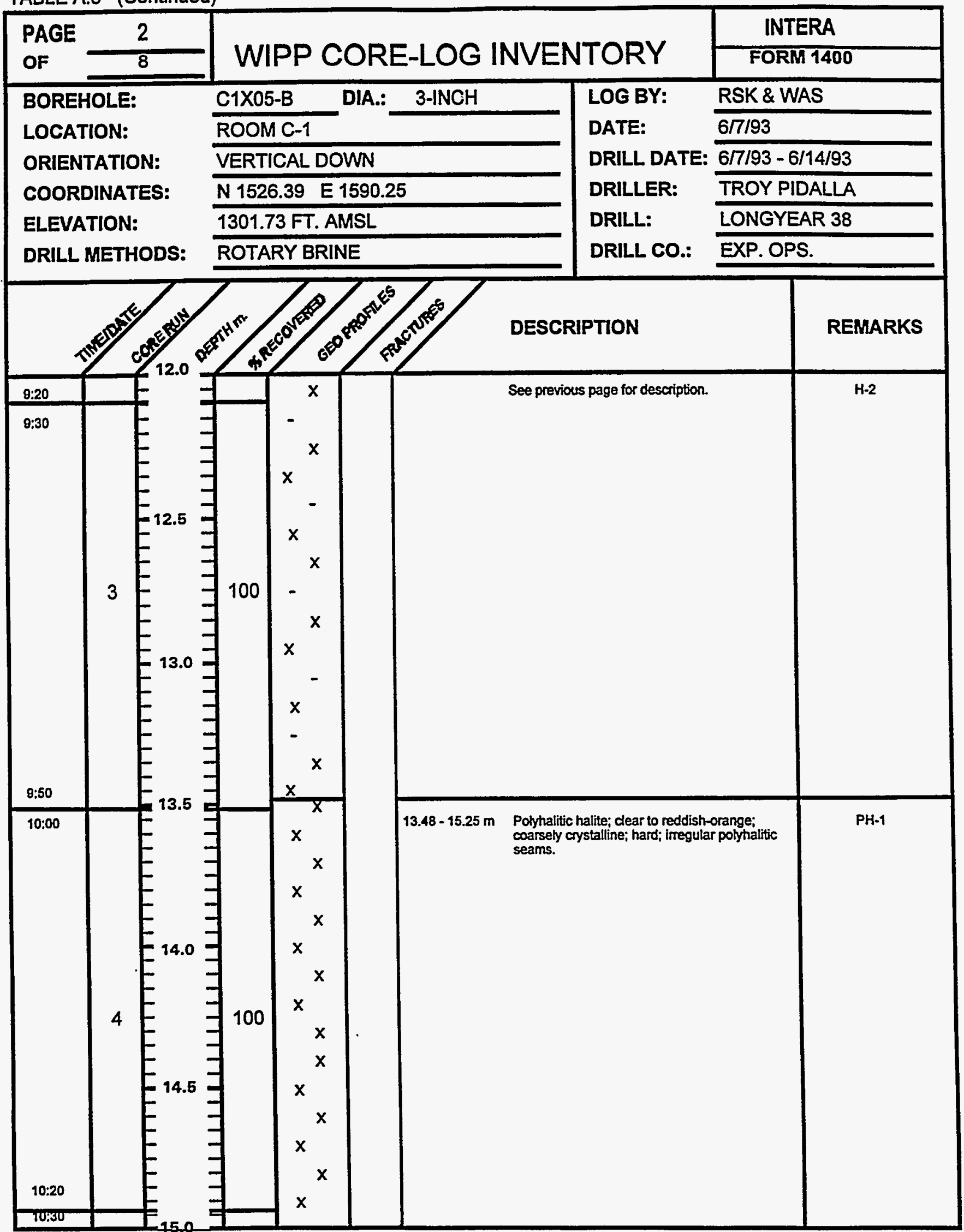


TABLE A.8 (Continued)

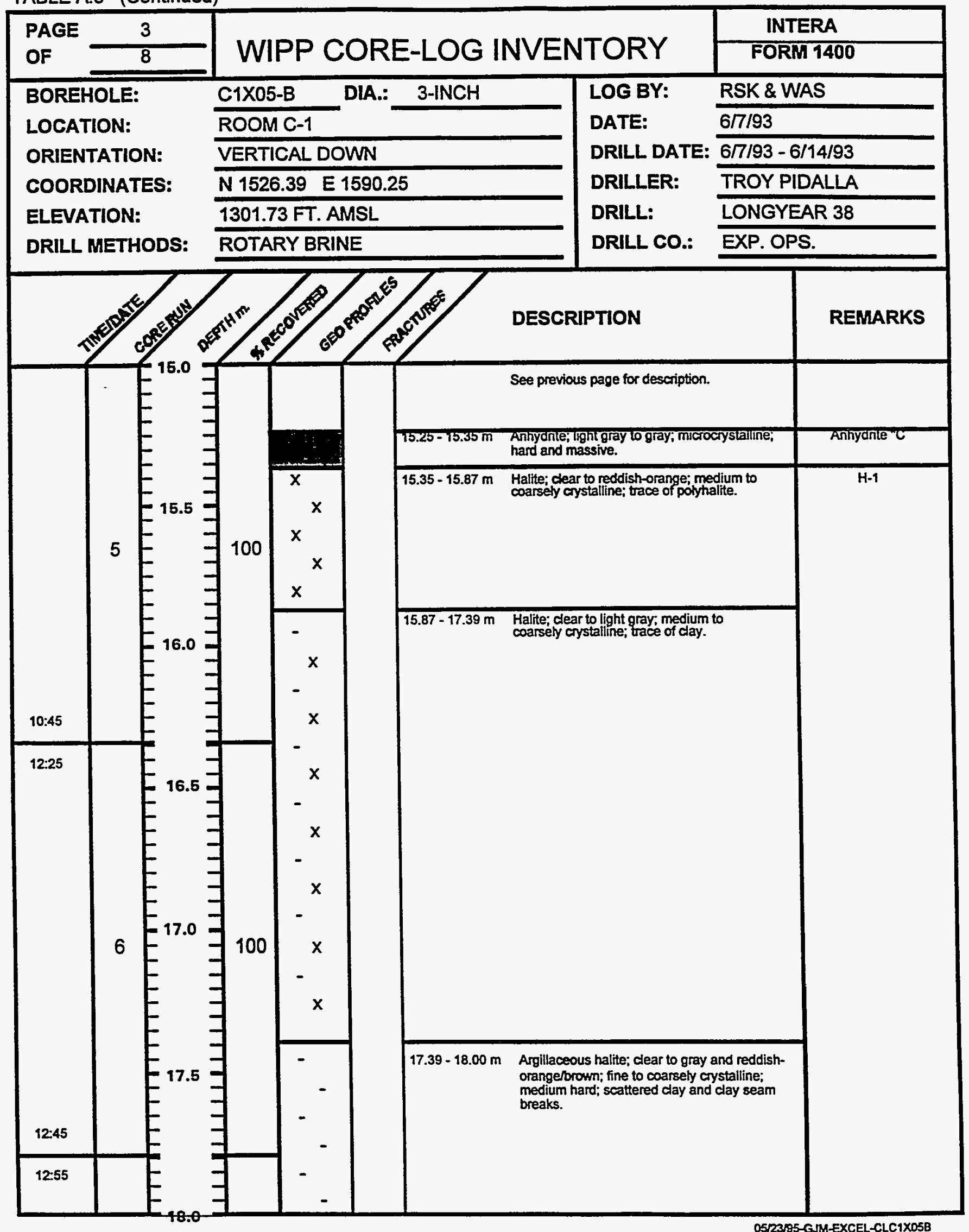


TABLE A.8 (Continued)

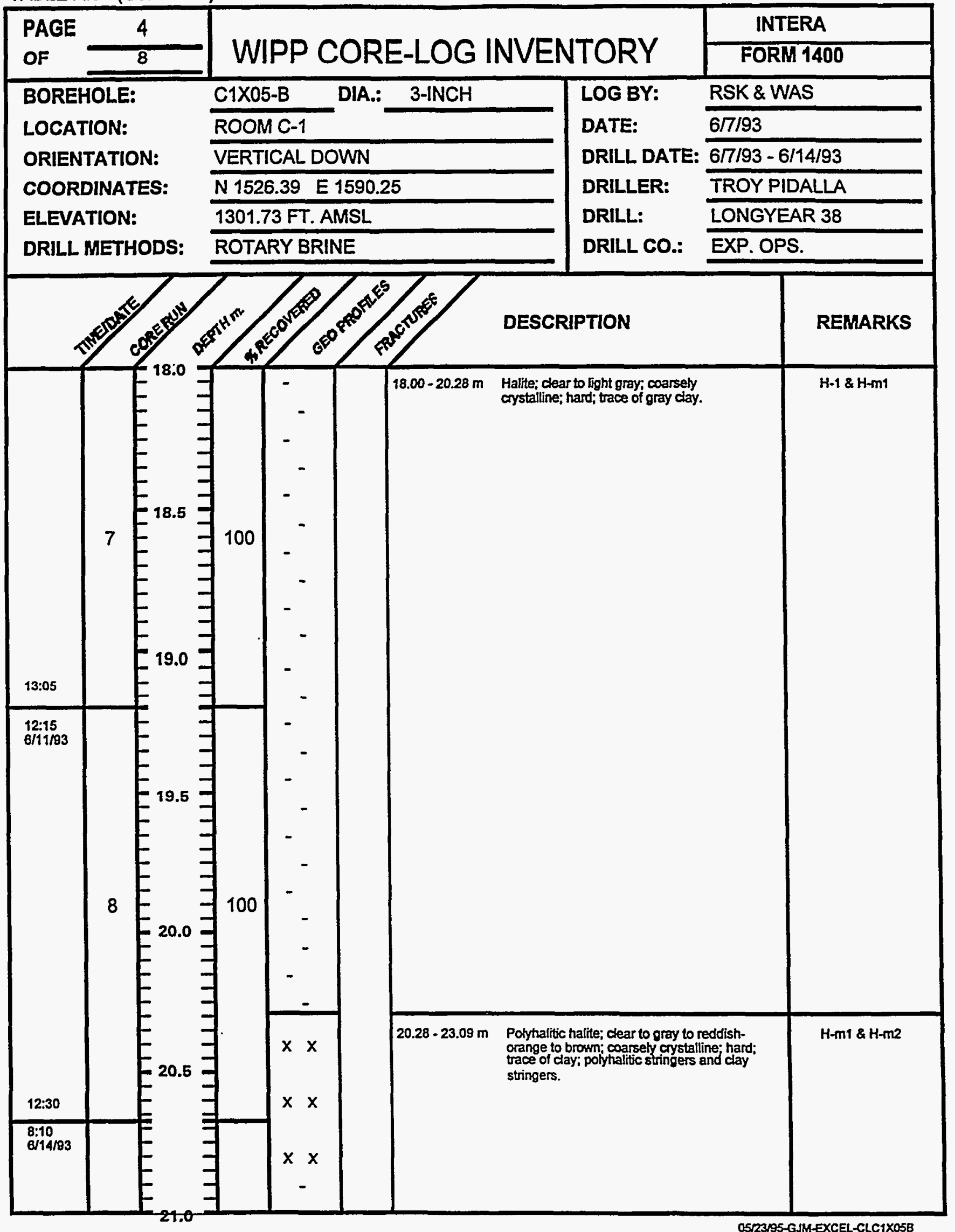


TABLE A.8 (Continued)

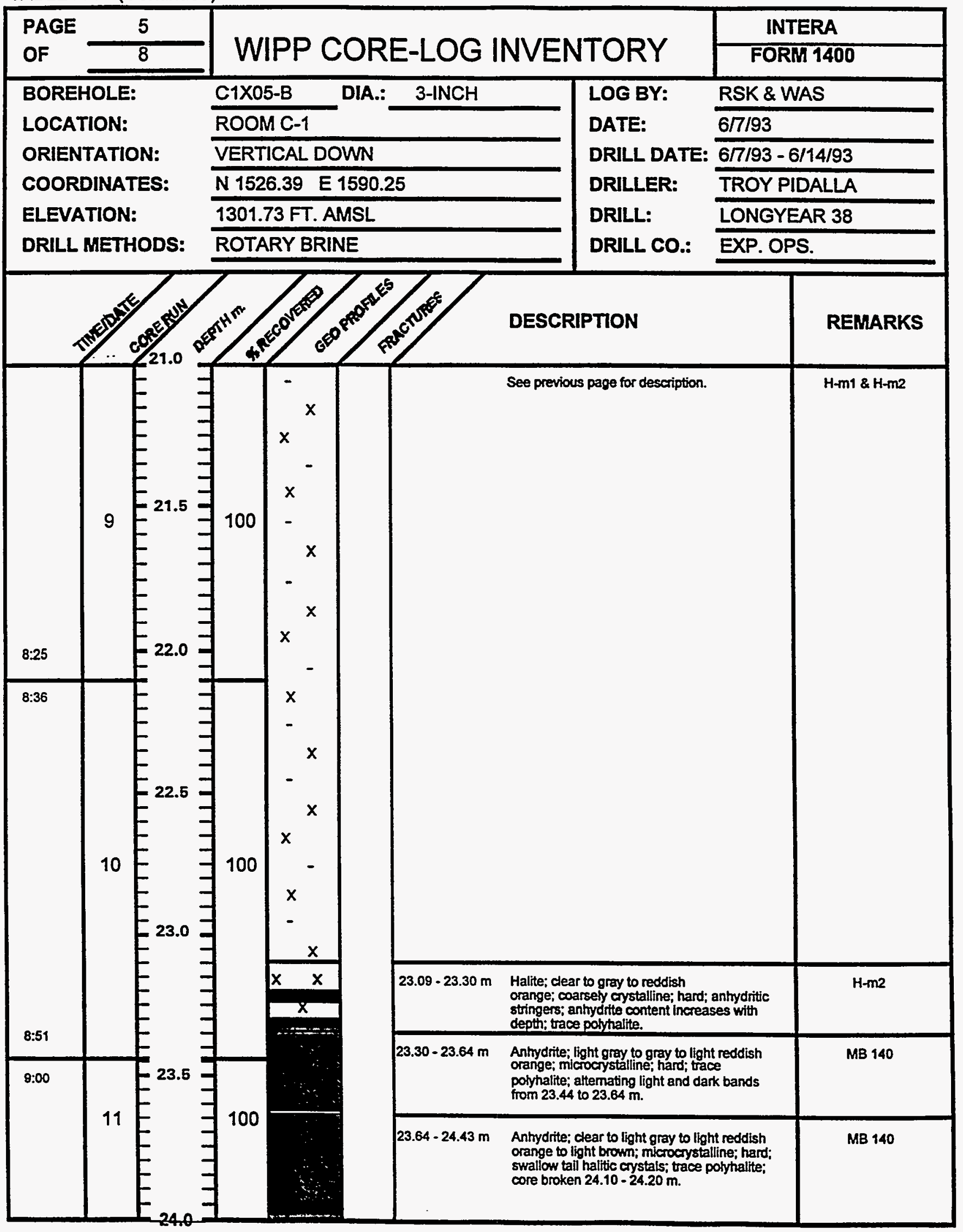


TABLE A.8 (Continued)

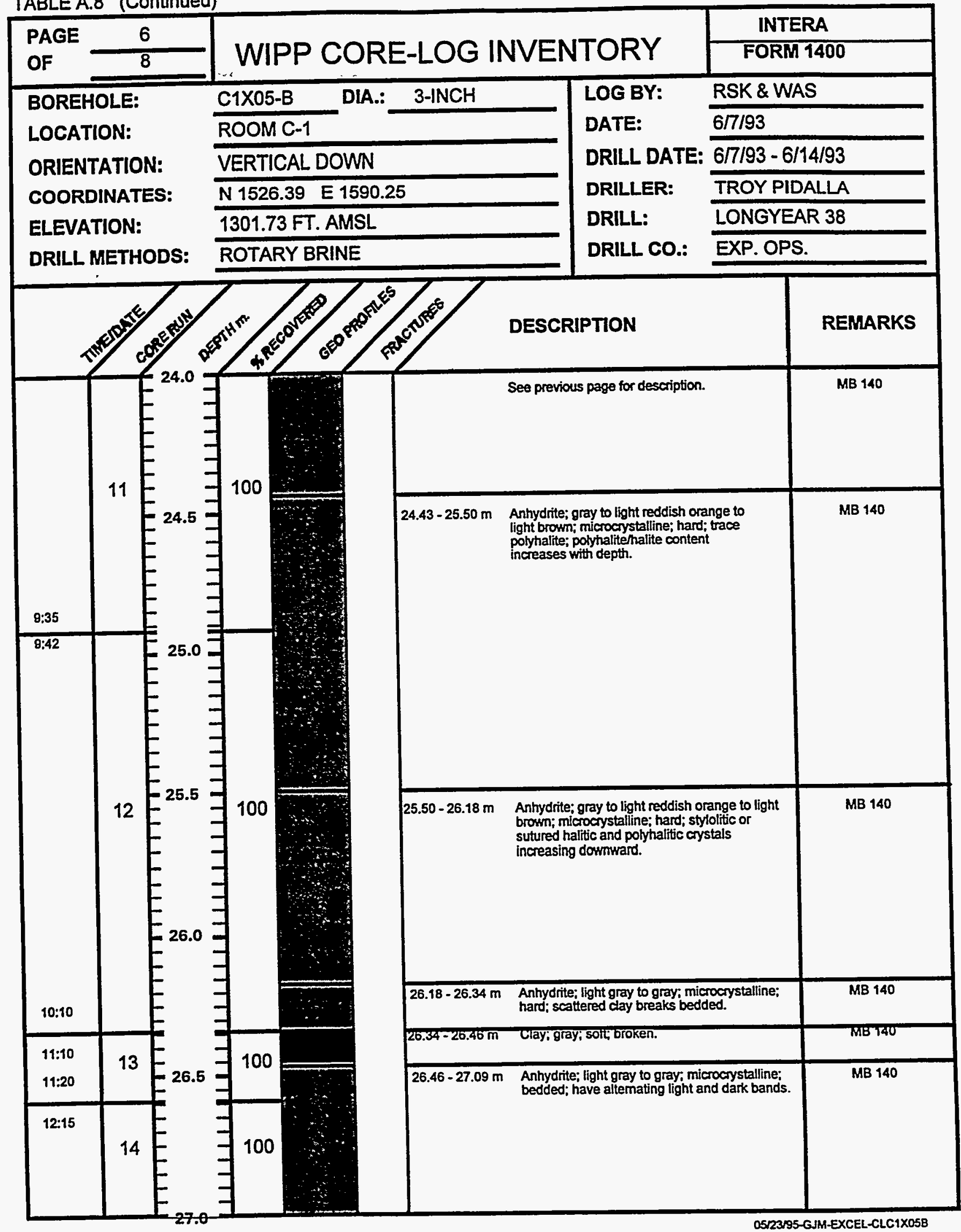


TABLE A.8 (Continued)

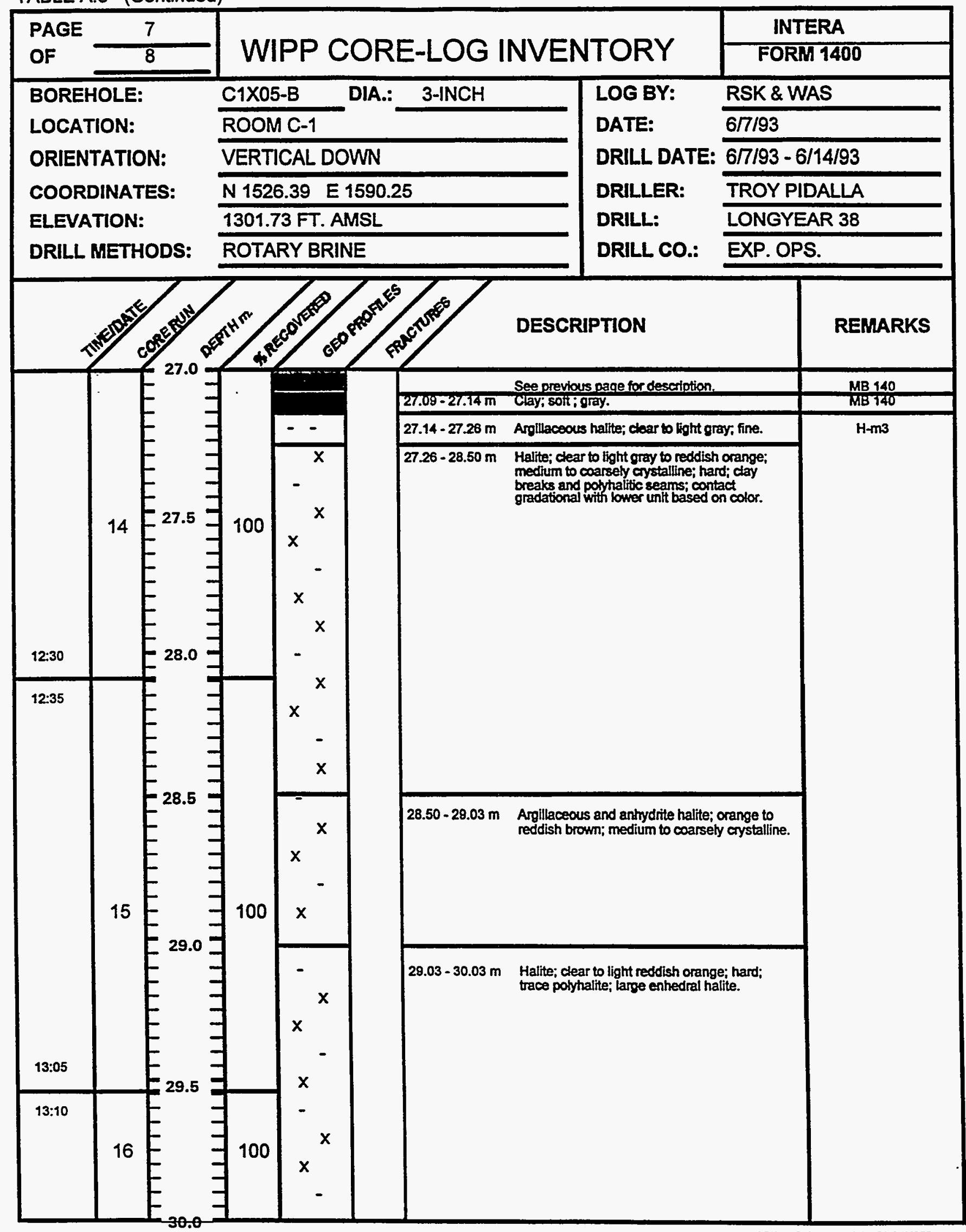


TABLE A.8 (Continued)

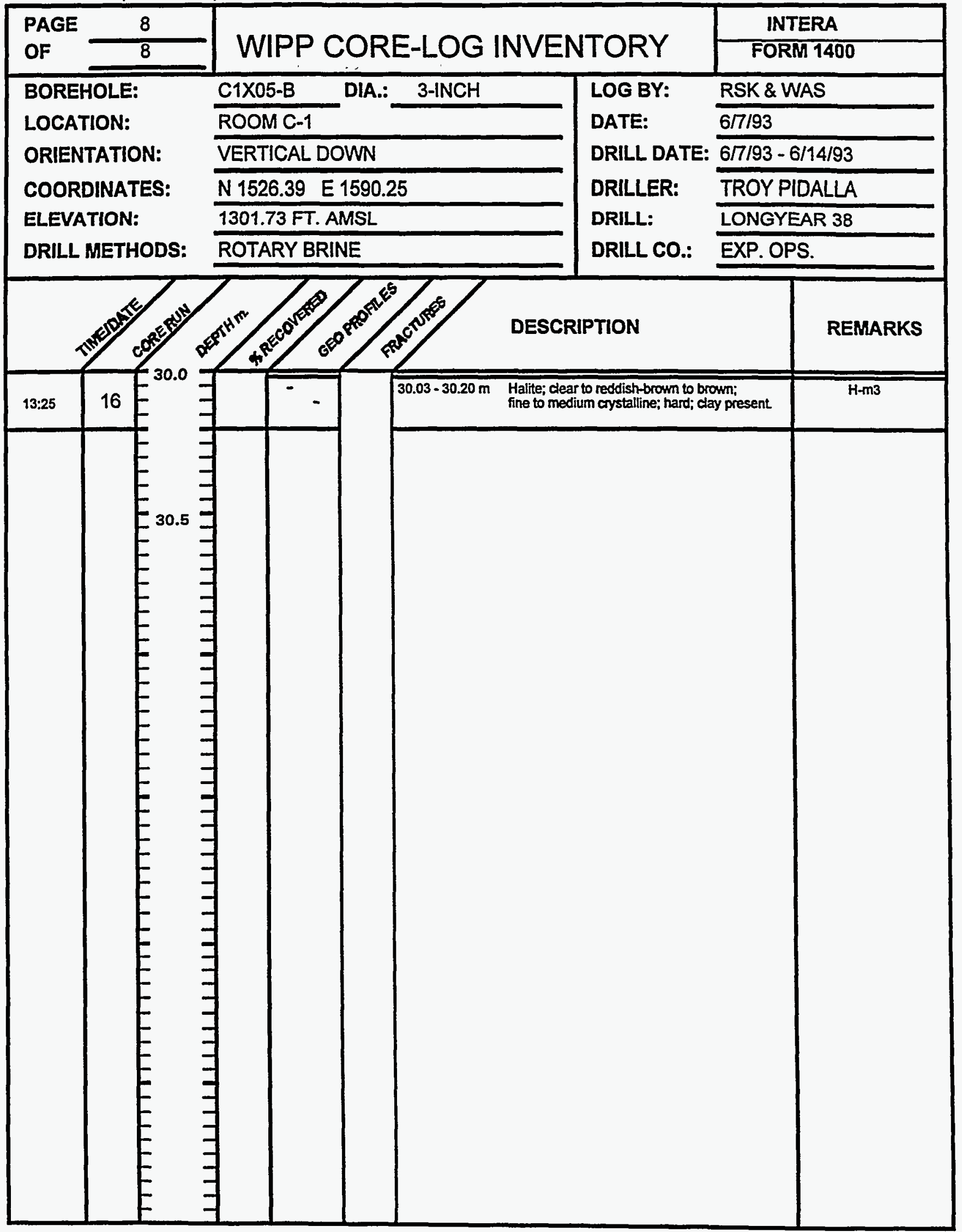


TABLE A.9

CORE DESCRIPTION OF BOREHOLE C1X06-A 
TABLE A. 9

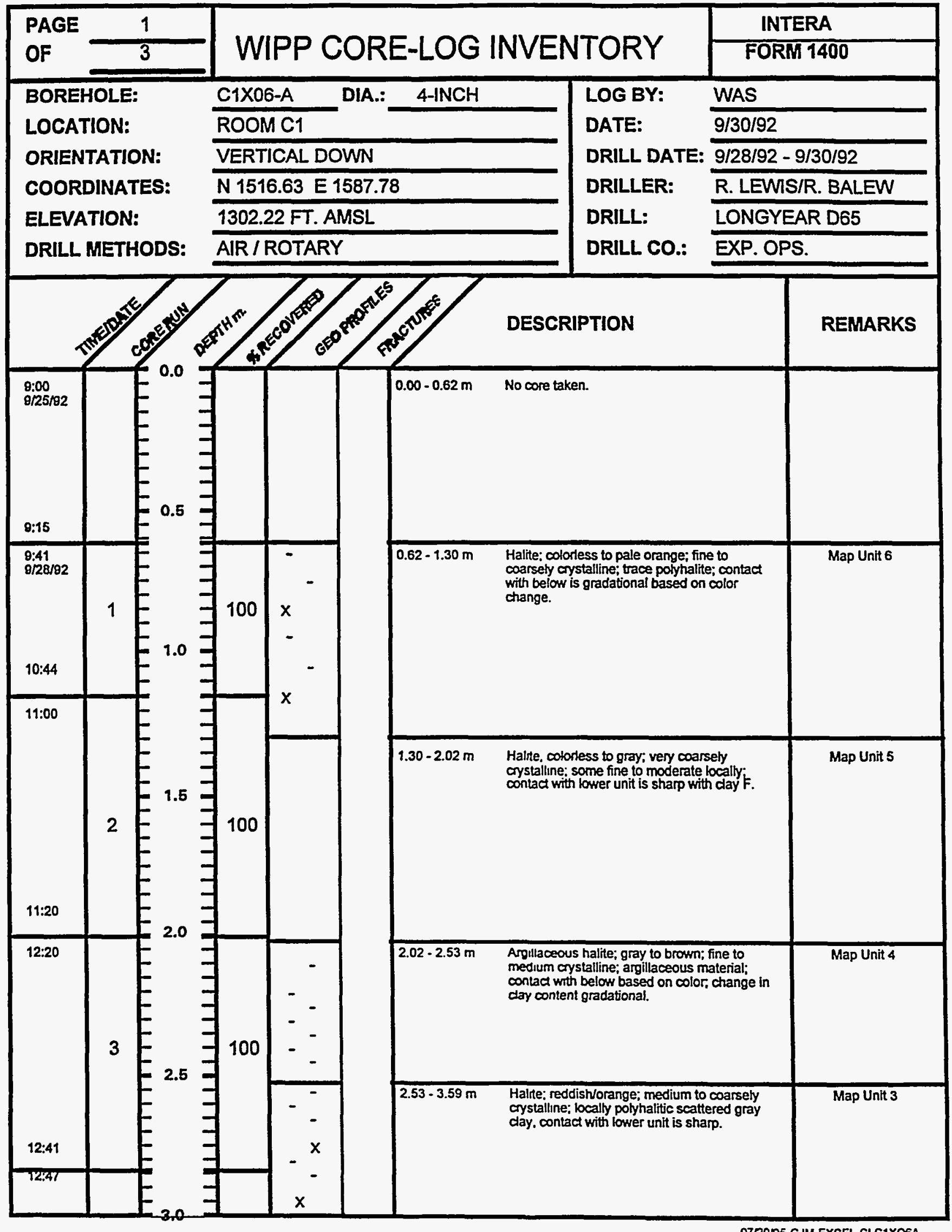


TABLE A.9 (Continued)

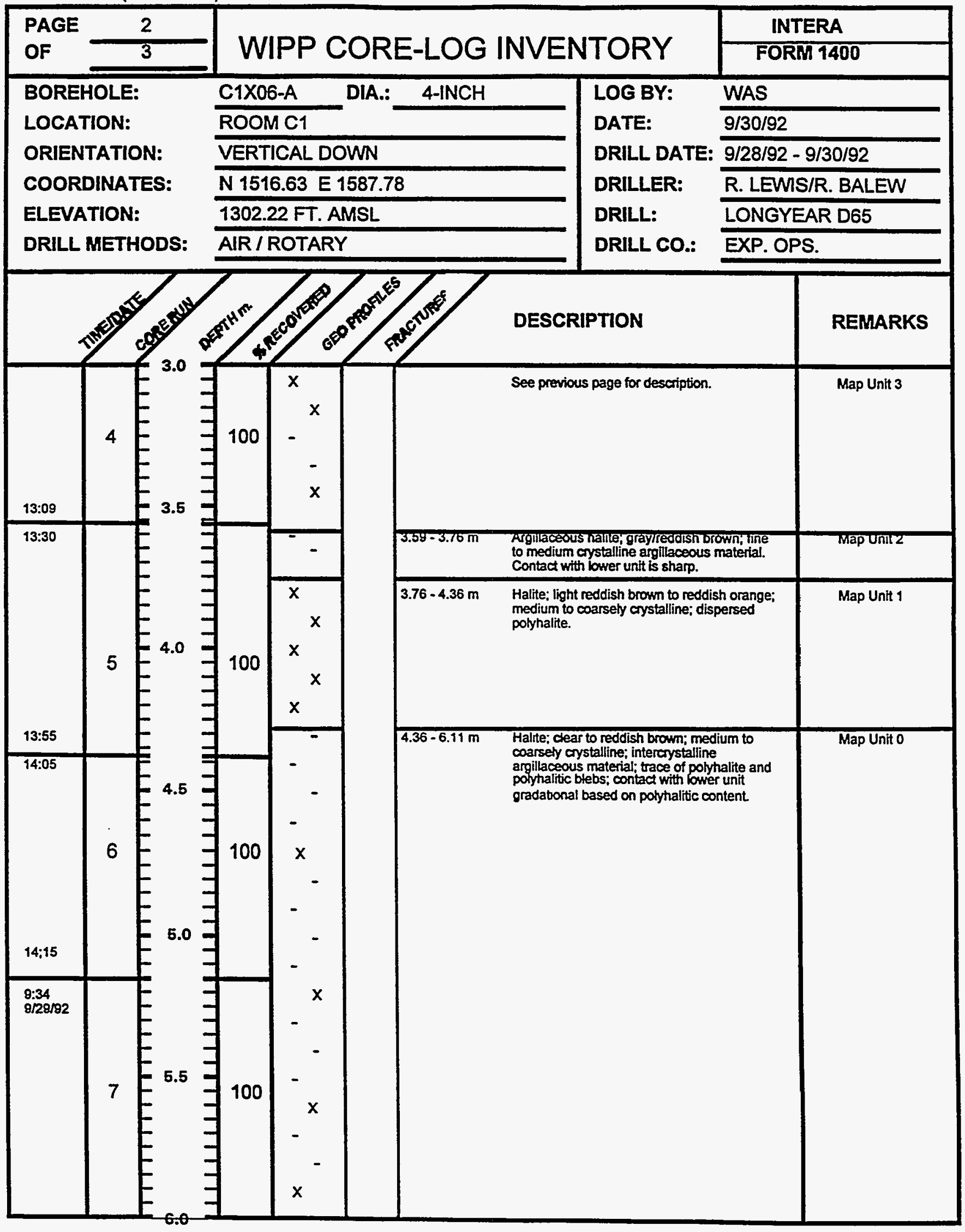


TABLE A.9 (Continued)

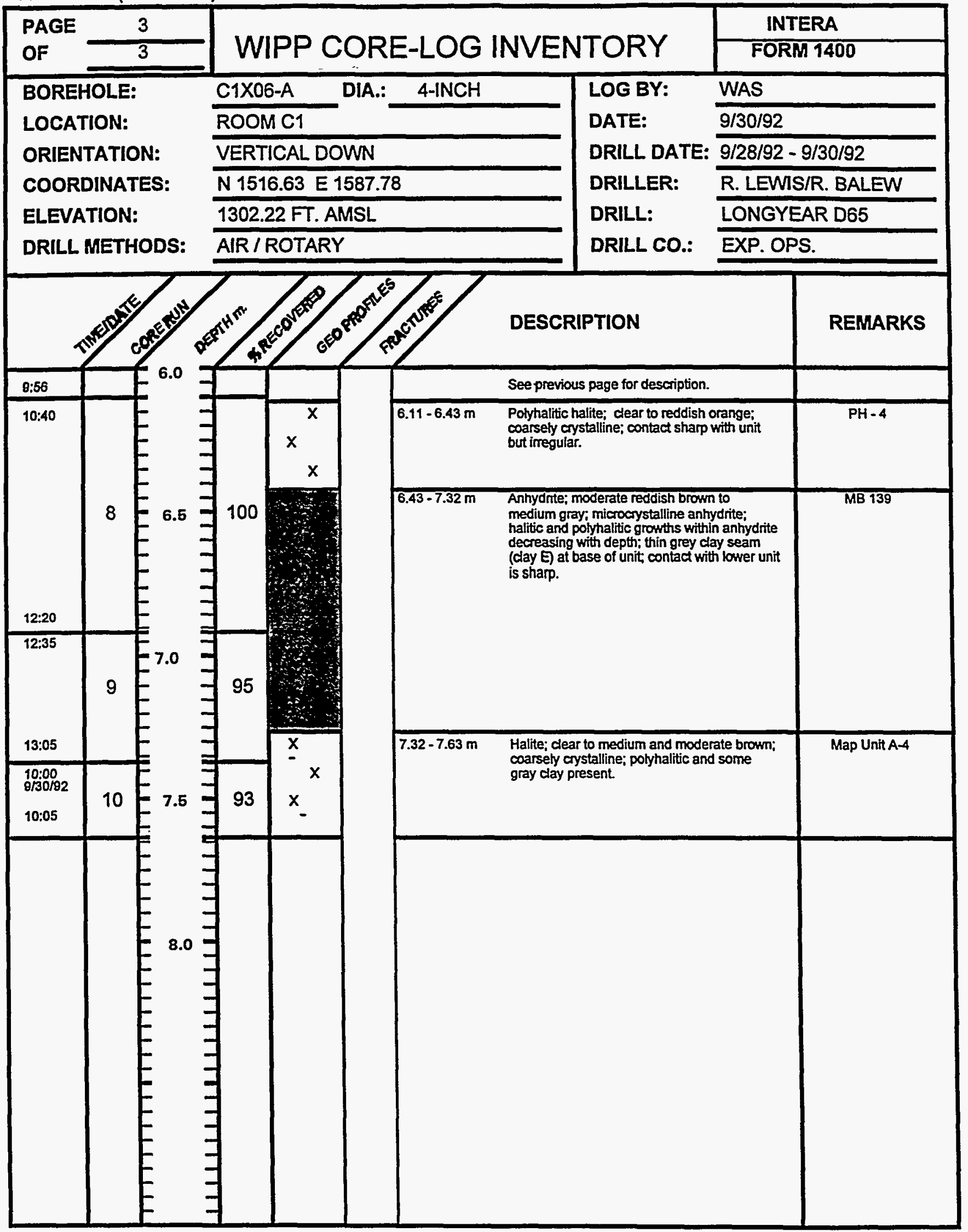


TABLE A. 10

CORE DESCRIPTION OF BOREHOLE C1X06-B 
TABLE A.10

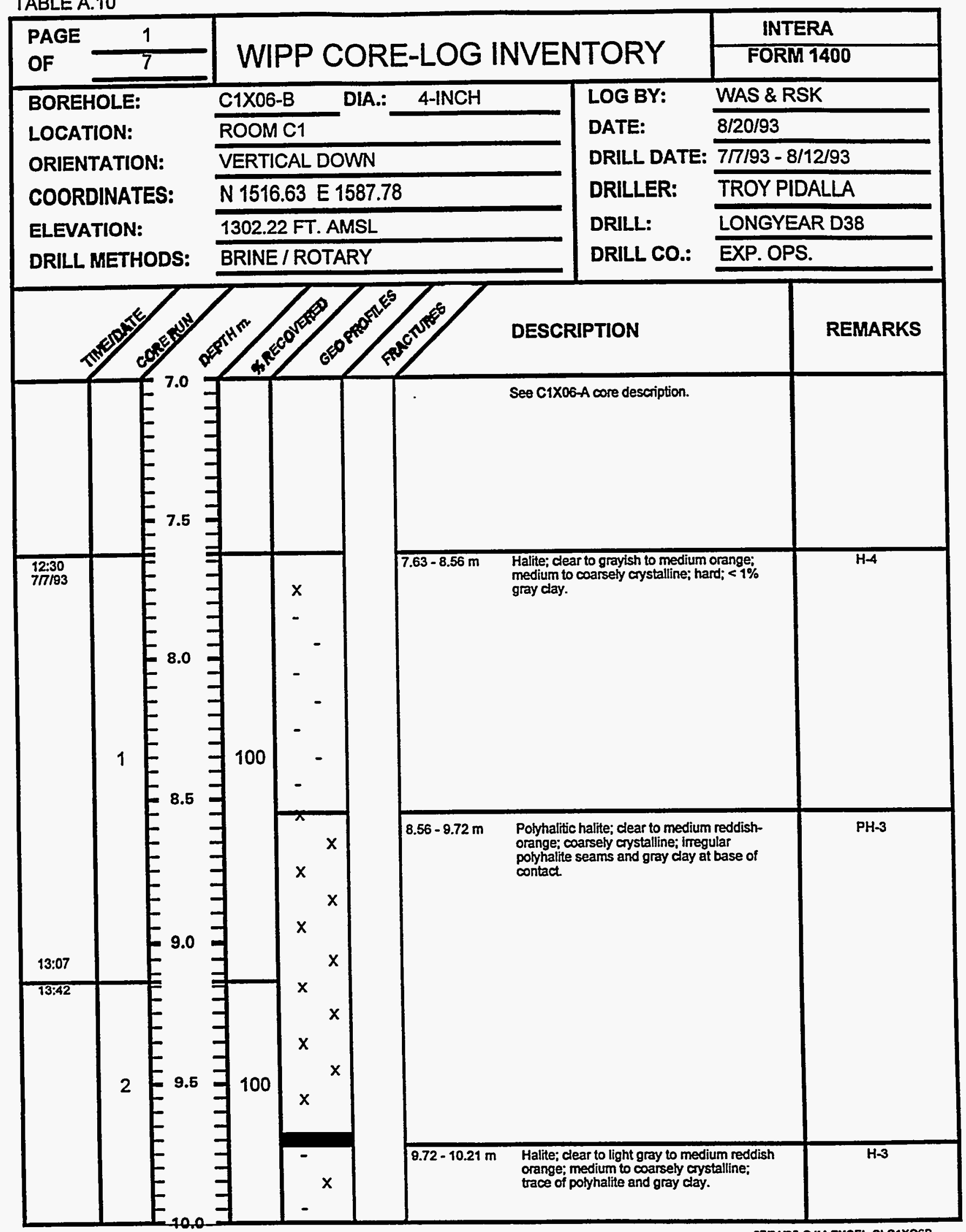


TABLE A.10 (Continued)

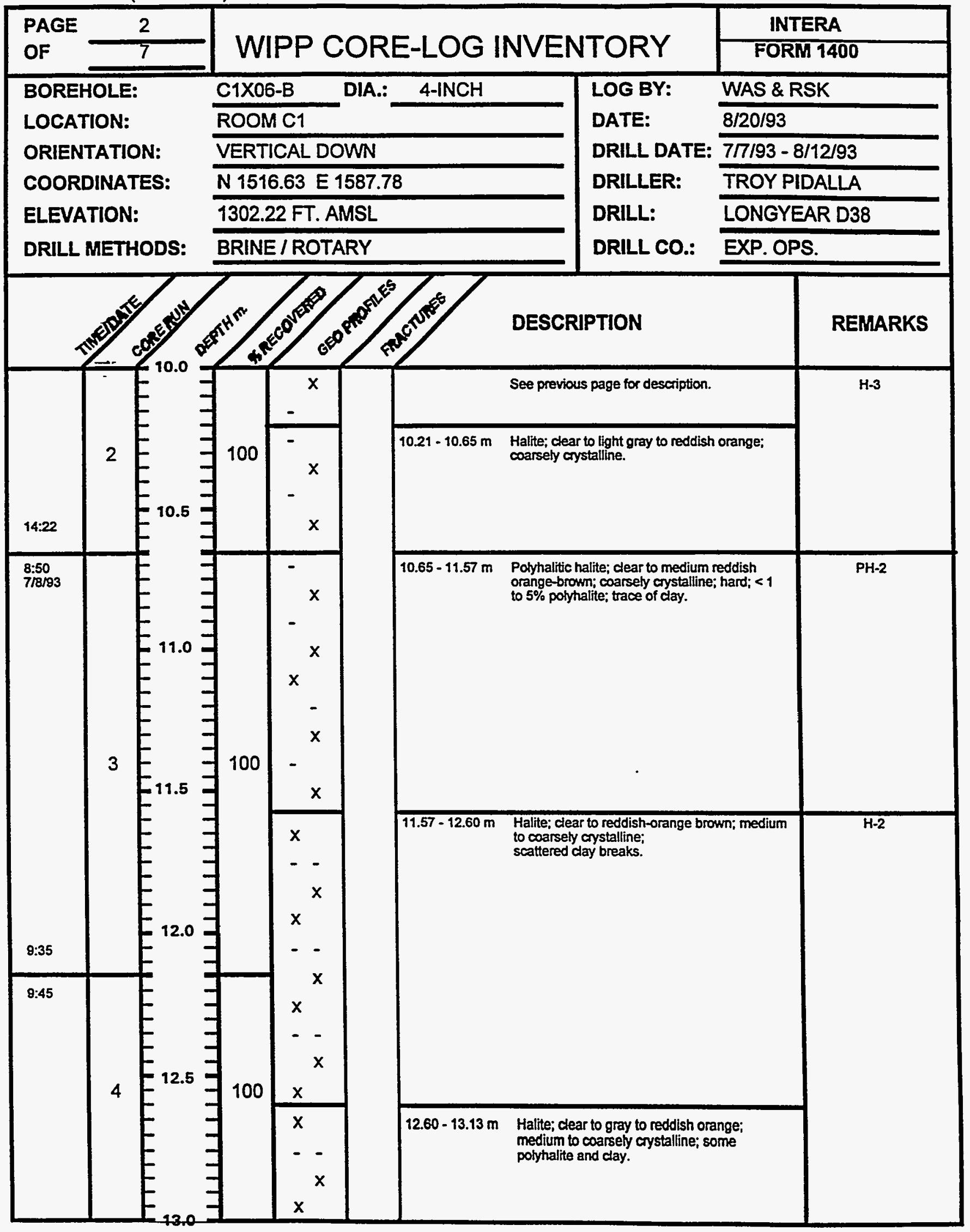


TABLE A.10 (Continued)

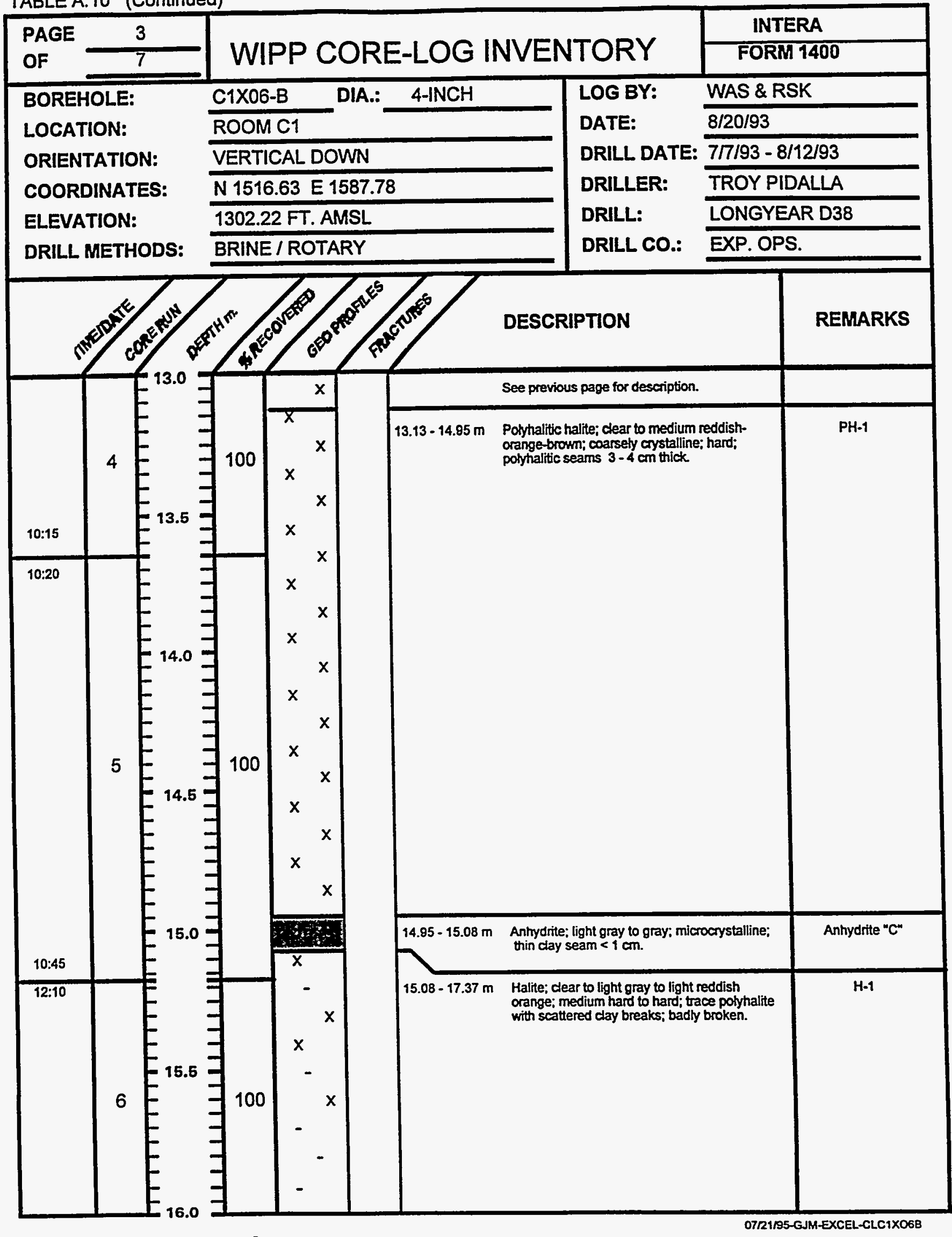


TABLE A.10 (Continued)

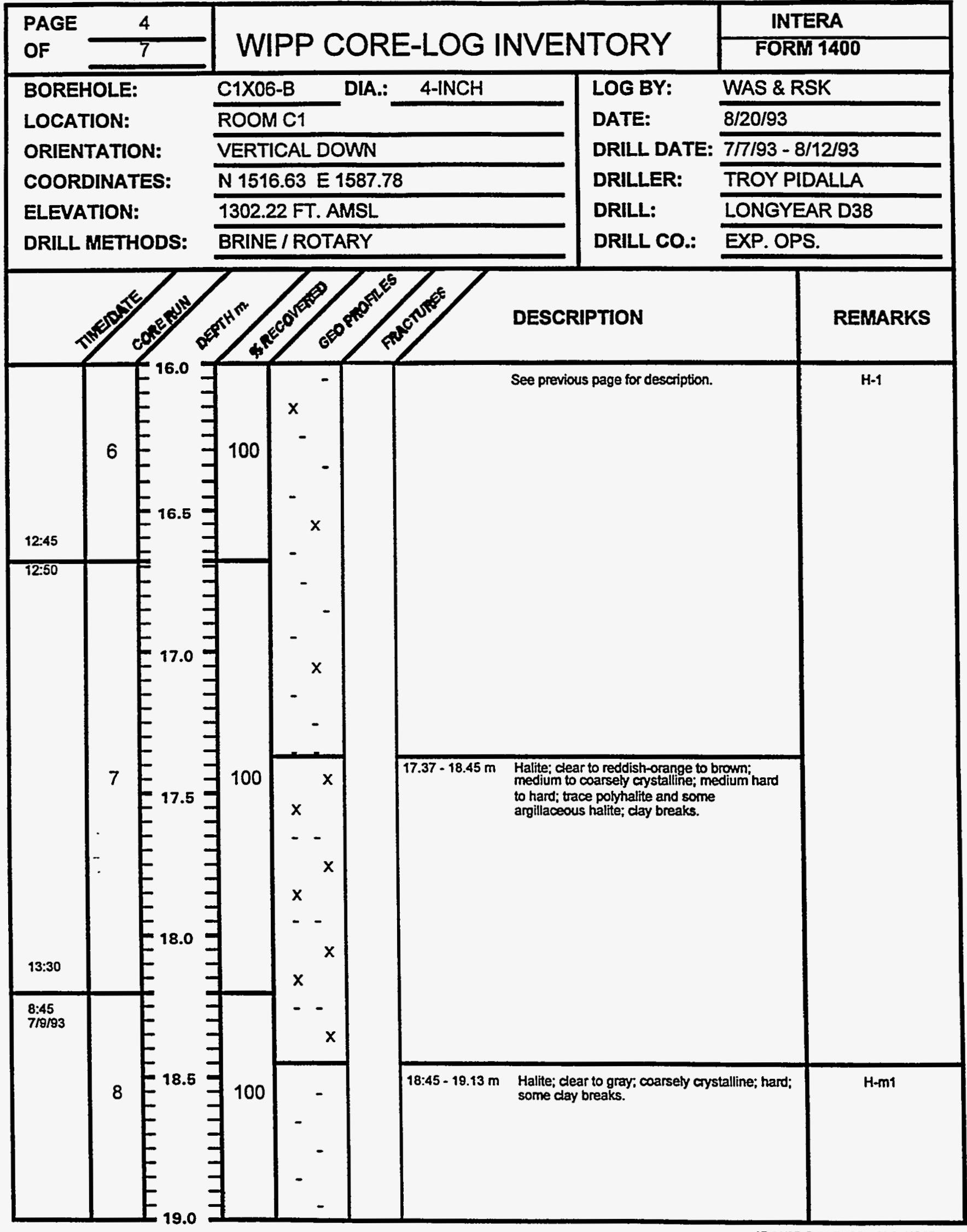

07/21/95-GJM-EXCEL_CLC1X06B 
TABLEA.10 (Continued)

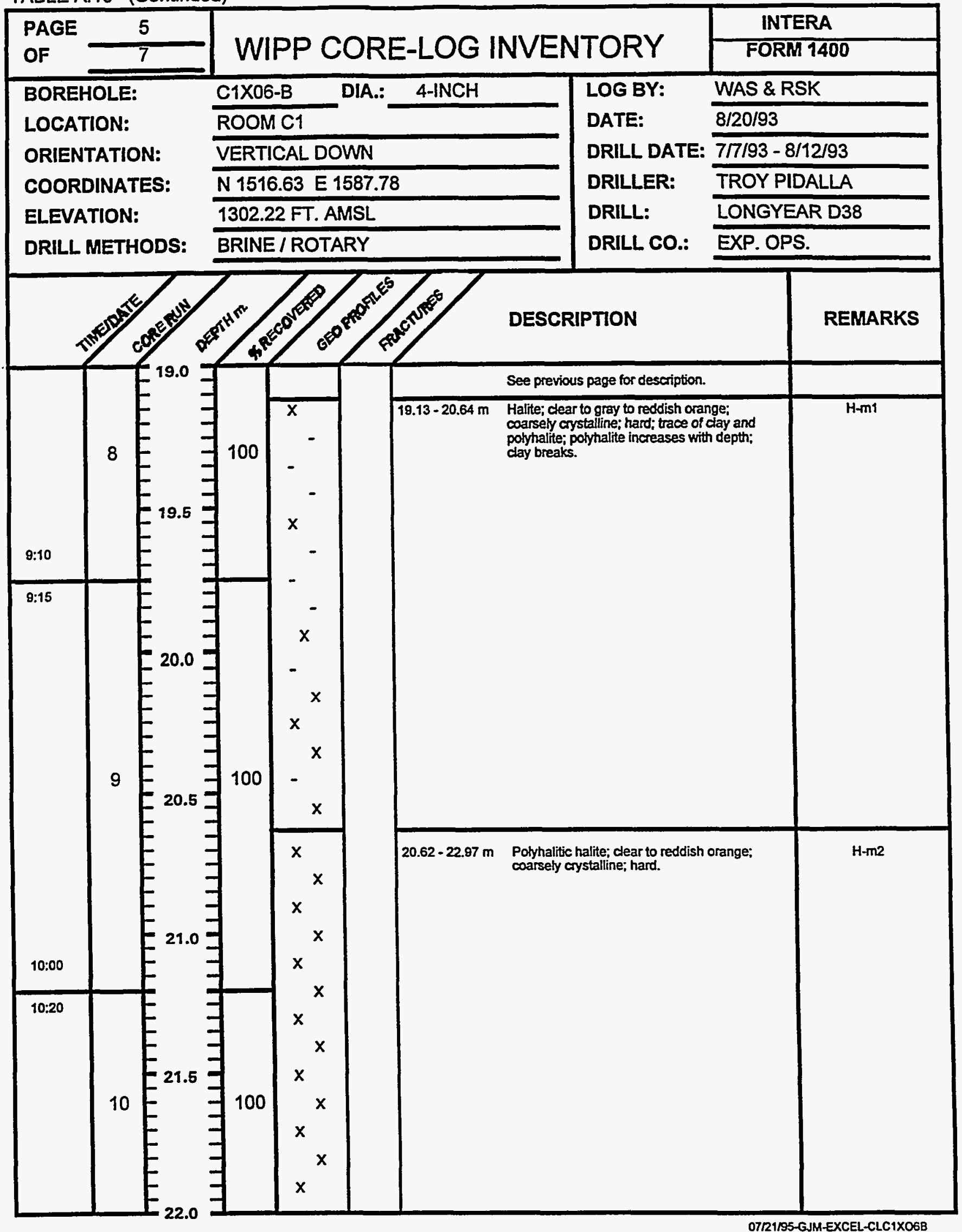


TABLE A.10 (Continued)

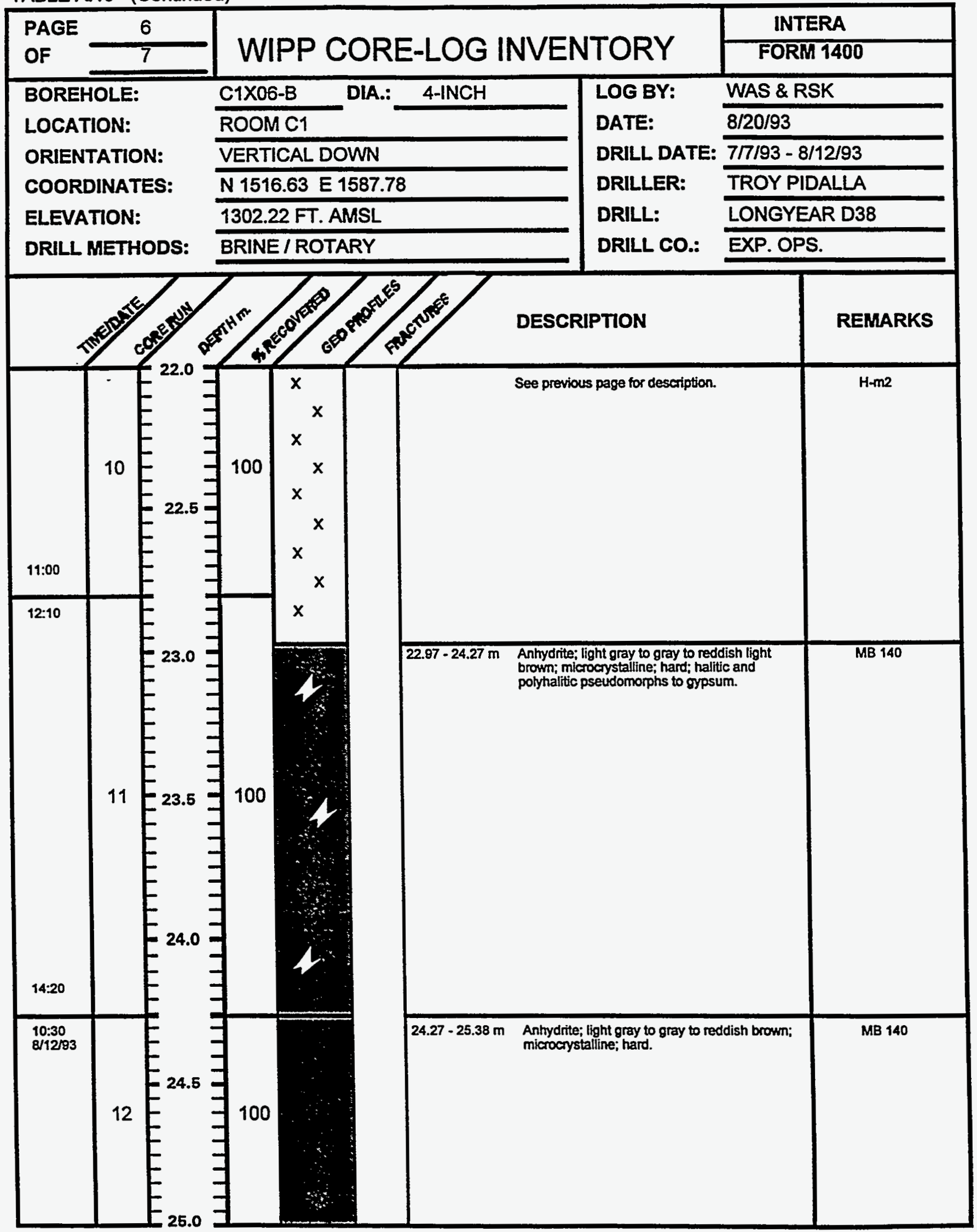


TABLE A.10 (Continued)

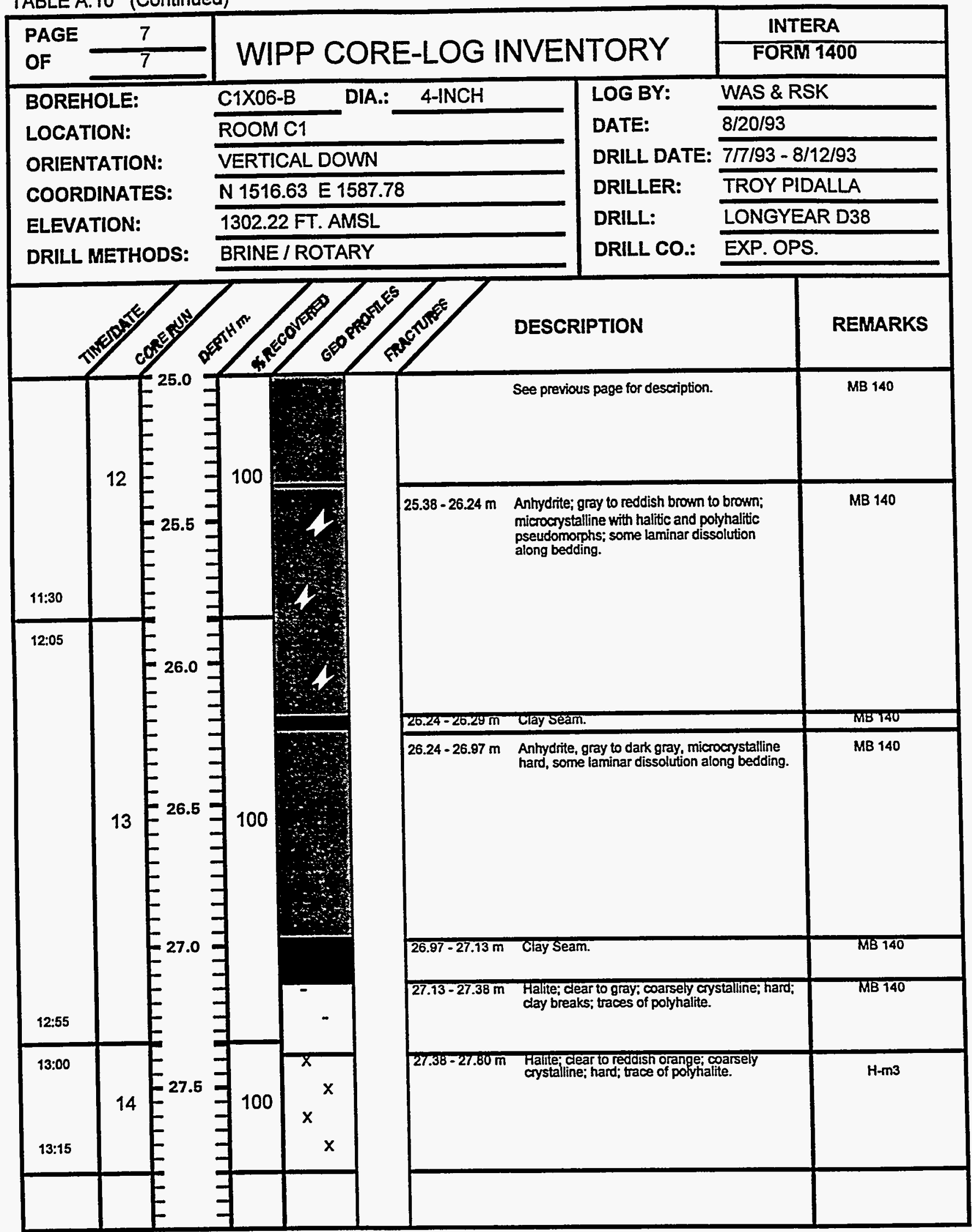


TABLE A.11

CORE DESCRIPTION OF BOREHOLE C1X10

A - 54 
TABLE A.11

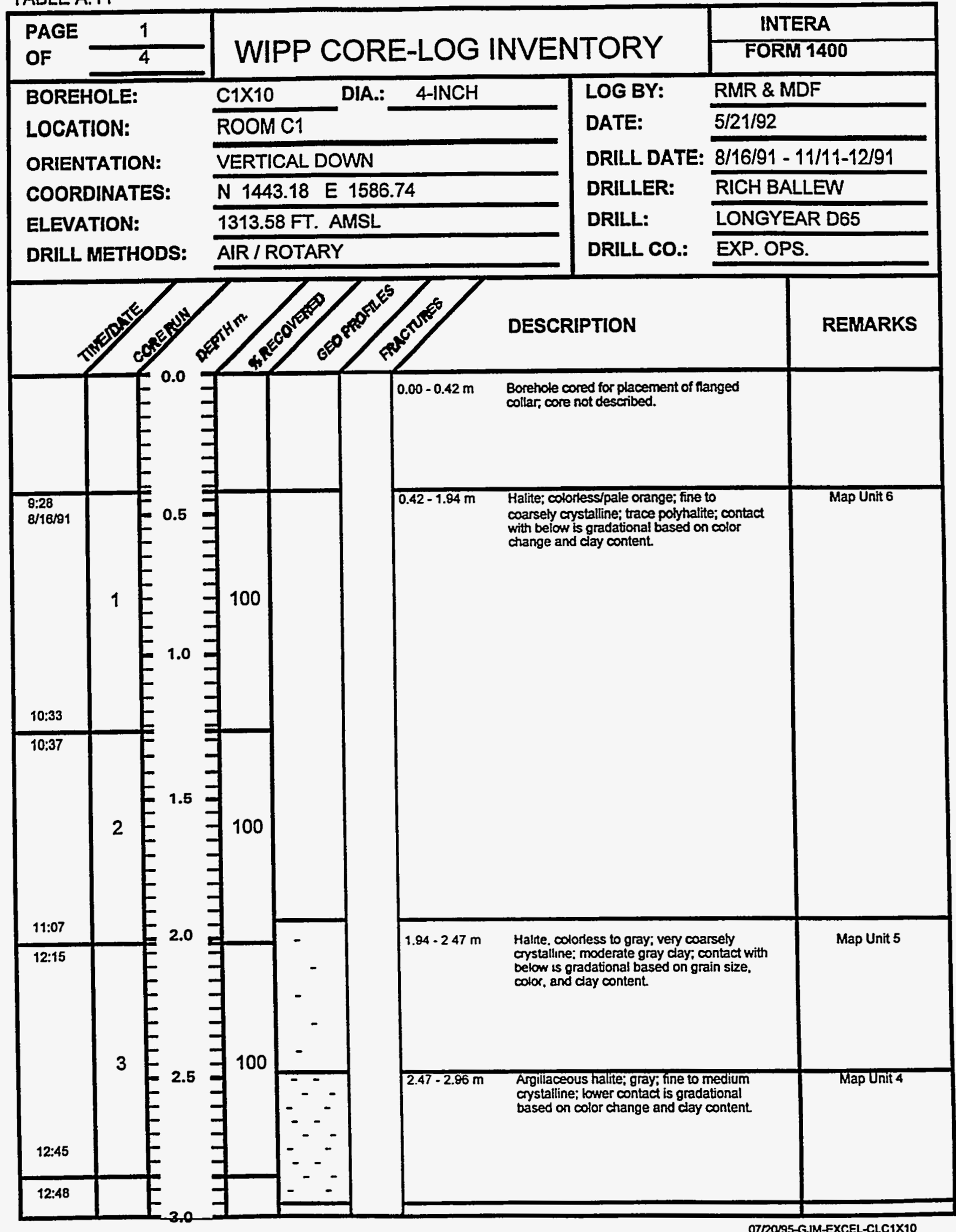


TABLE A.11 (Continued)

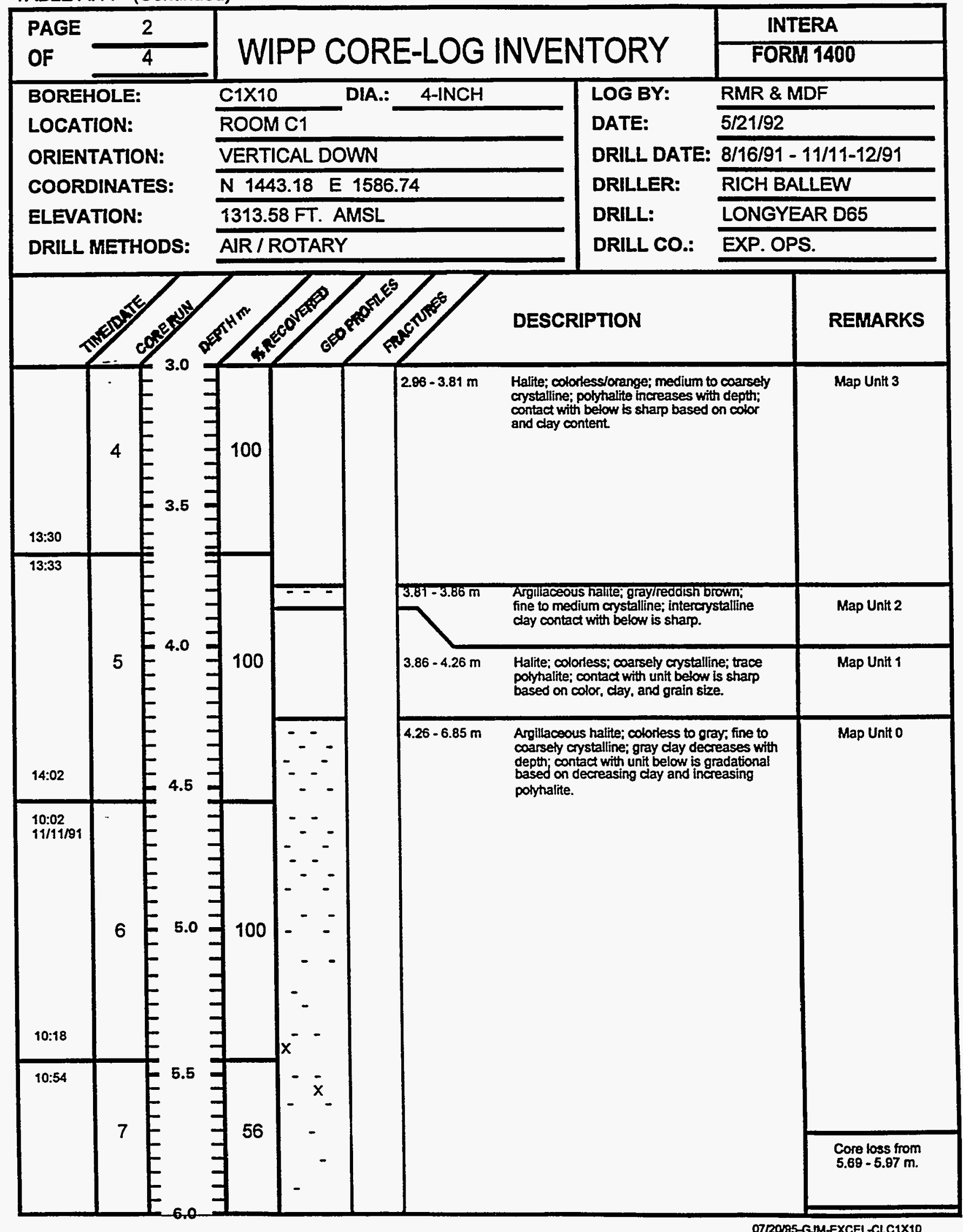


TABLE A.11 (Continued)

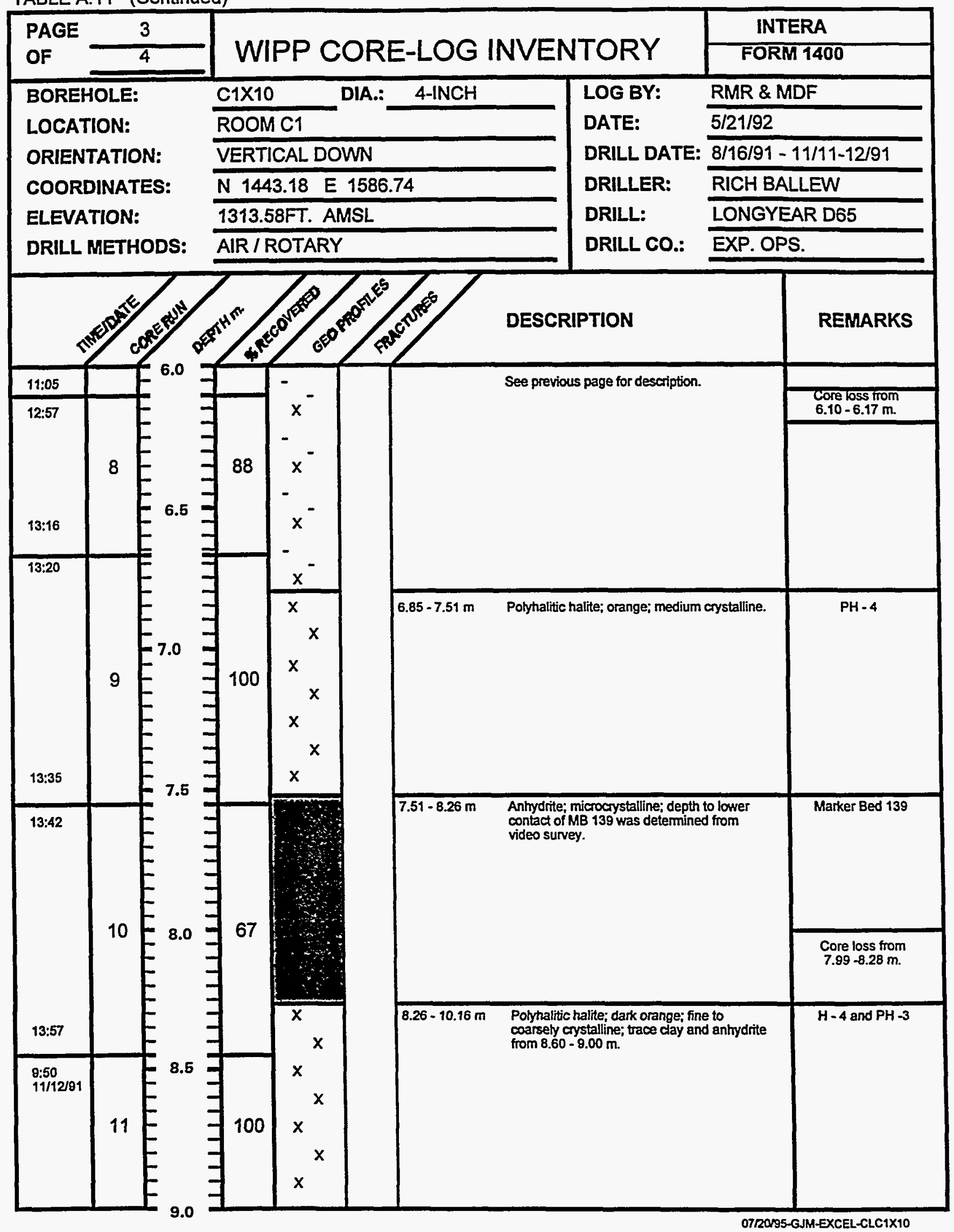


TABLE A.11 (Continued)

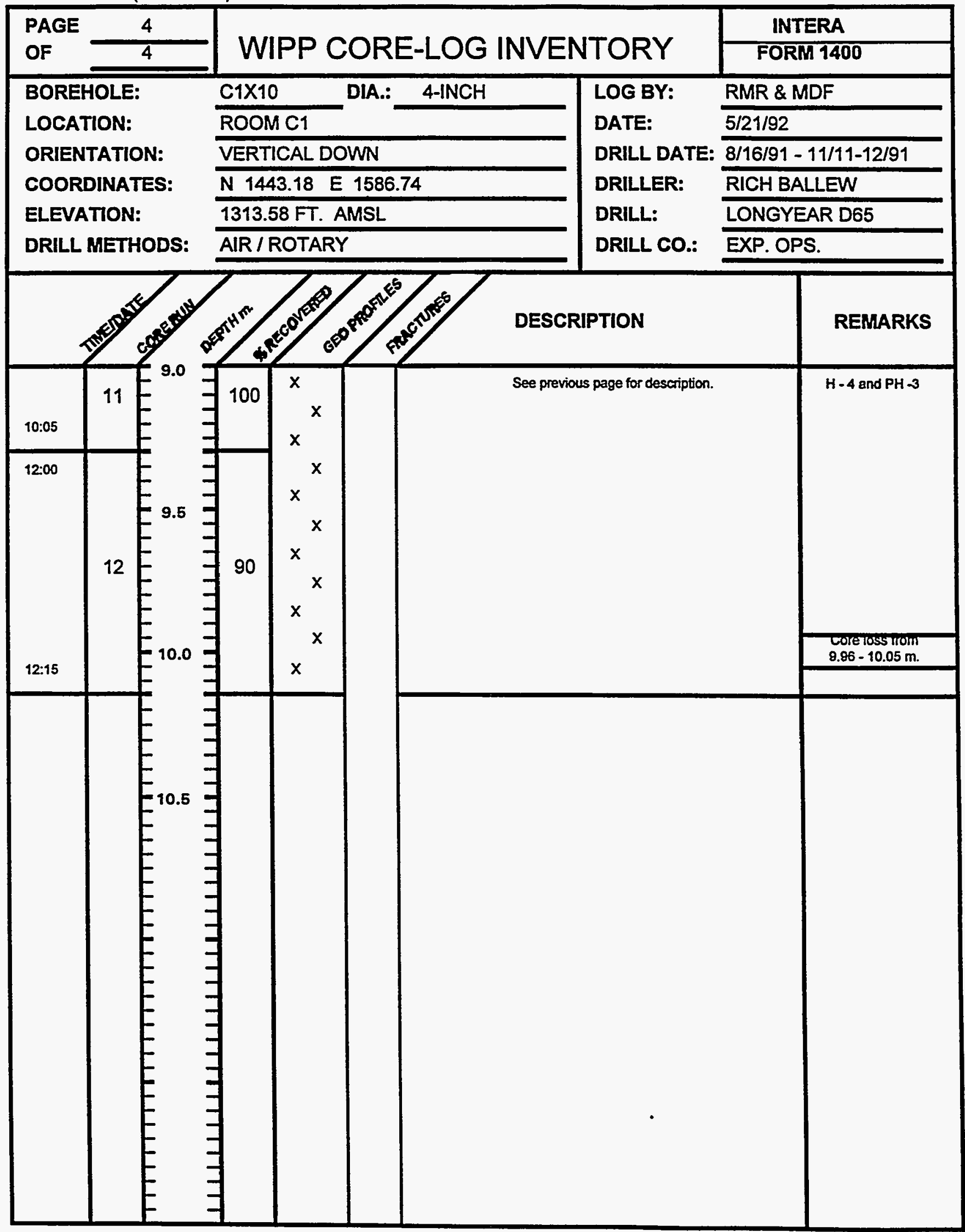


TABLE A.12

CORE DESCRIPTION OF BOREHOLE L4P51-C 
TABLE A.12

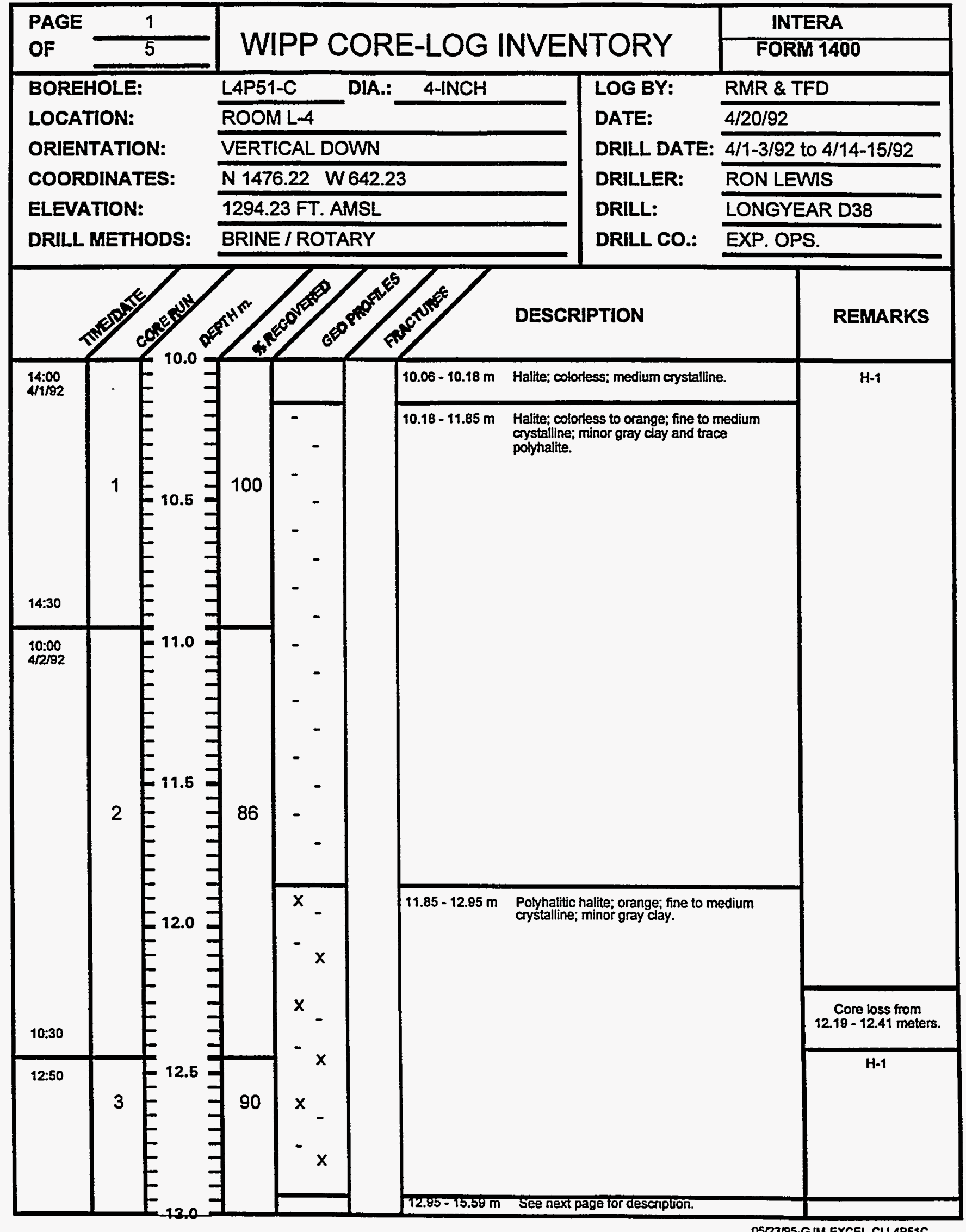


TABLE A.12 (Continued)

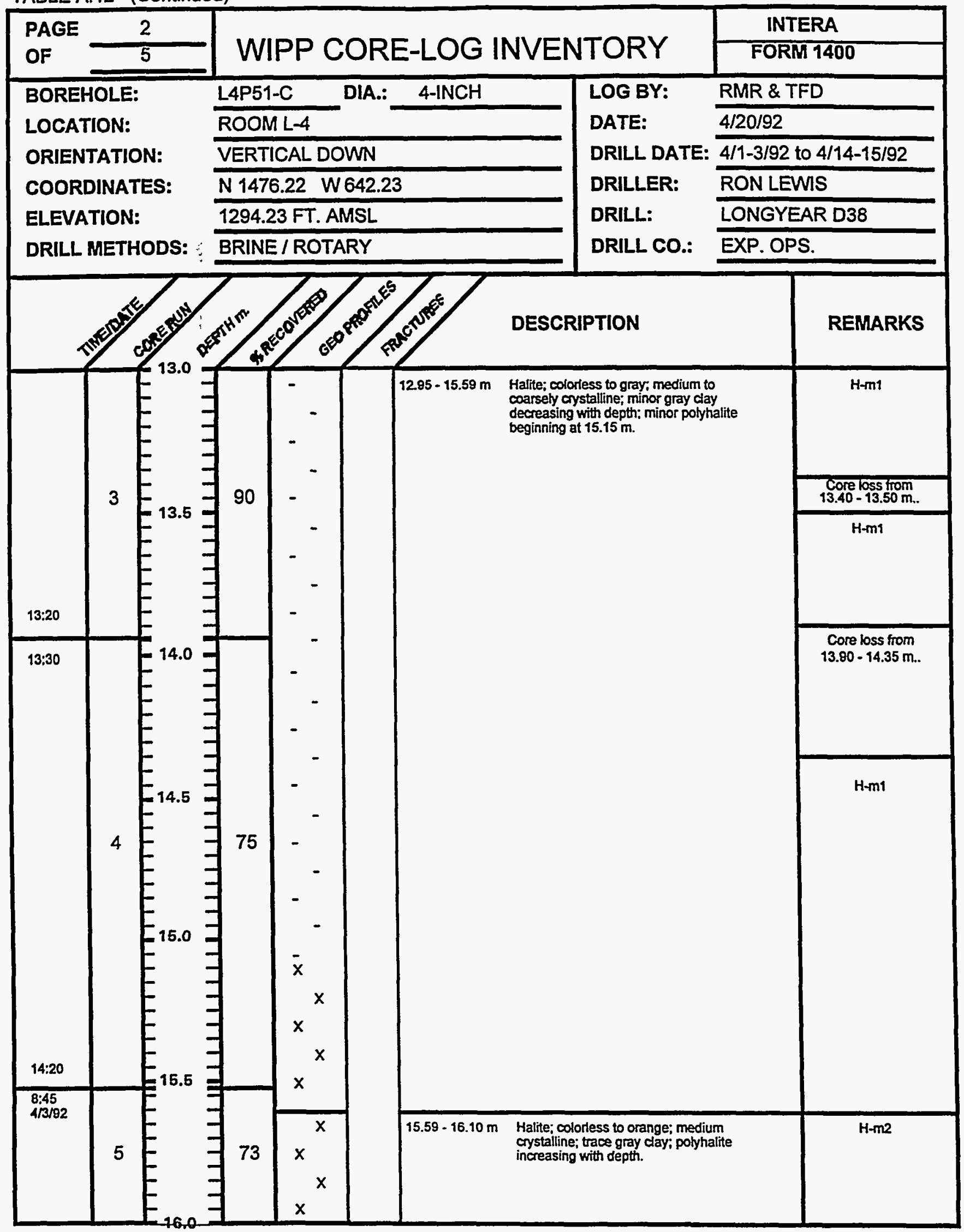


TABLE A.12 (Continued)

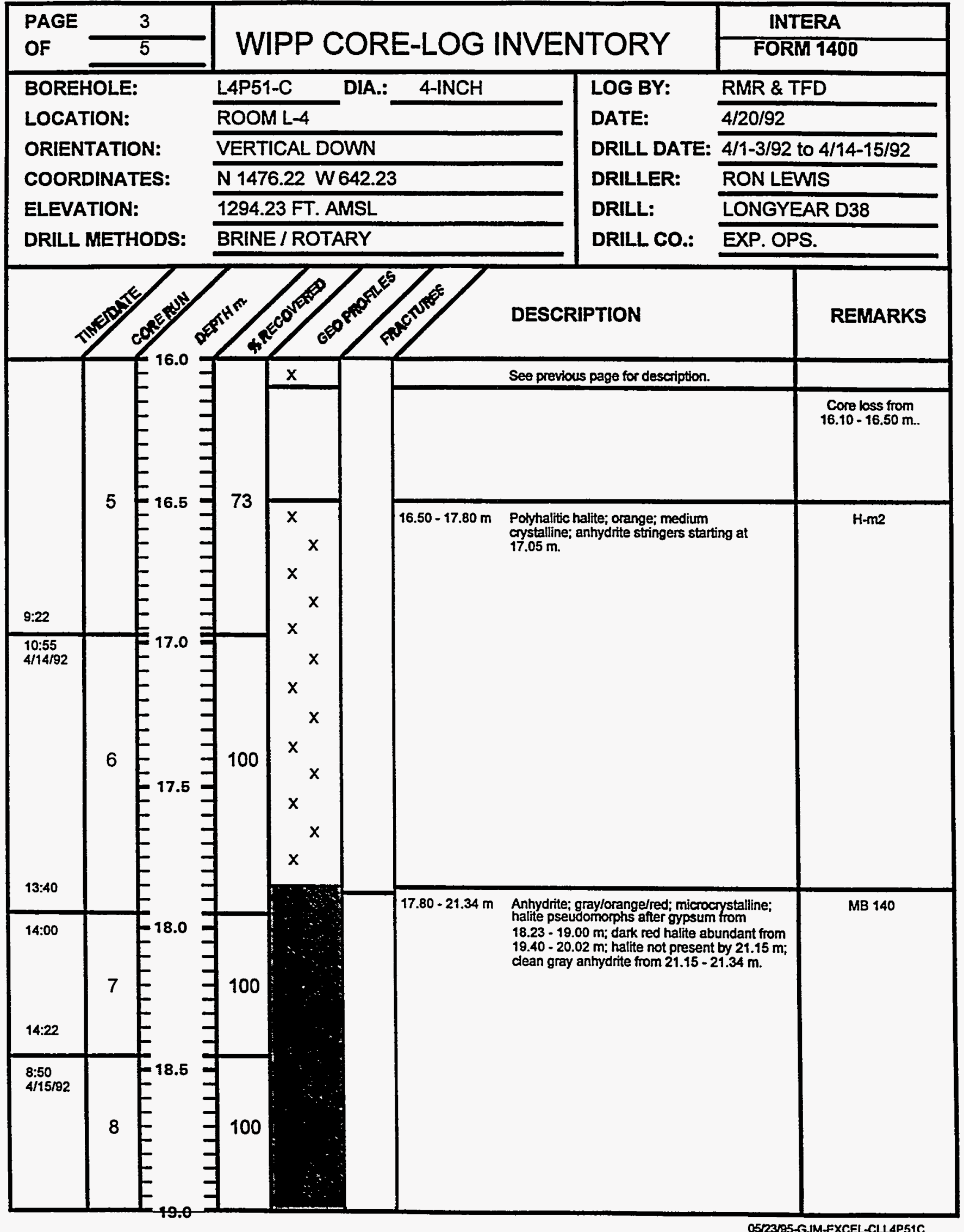


TABLE A.12 (Continued)

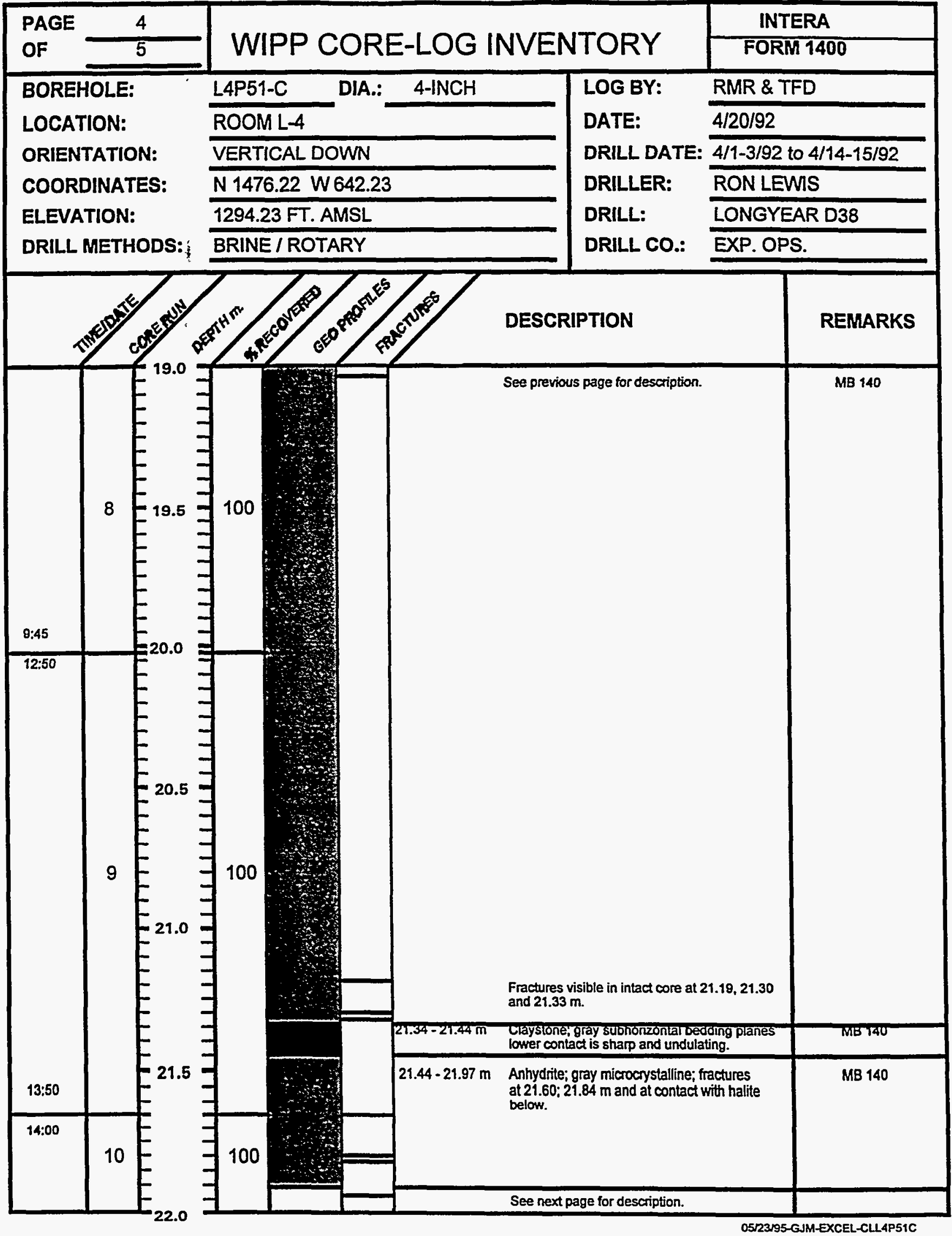


TABLE A.12 (Continued)

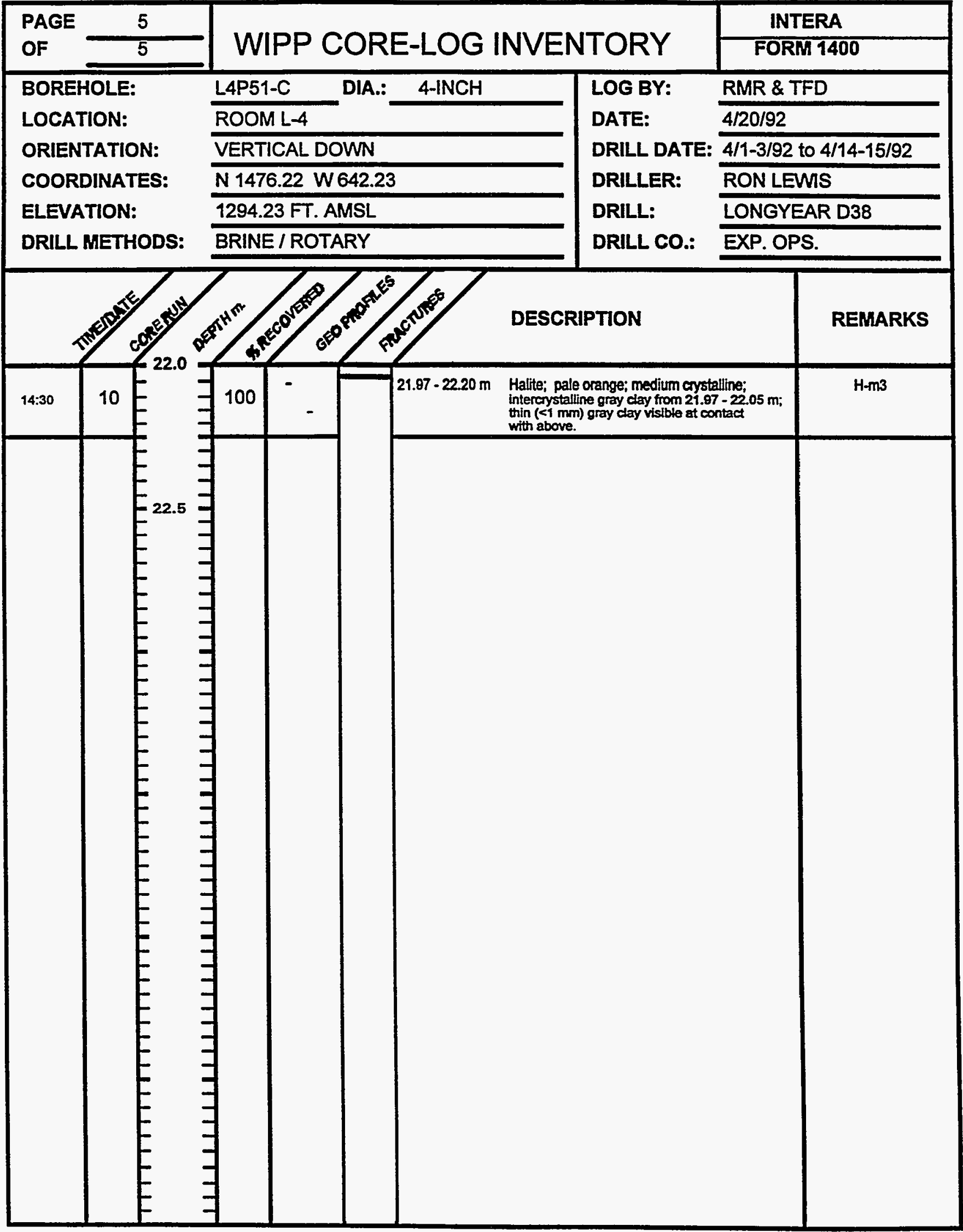


TABLE A.13

CORE DESCRIPTION OF BOREHOLE L4P51-D 
TABLE A.13 (Continued)

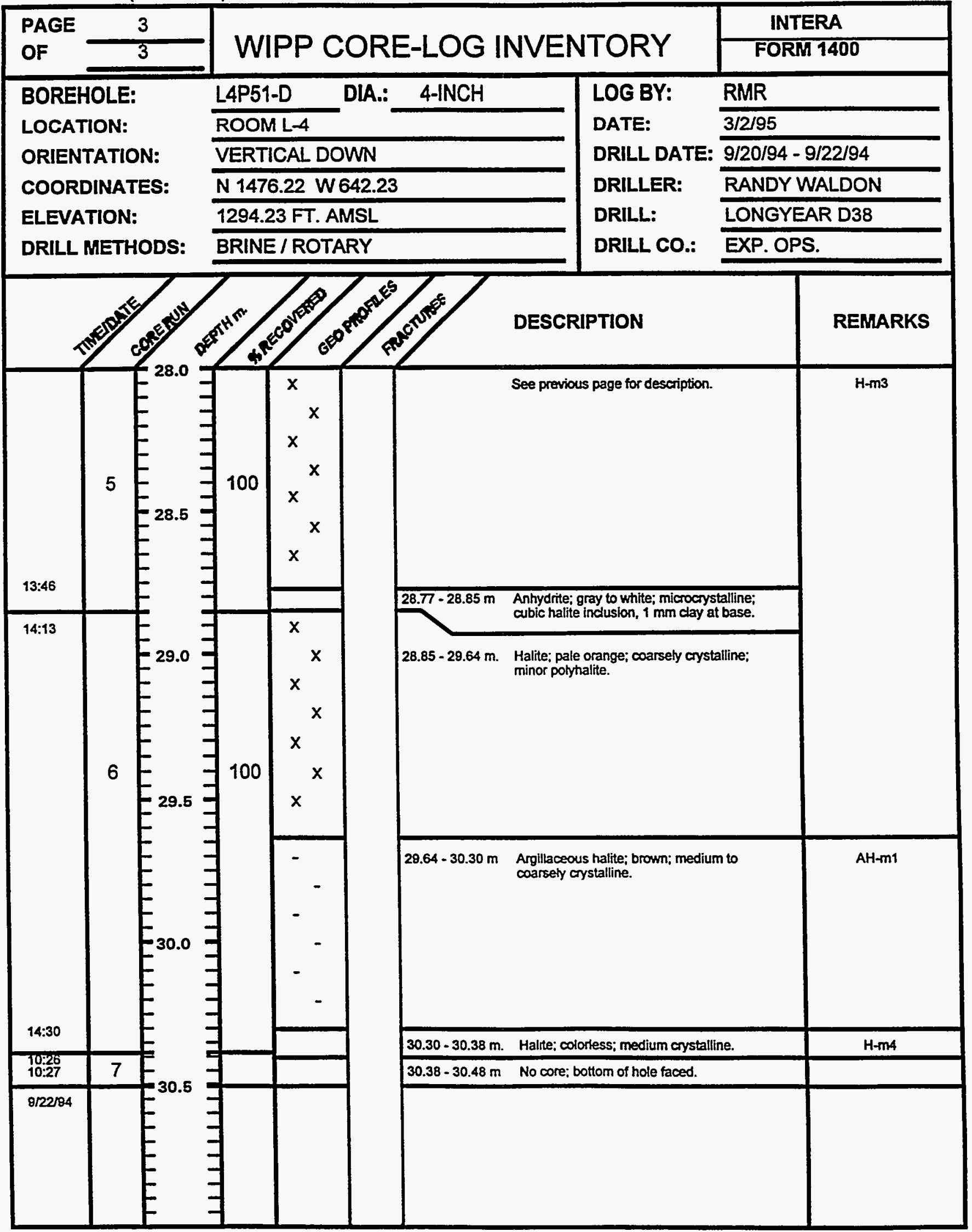


TABLE A.14

CORE DESCRIPTION OF BOREHOLE L4P52-B

A -69 
TABLE A.14

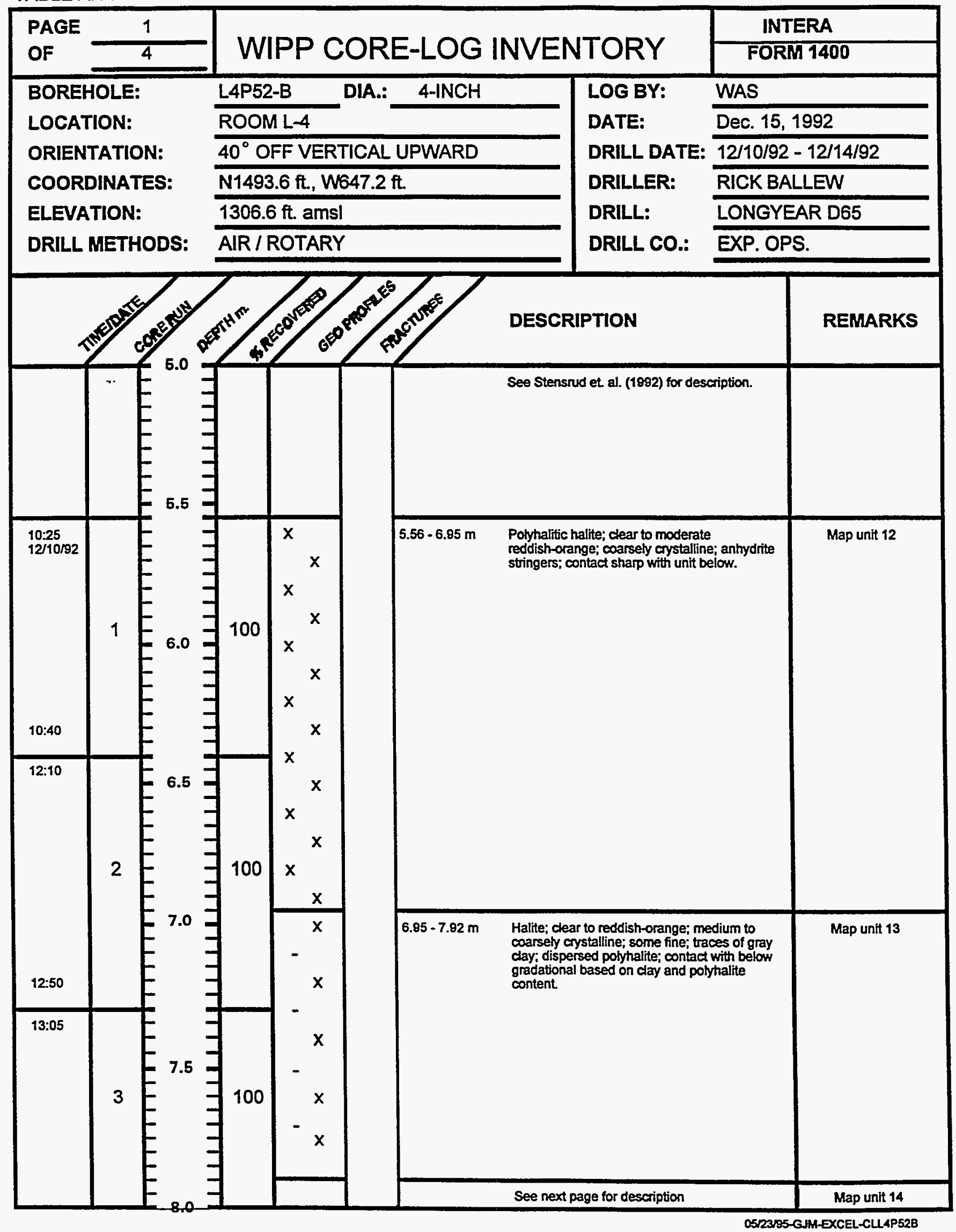


TABLE A.14 (Continued)

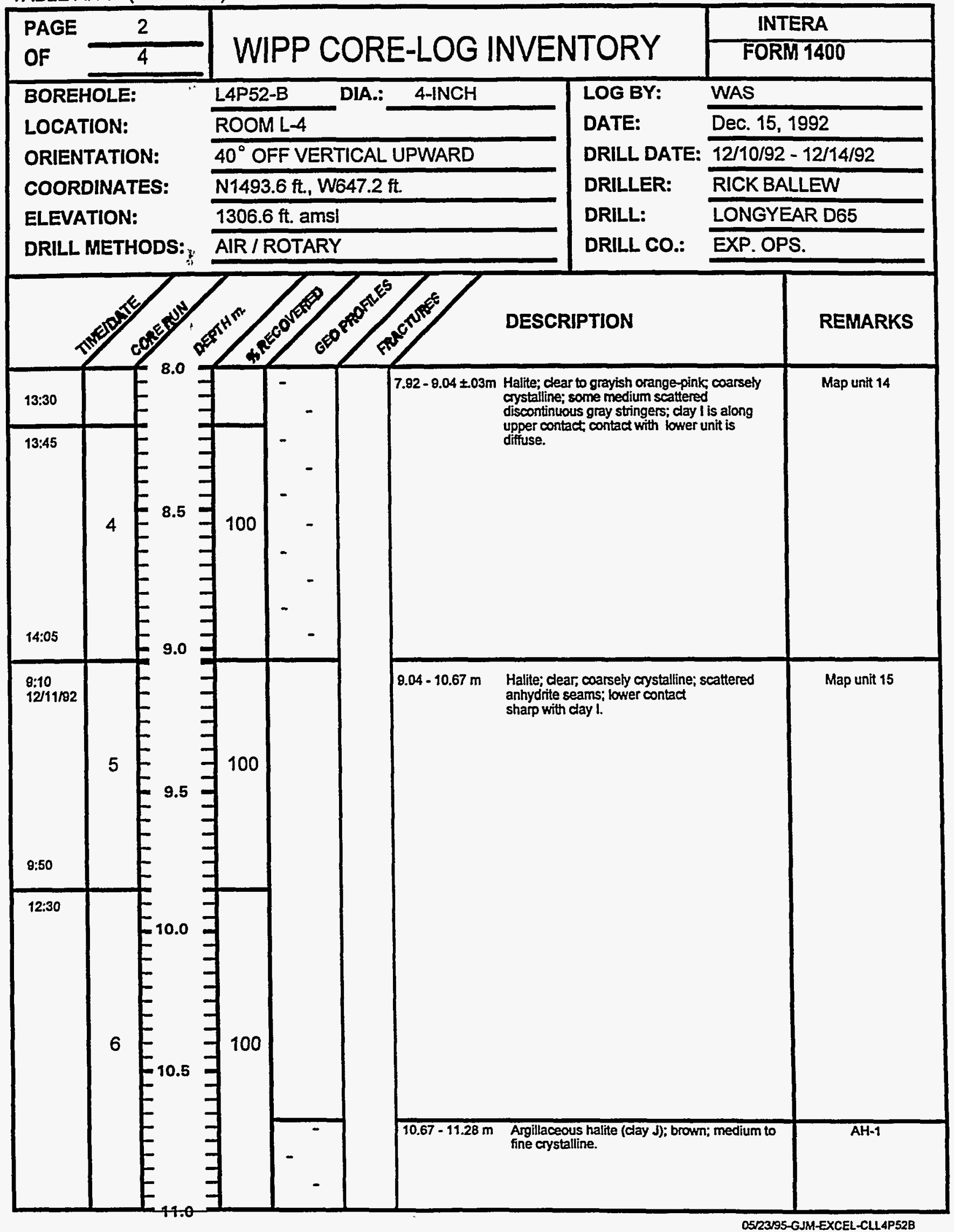


TABLE A.14 (Continued)

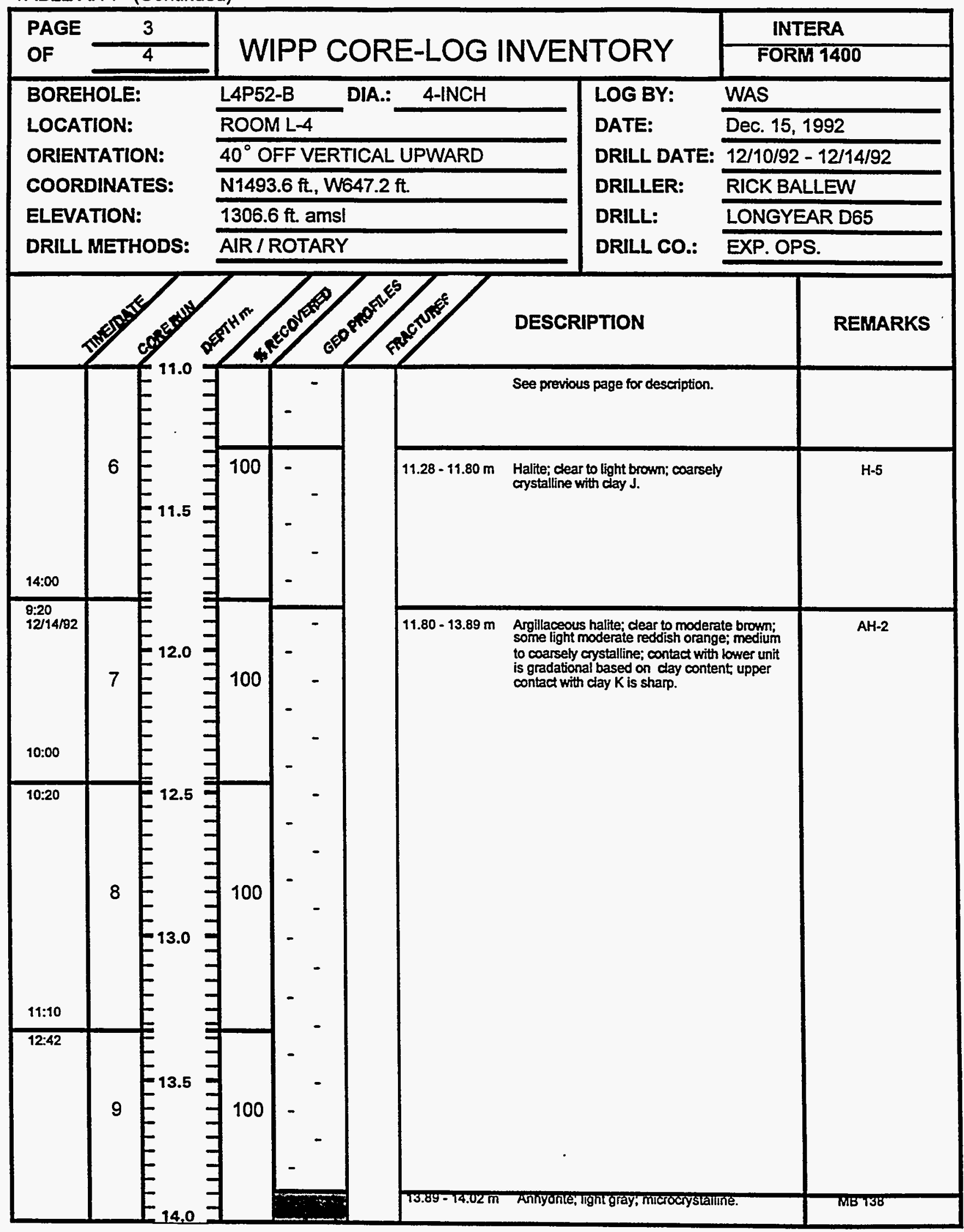


TABLE A.14 (Continued)

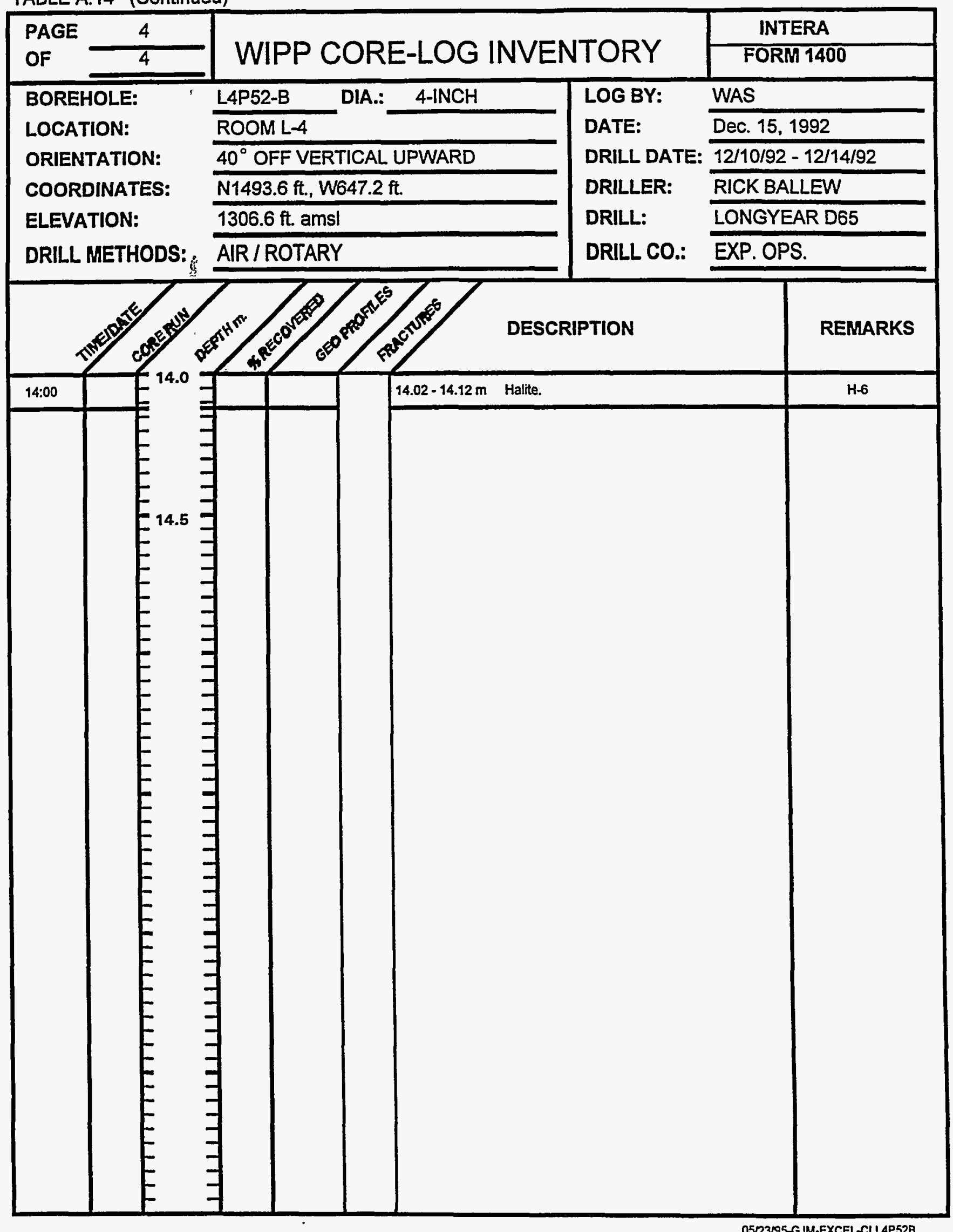




\section{WIPP \\ UC721 - DISTRIBUTION LIST \\ SAND97-1880}

\section{Federal Agencies}

US Department of Energy (4)

Office of Civilian Radioactive Waste Mgmt.

Attn: Deputy Director, RW-2

Acting Director, RW-10

Office of Human Resources \& Admin.

Director, RW-30

Office of Program Mgmt. \& Integ.

Director, RW-40

Office of Waste Accept., Stor., \& Tran.

Forrestal Building

Washington, DC 20585

Attn: Project Director

Yucca Mountain Site Characterization Office

Director, RW-3

Office of Quality Assurance

P.O. Box 30307

Las Vegas, NV 89036-0307

US Department of Energy

Albuquerque Operations Office

Attn: National Atomic Museum Library

P.O. Box 5400

Albuquerque, NM 87185-5400

US Department of Energy

Research \& Waste Management Division

Attn: Director

P.O. Box E

Oak Ridge, TN 37831

US Department of Energy (5)

Carlsbad Area Office

Attn: G. Dials

D. Galbraith

M. McFadden

R. Lark

J. A. Mewhinney

P.O. Box 3090

Carlsbad, NM 88221-3090

US Department of Energy

Office of Environmental Restoration and

Waste Management

Attn: M Frei, EM-30

Forrestal Building

Washington, DC 20585-0002
US Department of Energy (3)

Office of Environmental Restoration and Waste Management

Attn: J. Juri, EM-34, Trevion II

Washington, DC 20585-0002

US Department of Energy

Office of Environmental Restoration and

Waste Management

Attn: S. Schneider, EM-342, Trevion II

Washington, DC 20585-0002

US Department of Energy (2)

Office of Environment, Safety \& Health

Attn: C. Borgstrom, EH-25

R. Pelletier, EH-231

Washington, DC 20585

US Department of Energy (2)

Idaho Operations Office

Fuel Processing \& Waste Mgmt. Division 785 DOE Place

Idaho Falls, ID 83402

US Environmental Protection Agency (2)

Radiation Protection Programs

Attn: M. Oge

ANR-460

Washington, DC 20460

\section{Boards}

Defense Nuclear Facilities Safety Board

Attn: D. Winters

625 Indiana Ave. NW, Suite 700

Washington, DC 20004

Nuclear Waste Technical Review Board (2)

Attn: Chairman

J. L. Cohon

1100 Wilson Blvd., Suite 910

Arlington, VA 22209-2297 
State Agencies

Attorney General of New Mexico

P.O. Drawer 1508

Santa Fe, NM 87504-1508

Environmental Evaluation Group (3)

Attn: Library

7007 Wyoming NE

Suite F-2

Albuquerque, NM 87109

NM Environment Department (3)

Secretary of the Environment

Attn: Mark Weidler

1190 St. Francis Drive

Santa Fe, NM 87503-0968

NM Bureau of Mines \& Mineral Resources

Socorro, NM 87801

\section{Laboratories/Corporations}

Battelle Pacific Northwest Laboratories

Battelle Blvd.

Richland, WA 99352

Los Alamos National Laboratory

Attn: B. Erdal, INC-12

P.O. Box 1663

Los Alamos, NM 87544

Tech Reps, Inc. (3)

Attn: J. Chapman (1)

Loretta Robledo (2)

5000 Marble NE, Suite 222

Albuquerque, NM 87110

Westinghouse Electric Corporation (5)

Attn: Library

J. Epstein

J. Lee

B. A. Howard

R. Kehrman

P.O. Box 2078

Carlsbad, NM 88221

S. Cohen \& Associates

Attn: Bill Thuber

1355 Beverly Road

McLean, VA 22101
Golder Associates

Attn: T. W. Doe

$4104148^{\text {th }}$ Avenue, NE

Redmond, WA 98052

Duke Engineering and Services (9)

Attn: D. A. Chace (5)

G. A. Freeze

M. D. Fort

P. S. Domski

R. M. Roberts

1650 University Blvd NE Suite 300

Albuquerque, NM 87102

Duke Engineering and Services (2)

Attn: J. F. Pickens

9111 Research Blvd.

Austin, TX 78758

Duke Engineering and Services Inc. (2)

Attn: W. A. Stensrud

J. B. Palmer

1012 W. Pierce, Suite A

Carlsbad, NM 88220

National Academy of Sciences, WIPP Panel

Howard Adler

Oxyrase, Incorporated

7327 Oak Ridge Highway

Knoxville, TN 37931

Tom Kiess

Board of Radioactive Waste Management

GF456

2101 Constitution Ave.

Washington, DC 20418

Rodney C. Ewing

Department of Geology

University of New Mexico

Albuquerque, NM 87131

Charles Fairhurst

Department of Civil and Mineral Engineering

University of Minnesota

500 Pillsbury Dr. SE

Minneapolis, MN 55455-0220

B. John Garrick

PLG Incorporated

4590 MacArthur Blvd., Suite 400

Newport Beach, CA 92660-2027 
Leonard F. Konikow

US Geological Survey

431 National Center

Reston, VA 22092

Carl A. Anderson, Director

Board of Radioactive Waste Management

National Research Council

HA 456

2101 Constitution Ave. NW

Washington, DC 20418

Christopher G. Whipple

ICF Kaiser Engineers

1800 Harrison St., 7th Floor

Oakland, CA 94612-3430

John O. Blomeke

720 Clubhouse Way

Knoxville, TN 37909

Sue B. Clark

University of Georgia

Savannah River Ecology Lab

P.O. Drawer E

Aiken, SC 29802

Konrad B. Krauskopf

Department of Geology

Stanford University

Stanford, CA 94305-2115

Della Roy

Pennsylvania State University

217 Materials Research Lab

Hastings Road

University Park, PA 16802

David A. Waite

$\mathrm{CH}_{2} \mathrm{M}$ Hill

P.O. Box 91500

Bellevue, WA 98009-2050

Thomas A. Zordon

Zordan Associates, Inc. 3807 Edinburg Drive

Murrysville, PA 15668

\section{Universities}

University of New Mexico

Geology Department

Attn: Library

141 Northrop Hall

Albuquerque, NM 87131

University of Washington

College of Ocean \& Fishery Sciences

Attn: G. R. Heath

583 Henderson Hall, HN-15

Seattle, WA 98195

New Mexico Tech

Department of Geoscience

Attn: J. Wilson

Socorro, NM 87801

Texas A\&M University

Department of Geology

Attn: P. A. Domenico

College Station, TX 77843

University of Arizona

Department of Hydrology

Attn: S. P. Neuman

Tucson, AZ 85721

University of California

Lawrence Berkeley Laboratory

Earth Sciences Division

Attn: C. F. Tsang

1 Cyclotron Road

Berkeley, CA 94720

University of Kansas

Kansas Geological Survey

Attn: J. Butler

1930 Constant Ave.

Campus West

Lawrence, KS 66046

University of Wisconsin-Madison (2)

Department of Geology and Geophysics

Attn: M. P. Anderson

H. F. Wang

1215 Dayton St.

Madison, WI 53706 


\section{Libraries}

Thomas Brannigan Library

Attn: D. Dresp

$106 \mathrm{~W}$. Hadley St.

Las Cruces, NM 88001

Government Publications Department

Zimmerman Library

University of New Mexico

Albuquerque, NM 87131

New Mexico Junior College

Pannell Library

Attn: R Hill

Lovington Highway

Hobbs, NM 88240

New Mexico State Library

Attn: N. McCallan

325 Don Gaspar

Santa Fe, NM 87503

New Mexico Tech

Martin Speere Memorial Library

Campus Street

Socorro, NM 87810

WIPP Public Reading Room

Carlsbad Public Library

101 S. Halagueno St.

Carisbad, NM 88220

\section{Foreign Addresses}

Atomic Energy of Canada, Ltd.

Whiteshell Laboratories

Attn: B. Goodwin

Pinawa, Manitoba, CANADA ROE ILO

Environment Canada

National Water Research Institute

Canada Centre for Inland Lakes

Attn: K. S. Novakowski

867 Lakeshore Road

P.O. Box 5050

Burlington, Ontario, CANADA L7R 4A6

Francois Chenevier (2)

ANDRA

Route de Panorama Robert Schumann

B. P. 38

92266 Fontenay-aux-Roses, Cedex

FRANCE
Claude Sombret

Centre d'Etudes Nucleaires de la Vallee Rhone

CEN/VALRHO

S.D.H.A. B.P. 171

30205 Bagnols-Sur-Ceze

FRANCE

Commissariat a L'Energie Atomique

Attn: D. Alexandre

Centre d'Etudes de Cadarache

13108 Saint Paul Lez Durance Cedex

FRANCE

Bundesanstalt fur Geowissenschaften und

Rohstoffe (2)

Attn: M. Langer

K. Schelkes

Postfach 510153

D-30631 Hannover

GERMANY

WBI

Attn: W. Wittke

Henricistrasse 50

D-52072 Aachen

GERMANY

Bundesministerium fur Forschung und

Technologie

Postfach 200706

5300 Bonn 2

GERMANY

Institut fur Tieflagerung

Attn: K. Kuhn

Theodor-Heuss-Strasse 4

D-3300 Braunschweig

GERMANY

Gesellschaft fur Anlagen und Reaktorsicherheit (GRS)

Attn: B. Baltes

Schwertnergasse 1

D-50667 Cologne

GERMANY

Shingo Tashiro

Japan Atomic Energy Research Institute

Tokai-Mura, Ibaraki-Ken, 319-11

JAPAN 
Attn: A. Winberg

Ogardesvagen 4

S-433 30 Partille

SWEDEN

\section{GEOSIGMA AB}

Attn: P. Andersson

P. O. Box 894

S-751 08 Uppsala

SWEDEN

Netherlands Energy Research Foundation ECN

Attn: J. Prij

3 Westerduinweg

P.O. Box 1

1755 ZG Petten

THE NETHERLANDS

Svensk Karnbransleforsorjning AB

Attn: F. Karlsson

Project KBS (Karnbranslesakerhet)

Box 5864

S-102 48 Stockholm

SWEDEN

Nationale Genossenschaft fur die Lagenung

Radioaktiver Abfalle (2)

Attn: S. Vomvoris

P. Zuidema

Hardstrasse 73

CH-5430 Wettingen

SWITZERLAND

AEA Technology

Attn: J. H. Rees

D5W/29 Culham Laboratory

Abington, Oxfordshire OX14 3DB

UNITED KINGDOM

AEA Technology

Attn: W. R. Rodwell

044/A31 Winfrith Technical Centre

Dorchester, Dorset DT2 8DH

UNITED KINGDOM
D. W. Powers

HC 12

Box 87

Anthony, TX 79821
Internal

$\begin{array}{lll}\frac{\text { MS }}{1324} & \frac{\text { Org. }}{6115} & \text { P. B. Davies } \\ 1320 & 6831 & \text { E. J. Nowak } \\ 1322 & 6121 & \text { J. R. Tillerson } \\ 1328 & 6849 & \text { D. R. Anderson } \\ 1328 & 6848 & \text { H. N. Jow } \\ 1335 & 6801 & \text { M. Chu } \\ 1341 & 6832 & \text { J. T. Holmes } \\ 1395 & 6800 & \text { L. Shephard } \\ 1395 & 6821 & \text { M. Marietta } \\ 1324 & 6115 & \text { R. L. Beauheim (5) } \\ 1324 & 6115 & \text { A. R. Lappin } \\ 1330 & 6811 & \text { K. Hart (2) } \\ 1330 & 4415 & \text { NWM Library (20) } \\ 9018 & 8940-2 & \text { Central Technical Files } \\ 0899 & 4916 & \text { Technical Library (5) } \\ 0619 & 12690 & \text { Review and Approval Desk (2), } \\ & & \text { For DOE/OSTI }\end{array}$

AEA Technology

Attn: J. E. Tinson

B4244 Harwell Laboratory

Didcot, Oxfordshire OX11 ORA

UNITED KINGDOM 